Stephanie Schöber

\title{
Sprache im italienischen Erdkundeunterricht
}

Eine multiperspektivische Studie 
Stephanie Schöber

Sprache im italienischen Erdkundeunterricht 
Für meinen Vater Gerald, in Erinnerung an seinen

Einsatz für Sprachförderung,

Alphabetisierung und Grundbildung. 
Stephanie Schöber

\section{Sprache im italienischen Erdkundeunterricht}

Eine multiperspektivische Studie

Budrich UniPress Ltd.

Opladen • Berlin • Toronto 2017 
Bibliografische Information der Deutschen Nationalbibliothek

Die Deutsche Nationalbibliothek verzeichnet diese Publikation in der Deutschen Nationalbibliografie; detaillierte bibliografische Daten sind im Internet über http://dnb.d-nb.de abrufbar.

(C) 2017 Dieses Werk ist bei Budrich UniPress erschienen und steht unter folgender Creative

Commons Lizenz: http://creativecommons.org/licenses/by-nc-nd/3.0/de/

Verbreitung, Speicherung und Vervielfältigung erlaubt, kommerzielle Nutzung und Veränderung nur mit Genehmigung des Verlags Budrich UniPress.

(c)

Dieses Buch steht im OpenAccess Bereich der Verlagsseite zum kostenlosen Download bereit ( https://doi.org/10.3224/86388303)

Eine kostenpflichtige Druckversion (Printing on Demand) kann über den Verlag bezogen werden. Die Seitenzahlen in der Druck- und Onlineversion sind identisch.

$\begin{array}{ll}\text { ISBN } & 978-3-86388-743-8 \text { (PoD) } \\ \text { eISBN } & \mathbf{9 7 8 - 3 - 8 6 3 8 8 - 3 0 3 - 4} \text { (pdf) } \\ \text { DOI } & 10.3224 / 86388743\end{array}$

Umschlaggestaltung: Bettina Lehfeldt, Kleinmachnow - www.lehfeldtgraphic.de Verlag Budrich UniPress Ltd. http://www.budrich-unipress.de 


\section{Inhalt}

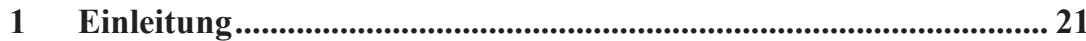

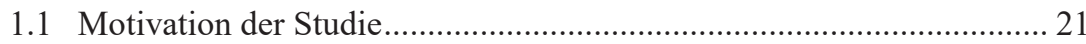

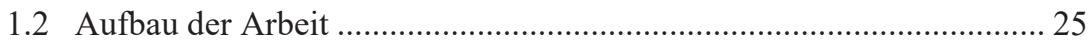

2 Theoretische Rahmung .................................................................. 29

2.1 Gesellschaftliche Sprachanforderungen und der Bildungsauftrag

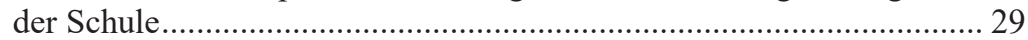

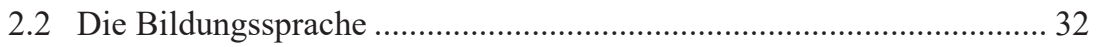

2.2.1 „Weiterentwicklung des Denkens als Denken fürs Sprechen“. 34

2.2.2 Merkmale der Bildungssprache ................................................ 38

2.2.3 Vermittlung und Erwerb der Bildungssprache......................... 40

2.3 Die Monolingualismus-Kontroverse ................................................ 42

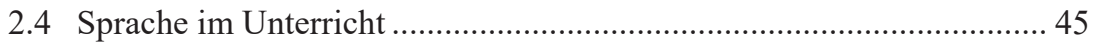

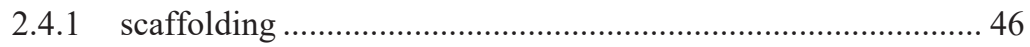

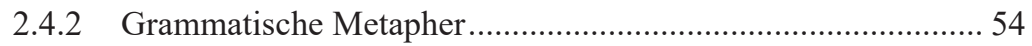

2.4.3 Sprachförderliche Lese- und Schreibdidaktik ........................... 56

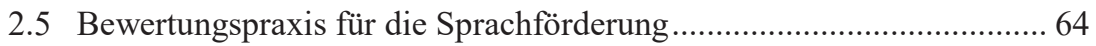

3 Überblick über den Forschungsstand zur Mehrsprachigkeit in der italienischen Schule und zur sprachlichen Gestaltung der Lehrbücher .................................................................................. 67

3.1 Sprachliche Heterogenität in der italienischen Schule ......................... 67

3.2 Das Unterrichtswerk und seine Schwächen als zentrales

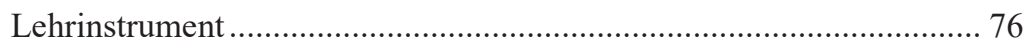

3.2.1 Kritik an Lehrmitteln und Bildungspolitik in der italienischen

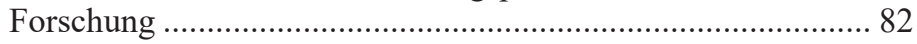

3.2.2 Der Wortschatz als Gegenstand der Lehrmittelforschung ........ 86

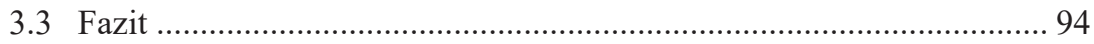

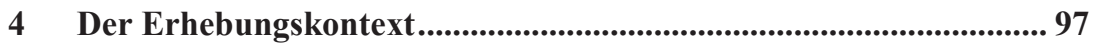

4.1 Das italienische Bildungssystem und die Lehrerausbildung ................ 97 
4.2. Die aktuellen Lehrpläne für das Fach Erdkunde in der achten Klasse und Richtlinien zur schulischen Sprachbildung......................... 99

4.2.1 Lernziele im Fach Erdkunde am Ende der Mittelschule ........... 99

4.2.2 Schulische Sprachbildung ..................................................... 101

4.3 Inhalte einer wirksamen Lehrerausbildung für sprachfördernde

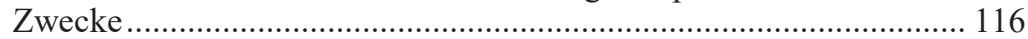

4.3.1 Sprach- und spracherwerbstheoretische Aspekte.................... 116

4.3.2 Pädagogische und sprachdidaktische Aspekte ....................... 121

4.4 Arbeitsbedingungen im Kontext sprachlicher Heterogenität in den beobachteten Klassen ............................................................ 126

5 Methodische Betrachtungen zur Studie ....................................... 129

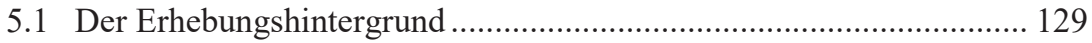

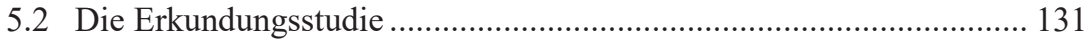

5.2.1 Auswahl der Erhebungsinstrumente zur Messung schriftlicher

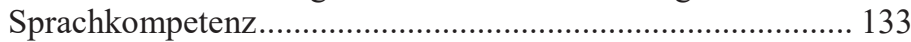

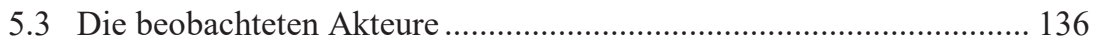

5.3.1 Informationsgewinnung anhand eines Fragebogens ............... 139

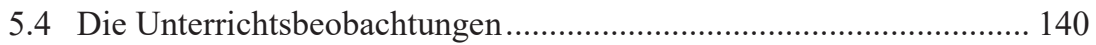

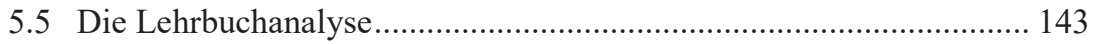

5.6 Erhebungsverfahren zur Ermittlung produktiver und rezeptiver schriftlicher Sprachleistungen der Lernenden .................................... 147

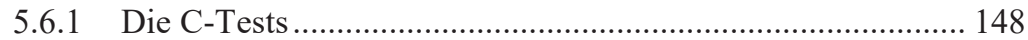

5.6.2 Weitere schriftliche Schülerdaten ......................................... 150

6 Vorstellung der beobachteten Klassen ............................................ 155

6.1 Auswahl der Schulen und allgemeine Informationen zur

Klassenzusammensetzung und zur Durchführung des

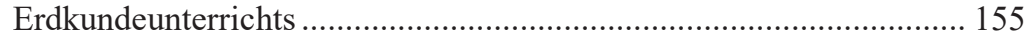

6.1.1 Schülerinnen und Schüler mit Lernbeeinträchtigungen .......... 157

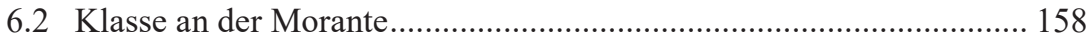

6.2.1 Lesegewohnheiten ............................................................ 159

6.2.2 Einstellung zum Erdkundeunterricht und zur Lehrkraft ......... 161

6.2.3 Schülerangaben zum Lehrbuch............................................ 164 


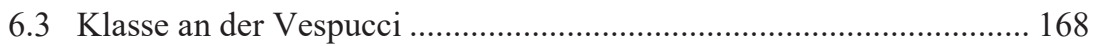

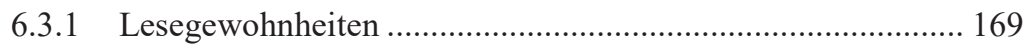

6.3.2 Einstellung zum Erdkundeunterricht und zur Lehrkraft ......... 171

6.3.3 Schülerangaben zum Lehrbuch.............................................. 174

6.4 Klasse III A der Pestalozzi ................................................................ 176

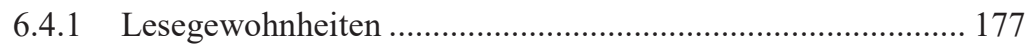

6.4.2 Einstellung zum Erdkundeunterricht und zur Lehrkraft ......... 179

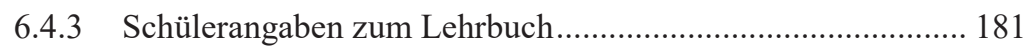

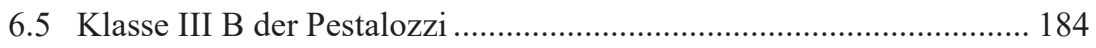

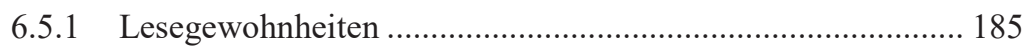

6.5.2 Einstellung zum Erdkundeunterricht und zur Lehrkraft ........ 187

6.5.3 Schülerangaben zum Lehrbuch........................................... 189

6.6 Klasse III C der Pestalozzi ................................................................ 192

6.6.1 Lesegewohnheiten ............................................................ 193

6.6.2 Einstellung zum Erdkundeunterricht und zur Lehrkraft ......... 195

6.6.3 Schülerangaben zum Lehrbuch............................................ 197

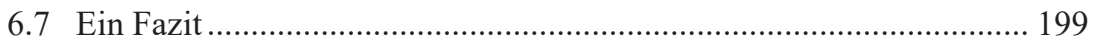

6.7.1 Sozioökonomischer Status .................................................... 206

$7 \quad$ Analyse der Unterrichtswerke .......................................................... 209

7.1 Die Bedeutung des Lehrwerks im beobachteten
Erdkundeunterricht......................................................................... 209

7.2 Präsentation der in der Erhebungsgruppe verwendeten Lehrwerke .... 211

7.2.1 Erster Eindruck zu den Lehrwerken ..................................... 213

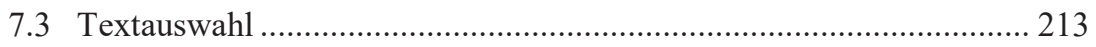

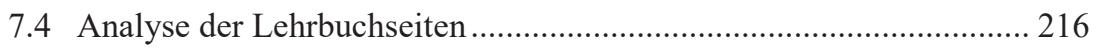

7.4.1 Das Nuovo Geolab der Schule Vespucci ............................... 216

7.4.2 Das Lehrbuch I nuovi territori dell'uomo an der Morante...... 231

7.4.3 Zaino in spalla der Klasse III A an der Schule Pestalozzi ...... 234

7.4.4 Das Geograficamente der Klasse III B der Schule Pestalozzi 260

7.4.5 Nuovo Geolibro der Klasse III C an der Pestalozzi ................ 263

7.5 Lehrbuchübergreifende Betrachtung und Gegenüberstellung der

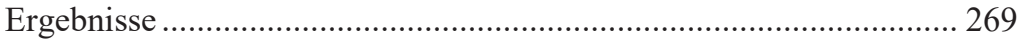

7.5.1 Der Fachwortschatz der untersuchten Lehrbuchseiten ........... 271 


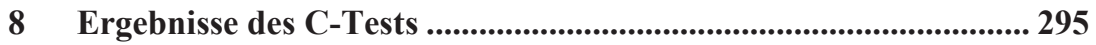

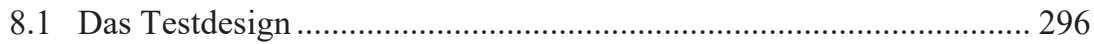

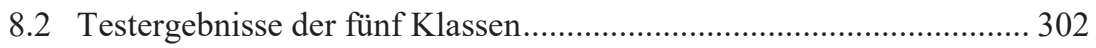

8.2.1 Erste Gegenüberstellung der Ergebnisse in den einzelnen

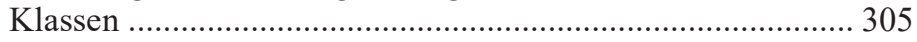

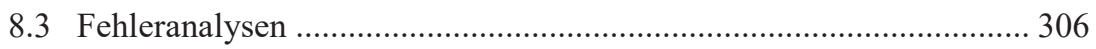

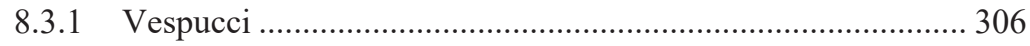

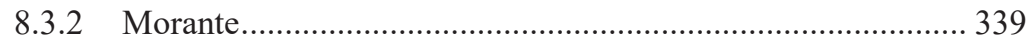

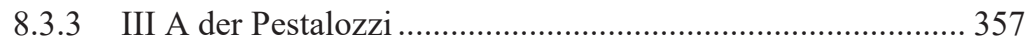

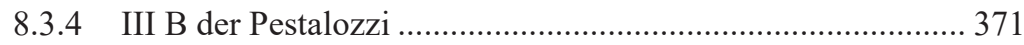

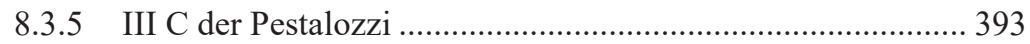

8.4 Klassenübergreifende Zusammenfassung der Testergebnisse ............ 414

9 Sprache im Fachunterricht: die Unterrichtsbeobachtungen......... 421

9.1 Rahmenbedingungen des beobachteten sprachlichen Handelns ........ 422

9.1.1 Zeitliche Organisation des Erdkundeunterrichts .................... 422

9.1.2 Lernförderliche Nutzung der Raumgestaltung....................... 424

9.1.3 Die beobachteten Formen der Unterrichtsgestaltung .............. 426

9.1.4 Mehrsprachigkeit im Unterricht.......................................... 452

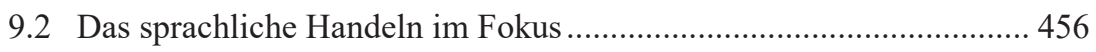

9.2.1 Beobachtetes sprachförderliches Lehrerhandeln im

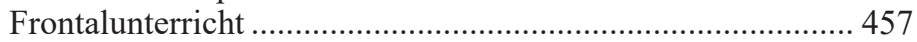

9.2.2 Sprachliche Herausforderungen und ihre Bewerkstelligung in den Abfragungen .............................................................. 461

9.2.3 Sprachbezogene Interaktion im beobachteten Erdkundeunterricht 470

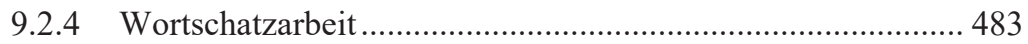

9.2.5 Sprachbezogenes Feedback der beobachteten Lehrpersonen.. 498

10 Schriftliche Textproduktionen der Lernenden............................. 509

10.1 Schülertexte der Klasse an der Morante. Die indische Region ............ 510

10.1.1 Schriftbild und graphische strukturelle Gestaltungsmittel ...... 511

10.1.2 Zusammensetzung des Wortschatzes.................................... 512 
10.1.3 Lexikogrammatikalische und morphosyntaktische Gestaltung 521

10.2 Die Lehrbuchseiten zur indischen Region........................................... 545

10.2.1 Zusammensetzung des Wortschatzes..................................... 545

10.2.2 Lexikogrammatikalische und syntaktische Eigenschaften...... 557

10.3 Vergleich der Schülertexte mit den Lehrbuchseiten ........................... 572

10.4 Schülertexte der Klasse an der Vespucci .......................................... 576

10.4.1 Schriftbild und graphische strukturelle Gestaltungsmittel ...... 577

10.4.2 Zusammensetzung des Wortschatzes.................................... 577

10.4.3 Grammatikalische und syntaktische Eigenschaften ................ 583

10.5 Inhaltlich-sprachlicher Vergleich der Texte beider Klassen ............... 596

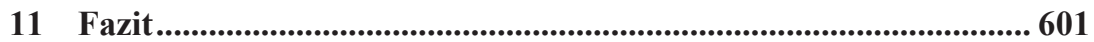

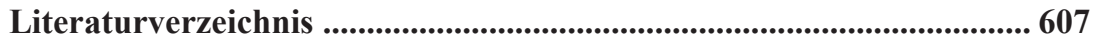

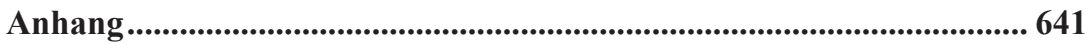

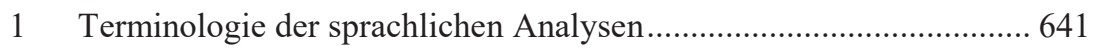

1.1 Abkürzungen der Wortarten .................................................. 641

1.2 Abkürzungen der verwendeten Fachsprachen nach dem Gradit

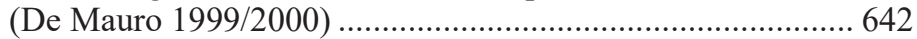

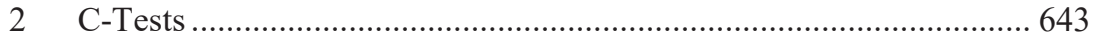

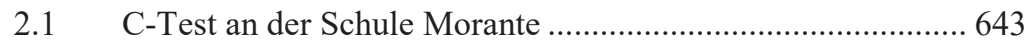

$2.2 \quad$ C-Test an der Schule Vespucci ................................................ 645

2.3 C-Test der Klasse III A an der Schule Vespucci ..................... 646

2.4 C-Test der Klasse III B an der Schule Vespucci...................... 648

2.5 C-Test der Klasse III C an der Schule Vespucci...................... 650

3 Zuordnung der Schüler-Aliasnamen aus Kapitel 9 ............................ 651

3.1 Klasse an der Vespucci ........................................................ 651

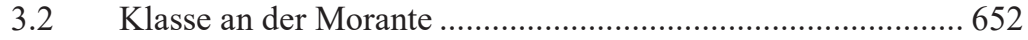

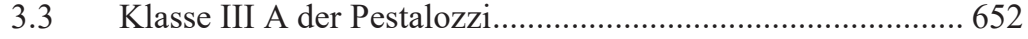

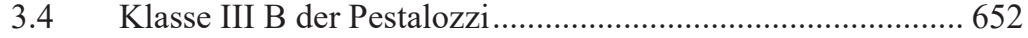

3.5 Klasse III C der Pestalozzi ...................................................... 653

4 Textproduktionen der Lernenden zu geographischen Regionen ......... 654 
4.1 Textbeispiele aus der Klasse an der Schule Morante............... 654

4.2 Textbeispiel aus der Klasse an der Schule Vespucci .............. 658

\section{Tabellenverzeichnis}

Kapitel 2

Tabelle 1: Merkmale der Bildungssprache z.T. nach Berendes, Dragon,

Weinert, Heppt \& Stanat (2013, S. 26) .....................................40

Kapitel 5

Tabelle 1: Phasen der Erhebung und Untersuchungsschritte 135

Tabelle 2: In die Studie involvierte Klassen 138

Tabelle 3: Angaben zur Anzahl der Lernenden und der beantworteten Fragebögen

Tabelle 4: Erhobene Schriftdaten

Kapitel 6

Tabelle 1: Zusammensetzung der Klassen

Tabelle 2: Einstellung zum Erdkundeunterricht und zur unterrichtenden Lehrkraft an der Morante

Tabelle 3: Einschätzung des Erdkundelehrbuchs, Lernende der Morante

Tabelle 4: Einstellung zum Erdkundeunterricht und zur unterrichtenden Lehrkraft an der Vespucci.

Tabelle 5: Anwendung von Lernstrategien, Vespucci 175

Tabelle 6: Lesegewohnheiten der Lernenden in der III A, Pestalozzi ..177

Tabelle 7: Bewertung des Erdkundelehrbuchs in der III A, Pestalozzi.181

Tabelle 8: Anwendung von Lernstrategien in der III A, Pestalozzi ......183

Tabelle 9: Einstellung zum Erdkundeunterricht und zur unterrichtenden

Lehrkraft, Klasse III B, Pestalozzi....

Tabelle 10: Bewertung des Erdkundelehrbuchs in der III B, Pestalozzi .

Tabelle 11: Anwendung von Lernstrategien in der III B, Pestalozzi ....191

Tabelle 12: Einstellung zum Erdkundeunterricht und zur

unterrichtenden Lehrkraft, Klasse III C, Pestalozzi.

Tabelle 13: Anwendung von Lernstrategien in der III C, Pestalozzi ....199 
Tabelle 14: Überblick über Angaben der Lernenden zum sprachlichen Hintergrund, Bildungsstand und zu den Lesegewohnheiten ...200

Tabelle 15: Auffassungen der Lernenden zum Lehrbuch, zum Schulund Erdkundeunterricht und zu den Lehrpersonen, alle Klassen 202

Tabelle 16: Lerngewohnheiten der Schülerinnen und Schüler. 204

Tabelle 17: Angaben zum sozioökonomischen Status, alle Klassen ....207 Kapitel 7

Tabelle 1: Informationen zu den verwendeten Lehrwerken.................212

Tabelle 2: Überblick über die untersuchten Lehrbuchseiten ................215

Tabelle 3: Terminologie der sprachlichen Analyse.............................217

Tabelle 4: Kürzel der Fachwortschatzbereiche und deutsche Übersetzungen .218

Tabelle 5: Untersuchungsergebnisse zu den Seiten 55 und $56 \mathrm{im}$ Nuovo Geolab (Carazzi \& Pizzetti 2010).... 224

Tabelle 6: Wörter des allgemeinen (CO) und des technisch-spezifischen Wortschatzes (TS) auf S. 56 im Nuovo Geolab (Carazzi \& Pizzetti 2010) .228

Tabelle 7: Untersuchungsergebnisse zu den Seiten 62, 68, 134 f., 319 und 320 im Nuovo Geolab (Carazzi \& Pizzetti 2010)

Tabelle 8: Untersuchungsergebnisse zu den Seiten aus Nuovi Territori dell'uomo (Bersezio 2005).. .231

Tabelle 9: Untersuchungsergebnisse zu den Seiten aus Zaino in spalla (Bastianelli, Rancati \& Maestri 2010) .234

Tabelle 10: Zusammensetzung der hervorgehobenen Wörter auf den untersuchten Seiten aller fünf Lehrbücher... 238

Tabelle 11: Hervorhebungen auf den untersuchten Seiten im Nuovo Geolab (Carazzi \& Pizzetti 2010).

Tabelle 12: Hervorhebungen auf den untersuchten Seiten im Nuovi Territori dell'uomo (Bersezio 2005). .244

Tabelle 13: Hervorhebungen auf den untersuchten Seiten im Zaino in spalla (Bastianelli et al. 2010) ...............................................246

Tabelle 14: Hervorhebungen auf den untersuchten Seiten im Geograficamente (Dinucci \& Dinucci 2008)

Tabelle 15: Hervorhebungen auf den untersuchten Seiten im Nuovo Geolibro (Forte \& Ubertazzi 2010) ......................................255

Tabelle 16: Untersuchungsergebnisse zu den Seiten aus Geograficamente (Dinucci \& Dinucci 2008) .260 
Tabelle 17: Untersuchungsergebnisse zu den Seiten aus Nuovo Geolibro (Forte \& Ubertazzi 2008) ........................................264

Tabelle 18: Fachwortschatz (Ts, Co Ts) aller untersuchten Seiten der Lehrbücher....

Tabelle 19: Fachwortschatzbereiche aller untersuchten Seiten der Lehrwerke

Tabelle 20: Fachwortschatz der untersuchten Seiten im Nuovi territori dell'uomo (Bersezio 2005).

Tabelle 21: Fachwortschatz auf den untersuchten Seiten im Nuovo Geolibro (Forte \& Ubertazzi 2008) .278

Tabelle 22: Stärkste Ts-Bereiche und Zusammensetzung des TsVorkommens nach Wortarten im Nuovo Geolibro (Forte \& Ubertazzi 2008) .280

Tabelle 23: Fachwortschatz auf den untersuchten Seiten im Geograficamente (Dinucci \& Dinucci 2008) .281

Tabelle 24: Stärkste Ts-Bereiche und Zusammensetzung des TsVorkommens nach Wortarten im Geograficamente (Dinucci \& Dinucci 2008)

Tabelle 25: Fachwortschatz auf den untersuchten Seiten im Zaino in spalla (Bastianelli et al. 2010) ...............................................284

Tabelle 26: Stärkste Ts-Bereiche und Zusammensetzung des TsVorkommens nach Wortarten im Zaino in spalla (Bastianelli et al. 2010)

Tabelle 27: Fachwortschatz auf den untersuchten Seiten im Nuovo Geolab (Carazzi \& Pizzetti 2010).

Tabelle 28: Stärkste Ts-Bereiche und Zusammensetzung des TsVorkommens nach Wortarten im Nuovo Geolab (Carazzi \& Pizzetti 2010)

Tabelle 29: Geographischer und wirtschaftlicher Fachwortschatz aller untersuchten Seiten

Tabelle 30: Geographischer Fachwortschatz und seine Vorkommnisse auf allen untersuchen Lehrbuchseiten.

Tabelle 31: Wirtschaftlicher Fachwortschatz und seine Vorkommnisse auf allen untersuchen Lehrbuchseiten

Kapitel 8

Tabelle 1: Ergebnisse des C-Tests an der Schule Vespucci .302

Tabelle 2: Ergebnisse des C-Tests an der Schule Morante 303 
Tabelle 3: Ergebnisse des C-Tests der Klasse III A, Pestalozzi.............303

Tabelle 4: Ergebnisse des C-Tests der Klasse III B, Pestalozzi ............304

Tabelle 5: Ergebnisse des C-Tests der Klasse III C, Pestalozzi..............304

Tabelle 6: Illustration des Testausgangs in allen fünf Lerngruppen......305

Tabelle 7: Getilgtes Wortvorkommen im C-Test der Vespucci mit Angaben zu den Falschlösungen...............................................306

Tabelle 8: Übersicht über die Texte 1 und 2 des C-Tests an der

Vespucci inklusive Lösungsvorschläge ....................................309

Tabelle 9: Übersicht über die Texte 3 und 4 des C-Tests an der

Vespucci inklusive Lösungsvorschläge

Tabelle 10: Häufige Fehler in den Texten 3 und 4 des C-Tests an der Vespucci

Tabelle 11: Übersicht über Text 5 des C-Tests an der Vespucci inklusive Lösungsvorschläge

Tabelle 12: Häufigste Fehler in Text 5 des C-Tests, Textauszug aus der Lehrbuchseite 68 (Carazzi \& Pizzetti 2010)

Tabelle 13: Getilgtes Wortvorkommen im C-Test der Vespucci nach

Wortarten mit Angaben zu den Falschlösungen ........................336

Tabelle 14: Häufigste Falschlösungen im C-Test an der Schule

Vespucci

Tabelle 15: Getilgte Wörter des C-Tests der Morante nach Gebrauchsmarken mit Angaben zu den Falschlösungen

Tabelle 16: Aus dem Lehrbuch entnommene Texte 1 bis 3 (Bersezio 2005, S. 59-61) des C-Tests an der Morante. 340

Tabelle 17: Häufigste Falschlösungen in den Texten 1 bis 3 des C-Tests an der Morante

Tabelle 18: C-Testtexte 4 und 5 aus dem Lehrbuch von Bersezio (2005,

S. 32 f.) inklusive Lösungsvorschläge (Morante)

Tabelle 19: Zu ergänzende Wörter im C-Test der Morante nach Wortart mit Angaben zu den Falschlösungen .........................................356

Tabelle 20: Getilgte Wörter des C-Tests der III A nach Gebrauchsmarken mit Angaben zu den Falschlösungen .357

Tabelle 21: Getilgtes Wortvorkommen des C-Tests der III A (Pestalozzi) nach Wortarten mit Angaben zu den Falschlösungen

Tabelle 22: Text 1 und 2 des Tests der Klasse III A, Pestalozzi

(Textgrundlage Bastianelli et al. 2010, S. 118).

Tabelle 23: Texte 3 bis 5 des C-Tests der Klasse III A, Pestalozzi

(Textgrundlage Bastianelli et al. 2010, S. 116, 149) .365 
Tabelle 24: Häufige Falschlösungen in den Texten 3 bis 5 des C-Test der Klasse III A (Textgrundlage Bastianelli et al. 2010, S. 116, 149).

Tabelle 25: Getilgte Wörter des C-Tests der Klasse III B (Pestalozzi) nach Gebrauchsmarken mit Angaben zu den Falschlösungen.372

Tabelle 26: Getilgtes Wortvorkommen des C-Tests der III B (Pestalozzi) nach Wortarten mit Angaben zu den Falschlösungen

Tabelle 27: Texte 1 bis 3 des C-Tests der Klasse III B, Pestalozzi

(Textgrundlage Dinucci \& Dinucci 2008, S. 238, 241).

Tabelle 28: Falschlösungen von mindestens fünf Lernenden in den ersten drei C-Testtexten der Klasse III B, Pestalozzi

Tabelle 29: Texte 4 und 5 des C-Tests der Klasse III B an der Schule Pestalozzi

Tabelle 30: Häufige Falschlösungen in den C-Testtexten 4 und 5 der

Klasse III B an der Pestalozzi .389

Tabelle 31: Getilgte Wörter des C-Tests der III C (Pestalozzi) nach Gebrauchsmarken mit Angaben zu den Falschlösungen

Tabelle 32: Getilgtes Wortvorkommen des C-Tests der III C (Pestalozzi) nach Wortarten mit Angaben zu den Falschlösungen

Tabelle 33: Wortvorkommen und Lösungsvorschläge der ersten drei

Texte des C-Tests der Klasse III C, Pestalozzi

Tabelle 34: Wortvorkommen und Lösungsvorschläge der Texte 4 und 5 des C-Tests der Klasse III C, Pestalozzi 406

Tabelle 35: Häufige Falschlösungen im vierten und fünften Text des C-

Test der Klasse III C, Pestalozzi..................................................410

Tabelle 36: Länge der Sätze mit Worttilgungen in den fünf C-Tests...416

Tabelle 37: Getilgte Ts-Wörter der fünf C-Tests mit Angaben zu den

Falschlösungen .418

Kapitel 9

Tabelle 1: Durchführung des Erdkundeunterrichts in den beobachteten Klassen.

Tabelle 2: Ergebnisse eines Worterklärungstests in der Klasse der

Schule Morante

Kapitel 10

Tabelle 1: Notenspiegel der Textproduktionen in der Klasse Morante511

Tabelle 2: Klasse Morante, Länge der Texte und Sätze sowie Gebrauchsmarken und Anteile der Wortschatzbereiche VDB 
und Fo am Gesamttext.

Tabelle 3: Fachsprachliches Wortvorkommen in den Schülertexten

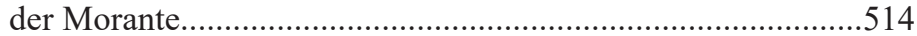

Tabelle 4: Phraseme in den Schülertexten der Morante ........................518

Tabelle 5: Prädikate der Schülertexte an der Morante ..........................525

Tabelle 6: Zusammensetzung des Wortschatzes auf den

Lehrbuchseiten zur indischen Region (Bersezio 2005) ............546

Tabelle 7: Zusammensetzung des Co-gekennzeichneten

Wortvorkommens auf den Lehrbuchseiten

Tabelle 8: Fachwortschatz auf den Lehrbuchseiten zur indischen Region (Bersezio 2005).

Tabelle 9: Phraseme der Lehrbuchseiten zur indischen Region (Bersezio 2005)

Tabelle 10: Konjunktionen der Lehrbuchseiten zur indischen Region (Bersezio 2005).

Tabelle 11: Worttypen der Konjunktionen mit Vorkommnissen .558

Tabelle 12: Prädikate der Lehrbuchseiten zur indischen Region (Bersezio 2005).

Tabelle 13: Mittelwerte sprachlicher Merkmale der Schülertexte und der

Lehrbuchseiten zur indischen Region (Bersezio 2005) ............574

Tabelle 14: Geographische Gebiete der Schülertexte (Vespucci) ........576

Tabelle 15: Text- und Satzlängen, Gebrauchsmarken und VDB- und FOAnteile am Gesamtvolumen der Schülertexte (Vespucci) .......577

Tabelle 16: Co-Wörter mit mindestens vier Tokens in den Schülertexten (Vespucci).

Tabelle 17: Fachwortschatzsprachliche Ausdrücke in den Schülertexten

(Vespucci). 580

Tabelle 18: Phraseme in den Schülertexten (Vespucci) .........................581

Tabelle 19: Konjunktionen in den Schülertexten (Vespucci)................583

Tabelle 20: Prädikate der Schülertexte (Vespucci) ..............................589

Tabelle 21: Partizipien, Gerundien und Infinitive in den Schülertexten

(Vespucci).

Tabelle 22: Klassenvergleich - Text- und Satzlängen,

Gebrauchsmarken

Tabelle 23: Klassenvergleich - Prädikate ... .598

Anhang

Tabelle 1: Terminologie der sprachlichen Analyse .641 
Tabelle 2: Im GRADIT (De Mauro 1999/2000) verwendete

Abkürzungen der Fachsprachen

\section{Abbildungsverzeichnis}

Kapitel 2

Abbildung 1: Designed-in scaffolding nach Hammond und Gibbons (2005, S. 13)

Abbildung 2: Interaktionales scaffolding nach Hammond und Gibbons (2005, S. 21)

Kapitel 6

Abbildung 1: Maximale Bücherzahl im Haushalt und maximale tägliche Lesezeit (in min), Morante

Abbildung 2: Maximale Bücherzahl im Haushalt und maximale tägliche Lesezeit (in min), Vespucci.

Abbildung 3: Maximale Bücherzahl im Haushalt und maximale tägliche Lesezeit (in min), III B Pestalozzi...

Abbildung 4: Maximale Bücherzahl im Haushalt und maximale tägliche Lesezeit (in min), III C, Pestalozzi

Kapitel 8

Abbildung 1: Textbeispiel aus dem C-Test der Schule Vespucci .........300

Abbildung 2: Textbeispiel ohne Lücken

Abbildung 3: Zusammensetzung der getilgten Wörter im C-Test der Vespucci

Abbildung 4: Sätze auf Seite 55 des Lehrbuchs von Carazzi und Pizzetti (2010) mit den häufigsten Fehlern

Abbildung 5: Der Lehrbuchseite 68 (Carazzi \& Pizzetti 2010) entnommener C-Testtext mit Angaben zu den Falschlösungen

Abbildung 6: Getilgtes Wortvorkommen nach Gebrauchsmarken und ihrem prozentualen Anteil im C-Test, Morante

Abbildung 7: Getilgtes Wortvorkommen im C-Test der Klasse III A (Pestalozzi) nach Gebrauchsmarken und ihren prozentualen Anteilen

Abbildung 8: Getilgtes Wortvorkommen nach Gebrauchsmarken und ihrem prozentualen Anteil im C-Test der Klasse III B, Pestalozzi 
Abbildung 9: Häufige Falschlösung im vierten C-Testtext der Klasse III B, der Lehrbuchseite 242 (Dinucci \& Dinucci 2008) entnommene Sätze

Abbildung 10: Der Lehrbuchseite 242 (Dinucci \& Dinucci 2008) entnommener letzter Satz im fünften C-Testtext der Klasse III B mit Anzahl der Falschlösungen

Abbildung 11: Zusammensetzung der getilgten Wörter im C-Test der III C (Pestalozzi)....

Abbildung 12: S. 122 des Lehrbuchs (Forte \& Ubertazzi 2008) entnommener Satz mit Hervorhebung der getilgten Wörter, Fehlerzahl und Übersetzung

Abbildung 13: Dem Lehrbuch entnommener Satz (Forte \& Ubertazzi 2008, S. 123) mit Hervorhebung der getilgten Wörter, Fehlerzahl und Übersetzung

Kapitel 9

Abbildung 1: Auszug Unterrichtsmitschrift III A, DIUs (I) ..................430

Abbildung 2: Auszug aus Unterrichtsmitschrift III B, DIUs (II)...........431

Abbildung 3: Transkriptauszug Regiolekt Frau Mancuso.....................436

Abbildung 4: Auszug aus Unterrichtsmitschrift Jugendsprache/Soziolekt Frau Cese (I)

Abbildung 5: Auszug aus Unterrichtsmitschrift Jugendsprache/Soziolekt Frau Cese (II)

Abbildung 6: Abfragung Herr Tigre, Asien .........................................440

Abbildung 7: Abfragung Frau Cese, zum Auswendiglernen ................443

Abbildung 8: Abfragung Frau Dante, zum Auswendiglernen................444

Abbildung 9: Auszug aus einem Unterrichtsgespräch an der Schule Vespucci, Mehrsprachigkeit

Abbildung 10: Abfragung Frau Rea, Pierpaolo, III A, Pestalozzi, Bestrebungen um bildungssprachlichen Ausdruck 461

Abbildung 11: Transkriptauszug Abfragung Frau Mancuso, Avas Versuch einer bildungssprachlichen Darstellung 462

Abbildung 12: Abfragung Frau Cese, Pamela, Bildungssprachversuche

Abbildung 13: Auszug aus Unterrichtsmitschrift, Abfragung Frau Cese, Registerwechsel eines Schülers aufgrund von Wortfindungsschwierigkeiten 468

Abbildung 14: Auszug aus Unterrichtsgespräch Klasse III A, Pestalozzi, Textarbeit 470 
Abbildung 15: Auszug aus Unterrichtsgespräch, Frau Rea, Costa, Textarbeit - Wortbedeutung

Abbildung 16: Auszug aus Unterrichtsgespräch Frau Rea, Bezug zur Lebenswelt der Schüler 474

Abbildung 17: Unterrichtsgespräch Frau Rea, Genozid. 475

Abbildung 18: Transkriptauszug zu Unterrichtsgespräch Frau Mancuso, neutrale und komplexe Lehrbuchsprache, lexikalische Schwierigkeiten

Abbildung 19: Unterrichtsgespräch Frau Mancuso, Mikro-scaffolding - PIL

Abbildung 20: Frau Mancuso, Vereinfachung/Erklärung der Lehrbuchtexte

Abbildung 21: Transkriptauszug Herr Tigre, Textarbeit - unbekannte Wörter und ihre Erklärungen

Abbildung 22: Transkriptauszug Herr Tigre, Textarbeit - Wortschatz492

Abbildung 23: Definitionsbeispiele aus dem Worterklärungstest an der Schule Morante.

Abbildung 24: Abfragung Frau Cese, Aufforderung zum genaueren Erklären

Abbildung 25: Textarbeit Klasse Vespucci, cioè .500

Abbildung 26: Transkriptauszug Klasse Morante, tipo (I) 501

Abbildung 27: Transkriptauszug Abfragung, Klasse Morante, tipo (II)

Abbildung 28: Gesprächsauszug III A, Pestalozzi, mündliches Ausdrucksvermögen - forse

Abbildung 29: Transkriptauszug Unterrichtsgespräch Herr Tigre, indirekt geäußerte Wissenslücke

Abbildung 30: Hausaufgabenbesprechung Frau Rea, III A, Pestalozzi, Betonung der Wichtigkeit des genauen Ausdrucks .504

Abbildung 31: Abfragung Frau Rea, III A, Pestalozzi, Unzufriedenheit mit Schüleräußerung (I)

Abbildung 32: Abfragung Frau Rea, III A, Pestalozzi, Unzufriedenheit mit Schüleräußerung (II).

Kapitel 10

Abbildung 1: Partzipvervendung von unito in verschieden Texten, Morante.

Abbildung 2: Textauszüge zum Partizip Perfekt formata/formate, 
Morante

Abbildung 3: Implizite Relativsätze einer Schülerin an der Morante ..534 Abbildung 4: Wortgetreue Übernahme des Lehrbuchtextes einschließlich der Partizipien, Schülerin A

Abbildung 5: Korrekte Verwendung eines bildungssprachlichen Begriffes, der nicht auf den entsprechenden Lehrbuchseiten erscheint

Abbildung 6: Wiederholungen von Lexemen aus derselben Wortfamilie

Abbildung 7: Gebrauch des generischen Substantivs cosa in den Schülertexten der Morante.

Abbildung 8: Passivkonstruktionen auf den Lehrbuchseiten zur indischen Region .564

Abbildung 9: Finalsätze mit Infinitiven auf den Lehrbuchseiten zur indischen Region

Abbildung 10: Bildungssprachliche Versuche

Anhang

Abbildung 1: Textproduktion der Schülerin M (Seite 1)

Abbildung 2: Textproduktion der Schülerin M (Seite 2)

Abbildung 3: Textproduktion der Schülerin B (Seite 1)

Abbildung 4: Textproduktion der Schülerin B (Seite 2)

Abbildung 5: Textproduktion der Schülerin E .658 


\section{Danksagung}

Mein aufrichtiger Dank für die anregende Kritik und geduldige Unterstützung gilt meinen beiden Doktormüttern Sabine Koesters Gensini und Ingrid Gogolin. Ebenfalls danken möchte ich meiner Familie, Ralph Hendriks, Friederike Anz, Letizia Mancuso, Peter van Oostrum, Mareike Köhne und Günter Voss sowie allen Schülerinnen, Schülern und Lehrkräften, die am Gelingen dieser Studie mitgewirkt haben und sich auch außerhalb des Unterrichts Zeit für zusätzliche Erklärungen nahmen und meine Fragen beantworteten. 


\section{Einleitung}

\subsection{Motivation der Studie}

Im Zentrum des vorliegenden Forschungsvorhabens stehen die sprachlichen Herausforderungen, mit denen Schülerinnen und Schüler in Italien am Ende der Sekundarstufe I (8. Klasse) im Fach Erdkunde konfrontiert sind. Nicht zuletzt die Ergebnisse internationaler Schulleistungsstudien wie PISA und IGLU sowie groß angelegte nationale Erhebungen auf der einen Seite und die $\mathrm{Zu}$ nahme von SchülerInnen, deren Erstsprache nicht die Unterrichtssprache ist (Cesareo 2014, S. 7; Bertozzi 2014, S. 15; MIUR - Ufficio di Statistica 2015), auf der anderen haben in den vergangenen Jahren verstärkt ins Bewusstsein gerückt, dass etliche Schülerinnen und Schüler nur unzureichend über eine sprachliche Grundbildung verfügen, die für den schulischen und beruflichen Erfolg und die aktive Teilhabe am gesellschaftlichen Leben in den westlichen Kulturen erforderlich ist (Colombo 2014; Gallina 2006). Die zentrale Rolle, die Sprache im Lern- und Entwicklungsprozess spielt, brachte Wittgenstein folgendermaßen auf den Punkt: „Die Grenzen meiner Sprache bedeuten die Grenzen meiner Welt" (Wittgenstein 2003 (1921), Satz 5.6).

So wurde mit der Veröffentlichung der Ergebnisse dieser Schulleistungsstudien eine Diskussion zu einem Aspekt ins Rollen gebracht, der Persönlichkeiten wie z.B. Wittgenstein (1977 (1926), 2001 (1953, posthum)) und Lombardo Radice (1931, 1959 (1936)) schon vor langer Zeit klar vor Augen stand: Eine jede Unterrichtsstunde ist zugleich Sprachunterricht. ${ }^{1}$ Da der Wissenstransfer und Kompetenzerwerb vorrangig über das Medium Sprache erfolgt, ist deutlich geworden, dass eventuelle Mängel in den Leistungen in nicht sprachlichen Fächern zum Teil auf Schwierigkeiten zurückzuführen sind, die sich in den einzelnen Disziplinen aus den Techniken ihrer sprachlichen Darbietung und Bewertung ergeben. Besondere Aufmerksamkeit hat dabei zunächst vor allem der Sprachgebrauch in Mathematik und den naturwissenschaftlichen Fächern erfahren (z.B. Guerriero 1988; Gallin \& Ruf 1990; Zambelli 1994; Leisen 1998; Maier Schweiger 1999; Gogolin \& Schwarz 2004, Ahrenholz 2010b), in denen die Ergebnisse vieler Teilnehmer an den PISAUntersuchungen besonders schlecht ausgefallen sind (OECD 2014).

Das Interesse an den sprachlichen Praktiken in diesen Fächern ist auch auf die Tatsache zurückzuführen, dass die mäßigen Leistungen mit der Vermittlung und Kontrolle von Lerninhalten einhergehen, die zu einem guten Teil fachsprachlich kommuniziert werden. Wird die Sprache in den Fachdidaktiken

1 Siehe diesbezüglich das Zitat von Lombardo-Radice zur Sprachbildung im Schulunterricht in Kap. 7.5, S. 269 f. 
thematisiert, so rückt primär die Terminologie ins Blickfeld, zumal sich diese im Mathematik- und im naturwissenschaftlichen Unterricht oft klar als Fachsprache ausmachen lässt und man im schulischen Kontext auf lexikalischer Ebene eine stete Zunahme und auch Überladung an fachwortschatzsprachlichen Ausdrücken beobachten kann (Graf \& Berck 1993, S. 25; Bascetta 1964).

Probleme mit dem vorrangigen Medium zur Inhaltsvermittlung und -bearbeitung in den nicht sprachlichen Fächern ergeben sich aber keineswegs ausschließlich oder vorrangig aus der Verwendung einer spezifischen Terminologie, die aus einem relativ überschaubaren Umfang von zu lernenden Fachausdrücken besteht (vgl. z.B. Lee 1996, S. 184; Ferreri 2005, S. 127 f.). Vielmehr geht es um die kognitive Herausforderung, die der Erwerb der Konzepte, die sich hinter den Fachausdrücken verbergen, mit sich bringt, und die Schwierigkeiten, die der Wortgebrauch generell verursachen kann. Das hängt direkt mit den spezifischen, im Kontext Schule verwendeten sprachlichen Registern zusammen, die potentiell sowohl produktive als auch rezeptive Hürden schaffen, die vor allem die Lesekompetenz und das Textverständnis sowie die schriftliche und mündliche Textproduktion betreffen und von den Lernenden unterschiedlich gemeistert werden. So betont auch Christie (2012, S. 96):

The technical language of subject specialisms like science is often unfamiliar and difficult, though it is misleading to suppose that students' difficulties in speaking, reading, or writing about science are primarily a matter of unfamiliar vocabulary, if by that we mean learning the meaning of words like "photosynthesis," "velocity," "peristalsis," or "atom." The issue is more complex, and here once again a functional grammar is helpful. The issue is one of the total pattern of discourse in which technical language is deployed and specialistic meanings are made, creating texts that are dense, abstract, and seemingly remote from lived experience as children know it. The abstractions are often built using grammatical metaphor.

Welche Merkmale diese schulsprachlichen Register aufweisen, ist bisher weder für das Deutsche noch für das Italienische umfassend belegt, weshalb diese Arbeit durch detaillierte sprachliche Analysen von Lehrbuchtexten und Diskursformen von Lehrkräften einen Beitrag zu ihrer genaueren Beschreibung leisten möchte. Während nach den naturwissenschaftlichen Fächern und Mathematik die Aufmerksamkeit unter den geisteswissenschaftlichen Fächern vor allem Geschichte galt (Deon 1997; Bozzone Costa 2003, S. 114), ist für diese Untersuchung das Unterrichtsfach Erdkunde gewählt worden, da sich Studien mit seiner sprachlichen Gestaltung bisher nur vereinzelt befassen ${ }^{2}$ und es sich

2 So gibt es etwa in Italien Untersuchungen zu diversen Erdkundebücher nach hauptsächlich inhaltlichen und fachdidaktischen Aspekten (Faccioli 1988a), über die Zusammensetzung des Wortschatzes in den Lehrwerken sowie die lexikalischen und Textverständniskompetenzen von Schülern verschiedener Klassenstufen (Miglietta \& Sobrero 2008, S. 210 f.) und über den Bestandteil von geographischen Fachwörtern im Gebrauchswortschatz und ihren Anteil in geographischen Schulbüchern (Ferreri 2005, S. 114 f.). Im angelsächsischen Raum beschäftigt sich eine Arbeit ausschließlich mit der Sprache im Erdkundeunterricht (Lee 1996) und bezieht dabei stärker als die italienischen Studien auch die Lehrpersonen und Lernenden 
um ein Fach handelt, dessen Inhalte sich aus ganz unterschiedlichen Wissensfeldern zusammensetzen, die von gesellschaftswissenschaftlichen Aspekten in z.B. Geschichte, Politik und Wirtschaft bis hin zu naturwissenschaftlichen wie der Geologie und Astronomie reichen.

Ausgangspunkt der Untersuchung war zunächst das Interesse an einer Bestandsaufnahme der sprachlichen Leistungen von Schülerinnen und Schülern, deren Muttersprache sich nicht mit der Unterrichtssprache deckt, sowie an der Weiterentwicklung dieser Fähigkeiten. Doch was im Vorfeld beispielsweise durch Klagen von Professoren und Professorinnen über die schwachen schriftsprachlichen Kenntnisse italienischer Studierender bereits auffällig war (vgl. z.B. Bozzone Costa 2003; S. 121; De Santis \& Gatta 2013; Piemontese \& Sposetti 2014), und sich beim Einlesen in die Materie immer deutlicher zeigte, ist, dass eben auch einsprachige Schülerinnen und Schüler zum Teil erhebliche Defizite bei der Bewältigung der sprachlichen Anforderungen haben. Die Registerunterschiede zwischen ihrem alltäglichen Sprachgebrauch und der Unterrichtssprache bzw. der in den Lehrmitteln benutzten Sprache können dabei so groß sein, dass sie den Lernerfolg beeinträchtigen (Battimelli 1997; Ferreri 2005, S. 113), zumal sich die Defizite während ihrer Schullaufbahn eher noch vermehren, als dass sie abgebaut werden (vgl. z.B. Piemontese; Cavaliere 1997, S. 223; Maas 2008, z.B. S. 630; Bosi et al. 2014, S. 104). Dadurch hat sich das Forschungsinteresse auf den Umgang aller Lernenden mit den sprachlichen Praktiken im Geographieunterricht ausgeweitet.

Den Kern dieser Studie bildet eine empirische Untersuchung in fünf Klassen an drei verschiedenen Mittelschulen in Rom, die über den Zeitraum November 2012 bis Juni 2013 durchgeführt wurde. Die Schulen befinden sich in einem recht zentralen Stadtviertel mit einem Einzugsgebiet der mittleren und unteren Mittelklasse und werden auch von Lernenden zugewanderter Familien besucht. Für die Erhebung ist das letzte Jahr der Sekundarstufe I ausgewählt worden, da bezüglich der sprachlichen Schwierigkeiten im Bildungssektor in den letzten Jahren vor allem der vorschulische und der Primarbereich erforscht wurde und deutlich geworden ist, dass die Schnittstellen an den Bildungsübergängen von besonderer Bedeutung für die Bildungslaufbahn der einzelnen Lernenden sind (Christie 2012, S. 190; Gogolin et al. 2011).

Auf einen solchen Übergang bereitet in Italien das achte Schuljahr vor, da auf fünf Jahre Grundschule eine dreijährige Mittelschule folgt, bevor sich die Schülerinnen und Schüler nach bestandener Abschlussprüfung für unter-

ein, allerdings aus einem Blickwinkel, der sich auf diskursanalytische und Genderunterschiede konzentriert. Aus dem deutschsprachigen Raum stammt eine Dissertation über die Verwendung der Erdkundebücher im Unterricht, welche sich auch mit sprachlichen Aspekten beschäftigt, aber den Großteil ihrer Erkenntnisse aus qualitativen Lehrerinterviews und Unterrichtsprotokollen gewinnt, ohne dass der effektive Unterricht beobachtet oder die Leistungen der Lernenden näher erforscht worden wären (Thöneböhn 1995). 
schiedlich ausgerichtete, mehr oder weniger stark berufsvorbereitende weiterführende Schulen zu entscheiden haben. Während die Grund- und Mittelschulen zunehmend in Form sogenannter istituti comprensivi gemeinsam geleitet werden und sich häufig im selben oder in benachbarten Gebäuden ansiedeln, gibt es große Unterschiede in den fachlichen Ausrichtungen und Leistungsanforderungen der zahlreichen Schultypen der Sekundarstufe II und wird zwischen den drei Schulformen Grund-, Mittel- und weiterführende Schule häufig ein übermäßiger Zuwachs der Ansprüche an die von den Lernenden zu erbringenden Leistungen beobachtet (Colombo \& Pallotti 2014).

Als besonders typisch für den Unterricht an italienischen Schulen kann angesehen werden, dass dieser stark lehrbuchzentriert gestaltet ist (Jafrancesco 2002; MIUR 2015), wobei die kursierenden Unterrichtswerke von unterschiedlichen Akteuren, nämlich Lehrpersonen, Universitätsdozenten und Wissenschaftlern, generell als komplex und den sprachlich-kognitiven Entwicklungsstand der Lernenden übersteigend eingestuft werden (Bertocchi 2003, S. 22; Bozzone Costa 2003, S. 113). So halten viele Lehrkräfte die sprachliche Gestaltung der Schulbücher für zu schwierig und nicht für eine eigenständige Lektüre der Schülerinnen und Schüler geeignet - selbst dann nicht, wenn das Thema der zu lesenden Texte bereits im Unterricht behandelt worden ist (Bertocchi 2003, S. 22). Die seit Jahrzehnten anhaltende Kritik an den Lehrbüchern hat allerdings kaum dazu geführt, dass sich die Lehrkräfte von ihnen lösten und ihren Unterricht freier gestalteten, was vermutlich in erster Linie an einer starken Traditionsverbundenheit liegt, durch die sich viele an den Erfahrungen der eigenen Schulzeit und den Praktiken ihrer Lehrer orientieren, welche sie als teaching models heranziehen (vgl. Zeichner 1986, S. 264 in Maas 2008, S. 238; De Mauro 1976b in ead. 1979, S. 76 u. S. 86). ${ }^{3}$

So besteht die gängige Praxis einer Erdkundestunde häufig in der Paraphrasierung von Lehrbuchtexten durch die Lehrkraft und dem mündlichen Abfragen einzelner Schülerinnen und Schüler zu den auf diese Weise vermittelten Inhalten. Daraus ergaben sich die folgenden forschungsleitenden Fragen:

1. Wie sind die verwendeten Unterrichtswerke sprachlich gestaltet?

2. Welche Diskrepanz besteht zwischen der sprachlichen Kompetenz der Schülerinnen und Schüler und dem im Lehrbuch verwendeten Register?

3. Auf welche Art schlägt die Lehrperson eine Brücke zwischen dem Unterrichtswerk und den Fähigkeiten der Lernenden?

4. Wie gestaltet sich die Kommunikation im Unterricht?

3 Weitere Gründe hierfür beruhen sicherlich auf Mängeln in der Lehrerausbildung, die sich kaum mit innovativeren Methoden der Unterrichtsgestaltung befasst, und, bei einer oft kritisierten Unterbezahlung der Lehrpersonen, auf ihrer unzureichenden Motivation für den zusätzlichen Arbeitsaufwand, der sich bei der Unterrichtsvorbereitung für sie daraus ergäbe. Dahingegen hat die deutsche Studie von Thöneböhn (1995, S. 204) ergeben, dass selbst Lehrkräfte, die stark lehrbuchzentriert vorgehen, sich von den Büchern abwenden, wenn ihnen der Aufbau oder die Behandlung eines bestimmten Themas nicht zusagt. 
Diese Studie hat das Anliegen, mit einem qualitativen Forschungsdesign möglichst genau abzubilden, was in den beobachteten Klassen sprachlich passiert und wie es in dieser Hinsicht um die Fähigkeiten der Schülerinnen und Schüler steht. Folglich werden schulbezogene sprachliche Herausforderungen und Leistungen aus verschiedenen Perspektiven betrachtet und zueinander in Bezug gesetzt. Insgesamt soll damit ein Beitrag zum Füllen einer bestehenden Forschungslücke geleistet werden, da es zu den konkreten Anforderungen an die Lernenden und ihrer Bewältigung abgesehen von den bereits angesprochenen großangelegten Leistungserhebungen bislang wenig Untersuchungen gibt, welche über die Befassung mit einzelnen sprachlichen Aspekten wie beispielsweise der Verwendung von Konnektoren durch die Lernenden hinausgehen (Harren 2015, S. 20 f.; De Mauro 1997, S. 26).

\subsection{Aufbau der Arbeit}

Das zweite Kapitel liefert als theoretische Rahmung Informationen zu den gesellschaftlichen Ansprüchen an die Sprachkompetenz, zu dem daraus für die Schulen resultierenden Bildungsauftrag und zur Beschaffenheit der schulsprachlichen Register samt ihrer kognitionspsychologischen Bedeutung und Erwerbsbedingungen. Dabei werden auch Überlegungen berücksichtigt, warum der Sprachausbau in der Zweitsprache lernenden Schülerinnen und Schülern zum Teil nicht gut gelingt, die unter dem Schlagwort „MonolingualismusKontroverse" (Gogolin 1994) zusammengefasst werden können. Im Weiteren befasst sich das Kapitel konkret mit der schulischen Sprachbildung. U.a. werden ein weitläufig rezipiertes Förderkonzept, der scaffolding-Ansatz (Wood, Bruner \& Ross 1976; Gibbons 2002; Hammond \& Gibbons 2005), und ,grammatische Metaphern“ (Halliday 1985) als ein entscheidendes, häufig auftretendes Merkmal bildungssprachlicher Texte vorgestellt und die Bedeutung der Lese- und Schreibdidaktik im Lernprozess erörtert. Außerdem wird auf die sprachbezogene Bewertungspraxis an den Bildungsinstitutionen eingegangen.

Im dritten Kapitel erfolgt zunächst ein historischer und dann ein gegenwartsorientierter Überblick über die unterschiedlichen, teils mehrsprachigen Ausgangslagen der Lernenden an italienischen Schulen und die Anzahl, Verteilung und Herkunft der Zugewanderten. Aufgrund der zentralen Rolle der Lehrwerke in der Unterrichtsgestaltung wird hiernach der Stand der Lehrmittelforschung umrissen, wobei auch auf den partikulären, polemischen Ton eingegangen wird, der diese charakterisiert. Zwei Schwerpunkte der Lehrmittelforschung werden dabei eigens thematisiert, und zwar die Lesbarkeitsforschung (Lucisano \& Piemontese 1988; Lumbelli 1989) und Untersuchungen zur Wortschatzverwendung. Letztere stützen sich teilweise auf das Grande dizionario italiano dell'uso (Großes italienisches Gebrauchswörterbuch) (De 
Mauro 1999/2000), das auf einer Gruppierung des italienischen Wortschatzes in unterschiedliche Kategorien gemäß ihrer Streuung und Frequenz in gesprochenen und geschriebenen Texten beruht und auch in der vorliegenden Studie verwendet wurde, so dass es ebenfalls vorgestellt wird.

Kapitel vier befasst sich mit wesentlichen Hintergrundinformationen zum Erhebungskontext. Es schildert die Lehrerausbildung in Italien, die geltenden Lehrpläne für das Fach Erdkunde in der Sekundarstufe I und die Verankerung der Sprachbildung in den Lehrplänen und ministerialen Richtlinien. Der Ausbau der lexikalischen Kompetenz als wichtige Komponente der sprachlichen Fähigkeiten und ihre Berücksichtigung in den Lehrplänen werden ebenso beschrieben wie die Divergenzen zwischen den darin enthaltenen Forderungen und der tatsächlichen Unterrichtspraxis sowie dem in der Lehrerausbildung Vermittelten. Desweiteren wird auf den registerbezogenen Sprachausbau eingegangen, der vor allem während der Schulzeit erfolgt und zu dessen Beschreibung das Konzept des spazio linguistico (De Mauro 1980; sprachlicher Raum) von Interesse ist. Als wichtige Voraussetzung für das Gelingen des Sprachausbaus wird die Entwicklung protoliterater Fähigkeiten im Vorschulalter erläutert und im Anschluss daran die Rolle von Sprachstandserhebungen und ihr eingeschränkter Einsatz in Italien beschrieben. Weitere Informationen über spracherwerbstheoretische und sprachpädagogische Aspekte und über die Arbeitsbedingungen in den während der Studie beobachteten Klassen an drei verschiedenen Bildungsinstitutionen schließen das Kapitel ab.

Im Folgenden werden die Erhebungsergebnisse vorgestellt, wozu das fünfte Kapitel zunächst die methodischen Überlegungen darlegt, die das Vorgehen geleitet haben, und das sechste Kapitel mit einer Präsentation der fünf beobachteten Klassen die Schilderung der Studie beginnt. Es stützt sich dabei auch auf Angaben der Lernenden, die anhand eines von ihnen bearbeiteten Fragebogens ermittelt wurden und ihren soziokulturellen und sprachlichen Hintergrund, ihre Einstellung zum Fach Erdkunde und seine Verfahrensweisen betreffen.

Das siebte Kapitel besteht aus einer sprachlichen Analyse ausgewählter Seiten der fünf verwendeten Erdkundebücher, die sich vorrangig auf die lexikalische Gestaltung konzentriert, aber auch andere Textmerkmale wie die Länge der Satzgefüge, die Zusammensetzung nach Wortarten und die Komplexität morphosyntaktischer Strukturen in den Blick nimmt.

Im achten Kapitel werden die Ergebnisse eines durchgeführten Sprachtests (C-Test; Raatz \& Klein-Brayley 1985; Grotjahn z.B. 2010, 2006) vorgestellt, der auf der Grundlage von Texten der Unterrichtswerke entwickelt wurde und auf dem Tilgungsprinzip beruht: Die Testteilnehmer mussten die zweite Hälfte jedes zweiten Wortes in mehreren kurzen Texten vervollständigen. Die Ergebnisse ermöglichen einen ersten Einblick in die Sprachkompetenz der Lernenden und geben Auskünfte über ihre sprachlichen Schwierigkeiten im Umgang 
mit dem Unterrichtswerk. Da es sich aufgrund der unterschiedlichen Lehrwerksverwendung um fünf verschiedene C-Tests handelt, deren Bearbeitung detailliert analysiert wurde, stellt dieses Kapitel einen Schwerpunkt der Arbeit dar und ergänzt die Auswertung der Lehrbuchseiten um Aspekte, die unter bildungssprachlichen und didaktischen Gesichtspunkten relevant sind.

Darauf folgt ein neuntes Kapitel, in dem anhand konkreter Beispiele aus der Unterrichtspraxis ein Bild der spracherzieherischen Rolle der Lehrkräfte und der mündlichen Leistungen der Schülerinnen und Schüler gezeichnet wird. Kapitel 10 hingegen stellt schriftliche Textproduktionen der Lernenden vor, die mit dem Auftrag verfasst wurden, dem Charakter eines Lehrbuchtextes ihrer Klassenstufe zu entsprechen, um die Lernenden zu einem möglichst bildungssprachlichen Schreibstil zu bewegen. Abschließend fasst das elfte Kapitel die einzelnen Ergebnisse der Untersuchung noch einmal zusammen, zieht ein Fazit und bietet Vorschläge für mögliche anknüpfende Untersuchungen.

Hierbei sei vorweggenommen, dass eine effiziente und durchgängige Sprachbildung in allen Fächern nicht nur aus ökonomischer Sicht als unabdingbares Requisit für eine bessere Beschäftigungstauglichkeit und aus soziopolitischer für eine aktive Teilhabe am gesellschaftlichen Leben von enormer Wichtigkeit ist, sondern dass die Sprachentwicklung aufs Engste mit der kognitiven Entwicklung verwoben ist und damit wesentlich zur Freisetzung von individuellen Potentialen und zur Persönlichkeitsbildung beiträgt (vgl. Maas 2008, S. 394). Um es mit den Worten von De Mauro zu sagen, kann Sprache gesehen werden ,come compagno ed ordinatore di tutta la nostra vita mentale ed emotiva, come filtro attraverso il quale passa tutta la nostra capacità di rapporto con gli altri“ (De Mauro 1976b in ead. 1979, S. 77; meine Übersetzung (im Folgenden m.Ü./M.Ü. abgekürzt): als Begleiterin und Ordnerin unseres ganzen mentalen und emotiven Lebens, als Filter, durch den all unsere Fähigkeit läuft, mit den anderen in Beziehung zu treten). 


\section{Theoretische Rahmung}

Die Sprachentwicklung aller Lernenden ist von primärer Wichtigkeit für erfolgreiche Schullaufbahnen und die Eingliederung in das berufliche und gesellschaftliche Leben. In diesem Kapitel werden theoretische Aspekte vorgestellt, welche die gesellschaftlichen Sprachanforderungen und den daraus resultierenden Bildungsauftrag der Schule illustrieren (2.1). Weil in der Schule andere Sprachregister verwendet werden, als die Mehrheit der Lernenden untereinander und in der alltäglichen Kommunikation nutzt, und diese mehrsprachigen und bildungsfernen Schülern und Schülerinnen Schwierigkeiten bei der Aneignung bereiten, werden diese Register (2.2), ihre kognitionspsychologische Bedeutung (2.2.1) und ihre sprachlichen Merkmale (2.2.2) vorgestellt. Darum, wie die schulsprachlichen Varietäten vermittelt und erworben werden, geht es im Unterkapitel 2.2.3. Im Anschluss daran werden Überlegungen in den Blick genommen, welche Gründe für Einschränkungen im Sprachausbau mehrsprachiger Schülerinnen und Schüler und Vorschläge für eine gerechtere Sprachbildung aufzeigen, die unter dem Schlagwort „MonolingualismusKontroverse" zusammengefasst werden können (2.3).

Der vierte Teil des Kapitels befasst sich konkreter mit dem schulischen Umgang mit Sprache (2.4) und stellt zunächst den scaffolding-Ansatz (2.4.1) als ein wesentliches Förderkonzept vor, welches sowohl auf einer unterrichtsplanenden Makroebene (2.4.1.1) als auch bei einer spontanen gerüstartigen Unterstützung des (sprachlichen) Lernens im Unterricht, also auf einer Mikroebene (2.4.1.2), zum Tragen kommt. Im Folgenenden wird ein wesentliches und sehr frequentes Merkmal der schulsprachlichen Register vorgestellt, um an einem konkreten Beispiel zu veranschaulichen, wo es im Sprachaneignungsprozess durch die Lernenden einer systematischen Förderung bedarf (2.4.2). Von besonderer Bedeutung sind außerdem die Lesedidaktik (2.4.3.1) und die in zwei weiteren Unterkapiteln beschriebene (2.4.3.2 und 2.4.3.2.1) Schreibdidaktik. Dasselbe gilt für die Bewertungspraxis der Bildungsinstitutionen, deren Skizzierung das Kapitel abschließt (2.5).

\subsection{Gesellschaftliche Sprachanforderungen und der Bildungsauftrag der Schule}

Die Notwendigkeit einer weitreichenderen Sprachbildungspraxis in der Institution Schule findet ihre Bestätigung in den mäßigen schriftkulturellen Leistungen, die viele Lernende bei Schulvergleichsstudien auf nationaler (Prove INVALSI) und auf internationaler Ebene (PISA) erzielen, sowie in den Sondierungen zur funktionalen Alphabetisierung, ALL (Adult Literacy and Life 
Skills). Piemontese und Sposetti (2014) erinnern daran, dass De Mauro in La cultura degli italiani (De Mauro 2010²) die für Italien von Gallina (2006) zusammengestellten Ergebnisse der Erhebung ALL folgendermaßen kommentiert: Italien befindet sich trotz ansteigender Beschulung unterhalb der Schwelle funktionaler Literalität, da nur 20 Prozent der Teilnehmer an ALL über ein Mindestmaß an Kompetenz im Lesen, Schreiben und Rechnen verfügen. Weiter ist es überraschend, dass 74 Prozent der Italiener trotz höherer Bildungsabschlüsse große Schwierigkeiten beim Verständnis oder Verfassen eines einfachen Textes zeigen (De Mauro 2010², S. 241 zit. in Piemontese \& Sposetti 2014, S. 9 f.). ${ }^{4}$

Dem steht auf der anderen Seite eine deutliche Zunahme der rezeptiven und produktiven Anforderungen im Schriftsprachlichen gegenüber, die seit einigen Jahren in den westlichen Kulturen zu beobachten ist und zu einem guten Teil aus der Verlagerung der ökonomischen Interessen vom industriellen Sekundärsektor zu wissensbasierten Wirtschaftszweigen resultiert (Cummins 2002, S. V). Dies hat zur Folge, dass heute selbst in den traditionellen Tätigkeitsfeldern profundere sprachliche und mathematische Kenntnisse vorausgesetzt werden als früher, um etwa einen Ausbildungsplatz im Handwerk zu erhalten. Genauso werden in der Industrie inzwischen für die computergesteuerte Bedienung von Maschinen und Arbeitsabläufen solide Kompetenzen im Schreiben und Lesen erwartet (OECD 2000, S. 8; Steffens 2005, S. 31 f.). Gleichzeitig hat die Weiterentwicklung der Kommunikationsformen auch vor der Arbeitswelt nicht haltgemacht, wodurch beispielsweise Mittteilungen immer seltener mündlich formuliert, sondern als E-Mails an die Kollegen, Vorgesetzten und anderen Abteilungen versendet werden (Döbert \& Hubertus 2000, S. 12, S. 18 f. mit Verweis auf Giese 1987). Daraus ergibt sich, dass laut wirtschaftswissenschaftlichen Untersuchungen die allgemeine Sprachkompetenz zwar kein Indikator für Beschäftigungstauglichkeit oder beruflichen Erfolg ist, schriftkulturelle Fähigkeiten aber eindeutig mit der Eingliederung in den Arbeitsmarkt korrelieren (Maas 2008, S. 667).

Maas beschreibt die sprachlichen Anforderungen an die Bürgerinnen und Bürger westlicher Kulturen wie folgt (ibid., S. 23 f.):

4 Auch Serianni (2010) verweist auf diese periodisch vehement beanstandete Situation des „Massen-Analphabetismus“, welche vielleicht zu relativieren, aber sicher nicht zu unterschätzen sei (Serianni 2010, S. X). Es ist möglich, dass sich der anscheinende Widerspruch zwischen hohen Bildungstiteln und niedrigen schriftkulturellen Fähigkeiten zu einem Teil durch die Notengebung der ItalienischlehrerInnen erklären lässt, wonach diese in der Vergabe von guten Noten auch für schwache Leistungen weitaus großzügiger seien als ihre Kollegen in den anderen Fächern (ibid., S. 43 f.). Deshalb könnte sich der Anteil der sogenannten Analphabeten di ritorno (zurück; der sekundäre Analphabetismus betrifft Personen, die alphabetisiert waren, aber diese Fertigkeit aufgrund unzureichender Praxis wieder verlernen (Hubertus 1995, S. 251)) in Wahrheit z.T. aus einer Gruppe von Personen zusammensetzen, die nie richtig literalisiert war, jedoch in der Schule trotzdem vorangekommen ist. 
Im Inneren der wohlfahrtsstaatlich organisierten (nationalen) Gesellschaften schreitet die soziale Integration voran, wird auf immer erweitertem Niveau reproduziert. Dadurch wächst der Anspruch an die einzelnen Mitglieder dieser Gesellschaften für eine erfolgreiche Partizipation. Das gilt insbesondere für die sprachlichen Verhältnisse, die durch exponentiell wachsende Anforderungen an die symbolische Kontrolle der Handlungsabläufe bestimmt sind: im politischen Raum, am Arbeitsplatz und nicht zuletzt auch in der privaten Organisation der Lebensformen. Die sprachlichen Verhältnisse werden dabei zunehmend auf dezentrierte schriftkulturelle Praxisformen ausgerichtet, denen gegenüber die lokal verfassten (intimen) gesprochenen Sprachformen eine Umwertung erfahren: Im Feld der Registerdifferenzierung sind sie die Ressourcen zum schriftkulturellen Ausbau.

Auf diese Anforderungen vorzubereiten, ist also ein wesentliches Erziehungsziel der Schule, in welcher auf dreierlei Art Sprache gelehrt wird: explizit im Mutter- und Fremdsprachenunterricht, in/durch Sprache im Fachunterricht und über Sprache (Halliday 1993, S. 112). Da viele Lerninhalte auf Textbasis vermittelt werden, aber auch die Lehrpersonen im Unterrichtsgespräch andere sprachliche Register wählen, als es die Kinder und Jugendlichen tun, wenn sie informell miteinander kommunizieren, kommen die Lernenden so mit sprachlichen Strukturen in Berührung, mit denen sie je nach sozialer Herkunft mehr oder weniger vertraut sind.

Es handelt sich hierbei um schichtspezifische Unterschiede im Sprachgebrauch, welche im Zuge der soziolinguistischen Studien ab den 1960er Jahren vor allem durch die Arbeiten von Basil Bernstein $(1959,1964,1971)$ mit seiner Unterscheidung zwischen einem restringierten und einem elaborierten Code einer breiteren Öffentlichkeit bewusst wurden (Pinto 1980, S. 94 f.), wobei diese Arbeiten jedoch aufgrund einseitiger Auslegungen schnell als politisch etwas inkorrekt in Verruf geraten sind (s. Weber 1973, S. 91; Maas 2008, S. 423). Eine auf den Beobachtungen des Soziologen Bernstein fußende differenziertere Hervorhebung der Registerunterschiede ist Jim Cummins zu verdanken, der in erster Linie durch seine Studien zum Bilingualismus bekannt geworden ist und in der pädagogischen und sprachwissenschaftlichen Forschung oft wegen seines Einsatzes für zweisprachige Erziehung erwähnt wird.

Für die Konzeptualisierung der sprachlichen Fähigkeiten der Lernenden im Verhältnis zur Schulsprache ist seine modulare Aufgliederung des Sprachwissens in BICS (Basic Interpersonal Communication Skills) und CALP (Cognitive Academic Language Proficiency) von großem Nutzen, weil die Einführung dieses Begriffspaares mit dem bislang vorherrschenden holistischen Sprachverständnis bricht, welches den Blick auf die Anforderungen und Schwierigkeiten des Sprachausbaus verstellt (vgl. Maas 2008, S. 640 f.). Während die BICS auch in einer Zweitsprache in der Regel innerhalb von wenigen Monaten bis zu zwei Jahren erworben werden, braucht es einen Zeitraum von fünf bis acht Jahren, bis der kognitive Sprachausbau, auf dem die CALP basiert, grundlegend erfolgt ist. In diesem Zusammenhang geht Cummins (1979a) von einer Interdependenz-Hypothese aus, nach der zunächst ein gewisser Schwellenwert 
in der kommunikativen Sprachfähigkeit (BICS) erreicht sein muss, damit der komplexere Ausbau (in der CALP) stattfinden kann.

Bevor w.u. erläutert wird, wie der kognitive Ausbau der Sprachfähigkeit dabei unabhängig von den idiomatisierten Sprachstrukturen, in denen er erfolgt, betrachtet werden kann, so dass nicht entscheidend ist, in welcher Sprache die Entwicklung der BICS vollzogen wird, sollen die in der Schule verwendeten Register detaillierter dargestellt werden.

\subsection{Die Bildungssprache}

Für die in der Schule verwendete Sprachvarietät gibt es verschiedene Bezeichnungen. Der auch von Cummins gebrauchte Begriff der academic language (Cummins 1979a) wird im Deutschen mit den Ausdrücken „Schulsprache“ und „Bildungssprache“ und im Italienischen mit italiano per lo studio (Favaro 2003, S. 13; Italienisch fürs Lernen) oder italiano per capire (Colombo \& Pallotti 2014; Italienisch fürs Verstehen) wiedergegeben. Gleichzeitig findet man diesen Sprachgebrauch aber auch als secret language betitelt (Lee 1996, S. 20), da er lange Zeit und im Grunde immer noch von den meisten Lehrkräften nicht explizit vermittelt, sondern seine Beherrschung stillschweigend vorausgesetzt wurde (Gantefort \& Roth 2010, S. 574 f.). Das resultiert zu einem guten Teil daraus, dass der Lehrkörper hauptsächlich der Mittelschicht entstammt und sein bildungsnaher familiärer Hintergrund u.a. durch die Vermittlung protoliterater Fähigkeiten (ibid., S. 576) und die Bekundung von Interesse für das in der Schule Erlernte (Maas 2008, S. 650 f.) einen sehr förderlichen Einfluss auf seine Sprachentwicklung und den Bildungserfolg hat. Deswegen sind ausgebaute Sprachkenntnisse hier der Normalfall und werden somit als fast ,natürlich“" vorausgesetzt (ibid., S. 186), bzw. wird die Auffassung vertreten, dass sich der Weg ihrer Aneignung in der Schule durch das Eintauchen ins bildungssprachliche Register (Favaro 2003, S. 13) von selbst gehen lässt schließlich haben die Lehrpersonen es sich auf diese Weise ja auch ziemlich ,automatisch" angeeignet.

So halten Gogolin und Lange (2010) fest (ibid., S. 11): „Auf der normativen Ebene ist mit Bildungssprache dasjenige Register bezeichnet, dessen Beherrschung von „erfolgreichen Schülerinnen und Schülern“ erwartet wird.“ Laut Wissenschaftlern wie Weber und Hattie hat diese ,schulische [...] Reproduktion der Machtverhältnisse in der Sprache“ (Maas 2008, S. 186) darüber hinaus zur Folge, dass der Mittelschicht angehörende Lernende bei gleichen Leistungen von den Lehrpersonen stets besser bewertet werden als ihre Altersgenossen aus den unteren sozialen Schichten (Hattie 2013, S. 17; Weber 1973, 
S. 38). ${ }^{5}$ Auf diese Weise setzt die Schule einen mächtigen Selektionsmechanismus in Gang, wenn sie sich nicht bemüht, das Sprachspektrum der bildungsferneren Schichten und der Lernenden mit Migrationshintergrund zu erweitern (Maas 2008, S. 186).

Der bei der Einführung des Hamburger Forschungsschwerpunktes FörMig (Förderung von Kindern und Jugendlichen mit Migrationshintergrund) ${ }^{6}$ empfohlene Begriff der Bildungssprache hat eine doppelte Bedeutung. Auf der einen Seite bezieht er sich auf die ,Sprache, in der Bildung in Einrichtungen und Institutionen gestaltet wird" (Gogolin \& Lange 2010, S. 9); auf der anderen Seite bezeichnet er eine ,spezielle Ausprägung des Deutschen [bzw. der jeweiligen Sprache, welche sich der Staat zu seiner Geschäftsführung gegeben hat]: Je weiter eine Bildungsbiographie fortgeschritten ist, desto mehr unterscheiden sich die sprachlichen Anforderungen in den Bildungseinrichtungen vom Repertoire der alltäglichen, mündlichen Sprachen“ (ibid.).

In der Schule werden permanent neue Inhalte erarbeitet, was oft in Form von Texten geschieht, die mit steigender Klassenstufe komplexer und häufig auch abstrakter werden, so dass die Lernenden über fundierte (v.a. konzeptionell schriftliche) sprachliche und kognitive Fähigkeiten verfügen müssen, um diese Inhalte verstehen, ordnen, miteinander vergleichen und schriftlich oder mündlich aushandeln zu können. Der Begriff Bildungssprache bezieht sich auf den Umgang mit Texten im weitesten Sinne und auf generelle Praxen des Wissenserwerbs und seiner Kommunikation. Damit geht er über den schulischen Sprachgebrauch hinaus (Bosisio 2003, S. 139), welcher für sich genommen als „Schulsprache“ bezeichnet wird (Gogolin \& Lange 2010, S. 12), ist aber auch dort „Ausdruck jener sprachlichen bzw. kommunikativen Anforderungen in fachlichen Lernkontexten, hinter denen sich komplexe Herausforderungen in der Verwendung von Sprache als kognitivem Werkzeug verbergen" (Vollmer

5 Vgl. in diesem Sinne Korte $\left(1993^{2}\right)$ mit Verweis auf Horkheimer (Schmidt \& Altwicker 1986) (Korte 1993², S. 147): „Die Wege, die zur Macht führen, sind in der bürgerlichen Welt nicht durch die Verwirklichung moralischer Werturteile, sondern durch die geschichtliche Anpassung an die Verhältnisse gekennzeichnet“. Desweiteren Maas (2008, S. 35) und Weber (1973, S. 99).

6 FörMig ist ein Modellprogramm, das von 2004 bis 2009 in zehn deutschen Bundesländern unter der wissenschaftlichen Begleitung des Instituts für International und Interkulturell Vergleichende Erziehungswissenschaft der Universität Hamburg durchgeführt worden ist. 2010 ist dort das FörMig Kompetenzzentrum eröffnet worden, das sich bis 2013 mit der Entwicklung und Förderung einer durchgängigen, sich auf die ganze Schullaufbahn erstreckende Sprachbildung beschäftigt hat, welche die unterschiedlichen Ausgangslagen von Schülerinnen und Schülern mit Migrationshintergrund berücksichtigt und für mehr Chancengleichheit im Bildungssystem sorgen möchte. Hierbei ist in verschiedenen Projekten eng mit anderen Ländern zusammengearbeitet worden. Die Expertise hat mehrere Bände zum erforschten Themenkomplex sowie verschiedene Unterrichtsmaterialien und Diagnoseinstrumente zum Erfassen sprachlicher Fähigkeiten sowohl im Deutschen als auch in nicht deutschen Erstsprachen herausgegeben. Seit 2013 läuft die Forschung unter dem Namen DiVER - Diversity in Education Research weiter. 
\& Thürmann 2010, S. 110). Es handelt sich also weniger um sprachliche Oberflächenphänomene als um komplexe „kognitive, textbezogene Fähigkeiten“ (Gantefort \& Roth 2010, S. 585), welche für eine effiziente Verwendung der bildungssprachlichen Register notwendig sind.

\subsection{1 „Weiterentwicklung des Denkens als Denken fürs Sprechen“}

Wissen kann nicht von der Sprache, über die es vermittelt wird, losgelöst werden. In diesem Sinne dient Sprache als vorrangiges Mittel zum Verständnis der Realität (Halliday 2007, S. 360 f.): ,Reality, the human experience of what is out there - and also of what is "in here", the reality inside the head - is not something readymade and waiting to be acquired. It has to be construed; and language is the primary means we use for construing it."

Gleichsam ist Sprache die prinzipielle Ressource, über die das Lernen stattfindet (ibid., S. 60): „Language is the most important instrument of human consciousness, as well as being the principal means by which we learn, whether we are thinking of common sense knowledge or of the knowledge that is taught in school." Wie sehr das schulische Lernen von der Sprachfähigkeit abhängt, beschreibt Corson im Beitrag Language Across the Curriculum für die von Spolsky herausgegebene Enzyklopädie Educational Linguistics (Corson 1999, S. 324):

Language plays a central role in learning. By engaging in purposeful talking and writing in curriculum areas, students assimilate new concepts. When they listen, talk, read, and write about what they are learning, they relate this to what they already know. Through talking and writing, language is linked to the thinking process. Language in use becomes a manifestation of the thinking that is taking place. As a result, by explaining and expressing their own interpretations of new items of learning across the curriculum, students clarify and increase their knowledge of the specialist concepts, they improve their understanding of the different forms of discourse used in each curriculum area. It follows that schools need to provide an environment in which students are encouraged to use language to explore concepts, to solve problems, to organize information, to share discoveries, to formulate hypotheses, and to explain personal ideas.

Die unauflösbare Verknüpfung von Sprache und Kognition wird auch mit dem Bild der Sprachentwicklung als Infrastruktur für die kognitive Entwicklung wiedergegeben, wobei nicht von einer harten biologischen Schwelle (s. Lenneberg 1967) im Spracherwerb auszugehen ist, aber dennoch hervorgehoben werden muss, dass der sprachliche Aneignungsprozess durch die vorhandenen kognitiven Strukturen determiniert wird (Maas 2008, S. 318):

Die Sprachentwicklung bildet im engeren Sinne die formale Seite (Infrastruktur) der kognitiven Entwicklung. Insofern ergibt sich eine enge Verschränkung von begrifflich-konzeptueller Entwicklung und formal-kommunikativ ausgeübter sprachlicher Entwicklung. In der Sprachentwicklung erschließen sich Potentiale, die kognitiv eröffnet werden, weil die Sprachstrukturen kognitiv verankert werden müssen (s.o. zur Grammatisierung). In anderer 
Hinsicht gilt aber auch: um den Anforderungen der sozialen Partizipation nachzukommen, müssen die kognitiven Ressourcen erweitert werden - wie D. Slobin [1987] es formuliert hat: Das Denken wird als Denken fürs Sprechen weiter entwickelt. Dadurch sind auch die Phasen der Entwicklung in diesem Feld bestimmt: Wo das Kind in Hinblick auf seine praktischen Erfahrungen und Handlungsspielräume komplexere kausale Zusammenhänge noch nicht überblickt, zwischen verschiedenen Ereignissen keine hierarchische Ordnung herstellen kann, in ihnen keine referenziellen Konstanten differenziert u. dgl., kann es auch die entsprechenden sprachstrukturellen Ressourcen nicht nutzen.

Der theoretische Hintergrund dieser Annahme ist im Werk des Psychologen Lev Vygotskij (v.a. 1934) dargelegt, welcher zum einen das Sprachlernen als Auseinandersetzung mit der sozialen Umgebung des Lernenden, also als einen sozial beeinflussten Prozess auffasst und diesen zum anderen von den Entwicklungsstadien des heranwachsenden Menschen konditioniert sieht. Weiter unten wird ausführlicher auf seine weitläufig rezipierte Theorie eingegangen, um zu erklären, wie der Sprachlernprozess von außen unterstützt werden kann. $^{7}$

Die besondere Bedeutung des Sprachausbaus für die Entwicklung kognitiver Kompetenzen tritt vor allen Dingen beim Schriftspracherwerb hervor. In dieser entscheidenden Phase der Sprachaneignung wird neben der Bildung metasprachlicher Kompetenzen das Wissen neu organisiert. Besonders Schreibpraktiken, welche für das schulische Lernen funktional sind, wirken sich dabei auf die kognitiven Fähigkeiten der Schülerinnen und Schüler aus, weil durch sie die erworbenen Kenntnisse neu verarbeitet und strukturiert werden (Guerriero 2014, S. 164; Halliday 1993, S. 109). ${ }^{8}$ Hierbei korrelieren die vier sprachlichen Grundfähigkeiten, die Produktion und Rezeption von gesprochenen und geschriebenen Texten, mit der „,kognitiven Mobilität“ (Guerriero 2014, S. 168) der Lernenden, welche Guerriero unter Bezugnahme auf Ferreri (2002) mit einigen Beispielen beschreibt (Guerriero 2014, S. 168): ,„.... [ cioè la loro maggiore o minore difficoltà di "movimento" cognitivo, per esempio nello spostarsi dalle proprie conoscenze al testo e viceversa, o a muoversi all'interno del testo, a cercare riscontri sul testo delle ipotesi formulate, a effettuare controlli delle informazioni. “(M.Ü.: das heißt, ihre größere oder geringere Schwierigkeit der kognitiven „Bewegung“, zum Beispiel beim Verlegen der eigenen Wissensbestände in den Text und umgekehrt oder beim Bewegen innerhalb des Textes, beim Suchen im Text nach Übereinstimmungen mit den formulierten Hypothesen, beim Kontrollieren der Informationen.)

So wirkt sich der Schreibprozess der Lernenden positiv auf ihre kognitive Entwicklung aus, weil er das Denken verfeinert und die Lernfähigkeit stärkt (ibid., S. 172, Hervorhebung im Original):

7 S. das Unterkapitel 2.4.1.

8 S.a. Ehlich (2007, S. 25): „Die Aneignung der (Mutter-)Sprache wird durch den Eintritt der Schrift erheblich und grundlegend umstrukturiert; das betrifft sowohl den Zugriff auf Sprache, die nun vergegenständlicht als Objekt zur Verfügung steht, die Formen der Sprachplanung und der Sprachrezeption wie das sprachliche Normbewusstsein.“ 
[L]a necessità di esplicitezza attraverso le risorse linguistiche incoraggia l'attivazione di connessioni tra idee e promuove l'esplorazione di affermazioni non analizzate. Se per Bereiter e Scardamaglia la creazione di nuovi contenuti attraverso la strategia della trasformazione delle conoscenze è il risultato di un'analisi condotta dallo scrivente sull'appropriatezza del suo testo rispetto alle sue intenzioni comunicative, Galbraith (1999) assume che i processi linguistici, applicati a unità di contenuto, generano unità di nuova conoscenza; la scrittura, dunque, in quanto trattamento semantico, lessicale, sintattico di idee assume un carattere epistemico: è questo un modello di scrittura definito come "costituzione di conoscenze".

(M.Ü.: Die Notwendigkeit von Explizität durch linguistische Ressourcen ermutigt die Aktivierung von Verbindungen zwischen Ideen und fördert die Exploration nicht analysierter Äußerungen. Wenn für Bereiter und Scardamaglia [1995] die Schöpfung neuer Inhalte durch die Strategie der Transformation der Kenntnisse das Resultat einer vom Schreibenden durchgeführten Analyse zur Angemessenheit seines Textes in Bezug auf seine Kommunikationsabsichten ist, geht Galbraith (1999) davon aus, dass die linguistischen Prozesse, die auf Inhaltseinheiten angewendet werden, Einheiten neuer Erkenntnisse generieren; das Schreiben nimmt also dadurch, dass es eine semantische, lexikalische und syntaktische Bearbeitung von Ideen ist, einen epistemischen Charakter an: Dies ist ein als „Bildung von Kenntnissen“ definiertes Modell des Schreibens.)

Auch Ehlich, der den Spracherwerb grob in drei wesentliche Phasen (0-3, 3-6 und 6-12 Jahre) unterteilt (Ehlich 2007, S. 34), betont die gnoseologische (erkenntnisbezogene) Funktion der Sprache, welche er zusammen mit der kommunitären und der interaktiven als die drei wesentlichen Funktionsbereiche der Sprache betrachtet, wobei die ersten beiden wesentlich weniger erforscht sind als die letzte. Demnach macht Sprache das Wissen formbar, beständig in der Zeit (scripta manent) und veränderbar (ibid., S. 18):

Sprache ist sowohl individuell wie für die jeweilige Sprechergruppe eine unabdingbare mentale Ressource. Erst die Versprachlichung verleiht Wissen die Komplexität und Plastizität, die Dauerhaftigkeit und Veränderbarkeit, die menschliches Wissen kennzeichnen. Es sind eher Strukturen des Lexikons, für die solche Aspekte von Sprache überhaupt ins Blickfeld gerückt werden.

Im bewussten produktiven und rezeptiven Umgang mit Texten, der sich in der dritten Phase der Sprachentwicklung mit der Aneignung der schriftkulturellen Fertigkeiten einstellt, wird einer „,er wichtigsten Prozessschritte überhaupt“ gesehen (ibid., S. 30). Desweiteren werde damit auch die Sprachreflexion stimuliert, welche für einen selbstständigen Sprachaneignungsprozess entscheidend ist (ibid., S. 37). Durch den modifizierten Zugriff auf Sprache, die nun nicht mehr nur oral und damit stark situationsgebunden auftritt, sondern ,vergegenständlicht als Objekt zur Verfügung“" steht (ibid., S. 25), werden Formen der Sprachplanung und -rezeption ausgebaut, verfestigt sich das sprachliche Normbewusstsein. Über die Bildung von metasprachlichem Wissen hinausgehend, entwickeln die Lernenden dadurch ein Bewusstsein für ihre Sprachfähigkeiten, welches eine Grundlage für die im schriftkulturellen Spracherwerb überaus relevante Selbstevaluation formt, auch wenn dieser Aspekt Ehlich zufolge in der Didaktik noch kaum beachtet wird (ibid.). 
Bezüglich der wesentlichen Rolle der Sprache in der kognitiven Entwicklung wird auch auf Gardners sieben menschliche Intelligenzen verwiesen (Stefinlongo 2002, S. 24 f.). Selbst wenn nach dieser Theorie Erwerb und Entwicklung der menschlichen Fähigkeiten in einem bestimmten Erkenntnisbereich ziemlich unabhängig von denen in anderen Erkenntnisbereichen verlaufen, sind bestimmte Fähigkeiten doch als prioritär und fundamental für jegliche kognitive, Erkenntnis betreffende und soziale Entwicklung aufzufassen, und hier allen voran die Sprache. Begründet wird dies folgendermaßen (Gardner 1993, S. 21 zit. in Stefinlongo 2002, S. 24): „Ciò dipende dal fatto che la lingua è, fra i sistemi di rappresentazione simbolica della realtà, il più duttile, potente $e$ inestricabilmente connesso con le attività cognitive dell'essere umano." (M.Ü.: Das hängt von der Tatsache ab, dass die Sprache unter den symbolischen Repräsentationssystemen der Realität das geschmeidigste, mächtigste und am unzertrennlichsten mit den kognitiven Tätigkeiten des Menschen verbundene ist.)

Dieses Primat kommt der als wichtigste der fünf „Fähigkeiten fürs Leben“ geltenden Sprachfähigkeit zu, bzw. genauer gesagt dem „uso dell'informazione contenuta in testi scritti o stampati al fine di agire in modo efficace nella società e comunità di riferimento, per raggiungere i propri obiettivi e sviluppare le proprie conoscenze e le proprie potenzialità" (CEDE 1999 zit. in Stefinlongo 2002, S. 25; m.Ü.: Gebrauch der in geschriebenen oder gedruckten Texten enthaltenen Information zum Zweck des wirksamen Handelns in der Bezugsgesellschaft oder -gemeinschaft, um die eigenen Ziele zu erreichen und seine Kenntnisse und Potenziale zu entwickeln). ${ }^{9}$

Die kognitiven und die bildungssprachlichen Kompetenzen stehen dabei in einer Wechselwirkung zueinander. Werden Erstere durch die Aneignung der Bildungssprache ausgebaut, so sind sie auf der anderen Seite in einer recht ausgeprägten Form erst einmal notwendig, damit der Aneignungsprozess der Bildungssprache überhaupt stattfinden kann. Beim wiederholten Resümieren, Analysieren, Interpretieren und Verfassen von Texten werden so „bestimmte Strategien oder Schemata entwickelt, die ab einem gewissen Zeitpunkt als prozedurales Wissen verfügbar sind“ (Gantefort \& Roth 2010, S. 577). Diese Fähigkeiten zur Strategie- und Schemabildung können als ,einzelsprachunabhängige Organisationszentren des sprachlichen Handelns" (ibid., S. 578) betrachtet werden. Sie basieren auf Komponenten der Cognitive Academic Language Proficiency (CALP), von denen Francis einige aufzählt und ebenfalls als sprachübergreifend charakterisiert (Francis 2000, S. 177 zit. in Gantefort \& Roth 2010, S. 578; Hervorhebung im Original):

9 Die anderen vier sind arithmetische und mathematische Fähigkeiten, Fähigkeit zum analytischen Denken und zur Formulierung und Lösung von Problemen, Teamfähigkeit und die Fähigkeit, Informations- und Kommunikationstechnologien zu nutzen (s. CEDE 1999 zit. in Stefinlongo 2002, S. 25). 
[...] core discourse competencies, text comprehension proficiencies, formal schemata, and organizational skills are not language bound. They are learned by means of language use in L1 or L2, or even by non-linguistic means. Subsequently, they are available for application to academic language tasks in either language. Rather than transfer from L1 to L2, or L2 to L1, (since CALP-type skills do not 'reside' or 'belong to' either in the strict sense), they are accessed from a CUP [Common Underlying Proficiency] that in essence is a kind of shared, non-verbal conceptual store.

Gegen eine Transfer-Vorstellung der literaten Fähigkeiten richtet sich auch Maas (2008, S. 649 f.) - vielleicht weil der Begriff des Transfers von Kompetenzen auf neue und andere Wissensbereiche oder Aufgaben aufgrund der Tatsache, dass es bei den schulisch vermittelten Inhalten immer mehr um ihre Applizierbarkeit auf die Arbeitswelt und lebenslanges (auch autonomes) Lernen geht, in den vergangenen Jahren zu einem internationalen Modewort in den Lehrplänen und ministerialen Richtlinien geworden ist. Schriftkulturelle Kompetenz ist eine für die Aneignung und Bearbeitung vieler Wissensbestände notwendige Ressource, und je ausgebauter sie ist, desto zielstrebiger erfolgt der Erwerb und die Verankerung neuer Erkenntnisse (ibid., Hervorhebung im Original):

In dieser Hinsicht hat das [...] Konzept des Transfers eine Schlüsselrolle. Trennt man dabei nicht systematisch orate von literaten Wissensbeständen, kann dieses Konzept den Blick mehr verstellen als leiten. Bei der gerade auch didaktisch verbreiteten Reduktion von Schrift auf ihre skribale Seite liegt die Vorstellung des Transfers gewissermaßen als der Mitnahme eines Werkzeugkastens nahe, den man auch für andere Aufgaben heranzieht als den bisher damit bewerkstelligten. Versteht man aber literates Wissen als die Nutzung aller verfügbaren Ressourcen, um komplexe Aufgaben zu lösen, die orat nicht lösbar sind, ergibt sich ein anderes Koordinatensystem. Erfolgreiche Leser zeichnen sich offensichtlich dadurch aus, dass sie alle „Register“ ihrer kognitiven Kompetenzen ziehen können und deren Management optimieren - schlechte Leser sind in dieser Hinsicht festgelegt: Sie bleiben beim linearen Dekodieren der Anfänger stecken, oder sie überspringen die Dekodierungsprobleme und raten auf der Basis von assoziativen Inferenzen aus ihrem Weltwissen. Erfolgreiche Lernkarrieren zeichnen sich schon früh dadurch aus, dass nicht etwa ganz andere Wissensbestände genutzt werden - sondern dass die gleichen Wissensbestände anders gemanagt werden (und dann allerdings auch weiter ausgebaut werden). Literate Kompetenz wird erworben, indem dieses komplexe Management der kognitiven Ressourcen gebootet wird - ontogenetisch im Regelfall im Ausgang von einer bestimmten oraten Wissenskompetenz aus, aber in seiner Struktur davon unabhängig. Sie braucht daher auch nicht „transferiert“ zu werden - wenn sie zugänglich ist, kann sie eben auch mit anders idiomatisierten sprachlichen Ausgangsstrukturen (die allerdings eine gewisse Gewöhnungsbedürftigkeit beim Kodieren haben) genutzt werden.

\subsubsection{Merkmale der Bildungssprache}

Das im englischsprachigen Raum in den klassischen Einwanderungsländern von Halliday, Cummins und anderen entwickelte und empirisch fundierte Kon- 
zept academic language ist bisher weder für das Deutsche noch für das Italienische empirisch abgesichert (Gogolin 2009a, S. 270; Ahrenholz 2010a, S. 33; Vollmer 2011, S. 2), aber es darf angenommen werden, dass etliche bildungssprachliche Strukturen sprachübergreifend gelten. So sind beispielsweise viele der für das Bildungsdeutsche zusammengetragenen Merkmale auch für das Italienische als typisch bildungssprachlich herausgestellt worden. Solche bildungssprachlichen Merkmale sind zum einen

- lexematisch-semantische wie eine möglichst genaue und explizite Wortwahl (Vollmer 2011, S. 6), differenzierende und abstrahierende Ausdrücke, Präfixverben und normierte Fachbegriffe (Gogolin \& Lange 2010, S. 48 nach Reich 2008, S. 13), Komposita, Derivate, Konversionen (Eckhardt 2008, S. 70 ff.), drei- oder mehrsilbige, nicht frequente Wörter (Butler, Lord, Stevens, Burrego \& Bailey 2004, S. 20)

und zum anderen

- syntaktische und textuelle Merkmale wie

die Unterbindung von Wiederholungen des bereits Erwähntem (Bryant 1999, S. 167), eine explizite Markierung von Kohäsion zur erkennbaren Verknüpfung von Ideen und Satzteilen, Sätzen und Abschnitten für einen logischen Aufbau der Text- oder Redestruktur und eine überzeugende Themengestaltung mit möglichst vollständigen Informationen bzw. Argumenten (Vollmer 2011, S. 6), hypotaktische Satzgefüge (Konjunktional- und Relativsätze, satzwertige Infinitive und Partizipien), unpersönliche Konstruktionen wie das Passiv oder „man“ (im Deutschen und si impersonale im Italienischen), Funktionsverbgefüge, ein ausgeprägter Nominalstil und umfangreiche Attribute (Gogolin \& Lange 2010, S. 48 nach Reich 2008, S. 13), wobei die Nomen oft durch adjektivierte Partizipien ergänzt sind (Neugebauer \& Nodari 1999, S. 166). Außerdem findet sich in bildungssprachlichen Texten meist ein ausgebautes Verbsystem, das die Tempora Präteritum, Plusquamperfekt, Futur I und Futur II sowie den Konjunktiv I einschließt (ibid.). ${ }^{10}$

Tabelle 1 fasst diese und einige weitere Merkmale stichwortartig zusammen.

10 Für eine tabellarische Übersicht über die von verschiedenen WissenschaftlerInnen aufgezeigten Merkmale von alltäglichem (BICS) gegenüber dem Bildungssprachgebrauch (CALP), auf die sich auch diese Darstellung u.a. stützt, vgl. Berendes, Dragon, Weinert, Heppt und Stanat (2013, S. 26). 


\begin{tabular}{|c|c|c|c|}
\hline $\begin{array}{l}\text { lexematisch-semanti- } \\
\text { sche Merkmale der Bil- } \\
\text { dungssprache }\end{array}$ & Studie & $\begin{array}{l}\text { syntaktische und textu- } \\
\text { elle Merkmale der Bil- } \\
\text { dungssprache }\end{array}$ & Studie \\
\hline präzise Wortwahl & $\begin{array}{l}\text { Vollmer }(2011, \\
\text { S. 6) }\end{array}$ & $\begin{array}{l}\text { keine Wiederholungen } \\
\text { von bereits Erwähntem }\end{array}$ & $\begin{array}{l}\text { Bryant (1999, } \\
\text { S. 167) }\end{array}$ \\
\hline $\begin{array}{l}\text { differenzierende, abs- } \\
\text { trahierende Ausdrücke }\end{array}$ & $\begin{array}{l}\text { Gogolin \& Lange } \\
\text { (2010, S. 48) nach } \\
\text { Reich (2008, S. 13) }\end{array}$ & $\begin{array}{l}\text { explizite Markierung der } \\
\text { Kohäsion }\end{array}$ & Vollmer (2011, S. 6) \\
\hline $\begin{array}{l}\text { keine deiktischen Ele- } \\
\text { mente }\end{array}$ & $\begin{array}{l}\text { Favaro }(2003, \\
\text { S. 15) }\end{array}$ & $\begin{array}{l}\text { ausgeprägte logische } \\
\text { Modifikation durch kom- } \\
\text { plexe Syntax und vielfäl- } \\
\text { tige Konnektoren }\end{array}$ & $\begin{array}{l}\text { Bernstein }(2010, \\
\text { S. } 53 \text {, S. } 65 ; 1971)\end{array}$ \\
\hline Präfixverben & ibid. & $\begin{array}{l}\text { hypotaktische Satzge- } \\
\text { füge (Konjunktional- und } \\
\text { Relativsätze, satzwer- } \\
\text { tige Infinitive und Partizi- } \\
\text { pien) }\end{array}$ & $\begin{array}{l}\text { Gogolin \& Lange } \\
\text { (2010, S. 48) nach } \\
\text { Reich (2008, S. 13) }\end{array}$ \\
\hline $\begin{array}{l}\text { drei- oder mehrsilbige, } \\
\text { niedrigfrequente Wör- } \\
\text { ter }\end{array}$ & $\begin{array}{l}\text { Butler et al. (2004, } \\
\text { S. 20) }\end{array}$ & Informationsverdichtung & $\begin{array}{l}\text { Deon (1997, } \\
\text { S. } 47 \text { f.) }\end{array}$ \\
\hline $\begin{array}{l}\text { normierte Fachbe- } \\
\text { griffe: Fach- und allge- } \\
\text { meiner Wortschatz mit } \\
\text { bestimmten Konnotati- }\end{array}$ & $\begin{array}{l}\text { ibid.; Ehlich (1999); } \\
\text { Piemontese \& } \\
\text { Cavaliere (1997, } \\
\text { S. 226) }\end{array}$ & $\begin{array}{l}\text { unpersönliche Konstruk- } \\
\text { tionen: Passiv, „man“ im } \\
\text { Deutschen und si imper- } \\
\text { sonale im Italienischen }\end{array}$ & $\begin{array}{l}\text { Gogolin \& Lange } \\
\text { (2010, S. 48) nach } \\
\text { Reich (2008, S. 13) }\end{array}$ \\
\hline \multirow[t]{5}{*}{$\begin{array}{l}\text { Komposita, Derivate, } \\
\text { Konversionen }\end{array}$} & $\begin{array}{l}\text { Eckhardt (2008, } \\
\text { S. } 70 \mathrm{ff} .)\end{array}$ & Funktionsverbgefüge & ibid. \\
\hline & & $\begin{array}{l}\text { ausgeprägter Nominal- } \\
\text { stil }\end{array}$ & \\
\hline & & $\begin{array}{l}\text { umfangreiche Attribute: } \\
\text { Nomen oft durch adjekti- } \\
\text { vische Partizipien er- } \\
\text { gänzt }\end{array}$ & $\begin{array}{l}\text { ibid., Neugebauer \& } \\
\text { Nodari (1999, } \\
\text { S. 166) }\end{array}$ \\
\hline & & $\begin{array}{l}\text { ausgeprägtes Verbsys- } \\
\text { tem mit großer Breite an } \\
\text { Tempora und Modi }\end{array}$ & ibid. \\
\hline & & $\begin{array}{l}\text { Dekontextualisierung, } \\
\text { vorherrschend deskrip- } \\
\text { tive Texte, selten narra- } \\
\text { tiv }\end{array}$ & Favaro (2013, S.15) \\
\hline
\end{tabular}

Tabelle 1: Merkmale der Bildungssprache z.T. nach Berendes, Dragon, Weinert, Heppt \& Stanat (2013, S. 26)

\subsubsection{Vermittlung und Erwerb der Bildungssprache}

Wie Vollmer hervorhebt, sind die „Schwierigkeiten im Erwerb [...] [der Bildungssprache] nicht solche von Regelaneignung allgemein, sondern solche des Erwerbs der spezifischen Sprachnormen und Sprachgebrauchsnormen des Systems Schule“ (Vollmer 2011, S. 2). Während alltagssprachliche Fähigkeiten also sowohl in der Erst- als auch in der Zweitsprache rasch erworben werden, handelt es sich bei der Aneignung der bildungssprachlichen Strukturen um einen langen Weg, bei dem es darum geht, möglichst viel und möglichst aktiv mit den bildungssprachlichen Mitteln zu arbeiten, also zu üben, um sich das oder die Register anzueignen (Guerriero 2014, S. 164): 
Praticare una pluralità di forme testuali mette utilmente in gioco la variabilità delle costruzioni testuali (lessico, organizzazione tematica, strategie comunicative) e serve a far sperimentare quanto il contesto pragmatico governi le scelte di chi scrive. In sintesi, lo "scrivere per comunicare", la pratica di differenti forme testuali contribuisce a sviluppare il controllo di un ampio spazio linguistico (De Mauro, 1980).

(M.Ü.: Eine Vielzahl von Textformen zu praktizieren, bringt auf nützliche Weise die Veränderbarkeit der Textkonstruktionen ins Spiel (Wortschatz, thematische Organisation, kommunikative Strategien) und dient dazu, die Schüler experimentieren zu lassen, wie sehr der pragmatische Kontext die Wahlmöglichkeiten des Schreibenden regiert. Zusammengefasst trägt das „Schreiben zum Kommunizieren“, die Praxis verschiedener Textformen zur Entwicklung der Kontrolle des sprachlichen Raumes (De Mauro 1980) ${ }^{11}$ bei.)

Guerriero betont bezüglich der Aneignung der schriftsprachlichen Kompetenz noch einmal die Wichtigkeit, sich in allen vier sprachlichen Fähigkeiten, also den rezeptiven und produktiven jeweils mündlichen und schriftlichen, zu üben, da diese nur gemeinsam effektiv weiterentwickelt werden können, anstatt sich beispielsweise auf die rezeptiven Fähigkeiten wie das Leseverständnis zu beschränken und die produktiven auszublenden (Guerriero 2014, S. 167 f.; Piemontese 2014, S. 9; Ehlich 2007, S. 25; Stefinlongo 2002, S. 15 f.). Dadurch wird klar, warum es so bedeutend ist, dass sich alle Lehrkräfte für eine ausgewogene Sprachbildung einsetzen, ohne die gesamte Arbeit einzig der Sprachlehrkraft zu überlassen. Nur so kommen die Lernenden mit möglichst vielen verschiedenen Text- und Diskursformen in Berührung und vervielfacht sich ihr Angebot an Übungsmöglichkeiten.

Auch wenn inzwischen verbreitet Einigkeit herrscht, dass die in der Schule verwendeten Sprachformen für alle schwächeren Lernenden zur Hürde werden können, sind diese Probleme doch vor allem durch die sprachliche Diversität der Schülerschaft in den Blick gerückt (s. Gogolin 1994), so dass es nicht verwunderlich ist, dass die pädagogische Diskussion um effiziente sprachliche Fördermaßnahmen in den Bildungsinstitutionen sich auch mit spezifischen Unterstützungsinitiativen für die allochthonen SchülerInnen wie muttersprachlichem Unterricht befasst und das von Monolingualismus geprägte sprachliche Selbstverständnis der Schule angreift. Das folgende Unterkapitel soll die wesentlichen Aspekte dieser Diskussion grob umreißen und sie mit dem sprachlichen Bildungsauftrag in Einklang bringen.

11 Dieses weitläufig rezipierte Konzept ist in Kap. 4.2.2.3 illustriert. 


\subsection{Die Monolingualismus-Kontroverse}

In ihrer Habilitationsschrift Der monolinguale Habitus der multilingualen Schule beschreibt Gogolin (1994), wie es an deutschen Schulen gängige Praxis war (und oft immer noch ist), das Verfügen über bildungsrelevante Fähigkeiten im Deutschen als quasi natürliche Ausstattung von SchülerInnen vorauszusetzen, anstatt auf die tatsächlich vorhandenen sprachlichen Fähigkeiten einzugehen und bildungsrelevante Fähigkeiten aufzubauen - egal in welcher Varietät des Deutschen oder anderer Sprachen sie entwickelt sind. Dieses Vorgehen fußt darauf, dass ,[d]ie Konzeptualisierung des Menschen als eines grundlegend einsprachigen [...] zutiefst in der westlichen Sprachauffassung verankert und offensichtlich kaum infrage zu stellen“" (Ehlich 2007, S. 26) ist, obwohl weltweit mehrsprachige Menschen in mehrsprachigen Nationen die Regel, nicht die Ausnahme sind (Brisk 2005, S.12). In den ca. 200 Staaten der Welt werden ungefähr 7.100 Sprachen gesprochen (Lewis, Simons \& Fennig 2016), wobei der statistisch häufigste Fall „Gesellschaften mit einer arbeitsteiligen Mehrsprachigkeit [sind], bei der auf den verschiedenen Registerebenen unterschiedliche Sprachen genutzt werden“ (Maas 2008, S. 23; Hervorhebung im Original).

Auf der apenninischen Halbinsel hat De Mauro ebenfalls schon in den 1970er Jahren den schulischen Monolingualismus moniert und seine historische Verwurzelung in den verschiedensten Kulturen verdeutlicht (De Mauro 1975a in ead. 1979, S. 125 f.). So zeigt er auf, wie etwa bereits in der Antike in China, Rom und Griechenland sowie im mittelalterlichen Europa überall Schüler dazu angehalten wurden, ein auf dem Schriftsprachlichen basierendes Modell einer ausgewählten Sprachform (literarisch-gehoben) zu erwerben, das aufs Engste mit Kultur, Religion, Nation und der herrschenden Klasse oder Kaste verbunden war. Der Mitbegründer der modernen italienischen Sprachwissenschaft, der später selbst das Amt des Bildungsministers bekleidete (Legislaturperiode 2000-2001), bezeichnet die schulische Sprachbildung, welche aus diesen historisch-kulturellen Wurzeln hervorgeht, als unilateralen „Drill zum Monolingualismus“, aus dem sich unsere Kulturen nur schwer befreien können, weil sie so sehr daran gewöhnt sind. ${ }^{12}$ Diese monolinguale Sprachbildung stellt für ihn mit Rückgriff auf Bourdieu und Passeron eine ,,pädagogische Gewalt" dar (ibid., S. 126), weil sie die Fähigkeiten derjenigen verfeinere, die mit einem bildungsnahen Kapital in die Schule kommen, während sie die bildungsferneren Schichten unweigerlich ausschließe (ibid.):

Il fenomeno è troppo esteso nel tempo e nello spazio, attraversa troppe epoche e troppe formazioni sociali per ridursi a frutto d'una scelta insipiente di singoli gruppi, i "padroni", la

12 Für eine Schilderung der gesellschaftlichen und (bildungs)politischen Umstände in Italien, die vermutlich zu dem im wissenschaftlichen Diskurs über die schulische Sprachbildung vorherrschenden polemischen Tenor geführt haben, vgl. die Ausführungen in Kap. 3.2.1. 
"borghesia", come qualcuno ha favoleggiato. Non basta condannarlo o proclamarne a parole il rifiuto per vederlo svanire. L'educazione linguistica ridotta ad addestramento monolinguistico ha radici profonde, e va considerata con grande impegno se vogliamo orientare diversamente l'educazione linguistica nella scuola.

(M.Ü.: Das Phänomen ist zu sehr in Zeit und Raum ausgebreitet, durch zu viele Epochen und zu viele soziale Formationen, um sich darauf zu reduzieren, einer dummen Wahl einzelner Gruppen zu entspringen, den „Besitzern“, der „Bourgeoisie“, wie jemand gefabelt hat. Es reicht nicht aus, es zu verdammen oder mit Worten die Ablehnung auszudrücken, um es verschwinden zu sehen. Die auf monolinguistischen Drill reduzierte Sprachbildung hat tiefe Wurzeln und muss mit großem Einsatz berücksichtigt werden, wenn wir die Sprachbildung in der Schule anders orientieren wollen.)

Die Selektionsfunktion der Schule nehme u.a. dadurch Gestalt an, dass die traditionelle Schulpolitik in der Absicht erfolge, nur einen kleinen Teil der Gesellschaft zu literalisieren, nämlich nur den, der für das (insbesondere ökonomische) Wohlergehen des Staates unabdingbar ist. Hierbei betont er, dass dieser Bildungsauftrag nicht wie in anderen Ländern vom Bürgertum bzw. der Mittelschicht ausgeführt wird, um die bildungsferneren Schichten zu beschulen und ihre Lesegewohnheiten zu stärken. Stattdessen bestehe dieser Teil der Gesellschaft in Italien auf der einen Seite selbst aus weniger gebildeten Personen und Analphabeten und auf der anderen Seite aus solchen, die versucht haben, die soziopolitische und wirtschaftliche Lenkung des Staates Ihresgleichen zu überlassen (De Mauro 1976a in ead. 1979, S. 227):

Abbiamo avuto un simulacro di borghesia [...] che non ha cercato l'egemonia fondata sulla costruzione d'una cultura scientifica capace di garantire e promuovere il consenso, ma ha cercato di tenersi stretto il potere e prepotere attraverso l'attuazione di un simulacro di politica scolastica. Verrebbe fatto di dire: in Italia non c'è stata, ancora stenta ad esserci una politica scolastica. Ma sarebbe sbagliato. C'è stata, c'è: è la politica scolastica volta a tenere il regime culturale di massa al livello di giri più basso possibile compatibilmente con le esigenze minime dello sviluppo produttivo.

(M.Ü.: Wir haben ein Götzenbild einer Bourgeoisie gehabt, [...], die keine Hegemonie gesucht hat, die sich auf die Konstruktion einer zur Garantie und Förderung des Konsenses fähigen wissenschaftlichen Kultur gründet, sondern die versucht hat, die Macht und Übermacht durch die Verwirklichung eines Götzenbildes von Schulpolitik fest zu umklammern. Es käme einem in den Sinn zu sagen: In Italien hat es keine Schulpolitik gegeben und macht sie immer noch einen kümmerlichen Versuch. Aber das wäre falsch. Es gab sie, es gibt sie: Es ist die Schulpolitik, welche darauf ausgerichtet ist, das kulturelle Massen-Regime auf dem Pegel der niedrigstmöglichen Umdrehungen zu halten, die mit den minimalen Anforderungen der Produktionsentwicklung kompatibel sind.)

Diesem Kontext entspringt auch sein negatives Urteil an anderer Stelle (De Mauro 1975b), das sich konkreter auf die schulische Sprachbildung bezieht. Hier wird eine verbreitete traditionelle Vorgehensweise beschrieben, nach der Lehrkräfte bei der Sprachbildung davon ausgingen, dass die Lernenden zu Beginn der Schullaufbahn bereits Aussprache, Wortschatz und Syntax des Italienischen beherrschten. Diese Lehrkräfte ignorierten somit die sprachliche Rea- 
lität breiter Bevölkerungsschichten Italiens und hätten geradezu eine eingeimpfte Phobie vor allem Sprachlichen, das ,vom italienischen Standard bürokratisch-politischen Schlages divergiert" (ibid. in ead. 1979, S. 215 f.; m.Ü.). Das resultiere aus ihrer schulischen und universitären Ausbildung, welche sie nicht zu einem anderen Blick auf die sprachliche Realität befähige. So hätten sie teils die besten Absichten, doch passten sich unbewusst der Funktion von Ausführenden des politischen Projekts an, die soziokulturelle Gliederung des Staates aufrechtzuerhalten (De Mauro 1975c in ead. 1979, S. 184). Ihr Verhalten habe zur Folge, dass nur diejenigen Lernenden gefördert werden, die bereits über solide Fähigkeiten in der Standardsprache verfügen, während es die bildungsferneren an den Rand dränge. Auf diese Weise werde bewusst ,la costruzione di un esercito di riserva poco o non qualificato, carne da cannone dello sfruttamento capitalistico e di ciò che Togliatti individuò come 'l'organizzazione reazionaria del consenso di massa' (Opere, v. III, p. 584). Il capolavoro del fascismo, e non del fascismo soltanto" (De Mauro 1975b in ead. 1979, S. 215 f.; m.Ü.: die Bildung eines nicht oder wenig qualifizierten Reserveheeres, Kanonenfutter der kapitalistischen Ausbeutung und dessen, was Togliatti [1973] als „reaktionäre Organisation des Massenkonsenses“ (Opere, Bd. III, S. 584) ausgemacht hat. Das Meisterstück des Faschismus und nicht nur des Faschismus; vorangetrieben).

De Mauro hat diese Auffassung zu einer Zeit vertreten, als die wirtschaftliche Lage in Italien stabiler war als heute und als Arbeiten, für die keine schulische und berufsvorbereitende Ausbildung nötig war, wie die der Erntehelfer, Küchenhilfen oder Handwerkslehrlinge noch größtenteils von Italienern verübt wurden. Heute werden diese Tätigkeiten immer mehr von Einwanderern verrichtet. In Analogie zur dargestellten selektionsfördernden Sprachbildungspolitik und Unterrichtspraxis kann die These aufgestellt werden, dass Schülerinnen und Schülern nun die Rolle derjenigen zugedacht wird, für die das Verfügen über sprachliche Fähigkeiten, die eine weitreichende gesellschaftliche Partizipation und die Übernahme von Leitungsfunktionen ermöglichen, nicht vorgesehen ist.

Im Falle der Zugewanderten eröffnet die Zwei- oder Mehrsprachigkeit, die sie durch ihre Lebenslage in den Bildungsprozess mitbringen, ein zusätzliches Argumentationsreservoir. Dieses speist sich aus einem Common Sense, also weitverbreiteten Ansichten, über Zweisprachigkeit, nach denen die Förderung der Sprache der Familie, wenn sich diese von der Schulsprache unterscheidet, die Sprachentwicklung in der Schulsprache behindere. Verbreitet ist die Vorstellung, dass die Sprachen sich den im Gehirn für Sprachwissen zugedachten Speicher sowie die Zeit, in der die Lernenden mit den jeweiligen Sprachen Kontakt haben, teilen müssen (Cummins 1981, S. 27 f.). So komme es zum Phänomen der ,doppelten Halbsprachigkeit“, nach dem Zweisprachige in keiner ihrer Sprachen über solide Fähigkeiten verfügen. 
Dieser im Alltagsverständnis fest verwurzelten, auch im pädagogischen Kontext verbreiteten Auffassung stehen zahlreiche Forschungsergebnisse entgegen, die sich mit unterschiedlichen Details des Konzepts befassen (Gogolin \& Neumann 2009). So ergeben Untersuchungen zur Frage, ob die Förderung der Zwei- oder Mehrsprachigkeit sich messbar negativ auf die Aneignung von Fähigkeiten in der Schul- und Unterrichtssprache auswirke, keine Bestätigung der Annahme. In Analysen bilingualer Unterrichtsmodelle zeigte sich z.B., dass die untersuchten Schülerinnen und Schüler keine geringeren Kompetenzen in der Schul- und Unterrichtssprache erreichten als einsprachig unterrichtete Lernende. Zugleich aber hatten die zweisprachig Unterrichteten sich in derselben Unterrichtszeit Kompetenzen in beiden Sprachen angeeignet und mithin einen Gewinn davongetragen (Söhn 2005; Gantefort 2013).

Für meine Untersuchung sind diese Erkenntnisse relevant, weil in den fünf beobachteten Klassen sowohl mehrsprachige als auch einsprachig italienische Schülerinnen und Schüler vertreten sind, deren alltagssprachliches Repertoire sich stark von der im Unterricht und in den Lehrmitteln verwendeten Sprache unterscheidet. Die hieraus resultierenden Verständnis- sowie generellen Schwierigkeiten bei der Aneignung der sprachlichen Kompetenzen, welche ihnen der Schulerfolg abverlangt, manifestierten sie während der Erhebung in der Rezeption und in der Produktion der Schul- und Unterrichtssprache. Daraus ergibt sich, dass die schulische Sprachbildung immer noch kein Garant für den Sprachausbau aller Lernenden ist. Eine Offenlegung ihrer historischen Entwicklung und der mit den Sprachbildungspraktiken möglicherweise verfolgten versteckten Absichten - so wie sie von De Mauro und anderen seit 50 Jahren vorangetrieben wird - kann zur Bewusstmachung der Divergenzen zwischen sprachbezogenen Fähigkeiten auf der einen Seite und den sprachlichen Herausforderungen und ihrer schulischen Vermittlung auf der anderen Seite führen und damit einen wichtigen Schritt in Richtung einer veränderten Praxis darstellen.

\subsection{Sprache im Unterricht}

Aufgrund der stark ausgeprägten Differenzen zwischen den sprachlichen Registern der Schule und dem alltäglichen Sprachgebrauch vieler Lernender kann nicht davon ausgegangen werden, dass Letztere den erforderlichen Sprachausbau von selbst und sozusagen nebenbei im herkömmlichen Fachunterricht vollziehen. Stattdessen haben die Lehrpersonen eine Möglichkeit, den Prozess maßgeblich zu unterstützen, indem sie sprachliches Lernen wirksam mit fachlichem Lernen verbinden und Ersteres zu einer Konstante ihrer Unterrichtsgestaltung machen (Gibbons 2002, S. 5 f.). Denn sprachliches Lernen funktioniert dann besonders gut, wenn es an authentische Bedeutungsaushandlungen 
und fachliches Lernen gebunden ist (ibid., S. 12). Ein weiterer wichtiger Faktor im Lernprozess ist die Eigenaktivität des oder der Lernenden, wodurch das Lernen vor allem dann erfolgreich ist, wenn Aufgaben in Angriff genommen werden, die alleine noch nicht gelöst werden können, sondern mit der Hilfe einer anderen Person bewältigt werden, bevor der/die Lernende dies zu einem späteren Zeitpunkt alleine schafft. Diese Form der Unterstützung wird in den nächsten Unterkapiteln genauer dargestellt, worauf ein Beispiel sprachlicher Merkmale der schriftlichen Texte folgt, bei deren Aneignung die Lehrkräfte behilflich sein sollten. Im Anschluss daran wird auf die Bedeutung einer wirksamen Lese- und Schreibdidaktik sowie sprachbezogenen Bewertungskultur eingegangen.

\subsection{1 scaffolding}

Eine gezielte Förderung des schriftkulturellen Sprachausbaus und die dazu nötige, nicht weniger komplexe und nur durch eine solide sprachwissenschaftliche Ausbildung zu erwerbende Fähigkeit, den Sprachstand der einzelnen Lernenden möglichst genau bestimmen zu können, stellen die Lehrpersonen vor eine enorm facettenreiche Herausforderung. Gerade Fachlehrkräfte fühlen sich für diese Aufgabe eher nicht zuständig und auch nicht kompetent genug (Duff 2005, S. 49; Atzeni \& Milia 2008, S. 288; Harren 2015, S. 128). Es handelt sich dabei aber um ein umfangreiches Projekt, das kontinuierliche Investitionen erfordert (Piemontese 2014, S. 9) und eine größere Aufsicht auf Erfolg hat, wenn sich alle Lehrpersonen daran beteiligen.

Während viele Fachlehrkräfte ein intuitives Gespür dafür haben, dass die Vermittlung des Fachwortschatzes für den Lernerfolg in ihren Fächern bedeutsam ist, überlassen sie die übrigen Aspekte der Sprachbildung in der Regel den Sprachlehrkräften (Gogolin 2009a, S. 276; Christie 2012, S. 10 f.). ${ }^{13}$ Das ist im Übrigen nicht verwunderlich, wenn man bedenkt, dass beispielsweise in den Lehrplänen in Italien zwar seit langem Spracherziehung von den Lehrkräften aller Schulfächer gefordert, die dazu nötige Ausbildung aber nicht erteilt wird. So stellt auch Nodari für den schweizerischen Schulkontext fest (Nodari 2010, S. 279):

13 S.a. Christie (2012), die in der fehlenden Konzentration der Fachlehrkräfte auf Sprachbildung einen der Gründe für die beobachteten Schwierigkeiten vieler Jugendlicher mit dem Sprachausbau und der Literalität sieht (Christie 2012, S. 106): ,[... [ the changing school demands met by young people in the transition to secondary school; the nature of adolescence and the pressures felt in growing up; 'a lack of capacity, time and will for middle and high school teachers to teach literacy within the content areas'; and 'few strategies provided pupils at the end of their third grade for dealing with the rapid shift from narrative to expository text' (Carnegie Council on Advancing Adolescent Literacy, [2009/] 2010 [...], p. 8)“. 
Die Forderung nach einer Sprachförderung in allen Fächern ruft bei Fachlehrern und Fachlehrerinnen unverzüglich die Frage hervor, wie das denn gehen soll. Diese Frage ist mindestens aus drei Gründen berechtigt: Erstens haben Fachlehrer in ihrer Ausbildung keine didaktischen Anleitungen dazu bekommen, wie die Fachsprachen innerhalb des Fachunterrichts gefördert werden können. Zweitens steht in keinem Schweizer Fachlehrplan, welche Ziele in Bezug auf die Fachsprache angestrebt werden sollen. Drittens enthalten Fachlehrmittel nur selten sprachfördernde Elemente, die die Sprachförderung in den Fachlektionen unterstützen. Die Notwendigkeit einer fächerübergreifenden Sprachförderung kollidiert somit mit der schulischen Realität und Tradition - ein Problem, das allein durch Empfehlungen oder Forderungen an die Fachlehrperson nicht gelöst werden kann.

Sprachdidaktische Förderkonzepte und entsprechendes linguistisches Hintergrundwissen werden auch in Deutschland und Italien nur in einigen wenigen Einzelfällen (z.B. Wiese \& Wiese 1998) innerhalb der Fachdidaktik vorgestellt (Ahrenholz 2010a, S. 4), sondern in der Sprachwissenschaft und Pädagogik vorangetrieben (s. z.B. Ahrenholz 2010b; Röhner \& Hövelbrinks 2013).

In der italienischen Sekundarstufe I besteht zwar zumindest für die Fächer Erdkunde und Geschichte der Vorteil, dass sie in der Regel von der Italienischlehrkraft erteilt werden, die über ein gewisses Maß an sprachwissenschaftlicher Ausbildung verfügt, doch auch diese benötigt für einen effizienten sprachförderlichen Unterricht mehr als ein fundiertes Grundlagenwissen. Ein konkretes Werkzeug für eine Vermittlung der Lehrinhalte, die ihre sprachliche Gestaltung angemessen berücksichtigt, ist das australische Konzept des scaffolding. Es ist in den letzten Jahren zunehmend auch in Europa rezipiert worden, so dass inzwischen erste Studien zum hiesigen Einsatz im Fachunterricht vorliegen (z.B. Quehl 2009).

Scaffolding bezeichnet eine Praxis, die offensichtlich im Unterricht als zentral erachtet wird. ${ }^{14}$ Im Zuge ihres intensiven Beforschens dieses Ansatzes sind Gibbons und Hammond auch der Frage nachgegangen, was die Praktiken des scaffolding sowohl erzieherisch als auch sprachwissenschaftlich konkret ausmacht und wie sie genauer erfasst werden können (Hammond \& Gibbons 2005, S. 6-8). Von Vygotskij selbst ist der Begriff zwar nicht verwendet worden, aber er steht für einen wesentlichen Bestandteil seiner Lerntheorie. Hiernach erfolgt das Lernen kollaborativ und interaktional (ibid., S. 8); das Unterrichten ist nicht nur Wissensvermittlung von einer Person an die andere, sondern ,a collaborative and negotiated social process, whereby knowledge is constructed between, rather than within, individuals" (Hammond \& Gibbons

14 Eingeführt worden ist die scaffolding-Metapher zur Beschreibung der Eltern-Kind-Kommunikation von Wood, Bruner und Ross (1976 zit. in Gibbons 2002, S. 10). Bruner beschreibt Eltern als expert scaffolders, von denen Lehrpersonen viel lernen können (MacLure 1999, S. 203 f.; s.a. Maas 2008, S. 192). Hierbei ist es wichtig zu betonen, dass Familien- und Gemeinschafts-Kontexte in der Sprachsozialisation oft als der prototype of effective learning environments (Cazden 1999, S. 64) angesehen werden, aber auch Eltern ihr Kind keineswegs nur implizit sprachförderlich beeinflussen, sondern dass in vielen Kulturen beobachtet wurde, wie Kinder z.B. ausdrücklich dazu aufgefordert werden, ein bestimmtes Wort oder einen Satz zu produzieren (ibid.). 
2005 , S. 9). Was wann gelernt und gelehrt werden kann, ist durch die menschliche Entwicklung vorgegeben, wobei eine Steuerung des Lernprozesses diesen optimieren kann. Besonders effektiv ist laut Vygotskij (Wygotski 1977 (1934)) solches Lernen, das innerhalb der „Zone der nächsten Entwicklung“ stattfindet. Das bedeutet, dass für die Bewerkstelligung einer Aufgabe die bisher erworbenen Fähigkeiten nicht ausreichen und die oder der Lernende auf Hilfe angewiesen ist. Von scaffolding kann dann gesprochen werden, wenn diese Hilfe so bereitgestellt wird, dass die Lernenden zu einem späteren Zeitpunkt in der Lage sind, die Aufgabe selbstständig zu bewerkstelligen (ibid.; Lantolf 2005, S. 338).

Aus diesem Grund plädieren Gibbons und andere dafür, dass die in der Schule gestellten Aufgaben zu komplex sein sollten, um von den Schülern allein gelöst werden zu können, aber innerhalb des Rahmens ihrer Möglichkeiten, sofern ihnen durch entsprechende Hilfestellung ein Gerüst geboten wird, das sie schützt und gleichzeitig den Ausbau ihrer Fähigkeiten ermöglicht.

Dieser als besonders wirksam betrachteten Lehr- und Lernmethode (Hammond \& Gibbons 2005, S. 8) liegt außerdem zugrunde, dass sie den Schülerinnen und Schülern zum einen eine Anwendung des Gelernten auf neue Kontexte ermöglicht, wodurch sie in ihrem Lernprozess nach und nach autonomer werden; zum anderen wird so gerade Lernenden, die Schwierigkeiten mit der Unterrichtssprache zeigen, geholfen (ibid.):

This emphasis on students' learning potential, and not simply on their current abilities, and the consequent raising of expectations of what is possible, seemed to us to be especially significant for students learning through the medium of their second language, where cognitive and conceptual understanding my outstrip English language development or, conversely, where abilities in English may constrain subject-specific learning.

Gibbons und Hammond stützen sich wie andere (z.B. van Lier 1996; Wells 1999) auf Ergebnisse verschiedener Studien, die eine wesentliche Voraussetzung für effektives Unterrichten in den Fähigkeiten der Lehrperson sehen, Unterrichtseinheiten sorgfältig zu planen und dabei aufgrund der divergierenden Leistungsprofile der Lernenden binnendifferenzierte Aufgaben zusammenzustellen. Auch ihre Eignung dafür, das Potential des lehrbaren Moments ganz und gar auszuschöpfen, spielt eine wesentliche Rolle. Das bedeutet, dass scaffolding auf einer Makro- und einer Mikroebene erfolgt. Auch wenn diese beiden zusammen ein Netzwerk bilden, ist ihre Unterscheidung durchaus sinnvoll, da es nicht so sehr um Aspekte der zwei Ebenen im Einzelnen geht, sondern um ihr Zusammenwirken, welches dieses System so effektiv macht (Hammond \& Gibbons 2005, S. 10 f.). ${ }^{15}$

15 Zuengler und Cole (2005, S. 312) sowie Pennycook (2001) verweisen zudem auf die generelle Nützlichkeit der Unterscheidung von Makro- und Mikroebene im gesamten Forschungsfeld der angewandten Linguistik. 
Bei der Analyse von Formen des scaffolding in verschiedenen Klassen und Fächern teilen die beiden Wissenschaftlerinnen van Liers Ansicht, dass die nützlichste pädagogische Theorie eine „reflexive Dimension der Praxis“ ist (van Lier 1994, S. 338 zit. in Hammond \& Gibbons 2005, S. 11; m.Ü.). Deshalb entstammen alle Kategorien ihres scaffolding-Modells der Auswertung von Unterrichtsdaten, und fußt ihr Ansatz durch den Einbezug der Lehrkräfte auf der Forschungsmethode der action research.

\subsubsection{Makro-scaffolding}

Für die Vorbereitung des Unterrichts sind auf Makroebene sieben Punkte relevant, deren Beachtung eine gute Unterrichtsplanung generell ausmachen sollte, die zum Zweck einer unterstützenden Förderung des Sprachausbaus allerdings in ihrer Totalität zu berücksichtigen sind, zumal sie sich gegenseitig bedingen (Hammond \& Gibbons 2005, S. 20):

- Kenntnisse und Erfahrungen, über die die Schülerinnen und Schüler schon verfügen;

- Auswahl der Aufgaben;

- Reihenfolge der Aufgaben;

- Zusammensetzung der Adressaten;

- semiotische Systeme;

- vermittelnde Texte (mediational texts);

- metasprachliches und metakognitives Bewusstsein.

Abbildung 1: Designed-in scaffolding nach Hammond und Gibbons (2005, S. 13)

Die Ermittlung des Wissensstandes der Lernenden bei der Einführung in ein neues Thema ist keineswegs eine innovative Lehrdisposition, beim scaffolding jedoch geschieht sie explizit (ibid., S. 14). Wichtig ist dabei immer der Blick auf den sprachlichen Raum, den die Lernenden aktuell beherrschen, und eine Einschätzung der zur Erarbeitung des neuen Lehrinhalts benötigten sprachlichen Mittel und Fähigkeiten. Dazu gehört bei mehrsprachigen Lernenden wenn möglich auch, die Fähigkeiten in der Erstsprache zu ermitteln und auf diese aufzubauen (u.a. Roth 2002; Szlabewski-Çavus 2002). Falls die Lernenden schon über Wissen verfügen, das sie alltagssprachlich mitteilen können, geht es darum, sie bei einer bildungssprachlicheren Artikulierung dieses Wissens zu unterstützen. Ein weiterer wesentlicher Punkt besteht darin, die Lernziele nicht nur vorher festzulegen, sondern die SchülerInnen darüber auch offen in Kenntnis zu setzen. Wong-Fillmore (1985) unterstreicht, dass dies gerade für in der Zweitsprache Lernende besonders wichtig ist, da es ihnen eine hilfreiche Orientierung beim Lernen neuer Inhalte und ihrer Versprachlichung 
ermöglicht, zumal sie mit den sprachlichen und kulturellen Praktiken der Institution Schule weniger vertraut sein können (Hammond \& Gibbons 2005, S. 14 f.).

Bezüglich der Sequenzierung der Aufgaben betonen die Autorinnen, dass ihre Bedeutung vor allem aus der Bewerkstelligung einer Aufgabe als Grundlage für die Ausführung der nächsten erwächst (ibid., S. 15):

Our analysis indicated that it was the relationship between sequential tasks, rather than choice of any one task, that was especially important in supporting the ESL [English as a Second Language] learners and in providing the context in which scaffolding occurred. The sequencing of the tasks thus became a larger activity, which took on significance beyond the learning outcomes of each individual task.

Ferner ermöglicht Variation bei der Zusammensetzung der Adressaten eine Binnendifferenzierung des Unterrichts mit unterschiedlichen Formen des scaffolding (die jedoch auf dasselbe Ziel orientiert sind) und gibt der Lehrperson die Gelegenheit, ihre Lernenden dazu zu bewegen, sich mit zunehmend komplexeren und anspruchsvolleren Aufgaben zu befassen. So kann sie beispielsweise zunächst im Klassenverband Hilfestellungen für die Bewerkstelligung einer Aufgabe an alle richten und während einer darauffolgenden Gruppenarbeitsphase die Unterstützung je nach den Schwierigkeiten den einzelnen Gruppen anpassen. Bestimmte Zusammensetzungen der Arbeitsgruppen im richtigen Moment führen außerdem zu einem selbstständigeren Arbeiten der Lernenden (ibid., S. 16).

Hammond und Gibbons haben während ihrer Unterrichtsbeobachtungen festgestellt, dass die Lehrkräfte sich für die Vermittlung von neuen Inhalten auf zusätzliche semiotische Systeme stützen, die für eine Reichhaltigkeit an Informationsträgern sorgen. Hiermit sind auf der einen Seite unterschiedliche Formen des Sprachgebrauchs gemeint (mündlich, schriftlich, von einem eher alltagssprachlichen Register bis zur Bildungs- und Fachsprache), aber auch weitere semiotische Unterstützung durch Wandplakate über Fachinhalte oder Tipps und Regeln für ihre Bearbeitung, Photos, Bilder, Diagramme, Graphen und mathematische Formeln. Darüber hinaus sorgten die Lehrpersonen für eine Kombination aus visueller und auditiver Unterstützung, indem sie beispielsweise Videos präsentierten oder mit dem Internet arbeiten ließen. Auch die taktile Komponente fand Einbezug, wenn die Lernenden in Aktivitäten involviert wurden, in denen sie Ergebnisse präsentieren oder Versuche durchführen sollten. Und schließlich wurden auch Momente körperlicher Bewegung und der Gebrauch von Gesten beobachtet.

Eine solche Reichhaltigkeit an semiotischen Botschaften erleichtert nicht nur ein besseres Verständnis der sprachlichen Strukturen, sondern beinhaltet auch Methoden wie das farbige Untermalen verschiedener Informationstypen in einem Geographietext. Das soll den Lernenden bei der Hypothesenbildung bezüglich des zu erwartenden Inhalts helfen. Eine weitere Taktik ist das vor- 
herige Besprechen von relevanten Ausdrücken eines Textes, die voraussichtlich nicht allen Schülerinnen und Schülern bekannt sind. Ihre Fixierung beispielsweise an der Tafel in Form eines semantischen Netzes, eines Wortfeldes, bevor mit der Bearbeitung eines neuen Textes begonnen wird, fördert das Textverständnis der Lernenden.

Für diese semiotische Praxis wurde der Term message abundancy geprägt (Gibbons 2003). Unterstützende graphische Informationsträger werden oft als Verständnis fördernder „Hintergrund“ für die sprachliche Aufmachung betrachtet, doch Gibbons und Hammond berichten, in verschiedenen Unterrichtsmomenten Verfahren beobachtet $\mathrm{zu}$ haben, die besser als eine gerade für ZweitsprachlernerInnen entscheidende ,symbiotic relationship between language and other modes of meaning, with the meaning potential being more explicitely foregrounded and integrated with the discourse" (Hammond \& Gibbons 2005, S. 17) angesehen werden können.

Mit dem vorletzten Punkt, mediational texts and artefacts, sind Unterrichtsaktivitäten und Texte angesprochen, über die Lernstrategien und -inhalte erworben werden. Die SchülerInnen beschäftigen sich eingehend und/oder periodisch innerhalb der Unterrichtseinheit mit ihnen, wobei sie eine Art Anker für den Erwerb neuen Wissens bilden. Das kann ein Arbeitsblatt zu den Vorgehensweisen beim Lösen bestimmter mathematischer Aufgaben sein, ein Physikexperiment zur Vermittlung fachlicher Konzepte oder eine Kurzgeschichte im Sprachunterricht, um sich in der literarischen Textanalyse, der Interpretation verschiedener Charaktere und Themen oder der Ermittlung der Erzählperspektive zu üben (ibid., S. 18).

Für die Durchführung sprachförderlichen Unterrichts erachten die Autorinnen eine solide sprachwissenschaftliche Ausbildung als fundamental, welche ihrer Auffassung nach an einer systemisch-funktionalen Linguistik (vor allem mit Blick auf die Notionen Genre und Register) ausgerichtet sein sollte (s.u.). Diese Sprachtheorie helfe den Lehrkräften sowohl bei der Ermittlung des Sprachstandes ihrer Lernenden als auch bei der Berücksichtigung sprachlicher Anforderungen der Unterrichtsmaterialien. Die folgenden im Unterricht beobachteten Beispiele zeigen, wie Lehrkräfte hierbei ihr sprachtheoretisches Wissen anwenden (ibid.):

$[R]$ eviewing previously learned work and building on those reviews to introduce new concepts and language; grounding the introduction of complex or abstract concepts by working systematically between concrete and more abstract linguistic realizations in classroom discourse; talking with students about appropriate language use in the context of curriculum learning; and building in opportunities for students to articulate what they had learned.

Dazu gehört, dass Sprache auch im Fachunterricht gelegentlich, aber kontinuierlich explizit gelehrt wird, was von einzelnen Wörtern und grammatischen Strukturen bis hin zu globaleren Textmerkmalen reichen sollte. Das dazugehörige Thematisieren der Sprache ist an sich sprachförderlich und hilft außerdem, eine Metasprache aufzubauen. So wird die Aufmerksamkeit der Lernenden auf 
die Rolle der Sprache im Lernprozess gelenkt, und gleichzeitig erfolgt eine Stärkung ihrer metasprachlichen Fähigkeiten. Diese gehen neben ihrer Relevanz im Sprachausbau auch mit einer metakognitiven Bewusstheit einher, denn Unterrichtsgespräche über die Absichten einer Lerneinheit entstanden oft im Zuge der Behandlung ihrer sprachlichen Gestaltung (ibid., S. 19):

Students who had participated in these programs were able to talk about their own learning processes in ways that were insightful and that enabled them to adjust their own behavior if necessary as they interacted with their peers and teachers.

\subsubsection{Mikro-scaffolding}

Die Differenzierung von Makro- und Mikroebene ist durch zwei unterschiedliche Arten des scaffolding gekennzeichnet. Während die bisher vorgestellten Aspekte des unterstützenden Unterrichts im Vorfeld geplant werden können, ergeben sich Interventionen auf der Mikroebene im Unterrichtsgespräch und müssen daher spontan von der Lehrkraft vorgenommen werden. Hier treten die Methoden des scaffolding deutlicher in Erscheinung; allerdings bildet das Wirken auf der Makroebene den Rahmen, in dem unterstützende Eingriffe auf der Mikroebene erst ihre volle Wirksamkeit entfalten können (ibid., S. 20). Abbildung 2 zeigt die einzelnen Handlungsweisen, die für das Mikro-scaffolding von Bedeutung sind:

- linking to prior experience, pointing forward;

- recapping/ meta comment;

- appropriating;

- recasting;

- cued elicitation;

- increasing prospectiveness;

- stop;

- chose over.

Abbildung 2: Interaktionales scaffolding nach Hammond und Gibbons (2005, S. 21)

Nicht nur auf Makroebene, sondern auch bei der Interaktion im Unterricht ist die Bezugnahme auf das von den Lernenden bereits erworbene Wissen und ihre Erfahrungen bedeutend. In diesem Zusammenhang wurde beobachtet, wie Lehrpersonen Bezüge zu außerschulischen Erfahrungen der Lernenden (Freizeitgestaltung und Zuhause) herstellen und an Lehr- und Lernaktivitäten vorausgehender Unterrichtsstunden erinnern, um die gerade verhandelten neuen Inhalte zu verankern. Von ähnlicher Relevanz ist auch der Verweis auf zukünftige Lerninhalte bzw. weitreichendere Referenzrahmen der Unterrichtsplanung, damit die SchülerInnen über Wert und Nutzen der Aufgaben besser informiert sind und ihre Lernmotivation gesteigert wird (Hammond \& Gibbons 2005, S. 21). Auch verschiedene andere WissenschaftlerInnen haben bezüglich 
des Lernerfolgs generell und den Sprachausbau insbesondere unterstrichen, dass den Schülerinnen und Schülern der Nutzen der Aufgaben deutlich gemacht werden muss, damit mehr Motivation geweckt und dadurch bessere Aussichten auf einen Lernerfolg geschaffen werden können (z.B. Peyer 2011, S. 102; Weber 1973, S. 94 bzgl. sprachlicher Aspekte).

Mit diesem ersten Punkt der Liste in Abbildung 2 ist der zweite verknüpft, der im Vorgehen der Lehrkräfte besteht, regelmäßig gegen Ende der Unterrichtseinheit die wesentlichen Erkenntnisse der erfolgten Interaktion zusammenzufassen. Dadurch erfahren die Lernenden eine Unterstützung beim Herausfiltern der Hauptlerninhalte der jeweiligen Unterrichtsstunden. Derartige Zusammenfassungen beziehen sich auf die drei Bereiche fachlicher Inhalt, sprachliches und metakognitives Wissen, wobei mit letzterem Erkenntnisse darüber gemeint sind, was den Lernprozess vorangetrieben hat (Hammond \& Gibbons 2005, S. 21).

Mit dem dritten Punkt der Appropriation und Neuformulierung von Schüleraussagen durch die Lehrkraft ist die sprach- und lernförderliche Maßnahme angesprochen, das Klassengespräch in die von den Lehrkräften gewünschte Richtung zu lenken und dabei aber die Lernenden aktiv in die Gestaltung und Entwicklung des Gesprächs einzubeziehen. Auch hier gilt wieder, dass diese Strategie erst dann ihre volle Wirkung entfalten kann, wenn die Interaktion regelmäßig von den Äußerungen der Lernenden ausgehend erfolgt und die Kontrolle der Lernenden über das Gespräch konstant erweitert wird (ibid., S. 23).

Mit einem getimten Elizitieren von präziseren und/oder umfangreicheren Schülerantworten, die sich in der verbreiteten Triade Lehrerfrage-Schülerantwort-Lehrerkommentar mit dem dritten Zug einstellen, wird den Lernenden Gelegenheit geboten, mehr zu sagen und explizit über ihre Beiträge und ihr Verständnis der verhandelten Inhalte zu reflektieren (ibid.). Das geschieht, wenn der dritte Zug nicht einfach in einem Werturteil zur Schüleräußerung besteht und damit die Interaktion beendet, sondern wenn darauf ein weiterer Beitrag der Schülerin oder des Schülers folgt. Bei derartigen Lehrerkommentaren wird also die Verantwortung für den Interaktionsfortgang an den/die Lernende(n) abgegeben und somit das Maß ihres Beitrags zur gemeinsamen Wissenskonstruktion gesteigert. Das erhöht ihre aktive Mitarbeit, die für die Effizienz des Lernprozesses und den Ausbau der sprachlichen Fähigkeiten so entscheidend ist.

Die letzten drei Punkte des interaktionalen scaffolding sind hiermit verflochten, wobei der von Wells (1996) übernommene Terminus increasing the prospectiveness illustrieren soll, wie die vorgestellte Gestaltung des dritten $\mathrm{Zu}-$ ges für eine Verlängerung des Gesprächs sorgen und zu umfangreicheren und produktiveren Bedeutungssequenzen führen kann. Dadurch wird gleichsam das Unterrichtstempo entschleunigt. 
Diese Ausführungen zum scaffolding-Ansatz sollten veranschaulicht haben, dass ihn mehr als Hilfestellungen wie das Aushändigen eines Arbeitsblattes mit Hintergrund- und erklärenden Informationen zum Unterrichtsthema ausmachen und seine Anwendung nicht bloß mit guten Unterricht gleichzusetzen ist: „In our view, scaffolding, unlike good teaching generally, is specific help that provides the intellectual 'push' to enable students to work at the outer limits of the ZPD [Zone of Proximal Development]" (Hammond \& Gibbons 2005, S. 25). Die Vorstellung dieses Ansatzes ist für die hier vorliegende Erhebung von Interesse, weil im Zuge der Unterrichtsbeobachtungen darauf geachtet wurde, ob und was für eine Art von Sprachbildung sprachwissenschaftlich ausgebildete Lehrkräfte im Fachunterricht betreiben und ob sich das sprachbezogene Lernen innerhalb der Zone der nächsten Entwicklung der Lernenden abspielt. ${ }^{16}$

Ein wesentliches Merkmal der schul- und bildungssprachlichen Register, das auch bei der Analyse der Lehrbücher, die in den fünf an der Studie teilnehmenden Klassen verwendet wurden, hervortrat, besteht in der Nominalisierung verbaler Prozesse. Aufgrund der Häufigkeit, mit der die Lernenden im Kontext Schule vor allem dort auf sie stoßen, wo das Erarbeiten von Lehrbuchtexten im Vordergrund des Unterrichtsgeschehens steht, und aufgrund der oftmals nicht implizit zu leistenden Aneignung wird es im folgenden Unterkapitel illustriert.

\subsubsection{Grammatische Metapher}

Im Spracherwerb können Momente der Regression beobachtet werden, wenn das sprachliche Repertoire erweitert wird, bevor es zu einer Verfestigung der neuen Strukturen kommt. Dies gilt insbesondere für den Erwerb skribaler Techniken (Halliday 1993). Dabei geht es nämlich nicht nur darum, ein neues Medium zu beherrschen, sondern es handelt sich um einen für die Lernenden vollkommen neuen Sprachgebrauch, der für eine andere Überlieferung des Wissens verantwortlich ist. Deshalb ist es nicht ungewöhnlich, dass Kinder, wenn sie mit dem Schreiben beginnen, eine semiotische Regression von bis zu drei Jahren aufweisen, welche sich in kurzen, schriftlichen Sätzen zeigt, die nicht miteinander verknüpft sind und oft auf die gleiche Weise beginnen, in der Regel mit der Nennung des Subjekts (ibid., S. 109 f.). Ein markantes Beispiel dafür, wie sehr schriftlicher und mündlicher Sprachgebrauch voneinander differieren, ist das, was Halliday „grammatische Metapher" nennt. Ihre Entwicklung ist in einer unterschiedlichen Perspektive auf die Realität vorgegeben (ibid., S. 111; Hervorhebung im Original): 
A written text is itself a static object (or has been until the event of computers): It is language to be processed synoptically. Hence it projects a synoptic perspective onto reality: It tells us to view experience like a text, so to speak. In this way writing changed the analogy between language and other domains of experience; it foregrounded the synoptic aspect, reality as object, rather than the dynamic aspect, reality as process, as the spoken word does. This synoptic perspective is then built into the grammar of the written language, in the form of grammatical metaphor: Processes and properties are construed as nouns, instead of verbs and adjectives. Where the spoken language says whenever an engine fails, because they can move very fast, ... happens if people smoke more, the written language writes in times of engine failure, rely on their great speed, ... is caused by increased smoking.

Diese für das Englische ermittelte Eigenschaft der Schriftsprache ist auch für die deutsche und die italienische Sprache mit ihren zahlreichen Nominalisierungen bezeichnend. Laut Halliday handelt es sich bei den beiden unterschiedlichen Möglichkeiten, dasselbe auszudrücken, nicht um synonyme Konstruktionen, da die prototypische Bedeutung eines Nomens eine Sache ist. Wenn also ein Prozess oder eine Eigenschaft als Nomen konstruiert werden, sind diese objektifiziert, worin das wesentliche Merkmal der grammatischen Metapher besteht (ibid.):

If there was no natural relationship between the semantics and the grammar, the difference between the two kinds of wording would be purely formal and ritualized; but there is such a natural relationship, and so the metaphor brings about a reconstrual of experience, in which reality comes to consist of things rather than doing and happening. ${ }^{17}$

Die grammatische Metapher wird von Halliday als letzter Schritt in einem Drei-Stufen-Modell der semiotischen Entwicklung gesehen (ibid.):

\section{(Protosprache $\rightarrow$ ) Generalisierung $\rightarrow$ Abstraktheit $\rightarrow$ Metapher}

Grammatische Generalisierung scheint hierbei der primäre Mechanismus, mit dem der Spracherwerb und -gebrauch sowie der Aufbau eines systematischen Weltwissens seinen Lauf nimmt. Die Fähigkeit zum Umgang mit grammatischer Abstraktheit ermöglicht den Beginn des schriftkulturellen Sprachausbaus und die Aneignung des schulischen Wissens in der Primarstufe. Grammatische Metaphern hingegen können erst in einem Alter von neun Jahren verarbeitet werden, wobei es etlichen Kindern auch erst zu einem späteren Zeitpunkt gelingt (Christie 2012, S. 3, S. 189). Sie sind der Schlüssel zum Lernerfolg in der Sekundarstufe und zur Aneignung von technischem und anderem

17 Der Anteil von Nomen übersteigt allerdings nicht nur in den Fachsprachen den der Verben. Dies ergibt sich automatisch in jedem sprachlichen Register (und sowohl in den schriftlichen als auch den mündlichen), da Verben i.d.R. mehrere Nomen regieren, bzw., auch wenn sie nach der Valenztheorie theoretisch nur ein- oder zweiwertig sind, oft aus pragmatisch-semantischen Gründen weitere nominale Ergänzungen an sich binden (s. Adamzik 2004, S. 153). Ihr Anteil steigt jedoch proportional zur Informationsdichte und zum Planungsgrad des (schriftlichen oder mündlichen) Textes. So wurde beispielsweise beobachtet, dass eine telefonische Unterhaltung zwischen Freunden weniger Substantive enthält als die Rede eines Politikers (Voghera 2008, S. 193). 
Fachwissen. Letzteres kann in seiner verschrifteten Form gar nicht ohne grammatische Metaphern konstruiert werden (Martin 1990 zit. in Halliday 1993, S. 111). Die grammatischen Metaphern dienten anfänglich dazu, eine Sprache für Technik und Naturwissenschaft zu entwickeln, deren Verwendung sich nach und nach auf fast jedes schriftsprachliche Register der Erwachsenenwelt ausgedehnt hat und vor allem in den institutionalisierten Registern der Staatsführung, Industrie, Finanzwelt, Wirtschaft u.a. auftritt (Halliday 1993, S. 112).

Erwachsene sind derart an diese Charakteristik der Schriftsprache gewöhnt, dass ihnen nicht mehr bewusst ist, wie sehr sie sich von der (mündlichen) Alltagssprache unterscheidet. ${ }^{18}$ Kinder bzw. Jugendliche hingegen bedürfen oft der Unterstützung, um ihr Lesen von Texten, die solche Formulierungen enthalten, nicht zu beeinträchtigen und vor allem, um selbst derartige Texte verfassen zu können. Das gewinnt gerade dann an Bedeutung, wenn das Verstehen von Texten eine zentrale Rolle einnimmt und Analysen der sprachlichen Beschaffenheit von Lehrmitteltexten die Notwendigkeit ergeben, die Lernenden durch eine sprachförderliche Lese- und Schreibpraxis bei der Herausbildung der für den Lernerfolg notwendigen Textkompetenz zu unterstützen. Das folgende Unterkapitel illustriert deshalb wesentliche Merkmale einer solchen Didaktik.

\subsubsection{Sprachförderliche Lese- und Schreibdidaktik}

\subsubsection{Lesedidaktik}

Weiter unten wird im Zuge der Analyse der in der Studie verwendeten Lehrbücher ausführlich auf die Lesbarkeit von Texten und Mittel ihrer Gestaltung, die das Verständnis erschweren können, eingegangen. ${ }^{19}$ Hier sei deshalb nur vorweggenommen, dass die Lehrbuchanalyse meiner Studie den italienischen Forschungsstand zur sprachlichen Komplexität der Lehrwerkstexte reflektiert, weshalb ein lese- und schreibdidaktisches Handlungswissen für alle Lehrkräfte von wesentlicher Bedeutung ist.

In Italien hat zunächst die beobachtete Divergenz zwischen den sprachlichen Anforderungen der Lernenden und ihren tatsächlichen Fähigkeiten sowie der im letzten Jahrzehnt festzustellende steigende Anteil von mehrsprachigen Schülerinnen und Schülern verstärkt für sprachwissenschaftlich-didaktische Vorschläge zur Erleichterung des Textverständnisses gesorgt. Diese finden

18 Vgl. Halliday (1993, S. 112; Hervorhebung im Original): „We are so familiar with wordings like prolonged exposure will result in rapid deterioration of the item (from a care label), he always credits his former big size with much of his career success (from a television magazine), that we forget how far these are from the language of daily life - or how far the language of daily life has had to evolve for these to become a part of it."

19 Siehe Kap. 7 zur Lehrbuchanalyse und das Unterkapitel 3.2 über den Forschungsstand zur sprachlichen Gestaltung der Lehrmittel. 
sich z.B. in dem von Grassi, Valentini und Bozzone Costa (2003) herausgegebenen Band L'italiano per lo studio nella scuola plurilingue: tra semplificazione e facilitazione (m.Ü.: Italienisch für das Lernen in der mehrsprachigen Schule: zwischen Vereinfachung und Erleichterung), in Lumbellis sprachwissenschaftlichen Beiträgen zum Textverständnis wie z.B. in Fenomenologia dello scrivere chiaro (1989; Phänomenologie des klaren Schreibens), in dem sie ihre zumindest in der Forschung weitläufig rezipierte Kategorisierung von Textverbindungselementen vorstellt, deren Fehlen oder unpassender Gebrauch das Textverständnis erheblich erschweren können ${ }^{20}$, und in dem von Desideri herausgegebenen Band La centralità del testo nelle pratiche didattiche (1991; m.Ü.: Die Zentralität des Textes in der didaktischen Praxis).

Dort wird ein theoretisches Modell des Textverständnisses präsentiert (Corno 1991), welches Letzteres als Prozess auffasst und in kleinschrittige Komponenten zerlegt. ${ }^{21}$ Demnach bedarf es, um einen Text verstehen zu können, einer Vielzahl von Fähigkeiten, nämlich des Erkennens von Zusammenhängen zwischen Texteinheiten, des Entwickelns einer Hypothese zur Textaussage, welche die einzelnen Texteinheiten zusammenhält, sowie der Fähigkeit zur Gliederung der einzelnen Aussagen nach dem Grad ihrer Relevanz und nicht zuletzt des Aufspürens der Kernaussage, um die herum eine Interpretationshypothese entwickelt werden kann (ibid., S. 48). Dabei vertritt Corno die Auffassung, das Textverständnis hänge von drei prinzipiellen Aktivitäten ab: Fokalisierung, Inferenzen-Bildung und Interpretation (ibid., S. 51 f.). Dieses Vorgehen wird durch drei zugrundeliegende kognitive Fähigkeiten ermöglicht, und zwar:

a) das Erkennen von relevanten Textaussagen,

b) ihre kohärente Verknüpfung,

c) das Konfrontieren mit dem eigenen Wissensspeicher (bagaglio di conoscenze, „Kenntnisgepäck“) zu ihrer Bewertung und eventuellen Verinnerlichung.

Alle drei Tätigkeiten werden von sogenannten Schemata/frames geleitet, welche beim Lesen aktiviert werden. Schemata sind eine Form von strukturierten Abbildungen des Erfahrenen und Erlernten im Gedächtnis, welche etwa beim Lesen zu einem bestimmten Thema wachgerufen werden und das Textverständnis ermöglichen. Corno betont, dass wir dann etwas Neues lernen, wenn wir uns der Aktivierung der Schemata und der Interferenzen, die wir beim Textverstehen anstellen, bewusst werden, da diese ansonsten bei uns bereits Vertrautem automatisch ablaufen (Corno 1991, S. 53-66).

Auf der Grundlage dieser Theorie wird für das schulische Textverständnis die Schlussfolgerung gezogen, dass Textarbeit die Qualität des Lernprozesses

20 Siehe hierzu die Ausführungen in Kap. 3.2.2.

21 Zur Didaktik des Textverständnisses, welche weniger auf das Ergebnis als auf den Prozess der Erschließung eines Textes konzentriert ist, vgl. auch Gensini (1991, S. 69-91). 
spürbar verbessert, wenn den Schülerinnen und Schülern diese Kenntnisse in einer „Verstehens-Didaktik“ (ibid., S. 66) vermittelt werden, bei der vor allem die Prozesse der Fokalisierung, der Inferenzen-Bildung und der Interpretation geübt werden. Außerdem verfeinerten bestimmte, stark von der Verstehensfähigkeit abhängende Textformen wie Notizen, Zusammenfassungen, Umschreibungen und Kommentare das Verständnis. Und schließlich seien dabei auch die Motivation, die sich aus dem Interesse für einen Gegenstand ergibt, und das Reflektieren des eigenen Lernprozesses entscheidend (ibid.):

La comprensione è attività che dipende dalla capacità di provare interesse. D'altra parte, si prova interesse nel momento in cui si va alla ricerca di spiegazioni. Per questa ragione, ogni espediente metacognitivo, di autovalutazione, di esplicito commento sulle proprie prestazioni fa progredire l'abilità nel comprendere testi.

(M.Ü.: Das Verständnis ist eine Tätigkeit, die von der Fähigkeit, Interesse zu verspüren, abhängt. Auf der anderen Seite verspürt man in dem Moment ein Interesse, in dem man sich auf die Suche nach Erklärungen macht. Aus diesem Grund treibt jedes metakognitive Mittel, der Selbstbeurteilung, der expliziten Bewertung der eigenen Leistungen, die Fähigkeit zum Textverständnis an.)

\subsubsection{Schreibdidaktik}

Mit der Lesekompetenz korreliert auch die andere Seite der geschriebenen Sprache, die Schreibkompetenz, sehr stark (Stefinlongo 2002, S. 15 f.). In diesem Zusammenhang ist schon festgehalten worden, wie es sich beim Schreiben um eine Praxis handelt, welche einen enormen Einfluss auf den Sprachausbau insgesamt und auch auf die kognitive Entwicklung hat (Guerriero 2014, S. 172; Stefinlongo 2001, S. 24). Deshalb wird ihr verstärkter Einsatz in der Schule gefordert, wobei nicht nur einzelne Sätze, sondern auch längere Texte einer großen Bandbreite von Gattungen und Thematiken verschriftet werden sollten.

Dafür ist eine veränderte Schreibdidaktik vonnöten (Guerriero 2014, S. 161, S. 164; Stefinlongo 2001, S. 86, S. 108; Gensini 1991, S. 182), zumal sich die Mängel der traditionellen Didaktik in den schriftlichen Abiturprüfungen und bei Zulassungstests an den Universitäten zeigen (De Santis \& Gatta 2012, S. 433). Die mäßige Qualität der dort verschrifteten Produkte weist vor allem auf Schwierigkeiten im Schriftsprachlichen hin, sobald das alltagssprachliche Register zum Formulieren des Textes nicht ausreicht (ibid., S. 431).

So ist in den letzten Jahren an den Universitäten eine Tendenz zur Einrichtung von Schreiblaboratorien erkennbar, in denen die Studierenden ihre skribalen Techniken ausbauen und verfeinern können. An einem solchen Schreibkurs teilnehmende Studierende wurden gebeten zu bewerten, was ihnen in der Schule über das Schreiben vermittelt worden ist. Das Ergebnis ist ein durchweg negativ ausfallendes Urteil über die dort erfahrene Förderung der Schreibkompetenz (Stefinlongo 2001, S. 37): 
'No, nelle scuole italiane non si insegna a scrivere, o si è geni o incapaci'; '[...] Solo eliminazione di errori grammaticali e sintattici più evidenti'; 'No, non credo di aver ricevuto una vera e propria preparazione'; 'No, solo temi di letteratura fatti a memoria o di attualità ripetendo ciò che si sente in TV'; 'No, non mi è stato insegnato a scrivere testi per la vita reale'; [...] 'No, tipo di scrittura molto scolastica e poco critica o personale. Eppoi non lo sapevano insegnare.'

(M.Ü.: ,Nein, in den italienischen Schulen wird das Schreiben nicht gelehrt, entweder ist man ein Genie oder unfähig'; , [...] Nur das Tilgen der evidentesten grammatischen und syntaktischen Fehler"; ,Nein, ich glaube nicht, eine wirkliche und passende Vorbereitung erhalten zu haben“; ,Nein, nur Literatur-Aufsätze, für die man auswendig lernt oder Aufsätze über aktuelle Themen, in denen man das wiederholt, was man im Fernsehen hört'; , Nein, mir ist nicht beigebracht worden, wie man Texte für das wahre Leben schreibt'; [...], Nein, eine sehr schulische Art des Schreibens und kaum kritisch oder persönlich. Und dann wussten sie es nicht zu lehren.')

Ähnlich wie die ehemaligen Schülerinnen und Schüler kommt auch die italienische Forschung über die herkömmliche (und immer noch weit verbreitete) Schreibdidaktik zu dem Ergebnis, dass über lange Zeit Unterricht und gesellschaftliches Leben zwei voneinander getrennte Sphären waren und Texte in der Schule allein in ihrer literarischen Dimension Beachtung fanden (z.B. Gensini 1991, S. 69).

Erst langsam gibt es dort eine praktisch-funktionalere Ausrichtung der Schreib- und Lesedidaktik, die auch von Zeitungsartikeln und Gebrauchstexten ausgeht und beispielsweise die Fähigkeit vermittelt, ein Arztrezept zu verstehen oder ein Formular auszufüllen (ibid.; Guerriero 2014, S. 161). Trotzdem besteht noch im Schuljahr 2014-2015 einer von zehn Punkten, welche die wesentlichen Resultate der nationalen Erhebung von Schülerleistungen in den Fächern Italienisch und Mathematik synthetisch präsentieren, in der Feststellung, „,nella prova di italiano gli allievi mostrano maggiore difficoltà ad affrontare testi espositivi, argomentativi e discontinui, ossia meno praticati nella quotidianità dell'attività scolastica" (INVALSI 2015; in der Italienischprüfung zeigen die Schüler größere Schwierigkeiten bei der Behandlung darlegender, argumentativer und diskontinuierlicher Texte, also bei den im Schulalltag weniger praktizierten).

Die traditionelle Schreibdidaktik mit ihrer Konzentration auf Orthographie, Grammatik und literarischen Ausdruck wirkt vor allem auf sprachlich weniger kompetente Lernende entwicklungshemmend. So wurde schon 1973 mit Blick auf die damals eben auf Aufsatzdidaktik reduzierte Schreibförderung betont (Weber 1973, S. 88):

,Es ist denkbar, daß der Aufsatzunterricht, der Sozialisationsstile und daraus resultierende Formen des Sprachverhaltens nicht zur Kenntnis nimmt, mit bestimmten Aufsatzformen vor allem Kinder der Unterschicht (aber nicht nur diese) dauernd überfordert, ohne sie sprachlich zu fördern. Es ist denkbar, daß die Kinder über Jahre hin mit Erlebniserzählungen gequält und für trockene und farblose Erlebnisberichte ohne richtigen Höhepunkt usw. mit schlechten Zensuren bestraft werden, obwohl sie vor und außerhalb ihrer Schulzeit nie Gelegenheit hatten, ihre Erlebnisse und Erfahrungen, selbst wenn sie einen richtigen Höhepunkt hatten, 
zu verbalisieren - zumindest nicht so, wie es Lehrer einer Mittelklassen-Institution für wünschenswert halten'. Es ist denkbar. Ist es denkbar? ${ }^{22}$

Auch wenn mittlerweile die für die Einführungsphase in den Schriftspracherwerb typischen Erlebnis-Aufsätze und Erzählungen ${ }^{23}$ in der Sekundarstufe durch andere Textformen ersetzt werden, kann es der Kompetenzentwicklung von mehrsprachigen Schülerinnen und Schülern dienen, zumindest gelegentlich Texte in ihrer Erstsprache verfassen zu dürfen (Gibbons 2002, S. 69). Das verhilft den betreffenden Lernenden potentiell zu mehr Vertrauen in ihre Fähigkeiten, was sich motivierend auf ihre Schreibpraxis auswirkt. ${ }^{24}$

Dass motivationale Aspekte wie für das Lernen generell, so auch für den Auf- und Ausbau der Schreibfähigkeit entscheidend sind, wird von vielen Seiten hervorgehoben (z.B. Weber 1973, S. 57; Peyer 2011, S. 102) und gilt vor allem für die Themenwahl (Müller 1997, S. 78). Deshalb sollten den Schülerinnen und Schülern Schreibimpulse oder -aufgaben gestellt werden, die sie ansprechen und ihnen verdeutlichen, dass Schreiben sinnvoll ist, weil es ,als Erschließen von Inhalten gesehen" (Maas 2008, S. 352 f.) und damit etwas bewerkstelligt werden kann, was im Mündlichen nicht zu schaffen ist (ibid., S. 652; Peyer 2011, S. 74).

Bei der Entwicklung einer umfangreicheren und funktionaleren Schreibkompetenz handelt es sich um einen langwierigen und oft mühseligen Prozess, dessen Nutzen gerade den FachlehrerInnen erkenntlich werden muss, damit sie bereit sind, die dafür notwendige Zeit und Geduld aufzubringen. Dass konzeptionelle Mündlich- und Schriftlichkeit zwei grundsätzlich verschiedene Dimensionen des Sprachgebrauch sind, wie zuvor mit den Hinweisen auf Hallidays Sprachtheorie schon angedeutet wurde, muss den Schülerinnen und Schülern explizit bewusst gemacht werden (Christie 2012, S. 220), damit konzeptionell mündliche Texte nicht weiterhin noch von Studierenden der Erstsemester verfasst werden, wie es beispielsweise De Santis und Gatta (2013) und Cacchione (2014) in Schreibproben beobachtet haben. ${ }^{25}$ Anderenfalls bleibt das

22 Für eine Beschreibung sprachlicher Auffälligkeiten der Aufsätze der Unterschichtkinder s. Weber (1973, S. 99).

23 Man beachte hierbei allerdings, dass gerade Erzählungen laut Gibbons zwar zu den ersten Schreibaufgaben gehören, die Lernende zu bewältigen haben, aber ironischerweise wahrscheinlich zu den komplexesten zu rechnen sind (Gibbons 2002, S. 55).

24 Im Übrigen weist Hinkel darauf hin, dass es relativ wenig Forschung zum schulischen Schreiben in der Zweitsprache gibt, was ihres Erachtens daran liegt, dass die Auswertung solcher Texte sehr zeitaufwändig ist, zumal sie nicht computeranalysiert werden können (Hinkel 2005b, S. 624 f.).

25 Neben etlichen typisch mündlichen Zügen ihrer Schreibproduktion ist außerdem auffällig, dass die Studierenden einen egozentrischen Schreibstil haben und ihre Werturteile und Einstellungen überschwänglich formulieren, wie es für Kinder normal ist, im Laufe der Sekundarstufe aber überwunden werden sollte (vgl. Cacchione 2014, S. 79; Christie 2012, S. 187, S. 209 f.; Lavinio 1991, S. 113). Auch rein formale Aspekte, die eigentlich in der Eingangsphase des Schriftspracherwerbs intensiv geübt und dadurch erworben werden, halten sie oft 
Schreibenlernen auf die Aneignung einer skribalen Technik reduziert, die an den heutzutage nötigen schriftkulturellen Sprachausbau bei Weitem nicht heranreicht (vgl. Maas 2008, S. 334 f., S. 650).

\subsection{Prozessorientierte Schreibdidaktik}

Wie in der Lesedidaktik wird daher auch in der Schreibdidaktik neben einer praxisnäheren Ausrichtung des Unterrichts für eine stärkere Konzentration auf den Prozess des Schreibens und weniger auf das fertige Produkt plädiert (z.B. Weber 1973, S. 101; Guerriero 1991, S. 168 f.; Müller 1997, S. 78; Czerniewska 1999; Duff 2005, S. 59).

Ende der 1970er, Anfang der 1980er Jahre ist ein Forschungsansatz zum Schreiben entwickelt worden, der in den englischsprachigen Ländern einen wesentlichen Einfluss auf die Gestaltung der Schreibdidaktik hatte, der process approach (Czerniewska 1999, S. 475). Mit Rückgriff auf Flower und Hayes (1980) betont Czerniewska die Komplexität unterschiedlicher kognitiver Aktivitäten, welche vor, während und nach dem Schreiben zum Tragen kommen (ibid.). ${ }^{26}$ Der von Flower und Hayes gesetzte Fokus hat das Erforschen der Aktivierung mentaler Strategien beim Schreiben stimuliert, was beispielsweise zu experimentellen Studien führte, in denen die Gedanken des Schreibenden beim Verfassen erfragt und Schreibprotokolle angefertigt wurden.

Das Zergliedern des Schreibprozesses in überschaubarere Einzelkomponenten soll den Lernenden den Erwerb einer soliden Schreibkompetenz erleichtern, indem es sie kognitiv entlastet (s. schon Weber 1973, S. 101). Für das Erstellen eines Schulaufsatzes etwa kann man die Schülerinnen und Schüler so zu einer Diskussion über das Aufsatzthema im Klassenverband oder in kleineren Gruppen auffordern, bevor man wesentliche Stichpunkte individuell notieren lässt und mit dem Verfassen einer ersten Version begonnen wird. Da-

nicht ein. So schreiben viele von ihnen beispielsweise nicht einheitlich in Schreibschrift, sondern vermischen Schreib- und Druckschrift (Stefinlongo 2001, S. 97-100), wenden - falls sie diese gelernt haben - die Zeichensetzungsregeln nicht an (Chiantera 2005, S. 205) und sind sehr sparsam mit der Verteilung von diakritischen Zeichen wie Akzenten (Stefinlongo 2001, S. 107) (wobei eine Ausnahme die Verwendung von Anführungszeichen darstellt, die an allen möglichen Stellen auftauchen, nur kaum in der wörtlichen Rede (ibid., S. 109)). Desweiteren fehlen in ihren Texten oft visuelle Orientierungshilfen wie ein gewisser Abstand zwischen Überschrift und Text und die Gliederung in Absätze: , , [...] i nostri corsisti hanno dimostrato di non possedere affatto questa sensibilità o di non aver ben presente la funzione di questa basilare forma di organizzazione e disposizione testuale, che, del resto, raramente viene spiegata a scuola “ (ibid., S. 94; M.Ü.: Unsere Kursteilnehmer haben gezeigt, dass sie überhaupt kein Gefühl dafür besitzen oder ihnen die Funktion dieser grundlegenden Form der textuellen Organisation und Disposition nicht präsent ist, die im Übrigen selten in der Schule erklärt wird).

26 Vgl. zum Schreibprozessmodell nach Flower und Hayes (1980) und einem darauf aufbauenden kognitiven Modell der Entwicklung der Schreibkompetenz auch Müller (1997, S. 24 ff.). 
nach bietet sich erneut eine Peer-Diskussion an, woraufhin der Aufsatz überarbeitet und anschließend ,druckreif“ gemacht und ,publiziert" werden kann (Czerniewska 1999, S. 475), wobei mit dem letzten Schritt entweder das Vortragen vor der Klasse oder die Abgabe an die Lehrperson, eine Veröffentlichung in der Schulzeitung oder in anderen Medien (Zeitschriften/Zeitungen oder Internet) verbunden ist, so dass es stets einen realen Empfänger des Schreibprodukts gibt.

Prozess-Theoretiker stellen unterschiedliche Sequenzierungen der einzelnen Komponenten auf (s. z.B. Graves 1983, Murray $1987^{2}$ ) und schließen daraus, dass sich der Schreibprozess nicht in starr festgelegten Handlungsabläufen abspielt, sondern eine komplex-dynamische Aktivität darstellt, die je nach Textsorte und Adressat variieren kann. Bei diesem Ansatz ist aus pädagogischer Sicht bedeutend, dass Schreiben als ein Handwerk konzipiert wird, das gelernt werden und über das man sich austauschen kann, um es sich möglichst geschickt anzueignen (Czerniewska 1999, S. 475). Wie im Handwerk gibt es dabei Meister - die Lehrpersonen -, welche es besser beherrschen und ihr Können an die „Lehrlinge“ weitergeben. Diese Auffassung ist also diametral zur in Italien immer noch anzutreffenden Ansicht ausgerichtet, Schreiben sei eine angeborene Gabe (Piemontese 2014, S. 10). ${ }^{27}$

Der process approach differenziert sich von anderen Ansätzen ferner in der Rollenzuschreibung der Lernenden und der Lehrperson. Hier werden die SchülerInnen als Schreibende (Autoren) betrachtet, die lernen, wie Erfahrenere mit Problemen wie der „Angst vorm weißen Blatt“ oder der Überarbeitung ihres Textes umgehen. Auf diese Weise sollen sie Verantwortung für ihr Schreibprodukt übernehmen, entscheiden, was und was wann und für wen geschrieben

27 „La seconda convinzione, altrettanto fallace ma diffusa e non estranea neppure ad alcuni addetti ai lavori, è che saper scrivere sia un dono, se non proprio divino, di natura. Non è infrequente ascoltare, a conclusione di certi discorsi, che saper scrivere non è da tutti, perché non è frutto solo di apprendimento (e insegnamento), perché è innanzitutto una dote innata. Prima che quest'idea cominciasse a essere scalfita, tanto sul piano teorico quanto nella pratica didattica, molti anni sono passati e molti danni sono stati fatti. Arrivare ad affermare che saper scrivere è, invece, un'arte oggettiva, come diceva don Lorenzo Milani, cioè il risultato di un processo consapevole, faticoso e teoricamente illimitato nel tempo, è stata una conquista lenta, di non poco conto e tuttora non del tutto ben accettata" (Piemontese 2014, S. 10; m.Ü.: Die zweite genauso irreführende, aber verbreitete und auch einigen Fachleuten nicht ferne Überzeugung ist, dass Schreiben eine, wenn nicht göttliche, so doch natürliche Gabe sei. Es ist nicht selten als Schlussfolgerung bestimmter Diskussionen zu hören, dass Schreibenkönnen nicht allen liegt, weil es nicht nur ein Ergebnis des Lernens (und Unterrichts) ist, weil es vor allem eine angeborene Gabe ist. Bevor begonnen wurde, diese Idee sowohl auf theoretischer Ebene als auch in der didaktischen Praxis anzufechten, sind viele Jahre vergangen und viele Schäden angerichtet worden. Dazu zu kommen zu behaupten, dass Schreibenkönnen hingegen eine objektive Kunst ist, wie Don Lorenzo Milani sagte, das heißt, das Resultat eines bewussten Prozesses, der mühsam und theoretisch zeitlich nicht begrenzt ist, war eine langsame Errungenschaft von nicht geringer Bedeutung und ist noch heute nicht vollständig akzeptiert). 
wird, und was mit dem fertigen Text geschieht. Aus dem Konzept der Lernenden als Eigentümer ihrer Schreibprodukte ist abzuleiten: ,[...] what the children own should be worth having, it should serve child-valued purposes" (Czerniewska 1999, S. 476).

Dieser Lernprozess sollte stattfinden, während die Lernenden mit dem Verfassen ihrer eigenen Texte beschäftigt sind, wobei ihnen eine größere Kontrolle über das Thema und den Adressaten eingeräumt wird. Die somit weniger dominante Lehrperson fungiert dabei Rat gebend und unterstützend; sie sorgt möglichst für eine lernförderliche Unterrichtsgestaltung, in der projektartige Aktivitäten wie Schreibwerkstätten und Schreibkonferenzen Platz finden (Czerniewska 1999, S. 476).

Viele der im Ansatz entwickelten didaktischen Vorschläge - das gemeinsame Verfassen eines Buches für jüngere SchülerInnen, Engagement für die Schülerzeitung, Briefe an berühmte AutorInnen, um herauszufinden, wie sie schreiben, das Anfertigen von Biographien über lokale Persönlichkeiten - sind nicht neu. Entscheidend wirken allerdings das tatsächliche Verschicken oder Veröffentlichen der Produkte im Gegensatz zum bloß simulierten Schreibanlass und die Zentralität, die das Verfassen von Texten im Unterricht einnimmt (ibid.). Aus letzterem Grund ist es sinnvoll, dass für prozessorientierte Schreibdidaktik von den Sprach- und den Fachlehrpersonen eine Zusammenarbeit angestrebt wird.

Obwohl der Kreativität der jungen Schreibenden auf diese Weise möglichst wenige Grenzen gesetzt werden sollen, sind die Lernenden doch nicht auf sich selbst gestellt, sondern werden in ihrem Vorgehen von der Lehrkraft unterstützt (ibid.). Dennoch ist der Ansatz kritisiert worden, weil vor allem bei der Themenwahl etwas zu viel Freiraum gelassen werde. Dadurch bestehe die Gefahr, dass schulisches Schreiben keine adäquate Vorbereitung auf die Schreibaufgaben darstellt, welche die Lernenden im späteren Leben erwarten (Czerniewska 1999, S. 476 f.). ${ }^{28}$ So argumentieren Vertreter der Genre-Theorie wie Reid (1987), es sei nicht ausreichend, dass Lernende sich „reellen“ Schreibanlässen und ,reellen“ Adressaten widmen, sondern sie bräuchten Unterstützung, um zu verstehen, auf welche Texte es im ,realen“ Leben ankommt und wie diese formuliert werden (Czerniewska 1999, S. 477).

In diesem Zusammenhang ist ein Blick auf Gibbons generell für Hintergrundwissen und praktische Anwendungsbeispiele zum sprachförderlichen Unterrichten ergiebiges Buch Scaffolding language, scaffolding learning (2002) nützlich, in dem der in Australien entwickelte Genre-Zyklus vorgestellt

28 Studien in den USA zeigen, dass der schulische Schreibunterricht mit seiner Konzentration auf Grammatik und überhaupt keiner oder einer geringen Einführung von Aktivitäten wie Planung und Brainstorming vor dem Schreibprozess oft nicht aufs akademische (Aufsatz)Schreiben vorbereitet (Duff 2005, S. 58 f.). Dadurch geschieht es, dass auch (vor allem in der Zweitsprache) Lernende, welche in der Schule zu den Besten zählten, an der Universität erst einmal English Writing-Klassen absolvieren müssen und dies als Demütigung erleben. 
wird, mit dem Lernende die Merkmale und Unterschiede der verschiedenen Textsorten verinnerlichen und im Verfassen entsprechender Texte unterstützt werden sollen. Ihnen derartige Regeln vorzugeben und sie bei der Textproduktion zu leiten, schränke die Kreativität und Freiheit der Lernenden nicht ein, sondern ermögliche es ihnen im Gegenteil, Erstere mithilfe dieses Werkzeuges zu entfalten (Gibbons 2002, S. 68). Gibbons motiviert ein derartiges Vorgehen desweiteren folgendermaßen (ibid.):

If students are to have real choices about what and how they write, they need to be shown what the range of options is. Otherwise, they will simply remain with what they know, writing about a limited range of things in the same way. And it is important to remember that the "rules" and conventions that govern different types of writing have not been imposed by linguists, but simply describe what these texts look like in the real world.

Zum Prozess-Ansatz gehört nicht zuletzt auch eine veränderte Bewertungspraxis, deren Vorstellung dieses Kapitel abschließt.

\subsection{Bewertungspraxis für die Sprachförderung}

Mit dem Prozess-Ansatz rücken in der Bewertungspraxis neue Aspekte in den Vordergrund. Zum einen geht es darum, die Lernenden durch eine Textbeurteilung in ihrer Fähigkeit der Selbstkontrolle zu stärken. Denn viele von ihnen wissen ohne eine explizite Hilfestellung gar nicht, was sie an ihren Texten verbessern können, wenn man einmal von offenkundigen orthographischen Fehlern oder der Zeichensetzung absieht (De Santis \& Gatta 2013, S. 425; Gibbons 2002, S. 74; Müller 1997, S. 77-81). ${ }^{29}$ Dazu tragen Bewertungsmethoden der Lehrkräfte bei, die neben sprachlichen Oberflächenphänomenen explizit auch den Aufbau des Textes und seinen Inhalt beurteilen.

Solch eine detaillierte Bewertung, die in Italien in der Regel höchstens im Sprachunterricht im Zuge von Aufsätzen zur Leistungskontrolle erfolgt, sollte auf andere Textproduktionen des Sprach- und auch im Fachunterricht ausgeweitet werden. Gerade Fachlehrerinnen und -lehrer - wenn sie die Lernenden in ihrem Unterricht überhaupt schreiben lassen - korrigieren in der Regel nicht sprachbezogen, was u.a. daran liegt, dass ihnen die nötige Kompetenz dazu fehlt (Duff 2005, S. 55). Hier gilt wieder, dass ihnen die Relevanz eines solchen Vorgehens sowie das erforderliche Wissen während der Ausbildung zu vermitteln wären.

29 Vgl. hierzu auch Ehlich (2007, S. 25): „Entsprechende Selbstevaluationen (Fehlersensibilität) sind für die förderdiagnostische Arbeit von höchster Relevanz, werden bisher aber kaum beachtet.“ 
Zum anderen sollte die Bewertung die Literalitätsentwicklung der Lernenden erschließen. ${ }^{30}$ Das ist allein schon deshalb ratsam, weil die SchülerInnen dadurch wirksamer in ihrem Sprachausbau unterstützt werden. Doch außerdem stellen profundere Analysen der Textproduktion für die Lehrperson eine gute Grundlage zur Ermittlung des aktuellen und individuellen Sprachstandes dar. Das ist eine wesentliche Voraussetzung für eine gezieltere Sprachförderung. In diesem Sinne empfiehlt es sich im Zuge einer prozessorientierten Schreibdidaktik nicht nur (aber gerade) für in der Zweitsprache lernende und einsprachige Schülerinnen und Schüler, die sich mit dem Schreiben schwerer tun, in die Bewertung einfließen zu lassen, wie gut das Schreibprodukt die Absichten seines Verfassers realisiert und welche Lernfortschritte bei der Textentwicklung jeweils erbracht wurden (Czerniewska 1999, S. 476).

Eine umfassende Bewertungspraxis ist nicht nur im Sinne der Förderung produktiver Textkompetenz ergiebig. Generell wird von verschiedenen Seiten für regelmäßige Leistungskontrollen plädiert, weil sie den am Lernprozess beteiligten Akteuren bescheinigen, wo sie stehen. Der Lehrperson erleichtert es die Planung ihres Unterrichts, indem sie genauere Informationen darüber erhält, welche Lerngegenstände noch nicht so gut beherrscht und deshalb wiederholt oder vertieft werden sollten (Weber 1973, S. 31), und wie es um den Sprachstand der Lernenden bestellt ist. Es geht also nicht darum, wie mit so manchem standardisierten Test eine Legitimierungsbasis $\mathrm{zu}$ finden, um das sprachliche Problem innerhalb der Verantwortung des schwächeren Lernenden anzusiedeln (Gibbons 2002 mit Verweis auf Cummins 2000, S. 123). Hingegen ist das Überprüfen der sprachlichen und fachlichen Fähigkeiten für die Lernenden selbst von Vorteil, da sie auf diese Weise eine Unterstützung dabei erfahren, selbständiger zu werden und eigenständiger lernen zu können. Dadurch kommen sie einem entscheidenden Bildungsziel näher, zumal ihnen auch Wege zur Selbstkontrolle aufgezeigt werden. Es empfiehlt sich dafür, ein Bewertungsklima zu schaffen, in dem möglichst wenig Prüfungsangst entsteht, der Leistungsdruck nicht zu stark wird und sich eventuelles Konkurrenzverhalten unter den Lernenden nicht nachteilig auf sie auswirkt.

Untrennbar von der Bewertungspraxis scheint in unserer westlichen Kultur die Benotung zu sein. In diesem Zusammenhang stuft Weber die Notenvergabe der SprachlehrerInnen als zu wohlwollend ein, wie im Übrigen auch für den italienischen Kontext festgestellt worden ist: Dort gilt gerade die Aufsatzbewertung als tendenziell labil und zu gutmütig, um das Selbstwertgefühl schon in anderen Fächern schlecht benoteter Lernender nicht weiter zu schwächen und ihnen die Eingliederung in den Arbeitsmarkt nicht zu erschweren. $\mathrm{Zu}$ Recht kommt die Frage auf, warum das die Aufgabe der Sprachlehrkräfte sein

30 Zur Erleichterung einer solchen Praxis finden sich in Gibbons (2002, S. 74) nützliche Informationen einschließlich einer Tabelle mit Fragen, die das Bewerten in verschiedenen Sprachbereichen leiten können. Weitere Empfehlungen zur Aufsatzbewertung finden sich z.B. auch schon in Weber (1973) und in Fröhler (2008). 
sollte (Serianni 2010, S. 42), und wird moniert, dass Letztere den Lernenden mit dieser Praxis keinen Gefallen tun (ibid., S. 44):

Si dice - e soprattutto si diceva negli anni Settanta - con un fortunato slogan: la scuola che boccia, boccia sé stessa. Ma sarà necessario distinguere tra scuola dell'obbligo e scuola superiore: nel primo segmento non è effettivamente pensabile che l'istruzione non conduca tutti gli alunni a certi obiettivi, senza abdicare all'inevitabile apparato sanzionatorio ma senza lasciare in dietro nessuno. Per il resto la scuola è davvero sconfitta non quando blocca chi non mostri l'attrezzatura e la disposizione necessarie per proseguire gli studi, bensì quando non discrimina il valore.

(M.Ü.: Man sagt - und vor allem sagte man in den Siebzigerjahren - mit einem geglückten Slogan: Die Schule, die durchfallen lässt, lässt sich selbst durchfallen. Aber es wird nötig sein, zwischen der Pflichtschule und der weiterführenden Schule zu unterscheiden: Im ersten Segment ist es tatsächlich nicht denkbar, dass die Schulbildung nicht alle Schüler zu bestimmten Zielen führt, ohne auf den unvermeidlichen Sanktionsapparat zu verzichten, aber ohne jemanden zurückzulassen. Im Übrigen ist die Schule nicht dann wirklich der Verlierer, wenn sie denjenigen aufhält, der nicht die nötige Ausrüstung und Disposition zeigt, um die Studien weiterzuführen, sondern wenn sie den Wert nicht unterscheidet.)

Dieser Umstand führt bei Schulabgängern mit guten Noten zu einer Überbewertung ihrer sprachlichen Fähigkeiten, welche dann mit den negativen Ergebnissen in Einstufungstests an den Universitäten kollidieren (s. Fn. 28, S. 63 zur amerikanischen Situation sowie z.B. Piemontese 2014, S. 10 und De Santis \& Gatta 2013, S. 430-434 über italienische Studierende). 


\section{3 Überblick über den Forschungsstand zur Mehrsprachigkeit in der italienischen Schule und zur sprachlichen Gestaltung der Lehrbücher}

In diesem Kapitel werden die unterschiedlichen und zum Teil mehrsprachigen Ausgangslagen der Lernenden an italienischen Schulen zunächst historisch und dann bezogen auf die gegenwärtige Situation vorgestellt sowie ein Überblick über die Anzahl, Verteilung und Herkunft der Zugewanderten gegeben (3.1). Verbunden mit den sprachlichen Schwierigkeiten vieler Lernender im Unterricht ist die Gestaltung der Lehrmittel, welche aufgrund ihrer Komplexität seit den 1980er Jahren ins Interesse der Forschung gerückt ist. Die Ergebnisse dieser Forschung (3.2) und der partikuläre Ton (3.2.1), in dem sie formuliert sind, werden beschrieben, um die wissenschaftliche Debatte über die schulische Sprachbildung und die Verwendung der Lehrmittel besser nachvollziehen und als theoretische Verankerung bei der Darstellung der vorliegenden Studie nutzen zu können. Desweiteren werden zwei Schwerpunkte der Lehrbuchforschung vorgestellt, und zwar Untersuchungen des verwendeten Wortschatzes (3.2.2) und der Lesbarkeit mittels dafür konzipierter Formeln sowie eines Katalogs von textimmanenten Verständnishürden (3.2.2.2). Viele Studien, welche sich auf die Wortschatzverwendung konzentrieren, bedienen sich dabei eines Gebrauchswörterbuchs des Italienischen, des GRADITs (De Mauro 1999/2000). Dieses ist auch in der vorliegenden Studie eingesetzt worden, weshalb in einem eigenen Unterkapitel seine Entstehung und die ihm zugrundeliegende Unterteilung des Wortschatzes nach seiner Verwendungsfrequenz erläutert wird (3.2.2.1).

\subsection{Sprachliche Heterogenität in der italienischen Schule}

Nachdem Italien erst seit dem Jahr 1976 eine positive Migrationsbilanz verzeichnet, also mehr Personen in das Land ein- als auswandern, ist der Zufluss von Migranten - und damit die Präsenz von ausländischen Lernenden in den italienischen Schulen - in den letzten zehn Jahren deutlich gestiegen. Dennoch stellt die Mehrsprachigkeit von Lernenden keineswegs ein neues Phänomen dar. Der Grund hierfür liegt in dem vergleichsweise langen Weg des Landes zur nationalen Einigung und zu einer einheitlichen Sprache für alle Staatsbürger, die stets eine Vielzahl von Sprachen und Dialekten gesprochen haben (Pinto 1980, S. 42).

Noch in den 1960er Jahren war die Erstsprache der Mehrheit der Bevölkerung und dem entsprechend auch der Lernenden keineswegs das Italienische 
im Sinne der Standardvariante, sondern einer der zahlreichen Dialekte, die vom Vulgärlatein abstammen und z.T. linguistisch mit anderen romanischen Sprachen wie dem Spanischen verwandter sind als mit dem Italienischen (vgl. De Mauro 1963). So erkannten viele Lehrkräfte die Bedeutung ihrer Aufgabe bei der sprachlichen Heranführung der Lernenden an die Nationalsprache, auch wenn ihre Unterrichtsmethoden von den heutigen Vorstellungen guten Unterrichts erheblich abwichen. Mehrheitlich unterbanden sie z.B. über Jahrzehnte strikt jeglichen Gebrauch der Dialekte (De Mauro 1976b in ead. 1979, S. 85 f.) $)^{31}$ und wollten sie die SchülerInnen an ein Italienisch gewöhnen, welches Züge einer Kunstsprache trug. Denn die Standardvariante war über einen langen Zeitraum fast ausschließlich eine literarische und nur wenigen Menschen zugängliche Sprache, die so gut wie gar nicht gesprochen wurde (vgl. De Mauro 1979², S. 27; Gensini 2005, S. 45; Villari 1909, S. 389 ff. zit. in Gensini 2005, S. 88-91). ${ }^{32}$

Auf diese Weise war nicht beabsichtigt, den Lernenden einen funktionalen Umgang mit der Sprache zu vermitteln, also einen, der sich an ihrem alltäglichen Gebrauch gemäß der Gesprächssituationen, dem Gegenstand und der Gesprächspartner orientiert. Sprachbildung bestand stattdessen hauptsächlich im Diktat von Wortlisten, die es auswendig zu lernen galt. Mit diesem Vorgehen war der Sprachunterricht sehr literarisch geprägt, was z.B. dazu führte, dass von den meisten Lehrkräften das Ersetzen allgemeinsprachlich nicht (negativ) konnotierter Wörter, welche durchaus aus der florentinischen literarischen Tradition stammen - also aus dem Sprachgebrauch, aus dem sich der italienische Sprachstandard entwickelt hat - durch gehobene oder literarische, wenn nicht verwaltungssprachliche Synonyme gefordert wurde. ${ }^{33}$ De Mauro bringt diese

31 Dies gilt vor allem für das Schriftliche; im Mündlichen verwendeten die Lehrkräfte z.T. selbst dialektale Formen, ohne dass es ihnen immer bewusst gewesen wäre. Gleichzeitig deckte die italienische Standardsprache über einen langen Zeitraum selbst den formaleren Schriftsprachbereich nicht vollständig ab, da gehobene Poesie und Literatur auch in den Dialekten zu einer gewissen Blüte gelangt waren (De Mauro 1979², S. 33).

32 Nirgendwo sonst in Europa hat eine Nationalsprache über so viele Jahrhunderte lang nur in der Literatur und als Sprache der Gebildeten bestanden wie in Italien (ibid., S. 27). Dadurch handelt es sich um eine Sprache, die über einen langen Zeitraum ausgesprochen statisch war, kaum phonetische Transformationen erlebte, da sie eben nur geschrieben wurde, und die noch einen Großteil des Wortschatzes aus dem 14. Jahrhundert auf transparente Weise enthält (ibid., S. 28). Auch die Vielzahl der Dialekte kennt Ihresgleichen weder in Europa noch im indogermanischen Sprachraum (abgesehen von Indien), wozu die geographische Beschaffenheit des Landes und die Phasen weniger blühenden wirtschaftlichen und gesellschaftlichen Austausches stark beigetragen haben. So konnten sich schon in vorrömischer Zeit verschiedenste Sprachen und Dialekte vollkommen unabhängig voneinander entwickeln (ibid., S. 17 f.).

33 Als Beispiele nennt De Mauro etwa die Bevorzugung von viso für faccia (Gesicht), indignarsi für arrabbiarsi (sich ärgern, wütend werden) oder trascorrere il giorno della festività natalizia (den Tag der weihnachtlichen Festivität verbringen) anstelle von passare il Natale (Weihnachten verbringen) (De Mauro 1976a in ead. 1979, S. 231). Und auch Weber (1973, 
Sprachdidaktik folgendermaßen auf den Punkt (De Mauro 1976b zit. in ead. 1979, S. 76):

Il punto dal quale volevo prendere le mosse è: a che cosa tendeva la vecchia cultura, basata su una tradizione scolastica tradizionale e retorica?

Che cosa abbiamo imparato noi, che ci siamo formati in una scuola tradizionale?

A scuola ci hanno insegnato essenzialmente questo: parlare e scrivere è una cosa che riguarda i poeti e i letterati, che serve per fare poesie e scrivere bei romanzi. In realtà nessuno ci ha spiegato che parlare e scrivere servono a cose molto più vitali.

(M.Ü.: Der Punkt, von dem ich ausgehen wollte, ist: Worauf zielte die alte Kultur, die auf einer traditionellen und rhetorischen schulischen Tradition beruht?

Was haben wir gelernt, die wir in einer traditionalen Schule ausgebildet wurden?

In der Schule haben sie uns im Wesentlichen dieses gelehrt: Sprechen und Schreiben sind eine Sache, die Poeten und Schriftsteller/Gebildete angeht, die man zum Dichten und Romaneschreiben braucht. In Wirklichkeit hat uns niemand erklärt, dass Sprechen und Schreiben viel vitaleren Dingen dient.)

Eine andere Form der Spracherziehung verfochten vehement einige Vorläufer unter Philosophen, Politikern und Pädagogen wie beispielsweise Vico (16681744), Ascoli (1829-1907), Wittgenstein (1889-1951), Giuseppe Lombardo Radice (1879-1938), Gramsci (1891-1937) und Don Milani (1923-1967) (s. De Mauro 2012; Gensini 2005, S. 37 f.) $)^{34}$, indem sie das linguistische Repertoire, mit dem die Lernenden die Schule erreichen, wertschätzten und als Ausgangspunkt für ihre spracherzieherischen Maßnahmen nahmen. Daneben ist ein erster entscheidender Moment für eine Umorientierung in der Sprachbildung durch die Wiederaufnahme und Weiterentwicklung dieser Prinzipien in den 1970er Jahren zu verzeichnen. AkademikerInnen und Lehrkräfte schlossen sich zum sogenannten GISCEL (Gruppo di intervento e studio nel campo dell'educazione linguistica; Interventions- und Studiengruppe im Bereich der schulischen Sprachbildung) zusammen und legten 1975 einen Text vor, der ihre Leitgedanken zum Ausdruck bringt. Er wird noch heute oft zitiert, wenn es um Fragen der schulischen Sprachbildung geht (z.B. Ferreri 1988, S.7-9; Lavinio 2005b, S. 152 f.), und hatte entscheidenden Einfluss auf einen Wandel

S. 112 f.) wettert gegen das „Prätentiöse“ und „Gekünstelte“ in der stilerzieherischen Schreibdidaktik.

34 S. La scienza nuova (Vico 2008 (1725)); Scritti sulla questione della lingua (Ascoli 1975 (1873)) und Relazione sul II ${ }^{\circ}$ tema proposto al IX Congresso Pedagogico Italiano (ead. 1874 in Raicich 1981); von Giuseppe Lombardo Radice Lezioni di didattica e ricordi di esperienza magistrale (1959 (1912)) und seine Aufsätze Accanto ai maestri aus den Jahren 1919-1930 (ead., 1931); die Gefängnishefte von Antonio Gramsci (1975); Esperienze pastorali (Milani 1958) und Lettera a una professoressa Milani/Scuola di Barbiana 1967). Wittgenstein beobachtete während seiner Tätigkeit von 1920 bis 1926 als Dorfschullehrer den restringierten und dialektalen Wortschatzgebrauch seiner Lernenden und versuchte ihn auszuweiten, was u.a. zur Veröffentlichung von einem Wörterbuch für Volksschulen (1926) führte. Außerdem ist davon auszugehen, dass die Lehrtätigkeit Einfluss auf sein philosophisches Denken nahm, was sich in seinem Werk Philosophische Untersuchungen (2001 (1953, posthum)) widerspiegelt (De Mauro 2012). 
bei der Formulierung sprachbildungsrelevanter Erziehungsziele in den Lehrplänen: Le 10 tesi dell'educazione linguistica democratica (GISCEL 1975; Die zehn Thesen der demokratischen Spracherziehung).

Da die Überzeugungen dieses Textes nichts von ihrer Bedeutung eingebüßt haben, zumal die dort formulierten Ziele noch immer nicht überall und vollständig in der schulischen Sprachbildung umgesetzt, geschweige denn in der Lehrerausbildung vermittelt werden (Gensini 2005, S. 48 f.), sollen im Folgenden drei wesentliche Forderungen der Dieci tesi wiedergegeben werden. Hierbei handelt es sich um das zweite bis vierte von insgesamt zehn Prinzipien der achten These, auf denen der Auffassung des GISCELs nach eine demokratische Sprachbildung fußt (GISCEL 1975):

VIII. Principi dell'educazione linguistica democratica

$[\ldots]$

1. $[\ldots]$

2. Lo sviluppo e l'esercizio delle capacità linguistiche non vanno mai proposti e perseguiti come fini a se stessi, ma come strumenti di più ricca partecipazione alla vita sociale e intellettuale: lo specifico addestramento delle capacità verbali va sempre motivato entro le attività di studio, ricerca, discussione, partecipazione, produzione individuale e di gruppo.

3. La sollecitazione delle capacità linguistiche deve partire dall'individuazione del retroterra linguistico - culturale personale, familiare, ambientale dell'allievo, non per fissarlo e inchiodarlo a questo retroterra, ma, al contrario, per arricchire il patrimonio linguistico dell 'allievo attraverso aggiunte e ampliamenti che, per essere efficaci, devono essere studiatamente graduali.

4. La scoperta della diversità dei retroterra linguistici individuali tra gli allievi dello stesso gruppo è il punto di partenza di ripetute e sempre più approfondite esperienze ed esplorazioni della varietà spaziale e temporale, geografica, sociale, storica, che caratterizza il patrimonio linguistico dei componenti di una stessa società: imparare a capire e apprezzare tale varietà è il primo passo per imparare a viverci in mezzo senza esserne succubi e senza calpestarla.

(M.Ü.: VIII. Prinzipien der demokratischen Spracherziehung

$[\ldots]$

1. $[\ldots]$

2. Die Entwicklung und die Ausübung der sprachlichen Fähigkeiten dürfen nie um ihrer selbst willen angeboten und verfolgt werden, sondern als Instrumente einer reicheren Teilhabe am sozialen und intellektuellen Leben: Die spezifische Ausbildung der Sprachfähigkeit muss immer im Zuge einer Aktivität des Lernens, Forschens, Diskutierens, Teilhabens und der individuellen sowie der Gruppenproduktion erfolgen.

3. Das Anregen der sprachlichen Fähigkeiten muss von einer Individualisierung des persönlichen, familiären und herkunftsspezifischen sprachlich-kulturellen Hintergrunds des Schülers ausgehen, nicht um ihn zu fixieren und an diesen Hintergrund zu ketten, sondern, im Gegenteil, um den Sprachbesitz des Schülers durch Zusätze und Erweiterungen zu bereichern, welche, um effektiv zu sein, bewusst graduiert werden müssen.

4. Die Entdeckung der Verschiedenheit des individuellen sprachlichen Hintergrunds zwischen Schülern derselben Gruppe ist der Ausgangspunkt für wiederholte und immer vertiefendere Erfahrungen und Erkundungen der räumlichen, zeitlichen, geographischen, sozialen und geschichtlichen Vielfalt, welche den Sprachbesitz der Mitglieder ein und derselben Gesellschaft kennzeichnet: Diese Vielfalt zu verstehen und wertschätzen zu lernen, ist der erste Schritt, um zu lernen, darin zu leben, ohne ihr Unterworfener zu sein oder sie zu missachten.) 
Dieser Ausschnitt aus den Zehn Thesen verdeutlicht, wie schon vor geraumer Zeit Aspekte zur Sprache gebracht wurden, die auch heute die Diskussion über eine Sprachbildung bestimmen, die den aktuellen Verhältnissen und Anforderungen angemessen Rechnung trägt - besonders, aber keineswegs nur, im Kontext einer sprachlich zunehmend heterogeneren Schülerschaft (s. z.B. Bertocchi 2009; Grassi et al. 2003; GISCEL 2004). So ist schon damals die Bedeutung der sprachlichen Fähigkeiten für eine aktive Teilnahme am gesellschaftlichen Leben unterstrichen worden. Desweiteren wurde die Umsetzung des wichtigen pädagogischen Grundsatzes erkannt, das individuelle sprachliche Kapital der Lernenden wertzuschätzen und darauf aufzubauen. Ein dritter Aspekt betrifft die Sprachbeschreibung, welche den im Common Sense, aber auch unter Didaktikern und Sprachwissenschaftlern verbreiteten monolithischen Charakter der Sprache zugunsten einer Registerdifferenzierung aufgibt. Die Vorteile einer solchen bewussten Differenzierung treten sowohl in der Diagnose der sprachlichen Fähigkeiten als auch in der didaktischen Tätigkeit, die am Sprachausbau der Lernenden orientiert ist, hervor.

So ist also der Umstand, dass in der Schule eine andere Sprache vermittelt wird als die Alltagssprache der Lernenden, von jeher die vorherrschende Situation an italienischen Schulen. Die Verschiedenartigkeit der sprachlichen Ausgangslagen der Schülerschaft ist im Laufe der Zeit gewachsen. Während in den ersten Jahrzehnten des letzten Jahrhunderts die Schulpflicht auf fünf Jahre Grundschulzeit beschränkt und inländische Mobilität kaum gegeben war, so dass sich die Lehrperson, ausgehend von einem oder einigen wenigen lokalen Dialekten, um eine Hinführung zum Italienischen zu bemühen hatte, sind ab den 1950er Jahren größere Migrationen von den Dörfern in die Städte und vor allem vom Süden in den industrialisierten Norden zu verzeichnen. ${ }^{35}$ Dadurch ist auch die Anzahl der von den Lernenden in einer Klasse gesprochenen Dialekte angestiegen. Außerdem wurden durch die Anhebung der allgemeinen Schulpflicht auf insgesamt acht Jahre breitere Bevölkerungsschichten erreicht als zuvor.

35 Es gab jedoch auch schon nach der Einheit Italiens um die Jahrhundertwende und vor allem im Jahrzehnt 1901-1911 im Zuge der Industrialisierung und der damit verbundenen Urbanisierung Migrationsbewegungen von den ländlichen und primär südlichen Regionen des Landes in den Norden (De Mauro 1979², S. 51-53, S. 63). Diese Faktoren haben entschieden zum Erwerb des Italienischen seitens größerer Bevölkerungsanteile beigetragen. Eine weitere wesentliche Rolle in der Verbreitung der Standardsprache spielte die Emigration: Da vor allem Analphabeten in die USA auswanderten, die untereinander einen Mix ihrer verschiedenen Dialekte versetzt mit einem rudimentären Englisch sprachen (aber keineswegs Italienisch) und auf die Unterstützung Schreibkundiger angewiesen waren, um den Kontakt zu den Daheimgebliebenen aufrechtzuhalten, erkannten sie die Bedeutung der Schulbildung und der Beherrschung der Sprache, wodurch sie darauf drängten, die in Italien gebliebenen Kinder in die Schule zu schicken (ibid., S. 60 ff.). 
Durch Mobilität, die Verbreitung von Massenmedien (Fernsehen und Radio), Wehrdienst und den stetigen, mit der Anhebung der Schulpflicht allgemeinen Anstieg der Beschulung hat sich das Italienische allmählich zu einer tatsächlich gesprochenen Sprache der Bevölkerung entwickelt und ist die Anzahl von Lernenden, welche ausschließlich mit einem Dialekt aufwachsen, stark zurückgegangen. Hinzu kommt, dass in den letzten Jahren eine fortschreitende Verflüssigung der Dialekte beobachtet wird, diese also immer mehr Elemente der Regiolekte und Standardsprache absorbieren (Simone 1998, S. 38). ${ }^{36}$ An die Stelle der dialektalen Sprachenvielfalt ist die Sprachenvielfalt durch Einwanderung getreten. Neben dem Italienischen werden im Land mindestens 33 weitere Sprachen gesprochen (Lewis, Simons \& Fennig 2016). Diese Pluralität nimmt noch zu, wenn man berücksichtigt, dass viele der Zugewanderten nicht die Standardsprachen ihrer Herkunftsländer sprechen, sondern unterschiedliche Dialekte und Varietäten.

Dem statistischen Dossier zur Einwanderung in Italien (Centro Studi e Ricerche IDOS 2014) ist zu entnehmen, dass 2014 knapp 5 Millionen Personen mit einem ausländischen Pass ihren Wohnsitz in Italien hatten $(8,1 \%$ der Gesamtbevölkerung) und die Präsenz von regulär Zugewanderten laut Schätzung noch um einige Hundert Personen höher lag (5.364.000 Menschen). Darunter befinden sich mehr als eine Million Minderjährige, von denen im Schuljahr 2013/2014 knapp 803.000 in italienische Schulen eingeschrieben waren, was neun Prozent der gesamten Schülerschaft entspricht (ibid., S. 1).

Dabei ist der Anteil von Kindern und Jugendlichen mit Migrationshintergrund in den italienischen Schulen seit der Jahrhundertwende stark angestiegen. Während ab Anfang der 1980er Jahre Kinder von Zugewanderten in den Schulen eingeschrieben sind, sie aber zunächst nur 0,06 Prozent der gesamten Schülerzahl ausmachten und ihr Anteil seit Mitte der 1990er Jahre um ca. 20 Prozent im Jahr gewachsen ist (Gattulo \& Leone 2001a, S. 4), lag er im Jahr 2003 bei 300.000. Er hatte sich damit im Vergleich zu den drei vorausgehenden Jahren verdoppelt (GISCEL 2004, S. 3). Von 2008 bis 2014 ist die Anzahl der Schülerinnen und Schüler mit Migrationshintergrund von 628.937 Einschreibungen um fast ein Drittel $(28 \%)$ angestiegen.

Die Verteilung der Migranten konzentriert sich zu einem Viertel auf die vier Provinzen Mailand, Brescia, Turin im Norden und Rom. In der Hauptstadt, wo auch die vorliegende empirische Studie durchgeführt worden ist, waren 2014 10,3 Prozent aller Zugewanderten, also über eine halbe Million, gemeldet. Während ihre Herkunft polyzentrischer Gestalt ist - 196 sind die Länder, aus denen sie stammen (Centro Studi e Ricerche IDOs 2014, S. 1) - und damit

36 Ihren Anfang nahm allerdings die Verflüssigung der Dialekte schon früher, und zwar zu Beginn des 20. Jahrhunderts, als immer mehr Menschen unterschiedlicher Dialekte in den Städten aufeinandertrafen und durch die Industrialisierung neue Wörter in das Italienische aufgenommen wurden, die auch die Dialekte übernahmen (ibid., S. 67). 
die Kultivierung zahlreicher verschiedener Sprachen verbunden ist, ist der Zufluss aus einigen wenigen Regionen besonders groß. So stammte 2014 über die Hälfte der Personen aus den fünf Ländern Rumänien, Albanien, Marokko, China und der Ukraine und zwei Drittel aller Einwanderer aus insgesamt zehn Ländern.

In den italienischen Klassenzimmern lassen sich inzwischen auch immer mehr SchülerInnen antreffen, welche in Italien geboren sind, aber eine andere Erstsprache haben als Italienisch. Unter den allochthonen Lernenden waren es 2010 in der Primarstufe zwischen 60 und 80 Prozent (in Städten wie Mailand, die unter den ersten Anlaufpunkten für Zugewanderte waren) (Favaro 2010a, S. 2). Ihr alltäglicher Sprachgebrauch unterscheidet sich kaum von dem der autochthonen Lernenden, jedoch fallen gerade unter ihnen im Laufe der Schulkarriere viele zurück, weil ihnen der für gute Schulleistungen in allen Fächern nötige Sprachausbau Schwierigkeiten bereitet (Favaro 2003, S. 13 f.). Eine in der Lombardei durchgeführte Studie von 2000 (ibid.) zeigt, dass 43 Prozent der SchülerInnen an den Mittelschulen, deren Erstsprache von der Unterrichtssprache differiert, über ausreichende oder gute Kenntnisse des Italienischen verfügen, während die der übrigen 57 Prozent als schwach eingestuft wurden. Die Konsequenzen spiegeln sich auch in den unterschiedlichen Versetzungsquoten von der Mittelschule auf die weiterführende Schule wider: Gegenüber den 95,5 Prozent der autochthonen Lernenden gelingt nur 86 Prozent der ausländischen Schülerinnen und Schüler die Versetzung (Favaro 2003, S. 13). ${ }^{37}$ Zudem kommen, nicht selten während des laufenden Schuljahrs, neue Lernende hinzu, welche oft noch gar kein Italienisch verstehen und sprechen. Wie sie in den schulischen Betrieb aufgenommen werden, ist von Region zu Region und von Schule zu Schule unterschiedlich geregelt. Gemein ist allen staatlichen Schulen, dass ihnen dafür keine spezifischen Ressourcen zur Verfügung stehen (Favaro 2001, S. 55). Neben einigen nationalen Schulen, welche von den allochthonen Gemeinschaften gegründet werden, wenn sie ihr Migrationsprojekt als transitorisch betrachten oder eine kulturelle Entwurzelung befürchten, und in denen die Lernenden in den Sprachen ihrer Herkunftsländer unterrichtet werden, gibt es einige Klassen, die „,monoethnisch“ zusammengesetzt sind, also beispielsweise alle Chinesischsprachigen in einer Klasse vereinen und alle Arabischsprachigen in einer anderen. Beide bilden allerdings eine Ausnahme zur vorherrschenden Situation der Beschulung in den staatlichen Schulen, in denen überwiegend nach dem Immersionsmodell gehandelt wird: Die Schülerinnen und Schüler werden einem Sprachbad ausgesetzt, in dem sie entweder schwimmen können oder untergehen (ibid., S. 56). Während manche

37 Es handelt sich hierbei um eine an Mailänder Schulen durchgeführte Studie, deren Abschlussbericht mithilfe der von Favaro (2003) gegebenen Quellenangabe nicht auffindbar war. Bezüglich des Verbleibs des Berichts verweisen die am Projekt beteiligten Akteure auf ihre Partner; wahrscheinlich ist er bei der Stadtverwaltung Mailand archiviert. 
Schulen einen sprachlichen Einstufungstest durchführen und/oder vorbereitende Einführungskurse ins Italienische anbieten, bevor die neu Hinzugekommenen am regulären Unterricht teilnehmen, werden sie anderswo direkt dem normalen Lehrbetrieb ausgesetzt. Generell bemüht man sich, die Lernenden nicht zu weit zurückzustufen, da es für ihre psychosoziale Entwicklung bedeutsam sei, dass sie mit möglichst gleichaltrigen Klassenkammeraden lernen. Dennoch kommt es nicht selten vor, dass allochthone Schülerinnen und Schüler die Bänke mit zwei Jahre jüngeren Klassenkameraden teilen (Favaro 2010b, S. 8).

In einem Land, dessen geringe Investitionen in Forschung und Bildung chronisch beklagt werden (z.B. Simone 1998, S. 39 f.; Renzi 1998, S. 54 f.; Piemontese 2014, S. 10) ${ }^{38}$, stehen an vielen Schulen keine Mittel zur Verfügung (oder werden keine freigesetzt), um den Neuankömmlingen zusätzliche Sprachförderungsmaßnahmen anzubieten. Teilweise wird deshalb auf ehrenamtliche Helfer und pensionierte Lehrpersonen zurückgegriffen, die als kulturelle Mediatoren fungieren und bei der Kommunikation mit den Familien helfen, Intensivkurse in Italienisch als Fremdsprache anbieten oder Neuankömmlinge während des regulären Unterrichts neben der Lehrkraft begleiten. Innerhalb des Lehrkörpers kommt diese Aufgabe auch den Unterstützungslehrkräften zu, welche eigentlich für die Inklusion und Begleitung Lernender mit Lernbeeinträchtigungen zuständig sind. Schulen mit einem konsistenteren Anteil von Kindern und Jugendlichen mit Migrationshintergrund können außerdem sogenannte facilitatori (Erleichterer) oder insegnanti su progetto (Projektlehrer) bei der Schulbehörde anfordern, welche entweder einige Stunden pro Woche parallel zum regulären Unterricht mit diesen SchülerInnen arbeiten oder ihre Eingliederung in den Klassenverband begleiten können. Während eine solche Unterstützung für viele Lernende einen Gewinn darstellt, da ihre Sprachbildung gradueller erfolgen und eine vertraulichere Atmosphäre geschaffen werden kann, weist sie allerdings auch Schwächen auf (Favaro 2001, S. 58):

I punti deboli di questo insegnante "aggiuntivo" sono rintracciabili soprattutto nella mancanza di competenza e di formazione specifica per l'insegnante dell'italiano come seconda lingua, nella precarietà e nell'ambiguità del ruolo e della collocazione e nei rischi di delega del "problema stranieri", da parte degli insegnanti di classe e della scuola, al collega distaccato.

38 Es kritisiert beispielsweise Piemontese ,l'insufficienza di investimenti adeguati nel campo dell'istruzione e della ricerca, fatte, invece, oggetto di sistematiche riduzioni di fondi e di tagli lineari. Nuovi insegnanti, migliori strutture, attrezzature e spazi più adeguati sembrano essere diventati un lusso che l'Italia di oggi-e forse ancora più quella di domani - non può permettersi“ “(Piemontese 2014, S. 10; m.Ü.: den Mangel an angemessenen Investitionen im Bereich der Bildung und der Forschung, die hingegen von systematischen Kürzungen der Mittel und linearen Einschnitten betroffen sind. Neue Lehrer, bessere Strukturen, Ausstattungen und angemessenere Räumlichkeiten scheinen ein Luxus geworden zu sein, den sich das heutige Italien - und vielleicht noch mehr das Italien von morgen - nicht erlauben kann). 
(M.Ü.: Die Schwachpunkte dieses ,hinzugefügten“ Lehrers sind vor allem im Fehlen der Kompetenz und einer spezifischen Ausbildung zum Lehrer für Italienisch als Zweitsprache, in der Präkarität und in der Ambiguität der Rolle und der Stellung und in den Delegierungsrisiken des „Ausländerproblems“" vonseiten der Lehrer der Klasse und der Schule an den externen Lehrer auszumachen.)

Schulen, die keinen Anspruch auf diese facilitatori und Projektlehrer erheben können, bauen oft auf gemeinnützige Träger, die nachmittags mit ehrenamtlich arbeitendem Personal Sprachkurse und Hausaufgabenhilfe anbieten. Besonders negativ macht sich die Kostenminimierung auch deshalb bemerkbar, weil Italienisch als Zweitsprache derzeit in Italien weder als Studien- noch als Unterrichtsfach existiert, sondern nur qualifizierende Kurse bestehen. In erster Linie werden diese von Personen besucht, welche später an privaten Sprachschulen unterrichten, während sich angehende und bereits im Dienst stehende Lehrpersonen der allgemeinbildenden Schulen in ihrer Aus- oder in Weiterbildungen gar nicht bzw. nur marginal mit diesem Themenkomplex befassen.

Die Anwesenheit von nicht in der Erstsprache Lernenden führt oft dazu, dass Lehrkräfte für die sprachlichen Herausforderungen, die sie und die verwendeten Lehrmittel an ihre Klassen stellen, sensibilisiert werden (Grassi et al. 2003, S. 7). Tendenziell trägt als ein weiterer Umstand zur Sprachsensibilisierung der im Interesse der vorliegenden Studie stehenden Erdkundelehrkräfte bei, dass sie in derselben Klasse auch die Fächer Italienisch und Geschichte erteilen. Aufgrund dieser verbindlich vorgeschriebenen Fächerkombination, welche eben Italienisch beinhaltet, kann davon ausgegangen werden, dass diese Lehrerinnen und Lehrer über eine vergleichsweise solidere sprachwissenschaftliche Ausbildung verfügen und ihren Unterricht in den drei Fächern auch nach sprachdidaktischen Aspekten koordinieren. Darüber hinaus besteht für sie durch die insgesamt zehn Wochenstunden, welche sie mit ihrer Fächerkombination in den einzelnen Klassen unterrichten, der Vorteil, einen größeren Zeitraum als die anderen Lehrpersonen mit den Lernenden zu verbringen und dadurch deren konkrete Sprachkompetenzen potentiell besser einschätzen zu können.

Die traditionelle Ausrichtung der schulischen Sprachbildung auf die literarisch-bürokratische Schriftsprachlichkeit der Standardvarietät (Gensini 2010, S. 156) schlägt sich bis heute sowohl im Stellenwert als auch in der Beschaffenheit der Lehrbücher nieder. Diese stehen fächerübergreifend im Mittelpunkt der Unterrichtsgestaltung, bei der es primär um eine gemeinsame Erarbeitung der Lehrbuchtexte geht. Dessen Inhalte werden anschließend zunächst von den Schülerinnen und Schülern in Eigenarbeit wiederholt und dann regelmäßig von den Lehrkräften in mündlichen Prüfungen abgefragt. Aufgrund dieser didaktischen Zentralität wird im Folgenden der Forschungsstand bezüglich der sprachlichen Gestaltung der Lehrmittel vorgestellt. 


\subsection{Das Unterrichtswerk und seine Schwächen als zentrales Lehrinstrument}

Rispetto agli altri paesi di cultura europea e d'oltreoceano, l'Italia si caratterizza generalmente per la sua cronica disattenzione per la fruibilità dei testi, vista in relazione agli obiettivi (informativi, formativi, regolativi, ecc.) e ai destinatari.

(M.Ü.: Im Vergleich zu den anderen Kulturländern Europas und Übersees zeichnet sich Italien generell durch seine chronische Unaufmerksamkeit für die Verwendbarkeit der Texte in Bezug auf die Absichten (informative, erzieherische/bildende, regulative etc.) und die Adressaten aus.)

(Piemontese \& Cavaliere 1997, S. 221)

Für die Erarbeitung der Lehrbuchtexte ist eine Lesekompetenz erforderlich, deren Ausbildung in den ministerialen Richtlinien für die Mittelschule für alle Fächer vorgeschrieben ist (MIUR 2012b): Die Lernenden sollen am Ende der Sekundarstufe I verschiedene Textsorten lesen können und sich Techniken wie das Unterstreichen, Stichwörter an den Rand schreiben ${ }^{39}$ und Notizen verfassen angeeignet haben, welche ihnen das Textverständnis erleichtern. Funktionale Texte sollen in alltäglichen Situationen gebraucht und darlegende Texte so erarbeitet werden können, dass sowohl ihr expliziter als auch ihr implizierter Inhalt klar ist. Die Lesekompetenz der Schülerinnen und Schüler ist so zu festigen, dass sie einen geübten Umgang mit den Lehrbüchern ermöglicht; das heißt, sie soll die Lernenden zur Informationsentnahme aus der Gesamtheit der Buchelemente befähigen (Inhaltsverzeichnis, Stichwortregister, Kapitel, Texte, Bilder, Diagramme usw.). Überdies sollen die Lernenden auch in der Lage sein, zu einem bestimmten Thema verschiedene Quellen zu vergleichen, die wesentlichen Informationen zu entnehmen und sie in Form von Listen, Schaubildern, Tabellen oder Zusammenfassungen zu ordnen.

Das Textverständnis muss für diverse Textsorten gefördert werden; neben literarischen Texten, die im Erdkundeunterricht (fast) keine Bedeutung haben, zählen deskriptive und argumentative dazu. Dieselben Textsorten sollen die Lernenden zusätzlich zum kreativen Schreiben auch selbst verfassen können (ibid., S. 34).

Die Umsetzung dieser Vorgaben ist jedoch aufgrund der Tatsache erschwert, dass die meisten Lehrwerke so komplizierte Texte enthalten, dass die Lehrkräfte ihren SchülerInnen eine eigenständige Bearbeitung gar nicht zutrauen (vgl. Bertocchi 2003, S. 22 in Bezug auf Geschichtsbücher der Sekundarstufe I und Piemontese \& Cavaliere 1997 sogar für die Lehrbücher der

39 Auch aus diesem Grund erachten es viele Lehrpersonen für wichtig, dass die Lehrbuchautoren von ihrer gängigen Praxis Abstand nehmen, ihre Texte mit zahlreichen fettgedruckten Wörtern und zusammenfassenden Schlüsselwörtern am Textrand auszustatten, da diese den Lernenden ihre Arbeit abnehmen. Das Textverständnis kann eher gesichert werden, wenn die Schülerinnen und Schüler diese Strukturierungstätigkeiten selbstständig ausüben müssen. S.a. Kap. 7 und Kap. 9. 
Grundschule $^{40}$ ). Diese Komplexität resultiert aus einer Akkumulation von Fachinhalten und einer sprachlichen Darbietung, die sich durch niedrigfrequenten, abstrakten und teilweise gehobenen Wortschatz, viele Fachtermini, grammatische Metaphern, eine ausgeprägte Hypotaxe, ein breites Spektrum an verbalen Tempora und Modi sowie einen im Vergleich zum Mündlichen stark erhöhten Passivgebrauch auszeichnet (Jafrancesco 2002, Favaro 2015, S. 6 f.). Beides, die inhaltliche Fülle und die Komplexität der Texte, ist vermutlich eine Konsequenz der langwährenden Auffassung der Autoren und Verleger, das Unterrichtswerk habe auch der Lehrkraft zu dienen, welche es als primäre Informationsquelle nutzt, um über die zu vermittelnden Fachinhalte zu verfügen, bzw. diese zumindest aufzufrischen (De Mauro 1997, S. 31). Denn gerade bezüglich der für die vorliegende Studie interessanten Lehrprofile mit drei Schulfächern ist eine vollständige Aneignung der zu lehrenden fachlichen Inhalte in Erdkunde, Geschichte und Italienisch nicht durch das Studium garantiert.

Wie in einem Beitrag von Arpaia et al. des GISCELs Piemont (1997) resümiert wird, konnten die SchulbuchautorInnen trotz der anhaltenden Kritik bislang nicht zu einer Veränderung der Lehrbuchgestaltung bewegt werden. Die Festlegung der Zielgruppe führe dazu, dass sich neben Bemühungen um eine sprachliche Darbietung, welche das Textverständnis der Lernenden gewährleistet, eine Art Diskurs der Lehrbuchautoren als Experten in den Text einflechte, der sich an andere Experten wie Verleger, Lehrbuchautoren, Dozenten, Lehrpersonen und Studierende richtet (ibid., S. 256). Die durch diese Zielgruppenorientierung entstehende „Ambiguität" und „Komplexität“ (ibid.) der Texte wird dann zu einem Problem, wenn die Lernenden im Umgang mit den Lehrmitteln so überfordert sind, dass sie bei der Texterschließung grundsätzlich auf die Hilfe der Lehrkräfte angewiesen sind und Schwierigkeiten zeigen, die Textinhalte mündlich oder schriftlich mit eigenen Worten wiederzugeben.

Mit der Textkomplexität ist außerdem ein oft als zu groß kritisierter Umfang der Unterrichtswerke verwoben. Denn sie bestehen in der Regel aus mehreren Hundert Seiten der Größe Din A4, die von den Lehrkräften im Laufe eines Schuljahrs so vollständig wie möglich behandelt werden. Hier liegt einer der Gründe, warum das Ausmaß der Unterrichtswerke zur kognitiven Überladung und Belastung der Lernenden führen kann (De Mauro 1997, S. 32). Auch durch textimmanente Aspekte kann dies verursacht werden, etwa durch die w.u. vorgestellten Mängel in der Verknüpfung von Textaussagen, welche die Lernenden bei der Unterscheidung wesentlicherer Informationen von weniger relevantem Detailwissen erheblich beeinträchtigen können.

40 Bei der 1993 durchgeführten Studie stand die Lesbarkeit von vier Grundschullehrbüchern im Fokus, welche Calò und Piemontese mithilfe der Lesbarkeitsformel GULPEASE, einer Berechnung, welcher Anteil des verwendeten Wortschatzes nicht im Gebrauchswortschatz (VDB) von De Mauro (1980) enthalten ist, und Lückentexten (jedes sechste Wort getilgt) für Lernende an drei römischen Schulen bestimmt haben. 
Schon in den 1960er Jahren gab in Italien die unverhältnismäßige Länge der Lehrbücher sowie die adressatenunangemessene Verwendung eines Wortschatzes, welcher die Fähigkeiten der Lernenden überstieg, Anlass zu Kritik aus den Forschungsreihen. Denn die benutzten Ausdrücke stammten oft aus niedrigfrequenten Bereichen des Wortschatzes und zeugten von einer Prädilektion der fachsprachlichen Klassifizierung. Gleichzeit erfolgte keine von pädagogischen Absichten geleitete systematische Erweiterung des Lerner-Wortschatzes. So wurde beispielsweise eine Untersuchung veröffentlicht, die ergab, dass Lesebücher für den Anfängerunterricht in den Vereinigten Staaten weniger als ein Drittel der Länge entsprechender italienischer Lehrwerke umfassten und weniger neue Wörter einführten. Diese stammten aus frequenter gebrauchten Wortschatzbereichen und wurden zudem drei- bis viermal öfter wiederholt als die in den italienischen Büchern verwendeten Ausdrücke (Bascetta 1964, S. 103 f.).

Auch nach der Umsetzung der Forderung, zwei unterschiedliche Ausgaben des Lehrwerks zu entwickeln, eine für die Lehrkraft und eine für die Lernenden, hat sich an der Dimension der Schülerausgabe nichts verändert. Dabei wurde bereits vor 20 Jahren dazu geraten, die Lehrbuchinhalte deutlich zu reduzieren: ,[C] i è necessaria una scuola che punti sulla qualità delle capacità acquisite dagli allievi e non sulla quantità di nozioni peregrine immagazzinate e di campi del sapere da cui quelle nozioni provengono" (De Mauro 1997, S. 32; m.Ü.: [W]ir brauchen eine Schule, welche auf die Qualität der von den Schülern erworbenen Fähigkeiten setzt und nicht auf die Quantität von abgelagerten eigenartigen Kenntnissen und Wissensfeldern, von denen diese Kenntnisse herrühren). Es ist daher denkbar, dass der fortwährende große Umfang der Lehrmittel von den Verlagen beabsichtigt wird. Diese profitieren schließlich davon, dass die Schulbücher in Italien von den Familien der Lernenden erworben werden müssen und sich mit größeren Ausmaßen höhere Einnahmen erzielen lassen. Außerdem könnte darin auch einer der Gründe liegen, warum sie so konzipiert sind, dass ihre Lebensdauer in der Regel nur fünf bis sechs Jahre beträgt (Simone 1997, S. 483).

Umfang und Komplexität der Unterrichtswerke werden auch darauf zurückgeführt, dass die Lehrbuchtexte normalerweise mittels einer Überarbeitung fachwissenschaftlicher Texte entstehen (Altichieri, Colmelet \& Deon 1997, S. 191). Dieses Vorgehen, bei dem die Texte nur in Ansätzen dem kognitiven Horizont der Lernenden angepasst werden, verleite dazu, sie mit Inhalten und interpretativen Thesen zu bestücken, welche dem gebildeteren Teil der Leserschaft zeigen sollen, dass seine AutorInnen die fachinhaltlichen Aspekte ihrer Materie besser beherrschen, als sie in einem für SchülerInnen bestimmten Text zum Ausdruck bringen können (Fortino, Stancati \& Vercillo 1997, S. 203). Die fehlende pädagogische Sensibilität der Lehrbuchautoren ist emblematisch für die Bedeutung pädagogisch-didaktischer Zielsetzungen und Konzepte in Italien, die auch in der Lehrerausbildung eher eine Nebenrolle 
spielen. So wird der Schreibstil vieler LehrbuchautorInnen als Anpassung an allgemein vorherrschende Gepflogenheiten interpretiert. Hierdurch zeige sich ihre Verbundenheit mit der dominanten Tradition, die einer ,demokratischen Art zu schreiben“ nicht dienlich sei (Arpaia et al. 1997, S. 257 f.; m.Ü.). Dort, wo generell Mängel in den schriftsprachlichen Kompetenzen beklagt werden, die u.a. auf die literarisch geprägte Tradition der Schreibdidaktik zurückzuführen sind, werden auch die LehrbuchautorInnen nicht ausgespart.

In einem anderen Kontext (Italienisch als Fremdsprache und die Umsetzung von Vorgaben des Europäischen Referenzrahmens für Sprachen, der im Sprachunterricht den Gebrauch authentischer Texte vorsieht) wird die sprachliche Komplexität des heutigen Italienischen folgendermaßen beschrieben (Vedovelli 2002, S. 80 f.):

[...] [T] eniamo presente le specifiche caratteristiche della lingua italiana, una lingua in movimento, in trasformazione, che proprio gli anni ottanta-novanta arriva ad una condizione di neostandardizzazione e di uso diffuso entro la nostra società. [...] L'italiano, per le sue dinamiche e tensioni in un processo di rinnovamento in quanto lingua d'uso, manifesta ancora oggi gravi carenze funzionali proprio nella struttura della comunicazione sociale [...]. La lingua italiana della comunicazione sociale, che coinvolge i testi della burocrazia, delle norme e delle leggi, degli avvisi al pubblico ecc., è ancora oggi una lingua ampiamente caratterizzata da tratti di formalità che la rendono lingua di una varietà alta e specialista, se non addirittura gergale e criptica.

(M.Ü.: Vergegenwärtigen wir uns die spezifischen Merkmale der italienischen Sprache, einer Sprache in Bewegung, in Umwandlung, die in den 80er und 90er Jahren den Zustand einer Neostandardisierung und eines verbreiteten Gebrauchs innerhalb unserer Gesellschaft erlangt. [...] Das Italienische, das sich wegen seiner Dynamiken und Spannungen in einem Prozess der Erneuerung als Gebrauchssprache befindet, zeigt noch heute schwerwiegende funktionale Mängel gerade in der Struktur der gesellschaftlichen Kommunikation. Die italienische Sprache der gesellschaftlichen Kommunikation, welche die Texte der Bürokratie, der Regeln und Gesetze, der öffentlichen Mitteilungen etc. betrifft, ist noch heute eine Sprache, die weitgehend durch formelle Züge bestimmt ist, die sie zur Sprache einer gehobenen und fachsprachlichen Varietät machen, wenn nicht gar zu einer jargonhaften und kryptischen.)

Viele Studien zu den Lehrwerken berufen sich nicht nur auf einen generellen Eindruck der untersuchten Werke, sondern stützen sich auf genauere sprachwissenschaftliche Analysen der Lehrbuchtexte, die Aufschluss über Aspekte wie den Aufbau des verwendeten Wortschatzes, die Satzlänge und andere Indikatoren für Textkomplexität geben. 1986 fand in Rom eine Tagung zum Thema Lesbarkeit und Textverständnis statt, bei der Ergebnisse der ersten Anwendungen von Lesbarkeitsformeln auf das Italienische vorgestellt wurden (De Mauro, Piemontese \& Vedovelli 1986), denen gemein war, dass Lehrbücher für den schulischen Unterricht grundsätzlich niedrige Werte erzielten (Arpaia et al. 1997, S. 241). Zehn Jahre später war die achte Nationaltagung des GISCELs dem Thema Sprache in den Lehrmitteln gewidmet. Dort wurden Studienergebnisse präsentiert (Tagungsband Calò \& Ferreri 1997), die auf empi- 
rischen Untersuchungen kleinerer Erhebungsgruppen (z.B. das Textverständnis der Lernenden aus insgesamt sechs Klassen bei Cavaliere (1993)) basieren, sich Analysemitteln wie der w.u. vorgestellten Lesbarkeitsformeln und der Wortschatzkategorisierung von De Mauro (1980) bedienen und die weitere Untersuchungen nach sich zogen (z.B. Brasca et al. 2014, La Grassa \& Troncarelli 2014).

Eine Studie des GISCELs in der Region Venetien (Verona) hat das Textverständnis von knapp 90 Schülerinnen und Schülern in sechs Klassen der Sekundarstufe II untersucht und sich dabei vor allem auf den Wortschatz konzentriert. Die Lernenden sollten in acht Auszügen aus insgesamt sechs Lehrbüchern für Geschichte und Literatur diejenigen Ausdrücke unterstreichen, welche ihnen Verständnisschwierigkeiten bereiten. Die Wortschatz- und Textkomplexität wurde danach beurteilt, wie viele Lernende bestimmte Ausdrücke unterstrichen, welche Bedeutung diese Ausdrücke für das Verständnis eines ganzen Absatzes oder Textes haben und wie viele Wörter insgesamt in einem Absatz oder Text unterstrichen wurden. Die GISCEL-Gruppe kommt zu dem Ergebnis, dass etwa 30 Prozent der SchülerInnen ihre Unterrichtswerke nicht oder falsch verstehen (Compagni, Lunardon, Mastella \& Sofia 1997, S. 264) und dass sich die Schwierigkeiten dort häufen, wo besonders viele Nominalisierungen auftreten und die syntaktische Komplexität zunimmt (ibid., S. 274).

In einer anderen Untersuchung (Deon 1997) werden einige Geschichtsbücher der Sekundarstufe I als schwieriger verständlich als die entsprechenden Bücher für die Sekundarstufe II bewertet und für die oft mittelmäßigen, wenn nicht schlechten Leistungen der Lernenden im Fach Geschichte verantwortlich gemacht (ibid., S. 45). Anhand eines Experiments, in dem Geschichtsexperten wissenschaftliche Texte umschreiben sollen, bis sie von Länge und Inhalt her einem Lehrbuchtext entsprechen, wird ein Urteil getroffen, dass generell für die Texte in den Unterrichtswerken gelte, und zwar, dass sie zwar kürzer sind und auf den ersten Blick lesbar wirken, aber eine hohe Informationsdichte aufweisen und Verständnisschwierigkeiten bereiten (ibid., S. 47 f.):

Quel che colpisce sono le medesime cose che colpiscono in una qualsiasi pagina manualistica: l'astrattezza, l'assenza di precisi riferimenti temporali e di luogo, la mancanza di notizie su concreti aspetti di vita quotidiana [...].

Il distillato finale è un concentrato che risulta significativo [...] solo se ha a monte la lettura e la comprensione profonda del testo combinato di partenza. Così come appare, è certo una pagina che presenta difficoltà di comprensione e quindi di studio.

(M.Ü.: Das, was auffällt, sind dieselben Sachen, die auf einer jedweden Lehrbuchseite auffallen: die Abstraktheit, die Abwesenheit konkreter zeitlicher und räumlicher Bezüge und das Fehlen von Anmerkungen über konkrete Aspekte des alltäglichen Lebens.

Das Enddestillat ist ein Konzentrat, das nur dann eine Bedeutung bekommt [...], wenn ihm eine Lektüre und ein profundes Verständnis des Ausgangstextes vorausgehen. So, wie es erscheint, ist es sicherlich eine Seite, die Verständnisschwierigkeiten und somit Lernschwierigkeiten bereitet.) 
Eine Studie über Physikbücher analysiert ihre sprachliche Gestaltung und bewertet sie negativ, da sie mit aller Kraft mit einem möglichst präzisen und nicht konnotierten Wortschatz ausgestattet seien. ${ }^{41}$ Dieser ist hauptsächlich fachsprachlicher Art, woraus eine lexikalische Armut bei gleichzeitiger Verwendung als überflüssig zu betrachtender Fachausdrücke entstehe. Diese Art der Buchgestaltung habe eine gravierende Konsequenz (Battimelli 1997, S. 178):

Ho indicato così una doppia povertà del manuale di fisica, imputabile ad uno svuotamento e ad un'assenza. Il prosciugamento del vocabolario utilizzato rende il testo noioso, la mancata esplicitazione delle regole di senso lo fa diventare incomprensibile. È appena il caso di sottolineare che, se è vero che il linguaggio è un potente strumento di unione/divisione, allora il fallimento dei manuali nel rendere accessibili le strutture fondanti del linguaggio scientifico li rende pesantemente corresponsabili di quella che deprechiamo come selezione scolastica. Non è detto che sia un prezzo necessario da pagare.

(M.Ü.: Ich habe so eine doppelte Armut des Physikbuchs aufgezeigt, welche einer Entleerung und einer Abwesenheit zuzuschreiben ist. Das Austrocknen des verwendeten Wortschatzes macht den Text langweilig, die fehlende Erklärung der Sinnregeln macht ihn unverständlich. Es ist angebracht zu unterstreichen, dass, wenn es wahr ist, dass die Sprache ein mächtiges Instrument der Vereinigung/Separation ist, das Scheitern der Unterrichtswerke beim Zugänglichmachen der tragenden Strukturen des wissenschaftlichen Sprachgebrauchs die Lehrbücher stark mitverantwortlich für das macht, was wir als schulische Selektion missbilligen. Es ist nicht gesagt, dass dieser Preis notwendigerweise zu zahlen ist.)

Zum Teil sind die Lehrwerke so komplex gestaltet, dass bei empirischen Studien zum Textverständnis der Lernenden etwa bei Philosophiebüchern eine eher für Studierende als für Schülerinnen und Schüler geeignete Konzeption festgestellt wurde (Fortino et al. 1997) und die Lehrbücher zum Teil im Studium weiter genutzt werden. ${ }^{42}$

Paradoxerweise entsteht ein Gutteil der sprachlichen Hürden, die nach wie vor in den Lehrwerken aufgespürt werden (Alfieri 2005, S. 409; Bertocchi

41 S. Battimelli (1997, S. 176; Hervorhebung im Original): „[I manuali] suggeriscono piuttosto l'idea che il linguaggio della fisica sia essenzialmente composto da una costellazione di termini rigidamente definiti, e da regole di assemblaggio dei medesimi che si riducono alle istruzioni formali di quello che chiamerò [...] "matematichese" La struttura di un manuale di fisica si presenta cosi come una ponderosa collezione di definizioni, teoremi e dimostrazioni, ed esso apparirà tanto più apprezzabile - perché più "rigoroso" - quanto più sarà pieno di simboli e equazioni e quanto meno spazio dedicherà alle parole" (M.Ü.: [Die Lehrbücher] suggerieren vor allem die Idee, dass die Sprache der Physik im Wesentlichen aus einer Konstellation starr definierter Termini und Regeln ihrer Zusammensetzung bestehe, die sich auf die formalen Anweisungen dessen reduzieren, was ich [...] „Mathematikisch“ [Mathematiksprache] nennen werde. So zeigt sich die Struktur eines Physiklehrbuchs als eine schwere Sammlung von Definitionen, Theoremen und Beweisen, und es erscheint umso schätzenswerter - weil ,rigoroser“ -, je mehr Symbole und Gleichungen es enthält und je weniger Raum den Wörtern gewidmet ist).

42 So wollten sie über zu viele Themen informieren und enthielten sie Interpretationsansätze, die nicht darauf verzichteten, den Erfahreneren unter den Lesern, wie den Lehrpersonen und Dozenten, ihr Wissen zu demonstrieren. Es bestehe also der pragmatische Fehler, dass die eingeschränkteren Kompetenzen der primären Empfänger des Textes, die Lernenden, beim Verfassen nicht berücksichtigt würden (Fortino et al., S. 203). 
2003, S. 20 f.), gerade dann, wenn versucht wird, den Umfang des Buches zu verringern. Denn dieser Prozess führt nicht automatisch zu einer effektiven Reduzierung der Inhalte, sondern, im Gegenteil, zu einer Informationsverdichtung, wodurch die Lesbarkeit und das Textverständnis zusätzlich beeinträchtigt werden können (Piemontese \& Cavaliere 1997, S. 22). Bevor weiter unten zwei wesentliche Instrumente zur sprachlichen Erforschung der Lehrwerke vorgestellt werden, sollen im Folgenden zunächst einige Hintergrundinformationen zu den gesellschaftlichen und (bildungs)politischen Umständen gegeben werden, unter denen die Lehrmittelforschung erfolgt. Es kann nämlich davon ausgegangen werden, dass sie die Ursache für die Polemik sind, die so bezeichnend für viele Veröffentlichungen dieser Forschungsrichtung ist.

\subsubsection{Kritik an Lehrmitteln und Bildungspolitik in der italienischen Forschung}

Il problema della leggibilità si [...] rivela particolarmente spinoso in presenza di fratture di tipo [...] socioculturali e socio-linguistiche. Le prime (legate alla sfasatura tra linguaggi speciali e linguaggio quotidiano) sono comuni a tutte le società ad alto tasso di terziarizzazione. Le seconde sono presenti specialmente in paesi a basso livello di scolarità. L'Italia ha il privilegio mondiale di essere in un regime di coppia massima dei due tipi di difficoltà: massima sofisticazione tecnologico-terziaria e massima persistenza di bassa scolarità.

In questo contesto è abbastanza comune trovarsi di fronte a libri di testo o manuali che dovrebbero essere diretti a ragazzi e che invece presentano difficoltà anche per un lettore esperto.

(M.Ü.: Das Problem der Lesbarkeit [...] offenbart sich als besonders heikel in Gegenwart von Brüchen [...] soziokultureller und soziolinguistischer Art. Die ersten (an die Zerfahrenheit zwischen Fachsprachen und Alltagssprache gebunden) sind allen Gesellschaften mit einem hohen Grad an Tertiarisierung gemein. Die zweiten sind vor allem in Ländern mit einem niedrigen Niveau an Beschulung präsent. Italien hat das weltweite Privileg, in einem Regime des Maximalpaares der beiden Schwierigkeitsarten zu sein: maximale technologisch-tertiäre Sophistizierung und maximaler Fortbestand einer niedrigen Beschulung.

In diesem Zusammenhang ist es nicht ungewöhnlich, Lehrwerke oder Handbücher vor sich $\mathrm{zu}$ haben, die an Kinder und Jugendliche gerichtet sein müssten und die stattdessen auch einem erfahrenen Leser Schwierigkeiten bereiten.)

(Lucisano \& Piemontese 1988, S. 110)

Seit mehr als vierzig Jahren gibt es in Italien einen in der allgemeinen Sprachwissenschaft verorteten Forschungszweig, der sich mit der Beschaffenheit der Lehrmittel und der schulischen Sprachbildung auseinandersetzt (s. als einen der ersten Bascetta 1964; außerdem z.B. De Mauro 1976b, 1979, 1980, 1985; Thornton 1984; Lumbelli 1984a, 1984b, 1989; Zambelli 1994; Calò \& Ferreri 1997; Lavinio 2005). In dieser Tradition wurden und werden vor allem ihre sprachlichen Mängel betont und der Umstand kritisiert, dass sie schlecht auf ihre primären Adressaten, die Lernenden, zugeschnitten sind. Außerdem wird 
den Verantwortlichen für die Lehrbuch- und Sprachbildungsgestaltung ein nachlässiges, wenn nicht gar beabsichtigtes Handeln unterstellt.

Das Interesse für den Gebrauchswert und die Lesbarkeit der Lehrmittel entwickelte sich zu einem Zeitpunkt, zu dem stärker ins Bewusstsein rückte, dass weite Teile der Bevölkerung nur über eine geringe Schulbildung verfügten und ihre sprachlichen Kompetenzen sie nicht befähigten, etwa amtliche Schreiben oder über das Lokalblatt hinausgehende Zeitungen zu verstehen. 1971 waren über fünf Prozent der Bevölkerung Analphabeten, und weitere 27 Prozent konnten zwar lesen und schreiben, hatten aber keinen Schulabschluss. Die Schulbildung der meisten (44,3\%) beschränkte sich auf den Besuch der Grundschule, weitere 14,7 Prozent verfügten über einen mittleren Schulabschluss, während weniger als 9 Prozent das Abitur erlangt bzw. studiert hatten und weniger als zwei Prozent der Bevölkerung einen akademischen Abschluss erreichten (ISTAT 2012). Das Sprachkapital breiter Bevölkerungsschichten divergierte stark von dem der führenden Schicht, so dass Erstere nur eingeschränkt am gesellschaftlichen Treiben teilhaben konnten. Die herkömmliche Sprachbildung in der Schule schien das Ziel zu verfehlen, ausreichend solide sprachliche Fähigkeiten herauszubilden, so dass eine Bewegung ins Rollen kam, die für ein radikales bildungspolitisches Umdenken eintrat. Diesem lag der Wunsch einer Demokratisierung der Schulbildung und der öffentlichen Kommunikation zugrunde, wodurch eine Brücke zu den sprachlichen Ausgangslagen der Mehrheit der Bevölkerung geschlagen werden sollte.

Zum einen wurde wiederentdeckt, was während des Faschismus in Vergessenheit geraten war, und zwar, dass die Vermittlung sprachlicher Fähigkeiten ausgehend von einer Valorisierung des bereits vorhandenen sprachlichen Kapitals in all seinen soziolinguistischen und dialektalen Varietäten erfolgen sollte (Gensini 2010). Zum anderen wurde eine Annäherung der Amtssprache an die Alltagssprache der Bevölkerung erstrebt. Infolgedessen begann man, die Sprache der Lehrmittel und der öffentlichen Kommunikation zu untersuchen, stieß auf Lexeme und Redewendungen, die dem Sprachgebrauch der Bevölkerung überhaupt nicht entsprachen, und bemühte sich um Neuformulierungen. Dies waren die Gründungsjahre des GISCELs und die Anfänge der italienischen Lesbarkeitsforschung (Vacca 1972; De Mauro, Piemontese \& Vedovelli 1986; Piemontese \& Lucisano 1988). Hier wurden Instrumente zur Analyse der Textkomplexität wie Lesbarkeitsformeln entwickelt und das Ermitteln eines italienischen Gebrauchswortschatzes vorangetrieben, Texte umgeschrieben und vereinfacht, seit 1980 erschien eine Verlagsreihe mit Sachbüchern in vereinfachter Sprache (Libri di base (Grundlagenbücher), Editori Riuniti) und ein Jahrzehnt später wurden Richtlinien für die Lesbarkeit und das Verständnis der Texte der öffentlichen Verwaltung formuliert (Piemontese \& Tiraboschi 1990; Zuanelli 1990; Piemontese 1993).

Der polemische Ton, welcher sich durch die wissenschaftlichen Veröffentlichungen zum schulischen Sprachunterricht und zu den Lehrbüchern zieht, ist 
möglicherweise der Tatsache zuzuschreiben, dass der Einsatz für eine Demokratisierung der Sprachbildung und öffentlichen Kommunikation zwar von vielen WissenschaftlerInnen und Lehrpersonen geteilt wurde, aber auf politischer Ebene und innerhalb der Schulbuchverlage nicht die erwünschte Resonanz erfuhr. So bemerken beispielsweise Lucisano und Piemontese (1988, S. 112):

T. De Mauro (1980) in Guida all'uso delle parole, polemizzando con i sostenitori del parlar difficile afferma che 'non pare esserci limite noto alla possibilità di oscurare un discorso e di rubare agli altri la possibilità di capirlo senza lunghe e spesso poco utili riflessioni' (p. 150).

(M.Ü.: T. De Mauro (1980) äußert in Guida all'uso delle parole mit den Verfechtern des Schwierigsprechens polemisierend, dass, es keine bekannte Grenze für die Möglichkeit zu geben scheint, einen Diskurs zu verdunkeln und den anderen die Möglichkeit zu rauben, ihn ohne lange und oft unnütze Überlegungen zu verstehen“ (S. 150).)

Obwohl die Lehrpläne Kerngedanken dieser neuen Sprachbildung aufnahmen und ein gutes Jahrzehnt lang die Entwicklung von Italienischlehrbüchern und Schulgrammatiken beobachtet werden konnte, die eine weniger literarisch und stärker gebrauchsorientierte Sprachbildung verfolgten, was sich in der Auswahl der Texte und der Darbietung der Themen niederschlug (s. z.B. Il libro d'italiano (Simone 1974)), flaute diese Modernisierungswelle zu Beginn der 1990er Jahre schon wieder ab (Arpaia et al. 1994, S. 258).

An der sprachlichen Gestaltung der Lehrmittel und anderer Gebrauchstexte änderte sich also nur wenig und nur vorübergehend, so dass der anfängliche Elan in eine andauernde Unzufriedenheit mit den soziopolitischen Gegebenheiten umschlug, welche in den Publikationen zur Sprachbildung und den Lehrmitteln bis heute explizit zum Ausdruck gebracht wird (Gensini 2005, S. 54; Piemontese 2014, S. 10). Der anhaltende anklagende Unterton in den wissenschaftlichen Arbeiten, der sich gegen Verleger, Lehrbuchautoren und (Bildungs-)Politiker richtet, ist auf diese Situation zurückzuführen. Ihre hier vorgenommene Schilderung in groben Zügen dient dem Zweck, Bewegründe für die in den w.o. zitierten und in den noch folgenden Textpassagen enthaltene Polemik besser nachvollziehen zu können, um den Blick auf die sprachbildungsbezogenen Aussagen darin nicht zu verstellen. Dazu zählt auch der in Kapitel 2.2 vorgestellte und scheinbar nicht leicht zu zerstreuende Verdacht, die führende Gesellschaftsschicht interessierte sich nicht für die Bildung und Emanzipierung der einfachen Bevölkerungsschichten, bzw. wollte diese bewusst so wenig gebildet wie nötig halten (De Mauro 1976a).

Die in den Zehn Thesen des GiSCELs (1975) getroffene und den Diskurs dieser Jahre über die schulische Sprachbildung bestimmende Wortwahl beinhaltet Begriffe wie „Demokratie“, „sprachliche Grundrechte“ und „Semianalphabetismus", die aus der zeitlichen Distanz heraus abgenutzt klingen mögen. In Educazione linguistica vent'anni dopo e oltre (Ferreri \& Guerriero 1998; 
Sprachbildung 20 Jahre später und darüber hinaus) gehen die Herausgeberinnen dem nach und kommen zu dem Schluss, dass weder die in den 1970er Jahren diskutierten Themen noch die mit ihnen verbundenen Schlagwörter an Aktualität eingebüßt haben (ibid., S. 9) ${ }^{43}$ :

Per le comunità di vecchio e nuovo insediamento sul territorio nazionale vale il principio della tutela delle loro lingue di origine; nei confronti dei figli diseguali della nostra società si opera ancora nel tentativo di attrezzarsi per riequilibrare le opportunità; per combattere il semianalfabetismo dentro e fuori la scuola si stenta a trovare soluzioni soddisfacenti. La rilettura di parole consunte, parole degli anni Settanta, fa riscoprire sensi perduti [...].

L'educazione linguistica riacquista, riconquista il suo spessore e se ne comprende la democraticità. Non legata al tempo che fu, l'espressione educazione linguistica democratica ha sapore di futuro: le distinzioni di lingua non possono costituire ostacolo al diritto di partecipazione alla vita individuale e collettiva; è necessario - oggi più di ieri - rimuovere gli ostacoli che si frappongono all'uguaglianza del diritto alla parola per tutti i membri di una comunità sociale.

(M.Ü.: Für die alt und neu eingesessenen Gemeinschaften auf nationalem Gebiet gilt das Prinzip des Schutzes ihrer Herkunftssprachen; gegenüber den ungleichen Söhnen unserer Gesellschaft versucht man immer noch, sich auszurüsten, um die Chancen wieder auszugleichen; um den Semianalphabetismus in und außerhalb der Schule zu bekämpfen, müht man sich ab, zufriedenstellende Lösungen zu finden. Das erneute Lesen abgenutzter Wörter, Wörter der Siebzigerjahre, lässt verlorene Bedeutungen wiederentdecken [...].

Die Spracherziehung gewinnt, erobert ihre Größe zurück, und man versteht ihren demokratischen Gehalt. Nicht an vergangene Zeiten gebunden, hat der Ausdruck demokratische Spracherziehung Zukunft: Die Sprachunterschiede dürfen kein Hindernis für das Recht der Teilhabe am individuellen und kollektiven Leben darstellen; es ist notwendig - heute mehr als damals - die Hindernisse, die sich zwischen die Gleichheit des Mitspracherechts für alle Mitglieder einer Gesellschaft stellen, zu beseitigen.

In demokratischen Ländern, in denen ein Recht auf Bildung und auf freie Meinungsäußerung herrscht, klingt es in den Ohren vieler grotesk, wenn eine demokratische Sprachbildung gefordert wird, weil davon ausgegangen wird, dass diese längst praktiziert werde. Doch so lange Selektionsmechanismen bestehen, die Lernenden etwa schon zu Beginn der Sekundarstufe I unterschiedliche Schulformen zuweisen und damit maßgeblich über ihre berufliche Zukunft mitentscheiden und in der Schule vor allem die vorankommen, die das nötige Bildungskapital bereits mitbringen (Passaponti 1980, S. 53 zit. in Piemontese \& Cavaliere 1997, S. 223), ist die Chancengleichheit im Bildungssystem nicht gegeben und damit sein demokratisches Selbstverständnis in Frage zu stellen (s.a. Maas 2008, S. 175 f., S. 422 f.).

Im Folgenden werden zwei wesentliche Untersuchungsgegenstände der Lehrbuchforschung vorgestellt: zum einen ihre Gestaltung auf lexikalischer Ebene und zum anderen Studien, die sich mit der Lesbarkeit der Texte befassen

43 S. Piemontese und Cavaliere als Beispiel für weitere AutorInnen, die mit großem zeitlichen Abstand zu den Zehn Thesen weiterhin Ausdrücke wie „Demokratie“ und „,demokratische Kommunikation“ im Zusammenhang mit der Sprachbildung verwenden (Piemontese \& Cavaliere 1997, S. 221). 
und im Zuge derer eine Forschungsmethode entwickelt wurde, mit der vor allem die Verknüpfung der Textaussagen untersucht wird.

\subsubsection{Der Wortschatz als Gegenstand der Lehrmittelforschung}

Fundamental für das Textverständnis ist eine solide lexikalische Kompetenz. Lange Zeit stand im Vordergrund der Spracherwerbsforschung die Aneignung morphosyntaktischer Fähigkeiten (Meara, Lightbown \& Halter 1997, S. 28), doch alles Wissen um Grammatik ist nichts, wenn ,in der aktuellen Sprechsituation nicht auf geeignetes lexikalisches Wissen zurückgegriffen werden kann. ,Das reine Satzschema ohne Worterfüllung ist ein genau so bestandsunfähiges Moment wie irgendeine Relation ohne Fundamente' (Bühler, 1982, S. 75)“ (Ott 1997, S. 29). In diesem Sinne kommt dem mentalen Lexikon ${ }^{44}$ eine zentrale Rolle sowohl bei der Produktion als auch bei der Rezeption von sprachlichen Äußerungen und im Spracherwerb bzw. -ausbau zu (Barrett 1999).

Ein Vorreiter italienischer Studien, die sich mit der lexikalischen Gestaltung von Lehrbuchtexten befassen, ist eine Untersuchung der 1960er Jahre, bei der in Geschichts-, Naturwissenschafts- und Lesebüchern für die Grundschule u.a. der verwendete Wortschatz analysiert wurde (Bascetta 1964). Registriert wurde eine Anhäufung von Fachausdrücken und einem literarisch geprägten Wortschatz; die Fachtermini waren vielmals nicht erklärend eingeführt und ihre (zirkuläre) Wiederholung unterblieb. Stattdessen wurden sie nur ein einziges Mal oder sehr selten verwendet, was aus didaktischer Perspektive wenig lernförderlich ist (ibid., S. 26). ${ }^{45}$ Der Verfasser dieser Studie stützt sich bei der

44 Dieser Begriff bezeichnet den Wortschatz, über den ein Sprecher verfügt. Einzelne Ausdrücke sind dabei nicht wie in einem Lexikon ,untereinander“ oder sequenziell abgespeichert, sondern miteinander vernetzt, z.B. durch semantische Wortfelder und Kollokationen, in denen die Wörter vermehrt auftreten. Das mentale Lexikon ist offensichtlich keine statische Einheit, sondern verändert seine Dimensionen im Laufe einer Sprachbiographie. Längst nicht alle Wörter, die ein Sprecher kennt, werden von ihm auch aktiv benutzt, so dass das rezeptive mentale Lexikon in der Regel wesentlich größer ist als das produktive. Aber auch in der Breite ist der Wortschatz hinsichtlich seiner einzelnen Bestandteile unterschiedlich ausgeprägt. Während z.B. für manche Wörter nur ihre Kernbedeutung bekannt ist, werden andere einschließlich aller oder vieler ihrer Bedeutungen beherrscht. Zum Auf- und Ausbau des mentalen Lexikons siehe auf Deutsch z.B. Das Lexikon im Spracherwerb (Meibauer \& Rothweiler 1999) und die vielen Beiträge hierzu in Lexikologie, Lexicology. Ein internationales Handbuch zur Natur und Struktur von Wörtern und Wortschätzen (Cruse 2002) sowie auf Italienisch Laudanner und Burani (1993), Baldi $\left(2008^{2}\right)$ und Kapitel 6.1 in Koesters Gensini (2009).

45 S. z.B. über einige Geschichtsbücher für die Grundschule (Bascetta 1964, S. 26; Hervorhebung im Original): „Dio solo sa a quale efficacia didattica possano mirare le liste di termini, e perché bambini di otto anni debbano ingozzare in meno di venti righe (v. F, p. 70): tunica, toga, mantello, pileo, galero, pètaso, peplo, palla, borchie; oppure la lista data in meno di 
Bewertung der Wortschatzkomplexität auf sein eigenes Sprachempfinden und nutzt außerdem in Ermangelung eines italienischen Äquivalents französische Untersuchungen zur Gebrauchsfrequenz des Wortschatzes (ibid., S. 100 ff.). So überträgt er den in französischen Lehrbüchern verwendeten Grundwortschatz ins Italienische und kontrolliert sein Vorkommen in den von ihm untersuchten Lehrwerken. Bascetta kommt zu dem Ergebnis, dass nur ein Bruchteil der verwendeten Wörter dem italienischen Grundwortschatz entspringt.

Aus der sich dann auch in Italien entwickelnden wortschatzbezogenen Frequenz- und Gebrauchsforschung ist ein sehr umfangreiches Gebrauchswörterbuch hervorgegangen, dessen Entstehung auf einem bedeutenden Korpus an geschriebenen und gesprochenen Texten unterschiedlicher Gattungen basiert, das Grande dizionario italiano dell'uso (Großes italienisches Gebrauchswörterbuch) (De Mauro 1999/2000). Da es sich hierbei um ein nützliches Instrument handelt, mit dessen Hilfe verschiedenste Lehrwerke auszugshaft analysiert wurden, und das auch in der vorliegenden Studie bei der Kategorisierung des Wortschatzes diente, den die von mir beobachteten Lernenden produzierten und der in ihren Erdkundelehrwerken enthalten ist, wird sein Aufbau im nächsten Unterkapitel beschrieben.

dieci righe $\mathrm{da} C$, p. 66: vestibolo, atrio, impluvio, tablinio, viridario, cubicolo, per non parlare poi di tutto il riassunto dell'Iliade e dell'Odissea in 40 righe $(F, p p$. 62-63) dove si leggono nell'ordine: Omero, Ilio, Iliade, Troia, Agamennone, Achille, Teti, Ulisse, Itaca, Penelope, Telemaco, Priamo, Ettore, Andromaca, Astianatte, Paride, Elena, Menelao, Patroclo, Vulcano; di questo passo non resta che proporre come libro di testo il vocabolario, perché lì c'è tutto (o quasi tutto) quello che bisognerebbe sapere... " (M.Ü.: Gott allein weiß, welche didaktische Wirksamkeit die Wörterlisten anstreben könnten und warum achtjährige Kinder dies in weniger als 20 Zeilen verdauen müssen (s. F, S. 70): Tunika, Toga, Umhang, Pileus, Galero, Petasos, Peplos, Palla, Metallverzierungen; oder die von C in weniger als zehn Zeilen gegebene Auflistung, S. 66: Vestibulum, Atrium, Impluvium, Tablinium, Viridarium, Cubiculum, um nicht von der ganzen Zusammenfassung der Ilias und Odyssee in vierzig Zeilen zu sprechen, wo man der Reihe nach lesen kann: Homer, Ilion, Ilias, Troja, Agamemnon, Achilleus, Thetis, Odysseus, Ithaka, Penelope, Telemachos, Priamos, Hektor, Andromache, Astyanax, Paris, Helena, Menelaos, Patroklos, Vulcanus; nach diesem Abschnitt bleibt nichts anderes übrig, als das Wörterbuch als Unterrichtswerk vorzuschlagen, denn dort gibt es alles (oder fast alles), was man wissen müsste...) und über den naturwissenschaftlichen Teil einiger Lehrwerke (ibid., S. 60 f.): „Debbo innanzi tutto ricordare la vana audacia, comune a molti autori di questi libri, di aver tentato di concentrare in quaranta pagine, la misura media in estensione della sezione di scienze nei sussidiari, la metà a dir poco dello scibile umano" (M.Ü.: Ich muss erst einmal an die einfältige Kühnheit erinnern, die vielen Autoren dieser Bücher gemein ist, versucht zu haben, auf vierzig Seiten, dem durchschnittlichen Ausmaß des naturwissenschaftlichen Teils der Lehrbücher, um untertrieben die Hälfte des menschlichen Wissens zu formulieren). 


\subsubsection{Die Wortschatzetikettierung im Gradit und ihr Nutzen für die Sprachbildungsforschung}

Das umfassendste Wörterbuch zum aktuellen Gebrauch der italienischen Sprache, in dem alle Lemmata korpusbasiert nach der Häufigkeit ihres Vorkommens und ihrer Streuung in unterschiedlichen Texttypen gesprochener und geschriebener Sprache mit einer Gebrauchsmarke versehen sind, ist das Grande dizionario italiano dell'uso (De Mauro 1999/2000), kurz GRADIT. Dieses umfangreiche Projekt nahm seine Anfänge in den 1970er Jahren und führte u.a. zur Entwicklung eines ersten Grundwortschatzes, der aus einer Liste von rund 6.700 Wörtern bestand (De Mauro 1980). Für seine Ermittlung stützte De Mauro sich auf das Frequenzwörterbuch der italienischen Sprache LIP von Bartolini, Tavaglini und Zampolli (1971) und berechnete den Gebrauchswert der Wörter als Produkt ihrer Frequenz und ihrer Streuung in unterschiedlichen Texten und Kommunikationssituationen. Zur selben Zeit entstand auch der erste Gebrauchswortschatz der „Disponibilität“, das heißt, von Ausdrücken, die nicht oder kaum geschrieben werden, aber den Italienischsprechenden dennoch zur Verfügung stehen.

Bei der für das GRADIT vorgenommenen Gruppierung des Wortschatzes wird von einem inneren Kern ausgegangen, der über 90 Prozent aller Wörter eines jeglichen Textes ausmacht (De Mauro 1998, S. 78), dem aus ca. 2.000 fundamentalen Wörtern bestehenden lessico fondamentale (Fo). Hierunter finden sich kurze Wörter wie Artikel, Präpositionen und viele Konjunktionen, Nomen und Verben, von Letzteren insbesondere die sehr polysemischen wie andare (gehen), avere (haben), dare (geben), dire (sagen) und fare (machen).

Der fundamentale Wortschatz (Fo) bildet zugleich den innersten Kern von ca. 7.000 Wörtern des Vocabolario di Base oder Grundwortschatzes, kurz VDB, zu dem auch die Gruppen des alto uso (AU, ca. 3000 Wörter), des hohen Gebrauchs, sowie der alta disponibilità (AD), der hohen Verfügbarkeit, gerechnet werden. Diese letzte Gruppe besteht aus Wörtern, die in gesprochenen und geschriebenen Texten des Korpus zwar nicht sehr stark vertreten sind und generell wenig verwendet werden, aber eng mit unserer Alltagswelt verknüpft sind. Sie werden daher oft gedacht und sollten jedem Sprecher zur Verfügung stehen (s. z.B. De Mauro 2003, S. 162). Beispiele hierfür sind die Lexeme forchetta (Gabel), parcheggio (Parkplatz), ragno (Spinne) und sugo (Soße).

Studien haben ergeben, dass der Großteil des fundamentalen Wortschatzes (Fo) von allen erwachsenen Sprechern des Italienischen beherrscht wird, die mindestens eine elementare Schulbildung von fünf Jahren genossen haben. Wer hingegen auch die mittlere Reife nach insgesamt acht Schuljahren erlangt hat, sollte zusätzlich den AU-Wortschatz beherrschen (De Mauro 1998a, S. 78). Deshalb ist es ein Bildungsauftrag der Schule, den gesamten Basiswortschatz zu vermitteln, was aber nach Meinung von Pädagogen und Sprachwissenschaftlern nicht ausreichend erreicht wird und vielleicht daran liegt, dass in 
den Lehrplänen zwar generell die Erweiterung des Wortschatzes verankert ist, aber nicht genauer expliziert ist, auf welche Weise dies geschehen soll (Ferreri 2005, S. 37).

$\mathrm{Um}$ den Grundwortschatz $(\mathrm{VDB}=\mathrm{FO}+\mathrm{AU}+\mathrm{AD})$ herum platziert sich eine sehr große Gruppe von fast 50.000 Wörtern, die dem allgemeinen Wortschatz (lessico comune, $\mathrm{CO}$ ) zugeordnet ist. Hierbei handelt es sich nach De Mauros Definition um allgemeinsprachliche Ausdrücke, die jedem, der eine mittlere oder höhere Schulbildung genossen hat, unabhängig von seinem Beruf und seiner geographischen Herkunft innerhalb Italiens bekannt sein und zum Gebrauch zur Verfügung stehen sollten (De Mauro 2005, S. 60).

Daran angrenzend befinden sich zum einen die als Fremdwörter gekennzeichneten Lexeme (esoterismi, Es), die selten gebrauchten (lessico di basso uso, $\mathrm{BU}$ ) sowie vorwiegend literarisch gebrauchte (lessico letterario, LE) und zum anderen die zahlreichen Fachwortschätze (lessico tecnico-specialistico, Ts), die nach ihrem jeweiligen Fachgebiet durch einen entsprechenden adjektivischen Zusatz in der Gebrauchsmarke gekennzeichnet sind. So ist etwa das Lemma fonema (Phonem) mit der Marke ,TS ling.“ (linguistischer Fachwortschatz) und equatore (Äquator) mit der Marke „TS geogr.“ (geographischer Fachwortschatz) aufgeführt.

Diesen beiden Beispielen ist außerdem eine andere häufig zu beobachtende Eigenschaft der Fachsprachenwörter gemein, nämlich, dass sie mit unterschiedlichen Bedeutungsakzentuierungen in mehreren Fachsprachen verwendet werden und/oder auch zum allgemeinen Wortschatz zählen. So ist equatore gleichzeitig ein Co-Lexem und Teil der Fachsprache der Biologie und wird fonema auch den Fachsprachen der Physiologie, der historischen Sprachwissenschaft und der Psychologie zugerechnet.

In Kapitel 7.4.1.1 findet sich zur Klassifizierung der in der Lehrbuchuntersuchung fokussierten sprachlichen Elemente u.a. eine Tabelle, in der alle vertretenen Gebrauchsmarken inklusive derer zahlreicher Fachsprachen mit den deutschen Übersetzungen aufgelistet sind.

Neben den niedrigfrequenten und fachwortschatzsprachlichen Wörtern können in den Lehrbüchern auch Ausdrücke zu Verständnisschwierigkeiten führen, die dem Grundwortschatz entstammen, aber in bestimmten Kontexten (z.B. dem Geschichts-, Erdkunde- oder naturwissenschaftlichen Unterricht) eine spezifische Bedeutung annehmen. Derartige Ausdrücke, derer polysemischen Gestalt sich nicht alle Lernenden bewusst sind, gehören zu dem, was Ehlich (1999) als ,alltägliche Wissenschaftssprache“ bezeichnet und was nicht nur für in der Zweitsprache Lernende oder in einer Fremdsprache Studierende, sondern auch in der Erstsprache zu einem Problem werden kann. Dies ist ebenfalls für das Italienische beobachtet worden (Piemontese \& Cavaliere 1997, S. 226; Ferreri 2005, S. 119, S. 146 f.; Favaro 2015). So ergaben Untersuchungen, dass die Lernenden weniger Verständnisschwierigkeiten mit monosemi- 
schen Wörtern aus dem Fachwortschatz wie beispielsweise acrocoro (Hochebene) oder clima monsonico (Monsunklima) haben als mit Ausdrücken des Grundwortschatzes, die mehrere verschiedene Bedeutungen tragen (Favaro 2015, S. 6), wie etwa carta (Papier, u.a. auch geogr. Karte; mit 9 verschiedenen Bedeutungen im GRADIT (1999/2000) eingetragen) und scala (Treppe, u.a. auch Maßstab; 11 Bedeutungen im GRADIT).

Ein Gebiet, auf dem die Kategorisierung des Gebrauchswortschatzes aus dem GRADIT genutzt wird, ist die Lehrbuchforschung. Mit Rückgriff darauf untersuchen beispielsweise Piemontese und Cavaliere die Lesbarkeit von Lesebüchern für die Primarstufe, indem sie u.a. den Anteil des verwendeten Wortschatzes berechnen, der nicht im VDB enthalten ist (Cavaliere 1993; Piemontese \& Cavaliere 1997). Die Gruppe Verona des GISCELs Venetien kommt durch Untersuchungen zu dem Ergebnis, dass SchülerInnen nicht nur der in den Lehrbüchern enthaltene Fachwortschatz, sondern auch der Grundwortschatz teilweise Schwierigkeiten bereitet (Compagni et al. 1997, S. 286 f.). Den schulischen Fachwortschatzerwerb unter die Lupe nehmend, analysieren Miglietta und Sobrero (2008) jeweils ein Kapitel aus zwei Unterrichtswerken mit naturwissenschaftlichen und eines mit geographischen Inhalten und stellen fest, dass viele der verwendeten Wörter nicht im Grundwortschatz (VDB) enthalten sind. Auch die lombardische Gruppe des GISCELs (Brasca et al. 2014, S. 80 f.) sowie La Grassa und Troncarelli (2014, S. 294-296) untersuchen u.a. Texte verschiedener Lehrbücher der Grund- und Mittelschule auf ihre Zusammensetzung aus Wörtern des VDBs und stellen fest, dass ein Anteil von 15 bis 19 Prozent aller verwendeten Wörter nicht hieraus stammt. Es handelt sich vor allem um fachsprachliche, für ganze Konzepte stehende Ausdrücke, deren Anteil deutlich über dem liegt, was eigentlich für einen fachspezifischen Text zu erwarten ist (ibid., S. 294 f. mit Verweis auf Ferreri 2005). Die Autoren kommen deshalb zu dem Ergebnis, dass die dadurch entstehende hohe Informationsdichte sowie Wortschatzlücken der Lernenden die Ursache für eine erhebliche Beeinträchtigung des Textverständnisses sein können, zumal die Ausdrücke und Konzepte zum Teil unangemessen oder gar nicht erklärt sind (ibid., S. 296). Vor allem wenn im Lehrbuch kein Glossar oder erklärende Fußnoten gegeben sind, sei es für die Lernenden unmöglich, die Bedeutung der Wörter eigenständig zu erschließen, so dass sie auf spezifische Eingriffe der Lehrkraft angewiesen seien, um den Wortschatzerwerb solider zu gestalten und den $\mathrm{Zu}-$ gang zum Erwerb des fachinhaltlichen Wissens nicht zu verstellen (ibid.).

Nach diesen Studien scheinen die Lehrwerke nach wie vor widerzuspiegeln, was Renzi 1997 in Bezug nicht nur auf die schulischen Lehrmittel folgendermaßen formuliert hat (Renzi 1997, S. 507):

Sono convinto che sottoposti alle stesse prove di comprensibilità, agli stessi esami di contenuto, molti libri non di testo, compresi dei libri di una certa fama, cadrebbero. In particolare la complicazione lessicale e quella sintattica sono un'antica eredità della nostra cultura $[\ldots]$. 
Non sono scritti in modo difficile, spesso inutilmente difficile, solo i libri di testo. I libri di testo non fanno che riflettere tendenze dominanti. Molta saggistica letteraria, filosofica, linguistica è altrettanto poco pianificata e poco chiara dei libri di scuola [...].

(M.Ü.: Ich bin davon überzeugt, dass viele Bücher, nicht nur die Lehrbücher, einschließlich der Bücher von gewissem Ansehen, durchfielen, wenn sie denselben Verständniskontrollen, denselben Prüfungen des Inhalts unterlägen. Insbesondere die lexikalische und die syntaktische Kompliziertheit sind ein antikes Erbe unserer Kultur [...].

Schwierig, oft unnötig schwierig sind nicht nur die Lehrwerke geschrieben. Die Lehrwerke reflektieren lediglich dominierende Tendenzen. Viele literarische, philosophische, linguistische Sachbücher sind genauso wenig strukturiert und klar wie die Schulbücher [...].)

\subsubsection{Untersuchungen zur Lesbarkeit}

Die Analyse des Wortschatzes, der einen Text ausmacht, ist ein wesentliches Kriterium zur Bestimmung seiner Lesbarkeit. Ein weiteres dafür eingesetztes Instrument aus der statistischen Linguistik sind sogenannte Lesbarkeitsformeln, deren Entwicklung ihren Ursprung zu Beginn des 19. Jahrhunderts in den Vereinigten Staaten nahm. Vor allem die dort von Flesch (1948) elaborierte Formel wurde einige Jahrzehnte später auch in Europa als Ausgangspunkt zum Aufstellen entsprechender Formeln für andere Sprachen als das Englische genommen (z.B. Richaudeau 1969 für das Französische, Togeby 1971 für das Dänische, Vacca 1972 und Vacca \& Franchina 1986 für das Italienische sowie Groeben 1978 für das Deutsche).

Die ersten italienischen Studien zur Verständlichkeit von Texten, die mit Lesbarkeitsformeln operierten (Fiorucci 1982; Thornton 1984), fanden also zeitgleich zur Entwicklung des Gebrauchswortschatzes statt. Durch diese Untersuchungen sowie in der Forschungstätigkeit der 1985 gegründeten universitären Arbeitsgruppe GULP (Gruppo Universitario Linguistico Pedagogico; universitäre linguistisch-pädagogische Gruppe) unter der Leitung von Corda Costa und De Mauro traten diverse Probleme bei der Anwendung der Formel und des Gebrauchswortschatzes hervor, die zur ihrer Weiterentwicklung führten.

Die 1988 daraus hervorgegangene Formel GULPEASE (Lucisano \& Piemontese 1988) basiert auf einer Untersuchung mit insgesamt 850 Lernenden der fünften, achten und 13. Klasse, bei der Texte aus Lehrbüchern dieser drei Klassenstufen aus den Bereichen Geschichte/Politik, Erzählungen/Literatur sowie Naturwissenschaft überprüft wurden. Für die Textanalyse wurde auf den Gebrauchswortschatz, die Flesch-Formel und auf linguistische Kriterien wie die Satz- und Wortlänge und die Anzahl von Eigennamen, Daten, Abkürzungen, Siglen und grammatischen Morphemen zurückgegriffen, um einen Lesbarkeitsindex zu entwerfen. Zum Überprüfen des Textverständnisses wurden anhand von drei Fragebögen Daten über den sozioökonomischen familiären Hintergrund der Lernenden, die Einschätzung der Textschwierigkeit durch die Lehrpersonen und die Lesegewohnheiten der Erhebungsgruppen (neben den 
Schülern auch eine Vergleichsgruppe von Erwachsenen) erfasst und Wortschatz-, Lücken- sowie Multiple-Choice-Tests zum Inhalt der Lehrbuchtexte durchgeführt. Bei den Wortschatztests mussten die Lernenden einen Satz bzw. eine Wortdefinition um das fehlende Wort ergänzen und unter vier möglichen Definitionen eines Wortes die passende auswählen. Auf diese Weise ist die GULPEASE-Formel entstanden, anhand der einerseits Aussagen über die Lesbarkeit eines Textes getroffen werden können und andererseits über den Schwierigkeitsgrad für die Lesenden (leicht lesbar: unabhängige Lektüre; mit Unterstützung lesbar: Schullektüre; nicht lesbar: „Frustrations-Level“ (Lucisano \& Piemontese 1988, S. 122)). Hierbei war dem Forschungsteam, das an der Entwicklung des Indexes beteiligt war, bewusst, dass die Auswahl der zugrundeliegenden Indikatoren problematisch ist. Zum Beispiel ist die Länge eines Satzgefüges nicht automatisch proportional zu seiner Schwierigkeit, die hingegen sehr vom hypotaktischen Aufbau und der Art der verwendeten Konnektoren abhängt. Aus diesem Grund wurde bestrebt, zur Optimierung der Formel auch auf weitere textimmanente Variablen einzugehen, deren Identifikation durch die Studien von Lucia Lumbelli vorangetrieben wurde (ibid., S. 113).

Lumbelli (1989) hat eine kognitivistische Untersuchungsmethode zur Bestimmung der Textkomplexität entwickelt, welche von der Einsicht ausgeht, dass zwischen den Kenntnissen des Autors und denen der Lesenden eine Asymmetrie besteht, die im Falle der Lehrwerke besonders groß ist. Sie bemüht sich daher nachzuvollziehen, welche Textmerkmale das Verständnis behindern können. Darauf hinweisend, dass Schwierigkeiten beim Leseverständnis oft an der Lexik festgemacht werden, anstatt beim Verfassen auch die Verwendung anderer sprachlicher Gestaltungsmittel zu kontrollieren, die einen Text kompliziert machen können (ibid., S. 20 f.), fasst sie diese in einer als offen zu betrachtenden Liste von sieben wesentlichen Kriterien zusammen. Davon sind gerade die ersten drei paradoxerweise nicht selten in Texten zu finden, um das Verständnis zu fördern, obwohl sie genau das Gegenteil bewirken. Allesamt sind sie als sprachlich-kognitive Hürden weniger leicht auszumachen als beispielsweise fachsprachlicher und niedrigfrequenter Wortschatz oder die Satzlänge, weil erfahrene und gebildete Leser sich nicht oder nur kaum an ihnen stören (ibid., S. 44-49):

- aggiunta relativizzante (relativierende Ergänzung) - ein oder mehrere Sätze, die für eine vereinfachende Darstellung eines Sachverhalts hinzugefügt werden (oft in Klammern) und ergänzende Informationen geben können, da der Autor befürchtet, seine Beschreibung könnte als unvollständig oder zu allgemein aufgefasst werden. Diese Ergänzungen sind allerdings oft nur für auf dem Gebiet erfahrene Leser und nicht für SchülerInnen verständlich;

- esempio difficile (schwieriges Beispiel) - Beispiele stellen normalerweise eine effektive Brückenfunktion zwischen abstrakten Informationen und 
dem lernenden Leser dar, sind also positiv, sofern sie keine Verständnisschwierigkeiten enthalten. Problematisch wird es, wenn der Bezug des Beispiels zum Vorausgehenden unklar bleibt und die im Beispiel enthaltenen Informationen und das, was sie erklären sollen, unverständlich sind. So können Beispiele das Textverständnis nicht unterstützen und/oder zusätzliche Schwierigkeiten beim Textverständnis verursachen;

- aggiunta problematizzante (problematisierende Ergänzung) - einzelne oder mehrere Wörter bzw. ganze Sätze, die ebenfalls oft in Klammern stehen und die Absicht verfolgen, das Vorausgehende klarer erscheinen zu lassen (z.B. welche präzise Bedeutung dem Genannten zuzuschreiben ist oder welche mögliche andere Bedeutung evtl. ausgeschlossen werden muss). Sie sind problematisch, wenn der Wissenshorizont des Lesers und seine Fähigkeit, die Ergänzung korrekt einzubeziehen, nicht bedacht oder falsch eingeschätzt wird. In diesem Fall wird den eventuell bereits vorhandenen Schwierigkeiten im Textverständnis eine weitere hinzugefügt;

- identità ostacolata (verhinderte Identität) - entsteht aus stilistischen Gründen, also nicht nur in Lehrbuch- und anderen gemeinverständlichen Texten, um Wiederholungen zu vermeiden. Problematisch wird es, wenn die intendierte Synonymität zweier Ausdrücke nicht erkannt wird, da hierfür Kenntnisse oder Mikro-Überlegungen nötig sind, die nur auf einer höheren Stufe kognitiver und linguistischer Fähigkeiten garantiert sind;

- nesso mal segnalato (schlecht signalisierte Verknüpfung) - resultiert ebenfalls oft aus stilistischen Gründen und ist eine unter- oder nebenordnende Verknüpfung, die geübten Lesern keine Schwierigkeiten bereitet, aber für schwächere Leser zum Hindernis werden kann, wenn sie die Verknüpfung nicht richtig deuten und dadurch das Textstück, das die Verknüpfung zusammenhalten sollte, anders lesen, als es beabsichtigt ist (,Una pioggia di ma, però, quindi, perciò, infatti, usati fuori posto, che dovrebbero essere sostituiti con termini più adatti." (ibid., S. 46; m.Ü.: Ein Regenguss von aber, jedoch, also, deshalb, in der Tat, die deplatziert gebraucht sind und durch angemessenere Ausdrücke ersetzt werden müssten));

- nesso non segnalato (nicht signalisierte Verknüpfung) - den Informationen in Sätzen folgen weitere Informationen, oft in einem neuen Absatz, ohne dass die Verknüpfung zum Vorausgehenden expliziert wird. Dieses Vorgehen ist nicht irreführend wie das des nesso mal segnalato, aber es unterstützt auch nicht das Textverständnis, da relativ große kognitive Anstrengungen erforderlich sein können, um die nicht gegebene Verknüpfung nachzuvollziehen;

- nesso distanziato (entfernte Verknüpfung) - zwei Informationen sind weit entfernt voneinander im Text formuliert, obwohl sie miteinander verbunden werden müssen. Damit dies gelingt, ist es erforderlich, die Textaussagen genau und kontinuierlich im Hinterkopf zu behalten, was gerade ungeübte Leser überfordert. 
Mithilfe dieses Katalogs an textimmanenten Verständnishürden sind in verschiedenen Studien Lehrbücher untersucht worden, die alle durch die hier vorgestellten Ursachen von Textkomplexität gekennzeichnet sind (s. z.B. Piemontese \& Cavaliere 1997; Fortino et al. 1997; Arpaia et al. 1997; Lavinio 2014).

\subsection{Fazit}

Das Vorhandensein der von Lumbelli ausgemachten sprachlichen Hürden beim Textverständnis konnte im Rahmen dieser Arbeit aufgrund des gewählten multiperspektivischen Blicks auf die sprachlichen Anforderungen und Leistungen im Fachunterricht nicht systematisch überprüft werden, wurde aber anhand einiger Beispiele für die fünf Unterrichtswerke belegt (s. Kap. 7). Eine sorgfältigere, vollständigere Analyse dieser sieben Hürden in den Lehrbuchtexten unterschiedlicher Fachrichtungen ist allerdings seinen zeitlichen Aufwand wert, um der Kritik an der sprachlichen Gestaltung der Lehrmittel eine weitere empirische Grundlage zu geben und die VerfasserInnen zu einer Überarbeitung und einer leserfreundlichen Veränderung ihres Schreibstils bei künftigen Publikationen anzuregen. Das ist auch deshalb erstrebenswert, da der Anteil zweisprachiger Schülerinnen und Schüler und sprachlich nicht so versierter autochthoner Lernender steigend ist und es nicht das Ziel sein kann, längerfristig eine Vereinfachung und Reduzierung der fachlichen und sprachlichen Inhalte im Unterricht anzustreben (z.B. Gibbons 2002, Favaro 2003, S. 16).

Was die übrigen in diesem Kapitel vorgestellten Kriterien der Textkomplexität anbelangt, die in den hier vorgestellten Studien hervorgetreten sind, ist festzuhalten, dass sie auch in den fünf von mir untersuchten Erdkundelehrwerken auftreten: Ein wesentliches Hindernis beim Textverständnis scheinen lexikalische Lücken zu sein. Diese nehmen vor allem dann zu, wenn die Texte nicht ausreichend aus Wörtern des fundamentalen (Fo) oder wenigstens des Gebrauchswortschatzes (VDB) zusammengesetzt sind, sondern einen erheblichen Anteil an fachsprachlichen Ausdrücken (Ts) und solchen aus dem allgemeinen Wortschatz (CO) aufweisen. Außerdem erschwert es das Textverständnis, wenn verschiedene komplexere Gestaltungsmerkmale gemeinsam auftreten, wie beispielsweise lange Sätze mit schlecht signalisierten Verknüpfungen, ein niedrigfrequenter Wortschatz, durch Appositionen oder Relativsätze ausgebaute Subjekte und andere Nominalgruppen, die für eine große Distanz zwischen Subjekt und Prädikat sorgen, und ein erhöhter Passivgebrauch.

Vor allem die unterschiedlichen Untersuchungen zur Verständlichkeit eines Textes verdeutlichen, dass es sich bei der Analyse sprachlicher Kompetenzen um eine vielschichtige Aufgabe handelt: Damit ein Text verstanden wird, reicht es nicht aus, die Länge seiner Sätze zu kontrollieren oder den Wortschatz 
zu bestimmen. Die Texte in naturwissenschaftlichen Lehrwerken etwa zeichnen sich oft durch kurze Sätze mit einem scheinbar einfachen Wortschatz aus, die dennoch schwierig zu verstehen sein können (Lucisano \& Piemontese 1988, S. 113). Deshalb ist es wichtig, dass u.a. auch der Textaufbau untersucht und darüber hinaus auf textexterne Merkmale wie den Schrifttyp, den Zeilenabstand und solche, welche die Lesenden charakterisieren, geachtet wird: In welchem Umfang wird gelesen? Was ist das Thema des Textes und wie verhält es sich zu den Interessen der Lesenden? Wie steht es um ihre Motivation, Konzentrationsfähigkeit, Lesekompetenz und ihr Hintergrundwissen (ibid., S. 110 mit Rückgriff auf Rye 1980)?

In meiner Studie spielt der Umgang mit den Lehrwerken eine zentrale Rolle bei der Beschreibung der sprachlichen Anforderungen, die an die beobachteten Lernenden gestellt werden, und dabei, wie sie ihnen gerecht werden, da die Unterrichtsgestaltung sich hauptsächlich auf die Erarbeitung der Lehrbuchtexte konzentrierte. Um so komplexe Gegenstände wie sprachliche Herausforderungen und Kompetenzen möglichst genau erfassen zu können, ist deswegen ein Ansatz gewählt worden, der sich dem Problem aus verschiedenen Richtungen nähert (s.a. Spolsky 1981, S. 17 f., 1995; McNamara 2001). Während sich die meisten im Vorfeld recherchierten italienischen Studien entweder nur mit der Gestaltung einiger Lehrwerke oder mit einzelnen Aspekten der sprachlichen Kompetenz wie dem Lernerwortschatz befassen und nur wenige Studien (GuLP; Piemontese \& Cavaliere 1997) beides in den Blick nehmen, ist für die vorliegende Erhebung entschieden worden,

- die verwendeten Lehrbücher zu analysieren und dabei vor allem mithilfe des GRADITs auf die Wortschatzverwendung zu achten, aber auch andere sprachliche Aspekte wie den Passivgebrauch, die Länge der Sätze und die Explizitheit von Verknüpfungen zwischen Satz- und Textaussagen zu berücksichtigen;

- den Erdkundeunterricht zu beobachten, um Erfahrungen über die Texterarbeitung einzuholen, die auf der einen Seite Auskunft über den rezeptiven und produktiven Umgang der Lernenden mit ihren Lehrwerken geben und auf der anderen Seite das Lehrerverhalten berücksichtige, um zu untersuchen, ob und ggf. wie sie den Textzugang zum Lehrbuch erleichtern und das Verständnis der Lernenden kontrollieren und garantieren;

- mithilfe eines C-Tests mit Auszügen aus den Lehrwerken das Textverständnis sowie produktive sprachliche Fähigkeiten der Lernenden zu überprüfen;

- anhand eines Fragebogens Informationen über die Lese- und Textlerngewohnheiten der Lernenden und darüber, wie sie ihr Erdkundebuch, den Unterricht und die Lehrperson bewerten, zu gewinnen;

- mithilfe einer Schreibaufgabe (lehrbuchähnliche Texterstellung) die produktiven (bildungs)sprachlichen Kompetenzen der Lernenden zu erfassen 
und Gemeinsamkeiten sowie Unterschiede in der sprachlichen Gestaltung auszumachen.

Bevor die Studie im Einzelnen vorgestellt wird, gibt das folgende Kapitel zunächst einige wesentliche Informationen über den Erhebungskontext. 


\section{Der Erhebungskontext}

In diesem Kapitel wird das italienische Bildungssystem in seinen für die Einordnung der Ergebnisse dieser Studie relevanten Zügen vorgestellt: die Lehrerausbildung (4.1), die geltenden Lehrpläne für das Fach Erdkunde (4.2.1) und die schulische Sprachbildung (4.2.2) sowie diesbezügliche ministeriale Richtlinien (4.2.2.1). Hieraus wird desweiteren mit der lexikalischen Kompetenz eine wesentliche Komponente der Sprachbildung umrissen (4.2.2.1.1). Das Unterkapitel 4.2.2.2 befasst sich mit den bestehenden Divergenzen zwischen ministerialen Vorgaben einerseits und Unterrichtspraxis sowie dem in der Lehrerausbildung Vermittelten andererseits. Im Folgenden wird auf den Registerausbau eingegangen, welcher während der Schulzeit vollzogen werden sollte. In diesem Zusammenhang ist das in Italien entwickelte Konzept des spazio linguistico (De Mauro 1980; sprachlicher Raum) von Interesse (4.2.2.3). Eine wesentliche Voraussetzung für das Gelingen des Sprachausbaus ist die Entwicklung protoliterater Fähigkeiten im Vorschulalter (4.2.2.3.1). Im Anschluss daran werden die Rolle von Sprachstandserhebungen und ihr eingeschränkter Einsatz in Italien erläutert (4.2.2.4). Welche spracherwerbstheoretischen (4.3.1) und sprachpädagogischen (4.3.2) Aspekte für die Lehrerausbildung von Bedeutung sind, wird in groben Zügen in 4.3 illustriert. Informationen über die Arbeitsbedingungen in den während der Studie beobachteten Klassen an drei verschiedenen Bildungsinstitutionen schließen das Kapitel ab (4.4).

\subsection{Das italienische Bildungssystem und die Lehrerausbildung}

Die Schulpflicht reicht in Italien von den fünf Jahren scuola elementare (Grundschule) über die drei Jahre der scuola media (Mittelschule) bis in die Sekundarstufe II, beträgt zehn Jahre, betrifft Kinder und Jugendliche im Alter von sechs bis 16 Jahren und hat entweder einen Abschluss der Sekundarstufe II oder eine abgeschlossene berufsqualifizierende Maßnahme von mindestens drei Jahren Dauer bis zum 18. Lebensjahr zum Ziel (MIUR 2012a). Dabei konzentriert sich die Erteilung des Erdkundeunterrichts hauptsächlich auf den Zeitraum des Mittelschulbesuchs (Sekundarstufe I); außerdem wird das Fach an einigen Schulen im letzten Jahr der Grundschule und - je nach Schultyp in einzelnen Jahren der Sekundarstufe II unterrichtet.

Die Erdkundelehrkraft unterrichtet in der Sekundarstufe I, also an der Mittelschule, in der Regel gleichzeitig Italienisch, Erdkunde und Geschichte. Was 
ihre Ausbildung anbelangt, so war als Zugangsqualifikation lange Zeit ein abgeschlossenes Studium fachaffiner Studienrichtungen (z.B. ein Physikstudium, um Physik, aber auch Mathematik zu unterrichten) ausreichend, so dass im heutigen Schulbetrieb noch immer Lehrende tätig sind, die während des Studiums nur wenige oder gar keine fachdidaktischen und pädagogischen Kenntnisse erworben haben. Gegen Ende der 1990er Jahre sind spezielle Lehramts-Aufbaustudiengänge eingerichtet worden, die von den SSIS (Scuola di specializzazione all'insegnamento secondario - Spezialisierungsschule für das Lehren im Sekundarstufen-Bereich) angeboten wurden und mit den Universitäten kooperierten. Diese Schulen von zweijähriger Dauer haben ihren Betrieb im akademischen Jahr 1999/2000 aufgenommen und 2008/2009 definitiv wieder eingestellt. Voraussetzung für die Teilnahme waren ein abgeschlossenes Studium und das Bestehen der Aufnahmeprüfung.

Seit dem akademischen Jahr 2011/2012 sind die SsIS durch das TFA (Tirocinio Formativo Attivo - bildendes aktives Praktikum) ersetzt, ein einjähriges Aufbaustudium an den Hochschulen, dessen Zugang durch Aufnahmeprüfungen und eine begrenzte Teilnehmerzahl reguliert wird und dessen Teilnahmebedingungen sowie Inhalte denen der SSIS ähneln: Neben fachdidaktischem, pädagogischem und inklusionsorientiertem Wissenserwerb müssen die angehenden Lehrpersonen ein Referendariat absolvieren und eine Abschlussarbeit verfassen. Nun sind auch die universitären Voraussetzungen zur Ausübung der Lehrtätigkeit stringenter geworden, indem während des Studiums je nach Unterrichtsfach bzw. Fächerkombination eine bestimmte Anzahl von bestandenen Prüfungen in genau festgelegten Modulen vorliegen muss. Dennoch beobachten sowohl Wissenschaftlerinnen und Wissenschaftler als auch Lehrkräfte mit Sorge, dass u.a. die sprachwissenschaftliche Ausbildung der Lehramtsanwärter nicht ausreichend ist (s. z.B. De Mauro 1998b, S. 27; Altieri Biagi 2005, S. 67 f.; Lavinio 2005, S. 152 f.). Denn nur die Sprachlehrkräfte und solche der geistes- und gesellschaftswissenschaftlichen Fächer müssen während des Studiums Kurse in Linguistik belegen, und selbst Lehrkräfte dieser Fächer haben oft keine fundierte sprachwissenschaftliche Ausbildung. ${ }^{46}$ Auch das Angebot an entsprechenden (finanzierten und wirksamen) Weiterbildungsmaßnahmen hält sich eher in Grenzen (Villarini 2001, S. 49 f.) ${ }^{47}$, so dass die einzelne Lehrkraft vor allem sich selbst überlassen ist, wenn sie sich angemessen auf die sprachlich heterogene Schülerschaft oder generell veränderte Unterrichtsbedingungen vorbereiten möchte.

46 S. hierzu auch Kap. 4.2.2: Bis vor einigen Jahren brauchten selbst Italienischlehrkräfte keine sprachwissenschaftlichen Prüfungen vorzuweisen (Lavinio 2005b, S. 153).

47 In der Sprachbildungsforschung wird jedoch die Auffassung vertreten, dass das, was die Lehrpersonen an Wissen für guten, sprachförderlichen Unterricht erwerben sollten, gar nicht durch Fortbildungsmaßnahmen geleistet werden könne, sondern im Laufe des Studiums entstehen und dort notwendigerweise zulasten anderer Ausbildungsbereiche gehen müsse (Maas 2008, S. 729). 
Hierzu muss gesagt werden, dass entsprechende Initiativen seitens der Lehrpersonen ein hohes Maß an Motivation erfordern, da die berufliche Lage vieler Lehrkräfte prekär ist, indem ihre Stellen zeitlich befristet sind und, auch wenn diese die Dauer eines Schuljahrs betragen, unklar ist, ob und wo sie im kommenden Schuljahr wiedereingestellt werden. Darüber hinaus beziehen auch fest angestellte Lehrpersonen ein im europäischen Vergleich für diese Berufsklasse niedriges Gehalt (Simone 1998, S. 39).

\subsection{Die aktuellen Lehrpläne für das Fach Erdkunde in der achten Klasse und Richtlinien zur schulischen Sprachbildung}

\subsubsection{Lernziele im Fach Erdkunde am Ende der Mittelschule}

Im Jahr 2012 sind neue Lehrpläne in Kraft getreten (MIUR 2012b), welche für das Fach Erdkunde in der dritten Klasse der Sekundarstufe I eine ganze Reihe von Kenntnissen und Kompetenzen voraussetzen. Auch wenn in ihnen nicht explizit auf die sprachlichen Anforderungen beim Erwerb und der Wiedergabe bzw. Anwendung geographischer Fachinhalte eingegangen wird, lassen sie doch erkennen, dass dafür ein hohes $\mathrm{Ma}$ an sprachlichen Fähigkeiten notwendig ist. So sollen die Lernenden sich nicht nur auf geographischen Karten verschiedenster Art orientieren können, sondern auch Italien, die europäischen Länder und die anderen Kontinente nach geopolitischen, wirtschaftlichen und sozialen Aspekten analysieren können und sich mit Problemen des Umweltschutzes auskennen. In den allgemeinen Richtlinien für die Spracherziehung in der Sekundarstufe I finden sich zudem Anweisungen hinsichtlich der Umsetzung sprachdidaktischer Aspekte in allen Fächern. Doch zunächst soll hier der gültige Lehrplan für das Fach Erdkunde vorgestellt werden.

In ihm sind Ziele für den Kompetenzerwerb bis zum Ende der Sekundarstufe I formuliert, welche die Orientierung auf geographischen Karten verschiedenster Art beinhalten. Zusammen mit der Fähigkeit, mithilfe unterschiedlicher Informationsträger wie aktuellen und historischen Photographien, Satellitenbildern, Schaubildern und statistischen Daten Kenntnisse über geographische Räume zu gewinnen, sollen die Lernenden imstande sein, angemessen über diese Räume kommunizieren zu können. Hierbei geht es nicht nur um physisch-geographische Aspekte, sondern auch um das kulturelle Erbe in Form von geschichtlichen, künstlerischen und architektonischen Errungenschaften und über den Einfluss des menschlichen Handelns auf die verschiedenen geographischen Regionen (ibid., S. 46-48).

Hierfür sind in den Lehrplänen Lernziele definiert, welche sich auf die folgenden Themenkomplexe beziehen (m.Ü.): 
- Orientierung;

- Sprache der „Geographizität“;

- Landschaft;

- Region und „territoriales System“.

Für den Erwerb von Orientierungsfähigkeiten ist das Erlernen des Kartenlesens vorgesehen, wobei die Kardinalpunkte bekannt sein, der Umgang mit dem Kompass gelernt werden und das Zurateziehen von Satellitenaufnahmen geübt werden soll.

Mithilfe der Sprache der „Geographizität“ sollen geographische Karten verschiedenster Art verhandelt und interpretiert werden. Hierzu müssen die Lernenden in der Lage sein, den Maßstab korrekt zu interpretieren, die verwendeten Symbole zu verstehen und mit geographischen Koordinaten zu arbeiten.

Bezüglich wesentlicher Merkmale der italienischen, europäischen und weltweiten Landschaften sollen die Lernenden dazu fähig sein, diese zu erkennen und miteinander zu vergleichen; außerdem sollen sie Kenntnisse über Themen und Probleme des Umweltschutzes sammeln und lernen, die Umwelt als kulturelles und natürliches Erbe aufzufassen, das es zu wertschätzen gilt. Dies kann durch die Entwicklung von (nicht näher definierten) Projekten zur Landschaftsvalorisierung geschehen.

Das Konzept der geographischen Region soll konsolidiert werden und ebenfalls auf Italien, Europa und die anderen Kontinente angewandt werden. Hierbei sind die Regionen nach geophysischen, geschichtlichen, klimatischen und wirtschaftlichen Gesichtspunkten vorzustellen. Die Lernenden sollen zudem über die Fähigkeit verfügen, die Beziehungen zwischen geographischen Regionen unter sozioökonomischen und demographischen Aspekten zu analysieren. Hierfür hat die Verwendung von Interpretationsmodellen zur Erklärung der territorialen Gestaltung wesentlicher Staaten sowie eine Bezugnahme auf ihre geschichtliche, politische und wirtschaftliche Entwicklung zu erfolgen. Ohne direkt darauf einzugehen, welche sprachlichen Mittel mit diesen Kompetenzen verbunden sind, ist doch an mehreren Stellen der Lernziele von der erstrebten Fähigkeit, diese geographischen Kompetenzen kommunizieren zu können, die Rede (ibid.).

Um so komplexe Kompetenzen, wie sie das Interpretieren von diversen Informationsträgern und das Konfrontieren von Ländern und Regionen auf soziopolitischer, geophysischer, gesellschaftlicher und wirtschaftlicher Ebene verlangen, erwerben und anwenden zu können, müssen die SchülerInnen über ein hohes Maß an Literalität verfügen, wie es beispielsweise auch von der OECD definiert ${ }^{48}$ und in Erhebungen wie PISA getestet wird. Dies schließt außer dem 
Versehen sprachlicher Elemente auch graphische und mathematische ein, wenn es beispielsweise darum geht, sich auf einer Karte zu orientieren, verschiedene Skalen miteinander zu vergleichen oder Schaubilder und Tabellen mit Zahlendaten und statistischen Angaben auszuwerten. Fachinhaltlich beziehen sich die Lernziele auf zum Teil recht komplizierte Zusammenhänge, die nur durch ein angemessenes Textverständnis und mit solider Unterstützung durch die Lehrkraft erschlossen werden können. Fundamental ist dabei ferner die Aneignung eines multidisziplinären Fachwortschatzes als Grundlage für Textkompetenz und produktive sprachliche Fähigkeiten.

\subsubsection{Schulische Sprachbildung}

Ende der 1960er Jahre hat Moffet (1968) die prioritäre Rolle von Sprache im Lernprozess unterstrichen und mit seiner Ansicht, dass unsere Denkfähigkeit von den vielen vorausgehenden Diskursen abhängig ist, in die wir involviert waren, motiviert. Damit hat er einen Weg für die Bemühungen um LAC (Language Across the Curriculum - also Sprachbildung in allen Fächern und Klassenstufen) und die formale Anerkennung dieses Konzepts einer umfassenderen Sprachbildung gebahnt, die im Jahr 1975 mit Bullocks Report A language for life (Department of Education and Science 1975) kam (Corson 1999). ${ }^{49}$

Seit 1991 ist in den italienischen Lehrplänen verankert, dass die Sprachbildung nicht nur Aufgabe der Italienischlehrerinnen und -lehrer sein soll, sondern den gesamten Lehrkörper angeht (Castellino \& Sgroi 2005, S. 364). Damit werden die Lehrpläne einer in den Zehn Thesen formulierten Forderung gerecht, die auf 1975 zurückgeht (GISCEL 1975). Wenn man berücksichtigt, dass Vorgaben zur Sprachbildung erst seit 1979 explizit in den Lehrplänen festgehalten sind, während dort zuvor nur literarische Lernziele formuliert waren (Castellino \& Sgroi 2005, S. 361 f.), und Italienischlehrkräfte im Studium keine einzige sprachwissenschaftliche Prüfung absolvieren mussten (Lavinio 2005b, S. 153), so ist dies äußerst fortschrittlich, auch wenn längst nicht davon

Competencies) nun folgende Definition zugrunde: ,Literacy is the ability to identify, understand, interpret, create, communicate and compute, using printed and written materials associated with varying contexts. Literacy involves a continuum of learning in enabling individuals to achieve their goals, to develop their knowledge and potential, and to participate fully in their community and wider society" (http://www.oecd.org/edu/innovation-education/adultliteracy.htm; zuletzt abgerufen am 03.11.2016).

49 Das Konzept LAC ist nicht zu verwechseln mit dem neueren, sich auch in Europa immer mehr verbreitetenden CLIL (Content and Language Integrated Learning), da es nicht um Fachunterricht in einer schulischen Fremdsprache, sondern um bewussten sprachbildungsförderlichen Unterricht in allen Fächern geht. LAC ist also Aufgabe aller Lehrpersonen, die entsprechend dafür ausgebildet sein müssen, und besteht nicht in einem Fokus auf Sprache als „Produkt" der Beschulung oder als etwas, das zu überprüfen ist, sondern als Lernprozess (Corson 1999, S. 326). 
ausgegangen werden kann, dass alle Lehrkräfte der nichtsprachlichen Fächer diesem Bildungsauftrag nachkommen.

Dass die Sprachbildung der Lernenden von den Lehrkräften aller Fächer wahrgenommen werden soll, ist zu einem Zeitpunkt vorgeschrieben worden, als es in Italien noch wenige SchülerInnen mit einer nicht italienischen Erstsprache gab. Die sprachlichen Leistungen der Lernenden sind immer im Verhältnis zu den schriftkulturellen Voraussetzungen für eine aktive Teilhabe am gesellschaftlichen Leben zu betrachten, welche keine festgesetzte Größe ausmachen, sondern sich gemeinsam mit der Gesellschaft im stetigen Wandel befinden. W.o. wurde bereits erläutert, wie stark die Alltagssprache der meisten Lernenden vom schulischen Sprachgebrauch divergiert.

Favaro (2003) listet für das Italienische folgende Merkmale der Sprache der Lehrpersonen und Lehrmittel auf und vergleicht sie mit den sprachlichen Charakteristiken von Schüleräußerungen: Die Beiträge der Lernenden enthalten in der Regel nicht mehr als eine Information und bestehen aus kurzen, einfachen Sätzen, welche vor allem parataktisch miteinander verknüpft sind, wobei der Gebrauch von Konjunktionen generell auf die einfachen, frequenten beschränkt ist, und als Subjekte oft deiktische Personalpronomen fungieren, wobei sich der Gebrauch von Nomen auf ihre Verwendung in Funktion einfacher Objekte (oder Ausdrücke wie ecco, c'è (das ist, es gibt)) reduziert und es nur selten zu einer Expansion durch zusätzliche nominale Syntagmen kommt. Auch das Verbsystem ist auf die einfachen Tempora des Präsens und Imperfekts und das zusammengesetzte, auch im mündlichen alltagssprachlichen Gebrauch vorherrschende des Perfekts begrenzt. Bei den Schülertexten handelt es sich um narrative, kontextualisierte Texte, auch dann, wenn ihr Zweck eigentlich darlegender Art ist.

Im Gegensatz dazu enthalten die bildungssprachlichen Äußerungen der Lehrbuchtexte und der Lehrenden stets mehrere Informationen, sind also durch eine weitaus höhere Informationsdichte gekennzeichnet, und die einzelnen Sätze sind oft durch hypotaktische Konjunktionen miteinander verknüpft. Die Verwendung des Passivs kommt entschieden häufiger vor als im mündlichen Sprachgebrauch, und die nominalen Syntagmen sind sowohl in ihrer Form als Subjekt als auch in der als Objekt oder adverbiale Bestimmung oft durch Attribute ausgebaut. Das Subjekt kann zudem z.B. durch einen Relativsatz umfangreich erweitert werden, woraus eine große, das Textverständnis mancher Leser erschwerende Distanz zwischen dem Nomen und dem Prädikat entsteht. Während die Negationen in den Schüleräußerungen einfacher Art sind, nehmen sie in den Lehrbuchtexten oft komplexere Gestalt an. Der Wortschatz ist abstrakt und spezifisch und schließt deiktische Elemente aus. Gleichsam ist die Textsorte vorherrschend deskriptiv und erklärend, selten narrativ und oft stark dekontextualisiert (vgl. Favaro 2003, S. 15). 


\subsubsection{Ministeriale Vorgaben zur Sprachbildung}

Neben den fachspezifischen Lernzielen sind in den Lehrplänen auch allgemeine, fachübergreifende formuliert. Hier wird konkreter auf die Sprachbildung eingegangen und, bezogen auf den ersten Zyklus, also die Primarstufe und die Sekundarstufe I (Grund- und Mittelschule), das Ziel einer ,kulturellen Grundalphabetisierung“" (MIUR 2012b, S. 24 f.; m.Ü.) statuiert. Dieses soll durch den Erwerb der Sprachregister und Kommunikationsformen gewährleistet werden, welche in unserer Kultur vorherrschen, wobei andere im Land verbreitete Kulturen nicht unbeachtet bleiben dürfen. Dieses Erziehungsziel wird in Hinblick auf die sprachlichen Grundlagen definiert (ibid., S. 25):

Si tratta di una alfabetizzazione culturale e sociale che include quella strumentale, da sempre sintetizzata nel "leggere, scrivere e far di conto", e la potenzia attraverso i linguaggi e i saperi delle varie discipline.

All'alfabetizzazione culturale e sociale concorre in via prioritaria l'educazione plurilingue e interculturale. La lingua materna, la lingua di scolarizzazione e le lingue europee, in quanto lingue dell'educazione, contribuiscono infatti a promuovere i diritti del soggetto al pieno sviluppo della propria identità nel contatto con l'alterità linguistica e culturale. L'educazione plurilingue e interculturale rappresenta una risorsa funzionale alla valorizzazione delle diversità e al successo scolastico di tutti e di ognuno ed è presupposto per l'inclusione sociale e per la partecipazione democratica.

(M.Ü.: Es handelt sich um eine kulturelle und soziale Alphabetisierung, welche die instrumentale, die schon immer im „Lesen, Schreiben und Rechnen“ zusammengefasst wird, beinhaltet und durch die Register und Inhalte der verschiedenen Fächer verstärkt.

Mit der kulturellen Alphabetisierung wirkt prioritär die mehrsprachige und interkulturelle Erziehung zusammen. Die Muttersprache, die Sprache der Beschulung und die europäischen Sprachen als schulische Fremdsprachen leisten nämlich einen Beitrag dazu, die Rechte des Subjekts zur vollen Entwicklung der eigenen Identität in Berührung mit der sprachlichen und kulturellen Alterität zu fördern. Die mehrsprachige und interkulturelle Erziehung stellt eine funktionale Ressource zur Valorisierung von Verschiedenheit und für den schulischen Erfolg aller und eines jeden dar und ist die Voraussetzung für soziale Inklusion und demokratische Teilhabe.)

Im zweiten Absatz dieser Definition wird ausdrücklich auf die Bedeutung der sprachlichen Fähigkeiten für die persönliche Entwicklung, Sozialisierung und eine aktive und demokratische Einbindung in das gesellschaftliche Leben eingegangen. Hierbei wird auch die wesentliche Rolle der Erstsprache und der Mehrsprachigkeit unterstrichen. Da aber in dem zitierten Kontext und an anderen Stellen der ministerialen Richtlinien immer wieder vorrangig oder auch ausschließlich die europäischen Sprachen benannt werden (was so ausgelegt werden kann, dass die nationale Schulpolitik um einen Einklang mit der europäischen Sprachpolitik bemüht ist), stellt sich die Frage, ob die Förderung von Mehrsprachigkeit sich nicht in erster Linie auf den auch von der Europäischen Union vorgesehenen Fremdsprachenerwerb in mindestens zwei weiteren Sprachen der Staatengemeinschaft bezieht. 
Bei der Darlegung der Bildungsziele im Bereich von Staatsbürgerschaft und Verfassung wird jedoch erneut das Sprachrepertoire aller SchülerInnen ins Auge gefasst und die Wichtigkeit der Wertschätzung aller sprachlichen Ausgangslagen der Lernenden unterstrichen (MIUR 2012b, S. 26):

[...] È attraverso la parola e il dialogo tra interlocutori che si rispettano reciprocamente, infatti, che si costruiscono significati condivisi e si opera per sanare le divergenze, per acquisire punti di vista nuovi, per negoziare e dare un senso positivo alle differenze così come per prevenire e regolare i conflitti.

La lingua italiana costituisce il primo strumento di comunicazione e di accesso ai saperi. La lingua scritta, in particolare, rappresenta un mezzo decisivo per l'esplorazione del mondo, l'organizzazione del pensiero e per la riflessione sull'esperienza e il sapere dell'umanità.

È responsabilità di tutti i docenti garantire la padronanza della lingua italiana, valorizzando al contempo gli idiomi nativi e le lingue comunitarie. Così intesa, la scuola diventa luogo privilegiato di apprendimento e di confronto libero e pluralistico.

(M.Ü.: [...] Durch das Wort und den Dialog zwischen Gesprächsteilnehmern konstruieren sich gemeinsame Bedeutungen und operiert man, um Meinungsverschiedenheiten aus dem Weg zu räumen, neue Standpunkte zu erwerben, um Verschiedenheiten auszuhandeln und ihnen einen positiven Sinn zu verleihen sowie Konflikten vorzubeugen und sie zu regeln.

Die italienische Sprache bildet das erste Instrument zur Kommunikation und zum Wissenserwerb. Vor allem die geschriebene Sprache stellt ein entscheidendes Mittel dar, um die Welt zu entdecken, die Gedanken zu ordnen und über die Erfahrung und das Wissen der Menschheit zu reflektieren.

Es liegt in der Verantwortung aller Lehrkräfte, das Beherrschen der italienischen Sprache zu garantieren, bei gleichzeitiger Valorisierung der Heimatsprachen und der Sprachen der Gemeinschaft. Eine so aufgefasste Schule wird zum privilegierten Ort des freien und pluralistischen Lernens und Konfrontierens.)

Bedeutend für die Bearbeitung der Fragestellungen dieser Arbeit sind hier vor allem die Betonung der gemeinsamen Aufgabe der Spracherziehung inklusive der Heranführung an die schulsprachlichen Register des Italienischen durch alle Lehrkräfte und die Wertschätzung der sprachlichen Heterogenität der Lernenden.

Auf diese beiden Aspekte wird in den Richtlinien an mehreren Stellen eingegangen. So findet z.B. die Verschiedenheit der Ausgangssprachen und sprachlichen Kompetenzen ausdrücklich Erwähnung und wird die Notwendigkeit eines gemeinsamen Einsatzes aller Lehrkräfte zur Entwicklung der sprachlichen Fähigkeiten eines jeden Lernenden unterstrichen. Gleichsam wird besondere Aufmerksamkeit für die nicht italienischen Lernenden gefordert, welchen bei einer vollständigen Integration mit der Vermittlung von Italienischkenntnissen zur Kommunikation und zum schulischen Wissenserwerb geholfen werden soll (MIUR 2012b, S. 26). Vor allem in Bezug auf Lernende, die mit ihren Familien erst kürzlich zugewandert sind, wird extra die Zuständigkeit aller Lehrkräfte für die Förderung ihrer sprachlichen Bildung und ein gemeinsamer didaktischer Plan betont (ibid.): „Questi alunni richiedono interventi differenziati che non devono investire il solo insegnamento della lingua italiana ma la progettazione didattica complessiva della scuola" (m.Ü.: Diese Schüler 
bedürfen differenzierter Interventionen, welche nicht nur den Italienischunterricht, sondern die gesamte didaktische Planung der Schule betreffen).

Vertieft werden diese Aspekte bei der Behandlung der Inhalte und Ziele des Italienischunterrichts. Aufgrund ihres sprachpolitisch und -pädagogisch relevanten Wertes sollen sie hier ebenfalls vorgestellt werden, zumal ein hoher Grad an Sensibilität für die Bedeutsamkeit der Sprachkompetenz auch daran erkennbar wird, dass die Richtlinien nicht nur allochthone, sondern auch autochthone Schülerinnen und Schüler in den Blick nehmen, deren Erstsprache zum Teil immer noch ein Dialekt ist oder deren Kompetenzen im Italienischen auf Registerebene (diastratisch) und unter diatopischen Aspekten stark variieren (MIUR 2012b, S. 28):

Lo sviluppo di competenze linguistiche ampie e sicure è una condizione indispensabile per la crescita della persona e per l'esercizio pieno della cittadinanza, per l'accesso critico a tutti gli ambiti culturali e per il raggiungimento del successo scolastico in ogni settore di studio. Per realizzare queste finalità estese e trasversali, è necessario che l'apprendimento della lingua sia oggetto di specifiche attenzioni da parte di tutti i docenti, che in questa prospettiva coordineranno le loro attività.

Nel nostro paese l'apprendimento della lingua avviene oggi in uno spazio antropologico caratterizzato da una varietà di elementi: la persistenza, anche se quanto mai ineguale e diversificata, della dialettofonia; la ricchezza e la varietà delle lingue minoritarie; la compresenza di più lingue di tutto il mondo; la presenza infine dell'italiano parlato e scritto con livelli assai diversi di padronanza e con marcate varianti regionali.

Tutto questo comporta che nell'esperienza di molti bambini e ragazzi l'italiano rappresenti una seconda lingua. La cura costante rivolta alla progressiva padronanza dell'italiano implica, dunque, che l'apprendimento della lingua italiana avvenga a partire dalle competenze linguistiche e comunicative che gli allievi hanno già maturato nell'idioma nativo e guardi al loro sviluppo in funzione non solo del miglior rendimento scolastico, ma come componente essenziale delle abilità per la vita.

(M.Ü.: Die Entwicklung umfangreicher und sicherer sprachlicher Kompetenzen ist eine unabkömmliche Bedingung für das persönliche Wachsen und für das vollständige Ausüben der Bürgerschaft, den kritischen Zugang zu allen kulturellen Feldern und für das Erreichen schulischen Erfolges in jedem Lernbereich. Um diese ausgedehnten und transversalen Ziele zu realisieren, ist es notwendig, dass das Erlernen der Sprache Objekt spezifischer Aufmerksamkeit aller Lehrpersonen ist, welche in dieser Hinsicht ihre Aktivitäten koordinieren.

In unserem Land erfolgt die Sprachaneignung heute in einem anthropologischen Raum, der von einer Vielzahl von Faktoren gekennzeichnet ist: das Fortbestehen der Dialektophonie, wenn auch ungleich und diversifiziert wie nie zuvor; der Reichtum und die Vielzahl der Minderheitensprachen; das gleichzeitige Vorhandensein mehrerer Sprachen aus der ganzen Welt; letztlich die Präsenz des gesprochenen und geschriebenen Italienisch mit ganz verschiedenen Beherrschungsgraden und markanten regionalen Varianten. All das führt dazu, dass in der Erfahrung vieler Kinder und Jugendlicher das Italienische eine Zweitsprache darstellt. Die konstante Pflege eines progressiven Beherrschens des Italienischen impliziert also, dass der italienische Spracherwerb ausgehend von den linguistischen und kommunikativen Kompetenzen erfolgt, über welche die Lernenden schon in ihrer Muttersprache verfügen, und ihre Entwicklung nicht nur in Funktion besserer schulischer Leistungen, sondern auch als essentialer Bestandteil der für das Leben relevanten Fähigkeiten betrachtet wird.) 
Und weiter heißt es zur schulischen Sprachbildung, dass sie aufgrund der Komplexität die Zusammenarbeit der Lehrkräfte aller Fächer notwendig mache, um jeder und jedem Lernenden ,die Gelegenheit zu geben, sich angemessen in das schulische Setting und die Lernwege einzufügen“ (ibid.; m.Ü.).

Im folgenden Unterkapitel soll eine der in den Lehrplänen erwähnten sprachlichen Komponenten, die schulische Wortschatzarbeit, genauer umrissen werden.

\subsection{Lexikalische Kompetenz}

Auffällig ist die Hervorhebung der Bedeutung des Wortschatzerwerbs. Dass seine Wichtigkeit betont und die Art, in der er erfolgen soll, genauer vorgestellt wird, erscheint auch deshalb von Belang, da der Wortschatzerwerb nicht nur in der Forschung lange Zeit ein Stiefkind war, sondern er dies für SchulbuchautorInnen und Lehrpersonen immer noch zu sein scheint (Revelli 2008, S. 345; Miglietta \& Sobrero 2008, S. 203).

So wird darauf hingewiesen, dass alle einzelnen sprachlichen Kompetenzen, also sowohl im Schriftlichen als auch im Mündlichen und auf rezeptiver wie produktiver Ebene, graduell und altersangemessen zu vermitteln und als permanentes Kontinuum zu betrachten sind, das vom Kindergarten bis zum Ende der Sekundarstufe II stets ausgebaut und dessen Grundlagen wiederholt werden müssen (MIUR 2012b, S. 30). Die Lehrpläne heben hierbei das Erfassen des jeweils verschieden gearteten Schülerwortschatzes als wesentlich hervor, um ihn effektiv erweitern zu können. Neben einer generellen, vor allem an den fachlichen Inhalten und Anforderungen ausgerichteten Wortschatzerweiterung steht also die individuelle lexikalische Förderung im Vordergrund, welche sich auch deshalb aufdränge, da sich eine allgemeine Verarmung des Wortschatzes bemerkbar mache (ibid., S. 29). Diese Auffassung wird von der sprachwissenschaftlichen Forschung geteilt, die ebenfalls einen Rückgang der lexikalischen Kompetenz unter SchülerInnen und Studierenden beobachtet (vgl. Serianni 2010, S. X):

Le polemiche che tanto spesso levano nei giornali sull'analfabetismo di massa che colpirebbe i nostri studenti sarebbe forse da relativizzare [...], ma non da sottovalutare. Non sono invenzioni dei giornali le mediocri prestazioni degli studenti italiani nelle rilevazioni internazionali e nei test d'accesso alle facoltà universitarie. In particolare colpisce la scarsa padronanza del lessico astratto [...] e la regressione del lessico meno usuale, ma non certo obsoleto $[\ldots]$.

(M.Ü.: Die Polemiken, die sich so oft in den Zeitungen hinsichtlich Massen-Analphabetismus auftun, der unsere Schüler und Studenten ereilte, wären vielleicht zu relativieren [...], aber nicht zu unterschätzen. Die mäßigen Leistungen der italienischen Schüler und Studenten in den internationalen Erhebungen und in den Zulassungstests zu den universitären Fakultäten sind keine Erfindungen der Zeitungen. Vor allem verblüfft das mangelnde Beherrschen des abstrakten Wortschatzes [...] und der Rückgang des weniger gebräuchlichen, aber sicher nicht obsoleten Wortschatzes [...]). 
Auch in einer anderen Studie wird bei Studierenden der Erstsemester der geisteswissenschaftlich-sprachlichen Fakultät eine fragile competenza lessicale (De Santis \& Gatta 2013, S. 430; zerbrechliche Wortschatzkompetenz) ${ }^{50}$ festgestellt und die häufige Verwendung von nichtssagenden Wörtern mit einer von Italo Calvino stammenden Metapher als fantasmi sonori (ibid., S. 429; Lautgespenster) beschrieben. Viele Studierende zeigen darüber hinaus geringe Fähigkeiten bei der Zusammenfassung von Texten und generell, sobald sie vom alltagssprachlichen Register in ein elaborierteres wechseln müssen (ibid., S 431).

Desweiteren wird die Bedeutung des Wortschatzes für den Wissenserwerb betont und legen die Lehrpläne konkret fest, welche Bereiche der Gebrauchswortschatz im dritten Schuljahr der Sekundarstufe I umfassen soll, nämlich alle Wörter der drei als Grundwortschatz definierten Kategorien des fundamentalen Wortschatzes (Fo), des Wortschatzes des hohen Gebrauchs (AU) und der hohen Verfügbarkeit (AD) in ihren verschiedenen Bedeutungen sowie spezifische Fachausdrücke der einzelnen Schulfächer (MIUR 2012b, S. 33).

Auch wenn es sich hierbei schon um einen recht genauen Hinweis handelt, um welche Art von Wörtern das mentale Lexikon der Lernenden erweitert werden soll und was die Verfasser von Unterrichtswerken bei der Erstellung ihrer Texte zu beachten haben, ist doch darauf hinzuweisen, dass von Seiten der Forschung seit geraumer Zeit darauf gedrängt wird, noch genauer festzulegen, welche Ausdrücke gelehrt werden sollten und wie eine wirksame Wortschatzdidaktik aussehen kann. In diesem Sinne fordert z.B. Ferreri (2005), dass es einen Plan der „lexikalischen Alphabetisierung“ (ibid., S. 37; m.Ü.) geben sollte, mit dessen Hilfe der Sprachinput verbessert werden kann, um den spontanen und gesteuerten Wortschatzerwerb der Lernenden voranzutreiben. Hierbei sollte der Lernerwortschatz sowohl quantitativ als auch qualitativ erweitert werden, indem die lexikalische Kompetenz der Lernenden durch gezielte didaktische Interventionen an Festigkeit und Tiefe gewinnt. Beispielsweise sollten also nicht nur die Grundbedeutung, sondern möglichst viele verschiedene Bedeutungen eines Wortes mit seinen kollokationalen Implikationen bekannt gemacht werden (ibid.).

Außerdem sollen die Lernenden im letzten Jahr der Sekundarstufe I über die Fähigkeit verfügen, ihren Wortschatz der Gesprächssituation, ihrem Gesprächspartner und der Textsorte nach angemessen zu wählen. Bei der Erschließung von ihnen unbekannten Ausdrücken haben sie über Kenntnisse der Wortbildungsregeln und der Beziehungen zwischen den Bedeutungen verschiedener Wörter zu verfügen, welche ihnen beim Verständnis unbekannter

50 Die Studie beruht auf der Auswertung von zwei schriftlichen Sprachtests für Studierende der Erstsemester des Studiengangs Sprachliche und kulturelle Mediation (m.Ü.) an der Universität Bologna (Erhebungspopolation: 153). Der erste überprüft sprachlich-grammatikalische Kenntnisse und der zweite lexikalische und textbezogene Kompetenzen. 
Ausdrücke behilflich sein könnten. Zuletzt ist ihnen auch der Gebrauch unterschiedlicher Arten von Wörterbüchern zu vermitteln, damit sie bei sprachlichen Problemen oder Zweifeln dort nachschlagen und aus den in den Wörterbüchern bereitgehaltenen Informationen die für sie relevanten herausfiltern können (MIUR 2012b, S. 35).

Für alle didaktischen Maßnahmen, welche der Wortschatzvermittlung und -festigung dienen, gilt, dass sie innerhalb eines ,uso vivo e reale della lingua, non attraverso forme di apprendimento meccanico-mnemonico" (ibid., S. 30; m.Ü.: lebendigen und realen Sprachgebrauchs, nicht durch mechanisch-mnemonische Formen des Lernens) zu erfolgen haben, was z.B. dadurch gewährleistet werden soll, dass der Wortschatz immer in einem Kontext vermittelt und darauf verzichtet wird, Wörterlisten zu erstellen, die es auswendig zu lernen gilt, ohne dass in den Lernenden ein Bewusstsein dafür geschärft wird, in welchen Kollokationen und Sprachregistern die Wörter auftreten können. ${ }^{51}$

Dieser prominente Raum, welcher der Wortschatzarbeit in den Richtlinien beigemessen wird, findet seine Berechtigung in dem Umstand, dass Sprache nun einmal aus Wörtern besteht und ohne ein solides mentales Lexikon kein angemessener Sprachausbau erfolgen kann. In diesem Sinne gilt die lexikalische Kompetenz als wichtiger Indikator sprachlicher Fähigkeiten im Allgemeinen (s. Pinto 1980, S. 33) und insbesondere der Lesekompetenz (Petermann 2010, S. 7 mit Verweis auf Moser, Stamm \& Hollenweger 2005):

Kinder mit Auffälligkeiten im Wortschatz haben deutlich häufiger Probleme in der Sprachwahrnehmung und im Sprachgedächtnis als Kinder ohne solche Auffälligkeiten. Der Umfang des Wortschatzes beeinflusst die Prozesse der Worterkennung und damit das flüssige Lesen entscheidend und stellt deshalb das größte Handicap für die literale Entwicklung dar (Moser, Stamm und Hollenweger, 2005).

\subsubsection{Divergenzen zwischen den ministeriellen Vorgaben und ihrer Um- setzung im Unterricht sowie den dafür in der Lehrerausbildung ver- mittelten Fähigkeiten}

Bei der vollständigen Umsetzung der von den Richtlinien und Lehrbüchern vorgegebenen Inhalte im Laufe eines Schuljahrs liegt ein generelles Problem darin, dass für die Vermittlung und Überprüfung eines umfangreichen Wissens und komplexer Kompetenzen nur eine begrenzte Zeit zur Verfügung steht. Dadurch werden Lehrkräfte grundsätzlich und nicht nur in Italien vor eine organisatorische Herausforderung gestellt, doch bezogen auf den italienischen Erhebungskontext werden an dieser Stelle mehrere miteinander verknüpfte

51 Hierzu lässt sich auch in Hinblick auf die Unterrichtsbeobachtungen dieser Studie anzweifeln, dass die im Erdkundeunterricht oft beobachtete Methode des Abfragens einzelner Schüler zu Lehrbuchinhalten, dem nicht selten das Auswendiglernen des Stoffs vorausgeht - also auch eine eher „mechanisch-mnemonische“ Art des Lernens -, eine effiziente Wortschatzerweiterung und -konsolidierung darstellt. 
Problematiken deutlich. Da sind zum einen die sowohl qualitativ als auch quantitativ hohen Ansprüche, die vonseiten der ministerialen Richtlinien an den fachlichen Kompetenzerwerb durch die Schülerinnen und Schüler gestellt werden. Zum anderen wird schon seit geraumer Zeit ein unverhältnismäßig großer Umfang der Lehrbücher kritisiert, der sich aus einer Anhäufung an Detailwissen ergibt und bei den Lernenden zu kognitiver Überladung führen kann (z.B. Bascetta 1964, S. 69 f.; De Mauro 1997, S. 28). Dass die qualitativen Ansprüche unter den quantitativen leiden, die dazu verführen, sich auf das Vermitteln und Überprüfen von Oberflächenwissen zu beschränken, scheint ein wesentlicher Grund dafür zu sein, warum die Lehrpläne oft innovativer sind als die konkrete Unterrichtspraxis (Sobrero 1993, S. 59; Bozzone Costa 2003, S. 113; Favaro 2010b, S. 11).

Bei den Divergenzen zwischen Vorgaben und Unterrichtspraxis handelt es sich nicht um eine länderspezifische Eigenart, doch in Italien wurde schon in den 1970er Jahren beobachtet, dass die traditionelle pädagogische Praxis deutlich hinter den Ministerialprogrammen zurückblieb, die selbst als noch weit entfernt vom „Ideal demokratischer Effizienz“ betrachtet wurden (De Mauro 1975 d in ead. 1979, S. 142; m.Ü.). Hierbei wurde intendiert, dass vielerorts Faktenwissen gelehrt und bezüglich der Sprachkompetenz vor allem auf einen literarisch gehobenen Ausdruck geachtet wurde, anstatt neben dem Oberflächenwissen auch kognitive Kompetenzen und sprachliche Fähigkeiten mit einem höheren Gebrauchswert zu vermitteln (ibid.). ${ }^{52}$ In Hinblick auf die Leistungen der Lernenden wurde so beobachtet, dass die SchülerInnen über weitaus weniger Fertigkeiten verfügen, als von den Richtlinien gefordert, und zwar in erster Linie, was die fachunabhängigen Kompetenzen kognitiver Art wie das Aufstellen von Hypothesen und die Bildung von Interferenzen, Vergleichen, Abstrahierungen und Generalisierungen betrifft (Simone 1998, S. 47)..$^{53}$

Konkret auf das Fach Erdkunde bezogen wurde unterstrichen, dass es in Italien im Vergleich zum europäischen Ausland stets Verspätungen in der Umsetzung didaktischer Neuerungen wie die für den Lernerfolg bedeutsame aktive und entdecken lassende Einbindung der Schülerinnen und Schüler gebe (Salvatori 1988, S. 58) und unter vielen Lehrkräften eine hartnäckige Tendenz verbreitet sei, zu den herkömmlichen Methoden zurückzukehren (Battisti 1988, S. 62). Auch wenn Verbesserungen in den Lehrplänen, im Wirken mancher Lehrkräfte und in einigen Lehrbüchern Lob ernten, wird doch betont: „,Ma

52 Siehe zu den Unterschieden und der Vermittlung von Oberflächenwissen und tiefgreifenderem Wissen das Unterkapitel 9.1.3.2.1, das diesem Thema gewidmet ist und herausstellt, welches Vorgehen in diesem Zusammenhang in den fünf Klassen beobachtet werden konnte.

53 Aus seinem Beitrag geht nicht hervor, wie Simone zu diesen Erkenntnissen kommt. Bei den Recherchen im Zuge dieser Arbeit fiel auf, dass in diesem Forschungsfeld in Italien in mehreren Fällen keine oder nur sehr geringe Angaben zu empirischen Verfahren des Erkenntnisgewinns gemacht werden, wobei diese allerdings für neuere Veröffentlichungen zunehmend die Grundlage bilden. 
occorre sottolineare con forza come il principale strumento didattico sia l'insegnante e come il discorso sulla formazione e sull'aggiornamento sia ancora tutto da fare" (Salvatori 1988, S.58; m.Ü.: Jedoch muss man vehement unterstreichen, dass die Lehrkraft das prinzipielle didaktische Instrument ist und dass der Diskurs über die Aus- und Fortbildung noch komplett zu führen ist).

Viele SprachwissenschaftlerInnen sind sich besonders in dem Punkt einig, dass die linguistischen und sprachbildungsbezogenen Ausbildungsbestandteile nicht genügend Substanz haben (vgl. De Mauro 1975d zit. in ead. 1979, S. 138, S. 184 f.; Salvatori 1988, S. 58; Alfieri 2005, S. 417; Lavinio 2005, S. 153; Serianni 2010, S. XII; Matozzi 2014, S. 396; Piemontese \& Sposetti 2014, S. 163, S. 165). Warum der Erwerb profunder sprachwissenschaftlicher Kenntnisse vonseiten der angehenden Lehrpersonen von großer Wichtigkeit ist und sich nicht auf Fortbildungsmaßnahmen reduzieren darf (Halliday 2007 (1990), S. 354 f.), wird im nächsten Kapitelabschnitt vertieft, der sich mit der Vermittlung funktionaler schriftkultureller Fertigkeiten befasst. Letztere sind notwendigerweise mit einem Sprachausbau verbunden, so dass sich hier ein sehr facettenreiches und komplexes sprachliches und sprachdidaktisches Feld auftut.

\subsubsection{Sprachausbau und Registerdifferenzierung im schulischen Kontext}

Die Beschreibung des im zweiten Kapitel behandelten Konzepts Bildungssprache sollte verdeutlicht haben, wie es sich dabei um ein sprachliches Register handelt, das über den Schulerfolg in allen Fächern hinaus auch im weiteren Leben von Bedeutung ist, da es die berufliche Eingliederung erleichtert und die Bürgerinnen und Bürger zu einer weitreichenderen Partizipation am gesellschaftlichen Leben befähigt. Sowohl die Schilderung einzelner Merkmale der Bildungssprache für das Italienische und das Deutsche als auch der sprachwissenschaftliche theoretische Hintergrund (konzeptionelle Schriftlichkeit) erklären, dass es sich um einen Sprachausbau handelt, der als universal zu begreifen ist. Zwar ist er immer an eine bestimmte Sprache gebunden, aber er bleibt übertragbar auf eventuelle Zweit- und Fremdsprachen (Cummins 1980; Francis 2000). Zur Veranschaulichung dieses Aspekts hat Cummins (1980, S. 87) das Bild eines Eisbergs mit zwei Spitzen verwendet: Die beiden Teile des Bergs, die aus dem Wasser ragen, stehen für die Oberflächenphänomene zweier Sprachen eines Sprechers, während diesen beiden eine einzige literate Sprachprofizienz zugrunde liegt, welche durch den Teil des Eisbergs abgebildet ist, der sich unterhalb der Wasseroberfläche befindet.

Wer also behauptet, im Aufsatzschreiben (und dem aus dieser Perspektive oft darauf reduzierten Sprachunterricht) nie gut gewesen zu sein, aber dies kein Problem im ,richtigen“"Leben darstelle, erzählt nicht die ganze Wahrheit, auch wenn dort nur selten Aufsätze verfasst werden. Das belegt beispielsweise die Tatsache, dass die Deutschnote sehr stark mit der Schulleistung allgemein kor- 
reliert (Maas 2008, S. 654). Diese Note ist gewiss oft kein angemessener Ausdruck einer Leistung, etwa, wenn sich Lehrkräfte an dem, was vom Standard abweicht, stören und in der Bewertung vor allem leicht Messbares wie orthographische und grammatikalische Fehler in den Blick nehmen (vgl. Gantefort \& Roth 2010, S. 588; Maas 2008, S. 654 f., S. 659). Außerdem werden bildungsferne und allochthone Lernende oft unter ihren Leistungen bewertet. ${ }^{54}$

So wie die Fertigkeiten, die den schriftkulturellen Sprachausbau betreffen, auf alle Sprachen angewandt werden können, die einem Sprecher zur Verfügung stehen, erlaubt ihm das Beherrschen verschiedener Register einer Sprache, ,prinzipiell beliebige Rollen in unterschiedlichen sozialen Subsystemen einnehmen zu können“, wie Weber mit Bezug auf Roeder und Bernsteins Forderung nach einer Kompensierung der schichtspezifischen Code-Beherrschung feststellt (Weber 1973, S. 43 f.). Hierbei bietet das Verfügen über eine große Variationsbreite die Möglichkeit, dasselbe mit unterschiedlichen sprachlichen Mitteln auszudrücken, die je nach Gesprächspartner und -situation angemessen gewählt werden können.

In Italien ist die Registerdifferenzierung der Sprache von Berruto in einem Modell abgebildet worden, das sich auf De Mauros Weiterentwicklung des von Wittgenstein übernommenen Konzepts (Banfi 1993b, S. 38) des spazio linguistico (De Mauro 1980; sprachlicher Raum) bezieht und diesen näher beschreibt, indem es das heutige Repertoire des Italienischen in seinen verschiedenen Erscheinungsformen in einem Koordinatensystem nach diatopischen, diastratischen, diamesischen und diaphasischen Dimensionen ${ }^{55}$ sortiert (Berruto 1993, S. 12). Je mehr Register wir verstehen und verwenden, desto größer ist das Gebiet, was wir im sprachlichen Raum bewandern.

Diese nützliche, die holistische Sprachauffassung auflösende Metapher ist von vielen WissenschaftlerInnen aufgegriffen worden (z.B. Cacchione 2014, S. 78; Guerriero 2014, S. 164; Bino et al. (GISCEL Sicilia 1997, S. 148); Felici, Giarè \& Villarini 1994, S. 480 f., 502; Banfi 1993b, S. 38; Vedovelli 1993,

54 Vgl. in dieser Hinsicht auch Brisk (2005, S. 18) mit Verweis auf Sizer (1992, 1995), welche in Bezug auf die Schwierigkeiten bei der Bewertung bildungssprachlicher Fähigkeiten anmerkt, dass dafür im Zuge einer sich kontinuierlich ausbreitenden Testkultur mehr und mehr standardisierte Tests verwendet werden, mit denen bestimmte bildungssprachliche Fertigkeiten nicht beurteilt werden, wie z.B. die Erstellung von Langzeitprojekten. Kritik an der Konzentration auf die leicht messbaren sprachlichen Aspekte ist desweiteren nicht erst in den letzten Jahren laut geworden, sondern wurde beispielsweise schon 1973 von Weber geübt (Weber 1973, S. 32): „Die Flucht in den Test bedeutet aber nichts anderes als die Flucht aus den Gesetzen der Sprache und den Kriterien ihrer Gestaltung. Mögen Schönschreiben, Rechtschreiben und Wortschatz vielleicht normbar, meßbar und testbar sein, sind sie schon Sprache? Erziehen wir zu Sprache, Denken, Bewußtsein oder zu Testbarkeit? Fallen wir nicht auf bloßes Wissen und Abfragbarkeit einseitig zurück?“

55 Diese Begriffe stehen in der oben aufgezählten Reihenfolge für geographische/regionale, soziokulturelle, mediumsabhängige (schriftlich vs. mündlich in unterschiedlichen Facetten) und stilistische Variationen im Sprachgebrauch. 
S. 24; Fibbi \& Vedovelli 1988, S. 32 f.) und bietet sich gut an, um den Spracherwerb der Zugewanderten zu beschreiben, da er keineswegs gradlinig erfolgt und ausschließlich auf die Aneignung des normativen italienischen Standards ausgerichtet ist (vgl. Fibbi \& Vedovelli 1988, S. 33 f.; Berretta 1990; Banfi 1993b, S. 46 f.). ${ }^{56}$

Es ist offensichtlich, dass sich niemand den ganzen sprachlichen Raum aneignen kann (geschweige denn auf einen Schlag), sondern dass der Spracherwerb immer an den sprachlichen Gepflogenheiten der unmittelbaren Umgebung orientiert ist (Wygotski 1977 (1934) zit. in Maas 2008, S. 292 f.). So beginnt die Eroberung des Raumes, wenn das Kind mit Gleichaltrigen und Kontaktpersonen wie z.B. Erziehenden im Kindergarten in Berührung kommt, wo es zu einer Erweiterung seiner kommunikativen Praxis gezwungen ist, und erfährt in der Regel in dem Moment eine exponentielle Erweiterung, in dem das Kind über eine solide Lesekompetenz verfügt, so dass es insbesondere im Schulunterricht lernt, sprachliche Äußerungen verschiedenster Register zu erfassen. ${ }^{57}$

\subsection{Entwicklung protoliterater Fähigkeiten}

Aufgrund der besonderen Bedeutung der protoliteraten Fähigkeiten für den späteren Sprachausbau und generell für den Bildungserfolg (Maas 2008, S. 352) ist deshalb in Deutschland zuletzt zu Recht viel Aufmerksamkeit auf die Sprachförderung im Elementarbereich gerichtet worden (Ehlich 2007, S. 45; Maas 2008, S. 667, Fn. 1188).

In dieser Phase werden Registerdifferenzierungen im kindlichen Spiel erprobt (z.B. Arzt und Krankenschwester: unterschiedliche Stimmlage, Art sich ausdrücken (Lantolf 2005, S. 137 f.)) und in der Regel gern Geschichten gehört, wobei die Kinder ihren Wortschatz erweitern und erste Erzählstrukturen

56 In diesem Zusammenhang wünschen sich die Autoren mehr Studien zum Sprachstand von Einwanderern und begründen ihr Desiderat neben dem Interesse an der Diagnose sprachlicher Fähigkeiten und Entwicklungsverläufe auch damit, dass am Sprachgebrauch von Migranten, der dialektale und regionale Elemente enthalten kann, auch das Fortschreiten der Standardisierung des Italienischen beobachtet werden kann (Felici, Giarè \& Villarini 1994, S. 502). Das ist auch deshalb interessant, weil verschiedene Sprachwissenschaftler eine fortschreitende Vermischung der einzelnen Varietäten beobachten (z.B. Sobrero 1998, S. 65).

57 Zur Korrelation von Lesefähigkeit und bildungssprachlicher Kompetenz vgl. auch Gogolin et al. (2007).

58 In dieser Note kritisiert Maas ferner, dass dafür appelliert wird, ohne dass dem auch ein sprachwissenschaftliches Gerüst gegeben wird. Vgl. zudem, dass für die Grundschule eine ähnliche Situation beschrieben wird (Maas 2008, S. 667 f.): „In praxisnäheren Diskussionen, wie sie sich z.B. in didaktisch orientierten Veröffentlichungen des Grundschulverbandes finden (z.B. in dem Heft „Deutsch als Zweitsprache“ seiner Zeitschrift „Grundschule aktuell“, Heft 92/2005), wird denn auch sehr deutlich herausgestellt, dass gerade auch bei ansonsten pädagogisch erfolgreicher Arbeit mit Migrantenkindern im sprachdidaktischen Bereich Ratlosigkeit herrscht. [...].“ 
verinnerlichen. Dies und ihre sich über das Geschichtenhören entwickelnde eigene Erzählfähigkeit treiben die „Dezentrierung gegenüber der kommunikativen Einbindung" voran, was zur Entfaltung der oraten Kommunikationsfähigkeit führt und außerdem den Grundstein für den sukzessiven schriftkulturellen Ausbau legt (Maas 2008, S. 348), der jetzt ebenfalls in ersten Schreibversuchen, welche oft die Schrift nur imitieren und noch keine Buchstaben als solche erkennen lassen, seine Anfänge nimmt. Auf diese Weise werden kategoriale Haltungen zur Schrift entwickelt (ibid., S. 312), die maßgeblich durch die Rolle, welche Bücher und andere Schriftmedien im Umfeld der Heranwachsenden spielen, beeinflusst sind. Gerade wo diese keinen bedeutenden Platz im Alltag einnehmen oder gar selten bzw. nie im Elternhaus gelesen werden, kommen Kindergarten und Grundschule eine wesentliche kompensatorische Funktion in der Gestaltung einer der Herausbildung protoliterater Fertigkeiten dienlichen Lernumgebung zu. Worauf es darüber hinaus aus didaktischer Perspektive in diesem Stadium der ersten, für die sprachliche Entwicklung entscheidenden Erfahrungen mit der Registervariation ankommt, formuliert Maas folgendermaßen (ibid., S. 312 f.; Hervorhebung im Original):

Kinder arbeiten sich an diesen Differenzen ab, spielerisch entlastet mit Gleichaltrigen (s.o. schon die Hinweise auf die Rollen-Spiele [so-tun-als-ob-Spiele]), aber u.U. auch konfliktreich im Umgang mit Erwachsenen, in Reaktion auf normative Vorgaben.

Die Konsequenzen für didaktisches Handeln liegen auf der Hand: Dieses muss von einer sorgfältigen Analyse der Strategien geleitet werden, die von Schülern verwendet werden bzw. für sie erreichbar sind, statt von normativen Annahmen über ,natürliche“ Verhältnisse in der Sprache und auch nicht von angenommenen kulturellen Zwangläufigkeiten. Das ist allerdings ein aufwendiges Prozedere, das vor allem eine entsprechend qualifizierte Ausbildung der Lehrenden verlangt.

Es darf also weder vorausgesetzt werden, dass Sprachausbau und eventueller Zweitspracherwerb ganz von alleine erfolgen und die soziokulturelle Ausgangslage dem Grenzen setzt, noch reicht es aus, die Oberflächenphänomene der Sprachproduktion wie die Gestaltung des kindlichen Wortschatzes zu beobachten. Stattdessen muss für eine genauere Interpretation und Diagnostik des sprachlichen Handelns der Kinder ein Fachwissen bei den Lehrpersonen herausgebildet werden, das Kenntnisse über wesentliche Aspekte des anfänglichen Sprachausbaus beinhaltet und ihre Relevanz für die sukzessive Entwicklung komplexerer Kompetenzen beachtet.

Hierbei sind die Erziehenden und Lehrenden (sowie die Erziehungsberechtigten) nicht allein für die Sprachbildung der Kinder verantwortlich, da diese maßgeblich auch miteinander und voneinander lernen. Die Vorteile des Lernens von Gleichaltrigen im Spiel liegen vor allem im wesentlich geringeren hierarchischen Gefälle zwischen den Gesprächspartnern, wodurch sich gerade schüchterne Kinder i.d.R. eher öffnen als einem Erwachsenen gegenüber. Im Vergleich zu sprachlichen Einbringungsmöglichkeiten in der Kindergartengruppe oder im Klassenverband steigen außerdem die Redeanteile des Kindes 
und damit seine Gelegenheiten, bereits Erworbenes anzuwenden und dadurch zu verfestigen sowie neue Kommunikationsmittel und -strukturen auszuprobieren.

\subsubsection{Sprachstandserhebungen}

Es ist schon darauf hingewiesen worden, dass für das Gelingen des Sprachausbaus ein sehr hohes Maß an Übungsmöglichkeiten an einer großen Bandbreite von Texten über einen ziemlich langen Zeitraum notwendig ist (Hammond \& Gibbons 2005, S. 10). Dieser Umstand ist es, welcher ein Umdenken der Lehrkräfte erfordert, auch wenn in Ansätzen ein geschärfteres Bewusstsein für die möglichen Komplikationen im Aneignungsprozess zu beobachten ist. Gerade in Klassen, in denen es mehrere Lernende mit sprachlichen Problemen gibt, ist es für die Lehrkraft fast unmöglich, deren Schwächen beispielsweise im Textverständnis nicht wahrzunehmen. Erschwert wird dies allerdings dadurch, dass die Lehrenden meist einen „Einheitsschüler" vor Augen haben, auf den sie ihren Unterricht ausrichten (Gantefort \& Roth 2010, S. 575). Stattdessen ist es aufgrund der unterschiedlichen sprachlichen Ausgangslagen der Lernenden von größter Bedeutung, ihren jeweiligen Sprachstand zu ermitteln, um ihn individuell zugeschnitten erweitern zu können.

In Deutschland hat zu diesem Zweck in den letzten Jahren die Entwicklung von soliden Sprachdiagnostik-Instrumenten wieder zugenommen. ${ }^{59}$ Beweggründe für eine gesteigerte Entwicklung solcher Instrumente im Vergleich zu Italien können in der unterschiedlich intensiven Reaktion auf die schlechten PISA-Ergebnisse gesehen werden (Döll \& Dirim 2011, S. 153 für Deutschland; Ferreri 2006 für Italien) sowie in der Eigenheit des deutschen Bildungssystems, an einem früheren Punkt der Schullaufbahn eine entscheidende Schnittstelle eingebaut zu haben: Während in der Bundesrepublik zum Ende der Grundschulzeit über den Fortgang der Schulkarriere in den unterschiedlichen (und verschiedenartig mit Prestige behafteten) Institutionen Gymnasium, Realschule und z.T. noch Hauptschule entschieden wird, wechseln die SchülerInnen in Italien nach der fünfjährigen Grundschulzeit zwar die Schule, werden aber bis zum Ende der Sekundarstufe I nicht nach ihren Leistungen gruppiert, sondern gemeinsam auf der Mittelschule unterrichtet.

59 Für einen Überblick über die Geschichte der Entwicklung von Diagnose-Instrumenten in Deutschland s. Döll und Dirim (2011, S. 153 f.). Die Autorinnen verdeutlichen u.a., dass Lehrkräfte schon in den 1970er Jahren ein Interesse an derartigen Instrumenten bekundet und somit ihre Entwicklung angestoßen haben. Allerdings erfüllten die Testverfahren oft nicht oder nur unzureichend die Testgütekriterien. Weitere Gründe, warum die Entwicklung von neuen Tests bis zum PISA-Schock geruht hat, waren die Auffassung, die Lernenden mit Migrationshintergrund würden sich die deutsche Sprache durch ihren Aufenthalt im Land von selbst aneignen und die verbreitete Ablehnung der Tests wegen ihres selektionsfördernden Charakters (ibid., S. 154). Zur Ethik der Testentwicklung vgl. z.B. Messick (1988) und McNamara (2001). 
Anders als in Deutschland, wo der schon länger zu verzeichnende Anteil von allochthonen Schülerinnen und Schülern der zweiten Generation und ihre nicht etwa gestiegenen, sondern sich eher verschlechterten Leistungen (Gogolin 2009a, S. 265), zum PISA-Schock beigetragen und die Entwicklung von Sprachstandsdiagnose-Instrumenten vorangetrieben haben, sind die Differenzen zwischen den ein- und mehrsprachigen Lernenden sowie zwischen den mehrsprachigen Lernenden erster und zweiter Generation in Italien nicht so groß (s. INVALSI 2015). Im Vergleich zum europäischen Ausland und den USA hat auch der Trend zu nationalen Erhebungen und Sprachtests erst später eingesetzt, so dass bisher keine Diagnose-Instrumente für die Primarstufe vorliegen. Es gibt zwar einen Sprachtest für Schulkinder, der bereits in den 1980er Jahren entwickelt wurde, das sogenannte Glottokit (Gensini \& Vedovelli 1981), doch wird es meines Wissens derzeit kaum eingesetzt. Hierbei handelt es sich um ein für die Lehrkräfte erarbeitetes Analyseverfahren, um mit wenig zeitlichem Aufwand wichtige Informationen zu bestimmten sprachlichen Fähigkeiten der Lernenden unterschiedlichen Alters zu gewinnen (ibid., S. 46). Die Entwicklung dieses Messverfahrens fällt noch in die psychometrischstrukturalistische (auch: moderne) Phase der Testentwicklung (Spolsky 1981, S. 13-20), welche mit objektiveren und reliableren Methoden an die Stelle der vorwissenschaftlichen subjektiven Einschätzung durch das persönliche Urteil des Prüfers trat. So werden mit dem Glottokit mündliche und schriftliche Kompetenzen wie flüssiges Sprechen (mit einem Index, der die korrekten Wörter berücksichtigt, die in einer Minute produziert werden), die Beherrschung des Grundwortschatzes, die Schreibmotorik (Einhalten der Linien und des Randes, Konstanz der Größe und Breite der Buchstaben pro hundert Buchstaben) und die Lesekompetenz (Korrektheit und Tempo des lauten Lesens, Fähigkeit des leisen Lesens) sowie das Auftreten eines altersunangemessenen (infantilen) und egozentrischen Sprachgebrauchs überprüft (Gensini \& Vedovelli 1981, S. 47).

Da zum einen die Zahl der zweisprachigen SchülerInnen in Italien jedoch steigend ist und sich die nationalen Erhebungen trotz vieler Proteste vonseiten der Lehrenden und Eltern mehr und mehr etablieren und zum anderen inzwischen auch Sprachtests für die Anfängerniveaus in Italienisch als Fremd/Zweitsprache entwickelt sind (Barki et al. 2003), ist es möglich, dass sich in den nächsten Jahren auch die Entwicklung sprachlicher Diagnoseinstrumente für den Primarbereich etablieren wird.

Die Verfahren zur Sprachstandserhebung können Entscheidungen über die Schulreife am Ende der Elementarstufe oder die Schulempfehlung in der vierten Klasse beeinflussen, weshalb darauf hingewiesen wird, dass solche Instrumente nicht zu derartigen Entscheidungszwecken missbraucht werden sollten (McNamara 2001, S. 341 f.), sondern dazu gedacht sind, die rechtzeitige und punktuelle Förderung der Lernenden zu ermöglichen, die entsprechende Unterstützung benötigen. 
Einen Überblick über die in den letzten Jahren entwickelten Testverfahren geben z.B. Ehlich et al. (2007) in dem im Auftrag des BMBF entwickelten Bericht Anforderungen an Verfahren der regelmäßigen Sprachstandsfeststellung als Grundlage für die frühe und individuelle Förderung von Kindern mit und ohne Migrationshintergrund sowie Döll und Dirim (2011). Abgesehen von einer Verbesserung der Testgüte ${ }^{60}$ und einer stärkeren Betonung der ethischen Komponente vor allem in Hinsicht auf ihre Anwendung, ist außerdem hervorzuheben, dass die neueren Instrumente im Gegensatz zu ihren Vorläufern zumindest zum Teil versuchen, die Gesamtsprachlichkeit der Schüler zu erfassen, indem sie nicht nur die Sprachkenntnisse im Deutschen, sondern auch in verschiedenen Erstsprachen testen, bzw. Sprachwechsel festhalten. ${ }^{61}$

\subsection{Inhalte einer wirksamen Lehrerausbildung für sprachfördernde Zwecke}

\subsubsection{Sprach- und spracherwerbstheoretische Aspekte}

So hilfreich die Entwicklung qualitativ hochwertiger und in der Anwendung praktischer Diagnoseinstrumente ist, bleibt eine solide sprachwissenschaftliche Ausbildung der angehenden Lehrkräfte von primärer Wichtigkeit, welche auf den Umgang mit der sprachlichen Heterogenität ihres schulischen Alltags vorbereitet. ${ }^{62}$ Neben umfangreichem Wissen über die Landessprache und ihr

60 Allerdings wird auch nach der Veröffentlichung der Anforderungen an Verfahren der regelmäßigen Sprachstandsfeststellung (BMBF 2007) weiter bemängelt, ,,dass Qualität und Menge der Verfahren nicht in gleichem Maße zunehmen“ (Döll \& Dirim 2011, S. 154 mit Verweis auf Ehlich 2009, S. 17).

61 Eine besondere Berücksichtigung der Kenntnisse in der Erstsprache ermöglicht HAVAS 5 Hamburger Verfahren zur Analyse des Sprachstands bei 5-Jährigen; der Cito-Sprachtest erfasst rezeptive Fähigkeiten im Deutschen und im Türkischen; die FörMig Instrumente Tulpenbeet und Bumerang sind für die Sprachen Deutsch, Russisch und Türkisch entwickelt worden. Abgesehen von HAVAS 5 werden die Sprachfähigkeiten aber einzelsprachlich erhoben und ermöglichen daher keine Aussagen über die Gesamtsprachlichkeit der Testpersonen, zu der auch Formen der Sprachmischung gehören. Selbst mit HAVAS 5 werden Sprachwechsel nur notiert und nicht weiter in die Interpretation der erhobenen Daten einbezogen (s. Döll \& Dirim 2011, S.159-162).

62 Vgl. auch Maas (2008, S. 682; Hervorhebung im Original): „Produktiver Ausgangspunkt für die weiteren Arbeiten sind vor allem Forschungen zur Sprachentwicklung, bei denen deutlich geworden ist, dass Kinder von ihren mitgebrachten Ressourcen aus zunehmend komplexere Sprachsysteme booten - und damit zugleich die entwickelten Strukturen zunehmend idiomatisieren. Für die fortschreitende Analyse bieten typologische Analysen den Horizont, die Strukturäquivalenzen zu definieren erlauben, innerhalb von denen auch das Management von mehrsprachigen Ressourcen verläuft. Hier ist ein unmittelbarer Anknüpfungspunkt für sprachdidaktische Aufgaben gegeben, bei denen die Lehrer für den Umgang mit sprachlicher 
Kontinuum an Varietäten muss ein sprachtypologisches und -strukturelles Wissen aufgebaut werden, das an die Stelle ursprünglich (als die mehrsprachigen Lernenden noch aus einigen wenigen Herkunftsländern kamen) ausreichender kontrastiver Kenntnisse tritt und eine Grundlage für die Analyse der sprachlichen Schülerleistungen und für binnendifferenzierendes Unterrichten bildet (Maas 2008, S. 416; S. 728). Desweiteren sind Kenntnisse zum kindlichen Sprach- und zum Zweitspracherwerb nützlich ${ }^{63}$, wie z.B. das Wissen um natürliche Erwerbssequenzen und Konzepte wie die teachability-Hypothese (Pienemann 1989), nach der die Lernenden sich neue, komplexere Strukturen zum einen nur aneignen können, wenn ihnen die einfacheren, auf die diese aufbauen, ausreichend geläufig sind, und zum anderen schneller lernen, wenn eine bestimmte Ordnung in der Vermittlung der sprachlichen Strukturen eingehalten wird. ${ }^{64}$

Sowohl kognitionspsychologische Grundlagen der angewandten Linguistik als auch soziolinguistische und -kulturelle Theorien sind als weitere unverzichtbare Inhalte des Lehramtsstudiums zu erwägen. Nach der Überwindung von nativistischen Vorstellungen, dass die menschliche Prädisposition zum Zweitspracherwerb wie zum Erstspracherwerb angeboren sei und der Erwerb natürlich, implizit verlaufe (vgl. Chomskys Konzept der Universalgrammatik), sofern angemessener sprachlicher Input zur Verfügung steht (s. Krashens Input-Hypothese), rückten die kognitiven Implikationen des Spracherwerbs in den Vordergrund. Seit einigen Jahren wird aber für eine stärkere Betonung soziolinguistischer Aspekte in der Sprachlehr- und -lernforschung plädiert ( $\mathrm{Zu}-$ engler \& Cole 2005, S. 312). Dieser Blickwinkel ist besonders hilfreich, wenn es um die pragmatischen Seiten des Sprachgebrauchs geht, da mit dem Aufkommen der Soziolinguistik der Schwerpunkt in Richtung Kommunikation und Sprache als funktionales Werkzeug verschoben wurde. Aber auch zur bisher vorherrschenden kognitiven Ausrichtung stellt er einen Brückenschlag dar, indem er die Ansichten zur menschlichen Entwicklung und zum Erwerb seines Wissens und seiner Fertigkeiten komplementiert (Hawkins 2005, S. 25 f.). So ruft Lantolf in Erinnerung, dass bereits Vygotskij und Luria hervorgehoben haben, Kognition sei keineswegs nur eine Sache des Gehirns, sondern ein Zusammenspiel der Person und ihrer Umwelt, die gemeinsam ein funktionales System bilden (Lantolf 2005, S. 343):

Heterogenität und nicht nur (wie beim herkömmlichen Fremdsprachenunterricht) für den Kontrast spezifischer Sprachen ausgebildet werden müssen."

63 Einen Überblick über die Geschichte der Spracherwerbsforschung geben z.B. Chini (2005), Hawkins (2005), Nunan (2005) und Gregg (1999) sowie über entsprechende Forschung in Italien Banfi (1993a).

64 Diese nunmehr seit Jahrzehnten bekannte Erkenntnis wird allerdings bisher weder weitläufig im Unterricht noch ausreichend bei der Erstellung von Lehrmaterialen berücksichtigt (Pica 2005, S. 268): „This finding has tremendous implications and applications to teaching decisions. Yet, as Cook (2001) has noted, even the most widely used, up-to-date textbooks, fail to follow the sequences that Pienemann has identified.“ 
Vygotsky conceived of development as a social and cultural process-a process in which the person and the world are necessarily connected in a dialectic and inseparable relationship, and thus, while heads are dearly implicated in thinking, they do not do their thinking alone; rather they are part of what Luria (1973) refers to as a functional system formed by the person and the world - a system in which cognition is at its core distributed between brain(s), people, artifacts, and features of the environment (see Salomon, 1993). On this view, then, although brains are inside of heads, minds extend beyond the head and into the world of cultural artifacts and social relationships. It is not without relevance that Vygotsky himself used the term "cultural development" to capture the formation of uniquely human forms of thinking.

Soziolinguistisches Wissen ist für Erziehende und Lehrpersonen in verschiedener Hinsicht von Interesse. Zum einen dient es dem Verständnis der bereits vorgestellten Auffassung von Sprachkompetenz als Beherrschung des sprachlichen Raumes, der sich gerade in Italien extrem varietätenreich und damit komplex darstellt (Banfi 1993b, S. 37). Neben einer dadurch möglichen positiveren Einstellung zu den unterschiedlichen Registern bildet es die Grundlage zur Vermittlung entsprechenden Hintergrundwissens an die Lernenden. Auch wenn deren primäre sprachliche Sozialisation (der Erstspracherwerb) spontan verläuft und in den meisten Fällen gelingt (Cazden 1999, S. 64), kann kein Zweifel daran bestehen, dass ihnen auf die Registerdifferenz bezogene explizite Kenntnisse behilflich sind, unter der Führung des Lehrpersonals die Bedeutung einer situationsangemessenen Sprachverwendung zu erfassen.

Eine weitere soziolinguistische Thematik, welche Lehrkräfte verinnerlichen und im Unterricht verhandeln sollten, ist die vom linguistischen Standpunkt aus vorausgesetzte Gleichwertigkeit aller sprachlichen Varietäten, von denen eine jede zu jedem Zeitpunkt ihres Bestehens vollständig funktional dazu ist, das auszudrücken, was ihre Sprecher versprachlichen wollen. Damit kann vermieden werden, dass mehrsprachige Schülerinnen und Schüler (sei es mit familiärem Migrationshintergrund oder auch im Dialekt sprachsozialisierte) sich für ihre Erstsprachen schämen oder diese verstecken und dadurch in ihrer sprachlichen Entwicklung und ihrem Ausbau gehemmt werden. Denn es wird immer wieder beobachtet, wie die positive Einstellung von Kindern zu ihrer vom Standard abweichenden Sprache beim Älterwerden dramatisch abnimmt (Edwards 1999, S. 198). In diesem Zusammenhang kann mit ihnen ein Gespräch über die unabdingbar miteinander verknüpften Dimensionen von Sprache und Macht ${ }^{65}$ geführt werden, mit denen auch die Prestigefrage einzelner Idiome zusammenhängt. Zu diesem Aspekt lassen sich Beobachtungen zur augenscheinlich widersprüchlichen Förderung der Mehrsprachigkeit auf Ebene der Europäischen Union anstellen, welche sich auf den schulischen Fremdsprachenunterricht konzentriert und die Mehrsprachigkeit, die aus anderen Erstsprachen der Lernenden resultiert, ignoriert.

65 Hierbei handelt es sich um eine vor allem im Bereich der critical linguistics entwickelte Theorie, welche seit einigen Jahren ebenfalls sowohl in der Spracherwerbsforschung als auch in der Sprachlehr- und -lernforschung stärker berücksichtig wird (Perera 1999, S. 18; Hawkins 2005, S. 25). 
Soziale Aspekte wie Schichtzugehörigkeit, Ethnizität und Gender ${ }^{66}$ werden oft als Ursachen für Schulversagen herangezogen, wobei vor allem die Rolle der sozialen Schicht als Erklärungsgrund herhält. Diese delikate Problematik läuft Gefahr, zu kurzsichtig betrachtet und als unumstößlicher Hinderungsgrund für schulischen Erfolg hingenommen zu werden (s. Maas 2008, S. 680 bzgl. der Leistungen von Lernenden mit Migrationshintergrund). Fundiertere Kenntnisse hierüber können die Lehrenden davon abbringen, der verbreiteten Defizithypothese zuzustimmen, welche, von der Divergenz zwischen sprachlichen Praktiken zuhause und denen der Schule ausgehend, die „Schuld“ am Schulversagen in der häuslichen Situation sieht, und sie stattdessen dazu bewegen, eher eine Differenzhypothese zu vertreten. Diese Hypothese unterstreicht zwar auf gleiche Weise die Schwelle zwischen häuslichen und schulischen Sprachpraktiken, sucht aber nach anderen Wegen, sie zu verringern. Sie richtet ihren kritischen Blick eher auf die Institution Schule, in der Praktiken, welche nicht dem traditionellen autochthonen Mittelklassendurchschnittsmaß entsprechen, zumeist ignoriert oder geringschätzig abgetan werden. Differenztheorien schlagen deshalb Strategien vor, wie die schulischen Kommunikationsnormen erweitert werden können, damit sie einer größeren Gruppe von Lernenden - im besten Falle allen - zugänglich werden (MacLure 1999, S. 202 f.).

Dabei geht es nicht nur um die allein schon schwierig genug in didaktisches Handeln umzusetzende Auffassung, dass die sprachliche Ausgangslage des Kindes oder Jugendlichen wertgeschätzt und auf sie aufgebaut werden soll (Perera 1999, S. 19), was erst einmal eine genauere Kenntnis der häuslichen Sprachsozialisation vonseiten der Lehrperson voraussetzt. Dabei wird verlangt, dass der Einblick in die Lebenswelt der Lernenden weitergeht und sich auch auf die Kultur und Identität der Lernenden ausweitet, damit diese Aspekte sprach- und entwicklungsförderlich in den Unterricht eingebunden werden können. Derartige Einsichten können durch Hausbesuche ${ }^{67}$ gewonnen werden,

66 In ihrer Studie zum Erdkundeunterricht beobachtet Lee, dass Jungen das Unterrichtsgespräch beherrschen und mehr Fachausdrücke in ihren Beiträgen verwenden als Mädchen (Lee 1996, S. 79). Es sei vorweggenommen, dass Ähnliches in dieser römischen Studie nicht festgestellt werden konnte. In allen fünf Klassen haben sich die Mädchen nicht weniger am Unterricht beteiligt als die Jungen, was auf ein kulturell erklärbares größeres Selbstbewusstsein der italienischen Mädchen zurückzuführen sein könnte oder auch einfach auf den Umstand, dass Mädchen in dieser Altersklasse den Jungen in der Regel in der Entwicklung etwas voraus sind und sich dadurch weniger passiv verhalten.

67 Bezüglich der Hausbesuche bleibt anzumerken, dass dieser Rat aus den UsA stammt, wo eine solche Praxis üblicher zu sein scheint als in Ländern wie Deutschland und Italien. Es sei jedoch erwähnt, dass eine der Lehrkräfte, die an dieser Studie mitgewirkt hat, einzelne SchülerInnen und ihre Eltern zuhause besucht und bei einem gemeinsamen Abendessen näher kennengelernt hat. Auf diese Weise kann ein Verhältnis aufgebaut werden, wie es bei formaleren Zusammenkünften (z.B. bei Elternsprechtagen) nur schwer möglich ist. Allerdings sollte nicht vergessen werden, wie es um die finanzielle Vergütung der Lehrkräfte in Italien 
wenn es nicht sogar möglich ist, die Familien in family literacy-Projekte einzubinden (Hawkins, S. 37 f.).

Dass Sprachbildung auch immer die soziale Entwicklung vorantreibt (Vedovelli 1993, S. 24), lässt sich gut mit dem Begriff der Sprachsozialisation fassen. Als Sozialisation wird der Prozess der Internalisierungen bezeichnet, durch die ein Individuum in eine bestimmte Kultur hineinwächst, indem es lernt, wie darin üblich zu sprechen, zu agieren, zu denken und zu fühlen (Cazden 1999, S. 63). Hierbei wird zwischen primärer und sekundärer Sprachsozialisation unterschieden, wobei die zweite von allen Personen, also auch von einsprachigen, durchlaufen wird, wenn sie während ihrer Schulpflicht mit dem Sprachausbau beginnen. Cazden hebt allerdings hervor, dass die sekundäre Sozialisierung nicht auf die Schulzeit beschränkt ist, sondern ein ganzes Leben lang andauert, da der sprachliche Raum eines Menschen keine konstante Größe ist. Stattdessen erweitert er sich im Laufe des Lebens (und verkleinert sich zum Teil auch, wenn bestimmte Sprachpraktiken nicht mehr vollzogen werden), weil sich das Registerbündel bei neuen Aufgaben, Situationen und Kontakt zu neuen Menschen erweitern kann (ibid.).

Während also in der ersten Phase der Spracherwerbsforschung und auch in den folgenden Jahren das Hauptaugenmerk auf der kognitiven Komponente lag und der/die Lernende vorrangig als isoliertes Individuum betrachtet wurde, gelingt mit dem soziolinguistischen Ansatz eine Beschreibung des dialektischen Zusammenwirkens von Individuum und Gesellschaft, welche für den Sprachausbau entscheidend ist und gleichzeitig die belangvolle Aufgabe der Sprache im generellen Sozialisationsprozess unterstreicht. Cazden verweist in diesem Zusammenhang auf Sapir, dem diese dialektische Dynamik bereits 1962 beachtenswert erschien: ,[I]n spite of the fact that language acts as a socializing and uniformizing force, it is at the same time the most potent single known factor for the growth of individuality" (Sapir 1962, S. 19 zit. in Cazden 1999, S. 65). Auch McKay hebt diese doppelte Funktion der Sprache hervor, die uns die Verbindung zu anderen Menschen ermöglicht und gleichzeitig für die Entwicklung von Individualität entscheidend ist, indem er Fasold (1984) zitiert (McKay 2005, S. 292):

As Fasold (1984) notes: The essence of sociolinguistics, in my view, depends on two facts about language that are often ignored in the field of linguistics. First, language varies - speakers have more than one way to say more or less the same thing.... Second, there is a critical purpose that language serves for its users that is just as important as the obvious one. It is obvious that language is supposed to be used for transmitting information and thoughts from one person to another. At the same time, however, the speaker is using language to make statements about who she is, what her group loyalties are, how she perceives her relationship to her hearer, and what sort of speech event she considers herself to be engaged in (p. ix.).

steht, weshalb die generelle Motivation zu solchen Aktivitäten, die Zeit beanspruchen und nicht bezahlt werden, eher gering sein dürfte. 


\subsubsection{Pädagogische und sprachdidaktische Aspekte}

Abgesehen von der Prägnanz einer umfassenderen sprachwissenschaftlichen Ausbildung darf auch die Vermittlung pädagogisch-didaktischen Wissens nicht zu kurz kommen. Darauf wird an dieser Stelle explizit hingewiesen, da auch während der Unterrichtsbeobachtungen im Zuge dieser Studie die Schwachstelle des Systems deutlich wurde, die darin liegt, dass lange Zeit ein abgeschlossenes Fachstudium die einzig nötige Voraussetzung für die Ausübung des Lehrberufs darstellte: Ein großes Potential an pädagogischen und didaktischen Maßnahmen zur Förderung des Lernens findet bisher wenig Einzug in die Gestaltung des Schulalltags (s.a. Jafrancesco 2002). Hierbei ist primär an die Unterrichtsführung zu denken.

Es ist seit langem bekannt, dass der Produktion im Spracherwerb und -ausbau eine wesentliche Bedeutung zukommt (vgl. Swains Output-Hypothese (1985); Christie 2012, S. 23), weil auf diese Weise sprachförderliche Handlungen ablaufen. Zum einen erhöht sich durch wiederholten Output der Sprachfluss, was zwar nicht automatisch zu einem korrekteren Sprechen führt, aber dafür sorgt, dass bestimmte Sprechakte automatisiert werden, so dass die kognitiven Kapazitäten entlastet und die Konzentration auf die Bewerkstelligung anderer Aufgaben gelenkt werden kann. Zum anderen wird den Lernenden im Moment der schriftlichen oder mündlichen Produktion sehr bewusst, welche sprachlichen Elemente ihnen noch Schwierigkeiten bereiten, wenn sie erfahren, dass sie etwas nicht so ausdrücken können, wie sie es möchten. Gleichsam stellt die Produktion von Output eine Gelegenheit dar, sprachliche Hypothesen auszuprobieren: Wenn sich die Lernenden nicht sicher sind, ob sie ein Wort richtig kennen oder es angemessen verwenden, können sie es schlicht ausprobieren und anhand der Reaktion des Gesprächspartners die Richtigkeit bzw. Fehlerhaftigkeit ihrer Hypothese überprüfen. ${ }^{68}$

Swain betont darüber hinaus, dass sich unsere Gedanken durch ihre Formulierung verändern können, wenn beispielsweise Ungereimtheiten offenbar werden (Swain 2005, S. 479), so dass Output also nicht nur auf die Sprachentwicklung, sondern darüber hinaus auf die Kognition Einfluss nimmt (ibid., S. 481):

The most significant new understanding for me in studying language production is just how very important it is as a cognitive tool - as a tool that mediates our thinking. Pursuing this idea widens greatly the second language learning and teaching research agenda.

68 Swain verweist in diesem Zusammenhang auf Studien von Loewe, die ergaben, dass ca. drei Viertel aller Äußerungen aufgrund der Reaktion des Gesprächspartners modifiziert bzw. verbessert werden (Swain 2005, S. 476). Im Austausch mit anderen werden einer/einem Lernenden also die Fehler und Lücken in der eigenen Sprachkompetenz eher bewusst. 
Trotz derartiger Erkenntnisse wird nicht nur in Italien, sondern auch in den Vereinigten Staaten und vielen anderen Ländern beobachtet, dass die Redeanteile der Lehrkraft die ihrer Lernenden exorbitant übersteigt. So scheint es eine verbreitete Praxis zu sein, dass zwei Drittel der Gesprächszeit in einer Schulstunde vom Output der Lehrperson bestimmt ist und nur ein Drittel auf die Lernenden entfällt (Mercer 1999, S. 316 mit Verweis auf Flanders 1970) ${ }^{69}$, wobei in manchen Studien Redeanteile der Lehrenden von bis zu 80 Prozent verzeichnet wurden (Nunan 2005, S. 227).

Ohne den lehrerzentrierten Unterricht pauschal als ineffizient hinstellen zu wollen, liegt auf der Hand, dass die aktive Mitarbeit der Schülerinnen und Schüler lernförderlich ist und zu einer erhöhten Motivation beiträgt (Battisti 1988, S. 62; Gueriero 2014, S. 163 f.). Da gerade für den Sprachausbau und die Verbesserung zweitsprachlicher Kenntnisse eine erhöhte produzierende Eigenaktivität der Lernenden maßgeblich ist, bieten sich im italienischen Kontext mehr schriftliche (Haus-)Aufgaben und vor allem generell der verstärkte Einsatz von Arbeitsformen wie Gruppen- und Partnerarbeit an, die bekanntermaßen von vielen Lehrpersonen zwar theoretisch als lernförderlich erachtet werden, in der praktischen Umsetzung aber zu gemischten Gefühlen führen (A. Helmke 2009², S. 212).

Margaret R. Hawkins hat einen aus sieben Schlüsselkonstrukten bestehenden Referenzrahmen für das Verstehen von Sprach- und Literalitätsentwicklung im Unterricht entworfen (Hawkins 2004 zit. in Hawkins 2005, S. 26 ff.), an dem sich die Lehrpersonen bei der Suche nach Maßnahmen für eine sprachförderliche Unterrichtsgestaltung orientieren kann. Er soll im Folgenden vorgestellt werden, wobei nur auf die ersten beiden und den letzten Punkt näher eingegangen wird, da die übrigen w.o. bereits behandelten Punkte wie die der Registerdifferenzen, der soziokulturellen und -linguistischen Aspekte (z.B. das Verhältnis von Sprache und Macht) und der Bedeutung des Sprachgebrauchs zur Herausbildung und Übermittlung von individuellen Persönlichkeitsmerkmalen darstellen (Hawkins 2005, S. 26 f.):

- Communities of Learners/Communities of Practice

A view of classrooms as environments that promote "scaffolded interaction", where "communities of learners" engage in cultural practices, with participants taking on various and different ("asymmetrical") roles over time. Knowledge is distributed across the community, not in the possession of any one individual member, thus available to all through activity-based interactions. (Brown, 1994; Lave, 1996; Rogoff, 1994)

- Zone of Proximal Development/Apprenticeship

69 Diese Erkenntnis ist Teil der sogenannten Zwei-Drittel-Regel: Zwei Drittel einer Schulstunde spricht jemand. Zwei Drittel dieser Zeit ist es die Lehrperson, und zwei Drittel ihrer Redeanteile bestehen aus Vorträgen und Fragen an die Schülerinnen und Schüler (Mercer 1999, S. 316). 
Learning as a process of "apprenticeship" where apprentices collaborate in (mediated) social practices with teachers and more expert peers, thus affording scaffolding, or support, in the process of acquiring/ constructing new forms of interaction, language, and thinking.

(Vygotsky, 1978, 1986; Wertsch, 1998)

- Identities/Positioning

The socially situated identities participants in social interaction take on at any given time are a complex integration of their diverse sociocultural experiences, the sociocultural experiences of others in the interaction, the structure and flow of language, participation and negotiation in the interaction, and the larger cultural and institutional settings within which the interactions take place. (Bernstein, 1990; Edwards \& Potter, 1992; Fairclough, 1992; Hanks, 1996; Schegloff, Ochs, \& Thompson,1996)

- Power/Status

Meaning and social relationships are interdependent. Social interactions are situated in specific local contexts, which are always part and parcel of larger social, institutional, and community contexts. Embedded are ideologies, beliefs, and values, which are carried out and reproduced through the unfolding social interactions.

(Foucault, 1980; Lemke, 1995)

- Multiple (Social) Languages

No language exists as a general thing. Rather, each language is comprised of many different "social languages," that is, different styles of language that communicate different socially situated identities (who is acting) and socially situated activities (what is being done). Every social language communicates in use, as it creates and reflects specific social contexts, socially situated identities integrally connected to social groups, cultures, and historical formations.

(Bakhtin, 1981; Gee, 1996)

- Multiple Literacies

Literacies are defined as the requisite knowledge and skills to send and interpret messages through multiple media and modes in (rapidly changing) local and global contextsand to align meanings within situated social practices.

(Cope \& Kalantzis, 1999; Kress \& Van Leeuwen, 2001)

- Classroom-as-Ecology

Classrooms viewed as ecological systems-the co-dependence of factors that together construct and define the nature of the learning that takes place.

Das Konstrukt Communities of Learners/Communities of Practice spielt der Forderung nach einer schülerzentrierteren Unterrichtsgestaltung zu. Hierbei werden die Lehrperson und die Lernenden als eine Gemeinschaft betrachtet, in der es nicht darum geht, das Wissensgefälle zwischen den am Unterrichtsprozess beteiligten Parteien hierarchisch zu untermauern und die Wissensvermittlung unilateral von der Lehrperson an die Lernenden gerichtet zu gestalten. Stattdessen wird Wissen gemeinsam konstruiert, wobei auch die Lernenden eine Expertenrolle übernehmen können.

Damit das gelingen kann, muss die Lehrperson Fähigkeiten der Lernenden erkennen, die im traditionellen Unterricht keine oder kaum eine Rolle spielen. Auch hier gilt ähnlich wie mit dem scaffolding-Ansatz wieder, dass man von der angelsächsischen Didaktik in den klassischen Einwanderungsländern ler- 
nen kann, die seit geraumer Zeit eine valorisierendere Haltung gegenüber unkonventionellen Schülerleistungen umsetzt (Maas 2008, S. 676, Fn. 54). Ein prominentes Beispiel sind Gedichte und Raps von Lernenden, in denen das Codeswitchen charakteristisch ist. Bei der Wertschätzung von Schülerwissen und -fähigkeiten geht es allerdings nicht darum, den Fokus vom Sprachausbau in eine „Wohlfühlzone“ zu verlagern, sondern es kann auch hier auf vielerlei Art sprach(ausbau)förderlich unterrichtet werden.

Konkret auf diese Studie und die sprachlichen Herausforderungen des Erdkundeunterrichts in der achten Klasse bezogen, sah der Lehrplan das Befassen mit den Herkunftsländern einiger SchülerInnen (oder ihrer Eltern) vor. Hierbei die entsprechenden Lernenden nicht zur Präsentation ihrer Länder oder zu einer Fragerunde heranzuziehen, ist eine verpasste Gelegenheit: Diejenigen, die sonst oft zu den eher leistungsschwächeren SchülerInnen zählen, könnten einen Wissensvorsprung gegenüber den anderen zeigen und damit ihr Selbstvertrauen stärken und eine Lernmotivation erhalten. ${ }^{70}$

Bezüglich des zweiten Punktes des Konzepts ist eine effiziente Form der gegenseitigen Unterstützung im Lernprozess die Partnerarbeit, bei der ein schwächerer Schüler vom Wissen und von den Erläuterungen eines Mitschülers profitiert. Ebenfalls geeignet sind diverse Arten der Gruppenarbeit, in der eine Aufgabe erarbeitet wird, die aus der Fachliteratur als „Lehren durch Lernen"-Szenario der scripted cooperation bekannt ist (vgl. A. Helmke 2009², S. 209): In leistungsheterogenen Gruppen erarbeiten die Lernenden z.B. gemeinsam einen Text. Zunächst liest jedes Gruppenmitglied den ganzen Text und wendet sich bei eventuellen Verständnisschwierigkeiten an die anderen. Anschließend stellen alle je einen Abschnitt vor, wobei die anderen Gruppenmitglieder das Vorgestellte auf Vollständigkeit und sprachliche und inhaltliche Korrektheit überprüfen.

Noch weiter in die Expertenrolle schlüpfen können die Lernenden, wenn jede Gruppe einen anderen Text liest und die wesentlichen Punkte, die in den einzelnen Gruppen zusammengetragen werden, in einer späteren Phase dem textunkundigen Klassenplenum vorgestellt werden. ${ }^{71}$

Mit dem letzten Punkt des Konstrukts ist eine Orientierungshilfe für sprachförderlichen Unterricht angesprochen, welche auf der Konzeption Unterrichtsgeschehens als ökologisches System fußt. Viele Lehrpersonen machen spätestens in ihren ersten selbst gestalteten Unterrichtsstunden die Erfahrung, dass es unmöglich ist, eine Stunde zu entwerfen und genau nach Vorstellung umzusetzen. Wer das Unterrichtsgeschehen als ökologisches System, als etwas

70 Dabei ist eine gewisse Sensibilität wünschenswert, da traumatische Erlebnisse der Lernenden oder ihrer Familien nicht ausgeschlossen werden können und niemand sich gezwungen fühlen sollte, von seiner Heimat, Kultur oder Religion zu berichten. Die Bereitschaft wird außerdem maßgeblich vom Klassenklima und von den Modalitäten der Gesprächsführung im Unterricht beeinflusst.

$71 \mathrm{Zu}$ dieser sogenannten information gap-Technik siehe z.B. Gibbons (2002, S. 27). 
Lebendiges, was nicht statisch, sondern in Bewegung und Transformation ist, betrachtet, bleibt offen für die Reaktionen der Lernenden und kann auf diese Weise die Vermittlung der Lerninhalte passender koordinieren. In diesem $\mathrm{Zu}$ sammenhang ist es für eine effektive Sprachförderung sehr wichtig, dass die Lehrpersonen in ihrem Handeln nicht so stark von ihren inhaltlichen Vorstellungen geleitet sind, dass sie Schüleräußerungen, die damit nicht kongruieren, tendenziell überhören. Derartige Beiträge der Lernenden sollten nicht ignoriert oder sofort verbessert werden. Stattdessen sollte die Lehrkraft nur einen Anstoß geben, damit sie sie eigenständig überdenken und modifizieren. Gibbons formuliert (2002, S. 38):

[T]here is considerable evidence that learners who have more opportunities to reflect on and improve their own communication receive more long-term benefits for language learning than those who have constantly solved communication problems for them by the teacher. $[\ldots]$

[I]t is important to respond to meaning. This involves really listening to what the students say, rather than waiting for the answer you would like them to give!

Weitere Merkmale des Ökosystems Klassenzimmer definiert Hawkins (2005, S. 28 f.) folgendermaßen:

[A]n ecological system is a living thing constantly shifting, with forces aligning and realigning to shape new landscapes. Thus, just as learner forms of language are not fixed but shift and develop through time, so do all other components within the ecology, constantly redesigning the interrelations between them and the meanings assigned to them. What this means, then, is that we cannot account for, say, a participant's "culture," or "identity," without acknowledging that at best we are describing interpretations at a particular moment in time.

Thus, research into English language learning in classrooms must account for not only discrete variables and components of language and language learning, but for ways in which these "pieces" come together to form a whole - an ecology within which it is the dynamics between and among variables that accounts for the learning that takes place.

Eine derart flexible und ergebnisoffene Haltung ist für viele Lehrpersonen vermutlich keine schnell und einfach zu erwerbende Fähigkeit, aber das theoretische Wissen über ihren pädagogischen Sinn sowie positive Erfahrungen mit dem Erproben ihrer Anwendung im Unterricht können entscheidend dazu beitragen, sie zu entwickeln. Dazu verhilft desweiteren ein regelmäßiges kritisches Hinterfragen des eigenen Handelns, was seinerseits durch Methoden vorangetrieben werden kann. Dazu zählen Videoaufzeichnungen des eigenen Unterrichts und neuerdings vermehrt angewandte wie gegenseitige Unterrichtsbesuche, die auf Eigeninitiative in kleinen Gruppen von drei bis vier Lehrpersonen erfolgen, die sich gegenseitig schätzen und vertrauen. 


\subsection{Arbeitsbedingungen im Kontext sprachlicher Heterogenität in den beobachteten Klassen}

Bezogen auf die italienische Situation ist zu vermerken, dass fachdidaktische sowie allgemein- und sonderpädagogische Kenntnisse auch in der derzeitigen Lehrerausbildung allein innerhalb eines einzigen Semesters im Lehramts-Aufbaustudium vermittelt werden. Wenn also ein guter Teil der heute unterrichtenden Lehrkräfte überhaupt keine pädagogische Ausbildung genossen und die letzte Generation von Lehrpersonen sich mit diesen Themen aus zeitökonomischen und organisatorischen Gründen nur marginal befasst hat, ist dies auch ein Erklärungsgrund für die von verschiedenen Wissenschaftlern beobachtete Tatsache, dass sich etliche Lehrpersonen nicht für die aktuellen Forschungsbefunde interessieren. Denn es ist wahrscheinlich, dass diejenigen, welche sich im Studium intensiv mit Veröffentlichungen von Relevanz für ihr künftiges Berufsfeld befasst haben, auch nach dem Abschluss des Studiums während ihrer Berufszeit eher versuchen, den aktuellen Forschungsstand weiterhin mitzuverfolgen. ${ }^{72}$ Von den in dieser Studie beobachteten Lehrkräften haben vier ihre Tätigkeit vor der Einführung der Lehramtsschulen SSIS (1999/2000) aufgenommen. Ihre Einstellungsqualifikation bestand also in einem abgeschlossenen Studium, in dem sie keine pädagogischen und fachdidaktischen Kenntnisse erworben haben. Ohne viel über ihr didaktisches Handeln vorwegzunehmen, mit dem sich das neunte Kapitel über die Unterrichtsbeobachtungen befasst, sei erwähnt, dass sie zwar alle um die Sprachbildung ihrer Lernenden auch im Erdkundeunterricht bemüht waren, ihn aber dennoch überwiegend traditionell lehrer- und lehrbuchzentriert gestalteten.

72 Hauptsächlich liegt das Problem im Desinteresse der Praktiker an den Ergebnissen der Theoretiker allerdings wohl in der Art und Weise, wie Letztere ihre Ergebnisse formulieren (Maas 2008, S. 668 Fn. 13; Hawkins 2005, S. 38 f.; Pica 2005, S. 277; Nunan 2005, S. 234), und in der Tatsache, dass die Lehrkräfte nur langsam und nur teilweise aktiv in die Forschungsprojekte einbezogen werden, obwohl sich diese Zusammenarbeit für beide Seiten als fruchtvoll erwiese. Auch Nunan (2005, S. 234) unterstreicht dieses Manko mit Verweis auf Beasley und Riordan (1981, S. 60) und Carr und Kemmis (1985, S. 6) und erläutert den Versuch zur Überwindung dieses Mankos mittels sogenannter action research in Australien. Diese wird direkt von den Lehrpersonen durchgeführt, ist aber nicht freigeblieben von Kritik (vor allem in Bezug auf die Reliabilität und Validität der Befunde) (Nunan 2005, S. 234 f.). Hawkins (2005, S. 38 f.) weist außerdem auf eine weitere in den Recherchen zumeist fehlende Stimme hin, nämlich die der Lernenden. Letztere werden zwar im Unterricht beobachtet, als Testteilnehmer und Informationsträger zu Sprachdaten u.Ä. herangezogen, die Interpretation ihrer Beiträge wird allerdings fast ausschließlich von anderen ohne ihren Einbezug vorgenommen. Erst die neueste Forschung beruht in einigen Bereichen auf einer gezielteren Einbindung der Lernenden, s. etwa Befragungen der TestteilnehmerInnen und zu den Strategien und Gründen für ihre Antworten (Nation 2001 mit Verweis auf Paribakht und Wesche (1996) oder Protokolle des „lauten Denkens“ während des Schreib- oder Leseprozesses (s. Lumbelli 1989, S. 42 f.). 
Keine der drei beobachteten Schulen Vespucci, Pestalozzi und Morante ${ }^{73}$ wies im Erhebungszeitraum die erforderliche Anzahl von mehrsprachigen SchülerInnen auf, um vom Bildungsministerium zusätzliche finanzielle Ressourcen für Sprachbildungsmaßnahmen bewilligt zu bekommen. ${ }^{74}$ Dafür muss die Schülerschaft nämlich zu mindestens 15 Prozent aus allochthonen Lernenden bestehen, und ihr Anteil machte in den von mir beobachteten Schulen nur maximal 11 Prozent (Schule Vespucci) aus. So wie bei dieser Schule handelt es sich allerdings auch bei den anderen beiden um exemplarische Bildungsinstitutionen, die auf sich selbst gestellt sind, wenn sie der sprachlichen Heterogenität der Lernenden gerecht werden und Letztere angemessen fördern möchten. Denn in allen beobachteten Klassen waren mehrsprachige Lernende unterschiedlicher Herkunftsländer (und auch einsprachig Italienische) vertreten, welche einer sorgfältigen Sprachförderung bedurften.

In den Klassen an den Schulen Vespucci und Morante gab es neben weiteren Lernenden, die mit ihren Familien aus dem Ausland nach Italien gezogen waren, jeweils zwei, die erst während der Mittelschule in das Land einreisten und zu dem Zeitpunkt noch kein Wort Italienisch sprachen. Erschwerend kam außerdem hinzu, dass es sich dabei um Kinder handelte, die aus Ländern wie China und Ägypten kamen, in denen typologisch nicht mit dem Italienischen verwandte Sprachen gesprochen und andere Schriftsysteme benutzt werden. Sie wurden direkt in den normalen Schulbetrieb aufgenommen, und bei ihrer Inklusion in den Klassenverband konnten sich die beobachteten Lehrkräfte nur auf die Mithilfe einer Unterstützungslehrkraft verlassen, welche den beiden Klassen aufgrund der Anwesenheit von Lernenden mit Lernbeeinträchtigungen zugeteilt waren. Davon abgesehen, dass dies eigentlich nicht in den $\mathrm{Zu}-$ ständigkeitsbereich der Unterstützungslehrkraft fällt, war eine von ihnen zunächst mit neun Wochenstunden nur die Hälfte der maximal vorgesehenen Zeit anwesend, so dass die beobachteten Lehrkräfte der Aufgabe überwiegend allein gerecht werden mussten.

Während an der Schule Morante zumindest periodisch am Nachmittag ein von ehrenamtlichen Helfern durchgeführter Sprachunterricht stattfand, bestand an der Schule Vespucci kein solches Angebot. Nach Angaben der Lehrkräfte stehen Schulen wie ihre vor einer nur mit Mühe und Erfindungsgeist zu

73 Die Namen der beobachteten Schulen und Lehrkräfte sind durch Aliasnamen ersetzt worden.

74 Das Gesetz 144/1997 (MIUR) sieht vor, dass Bildungsinstitutionen finanzielle Zuschüsse erhalten, um mit schulinternem (i.d.R. dafür nicht ausgebildetem) Personal zusätzliche Aufgaben wie beispielsweise Sprachkurse abzudecken. Auf schulexterne Unterstützung kann hingegen nur zugegriffen werden, wenn der Antrag einer Bildungsinstitution für ein entsprechendes Projekt von der Region bewilligt wird. Hierfür wurden bisher pro Projekt von einer Laufzeit von zwei Jahren ca. 3.000 Euro bereitgestellt, wobei die Zahlungen spät erfolgten und ohnehin nur Initiativen von Schulen in „Risikogebieten“ und mit einem erheblich höheren Anteil von mehrsprachigen Lernenden als in den von mir beobachteten genehmigt wurden. 
bewältigenden Herausforderung, seitdem mit der nach der damaligen Ministerin benannten Schulreform Gelmini (Gelmini 2009) finanzielle Kürzungen in Kraft traten, das Unterrichten im Team abgeschafft und die Arbeitszeiten reduziert wurden. Darüber hinaus lägen alle drei Schulen in einem Einzugsgebiet der unteren Mittelschicht und der Arbeiterschaft, in denen sich Familienangehörige der Lernenden wesentlich seltener für ehrenamtliche Tätigkeiten wie Sprach- oder Nachhilfeunterricht bereit erklärten als in Einzugsgebieten mit finanziell besser gestellten Familien.

Die Lehrkraft an der Schule Vespucci hat aufgrund ihrer mehrjährigen Tätigkeit an dieser Schule, während der sie immer wieder mit Lernenden konfrontiert war, die noch gar kein Italienisch sprachen, aus eigener Initiative eine Qualifikation als Lehrkraft für Italienisch als Zweit- und Fremdsprache erworben. Zusammen mit einer beobachteten Kollegin sind sie die einzigen, die während des beobachteten Unterrichts Settings wie Partner- oder Gruppenarbeit einrichteten. Es ist also davon auszugehen, dass die Lehrkräfte dieser Studie ohne ein im Studium erworbenes pädagogisches Handlungswissen so unterrichteten, wie sie es selbst während ihrer Schulzeit erlebt haben (Konzentration auf den Erwerb und das Abfragen von Lehrbuchinhalten) und eher intuitiv auf die sprachlichen und lernbezogenen Schwierigkeiten der Schülerinnen und Schüler reagierten.

Bevor in Kapitel 6 die einzelnen Klassen näher vorgestellt und die anhand eines Fragebogens gewonnenen Informationen über den sprachlichen Hintergrund der Lernenden sowie ihre Einschätzungen in Bezug auf den Erdkundeunterricht und das Lernverhalten wiedergegeben werden, erläutert das folgende Kapitel die in der Studie eingesetzten Untersuchungsmethoden. 


\section{Methodische Betrachtungen zur Studie}

In diesem Kapitel wird das methodische Vorgehen zur Datengewinnung und auswertung vorgestellt. Zunächst beleuchten einige Informationen den Erhebungshintergrund (5.1), wobei eine kurze Präsentation der im Vorfeld durchgeführten Erkundungsstudie und der daraus resultierenden Einsichten in eine möglichst ergiebige Studiendurchführung (5.2) der Beschreibung der Erhebungsgruppe vorausgeht (5.3). In diesem Zusammenhang werden auch die Auswahl der neben den Unterrichtsbeobachtungen eingesetzten Erhebungsinstrumente zur Messung (bildungs)sprachlicher Kompetenzen (5.2.1) und der Einsatz eines Fragebogens zur Gewinnung von sprachlichen und weiteren Hintergrundinformationen über die Lernenden (5.3.1) illustriert. Es folgt eine Darstellung der Art und Weise, in der die Unterrichtsbeobachtungen vorgenommen wurden, um das sprachliche Handeln der einzelnen Akteure in den Blick zu nehmen (5.4). Hierbei sind die Texte der Lehrwerke in der Vermittlung von Lerninhalten durch die Lehrperson und bei ihrer Widergabe durch die Lernenden im Zuge regelmäßiger mündlicher Leistungskontrollen zentral, die jedoch in der Forschung und von vielen Lehrpersonen als sprachlich kompliziert eingestuft werden (Compagni et al. 1997; Deon 1997). Da ihr Schwierigkeitsgrad zum Teil als so hoch eingestuft wird, dass an eine eigenständige Erarbeitung durch die Lernenden nicht zu denken ist (z.B. Bertocchi 2003; Piemontese \& Cavaliere 1997), verbinden sich hiermit auch die Fragen, ob bzw. wie die Lehrpersonen eine Brückenfunktion übernehmen, um den Lernenden die Texte zugänglich zu machen, und ob bzw. wie sie dabei die Lernenden in ihrem sprachlichen Ausdrucksvermögen fördern. Ein weiteres Unterkapitel beschäftigt sich mit den Modalitäten der sprachlichen Analyse der Lehrmittel (5.5). Im Anschluss daran werden die ausgewählten Ermittlungsverfahren produktiver und rezeptiver schriftsprachlicher Leistungen der Schülerinnen und Schüler vorgestellt (5.6), welche aus lehrbuchbasierten C-Tests (5.6.1) sowie zusätzlichen schriftlichen Textproduktionen der Lernenden bestehen (5.6.2).

\subsection{Der Erhebungshintergrund}

Wie eingangs vorgestellt wurde, ist sprachliche Förderung im Fachunterricht in Italien in den Lehrplänen festgelegt, und ist man sich über die Bedeutung einer in diesem Sinne durchgängigen Sprachbildung in allen Fächern und Klassenstufen einig. Was allerdings sprachlich im Fachunterricht gefordert ist und wie dort tatsächlich kommuniziert wird, ist nicht nur in Italien bisher kaum empirisch beforscht (Ahrenholz 2013, S. 87; Harren 2015, S. 2). Wenn es Stu- 
dien gibt, so konzentrieren sie sich meist auf die Sprachkompetenz von zweisprachigen Schülerinnen und Schülern (z.B. Schmölzer-Eibinger 2011; Schmölzer-Eibinger \& Weidacher 2007), und dabei vor allem auf den schriftlichen Sprachgebrauch (Harren 2015, S. 30, S. 95). ${ }^{75}$ Allerdings ist schon betont worden, dass auch viele in der Erstsprache lernende Schülerinnen und Schüler sprachbezogenen Lernbedarf haben, zumal die sprachlichen Anforderungen für schulischen Lernerfolg, die Entwicklung der Persönlichkeit und die Partizipation am gesellschaftlichen Leben sowie am Arbeitsmarkt nicht zu unterschätzen sind (De Mauro 1976b).

Während der (schulische) Spracherwerb in seinen Anfängen sehr intensiv untersucht wurde (z.B. Cummins 1979b, 1980, 1981; Halliday 1993, 2002, 2007; Perdue 1993; Chini 2000; für einen Überblick Ehlich, Bredel \& Reich (BMBF 2008) und bezogen auf den kindlichen Zweitspracherwerb und seine Förderung z.B. Ahrenholz 2006) ${ }^{76}$, besteht ein wesentlich geringeres wissenschaftliches Interesse am weiteren Spracherwerb, vor allem am erstsprachigen. Ein Grund hierfür kann in der noch verbreiteten Annahme gesehen werden, dass dieser bezogen auf den mündlichen Sprachgebrauch natürlich erfolgt und in den meisten Fällen auch gelingt (s. Maas 2008, S. 186; Gogolin 2009b, S. 98; Koesters Gensini 2010, S. 112; Harren 2015, S. 95). Hinsichtlich der sprachorientierten Unterrichtsforschung ist zu vermuten, dass sie neben möglichen Finanzierungsschwierigkeiten auch wegen des erheblichen Zeitaufwandes, der insbesondere mit den Transkriptionen und Auswertungen von Sprachproduktionen verbunden ist, kaum betrieben wird. Diese können zwar zumindest teilweise von Computerprogrammen unterstützt werden, müssen für viele Aspekte aber weiterhin manuell erfolgen (Meara et al. 1997, S. 32 ff.; Hinkel 2005, S. 624 f.). Gerade die Untersuchung des mündlichen Sprachgebrauchs in der Schule kann sich dabei nicht auf einen elaborierten theoretischen Rahmen stützen, sondern das Mündliche wird nach wie vor primär in Opposition

75 Davon ausgenommen sind zwei aktuelle italienische Studien, die sich mit der mündlichen narrativen Textkompetenz von Zwölf- bis 14-Jährigen im Vergleich zu Erwachsenen (Giuliano 2014) und den Lernerleistungen in der mündlichen Abschlussprüfung der Sekundarstufe I (achtes Schuljahr) (Milia et al. 2014) befassen. S.a. die Studie von Harren (2015) über sprachliche Anforderungen im Biologieunterricht.

76 Die Anfänge des Spracherwerbs werden oft in der Produktion der ersten Wörter gesehen und wurden zusammen mit der Entwicklung morphologischer Fähigkeiten bereits im 19. Jahrhundert von verschiedenen Wissenschaftlern in Tagebuchstudien (z.B. Ament 1899; Stern \& Stern 1928) festgehalten (s. Meibauer \& Rothweiler 1999, S. 9). Im Folgenden befassen sich Forschungsarbeiten mit dem Aufbau und Umfang des kindlichen Wortschatzes (Augst 1984; Wagner 1985) und dem Erwerb der grammatischen Flexion (z.B. Clahsen 1982, 1988; Clahsen, Rothweiler \& Woest 1990). Seit den 1980er Jahren zieht eine Zunahme wissenschaftlicher Untersuchungen zum Wortschatzerwerb in der angloamerikanischen und australischen Forschung auch Studien im deutsch- und italienischsprachigen Raum nach sich (s. Meibauer \& Rothweiler 1999, S. 9; Koesters 2009a; Chini 2005 für einen Überblick über die internationale Zweitspracherwerbsforschung und den italienischen Kontext). 
zum Schriftlichen betrachtet und mit dessen grammatischen Bezeichnungen beschrieben (Harren 2015, S. 24).

Die sprachliche Interaktion im Unterricht erfolgt auf verschiedenen Ebenen (sprechend und zuhörend, lesend und schreibend sowie in unterschiedlichen Registern) und setzt bei den Akteuren Sprachkompetenz in ihrer ganzen Vielschichtigkeit voraus. Aus diesem Grund wurde mit der vorliegenden Studie angestrebt, zu möglichst vielen unterschiedlichen Aspekten der sprachlichen Herausforderungen im Fachunterricht und dazu, wie die Lernenden ihnen begegnen, Daten zu gewinnen und auszuwerten. Gegen eine Konzentration auf ein einzelnes Sprachphänomen wie z.B. die Lexik spricht zudem, dass dieses nur eingeschränkt und nur theoretisch trennbar ist von den anderen Sprachphänomenen, die sich in der Regel gegenseitig bedingen. Beim Beispiel des Wortschatzes bleibend, bedeutet dies, dass die lexikalische Kompetenz auch grammatikalisches Wissen und Fähigkeiten einschließt, um einen Ausdruck angemessen verstehen und verwenden zu können (Halliday 2005; Nation 2001).

Das folgende Unterkapitel illustriert die erste Eingangsphase der Erhebung.

\subsection{Die Erkundungsstudie}

In Vorbereitung auf die Erhebung ist zunächst eine Erkundung durchgeführt worden, in der über mehrere Monate (Januar bis Juni 2012) der Erdkundeunterricht einer Klasse beobachtet wurde. Der Kontakt zur Lehrperson ist privat vermittelt worden; es handelt sich also nicht um eine systematische Auswahl.

Diese Erkundung diente vor allem dem Zweck, sich mit dem italienischen Schulbetrieb vertraut zu machen und die Brauchbarkeit einzelner Erhebungsinstrumente zu überprüfen. Ein erheblicher Unterschied zum deutschen Schulsystem besteht in dem oben beschriebenen gemeinsamen Unterrichten aller Schülerinnen und Schüler in der Sekundarstufe I, deren drittes Jahr, in welches diese Studie fällt, für die Lernenden ein entscheidendes Schuljahr darstellt, da es mit einer Abschlussprüfung endet, deren Ergebnis von großem Einfluss auf die Wahl der weiterführenden Schule ist. Schulsystembedingt, durch das gemeinsame Unterrichten der Lernenden von der sechsten bis zur neunten Klasse an der Mittelschule, kann es also zu einer ausgeprägten Leistungsheterogenität innerhalb der Klassen kommen, welche in allen Fächern geschlossen im Verband unterrichtet werden, so dass Unterrichtsniveau und -tempo sich notwendigerweise an den unterschiedlichen Schülerleistungen orientieren müssen.

Ein zweiter wesentlicher Aspekt, in dem das italienische Schulwesen zum deutschen differiert, ist der Umstand, dass in der Sekundarstufe I die drei Fächer Italienisch, Erdkunde und Geschichte in der Regel von derselben Lehrkraft erteilt werden. Neben dem eventuellen Nachteil, der daraus für die Ler- 
nenden erwachsen kann, wenn sie mit der Lehrkraft nicht so gut zurechtkommen, hat diese Regelung den offensichtlichen Vorteil, dass die Lehrkraft durch die zehn Stunden, die sie wöchentlich in einer Klasse unterrichtet ${ }^{77}$, mehr Zeit mit den Lernenden verbringt, diese dadurch besser kennt und somit theoretisch ihre sprachlichen Fähigkeiten und Förderbedarfe auch besser als die übrigen Lehrpersonen einschätzen kann.

Bezüglich der Unterrichtsgestaltung hat sich in dieser ersten Phase der Erhebung herausgestellt, dass sie der gängigen Beschreibung des italienischen Schulunterrichts entspricht, d.h. lehrerzentrierter Frontalunterricht (Jafrancesco 2002) mit seiner Konzentration auf das Vermitteln von Lehrbuchinhalten (welche von den Schülern oft auswendig gelernt werden) und permanenten Prüfungen durch die Lehrkraft in den sogenannten interrogazioni (Abfragungen). Um in Erfahrung zu bringen, ob es sich dabei nur um ein individuelles Festhalten an der traditionellen Lehrpraxis bei ansonsten veränderten Unterrichtsbedingungen handelte, erfolgte ergänzend die Teilnahme an Unterrichtsstunden anderer in derselben Klasse unterrichtender Lehrkräfte, die jedoch eine ganz ähnliche Vorgehensweise zeigten.

Durch das Vertrautwerden mit der Schulorganisation und Unterrichtsgestaltung konnte die Aufmerksamkeit bei der auf die Erkundungsstudie folgenden empirischen Untersuchung gezielt auf das sprachliche Handeln gerichtet werden. Von nicht geringerer Bedeutung ist zudem, dass dank der Erkundung die zur Durchführung bestimmter Erhebungsverfahren erforderlichen Zeiten und die Akzeptanz ihrer Einbettung in den Unterricht, ohne dessen normalen Verlauf zu sehr zu stören, besser eingeschätzt werden konnten. So sind gerade die letzten Monate des achten Schuljahrs von verschiedenen Leistungskontrollen und Simulationen der bevorstehenden Abschlussprüfung geprägt, die viel Zeit in Anspruch nehmen, so dass wenig Freiraum für außerplanmäßige Aktivitäten bleibt. Deshalb und um zu vermeiden, in der Endphase der Beobachtungen und Datensammlung unter Zeitdruck zu geraten, wurde die Durchführung der tatsächlichen Erhebung auf den Schuljahresbeginn festgelegt.

77 Seit der 2009/2010 erfolgten Reform Gelmini (Gelmini 2009) sind die vorher sieben Wochenstunden für Italienisch und die je zwei für Geschichte und Erdkunde um eine Stunde gekürzt worden. Die Verteilung von neun dieser Stunden auf die drei Fächer wird durch die Reform nicht näher festgelegt, sondern kann nach Gutdünken und Bedarf von den Lehrkräften gehandhabt werden, während die zehnte Stunde für vertiefenden Unterricht vorgesehen ist. Es ist üblich, dass weiterhin sieben Stunden Italienischunterricht erfolgt und anstatt Erdkunde eher Geschichte zweistündig unterrichtet wird. Vier der fünf Lehrkräfte, die an der Studie mitgewirkt haben, erteilten aber schon vor Beginn der Unterrichtsbeobachtungen überwiegend zwei Wochenstunden Erdkunde und fuhren damit auch während des beobachteten Zeitraums fort. Hinsichtlich der im Einzelnen gewählten Praxis der jeweiligen Lehrkräfte s. Tabelle 2 w.u. 


\subsubsection{Auswahl der Erhebungsinstrumente zur Messung schriftlicher Sprachkompetenz}

Während der Erkundungsstudie fiel wegen der Fokussierung in der Unterrichtsgestaltung auf das Lehrwerk ferner die Entscheidung, erstens einen darauf basierenden C-Test durchzuführen, um Aufschluss über die Fähigkeiten der Lernenden im Umgang mit dem Buch zu gewinnen, und zweitens eine weitere schriftsprachliche Erhebung vorzunehmen, bei der die Lernenden nicht nur wie im C-Test einzelne Wörter ergänzen, sondern einen ganzen Text mit Lehrbuchcharakter verfassen sollten.

Dass die Wahl geeigneter Erhebungsinstrumente für die schriftsprachlichen Leistungen zugunsten des C-Tests sowie einer Schriftproduktion mit besonderer Konzentration auf die lexikalische Kompetenz getroffen wurde, hat mehrere Gründe. Es sind vor allem das Anliegen, die sprachlichen Fähigkeiten bezogen auf die konkreten schulischen Anforderungen zu messen, und die generelle Schwierigkeit, ein Konstrukt zu entwickeln, das möglichst viele Facetten der sprachlichen Fähigkeiten abbildet. ${ }^{78}$ In einem Test kann immer nur ein Ausschnitt der tatsächlichen Fertigkeiten einer Person überprüft werden (s. Bachmann \& Palmer 1996, 2010), und „Kompetenz“ ist nur vermittelt, über die erbrachte Leistung, zu erschließen (McNamara 1996, 1997, 1999 und 2001, S. 337 mit Bezug auf Bachmann 1990). Gleichzeitig ist jede Messung immer eine Momentaufnahme. Sprachliche Fähigkeiten hingegen sind keine stabile Größe, sondern variieren, beispielweise nach „Tagesform“, Gesprächspartner und -situation oder sich verändernden schulischen, beruflichen oder privaten Erfahrungshorizonten. Zudem sind für das Italienische, abgesehen vom Bereich der Zertifizierung von Sprachkenntnissen in Italienisch als Zweit- oder Fremdsprache für Erwachsene, nur wenige Sprachtests entwickelt.

Zum Erheben der sprachlichen Fähigkeiten von Testpersonen im schulpflichtigen Alter sind dies die nationalen Schulleistungserhebungen Prove INVALSI in den Fächern Mathematik, Naturwissenschaften und Italienisch (s. INVALSI 2015) für bestimmte Klassenstufen von der Primarstufe bis in die Sekundarstufe II $^{79}$ und das von Gensini und Vedovelli 1981 entwickelte und w.o. vorgestellte Glottokit (Gensini \& Vedovelli 1981). Letzteres ist nicht gewählt

78 Dies gilt auch für Tests, welche die komplexe Größe Wortschatz erfassen wollen (s. z.B. Schmitt, Schmitt \& Clapham 2001, S. 59-61), zumal sich die meisten Wortschatztests, vor allem im frühkindlichen und im fremdsprachlichen Wortschatzerwerb, auf das Ausmaß beschränken und dabei die Breite, also in wie vielen Bedeutungen ein Wort bekannt ist, außer Acht lassen (Koesters Gensini 2010, S. 111).

79 Die nach dem Schulforschungsinstitut, das sie entwickelt und auswertet, benannten standardisierten Tests werden alljährlich durchgeführt und regelmäßig von zahlreichen Eltern und Lehrern boykottiert, indem die Schülerinnen und Schüler direkt dazu aufgefordert werden, am Tag ihrer Durchführung nicht in der Schule zu erscheinen (s. z.B. den aus diesem und anderen bildungspolitischen Gründen veranstalteten Generalstreik der Lehrkräfte und Schülerschaft am 6.5.2015). 
worden, da mit ihm überwiegend Fähigkeiten gemessen werden, die nicht im Interesse dieser Arbeit standen, wie das flüssige und korrekte Sprechen, bei dem die richtig verwendeten und ausgesprochenen Wörter pro Minute gezählt werden, und das Einhalten der Rechtschreibregeln.

Im Vergleich zum Glottokit und dem Multiple-Choice-Test, mit dem im sprachlichen Bereich der INVALSI-Prüfungen vor allem das Textverständnis überprüft wird, bot sich der C-Test für meine Studie besser an, da mit ihm das Verständnis von Auszügen aus den im Unterricht verwendeten Lehrwerken überprüft und gleichzeitig Informationen über die produktive Sprachkompetenz der Lernenden gewonnen werden konnten. Weil sich nur wenige Studien zum Schulunterricht auf die sprachlichen Leistungen aller Lernenden (s.o., Kap. 5.1 $)^{80}$ konzentrieren, schien es anstatt des Einsatzes standardisierter Sprachtests interessanter, die tatsächlichen sprachlichen Herausforderungen im Fachunterricht in den Blick zu nehmen. Und deren Bewältigung erfordert weit mehr als eine solide lexikalische Kompetenz.

Bezogen auf den italienischen Schulkontext liegen meines Wissens bislang noch keine Studien vor, die Lehrbuchtexte, ihre Behandlung und darüber hinausgehende sprachbezogene Aspekte in der Unterrichtskommunikation sowie schriftliche Textproduktionen der Schülerinnen und Schüler untersuchen, so dass die Erhebungsweise dieser Arbeit ein Novum in der sprachbezogenen italienischen Unterrichtsforschung darstellt. Auch ist mir der Einsatz des CTests in Italien weder zur Ermittlung sprachlicher Schülerfähigkeiten noch der Lesekompetenz bzw. Textschwierigkeit bekannt, für die in der lehrbuchbezogenen italienischen Forschung bislang maximal Multiple-Choice-Tests zum Textverständnis verwendet wurden. Für die Beschreibung der lexikalischen Kompetenz der Lernenden wurden auch Lückentexte verwendet, allerdings ohne die lexikalische Gestaltung des gesamten Textes mithilfe der Gebrauchswortschatzkategorien des GRADITs zu beschreiben und ohne das sprachliche Umfeld der Lücken zu analysieren.

Zusätzlich zum C-Test auch eine längere schriftliche Textproduktion anfertigen zu lassen, um mit dem Auftrag, so über eine geographische Region zu schreiben, wie es im Lehrbuch stehen könnte, neben einem generellen Einblick in die Sprachkompetenz der Lernenden auch etwas über ihre produktiven bildungssprachlichen Fähigkeiten zu erfahren, beruht auf der Annahme, dass ihnen bildungssprachliches Formulieren im Schriftlichen leichter fallen sollte als im Mündlichen. Denn beim Schreiben steht ihnen mehr Zeit zum Abwägen angemessener sprachlichen Formen und Strukturen zur Verfügung. ${ }^{81}$

80 S. z.B. Harren (2015, S. 21): Sprachliches Lernen im Fachunterricht wird meist innerhalb der Zweitsprachenerwerbsforschung untersucht, wobei auch die muttersprachlichen Lernenden in die Analysen einbezogen werden, die sich allerdings in der Regel auf die Schriftsprache beziehen.

81 Mit den Einzelheiten der schriftsprachlichen Erhebungen befasst sich das Unterkapitel 5.6. 
Bevor die Erhebungsgruppe und die Analysen der einzelnen Untersuchungen vorgestellt werden, dient Tabelle 1 einer besseren Veranschaulichung, indem sie bereits einem Überblick über die einzelnen Phasen der Vorbereitung und Durchführung der Erhebung einschließlich des methodischen Vorgehens gibt.

\begin{tabular}{|c|c|c|c|c|c|c|}
\hline $\begin{array}{l}\text { Untersu- } \\
\text { chungsphasen } \\
\text { und -gegen- } \\
\text { stände }\end{array}$ & Vorgehen & Zeitraum & Instrumente & Vorgehen & $\begin{array}{l}\text { Zeit- } \\
\text { raum }\end{array}$ & Instrumente \\
\hline \multirow[t]{3}{*}{ 1) Erkundung } & $\begin{array}{l}\text { Unterrichts- } \\
\text { beobach- } \\
\text { tungen: } \\
\text { Erdkunde }\end{array}$ & $\begin{array}{l}\text { 2. Schul- } \\
\text { halbjahr } \\
2011- \\
2012\end{array}$ & $\begin{array}{l}\text { Mitschrift } \\
\text { (handschrift- } \\
\text { liche Noti- } \\
\text { zen) }\end{array}$ & 1) C-Test & $\begin{array}{l}\text { 1) } \\
\text { März } \\
2012\end{array}$ & $\begin{array}{l}\text { 1) konstru- } \\
\text { iert mit be- } \\
\text { handelten } \\
\text { Lehrbuch- } \\
\text { texten }\end{array}$ \\
\hline & & & & $\begin{array}{l}\text { 2) schriftli- } \\
\text { che Text- } \\
\text { produk- } \\
\text { tionen der } \\
\text { Lernen- } \\
\text { den }\end{array}$ & $\begin{array}{l}\text { 2) } \\
\text { Mai } \\
2012\end{array}$ & $\begin{array}{l}\text { 2) Auftrag: } \\
\text { lehrbuch- } \\
\text { ähnliche } \\
\text { Texte über } \\
\text { eine geo- } \\
\text { graphische } \\
\text { Region ver- } \\
\text { fassen }\end{array}$ \\
\hline & $\begin{array}{l}\text { Unterrichts- } \\
\text { beobach- } \\
\text { tungen: } \\
\text { Englisch, } \\
\text { Mathe, } \\
\text { Technik }\end{array}$ & Mai 2012 & $\begin{array}{l}\text { Mitschrift } \\
\text { (handschrift- } \\
\text { liche Noti- } \\
\text { zen) }\end{array}$ & & & \\
\hline $\begin{array}{l}\text { 2) Auswahl der } \\
\text { Schulen und } \\
\text { Klassen, Ge- } \\
\text { nehmigung der } \\
\text { Studie }\end{array}$ & $\begin{array}{l}\text { Internet- } \\
\text { Recherche }\end{array}$ & $\begin{array}{l}\text { August- } \\
\text { Oktober } \\
2012\end{array}$ & & & & \\
\hline \multirow[t]{3}{*}{$\begin{array}{l}\text { 3) empirische } \\
\text { Studie }\end{array}$} & $\begin{array}{l}\text { Unterrichts- } \\
\text { be- } \\
\text { obachtun- } \\
\text { gen: } \\
5 \text { Klassen } \\
\text { an } 3 \text { Schu- } \\
\text { len }\end{array}$ & $\begin{array}{l}\text { Novem- } \\
\text { ber } 2012 \\
\text { bis Juni } \\
2013\end{array}$ & $\begin{array}{l}\text { Audiogra- } \\
\text { phien, Mit- } \\
\text { schrift }\end{array}$ & $\begin{array}{l}\text { 1) Tran- } \\
\text { skription } \\
\text { der Audio- } \\
\text { graphien }\end{array}$ & $\begin{array}{l}12 / \\
2012 \\
\text { bis } \\
12 / \\
2014\end{array}$ & $\begin{array}{l}\text { Wort- } \\
\text { schatzana- } \\
\text { lyse GRA- } \\
\text { DIT, Ver- } \\
\text { ständnis- } \\
\text { hürden } \\
\text { (Lumbelli } \\
\text { 1989) }\end{array}$ \\
\hline & & & & $\begin{array}{l}\text { 2) Ana- } \\
\text { lyse der } \\
\text { sprachbe- } \\
\text { zogenen } \\
\text { Interaktion }\end{array}$ & & \\
\hline & $\begin{array}{l}\text { Lehrbuch- } \\
\text { analyse }\end{array}$ & $\begin{array}{l}\text { Septem- } \\
\text { ber } 2013\end{array}$ & $\begin{array}{l}\text { Digitalisie- } \\
\text { rung der }\end{array}$ & & & \\
\hline
\end{tabular}




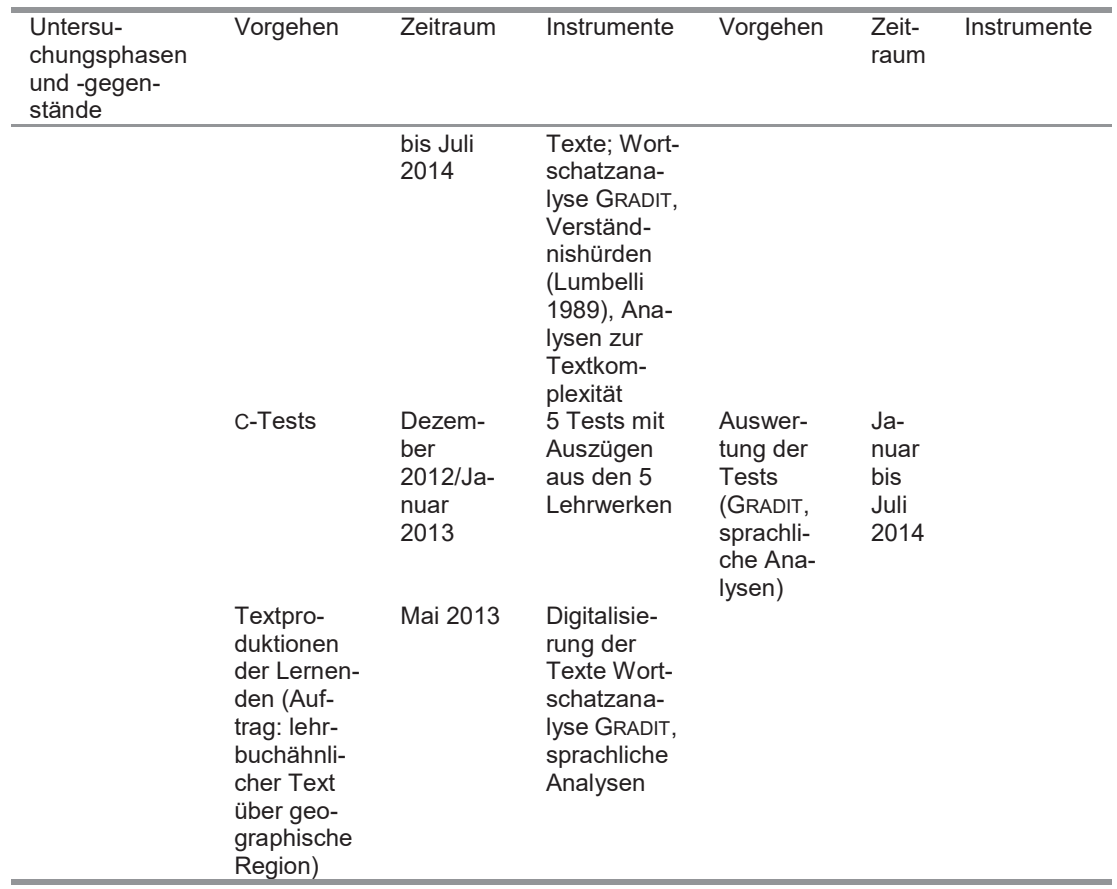

Tabelle 1: Phasen der Erhebung und Untersuchungsschritte

\subsection{Die beobachteten Akteure}

Die während der Erkundungsstudie beobachteten sprachlichen Leistungen der Lernenden legen Zusammenhänge zwischen sozialer Herkunft und Bildungserfolg nahe, weshalb für die Durchführung der tatsächlichen Studie ein Stadtteil mit einem schulischen Einzugsgebiet von Familien der unteren Mittelschicht gewählt wurde, in dem ein mittlerer Leistungsdurchschnitt sowie eine sprachlich heterogener zusammengesetzte Schülerschaft zu erwarten sind. ${ }^{82}$

82 Für das zunehmend multikulturelle Rom liegen keine Daten über den sprachlichen und sozioökonomischen Hintergrund der im Einzugsgebiet der Schulen oder in den verschiedenen Stadtteilen wohnenden Familien vor. Dennoch ist die Bevölkerung je nach Zugehörigkeit zu den verschiedenen Gesellschaftsschichten überwiegend traditionell in unterschiedlichen Stadtvierteln distribuiert und besteht unter den Einwohnern der Stadt ein Wissen über die schichtspezifische Zusammensetzung in den einzelnen Stadtteilen, dass sich die Unterrichtsforschung bei der Auswahl der Schulen zunutze macht (s. Piemontese \& Cavaliere 1997, 
Anhand der Beobachtungen und anschließenden Analysen von sprachlichen Handlungsabläufen im Fachunterricht, den sprachbezogenen Herausforderungen, vor denen Lernende stehen, und ihrer Reaktion darauf, soll das Sprachrepertoire der einzelnen Akteure (Lehrpersonen und Lernende) möglichst genau beschrieben werden. Dies gilt ebenfalls für die sprachliche Beschaffenheit der jeweils verwendeten Lehrmittel, so dass der Erhebung insgesamt ein qualitatives Forschungsdesign zugrunde liegt.

Die Entscheidung, fünf verschiedene Klassen zu beobachten, wurde getroffen, um zu verhindern, dass Informationen über eine Klasse gesammelt werden, welche sich in den sprachlichen Fähigkeiten sowie in den eingesetzten Methoden und Ressourcen erheblich von der durchschnittlichen Unterrichtsgestaltung anderer achten Klassen unterscheidet. So wurden während des Beobachtungszeitraums Übereinstimmungen in der Unterrichtsführung der fünf Klassen festgestellt, die sich mit der Beschreibung des herkömmlichen italienischen Unterrichts in der Forschung (Jafrancesco 2002) decken. Deshalb kann davon ausgegangen werden, dass die in dieser Studie beobachteten Klassen eine typische Gestaltung des Unterrichtsgeschehens im Fach Erdkunde widerspiegeln. Aufgrund der Bandbreite der Untersuchungsgegenstände und der zur Verfügung stehenden technischen und personellen Mittel war eine Ausdehnung auf mehr als fünf Klassen nicht möglich. ${ }^{83}$

Die mit dieser Studie gewonnenen Einsichten in die sprachliche Interaktion im Fachunterricht und die dabei verwendeten Lehrmittel bestätigen den Forschungsstand zur Lehrmittelgestaltung (Calò \& Ferreri 1997; Cacia 2014; La Grassa \& Troncarelli 2014) und zu den sprachlichen Leistungen der Lernenden (Amenta \& Cappadonna 2014; Giuliano 2014; Milia et al. 2014). Zudem vertieft die Studie den Forschungsstand, indem sie die verschiedenen Einflussgrößen sprachlichen Lehrens und Lernens im Rahmen des Erdkundeunterrichts zueinander in Bezug setzt. Das sprachliche Handeln der Lernenden wird dabei gründlicher und sowohl für literate als auch für orate Sprachkompetenzen beschrieben. So stellte sich die Verknüpfung einer qualitativen Lehrbuchanalyse mit darauf basierenden C-Tests als geeignetes Vorgehen heraus, um das Textverständnis zu überprüfen und gleichzeitig Informationen über die Sprachkompetenz der Lernenden zu gewinnen. Denn es ermöglicht eine relativ zeitökonomische Überprüfung der (sprach)lernförderlichen Gestaltung der Lehrmittel, welche auf weitere Unterrichtswerke dieser und anderer Fachrichtungen übertragbar ist. Außerdem laden die Erkenntnisse über die Sprachkompetenzen der Lernenden und die Sprachförderung der Lehrkräfte im Fachunterricht

S. 227). Für die Kontrolle der Übereinstimmung sozioökonomischer Hintergründe der Lernenden mit den vorliegenden Einschätzungen des Einzugsgebiets wurden für den Fragebogen an die Lernenden entsprechende Items entwickelt (s.u. und Kap. 6).

83 Beispielsweise war die Anwesenheit der Beobachterin während der Stunden unbedingt erforderlich, da die Unterrichtsstunden nur teilweise audio- und in keinem Fall videographiert werden konnten (zum Videographieren stand keine entsprechende Ausstattung zur Verfügung, und das Audioaufzeichnen war an einer der drei Schulen nicht gestattet). 
dazu ein, sie mit größeren Erhebungsgruppen oder ähnlich gestalteten Einzelstudien weiter zu beforschen, um Qualitäten und Schwachpunkte der Sprachförderung in den Bildungsinstitutionen aufzudecken.

$\mathrm{Zu}$ den Schulen der beforschten Klassen bestand im Vorfeld kein Kontakt. Leitend waren bei der Auswahl ihre Internetporträts, in denen sie sich mit einer interkulturellen Ausrichtung und Erfahrung mit der Inklusion von in der Zweitsprache lernenden Kindern und Jugendlichen präsentierten. Die drei kontaktierten SchulleiterInnen haben ihr Einverständnis mit der geplanten Erhebung erklärt und nach bereitwilligen Erdkundelehrkräften in ihren Kollegien gesucht. Hierbei wurden die Lehrkräfte zunächst nur darüber informiert, dass es sich um Studien für ein Dissertationsvorhaben in der allgemeinen Sprachwissenschaft handelte. Die fünf disponiblen Lehrkräfte (an zwei Schulen eine Lehrerin bzw. ein Lehrer und an der dritten insgesamt drei Lehrerinnen) verfügten über eine langjährige Unterrichtserfahrung. Zu Beginn der Beobachtungen wurden sie darüber in Kenntnis gesetzt, dass das Forschungsinteresse den sprachlichen Anforderungen und Fähigkeiten der Lernenden im Fach Erdkunde galt und gebeten, ihren Unterricht planmäßig zu gestalten.

Der Beobachtungszeitraum erstreckt sich vom Herbst 2012 bis zum Ende des Schuljahrs im Juni 2013. Tabelle 2 veranschaulicht die Erhebungsgruppe samt Lehrkräften, die Anzahl von wöchentlich erteilten Erdkundestunden und die Art, in der die Dokumentation des Unterrichtsgeschehens stattfand. Hierbei sind für die an der Studie beteiligten Schulen und Lehrpersonen Aliasnamen gewählt worden und bei der Anzahl der Lernenden im Falle von Abwesenheit einzelner Schüler über den gesamten Erhebungszeitraum zwei Werte angegeben.

\begin{tabular}{|c|c|c|c|c|c|}
\hline Schule & Klasse & Lehrperson & $\begin{array}{l}\text { Anzahl der Schü- } \\
\text { lerlnnen }\end{array}$ & $\begin{array}{l}\text { Wochenstun- } \\
\text { den }\end{array}$ & $\begin{array}{l}\text { Aufzeichnun- } \\
\text { gen }\end{array}$ \\
\hline Vespucci & 1 & $\begin{array}{l}\text { Frau } \\
\text { Mancuso }\end{array}$ & $\begin{array}{l}14 \\
\text { (13 beobachtet) }\end{array}$ & $\begin{array}{l}2 \text { (Doppel- } \\
\text { stunde) }\end{array}$ & $\begin{array}{l}\text { Audio-, hand- } \\
\text { schriftlich }\end{array}$ \\
\hline Morante & 1 & Herr Tigre & $\begin{array}{l}18 \\
\text { (17 beobachtet) }\end{array}$ & 2 & $\begin{array}{l}\text { Audio-, hand- } \\
\text { schriftlich }\end{array}$ \\
\hline \multirow[t]{4}{*}{ Pestalozzi } & 3 & & & & \\
\hline & III A & Frau Rea & 19 & 2 & \multirow{3}{*}{$\begin{array}{l}\text { handschrift- } \\
\text { lich } \\
\text { handschrift- } \\
\text { lich } \\
\text { handschrift- } \\
\text { lich }\end{array}$} \\
\hline & III B & Frau Cese & 15 & $1-2$ & \\
\hline & III C & Frau Dante & 21 & 1 (selten 2) & \\
\hline
\end{tabular}

Tabelle 2: In die Studie involvierte Klassen

Wie der Tabelle zu entnehmen ist, variieren die Klassengrößen mit einem Minimum von 14 bzw. 15 Lernenden in den Klassen an der Vespucci und in der III B der Pestalozzi und einem Maximum von 21 in der Klasse III C der Pestalozzi stark. Die Ursachen hierfür liegen wahrscheinlich in dem Umstand, dass es sich bei der Klasse an der Vespucci um die einzige ganztags beschulte 
des Jahrgangs handelte. Die Option, ihre Kinder anstelle der normalen Unterrichtsdauer bis um 14.00 Uhr an einem verlängerten Schulunterricht teilnehmen zu lassen, war den Familien freigestellt. So wurde das Angebot von 14 Lernenden genutzt, von denen jedoch einer dem Unterricht über den gesamten Zeitraum der Beobachtungen fernblieb. An der Schule Pestalozzi bestand der Jahrgang aus insgesamt sieben Klassenzügen, welche zu Beginn der Mittelschule numerisch ungefähr gleichstark waren. Aufgrund einiger Schuljahreswiederholungen und Versetzungen in Parallelklassen bestand die Klasse III B im dritten Jahr der Mittelschule nur noch aus 15 Lernenden.

\subsubsection{Informationsgewinnung anhand eines Fragebogens}

Um Hintergrundinformationen über die Lernenden zu erhalten, ist im Zuge der Unterrichtsbeobachtungen in allen Klassen ein Fragebogen verteilt worden, mit dessen Hilfe Einsichten in den häuslichen Sprachgebrauch und den familiären sozioökonomischen Status gewonnen wurden. Außerdem wurden damit Einsichten über die Lernstrategien, das Leseverhalten und den Schulerfolg (anhand von Angaben über eventuelle Schuljahreswiederholungen sowie indirekt durch die für die Hausaufgaben benötigte Zeit und den für die Sekundarstufe II gewählten Schultyp) gewonnen sowie über ihre Einstellung zum Erdkundeunterricht, der Lehrkraft und dem Unterrichtswerk. Es handelt sich um einen umfangreichen Fragebogen (17 Seiten), dessen Bearbeitung während der Unterrichtszeit erfolgte, wobei sich die Lernenden mit eventuellen inhalts- oder sprachbezogenen Fragen an mich und die jeweiligen Lehrkräfte wenden konnten.

Bei der Entwicklung des Fragebogens wurden der PISA-Hintergrundfragebogen und der Fragebogen von Achterberg (2005) zur Erhebung des sprachlichen und familiären Hintergrunds slavophoner Einwanderer in Deutschland zu Hilfe genommen. Diese Wahl wurde getroffen, da der PISA-Fragebogen innerhalb einer langwährenden Tradition der international vergleichenden Schulforschung entwickelt wurde, deren wissenschaftlicher Erkenntnisstand bei der Erstellung berücksichtigt wurde, beispielsweise über Indikatoren für die Messbarkeit der sozioökonomischen Verhältnisse und die Beschreibung der Lernund Lesegewohnheiten. Der von Achterberg konzipierte Fragebogen hingegen diente deshalb als Vorbild, weil er den sprachlichen Hintergrund der Befragungsgruppe besonders gründlich erfasst. So wird dort etwa nicht nur nach der „Muttersprache“ oder der zuhause gesprochenen Sprache gefragt, sondern es werden die im Haushalt wohnenden Familienmitglieder inklusive ihrer Erstsprachen und der jeweilig vorwiegenden Kommunikationssprache zwischen ihnen und der befragten Person ermittelt. 
Die meisten Schülerinnen und Schüler haben den Fragebogen ausgefüllt, da auch bei der Verteilung nicht anwesende Lernende später durch die Lehrpersonen zu seiner Beantwortung angehalten wurden. Weil einige Lernende jedoch aus krankheitsbedingten und privaten Gründen über einen längeren Zeitraum fehlten, ist der Fragebogen von insgesamt 75 der 87 Lernenden beantwortet worden:

\begin{tabular}{lll}
\hline Klasse & $\mathrm{n}$ Lernende & $\mathrm{n}$ beantworte Fragebögen \\
\hline Morante & 18 & 15 \\
Vespucci & 14 & 12 \\
Pestalozzi III A & 19 & 18 \\
Pestalozzi III B & 15 & 12 \\
Pestalozzi III C & 21 & 18 \\
\hline
\end{tabular}

Tabelle 3: Angaben zur Anzahl der Lernenden und der beantworteten Fragebögen

\subsection{Die Unterrichtsbeobachtungen}

Vor dem Beginn der Unterrichtsbeobachtungen sind erst die Schulleitungen und anschließend die Eltern schriftlich über das geplante Vorhaben informiert worden. In den Klassen, in denen der Unterricht audiographiert werden konnte (Vespucci und Morante), ist dies im Vorfeld von den Erziehungsberechtigten genehmigt worden.

Die Lehrpersonen wurden nicht für die Unterrichtsbeobachtungen instruiert, sondern gebeten, ihren Unterricht wie vorgesehen durchzuführen. Lernende und Lehrkräfte haben sich rasch an die Anwesenheit einer Beobachterin und die Aufzeichnungen gewöhnt. Nur in seltenen Fällen kam es zu einer direkten Ansprache und dadurch zu einem kurzzeitigen Involvieren in das Unterrichtsgeschehen, etwa indem die Lehrpersonen Fragen nach schulbezogenen Praktiken u.Ä. (bisweilen fachinhaltlich relevanten Gegebenheiten) in Deutschland stellten oder in zwei Klassen die Lehrkraft vorlaute Schüler darauf hinwies, dass sie aufgenommen wurden. In einer Klasse bin ich von der Lehrkraft aufgrund ihrer außerplanmäßigen Abwesenheit zweimal dazu eingeladen worden, die Stunden zusammen mit der Unterstützungslehrkraft zu leiten und nach eigenem Ermessen für diese Studie zu nutzen.

Während der beobachteten Stunden habe ich im Klassenraum unterschiedliche Plätze eingenommen, in der Regel an den hinteren Tischen, um von dort möglichst die gesamte Klasse beobachten zu können, ohne dabei zu sehr aufzufallen. Nur wenn sich der Hauptteil des Unterrichtsgeschehens im vorderen Bereich des Klassenzimmers abspielte, etwa weil die Lehrkraft einzelne Schülerinnen und Schüler nach vorne an ihr Pult bat, wurden auch die Beobachtungen von dort getätigt, da von den hinteren Reihen aus das Gespräch zwischen 
der Lehrkraft und den einzelnen Schülerinnen und Schülern nicht vollständig mitverfolgt werden konnte.

Die sprachbezogene Interaktion im Erdkundeunterricht über einen Zeitraum zu beobachten, der sich fast auf ein ganzes Schuljahr erstreckt, resultiert aus dem Anliegen, sie in einem größeren Zusammenhang zu erfassen und zu untersuchen. Hiermit sollte ein Beitrag zur Füllung einer bildungssprachlichen Forschungslücke geleistet werden, da:

[...] es sich bei der empirischen Untersuchung mündlicher Kommunikation um einen sehr jungen Wissenschaftszweig handelt. Außerdem sind empirische Forschungsarbeiten zur Förderung mündlicher Teilkompetenzen und besonders des Sprechens ein Desiderat (vgl. z.B. Schneider et al. 2013). Ungeachtet des Mangels an Forschung: Die Förderung mündlicher Kompetenzen ist von immenser Wichtigkeit (Harren 2015, S. 95).

In allen fünf Klassen ist das Unterrichtsgeschehen schriftlich festgehalten worden, wobei besonderes Augenmerk auf die Beiträge gerichtet war, die sich explizit mit Sprache und Sprachförderung beschäftigten oder in denen sprachliche Hürden offensichtlich wurden. Dabei ist jedoch stets angestrebt worden, so neutral und so umfassend wie möglich das gesamte Unterrichtsgeschehen zu dokumentieren. So wurden die Namen der GesprächsteilnehmerInnen und ihre Äußerungen notiert, gegebenenfalls Angaben zu Parallelgesprächen, gleichzeitigem bzw. leisem/lautem Sprechen, zur Änderung der Tonlage und zu anderen akustischen Auffälligkeiten wie ein erhöhter Lärmpegel gemacht.

Vor allem bei der Lehrkraft und zum Teil bei den mündlich abgefragten Lernenden kam es gelegentlich zu langen Redebeiträgen mit einem hohen Sprechtempo, so dass nicht jedes Wort erfasst wurde. In diesen Fällen ist versucht worden, nur den Inhalt der Redebeiträge zu dokumentieren und die Aufmerksamkeit auf Sprachbezogenes zu lenken, was möglichst vollständig und wortgetreu vermerkt wurde. Abgesehen von dem Aufwand, der mit dieser Erhebungstechnik verbunden ist, steht außer Frage, dass durch Audio- oder besser noch Videographien das Gesprächsverhalten einer Vielzahl von Akteuren umfassender festgehalten werden kann. Von Vorteil war beim handschriftlichen Protokollieren des Unterrichts die Konzentration auf das Lehrbuch, dessen Inhalte entweder von der Lehrkraft in enger Anlehnung an das Buch vorgestellt oder von den Lernenden in mündlichen Abfragungen wiedergegeben wurden. Da Letztere in ihrem Sprachgebrauch von dem des Lehrbuchs deutlich abwichen, wurden sie von der Lehrkraft des Öfteren auf sprachliche Fehler oder Ungenauigkeiten hingewiesen, welche mühelos dokumentiert werden konnten. Mit dem Lehrwerk als Leitfaden der Unterrichtsgestaltung war also das handschriftliche Erfassen der Interaktion besser ausführbar, als es beispielsweise während eines freien Unterrichtsgesprächs zur Erarbeitung von Lerninhalten oder Gruppenarbeitsphasen der Fall gewesen wäre.

Direkt nach den beobachteten Stunden wurden die Mitschriften durchgelesen, teilweise ergänzt und besondere Auffälligkeiten hervorgehoben und manchmal kommentiert. Mithilfe der Audiographien fand eine Anfertigung 
vollständiger Analysetranskripte der gesamten Unterrichtskommunikation statt, wofür die Audiodateien mehrere Male angehört und in einigen schwerer verständlichen Fällen der Rat einer kompetenten Muttersprachlerin hinzugezogen wurde. Dieses wiederholte Anhören ist sehr nützlich, um auch Daten zu erfassen, die sich anfangs der Aufmerksamkeit entziehen. Bei der Transkription in Alphabetschrift wurden Längen und Verzögerungen in der Aussprache notiert und das gleichzeitige Sprechen verschiedener Gesprächsteilnehmer und Sprechqualitäten wie Veränderungen im Tonfall und der Lautstärke markiert.

Die Mitschriften und Transkripte der Unterrichtsgespräche sind nach unterrichtsorganisierenden Merkmalen durchleuchtet worden, an denen sich eine für das Makro-scaffolding typische Vorgehensweise der Lehrkräfte erkennen lässt. ${ }^{84}$ Desweiteren wurde auf überindividuelle Regelmäßigkeiten in der ähnlichen Unterrichtsgestaltung (Lehrervortrag und Abfragungen der Lernenden zu Lehrbuchinhalten) geachtet sowie auf die subtilen Unterschiede im sprachförderlichen Lehrerhandeln.

Bezüglich beobachteter interrogazioni ergab sich für die Analyse die Frage, welche sprachlichen und kognitiven Anforderungen dabei an die Lernenden gestellt werden: Richtet die Lehrkraft punktuelle Fragen an die SchülerInnen, die sie mit einigen wenigen Worten beantworten können, oder sind die Fragen offener gehalten? Handelt es sich um Fragen, für deren Beantwortung ein Auswendiglernen von Ausdrücken und Konzepten ausreicht, oder müssen die Lernenden kognitiv anspruchsvollere Aufgaben lösen?

Ferner ist die Aufmerksamkeit auf solche Momente gerichtet worden, in denen sich die Gesprächsbeiträge der an der Unterrichtskommunikation beteiligten Personen explizit auf die sprachliche Beschaffenheit der Lerninhalte konzentrierten, und auf Sequenzen, in denen sprachliche Schwierigkeiten von Lernenden offenkundig waren. Da sich das beobachtete sprachliche Handeln so gut wie ausschließlich mündlich abspielte, bot sich eine Konzentration auf den verwendeten Wortschatz an. Längere Schüleräußerungen, die aus mehr als ein paar Wörtern oder kurzen Sätzen bestanden, waren seltener zu beobachten. Darüber hinaus macht die Flüchtigkeit der gesprochenen Sprache eine Konzentration auf größere sprachliche Einheiten, syntaktische Strukturierungen und Gestaltungsmittel der Textkohärenz schwierig. So bezieht sich auch sprachliches Lehrerfeedback in der Regel auf kleinere Einheiten (vgl. Harren 2015, S. 247 f. und Kap. 9).

Bei der Wortschatzverwendung wurden nicht nur Fachtermini beachtet, sondern ebenso Ausdrücke aus dem allgemeinen und dem gehobenerem Wortschatz, welche der Bildungssprache zugeschrieben werden können. Für das Deutsche liegen bislang keine Gebrauchswörterbücher mit Unterteilungen des Wortschatzes nach Art, Frequenz und Feld ihrer Verwendung vor, die auf den 
Analysen umfangreicher Korpora des mündlichen und schriftlichen Sprachgebrauchs basieren (Koesters Gensini 2010, S. 116 f.). ${ }^{85}$ In Italien hingegen gibt es eine lange Tradition der wortschatzbezogenen Frequenz- und Gebrauchsforschung, aus der als umfassendste Kategorisierung das von De Mauro (1999/2000) entwickelte GRADIT hervorgeht, das Grande dizionario italiano dell'uso (Großes italienisches Gebrauchswörterbuch), hervorgeht. Dieses bildete die Grundlage für die Bestimmung des in den Lehrbüchern und von den Lehrpersonen und Lernenden verwendeten Wortschatzes.

Im Mittelpunkt der sprachlichen Untersuchung steht der Wortschatz auch deshalb, da seine Bedeutung für die allgemeine Sprachkompetenz unumstritten ist (z.B. Laufer \& Nation 1995, S. 316; Bates \& Goodman 1997, Schmitt et al. 2001, S. 55). Er gilt insbesondere in den Fachsprachen als zentral (Harren 2005, S. 105), und in Hinblick auf seinen Einfluss auf die Lesekompetenz ist nachgewiesen worden, dass ein kompetentes Verfügen über einen umfangreichen Wortschatz das Textverständnis maßgeblich erleichtert. So sehen WissenschaftlerInnen in dem oft wesentlich geringeren Umfang des Unterrichtssprachenwortschatzes von zweisprachigen Lernenden den ausschlaggebenden Grund für ihr im Vergleich zu vielen in der Erstsprache Lernenden geringeres Leseverstehen (Duff 2005, S. 49), zumal beim Lesen weniger kognitive Kapazitäten für den Verstehensprozess freigesetzt werden können, wenn sie für das Erfassen unbekannter oder kaum bekannter Wörter beansprucht werden.

\subsection{Die Lehrbuchanalyse}

Aufgrund der dominanten Rolle des Lehrwerks in der Unterrichtsgestaltung an italienischen Schulen allgemein und somit auch im Erdkundeunterricht sind Auszüge aus den verwendeten Lehrbüchern sorgfältig analysiert worden, wobei der Fokus auf den sprachlichen Gestaltungsmitteln lag und die übrigen As-

85 Es gibt auch nur ein Häufigkeitswörterbuch (Tschirner \& Jones 2006), wenn man von Kaedings Häufigkeitswörterbuch der deutschen Sprache absieht, das Ende des 19. Jahrhunderts veröffentlicht wurde (Kaeding 1896). So stützt sich Harren beispielsweise bei der lexikalischen Analyse der von ihr beobachteten Unterrichtskommunikation auf die Wortschatzeinteilung im Duden in Fachsprachen, Fremdwörter; Hochsprache/,,allgemeinsprachlich“" und „gehoben“ sowie „umgangssprachlich“/,familiär“ (Duden 1999) und verweist außerdem auf die für die DESI-Studie (s. DESI-Konsortium 2008) vorgenommene Einteilung zur Einschätzung des Schüler-Kompetenzniveaus in Wörter geringerer und größerer Schwierigkeit: Basiswörter (Niveau 1), gebräuchlicher Wortschatz - Konkreta/Abstrakta (Niveau 2), seltene Wörter - Konkreta, Fach- und Fremdwörter, Redensarten (Niveau 3) (Harren 2015, S.97 mit Verweis auf Willenberg 2007a, S. 151 f. zur Wortschatzeinteilung in der DESI-Studie). 
pekte wie Bildmaterial, Graphiken, Tabellen und Layout nur am Rande einbezogen wurden. Nachfolgend stelle ich die Auswahl von Geographielehrbüchern vor, die in den untersuchten Klassen verwendet wurden:

- Inuovi territori dell'uomo. 3B, Regioni e paesi extraeuropei (Bersezio 2005 (De Agostini))

- Il nuovo Geolab 3. I continenti extraeuropei. Manuale e Dossier (Carazzi \& Pizzetti 2010 (Mondadori))

- Zaino in spalla 3. Il mondo e i paesi extraeuropei (Bastianelli, Rancati \& Maestri 2010 (Sansoni))

- Geograficamente 3. Noi cittadini del mondo (Dinucci \& Dinucci 2008 (Zanichelli))

- Nuovo Geolibro 3

(Forte \& Ubertazzi 2008 (De Agostini))

Aus den für das Unterrichtsprogramm eines Schuljahrs konzipierten umfangreichen Lehrwerken (ca. 300 Seiten im Format Din A4) ist für die Analyse eine Seitenauswahl getroffen worden, welche sich aus den folgenden Kriterien ergab: In jeder Klasse sollte ein C-Test durchgeführt werden, der mithilfe von Textauszüge aus den jeweiligen Lehrbucheinheiten entwickelt wurde, die kurz zuvor Gegenstand des Erdkundeunterrichts waren. Die für diese Tests jeweils aus den fünf Unterrichtswerken ausgewählten Seiten bilden den Kern der sprachlichen Lehrbuchanalyse, weil sich daraus Kriterien für die Beurteilung der Schülerleistungen im C-Test ergeben. Desweiteren war ein gewisses Maß an inhaltlicher und dadurch sprachlicher Vergleichbarkeit zwischen den analysierten Seiten der verschiedenen Bücher beabsichtigt (das mit den C-Testseiten nicht gegeben war, da kurz vor Durchführung der Tests in den einzelnen Klassen unterschiedliche Themen behandelt wurden), weshalb darauf geachtet wurde, aus je mindestens zwei Büchern Seiten zu extrahieren, die dasselbe Thema behandeln. Außerdem wurden für alle fünf Lehrbücher sowohl landeskundliche als auch sozioökonomische bzw. -kulturelle Themenbereiche abgedeckt. $^{86}$

Wie oben verdeutlicht, korreliert die Größe des Wortschatzes stark mit der Sprachkompetenz und ist er maßgeblich für die Sicherung des Textverständnisses verantwortlich. Studien belegen, dass die Erweiterung des Lernerwortschatzes im Schulalter vor allem durch die Schriftsprache und somit durch die Lektüre der Lehrwerke vorangetrieben wird (Harren 2015 mit Verweis auf Nippold 2007, S. 25 f.; De Renzo 2005, S. 225). Deshalb wurde auch bei der Lehrbuchanalyse der Schwerpunkt auf die Wortschatzuntersuchung gesetzt. Das Wortvorkommen ist mit grammatisch-syntaktischen Kategorien sowie mit den GRADIT-basierten Gebrauchsmarken versehen worden und ist durch eine 
stark ausgeprägte Verwendung von Lexemen, die über den Basiswortschatz hinausgehen, gekennzeichnet.

Der auf der Verwendung des GRADITs fußende Analyseansatz hat sich in der empirischen Wortschatzforschung bereits bewährt: Das Beherrschen des Grundwortschatzes VDB (Vocabolario di Base), der sich in die drei Gruppen FO, AU und AD gliedert, wurde in mehreren Untersuchungen überprüft, um die Größe des mentalen Lexikons verschiedener Populationen zu erfassen. Zu nennen sind hier die drei Magisterarbeiten von Marina Boni, Maria Stefania Tomassi und Alessandro Franciotti, deren Recherchen und Resultate De Mauro (1986, S. 56; 1991², S. 149-183; 1995b, S. 81 f.) und Ferreri (2005, S. 32 f.) referieren. Die kalabrische Sektion des GISCELs (De Renzo 2008) kontrolliert mit einer Erhebung, ob Lernende der Primar- und der Sekundarstufe über Lexeme verfügen, die dem knapp 50.000 Lemmata umfassenden allgemeinen Wortschatz (CO) zugeteilt sind, sich aber in größerer Nähe zum inneren Kern des VDBs befinden und damit frequenter gebraucht werden sollten als andere Ausdrücke des umfangreichen Co-Wortschatzes. Wörter aus diesem Bereich des Gebrauchswortschatzes sind Lernenden zwar zum Teil schon in der Grundschule bekannt, werden von Abiturienten und Abiturientinnen allerdings in größerem Umfang nicht besonders gut beherrscht: Nur eine gute Hälfte der untersuchten Wörter (53 von 100) ist 75 Prozent der Befragten bekannt, die anderen weitaus wenigeren (ibid., S. 197). Die Sektion Venetien des GISCELs (Colmelet et. al. 2008) hat eine der in der italienischen Schulforschung seltenen Analysen von schriftlichen Textproduktionen der Lernenden vorgenommen, in der eine Unterteilung des Wortvorkommens in Lemmata der einzelnen Kategorien des Grundwortschatzes sowie in darüber hinausgehende Ausdrücke erfolgt ist (ibid., S. 319 ff.). De Renzo (2005, S. 225) stellt außerdem Studien vor, in denen für mehrere Schulklassen nachgewiesen wurde, dass das mentale Lexikon der Lernenden auch Fremdwörter beinhaltet, die nicht zum VDB gerechnet werden. Wie schon w.o. geschildert, ist die Kategorisierung des Gebrauchswortschatzes aus dem GRADIT auch in der Lehrbuchforschung genutzt worden (Piemontese \& Cavaliere 1997; Compagni et al. 1997, S. 286 f.; Miglietta \& Sobrero 2008; La Grassa \& Troncarelli 2014, S. 294 ff.). ${ }^{87}$

Meine Untersuchung unterscheidet sich von den anderen darin, dass sie nicht nur jeweils den Wortschatzgebrauch der Lehrbücher, der Lehrkräfte oder der Lernenden untersucht, sondern alle drei Komponenten berücksichtigt und zueinander in Bezug setzt, um die tatsächlichen sprachbezogenen Anforderungen und Leistungen der Lernenden im Fachunterricht detaillierter erkennen und beschreiben zu können.

Damit zutreffende Aussagen über die sprachliche Gestaltung und Komplexität der Unterrichtswerke getroffen werden können, sollte eine möglichst 
große Anzahl von Textauszügen aus unterschiedlichen Themeneinheiten untersucht werden. Für den zur Lesbarkeit und Komplexität von italienischen Texten entwickelten GULPEASE-Index (Lucisano \& Piemontese 1988; Piemontese 1996) etwa gilt, dass mit ihm berechnete Ergebnisse als valide betrachtet werden, wenn Textauszüge von je 100 Wörtern untersucht wurden, die einem Ausmaß von 30 Prozent des Gesamttextes entsprechen - oder mindestens zehn Prozent, um noch als aussagekräftig gelten zu können. Die hier vorgenommene Analyse konzentriert sich auf einen Umfang von 39 Seiten, was drei Hauptgründe hat. Der erste und primäre ist zeitökonomischer Art, da die einzelnen Seiten für die Untersuchung zunächst manuell digitalisiert werden mussten und danach ebenfalls manuell eine Wortschatzanalyse nach der im GRADIT vorgenommen Einteilung durchgeführt wurde. Desweiteren stieße die Möglichkeit, quantitative Aussagen über das Wortvorkommen in den fünf Lehrwerken treffen zu können, auf wissenschaftliches Interesse, wie sich etwa an dem Wunsch zeigt, in den Lehrplänen genauer zu verorten, welchen Wortschatz die Schüler lernen sollen (Ferreri 2005, S. 37; Compagni et al. 1997, S. 262). Hierzu wären quantitative Studien zur Wortschatzverwendung in den eingesetzten Lehrmitteln aller Fachrichtungen hilfreich; dennoch bleibt in meiner Arbeit die qualitative, möglichst punktuelle Erhebung einzelner sprachlicher Aspekte und ihrer Wirkung auf die beobachteten Akteure im Vordergrund.

Darüber hinaus kann davon ausgegangen werden, dass in den einzelnen Lehrbüchern durch das mit den Lehrplänen vorgegebene Themenspektrum ein ähnlicher Wortschatz verwendet wird, so dass die Anwendung der GULPEASEFormel zu einem validen Ergebnis führen dürfte, obwohl mit den untersuchten 39 Seiten nur insgesamt ein Umfang von etwas mehr als den für die Validität der GULPEASE-Berechnung erforderlichen zehn Prozent eines Lehrbuchs abgedeckt wird ${ }^{88}$ Auf eine Verwendung des GULPEASE-Indexes wie in einigen Studien zur Berechnung der Schwierigkeit von Lehrbuchtexten (z.B. Cacia 2014) wurde im Zuge dieser Studie verzichtet, da die Beschreibung der Textkomplexität anhand einer genauen Wortschatzanalyse erfolgt und der Wortschatz bei Weitem als bester einzelner Prädiktor für Textschwierigkeit gilt (Harrison 1999, S. 429). Überdies sind zusätzliche Eigenschaften des Textes (Satzlänge, Verknüpfungen, Dichte usw.) untersucht worden.

Lesbarkeitsformeln stellen ein praktisches, da schnell applizierbares Instrument dar, weisen jedoch einige Schwächen auf (z.B. ibid., S. 428 f.). So sagt die lexikalische Variabel, die auf einer Buchstaben- oder Silbenzählung basiert, nur bedingt etwas über die Wortfrequenz aus, ist die Satzlänge ein ziemlich grobes Maß für syntaktische Komplexität und wird die Textorganisation

88 Für Anweisungen zur Berechnung italienischer Texte mit der GULPEASE-Formel s. Lucisano und Piemontese (1989) oder Piemontese (1996). Ferner steht im Internet ein Dienst zur Verfügung, um die Lesbarkeit eigens ausgewählter Textproben kontrollieren zu lassen (in der Rubrik Leggibilità auf www.corrige.it, Stand: 18.10.2016). 
ignoriert (würden die Sätze wild gemischt, ergäbe sich meist ein schlecht lesbarer Text, das Ergebnis der Formel bliebe aber gleich). Aus diesem Grund wird selbst von den Entwicklern dieser italienischen Lesbarkeitsformel unterstrichen, dass zur Bestimmung der Textschwierigkeit zusätzlich qualitative Analysen erfolgen sollten, die - beispielsweise mithilfe des oben vorgestellten Katalogs von Verständnishürden (Lumbelli 1989) - sprachliche Problemstellen in den Texten ausmachen, die mit dem GULPEASE-Index nicht erfasst werden (Lucisano \& Piemontese 1988, S. 112 f.).

Zusätzlich zu den GRADIT-gestützten Analysen wurden in der vorliegenden Lehrbuchstudie der Aufbau der Texte und einige ihrer Eigenschaften wie die Länge und Dichte der einzelnen Sätze ermittelt, um auf ihre Komplexität schließen zu können. Hierbei lag die Aufmerksamkeit insbesondere der Verwendung von als typisch bildungssprachlich geltenden Mitteln wie Nominalisierungen und ausgebauten Attribut- und Adverbialsätzen. Da die Lehrbuchanalyse zwar einen wesentlichen, aber eben nur einen Teil dieser Arbeit darstellt, wurde auf eine punktuelle Untersuchung weiterer sprachlicher Gestaltungsmittel, die - bzw. deren Nichtvorhandensein - potentiell Verständnishürden schaffen können, verzichtet. Allerdings wurden solche Textstellen identifiziert und beschrieben, in denen das Textverständnis mit größerer Wahrscheinlichkeit dadurch beeinträchtigt sein kann, dass sich verschiedene Indizien für mögliche Textkomplexität verdichten. $\mathrm{Zu}$ diesen Indizien zählen die Länge der Sätze, ein Vermeiden von Wortwiederholungen, welches zur Verwendung von synonymen Formen (v.a. Pronomina) führt, deren Bezugswörter nicht immer eindeutig sind, und das Explizieren von kausalen, konzessiven oder ähnlichen Zusammenhängen. Hier traten auch einige der von der Forschung aufgezeigten Verständnishürden in Erscheinung, die sich aus der Art der Informationsverknüpfung ergeben (Lumbelli 1989) und Teil meiner Untersuchungen sind.

Vervollständigt werden die Analysen durch Informationen zur Bewertung und Handhabung der Lehrbücher seitens der Lehrpersonen und Lernenden. Die von den Lehrenden stammenden Angaben wurden mittels mündlicher Befragungen und die Urteile der Schülerinnen und Schüler mithilfe entsprechender Items des oben vorgestellten Fragebogens gewonnen.

\subsection{Erhebungsverfahren zur Ermittlung produktiver und rezeptiver schriftlicher Sprachleistungen der Lernenden}

Im Zuge dieser Studie wurden verschiedene schriftsprachliche Daten der Lernenden ermittelt, um weitere Einsichten in ihren produktiven und rezeptiven 
Umgang mit dem Lehrwerk und ein genaueres Bild ihrer Sprachkompetenz zu gewinnen. Auf diese Weise wurden die aus den Unterrichtsbeobachtungen gezogenen Schlüsse über die mündlichen Sprachkompetenzen der Lernenden um die schriftbezogenen ergänzt. Im Folgenden werden zunächst Informationen zum C-Test, seiner Vorbereitung, Durchführung und Auswertung (5.6.1) und anschließend zu den übrigen gesammelten Textproduktionen der Lernenden (5.6.2) bereitgestellt, von denen die einen größeren Ausmaßes sind und die anderen aus Definitionen einiger Begriffe bestehen, deren Verfassen in beiden Fällen die Verwendung eines bildungssprachlichen Registers vorsah.

Hierzu muss angemerkt werden, dass Lerninhalte im Unterricht während des Beobachtungszeitraums kaum schriftlich fixiert wurden. So ist in allen Klassen mit einigen seltenen Ausnahmen nie schriftlich gearbeitet worden und wurden die Hefte der Lernenden nicht überall kontrolliert. Wenn dies geschah, dann eher oberflächlich beim Abfragen einzelner SchülerInnen, die für die Benotung zusätzlich auch ihr Heft kurz vorlegen sollten.

\subsubsection{Die C-Tests}

Beim C-Test (Raatz \& Klein-Brayley 1985) handelt es sich um ein bekanntes und mittlerweile für eine Vielzahl von Sprachen validiertes Screening-Verfahren, das vor allem zur Ermittlung von Fähigkeiten in einer Zweit- oder Fremdsprache eingesetzt wird. Das bedeutet, es erhebt nicht den Anspruch, detaillierte Aussagen über den Sprachstand der Testteilnehmenden treffen zu können. Es bewertet jedoch ganz unterschiedliche, miteinander verwobene Aspekte der Sprachkompetenz, wie den Wortschatz, grammatikalisch-syntaktische Fähigkeiten, Textkompetenz, und ermöglicht damit eine globale Einschätzung von Sprachkenntnissen.

Neben traditionellen Anwendungsbereichen, in denen allgemeinsprachliche Fähigkeiten getestet werden, wie Einstufungstests zur Teilnahme an Sprachkursen verschiedener Niveaustufen oder im Vorfeld differenzierterer Sprachstandsanalysen, kommt der C-Test in den letzten Jahren auch zunehmend beim Ermitteln registerbezogener Kompetenzen (z.B.im Wirtschaftsdeutschen; s. Grotjahn 2006, 2010), in der empirischen Unterrichtsforschung (Ahrenholz 2013) und vereinzelt sogar als didaktisches Lehrmittel durch Lehrkräfte zum Einsatz. So werden beispielsweise Arbeitsblätter erstellt, die anhand eines C-Tests dazu dienen zu kontrollieren, wie gut die Lernenden die sprachlichen Mittel eines zuvor behandelten Lerninhaltes beherrschen. Hierbei ist abweichend vom klassischen Tilgungsprinzip auch das ausschließliche Tilgen von Fachwörtern oder grammatischen Strukturen (z.B. alle konjugierten Verbformen) möglich (vgl. Harsch-Schröder 2014; Baur \& Spettmann 2007).

Der C-Test beruht auf dem Worttilgungsprinzip, durch welches die Textredundanz reduziert wird. Für eine erfolgreiche Testbearbeitung müssen über die 
lexikalische Kompetenz hinausgehende Sprachfähigkeiten wie grammatikalisch-syntaktische eingesetzt werden, wenn es etwa um das Vervollständigen einer Verbform geht. Auch die rezeptiven Sprachkompetenzen werden überprüft, weil eine korrekte Auflösung der getilgten Wörter jeweils auf dem Verständnis der unmittelbaren sprachlichen Umgebung des zu ergänzenden Wortes fußt und teils mehr, teils weniger stark auch vom Verständnis des Makrokontextes abhängt. Der klassische C-Test besteht aus fünf kleinen, jeweils 25 Lücken enthaltenden Texten (Grotjahn 2002). Es werden oft auch kürzere Versionen entwickelt, doch für diese Studie sind das herkömmliche Format und sein Tilgungsprinzip gewählt worden, wonach bei jedem zweiten Wort die zweite Hälfte fehlt. Die Vervollständigung dieser Wörter erfolgt, ohne dass die Testteilnehmenden wissen, dass immer exakt die Hälfte eines Wortes von der Tilgung betroffen ist. Um das Textverständnis zu erleichtern, sind die einzelnen Texte mit einer Überschrift versehen und bleiben jeweils der erste und letzte Satz von den Worttilgungen ausgespart.

Die prinzipielle Anwendbarkeit von C-Tests ist für das Italienische empirisch überprüft (vgl. Grotjahn, Tönshoff \& Hohenbleicher 1994). Während der Erkundungsstudie zeigte sich, dass die Verwendung von Texten, deren schulische Bearbeitung länger zurücklag, zu einer extrem hohen Fehlerquote führte. Daher wurden die C-Tests für die empirische Studie aus Textmaterial konstruiert, das unmittelbar vor der Testdurchführung im Unterricht behandelt wurde. Die Schülerinnen und Schüler erhielten eingangs eine Einführung in die Testbearbeitungen, bei der die Vorgehensweise anhand von Beispielsätzen mit getilgten Wörtern an der Tafel gemeinsam erprobt wurde.

Die Auswertung der mithilfe der C-Tests gewonnenen Daten begann mit einem Abgleich der Lösungsvorschläge der TestteilnehmerInnen mit den im Original verwendeten Ausdrücken. Für eine genauere Einschätzung des Schwierigkeitsgrades bei der Bearbeitung sind sowohl die halbierten Wörter, die es zu ergänzen galt, als auch alle anderen Wörter, aus denen die Tests bestanden, mit den im GRADIT angegebenen Gebrauchsmarken versehen worden.

Im Anschluss daran wurde eine Beschreibung der einzelnen Falschlösungen (ausbleibende Vervollständigung, lexikogrammatkalisch oder morphosyntaktisch nicht korrekte Ergänzungen usw.) vorgenommen und mit Erklärungshypothesen darüber verbunden, was das korrekte Schließen einzelner Lücken verhindert hat, beispielweise eine syntaktisch komplexe Satzstruktur oder das vermehrte Auftreten von Fach- bzw. selten gebrauchten Wörtern. Es ist auch nicht auszuschließen, dass mangelnde Konzentration, Aufregung oder andere Merkmale der Testsituation zu Fehlleistungen führten. Den Lernenden war vorab versichert worden, dass vom Testergebnis kein Einfluss auf ihre Benotung ausgehe und es den Lehrpersonen nicht bekannt gemacht werde. So wird gerade bei einfachen, als hinlänglich bekannt vorausgesetzten Wörtern, die zu vervollständigen waren, und einem an Komplexität überschaubaren Mikrokon- 
text der Lücken die Hypothese aufgestellt, dass die Ursache der Falschlösungen voraussichtlich in mangelnder Konzentration oder im Nichtverfügen über bestimmte Lösungsstrategien bzw. ihrem Nichtbeachten zu suchen ist. Unter diesen Strategien versteht man z.B. die Berücksichtigung des Makrokontextes, anstatt beim Ausfüllen nur die ein, zwei der Lücke unmittelbar vorausgehenden Wörter zu berücksichtigen.

Ein genaueres Bestimmen der Fehlerquellen kann erreicht werden, indem man alle oder einige Testteilnehmer einzeln zur Testbearbeitung befragt, wobei die Zuverlässigkeit der Angaben steigt, wenn dies sofort im Anschluss an die Testdurchführung erfolgt (z.B. Schmitt et al. 2001, S. 72 f.). Aufgrund der knapp bemessenen Unterrichtszeit, die zur Vermittlung der vom Lehrplan vorgeschriebenen Lerninhalte zur Verfügung stand, war dies jedoch nicht möglich.

\subsubsection{Weitere schriftliche Schülerdaten}

In zwei der fünf Klassen wurden von den Schülerinnen und Schülern Textproduktionen erhoben, mit denen sowohl die Darstellungskompetenz bezogen auf fachinhaltliche Aspekte als auch sprachliche und insbesondere bildungssprachliche Fähigkeiten ermittelt wurden. Anhand dieser im Durchschnitt mindestens eine handschriftliche Seite langen Texte konnten genauere Einsichten in die produktive Sprachkompetenz in Bezug auf die in der Schule erwünschten Sprachregister und ihre Einhaltung gewonnen werden.

Die Erstellung dieses Korpus beruht auf dem Umstand der vorhandenen Forschungslücke im Bereich des bildungssprachlichen Handelns (Harren 2015, S. 30-33), von dem das registerangemessene Verfassen von Texten einen Ausschnitt darstellt. So zählt der beobachtete Erdkundeunterricht in Italien zwar zu den traditionell mündlichen Fächern, doch dafür in den Lehrplänen als Bildungsziel die Fähigkeit eines multidisziplinären (physisch, sozioökonomisch, kulturell, ökologisch usw.) Kommunizierens über geographische Räume, vorgesehen (MIUR 2012b, S. 46-48), ohne dass dort expliziert wird, ob dies orat oder literat geleistet werden soll.

Dennoch wird in den Lehrplänen und ministerialen Richtlinien zur Schulbildung die Bedeutung des Sprachausbaus hervorgehoben, den die Lehrenden aller Fächer als ihre Aufgabe zu betrachten haben, und über den hinlänglich bekannt ist, dass er nur wirksam erfolgen kann, wenn die sprachlichen Kompetenzen auf allen vier Ebenen des Sprachgebrauchs, also mündlich, schriftlich, produktiv und rezeptiv, weiterentwickelt werden (Guerriero 2014, S. 167 f.; Piemontese 2014, S. 9; Ehlich 2007, S. 25). In diesem Sinne ist zumindest von einer Lehrkraft der beobachteten Klassen eine schriftliche Leistungskontrolle erstellt worden, die im Zeichen des Arbeitsauftrags der von mir erbetenen Textproduktion stand. 
Diese zusätzlich zum C-Test erhobenen Schriftdaten stammen nur aus zwei der fünf Klassen, weil davon auszugehen ist, dass die Produktionsleistungen eines guten Drittels der Erhebungsgruppe die schriftsprachlichen Fähigkeiten aller Lernenden angemessen widerspiegeln. ${ }^{89}$

In der Klasse an der Schule Vespucci sind die Lernenden unter Absprache mit der Lehrkraft in ihrer Abwesenheit von mir und der Unterstützungslehrkraft dazu angehalten worden, einen Text über ein Land, eine Region oder einen Kontinent ihrer Wahl zu verfassen und, wie im Unterricht mündlich erprobt und von den Lehrbuchtexten gewohnt, nach geographischen, politischen, wirtschaftlichen und soziokulturellen sowie gegebenenfalls geschichtlichen Aspekten vorzustellen. Der Text war zudem mit einer persönlichen Stellungnahme zu beenden, bei der es um die Frage ging, ob sie sich vorstellen könnten, dort für eine gewisse Zeit zu leben. Damit die Lernenden ein möglichst bildungssprachliches Register wählten, ist ihnen in beiden Klassen vorgegeben worden, den Text möglichst so zu gestalten, wie er in einem Erdkundebuch für die achte Klasse stehen könnte.

Die freie Wahl eines geographischen Raumes und die abschließende persönliche Beurteilung sollten es den SchülerInnen erleichtern, über etwas zu schreiben, das sie hinreichend kennen und eventuell mögen, um zu verhindern, dass fachinhaltliche Lücken die Schreibkompetenz zu sehr beeinflussen. AuBerdem verband sich damit die Erwartung einer höheren Motivation für die Bewältigung der Aufgabe. Aus demselben Grund wurden sie darauf hingewiesen, dass dieser Arbeitsauftrag, den sie so aus dem Geographieunterricht kaum kannten, als hilfreiche Vorbereitung auf die Abschlussprüfung gesehen werden könne, in der sie sowohl eine schriftliche Textproduktion (im Fach Italienisch) als auch eine mündliche Prüfung über das in den drei Jahren der Sekundarstufe I erworbene geographische Fachwissen erwartete.

In der zweiten Klasse (Morante) war der Lehrer damit einverstanden, die Lernenden einen solchen Text verfassen zu lassen, wollte die Produktion jedoch zur Bewertung nutzen und bestand deswegen darauf, alle zu der vorweg im Unterricht behandelten Region schreiben zu lassen. Diese Leistungskontrolle wurde angekündigt, so dass die Schülerinnen und Schüler wussten, dass sie sich auf ein Überprüfen ihrer Kenntnisse der Indischen Region (Indien, Pakistan, Nepal) vorzubereiten hatten. Auch hier wurden sie dazu angehalten,

89 Dass sie nur in zwei der fünf Klassen erhoben wurden, hat v. a. auch die Ursache, dass selbst bei einer vollständigen Erhebung solcher Daten aller beobachteten Lernenden aufgrund der gewählten approfundierten qualitativen Analyse der Texte eine Auswahl erfolgt wäre, deren Kriterien aufzustellen, nicht unproblematisch ist. Darüber hinaus erlaubte es der straffe Zeitplan der Lehrpersonen nicht, nach der Durchführung des C-Tests und der Bearbeitung der Fragebögen noch weitere Unterrichtszeit zur Verfügung zu stellen. Das gewählte Vorgehen hat zudem den Vorteil, dass die Daten dort erhoben wurden, wo auch Audiographien zur Interaktion im Unterricht vorliegen, so dass in eventuell anknüpfenden Untersuchungen die Analyse der mündlichen Sprachkompetenz im Verhältnis zur schriftlichen vertieft werden kann. 
den Text mit einem persönlichen Urteil über einen eventuellen Aufenthalt in der Region abzuschließen.

Die produzierten Texte sind weniger auf die inhaltliche Vollständigkeit als vielmehr auf die sprachliche Gestaltung untersucht worden. Hierbei standen der Wortschatz und seine Zusammensetzung aus den verschiedenen Gebrauchsfeldern wieder im Fokus der Analyse. Überdies wurden die Produkte einerseits nach typischen Merkmalen bildungssprachlicher Schrifttexte wie Nominalisierungen, langen Satzgefügen, unterordnenden Konjunktionen und Passivgebrauch untersucht. Andererseits wurde auch auf die Verwendung konzeptionell mündlicher Sprachmittel und Einflüsse des Soziolekts geachtet. Darüber hinaus sind die Satzlängen berechnet und auch formale, textexterne Gestaltungsmittel wie das Schriftbild, die folgerichtige Gliederung der Texte in Abschnitte und das Vorhandensein einer Überschrift beachtet worden.

In der Klasse an der Morante konnten die Schülertexte, die sich alle mit derselben geographischen Region befassen, zudem mit den entsprechenden Lehrbuchseiten abgeglichen werden. So wurden Gemeinsamkeiten und Unterschiede im Wortschatzgebrauch und der Konstruktion und Komplexität der Sätze ermittelt. Weiter wurde hierbei untersucht, was während der mündlichen Abfragungen in allen fünf Klassen aufgefallen war, und zwar ob die Lerninhalte von Schülerinnen und Schülern auswendig gelernt und wortwörtlich wiedergegeben werden oder ob sie Fähigkeiten des Paraphrasierens zeigen.

In zwei Klassen wurde zusätzlich eine kürzere schriftliche Arbeit angeregt, bei der die Lernenden Definitionen zu im Erdkundeunterricht ausführlich behandelten und teilweise schon im vorherigen Schuljahr eingeführten Konzepten und Fachwörtern (z.B. Bruttoinlandsprodukt, Hochebene) verfassen sollten. Diese Daten wurden erhoben, weil es sich auch hierbei um ein Lernziel im Erdkundeunterricht handelt (MIUR 2012b) und beobachtet wurde, dass die SchülerInnen gelegentlich von ihren Lehrkräften aufgefordert wurden, ein bestimmtes Konzept oder einen Begriff zu erklären. Demnach geht es um eine weitere konkrete sprachliche Anforderung im Fachunterricht, deren Beherrschung für diese Studie auch in schriftlicher Form erhoben wurde, um den Lernenden durch die im Vergleich zur mündlichen Interaktion in größerem Ausmaß zur Verfügung stehende Zeit eine Gelegenheit zu geben, ihre Äußerung nicht nur inhaltlich, sondern auch auf Registerebene angemessen zu gestalten. Damit wurden möglichst bildungssprachliche Formulierungen von den Schülerinnen und Schülern bezweckt.

Darüber hinaus schien die Erhebung dieser Daten von Interesse, weil mit ihnen die lexikalische Kompetenz der Lernenden genauer erfasst werden konnte. Denn ein Wort zu kennen und es verwenden zu können, bedeutet weit mehr als zu wissen, wie es ausgesprochen (und geschrieben) wird. So umschließt es ein detaillierteres Wissen über zahlreiche weitere Aspekte wie die grammatikalische Kategorie, der es angehört, und seine grammatikalisch kor- 
rekte Verwendung im Satz mit angemessenen Kollokationen. Auch das Erkennen anderer Wörter derselben Wortfamilie sowie die Fähigkeit, sie unter der Anwendung von Wortbildungsregeln (mithilfe von Affixen) bilden zu können, registerspezifische Einschränkungen im Wortgebrauch, welche kulturelle, geographische, stilistische und andere Ursachen haben können, zu kennen und möglichst über die gesamte Spannbreite seiner Bedeutungen und Assoziationen zu verfügen, zählen zur lexikalischen Kompetenz (Nation 2001, S. 397). So wurde während des Unterrichts an mehreren Stellen beobachtet, dass die SchülerInnen auf Fragen nach Wortbedeutungen einsilbig antworteten, etwa mit einem Synonym, woraus nicht ersichtlich wurde, ob sie das Konzept, das mit einem bestimmten Begriff verbunden wird, tatsächlich verstanden hatten. Gleichzeitig forderten die Lehrkräfte sie nur in manchen Fällen zu einer ausführlicheren Erklärung auf.

In der Klasse an der Schule Vespucci war die Lehrerin in der Stunde, in der die Wortdefinitionen angefertigt wurden, abwesend, so dass der Auftrag in Eigenregie zusammen mit der Unterstützungslehrkraft erteilt wurde. Nach der schriftlichen Formulierung sind die Schülerinnen und Schüler angeregt worden, in Gruppen von ca. vier Personen die eigenen Definitionen zu präsentieren, ihre Stärken und Schwächen zu erörtern und gemeinsam die beste Definition auszuwählen, um sie dem Klassenplenum vorzustellen.

In den schriftlichen Erhebungen, welche den Lehrkräften als Leistungskontrolle dienten, sind abgesehen von den zu untersuchenden Sprachleistungen der Lernenden auch die Bewertungsmodalitäten von Interesse, weil sich diese sprachförderlich auswirken können. Wie gehen die Lehrerinnen und Lehrer auf sprachliche Eigenheiten der Testbearbeitung ein? Unterstreichen oder korrigieren sie etwas und wenn ja, orthographische, grammatikalische und Zeichensetzungsfehler oder auch Ausdrucks- und Textaufbauschwächen? Bieten sie sprachliche Lösungsvorschläge an? Diesen Fragen wurde hinsichtlich der Klasse an der Morante bei der Auswertung der Textproduktionen zur Region Indien und der Wortdefinitionen, die beide von der Lehrkraft korrigiert und benotet wurden, nachgegangen.

Bevor das nächste Kapitel, das die Zusammensetzung der einzelnen Klassen auf der Grundlage der Fragebogenergebnisse beschreibt und über die Lernund Lesegewohnheiten der Lernenden sowie ihre Einstellung zum Erdkundeunterricht informiert, zeigt Tabelle 4 abschließend, welche schriftlichen Daten in den jeweiligen Klassen gesammelt wurden ( $\mathrm{LK}=$ Leistungskontrolle).

\begin{tabular}{llll}
\hline Klasse & C-Test & Definitionen & Textproduktionen \\
\hline Vespucci & $*$ & ${ }^{*}$ & ${ }^{*}$ \\
Morante & ${ }^{*}$ & ${ }^{*}($ LK Lehrperson) & ${ }^{*}($ LK Lehrperson $)$ \\
Pestalozzi III A & $*$ & & \\
Pestalozzi III B & ${ }^{*}$ & & \\
Pestalozzi III C & $*$ & & \\
\hline
\end{tabular}

Tabelle 4: Erhobene Schriftdaten 


\section{$6 \quad$ Vorstellung der beobachteten Klassen}

Dieses Kapitel liefert Informationen über die Zusammensetzung der Erhebungsgruppe, die Einstellung der Schülerinnen und Schüler zu wesentlichen Aspekten des Erdkundeunterrichts und ihr Lese- und Lernverhalten. Nach einer einführenden Schilderung der Klassengrößen, der Anzahl der mehrsprachigen Lernenden und der Wochenstunden, in denen das Fach Erdkunde jeweils erteilt wurde (6.1), erfolgen eine kurze Beschreibung der Inklusion von Lernenden mit Lernbeeinträchtigungen (6.1.1) und die jeweilige Vorstellung der fünf Klassen (6.2 bis 6.6). Hierbei werden zunächst der sprachliche und familiäre Hintergrund der Lernenden $(6.2 .1,6.3 .1,6.4 .1,6.5 .1,6.6 .1)$ und ihre Lesegewohnheiten in der Freizeit $(6.2 .2,6.3 .2,6.4 .2,6.5 .2,6.6 .2)$ geschildert. Im Anschluss daran werden die Einstellung der Lernenden zum Erdkundeunterricht sowie ihre Ansichten über die unterrichtende Lehrkraft (6.2.3, 6.3.3, 6.4.3, 6.5.3, 6.6.3) und das Unterrichtswerk (6.2.4., 6.3.4, 6.4.4, 6.5.4, 6.6.4) vorgestellt. Im Zusammenhang mit der Nutzung des Lehrbuchs sind auch die Lernstrategien zu betrachten, derer sich die Lernenden unterschiedlich häufig und intensiv bedienen, was in den Unterkapiteln 6.2.5, 6.3.5, 6.4.5, 6.5.5 und 6.6.5 referiert wird. 6.7 bildet mit einem Fazit, in dem einige wesentliche Angaben der Lernenden und Gemeinsamkeiten und Unterschiede der einzelnen Klassen in tabellarischen Übersichten präsentiert werden, den Abschluss des Kapitels. Im Zuge dessen wird außerdem ein Überblick über den sozioökonomischen Status aller Familien gegeben (6.7.1).

\subsection{Auswahl der Schulen und allgemeine Informationen zur Klassenzusammensetzung und zur Durchführung des Erdkundeunterrichts}

Im Verlaufe dieser Studie wurde der Erdkundeunterricht von November/Dezember 2012 bis zum Ende des Schuljahrs im Juni 2013 in fünf achten Klassen an drei römischen Mittelschulen beobachtet, die in diesem Kapitel vorgestellt 
werden sollen. ${ }^{90}$ An der Schule Vespucci war die Klasse von Prof.ssa ${ }^{91}$ Mancuso und an der Morante die von Prof. Tigre beteiligt, die beide zwei Wochenstunden Erdkundeunterricht erteilten. An der Schule Pestalozzi wurden die drei Klassen der Lehrerinnen Rea (III A), Cese (III B) und Dante (III C) beobachtet, von denen die erste das Fach wöchentlich zweistündig unterrichtete, Prof.ssa Cese ein- bis zweistündig und Prof.ssa Dante nur selten zweistündig.

Die unterschiedlichen Klassengrößen - 14 SchülerInnen in der Ganztagsschulklasse an der Vespucci und 15 in der Klasse III B (Pestalozzi) gegenüber 18 an der Morante, 19 in der III A der Pestalozzi und 21 in ihrer Parallelklasse III C - resultieren aus dem fakultativen (allerdings aufgrund des Mittagsessens kostenpflichtigen) Angebot der Ganztagsbeschulung (Vespucci), das vor allem von Familien genutzt wird, die mittags keine Möglichkeit haben, ihre Kinder zu betreuen, sowie an der Pestalozzi aus dem Umstand, dass sich die Klassengröße der III B, welche zunächst den Parallelklassen entsprach, im Laufe der Zeit so reduziert hatte, dass die Lernenden eine Umverteilung auf die sechs Parallelklassen befürchteten, zu der es jedoch nicht kam.

Im Ganzen bestand die Erhebungsgruppe aus 85 Schülerinnen und Schülern. Darunter befanden sich mindestens 28 mit Migrationshintergrund, also knapp 33 Prozent. ${ }^{92}$ Dieser Anteil war in einigen Klassen allerdings ausgeprägter als in anderen. Wie sie sich im Einzelnen zusammensetzten, zeigt Tabelle $1(\mathrm{MH}=$ Migrationshintergrund; Angaben in Klammern: Anzahl der während des Beobachtungszeitraums tatsächlich Anwesenden).

\begin{tabular}{lrrrrr}
\hline Klassen & n Lernende & n Befragte & Mädchen & Jungen & mit MH \\
\hline Morante & $18(17)$ & 15 & 9 & 6 & 8 \\
Vespucci & $14(13)$ & 12 & 8 & 4 & 8 \\
III A & 19 & 18 & 12 & 6 & 2 \\
III B & 15 & 12 & 7 & 5 & 4 \\
III C & 21 & 18 & 10 & 8 & 6 \\
\hline
\end{tabular}

Tabelle 1: Zusammensetzung der Klassen

90 Wie bereits im Kapitel zum methodischen Vorgehen erwähnt (s. Kap. 5.3), war es mein Anliegen, Schulen auszuwählen, deren Einzugsgebiet aus Familien der Mittelschicht besteht, auch wenn für Rom keine genauen Angaben über die sozioökonomische Lage der Einwohner einzelner Stadtteile vorliegen. Der Leistungsstand der SchülerInnen sollte sich im Mittelfeld befinden und die Zusammensetzung der Klassen sprachlich heterogen sein. Hierfür wurden die Profile verschiedener Mittelschulen im Internet gesichtet und solche ausgewählt, die mit einer interkulturellen Ausrichtung und Erfahrung bei der Inklusion von in der Zweitsprache lernenden Kindern und Jugendlichen warben. Im Folgenden werden für die Schulen, Klassen und Lehrpersonen Aliasnamen verwendet.

91 Prof.ssa und Prof. sind die Abkürzungen der in Italien gängigen Anreden für Lehrpersonen der Sekundarstufe, professoressa und professore. Ich verwende diese Titel hier, weil sie sich auch w.u. in den Auszügen aus der Unterrichtskommunikation ergeben.

92 Diese Angaben resultieren aus den Ergebnissen der Fragebögen, an denen aus Abwesenheitsgründen nicht alle Schüler teilgenommen haben. Da auch während des gesamten Zeitraums der Unterrichtsbeobachtungen einige Lernende dem Unterricht fernblieben, wurden keine Angaben zu ihrer Herkunft ermittelt. 


\subsubsection{Schülerinnen und Schüler mit Lernbeeinträchtigungen}

In allen fünf Klassen befanden sich SchülerInnen mit verschiedenen Lernbeeinträchtigungen, wobei es in Italien üblich ist, dass ihnen stundenweise während des Unterrichts eine zusätzliche Lehrkraft (insegnante di sostegno, Unterstützungslehrkraft) zur Seite steht, die sich gezielt um sie kümmert. Dies ist der Kern des Inklusionsprinzips, das Italien in der Position eines europäischen Vorreiters im allgemeinen Bildungswesen schon seit den 1970er Jahren verfolgt, indem es alle schulpflichtigen Heranwachsenden ungeachtet einer eventuellen Lernbeeinträchtigung inkludierend unterrichtet, anstatt diese Gruppe der Lernenden in Förder- oder Sonderschulen auszugliedern. Die Wochenstundenzahl, welche die sostegno-LehrerInnen mit ihnen verbringen, richtet sich nach dem Ausmaß der Beeinträchtigung. So war in der Schule Vespucci im Erdkundeunterricht konstant eine zweite betreuende Lehrkraft anwesend, die sich gezielt um eine Schülerin kümmerte. In der Morante war häufig eine solche zweite Lehrkraft in den Erdkundestunden zugegen, die sich nicht nur um eine muttersprachliche Schülerin mit einer Lernbeeinträchtigung bemühte, sondern auch um einen chinesischen Schüler, der erst seit kurzem in Italien lebte und die Landessprache noch nicht beherrschte. Für diesen und weitere SchülerInnen mit erheblichen sprachlichen Schwierigkeiten fand außerdem gelegentlich parallel zum normalen Schulunterricht und auch an den Nachmittagen Italienischunterricht in einer Kleingruppe statt.

Die sostegno-Lehrkräfte sollen sich nicht nur um die SchülerInnen mit Lernbeeinträchtigungen kümmern, sondern mit der Fachlehrkraft im Team unterrichten, um ein integratives Lehr- und Lernklima entstehen zu lassen, das für die gesamte Klasse geeignet ist. Dennoch zogen es die beobachteten sostegno-Lehrerinnen vor, sich im Hintergrund des Unterrichtsgeschehens zu halten und dessen Lenkung der Fachlehrkraft zu überlassen. An der Vespucci richtete sich die Unterstützungslehrerin hin und wieder an die ganze Klasse und übernahm den Unterricht in Abwesenheit der Lehrkraft. An der Morante hielt sich die Unterstützungslehrerin stets zurück, und an der Pestalozzi hingegen wurden die betreuten Schülerinnen und Schüler ab und zu aus dem Erdkundeunterricht geholt, um entweder alleine oder in Kleingruppen mit Lernenden aus ihren Parallelklassen in einem anderen Raum unterrichtet zu werden.

Im Folgenden werden die beobachteten Lernenden klassenweise und hauptsächlich anhand ihrer eigenen, mithilfe eines Fragebogens ${ }^{93}$ ermittelten Angaben vorgestellt. Zunächst werden Aspekte ihres familiären Hintergrunds illustriert.

93 Den Fragebogen im Original und in der deutschen Übersetzung sowie tabellarische Überblicke über die Antworten der Lernenden in den einzelnen Klassen stelle ich Interessierten gerne auf Anfrage zur Verfügung. Selbiges gilt für die Wortschatzanalysen der Lehrbuchseiten, CTests und Schülertexte. 


\subsection{Klasse an der Morante}

An der Schule Morante bestand die beobachtete Gruppe aus 18 Schülerinnen und Schülern, von denen 16 an der Umfrage teilnahmen. Es sind sechs Jungen und zehn Mädchen, von denen eines die ersten beiden Jahre der Mittelschule wiederholt hatte. Ein weiteres Mädchen war während der Fragebogenbeantwortung krank, und es ergab sich keine Möglichkeit, ihn zu einem späteren Zeitpunkt von ihr bearbeiten zu lassen. Der 18. Schüler fehlte während des gesamten Erhebungszeitraums aus persönlich-familiären Gründen.

Sieben Lernende stammten aus Familien, die nach Italien eingewandert waren, und ein Schüler hatte einen ausländischen Vater. Von diesen Kindern wurden zwei im Ausland geboren: ein chinesischer Schüler, der erst im Alter von 15 Jahren nach Italien kam, sowie ein Mädchen ägyptischer Herkunft, deren Familie nach Italien zog, als sie acht Jahre alt war. Ein weiteres Mädchen, mit chinesischen Eltern, wurde zwar in Italien geboren, zog aber im Alter von fünf Jahren für einige Zeit nach China und wurde dort eingeschult. Sie gab an, Italienisch zunächst wieder verlernt zu haben. Diese Schülerin war von den in Italien geborenen auch die einzige, die mit dem Lesen und Schreiben nicht auf Italienisch begonnen hatte.

Die Lernenden mit Migrationshintergrund sprachen zuhause nur oder überwiegend die Erstsprache der Eltern (Arabisch, Philippinisch, Bengalisch und Chinesisch) bzw. im Falle einer philippinischen Schülerin sowohl deren Erstsprache als auch ihre Zweitsprache Englisch. Einige gaben außerdem an, dass sie mit den Geschwistern Italienisch sprachen (vgl. Fragebogen (im Folgenden FB abgekürzt), Nr. 14-21). Die Unterrichtssprache in dieser Klasse war vonseiten der Schülerinnen und Schüler stark durch römische Umgangssprache geprägt, was von Prof. Tigre in einigen Fällen kritisiert und korrigiert wurde. Die Lernenden zeigten zwar Schwierigkeiten, sich in der Standardsprache oder gar bildungssprachlich zu artikulieren, jedoch wurden bei denen mit Migrationshintergrund (abgesehen von dem neu dazugestoßenen chinesischen Schüler) bei der Verwendung des umgangssprachlichen römischen Registers kaum Unterschiede zu ihren einsprachigen Mitschülerinnen und -schülern wahrgenommen. Diesen Eindruck teilten auch Prof. Tigre sowie die in der Klasse unterrichtende Unterstützungslehrkraft.

Drei italienische Lernende waren Einzelkinder, alle anderen hatten ein bis zwei Geschwister. Neun von 16 besaßen ein Einzelzimmer, und zehn von 16 gaben an, in ihrer Wohnung einen ruhigen Ort zum Lernen und zum Anfertigen der Hausaufgaben zu finden. Nur einer Schülerin, die in ihrer Wohnung keine Ruhe hatte, stand dafür auch kein Schreibtisch zur Verfügung.

Unter den Eltern dieser SchülerInnen gab es keine, die beide einen Universitätsabschluss vorweisen konnten; insgesamt hatten nur drei Elternteile ein Studium absolviert und weitere sieben Elternteile das Abitur (vgl. FB, Nr. 9 
und Nr. 13). Auf die Frage nach der Wahl einer weiterführenden Schule gab nur eine einzige Schülerin an, ein Lyzeum (Gymnasium) besuchen zu wollen, während sich alle anderen für die weniger anspruchsvollen technischen und berufsvorbereitenden Institute entschieden.

\subsubsection{Lesegewohnheiten}

Mindestens die Hälfte der Lernenden dieser Klasse las in ihrer Freizeit entweder gar nicht oder weniger als dreißig Minuten am Tag. Als sie klein waren, wurde vier von ihnen regelmäßig vorgelesen, den anderen nur gelegentlich, bzw. machten sie dazu keine Angaben (FB, Nr. 55). Vier Lernende stammten aus Haushalten mit mehr als hundert Büchern, acht Haushalte wiesen maximal 25 auf und nur in fünf befanden sich Klassiker unter den Büchern (vgl. FB, Nr. 21 und 23 f.). Abbildung 1 erläutert diese Angaben zur maximalen täglichen Lesezeit (in Minuten) und zur Menge an Büchern im Haushalt (ebenfalls $\max$.).

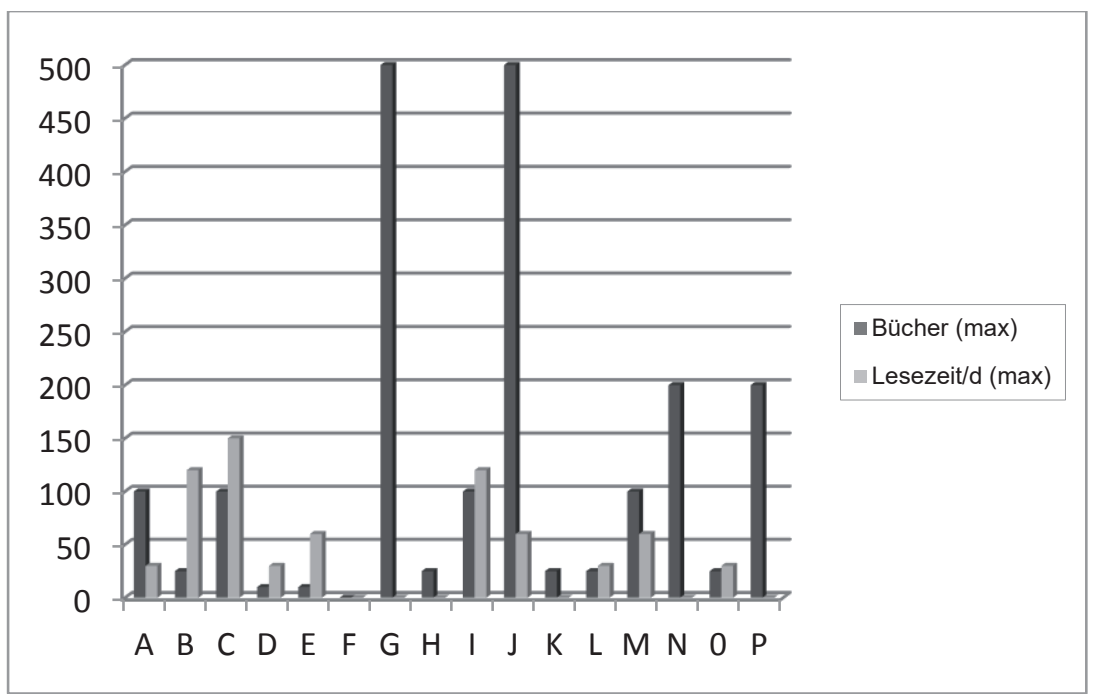

Abbildung 1: Maximale Bücherzahl im Haushalt und maximale tägliche Lesezeit (in min), Morante

Bis auf drei Haushalte verfügten alle über einen Computer und Internetzugang, davon nur drei auch über Lernsoftware. Stattdessen gaben alle SchülerInnen an, ein Wörterbuch und bis auf drei, nützliche Bücher zum Anfertigen der Hausaufgaben und zum Lernen zu besitzen. Sieben Lernende berichteten, dass sie in ihrer Freizeit nur lasen, wenn sie es mussten, und für lediglich drei war 
Lesen eine der Lieblingsbeschäftigungen. Trotzdem hielten alle bis auf drei Lesen nicht für Zeitverschwendung.

Fünf Lernende, darunter drei Jungen, tauschten gerne Bücher mit ihren Freunden, und als einzige dieser Klasse gaben vier der fünf an, dass sie gerne mit anderen über das Gelesene redeten (FB, Nr. 25). Für die meisten Kinder dieser Klasse schien das Lesen also eine Freizeitbeschäftigung zu sein, der sie, wenn überhaupt, kaum oder nur selten und im Privaten nachkamen, ohne sich darüber mit ihren Freunden oder Familien auszutauschen. Danach befragt, welche Texte sie wie oft lesen, gaben neun Lernende an, so gut wie nie Zeitschriften zu lesen. Die meisten lasen am häufigsten Comics, nämlich zwölf mehrmals wöchentlich. Belletristik las nur eine Schülerin mehrmals in der Woche und drei einige Male im Monat. Die meisten (10) lasen entweder nie oder höchstens ein paarmal im Jahr Zeitung, doch vier mehrmals im Monat (3) bzw. mehrmals wöchentlich (1).

Insgesamt lasen diejenigen, die mehr lasen, alle genannten Textsorten häufiger, während es wenige Lernende gab, die eine bestimmte Sorte sehr oft lasen und die anderen gar nicht oder kaum. Eine Schülerin las bspw. mehrmals wöchentlich Zeitschriften und Romane, aber kaum andere Textsorten, und eine weitere Schülerin ebenfalls oft Zeitschriften und kaum andere Textsorten. Nur drei Lernende, ausschließlich Jungen, lasen wenigstens mehrmals im Monat mehr als zwei Textsorten. Darunter ist auch der chinesische Schüler. Als einziger las er mehrmals wöchentlich Zeitung, was möglicherweise auch damit zusammenhängt, dass er ein Jahr älter war als seine Mitschüler (FB, Nr. 26).

Bibliotheken suchten diese Lernenden kaum auf, um Bücher für die Freizeitgestaltung auszuleihen: die überwiegende Mehrheit nie oder nur ein paarmal jährlich. Von sechs Lernenden lieh nur einer ungefähr einmal pro Monat Bücher für die Schule aus, die anderen weder zu schulischen Zwecken noch zur Freizeitgestaltung. Sie besuchten Bibliotheken auch nicht aus anderen Gründen, etwa um dort zu lesen, Hausaufgaben zu machen oder den Internetzugang zu nutzen. Auf einer Skala von 1 (nie) bis 5 (mehrmals pro Woche) war zu bewerten, wozu sie Bibliotheken nutzen. Die meisten Punkte bekam dabei mit 44 von möglichen 80 das Lesen von Büchern, gefolgt von der Internetnutzung (41 Punkte) und vom Bücherausleihen (38 Punkte). Nur vier Lernende gaben an, dass sie wenigstens mehrmals im Monat Bücher ausliehen (FB, Nr. 47).

In ihrer Schule befand sich außerdem eine Bücherei, die zudem von ihrem Erdkundelehrer, Prof. Tigre, geleitet wurde. Dieser ermutigte sie regelmäßig, Bücher zum Lesen in der Freizeit auszuleihen, und die Schüler bekamen hier wie auch in zwei der beobachteten Klassen an der Schule Pestalozzi noch während der Unterrichtszeit allein oder in Zweiergruppen die Gelegenheit, Bücher dorthin zurückzubringen und neue zu entleihen. Von 15 Lernenden, die auf die Frage antworteten, ob und wie oft sie eine Bücherei außerhalb der Schule auf- 
suchten, gaben sieben an, dass sie dort nie oder nur ein paarmal im Jahr hingingen. Lediglich drei Schüler taten es monatlich und zwei mehrmals wöchentlich.

Wesentlich häufiger als Printmedien lasen die Lernenden im Internet. Dabei waren sie vor allem in den sozialen Netzwerken aktiv. So gaben zwölf der 16 Lernenden an, mehrmals die Woche zu chatten, und zehn, sich an Gruppendiskussionen in Internetforen oder beispielsweise auf Facebook zu beteiligen. Jeweils vier weitere SchülerInnen machten beides ein oder mehrmals pro Monat. Die Hälfte der Lernenden benutzte aber auch wenigstens mehrmals im Monat ein Internetwörterbuch (der chinesische Schüler als einziger mehrmals pro Woche), und sieben Lernende konsultierten genauso oft Online-Nachschlagewerke. Insgesamt zwölf Schülerinnen und Schüler lasen mehrmals in der Woche oder im Monat Nachrichten im Internet, zehn informierten sich dort genauso häufig über bestimmte Themen, und acht Lernende nutzten das Internet außerdem, um sich über praktische Dinge wie Veranstaltungstermine, Spielanleitungen oder Rezepte zu erkundigen (FB, Nr. 27). ${ }^{94}$

\subsubsection{Einstellung zum Erdkundeunterricht und zur Lehrkraft}

Um die Einstellung der Lernenden zum Fach Erdkunde und der unterrichtenden Lehrkraft besser einordnen zu können, wurden die Kinder auch danach befragt, was sie generell von der Schule hielten und was für ein Verhältnis sie zu den Lehrkräften hatten. In dieser Klasse waren die Lernenden zwar größtenteils der Auffassung, dass die Schule keine Zeitverschwendung sei und sie auf ihr späteres Leben vorbereite, aber nur die gute Hälfte (9) war vollkommen davon überzeugt, dass die Schule sie auf ihre berufliche Zukunft vorbereite, und nur zwei Lernende schrieben der Schule uneingeschränkt die Qualität zu, ihnen bisher dabei geholfen zu haben, Entscheidungen bewusster zu treffen. Weitere fünf stimmten dem immerhin zu, während die übrigen kaum oder gar nicht dieser Auffassung waren.

Danach befragt, wie sie überwiegend von ihren Lehrkräften behandelt werden, waren sie vor allem der Ansicht, dass diese gut mit ihnen umgingen (42 von möglichen 60 Punkten; vgl. FB, Nr. 34); fünf Lernende fanden das eher nicht. Mit etwa derselben Zustimmung (40 Punkte) äußerten die meisten Lernenden, dass sie sich mit ihren Lehrpersonen größtenteils verstanden; demgegenüber stehen sieben, die gar nicht oder kaum dieser Meinung waren. 38

94 In allen drei Schulen gibt es einen Computerraum mit Internetzugang. Dieser wurde während des Zeitraums der Unterrichtsbeobachtungen von zwei Klassen (an den Schulen Morante und Vespucci) im Erdkundeunterricht genutzt: Eine Klasse recherchierte zu einigen im Lehrbuch knapper behandelten Ländern und die andere digitalisierte einen handschriftlich verfassten Text. Beide Unterrichtseinheiten im Computerraum bestanden aus zwei bzw. drei Erdkundestunden. Zu den mit dem Fragebogen erfassten Aktivitäten im Internet nutzen die Lernenden allerdings ihre privaten Computer und andere außerschulische PCs wie die in den Büchereien. 
Punkte vergaben die SchülerInnen ferner für die Bereitschaft der Lehrpersonen, sie zu fördern und zu unterstützen, wenn sie zusätzliche Hilfe benötigten. Auch hier waren wieder fünf Lernende kaum oder gar nicht dieser Ansicht. Ihr Verhältnis zu den Lehrkräften und deren Behandlung stuften die SchülerInnen an der Morante positiver ein als das ihnen vom Lehrpersonal geschenkte Interesse und deren Fähigkeit, ihnen richtig zuzuhören. ${ }^{95}$

Danach befragt, wie nützlich ihnen konkret der Erdkundeunterricht erschien, schnitt dieser insgesamt etwas besser ab als ihr Schulunterricht im Ganzen. Während sie Letzteren im Mittelwert mit 34 Punkten bewerteten, gaben sie dem Erdkundeunterricht 36. Im Verhältnis zum Schulunterricht insgesamt hielten sie ihn noch weniger für Zeitverschwendung oder für nicht ergiebig als Vorbereitung auf ihr späteres Leben. Auch half er ihnen etwas besser, Entscheidungen bewusst zu treffen, als es der schulische Unterricht im Ganzen leistete; nur in Bezug auf ihre berufliche Zukunft sahen sie in ihm einen geringeren Nutzen als in allen Fächern zusammen betrachtet (FB, Nr. 37).

Eine ebenfalls positivere Wertung wurde der Erdkundelehrkraft im Vergleich zur Gesamtheit der in der Klasse unterrichtenden Lehrpersonen zuteil. Hierbei sei wiederholt, dass Prof. Tigre wie alle beobachteten Erdkundelehrkräfte auch Italienisch und Geschichte mit insgesamt zehn Wochenstunden in der Klasse unterrichtete, so dass die Lernenden mit ihm deutlich mehr Zeit verbrachten als mit allen übrigen Lehrkräften. Hinsichtlich des Verhältnisses zwischen Lernenden und Lehrkraft fiel die Bewertung durchschnittlich um einiges höher aus als bezüglich der Nützlichkeit des Unterrichts, nämlich um 10 Punkte. Vor allem das Interesse Prof. Tigres für sie und das, was sie dachten, bewerteten sie als deutlich ausgeprägter als das der anderen Lehrkräfte; selbiges für seine Bereitschaft, sie zusätzlich zu fördern und zu unterstützen, wenn sie etwas nicht verstanden (12 Punkte mehr für alle drei Aspekte; FB, Nr. 39). In Tabelle 2 sind die Angaben der Lernenden zum Erdkundeunterricht und zur Lehrkraft abgebildet. Die Zahlenwerte stehen für , überhaupt nicht einverstanden“ (1), „kaum einverstanden“ (2), ,einverstanden“ (3) und „,vollkommen einverstanden“ (4), U für Erdkundeunterricht, LP für die Erdkunde unterrichtende Lehrperson und der Gedankenstrich für „ohne Angabe“.

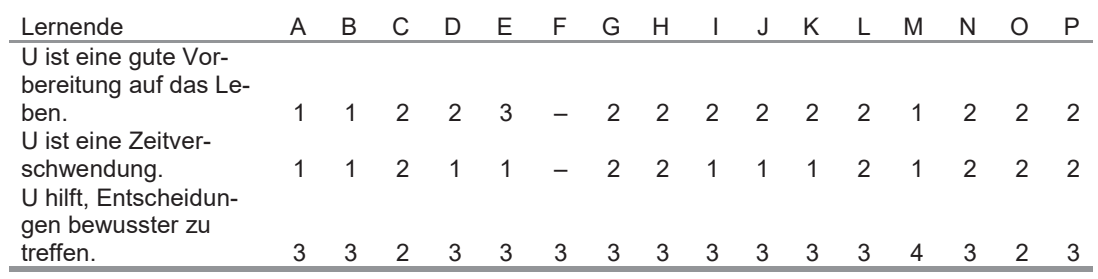

95 Eine Lernende (N) gibt außer dem Zahlenwert in diesem Zusammenhang an, dass die Lehrkraft ihr oft sage, still zu sein. 


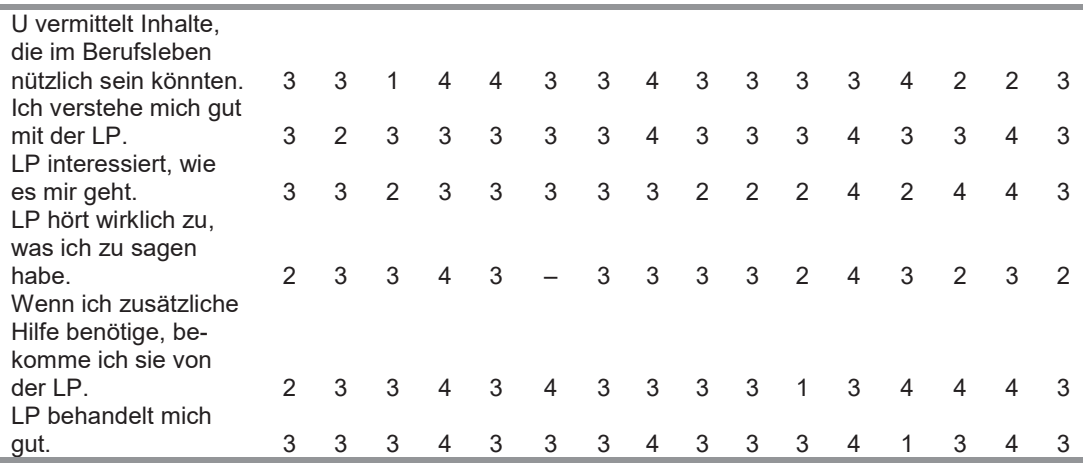

Tabelle 2: Einstellung zum Erdkundeunterricht und zur unterrichtenden Lehrkraft an der Morante

Der Lehrer unterrichtete sein Fach nach eigenen Angaben sehr gerne - im Gegensatz zu vielen seiner Kolleginnen und Kollegen und auch zu den anderen vier in dieser Studie beobachteten, die geschlossen den Schwerpunkt auf das Fach Italienisch legten und danach Geschichte bevorzugten.

Während der Unterrichtsbeobachtungen wurde diese Klasse als sehr lebhaft wahrgenommen. Es war selten ganz still, die Schülerinnen und Schüler führten zum einen Nebengespräche untereinander und beteiligten sich zum anderen am Unterrichtsgespräch zumeist, ohne darauf warten zu müssen, dass ihnen das Rederecht erteilt wurde. Das Klassenklima schien überwiegend freundlich und humorvoll, wobei ein Teil der Lernenden im Fragebogen angab, den Erdkundeunterricht etwas langweilig zu finden und sich mehr praktische Einbindung und eine andere Art der Erklärung fachlicher Inhalte zu wünschen (FB, Nr. 52 f.). Die während der Beobachtung gewonnenen Eindrücke stimmen mit den Einschätzungen der Lernenden überein, die zugaben - obgleich es ihrer Auffassung nach nur gelegentlich vorkam -, dass sie ihrem Lehrer nicht zuhörten und im Klassenzimmer oft Lärm und Unruhe herrschten. Nur ab und zu hatten sie jedoch Schwierigkeiten, gut zu arbeiten, und manchmal dauerte es recht lange, bis der eigentliche Unterricht begann und bis im Klassenzimmer Ruhe herrschte (FB, Nr. 36).

Der Erdkundeunterricht bestand vorwiegend aus Abfragungen, zu denen sich nur selten Freiwillige meldeten, so dass die Lehrkraft in der Regel zwei Lernende pro Stunde dazu aufforderte. Nicht alle verfolgten die Abfragungen aufmerksam mit, jedoch stieg ihre Konzentration auf das Unterrichtsgeschehen gegen Ende des Schuljahres mit dem Beginn der Vorbereitungsphase auf die Abschlussprüfung.

Zum Unterrichtsverlauf gaben sie im Mittelwert an, dass der Lehrer sie oft dazu aufforderte, die Bedeutung eines Textes aus dem Lehrwerk zu erläutern. Dabei stellte er auch oft Fragen, die ihnen dabei behilflich waren, den Text besser zu verstehen. Nur manchmal gab er ihnen allerdings genug Zeit, sich 
eine Antwort zu überlegen, hingegen riet sie ihnen oft, einen Autor oder ein bestimmtes Buch zu lesen (FB, Nr. 38). Dass er das oft tat, kann anhand der Stundenaufzeichnungen nicht bestätigt werden. Es kam zwar tatsächlich gelegentlich vor, aber dieser Eindruck der Schüler rührt vielleicht ehe daher, dass Prof. Tigre in seiner Funktion als Italienischlehrer einen Überblick über geeignete Lektüre für die Lernenden hatte und ihm daran lag, die Leselust der Lernenden zu wecken oder aufrecht zu erhalten. Wahrscheinlich wurde das Antwortverhalten der Lernenden bezüglich der Leseempfehlungen dadurch beeinflusst, dass der Lehrer ihnen im Italienischunterricht häufiger Lektürevorschläge machte.

Die Aufzeichnungen deckten sich hingegen mit den Auffassungen der SchülerInnen, dass Herr Tigre sie oft nach ihrer Meinung zu bestimmten Texten fragte und ihnen ab und zu dabei half, Bezüge zwischen dem Gelesenen und der eigenen Lebenswelt herzustellen. Gleichermaßen zeigte er ihnen häufig, wie sie die Inhalte mit bereits erworbenem Wissen verknüpfen konnten und Letzteres vertieft wurde. Hin und wieder erteilte er schriftliche Hausaufgaben, nie aber den Auftrag einer Zusammenfassung der gelesenen Texte, und insgesamt hatten die Lernenden das Gefühl, etwas zu viel Zeit für die Hausaufgaben zu benötigen (s. FB, Nr. 38): Die dafür aufgebrachte Zeit variierte bei den Lernenden stark und reichte von einer halben Stunde bis zu zwei Stunden, wobei sie im Durchschnitt eine Stunde und 23 Minuten betrug (vgl. FB, Nr. 46). Dieser für ein Nebenfach recht große und je nach Schüler sehr unterschiedliche Zeitaufwand resultiert aller Wahrscheinlichkeit nach daraus, dass sich die Lernenden stets darauf vorbereiteten, abgefragt zu werden. Es verhielt sich in den anderen vier Klassen, die an der Studie teilnahmen, aus dem gleichen Grund sehr ähnlich.

Auf die Frage, wie ihr der Erdkundeunterricht im Vergleich zu den anderen „mündlichen“ Schulfächern gefiel, gab die Klasse an der Morante zur Antwort, dass sie ihn sehr mochte, auch wenn hier die Meinungen erneut weit auseinandergingen (FB, Nr. 53). Das Fach wurde gegenüber Mathematik, Naturwissenschaft, Italienisch, Geschichte, Englisch und Spanisch bzw. Französisch als etwas leichter eingestuft (FB, Nr. 54).

\subsubsection{Schülerangaben zum Lehrbuch}

Die Lehrwerksbewertung der Lernenden fällt etwas schlechter aus als in den anderen Klassen. Es handelt sich hier um ein relativ altes Unterrichtswerk (Bersezio 2005 aus dem Verlag De Agostini). Durchschnittlich hielten die Lernenden die Darbietung der Inhalte für einigermaßen interessant und vertraten sie die Ansicht, dass die Illustrationen für ein besseres Verstehen und Behalten der Inhalte hilfreich seien. Sie erachteten die Texte nicht als leicht verständlich, 
die Aufgaben jedoch als das Verständnis unterstützend. ${ }^{96}$ Während sie die verwendeten Ausdrücke sowie die Textlänge für ihrem Alter und ihren Fähigkeiten angemessen hielten, bewerteten sie die Schreibweise des Buches als nicht klar und die Erklärungen zu neu eingeführten Fachtermini als mangelhaft. Über ein Viertel der Lernenden vertrat die Meinung, dass die Texte leichter zu verstehen wären, wenn sie in einer anderen Sprache geschrieben wären (s. FB, Nr. 42), wobei eine Lernende (P) neben dem Zahlenwert vermerkte, dass sie das Buch leichter verstehen würde, wenn es im römischen Dialekt geschrieben wäre. Auch wenn dieser Hinweis wahrscheinlich als Scherz zu verstehen ist, deutet er doch darauf hin, dass die Lernende einen Unterschied zwischen ihrer Alltagssprache und der im Lehrbuch verwendeten Sprache wahrnahm, der ihr das Verständnis des Lehrbuchs nicht erleichterte.

In Tabelle 3 sind diese Lehrwerkseinschätzungen der Lernenden wiedergegeben, wobei LB für Erdkundelehrbuch und Ws für Wortschatz steht und die Bewertungsskala wieder von 1 bis 4 mit ,überhaupt nicht einverstanden“, „kaum einverstanden“, „einverstanden“ und „vollkommen einverstanden“ reicht.

\begin{tabular}{|c|c|c|c|c|c|c|c|c|c|c|c|c|c|c|c|c|}
\hline Lernende & A & B & C & D & $\mathrm{E}$ & $\mathrm{F}$ & G & $\mathrm{H}$ & I & $\mathrm{J}$ & $\mathrm{K}$ & $\mathrm{L}$ & M & $\mathrm{N}$ & & $\mathrm{P}$ \\
\hline $\begin{array}{l}\text { LB bietet die Inhalte auf interessante Weise } \\
\text { dar. } \\
\text { Die Abbildungen helfen mir, die Inhalte besser }\end{array}$ & 2 & 2 & 2 & 3 & 3 & 1 & 3 & 4 & 3 & 3 & 1 & 4 & 3 & 3 & & \\
\hline zu verstehen und zu behalten. & 3 & 3 & 3 & 3 & 4 & - & 3 & 3 & 4 & 4 & 2 & 3 & 3 & 3 & 2 & \\
\hline Die Texte sind leicht zu verstehen. & 3 & 2 & 1 & 2 & 3 & 2 & 3 & 3 & 3 & 3 & 2 & 2 & 3 & 3 & 1 & 2 \\
\hline Die Ü & & 2 & 2 & 2 & 2 & - & 3 & 3 & 2 & 2 & 3 & 4 & 3 & * & 1 & \\
\hline $\begin{array}{l}\text { rt. } \\
\text { inem Alter und mei- }\end{array}$ & & 2 & 1 & 2 & 3 & - & 3 & 3 & 3 & 3 & 2 & 3 & 3 & 3 & 1 & 2 \\
\hline nen & & 2 & 1 & 2 & 3 & 2 & 3 & 3 & 3 & 3 & 3 & 3 & 4 & 4 & 1 & 2 \\
\hline ahen die richtige I änge & 3 & 3 & 1 & 1 & 3 & 4 & 3 & 3 & 3 & 3 & 3 & 3 & 4 & 3 & 1 & \\
\hline $\begin{array}{l}\text { Neue Fachwörter sind gut erklärt. } \\
\text { Wenn LB nicht in Italienisch, sondern in einer an- } \\
\text { deren Sprache geschrieben wäre, würde ich es }\end{array}$ & & 1 & 1 & 2 & 2 & 3 & 3 & 2 & 2 & 2 & 4 & 3 & 3 & 2 & 2 & 3 \\
\hline besser verstehen. & 1 & 2 & 3 & 2 & 1 & 2 & 1 & 1 & 4 & 4 & 1 & 2 & 1 & 1 & 4 & 4 \\
\hline
\end{tabular}

Tabelle 3: Einschätzung des Erdkundelehrbuchs, Lernende der Morante *s. Fn.

\subsubsection{Lernstrategien}

Die Lernenden wurden auch danach befragt, wie nützlich sie auf einer Skala von 1 (überhaupt nicht nützlich) bis 6 (absolut nützlich) sieben bestimmte Techniken einstufen, um die Lehrbuchtexte zu verstehen und sich ihren Inhalt einzuprägen. Eine der 16 Lernenden beantwortete diese Frage nicht. Die effektivste Technik ist den Angaben der anderen zufolge das Zusammenfassen der Texte mit eigenen Worten (75 von 90 möglichen Punkten). Lediglich der chinesische Schüler bewertete diese Strategie als überhaupt nicht nützlich, wobei

96 Nur eine Lernende (N) gab anstelle des Zahlenwertes an, dass sie die Aufgaben nicht mache. 
nicht klar ist, ob er seine Angaben auf die italienischen Lehrwerke bezog, für dessen Verständnis seine Kenntnisse noch nicht ausreichten und die er noch nicht paraphrasieren konnte, oder auf seine chinesischen Erdkundebücher.

An zweiter Stelle folgt für die meisten Lernenden das Unterstreichen von Schlüsselwörtern und wichtigen Konzepten, was nur zwei Lernende nicht besonders nützlich fanden (67 Punkte). An dritter Stelle steht die Strategie der Konzentration auf die leichter verständlichen Textstücke (59 Punkte), wobei zwei Lernende angaben, dass dies vollkommen und weitere fünf, dass es sehr nützlich sei. Auf der anderen Seite waren aber auch sechs der Auffassung, dass diese Strategie wenig oder nur einigermaßen hilfreich sei.

Ähnlich viele Punkte erhielt die Technik, das Gelesene mit jemandem zu besprechen (57 Punkte). Hierzu gab niemand an, dass es überhaupt nicht nützlich sei, aber viele hielten es nur für bedingt förderlich. Auch andere Lernstrategien wie jemandem den Text vorlesen (41) und zweimal schnell lesen (40) erachteten die meisten für weniger hilfreich als die zuerst genannten Strategien. Für das Vorlesen gaben außerdem acht und für das zweitmalige schnelle Lesen sieben Lernende an, dass diese Strategien überhaupt nicht oder nur geringfügig nützlich seien (vgl. FB, Nr. 41).

Neun von 15 Lernenden lasen einen Text zum Lernen außerdem grundsätzlich laut, was mit der Vorbereitung der mündlichen Abfragungen in Zusammenhang gestanden und dazu beigetragen haben dürfte, dass einige die Formulierungen der Texte wortwörtlich übernahmen. Insgesamt zehn SchülerInnen wiederholten so auch das Gelesene laut, wobei unter ihnen dieselben Lernenden waren, die immer laut lasen. Eine weitere Schülerin las zwar leise, wiederholte dann aber laut. Lediglich vier Lernende dieser Klasse wiederholten den Text nicht laut und zwei von ihnen weder laut noch leise, während andere Lernende beide Strategien anwandten (s. FB, Nr. 47). Sechs SchülerInnen machten sich beim Lernen Notizen über die Textinformationen und vier schrieben ganze Zusammenfassungen. Da sie angaben, recht lange für die Hausaufgaben zu brauchen, ist davon auszugehen, dass sie den Text zunächst langsamer und vielleicht auch mehrmals lasen und dann versuchten, seinen Inhalt zu wiederholen. Die Hälfte der Lernenden ( 7 bei 2 Enthaltungen) ließ sich hierbei von jemandem abfragen.

Auf die abschließende offene Frage, was sie darüber hinaus noch machten, um die Texte zu lernen, gab lediglich eine Schülerin etwas an, und zwar, dass sie wichtige Aspekte unterstrich.

Ferner antworteten 15 von 16 anhand einer Skala, die von 1 (fast nie) bis 4 (fast immer) reicht, auf 13 Fragen, die ihre allgemeine Vorgehensweise beim Lernen betrafen (vgl. FB, Nr. 28). Mit 48 von möglichen 60 Punkten stimmten sie am meisten der Aussage zu, dass sie beim Lernen versuchten, die wichtigsten Textaussagen zu behalten. Fast genauso viele Punkte (46) gaben sie dem Versuch, möglichst viele Einzelheiten zu behalten, und der Überprüfung, ob sie das, was sie lasen, auch verstanden (47). 
Mit 29 Punkten stimmten sie am wenigsten von allen Aussagen der zu, dass sie einen Text so oft lasen, bis sie ihn auswendig kannten, wobei vier eingestanden, dies oft zu tun. Es kann davon ausgegangen werden, dass eine von den Lehrkräften vermittelte negative Sicht auf das Auswendiglernen das Antwortverhalten insgesamt beeinflusst hatte und die Angaben der Lernenden deshalb nicht ganz wahrheitsgetreu waren. Während der Unterrichtsbeobachtungen und bei der Durchsicht schriftlicher Tests fiel nämlich auf, dass viele Lernende sowohl in mündlichen Abfragungen als auch in Textproduktionen Ausdrücke oder auch ganze Sätze wortwörtlich aus dem Lehrbuch übernahmen. Und da ihre Leistungen von der Lehrkraft trotzdem positiv bewertet wurden, besteht der Eindruck, dass sie häufiger auswendig lernten, als sie eingestanden. ${ }^{97}$

Auch wenn die Lernenden von verschiedenen Lehrkräften daran erinnert wurden, dass es bei der Abschlussprüfung wichtig sei, die verschiedenen fachlichen Inhalte miteinander in Verbindung bringen zu können, vergaben sie ebenfalls wenig Punkte für die Strategien, das zu Lernende mit dem in anderen Fächern erworbenen Wissen (31) bzw. eigenen Erfahrungen (32) zu verknüpfen. Trotzdem bekundeten immerhin fünf Lernende, dass sie oft oder sogar fast immer versuchten, die Inhalte verschiedener Fächer miteinander in Verbindung zu setzen, und vier, dass sie Gelerntes oft oder fast immer mit den eigenen Erfahrungen verknüpften.

Durchschnittlich gaben die Lernenden für diese sieben der 13 Strategien an, sie selten anzuwenden:

- das Gelernte mit Inhalten anderer Fächer in Verbindung setzen;

- wiederholtes Lesen zum Auswendiglernen;

- mehrmaliges Lesen;

- Hinterfragen, wie das Gelesene außerhalb der Schule nützlich sein könnte;

- Versuch, nicht verstandene Konzepte zu bemerken;

- Versuch, Unverstandenes mit zusätzlichen Hilfsmitteln wie anderen Texten oder Nachschlagewerken zu klären.

Durchschnittlich verfuhren sie hingegen oft folgendermaßen:

- Versuch, alle Informationen des Textes zu behalten;

- mit der Kontrolle dessen zu beginnen, was genau gelernt werden soll;

- Versuch, sich möglichst viele Details zu merken;

- Kontrolle, ob das Gelesene verstanden wird;

- die wichtigsten Punkte des Textes zu behalten;

- Hinterfragen, welche Bedeutung das Gelesene im wahren Leben hat.

97 Vgl. hierzu die Kapitel 9 (Unterrichtsbeobachtungen) und 10 (schriftliche Textproduktionen der Lernenden). 


\subsection{Klasse an der Vespucci}

In der Klasse an der Schule Vespucci waren während der Unterrichtsbeobachtungen 13 von 14 Lernenden anwesend, während einer aus Krankheitsgründen dauerhaft fehlte. Unter den zwölf Lernenden, welche den Fragebogen ausgefüllten, sind acht Mädchen und vier Jungen, von denen eine italienische und eine in Italien geborene Schülerin peruanischer Herkunft das zweite Mittelschuljahr wiederholt hatten. In dieser Klasse hatte gute die Hälfte der beobachteten Schülerinnen und Schüler einen Migrationshintergrund, nämlich insgesamt sieben. Was die geographische Herkunft der Familien anbelangt, so waren vier Elternpaare italienischstämmig und sieben Elternpaare aus dem Ausland nach Italien immigriert. Eine Familie bestand aus einem italienischen und einem ausländischen Elternteil, wobei es sich bei seinem Herkunftsland nicht um ein typisches Einwanderungsland handelte, sondern um die Schweiz, wahrscheinlich die italienische Schweiz, da keine Angaben über einen bilingualen familiären Sprachgebrauch vorliegen. Zwei der Schüler, die an der Umfrage teilnahmen, wurden nicht in Italien geboren. Eine Schülerin war im Alter von zehn Jahren aus Rumänien ins Land gekommen und ein weiterer mit elf aus Ecuador (vgl. FB, Nr. 14 und 15). Ihrem umgangssprachlichen Gebrauch des Italienischen merkte man kaum an, dass es sich dabei um eine Zweitsprache handelte, aber nach Aussage von Prof.ssa Mancuso hatten sie mitunter erhebliche Schwierigkeiten mit der Verarbeitung und Verwendung der Schulsprache - ein Problem, das auch weitere Schülerinnen und Schüler dieser Klasse gemein hatten, zumal in vielen Elternhäusern vorwiegend und teilweise ausschließlich andere Sprachen gesprochen wurden als Italienisch. Außerdem ist ihren Berichten nach davon auszugehen, dass sich dieser allochthone Sprachgebrauch größtenteils auch in der familiären Sozialisation fortsetzte. Außer Italienisch wurde in den Familien Rumänisch, Spanisch, Bengalisch und Englisch gesprochen (FB, Nr. 16-20), und in vielen Fällen war die Unterrichtssprache also nicht die Erstsprache der SchülerInnen, auch wenn sie von klein auf mit dieser Sprache in Berührung gekommen waren.

Acht der Lernenden hatten ein bis zwei Geschwister, vier waren Einzelkinder. Ebenfalls acht hatten ein eigenes Zimmer, und alle zwölf gaben an, zuhause einen Schreibtisch und einen ruhigen Platz zum Lernen zu haben. Nur in einem Haushalt gab es weder einen Computer noch einen Internetanschluss. Zwar verfügten nur zwei Haushalte über Lernsoftware, aber in zehn von zwölf gab es ein Wörterbuch und in acht weiteren nützliche Bücher für das Anfertigen der Hausaufgaben und zum Lernen.

Von den verschiedenen weiterführenden Schultypen wählte auch in dieser Klasse nur eine Schülerin ein Lyzeum, wobei es um die Schulbildung der Eltern ähnlich wie an der Morante bestellt war: Es gab keine Eltern, die beide 
einen Universitätsabschluss hatten; lediglich drei Elternteile hatten ein Studium und weitere sieben das Abitur absolviert.

Desweiteren ergibt sich aus der Bearbeitung des Fragebogens, dass die Hälfte aller Lernenden zusätzlichen Unterricht in Form von Nachhilfe und Hausaufgabenbetreuung nahm. Dieser beschränkte sich nur bei einem Schüler auf ein einziges Fach (Mathematik); die anderen wurden gleich in mehreren Fächern zusätzlich außerschulisch unterstützt (FB, Nr. 31). Es ist anzunehmen, dass sich dieses zusätzliche Angebot, das von der Lehrperson empfohlen worden war, gerade für SchülerInnen mit Schwierigkeiten im Umgang mit der Unterrichtssprache positiv auf die Sprachbildung im Speziellen und die Allgemeinbildung im Ganzen auswirkte. Zudem offenbarte es den Ehrgeiz der Lernenden (bzw. ihrer Eltern), die schulischen Leistungen zu verbessern.

Der unterstützende Unterricht wurde in einer Einrichtung in der Nähe der Schule erteilt, in der die Lernenden an mehreren Wochentagen nach dem Unterricht betreut wurden, um ihre Hausaufgaben anzufertigen und für Tests und Klassenarbeiten zu lernen. Dort konnten sie auch unter Anleitung Gegenstände des Schulstoffs wiederholen oder vertiefen, die sie noch nicht so gut verstanden hatten. Auffällig ist, dass ein solches Angebot gerade von denjenigen Lernenden wahrgenommen wurde, die bereits ganztägigen Schulunterricht hatten, während ihre Altersgenossen in den anderen beobachteten Klassen außerschulische Fördermaßnahmen nicht in demselben Ausmaß in Anspruch nahmen, obwohl sie dafür mehr Zeit gehabt hätten.

\subsubsection{Lesegewohnheiten}

Fast in der Hälfte aller Haushalte (fünf) gab es maximal 25 Bücher, nirgendwo mehr als 500, und nur zwei Lernenden in dieser Klasse war früher regelmäßig vorgelesen worden (FB, Nr. 23 und 55). Alle vier befragten Jungen gaben an, in ihrer Freizeit entweder gar nicht oder maximal eine halbe Stunde zu lesen. Bei den Mädchen waren es fünf von acht (FB, Nr. 24). Die Angaben sind in Abbildung 2 dargestellt.

Auch an der Schule Vespucci gab es eine Bücherei. Schulexterne Bibliotheken suchten die befragten Lernenden kaum auf: sieben SchülerInnen nie, vier ein paarmal im Jahr und nur ein Schüler, der noch nicht lange in Italien lebte, einmal im Monat (FB, Nr. 49). Danach befragt, was sie wie oft in einer Bücherei machten, gaben die Lernenden an, am meisten den Internetzugang zu nutzen (39 von 65 möglichen Punkten). Der Schüler, der zuhause keinen Internetzugang hatte, griff mehrmals wöchentlich in der Bücherei darauf zu. An zweiter und dritter Stelle folgten das Lesen von Zeitschriften und das Lernen von Dingen, die nichts mit der Schule zu tun hatten, beispielsweise Lektüre über Hobbies wie Sport und Musik. Am seltensten liehen die Lernenden Bücher für die Freizeit aus. Schon etwas öfter, aber durchschnittlich auch nur ein 
paarmal im Jahr, liehen sie Bücher aus, die sie für die Schule brauchten. Nur drei der Lernenden gingen einmal pro Monat bis mehrmals die Woche in die Bücherei, um dort ihre Hausaufgaben zu erledigen, während die meisten das nie oder nur sehr selten taten.

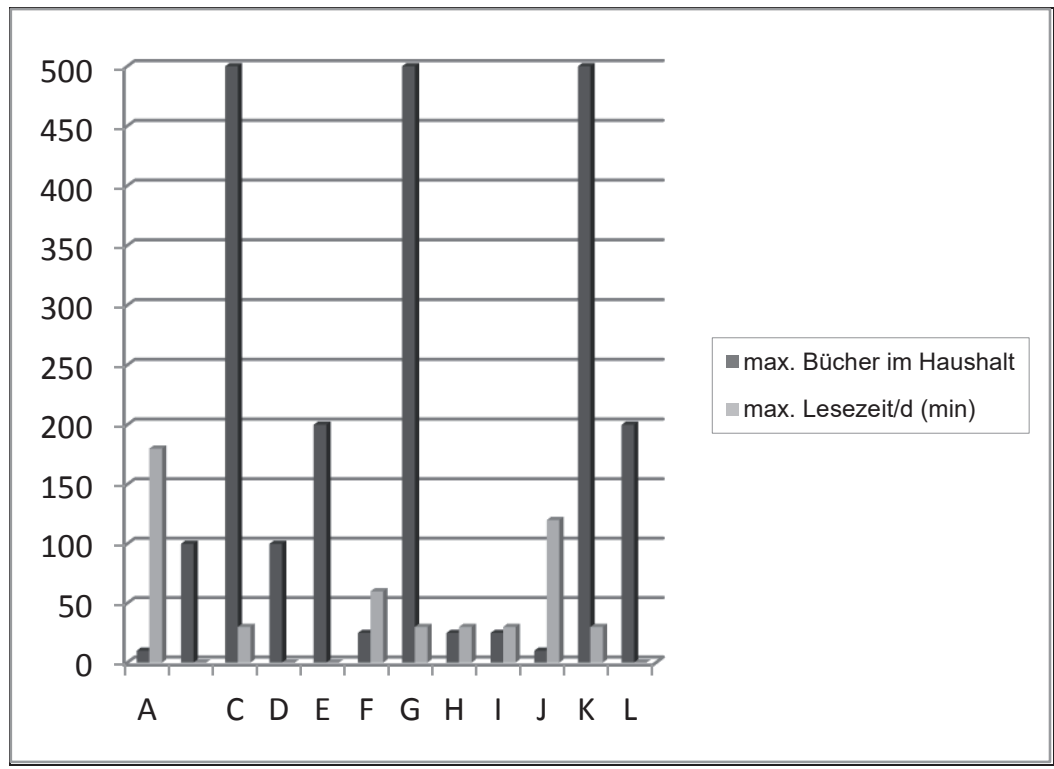

Abbildung 2: Maximale Bücherzahl im Haushalt und maximale tägliche Lesezeit (in min), Vespucci

Im Mittelwert stimmten die Schülerinnen und Schüler der Aussage zu, dass sie nur lasen, wenn sie dazu verpflichtet wurden. Drei betonten sogar, dass sie dem vollkommen zustimmen. So stellte das Lesen auch nur für drei Lernende eine der Lieblingsfreizeitbeschäftigungen dar. Fünf Lernenden fiel es schwer, ein angefangenes Buch zu beenden, vier gaben zu, dass sie Mühe hatten, länger als ein paar Minuten still zu sitzen und zu lesen, und nur vier freuten sich, wenn sie ein Buch geschenkt bekamen. Fünf Lernenden gefiel es recht gut, Bücher mit Freunden zu tauschen; lediglich drei redeten gerne mit anderen über das, was sie lasen. Außerdem gaben neun an, dass sie nur zur Gewinnung benötigter Informationen lasen (FB, Nr. 25).

Gefragt nach der Art der Texte, die sie lasen, ergibt sich, dass die meisten am liebsten Zeitschriften lasen, nämlich acht von ihnen mehrmals monatlich bzw. wöchentlich. Mit zwölf Punkten Abstand folgt an zweiter Stelle das Lesen von Zeitungen; zwei taten dies mehrmals wöchentlich, alle anderen höchstens einmal im Monat und drei nie. Auch Comics wurden in dieser Klasse nicht viel gelesen, nämlich nur von zweien mehrmals im Monat, während sechs sie 
nie lasen. Gleichermaßen gaben fünf Lernende an, Romane und Kurzgeschichten nie oder nur sehr selten zu lesen (FB, Nr. 26).

Etwas anders verhielt es sich mit ihren Leseaktivitäten im Internet. Von acht Möglichkeiten, im Internet zu lesen und zum Teil zu schreiben, welche die Lernenden auf einer Skala von 1 bis 5 nach der Häufigkeit, mit der sie diesen Tätigkeiten nachgingen, bewerten sollten, nutzte eine Schülerin alle mehrmals wöchentlich, wenn nicht täglich und vergab insgesamt 38 von 40 möglichen Punkten. Ein weiterer Schüler verteilte 36 Punkte und nutzte sieben Möglichkeiten ähnlich oft, las nur wesentlich seltener Nachrichten. Durchschnittlich vergaben die Lernenden insgesamt für alle Aktivitäten 29 Punkte. Nur der Schüler, der zuhause keinen Internetanschluss hatte, ging allen Aktivitäten fast nie oder nur selten nach (18 Punkte).

Am häufigsten chatteten die Lernenden (51 Punkte) und beteiligten sich an Foren und Diskussionen auf Facebook (49 Punkte), aber auch sehr oft suchten sie im Internet nach Informationen zu einem bestimmten Thema (45 Punkte). Öfter als E-Mails zu schreiben benutzten sie außerdem Online-Wörterbücher und -Enzyklopädien (41 und 44 Punkte).

\subsubsection{Einstellung zum Erdkundeunterricht und zur Lehrkraft}

Der Erdkundeunterricht fand nach der Mittagspause statt, in einer Doppelstunde zu Wochenbeginn. Die kleinste an der Studie teilnehmende Lerngruppe setzte sich aus recht disziplinierten Schülerinnen und Schülern zusammen, wodurch es im Klassenraum selbst dann ruhig blieb, wenn die Lehrkräfte ihn vorübergehend verließen. Die Lernenden erweckten den Eindruck, konzentriert zu arbeiten, wobei von allen beobachteten Klassen nur in dieser regelmäßig Gruppenarbeitsphasen durchgeführt wurden. Auch ihrer eigenen Meinung nach kam es im Erdkundeunterricht nur gelegentlich vor, dass der Lehrkraft keine Aufmerksamkeit geschenkt wurde, Lärm und Unordnung herrschten und Prof.ssa Mancuso lange warten musste, bevor es in der Klasse still wurde. Ebenfalls konnten die Schülerinnen und Schüler nur ab und zu nicht konzentriert arbeiten und verging gelegentlich viel Zeit, bis der eigentliche Unterricht begann (vgl. FB, Nr. 36).

Nur zwei der Lernenden erachteten den Schulunterricht grundsätzlich als keine gute Vorbereitung auf ihr Leben, und eine von ihnen hielt ihn für komplette Zeitverschwendung; die anderen waren gegenteiliger Meinung und sich fast geschlossen (zwei Ausnahmen) darin einig, dass er ihnen dabei half, Entscheidungen bewusster zu treffen, und dass er ihnen Inhalte vermittelte, die für ihre berufliche Laufbahn von Nutzen sein könnten (FB, Nr. 33). Die Lernenden schienen mit ihrem Verhältnis zu den Lehrkräften ganz zufrieden zu sein, denn sie fühlten sich von ihnen ausnahmslos gut behandelt. Ebenfalls einstimmig 
vertraten sie ferner die Auffassung, dass ihnen die Lehrkräfte zusätzliche Unterstützung zukommen ließen, wenn diese benötigt wurde, und dass sie sich mit ihnen gut verstanden (eine Ausnahme). Es gab aber auch fünf Lernende, die das Gefühl hatten, die Lehrkräfte interessierten sich kaum für sie und ihre Meinung (FB, Nr. 34).

Auch in der Klasse an der Vespucci wurde das Verhältnis zur Erdkundelehrkraft im Großen und Ganzen besser bewertet als das zu den anderen unterrichtenden Lehrerinnen und Lehrern. Allerdings fiel der Unterschied etwas kleiner aus als an der Schule Morante und fühlten sich zwei Lernende von Prof.ssa Mancuso ein bisschen schlechter behandelt als von den anderen Lehrkräften (FB, Nr. 39). Auf der anderen Seite waren sie noch mehr als ihre Altersgenossen in den anderen Klassen der Ansicht, dass ihre Lehrerin ihnen zusätzliche Unterstützung bot, wenn sie diese benötigten (FB, Nr. 39). Auf die offenen Fragen, was ihnen am Erdkundeunterricht gefiel und was nicht, gaben viele Lernende an, dass Prof.ssa Mancuso gut, vertiefend und wenn nötig geduldig wiederholend erklärte. Einige waren aber auch der Auffassung, dass sie manchmal schrie und sich zu schnell ärgerte, doch die meisten hätten nichts an ihrem Unterricht verändern wollen (FB, Nr. 50-52).

Im Mittelwert gefiel der Klasse der Geographieunterricht nicht ganz so gut wie den Lernenden an der Morante (4,2 zu 4,8; vgl. FB, Nr. 53). Während sie ihn als leichter bzw. etwas leichter als den Unterricht in Mathematik, Naturwissenschaften, Italienisch und in den Fremdsprachen einstufte, erachtete sie Geschichte als gleichermaßen anspruchsvoll. Was seine Nützlichkeit anbelangt, schätzten die Lernenden sie ähnlich ein wie die des Schulunterrichts insgesamt, was an gleichen Durchschnittswerten abzulesen ist (vgl. FB, Nr. 37). Tabelle 4 fasst einen Teil ihrer Angaben zusammen.

\begin{tabular}{|c|c|c|c|c|c|c|c|c|c|c|c|c|c|}
\hline Lernende & A & B & C & D & $E$ & $\mathrm{~F}$ & G & $\mathrm{H}$ & I & $\mathrm{J}$ & $\mathrm{K}$ & $\mathrm{L}$ & $\varnothing$ \\
\hline $\begin{array}{l}\mathrm{U} \text { ist eine gute Vorberei- } \\
\text { tung auf das Leben. }\end{array}$ & 3 & 1 & 2 & 2 & 3 & 2 & 2 & 1 & 3 & 2 & 1 & 2 & 2 \\
\hline $\begin{array}{l}U \text { ist Zeitverschwendung. } \\
\mathrm{U} \text { hilft, Entscheidungen }\end{array}$ & 2 & 1 & 1 & 1 & 1 & 2 & 1 & 1 & 1 & 1 & 1 & 1 & 1 \\
\hline $\begin{array}{l}\text { bewusster zu treffen. } \\
\text { U vermittelt Inhalte, die im } \\
\text { Berufsleben nützlich sein }\end{array}$ & 2 & 4 & 4 & 1 & 1 & 3 & 2 & 3 & 2 & 2 & 4 & 2 & 3 \\
\hline $\begin{array}{l}\text { könnten. } \\
\text { Ich verstehe mich gut mit }\end{array}$ & 4 & 4 & 3 & 1 & 3 & 3 & 3 & 4 & 3 & 3 & 4 & 3 & 3 \\
\hline $\begin{array}{l}\text { der LP. } \\
\text { LP interessiert, wie es mir }\end{array}$ & 3 & 3 & 3 & 4 & 4 & 3 & 4 & 3 & 3 & 4 & 4 & 3 & 3 \\
\hline $\begin{array}{l}\text { geht. } \\
\text { LP hört wirklich zu, was }\end{array}$ & 3 & 3 & 3 & 4 & 4 & 3 & 3 & 3 & 3 & 3 & 4 & 2 & 3 \\
\hline $\begin{array}{l}\text { ich zu sagen habe. } \\
\text { Wenn ich zusätzliche Hilfe } \\
\text { benötige, bekomme ich }\end{array}$ & 2 & 3 & 4 & 4 & 4 & 2 & 3 & 3 & 3 & 3 & 4 & 2 & 3 \\
\hline sie von der LP. & 3 & 3 & 4 & 4 & & & 3 & 4 & 3 & 4 & 4 & 3 & 4 \\
\hline LP behandelt mich gut. & 3 & 3 & 4 & 4 & 3 & 3 & - & 3 & 4 & 3 & 4 & 4 & 3 \\
\hline
\end{tabular}

Tabelle 4: Einstellung zum Erdkundeunterricht und zur unterrichtenden Lehrkraft an der Vespucci 
Während des Unterrichts fragte die Lehrerin oft nach der Bedeutung eines Textes und stelle diesbezüglich verständnisleitende Fragen. Außerdem gab sie ihren Schülerinnen und Schülern in der Regel genügend Zeit, um eine Antwort vorzubereiten. Auch für diese Klasse steht die Aussage der Lernenden, wie oft die Lehrkraft einen Autor oder ein bestimmtes Buch empfahl, im Widerspruch zum beobachteten Unterrichtsgeschehen: Nach Meinung der SchülerInnen machte ihre Lehrerin so gut wie immer Lektürevorschläge. Wieder drängt sich die Annahme auf, dass dieser Eindruck darauf zurückzuführen ist, dass Prof.ssa Mancuso ihnen auch Italienischunterricht erteilte und in diesem Fach wahrscheinlich häufiger Leseempfehlungen gab (FB, Nr. 38).

Bei der Textarbeit hatten die Lernenden den Eindruck, oft aufgefordert zu werden, ihre Meinung zu äußern. Aber Prof.ssa Mancuso half ihnen dabei nur gelegentlich, die Textinhalte mit ihrer Lebenswelt in Verbindung zu bringen. Oft hingegen zeigte sie, wie das Gelesene mit bereits erworbenem Wissen zusammenhängt und dieses vertieft. Die Schülerinnen und Schüler gaben an, im Fach Erdkunde oft schriftliche Hausaufgaben zu bekommen, wobei es sich nur $\mathrm{ab}$ und zu um die Zusammenfassung eines Textes aus dem Lehrwerk handelte. Dabei waren die Lernenden durchschnittlich nur selten in der Lage, ihre Hausaufgaben mit geringerem Zeitaufwand zu erledigen (vgl. FB, Nr. 38). Bei einem Mittelwert von 1,5 Stunden für die Bewältigung der Hausaufgaben brauchten die Lernenden hierfür etwas länger als in den anderen vier Klassen (FB, Nr. 46).

Abfragungen und Klassenarbeiten betreffend, ergab sich aus der Bearbeitung des Fragebogens, dass Prof.ssa Mancuso schriftliche Leistungsnachweise verlangte, bei denen sie darauf achtete, dass selbstständig und konzentriert gearbeitet wurde. Bei der Durchführung von Klassenarbeiten vergewisserte sie sich zunächst, ob die Fragen auch von allen verstanden wurden. Außerdem erklärte sie die Gewichtung der einzelnen Aufgaben bei der Bewertung und gab den Schülern die Möglichkeit, Fragen zu stellen. Im Anschluss an die Arbeit erfolgte eine gemeinsame Besprechung derselben. Durchschnittlich gaben die Lernenden an, manchmal das Gefühl zu haben, eine Antwort zu wissen, aber nicht die richtigen Worte zu finden, was an der Zweisprachigkeit einiger Lernender gelegen haben könnte. Allerdings fanden nur zwei von zwölf, dass es ihnen leichter fiele, in einer anderen Sprache als Italienisch zu antworten. Weiter verteilte die Lehrerin Noten für die Klassenarbeiten, deren Korrektur die SchülerInnen anfertigten, wobei sie die Gestaltung der Klassenarbeiten für angemessen in Bezug auf ihre Fähigkeiten und Vorbereitung hielten. Hinsichtlich der Abfragungen gaben sie an, gelegentlich Angst davor zu haben, nervös zu sein und sich unbehaglich zu fühlen (s. FB, Nr. 40). 


\subsubsection{Schülerangaben zum Lehrbuch}

Die Bewertung des Lehrwerks (Carazzi \& Pizzetti 2010) durch die SchülerInnen fiel im Ganzen positiv aus und deckte sich fast vollständig mit derjenigen der Klasse III A an der Schule Pestalozzi: Diese beiden Klassen waren etwas zufriedener mit ihrem Unterrichtswerk als die anderen drei. Im Einzelnen waren die SchülerInnen an der Vespucci der Meinung, das Buch präsentiere die Lerninhalte auf ansprechende Weise und verfüge über Photos und Illustrationen, die das Verstehen und Behalten des Lernstoffs unterstützen. Die Texte wären einfach zu verstehen, klar und deutlich geschrieben und weder zu lang noch zu kurz. Der verwendete Wortschatz entspräche ihrem Alter und ihren Fähigkeiten, und neu eingeführte Fachtermini seien gut erklärt. Ebenso hielten sie die Übungen für nützlich. Ähnlich wie in der Klasse Morante und in einer weiteren Lerngruppe der Pestalozzi behaupteten manche Lernenden, dass sie den Stoff besser verständen, wenn das Buch in einer anderen Sprache geschrieben wäre (vgl. Fragebogen, Nr. 42).

Wenn man allerdings die Antworten auf die beiden offenen Fragen, was ihnen am Erdkundebuch gefiel, bzw. nicht gefiel, betrachtet, so zeigt sich doch, dass einige die Übungen nicht mochten, die Texte für zu lang und den verwendeten Wortschatz für zu kompliziert hielten. Während in allen Klassen die Mehrheit der Lernenden auf die erste offene Frage nach den Qualitäten des Lehrbuchs antwortete, dass ihr die Illustrationen gefielen, äußerten an der Vespucci einige SchülerInnen auch ihre Zufriedenheit mit den Schilderungen über die Länder und mit dem geschichtlichen Teil sowie den verschiedenen geographischen Karten (s. FB, Nr. 43 f.).

\subsubsection{Lernstrategien}

Am nützlichsten zum Verstehen der Lehrbuchtexte und zum Behalten ihres Inhalts erschien den Lernenden das Unterstreichen von Schlüsselwörtern und wichtigen Konzepten (69 von 72 möglichen Punkten) und das Zusammenfassen der Texte mit eigenen Worten (66 Punkte).

Viele hielten es außerdem für sehr förderlich, sich auf die Teile des Textes zu konzentrieren, die leicht zu verstehen waren (55 Punkte), wobei nur ein einziger Schüler diese Auffassung gar nicht teilte. Am wenigsten nützlich war ihrer Meinung nach das schnelle zweimalige Lesen des Textes (27 Punkte). Ebenfalls für wenig nützlich hielten sie das Gespräch über das Gelesene mit einer anderen Person (35 Punkte) (FB, Nr. 41).

Die Hälfte der Schülerinnen und Schüler gab an, die Texte beim Lernen laut zu lesen, und alle außer zwei wiederholten sie laut, wobei eine Schülerin sowohl laut als auch leise wiederholte. Lediglich drei Lernende fertigten beim Lernen der Textinhalte Notizen an; einer davon und zwei weitere schrieben 
eine Zusammenfassung. Die Hälfte der Lernenden ließ sich außerdem von jemandem abfragen, und bei der offenen Frage, wie sie zusätzlich beim Lernen der Texte noch vorgingen, schrieben zwei, dass sie wichtige Aspekte unterstrichen (FB, Nr. 45). Beim generellen schulischen Lernen gaben fast alle an, dass sie kontrollierten, ob sie das Gelesene verstanden hatten. Außerdem versuchten sie oft, die wichtigsten Textinformationen zu behalten, und lasen den Text mehrmals. Dabei bemühten sie sich, darauf zu achten, ob ihnen bestimmte Konzepte unklar waren.

Am seltensten versuchten sie, das Gelesene mit ihren persönlichen Erfahrungen in Verbindung zu bringen: Fünf Lernende machten dies nie und sieben sehr selten. Ähnlich selten fragten sie sich, welche Bedeutung das Gelesene für ihr Leben haben könnte, und nur etwas öfter, ob es ihnen außerhalb der Schule nützlich sein könnte. Darüber hinaus versuchten drei Lernende nie und fünf nur selten, das Gelesene mit den Inhalten anderer Fächer in Verbindung zu setzen.

Mehr als die Hälfte (7) bemühte sich nie bzw. selten, mithilfe zusätzlicher Informationen Verständnisprobleme zu klären. Viele begannen das Lernen mit der Frage, was genau sie eigentlich lernen sollten, aber es gab auch jeweils zwei, die sich diese Frage nie bzw. nur selten stellten. Diese Angaben auf einer Bewertungsskala von 1 bis 4 (fast nie, selten, oft, fast immer), die Durchschnitts- $(\varnothing)$ und die insgesamt vergebenen Werte $(P)$ zeigt Tabelle 5.

\begin{tabular}{|c|c|c|c|c|c|c|c|c|c|c|c|c|c|c|}
\hline Während ich lerne... & A & B & C & $\mathrm{D}$ & $\mathrm{E}$ & $\mathrm{F}$ & G & $\mathrm{H}$ & $\mathrm{I}$ & $\mathrm{J}$ & $\mathrm{K}$ & $\mathrm{L}$ & $\varnothing$ & $\mathrm{P}$ \\
\hline $\begin{array}{l}\text { versuche ich, alle Textinformationen zu be- } \\
\text { halten. } \\
\text { beginne ich damit zu überprüfen, was genau }\end{array}$ & 2 & 4 & 1 & 2 & 2 & 3 & 3 & 4 & 1 & 2 & 3 & 3 & 3 & 30 \\
\hline & 1 & 4 & 1 & 3 & 3 & 4 & 3 & 4 & 2 & 3 & 2 & 3 & 3 & 33 \\
\hline & 3 & 4 & 1 & 2 & 2 & 3 & 4 & 4 & 2 & 3 & 4 & 2 & 3 & 34 \\
\hline $\begin{array}{l}\text { chern Gelernten zu verknüpfen. } \\
\text { lese ich den Text so oft, dass ich inn aus- }\end{array}$ & 1 & 2 & 1 & 3 & 2 & 3 & 2 & 1 & 2 & 3 & 4 & 3 & 2 & 27 \\
\hline $\begin{array}{l}\text { wendig kenne. } \\
\text { kontrolliere ich, ob ich das, was ich lese, ver- }\end{array}$ & 1 & 3 & 3 & 4 & 3 & 2 & 3 & 3 & 3 & 2 & 2 & 3 & 3 & 32 \\
\hline stehe & 2 & 4 & 3 & 4 & 3 & 4 & 4 & 4 & 4 & 4 & 4 & 2 & 4 & 42 \\
\hline & 3 & 3 & 3 & 3 & 3 & 3 & 3 & 4 & 4 & 4 & 3 & 2 & 3 & 38 \\
\hline & 2 & 2 & 1 & 1 & 2 & 4 & 3 & 1 & 2 & 2 & 4 & 2 & 2 & 26 \\
\hline $\begin{array}{l}\text { noch nicht ganz verstanden habe. } \\
\text { versuche ich, die Inhalte besser zu verste- } \\
\text { hen, indem ich sie mit meinen Erfahrungen }\end{array}$ & 2 & 3 & 3 & 4 & 3 & 3 & 4 & 2 & 2 & 3 & 4 & 3 & 3 & 36 \\
\hline $\begin{array}{l}\text { in Verbindung bringe. } \\
\text { merke ich mir die wichtigsten Punkte des }\end{array}$ & 1 & 2 & 1 & 1 & 1 & 3 & 2 & 1 & 2 & 2 & 2 & 2 & 2 & 20 \\
\hline $\begin{array}{l}\text { Textes. } \\
\text { frage ich mich, von welcher Bedeutung die }\end{array}$ & 3 & 4 & 3 & 4 & 3 & 4 & 3 & 4 & 4 & 4 & 2 & 3 & 3 & 41 \\
\hline $\begin{array}{l}\text { Textinfos für das wirkliche Leben sind. } \\
\text { und merke, dass ich etwas nicht verstehe, }\end{array}$ & 1 & 2 & 1 & 1 & 1 & 1 & 1 & 1 & 3 & 3 & 4 & 3 & 2 & 22 \\
\hline suche ich nach zusätzlichen Informationen. & 1 & 2 & 1 & 3 & 3 & 4 & 2 & 1 & 2 & 3 & 3 & 2 & 2 & 27 \\
\hline
\end{tabular}

Tabelle 5: Frequenz der angewandten Lernstrategien, Vespucci 


\subsection{Klasse III A der Pestalozzi}

Obgleich die Schule Pestalozzi auf ihrer Webseite stärker als die anderen Schulen dieser Studie mit einem interkulturellen Profil warb, das sich besonders um Lernende mit Migrationshintergrund bemühte, gestaltete sich die sprachliche Zusammensetzung in den drei Klassen der Schule Pestalozzi etwas homogener als in den beiden bisher vorgestellten.

In der Klasse III A befanden sich 19 Schülerinnen und Schüler, von denen 18 an der Umfrage teilnahmen. Nur in einer Familie waren beide Elternteile nicht italienischer, sondern rumänischer Herkunft, und in einer weiteren Familie stammte ein Elternteil aus Santo Domingo. Die Tochter der ersten Familie wurde in Rumänien geboren und kam im Alter von sechs Jahren nach Italien. Demnach sprach man auch nur in diesen beiden Familien neben dem Italienischen noch Rumänisch bzw. Spanisch (vgl. FB, Nr. 14-17). Was den Bildungshorizont der Eltern betrifft, waren hier mehr Erziehungsberechtigte mit einem universitären oder zumindest gymnasialen Abschluss vertreten: Von sechs Elternpaaren waren in zweien jeweils beide Akademiker und vier hatten das Abitur; desweiteren hatte in je fünf Familien ein Elternteil ein Studium bzw. das Abitur absolviert (s. FB, Nr. 9 und 13).

In dieser Klasse waren im Großen und Ganzen die leistungsstärkeren Lernenden des Jahrgangs vertreten, weil bei der Zusammensetzung der Klassen zu Beginn des ersten Jahres der Mittelschule eine Lerngruppe gebildet worden war, deren Leistungsniveau etwas höher lag als das der Parallelklassen, so dass ein anderes Lerntempo herrschen konnte. Theoretisch hatten die Eltern zwar keinen Einfluss darauf, welcher Klasse ihre Kinder zugeteilt wurden, praktisch versuchten sie nach Aussage einiger Lehrpersonen jedoch sehr wohl und teilweise mit Erfolg, in die Entscheidung der Klassenzuteilung einbezogen zu werden. So verwundert es auch nicht, dass sich in dieser Klasse gleich zwölf SchülerInnen befanden, die ihre schulische Laufbahn auf einem Lyzeum fortsetzen wollten (vgl. FB, Nr. 56). Es gab allerdings auch drei Lernende, die ein Schuljahr wiederholt hatten, eine davon zweimal. Dieser Schülerin war eine Lernbeeinträchtigung attestiert, weshalb sie eine Unterstützungslehrkraft hatte, die aber im Erdkundeunterricht nicht anwesend war. Sie selbst erschien nur unregelmäßig zum Unterricht.

In der Klasse gab es vier Einzelkinder; die meisten hatten einen Bruder oder eine Schwester und zwei Lernende zwei bzw. drei Geschwister. Neun hatten ein Einzelzimmer, und nur ein Schüler gab an, in seiner Wohnung keinen ruhigen Platz zum Lernen zu finden. Alle Kinder wohnten in Haushalten mit einem PC und Internetzugang, fünf von ihnen stand außerdem Lernsoftware zur Verfügung. In allen Haushalten gab es ein Wörterbuch, und nur drei wiesen darüber hinaus keine nützlichen Bücher zur Bewerkstelligung der 
Hausaufgaben auf (FB, Nr. 21). Dass der sozioökonomische Status der Familien höher als in den anderen bisher vorgestellten Klassen lag, ist daher zu vermuten, dass nur in dieser Klasse alle Familien ein oder mehrere Autos besaßen, fünf von ihnen eine Haushaltshilfe beschäftigten und im Gegensatz zu den anderen Klassen die meisten über eine Spülmaschine und eine Klimaanlage verfügten (ibid.). ${ }^{98}$

\subsubsection{Lesegewohnheiten}

Nach ihrem freizeitlichen Leseverhalten befragt, gab über ein Viertel der Lernenden an, dass es gar nicht zum eigenen Vergnügen las, und ein weiteres, dass dies maximal 30 Minuten am Tag geschah. Es handelt sich hier um vier von sechs Jungen und sechs von zwölf Mädchen (FB, Nr. 24). Unter den übrigen fünf Lernenden, die mindestens eine Stunde am Tag lasen, sind vier Mädchen. Sieben Lernenden wurde früher regelmäßig vorgelesen, dreien nie, fast nie, bzw. wurde keine Angabe dazu gemacht, und den übrigen gelegentlich (FB, Nr. 55).

Bei sieben SchülerInnen gab es maximal 25 Bücher im Elternhaus, bei vieren 26 bis 100 und bei sechsen zwischen 100 und 500 (vgl. FB, Nr. 23). Danach gefragt, welche Textsorten sie wie oft lasen, gaben die meisten an, am häufigsten Zeitschriften und ähnlich oft Belletristik zu lesen. Erstere wurden von sechs Lernenden mehrmals im Monat bzw. in der Woche und von einem gar nicht gelesen. Romane und Erzählungen lasen fünf Lernenden mehrmals in der Woche bzw. im Monat und drei Lernende gar nicht. Viele von ihnen (10) lasen auch keine Comics und 15 Sachbücher nie oder nur selten im Jahr. Zeitungen lasen diese Jugendlichen etwas öfter als ihre Altersgenossen der anderen Klassen, nämlich nur drei von ihnen nie und fünf mehrmals pro Monat bzw. pro Woche. Lediglich ein Schüler las alle Textsorten nie bzw. nicht mehr als ein paarmal im Jahr (FB, Nr. 26). In Tabelle 6 sind die Angaben der Lernenden auf einer Skala von 1 bis 5 (nie, manchmal pro Jahr, ca. einmal im Monat, mehrmals im Monat und mehrmals in der Woche) sowie die Durchschnittswerte und Gesamtpunktzahlen (P) wiedergegeben.

\begin{tabular}{lcccccccccccccccccccc}
\hline Wie oft liest du...? & $\mathrm{A}$ & $\mathrm{B}$ & $\mathrm{C}$ & $\mathrm{D}$ & $\mathrm{E}$ & $\mathrm{F}$ & $\mathrm{G}$ & $\mathrm{H}$ & $\mathrm{I}$ & $\mathrm{J}$ & $\mathrm{K}$ & $\mathrm{L}$ & $\mathrm{M}$ & $\mathrm{N}$ & $\mathrm{O}$ & $\mathrm{P}$ & $\mathrm{Q}$ & $\mathrm{R}$ & $\varnothing$ & $\mathrm{P}$ \\
\hline Zeitschriften & 4 & 3 & 5 & 4 & 4 & 4 & 3 & 2 & 2 & 2 & 4 & 2 & 1 & 3 & 3 & 3 & 2 & 3 & 3 & 54 \\
Comics & 4 & 1 & 1 & 1 & 1 & 1 & 1 & 2 & 1 & 2 & 3 & 2 & 1 & 1 & 2 & 4 & 1 & 2 & 2 & 31 \\
Belletristik (Romane, Er- & & & & & & & & & & & & & & & & & & & & \\
zählungen, Geschichten) & 3 & 2 & 3 & 4 & 2 & 3 & 5 & 3 & 4 & 3 & 3 & 5 & 3 & 2 & 1 & 5 & 1 & 1 & 3 & 53 \\
Sachbücher & 2 & 1 & 2 & 1 & 2 & 1 & 2 & 3 & 1 & 3 & 1 & 2 & 2 & 1 & 1 & 3 & 1 & 1 & 2 & 30 \\
Zeitungen & 5 & 3 & 4 & 2 & 2 & - & 2 & 2 & 1 & 1 & 3 & 2 & 1 & 3 & 4 & 4 & 2 & 5 & 3 & 46 \\
\hline
\end{tabular}

Tabelle 6: Lesegewohnheiten der Lernenden in der III A, Pestalozzi 
Durchschnittlich gaben die Lernenden zwar an, dass sie nicht nur dann lasen, wenn sie dazu aufgefordert wurden oder sich Informationen einholen wollten, und ferner, dass es nicht schwierig wäre, ein begonnenes Buch zu beenden oder mehr als ein paar Minuten still zu sitzen und zu lesen, aber das Lesen zählte auch nicht gerade zu ihren liebsten Freizeitbeschäftigungen. So freute sich bei einer Enthaltung die Hälfte der Lernenden (8) auch nicht, wenn sie ein Buch geschenkt bekam. Mehr als in den anderen bisher vorgestellten Klassen gefiel es ihnen aber, ihre Meinung zu dem, was sie lesen, zu äußern, und etwas mehr als die Hälfte (acht bei einer Enthaltung) tauschte ihre Bücher gerne mit Freunden (FB, Nr. 25).

Auch an der Schule Vespucci gab es eine Bücherei, welche die Lernenden zum Teil nutzten. Im Gegensatz dazu, wie es in der Klasse III B und an der Morante üblich war, bekamen die Lernenden in der III A allerdings keine Erlaubnis, die Bücherei während des Erdkundeunterrichts aufzusuchen. Fast die Hälfte der Lernenden (7) gab an, nie eine schulexterne Bücherei zu besuchen, und weitere sieben gingen nur ein paarmal im Jahr dorthin, obwohl sich im Einzugsgebiet der Schule eine Stadtbücherei befand. Am häufigsten wurden schulexterne Büchereien von drei Jungen aufgesucht, die das einmal im Monat machten (vgl. FB, Nr. 49). Das von den Lernenden durchschnittlich meistgenutzte Bibliotheksangebot (ca. einmal pro Monat) bestand im Internetzugang. Alle anderen Aktivitäten verfolgten sie im Mittelwert nur ein paarmal pro Jahr. Hierbei stand an erster Stelle das Lernen von Dingen, die nichts mit der Schule zu tun hatten, an zweiter das Bücherlesen, an dritter das Ausleihen zu schulischen Zwecken und mit fast derselben Häufigkeit das Ausleihen zur Freizeitgestaltung. Danach folgte das Lesen von Zeitungen und Zeitschriften und ganz zum Schluss das Hausaufgabenmachen. Nur je ein Lernender entlieh Bücher zur Freizeit- oder zur schulischen Lektüre mehrmals im Monat, alle anderen wesentlich seltener bzw. nie. Fünf Lernende gingen nie in die Bibliothek oder machten dies nur zur Internetnutzung oft und für alle anderen Beschäftigungen höchstens ein paarmal im Jahr (FB, Nr. 47).

Abgesehen von den Printmedien lasen die Lernenden auch im Internet. Von 90 möglichen Punkten für die größte Häufigkeit gaben auch sie gemeinsam am meisten dem Chatten (76 Punkte). Ebenfalls sehr oft nahmen sie an Gruppengesprächen in Foren oder auf Facebook teil (71 Punkte), sieben von ihnen sogar mehrmals am Tag. An dritter Stelle folgte mit einem gewissen Abstand das Suchen nach Informationen über ein bestimmtes Thema (61 Punkte). Sieben Lernende schrieben fast nie E-Mails, alle anderen häufiger und drei von ihnen sogar mehrmals am Tag. Am seltensten nutzen sie das Internet zum Konsultieren eines Wörterbuchs (44 Punkte). Selten erkundigten sie sich nach Veranstaltungsterminen und anderen Informationen praktischer Art sowie nach Einträgen in Online-Enzyklopädien. Insgesamt ein Drittel der Lernenden widmete sich all diesen Tätigkeiten im Internet durchschnittlich mehrmals wöchentlich, die anderen weniger, aber keiner seltener als mehrmals im Monat. Nur zwei 
Lernende kannten eine der Aktivitäten nicht: Einer wusste nicht, was ein Online-Wörterbuch und der andere nicht, was eine Enzyklopädie wie Wikipedia ist.

15 von 18 Schülerinnen und Schülern dieser Klasse gingen einer oder mehreren der vorgestellten Internettätigkeiten nie oder fast nie nach, aber nur eine Schülerin übte jede nie bzw. nur sehr selten aus. Alle anderen waren in einem bis drei Bereichen weniger aktiv. Die meisten Lernenden (10) benutzten nie oder nur selten ein Online-Wörterbuch, und auch E-Mails schrieben sieben äuBerst selten oder nie. Jeweils sechs Lernende lasen im Internet nie Nachrichten oder suchten nach Veranstaltungsterminen und ähnlichen Informationen praktischer Art. Es gibt keine Tätigkeit, der alle Lernenden mindestens ein paarmal pro Monat nachkamen, doch nur zwei von ihnen nutzten Online-Nachschlagewerke kaum, und eine Lernende suchte im Internet nie nach Informationen. Diese Lernende ist auch die einzige, die das Internet nur zum Pflegen sozialer Kontakte und nicht zum Lernen nutzte, während alle anderen zwar öfter in sozialen Netzwerken aktiv waren und chatteten, aber das Internet auch zu lernorientierten Zwecken nutzten (s. FB, Nr. 27).

\subsubsection{Einstellung zum Erdkundeunterricht und zur Lehrkraft}

Während der zwei Wochenstunden Erdkunde, die methodisch recht vielfältig gestaltet waren, herrsche nach Angabe der Lernenden Ruhe im Klassenzimmer (FB, Nr. 36), was sich mit den Beobachtungen des Unterrichts deckt. Darüber hinaus wurde die Mitarbeit der Lernenden als rege wahrgenommen. Wie an der Vespucci gab es aber auch hier einige Lernende, die mit Prüfungsangst kämpften, nervös waren und ihr Leistungspotential deshalb kaum ganz entfalten konnten. Bezüglich des Lernklimas sagten die Befragten ansonsten aus, dass es nur gelegentlich vorkam, dass die Klasse der Lehrkraft nicht zuhörte, es laut war, Unordnung herrschte und die Lehrerin lange warten musste, bevor Ruhe einkehrte. Nie oder fast nie sei die Lernatmosphäre so schlecht gewesen, dass sie nicht mehr in der Lage waren, gut zu arbeiten, oder dass sehr viel Zeit verging, bevor der eigentliche Unterricht beginnen konnte (s. FB, Nr. 36).

Auf die Frage, wie ihr der Unterricht gefiel, antwortete die Klasse recht positiv. Mit durchschnittlich 3,3 Punkten, denen die Wertung ,ganz gut" entspricht, liegt das Ergebnis zwar unter dem der beiden bisher vorgestellten Klassen, aber es bleibt zu berücksichtigen, dass die Antworten auf diese Frage höchst unterschiedlich ausfielen (FB, Nr. 53). Die Lernenden stimmten grundsätzlich darin überein, dass der Schulunterricht es weder versäume, eine Vorbereitung auf das Leben zu sein, noch Zeitverschwendung sei, auch wenn zwei Lernende sich durch ihn nicht ausreichend auf das Leben vorbereitet fühlten. Je 14 von 18 Lernenden hatten das Gefühl, dass der Unterricht ihnen dabei 
half, Entscheidungen bewusster zu treffen, und sie Dinge lernten, die im Berufsleben nützlich sein könnten (FB, Nr. 33).

Im Durchschnitt gab die gesamte Klasse an, sich gut mit den Lehrpersonen zu verstehen und von ihnen auch gut behandelt zu werden. Allerdings hatten sie gleichzeitig den Eindruck, dass die Lehrerinnen und Lehrer sich nicht besonders für sie und ihre Meinung interessierten und ihnen auch nicht genau zuhörten. Nur sieben Lernende bewerten das Interesse der Lehrkräfte positiver und neun empfanden im Unterrichtsgespräch die Aufmerksamkeit, die ihnen gewidmet wurde, als ausreichend (ibid., Nr. 34).

Konkret auf den Erdkundeunterricht bezogen, hielten sie diesen im Mittelwert für gleich vorbereitend auf ihr späteres Leben und geringfügig mehr für Zeitverschwendung als den Schulunterricht generell. Allerdings waren sie sich auch hier im Durchschnitt einig, dass er nützlich war. Nur sechs Lernende rechneten dem Erdkundeunterricht das Verdienst zu, das bewusstere Treffen von Entscheidungen zu schulen, und 13 Lernende gingen davon aus, dass sie die dort erworbenen Kenntnisse und Fähigkeiten im späteren Beruf anwenden könnten, so dass sie den Schulunterricht insgesamt betrachtet als förderlicher einschätzten (FB, Nr. 37). Während die Klasse Geographie als leichter bewertete als den Unterricht in Mathematik und Naturwissenschaften und als etwas leichter als den in Italienisch und Geschichte, hielten sie ihn für schwieriger als den Englisch- und Spanischunterricht (s. FB, Nr. 54).

Ihre Einschätzungen des Verhältnisses zur Lehrerin decken sich mit denen der anderen Klassen: Sie verstanden sich gut mit ihr und hatten das Gefühl, diese interessierte sich dafür, wie es ihnen ging. Außerdem hörte sie sich die Schülerbeiträge aufmerksam an und gab zusätzliche Unterstützung, sofern diese benötigt wurde. Somit bewerteten sie das Verhältnis zu Prof.ssa Rea insgesamt in allen Punkten besser als das zu ihren anderen Lehrkräften. Lediglich das Interesse für das Befinden der Lernenden stuften sie zwar als ausgeprägter, aber immer noch recht gering ein (FB, Nr. 39). ${ }^{99}$

Auf die offenen Fragen, was ihnen am Unterricht gut und was weniger gut gefiel oder was sie anders machen würden, antworteten viele Lernende, dass sie Prof.ssa Reas Art zu erklären mochten, da die Inhalte einfach zu verstehen waren (eine Lernende war derselben Auffassung, betonte aber, dass sie trotzdem nicht alles verstand), sie Beispiele lieferte und außerdem bekannt gab, was sie von ihnen in den Abfragungen erwartete. Manchen war das Unterrichtstempo zu schnell, die Menge an Hausaufgaben zu groß und das viele Lernen zu aufwendig (eine Schülerin störte es, dass sie, wenn sie etwas nicht verstand, nicht einfach die Seite des Lehrbuchs angeben durfte, sondern die genaue Textstelle nennen sollte). Auch das Anfertigen von geographischen Karten sowie das Schreiben von Zusammenfassungen mochten nicht alle (FB, Nr. 50-52).

99 Wie zuvor stehen U und LP für Erdkundeunterricht und Lehrperson und die Zahlenangaben entsprechen Werten auf einer Skala von 1 bis 4 (überhaupt nicht einverstanden, kaum einverstanden, einverstanden und vollkommen einverstanden). 
Während des Unterrichts fragte die Lehrerin oft nach der Bedeutung eines Textes. Hierbei stellte sie verständnisleitende Fragen, gab den Schülern aber eher selten ausreichend Zeit, um eine Antwort vorzubereiten. Ab und zu riet sie zur Lektüre eines bestimmten Autors oder eines Buchs, und oft waren die Lernenden aufgefordert, ihre Meinung zu einem Text zu äußern. Schriftliche Hausaufgaben verteilte sie fast immer, wobei es sich oft um eine Textzusammenfassung handelte. Dabei gelang es den Schülern nur manchmal, die Hausaufgaben mit geringem Zeitaufwand zu erledigen (vgl. FB, Nr. 38). Durchschnittlich benötigten sie dafür 1,5 Stunden. Die Klasse III A ist die einzige Klasse, in der beobachtet wurde, dass abgesehen von den Textzusammenfassungen noch verschiedene andere Arten von schriftlichen Hausaufgaben wie z.B. das Beantworten der Fragen und Übungen im Lehrwerk gestellt wurden.

Bei schriftlichen Abfragungen kontrollierte die Lehrperson, dass die Lernenden nicht voneinander abschrieben und informierte sie im Vorfeld der Tests manchmal, wie diese und eventuell auch die einzelnen Fragen oder Aufgaben bewertet wurden. Auch eine anschließende Besprechung des Tests fand nach Angaben der Lernenden nur gelegentlich statt. Sie wurden gefragt, ob alle Aufgaben klar waren, und es war ihnen erlaubt, Fragen zum Test zu stellen. Viele gaben an, manchmal das Gefühl zu haben, eine Antwort zu wissen, aber nicht in Worte fassen zu können. Sie fühlten sich ausreichend auf die Tests vorbereitet, die von der Lehrperson benotet wurden. Grundsätzlich musste eine Berichtigung angefertigt werden. Sie gaben zu, dass sie vor den Abfragungen nervös waren und sich unwohl fühlten. Dass es ihnen nicht leichter gefallen wäre, in einer anderen Sprache als Italienisch zu antworten, ergibt sich aus der Tatsache, dass sie fast ausnahmslos erstsprachig italienisch waren (FB, Nr. 40).

\subsubsection{Schülerangaben zum Lehrbuch}

Bezüglich der Qualität ihres Lehrwerks (Bastianelli \& Rancati 2010) hielten sie seine Aufmachung für ansprechend und betrachteten die Illustrationen als Verständnishilfe und Gedächtnisstütze. Die Übungen wären nützlich und die angemessen langen Texte leicht verständlich und klar geschrieben. Der verwendete Wortschatz entspräche ihrem Alter und ihren Fähigkeiten, wobei neue Fachtermini gut eingeführt würden (FB, Nr. 42).

Tabelle 7 führt diese Beurteilung der Lernenden auf $(\mathrm{LB}=$ Erdkundelehrbuch, Ws $=$ Wortschatz), wobei die Bewertungsskala wieder von 1 bis 4 mit ,überhaupt nicht einverstanden“, ,kaum einverstanden“, ,einverstanden“ und ,,vollkommen einverstanden" reicht.

\begin{tabular}{llllllllllllllllllll} 
Lernende & A & B & C & D & E & F & G & H & I & J & K & L & M & N & O & P & Q & R \\
\hline $\begin{array}{l}\text { LB bietet die Inhalte } \\
\begin{array}{l}\text { auf interessante } \\
\text { Weise dar. }\end{array}\end{array}$ & 3 & & 2 & 3 & 2 & 3 & 2 & 2 & 3 & 2 & 3 & 2 & 4 & 3 & 2 & 2 & 3 & 2 & 3 \\
\hline
\end{tabular}




\begin{tabular}{|c|c|c|c|c|c|c|c|c|c|c|c|c|c|c|c|c|c|c|}
\hline $\begin{array}{l}\text { Die Abbildungen } \\
\text { helfen mir, die In- } \\
\text { halte besser zu ver- } \\
\text { stehen und zu be- } \\
\text { halten. }\end{array}$ & 3 & 3 & 2 & 3 & 1 & - & 2 & 3 & 3 & 4 & 3 & 4 & 2 & 3 & 3 & 3 & 2 & 3 \\
\hline Die Texte sind leicht & & & & & & & & & & & & & & & & & & \\
\hline $\begin{array}{l}\text { zu verstehen. } \\
\text { Die Übungen im LB }\end{array}$ & 3 & 3 & 4 & 3 & 2 & 1 & 3 & 2 & 2 & 3 & 2 & 4 & 3 & 3 & 2 & 3 & 3 & 3 \\
\hline $\begin{array}{l}\text { sind nützlich. } \\
\text { Die Texte sind klar }\end{array}$ & 3 & 3 & 3 & 2 & 3 & 3 & 3 & 3 & 4 & 2 & 4 & 4 & 4 & 4 & 2 & 4 & 2 & 3 \\
\hline $\begin{array}{l}\text { formuliert. } \\
\text { Der verwendete Ws } \\
\text { ist meinem Alter und } \\
\text { meinen Fähigkeiten }\end{array}$ & 3 & 3 & 3 & 3 & 2 & 1 & 2 & 3 & 3 & 3 & 3 & 4 & 3 & 3 & 3 & 3 & 3 & 3 \\
\hline $\begin{array}{l}\text { angemessen. } \\
\text { Die Texte haben die }\end{array}$ & 3 & 3 & 3 & 3 & 3 & 1 & 3 & 2 & 3 & 3 & 3 & 4 & 3 & 4 & 3 & 3 & 3 & 3 \\
\hline & 3 & 3 & 3 & 2 & 3 & 2 & 2 & 2 & 2 & 4 & 3 & 3 & 3 & 3 & 3 & 3 & 3 & 3 \\
\hline $\begin{array}{l}\text { sind gut erklärt. } \\
\text { LB wäre in einer an- } \\
\text { deren Sprache als } \\
\text { Italienisch besser }\end{array}$ & 3 & 2 & 3 & 3 & 3 & 1 & 2 & 3 & 3 & 2 & 4 & 2 & 3 & 3 & 4 & 3 & 4 & 3 \\
\hline verständlich. & 1 & 1 & 2 & 1 & 2 & 1 & 1 & 1 & 1 & 1 & 1 & 1 & 1 & 1 & 1 & 1 & 1 & 1 \\
\hline
\end{tabular}

Tabelle 7: Bewertung des Erdkundelehrbuchs in der III A, Pestalozzi

Auf die Fragen, was ihnen am Lehrwerk besonders, bzw. gar nicht gefiel, antworten sie sehr unterschiedlich. Das Spektrum reicht von den Illustrationen, Photos und geographischen Karten über die Einführungen in ein neues Thema, die Vorstellung der geographischen Regionen und die Übungen bis zum verwendeten Wortschatz. Dennoch kritisierten sie gleichzeitig, dass die Texte zum Teil zu lang wären und der Wortschatz zu komplex (s. FB, Nr. 43 und 44). Die Lehrerin versuchte auch nur ab und zu, Berührungspunkte zwischen den Inhalten der Lehrbuchtexte und der Lebenswelt der Lernenden herzustellen, aber oft erläuterte sie, wie das Gelernte an zuvor erworbenes Wissen anknüpfte und Letzteres dadurch vertieft werden konnte (FB, Nr. 38).

\subsubsection{Lernstrategien}

Durchschnittlich hielten die Lernenden der Klasse III A an der Pestalozzi das Zusammenfassen mit eigenen Worten für die nützlichste Strategie zum Verstehen und Lernen der Lehrbuchtexte (83 von 108 möglichen Punkten). An zweiter Stelle sahen sie das Unterstreichen von Schlüsselwörtern und wichtigen Konzepten (77 Punkte). Danach folgten mit jeweils ca. 10 Punkten Abstand das anfängliche langsame Lesen des Textes und daran anschließend ein weiteres oder mehrmaliges schnelleres Lesen (67 Punkte) sowie das laute Vorlesen des Textes, während eine Person zuhört (66 Punkte). Mit deutlichem Abstand für am wenigsten hilfreich hielten sie die Strategie, den Text zweimal schnell zu lesen (33 Punkte). 
Zwei Lernende stuften drei der Techniken als wesentlich weniger nützlich ein als ihre Klassenkammeraden, und zwar sowohl das Zusammenfassen mit eigenen Worten als auch das erste langsame und dann wiederholende schnellere Lesen sowie das laute Vorlesen des Textes. Stattdessen erachteten sie es für am nützlichsten, sich auf die einfachen Abschnitte des Textes zu konzentrieren, womit sie sich klar von der Mehrheit abgrenzten. Denn neben diesen beiden Schülern gab es nur zwei weitere, welche diese Strategie auch sehr nützlich fanden, während die meisten sie zusammen mit dem zweimaligen schnellen Lesen von allen Strategien am schlechtesten bewerteten (s. FB, Nr. 41). Danach befragt, wie sie beim Lernen generell mit welcher Häufigkeit vorgingen, gaben sie an, durchschnittlich am meisten zu versuchen, sich die wichtigsten Aussagen eines Textes einzuprägen (56 von 72 möglichsten Punkten). Fast genauso oft waren sie bemüht, sich alle Informationen eines Textes zu merken (55 Punkte). An dritter Stelle folgten das mehrmalige Lesen eines Textes (52 Punkte) und der Versuch, sich an so viele Details wie möglich zu erinnern (50 Punkte). Den Text so oft zu lesen, bis sie ihn auswendig kannten, machten die Lernenden am seltensten (30 Punkte; nur eine Schülerin tat es oft und ein Schüler fast immer). Ebenfalls seltener versuchten sie, Konzepte mit ihren eigenen Erfahrungen zu verknüpfen, was nur drei Lernende oft und eine fast immer machten (36 Punkte).

Drei Lernende gaben an, dass sie beim Lernen alle Techniken anwandten, die meisten verzichteten auf eine bis zwei, und zwar vor allem auf das Auswendiglernen durch häufiges Lesen, das Bemerken von noch nicht verstandenen Konzepten und das Suchen zusätzlicher Informationen bei Unklarheiten. Nur vier Lernende wandten fünf bis acht der Techniken nie an. Die Schülerin mit der Lernbeeinträchtigung verfolgte die wenigsten Strategien.

Während ein Schüler beim Lernen alle Strategien und ganze neun davon immer einsetzte, nutzten die anderen im Durchschnitt nur zwei Strategien immer und acht von ihnen maximal eine (FB, Nr. 28). Tabelle 8 fasst die Angaben zusammen; die Zahlenwerte von 1 bis 4 stehen auf der Bewertungsskala für „fast nie“, „selten“, „oft“ und „,fast immer“.

\begin{tabular}{|c|c|c|c|c|c|c|c|c|c|c|c|c|c|c|c|c|c|c|c|c|}
\hline $\begin{array}{l}\text { Lernende/Während ich } \\
\text { lerne... }\end{array}$ & A & B & C & D & $E$ & $\mathrm{~F}$ & G & $\mathrm{H}$ & 1 & $\mathrm{~J}$ & $\mathrm{~K}$ & L & $M$ & $\mathrm{~N}$ & $\mathrm{O}$ & $P$ & $Q$ & $\mathrm{R}$ & $\varnothing$ & $P$ \\
\hline $\begin{array}{l}\text { versuche ich, alle Textinfor- } \\
\text { mationen zu behalten. } \\
\text { beginne ich damit zu überprü- } \\
\text { fen, was genau ich lernen }\end{array}$ & 3 & 3 & 3 & 3 & 4 & 3 & 2 & 3 & 2 & 3 & 3 & 4 & 4 & 2 & 3 & 3 & 3 & 4 & 3 & 55 \\
\hline $\begin{array}{l}\text { muss. } \\
\text { versuche ich, möglichst viele }\end{array}$ & 3 & 3 & 4 & 4 & 3 & - & 3 & 2 & 2 & 2 & 4 & 2 & 4 & 3 & 2 & 3 & 2 & 3 & 3 & 49 \\
\hline $\begin{array}{l}\text { Details zu behalten. } \\
\text { versuche ich, Inhalte mit dem } \\
\text { in anderen Fächern Gelern- }\end{array}$ & 4 & 3 & 3 & 2 & 4 & 2 & 1 & 3 & 1 & 3 & 2 & 4 & 3 & 2 & 3 & 3 & 3 & 4 & 3 & 50 \\
\hline $\begin{array}{l}\text { ten zu verknüpfen. } \\
\text { lese ich den Text so oft, dass }\end{array}$ & 2 & 2 & 2 & 2 & 4 & 1 & 2 & 3 & 2 & 4 & 2 & 1 & 3 & 2 & 2 & 3 & 2 & 2 & 2 & 41 \\
\hline $\begin{array}{l}\text { ich ihn auswendig kenne. } \\
\text { kontrolliere ich, ob ich das, }\end{array}$ & 2 & 2 & 1 & 2 & 3 & 1 & 1 & 2 & 1 & 1 & 1 & 2 & 4 & 1 & 2 & 2 & 1 & 1 & 2 & 30 \\
\hline was ich lese, verstehe. & 3 & 3 & 3 & 4 & 3 & 2 & 2 & 3 & 4 & 4 & 4 & 3 & 4 & 4 & 3 & 3 & 4 & 3 & 3 & 49 \\
\hline
\end{tabular}




\begin{tabular}{|c|c|c|c|c|c|c|c|c|c|c|c|c|c|c|c|c|c|c|c|c|}
\hline $\begin{array}{l}\text { lese ich den Text mehrmals. } \\
\text { frage ich mich, wie die Infor- } \\
\text { mationen außerhalb der }\end{array}$ & 2 & 3 & 2 & 3 & 4 & 2 & 3 & 3 & 3 & 3 & 3 & 3 & 4 & 3 & 2 & 3 & 3 & 3 & 3 & 52 \\
\hline $\begin{array}{l}\text { Schule nützlich sein könnten. } \\
\text { versuche ich, Konzepte zu } \\
\text { bemerken, die ich noch nicht }\end{array}$ & 2 & 4 & 2 & 3 & 4 & 1 & 2 & 2 & 1 & 2 & 2 & 1 & 4 & 2 & 3 & 3 & 3 & 2 & 2 & 43 \\
\hline $\begin{array}{l}\text { ganz verstanden habe. } \\
\text { versuche ich, die Inhalte bes- } \\
\text { ser zu verstehen, indem ich } \\
\text { sie mit meinen Erfahrungen in }\end{array}$ & 3 & 3 & 1 & 2 & 3 & 1 & 1 & 3 & 3 & 4 & 1 & 4 & 4 & 3 & 1 & 4 & 3 & 3 & 2 & 47 \\
\hline $\begin{array}{l}\text { Verbindung bringe. } \\
\text { merke ich mir die wichtigsten }\end{array}$ & 1 & 4 & 2 & 2 & 3 & 1 & 1 & 3 & 1 & 3 & 3 & 1 & 2 & 1 & 2 & 2 & 2 & 2 & 2 & 36 \\
\hline $\begin{array}{l}\text { Punkte des Textes. } \\
\text { frage ich mich, von welcher } \\
\text { Bedeutung die Textinfos für }\end{array}$ & 3 & 4 & 3 & 4 & 3 & 1 & 3 & 3 & 3 & 4 & 4 & 3 & 4 & 3 & 2 & 3 & 3 & 3 & 3 & 56 \\
\hline $\begin{array}{l}\text { das wirkliche Leben sind. } \\
\text { und merke, dass ich etwas } \\
\text { nicht verstehe, suche ich } \\
\text { nach zusätzlichen Informatio- }\end{array}$ & 2 & 4 & 3 & 3 & 4 & 1 & 1 & 2 & 4 & 3 & 3 & 1 & 4 & 2 & 4 & 2 & 3 & 3 & 3 & 49 \\
\hline nen. & 3 & 1 & 2 & 1 & 2 & 1 & 2 & 3 & 1 & 2 & 3 & 2 & 3 & 4 & 2 & 3 & 1 & 2 & 2 & 38 \\
\hline
\end{tabular}

Tabelle 8: Anwendung von Lernstrategien in der III A, Pestalozzi

Die Mehrheit der Lernenden (11) las beim Lernen laut, acht lasen leise und einer davon sowohl laut als auch leise. 13 wiederholten das Gelernte nur laut, drei nur leise und zwei auf beide Weisen. Elf machten sich beim Lernen keine Notizen und nur sechs schrieben Zusammenfassungen. Außerdem ließen sich zwei Drittel der Kinder (12) von jemandem abfragen. Im Vergleich zu den anderen Klassen gaben hier viele an, was sie zusätzlich noch taten: Neben Übungen und dem Anfertigen von Schaubildern trug eine Schülerin jemandem die Unterrichtsstunde wiederholend vor und ließ sich eine andere ihre Fehler und das, was sie noch nicht so gut verstanden hatte, erläutern (FB, Nr. 45).

\subsection{Klasse III B der Pestalozzi}

Die Klasse III B der Schule Pestalozzi war aus insgesamt 15 Schülerinnen und Schülern zusammengesetzt, von denen 14 den Fragebogen ausfüllten. Auch hier war die Mehrheit, nämlich zehn Lernende, italienischer Herkunft, und eine Schülerin hatte einen italienischen Vater und eine kolumbianische Mutter. Eine Lernende wies innerhalb der Familie einen polnischen und zwei einen ägyptischen Migrationshintergrund auf; es handelte sich bei zwei von ihnen um Zugewanderte der zweiten Generation. Eine Schülerin ägyptischer Herkunft wurde in Ägypten geboren, zog aber schon im Alter von zwei Jahren mit ihrer Familie nach Italien. So wurde in zwei Familien neben Italienisch auch Arabisch gesprochen, in einer Polnisch und in einer weiteren Spanisch (FB, Nr. 13, 14 und 16). Von vier Paaren hatten beide Elternteile einen akademischen Abschluss und von vier weiteren beide das Abitur. Hinzu kommen zwei 
Familien, in denen einer ein Studium absolviert hatte und drei, in denen einer das Abitur gemacht hatte. Die absolute Mehrheit in dieser Klasse (12) wollte nach der Mittelschule ein Lyzeum besuchen, nur zwei entschieden sich für ein technisches und ein berufsvorbereitendes Institut (FB, Nr. 56).

Drei Schülerinnen, die alle italienischer Herkunft waren, hatten das erste Jahr der Mittelschule wiederholt. Ein Schüler war lernbeeinträchtigt und wurde von einem sostegno-Lehrer unterstützt, allerdings nicht im Erdkundeunterricht. Dieser Schüler beantwortete nur einen Teil des Fragebogens.

Außer zwei Einzelkindern hatten alle ein bis drei Geschwister. Acht Lernende besaßen ein Einzelzimmer, und bei einer Enthaltung gaben alle an, zuhause einen ruhigen Platz zum Lernen und für die Hausaufgaben zu finden. Alle Haushalte verfügten über einen Computer, Internetzugang und ein Wörterbuch sowie weitere für die Schularbeit nützliche Bücher. Die Hälfte der SchülerInnen besaß außerdem Lernsoftware (FB, Nr. 21).

\subsubsection{Lesegewohnheiten}

Drei Lernende gaben an, in ihrer Freizeit nie zu lesen, einer maximal eine halbe Stunde, die meisten (8) zwischen dreißig Minuten und einer Stunde, und lediglich ein Schüler und eine Schülerin lasen ein bis zwei Stunden am Tag (FB, Nr. 23). Nur sechsen hatte man früher oft vorgelesen, den anderen selten oder nie (FB, Nr. 55). Ein Haushalt verfügte über maximal zehn Bücher, fünf weitere über maximal 100, zwei über 100 bis 200 und die anderen fünf über bis zu 500 Bücher (s. Abb. 3). In allen bis auf drei Haushalten war auch klassische Literatur vorhanden (FB, Nr. 21).

Dass Lesen Zeitverschwendung wäre, sie es nur taten, wenn sie mussten oder um bestimmte Informationen zu erhalten, dass es ihnen schwerfiel, länger als ein paar Minuten still zu sitzen und ein Buch zu lesen oder eines zu beenden, meinte der Durchschnitt der Befragten nicht. Allerdings war die Lektüre auch nicht ihre Lieblingsbeschäftigung, gingen sie weder sehr gerne in eine Buchhandlung, noch freuten sie sich, wenn sie Bücher geschenkt bekamen oder tauschten sie gern mit ihren Freunden. Interessanterweise gefiel es ihnen hingegen etwas mehr, ihre Meinung über das Gelesene mitzuteilen. Sieben Lernende lasen ihren Angaben zufolge jedoch recht bzw. sehr gerne. Darunter sind drei Mädchen und vier Jungen (FB, Nr. 25).

Danach befragt, welche Textsorten sie wie oft lasen, erklärten die Lernenden, dass sie durchschnittlich am häufigsten Comics lasen, nämlich einmal im Monat, wobei drei sie mehrmals wöchentlich und weitere drei sie mehrmals monatlich lasen. Fast genauso oft lasen sie im Durchschnitt Belletristik, Zeitschriften und Zeitungen, nur Sachbücher lasen sie seltener. Unter ihnen befanden sich acht, die alle Textsorten wenigstens ein paarmal im Jahr lasen, davon zwei alle Textsorten mindestens einmal im Monat. Jeweils drei gaben an, nie 
Zeitschriften und Zeitungen zu lesen, während die anderen Textsorten je nur von zweien nie gelesen wurden. Der lernbeeinträchtigte Schüler las außer Comics einmal pro Monat keine weiteren Printmedien. Auch ein anderer Schüler las alle Textsorten entweder nur ein paarmal pro Jahr oder maximal einmal pro Monat (FB, Nr. 26).

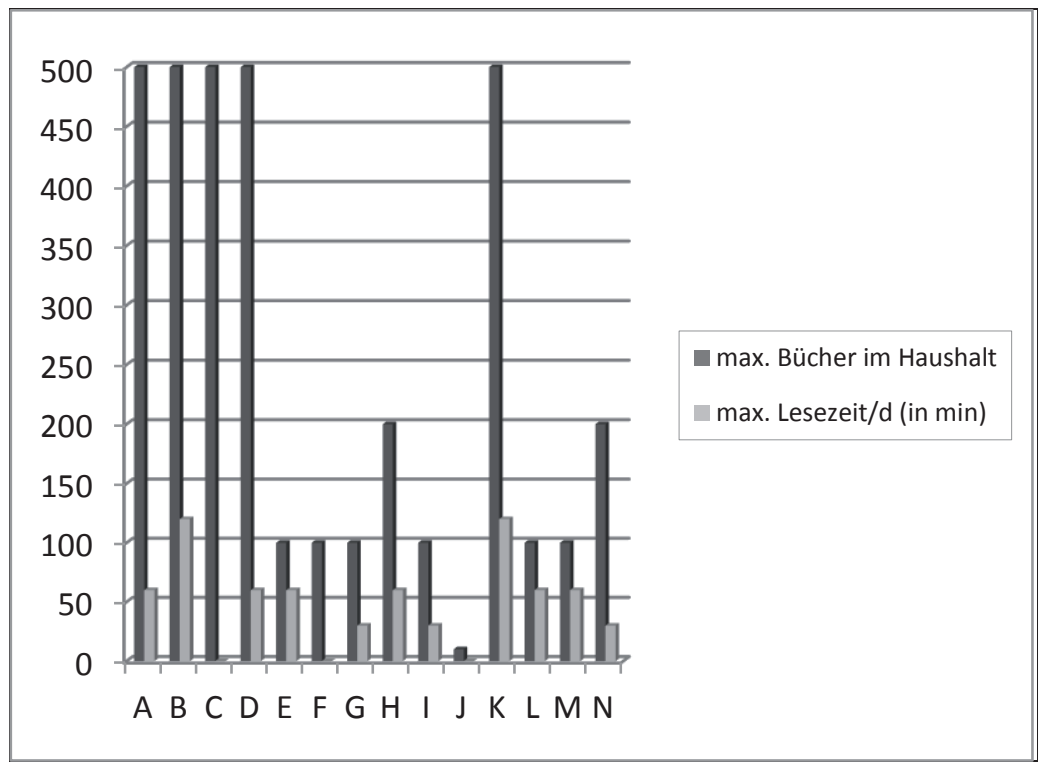

Abbildung 3: Maximale Bücherzahl im Haushalt und maximale tägliche Lesezeit (in min), III B, Pestalozzi

Was die Häufigkeit ihrer Lese- und Schreibaktivitäten im Internet betrifft, so waren die Lernenden dieser Klasse durchschnittlich am meisten an Gruppendiskussionen in Foren und sozialen Netzwerken beteiligt, nämlich mehrmals pro Woche. Gleich an zweiter Stelle folgte mit derselben durchschnittlichen Häufigkeit das Chatten. Ebenfalls mehrmals in der Woche suchten sie im Internet auch nach Informationen über ein bestimmtes Thema oder praktischer Art wie Veranstaltungsterminen. Den anderen vier Aktivitäten, also dem Konsultieren von Wörterbüchern und Nachschlagewerken, dem E-Mailschreiben und dem Nachrichtenlesen, kamen sie durchschnittlich etwas seltener nach.

Während ein Schüler angab, mehrmals am Tag in all diesen Bereichen im Internet aktiv zu sein, und der lernbeeinträchtigte Schüler nur einmal pro Monat, machten alle anderen maximal zwei der acht Dinge nur einmal monatlich (FB, Nr. 27).

Mit neun gaben die meisten Befragten an, nie eine Bücherei außerhalb der Schule zu besuchen, einer ein paarmal im Jahr und weitere drei nur ca. einmal 
im Monat. Lediglich eine Schülerin ging mehrmals wöchentlich dorthin (FB, Nr. 49). Durchschnittlich suchten die Lernenden am häufigsten (mehrmals im Monat) eine Bibliothek auf, um dort den Internetzugang zu nutzen und Dinge zu lernen, die nichts mit der Schule zu tun hatten. Einmal im Monat hingegen entliehen sie Bücher für die Freizeit, erledigten dort ihre Hausaufgaben oder lernten für die Schule und lasen Bücher, Zeitschriften und Zeitungen. Der lernbeeinträchtigte Schüler gab an, nie eine Bücherei aufzusuchen, und eine weitere Schülerin nur, um dort ein paarmal im Jahr Bücher auszuleihen. Zwei Schülerinnen und ein Schüler hingegen nutzten die Bücherei zu all diesen Zwecken wenigstens einige Male im Monat (FB, Nr. 47).

\subsubsection{Einstellung zum Erdkundeunterricht und zur Lehrkraft}

Von allen beobachteten Klassen der Schule Pestalozzi gefiel dieser Klasse der Erdkundeunterricht im Vergleich zu den anderen Fächern mit einer Benotung von 4,5, die ,sehr“ entspricht, am besten. Dabei stuften sie das Fach im Vergleich zu den anderen Schulfächern als leichter ein als alle anderen Klassen, die an der Umfrage teilnahmen. Im Einzelnen hielten sie es für etwas leichter als Mathematik und Englisch und für deutlich leichter als Naturwissenschaften, Italienisch, Geschichte und Spanisch (vgl. FB, Nr. 53 f.).

Ihre Bewertung der Nützlichkeit des Erdkundeunterrichts stimmt mit der in der Klasse der Vespucci überein: Sie waren der Auffassung, dass der Unterricht sie auf ihr Leben nach der Schule vorbereitete und keine Zeitverschwendung war. Im Gegenteil, Geographie half ihnen, Entscheidungen bewusster zu treffen, und vermittelte ihnen Inhalte, die im Berufsleben von Bedeutung sein könnten (s. FB, Nr. 37).

Bezüglich ihrer generellen Einstellung zum schulischen Unterricht und den Lehrkräften ist den Antworten des Fragebogens zu entnehmen, dass sich die Lernenden grundsätzlich mit ihren Lehrkräften verstanden, sich von ihnen gut behandelt fühlten und zusätzliche Förderung erhielten, wenn sie benötigt wurde. Auch diese Klasse hatte aber weniger das Gefühl, die Lehrkräfte interessierten sich für sie und hörten ihnen aufmerksam zu (s. FB, Nr. 34). Die Bewertung des Verhältnisses zur Erdkundelehrkraft war zwar nicht positiver, aber die Lernenden sprachen ihr zu, sich ein wenig mehr für sie zu interessieren und ihnen zuzuhören. Außerdem habe sie ihnen auch mehr zusätzliche Unterstützung zukommen lassen (s. FB, Nr. 39).

\begin{tabular}{|c|c|c|c|c|c|c|c|c|c|c|c|c|c|c|c|}
\hline Lernende & A & B & C & D & $\mathrm{E}$ & $\mathrm{F}$ & G & $\mathrm{H}$ & I & $\mathrm{J}$ & $\mathrm{K}$ & $\mathrm{L}$ & M & $\mathrm{N}$ & $\varnothing, P$ \\
\hline $\begin{array}{l}U \text { ist eine gute Vorbe- } \\
\text { reitung auf das Leben. } \\
U \text { ist Zeitverschwen- }\end{array}$ & 2 & 1 & 3 & 2 & 3 & 1 & 1 & 2 & 2 & 3 & 1 & 2 & 1 & 2 & $2(26)$ \\
\hline $\begin{array}{l}\text { dung. } \\
\text { U hilft, Entscheidungen }\end{array}$ & 2 & 1 & 3 & 2 & 2 & 2 & 2 & 2 & 2 & 2 & 1 & 1 & 2 & 2 & $2(26)$ \\
\hline bewusster zu treffen. & 1 & 2 & 1 & 2 & 2 & 2 & 3 & 2 & 3 & 2 & 2 & 3 & 3 & 2 & $2(30)$ \\
\hline
\end{tabular}




\begin{tabular}{|c|c|c|c|c|c|c|c|c|c|c|c|c|c|c|c|}
\hline $\begin{array}{l}\text { U vermittelt Inhalte, die } \\
\text { im Berufsleben nützlich }\end{array}$ & & & & & & & & & & & & & & & \\
\hline $\begin{array}{l}\text { sein könnten. } \\
\text { Ich verstehe mich gut }\end{array}$ & 2 & 4 & 2 & 3 & 2 & 4 & 3 & 3 & 3 & 2 & 3 & 3 & 3 & 1 & $3(38)$ \\
\hline $\begin{array}{l}\text { mit der LP. } \\
\text { LP interessiert, wie es }\end{array}$ & 4 & 3 & 3 & 3 & 3 & 3 & 3 & 3 & 3 & 3 & 3 & 3 & 3 & 3 & $3(43)$ \\
\hline $\begin{array}{l}\text { mir geht. } \\
\text { LP hört wirklich zu, was }\end{array}$ & 4 & 3 & 3 & 3 & 3 & 1 & 1 & 3 & 3 & 3 & 3 & 3 & 3 & 3 & $3(39)$ \\
\hline $\begin{array}{l}\text { ich zu sagen habe. } \\
\text { Wenn ich zusätzliche } \\
\text { Hilfe benötige, be- } \\
\text { komme ich sie von der }\end{array}$ & 4 & 3 & 3 & 3 & 3 & 2 & 2 & 3 & 3 & 3 & 2 & 3 & 3 & 2 & $3(39)$ \\
\hline LP. & 4 & 3 & 3 & 3 & 3 & 3 & 3 & 3 & 3 & 3 & 3 & 3 & 2 & 4 & $3(43)$ \\
\hline LP behandelt mich gut. & 4 & 3 & 3 & 3 & 3 & 3 & 3 & 3 & 3 & 3 & 3 & 3 & 3 & 3 & $3(43)$ \\
\hline
\end{tabular}

Tabelle 9: Einstellung zum Erdkundeunterricht und zur unterrichtenden Lehrkraft, Klasse III B, Pestalozzi

Am Erdkundeunterricht mochten viele Lernende die Art der Lehrkraft, die Inhalte zu erklären, und dass sie nicht müde wurde, bereits Behandeltes erneut $\mathrm{zu}$ erklären. Einige hätten lieber öfter Exkursionen unternommen und viele fanden, dass der Unterricht zu sehr aus Abfragungen bestand (FB, Nr. 50-52). Der Erdkundeunterricht bereitete sie geringfügig besser auf ihr Leben vor als der restliche Unterricht und wurde von ihnen genauso wenig als Zeitverschwendung betrachtet, doch gleichzeitig trug er etwas weniger zum bewussten Treffen von Entscheidungen und zur Vorbereitung auf die Berufswelt bei (FB, Nr. 37).

Den Lernenden war bewusst, dass sie Prof.ssa Cese gelegentlich nicht zuhörten und Lärm und Chaos herrschten, so dass man nicht gut arbeiten konnte. Die Lehrkraft habe manchmal lange warten müssen, bevor Ruhe einkehrte und auch, bevor mit dem eigentlichen Unterricht begonnen werden konnte. In dieser Hinsicht decken sich die Angaben mit den gemachten Beobachtungen. So zeigten neben Prof.ssa Cese auch verschiedene andere Lehrpersonen gelegentlich Schwierigkeiten, die Klasse zum konzentrierten Mitarbeiten zu bewegen, und der Erdkundeunterricht wurde so gut wie nie pünktlich begonnen. Prof.ssa Cese erklärte die Lebhaftigkeit ihrer Schülerinnen und Schüler, die sie seit dem ersten Jahr der Mittelschule begleitete, damit, dass die Klasse gerade, etwas spät und dadurch umso heftiger, einen pubertären Entwicklungsschub erfuhr. Der habe andererseits dafür gesorgt, dass die Lernenden nun damit begannen, sich für gesellschaftliche und politische Fragen zu interessieren und eine eigene Meinung zu diesen Themen zu entwickeln und zu äußern.

Die Erdkundestunden vergingen überwiegend damit, dass die Lernenden in zwei Zweiergruppen abgefragt wurden, wozu sie am Lehrerpult standen. Diese Abfragungen konnten sich auch bis in die Pause erstrecken. Gegen Ende der Stunde wurden neue Themen behandelt oder das Erschließen neuer Texte als Hausaufgabe aufgetragen (FB, Nr. 36). Hierzu wurden die Lernenden in der nächsten Stunde abgefragt, wobei diese Form der Leistungskontrolle u.a. auch 
dazu diente, Inhalte, die noch nicht so gut verstanden wurden, besser zu erklären und zu vertiefen sowie auf Fragen der SchülerInnen einzugehen. Während es in der Klasse III A der Pestalozzi und auch in der Klasse an der Vespucci während der Abfragungen im Klassenzimmer still war, herrschte hier ähnlich wie an der Schule Morante recht große Unruhe, und nur ein Teil der Klasse erweckte den Eindruck, das Unterrichtsgeschehen mitzuverfolgen. Dadurch war es in den ersten Bankreihen auch besser möglich, den sich am Pult abspielenden Abfragungen zu folgen als von den hinteren Reihen aus.

Während des Unterrichts kam es nach Angaben der Befragten oft vor, dass die Lehrkraft Fragen zum Text stellte, um das Verständnis zu erleichtern, und dass sie ihre Schüler bat, die Bedeutung eines Textes zu erklären sowie ihre Meinung dazu zu äußern. Generell hätten sie genug Zeit gehabt, eine Antwort vorzubereiten, und auch wenn die Lehrerin nur gelegentlich darauf verwiesen habe, wie behandelte Inhalte mit der persönlichen Lebenswelt der Jugendlichen zusammenhingen, erklärte sie doch, wie neu erworbenes Wissen mit schon Bekanntem verbunden und dadurch vertieft werden konnte. Desweiteren formulierte sie Leseempfehlungen und gab fast immer schriftliche Hausaufgaben, wobei es sich in der Regel um die Zusammenfassung eines Textes handelte (vgl. FB, Nr. 38).

\subsubsection{Schülerangaben zum Lehrbuch}

In dieser Klasse gab es im Fach Erdkunde keine schriftlichen Leistungskontrollen, wodurch sich das große Gewicht der mündlichen Überprüfungen in der Unterrichtsgestaltung zum Teil erklären lässt. Dadurch gewann für die Lernenden das Lehrwerk noch an Bedeutung, da sie die Abfragungen mit dem Lesen und Lernen seiner Texte vorbereiteten. Ihre Bewertung des Buches (Dinucci \& Dinucci 2008) fiel fast genauso positiv aus wie in der Klasse III A für das dort benutzte Lehrwerk: Die Darbietung der Inhalte sei interessant, die Bilder verständnisfördernd und die angemessen langen und klar geschriebenen Texte enthielten einen für ihr Alter und Können geeigneten Wortschatz, wobei neue Fachwörter gut eingeführt würden. Nur die Übungen unterstützten das Lernen nicht (vgl. FB, Nr. 42).

\begin{tabular}{|c|c|c|c|c|c|c|c|c|c|c|c|c|c|c|}
\hline Lernende & A & B & C & D & $\mathrm{E}$ & $\mathrm{F}$ & G & $\mathrm{H}$ & I & $\mathrm{J}$ & $\mathrm{K}$ & L & M & $\mathrm{N}$ \\
\hline $\begin{array}{l}\text { LB bietet die Inhalte auf inte- } \\
\text { ressante Weise dar. } \\
\text { Die Abbildungen helfen mir, die }\end{array}$ & 3 & 3 & 3 & 3 & 2 & 4 & 3 & 2 & 3 & 1 & 3 & 3 & 3 & 2 \\
\hline $\begin{array}{l}\text { Inhalte besser zu verstehen } \\
\text { und zu behalten. } \\
\text { Die Texte sind leicht zu verste- }\end{array}$ & 3 & 3 & 3 & 3 & 3 & 3 & 3 & 3 & 3 & 3 & 3 & 2 & 2 & 2 \\
\hline $\begin{array}{l}\text { hen. } \\
\text { Die Übungen im LB sind nütz- }\end{array}$ & 3 & 3 & 3 & 3 & 3 & 3 & 3 & 3 & 3 & 2 & 3 & 4 & 2 & 3 \\
\hline lich. & 3 & 2 & 2 & 2 & 2 & 3 & 3 & 3 & 2 & 1 & 3 & 1 & 3 & 2 \\
\hline Die Texte sind klar formuliert. & 3 & 3 & 3 & 3 & 3 & 3 & 3 & 3 & 3 & 4 & 3 & 3 & 23 & 1 \\
\hline
\end{tabular}




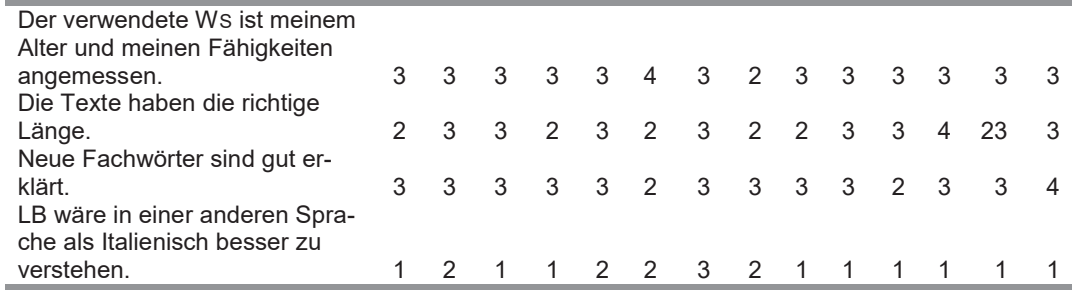

Tabelle 10: Bewertung des Erdkundelehrbuchs in der III B, Pestalozzi

Wenn man diese Bewertungen mit den Antworten auf die Frage, was ihnen am Buch konkret gefiel und was nicht, vergleicht, zeigt sich wieder ein leicht differenziertes Bild, indem sie als positiv fast ausschließlich die Illustrationen hervorhoben, die Texte hingegen oft als zu lang bewerteten und z.T. außerdem die geschichtlichen und vertiefenden Texte nicht mochten (s. FB, Nr. 43 f.).

\subsubsection{Lernstrategien}

Danach befragt, wie sie sich im Fach Erdkunde die Lehrbuchtexte aneigneten, resultiert aus den Antworten, dass sie es durchschnittlich am nützlichsten fanden, Schlüsselwörter und wichtige Konzepte zu unterstreichen (67 von 84 möglichen Punkten), wofür die meisten Höchstwerte von fünf bzw. sechs Punkten verteilten (Skala von 1 bis 6), während ein Schüler dies überhaupt nicht hilfreich fand. An zweiter Stelle folgte das Zusammenfassen mit eigenen Worten (63 Punkte), wofür alle mindestens mittlere Punktzahlen (3) vergaben und drei die maximale Punktzahl (6). Mit etwas Abstand folgte an dritter Stelle ein erstes gründliches und ein nachfolgendes schnelleres Lesen des Textes (54 Punkte).

Am wenigsten förderlich beurteilten sie mit deutlichem Abstand zu allen anderen Strategien das schnelle zweimalige Lesen des Textes (28 Punkte), obgleich ein Schüler dafür fünf Punkte vergab. Geteilter Meinung waren sie bei der Ergiebigkeit des lauten Vorlesens, während eine andere Person zuhörte: Acht Lernenden war diese Strategie maximal drei Punkte wert, den anderen mehr und zum Teil sogar die Höchstpunktzahl.

Mehr als die Hälfte der Klasse (8) vergab für keine Strategie den maximalen Wert; von den übrigen hielten drei vier bzw. drei Strategien für besonders effektiv. Hierbei ist sechsen gemein, dass sie das Zusammenfassen für äußerst nützlich hielten. Während die Hälfte auf der anderen Seite auch keine Minimalpunktzahl von 1 vergab, bewertete ein Schüler vier Strategien mit 1 und eine weitere Schülerin drei. Lediglich für das Zusammenfassen und die Kon- 
zentration auf die leicht verständlichen Abschnitte vergab niemand den niedrigsten Wert. Abgesehen von dreien hielten es alle anderen auch für förderlich, nach dem Lesen mit jemandem über den Text zu sprechen (FB, Nr. 41). ${ }^{100}$

\begin{tabular}{|c|c|c|c|c|c|c|c|c|c|c|c|c|c|c|c|}
\hline Während ich lerne... & $A B$ & B & C & D & $\mathrm{E}$ & $\mathrm{E}$ & $\mathrm{F}$ & G & $\mathrm{H}$ & 1 & $\mathrm{~J}$ & $\mathrm{~K}$ & $\mathrm{~N}$ & $\varnothing$ & $\mathrm{P}$ \\
\hline versuche ich, alle Textinformationen zu & & & & & & & & & & & & & & & \\
\hline $\begin{array}{l}\text { behalten. } \\
\text { beginne ich damit zu überprüfen, was ge- }\end{array}$ & 3 & 3 & 3 & 3 & 3 & 3 & 1 & 3 & 2 & 2 & 3 & 3 & 3 & 3 & 38 \\
\hline $\begin{array}{l}\text { nau ich lernen muss. } \\
\text { versuche ich, möglichst viele Details zu }\end{array}$ & 3 & 3 & 3 & 3 & 3 & 4 & 3 & 4 & 2 & 2 & 4 & 3 & 2 & 2 & 41 \\
\hline $\begin{array}{l}\text { behalten. } \\
\text { versuche ich, Inhalte mit dem in anderen }\end{array}$ & 3 & - & 3 & 2 & 3 & 2 & 1 & 4 & 3 & 3 & 3 & 3 & 4 & 3 & 37 \\
\hline $\begin{array}{l}\text { Fächern Gelernten zu verknüpfen. } \\
\text { lese ich den Text so oft, dass ich inn aus- }\end{array}$ & 2 & 2 & 3 & 4 & 2 & 3 & 2 & 2 & 4 & 3 & 3 & 3 & 4 & 2 & 39 \\
\hline & 12 & 2 & 2 & 1 & 2 & 1 & 2 & 2 & 2 & 1 & 1 & 2 & 3 & 1 & 23 \\
\hline verstehe. & 4 & 4 & 3 & 3 & 3 & 3 & 3 & 3 & 4 & 3 & 4 & 3 & 4 & 4 & 48 \\
\hline $\begin{array}{l}\text { lese ich den Text mehrmals. } \\
\text { frage ich mich, wie die Informationen au- }\end{array}$ & 42 & 2 & 3 & 2 & 3 & 2 & 3 & 2 & 3 & 1 & 3 & 2 & 4 & 3 & 37 \\
\hline $\begin{array}{l}\text { Berhalb der Schule nützlich sein könnten. } \\
\text { versuche ich, Konzepte zu bemerken, die }\end{array}$ & 43 & 3 & 3 & 1 & 2 & 2 & 3 & 1 & 2 & 3 & 1 & 2 & 4 & 3 & 34 \\
\hline $\begin{array}{l}\text { ich noch nicht ganz verstanden habe. } \\
\text { versuche ich, die Inhalte besser zu ver- }\end{array}$ & 23 & 3 & 3 & 1 & 3 & 2 & 3 & 3 & 4 & 2 & 2 & 2 & 3 & 4 & 37 \\
\hline $\begin{array}{l}\text { stehen, indem ich sie mit meinen Erfah- } \\
\text { rungen in Verbindung bringe. } \\
\text { merke ich mir die wichtigsten Punkte des }\end{array}$ & 22 & 2 & 2 & 2 & 2 & 2 & 2 & 2 & 2 & 1 & 2 & 2 & 3 & 3 & 29 \\
\hline Textes. & 33 & 3 & 3 & 3 & 3 & 4 & 3 & 2 & 3 & 1 & 4 & 3 & 3 & 2 & 40 \\
\hline $\begin{array}{l}\text { sind. } \\
\text { und merke, dass ich etwas nicht ver- }\end{array}$ & 13 & 3 & 2 & 2 & 3 & 3 & 4 & 3 & 2 & 1 & 2 & 3 & 4 & 4 & 37 \\
\hline $\begin{array}{l}\text { stehe, such } \\
\text { mationen. }\end{array}$ & 13 & 3 & 2 & 3 & 3 & 2 & 3 & 2 & 2 & 2 & 2 & 3 & 3 & 2 & 33 \\
\hline
\end{tabular}

Tabelle 11: Anwendung von Lernstrategien in der Klasse III B, Pestalozzi

Was ihr generelles Vorgehen beim Lernen anbelangt, so gaben alle Lernenden an, dass sie oft oder immer kontrollierten, ob sie das, was sie lasen, auch verstanden. Ähnlich sieht es mit der Technik aus, das Lernen mit einer Kontrolle dessen zu beginnen, was genau eigentlich gelernt werden sollte. Lediglich vier SchülerInnen gaben hierfür an, es nur selten zu tun. Die meisten versuchten oft, die wichtigsten Textaussagen zu behalten; nur einer fast nie und zwei weitere selten. Ebenfalls oft bedienten sie sich mit absteigender durchschnittlicher Frequenz der Techniken, das Gelernte möglichst mit dem in anderen Fächern erworbenen Wissen zu verbinden, alle Textinformationen zu behalten, so viele Details wie möglich zu behalten, den Text mehrmals zu lesen, zu bemerken, wenn Konzepte nicht verstanden wurden, und sich nach der Bedeutung des Gelernten für das wahre Leben zu fragen.

100 Die Skala in Tabelle 11 reicht von 1 bis 4: „fast nie“, „selten“, „oft“ und „fast immer“. 
Im Mittelwert selten hingegen verfolgten die Lernenden mit abnehmender Häufigkeit die Strategien, sich nach der außerschulischen Nützlichkeit des Gelernten zu fragen, das Gelernte mit eigenen Erfahrungen zu verbinden und den Text so oft zu lesen, bis sie ihn auswendig kannten. Wobei ein Schüler angab, Letzteres oft zu tun.

Sechs der 14 SchülerInnen verwendeten alle Techniken mit einer gewissen Häufigkeit; gleichzeitig gaben vier an, keine einzige immer anzuwenden. Dagegen verzichteten vier auf drei bis sechs der Techniken nie (FB, Nr. 28). Zehn lasen beim Lernen laut und fünf leise, eine von ihnen sowohl laut als auch leise. Bis auf zwei wiederholten alle den Textinhalt laut, wobei zwei das teils laut, teils leise machten. Die Mehrheit (10) fertigte außerdem beim Lernen Notizen an, und alle bis auf einen schrieben Zusammenfassungen der Texte. Nur fünf SchülerInnen gaben außerdem an, sich von jemandem abfragen zu lassen (FB, Nr. 45).

\subsection{Klasse III C der Pestalozzi}

Die letzte Klasse, die an der Studie beteiligt war, die Klasse III C der Pestalozzi, bestand aus 21 Schülern, von denen 19 den Fragebogen beantworteten. Die Eltern von 13 dieser Lernenden waren Italiener; drei Schülerinnen und zwei Schüler hatten Eltern peruanischer, bengalischer und rumänischer Herkunft. Zwei Lernende wurden in Rumänien geboren und kamen im Alter von vier bzw. zwei Jahren nach Italien. Ein Schüler übersiedelte als Säugling mit seiner Familie von Bangladesch nach Italien. Außerdem gehörten zwei peruanische Schwestern zur Klasse, von denen einer eine Lernbeeinträchtigung attestiert war. Ihr stand eine Unterstützungslehrkraft zur Seite, aber nicht im Fach Erdkunde.

Nur in zwei Familien war die am meisten gesprochenen Sprache nach Angaben der Lernenden nicht Italienisch, sondern Spanisch bzw. Rumänisch. In drei weiteren Familien wurde neben dem Italienischen auch Rumänisch und Bengalisch gesprochen (vgl. FB, Nr. 14-16).

Die Bildungsabschlüsse der Eltern waren mit nur einem Elternpaar mit einem Hochschulabschluss und zwei weiteren Elternteilen mit einem solchen sowie fünf Elternpaaren und acht Elternteilen mit dem Abitur die niedrigsten aller beobachteten Klassen der Pestalozzischule (jedoch höher als die der Eltern der Vespucci), und auch die Anzahl der Lernenden, welche die Schule an einem Lyzeum fortsetzen wollten, war mit acht am geringsten (s. FB, Nr. 9, 13 und 56). 


\subsubsection{Lesegewohnheiten}

Bezüglich des Bücherbestands in den Elternhäusern und des Leseverhaltens der Lernenden lässt sich festhalten, dass in knapp einem Viertel aller Haushalte maximal 25 Bücher und in weiteren neun zwischen 26 und 200 vorhanden waren. Allerdings handelte es sich gleichzeitig um die einzige Klasse mit Haushalten, in denen sich mehr als 500 Bücher befanden (3). Fast die Hälfte aller Lernenden gab an, nie zum persönlichen Vergnügen zu lesen und weitere fünf nur maximal 30 Minuten am Tag (vgl. FB, Nr. 23 f.). Lediglich dreien wurde früher regelmäßig vorgelesen, weiteren neun immerhin gelegentlich ( $\mathrm{FB}$, Nr. 55).

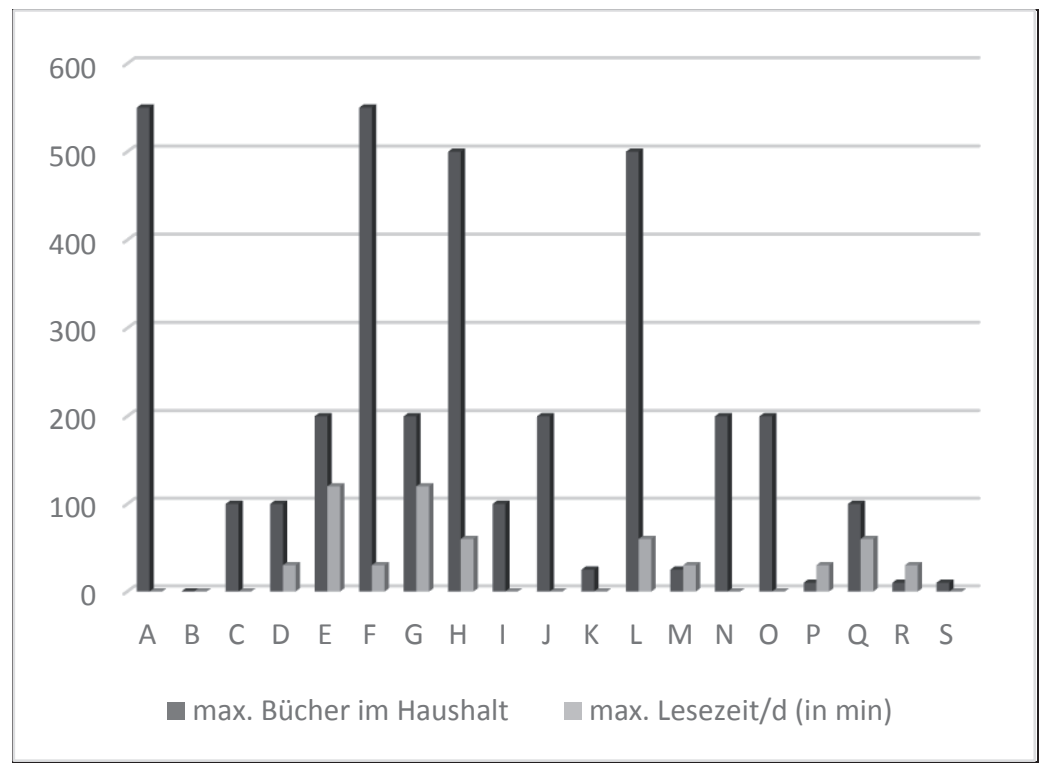

Abbildung 4: Maximale Bücherzahl im Haushalt und maximale tägliche Lesezeit (in min), III C, Pestalozzi

Diese Klasse stimmte durchschnittlich vor allem darin überein, nur zu lesen, um Informationen zu erhalten, die sie benötigte, und wenn sie dazu aufgefordert wurde. Außerdem fiel es vielen Lernenden schwer, ein Buch zu beenden und überhaupt länger als ein paar Minuten still zu sitzen und zu lesen. Lediglich zwei stimmten Letzterem überhaupt nicht zu. Auf der anderen Seite betrachteten vier Schülerinnen das Lesen als eine bevorzugte Freizeitbeschäftigung.

13 Befragte stimmten der Aussage, gerne mit jemandem über das, was sie lasen, zu reden, überhaupt nicht oder kaum zu. Etwas weniger als die Hälfte 
teilte gerne ihre Meinung über das Gelesene mit, aber nur vier Schülerinnen mochten es, Bücher mit Freunden zu tauschen (FB, Nr. 25).

Am häufigsten lasen die Kinder dieser Klasse Comics, nämlich durchschnittlich einmal im Monat, wobei sechs sie mehrmals monatlich bzw. wöchentlich lasen und zwei Mädchen sowie ein Junge nie. An zweiter Stelle folgten Zeitschriften und Romane sowie Erzählungen. Zwei Mädchen lasen mehrmals pro Woche Zeitschriften und fünf Lernende nie. Belletristik wurde von drei Lernenden, allesamt Mädchen, nie gelesen. Sachbücher wurden im Mittelwert nur wenige Male im Jahr, von neun Lernenden gar nicht und nur von einem Schüler wesentlich öfter (mehrmals pro Monat) gelesen. Ferner gab über ein Drittel (7) an, nie Zeitung zu lesen, indessen je drei Lernende mehrmals pro Woche bzw. mehrmals im Monat.

Den Angaben der SchülerInnen zufolge lasen drei Mädchen jeweils nur eine Textsorte: eine nur Zeitschriften (mehrmals wöchentlich) und die anderen beiden nur Comics (mehrmals pro Woche und einige Male im Jahr). Mit Abstand am meisten las ein Mädchen, das Zeitschriften, Zeitungen und Comics mehrmals in der Woche, Belletristik mehrmals im Monat und Sachbücher ungefähr einmal im Monat las (FB, Nr. 26).

Ähnlich wie in den anderen Klassen waren auch hier die Lese- und Schreibaktivitäten der Lernenden im Internet etwas ausgeprägter: Im Mittelwert am häufigsten, nämlich mehrmals in der Woche, chatteten sie und suchten in Online-Nachschlagewerken nach Informationen. Insgesamt ähnlich oft, aber durchschnittlich nur mehrmals im Monat, nahmen sie an Diskussionen in Internetforen teil. Fünf Lernende machten das täglich, wiederum fünf hingegen nur ein paarmal im Jahr, eine Schülerin nie, und eine weitere wusste gar nicht, was das ist. Sechs der Internetaktivitäten ging diese Klasse durchschnittlich ca. einmal im Monat nach, und zwar mit absteigender Frequenz dem Suchen von Informationen über ein bestimmtes Thema, dem Suchen nach praktischen Informationen, dem Nachrichtenlesen, dem Benutzen eines Wörterbuchs und dem Schreiben von E-Mails.

Insgesamt übten elf der Lernenden mindestens eine Tätigkeit nie oder fast nie aus; für zwei Mädchen gilt das bezogen auf alle Aktivitäten, wenn man bei der einen von der Informationssuche zu einem bestimmten Thema (mehrmals pro Monat) einmal absieht (FB, Nr. 27).

Abgesehen von zwei Schülerinnen, die ein paarmal im Jahr eine schulexterne Bücherei besuchten, tat das aus dieser Klasse keiner (FB, Nr. 49). In der Schulbücherei nutzten die Lernenden vor allem den Internetzugang. So waren elf Lernende mehrmals pro Woche deshalb dort und einer von ihnen nur aus diesem Grund, während sechs das Internet in der Bücherei nie benutzten. Im Mittelwert am zweithäufigsten gingen die Lernenden in eine Bibliothek, um Dinge zu lernen, die nichts mit der Schule zu tun hatten. Fast genauso oft lasen sie dort, allerdings nur drei von ihnen mehrmals wöchentlich und vier nie. Am seltensten nutzten sie eine Bibliothek, um Bücher für die Freizeitgestaltung 
auszuleihen, nämlich neun Lernende, also fast die Hälfte der Befragten, nie. Häufiger, aber durchschnittlich auch nur ein paarmal pro Jahr, entliehen sie Bücher für die Schule. Ebenso selten lasen sie dort Zeitschriften oder Zeitungen (FB, Nr. 47).

\subsubsection{Einstellung zum Erdkundeunterricht und zur Lehrkraft}

In dieser Klasse fand der Erdkundeunterricht nur einmal wöchentlich statt, da die Lehrerin die zusätzliche Stunde dazu nutzte, gesellschaftliche Themen zu erörtern, wie es auch in der Abschlussprüfung von den Lernenden verlangt werden konnte. Eine typische Unterrichtsstunde begann mit der Abfragung von vier Lernenden in Zweiergruppen, die dabei, anders als in den anderen Klassen, an ihren Plätzen bleiben durften. Im restlichen Verlauf der Stunde vermittelte die Lehrerin neue Inhalte, indem sie den Schülern Texte aus dem Lehrwerk zusammenfasste. Diese Texte sollten dann zuhause nachgelesen und gelernt werden. Nach Angaben der SchülerInnen gefiel ihnen der Erdkundeunterricht im Vergleich zu den anderen Fächern relativ gut. Ihr Urteil fiel also etwas schlechter aus als in den meisten anderen beobachteten Klassen (s. FB, Nr. 53). Sie hielten das Fach für leichter als Mathematik und Naturwissenschaften, etwas leichter als Italienisch und Geschichte und gleich schwer wie Englisch und Spanisch (FB, Nr. 54). Negativer fiel auch ihre Bewertung der Bedeutung des Erdkundeunterrichts aus. Denn auch wenn sie ihn nicht für komplette Zeitverschwendung hielten, sondern davon ausgingen, dass er sie einigermaßen auf ihr Leben nach der Schule vorbereitete, half er ihnen nicht dabei, Entscheidungen bewusster zu treffen, und sahen sie in ihm auch keinen Nutzen für ihre berufliche Zukunft (vgl. FB, Nr. 37).

Bei der Bewertung ihres Verhältnisses zur Lehrperson zeigte sich allerdings wieder das gleiche positive Bild, das schon für die anderen Klassen der Studie beobachtet werden konnte. Die SchülerInnen gaben an, dass sie sich mit der Lehrerin gut verstanden und das Gefühl hatten, diese interessiere sich für sie und ihre Meinungen. Außerdem bot Prof.ssa Dante zusätzliche Hilfe, wenn diese erforderlich war. Sie fühlten sich somit gut behandelt (FB, Nr. 39).

\begin{tabular}{llllllllllllllllllll}
\hline Lernende & $\mathrm{A}$ & $\mathrm{B}$ & $\mathrm{C}$ & $\mathrm{D}$ & $\mathrm{E}$ & $\mathrm{F}$ & $\mathrm{G}$ & $\mathrm{H}$ & $\mathrm{I}$ & $\mathrm{J}$ & $\mathrm{K}$ & $\mathrm{L}$ & $\mathrm{M}$ & $\mathrm{N}$ & $\mathrm{O}$ & $\mathrm{P}$ & $\mathrm{Q}$ & $\mathrm{R}$ & $\mathrm{S}$ \\
\hline $\begin{array}{l}\text { LB bietet die Inhalte auf } \\
\text { interessante Weise dar. }\end{array}$ & 2 & 3 & 1 & 3 & 2 & 3 & 2 & 3 & 2 & 2 & 2 & 1 & 2 & 1 & 3 & 2 & 1 & 2 & 3 \\
$\begin{array}{l}\text { Die Abbildungen helfen } \\
\text { mir, die Inhalte besser zu }\end{array}$ & & & & & & & & & & & & & & & & & & & \\
$\begin{array}{l}\text { verstehen und zu behal- } \\
\text { ten. }\end{array}$ & 1 & 1 & 1 & 2 & 1 & 2 & 1 & 1 & 2 & 2 & 3 & 3 & 1 & 1 & 1 & 1 & 1 & 1 & 4 \\
$\begin{array}{l}\text { Die Texte sind leicht zu } \\
\text { verstehen. }\end{array}$ & 2 & 2 & 3 & 2 & 2 & 4 & 3 & & 2 & 2 & 3 & 1 & 1 & 4 & 3 & 2 & 3 & 3 & 4 \\
$\begin{array}{l}\text { Die Übungen im LB sind } \\
\text { nützlich. }\end{array}$ & 3 & 3 & 2 & 2 & 2 & 4 & 3 & 2 & 3 & 2 & 3 & 1 & 2 & 4 & 3 & 3 & 3 & 3 & 2 \\
$\begin{array}{l}\text { Die Texte sind klar for- } \\
\text { muliert. }\end{array}$ & 3 & 4 & 4 & 4 & 3 & 3 & 4 & 3 & 4 & 4 & 2 & 1 & 3 & 4 & 3 & 3 & 3 & 3 & 4 \\
\hline
\end{tabular}




\begin{tabular}{|c|c|c|c|c|c|c|c|c|c|c|c|c|c|c|c|c|c|c|c|}
\hline $\begin{array}{l}\text { Der verwendete Ws ist } \\
\text { meinem Alter und mei- } \\
\text { nen Fähigkeiten ange- } \\
\text { messen. }\end{array}$ & 3 & 3 & 4 & 3 & 2 & 1 & 3 & 3 & 3 & 3 & 3 & 1 & 2 & 4 & 3 & 3 & 2 & 3 & \\
\hline $\begin{array}{l}\text { Die Texte haben die rich- } \\
\text { tige Länge. } \\
\text { Neue Fachwörter sind }\end{array}$ & 3 & 3 & 4 & 3 & 3 & 1 & 4 & 3 & 4 & 4 & 3 & 1 & 2 & 4 & 3 & 3 & 2 & 3 & \\
\hline $\begin{array}{l}\text { gut erklärt. } \\
\text { LB wäre in einer anderen } \\
\text { Sprache als Italienisch }\end{array}$ & 3 & 4 & 4 & 3 & 3 & 1 & 4 & 3 & 4 & 4 & 4 & 1 & 2 & 4 & 3 & 3 & 3 & 3 & \\
\hline sser zu verstehen. & 4 & 4 & 4 & 3 & 3 & 3 & 4 & 4 & 3 & 4 & 3 & 1 & 2 & 4 & 3 & 3 & 3 & 3 & \\
\hline
\end{tabular}

Tabelle 12: Einstellung zum Erdkundeunterricht und zur unterrichtenden Lehrkraft, Klasse III C, Pestalozzi

Auch in dieser Klasse gefiel den Lernenden am Erdkundeunterricht vor allem die Weise, auf welche Prof.ssa Dante die Lerninhalte erklärte, auch wenn einer ihre Schilderungen zwar verständlich, aber auch anstrengend fand. Mehrere störte das Anfertigen der Zusammenfassungen, und zwei Jungen italienischer Herkunft gaben an, Schwierigkeiten mit den Texten zu haben bzw. die Namen der Schlüsselwörter ändern zu wollen, da sie unmöglich einzuprägen seien. Eine Schülerin wollte lieber nur eine Lehrbuchseite pro Mal lernen anstatt mehrere (FB, Nr. 50-52).

Die Angaben der Lernenden zum Erdkundeunterricht und der Lehrkraft Prof.ssa Dante decken sich in allen bis auf einen Punkt mit denen über den Schulunterricht und die Lehrpersonen insgesamt, wobei die einzelnen Aussagen bezogen auf die Nützlichkeit von Erdkunde noch etwas mehr Zuspruch erhielten, sie das Fach also noch mehr als Zeitverschwendung und kaum auf das Leben nach der Schule vorbereitend sahen. Etwas weniger Bedeutung maßen sie ihm gleichsam als Hilfe beim Treffen von Entscheidungen und für ihr späteres Berufsleben bei (FB, Nr. 33). Auf der anderen Seite schien Prof.ssa Dante durchschnittlich etwas beliebter als die übrigen Unterrichtenden der Klasse zu sein, wobei vor allem ihr Interesse an den Lernenden positiver bewertet wurde (FB, Nr. 34).

Während des Erdkundeunterrichts kam es zwar ab und zu vor, dass sie ihr nicht zuhörten und ein hoher Lärmpegel und Unordnung herrschten, so dass Prof.ssa Dante manchmal lange warten musste, bevor im Klassenzimmer Ruhe einkehrte. Allerdings schien sich diese Aspekte nicht sehr negativ auf das Lernklima auszuwirken, denn die SchülerInnen hatten trotzdem das Gefühl, konzentriert arbeiten zu können, und dass nie viel Zeit verging, bevor der eigentliche Unterricht begann (vgl. FB, Nr. 36).

Ferner vermerkten sie, auch schriftlich abgefragt zu werden, wobei die Lehrerin darauf Acht gegeben habe, dass man selbstständig und konzentriert arbeitete. Nur ab und zu seien die Lernenden dabei vorher über die Gewichtung der einzelnen Fragen und die Bewertung des Tests in Kenntnis gesetzt worden, und auch eine Besprechung der Aufgaben oder des Testverlaufs sei im An- 
schluss daran nur gelegentlich erfolgt. Die Lernenden durften aber Fragen stellen, wenn sie etwas nicht verstanden hatten, und wurden dazu von der Lehrerin auch aufgefordert. Außerdem hatten sie sowohl bei den Tests als auch bei den Abfragungen durchaus manchmal das Gefühl, eine Antwort zu wissen, aber nicht in Worte fassen zu können. Die Tests wurden bewertet und die Note den Lernenden mitgeteilt, welche eine Berichtigung anfertigen mussten. Nach ihren Angaben waren sie im Durchschnitt bei den Tests und Abfragungen recht häufig nervös und fühlten sich unwohl. Alles in allem dachten sie dennoch, dass die Leistungsanforderungen ihren Fähigkeiten entsprachen. Aus der Situation der Mehrsprachigkeit einiger Schüler resultierte wahrscheinlich ihre Einschätzung, dass es für sie $\mathrm{ab}$ und zu einfacher gewesen wäre, in einer anderen Sprache als Italienisch zu antworten (s. FB, Nr. 40).

\subsubsection{Schülerangaben zum Lehrbuch}

Was das Lehrwerk (Forte \& Ubertazzi 2008) betrifft, das auch hier einen hohen Stellenwert genoss, zeigte sich zumindest im Durchschnitt die gleiche Gesamteinschätzung wie in der Klasse der Vespucci: Die Schüler beurteilten die Darbietung als interessant, waren der Auffassung, die Abbildungen halfen ihnen beim Verständnis und beim Behalten des Lernstoffs und die Übungen waren dafür auch nützlich. Die Texte fanden sie weder zu lang noch zu kurz, geeignet für ihre Altersstufe und klar verständlich, wobei Letzteres auch für die Einführung neuer Fachausdrücke gilt.

Sie waren nicht der Meinung, dass sie ihr Lehrwerk besser verstehen würden, wenn es in einer anderen Sprache geschrieben wäre (FB, Nr. 42). Während fast alle Lernenden bei den offenen Fragen zum Lehrwerk betonten, dass ihnen vor allem die Illustrationen und Landkarten gefielen, wurde von einigen auch noch einmal hervorgehoben, dass die Texte klar verständlich seien. Auf der anderen Seite fand eine Mehrheit die Texte zu kompliziert und auch zu lang (FB, Nr. 43 f.). Es ist also davon auszugehen, dass die Lernenden über unterschiedlich gute bildungssprachliche Kompetenzen verfügten und deshalb teils gut mit dem Lehrbuch zurechtkamen, teils überfordert waren.

Während des Unterrichts forderte die Lehrerin sie oft dazu auf, die Bedeutung eines Textes zu erklären und stellte dabei Fragen, die das Textverständnis leiteten und erleichterten. Die Lernenden waren der Meinung, dass ihnen dabei nur gelegentlich ausreichend Zeit gegeben wurde, eine Antwort vorzubereiten. Leseempfehlungen habe es ab und zu gegeben, genauso wie Hilfestellungen, um das neu erworbene Wissen mit der eigenen Lebenswelt in Verbindung zu bringen. Oft hingegen hätte die Lehrerin sie aufgefordert, ihre Meinung zu einem Text zu äußern, und ihnen gezeigt, wie sie das neue mit dem bereits erworbenen Wissen verknüpfen konnten und es so vertieften. 
Schriftliche Hausaufgaben bekamen die Lernenden ihren Angaben zufolge fast immer, wobei es sich oft um die Zusammenfassung von Texten handelte. Nur ab und zu hatten sie das Gefühl, die Hausaufgaben relativ zügig bewerkstelligen zu können (s. FB, Nr. 38). Dabei gaben nur vier Lernende als Zeitspanne, die sie durchschnittlich für die Hausaufgaben benötigen, weniger als eine Stunde an (min. 20 Minuten), während sieben dafür mindestens zwei Stunden und sogar bis zu dreieinhalb Stunden brauchten. Im Durchschnitt betrug die aufgewendete Zeit 1,5 Stunden.

\subsubsection{Lernstrategien}

Beim generellen, nicht fachspezifischen Lernen war die im Mittelwert von den Lernenden am häufigsten verfolgte Strategie der Versuch, die wichtigsten Textaussagen zu behalten (64 von 76 möglichen Punkten). An zweiter Stelle folgte, mit dem Überprüfen zu beginnen, was konkret gelernt werden sollte (61 Punkte). Sich an möglichst viele Details zu erinnern (59 Punkte), erfuhr fast denselben Zuspruch wie die drei Strategien, sich an die wichtigsten Textaussagen zu erinnern, mehrmals zu lesen und zu kontrollieren, ob das Gelesene auch verstanden wurde (57 Punkte). Am seltensten versuchten die Lernenden, den Stoff besser zu verstehen, indem sie ihn mit den eigenen Erfahrungen verknüpften (38 Punkte). Fast genauso selten fragten sie sich, welchen Nutzen die Informationen außerhalb der Schule und welche Bedeutung sie im wahren Leben haben könnten (40 Punkte).

Ferner geben sie an, den Text nur selten so oft zu lesen, bis sie ihn auswendig kannten (43 Punkte), wobei lediglich drei Lernende vermerkten, das nie zu tun, und sechs hingegen oft bis fast immer. Zwei Drittel (13) suchte nie bzw. selten nach zusätzlichen Informationen, wenn etwas nicht verstanden wurde.

Sechs Lernende wandten alle Strategien zumindest gelegentlich an, während die anderen mindestens eine Strategie nie nutzten (FB, Nr. 28). ${ }^{101}$

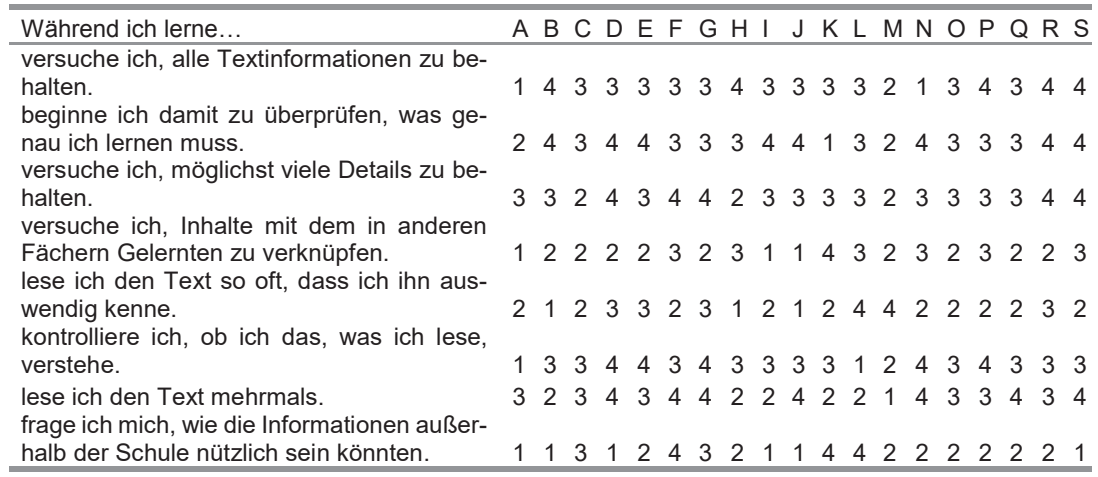

101 Die Skala in Tabelle 13 reicht von 1 bis 4: „fast nie“, „selten“, „oft“ und „fast immer“. 


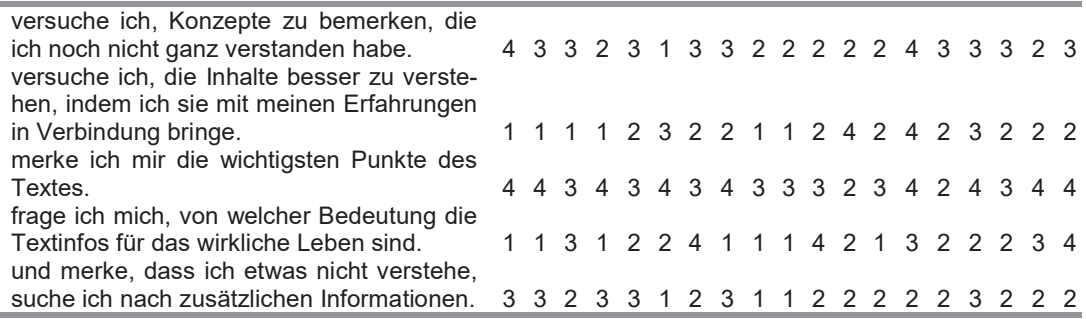

Tabelle 13: Anwendung von Lernstrategien in der Klasse III C, Pestalozzi

Um Lehrbuchtexte zu verstehen und ihren Inhalt zu behalten, fanden die SchülerInnen es am nützlichsten, Schlüsselwörter und wichtige Konzepte zu unterstreichen. Dieser Strategie gaben sie durchschnittlich fünf von sechs Punkten (insg. 97 von mögl. 114). An zweiter Stelle folgte das Zusammenfassen des Textes mit eigenen Worten (88 Punkte) und an dritter ein erstes langsames und dann (ggf. mehrmaliges) schnelleres Lesen des Textes (77 Punkte, durchschnittlich 4). Ebenfalls als recht förderlich bewerteten sie die Konzentration auf die leicht verständlichen Textteile (73 Punkte) und das Vorgehen, jemandem den Text laut vorzulesen (70 Punkte). Mit einem gewissen Abstand hielten sie die Technik, über das Gelesene mit jemandem zu sprechen, durchschnittlich für weniger produktiv (3, insgesamt 59 Punkte), und am unergiebigsten erschien es ihnen, den Text zweimal schnell zu lesen (49 Punkte). Zwei Schülerinnen stuften keine der Strategien als besonders nützlich ein, sondern verteilten für alle nur einen bis maximal drei Punkte. Auf der anderen Seite gab es sieben Lernende, die mindestens zwei Strategien mit sechs Punkten bewerteten (FB, Nr. 43).

Die Mehrheit der Lernenden (13) las einen Text zum Lernen laut; zwei Schülerinnen gaben an, sowohl laut als auch leise zu lesen. Nur drei lasen den Text nicht laut, und sechs wiederholten leise, davon vier sowohl laut als auch leise. Die meisten Schülerinnen und Schüler (13) machten sich Notizen, und so gut wie alle (18) schrieben außerdem eine Zusammenfassung. Zusätzlich ließ sich fast die Hälfte (9) von jemandem abfragen. Eine Schülerin gab ferner an, sich oft von ihrer Schwester helfen zu lassen, und ein Schüler, den Text zwei-, dreimal nachzuschreiben (FB, Nr. 45).

\subsection{Ein Fazit}

Abschließend lässt sich festhalten, dass die Lernenden aller Klassen im Durchschnitt recht ähnliche Lerngewohnheiten und Ansichten über die Schule und ihre Lehrpersonen, das Fach Erdkunde, die unterrichtende Lehrkraft und das 
verwendete Lehrwerk hatten. Im Großen und Ganzen waren sie mit dem Erdkundeunterricht zufrieden, was wahrscheinlich am meisten daran liegt, dass sie ihren Lehrer bzw. ihre Lehrerin schätzten.

Im Folgenden werden drei Tabellen vorgestellt, die einige wichtige Ergebnisse aus der Bearbeitung der Fragebögen wiederholen. Die erste (Tab. 14) zeigt neben den Angaben zum sprachlichen Hintergrund und zur Schullaufbahn (Schuljahreswiederholungen und Schulwahl) die Lesegewohnheiten der Lernenden, wobei die Abkürzungen $\mathrm{WH}, \mathrm{L}_{1}, \mathrm{P}$. und $\mathrm{Zs}$ für Wiederholung(en), Erstsprache, Pestalozzi und Zeitschriften stehen.

\begin{tabular}{|c|c|c|c|c|c|c|}
\hline Klassen & Morante & Vespucci & III AP. & III B P. & III C P. & Kommentar \\
\hline $\mathrm{n}$ Lernende & 18 & 14 & 19 & 15 & 21 & \\
\hline n beobachtete Lernende & 17 & 13 & 19 & 15 & 21 & \\
\hline $\mathrm{n}$ befragte Lernende & 16 & 12 & 18 & 14 & 19 & \\
\hline $\begin{array}{l}\text { n WH Schuljahre } \\
\text { n Lernbeeinträchtigun- }\end{array}$ & 1 & 2 & 3 & 3 & 2 & \\
\hline gen & 1 & 1 & 1 & 1 & 1 & \\
\hline n Lernende L1 nicht It. & 7 & 7 & 2 & 3 & 5 & \\
\hline n Einschulung nicht in It. & 2 & 2 & 0 & 0 & 0 & \\
\hline $\begin{array}{l}\text { n Lyzeum } \\
\text { Lesegewohnheiten: }\end{array}$ & $1(6 \%)$ & $1(8 \%)$ & $12(67 \%)$ & $12(86 \%)$ & $8(42 \%)$ & \\
\hline$\overline{\mathrm{n} \text { nie freiwillig lesen }}$ & $6(38 \%)$ & $4(33 \%)$ & $5(28 \%)$ & $3(21 \%)$ & $9(47 \%)$ & $\mathrm{Nr} 25 \mathrm{~m}$ \\
\hline n sehr gerne lesen & $3(19 \%)$ & $3(25 \%)$ & $7(39 \%)$ & $7(50 \%)$ & $4(21 \%)$ & 3 und 4$)$ \\
\hline$\varnothing$ lesen & 1,75 & 1,8 & 2,2 & 2,3 & 1,7 & Nr. 25, 2 \\
\hline $\begin{array}{l}\varnothing \text { häufigste Lektüre } \\
\text { Bücherei: }\end{array}$ & ZS & ZS & ZS & Comics & Comics & \\
\hline$\overline{\mathrm{n} \text { schulextern nie }}$ & $4(25 \%)$ & $7(58 \%)$ & $7(39 \%)$ & $9(64 \%)$ & $17(89 \%)$ & \\
\hline $\begin{array}{l}\varnothing \text { Besuche } \\
\text { Internet: } \varnothing \text { häufigste } \\
\text { Aktivitäten }\end{array}$ & $\begin{array}{l}\text { Chat } \\
\text { Diskus- } \\
\text { sionen } \\
\text { Enzyklo- } \\
\text { pädien }\end{array}$ & $\begin{array}{l}\text { Chat } \\
\text { Diskus- } \\
\text { sionen } \\
\text { Infos su- } \\
\text { chen }\end{array}$ & $\begin{array}{l}2 \\
\text { Diskussi- } \\
\text { onen } \\
\text { Chat } \\
\text { Nachrich- } \\
\text { ten }\end{array}$ & $\begin{array}{l}1,8 \\
\text { Chat } \\
\text { Diskussi- } \\
\text { onen } \\
\text { Infos su- } \\
\text { chen }\end{array}$ & $\begin{array}{l}1,1 \\
\text { Chat, En- } \\
\text { zyklopädien } \\
\text { Diskussio- } \\
\text { nen } \\
\text { Infos su- } \\
\text { chen }\end{array}$ & Nr. 49 \\
\hline
\end{tabular}

Tabelle 14: Überblick über Angaben der Lernenden zum sprachlichen Hintergrund, Bildungsstand und zu den Lesegewohnheiten

In jeder Klasse gab es einige Lernende, die zuhause neben Italienisch andere Sprachen pflegten. Zum Teil sprachen sie diese auch vorherrschend oder sogar ausschließlich. Fast alle wurden in Italien geboren, ein paar wanderten als Säuglinge oder Kleinkinder ein, und nur je zwei Lernende mit Migrationshintergrund in den Klassen an der Vespucci und an der Morante wurden noch vor dem Umzug ihrer Familien nach Italien in anderen Ländern eingeschult und begannen somit in anderen Sprachen als Italienisch zu lesen und schreiben.

In allen fünf Klassen gab es ein bis drei Lernende, die ein Schuljahr wiederholt hatten bzw. gerade wiederholten und je eine Schülerin oder einen Schüler mit einer Lernbeeinträchtigung, denen ein Unterstützungslehrer zugeteilt war. In einigen Klassen hatten nur sehr wenige Eltern studiert bzw. das Abitur 
gemacht, so dass es durchaus SchülerInnen gab, die aus Familien stammten, in denen sie die ersten waren, die sich dafür entscheiden, ihre Schullaufbahn auf einem Lyzeum fortzusetzen. Der Anteil, der ein Lyzeum und damit den anspruchsvolleren Schultyp wählte, war in den Klassen der Schulen Vespucci und Morante mit sechs und acht Prozent deutlich niedriger als in den Klassen an der Schule Pestalozzi, wo er mindestens fünfmal so hoch lag (Klasse III C) und bis zu 86 Prozent in der Klasse III B reichte.

Bezüglich ihres Leseverhaltens lässt sich festhalten, dass in den Klassen ein Teil der Lernenden nie freiwillig las: von 21 Prozent in der Klasse III B der Schule Pestalozzi bis zu 47 in der Klasse III C derselben Schule. Auf der anderen Seite machte der Anteil der Lernenden, die der Aussage „Lesen ist eine meiner Lieblingsbeschäftigungen“ zustimmten oder vollkommen zustimmten (3 bzw. 4), nur ca. 20 Prozent in den Klassen an der Schule Morante und III C der Pestalozzi aus und lag mit knapp 40 Prozent in der Klasse III A und 50 Prozent in der Klasse III B deutlich höher als in den übrigen beobachteten Lerngruppen. Im Durchschnitt lasen die SchülerInnen aller Klassen aber nicht besonders gerne und noch am häufigsten Zeitschriften oder Comics. Von einem Viertel der Lernenden an der Morante bis zu 90 Prozent in der Klasse III C an der Pestalozzi besuchte nie eine schulexterne Bücherei. Im Mittelwert gingen die SchülerInnen der Klasse III C auch nie oder fast nie in die Schulbücherei, die Klassen III A und III B sowie die der Vespucci manchmal im Jahr und nur die Klasse an der Morante ca. einmal pro Monat.

Was ihre Lese- und Schreibtätigkeiten im Internet anbelangt, so waren die Lernenden aller Klassen am häufigsten in sozialen Netzwerken aktiv, chatteten und beteiligten sich an Gruppendiskussionen. Nur in der Klasse III B der Vespucci wurde mit durchschnittlich derselben Häufigkeit auch das Angebot an Online-Nachschlagewerken genutzt. Erst an dritter Stelle folgten in vier Klassen Aktivitäten, die wahrscheinlich vor allem durch schulische Arbeitsaufträge bedingt waren: Recherchieren von Informationen über ein bestimmtes Thema sowie das Konsultieren von Enzyklopädien. Lediglich in der Klasse III A der Pestalozzi stand an dritter Stelle das Nachrichtenlesen, was ein Interesse am gesellschaftlich-politischen Leben offenbart.

Die Tabelle 15 fasst ihre Einstellung zum Unterricht und zum Lehrbuch sowie ihr Verhältnis zu den Lehrpersonen im Allgemeinen und zur Erdkundelehrkraft im Spezifischen zusammen (P. steht für Pestalozzi).

\begin{tabular}{lllllll}
\hline Klassen & Morante & $\begin{array}{l}\text { Ve- } \\
\text { spucci }\end{array}$ & III A P. & III B P. & III C P. & Kommentar \\
\hline Lehrbuch & & & & & & $\begin{array}{l}\text { Nr. 42 } \\
\text { 2: kaum einver- } \\
\text { standen, 3: ein- }\end{array}$ \\
$\begin{array}{l}\text { Ø interessante Darbietung } \\
\text { der Themen }\end{array}$ & 2,5 & 3,1 & 2,6 & 2,7 & 2,8 & verstanden \\
$\begin{array}{l}\varnothing \text { Texte leicht verständlich } \\
\varnothing \text { WS Alter und Kompeten- }\end{array}$ & 2,4 & 2,8 & 2,7 & 2,9 & 2,4 & \\
\begin{tabular}{l} 
zen angemessen \\
\hline
\end{tabular} & 2,6 & 3,1 & 2,9 & 3 & 2,5 & \\
\hline
\end{tabular}




\begin{tabular}{|c|c|c|c|c|c|c|}
\hline Klassen & Morante & $\begin{array}{l}\text { Ve- } \\
\text { spucci }\end{array}$ & III AP. & III B P. & III C P. & Kommentar \\
\hline $\begin{array}{l}\varnothing \text { neuer Fach-Ws gut er- } \\
\text { klärt }\end{array}$ & 2,4 & 3,4 & 2,8 & 2,9 & 2,7 & \\
\hline \multicolumn{7}{|l|}{$\begin{array}{l}\text { Erdkundeunterricht } \\
\text { wie er im durchschnittlich }\end{array}$} \\
\hline $\begin{array}{l}\text { gefällt } \\
\text { schriftliche Tests } \\
\varnothing \text { Angst vor den Abfragun- }\end{array}$ & $\begin{array}{l}3,9 \\
\text { ja }\end{array}$ & $\begin{array}{l}4,5 \\
\text { ja }\end{array}$ & $\begin{array}{l}3,3 \\
\text { ja }\end{array}$ & $\begin{array}{l}3,4 \\
\text { nein }\end{array}$ & $\begin{array}{l}3,3 \\
\text { manchmal }\end{array}$ & $\begin{array}{l}\text { Nr. } 53 \text {, Skala 1-6 } \\
\text { Nr. } 40\end{array}$ \\
\hline $\begin{array}{l}\varnothing \text { Antwort zu wissen, aber } \\
\varnothing \text {. }\end{array}$ & 3 & 3 & 1 & 1 & 3 & 3: manchmal \\
\hline $\begin{array}{l}\text { Worte nicht zu finden } \\
\varnothing \text { Tests und Abfragungen }\end{array}$ & 1 & 3 & 1 & 2 & 1 & 1: ja, 2: nein \\
\hline $\begin{array}{l}\text { den Fähigkeiten angemes- } \\
\text { sen }\end{array}$ & 1 & 1 & 1 & 1 & 1 & \multirow{3}{*}{$\begin{array}{l}\text { Nr. 38, 1: nie, 2: } \\
\text { manchmal, 3: } \\
\text { oft, 4: fast imme }\end{array}$} \\
\hline $\begin{array}{l}\varnothing \text { schriftl. Zusammenfas- } \\
\text { sungen der Texte } \\
\varnothing \text { genug Zeit zum Antwor- }\end{array}$ & 1,4 & 1,8 & 2,9 & 3,9 & 3,4 & \\
\hline ten & 2,5 & 2,9 & 2,7 & 2,5 & 2,6 & \\
\hline$\varnothing$ Lärm und Chaos & 2,3 & 2,2 & 1,8 & 2,4 & 2,2 & Nr. 36 \\
\hline$\varnothing$ Lernende hören nicht zu & 2,7 & 2 & 2,2 & 2,4 & 2,1 & \\
\hline Erdkundelehrkraft & & & & & & Nr. 39 \\
\hline$\varnothing$ Verhältnis zu ihr & 3,1 & 3,4 & 3,2 & 3,1 & 3,3 & Skala 1-4 \\
\hline $\begin{array}{l}\varnothing \text { Behandlung von ihr } \\
\varnothing \text { ihr Interesse an den Ler- }\end{array}$ & 3,1 & 3,5 & 3,2 & 3,1 & 3,1 & \\
\hline $\begin{array}{l}\text { nenden } \\
\text { unterrichtende Lehrper- } \\
\text { sonen allgemein }\end{array}$ & 2,9 & 3,2 & 2,4 & 2,8 & 2,8 & Nr. 34 \\
\hline$\varnothing$ Verhältnis zu innen & 2,5 & 3,2 & 2,9 & 3,1 & 3,1 & Skala 1-4 \\
\hline $\begin{array}{l}\varnothing \text { Behandlung von innen } \\
\varnothing \text { ihr Interesse an den Ler- }\end{array}$ & 2,8 & 3,6 & 3,2 & 3,1 & 3,1 & \\
\hline nenden & 2,1 & 2,6 & 2,1 & 2,4 & 2,4 & \\
\hline
\end{tabular}

Tabelle 15: Auffassungen der Lernenden zum Lehrbuch, zum Schul- und Erdkundeunterricht und zu den Lehrpersonen, alle Klassen

Durchschnittlich differieren die Bewertungen der Lernenden hinsichtlich der Darbietung ihres Lehrbuchs nur um 0,6 Punkte, wobei alle darin übereinstimmten, dass es recht interessant sei. Dasselbe dachten die Klassen III A und III B an der Pestalozzi und die an der Vespucci über die Verständlichkeit der Texte, während die Schülerinnen und Schüler an der Morante und in der Klasse III C der Aussage, dass die Texte leicht verständlich seien, im Mittelwert nicht zustimmten. Alle hielten jedoch den verwendeten Wortschatz für ihrem Alter und ihren Fähigkeiten angemessen. Was die Einführung von neuem Fachwortschatz betrifft, so unterscheiden sich die Mittelwerte der einzelnen Klassen etwas mehr: An der Schule Morante, wo die Lernenden alle bisher vorgestellten Aspekte des Buches am schlechtesten bewerteten, waren sie nicht der Auffassung, dass der Fachwortschatz gut erklärt werde $(2,4)$. In den anderen Klassen dachten sie das schon, wobei der Wert mit 3,4 in der Klasse an der Vespucci, die es gewohnt war, die Texte in Form von Gruppenarbeit zu erschließen, mit einem Abstand von 0,5 Punkten am höchsten war. 
Alle Klassen mochten den Erdkundeunterricht im Verhältnis zu den anderen mündlichen Fächern recht gerne und vergaben dafür auf einer Skala von 1 bis 6 Werte zwischen 3,3 und 4,5 (3: ganz gut, 4: mehr ja als nein, 5: sehr). An der Schule Vespucci verteilten alle drei Klassen niedrigere Noten $(3,3$ und 3,4) als an den beiden anderen Schulen, von denen der Erdkundeunterricht vor allem den Lernenden der Vespucci sehr gefiel. Es ist möglich, dass diese bessere Bewertung damit zusammenhängt, dass die Lernenden hier durch die Gruppenarbeitsphasen am Unterrichtsverlauf aktiver beteiligt waren als in den anderen Klassen.

Fast in allen Klassen wurden im Fach Erdkunde schriftliche Tests durchgeführt. Bezüglich der Fähigkeit, ihr geographisches Wissen zu versprachlichen, gaben die Lernenden aus drei Klassen (Morante, III C und III A Pestalozzi) durchschnittlich an, dass es durchaus vorkam, dass sie das Gefühl hatten, eine Antwort zu kennen, aber nicht die richtigen Worte zu finden. In der Klasse Vespucci war das nur gelegentlich und in der Klasse III B Pestalozzi nicht der Fall. Dennoch hielten die Lernenden im Mittelwert die schriftlichen Tests und Abfragungen für ihren Fähigkeiten angemessen.

Vor den Abfragungen hatten die Lernenden aller Klassen im Durchschnitt Angst, in den Klassen III A und III B der Pestalozzi etwas mehr als in den anderen.

Nach Angaben der Lernenden erteilten die fünf beobachteten Lehrkräfte unterschiedlich oft die Aufgabe, schriftliche Zusammenfassungen der behandelten Texte anzufertigen. So sei es an der Morante nie und an der Vespucci nur selten vorgekommen, in den Klassen III A und III C der Schule Pestalozzi oft verlangt worden und in der Klasse III B so gut wie immer. Während der Unterrichtsbeobachtungen konnte allerdings nicht bemerkt werden, dass Lernende diese Zusammenfassungen vorlesen oder der Lehrkraft zeigen sollten, noch fanden Heftkontrollen statt, außer im Falle von Prof.ssa Cese, die sich jeweils die Hefte der in einer Stunde abgefragten Lernenden vorlegen ließ. Deshalb bleibt unklar, mit welcher Gewissenhaftigkeit die Lernenden solche Zusammenfassungen schrieben.

In allen fünf Klassen hatten die Lernenden ferner durchschnittlich das Gefühl, oft genug Zeit zu haben, um eine Antwort vorzubereiten, und dass gelegentlich im Erdkundeunterricht Unruhe und Chaos herrschte, wobei dies nach Angaben der Lernenden in der Klasse III A der Pestalozzi am seltensten vorkam. Auch passierte es in allen Klassen ab und zu, dass die Lernenden der Lehrkraft nicht zuhörten, was in der Klasse an der Schule Morante etwas häufiger war als in den anderen Klassen. Diese Aussage der Schülerinnen und Schüler deckt sich auch mit dem während der Unterrichtsbeobachtungen gewonnenen Eindruck.

Um nur 0,3 Punkte unterscheidet sich die Bewertung des Verhältnisses zwischen der Lehrkraft und den Lernenden der beobachteten Klassen: Sie hatten alle das Gefühl, sich gut mit ihr zu verstehen und von ihr gut behandelt zu 
werden, wobei die Klasse an der Schule Vespucci sich sogar sehr gut behandelt fühlte. Etwas weniger Zuspruch erhielten die Erdkundelehrkräfte für ihr Interesse an den Lernenden, welches wiederum von den Lernenden der Vespucci höher eingestuft wurde als in den übrigen Klassen, die dieses schlechter bewerteten als die Qualität der Beziehung zur Lehrkraft. Letztere lag aber für die Erdkundelehrkräfte in allen Klassen etwas höher als die zu den unterrichtenden Lehrpersonen allgemein; lediglich die Lernenden in der Klasse III B an der Pestalozzi bewerteten sie gleich. An dieser Schule stuften alle drei Klassen im Mittelwert außerdem die Behandlung durch die Erdkundelehrkräfte im Vergleich zu den anderen LehrerInnen als gleich ein, während die Lernenden der Vespucci sich von den anderen geringfügig besser und die an der Morante geringfügig schlechter behandelt fühlten. Das Interesse an ihnen schätzten diese aber in Bezug auf ihre Erdkundelehrkräfte im Durchschnitt geschlossen als höher ein, wobei drei der Klassen (Morante, Pestalozzi III B und III C) auf der Skala von 1 bis 4 der Erdkundelehrkraft einen Punkt mehr gaben als den anderen Lehrpersonen.

Was die Lerngewohnheiten der Schülerinnen und Schüler anbelangt, zeigt sich ebenfalls ein ähnliches Bild. Auch wenn die durchschnittlich zur Anfertigung der Hausaufgaben benötigte Zeit zwischen den Lernenden einer Klasse recht stark differierte, brauchten doch alle Klassen dafür im Mittelwert zwischen einer Stunde und 20 Minuten und einer Stunde und 40 Minuten. Diese und weitere Angaben sind der Tabelle $16 \mathrm{zu}$ entnehmen $(\mathrm{K}=$ Kommentar).

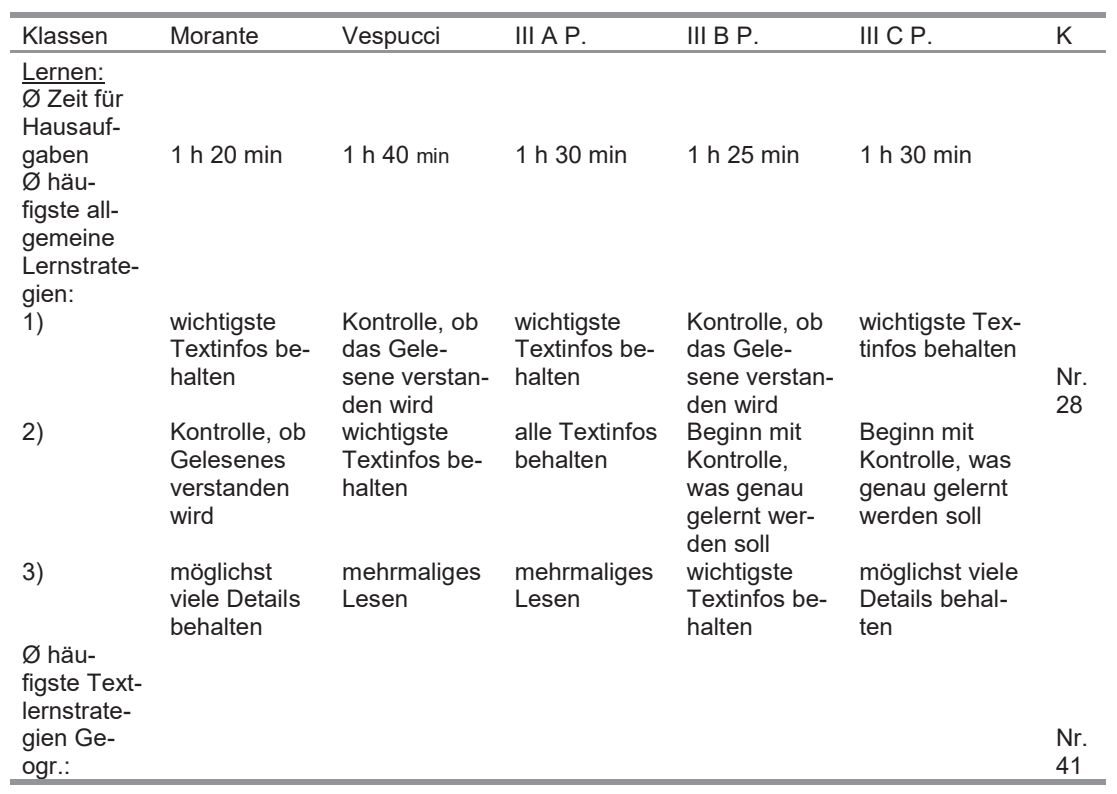




\begin{tabular}{|c|c|c|c|c|c|c|}
\hline Klassen & Morante & Vespucci & III A P. & III B P. & III C P. & $\mathrm{K}$ \\
\hline 1) & $\begin{array}{l}\text { zusammen- } \\
\text { fassen }\end{array}$ & $\begin{array}{l}\text { unterstrei- } \\
\text { chen }\end{array}$ & $\begin{array}{l}\text { zusammen- } \\
\text { fassen }\end{array}$ & $\begin{array}{l}\text { unterstrei- } \\
\text { chen }\end{array}$ & unterstreichen & \\
\hline 2) & $\begin{array}{l}\text { unterstrei- } \\
\text { chen }\end{array}$ & $\begin{array}{l}\text { zusammen- } \\
\text { fassen }\end{array}$ & $\begin{array}{l}\text { unterstrei- } \\
\text { chen }\end{array}$ & $\begin{array}{l}\text { zusammen- } \\
\text { fassen }\end{array}$ & $\begin{array}{l}\text { zusammenfas- } \\
\text { sen }\end{array}$ & \\
\hline 3) & $\begin{array}{l}\text { Konzentra- } \\
\text { tion auf leicht } \\
\text { Verständli- } \\
\text { ches }\end{array}$ & $\begin{array}{l}\text { Konzentra- } \\
\text { tion auf leicht } \\
\text { Verständli- } \\
\text { ches }\end{array}$ & $\begin{array}{l}\text { erstes lang- } \\
\text { sames, dann } \\
\text { schnelleres } \\
\text { Lesen }\end{array}$ & $\begin{array}{l}\text { erstes lang- } \\
\text { sames, dann } \\
\text { schnelleres } \\
\text { Lesen }\end{array}$ & $\begin{array}{l}\text { erstes langsa- } \\
\text { mes, dann } \\
\text { schnelleres Le- } \\
\text { sen }\end{array}$ & \\
\hline $\mathrm{n}$ nur laut & & & & & & \\
\hline & $7(44 \%)$ & $6(50 \%)$ & 10 (56 \%) & $9(64 \%)$ & $11(58 \%)$ & 45 \\
\hline $\begin{array}{l}\text { wiederho- } \\
\text { len }\end{array}$ & $8(50 \%)$ & $9(75 \%)$ & $13(72 \%)$ & $10(71 \%)$ & $12(63 \%)$ & \\
\hline n Notizen & $6(38 \%)$ & $3(25 \%)$ & $7(39 \%)$ & $10(71 \%)$ & $13(68 \%)$ & \\
\hline sung & $4(25 \%)$ & $2(17 \%)$ & $6(33 \%)$ & $13(93 \%)$ & $18(95 \%)$ & \\
\hline
\end{tabular}

Tabelle 16: Lerngewohnheiten der Schülerinnen und Schüler

Nach der Häufigkeit befragt, mit der sie beim generellen Lernen bestimmte Verfahren anwandten, ergaben sich wenig Unterschiede in den drei Techniken, die in allen Klassen mit der größten Frequenz verfolgt wurden: Drei Klassen konzentrierten sich am häufigsten darauf, die wichtigen Textaussagen zu behalten, während die anderen beiden am meisten kontrollierten, ob sie das Gelesene auch verstanden hatten. Diese Strategie folgte an der Morante an zweiter Stelle; in den anderen Klassen bemühten sich die SchülerInnen am zweitintensivsten darum, wichtige bzw. alle Textinformationen zu behalten oder zunächst zu kontrollieren, was genau sie eigentlich lernen sollten. An dritter Stelle stand in zwei Klassen (Morante, III C Pestalozzi) das Behalten möglichst vieler Details, in einer anderen (III B Pestalozzi) das Behalten der wichtigsten Textaussagen und in den Klassen an der Vespucci und III A der Pestalozzi das mehrmalige Lesen des Textes. So ist in allen Klassen unter den am häufigsten beim Lernen angewandten Techniken der Versuch zu verzeichnen, die wichtigsten Textinformationen zu behalten, was in drei der fünf Klassen außerdem davon ergänzt wird, sich möglichst alle Informationen oder gar alle Details zu merken.

Eine Strategie hierfür kann das mehrmalige Lesen des Textes sein, das zwei der Klassen als eine der drei häufigsten Vorgehensweisen angaben (Vespucci, III A Pestalozzi). In drei Klassen (Morante, Vespucci und III B Pestalozzi) wurde außerdem mit großer Häufigkeit kontrolliert, ob das Gelesene auch verstanden wurde. Die Klassen III B und III C der Pestalozzi kontrollierten darüber hinaus öfter als die anderen Klassen zunächst, was genau sie lernen sollten. Insgesamt befanden sich also in vier der fünf Klassen unter den drei am häufigsten eingeschlagenen Wege Metastrategien, um den Lernprozess zu überwachen und zu steuern. Überall wurde der Schwerpunkt ansonsten auf den Erwerb des Lerninhalts, also den Textgegenstand, gesetzt. 
Nach der Nützlichkeit von sieben verschiedenen Strategien beim verstehenden Lesen und Lernen eines Textes aus dem Erdkundelehrbuch befragt, setzten die Lernenden aller fünf Klassen das Zusammenfassen mit eigenen Worten und das Unterstreichen von Schlüsselwörtern und wichtigen Konzepten jeweils an erste bzw. an zweite Stelle. Danach fanden es die Lernenden der Klassen Morante und Vespucci im Mittelwert am förderlichsten, sich auf die leicht verständlichen Textabschnitte zu konzentrieren, während die Klassen an der Schule Pestalozzi ein erstes langsames Lesen des Textes und ein darauffolgendes, gegebenenfalls mehrmaliges schnelleres Lesen für ergiebiger hielten.

Lediglich in der Klasse an der Schule Morante las weniger als die Hälfte der Lernenden die Texte ausschließlich laut beim Lernen; in den anderen Klassen waren es mindestens 50 Prozent und in der Klasse III B der Pestalozzi sogar 64 Prozent der Schüler. Auch das Wiederholen der Textinhalte gestalteten die meisten nur laut, und zwar von einem Minimum der Hälfte der Lernenden an der Morante bis zu 75 Prozent der Lernenden an der Vespucci, was wahrscheinlich an den steten mündlichen Abfragungen in den meisten Schulfächern liegt.

Was das Anfertigen von Notizen beim Lernen anbelangt, so ergibt sich ein sehr unterschiedliches Bild: An der Schule Vespucci tat dies nur ein Viertel der befragten SchülerInnen, an der Morante und in der Klasse III A der Pestalozzi 38 bzw. 39 Prozent, aber in den anderen beiden Klassen dieser Schule waren es mehr als doppelt so viele, nämlich 68 Prozent in der Klasse III C und 71 in der Klasse III B.

Für eine weitere schriftsprachliche Aktivität war der Unterschied zwischen den Klassen noch größer, denn dort, wo mehr Notizen angefertigt wurden, schrieben über 90 Prozent der Schülerinnen und Schüler Zusammenfassungen der Texte, die sie lernen sollten. Das machten in den anderen drei Klassen höchstens 33 Prozent der Lernenden, an der Schule Vespucci sogar nur 17 Prozent.

\subsubsection{Sozioökonomischer Status}

Bezüglich des sozioökonomischen Status lässt sich außerdem zusammenfassend festhalten, dass die meisten Familien der Lernenden mithilfe ihrer Angaben im Fragebogen wahrscheinlich der unteren Mittelschicht zugerechnet werden können. So waren in der großen Mehrheit der Haushalte ein PC mit Internetzugang, Flachbildschirmfernseher, mehrere Handys und Einzelzimmer für die Kinder (z.T. auch, wenn diese Geschwister hatten) vorhanden. In vielen Familien waren beide Elternteile berufstätig, dennoch leisteten sich die wenigsten eine Haushaltshilfe, und auch über eine Klimaanlage verfügte trotz der oft sehr hohen Temperaturen in den Sommermonaten nur insgesamt die Hälfte 
der Haushalte. Ebenso selten kam es vor, dass Haushalte mehr als ein Auto besaßen. Vor allem diese letzten drei Aspekte (Haushaltshilfe, Klimaanlage und mehr als ein Auto) könnten als Indikatoren für eine Zugehörigkeit zur oberen Mittelschicht gerechnet werden.

Demnach decken sich die Informationen der Lernenden über die sozioökonomischen Verhältnisse in ihren Familien mit den allgemeinen Einschätzungen des Einzugsgebiets der Schule und dem, was die Lehrkräfte mitteilten. Abschließend fasst Tabelle 17 wesentliche Informationen über die sozioökonomische Lage der Familien zusammen (o.A. = ohne Angabe).

\begin{tabular}{lrrrrr}
\hline Klassen & Morante & Vespucci & III A P. & III B P. & III C P. \\
\hline Schulbildung Mütter: & & & & & \\
mind. Abitur & $5(16)$ & $9(12)$ & $10(18)$ & $11(14)$ & $9(19)$ \\
n Lernenden unbekannt & $4(16)$ & $0(12)$ & $2(18)$ & $1(14)$ & $5(19)$ \\
berufstätige Mütter & $10(16)$ & $9(12)$ & $16(18)$ & $12(14)$ & $13(19)$ \\
o.A. & $1(16)$ & $1(12)$ & $0(18)$ & $0(14)$ & $1(19)$ \\
Schulbildung Väter: mind. Abitur & $6(16)$ & $9(11)$ & $12(18)$ & $10(13)$ & $12(19)$ \\
unbekannt/o.A. & $4(16)$ & $2(11)$ & $2(18)$ & $1(13)$ & $0(19)$ \\
berufstätige Väter & $15(16)$ & $9(11)$ & $16(18)$ & $13(13)$ & $19(19)$ \\
o.A. & $1(16)$ & $0(11)$ & $1(18)$ & $0(13)$ & $0(19)$ \\
PC & $13(16)$ & $11(12)$ & $18(18)$ & $12(13)$ & $16(19)$ \\
o.A. & $0(16)$ & $0(12)$ & $0(18)$ & $1(13)$ & $0(19)$ \\
Internetzugang & $15(16)$ & $11(12)$ & $18(18)$ & $12(13)$ & $16(19)$ \\
o.A. & $0(16)$ & $0(12)$ & $0(18)$ & $1(13)$ & $0(19)$ \\
Fernseher mit Flachbildschirm & $12(16)$ & $9(12)$ & $17(18)$ & $11(13)$ & $15(19)$ \\
o.A. & $2(16)$ & $0(12)$ & $0(18)$ & $1(13)$ & $1(19)$ \\
Spülmaschine & $11(16)$ & $6(12)$ & $11(18)$ & $7(13)$ & $11(19)$ \\
o.A. & $1(16)$ & $0(12)$ & $0(18)$ & $1(13)$ & $0(19)$ \\
Auto(s) & $10(16)$ & $11(12)$ & $18(18)$ & $13(13)$ & $16(19)$ \\
O.A. & $0(16)$ & $0(12)$ & $0(18)$ & $0(13)$ & $1(19)$ \\
mehr als ein Auto & $2(16)$ & $6(12)$ & $9(18)$ & $5(13)$ & $7(19)$ \\
Haushaltshilfe & $1(16)$ & $2(12)$ & $5(18)$ & $5(13)$ & $4(19)$ \\
o.A. & $1(16)$ & $0(12)$ & $0(18)$ & $1(13)$ & $1(19)$ \\
Klimaanlage & $6(16)$ & $6(12)$ & $13(18)$ & $8(13)$ & $11(19)$ \\
o.A. & $2(16)$ & $0(12)$ & $0(18)$ & $1(13)$ & $0(19)$ \\
\hline
\end{tabular}

Tabelle 17: Angaben zum sozioökonomischen Status, alle Klassen

Nachdem die fünf Klassen vorgestellt sind, werden im folgenden Kapitel zunächst die verwendeten Lehrmittel anhand einer detaillierten Analyse ihrer sprachlichen Gestaltung präsentiert. Drei Kapitel über die Ergebnisse des auf den Lehrbuchtexten basierenden C-Tests und die Einsichten in die sprachlichen Herausforderungen des Erdkundeunterrichts, die durch die Unterrichtsbeobachtungen und die schriftlichen Textproduktionen der Lernenden gewonnen wurden, werden das Ganze abrunden. 


\section{Analyse der Unterrichtswerke}

\subsection{Die Bedeutung des Lehrwerks im beobachteten Erdkundeunterricht}

Im Folgenden sollen die Lehrwerke untersucht werden, welche in den fünf Klassen, die an der empirischen Studie mitgewirkt haben, verwendet wurden. Wie bereits erwähnt, spielen Lehrbücher im Unterricht in Italien eine zentrale Rolle, da sie die Hauptgrundlage für die Vermittlung von Wissen und Fertigkeiten bilden (s. Piemontese \& Cavaliere 1997, S. 236 zu ihrer Bedeutung bereits in der Grundschule; Jafrancesco 2002; MIUR 2015). Dennoch ist mir keine landeseigene Forschung zu ihrem Einsatz bekannt, was vielleicht darauf zurückzuführen ist, dass die Konzentration auf sie in einem vorherrschend traditionsverbundenen Unterricht so unumstritten ist. ${ }^{102}$ Weitere sprachliche Untersuchungen der italienischen Lehrwerke hingegen sind w.o. beim Umreißen des Forschungsstands referiert worden. ${ }^{103}$

Im Beobachtungszeitraum wurden die Lehrbuchinhalte zum einen während des Unterrichts gemeinsam erarbeitet, indem Texte aus dem Lehrwerk gelesen oder von der Lehrperson vorgestellt wurden. ${ }^{104} \mathrm{Zum}$ anderen bestand die am häufigsten erteilte Hausaufgabe darin, diese Texte zu wiederholen oder neue Texte zu erarbeiten, was oft der Vorbereitung einer - in der Regel individuellen und benoteten - mündlichen Überprüfung des Gelesenen durch die Lehrkraft diente. Gelegentlich wurden desweiteren während der Unterrichtszeit in Einzel- oder Partnerarbeit oder als Hausaufgabe gezielt Übungen aus den Lehrwerken bearbeitet. Auf zusätzliches didaktisches Material wie z.B. kopierte Arbeitsblätter stützten sich die beobachteten Lehrkräfte im Fach Erdkunde nur äußerst selten, nämlich nur in zwei Fällen. ${ }^{105}$ Nach eigenen Angaben versuch-

102 Unter den Ausnahmen s. bspw. die 1995 im Projekt Youth and History erhobenen Daten zum Geschichtsbewusstsein europäischer Jugendlicher, an dem auch Italien beteiligt war (s. Angvik \& von Borris 1997). Allerdings beziehen sich die Ergebnisse lediglich auf Geschichtslehrbücher und wurden zudem nur am Rande miterhoben. Auch in der deutschsprachigen Forschung wird referiert, dass Studien zur Schulbuchwirkung und -nutzung sowie zu den Erwartungen, die mit ihm verbunden werden, weiterhin sehr selten sind (Angvik 2012, S. 62; Oelkers 2010 zit. in Doll, Frank, Fickermann \& Schwippert 2012, S. 9 f.). Zu den Ausnahmen zählen die Beiträge in dem von Doll et al. (2012) herausgegebenen Tagungsband Schulbücher im Fokus. Nutzungen, Wirkungen und Evaluation.

103 S. v.a. Kapitel 3.

104 S. hierzu das Kapitel 9 über die Unterrichtsbeobachtungen.

105 Eine Lehrerin las einen das Unterrichtsthema vertiefenden Zeitungsartikel vor und besprach ihn mit den Lernenden. Eine weitere stellte Texte aus anderen Lehrwerken zusammen, die 
ten sie, während des Schuljahrs das Lehrwerk komplett durchzuarbeiten, wobei aus Zeitgründen auf einige Länder bzw. einen ganzen Kontinent verzichtet werden musste. Einige von ihnen beklagten die Diskrepanz zwischen der Fülle an Stoff und der knapp bemessenen Zeit, die zu dessen Vermittlung zur Verfügung steht. ${ }^{106}$ Dies könnte ein Grund dafür sein, warum die Lehrpersonen kein zusätzliches didaktisches Material verwendeten.

Es ist anzunehmen, dass die Tradition des auf das Lehrbuch konzentrierten Unterrichts in Italien sehr fest verankert ist und die Lehrkräfte in dieser Hinsicht ihren Unterricht so gestalten, wie sie ihn als Lernende auch selbst erfahren haben (Hattie 2013, S. 131 f.). Dies wird auch in Gesprächen darüber, wie die eigene Schulzeit erlebt worden ist, immer wieder deutlich. Im dritten Schuljahr der Mittelschule kommt zudem die Vorbereitung auf die Abschlussprüfung hinzu, deren Bestehen die Voraussetzung für die Versetzung an eine weiterführende Schule bildet. Erdkunde ist dabei ein mündliches Prüfungsfach, in dem die Schülerinnen und Schüler für eine positive Bewertung den Stoff des letzten Schuljahrs und somit den Inhalt ihres Unterrichtswerks beherrschen sollten.

Zunächst werden die jeweils verwendeten Lehrwerke vorgestellt und Umfang und Aufbau sowie ihre graphische Aufmachung beschrieben (7.2). Anschließend werden die für die sprachwissenschaftliche und sprachdidaktische Analyse bearbeiteten Seiten der Lehrbücher aufgelistet und eine Begründung der Auswahlkriterien vorgebracht (7.3). Diesen anfänglichen Schilderungen folgt die Untersuchung der Texte, wobei als solche auch die graphischen Elemente wie etwa thematische Karten und Schaubilder verstanden werden, auf die im Rahmen dieser Arbeit allerdings nur am Rande eingegangen werden kann. ${ }^{107}$ Hierbei werden zunächst die einzelnen Texte auf ihren Aufbau und Qualitäten wie Länge, Satzdichte und -komplexität unter besonderer Berücksichtigung der bildungssprachlichen Elemente untersucht. Ebenso wird eine Klassifizierung des Wortschatzes nach grammatischen Kategorien und nach der Häufigkeit seines Vorkommens im italienischen Sprachgebrauch vorgenommen (7.4.1 bis 7.4.5). Ein weiteres Unterkapitel (7.4.3.1) befasst sich nach der Vorstellung der ersten drei Bücher mit einer Analyse der graphisch hervorgehobenen Wörter aller fünf Lehrwerke. Danach erfolgt eine Gegenüberstellung der Ergebnisse für die einzelnen Bücher (7.5), bei der vorrangig der verwendete Fachwortschatz näher analysiert und verglichen wird (7.5.1). Dies schließt für zwei der vertretenen Fachsprachen, die geographische und die

für den Einsatz in einer Gruppenarbeit zur Vorstellung einzelner Länder bestimmt waren, da die entsprechenden Länder im verwendeten Unterrichtswerk nur knapp umrissen werden.

106 Es ist möglich, dass die Lehrpersonen den Zeitdruck im Augenblick als noch störender als gewöhnlich empfanden, da nach den neuen Lehrplänen nur noch eine Wochenstunde für das Fach Erdkunde vorgesehen war, und sie es bisher gewohnt waren, zwei Wochenstunden zu unterrichten.

107 Für eine Analyse der Bildelemente in Unterrichtswerken sei z.B. auf ein Handbuch zur Bilddidaktik (Lieber 2008) sowie Lieber (2012) und Heinze \& Matthes (2010) verwiesen. 
wirtschaftliche, eine Untersuchung dazu ein, welche der Ausdrücke in mehreren Lehrbüchern auftreten und wie oft (7.5.2). Einschätzungen der Lehrwerke durch die SchülerInnen (s. Fragebogen) auf der einen Seite und durch die Lehrenden auf der anderen Seite sind gelegentlich in die Unterkapitel eingestreut; auf eine Wiederholung und Vertiefung der mithilfe des Fragebogens gewonnen Schülerangaben und der aus mündlichen Befragungen der Lehrkräfte gewonnenen Erkenntnisse in einem eigenen Unterkapitel wird aufgrund des großen Umfangs, den die sprachlichen Analysen einnehmen, verzichtet. Konkrete, im Unterricht geleistete Wortschatzarbeit, die aus der Erarbeitung von Lehrbuchtexten resultierte, wird hingegen im Kapitel 9 zu den Unterrichtsbeobachtungen thematisiert.

\subsection{Präsentation der in der Erhebungsgruppe verwendeten Lehrwerke}

Italienische Lehrkräfte haben das Recht, selbst zu bestimmen, welches Lehrwerk sie in ihrem Unterricht verwenden (MIUR 2014, S. 2). ${ }^{108}$ Im Normalfall, wenn sie keine Klasse übernehmen, die den Dreijahreszyklus bereits mit einer anderen Lehrkraft begonnen hat, unterrichten sie eine Klasse an der Mittelschule in den Fächern Italienisch, Erdkunde und Geschichte über den gesamten Zeitraum von drei Jahren und wählen daher aus dem Angebot der Schulbuchverlage drei sie ansprechende, möglichst aufeinander aufbauende Geographiebücher, also ein Lehrwerk pro Schuljahr, eventuell mit Zusatzmaterial.

Dies ist der Fall bei den Lehrpersonen, die an meiner Studie mitgewirkt haben, auch wenn eine von ihnen mehrmals äußerte, dass sie ihr Unterrichtswerk zwar für sehr gut, aber veraltet hielt und es mit dem nächsten Dreijahreszyklus gegen ein moderneres eintauschen wollte. Eine weitere Lehrkraft wechselte nach Ablauf dieser Studie ebenfalls das Buch, auch wenn das von ihr verwendete Unterrichtswerk (Bastianelli, Rancati \& Maestri 2010) aus dem Verlag Sansoni neueren Datums war. Gespräche mit den an der Studie beteiligten Lehrenden ergaben, dass sie ihr Lehrwerk sorgfältig auswählten und über Jahre benutzten. Dabei konnte es passieren, dass sie es zwar für sehr geeignet hielten, aber der Behandlung der immer gleichen Texte überdrüssig wurden. Ein zusätzlicher Ansporn, das Lehrwerk zu wechseln, ist sowohl

108 Hierbei sind sie in der Wahl durch die geltenden Lehrpläne eingeschränkt und müssen sich an ministeriale Vorgaben halten, was den Höchstbetrag anbelangt, den die Familien für alle Bücher eines Schuljahrs entrichten sollen. Desweiteren wird von den Lehrpersonen in der Schulversammlung gegen Ende des Schuljahrs festgelegt, welche Lehrbücher in den von ihnen unterrichteten Fächern verwendet werden (MIUR 2014). Das Gesetz aus dem Jahr 2012, dass ein Lehrbuch in den Schulen der Sekundarstufe nach seiner Einführung sechs Jahre lang benutzt werden muss, wurde im Schuljahr 2014/2015 wieder abgeschafft (ibid.). 
durch Bemühungen von Verlagsvertretern gegeben, die gegen Ende des Schuljahrs ihre Klassiker und Neuerscheinungen an den Schulen anpreisen, als auch den konkreten bildungspolitischen Veränderungen 2009/2010 (Gelmini 2009, S. 3) geschuldet: Die bereits erwähnte Reduzierung der für das Fach Erdkunde vorgesehenen Unterrichtsstunden auf eine, nur wahlweise (und dadurch nicht jährliche, sondern allenfalls periodische) zwei Wochenstunden hatte zur Folge, dass die für zwei Stunden konzipierten Erdkundebücher stofflich zu umfangreich wurden. Im Allgemeinen lässt sich feststellen, dass der italienische Schulbuchvertrieb ein florierendes Geschäft ist, bei dem in geringen Zeitabständen neue Ausgaben der im Handel erhältlichen Bücher vermarktet werden. ${ }^{109}$

In den fünf beobachteten Klassen wurden fünf verschiedene Lehrwerke aus vier Verlagen eingesetzt. An der Schule Morante wurde das durch die Lehrkraft als gut, aber veraltet eingestufte Lehrwerk I nuovi territori dell'uomo. 3B, Regioni e paesi extraeuropei von Lorenzo Bersezio (2005) aus dem Schulbuchverlag De Agostini verwendet, an der Vespucci das Buch Il nuovo Geolab 3. I continenti extraeuropei. Manuale e Dossier von Maria Carazzi und Luisella Pizzetti (2010) aus dem Verlag Edizioni Scolastiche Bruno Mondadori (Mailand, Turin) und an der Pestalozzi die Bücher Zaino in spalla 3. Il mondo e i paesi extraeuropei von Elisabetta Bastianelli, Sergio Rancati und Paola Maestri (2010) aus dem Verlag Sansoni in der Klasse III A, Geograficamente 3. Noi cittadini del mondo von Manlio und Federico Dinucci (2008), erschienen im Verlag Zanichelli, in der Klasse III B und das Nuovo Geolibro 3 von Gioacchino Forte und Milli Tanara Ubertazzi (2008), wie das Lehrwerk von Bersezio (2005) ebenfalls aus dem Verlag De Agostini, in der Klasse III C. Der Übersicht halber sind die einzelnen Bücher in Tabelle 1 mit Angaben zu ihrem Umfang, dem Erscheinungsjahr und eventuellem Zusatzmaterial für die Lernenden sowie seiner Verwendung im Unterricht aufgeführt.

\begin{tabular}{|c|c|c|c|c|c|c|}
\hline Lehrwerk und AutorInnen & Klasse & $\begin{array}{l}\text { Erschei- } \\
\text { nungsjahr }\end{array}$ & $\begin{array}{l}\text { Format } \\
\text { in } \mathrm{cm}\end{array}$ & $\begin{array}{l}\text { Seiten- } \\
\text { anzahl }\end{array}$ & $\begin{array}{l}\text { Zusatz- } \\
\text { material }\end{array}$ & $\begin{array}{l}\text { Zusatzmat. } \\
\text { verwendet }\end{array}$ \\
\hline $\begin{array}{l}\text { I nuovi territori dell'uomo. } \\
\text { 3B, Regioni e paesi extraeu- } \\
\text { ropei (Bersezio) } \\
\text { II nuovo Geolab 3. I conti- } \\
\text { nenti extraeuropei. Manuale }\end{array}$ & Morante & 2005 & $\begin{array}{l}23 x \\
28,5\end{array}$ & 264 & $\begin{array}{l}3 A^{*} \\
\text { Atlas }\end{array}$ & nein \\
\hline $\begin{array}{l}\text { e Dossier (Carazzi \& Piz- } \\
\text { zetti) } \\
\text { Zaino in spalla 3. II mondo e } \\
\text { i paesi extraeuropei }\end{array}$ & Vespucci & 2010 & $\begin{array}{l}21,5 x \\
28,5\end{array}$ & 359 & Atlas & $\begin{array}{l}\text { gelegent- } \\
\text { lich }\end{array}$ \\
\hline $\begin{array}{l}\text { (Bastianelli, Rancati \& Mae- } \\
\text { stri) }\end{array}$ & $\begin{array}{l}\text { III A } \\
\text { Pestalozzi }\end{array}$ & 2010 & $\begin{array}{l}22,5 x \\
28,5\end{array}$ & 400 & Atlas & nein \\
\hline
\end{tabular}

109 Ein Beispiel hierfür ist das in der Klasse III A verwendete Buch Geograficamente von 2008 (Dinucci \& Dinucci 2008), zu dem es auch eine Ausgabe für die Lehrkraft mit Tipps zur Unterrichtsgestaltung gibt. 2009 ist eine CD-ROM mit Powerpoint-Präsentationen erschienen, die im Unterricht eingesetzt werden können, und 2010 eine neue, verkürzte Ausgabe mit dem Titel Geograficamente. Essenziale. 


\begin{tabular}{|c|c|c|c|c|c|c|}
\hline Lehrwerk und AutorInnen & Klasse & $\begin{array}{l}\text { Erschei- } \\
\text { nungsjahr }\end{array}$ & $\begin{array}{l}\text { Format } \\
\text { in } \mathrm{cm}\end{array}$ & $\begin{array}{l}\text { Seiten- } \\
\text { anzahl }\end{array}$ & $\begin{array}{l}\text { Zusatz- } \\
\text { material }\end{array}$ & $\begin{array}{l}\text { Zusatzmat. } \\
\text { verwendet }\end{array}$ \\
\hline $\begin{array}{l}\text { Geograficamente 3. Noi cit- } \\
\text { tadini del mondo }\end{array}$ & III B & & $23 x$ & & & \\
\hline (Dinucci \& Dinucci) & Pestalozzi & 2008 & 28,5 & 360 & Atlas & nein \\
\hline Nuovo Geolibro 3 & III C & & $23 x$ & & & \\
\hline (Forte \& Ubertazzi) & Pestalozzi & 2008 & 28,5 & 456 & Atlas & nein \\
\hline
\end{tabular}

Tabelle 1: Informationen zu den verwendeten Lehrwerken

*I nuovi Territori dell'uomo. 3A, Territori e culture del mondo (Bersezio 2005)

\subsubsection{Erster Eindruck zu den Lehrwerken}

Alle fünf Bücher präsentieren sich sehr farbenfroh und mit einem auf die spezifische Altersgruppe zugeschnittenen Layout: Auf jeder Seite finden sich graphische Elemente wie Photos, Schaubilder, Tabellen, geographische und thematische Karten, die neben ihrem Informationscharakter auch der Auflockerung dienen. Die Texte sind durch die Verwendung von verschiedenen Schrifttypen und -größen sowie durch die unterschiedliche farbliche Gestaltung bzw. Hintergrunduntermalung gekennzeichnet.

Auf den ersten Blick scheinen Gestaltungskriterien zur Förderung der Motivation der Lernenden solchen, die einer übersichtlicheren Strukturierung dienlich wären, vorgezogen zu sein. So gibt es z.B. manchmal keine Seitenzahlangaben, da der dafür vorgesehene Platz von graphischen Elementen eingenommen ist, und auf einer Seite finden sich in der Regel eine Vielzahl an verschiedenen Gestaltungselementen (zwei oder mehr Texte, Bilder, Schaubilder usw.). ${ }^{110}$

\subsection{Textauswahl}

Der Fokus dieser Analyse liegt auf der Verwendung des Wortschatzes in den Lehrbüchern, da seine zentrale Bedeutung für die Aneignung von bildungssprachlichen Fertigkeiten im Allgemeinen und fachspezifischen Kompetenzen im Besonderen nicht zu unterschätzen ist. Ohne die Wörter verstehen und ver-

110 Im Internet stehen einige Seiten dieser Bücher zur Ansicht bereit: z.B. Zaino in Spalla (Bastianelli, Rancati \& Maestri 2010) unter dem Link http://www.scuolabook.it (zuletzt abgerufen am 20.01.2017). Wie bereits erwähnt, kann hier auf den interessanten Aspekt rund um das Layout der Lehrwerke nicht näher eingegangen werden. Zu der Funktion strukturierender Elemente im Laufe des Kapitels mehr, beispielsweise zum umstrittenen Fettdrucken einzelner Wörter oder Wortgruppen in den Texten. 
wenden zu können, mit denen bildungssprachliche komplexe Strukturen geformt werden, kann man auch Letztere nicht verstehen, geschweige denn selbst produzieren.

Hierzu wurde das derzeitig umfassendste Wörterbuch zum Gebrauch der italienischen Sprache genutzt, das GRADIT (Grande dizionario italiano dell'uso, De Mauro 1999/2000). An dieser Stelle sei wiederholt, dass alle darin enthaltenen Lemmata mit einer Gebrauchsmarke ausgestattet sind, die gemäß ihrer Häufigkeit und Streuung innerhalb der unterschiedlichen mündlichen und schriftlichen Texttypen des Korpus ermittelt wurde. Diese Gruppierung des Wortschatzes geht von einem Kern von ca. 7.000 häufig gebrauchten Wörtern aus, dem Vocabolario di Base (VDB) oder Grundwortschatz, der sich seinerseits aus einem innersten Kern, dem ca. 2.000 Wörter umfassenden fundamentalen Wortschatz mit der Marke Fo, zusammensetzt. Die Fo-gekennzeichneten Wörter machen um die 90 Prozent eines jeden italienischen Textes aus (De Mauro 1998, S. 78). Um ihn herum schichten sich zwei weitere sehr häufig gebrauchte Wortschatzgruppen, die des alto uso (AU, hoher Gebrauch, ca. 3.000 Wörter) sowie die der alta disponibilità (AD, hohe Verfügbarkeit). ${ }^{111}$

Um hingegen auf zwischentextliche Differenzen eingehen zu können, ist bei der Auswahl der Texte darauf geachtet worden, dass sich je mindestens zwei von ihnen aus zwei verschiedenen Lehrbüchern mit demselben Thema befassen. Das Herzstück dieser Analyse bilden diejenigen Seiten, aus denen die C-Tests entwickelt wurden. Die Seitenauswahl stützt sich darauf, welche Texte kurz vor der Durchführung der C-Tests in den einzelnen Klassen behandelt wurden. Aus diesem Grund konnte für einen der Texte kein Vergleichstext aus einem anderen Unterrichtswerk herangezogen werden, da sich die Klasse III C im Zeitraum vor der Durchführung des C-Tests mit der Türkei beschäftigte, die nur in diesem Lehrwerk vorgestellt ist, während das Land in den anderen vier Büchern zum europäischen Kontinent gezählt wird und daher Bestandteil der Lehrbuchausgabe für das zweite Jahr der Mittelschule ist. Ein letztes Auswahlkriterium besteht in der Art des Textes, indem darauf geachtet wurde, dass aus jedem Lehrwerk sowohl landeskundliche als auch sozioökonomische und/oder soziokulturelle Themen vertreten sind.

Tabelle 2 gibt einen genauen Überblick über alle analysierten Lehrbuchseiten, wobei $x$ in der Spalte „C-Test“ dafür steht, dass Textauszüge der Seite für die Testentwicklung verwendet wurden. Wenn einzelne Texte nicht auf einer

111 Den zuletzt genannten Wortschatzbereich der hohen Verfügbarkeit formen in den gesprochenen und geschriebenen Texten des Korpus zwar nicht so stark vertretene und generell seltener verwendete Wörter, die aber eng mit der Alltagswelt der italienischen Sprachgemeinschaft verknüpft sind. Daher werden sie oft gedacht und sollten jedem Sprecher zur Verfügung stehen (s. De Mauro 2003, S. 162; De Renzo 2005, S. 220). Für Beispiele aus diesem Wortschatzbereich s. das Unterkapitel 3.2.2.1 zur Wortschatzbeschreibung des GRADITs, die Analysen in diesem Kapitel sowie die Kapitel 8 und 10. 
Seite enden, sondern sich über mehrere Seiten erstrecken, wurden diese als Einheit zusammen untersucht.

\begin{tabular}{|c|c|c|c|c|}
\hline Klasse & Seite(n) & C-Test & Titel & Thema \\
\hline \multirow[t]{6}{*}{ Vespucci } & 55 & $x(2)$ & $\begin{array}{l}\text { Ricchezza e qualità della vita. } \\
\text { II PIL e il PIL pro capite }\end{array}$ & $\begin{array}{l}\text { Wirtschaft (Lebensqualität, } \\
\text { BIP) }\end{array}$ \\
\hline & 56 & $x(2)$ & $\begin{array}{l}\text { La mortalità infantile: un indica- } \\
\text { tore per misurare la povertà. } \\
\text { L'ISU: un indicatore per misu- } \\
\text { rare lo sviluppo }\end{array}$ & $\begin{array}{l}\text { Wirtschaft (Säuglingssterb- } \\
\text { lichkeit als Indikator für Ar- } \\
\text { mut, Human Development In- } \\
\text { dex) }\end{array}$ \\
\hline & 62 & & $\begin{array}{l}\text { Economia mondiale. Distribu- } \\
\text { zione della ricchezza - Nord e } \\
\text { Sud del Mondo }\end{array}$ & $\begin{array}{l}\text { Weltwirtschaft (Wohlstand - } \\
\text { Norden und Süden der Welt) }\end{array}$ \\
\hline & 68 & $x$ & $\begin{array}{l}\text { Le risorse energetiche: Paesi } \\
\text { consumatori e paesi produttori; } \\
\text { L'opzione dell'energia nucleare }\end{array}$ & $\begin{array}{l}\text { Wirtschaft/Umwelt (Energie- } \\
\text { ressourcen, Kernenergie) }\end{array}$ \\
\hline & $134 \mathrm{f}$. & & Africa meridionale & Südafrika (Landeskunde) \\
\hline & $319 \mathrm{f}$. & & $\begin{array}{l}\text { Egitto: Gli insediamenti; L'eco- } \\
\text { nomia }\end{array}$ & $\begin{array}{l}\text { Ägypten (Bevölkerung, Wirt- } \\
\text { schaft) }\end{array}$ \\
\hline Tot. Seiten & 8 & & & \\
\hline \multirow[t]{5}{*}{ Morante } & $58-60$ & & Africa: La regione meridionale & Südafrika (Landeskunde) \\
\hline & 61 & & $\begin{array}{l}\text { Africa: L'identità demografica; } \\
\text { Approfondimento }\end{array}$ & $\begin{array}{l}\text { Afrika (Bevölkerung, „Vertie- } \\
\text { fung“) }\end{array}$ \\
\hline & 32 & & $\begin{array}{l}\text { Egitto: Demografia + Modelli } \\
\text { urbani; Popoli e culture }\end{array}$ & $\begin{array}{l}\text { Ägypten (Bevölkerung, Urba- } \\
\text { nistik; Völker und Kulturen) }\end{array}$ \\
\hline & 33 & & $\begin{array}{l}\text { Egitto: Risorse ed economia; } \\
\text { Saper fare: Leggiamo un'im- } \\
\text { magine }\end{array}$ & $\begin{array}{l}\text { Ägypten (Ressourcen und } \\
\text { Wirtschaft; „,Gewusst wie“: } \\
\text { Wir lesen ein Schaubild) }\end{array}$ \\
\hline & $82 \mathrm{f}$. & & $\begin{array}{l}\text { Asia - storia: Terre di antiche } \\
\text { civiltà e di recenti conflitti }\end{array}$ & $\begin{array}{l}\text { Asien - Geschichte (antike } \\
\text { Kulturen und Dekolonialisie- } \\
\text { rung) und jüngste Konflikte }\end{array}$ \\
\hline Tot. Seiten & 8 & & & \\
\hline \multirow[t]{6}{*}{$\begin{array}{l}\text { Pestalozzi, } \\
\text { III A }\end{array}$} & 87 & & $\begin{array}{l}\text { L'uomo ha bisogno di energia } \\
\text { per lavorare }\end{array}$ & $\begin{array}{l}\text { Wirtschaft/Umwelt (Energie- } \\
\text { ressourcen) }\end{array}$ \\
\hline & $116 \mathrm{f}$ & $x$ & $\begin{array}{l}\text { Le potenze economiche mon- } \\
\text { diali }\end{array}$ & $\begin{array}{l}\text { Wirtschaftliche Weltmächte } \\
\text { und internationale Handelsor- } \\
\text { ganisationen }\end{array}$ \\
\hline & 118 & $x(2)$ & $\begin{array}{l}\text { Sviluppo e sottosviluppo tra } \\
\text { Nord e Sud del Mondo }\end{array}$ & $\begin{array}{l}\text { Weltwirtschaft (Wohlstand - } \\
\text { Norden und Süden der Welt, } \\
\text { BIP) }\end{array}$ \\
\hline & 149 & $x(2)$ & $\begin{array}{l}\text { Le dinamiche demografiche } \\
\text { oggi }\end{array}$ & $\begin{array}{l}\text { Demographie (Geburten- und } \\
\text { Sterberate) }\end{array}$ \\
\hline & 182 & & Le lingue nel mondo & $\begin{array}{l}\text { Kultur (Sprachfamilien und } \\
\text { ihre Verbreitung) }\end{array}$ \\
\hline & 206 & & $\begin{array}{l}\text { Asia: uno sguardo d'insieme; } \\
\text { posizione }\end{array}$ & $\begin{array}{l}\text { Asien (Einleitung, geogr. Ein- } \\
\text { ordnung) }\end{array}$ \\
\hline Tot. Seiten & 8 & & & \\
\hline \multirow[t]{4}{*}{$\begin{array}{l}\text { Pestalozzi, } \\
\text { III B }\end{array}$} & $52 \mathrm{f}$. & $x$ & $\begin{array}{l}\text { II mosaico dei popoli - Le lin- } \\
\text { gue }\end{array}$ & $\begin{array}{l}\text { Kultur (Sprachfamilien und } \\
\text { ihre Verbreitung) }\end{array}$ \\
\hline & 238 & $\mathrm{x}$ & Asia - introduzione & Asien (Einleitung) \\
\hline & $239-241$ & $x(2)$ & $\begin{array}{l}\text { Asia - morfologia, clima e am- } \\
\text { biente, popolazione, economia } \\
\text { e società }\end{array}$ & $\begin{array}{l}\text { Asien (Landeskunde, Klima, } \\
\text { Bevölkerung, Wirtschaft und } \\
\text { Gesellschaft) }\end{array}$ \\
\hline & $242 \mathrm{f}$. & $x$ & $\begin{array}{l}\text { Asia occidentale - Medio } \\
\text { Oriente }\end{array}$ & $\begin{array}{l}\text { Mittlerer Osten (Landes- } \\
\text { kunde) }\end{array}$ \\
\hline Tot. Seiten & 8 & & & \\
\hline
\end{tabular}




\begin{tabular}{|c|c|c|c|c|}
\hline Klasse & Seite(n) & C-Test & Titel & Thema \\
\hline \multirow{4}{*}{$\begin{array}{l}\text { Pestalozzi, } \\
\text { III C }\end{array}$} & 105 & $\mathrm{x}$ & Asia-introduzione & Asien (Einleitung) \\
\hline & $106 \mathrm{f}$. & $\mathrm{x}$ & Asia - posizione, aspetti fisici & Asien (Landeskunde) \\
\hline & $120 \mathrm{f}$. & $x$ & $\begin{array}{l}\text { Asia Occidentale - enciclope- } \\
\text { dia (Le vie del petrolio); } \\
\text { Civiltà e storia; Relazioni inter- } \\
\text { nazionali }\end{array}$ & $\begin{array}{l}\text { westliches Asien (Wirtschaft: } \\
\text { Erdöl, Kulturen und Ge- } \\
\text { schichte, internationale Be- } \\
\text { ziehungen) }\end{array}$ \\
\hline & $122 \mathrm{f}$. & $x(2)$ & $\begin{array}{l}\text { Turchia - territorio e clima, or- } \\
\text { ganizzazione dello Stato, po- } \\
\text { polazione, società e economia, } \\
\text { aspetti geopolitici; enciclopedia } \\
\text { (La questione curda) }\end{array}$ & $\begin{array}{l}\text { Türkei (Landeskunde und } \\
\text { Klima, politische Ordnung, } \\
\text { Bevölkerung, Wirtschaft, geo- } \\
\text { politische Aspekte, die Situa- } \\
\text { tion der Kurden) }\end{array}$ \\
\hline
\end{tabular}

Tabelle 2: Überblick über die untersuchten Lehrbuchseiten

\subsection{Analyse der Lehrbuchseiten}

\subsubsection{Das Nuovo Geolab der Schule Vespucci}

Die einzelnen Seiten werden nun bezüglich ihrer Aufmachung, ihres Wort- und Bildanteils und ihrer textuellen Gestaltung vorgestellt, wobei nach der durch die Tabelle festgelegten Reihenfolge vorgegangen wird und zunächst die ersten beiden untersuchten Seiten aus dem Unterrichtswerk Nuovo Geolab (Carazzi \& Pizzetti 2010) ausführlicher und danach die anderen in tabellarischer Form beschrieben werden.

Bei den ersten beiden Seiten handelt es sich um Texte zur Vermittlung sozioökonomischer Kenntnisse. Hierbei geht es um die Wiederholung und Vertiefung einiger Konzepte, die für das Verstehen bestimmter wirtschaftlicher Zusammenhänge erforderlich sind. Daher werden sie auch unter der Oberüberschrift Le parole per capire (Die Wörter zum Verstehen) gefasst. Alle Texte auf den Seiten 55 und 56 sind im linksbündigen Flattersatz gedruckt, was den Lernenden mit schwächerer Lesekompetenz das Lesen im Vergleich zu Texten, die im Blocksatz verfasst sind, erleichtert (Döbert \& Hubertus 2000 S. 97).

Den Texten ist eine zehnzeilige Einleitung zum Thema Wohlstand und Lebensqualität vorangestellt, an der auffällig ist, dass die Verfasser des Öfteren in der ersten Person Plural formulieren (s. konjugierte Verbformen, Personalpronomen und pronominale Adjektive). Sie betonen, dass die folgenden Konzepte des Bruttoinlandsprodukts, der Säuglingssterblichkeit und des Human Development Index nicht neu eingeführt werden, sondern schon im vorangegangenen Schuljahr vermittelt worden sind und nun nicht mehr im europäischen, sondern im weltweiten Vergleich einzelner Länder Anwendung finden sollen (Carazzi \& Pizzetti 2010, S. 55; m.Ü.): ,Noi europei siamo abituati [wir 
Europäer sind es gewöhnt] [...] li abbiamo già incontrati e usati [wir sind ihnen schon begegnet und haben sie benutzt] [...] nostro continente [unser Kontinent]. Ora gli indicatori ci servono per confrontare situazioni di cui abbiamo un 'idea più vaga [Jetzt brauchen wir die Indikatoren um Situationen zu vergleichen, von denen wir eine vagere Idee haben] [...].“

Das Lesen und Lernen motivierend, schließt die Einleitung mit einer Begründung, warum diese Indikatoren jetzt wiederholt werden, und zeichnet sich an dieser Stelle als leserfreundlicher aus, als es auf den untersuchten Seiten der Lehrwerke sonst üblich ist.

\subsubsection{Zur Klassifizierung der sprachlichen Elemente}

Bevor jetzt auf die sprachlichen Elemente dieser Seite genauer eingegangen wird, sei erklärt, nach welchen Gesichtspunkten die Texte analysiert wurden. Dadurch dass die Sprache mithilfe des GRADITs untersucht wurde, sind die dort verwendeten Abkürzungen, die große Ähnlichkeiten mit den traditionellen deutschen grammatikalischen Bezeichnungen aufweisen, übernommen worden. Tabelle 3 gibt einen Überblick über die verwendete Terminologie, wobei Ws für Wortschatz steht. ${ }^{112}$

\begin{tabular}{|c|c|c|c|}
\hline Abkürzung & übersetzte Bedeutung & $\begin{array}{l}\text { Abkürzungen, Ge- } \\
\text { brauchsmarken }\end{array}$ & übersetzte Bedeutung \\
\hline s.m. & maskulines Substantiv & part.pres.v.tr./intr. & Partizip Präsens \\
\hline s.f. & feminines Substantiv & pron.dimostr. & Demonstrativpronomen \\
\hline pl. & Plural & pron.pers. & Personalpronomen \\
\hline art.det. & bestimmter Artikel & pron.rel. & Relativpronomen \\
\hline art.indet. & unbestimmter Artikel & pron.indef. & Indefinitpronomen \\
\hline agg. & Adjektiv & conf. & Konfix \\
\hline agg.num.ord. & Ordinalzahl & var. (s.m./f.) & $\begin{array}{l}\text { Variante (eines No- } \\
\text { mens) }\end{array}$ \\
\hline agg.num.card. & Kardinalzahl & var.posiz. & $\begin{array}{l}\text { positionsbedingte Vari- } \\
\text { ante }\end{array}$ \\
\hline agg.dimostr. & Demonstrativadjektiv & el.inf.p. & $\begin{array}{l}\text { Element eines Infinitiv } \\
\text { Passivs }\end{array}$ \\
\hline agg.poss. & Possessivadjektiv & el.loc. & $\begin{array}{l}\text { Element eines Phra- } \\
\text { sems }\end{array}$ \\
\hline agg.indef. & indefinites Adjektiv & el.n.p. & $\begin{array}{l}\text { Element eines Eigenna- } \\
\text { mens }\end{array}$ \\
\hline n.p. & Eigenname & el.CO/el.FO, etc. & $\begin{array}{l}\text { Element eines Phra- } \\
\text { sems aus dem entspre- } \\
\text { chenden Teil des WS }\end{array}$ \\
\hline sigla & Sigle & FO & $\begin{array}{l}\text { fundamentaler Wort- } \\
\text { schatz }\end{array}$ \\
\hline n.p./sigla & Eigenname/Sigle & $A U$ & hoher Gebrauch \\
\hline prep. & Präposition & $A D$ & hohe Disponibilität \\
\hline avv. & Adverb & $\mathrm{CO}$ & allgemeiner Wortschatz \\
\hline abbr. & Abkürzung & ES & Exotismus/Fremdwort \\
\hline
\end{tabular}

112 Zwecks dieser Untersuchung sind den Abkürzungen des GRADITs einige weitere hinzugefügt worden: inf.v.tr./intr., ger.v.tr./intr., el.Co/FO etc., el.n.p., n.p./sigla. 


\begin{tabular}{|c|c|c|c|}
\hline Abkürzung & übersetzte Bedeutung & $\begin{array}{l}\text { Abkürzungen, Ge- } \\
\text { brauchsmarken }\end{array}$ & übersetzte Bedeutung \\
\hline simb. & Symbol & $\mathrm{BU}$ & niedriger Gebrauch \\
\hline loc.agg. & adjektivales Phrasem & LE & literarischer Gebrauch \\
\hline loc.cong. & $\begin{array}{l}\text { konjunktionales Phra- } \\
\text { sem }\end{array}$ & ES & Exotismus \\
\hline loc.s.f./m. & nominales Phrasem & TS & $\begin{array}{l}\text { technisch-spezifischer } \\
\text { WS }\end{array}$ \\
\hline loc.avv. & adverbiales Phrasem & (FO, AU etc.) fig. & figurative Bedeutung \\
\hline loc.v. & verbales Phrasem & (FO, AU etc.) estens. & $\begin{array}{l}\text { ausgeweitete Bedeu- } \\
\text { tung }\end{array}$ \\
\hline v.tr. & transitives Verb & Beispiele für TS: & \\
\hline v.intr. & intransitives Verb & TS geogr. & geographischer WS \\
\hline inf.v.tr./intr. & Infinitiv Präsens & TS econ. & wirtschaftlicher WS \\
\hline inf.p.v.tr. & Infinitiv Passiv & TS dir.intern. & $\begin{array}{l}\text { WS internationales } \\
\text { Recht }\end{array}$ \\
\hline inf.pass.v.tr./intr. & Infinitiv Perfekt & TS ling. & linguistischer WS \\
\hline ger.v.tr./intr. & Gerundium & TS scient. & $\begin{array}{l}\text { naturwissenschaftlicher } \\
\text { WS }\end{array}$ \\
\hline part.pass.v.tr./intr. & Partizip Perfekt & TS stor. & geschichtlicher WS \\
\hline
\end{tabular}

Tabelle 3: Terminologie der sprachlichen Analyse

Der technisch-spezifische Wortschatz ist in verschiedene Wissensgebiete unterteilt; welchen Gebieten der Fachwortschatz der untersuchten Seiten im Einzelnen zugeordnet ist, zeigt die nachstehende Liste einschließlich der Übersetzungen (Tabelle 4).

Es kommt durchaus vor, dass bestimmte Wörter in verschiedenen Fachgebieten benutzt werden, was im GRADIT durch Marken wie z.B. Ts econ., sociol. gekennzeichnet wird und hier so übernommen ist. Gleichermaßen weist ein Lemma manchmal zwei Gebrauchsmarken auf, z.B. das Adjektiv musulmano (Dinucci \& Dinucci 2008, S. 243; muslimisch) ${ }^{113}$ die Marken Co und Ts relig., welche dann beide in den Auswertungen berücksichtigt wurden.

\begin{tabular}{ll}
\hline Technisch-spezifischer & \\
Wortschatz & Übersetzung \\
\hline TS agr. & Agrarwissenschaft \\
TS amin.az. & Betriebsverwaltung \\
TS antrop. & Anthropologie \\
TS archeol. & Archäologie \\
TS arm. & Waffen, Bewaffnungen \\
TS astrol. & Astrologie \\
TS astron. & Astronomie \\
TS biochim. & Biochemie \\
TS biol. & Biologie \\
TS bot. & Botanik \\
TS bot.com. & allgemeiner Begriff aus der Botanik \\
TS burocr. & Bürokratie \\
TS cartogr. & Kartographie \\
\hline
\end{tabular}

113 Dieser Fall ist zu unterscheiden von dem der Lemmata, deren einzelnen Bedeutungen je verschiedene Gebrauchsmarken zugeteilt sind. 


\begin{tabular}{ll}
\hline Technisch-spezifischer & \\
Wortschatz & Übersetzung \\
\hline TS chim. & Chemie \\
TS dir. & Rechtswissenschaften \\
TS dir.intern. & internationales Recht \\
TS dir.amm. & Verwaltungsrecht \\
TS ecol. & Ökologie \\
TS econ. & Wirtschaft \\
TS etnol. & Ethnologie \\
TS fin. & Finanzwesen \\
TS fis. & Physik \\
TS mat. & Mathematik \\
TS geofis. & Geophysik \\
TS geogr. & Geographie \\
TS geol. & Geologie \\
TS idrol. & Hydrologie \\
TS industr. & Industrie \\
TS inform. & Informatik \\
TS let. & Literatur \\
TS ling. & Linguistik \\
TS mar. & Marine \\
TS metall. & Hüttenwesen \\
TS meteor. & Wetterkunde \\
TS metrol. & Maß- und Gewichtslehre \\
TS miner. & Mineralogie \\
TS monet. & Währungen \\
TS paleont. & Paläontologie \\
TS polit. & Politik \\
TS pubbl. & Werbung \\
TS relig. & Religion \\
TS scient. & (natur)wissenschaftlicher Wortschatz \\
TS sociol. & Soziologie \\
TS sport & Sport \\
TS stat. & Statistik \\
TS stor. & Geschichte \\
TS tecn. & Technik \\
TS topogr. & Topographie \\
TS urban. & Vulkanologistik \\
TS vulcanol. & \\
TS zool.com. & \\
TS zoot. & Viehzucht \\
\hline
\end{tabular}

Tabelle 4: Kürzel der Fachwortschatzbereiche und deutsche Übersetzungen

Als Untersuchungseinheit ist aus praktischen Gründen das graphische Wort gewählt worden, also das, was zwischen zwei Leerzeichen steht. Nicht als 
Wort gelten dabei Zahlen, einzelne Buchstaben, die der Kapitelgliederung dienen und Symbole, die keinen eigenen Eintrag im GRADIT haben, z.B. das Prozentzeichen (\%). ${ }^{114}$

In bestimmten Fällen ist die Entscheidung zugunsten des graphischen Wortes problematisch. Betroffen sind im Einzelnen mehrgliedrige Verbformen, Wörter, die durch einen Bindestrich verbunden sind, Zusammenziehungen durch Apostrophierung, die im Italienischen recht häufig vorkommen ${ }^{115}, \mathrm{Zu}-$ sammenziehungen von Verbformen und klitischen Pronomen (z.B. compramelo (kauf ihn/es mir)) sowie Phraseme.

Um komplexe Verbformen wie beispielsweise è stato tramandato (es ist überliefert worden) nicht als drei konjugierte Verben zu zählen, wurden die ersten beiden Formen als Hilfsverb und nur die letzte Form als konjugiertes transitives (bzw. intransitives) Verb gekennzeichnet. Generell wurden nur konjugierte Verbformen als v.tr. oder v.intr. kategorisiert und damit von gesondert gekennzeichneten Infinitiven, Partizipien und Gerundien differenziert.

Bindestrichwörter sind als Lemmata nicht im GRADIT vertreten, sondern manchmal in zusammengesetzter Form ohne Bindestrich und manchmal nur als einzelne Wörter. So ist z.B. sub-sahariana (Bastianelli, Rancati \& Maestri 2010, S. 116; subsaharisch) in der Form subsahariano im GRADIT eingetragen, wohingegen medio-alto (Dinucci \& Dinucci 2008, S. 243; mittelhoch, bzw. mittel bis hoch) nur zwei eigene Einträge für die beiden Adjektive medio (AU) und alto (Fo) hat. Da die beiden Ausdrücke oft derselben Wortklasse angehören, sind sie bei gleicher Gebrauchsmarke nur einmal gezählt worden, bei unterschiedlichen Gebrauchsmarken hingegen zweimal.

Bei den Zusammenziehungen ist nie das grammatikalische Morphem, sondern immer das lexikalische mit der größeren semantischen Bedeutung klassifiziert worden. So steht z.B. für dall'Iran (aus dem/vom Iran) n.p. (Eigenname) für den Iran und nicht etwa prep.art. (artikulierte Präposition) ${ }^{116}$, und für compralo $=$ compra (imp.v.tr., kaufe) + lo (pron.pers., ihn/es) imp.v.tr. für den Imperativ von „kaufen“ und nicht pron.pers.

Ähnlich wie bei den komplexen Verbformen sind auch die einzelnen Elemente, die einen Phraseologismus formen, nicht wie autonome Phraseme mit

114 In der digitalen Ausgabe des GRADITs gelangt man aber über percento (Prozent) zu per cento, dem Adektivalphrasem „Prozent“, das wie alle anderen Phraseme mit dem Bestandteil cento unter cento aufgeführt ist und auch den Hinweis auf das dazugehörige Symbol liefert. Dieser recht komplizierte Weg hat auch den Nachteil, dass das Symbol \% im Gegensatz zu anderen Symbolen wie $k m q\left(\mathrm{~km}^{2}\right)$ nicht mit einer Gebrauchsmarke versehen ist.

115 Sie betreffen Artikel und Zusammenziehungen aus Artikeln und Präpositionen, wenn das Folgewort mit einem Vokal beginnt, z.B. l'altezza, dell'uomo.

116 Dadurch ist immer das Wort nach dem Apostroph und nicht die Präposition oder der Artikel klassifiziert, außer in einem Fall, in dem das graphische Wort dell' $8-10 \%$ lautet (Bastianelli, Rancati \& Maestri 2010, S. 116). Dadurch dass weder Zahlen noch das Prozentzeichen in die Analyse mit eingegangen sind, wurde die Entscheidung getroffen, hier die artikulierte Präposition als Untersuchungseinheit zu zählen. 
einer eigenen Gebrauchsmarke versehen worden, sondern immer nur das erste Element der Wortgruppe, aus welcher der Phraseologismus besteht, während alle folgenden Wörter als Wortart el.loc. und z.B. mit der Gebrauchsmarke el.Co erscheinen, wie im Falle von punto di vista (Standpunkt), bei dem das nominale Phrasem für punto die Zuordnung loc.s.m.Co erhalten hat, und die weiteren Elemente di und vista jeweils el.loc.s.m. und el.Co. ${ }^{117}$

Darüber hinaus kommt es bei einigen weiteren graphischen Wörtern vor, dass sie doppelt klassifiziert und mit zwei Gebrauchsmarken ausgestattet sind. Der Fall tritt ein, wenn sie durch einen Schrägstrich miteinander verbunden sind, wie z.B. bei Angaben zur Bevölkerungsdichte, $a b . / \mathrm{km}^{2}$ (Bersezio 2005, S. 58; Einw. $/ \mathrm{km}^{2}$ ), was als abbr.s.m. + simb. und AU + Ts metrol. definiert ist.

Generell wurden alle Wörter einer Seite untersucht, also auch Angaben zum Kapitel oder zu einer Kapiteleinheit, Kommentare zu graphischen Elementen und darin enthaltenen Wörtern, mit Ausnahme reiner Toponyme in Land- und thematischen Karten.

Als Satz gilt alles bis zu einem Punkt, wobei dadurch in Einzelfällen, vor allem bei den Bildbeschriftungen, zu den Sätzen auch solche zählen, die kein Prädikat aufweisen (s. Fabbrica ad alta tecnologia in Giappone. (Forte \& Ubertazzi 2008, S. 241; Hochtechnologiefabrik in Japan.)).

\subsection{Kategorisierungsschwierigkeiten bei den Eigennamen}

Zwei Wortarten sind im GRADIT nicht mit einer Gebrauchsmarke versehen, nämlich Eigennamen und Siglen. Während Letztere, wenn sie aus anderen Sprachen stammen, wie WTO (World Trade Organization), die Marke Es tragen und die italienischen Siglen zumindest teilweise ausgeschrieben als Phraseme mit Gebrauchsmarke aufgeführt sind, ist es bei den Eigennamen so, dass einige Toponyme gar keinen Eintrag haben, wohl aber die dazugehörigen Adjektive, und zwar mit Gebrauchsmarke. Beispielsweise erscheint Asia (Asien) in diesem Wörterbuch nicht als Lemma, asiatico (AD; asiatisch) aber schon. In der vorliegenden Untersuchung sind alle Toponyme als solche gekennzeichnet und ohne Gebrauchsmarke aufgelistet, auch wenn sie im GRADIT keinen Eintrag als Eigenname haben; allerdings war ihre Zuordnung in einigen Fällen problematisch.

Eine Besonderheit bilden etwa diejenigen Staaten und allgemein Ortsnamen, die aus mehreren graphischen Wörtern bestehen. Viele von ihnen sind nicht als Lemmata im GRADIT aufgeführt, jedoch haben einige schätzungsweise aufgrund ihrer Mehrgliedrigkeit eine Gebrauchsmarke erhalten, wie z.B. Co bei Stati Uniti d'America (Vereinigte Staaten von Amerika), wobei die

117 Von der ursprünglichen Absicht, jedes graphische Wort einer Wortklasse zuzuordnen und mit einer Gebrauchsmarke zu versehen, ist abgesehen worden, da es bei den Phraseologismen und Eigennamen zum Teil schwierig und bei Ersteren oftmals auch sinnlos ist, eben weil ihre Bedeutung über die Summe der Bedeutungen ihrer Bestandteile hinausgeht. 
Sigle UsA im Gegensatz zur Sigle RfT (Repubblica Federale Tedesca, Bundesrepublik Deutschland) auch nicht als solche aufgeführt ist, sondern als Substantiv Plural (und nicht als Eigenname!) mit Kennzeichnung durch die Marke Co. Das gleiche gilt sowohl für das Vereinigte Königreich (Großbritanniens) (Regno Unito, CO), ein Land, welches im GRADIT nur so und nicht auch als Gran Bretagna (Großbritannien) zu finden ist, als auch für die ehemalige Sowjetunion, als Nomen gekennzeichnet unter URSS (CO) und als Phraseme Unione Sovietica und Unione delle Repubbliche Socialiste Sovietiche (beide auch $\mathrm{Co}$ ) vertreten. Auf der anderen Seite hat das im Italienischen zweigliedrige Neuseeland (Nuova Zelanda) einen Eintrag, und zwar als Eigenname, also ohne Gebrauchsmarke.

Der Einheitlichkeit halber sind daher in dieser Untersuchung alle offiziellen Staatsnamen und im allgemeinen Sprachgebrauch übliche Abweichungen davon, wie z.B. Israel für das offizielle „Staat Israel“, als Eigenname ohne Gebrauchsmarke aufgelistet, bzw. als n.p./sigla gekennzeichnet, wie im Fall der USA.

Eine weitere Schwierigkeit bei der Kategorisierung stellen geographische Bezeichnungen dar, die aus einem allgemeinen Wort oder einem geographischen Adjektiv und einem Eigennamen bestehen. Während sie im Deutschen - einer Sprache, die, anders als das Italienische, stark zur Kompositabildung neigt - oft durch zusammengesetzte Nomen wiedergegeben werden, wie beispielsweise „Uralgebirge“, oder als Eigennamen durch die Großschreibung klar als solche zu erkennen sind (z.B. Holsteinische Schweiz), gibt es auf diesem Gebiet im Italienischen keine klare Regelung der Groß- bzw. Kleinschreibung (vgl. z.B. Serianni 2007, S. 63 f.) ${ }^{118}$, so dass sich für „Ostasien“ sowohl die Form Asia orientale als auch Asia Orientale finden lässt. Gerade in diesem letzten Fall ist die Groß- bzw. Kleinschreibung von Buch zu Buch verschieden und teilweise nicht einmal in ein und demselben Lehrbuch konsequent eingehalten (s. Forte \& Ubertazzi 2008, S. 106: Asia Orientale, aber auf der nächsten Seite Africa orientale). Außerdem ist Italia meridionale (Süditalien) im GRADIT als Phrasem eingetragen, was nicht auch für den meridionalen Teil anderer Länder gilt. Hier wurde die Entscheidung getroffen, Länder und Kontinente als Eigennamen zu werten, aber dazugehörige positionsangebende Adjektive nicht als ihre Elemente, obwohl die deutschen Äquivalente oft aus nur einem Wort bestehen (vgl. Osteuropa, Südostasien). Also ist Asia Orientale/Asia orientale als $A s i a=$ n.p. und orientale = agg. AU klassifiziert worden.

Natürlich wäre es wünschenswert, für alle in einer Sprache mit einer gewissen Häufigkeit benutzten Wörter eine Gebrauchsmarke ermitteln zu können, und gerade auch in einer Studie zum Sprachgebrauch im Schulfach Erd-

118 Diese Schwierigkeit stellt sich auch bei den Bezeichnungen für historische Ereignisse wie z.B. den Zweiten Weltkrieg, den Golfkrieg und die Römischen und anderen antiken Reiche, die nur zum Teil im GRADIT eingetragen sind (als Phraseme). 
kunde, in dem Toponyme eine große Rolle spielen, interessiert, welchem Bereich des italienischen Gebrauchswortschatzes sie zuzuteilen sind. Jedoch habe ich aus Gründen der Einheitlichkeit entschieden, alle klar definierbaren Toponyme und sonstigen geographischen Eigennamen, z.B. die der Gewässer wie Ozeane, Meere und Golfe, als n.p. zu klassifizieren, auch wenn sie entweder gar nicht oder aber als Phrasem bzw. geographisches Adjektiv im GRADIT auftreten und dadurch in meinen Listen auf einige durch das Wörterbuch vorgegebene Gebrauchsmarken verzichtet werden musste.

Darüber hinaus sind auch die Exotismen in der überwiegenden Mehrheit nicht mit einer Gebrauchsmarke ausgestattet, sondern nur mit der Marke Es, die im GRADIT gebraucht wird für ,vocaboli avvertiti come stranieri, esotismi non addattati e non inseriti nella morfologia italiana" (De Mauro 1999/2000; m.Ü.: Wörter, die als fremd empfunden werden, Exotismen, die der italienischen Morphologie nicht angepasst und nicht in sie eingefügt sind). Hierbei handelt es sich um eine Kategorie von Wörtern, die den Wandel der Zeit und der kulturellen Gepflogenheiten stärker verzeichnen als andere, wobei ihre Bedeutungen schnell Bestandteil der aufnehmenden Sprache und der dazugehörigen Kultur werden können, so dass sie den Charakter ihrer Fremdartigkeit verlieren. So wurde darauf hingewiesen (De Renzo 2005, S. 227 f.), dass die im GRADIT aufgeführten Fremdwörter keine oder maximal eine Fachwortschatzmarke aufweisen, obwohl sie beispielsweise in den Tageszeitungen stark vertreten sind und auch eine Studie mit Grundschulkindern gezeigt hat, dass ihnen die Bedeutung vieler Fremdwörter bekannt ist. Deshalb liegt es nahe anzunehmen, dass ein Teil der Exotismen de facto inzwischen zum Basiswortschatz oder zumindest zum allgemeinen Wortschatz gerechnet werden kann, was allerdings für die Fremdwörter auf den hier analysierten Seiten (z.B. afrikaans (Bersezio 2005, S. 61)) nicht gelten dürfte.

Auf die eben erwähnten Klassifizierungsabweichungen vom GRADIT sowie einige Auffälligkeiten in der sprachlichen Gestaltung wird in den Wörterlisten und Tabellen im Kommentarfeld hingewiesen. ${ }^{119}$

\subsubsection{Die Seiten 55 und 56 im Nuovo Geolab 3}

Etwas mehr als drei Viertel der Seite 55 nimmt unter der besprochenen Einleitung zum Thema ein Kasten ein, in dem sich ein Text zum Bruttoinlandsprodukt sowie ein Kreisdiagramm zur weltweiten „Produktion“ des Wohlstands, eine Tabelle mit dem Bruttoinlandsprodukt pro Kopf mehrerer Länder und ein Photo befinden, auf dem verschiedene Währungen abgebildet sind. Sowohl das Kreisdiagramm als auch das Photo werden von mehreren das Verständnis unterstützenden Sätzen begleitet.

119 Die vollständige Kategorisierung des Wortvorkommens auf den analysierten Lehrwerkseiten stelle ich Interessierten auf Anfrage gerne zur Verfügung. 
Insgesamt besteht die Seite aus 455 Wörtern, die sich überwiegend auf die beiden Texte der Einleitung und zum BIP verteilen, und von denen 390 die Sätze dieser Seite bilden. Der kürzeste Satz ist aus zwölf und der längste aus 45 Wörtern zusammengesetzt, bei einer mittleren Satzlänge von 24 Wörtern. Zwei Sätze weisen einen Doppelpunkt auf und liegen mit einer Länge von 25 und 30 Wörtern über dem Durchschnitt. Im Haupttext, der aus 187 Wörtern besteht, sind fünf Wörter fettgedruckt, also knapp 3 Prozent. ${ }^{120}$

Wie für einen deskriptiven schriftlichen Text im Gegensatz zu mündlichen Texten üblich, ist der Anteil an verbalen Formen recht gering (s. z.B. Giuliani, Iacobini \& Thornton 2005, S. 211 f.) und die lexikalische Dichte aufgrund einer hohen Anzahl an Substantiven sehr groß. Es handelt sich hierbei um die Wortart mit dem größten Anteil am Wortvorkommen: inklusive Phrasemen (3), Eigennamen (32), Pronomen (19) und Siglen (12) insgesamt 157 Wörter. Zählt man noch die Elemente der Phraseme und Eigennamen hinzu, sind es 172 Wörter und damit 38 Prozent aller Wörter. Zuzüglich der Adjektive (49), Adjektivalphraseme (8) einschließlich ihrer Elemente (8), Artikel (36) und artikulierten Präpositionen (26) sind es 299 Wörter (66 \%).

Allerdings wiederholen sich viele Wörter im Text, was bei Wortarten wie Artikeln, Präpositionen und Konjunktionen selbstverständlich ist. Aber auch diverse Nomen zum Beispiel treten mehrmals im Text auf, so dass 91 substantivische Wortvorkommnisse auf 58 Typen zurückgehen, wobei 18 Wörter mindestens zweimal und davon je vier Wörter drei- bis viermal und fünf- bis sechsmal gebraucht werden. In Tabelle 5 sind diese und die folgenden Analyseergebnisse für die Seiten 55 und 56 illustriert.

\begin{tabular}{lrlrl}
\hline Seite/Kommentar & 55 & Kommentar & 56 & Kommentar \\
\hline Tot. Wörter & 455 & 417 & \\
n Sätze & 16 & 12 & \\
kürzester Satz & 12 & 14 & \\
längster Satz & 45 & 53 & \\
$\varnothing$ Satzlänge & 24 & 29 & \\
Doppelpunkte & 2 & & auch in Überschrift \\
fettgedruckte Wörter & 5 & PIL pro capite, reddito me- & 18 & $4+5$ (Tot. 5 Phraseme) \\
& & dio & 167 & $40 \%$ \\
Substantive u.Ä. & 180 & $38 \%$ & 272 & $65 \%$ \\
Substantive plus Adj. und Art. & 299 & $66 \%$ & 17 & adj., adv., subst., präp., \\
Phraseme & 19 adj., adv., subst., verb. & & verb. Phraseme \\
& & Phraseme; CO, 3 TS & 24 & \\
el.loc. & 21 & 12 & 6 Types (FO) \\
Konjunktionen & 28 9 Types (8 FO, 1 AU) & 1 & essere solito \\
loc.v. & 2 mettere in relazione, fare & & \\
konjugierte Verben & & parte & 33 & \\
Imperative & 30 & & 0 & \\
\hline
\end{tabular}




\begin{tabular}{lrlrl}
\hline Seite/Kommentar & 55 & Kommentar & 56 & Kommentar \\
\hline$\varnothing$ konj. Verben (inkl. Impera- & 1,9 & 2,7 & \\
tive) pro Satz & 0 & & 1 & \\
Gerundien & 12 & & 13 & \\
Infinitive & 3 & 1 attributivisch, 2 satzwertig & 7 & \\
Partizipien & 285 & $63 \%$ & 258 & $62 \%$ \\
FO & 0 & & 0 & \\
el.FO & 0 & & 0 & \\
FO TS & 18 & 25 & \\
AU & 2 & & 5 & \\
AU TS & 5 & 5 & \\
AD & 310 & $68 \%$ & 293 & $70 \%$ \\
Tot. VdB & 0 & & 0 & \\
BU & 48 & 40 & \\
CO & 3 & & 3 & \\
CO TS & 17 & 8 & \\
el.CO & 18 & 33 & \\
TS & 4 & 16 & \\
el.TS & 100 & $22 \%$ & 100 & $24 \%$ \\
Tot. restl. Marken & 410 & $30 \%$ & $94 \%$ & $94 \%$ \\
Tot. Marken insg. & & & & \\
\hline
\end{tabular}

Tabelle 5: Untersuchungsergebnisse zu den Seiten 55 und 56 im Nuovo Geolab (Carazzi \& Pizzetti 2010)

Die Anzahl konjugierter Verben beträgt 30; zwei davon bilden verbale Phraseme. Bei einer Gesamtzahl von 16 Sätzen verteilen sich also durchschnittlich knapp zwei konjugierte Verbformen auf jeden Satz $(1,9)$, zu denen insgesamt zwölf Infinitive und drei Partizipien kommen.

Betrachtet man hingegen die Einteilung der Wörter in die verschiedenen Gruppen des Wortschatzes, so ergibt sich, dass, wie zu erwarten war, der Großteil der Wörter dem Bereich des fundamentalen Wortschatzes (FO) entstammt, nämlich 285 Wörter (63 \%). Mit den 18 Wörtern des hohen Gebrauchs (AU) und den fünf der hohen Disponibilität (AD) sowie den beiden als AU Ts astron. und AU Ts econ. doppelt markierten Wörtern besteht der Anteil an Wörtern aus dem Grundwortschatz aus 310. Das entspricht 68 Prozent und damit einem Wert, der deutlich unter dem für einen Text als typisch angesehenen Anteil von um die 90 Prozent aus dem Bereich des Vocabolario di Base liegt. Der Wert ist auf der untersuchten Seite wesentlich niedriger, auch wenn man als Richtgröße nicht nur den vom Verfasser des GRADITs angegebenen Wert von etwa 90 Prozent an fundamentalen Wörtern in gesprochenen und geschriebenen Texten (De Mauro 1999/2000, Bd. 1, S. XX) und ca. 96 Prozent für die fundamentalen Wörter und die des hohen Gebrauchs heranzieht, sondern auch geringere Angaben wie 80 Prozent Fo-Wortschatz in fachspezifischen Texten (De Renzo 2011, S. 188) berücksichtigt.

Von den übrigen Wörtern entstammen 48 dem allgemeinen Wortschatz (CO), drei sind doppelt markiert als allgemein und technisch-spezifisch (CO Ts 
scient., Co Ts monet.) und weitere 18 Wörter sind rein fachwortschatzsprachlicher Art. Im Einzelnen sind sie aus dem wirtschaftlichen (11), wirtschaftlichsoziologischem (1), physikalischem (1), geographischem (4) und statistischem Bereich (1) zusammengesetzt. Es handelt sich dabei allerdings nicht um 18 verschiedene Ausdrücke, sondern die Mehrheit von ihnen wiederholt sich im Text, wie z.B. ricchezza (Reichtum/Wohlstand), vertreten mit fünf Vorkommnissen, und indicatore (Indikator) mit neun Vorkommnissen. Zusätzliche 21 Wörter sind Elemente von Phraseologismen, die ebenfalls den Bereichen CO und Ts zugeteilt sind. ${ }^{121}$

Seite 56 ist mit zwei untereinander angeordneten Kästen gestaltet, in denen sich jeweils ein Text und ein Schaubild befinden. Der Text des oberen Kastens ist 146 Wörter lang, handelt von der Säuglingssterblichkeit, und neben ihm steht eine Tabelle, in der die Bruttoinlandsprodukte pro Kopf verschiedener Länder zusammen mit ihrer Säuglingssterblichkeitsrate erfasst sind. Sieben Wörter im Text sind fettgedruckt, also knapp 5 Prozent.

Der zweite Kasten besteht aus einem 225 Wörter langen Text zum Human Development Index (HDI) und elf fettgedruckten Wörtern (wieder ca. $5 \%$ ), unter denen sich ein dreigliedriges Phrasem (speranza di vita, Lebenserwartung) sowie die italienische Sigle für den HDI einschließlich ihrer Erklärung befinden. Bei dessen Sigle ISU wie auch bei der Sigle PIL (deutsch BIP) handelt es sich in der ausgeschriebenen Form um Phraseologismen, welche im GRADIT mit einer Ts-Gebrauchsmarke (TS econ. für PIL und Ts stat., polit. für ISU) versehen sind. Diese beiden Beispiele veranschaulichen gut, dass auf den untersuchten Seiten ein gewisser Anteil des Wortvorkommens ohne Gebrauchsmarke (da er sich im Text beispielsweise als Siglen und nicht als ihre ausgeschriebenen Varianten präsentiert) den seltener und nur fachspezifisch benutzten Bereichen des Wortschatzes angehört und für die Lernenden dadurch oft schwieriger zu verstehen, zu behalten und aktiv zu verwenden ist. Zu diesem Aspekt sei auf den letzten Kapitelabschnitt (7.5) sowie auf eine Gegenüberstellung der Begriffsdefinition des BIPs seitens der Lernenden und der Lehrwerke und Lehrenden (Kapitel 9) verwiesen. Zur Veranschaulichung des HDIIndikators gibt es unter dem Text eine thematische Karte, welche als Weltkarte den entsprechenden Wert für alle Länder zeigt. Insgesamt zählt diese Seite 417 Wörter, von denen sich 350 auf zwölf Sätze verteilen. Der kürzeste Satz ist 14 Wörter, der längste Satz 53 Wörter lang, und die durchschnittliche Länge beträgt 29 Wörter. Auch auf dieser Seite sind einige Sätze (3) dadurch, dass sie Doppelpunkte beinhalten, überdurchschnittlich lang.

Der Anteil an Substantiven, Pronomen, Siglen, Eigennamen und nominalen Phrasemen liegt hier bei 146 Wörtern und somit bei 35 Prozent. Zählt man die Elemente der Eigennamen und Phraseme hinzu, sind es 167 Wörter (40 \%);

121 Eine genauere Betrachtung des technisch-spezifischen Wortschatzes und der Phraseme erfolgt in den Unterkapiteln 7.5.1 f. im Vergleich zu den anderen Texten sowie im Kapitel zu den C-Tests. 
inklusive der Adjektive (46), adjektivalen Phraseologismen und ihrer Elemente (2), Artikel (40) und artikulierten Präpositionen (17) 272 Wörter (65 \%).

In den zwölf Sätzen sind 32 Prädikate und ein prädikatives Verbalphrasem enthalten; also kommen auf jeden Satz durchschnittlich fast drei konjugierte Verbformen $(2,7)$ und außerdem insgesamt zwölf Infinitive, ein Gerundium und sieben Partizipien.

Bezüglich der Häufigkeit, mit der die auf dieser Seite verwendeten Wörter im gesprochenen und geschriebenen Italienischen auftreten, lässt sich festhalten, dass 258 Wörter aus dem Bereich des fundamentalen Wortschatzes (Fo) stammen, 30 aus dem des hohen Gebrauchs (AU) (inklusive fünf Vorkommnisse eines Worttyps, der als AU TS econ. doppelt markiert ist) und fünf aus dem der hohen Verfügbarkeit (AD). Also sind hier 293 Wörter aus dem Bereich des Basiswortschatzes und damit nur 70 Prozent vertreten. Von den übrigen 124 Wörtern sind 24 Eigennamen sowie Siglen und 40 Teil des allgemeinen Wortschatzes (CO), drei tragen die doppelte Marke Co Ts scient./Co Ts med., weitere acht sind Elemente aus Co-Phraseologismen, und 33 Wörter entstammen den technisch-spezifischen Wortschätzen. Im Detail sind es zwölf des Ts econ., zwei des Ts econ., sociol., zwei des Ts fis., mat., 15 des Ts stat. und zwei des Ts stat., polit., mit insgesamt 16 Elementen. Aber auch bei den 100 Co- und TS-gekennzeichneten Wörtern handelt es sich wieder nicht um entsprechend viele verschiedene Worttypen, sondern einige dieser Ausdrücke sind mit mehreren Vorkommnissen vertreten, wie Tabelle 6 veranschaulicht: Am Beispiel aller 26 Worttypen aus dem Bereich des allgemeinen Wortschatzes (CO) und der 13 aus den Ts-Wortschätzen wird zum einen deutlich, wie sie durch ihr vermehrtes Vorkommen mindestens 72 Wörter abdecken, die über den Basiswortschatz hinausgehen, und zum anderen, dass es im Bereich des allgemeinen Wortschatzes, der ca. 47.000 Wörter umfasst, seltener ist, dass ein und dasselbe Wort mehrmals verwendet wird ${ }^{122}$. Dadurch unterscheidet sich dieser Teil des Gebrauchswortschatzes stark vom 7.000 Wörter umfassenden Grundwortschatz, dessen innersterer Kern der ca. 2.000 häufigsten Wörter des Italienischen allein schon um die 90 Prozent eines Textvolumens ausmacht.

Auch im Ts-Bereich sind Wiederholungen relativ frequent (vgl. auch Seite 55), was mit der Natur des Fachwortschatzes und der Texte, in denen er hier verwendet wird, erklärbar ist: Es geht um die Behandlung sozioökonomischer Konzepte, deren Bezeichnungen aus dem Fachwortschatz nicht gut durch Synonyme wiedergegeben werden können, so dass sie mit mehreren Vorkommnissen im Text erscheinen. Das entspricht der deskriptiven und klassifizierenden Funktion der fachwortschatzsprachlichen Elemente, bspw. einen Gegenstand oder Sachverhalt exakt zu bezeichnen, während VDB- und Co-Wörter

122 Konkret ist das bei den Co-Wörtern auf dieser Seite nur bei zwei Wörtern der Fall, bei denen man sich sowieso wundern kann, warum sie dem allgemeinen Wortschatz und nicht dem fundamentalen zugeteilt sind: der maskuline Pluralartikel $i / g l i$ und das Reflexivpronomen $s i$ (sich) der dritten Person Singular und Plural. 
überwiegend polysemisch sind und durch sinnverwandte Wörter ersetzt werden können.

\begin{tabular}{|c|c|c|c|c|}
\hline Worttyp & Wortart & Marke & Tokens & Kommentar/Übersetzung \\
\hline rapportato & part.pass. & $\mathrm{CO}$ & 1 & bezogen (auf) \\
\hline abitativo & agg. & $\mathrm{CO}$ & 1 & Wohn- \\
\hline potabile & agg. & $\mathrm{CO}$ & 1 & trinkbar \\
\hline squilibrato & agg. & $\mathrm{CO}$ & 1 & gestört/unausgeglichen \\
\hline ristretto & agg. & $\mathrm{CO}$ & 1 & beschränkt \\
\hline elevato & agg. & $\mathrm{CO}$ & 2 & hoch/erhaben \\
\hline equilibrato & agg. & $\mathrm{CO}$ & 1 & ausgeglichen \\
\hline determinato & agg. & $\mathrm{CO}$ & 1 & bestimmt \\
\hline tendente & agg. & $\mathrm{CO}$ & 1 & tendierend \\
\hline i/gli & art.det. & $\mathrm{CO}$ & 6 & die \\
\hline completamente & avv. & $\mathrm{CO}$ & 1 & vollkommen \\
\hline $\begin{array}{l}\text { contemporanea- } \\
\text { mente }\end{array}$ & avv. & $\mathrm{CO}$ & 1 & gleichzeitig \\
\hline esservi & inf.v.procompl. & $\mathrm{CO}$ & 1 & da sein, geben \\
\hline aspettarsi & $\begin{array}{l}\text { inf.v.pro- } \\
\text { nom.intr. }\end{array}$ & $\mathrm{CO}$ & 1 & erwarten \\
\hline pro capite & loc.agg.inv. & $\mathrm{CO}$ & 1 & pro Kopf \\
\hline in particolare & loc.avv. & $\mathrm{CO}$ & 1 & besonders \\
\hline vicino a & loc.prep. & $\mathrm{CO}$ & 1 & in der Nähe von \\
\hline livello di vita & loc.s.m. & $\mathrm{CO}$ & 1 & Lebensstandard \\
\hline essere solito & loc.v. & $\mathrm{CO}$ & 1 & gewohnt sein, pflegen \\
\hline si & pron.pers. & $\mathrm{CO}$ & 6 & $\begin{array}{l}\text { sich; } 3 \text { davon Teil eines } \\
\text { v.pronom.intr. }\end{array}$ \\
\hline sopravvivenza & s.f. & $\mathrm{CO}$ & 1 & Überleben \\
\hline incidenza & s.f. & $\mathrm{CO}$ & 1 & Einfluss \\
\hline disuguaglianza & s.f. & $\mathrm{CO}$ & 1 & Ungleichheit \\
\hline scala & s.f. & $\mathrm{CO}$ & 1 & Skala, Sequenz \\
\hline trovarsi & v.pronom.intr. & $\mathrm{CO}$ & 1 & sich befinden \\
\hline verificarsi & v.pronom.intr. & $\mathrm{CO}$ & 1 & sich ereignen \\
\hline Tot. CO & 26 & Tot. Tokens & 37 & \\
\hline povertà & s.f. & TS econ. & 6 & Armut \\
\hline ricchezza & s.f. & TS econ. & 2 & Reichtum \\
\hline indicatore & s.m. & TS econ. & 4 & Indikator \\
\hline reddito & s.m. & TS econ. & 1 & Einkommen \\
\hline sviluppo & s.m. & $\begin{array}{l}\text { TS econ., } \\
\text { sociol. }\end{array}$ & 2 & Entwicklung \\
\hline valore & s.m. & TS fis., mat. & 2 & Wert \\
\hline mortalità infantile & loc.s.f. & TS stat. & 6 & Kindersterblichkeit \\
\hline speranza di vita & loc.s.f. & TS stat. & 2 & Lebenserwartung \\
\hline graduatoria & s.f. & TS stat. & 2 & Rangfolge \\
\hline indice & s.m. & TS stat. & 5 & Index \\
\hline $\begin{array}{l}\text { Indice di Sviluppo } \\
\text { Umano }\end{array}$ & loc.s.m. & $\begin{array}{l}\text { TS stat., } \\
\text { polit. }\end{array}$ & 2 & Human Development Index \\
\hline Tot. TS & 13 & Tot. Tokens & 37 & \\
\hline Tot. $\mathrm{CO}+\mathrm{TS}$ & 41 & Tot. Tokens & $70(-3)$ & \\
\hline
\end{tabular}

Tabelle 6: Wörter des allgemeinen (CO) und des technisch-spezifischen Wortschatzes (Ts) auf S. 56 im Nuovo Geolab (Carazzi \& Pizzetti 2010) 


\subsubsection{Die übrigen untersuchten Seiten des Nuovo Geolab}

Die sprachliche Zusammensetzung der weiteren untersuchten Seiten des Nuovo Geolab wird aus Tabelle 7 ersichtlich; die in den Kommentarspalten angegebenen Prozentwerte beziehen sich dabei auf den jeweiligen Anteil einer Untersuchungseinheit am Gesamtwortvorkommen der einzelnen Seiten.

\begin{tabular}{|c|c|c|c|c|c|c|c|c|c|c|}
\hline $\begin{array}{l}\text { Seite/Kom- } \\
\text { mentar (K) }\end{array}$ & 62 & K1 & 68 & K2 & $134 \mathrm{f}$. & K3 & 319 & K4 & 320 & K5 \\
\hline $\begin{array}{l}\text { Tot. Wör- } \\
\text { ter }\end{array}$ & 298 & & 335 & & 422 & $\begin{array}{l}\text { Feh- } \\
\text { ler: } 3 \\
\text { Wör- } \\
\text { ter } \\
\text { dop- } \\
\text { pelt }\end{array}$ & 234 & & 279 & \\
\hline Sätze & 9 & & 17 & & 18 & & 13 & $\begin{array}{l}\text { Groß- } \\
\text { schrei- } \\
\text { bung } \\
\text { nach ,:." > } \\
\text { neuer } \\
\text { Satz }\end{array}$ & 14 & \\
\hline $\begin{array}{l}\text { kürzester } \\
\text { Satz }\end{array}$ & 10 & & 4 & $\begin{array}{l}\text { Bildbe- } \\
\text { schrei- } \\
\text { bung; im } \\
\text { Text: } 8\end{array}$ & 9 & & 4 & $\begin{array}{l}\text { Aufgabe; } \\
\text { im Text: } \\
10\end{array}$ & 5 & $\operatorname{im}_{\text {Text }}$ \\
\hline längster & 45 & & 46 & & 49 & & 29 & & 30 & \\
\hline $\begin{array}{l}\text { Satz } \\
\varnothing \text { Satz- } \\
\text { länge }\end{array}$ & 25 & & 18 & & 21 & & 15 & $\begin{array}{l}\text { Aufga- } \\
\text { ben: } \\
\text { kurze } \\
\text { Fragen }\end{array}$ & 16 & \\
\hline $\begin{array}{l}\text { Doppel- } \\
\text { punkte }\end{array}$ & 2 & & 0 & & 4 & & 1 & & 0 & \\
\hline $\begin{array}{l}\text { fettge- } \\
\text { druckte } \\
\text { Wörter }\end{array}$ & 9 & & 31 & $10+21$ & 49 & $14+35$ & 8 & $\begin{array}{l}2 \text { Phra- } \\
\text { seme }\end{array}$ & 25 & $19+6$ \\
\hline $\begin{array}{l}\text { Substan- } \\
\text { tive u.Ä. }\end{array}$ & 104 & $35 \%$ & 143 & $43 \%$ & 195 & $46 \%$ & 91 & $39 \%$ & 112 & $40 \%$ \\
\hline $\begin{array}{l}\text { Substan- } \\
\text { tive plus } \\
\text { Adj. und } \\
\text { Art. }\end{array}$ & 200 & $67 \%$ & 224 & $67 \%$ & 316 & $75 \%$ & 164 & $70 \%$ & 194 & $70 \%$ \\
\hline Phraseme & 10 & alle CO & 20 & $\mathrm{CO}+\mathrm{TS}$ & 6 & $\begin{array}{l}\mathrm{CO}+ \\
\mathrm{TS}\end{array}$ & 3 & $\mathrm{CO}+\mathrm{TS}$ & 11 & $\begin{array}{l}\mathrm{CO}+ \\
\mathrm{TS}\end{array}$ \\
\hline el.loc. & 12 & & 23 & & 6 & $\mathrm{x}$ & 3 & & 13 & \\
\hline $\begin{array}{l}\text { Konjunkti- } \\
\text { onen }\end{array}$ & 18 & $\begin{array}{l}7 \text { Ty- } \\
\text { pes } \\
\text { (FO); } \\
11 e\end{array}$ & 22 & $\begin{array}{l}2 \mathrm{CO} \\
\text { (pur) }\end{array}$ & 22 & $\begin{array}{l}\text { nur } \\
\text { FO; } 4 \\
\text { Types }\end{array}$ & 13 & $\begin{array}{l}5 \text { Types; } \\
1 \text { CO } \\
\text { (pur) }\end{array}$ & 20 & $\begin{array}{l}5 \text { Ty- } \\
\text { pes; } 2 \\
\text { AU } \\
\text { (però) }\end{array}$ \\
\hline loc.v. & 1 & $\begin{array}{l}2 \\
\text { el.loc.v. }\end{array}$ & 0 & & 1 & & 0 & & 0 & \\
\hline $\begin{array}{l}\text { konjugierte } \\
\text { Verben }\end{array}$ & 23 & & 26 & & 27 & & 17 & & 18 & \\
\hline Imperative & 1 & & 0 & & 0 & & 2 & & 1 & \\
\hline $\begin{array}{l}\varnothing \text { konju- } \\
\text { gierte Ver- } \\
\text { ben inkl. }\end{array}$ & 2,9 & & 1,5 & & 1,5 & & 1,5 & & 1,4 & \\
\hline
\end{tabular}




\begin{tabular}{|c|c|c|c|c|c|c|c|c|c|c|}
\hline $\begin{array}{l}\text { Seite/Kom- } \\
\text { mentar (K) }\end{array}$ & 62 & K1 & 68 & K2 & $134 \mathrm{f}$. & K3 & 319 & K4 & 320 & K5 \\
\hline \multicolumn{11}{|l|}{$\begin{array}{l}\text { Imperative } \\
\text { pro Satz }\end{array}$} \\
\hline Gerundien & 1 & & 6 & $x$ & 0 & & 1 & $\begin{array}{l}\text { Prät.: } \\
\text { avendo } \\
\text { passato }\end{array}$ & 1 & \\
\hline Infinitive & 5 & & 3 & $x$ & 0 & & 2 & & 3 & \\
\hline Partizipien & 2 & & 2 & $x$ & 5 & & 1 & & 2 & \\
\hline $\mathrm{FO}$ & 188 & $63 \%$ & 180 & $54 \%$ & 236 & $56 \%$ & 155 & $66 \%$ & 167 & $60 \%$ \\
\hline el.FO & 0 & & 0 & & 0 & & 0 & & 0 & \\
\hline FO TS & 0 & & 0 & & 1 & & 0 & & 0 & \\
\hline $\mathrm{AU}$ & 27 & & 26 & & 26 & & 18 & & 30 & \\
\hline AU TS & 2 & & 0 & & 0 & & 0 & & 1 & \\
\hline$A D$ & 2 & & 7 & & 7 & & 1 & & 8 & \\
\hline Tot. VdB & 217 & $73 \%$ & 210 & $63 \%$ & 270 & $64 \%$ & 174 & $74 \%$ & 206 & $74 \%$ \\
\hline $\mathrm{BU}$ & 0 & & 1 & $\begin{array}{l}\text { consuma- } \\
\text { tori (agg.) }\end{array}$ & 0 & & 0 & & 0 & \\
\hline $\mathrm{CO}$ & 41 & & 56 & & 44 & & 27 & $\begin{array}{l}\text { inkl. Wort } \\
\text { o.M. } \\
\text { (geocom- } \\
\text { petenza: } \\
\text { compe- } \\
\text { tenza = } \\
\text { CO) }\end{array}$ & 27 & \\
\hline CO TS & 2 & & 3 & & 2 & & 1 & & 2 & \\
\hline el.CO & 12 & & 13 & & 4 & & 0 & & 4 & \\
\hline TS & 5 & & 13 & & 18 & $\begin{array}{l}\text { ohne } \\
\text { Sigle } \\
\text { o. M. } \\
\text { (ISU: } \\
\text { als } \\
\text { Phra- } \\
\text { sem } \\
\text { TS) }\end{array}$ & 9 & & 14 & $\begin{array}{l}\text { ohne } \\
2 \\
\text { Siglen } \\
\text { o. M. } \\
\text { (als } \\
\text { Phra- } \\
\text { seme } \\
\text { TS) }\end{array}$ \\
\hline el.TS & 0 & & 10 & & 1 & & 3 & & 9 & \\
\hline $\begin{array}{l}\text { Tot. restl. } \\
\text { Marken }\end{array}$ & 60 & $20 \%$ & 96 & $29 \%$ & 69 & $16 \%$ & 40 & $17 \%$ & 56 & $20 \%$ \\
\hline $\begin{array}{l}\text { Tot. Mar- } \\
\text { ken insg. }\end{array}$ & 277 & $93 \%$ & 306 & $92 \%$ & 408 & $97 \%$ & 214 & $91 \%$ & 262 & $94 \%$ \\
\hline
\end{tabular}

Tabelle 7: Untersuchungsergebnisse zu den Seiten 62, 68, 134 f., 319 und 320 im Nuovo Geolab (Carazzi \& Pizzetti 2010)

Durchschnittlich befinden sich auf allen acht Seiten dieses Lehrbuchs 305 Wörter (2.440 insgesamt). Während für die Beschreibungen der Abbildungen in der Regel nur wenige verwendet werden und bei Frage- oder Aufgabenstellungen oft das Prädikat und Satzglieder der ersten Frage in den folgenden impliziert werden, sind sie in den Texten nur in Ausnahmefällen in sehr kurzen Sätzen angeordnet. Im Mittelwert sind die Sätze 21 Wörter lang und weisen durchschnittlich knapp zwei konjugierte Verbformen $(1,9)$ auf, an die sich vor allem Infinitive, aber auch Gerundien und Partizipien anschließen.

Der Anteil an Substantiven inklusive Artikel und Adjektive liegt bei 69 Prozent, und den gleichen Prozentwert erzielt auch der Anteil an Wörtern aus 
dem Basiswortschatz (VDB) am Gesamtvorkommen der Wörter aller Seiten. Der niedrigste Wert liegt hier bei 63 Prozent und der höchste bei 74 Prozent, womit Letzterer immer noch deutlich geringer ist als die ca. 96 Prozent, zu denen ein jeder Text aus dem Basiswortschatz bestehen soll.

Im Durchschnitt sind nur 93 Prozent der Wörter einer Seite mit einer Gebrauchsmarke versehen; bei den restlichen sieben Prozent handelt es sich zum einen um Eigennamen und zum anderen um Siglen. Hierbei haben die Seiten $134 \mathrm{f}$. mit landeskundlichem Inhalt und dadurch mit vielen geographischen Eigennamen mit 20 Prozent den höchsten Anteil an markenlosen Ausdrücken und die Seiten 55, 68 und 319 mit mehr theoretisch-konzeptuellem Inhalt zu sozioökonomischen und energetischen Aspekten den geringsten Anteil an Wörtern, die nicht mit einer Gebrauchsmarke versehen sind (93-94 \%).

\subsubsection{Das Lehrbuch I nuovi territori dell'uomo an der Morante}

Einen Überblick über die sprachliche Gestaltung der insgesamt 2.364 Wörter auf den untersuchten Seiten des in der Klasse der Schule Morante verwendeten Unterrichtswerks ermöglicht die Tabelle 9 (K steht für Kommentar).

\begin{tabular}{|c|c|c|c|c|c|c|c|c|c|c|}
\hline Seite/K & 32 & K1 & 33 & K2 & $58-60$ & K3 & 61 & K4 & $82 \mathrm{f}$. & K5 \\
\hline $\begin{array}{l}\text { n Wör- } \\
\text { ter }\end{array}$ & 364 & & 377 & & 582 & $\begin{array}{l}\text { Fehler: ein } \\
\text { Adj. zu viel }\end{array}$ & 386 & & 654 & \\
\hline Sätze & 13 & & 19 & & 29 & & 20 & & 28 & \\
\hline $\begin{array}{l}\text { kürzes- } \\
\text { ter Satz }\end{array}$ & 11 & & 5 & $\begin{array}{l}\text { Bildbe- } \\
\text { schrei- } \\
\text { bung; im } \\
\text { Text: } 8\end{array}$ & 7 & im Text & 9 & & 5 & $\begin{array}{l}\text { im } \\
\text { Text }\end{array}$ \\
\hline $\begin{array}{l}\text { längster } \\
\text { Satz }\end{array}$ & 53 & & 27 & & 33 & & 33 & & 43 & \\
\hline $\begin{array}{l}\varnothing \text { Satz- } \\
\text { länge }\end{array}$ & 27 & & 17 & & 17 & & 18 & & 21 & \\
\hline $\begin{array}{l}\text { Doppel- } \\
\text { punkte }\end{array}$ & 3 & & 3 & & 1 & & 1 & & 3 & \\
\hline $\begin{array}{l}\text { fettge- } \\
\text { druckte } \\
\text { Wörter }\end{array}$ & 3 & $\begin{array}{l}3+0 ; \\
\text { Eigen- } \\
\text { namen } \\
\text { (einer } \\
\text { zwei- } \\
\text { teilig) }\end{array}$ & 4 & $0+2+2+0$ & 14 & $3+3+2+4+2$ & 13 & $0+11+2+0$ & 19 & $8+2+9$ \\
\hline $\begin{array}{l}\text { Sub- } \\
\text { stantive } \\
\text { u. A. }\end{array}$ & 112 & $31 \%$ & 145 & $41 \%$ & 265 & $46 \%$ & 129 & $33 \%$ & 262 & $40 \%$ \\
\hline $\begin{array}{l}\text { Sub- } \\
\text { stantive } \\
\text { plus } \\
\text { Adj. } \\
\text { und Art. }\end{array}$ & 223 & $61 \%$ & 267 & $73 \%$ & 436 & $75 \%$ & 258 & $67 \%$ & 450 & $69 \%$ \\
\hline $\begin{array}{l}\text { Phra- } \\
\text { seme }\end{array}$ & 10 & $\begin{array}{l}5 \text { abbr. } \\
\text { (a.C.) }\end{array}$ & 11 & & 14 & & 2 & & 15 & $\begin{array}{l}2 \text { abbr. } \\
\text { (a.C.) }\end{array}$ \\
\hline el.loc. & 6 & & 15 & & 16 & & 2 & & 21 & \\
\hline
\end{tabular}




\begin{tabular}{|c|c|c|c|c|c|c|c|c|c|c|}
\hline Seite/K & 32 & K1 & 33 & K2 & $58-60$ & K3 & 61 & K4 & $82 \mathrm{f}$. & K5 \\
\hline $\begin{array}{l}\text { Kon- } \\
\text { junktio- } \\
\text { nen }\end{array}$ & 25 & $\begin{array}{l}9 \text { Ty- } \\
\text { pes; } 2 \\
\text { CO }\end{array}$ & 17 & $\begin{array}{l}5 \text { Types; } \\
1 \text { loc. } \\
\text { cO } \\
\text { (dopo } \\
\text { che) }\end{array}$ & 24 & 6 Types & 24 & 6 Types & 31 & $\begin{array}{l}5 \text { Ty- } \\
\text { pes; } 1 \\
\text { AU } \\
\text { (però) }\end{array}$ \\
\hline loc.v. & 2 & $\begin{array}{l}1: 2 \\
\text { elloc }\end{array}$ & 0 & & 0 & & 0 & & 2 & \\
\hline $\begin{array}{l}\text { konju- } \\
\text { gierte } \\
\text { Verben }\end{array}$ & 29 & & 25 & & 40 & & 37 & & 44 & \\
\hline $\begin{array}{l}\text { Impera- } \\
\text { tive }\end{array}$ & 0 & & 1 & & 1 & & 0 & & 0 & \\
\hline $\begin{array}{l}\varnothing \text { kon- } \\
\text { jugierte } \\
\text { Verben } \\
\text { inkl. Im- } \\
\text { perative } \\
\text { pro } \\
\text { Satz }\end{array}$ & 2,2 & & 1,4 & & 1,4 & & 2 & & 1,6 & \\
\hline $\begin{array}{l}\text { Gerun- } \\
\text { dien }\end{array}$ & 0 & & 1 & & 0 & & 0 & & 1 & \\
\hline $\begin{array}{l}\text { Infini- } \\
\text { tive }\end{array}$ & 2 & & 4 & & 1 & & 2 & $\begin{array}{l}2 \\
\text { inf.pass. }\end{array}$ & 4 & \\
\hline $\begin{array}{l}\text { Partizi- } \\
\text { pien }\end{array}$ & 2 & & 2 & & 3 & & 2 & & 0 & \\
\hline FO & 237 & $65 \%$ & 220 & $58 \%$ & 325 & $56 \%$ & 234 & $61 \%$ & 372 & $57 \%$ \\
\hline el.FO & 0 & & 0 & & 0 & & 0 & & 0 & \\
\hline FO TS & 1 & & 0 & & 1 & & 1 & & 2 & \\
\hline $\mathrm{AU}$ & 32 & & 36 & & 60 & & 39 & & 48 & \\
\hline AU TS & 0 & & 2 & & 0 & & 0 & & 0 & \\
\hline$A D$ & 3 & & 8 & & 8 & & 5 & & 14 & \\
\hline AD TS & 0 & & 0 & & 0 & & 1 & & 0 & \\
\hline $\begin{array}{l}\text { Tot. } \\
\text { VdB }\end{array}$ & 273 & $75 \%$ & 266 & $71 \%$ & 394 & $68 \%$ & 280 & $73 \%$ & 436 & $67 \%$ \\
\hline $\mathrm{BU}$ & 0 & & 0 & & 0 & & 0 & & 0 & \\
\hline $\mathrm{CO}$ & 42 & & 54 & & 66 & & 69 & & 91 & \\
\hline el.CO & 4 & & 8 & & 12 & & 2 & & 20 & \\
\hline CO TS & 0 & & 3 & & 5 & & 2 & & 1 & \\
\hline $\begin{array}{l}\text { el.CO } \\
\text { TS }\end{array}$ & 0 & & 0 & & 0 & & 0 & & 0 & \\
\hline TS & 11 & & 21 & & 19 & & 18 & & 16 & $\begin{array}{l}\text { dop- } \\
\text { pelte } \\
\text { Marke } \\
\text { (TS } \\
\text { und } \\
\text { ES): } \\
\text { cricket }\end{array}$ \\
\hline el.TS & 2 & & 7 & & 4 & & 1 & & 0 & \\
\hline LE & 0 & & 0 & & 0 & & 1 & genti & 0 & \\
\hline ES & 0 & & 0 & & 0 & & 2 & $\begin{array}{l}\text { doppelte } \\
\text { Marke } \\
\text { (TS und } \\
\text { ES): kraal }\end{array}$ & 2 & $\begin{array}{l}\text { dop- } \\
\text { pelte } \\
\text { Marke } \\
\text { (TS } \\
\text { und } \\
\text { ES): } \\
\text { cricket }\end{array}$ \\
\hline
\end{tabular}




\begin{tabular}{|c|c|c|c|c|c|c|c|c|c|c|}
\hline Seite/K & 32 & K1 & 33 & K2 & $58-60$ & K3 & 61 & K4 & $82 \mathrm{f}$. & K5 \\
\hline $\begin{array}{l}\text { nicht im } \\
\text { GRADIT } \\
\text { (ES; TS } \\
\text { etnol.) }\end{array}$ & 1 & $\begin{array}{l}\text { ezba- } \\
\text { hin }\end{array}$ & 0 & & 0 & & 4 & & 0 & \\
\hline $\begin{array}{l}\text { Tot. } \\
\text { restl. } \\
\text { Marken }\end{array}$ & 59 & $16 \%$ & 93 & $25 \%$ & 106 & $18 \%$ & 95 & $25 \%$ & 128 & $20 \%$ \\
\hline $\begin{array}{l}\text { Tot. } \\
\text { Marken } \\
\text { insg. }\end{array}$ & 332 & $91 \%$ & 359 & $95 \%$ & 500 & $86 \%$ & 375 & $98 \%$ & & $87 \%$ \\
\hline
\end{tabular}

Tabelle 8: Untersuchungsergebnisse zu den Seiten aus Nuovi Territori dell'uomo (Bersezio 2005)

Durchschnittlich befinden sich auf den analysierten Seiten des Lehrwerks von Bersezio (2005) 295 Wörter. Sie entstammen zu mittleren 71 Prozent dem Basiswortschatz, wobei dieser mit 75 Prozent auf Seite 32 (Bevölkerung und Urbanistik; Völker und Kulturen in Ägypten) am höchsten und auf Seite $82 \mathrm{f}$. (Asiens Geschichte und jüngste Konflikte) mit nur 66 Prozent am niedrigsten ist. Der Anteil aus dem weniger häufig gebrauchten Wortschatz beläuft sich in den Texten auf 21 Prozent, so dass durchschnittlich acht Prozent des Wortvorkommens keine Gebrauchsmarke aufweisen. Am meisten Wörter ohne Marke gibt es mit 14 Prozent auf den Seiten 58 bis 60, was aufgrund des landeskundlichen Inhalts (Präsentation Südafrikas) am erhöhten Vorkommen von Eigennamen liegt. Nur zwei Prozent markenlose Ausdrücke lassen sich hingegen auf Seite 61 finden, wo es um die Demographie Südafrikas geht und sich darüber hinaus ein Text zur Vertiefung des behandelten Themas befindet (die Beschreibung eines Dorfes der Zulu; solche Kästen unter dem Titel approfondimento findet man in diesem Lehrbuch in jedem Kapitel).

Die durchschnittliche Satzlänge beträgt 20 Wörter, wobei auf jeden Satz ca. 1,7 konjugierte Verbformen entfallen. Der Mittelwert des Anteils an einund mehrgliedrigen Substantiven zuzüglich Adjektive und Artikel am gesamten Wortvorkommen liegt bei 69 Prozent.

Auffällig an den untersuchten Seiten sind ein Konstruktionsfehler durch ein Possessivadjektiv, das auf Seite 59 im ersten Satz durch das folgende Genitivattribut „der Region Südafrikas“ (m.Ü.) grammatikalisch-syntaktisch nicht korrekt platziert ist: „La sua situazione climatico-ambientale della regione dell'Africa meridionale è molto varia. “ (Bersezio 2005, S. 59; m.Ü.: Ihre klimatische und umweltbezogene Situation der Region Südafrikas ist sehr unterschiedlich). Ebenfalls auffällig ist die Verwendung des Wortes genti (ibid., S. 61; Volksstämme) mit der Marke $\mathrm{LE}^{123}$ und die doppelte Markierung eines Fremdworts. Es ist nämlich sehr selten, dass ihnen noch eine zusätzliche Marke

123 Im GRADIT lautet die Beschreibung des literarisch gebrauchten Teils des Wortschatzes (LE): „vocaboli di uso solo letterario, noti a chi ha più dimestichezza con i classici della nostra letteratura" (m.Ü.: Wörter rein literarischen Gebrauchs, welche denjenigen bekannt sind, denen die Klassiker unserer Literatur geläufiger sind). 
zugeteilt ist, die mehr über ihre Verwendung aussagt. Hierbei handelt es sich um das Wort kraal (ibid.), das im GRADIT als Exotismus (ES) dem ethnologischen Fachwortschatz zugeteilt ist und den kreisförmig angeordneten Pferch in Dörfern afrikanischer Stämme bezeichnet.

\subsubsection{Zaino in spalla der Klasse III A an der Schule Pestalozzi}

Die acht untersuchten Seiten des in der III A verwendeten Lehrbuchs beinhalten durchschnittlich 274 Wörter (insg. 2.202 Wörter), die zu 67 Prozent aus ein- und mehrgliedrigen Substantiven, Adjektiven und Artikeln bestehen. Mittlere 74 Prozent des Wortvorkommens entspringen dem Basiswortschatz und 21 Prozent den weniger häufigen Wortschatzbereichen. Die Seiten 206 f., die zu Kapitelbeginn eine Einführung in den asiatischen Kontinent geben und eine geographische Einordnung vornehmen, weisen mit 65 Prozent den geringsten Basiswortschatzanteil auf, während Seite 182 mit ihrem soziokulturellen Inhalt zu den verschiedenen Sprachfamilien der Welt und ihrer Verbreitung den höchsten Basiswortschatzanteil (80 \%) verzeichnet.

Insgesamt sind durchschnittlich 94 Prozent der Ausdrücke mit einer Gebrauchsmarke versehen, wobei fünf Seiten Werte von mindestens 97 Prozent (eine sogar $100 \%$ ) registrieren und nur drei Seiten mit 86 bis 87 Prozent geringere Werte. Es handelt sich hier um die schon erwähnten Seiten $206 \mathrm{f}$. mit landeskundlichem Inhalt sowie um einen Text, der sich mit internationalen Handelsabkommen beschäftigt, deren Namen als Siglen bzw. ausgeschrieben als Eigennamen keine Gebrauchsmarken zugeteilt sind.

Die mittlere Anzahl an Sätzen pro Seite beträgt zehn bei einer Länge von 24 Wörtern, die durch 2,4 konjugierte Verbformen zusammengehalten werden. Diese und weitere Untersuchungsergebnisse sind in tabellarischer Form dargestellt (Tab. 9; K steht für Kommentar), woran sich die Vorstellung einiger sprachlicher Besonderheiten des Lehrbuchs anschließt.

\begin{tabular}{|c|c|c|c|c|c|c|c|c|}
\hline Seite/K & $87 \quad \mathrm{~K} 1$ & $\begin{array}{rl}116 & K 2 \\
\text { f. } & \end{array}$ & $118 \mathrm{~K} 3$ & 149 & K4 & 182 & K5 & $\begin{array}{rl}206 & K 6 \\
f . & \end{array}$ \\
\hline $\begin{array}{l}\text { n Wör- } \\
\text { ter }\end{array}$ & 248 & 598 & 326 & 404 & & 352 & & 274 \\
\hline Sätze & 9 & 12 & 19 & 10 & & 17 & & 11 \\
\hline $\begin{array}{l}\text { kürzes- } \\
\text { ter } \\
\text { Satz }\end{array}$ & 12 & 5 & 5 & 5 & $\begin{array}{l}\text { Bildbe- } \\
\text { schrei- } \\
\text { bung } \\
\text { (ohne } \\
\text { Prädi- } \\
\text { kat); im } \\
\text { Text: } \\
20\end{array}$ & 3 & $\begin{array}{l}\text { Auf- } \\
\text { gabe; } \\
\text { im } \\
\text { Text: } \\
11\end{array}$ & 8 \\
\hline $\begin{array}{l}\text { längs- } \\
\text { ter } \\
\text { Satz }\end{array}$ & 36 & 46 & 46 & 201 & & 35 & & 41 \\
\hline
\end{tabular}




\begin{tabular}{|c|c|c|c|c|c|c|c|c|c|c|c|c|}
\hline Seite/K & 87 & K1 & $\begin{array}{r}116 \\
\text { f. }\end{array}$ & K2 & 118 & K3 & 149 & K4 & 182 & K5 & $\begin{array}{r}206 \\
\text { f. }\end{array}$ & K6 \\
\hline $\begin{array}{l}\text { ø Satz- } \\
\text { länqe }\end{array}$ & 22 & & 23 & & 23 & & 35 & & 17 & & 23 & \\
\hline $\begin{array}{l}\text { Dop- } \\
\text { pel- } \\
\text { punkte }\end{array}$ & 2 & & 0 & & 0 & & 2 & & 1 & & 2 & \\
\hline $\begin{array}{l}\text { fettge- } \\
\text { druckte } \\
\text { Wörter }\end{array}$ & 9 & & 23 & $\begin{array}{l}12+13+ \\
\text { Fragen }\end{array}$ & 23 & $\begin{array}{l}\text { plus } \\
\text { Fra- } \\
\text { gen }\end{array}$ & 18 & $\begin{array}{l}\text { plus } \\
\text { Fragen }\end{array}$ & 5 & $\begin{array}{l}\text { plus } \\
\text { Fra- } \\
\text { gen }\end{array}$ & 22 & $10+12$ \\
\hline $\begin{array}{l}\text { Sub- } \\
\text { stan- } \\
\text { tive } \\
\text { u.Ä. }\end{array}$ & 98 & $40 \%$ & 249 & $42 \%$ & 116 & $36 \%$ & 134 & $33 \%$ & 127 & $36 \%$ & 114 & $42 \%$ \\
\hline $\begin{array}{l}\text { Sub- } \\
\text { stan- } \\
\text { tive } \\
\text { plus } \\
\text { Adj. } \\
\text { und } \\
\text { Art. }\end{array}$ & 164 & $66 \%$ & 425 & $71 \%$ & 215 & $66 \%$ & 266 & $66 \%$ & 223 & $63 \%$ & 191 & $70 \%$ \\
\hline $\begin{array}{l}\text { Phra- } \\
\text { seme }\end{array}$ & 7 & & 15 & & 8 & & 15 & & 5 & & 8 & \\
\hline el.loc. & 7 & & 20 & & 11 & & 21 & & 5 & & 10 & \\
\hline $\begin{array}{l}\text { Kon- } \\
\text { junktio- } \\
\text { nen }\end{array}$ & 16 & $\begin{array}{l}\text { nur } \\
\text { FO, } \\
4 \text { Ty- } \\
\text { pes }\end{array}$ & 29 & $\begin{array}{l}6 \mathrm{Ty}- \\
\text { pes; } 1 \\
\text { loc. CO } \\
\text { (per di } \\
\text { più) } \\
+1 \mathrm{AU}\end{array}$ & 21 & $\begin{array}{l}6 \text { Ty- } \\
\text { pes } \\
\text { (FO) }\end{array}$ & 18 & $\begin{array}{l}6 \text { Ty- } \\
\text { pes; } 2 \\
\text { loc. Co } \\
\text { (di con- } \\
\text { se- } \\
\text { guenza, } \\
\text { per cui) }\end{array}$ & 22 & $\begin{array}{l}\text { nur } \\
\text { FO, } 9 \\
\text { Ty- } \\
\text { pes }\end{array}$ & 19 & $\begin{array}{l}\text { nur } \\
\text { FO, } 2 \\
\text { Types }\end{array}$ \\
\hline loc.v. & 1 & & 2 & & 0 & & 0 & & 0 & & 0 & \\
\hline $\begin{array}{l}\text { konju- } \\
\text { gierte } \\
\text { Verben }\end{array}$ & 21 & $\begin{array}{l}1 \text { Prä- } \\
\text { dikat } \\
\text { im } 1 . \\
\text { Satz } \\
\text { impli- } \\
\text { ziert }\end{array}$ & 39 & & 19 & $\begin{array}{l}2 \text { Prä- } \\
\text { dikate } \\
\text { impli- } \\
\text { ziert } \\
\text { (Bild- } \\
\text { be- } \\
\text { schrei- } \\
\text { bung } \\
\text { und } \\
\text { Auf- } \\
\text { gabe) }\end{array}$ & 38 & $\begin{array}{l}1 \text { Prädi- } \\
\text { kat in } \\
\text { Bildbe- } \\
\text { schrei- } \\
\text { bung } \\
\text { impli- } \\
\text { ziert }\end{array}$ & 27 & $\begin{array}{l}1 \\
\text { Prä- } \\
\text { dikat } \\
\text { in } \\
\text { Auf- } \\
\text { gabe } \\
\text { impli- } \\
\text { ziert }\end{array}$ & 18 & \\
\hline $\begin{array}{l}\text { Impe- } \\
\text { rative }\end{array}$ & 0 & & 5 & & 2 & & 0 & & 2 & & 0 & \\
\hline $\begin{array}{l}\varnothing \text { konj. } \\
\text { Verben } \\
\text { (inkl. } \\
\text { Impe- } \\
\text { rative) } \\
\text { pro } \\
\text { Satz }\end{array}$ & 2,3 & & 3,8 & & 1,2 & & 3,8 & & 1,7 & & 1,6 & \\
\hline $\begin{array}{l}\text { Gerun- } \\
\text { dien }\end{array}$ & 0 & & 4 & & 1 & & 0 & & 1 & & 0 & \\
\hline $\begin{array}{l}\text { Infini- } \\
\text { tive }\end{array}$ & 7 & $\begin{array}{l}1 \\
\text { inf.p. }\end{array}$ & 8 & & 5 & & 4 & & 4 & & 3 & $\begin{array}{l}2 \\
\text { inf.p. }\end{array}$ \\
\hline $\begin{array}{l}\text { Partizi- } \\
\text { pien }\end{array}$ & 1 & & 0 & & 2 & & 0 & & 7 & & 1 & \\
\hline FO & 173 & $70 \%$ & 365 & $61 \%$ & 205 & $63 \%$ & 270 & $67 \%$ & 256 & $73 \%$ & 154 & $56 \%$ \\
\hline
\end{tabular}




\begin{tabular}{|c|c|c|c|c|c|c|c|c|c|c|c|c|}
\hline Seite/K & 87 & K1 & $\begin{array}{r}116 \\
f .\end{array}$ & K2 & 118 & K3 & 149 & K4 & 182 & K5 & $\begin{array}{r}206 \\
\mathrm{f} .\end{array}$ & K6 \\
\hline el.FO & 0 & & 0 & & 0 & & 0 & & 0 & & 0 & \\
\hline FO TS & 2 & & 0 & & 1 & & 0 & & 0 & & 1 & \\
\hline $\mathrm{AU}$ & 14 & & 48 & & 29 & & 28 & & 23 & & 18 & \\
\hline AU TS & 1 & & 1 & & 3 & & 0 & & 1 & & 1 & \\
\hline$A D$ & 3 & & 6 & & 1 & & 5 & & 2 & & 3 & \\
\hline AD TS & 1 & $\begin{array}{l}\text { vege- } \\
\text { tali }\end{array}$ & 0 & & 0 & & 0 & & 0 & & 0 & \\
\hline $\begin{array}{l}\text { Tot. } \\
\text { VdB }\end{array}$ & 194 & $78 \%$ & 420 & $70 \%$ & 240 & $74 \%$ & 303 & $75 \%$ & 282 & $80 \%$ & 177 & $65 \%$ \\
\hline $\mathrm{BU}$ & 0 & & 0 & & 0 & & 0 & & 0 & & 0 & \\
\hline $\mathrm{CO}$ & 28 & & 52 & & 50 & & 53 & & 36 & & 36 & \\
\hline CO TS & 3 & & 3 & & 3 & & 5 & & 2 & & 2 & \\
\hline el.CO & 3 & & 12 & & 5 & & 15 & & 4 & & 7 & \\
\hline ES & 0 & & 12 & & 0 & & 0 & & 0 & & 0 & \\
\hline TS & 16 & & 11 & & 19 & & 10 & & 19 & & 11 & \\
\hline $\begin{array}{l}\text { el.TS } \\
\text { Tot. } \\
\text { restl. } \\
\text { Mar- }\end{array}$ & 4 & & 7 & & 6 & & 7 & & 1 & & 3 & \\
\hline $\begin{array}{l}\text { ken } \\
\text { Tot. } \\
\text { Mar- } \\
\text { ken }\end{array}$ & 54 & $22 \%$ & 97 & $16 \%$ & 81 & $25 \%$ & 90 & $22 \%$ & 62 & $18 \%$ & 57 & $21 \%$ \\
\hline insg. & 248 & $100 \%$ & 517 & $86 \%$ & 321 & $98 \%$ & 393 & $97 \%$ & 344 & $98 \%$ & 236 & $86 \%$ \\
\hline
\end{tabular}

Tabelle 9: Untersuchungsergebnisse zu den Seiten aus Zaino in spalla (Bastianelli, Rancati \& Maestri 2010)

Die durchschnittliche Satzlänge ist mit 35 Wörtern auf Seite 149 so hoch, da ein Satz ganze 201 Wörter lang ist. Der Satz behandelt die aktuelle demographische Entwicklung, beschreibt ihre Phasen und den bestimmten Verlauf, den sie nehmen. Die vier einzelnen Phasen werden nach einem Doppelpunkt unter Gliederungsstrichen vorgestellt, die durch ein Semikolon voneinander getrennt sind, so dass dieses lange Satzgefüge erst nach dem vierten Gliederungspunkt endet. Insgesamt weist jeder der einzelnen Punkte abgesehen von vielen $\mathrm{Ne}-$ bensätzen ein weiteres Semikolon auf (Bastianelli, Rancati \& Maestri 2010, S. 149; Hervorhebung im Original):

Secondo alcuni studiosi, l'andamento demografico di un Paese segue delle fasi precise:

- inizialmente la natalità è alta ma simile alla mortalità, perché quest'ultima è molto elevata a causa delle precarie condizioni di vita; la popolazione aumenta leggermente (saldo naturale positivo); è quanto si verifica attualmente nei Paesi sottosviluppati, come la Sierra Leone in Africa, Haiti in America centrale, il Bangladesh in Asia;

- in una fase successiva, grazie ai progressi in campo medico, diminuisce la mortalità e aumenta la durata media della vita, mentre non muta la natalità che rimane alta; è ciò che accade oggi in Senegal, uno dei Paesi in via di sviluppo, in cui si registra una morte ogni quattro nascite e di conseguenza un incremento demografico molto sostenuto;

- durante la fase seguente diminuisce la natalità, con l'applicazione di sistemi di controllo delle nascite; in molti Paesi europei e negli Stati Uniti la maggioranza delle coppie tende ad 
avere pochi figli (meno di due per coppia), di conseguenza l'incremento demografico è molto basso, con valori spesso vicino allo $0 \%$;

- nella fase finale la mortalità supera la natalità e la popolazione diminuisce; è ciò che accade oggi in Paesi come la Germania e l'Italia, in cui il saldo naturale è inferiore allo $0 \%$, cioè è negativo, perché le morti superano le nascite.

(M.Ü.: Laut einigen Wissenschaftlern folgt die demographische Entwicklung eines Landes präzisen Phasen:

- anfänglich ist die Geburtenrate hoch, aber ähnelt der Sterberate, weil Letztere aufgrund prekärer Lebensbedingungen sehr hoch ist; die Bevölkerung wächst langsam (positive Geburtenbilanz); es ist das, was derzeit in den unterentwickelten Ländern wie Sierra Leone in Afrika, Haiti in Zentralamerika, Bangladesch in Asien passiert;

- in einer nächsten Phase sinkt die Sterberate dank medizinischer Fortschritte und steigt die durchschnittliche Lebenserwartung, während sich die Geburtenrate, die hoch bleibt, nicht verändert; es ist das, was derzeit in Senegal, einem der Entwicklungsländer, passiert, in dem ein Todesfall bei vier Geburten und demzufolge ein sehr starkes demographisches Wachstum verzeichnet wird;

- während der folgenden Phase sinkt die Geburtenrate mit der Anwendung von Systemen zur Geburtenkontrolle; in vielen europäischen Ländern und in den Vereinigten Staaten tendiert die Mehrheit der Paare dazu, wenige Kinder zu haben (weniger als zwei pro Paar), demzufolge ist das demographische Wachstum bei Werten die oft nahe an $0 \%$ liegen, sehr niedrig;

- in der Schlussphase übersteigt die Sterberate die Geburtenrate und die Bevölkerung nimmt ab; es ist das, was derzeit in Ländern wie Deutschland und Italien passiert, in denen die Geburtenrate kleiner als $0 \%$ ist, also negativ, weil die Todesfälle die Geburten übersteigen.)

Aus dem gesteigerten Gebrauch von komplexen Verbgefügen (große Anzahl von Gerundien, Infinitiven und Partizipien) auf den ausgewählten Seiten dieses Lehrwerks lässt sich bereits folgern, dass kompliziertere syntaktische Strukturen vorliegen: Im Vergleich zu den untersuchten Texten aus dem Lehrbuch von Bersezio (2005), in denen insgesamt 19 dieser Formen erscheinen (13 Infinitive, zwei Gerundien und neun Partizipien), sind es hier mehr als doppelt so viele, nämlich 31 Infinitive (von denen drei passivischer Art sind), sechs Gerundien und elf Partizipien.

Eine stilistische Besonderheit von marginalerer Bedeutung betrifft den Ausdruck ,Neue Welt", der als Nominalphrasem nuovo mondo einen Eintrag im GRADIT hat und dort mit der Gebrauchsmarke Co versehen ist. Von den Autoren wird er jedoch doppelt hervorgehoben, einmal durch die Großschreibung beider Elemente und außerdem durch Anführungszeichen (Bastianelli, Rancati \& Maestri 2010, S. 149).

Desweiteren ist der Anteil von fettgedruckten Wörtern mit insgesamt 100, was einem Prozentwert von 4,5 entspricht, höher als in den anderen bisher untersuchten Lehrwerken. Während des Unterrichts hat sich die Lehrerin mehrmals dazu geäußert, dass sie von dieser graphischen Art der Lernhilfe wenig hält. Sie vertritt nämlich die auch in der Lehrbuch- und erziehungssprachwissenschaftlichen Forschung vertretene Auffassung (Lavinio 2014, S. 374), dass 
die Lesenden mehr lernen und besser behalten, wenn sie die Kernaussagen eines Textes eigenständig herausarbeiten müssen. Da von den beobachteten Lehrkräften nicht nur sie die Hervorhebung von Ausdrücken und Wortgruppen in den Lehrbuchtexten negativ bewertete und sich in allen fünf untersuchten Büchern fettgedruckte Wörter befinden (allerdings mit Unterschieden in Auswahl und Anzahl), befasst sich das folgende Unterkapitel mit ihrer Schilderung, bevor die Untersuchungsergebnisse zu den letzten beiden Lehrbüchern vorgestellt werden.

\subsubsection{Hervorhebungen auf den untersuchten Seiten der fünf Lehrbücher}

Auf den untersuchten Lehrbuchseiten sind in jedem Lehrwerk mindestens 34 und maximal 96 Wörter hervorgehoben (in drei Büchern weniger als 50 und in den anderen zwei mehr als 70). In vier Lehrwerken handelt es sich hierbei um einen erheblichen Anteil von Mehrwortgruppen, zu denen auch Phraseme gerechnet wurden. Lediglich im Buch der Klasse Vespucci (Nuovo Geolab) machen diese Gruppen etwas weniger als die Hälfte aller Hervorhebungen und im Buch an der Morante sogar weniger als ein Viertel aus.

Auf allen untersuchten Seiten handelt es sich bei den meisten dieser Wörter um Nomen und geographische Eigennamen. Diese sind nicht selten durch Adjektive ergänzt. In einigen Fällen werden aber auch ganze Sätze hervorgehoben, so dass sich in drei Büchern unter den fettgedruckten Wörtern auch konjugierte Verbformen befinden.

Unterschiede gibt es ebenfalls in der Zusammensetzung nach Gebrauchsmarken, wobei allen Büchern gemein ist, dass sich unter den hervorgehobenen Wörtern sowohl Ausdrücke aus dem fundamentalen Wortschatz (FO) und dem VDB, als auch solche aus den weniger frequenten Wortschatzbereichen antreffen lassen, die mit den Marken Co und Ts gekennzeichnet sind.

Diese und weitere Angaben sind aus Tabelle 10 zu entnehmen, die zunächst einen Überblick über das hervorgehobene Wortvorkommen verschafft.

\begin{tabular}{lrrrrr}
\hline Klassen/Hervorhebungen & Vespucci & Morante & III A P. & III B P. & III C P. \\
\hline $\begin{array}{l}\text { Hervorhebungen } \\
\text { (Wörter und Wortgruppen) }\end{array}$ & 73 & 34 & 43 & 96 & 48 \\
davon mehr als ein Wort & & & & & \\
(inkl. Phraseme) & 33 & 8 & 34 & & 28 \\
Nomen (eingliedrig) & 49 & 13 & 36 & 81 & 26 \\
Adjektive & 16 & 6 & 23 & 55 & 26 \\
Verben & 0 & 0 & 4 & 7 (inkl. 1 & 1 inf.v.intr. \\
& & & & v.aus.) & 18 \\
geographische Eigennamen & 30 & 19 & 6 & 33 & 1 \\
Phraseme & 10 & 0 & 9 & 5 & 21 \\
Tot. FO & 26 & 2 & 44 & 115 & 34 \\
Tot. VdB & 49 & 4 & 58 & 163 & 15 \\
Tot. CO & 17 & 8 & 19 & 23 & 9 \\
Tot. TS & 20 & 6 & 13 & 22 & \\
\hline
\end{tabular}




\begin{tabular}{lrrrrr}
\hline Klassen/Hervorhebungen & Vespucci & Morante & III A P. & III B P. & III C P. \\
\hline ES & 0 & 1 & 0 & 0 & 0 \\
\hline
\end{tabular}

Tabelle 10: Zusammensetzung der hervorgehobenen Wörter auf den untersuchten Seiten aller fünf Lehrbücher

Im Folgenden wird der hervorgehobene Wortschatz etwas genauer beschrieben. Im Nuovo Geolab (Carazzi \& Pizzetti 2010, Verlag Mondadori), das in der Klasse an der Schule Vespucci benutzt wurde, sind diese in Tabelle 11 aufgelisteten 73 Wörter und Wortgruppen evidenziert:

\begin{tabular}{|c|c|c|c|c|c|}
\hline $\begin{array}{l}\text { Seite, Titel, } \\
\text { n Wörter }\end{array}$ & $\begin{array}{l}\text { Wort/Wort- } \\
\text { gruppe }\end{array}$ & Wortart & Marke & Übersetzung & Kommentar \\
\hline S.55 & keine(s) & & & & \\
\hline $\begin{array}{l}\text { Ricchezza e } \\
\text { qualità della } \\
\text { vita } \\
\text { (113 Wörter) }\end{array}$ & & & & $\begin{array}{l}\text { Reichtum und } \\
\text { Lebensqualität }\end{array}$ & \\
\hline S. 55 & Pil pro capite & $\begin{array}{l}\text { sigla } \\
\text { (loc.s.m.) } \\
+ \\
\text { loc.agg.inv }\end{array}$ & $\begin{array}{l}\text { TS econ. } \\
+\mathrm{CO}\end{array}$ & Pro-Kopf-BIP & neues Konzept \\
\hline $\begin{array}{l}\text { Il Pil e il Pil } \\
\text { pro capite } \\
\text { (307 Wörter) } \\
\text { S. } 56\end{array}$ & reddito medio & $\begin{array}{l}\text { s.m. + } \\
\text { agg. }\end{array}$ & $\begin{array}{l}\text { AU TS } \\
\text { econ. + } \\
\text { AU }\end{array}$ & $\begin{array}{l}\text { Durchschnitts- } \\
\text { einkommen }\end{array}$ & $\begin{array}{l}\text { dient als zweite De- } \\
\text { finition des Kon- } \\
\text { zepts }\end{array}$ \\
\hline \multirow[t]{3}{*}{$\begin{array}{l}\text { La mortalità } \\
\text { infantile: un } \\
\text { indicatore per } \\
\text { misurare la } \\
\text { povertà } \\
\text { (133 Wörter) }\end{array}$} & livello di povertà & $\begin{array}{l}\text { s.m. + } \\
\text { prep. + } \\
\text { s.m. }\end{array}$ & $\begin{array}{l}\text { CO TS } \\
\text { scient. + } \\
\text { FO + TS } \\
\text { econ. }\end{array}$ & $\begin{array}{l}\text { Grad der Ar- } \\
\text { mut }\end{array}$ & $\begin{array}{l}\text { zweites Nomen } \\
\text { sollte bekannt sein } \\
\text { (Armut: povertà } \\
\text { (CO), hat hier ver- } \\
\text { wandte, aber spezi- } \\
\text { fische Bedeutung }\end{array}$ \\
\hline & povertà estrema & s.f. + agg. & $\begin{array}{l}\text { TS econ. } \\
\text { + FO }\end{array}$ & extreme Armut & \\
\hline & $\begin{array}{l}\text { mortalità infan- } \\
\text { tile }\end{array}$ & loc.s.f. & TS stat. & $\begin{array}{l}\text { Säuglings- } \\
\text { sterblichkeit }\end{array}$ & $\begin{array}{l}\text { Begriff wird in den } \\
\text { folgenden Sätzen } \\
\text { zweimal wiederholt, } \\
\text { doch vor seiner Er- } \\
\text { klärung befinden } \\
\text { sich } 35 \text { Wörter, die } \\
\text { u.a. erst Ursachen } \\
\text { nennen, die zur } \\
\text { Säuglingssterblich- } \\
\text { keit führen }\end{array}$ \\
\hline \multicolumn{6}{|l|}{ S. 56} \\
\hline \multirow{3}{*}{$\begin{array}{l}\text { L'Isu: un indi- } \\
\text { catore per mi- } \\
\text { surare lo svi- } \\
\text { luppo } \\
\text { ( } 218 \text { Wörter) }\end{array}$} & $\begin{array}{l}\text { situazione so- } \\
\text { ciale }\end{array}$ & s.f. + agg. & $2 \mathrm{FO}$ & soziale Lage & $\begin{array}{l}\text { Tafelbild Wortfeld } \\
\text { sociale }\end{array}$ \\
\hline & speranza di vita & loc.s.f. & TS stat. & $\begin{array}{l}\text { Lebenserwar- } \\
\text { tung }\end{array}$ & $\begin{array}{l}\text { bereits eingeführtes } \\
\text { Konzept; erneut er- } \\
\text { klärt }\end{array}$ \\
\hline & istruzione & s.f. & $\mathrm{AU}$ & Bildung(sgrad) & $\begin{array}{l}\text { livello di istruzione } \\
\text { (FO fig. + FO) }\end{array}$ \\
\hline
\end{tabular}




\begin{tabular}{|c|c|c|c|c|c|}
\hline $\begin{array}{l}\text { Seite, Titel, } \\
\text { n Wörter }\end{array}$ & $\begin{array}{l}\text { Wort/Wort- } \\
\text { gruppe }\end{array}$ & Wortart & Marke & Übersetzung & Kommentar \\
\hline & $\begin{array}{l}\text { Isu (Indice di } \\
\text { Sviluppo } \\
\text { Umano) }\end{array}$ & $\begin{array}{l}\text { sigla + } \\
\text { loc.s.m. }\end{array}$ & $\begin{array}{l}\text { TS stat., } \\
\text { polit. }\end{array}$ & $\begin{array}{l}\text { Human Deve- } \\
\text { lopment Index }\end{array}$ & $\begin{array}{l}\text { Begriff auch in der } \\
\text { Überschrift enthal- } \\
\text { ten und mit dem ge- } \\
\text { samten Text erklärt }\end{array}$ \\
\hline \multicolumn{6}{|r|}{ 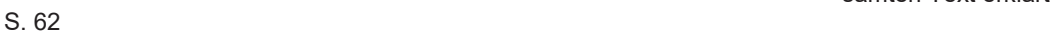 } \\
\hline \multirow{5}{*}{$\begin{array}{l}\text { Come è di- } \\
\text { stribuita la } \\
\text { ricchezza nel } \\
\text { mondo } \\
\text { (172 Wörter) }\end{array}$} & Pil pro capite & $\begin{array}{l}\text { sigla } \\
\text { (loc.s.m.) } \\
+ \\
\text { loc.agg.inv }\end{array}$ & $\begin{array}{l}\text { TS econ. } \\
+\mathrm{CO}\end{array}$ & Pro-Kopf-BIP & $\begin{array}{l}\text { baut auf S. } 55 \text { ein- } \\
\text { geführtes Konzept } \\
\text { auf }\end{array}$ \\
\hline & prezzi delle & s.m. + & $2 \mathrm{FO}+$ & Warenpreise & \\
\hline & merci & $\begin{array}{l}\text { prep.art. + } \\
\text { s.f. }\end{array}$ & $\mathrm{AU}$ & & \\
\hline & distribuzione & s.f. + & $A U+F O$ & Verteilung des & \\
\hline & della ricchezza & $\begin{array}{l}\text { prep.art. + } \\
\text { s.f. }\end{array}$ & $\begin{array}{l}\text { + TS } \\
\text { econ. }\end{array}$ & Reichtums & \\
\hline S. 68 & & & & $\begin{array}{l}\text { Thema auch } \\
\text { im Technikun- } \\
\text { terricht im sel- } \\
\text { ben Schuljahr } \\
\text { behandelt }\end{array}$ & \\
\hline $\begin{array}{l}\text { Le risorse } \\
\text { energetiche }\end{array}$ & $\begin{array}{l}\text { più alto livello di } \\
\text { consumo }\end{array}$ & $\begin{array}{l}\text { avv. + } \\
\text { agg. + } \\
\text { s.m. + } \\
\text { prep. + } \\
\text { s.m. }\end{array}$ & $\begin{array}{l}2 \mathrm{FO}+ \\
\mathrm{FO} \text { fig. }+ \\
\mathrm{FO}+\mathrm{AU}\end{array}$ & $\begin{array}{l}\text { der höchste } \\
\text { Grad an Ver- } \\
\text { brauch }\end{array}$ & \\
\hline \multirow{6}{*}{$\begin{array}{l}\text { Paesi consu- } \\
\text { matori e } \\
\text { paesi produt- } \\
\text { tori } \\
\text { (134 Wörter) }\end{array}$} & Usa & n.p. & & USA & $\begin{array}{l}\text { einfache Wörter, } \\
\text { aber sprachliches } \\
\text { Umfeld komplex }\end{array}$ \\
\hline & petrolio & s.m. & $\mathrm{FO}$ & Erdöl & \\
\hline & Europa & n.p. & & Europa & \\
\hline & Giappone & n.p. & & Japan & $\begin{array}{l}\text { Länder sollten hier } \\
\text { m.E. nicht hervorge- } \\
\text { hoben werden }\end{array}$ \\
\hline & Cina & n.p. & & China & \\
\hline & carbone & s.m. & FO & Kohle & \\
\hline \multicolumn{6}{|l|}{ S. 68} \\
\hline \multirow{9}{*}{$\begin{array}{l}\text { L'opzione } \\
\text { dell'energia } \\
\text { nucleare } \\
\text { (139 Wörter) }\end{array}$} & $\begin{array}{l}\text { energia nu- } \\
\text { cleare }\end{array}$ & loc.s.f. & TS fis. & Kernenergie & $\begin{array}{l}\text { Kontext komplex } \\
\text { (inhaltlich) }\end{array}$ \\
\hline & Francia & n.p. & & Frankreich & \\
\hline & Giappone & n.p. & & Japan & \\
\hline & Germania & n.p. & & Deutschland & \\
\hline & Russia & n.p. & & Russland & \\
\hline & Corea del Sud & n.p. & & Südkorea & \\
\hline & $\begin{array}{l}\text { combustibili ve- } \\
\text { getali }\end{array}$ & $\begin{array}{l}\text { s.m. + } \\
\text { agg. }\end{array}$ & $\begin{array}{l}\text { TS chim. } \\
+A D\end{array}$ & $\begin{array}{l}\text { nachwach- } \\
\text { sende/pflanzli- } \\
\text { che Rohstoffe }\end{array}$ & $\begin{array}{l}\text { Überschrift passt } \\
\text { nicht mehr zum In- } \\
\text { halt }\end{array}$ \\
\hline & legna & s.f. & $A D$ & Holz & \\
\hline & $\begin{array}{l}\text { escrementi di } \\
\text { animali }\end{array}$ & $\begin{array}{l}\text { s.m. + } \\
\text { prep. + } \\
\text { s.m. }\end{array}$ & $\begin{array}{l}\mathrm{CO}+2 \\
\mathrm{FO}\end{array}$ & $\begin{array}{l}\text { tierische Ex- } \\
\text { kremente }\end{array}$ & \\
\hline
\end{tabular}




\begin{tabular}{|c|c|c|c|c|c|}
\hline $\begin{array}{l}\text { Seite, Titel, } \\
\text { n Wörter }\end{array}$ & $\begin{array}{l}\text { Wort/Wort- } \\
\text { gruppe }\end{array}$ & Wortart & Marke & Übersetzung & Kommentar \\
\hline & $\begin{array}{l}\text { pannelli solari } \\
\text { vento }\end{array}$ & $\begin{array}{l}\text { loc.s.m. } \\
\text { s.m. }\end{array}$ & $\begin{array}{l}\text { TS techn. } \\
\text { FO }\end{array}$ & $\begin{array}{l}\text { Solarzellen } \\
\text { Wind }\end{array}$ & \\
\hline & maree & s.f. & $\mathrm{AD}$ & $\begin{array}{l}\text { Gezeiten/See- } \\
\text { gang }\end{array}$ & $\begin{array}{l}\text { Energiegewinnung } \\
\text { wird nicht erklärt }\end{array}$ \\
\hline \multicolumn{6}{|l|}{ S. 134} \\
\hline $\begin{array}{l}\text { L'Africa meri- } \\
\text { dionale o au- } \\
\text { strale }\end{array}$ & $\begin{array}{l}\text { gruppo etnico } \\
\text { omogeneo }\end{array}$ & $\begin{array}{l}\text { loc.s.m. + } \\
\text { agg. }\end{array}$ & $\begin{array}{l}\text { TS sociol. } \\
+\mathrm{CO}\end{array}$ & $\begin{array}{l}\text { homogene } \\
\text { Volksgruppe }\end{array}$ & \\
\hline \multirow{4}{*}{$\begin{array}{l}\text { Gli stati } \\
\text { dell'area } \\
\text { (74 Wörter) }\end{array}$} & Malawi & n.p. & & Malawi & \\
\hline & Zimbabwe & n.p. & & Simbabwe & \\
\hline & $\begin{array}{l}\text { Mozambico, } \\
\text { Swaziland, } \\
\text { Zambia, Angola, } \\
\text { Namibia, Bo- } \\
\text { tswana, Suda- } \\
\text { frica, Lesotho }\end{array}$ & 8 n.p. & & $\begin{array}{l}\text { Mosambik, } \\
\text { Swasiland, } \\
\text { Sambia, An- } \\
\text { gola, Namibia, } \\
\text { Botswana, } \\
\text { Südafrika, } \\
\text { Lesotho }\end{array}$ & \\
\hline & $\begin{array}{l}\text { Madagascar, } \\
\text { Seychelles, } \\
\text { Mauritius (e) } \\
\text { Comore }\end{array}$ & 4 n.p. & & $\begin{array}{l}\text { Madagaskar, } \\
\text { Seychellen, } \\
\text { Mauritius, } \\
\text { Komoren }\end{array}$ & \\
\hline S. $134 \mathrm{f}$. & & & & & \\
\hline \multirow{15}{*}{$\begin{array}{l}\text { Territorio, po- } \\
\text { polazione e } \\
\text { risorse } \\
\text { ( } 247 \text { Wörter) }\end{array}$} & vasti altipiani & $\begin{array}{l}\text { agg. + } \\
\text { s.m. }\end{array}$ & $F O+A D$ & $\begin{array}{l}\text { weite Hoch- } \\
\text { ebenen }\end{array}$ & $\begin{array}{l}\text { langer Text, zu viele } \\
\text { verschiedene The- } \\
\text { matiken }\end{array}$ \\
\hline & (I')Orange & n.p. & & Oranje (Fluss) & \\
\hline & Zambesi & n.p. & & $\begin{array}{l}\text { Sambesi } \\
\text { (Fluss) }\end{array}$ & \\
\hline & Niassa & n.p. & & $\begin{array}{l}\text { Malawisee } \\
\text { (auch Nya- } \\
\text { sasee ge- } \\
\text { nannt) }\end{array}$ & \\
\hline & savana & s.f. & $\begin{array}{l}\text { CO TS } \\
\text { geogr. }\end{array}$ & Savanne & \\
\hline & aree desertiche & s.f. + agg. & $\begin{array}{l}\text { TS geogr. } \\
+\mathrm{CO}\end{array}$ & $\begin{array}{l}\text { Wüstenge- } \\
\text { biete }\end{array}$ & \\
\hline & $\begin{array}{l}\text { clima mediterra- } \\
\text { neo }\end{array}$ & loc.s.m. & $\begin{array}{l}\text { TS me- } \\
\text { teor., ge- } \\
\text { ogr. }\end{array}$ & $\begin{array}{l}\text { Mittelmeer- } \\
\text { klima }\end{array}$ & \\
\hline & Sudafrica & n.p. & & Südafrika & \\
\hline & $\begin{array}{l}\text { densità demo- } \\
\text { grafica }\end{array}$ & s.f. + agg. & $\begin{array}{l}\text { TS stat. + } \\
\text { CO }\end{array}$ & $\begin{array}{l}\text { Bevölkerungs- } \\
\text { dichte }\end{array}$ & \\
\hline & $\begin{array}{l}\text { colonizzazione } \\
\text { portoghese }\end{array}$ & s.f. + agg. & $C O+A D$ & $\begin{array}{l}\text { portugiesische } \\
\text { Kolonisation }\end{array}$ & \\
\hline & colonia francese & s.f. + agg. & $A U+F O$ & $\begin{array}{l}\text { französische } \\
\text { Kolonie }\end{array}$ & \\
\hline & Sudafrica & n.p. & & Südafrika & \\
\hline & Rhodesia & n.p. & & Rhodesien & \\
\hline & $\begin{array}{l}\text { segregazione } \\
\text { razziale }\end{array}$ & loc.s.f. & $\mathrm{CO}$ & $\begin{array}{l}\text { Rassentren- } \\
\text { nung }\end{array}$ & \\
\hline & $\begin{array}{l}\text { risorse minera- } \\
\text { rie }\end{array}$ & s.f. + agg. & $\begin{array}{l}\mathrm{AU}+\mathrm{TS} \\
\text { miner. }\end{array}$ & Bodenschätze & \\
\hline
\end{tabular}




\begin{tabular}{|c|c|c|c|c|c|}
\hline $\begin{array}{l}\text { Seite, Titel, } \\
\text { n Wörter }\end{array}$ & $\begin{array}{l}\text { Wort/Wort- } \\
\text { gruppe }\end{array}$ & Wortart & Marke & Übersetzung & Kommentar \\
\hline & $\begin{array}{l}\text { diamanti, oro, } \\
\text { carbone, petro- } \\
\text { lio }\end{array}$ & 4 s.m. & $\begin{array}{l}\mathrm{AD}+3 \\
\mathrm{FO}\end{array}$ & $\begin{array}{l}\text { Diamanten, } \\
\text { Gold, Kohle, } \\
\text { Erdöl }\end{array}$ & \\
\hline & uranio & s.m. & TS chim. & Uran & \\
\hline & sussistenza & s.f. & $\mathrm{CO}$ & Subsistenz & loc.: TS agr. \\
\hline & mais & s.m. & $\mathrm{CO}$ & Mais & \\
\hline & patate & s.f. & FO & Kartoffeln & \\
\hline & $\begin{array}{l}\text { esportazione: } \\
\text { caffè }\end{array}$ & s.f. + s.f. & $\begin{array}{l}\mathrm{AU}+\mathrm{TS} \\
\text { bot.com. }\end{array}$ & Export: Kaffee & \\
\hline & tè $(e)$ cotone & 2 s.m. & $\begin{array}{l}\text { TS } \\
\text { bot.com. } \\
+ \text { AU }\end{array}$ & $\begin{array}{l}\text { Tee (und) } \\
\text { Baumwolle }\end{array}$ & \\
\hline \multicolumn{6}{|l|}{ S. 319} \\
\hline $\begin{array}{l}\text { Gli insedia- } \\
\text { menti }\end{array}$ & 74 milioni & s.m. & FO & 74 Millionen & \\
\hline [Ägypten] & gruppo etnico & loc.s.m. + & TS sociol. & homogene & \\
\hline (151 Wörter) & $\begin{array}{l}\text { omogeneo } \\
\text { religione isla- } \\
\text { mica }\end{array}$ & $\begin{array}{l}\text { agg. } \\
\text { s.f. + agg. }\end{array}$ & $\begin{array}{l}+\mathrm{CO} \\
\mathrm{FO}+\mathrm{CO} \\
\text { TS relig. }\end{array}$ & $\begin{array}{l}\text { Volksgruppe } \\
\text { islamische Re- } \\
\text { ligion }\end{array}$ & \\
\hline & I/ Cairo & n.p. & & Kairo & \\
\hline \multicolumn{6}{|l|}{ S. 320} \\
\hline \multirow{11}{*}{$\begin{array}{l}\text { L'economia } \\
\text { (163 Wörter) }\end{array}$} & (L')agricoltura & s.f. & $\mathrm{FO}$ & Landwirtschaft & \\
\hline & $\begin{array}{l}\text { elevata produtti- } \\
\text { vità }\end{array}$ & agg. + s.f. & $\mathrm{FO}+\mathrm{CO}$ & $\begin{array}{l}\text { hohe Produkti- } \\
\text { vität }\end{array}$ & \\
\hline & $\begin{array}{l}\text { frumento, orzo } \\
\text { (e) fagioli }\end{array}$ & 3 s.m. & $\begin{array}{l}C O+A D \\
+A U\end{array}$ & $\begin{array}{l}\text { Weizen, } \\
\text { Gerste (und) } \\
\text { Bohnen }\end{array}$ & $\begin{array}{l}\text { Fettdruck m.E. nicht } \\
\text { erforderlich }\end{array}$ \\
\hline & mais & s.m. & $\mathrm{CO}$ & Mais & \\
\hline & riso & s.m. & $A U$ & Reis & \\
\hline & cotone & s.m. & $\mathrm{AU}$ & Baumwolle & \\
\hline & $\begin{array}{l}\text { canna da zuc- } \\
\text { chero }\end{array}$ & loc.s.f. & $\begin{array}{l}\text { TS } \\
\text { bot.com. }\end{array}$ & Zuckerrohr & \\
\hline & petrolio & s.m. & $\mathrm{FO}$ & Erdöl & \\
\hline & $\begin{array}{l}\text { industria side- } \\
\text { rurgica, petrol- } \\
\text { chimica (e) chi- } \\
\text { mica }\end{array}$ & s.f. & $\begin{array}{l}\text { FO + CO } \\
+ \text { CO TS } \\
\text { chim. + } \\
\text { (FO) + } \\
\text { AU }\end{array}$ & $\begin{array}{l}\text { Stahlindustrie, } \\
\text { petrochemi- } \\
\text { sche und che- } \\
\text { mische Indust- } \\
\text { rie }\end{array}$ & \\
\hline & settore turistico & $\begin{array}{l}\text { s.m. + } \\
\text { agg. }\end{array}$ & $\begin{array}{l}A D \text { fig. + } \\
A D\end{array}$ & $\begin{array}{l}\text { Tourismus- } \\
\text { branche }\end{array}$ & $\begin{array}{l}\text { Wertung im Text } \\
\text { (purtroppo; leider) }\end{array}$ \\
\hline & attentati & s.m. & FO & Attentate & \\
\hline
\end{tabular}

Tabelle 11: Hervorhebungen auf den Untersuchten Seiten im Nuovo Geolab (Carazzi \& Pizzetti 2010)

In diesem Lehrbuch werden auf den untersuchten Seiten die zweitmeisten Hervorhebungen verzeichnet. Nur in einem kurzen Text gibt es gar keine fettgedruckten Wörter, in den anderen sind es mindestens zwei Ausdrücke bzw. Wortgruppen und bis zu 22. Diese große Anzahl kommt auch dadurch zustande, dass in einigen Texten beispielsweise alle Anbauprodukte (Carazzi \& Pizzetti 2010, S. 320; s.a. S. 134 f.) und im Text über den Süden Afrikas alle Länder dieses Teils des Kontinents (ibid. S. 134) hervorgehoben sind. 
In beiden Fällen handelt es sich um Hervorhebungen, die verzichtbar sind, zumal die Begriffe Glieder einer Aufzählung darstellen, so dass keine allzu hohe kognitive Leistung vorausgesetzt zu werden braucht, um den Textinhalt auch ohne Hervorhebungen zu erfassen.

33 der Hervorhebungen bestehen aus mehr als einem Wort. Hierbei handelt es sich meistens um Substantive, und fast die Hälfte der Hervorhebungen betreffen Eigennamen. In diesem Lehrbuch sind auf den untersuchten Seiten auBerdem die meisten Phraseme enthalten (10). Insgesamt 37 Ausdrücke werden niedrigfrequent gebraucht und sind mit den Marken Co, Co Ts und Ts im GRADIT verzeichnet. Hierunter befinden sich zwei wirtschaftliche Fachbegriffe, die in den Texten (ibid., S. 55 f.) eingeführt werden. Einer von ihnen, das Pro Kopf-Bruttoinlandsprodukt, wird dabei in einem späteren Text (ibid., S. 62) wieder aufgegriffen. Die Hervorhebung bringt zum einen seine Relevanz für die Behandlung in dem dort eingeführten wirtschaftlichen Kontext zum Ausdruck und unterstützt zum anderen wahrscheinlich den mnemotechnischen Lernprozess der SchülerInnen.

Dass es sich für die Lernenden um eine komplexe Thematik handelt, haben die Unterrichtsbeobachtungen ergeben, da sie nicht imstande waren, das mit dem Human Development Index verbundene Konzept zu erklären.

An dieser Stelle wurde deutlich, dass eine hohe Konzentration von fachsprachlichen Ausdrücken in komplexeren grammatikalisch-syntaktischen Strukturen das Textverständnis der Lernenden beeinträchtigen kann und in einem solchen sprachlichen Umfeld auch Wörter des fundamentalen Wortschatzes potentielle Hürden darstellen. In diesem konkreten Beispiel betrifft das etwa sociale (Fo; sozial). Das Adjektiv wurde von der Lehrkraft im Unterrichtsgespräch gemeinsam mit der Klasse erarbeitet, indem sie dazu als Tafelbild ein Bedeutungsnetz erstellen ließ. Hierbei sei erwähnt, dass das dem GRADIT zugrundeliegende Korpus auf dem Sprachgebrauch der Erwachsenen beruht und in einigen Fällen nicht davon ausgegangen werden kann, dass die Bedeutungen bestimmter Wörter den Kindern und jungen Heranwachsenden ebenso geläufig sind wie den Erwachsenen.

Analysiert man den inhaltlichen Bezug zwischen den Überschriften der Texte und den fettgedruckten Wörtern, so sticht hervor, dass im Text L'opzione dell'energia nucleare auch Begriffe hervorgehoben sind, die besser unter einer anderen Überschrift geführt wären, und zwar diejenigen, welche erneuerbare Energien bezeichnen. Eine ähnliche Situation ist auf den Seiten 134 f. gegeben, auf denen sich ein relativ langer Text (247 Wörter) befindet, der sich unter der Überschrift Territorio, popolazione e risorse mit dem Süden Afrikas befasst und dabei so unterschiedliche Aspekte wie die physische Geographie der Region, ihre soziohistorische Entwicklung, die Klimata, die Bodenschätze und die Anbauprodukte aufgreift. Alternativ zur Hervorhebung der 22 Wortgruppen könnte der Text in Abschnitte mit einzelnen Überschriften unterteilt werden, was dem Textverständnis vielleicht dienlicher wäre. 
Im Buch I nuovi territori dell'uomo (Bersezio 2005, Verlag De Agostini), das in der Klasse an der Schule Morante benutzt wurde und von allen fünf untersuchten Lehrwerken das älteste ist, sind die wenigsten Wörter und Wortgruppen hervorgehoben, nämlich insgesamt nur die in Tabelle 12 aufgelisteten 34.

\begin{tabular}{|c|c|c|c|c|c|}
\hline $\begin{array}{l}\text { Seite/Überschrif- } \\
\text { ten/n Wörter }\end{array}$ & $\begin{array}{l}\text { Wort/Wort- } \\
\text { gruppe }\end{array}$ & Wortart & $\begin{array}{l}\text { Gebrauchs- } \\
\text { marke }\end{array}$ & Übersetzung & Kommentar \\
\hline S. 32 & II Cairo & n.p. & & Kairo & \\
\hline $\begin{array}{l}\text { Demografia e } \\
\text { modelli urbani } \\
155 \text { Wörter } \\
\text { S. } 33\end{array}$ & $\begin{array}{l}\text { Alessan- } \\
\text { dria }\end{array}$ & n.p. & & Alexandria & \\
\hline \multicolumn{6}{|l|}{$\begin{array}{l}\text { Risorse ed eco- } \\
\text { nomia (Einlei- } \\
\text { tung }+3 \text { Texte) }\end{array}$} \\
\hline \multirow{2}{*}{$\begin{array}{l}\text { L'agricoltura } \\
\text { (129 Wörter) }\end{array}$} & cotone & s.m. & $\mathrm{AU}$ & Baumwolle & \\
\hline & datteri & s.m. & $\mathrm{CO}$ & Datteln & \\
\hline \multirow[t]{2}{*}{$\begin{array}{l}\text { L'industria } \\
\text { (53 Wörter) }\end{array}$} & idrocarburi & s.m. & CO TS chim. & $\begin{array}{l}\text { Kohlenwas- } \\
\text { serstoff }\end{array}$ & $\begin{array}{l}\text { zwei im Fol- } \\
\text { genden ge- } \\
\text { nannt (Erdöl } \\
\text { und Erdgas) }\end{array}$ \\
\hline & \multicolumn{5}{|c|}{ S. 33} \\
\hline $\begin{array}{l}\text { II terziario } \\
\text { (91 Wörter) } \\
\text { S. } 58\end{array}$ & keine & & & & \\
\hline $\begin{array}{l}\text { La regione meri- } \\
\text { dionale [Afrika] } \\
\text { (82 Wörter) } \\
\text { S. } 59\end{array}$ & $\begin{array}{l}\text { keine Her- } \\
\text { vorhebun- } \\
\text { gen }\end{array}$ & & & & \\
\hline \multicolumn{6}{|l|}{$\begin{array}{l}\text { L'identità geo- } \\
\text { grafica (2 Texte) }\end{array}$} \\
\hline $\begin{array}{l}\text { II territorio } \\
\text { ( } 38 \text { Wörter) }\end{array}$ & $\begin{array}{l}\text { Monti dei } \\
\text { draghi }\end{array}$ & n.p. & & Drakensberge & \\
\hline S. 59 & & & & & $\begin{array}{l}\text { Text geht auf } \\
\text { S. } 60 \text { weiter }\end{array}$ \\
\hline \multirow[t]{2}{*}{$\begin{array}{l}\text { Coste e isole } \\
\text { (100 Wörter) }\end{array}$} & $\begin{array}{l}\text { poco arti- } \\
\text { colate }\end{array}$ & avv. + agg. & $\begin{array}{l}\text { FO + TS ge- } \\
\text { ogr. }\end{array}$ & $\begin{array}{l}\text { kaum ungrad- } \\
\text { liniger (Küs- } \\
\text { tenverlauf) }\end{array}$ & $\begin{array}{l}\text { direkter Bezug } \\
\text { zur Überschrift }\end{array}$ \\
\hline & $\begin{array}{l}\text { Mada- } \\
\text { gascar }\end{array}$ & n.p. & & Madagaskar & \\
\hline \multirow{2}{*}{$\begin{array}{l}\text { S. } 59 \\
\text { Osserva la carta } \\
\text { (74 Wörter) }\end{array}$} & & & & & \\
\hline & Namib & n.p. & & Namib & \\
\hline \multicolumn{6}{|l|}{$\begin{array}{l}\text { S. } 60 \text { (Fortset- } \\
\text { zung S. } 59 \text { ) }\end{array}$} \\
\hline & $\begin{array}{l}\left(I^{\prime}\right) \text { Isola } \\
\text { Maurizio }\end{array}$ & n.p. & & $\begin{array}{l}\text { ((die) Insel) } \\
\text { Mauritius }\end{array}$ & \\
\hline \multicolumn{6}{|l|}{ S. 60} \\
\hline $\begin{array}{l}\text { Le acque interne } \\
\text { ( } 55 \text { Wörter) }\end{array}$ & Zambesi & n.p. & & $\begin{array}{l}\text { Sambesi } \\
\text { (Fluss) }\end{array}$ & \\
\hline
\end{tabular}




\begin{tabular}{|c|c|c|c|c|c|}
\hline $\begin{array}{l}\text { Seite/Überschrif- } \\
\text { ten/n Wörter }\end{array}$ & $\begin{array}{l}\text { Wort/Wort- } \\
\text { gruppe }\end{array}$ & Wortart & $\begin{array}{l}\text { Gebrauchs- } \\
\text { marke }\end{array}$ & Übersetzung & Kommentar \\
\hline & $\begin{array}{l}\text { Cascate } \\
\text { Vittoria } \\
\text { Orange }\end{array}$ & $\begin{array}{l}\text { n.p. } \\
\text { n.p. }\end{array}$ & & $\begin{array}{l}\text { Viktoria-Was- } \\
\text { serfälle } \\
\text { Oranje (Fluss) }\end{array}$ & \\
\hline \multicolumn{6}{|l|}{ S. 60} \\
\hline $\begin{array}{l}\text { La regione de- } \\
\text { sertica } \\
\text { ( } 39 \text { Wörter) }\end{array}$ & Kalahari & n.p. & & Namib & $\begin{array}{l}\text { dieselben } \\
\text { Wörter wie auf } \\
\text { der vorausge- } \\
\text { henden Seite }\end{array}$ \\
\hline & \multicolumn{4}{|c|}{ S. 61} & \\
\hline \multirow[t]{8}{*}{$\begin{array}{l}\text { L'identità demo- } \\
\text { grafica } \\
\text { (135 Wörter) }\end{array}$} & boscimani & s.m. & TS etnol. & $\begin{array}{l}\text { San (Busch- } \\
\text { mann/Bosjes- } \\
\text { man) }\end{array}$ & \\
\hline & ottentotti & s.m. & TS etnol. & $\begin{array}{l}\text { Khoikhoi (Hot- } \\
\text { tentotten) }\end{array}$ & \\
\hline & $\begin{array}{l}\text { scarsa- } \\
\text { mente po- } \\
\text { polati }\end{array}$ & avv. + agg. & $2 \mathrm{CO}$ & $\begin{array}{l}\text { dünn besie- } \\
\text { delte }\end{array}$ & \\
\hline & bantu & agg. & TS etnol. & Bantu & \\
\hline & malgasci & s.m. & $\mathrm{CO}$ & Madagassen & \\
\hline & $\begin{array}{l}\text { (l')afri- } \\
\text { caans }\end{array}$ & s.m. & ES ingl. & Afrikaans & $\begin{array}{l}\text { Markenzusatz } \\
\text { im GRADIT } \\
\text { nicht korrekt }\end{array}$ \\
\hline & boeri & s.m. & & Buren & \\
\hline & $\begin{array}{l}\text { piccoli vil- } \\
\text { laggi rurali }\end{array}$ & $\begin{array}{l}\text { agg. }+ \text { s.m. }+ \\
\text { agg. }\end{array}$ & $\mathrm{FO}+2 \mathrm{CO}$ & $\begin{array}{l}\text { kleine ländli- } \\
\text { che Dörfer }\end{array}$ & \\
\hline $\begin{array}{l}\text { Approfondi- } \\
\text { mento: Visitiamo } \\
\text { un villaggio zulu } \\
\text { ( } 73 \text { Wörter) }\end{array}$ & zulu & s.m. & TS etnol. & Zulu & \\
\hline S. 82 & xhosa & s.m. & TS etnol. & Xhosa & \\
\hline \multirow{6}{*}{$\begin{array}{l}\text { S. } 82 \\
\text { La storia del ter- } \\
\text { ritorio: Terre di } \\
\text { antiche civiltà e } \\
\text { di recenti conflitti } \\
\text { (143 Wörter) }\end{array}$} & $\begin{array}{l}\text { Mesopota- } \\
\text { mia }\end{array}$ & n.p. & & Mesopotamien & \\
\hline & $\begin{array}{l}\text { Penisola } \\
\text { dell'Anato- } \\
\text { lia }\end{array}$ & $\begin{array}{l}\text { s.f. + prep.art. } \\
+ \text { n.p. }\end{array}$ & $\mathrm{CO}$ & $\begin{array}{l}\text { anatolische } \\
\text { Halbinsel }\end{array}$ & \\
\hline & $\begin{array}{l}\text { altopiano } \\
\text { dell'Iran }\end{array}$ & s.m. + n.p. & & $\begin{array}{l}\text { Iranische } \\
\text { Hochebene }\end{array}$ & \\
\hline & India & n.p. & & Indien & \\
\hline & Cina & n.p. & & China & \\
\hline & Mongolia & n.p. & & Mongolien & \\
\hline
\end{tabular}

Tabelle 12: Hervorhebungen auf den untersuchten Seiten im Nuovi territori dell'uomo (Bersezio 2005)

Wie der Tabelle zu entnehmen ist, sind in zwei Texten (eine Einleitung) zur Wirtschaft der Region keine Wörter hervorgehoben (Bersezio 2005, S. 33) und in der Einleitung zur südlichen Region Afrikas (ibid., S. 58) ebenfalls nicht. 
Allerdings ist hier der gesamte kurze Text mit einer höheren Schriftgröße abgedruckt. Auf den Fettdruck in den übrigen Texten verteilen sich überwiegend geographische Eigennamen (19), wobei nur wenige von ihnen pro Text verzeichnet werden, und darüber hinaus 13 eingliedrige Substantive und sechs Adjektive. In diesem Buch werden nicht nur insgesamt die wenigsten Hervorhebungen verzeichnet, sondern unter ihnen befinden sich auch die wenigsten Wortgruppen: Weniger als ein Viertel (8) der Hervorhebungen überschreiten die Länge eines graphischen Wortes, während es in den anderen vier Lehrwerken mindestens 28 und bis zu 57 sind.

Zehn der 14 niedrigfrequenten Wörter (CO, Co Ts, Ts und Es) verteilen sich auf zwei Texte, die sich mit der Demographie der Region befassen und diese anhand eines Beispiels (Zulu-Dorf) in einem weiteren Text vertiefen. Alle fachwortschatzsprachlichen Hervorhebungen stammen aus dem ethnologischen Bereich, so dass hier wie auch in den übrigen Texten eine hohe Kongruenz zwischen den Textüberschriften und dem semantischen Gehalt der hervorgehobenen Wörter beobachtet werden kann.

Die Überschriften geben generell präzise an, worum es in den relativ kurzen Texten geht, was eine zusätzliche Hervorhebung von Textelementen unnötig zu machen scheint. Im Übrigen ist die im Verhältnis zu den anderen untersuchten Texten große Anzahl von Hervorhebungen in den beiden Texten demographischen und ethnologischen Inhalts wahrscheinlich darauf zurückzuführen, dass es sich bei den fettgedruckten Ausdrücken um Lehnwörter und in einem Fall sogar um ein Fremdwort handelt (afrikaans; Bersezio 2005, S. 61), welche die Lernenden mit hoher Wahrscheinlichkeit zum ersten Mal lesen.

Im Lehrbuch Zaino in spalla (Bastianelli et al. 2010; Verlag Sansoni) sind auf den untersuchten Seiten 43 Wörter bzw. Wortgruppen hervorgehoben und damit nach dem Lehrbuch von Bersezio (2005) die wenigsten. Tabelle 13 veranschaulicht diese Hervorhebungen.

\begin{tabular}{|c|c|c|c|c|c|}
\hline $\begin{array}{l}\text { Seite/Überschrif- } \\
\text { ten/n Wörter }\end{array}$ & $\begin{array}{l}\text { Wort/ } \\
\text { Wortgruppe }\end{array}$ & Wortart & Marke & Übersetzung & Kommentar \\
\hline \multirow{7}{*}{$\begin{array}{l}\text { S. } 87 \\
\text { L'uomo ha biso- } \\
\text { gno di energia } \\
\text { per lavorare } \\
\text { (137 Wörter) }\end{array}$} & & & & & \multirow{7}{*}{$\begin{array}{l}\text { fonti: energeti- } \\
\text { che impliziert, } \\
\text { Marke der loc. } \\
\text { fonti: energeti- } \\
\text { che impliziert, } \\
\text { Marke der loc. }\end{array}$} \\
\hline & forza & s.f. & $\mathrm{FO}$ & Kraft & \\
\hline & lavoro & s.m. & FO & Arbeit & \\
\hline & $\begin{array}{l}\text { fonti non rin- } \\
\text { novabili }\end{array}$ & $\begin{array}{l}\text { s.f. + avv. + } \\
\text { agg. }\end{array}$ & $\begin{array}{l}\text { TS ecol. + } \\
\text { FO + CO }\end{array}$ & $\begin{array}{l}\text { nicht erneuerbare } \\
\text { (fossile) Energie- } \\
\text { quellen }\end{array}$ & \\
\hline & inquinamento & s.m. & TS ecol. & Verschmutzung & \\
\hline & nucleare & s.m. & $\mathrm{CO}$ & Atomkraft & \\
\hline & $\begin{array}{l}\text { fonti rinnova- } \\
\text { bili }\end{array}$ & s.f. + agg. & $\begin{array}{l}\text { TS ecol. + } \\
\text { CO }\end{array}$ & $\begin{array}{l}\text { erneuerbare } \\
\text { Energiequellen }\end{array}$ & \\
\hline \multicolumn{6}{|l|}{ S. $116 \mathrm{f}$. } \\
\hline $\begin{array}{l}\text { Il sistema econo- } \\
\text { mico mondiale. }\end{array}$ & $\begin{array}{l}\text { maggiori pro- } \\
\text { duttori }\end{array}$ & agg. + s.m. & $\mathrm{FO}+\mathrm{AU}$ & $\begin{array}{l}\text { größte Produzen- } \\
\text { ten }\end{array}$ & \\
\hline
\end{tabular}




\begin{tabular}{|c|c|c|c|c|c|}
\hline $\begin{array}{l}\text { Seite/Überschrif- } \\
\text { ten/n Wörter }\end{array}$ & $\begin{array}{l}\text { Wort/ } \\
\text { Wortgruppe }\end{array}$ & Wortart & Marke & Übersetzung & Kommentar \\
\hline \multicolumn{6}{|l|}{$\begin{array}{l}\text { Le potenze eco- } \\
\text { nomiche mondiali } \\
\text { (180 Wörter) }\end{array}$} \\
\hline & $\begin{array}{l}\text { manufatti ad } \\
\text { alto conte- } \\
\text { nuto tecnolo- } \\
\text { gico }\end{array}$ & $\begin{array}{l}\text { s.m. + prep. } \\
\text { + agg. + s.m. } \\
\text { + agg. }\end{array}$ & $\begin{array}{l}\mathrm{CO}+2 \mathrm{FO} \\
+\mathrm{AU}\end{array}$ & $\begin{array}{l}\text { Fabrikwaren mit } \\
\text { hohem technolo- } \\
\text { gischen Gehalt }\end{array}$ & \\
\hline & $\begin{array}{l}\text { Paesi orien- } \\
\text { tali }\end{array}$ & s.m. + agg. & $\mathrm{FO}+\mathrm{AU}$ & östliche Länder & $\begin{array}{l}\text { morgenlän- } \\
\text { disch }\end{array}$ \\
\hline & $\begin{array}{l}\text { fortissima } \\
\text { crescita } \\
\text { dell'economia }\end{array}$ & $\begin{array}{l}\text { agg. + s.f. + } \\
\text { prep.art. + } \\
\text { s.f. }\end{array}$ & $\begin{array}{l}\mathrm{FO}+\mathrm{AD}+ \\
\mathrm{AU}\end{array}$ & $\begin{array}{l}\text { sehr starkes Wirt- } \\
\text { schaftswachstum }\end{array}$ & \\
\hline \multicolumn{6}{|l|}{ S. 117} \\
\hline \multirow{5}{*}{$\begin{array}{l}\text { Gli altri protago- } \\
\text { nisti dell'econo- } \\
\text { mia mondiale } \\
\text { (118 Wörter) }\end{array}$} & Brasile & n.p. & & Brasilien & \\
\hline & $\begin{array}{l}\text { Paesi dei Ca- } \\
\text { raibi }\end{array}$ & $\begin{array}{l}\text { s.m. + } \\
\text { prep.art. + } \\
\text { n.p. }\end{array}$ & $\begin{array}{l}2 \mathrm{FO}+ \\
\text { n.p. }\end{array}$ & $\begin{array}{l}\text { Länder der Kari- } \\
\text { bik }\end{array}$ & \\
\hline & Stati africani & s.m. + agg. & $\mathrm{FO}+\mathrm{AU}$ & $\begin{array}{l}\text { afrikanische Staa- } \\
\text { ten }\end{array}$ & \\
\hline & $\begin{array}{l}\text { medio-orien- } \\
\text { tale }\end{array}$ & agg. + agg. & $2 \mathrm{AU}$ & $\begin{array}{l}\text { des Nahen Os- } \\
\text { tens }\end{array}$ & \\
\hline & $\begin{array}{l}\text { Paesi } \\
\text { dell'Africa } \\
\text { sub-saha- } \\
\text { riana }\end{array}$ & $\begin{array}{l}\text { s.m. + } \\
\text { prep.art. + } \\
\text { n.p. + pref. + } \\
\text { agg. }\end{array}$ & $\begin{array}{l}\text { FO + n.p. } \\
+ \text { TS ge- } \\
\text { ogr. }\end{array}$ & $\begin{array}{l}\text { afrikanische Län- } \\
\text { der südlich der } \\
\text { Sahara }\end{array}$ & \\
\hline \multirow{7}{*}{$\begin{array}{l}\text { S. } 118 \\
\text { Sviluppo e sotto- } \\
\text { sviluppo tra Nord } \\
\text { e Sud del Mondo } \\
\text { (227 Wörter) }\end{array}$} & & & & & \\
\hline & $\begin{array}{l}\text { crescita eco- } \\
\text { nomica }\end{array}$ & loc.s.f. & TS econ. & $\begin{array}{l}\text { Wirtschaftswachs- } \\
\text { tum }\end{array}$ & \\
\hline & $\begin{array}{l}\text { condizioni di } \\
\text { vita della po- } \\
\text { polazione }\end{array}$ & $\begin{array}{l}\text { s.f. + prep. + } \\
\text { s.f. + } \\
\text { prep.art. + } \\
\text { s.f. }\end{array}$ & $4 \mathrm{FO}+\mathrm{AU}$ & $\begin{array}{l}\text { Lebensbedingun- } \\
\text { gen der Bevölke- } \\
\text { rung }\end{array}$ & \\
\hline & $\begin{array}{l}\text { Paesi svilup- } \\
\text { pati }\end{array}$ & s.m. + agg. & $\mathrm{FO}+\mathrm{CO}$ & $\begin{array}{l}\text { entwickelte Län- } \\
\text { der }\end{array}$ & \\
\hline & $\begin{array}{l}\text { Paesi sotto- } \\
\text { sviluppati }\end{array}$ & s.m. + agg. & $\mathrm{FO}+\mathrm{CO}$ & $\begin{array}{l}\text { unterentwickelte } \\
\text { Länder }\end{array}$ & \\
\hline & $\begin{array}{l}\text { Paesi a svi- } \\
\text { luppo inter- } \\
\text { medio }\end{array}$ & $\begin{array}{l}\text { s.m. + prep. } \\
\text { + s.m. + agg. }\end{array}$ & $\begin{array}{l}2 \text { FO + TS } \\
\text { econ., } \\
\text { sociol. + } \\
\text { CO }\end{array}$ & $\begin{array}{l}\text { Länder mittleren } \\
\text { Entwicklungsstan- } \\
\text { des }\end{array}$ & \\
\hline & $\begin{array}{l}\text { divario tra i } \\
\text { paesi ricchi e } \\
\text { quelli poveri }\end{array}$ & $\begin{array}{l}\text { s.m. + prep. } \\
+ \text { art. + s.m. } \\
+ \text { agg. + } \\
\text { cong. + } \\
\text { pron. + agg. }\end{array}$ & $\begin{array}{l}\mathrm{CO}+\mathrm{FO}+ \\
\mathrm{CO}+5 \mathrm{FO}\end{array}$ & $\begin{array}{l}\text { Gefälle zwischen } \\
\text { den reichen Län- } \\
\text { dern und den ar- } \\
\text { men }\end{array}$ & \\
\hline \multirow{3}{*}{$\begin{array}{l}\text { S. } 149 \\
\text { Le dinamiche de- } \\
\text { mografiche oggi } \\
\text { (261 Wörter) }\end{array}$} & & & & & \\
\hline & $\begin{array}{l}\text { tasso di mor- } \\
\text { talià }\end{array}$ & loc.s.m. & TS stat. & Sterberate & \\
\hline & $\begin{array}{l}\text { tasso di nata- } \\
\text { lità }\end{array}$ & loc.s.m. & TS stat. & Geburtenrate & \\
\hline
\end{tabular}




\begin{tabular}{|c|c|c|c|c|c|}
\hline $\begin{array}{l}\text { Seite/Überschrif- } \\
\text { ten/n Wörter }\end{array}$ & $\begin{array}{l}\text { Wort/ } \\
\text { Wortgruppe }\end{array}$ & Wortart & Marke & Übersetzung & Kommentar \\
\hline & $\begin{array}{l}\text { saldo natu- } \\
\text { rale }\end{array}$ & loc.s.m. & TS stat. & Geburtenbilanz & \\
\hline & $\begin{array}{l}\text { natalità è } \\
\text { alta, ma si- } \\
\text { mile alla mor- } \\
\text { talità }\end{array}$ & $\begin{array}{l}\text { s.f. + v.intr. + } \\
\text { agg. + } \\
\text { prep.art. + } \\
\text { s.f. }\end{array}$ & $\begin{array}{l}\mathrm{FO}+\mathrm{CO} \\
\mathrm{TS} \text { stat. }+ \\
5 \mathrm{FO}+\mathrm{CO}\end{array}$ & $\begin{array}{l}\text { Geburtenrate ist } \\
\text { hoch, aber der } \\
\text { Sterberate ähn- } \\
\text { lich }\end{array}$ & \\
\hline & $\begin{array}{l}\text { diminuisce la } \\
\text { mortalità } \\
\text { diminuisce la } \\
\text { natalità }\end{array}$ & $\begin{array}{l}\text { v.intr. + art. } \\
\text { + s.f. } \\
\text { v.intr. + art. } \\
\text { + s.f. }\end{array}$ & $\begin{array}{l}\mathrm{AU}+\mathrm{FO}+ \\
\mathrm{CO} \\
\mathrm{AU}+\mathrm{FO}+ \\
\mathrm{CO} \text { TS } \\
\text { stat. }\end{array}$ & $\begin{array}{l}\text { die Sterberate } \\
\text { sinkt } \\
\text { die Geburtenrate } \\
\text { sinkt }\end{array}$ & \\
\hline & $\begin{array}{l}\text { la mortalità } \\
\text { supera la na- } \\
\text { talità }\end{array}$ & $\begin{array}{l}\text { art. + s.f. + } \\
\text { v.tr. + art. + } \\
\text { s.f. }\end{array}$ & $\begin{array}{l}\mathrm{FO}+\mathrm{CO}+ \\
2 \mathrm{FO}+\mathrm{CO} \\
\text { TS stat. }\end{array}$ & $\begin{array}{l}\text { die Sterberate } \\
\text { übertrifft die Ge- } \\
\text { burtenrate }\end{array}$ & \\
\hline \multicolumn{6}{|l|}{ S. 182} \\
\hline \multirow{3}{*}{$\begin{array}{l}\text { Le lingue nel } \\
\text { mondo } \\
\text { (165 Wörter) }\end{array}$} & $\begin{array}{l}\text { come organi- } \\
\text { smi viventi }\end{array}$ & $\begin{array}{l}\text { avv. + s.m. + } \\
\text { agg. }\end{array}$ & $\begin{array}{l}\mathrm{FO}+\mathrm{AU}+ \\
\mathrm{CO}\end{array}$ & $\begin{array}{l}\text { wie lebendige Or- } \\
\text { ganismen }\end{array}$ & \\
\hline & $\begin{array}{l}\text { continenti in- } \\
\text { teri }\end{array}$ & s.m. + agg. & $2 \mathrm{FO}$ & ganze Kontinente & \\
\hline & lingue locali & s.f. + agg. & $\mathrm{FO}+\mathrm{AU}$ & Lokalsprachen & \\
\hline \multicolumn{6}{|l|}{ S. $206 \mathrm{f.}$} \\
\hline \multirow[t]{5}{*}{$\begin{array}{l}\text { Le regioni } \\
\text { dell'Asia } \\
\text { ( } 41 \text { Wörter) }\end{array}$} & $\begin{array}{l}\text { Medio } \\
\text { Oriente }\end{array}$ & loc.s.m. & $\mathrm{CO}$ & $\begin{array}{l}\text { Naher und Mittle- } \\
\text { rer Osten }\end{array}$ & $\begin{array}{l}\text { im Deutschen } \\
\text { unterscheiden } \\
\text { sich die Län- } \\
\text { der des Mittle- } \\
\text { ren Osten von } \\
\text { denen im Itali- } \\
\text { enischen }\end{array}$ \\
\hline & $\begin{array}{l}\left(l^{\prime}\right) \text { Asia cen- } \\
\text { tro-settentrio- } \\
\text { nale }\end{array}$ & n.p. + agg. & $\mathrm{CO}$ & Nord-Zentralasien & \\
\hline & $\begin{array}{l}\text { regione in- } \\
\text { diana }\end{array}$ & n.p. & & indische Region & \\
\hline & $\begin{array}{l}\text { (l')Asia orien- } \\
\text { tale }\end{array}$ & n.p. + agg. & $A U$ & Ostasien & \\
\hline & $\begin{array}{l}\text { Sud-Est asia- } \\
\text { tico }\end{array}$ & s.m. + agg. & $\begin{array}{l}\text { CO TS ge- } \\
\text { ogr. + AD }\end{array}$ & Südostasien & \\
\hline \multirow{7}{*}{$\begin{array}{l}\text { L'ambiente natu- } \\
\text { rale } \\
\text { (126 Wörter) }\end{array}$} & $\begin{array}{l}\text { catene mon- } \\
\text { tuose }\end{array}$ & loc.s.m. & TS geogr. & $\begin{array}{l}\text { Bergkette/Ge- } \\
\text { birgszug }\end{array}$ & \\
\hline & tavolati & s.m. & TS geogr. & Deckgebirge & \\
\hline & pianure & s.f. & FO & Ebene & \\
\hline & $\begin{array}{l}\text { catene mon- } \\
\text { tuose molto } \\
\text { elevate }\end{array}$ & $\begin{array}{l}\text { loc.s.m. + } \\
\text { avv. + agg. }\end{array}$ & $\begin{array}{l}\text { TS geogr. } \\
+\mathrm{FO}+\mathrm{CO}\end{array}$ & $\begin{array}{l}\text { sehr hohe Berg- } \\
\text { ketten }\end{array}$ & \\
\hline & grandi fiumi & agg. + s.m. & $2 \mathrm{FO}$ & große Flüsse & \\
\hline & climi & s.m. & FO & Klimata & $\begin{array}{l}\text { das entschei- } \\
\text { dende Wort } \\
\text { nicht fettge- } \\
\text { druckt (varia- } \\
\text { zione) }\end{array}$ \\
\hline & ambienti & s.m. & TS ecol. & natürliche Umwelt & \\
\hline
\end{tabular}

Tabelle 13: Hervorhebungen auf den untersuchten Seiten im Zaino in spalla (Bastianelli et al. 2010) 
Hier finden sich in jedem Text mindestens zwei Hervorhebungen, die zu über drei Vierteln (34 von 40) aus mehr als einem graphischen Wort bestehen. Anders als in dem zuletzt vorgestellten Buch handelt es sich dabei zum Teil um ganze Sätze inklusive der Prädikate (4). So lassen sich in diesem Buch mehrere hervorgehobene Wortgruppen registrieren, die aus fünf oder mehr Wörtern bestehen.

Der Großteil der Wörter stammt aus dem Basiswortschatz, aber 22 Wörter sind niedrigfrequent und tragen die Marken Co, Co Ts und Ts. Diese verteilen sich auf alle Texte, wobei die Konzentration in den Texten auf den Seiten 118 und 149 besonders hoch ist. Die am häufigsten unter den Hervorhebungen zu findenden fachsprachlichen Ausdrücke stammen aus den Bereichen der Geographie, der Wirtschaft und der Statistik. Mit Abstand am geringsten im Vergleich zu den anderen Lehrbüchern ist hier der Anteil an fettgedruckten geographischen Eigennamen (6), während die meisten Hervorhebungen Substantive betreffen, die oft durch Adjektive ergänzt sind und neun Phraseme beinhalten.

In dem in der Klasse III B benutzten Erdkundebuch Geograficamente (Dinucci \& Dinucci 2008; Verlag Zanichelli) sind auf den untersuchten Seiten mit Abstand die meisten Wörter und Wortgruppen hervorgehoben, nämlich die in Tabelle 14 aufgelisteten 96:

\begin{tabular}{|c|c|c|c|c|c|}
\hline $\begin{array}{l}\text { Seite/Über- } \\
\text { schriften/n } \\
\text { Wörter }\end{array}$ & $\begin{array}{l}\text { Wort/ } \\
\text { Wortgruppe }\end{array}$ & Wortart & $\begin{array}{l}\text { Ge- } \\
\text { brauchs- } \\
\text { marke }\end{array}$ & Übersetzung & Kommentar \\
\hline S. 52 & & & & & \\
\hline \multirow{8}{*}{$\begin{array}{l}\text { Le lingue: } \\
\text { Quante e } \\
\text { quali sono le } \\
\text { lingue? } \\
\text { (171 Wörter) }\end{array}$} & circa 6800 & avv. & FO & ca. 6.800 & \\
\hline & famiglie linguistiche & loc.s.f. & TS ling. & Sprachfamilie & \\
\hline & lingua madre loc.s.f. & & TS ling. & Muttersprache & $\begin{array}{l}\text { nicht Haupt- } \\
\text { bedeutung }\end{array}$ \\
\hline & indoeuropea & agg. & TS ling. & $\begin{array}{l}\text { indogerma- } \\
\text { nisch }\end{array}$ & \\
\hline & afro-asiatica & s.f. & $\mathrm{CO}$ & Afroasiatische & \\
\hline & sino-tibetana & s.f. & TS ling. & Sinotibetische & \\
\hline & uralo-altaica & s.f. & TS ling. & Uralaltaische & \\
\hline & 225 sono ufficiali & v.intr. + agg. & $2 \mathrm{FO}$ & $\begin{array}{l}225 \text { sind offizi- } \\
\text { ell (Amtsspra- } \\
\text { chen) }\end{array}$ & $\begin{array}{l}\text { lingua uffi- } \\
\text { ciale: loc.s.f. } \\
\text { TS ling.dir. } \\
\text { (Amtsspra- } \\
\text { che) }\end{array}$ \\
\hline \multicolumn{6}{|l|}{ S. 53} \\
\hline $\begin{array}{l}\text { Quali sono } \\
\text { le lingue più } \\
\text { parlate? }\end{array}$ & cinese & s.m. & $A U$ & Chinesisch & \\
\hline \multirow[t]{4}{*}{ (125 Wörter) } & (I) inglese & s.m. & FO & Englisch & \\
\hline & (I')hindi & s.m. & TS ling. & Hindi & \\
\hline & spagnolo & s.m. & TS ling. & Spanisch & \\
\hline & russo & s.m. & FO & Russisch & \\
\hline
\end{tabular}




\begin{tabular}{|c|c|c|c|c|c|}
\hline $\begin{array}{l}\text { Seite/Über- } \\
\text { schriften/n } \\
\text { Wörter }\end{array}$ & $\begin{array}{l}\text { Wort/ } \\
\text { Wortgruppe }\end{array}$ & Wortart & $\begin{array}{l}\text { Ge- } \\
\text { brauchs- } \\
\text { marke }\end{array}$ & Übersetzung & Kommentar \\
\hline & la metà delle lingue & $\begin{array}{l}\text { art. + s.f. + } \\
\text { prep.art. + } \\
\text { s.f. }\end{array}$ & $4 \mathrm{FO}$ & $\begin{array}{l}\text { die Hälfte der } \\
\text { Sprachen }\end{array}$ & \\
\hline & multilinguismo & s.m. & $\begin{array}{l}\text { CO TS } \\
\text { ling. }\end{array}$ & $\begin{array}{l}\text { Mehrsprachig- } \\
\text { keit }\end{array}$ & \\
\hline \multicolumn{6}{|l|}{ S. 238} \\
\hline \multirow[t]{4}{*}{$\begin{array}{l}\text { Asia } \\
\text { (114 Wörter) }\end{array}$} & Mar Glaciale Artico & n.p. & & $\begin{array}{l}\text { Arktischer } \\
\text { Ozean }\end{array}$ & \\
\hline & Oceano Pacifico & n.p. & & $\begin{array}{l}\text { Pazifischer } \\
\text { Ozean }\end{array}$ & \\
\hline & $\begin{array}{l}\text { grandi stati insulari } \\
\text { di Giappone, Filip- } \\
\text { pine e Indonesia }\end{array}$ & $\begin{array}{l}\text { agg. + s.m. } \\
+ \text { agg. }+ \\
\text { prep. }+2 \\
\text { n.p. + cong. } \\
+ \text { n.p. }\end{array}$ & $\begin{array}{l}2 \mathrm{FO}+\mathrm{CO} \\
+\mathrm{FO}+2 \\
\text { n.p. }+ \\
\text { cong. }+ \\
\text { n.p. }\end{array}$ & $\begin{array}{l}\text { große Insel- } \\
\text { staaten wie } \\
\text { Japan, die } \\
\text { Philippinen } \\
\text { und Indone- } \\
\text { sien }\end{array}$ & \\
\hline & $\begin{array}{l}\text { penisole indocinese, } \\
\text { indiana e araba }\end{array}$ & $\begin{array}{l}\text { s.f. }+2 \text { agg. } \\
+ \text { cong. }+ \\
\text { agg. }\end{array}$ & $\begin{array}{l}\mathrm{FO}+\mathrm{CO} \\
+\mathrm{TS} \text { etnol. } \\
+\mathrm{AU}+2 \\
\mathrm{FO}\end{array}$ & $\begin{array}{l}\text { indochinesi- } \\
\text { sche, indische } \\
\text { und arabische } \\
\text { Halbinsel }\end{array}$ & \\
\hline \multicolumn{6}{|l|}{ S. 239} \\
\hline \multirow[t]{3}{*}{$\begin{array}{l}\text { Rilievo } \\
\text { (146 Wörter) }\end{array}$} & fascia settentrionale & s.f. + agg. & $2 \mathrm{AU}$ & $\begin{array}{l}\text { nördlicher } \\
\text { Streifen }\end{array}$ & $\begin{array}{l}\text { in drei ein- } \\
\text { zelne Ab- } \\
\text { sätze unter- } \\
\text { gliedert, die } \\
\text { jeweils mit } \\
\text { diesen Wör- } \\
\text { tern begin- } \\
\text { nen }\end{array}$ \\
\hline & fascia centrale & s.f. + agg. & $A U+F O$ & $\begin{array}{l}\text { zentraler Strei- } \\
\text { fen }\end{array}$ & \\
\hline & fascia meridionale & s.f. + agg. & $2 \mathrm{AU}$ & $\begin{array}{l}\text { südlicher } \\
\text { Streifen }\end{array}$ & \\
\hline \multicolumn{6}{|l|}{ ibid. } \\
\hline \multirow[t]{6}{*}{$\begin{array}{l}\text { Idrografia } \\
\text { (267 Wörter) }\end{array}$} & zone desertiche & s.f. + agg. & $\mathrm{FO}+\mathrm{CO}$ & $\begin{array}{l}\text { Wüstenge- } \\
\text { biete }\end{array}$ & \\
\hline & $\begin{array}{l}\text { versanti dei fiumi } \\
\text { asiatici }\end{array}$ & $\begin{array}{l}\text { s.m. + } \\
\text { prep.art. + } \\
\text { s.m. + agg. }\end{array}$ & $\begin{array}{l}\text { TS geogr. } \\
+2 \mathrm{FO}+ \\
A D\end{array}$ & $\begin{array}{l}\text { Abhänge der } \\
\text { asiatischen } \\
\text { Flüsse }\end{array}$ & \\
\hline & versante artico & s.m. + agg. & $\begin{array}{l}\text { TS geogr. } \\
+A D\end{array}$ & $\begin{array}{l}\text { arktischer Ab- } \\
\text { hang }\end{array}$ & \\
\hline & $\begin{array}{l}\text { versante del Pa- } \\
\text { cifico }\end{array}$ & $\begin{array}{l}\text { s.m. + } \\
\text { prep.art. }+ \\
\text { n.p. }\end{array}$ & $\begin{array}{l}\text { TS geogr. } \\
\text { + FO + n.p. }\end{array}$ & $\begin{array}{l}\text { Abhang des } \\
\text { Pazifiks }\end{array}$ & \\
\hline & $\begin{array}{l}\text { versante } \\
\text { dell'oceano Indiano }\end{array}$ & $\begin{array}{l}\text { s.m. + } \\
\text { prep.art. + } \\
\text { n.p. }\end{array}$ & $\begin{array}{l}\text { TS geogr. } \\
+ \text { FO + n.p. }\end{array}$ & $\begin{array}{l}\text { Abhang des } \\
\text { Indischen } \\
\text { Ozeans }\end{array}$ & \\
\hline & laghi principali & s.m. + agg. & $2 \mathrm{FO}$ & $\begin{array}{l}\text { bedeutendste } \\
\text { Seen }\end{array}$ & \\
\hline \multicolumn{6}{|l|}{ S. 240} \\
\hline \multirow[t]{2}{*}{$\begin{array}{l}\text { Clima e am- } \\
\text { biente } \\
\text { (389 Wörter) }\end{array}$} & $\begin{array}{l}\text { grande varietà di } \\
\text { climi e vegetazioni }\end{array}$ & $\begin{array}{l}\text { agg. + s.f. + } \\
\text { prep. S.m. }+ \\
\text { cong. + s.f. }\end{array}$ & $\begin{array}{l}2 \mathrm{FO}+\mathrm{AU} \\
+3 \mathrm{FO}+ \\
\mathrm{AU}\end{array}$ & $\begin{array}{l}\text { große Vielfäl- } \\
\text { tigkeit an Kli- } \\
\text { mata und Ve- } \\
\text { gatation }\end{array}$ & \\
\hline & zona siberiana & s.f. + agg. & $\mathrm{FO}+\mathrm{CO}$ & $\begin{array}{l}\text { sibirisches } \\
\text { Gebiet }\end{array}$ & \\
\hline
\end{tabular}




\begin{tabular}{|c|c|c|c|c|c|}
\hline $\begin{array}{l}\text { Seite/Über- } \\
\text { schriften/n } \\
\text { Wörter }\end{array}$ & $\begin{array}{l}\text { Wort/ } \\
\text { Wortgruppe }\end{array}$ & Wortart & $\begin{array}{l}\text { Ge- } \\
\text { brauchs- } \\
\text { marke }\end{array}$ & Übersetzung & Kommentar \\
\hline & $\begin{array}{l}\text { località abitata dove } \\
\text { si registra la più } \\
\text { bassa temperatura } \\
\text { invernale }\end{array}$ & $\begin{array}{l}\text { s.f. + agg. + } \\
\text { cong. + } \\
\text { pron.pers. + } \\
\text { v.tr. + art. + } \\
\text { avv. + agg. + } \\
\text { s.f. + agg. } \\
\text { s.f. + } 3 \text { agg. }\end{array}$ & $\begin{array}{l}2 \mathrm{FO}+\mathrm{CO} \\
+\mathrm{FO}+\mathrm{CO} \\
+\mathrm{AD}+3 \\
\mathrm{FO}+\mathrm{FO} \\
\mathrm{TS} \text { meteor. } \\
+\mathrm{AU} \\
3 \mathrm{FO}+1 \\
\mathrm{AU}\end{array}$ & $\begin{array}{l}\text { bewohnter } \\
\text { Ort, in dem die } \\
\text { niedrigste } \\
\text { Wintertempe- } \\
\text { ratur verzeich- } \\
\text { net wird } \\
\text { trockenes kon- } \\
\text { tinentales Ge- } \\
\text { biet }\end{array}$ & $\begin{array}{l}\text { viele Wörter } \\
\text { hervorgeho- } \\
\text { ben }\end{array}$ \\
\hline & $\begin{array}{l}\text { zone di alta mon- } \\
\text { tagna }\end{array}$ & $\begin{array}{l}\text { s.f. + prep. + } \\
\text { agg. + s.f. }\end{array}$ & $4 \mathrm{FO}$ & $\begin{array}{l}\text { Gebiete der } \\
\text { Hochgebirge }\end{array}$ & \\
\hline & zona subtropicale & s.f. + agg. & $\mathrm{FO}+\mathrm{CO}$ & $\begin{array}{l}\text { subtropisches } \\
\text { Gebiet }\end{array}$ & \\
\hline & fascia tropicale & s.f. + agg. & $\mathrm{AU}+\mathrm{CO}$ & $\begin{array}{l}\text { tropischer } \\
\text { Streifen }\end{array}$ & \\
\hline & fascia equatoriale & s.f. + agg. & $\begin{array}{l}\text { AU + TS } \\
\text { geogr. }\end{array}$ & Äquatorzone & \\
\hline & foreste pluviali & loc.s.f. & TS geogr. & Regenwälder & \\
\hline & deforestazine & s.f. & $\mathrm{CO}$ & Waldrodung & \\
\hline ibid. & $\begin{array}{l}\text { inquinamento atmo- } \\
\text { sferico }\end{array}$ & s.f. + agg. & $\begin{array}{l}\text { TS ecol- + } \\
\text { CO }\end{array}$ & $\begin{array}{l}\text { Luftver- } \\
\text { schmutzung }\end{array}$ & \\
\hline $\begin{array}{l}\text { Popolazione } \\
\text { (181 Wörter) }\end{array}$ & regione più popolata & $\begin{array}{l}\text { s.f. + avv. + } \\
\text { agg. }\end{array}$ & $2 \mathrm{FO}+\mathrm{CO}$ & $\begin{array}{l}\text { am dichtesten } \\
\text { besiedelte Re- } \\
\text { gion }\end{array}$ & \\
\hline & $\begin{array}{l}\text { oltre } 4 \text { miliardi di } \\
\text { abitanti }\end{array}$ & $\begin{array}{l}\text { prep. + s.m. } \\
+ \text { prep. + } \\
\text { s.m. }\end{array}$ & $2 \mathrm{FO}+\mathrm{AU}$ & $\begin{array}{l}\text { über vier Milli- } \\
\text { arden Einwoh- } \\
\text { ner }\end{array}$ & \\
\hline & aree più abitate & $\begin{array}{l}\text { s.f. + avv. + } \\
\text { agg. }\end{array}$ & $\begin{array}{l}\text { TS geogr. } \\
+\mathrm{FO}+\mathrm{CO}\end{array}$ & $\begin{array}{l}\text { am dichtesten } \\
\text { besiedeltste } \\
\text { Gebiete }\end{array}$ & \\
\hline & $\begin{array}{l}\text { durata media della } \\
\text { vita }\end{array}$ & $\begin{array}{l}\text { s.f + agg. + } \\
\text { prep.art. + } \\
\text { s.f. }\end{array}$ & $\begin{array}{l}\mathrm{FO}+2 \mathrm{AU} \\
+2 \mathrm{FO}\end{array}$ & $\begin{array}{l}\text { durchschnittli- } \\
\text { che Lebens- } \\
\text { dauer }\end{array}$ & \\
\hline & popolazione urbana & s.f. + agg. & $2 \mathrm{AU}$ & $\begin{array}{l}\text { städtische Be- } \\
\text { völkerung }\end{array}$ & \\
\hline & $\begin{array}{l}\text { enormi agglomerati } \\
\text { urbani }\end{array}$ & $\begin{array}{l}\text { agg. }+ \\
\text { loc.s.m. }\end{array}$ & $\begin{array}{l}\text { FO + TS } \\
\text { urban. }\end{array}$ & $\begin{array}{l}\text { enorme Bal- } \\
\text { lungsräume }\end{array}$ & \\
\hline \multirow{9}{*}{$\begin{array}{l}\text { S. } 241 \\
\text { Economi e } \\
\text { società } \\
\text { ( } 281 \text { Wörter) }\end{array}$} & & & & & \\
\hline & (L')agricoltura & s.f. & FO & Landwirtschaft & \\
\hline & Cina & n.p. & & China & \\
\hline & India & n.p. & & Indien & \\
\hline & $\begin{array}{l}\text { al primo posto nel } \\
\text { mondo }\end{array}$ & $\begin{array}{l}\text { prep.art. + } \\
\text { agg. + s.m. } \\
\text { + prep.art. + } \\
\text { s.m. }\end{array}$ & $5 \mathrm{FO}$ & $\begin{array}{l}\text { an erster } \\
\text { Stelle in der } \\
\text { Welt }\end{array}$ & \\
\hline & $\begin{array}{l}\text { riso, grano, tè, } \\
\text { cotone }\end{array}$ & 4 s.m. & $\begin{array}{l}\mathrm{AU}+2 \mathrm{FO} \\
+\mathrm{AU}\end{array}$ & $\begin{array}{l}\text { Reis, Ge- } \\
\text { treide, Tee, } \\
\text { Baumwolle }\end{array}$ & \\
\hline & (l')allevamento & s.m. & FO & Viehzucht & \\
\hline & pesca & s.f. & $A U$ & Fischfang & \\
\hline & ricca di minerali & $\begin{array}{l}\text { agg. + prep. } \\
+ \text { s.m. }\end{array}$ & $3 \mathrm{FO}$ & $\begin{array}{l}\text { reich an Bo- } \\
\text { denschätzen }\end{array}$ & \\
\hline
\end{tabular}




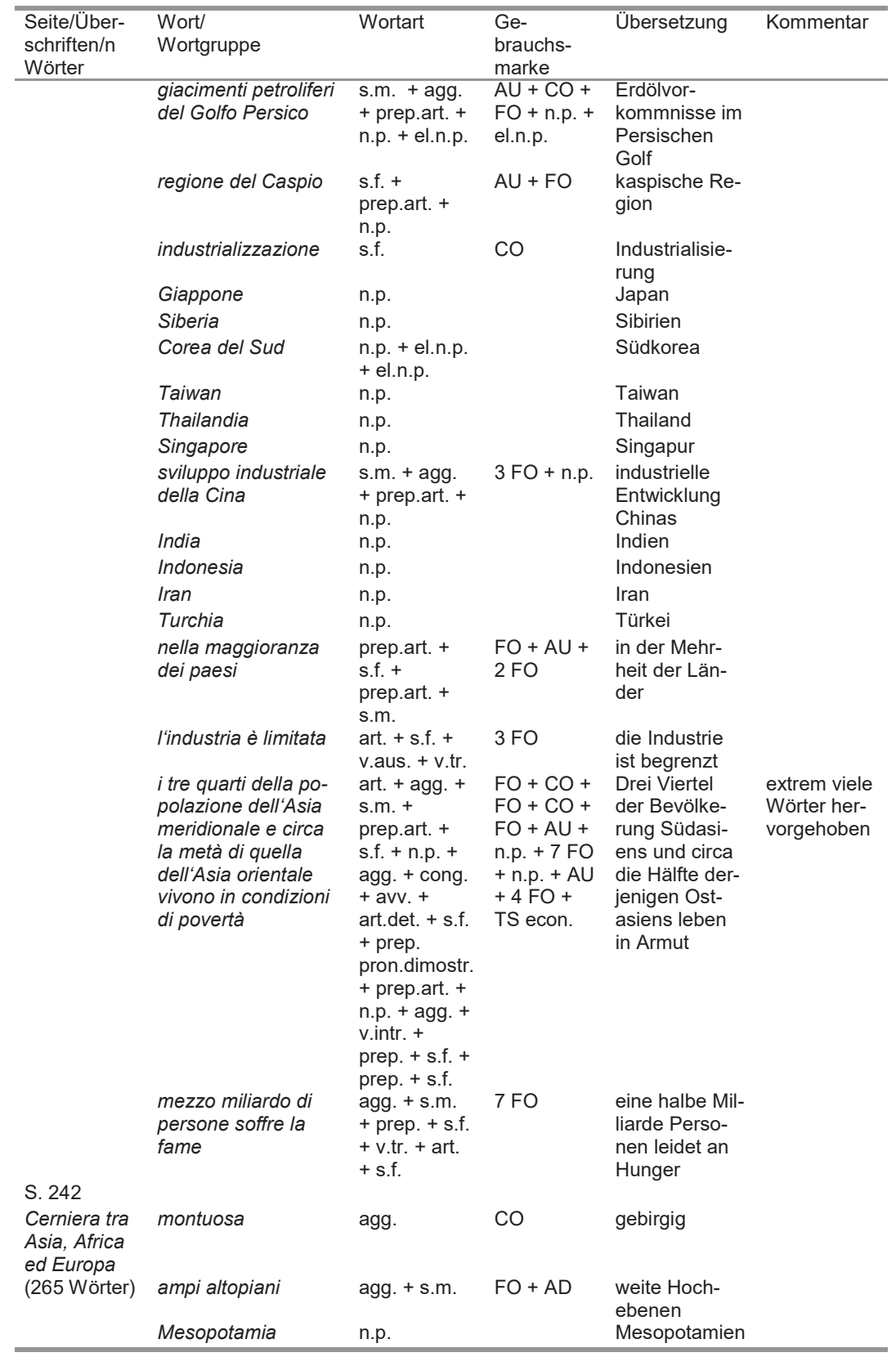




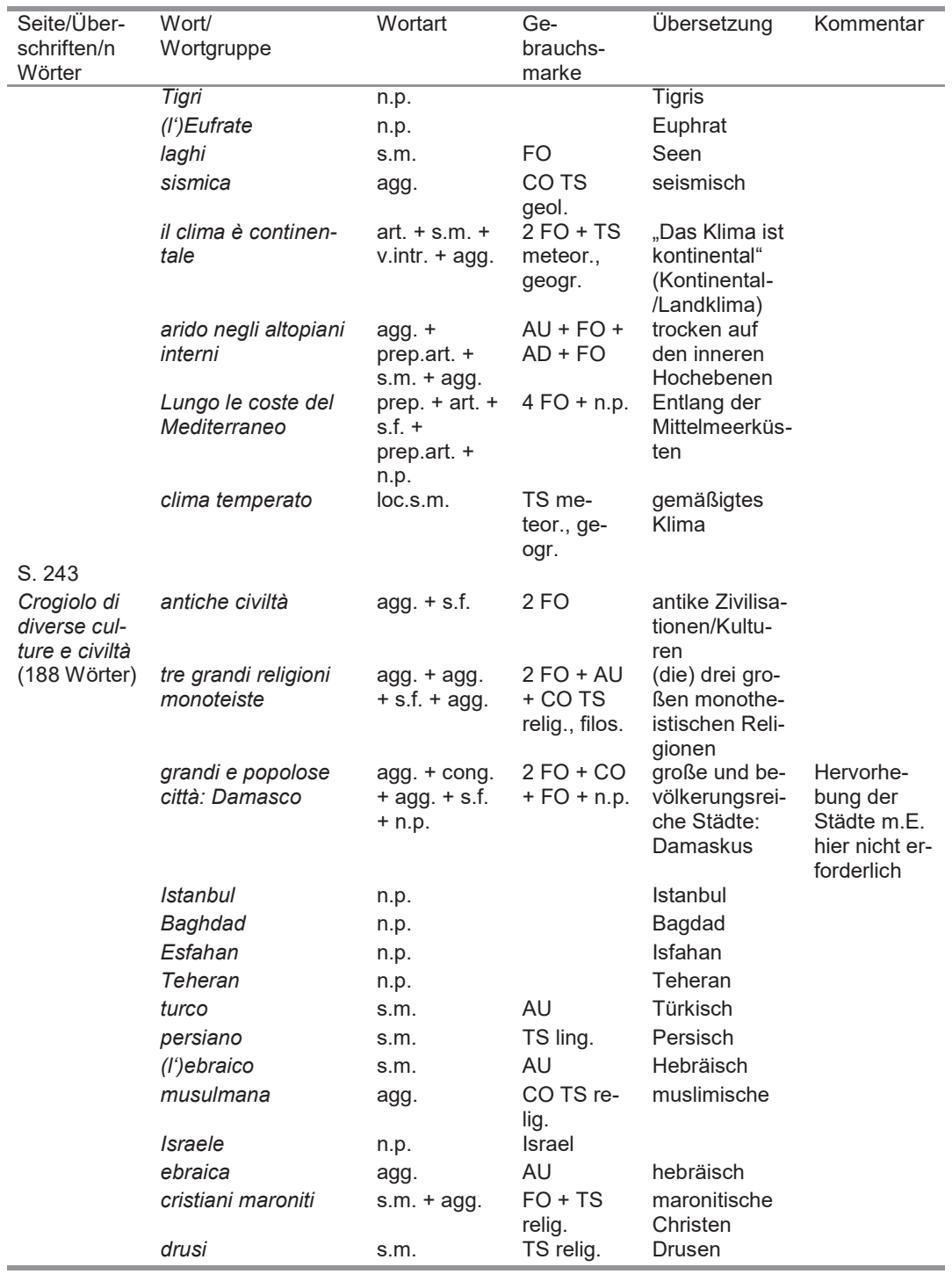

Tabelle 14: Hervorhebungen auf den untersuchten Seiten im Geograficamente (Dinucci \& Dinucci 2008)

Die längste Wortgruppe befindet sich mit 21 graphischen Wörtern auf Seite 241 (Dinucci \& Dinucci 2008), wobei eine Hervorhebung von fünf von ihnen, nämlich tre quarti und condizioni di povertà (bzw. nur povertà), vermutlich 
ausreichten, um denselben Zweck zu erfüllen. Ähnliches gilt für eine Wortgruppe im Text über das Klima und die natürliche Umgebung Asiens auf S. 240.

Ein großer Teil der fettgedrückten Wörter, ein gutes Drittel, sind geographische Eigennamen. Deren Akzentuierung ist m. E. in manchen Fällen nicht erforderlich, beispielsweise im Text auf S. 243, in dem einige Städte hervorgehoben werden, nachdem im Fettdruck bereits auf bevölkerungsreiche Städte aufmerksam gemacht wurde. Ebenfalls auffällig ist die Hervorhebung zahlreicher Ländernamen auf Seite 241 zur wirtschaftlichen Lage der Region. Neben den Toponymen sind dort auch sehr viele Bodenschätze und Anbauprodukte hervorgehoben, so dass die hohe Gesamtzahl an fettgedruckten Wörtern zu einem guten Teil darauf zurückzuführen ist: Insgesamt 76 graphische Wörter sind allein auf dieser Seite hervorgehoben. Unter ihnen befinden sich darüber hinaus verschiedene Prädikate, von denen insgesamt sieben fettgedruckt erscheinen.

Die meisten Begriffe stammen auch auf den Seiten dieses Buches aus dem Basiswortschatz. Dennoch befinden sich unter den Hervorhebungen je 22 Wörter, die dem allgemeinen Wortschatz (Co) oder dem fachsprachlichen (Ts) entspringen. Letztere stammen hauptsächlich aus dem sprachwissenschaftlichen und dem geographischen Fachwortschatz. Unter den sprachwissenschaftlichen Ausdrücken befindet sich bspw. lingua madre (Dinucci \& Dinucci 2008, S. 52; Sprachmutter (Ts ling.) und Muttersprache). Dieser Begriff wird im Text nicht in seiner Hauptbedeutung, Muttersprache, verwendet, sondern in der weniger geläufigen fachsprachlichen „Mutter“ der Sprachen einer Sprachfamilie, also beispielsweise dem Lateinischen als Mutter der romanischen Sprachen. Obwohl der Begriff in einem recht kurzen, aus 14 graphischen Wörtern bestehenden Satz verwendet wird, was das Textverständnis erleichtert, ist Letzteres möglicherweise dadurch beeinträchtigt, dass der Text sich grammatikalisch-syntaktisch komplex gestaltet.

So ist das Prädikat passivisch konstruiert und erscheint der Begriff Muttersprache als Bestandteil eines Partizipsatzes (zudem geformt mit einem Partizip Präsens und nicht mit dem häufiger gebrauchten Partizip Perfekt). Zwar folgt im Text später ein konkretes Beispiel für den Begriff, jedoch ist auch dieses sprachlich nicht einfach gestaltet, da es ganze vier satzwertig gebrauchte Partizipien enthält und im selben Satzgefüge neue Informationen liefert (einzelne konkrete Mitglieder einer Sprachfamilie).

Zuletzt sollen noch die fettgedruckten Wörter im Lehrbuch Nuovo Geolibro (Forte \& Ubertazzi 2010, Verlag Mondadori) vorgestellt werden, das in der Klasse III C benutzt wurde. Hier sind 48 Wörter und Wortgruppen hervorgehoben, von denen über die Hälfte aus mehr als einem graphischen Wort (28, nur ein Phrasem) und Substantiven (26) besteht. Mit insgesamt 18 machen auch die Toponyme einen stattlichen Anteil (ein gutes Drittel) aller Hervorhe- 
bungen aus. Konjugierte Verbformen sind hier nicht unter den Hervorhebungen $\mathrm{zu}$ verzeichnen, stattdessen jedoch ein Infinitiv, und zwar entrare (nell'Unione Europea) ((der Europäischen Union) beitreten).

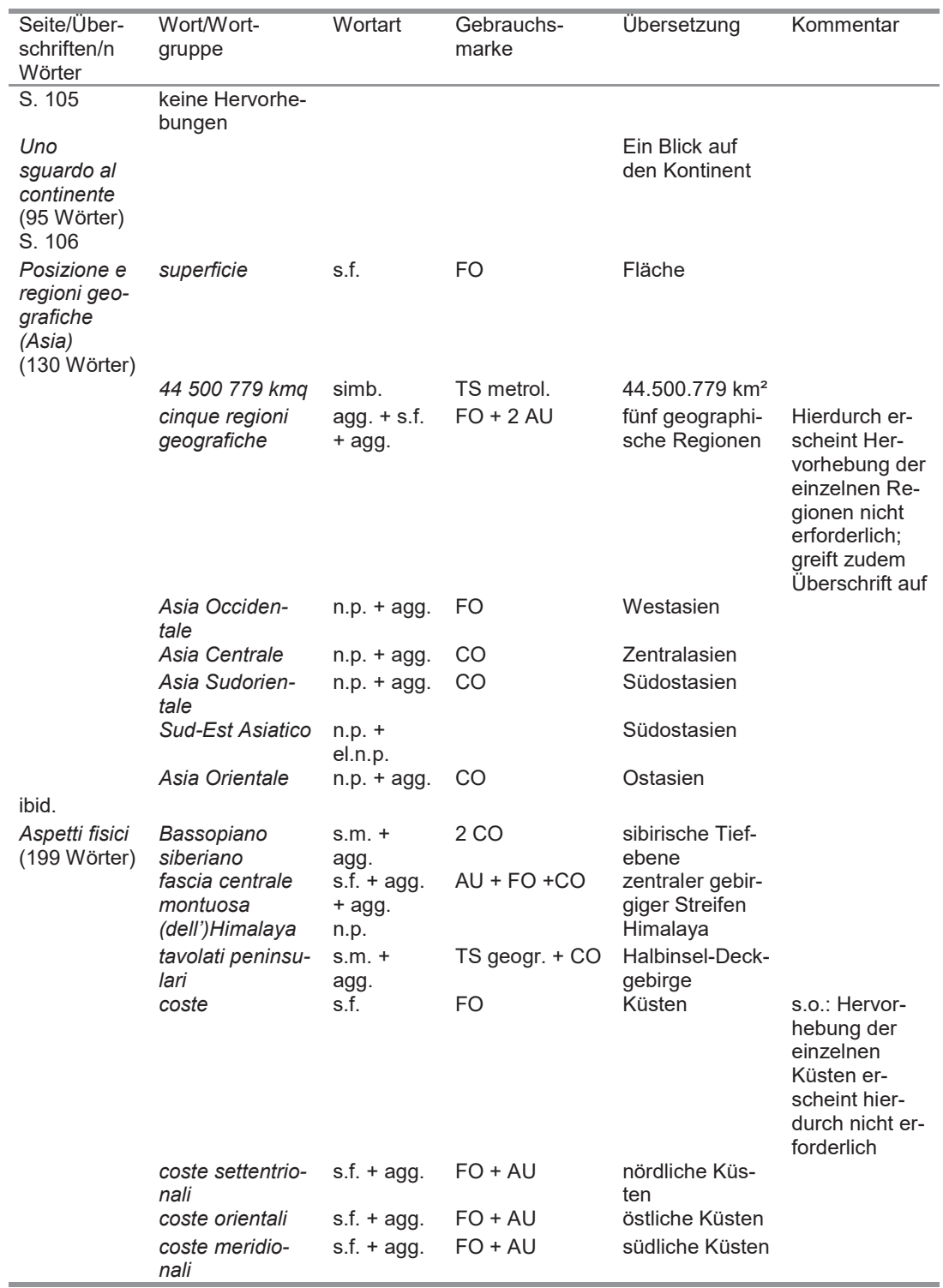




\begin{tabular}{|c|c|c|c|c|c|}
\hline $\begin{array}{l}\text { Seite/Über- } \\
\text { schriften/n } \\
\text { Wörter }\end{array}$ & $\begin{array}{l}\text { Wort/Wort- } \\
\text { gruppe }\end{array}$ & Wortart & $\begin{array}{l}\text { Gebrauchs- } \\
\text { marke }\end{array}$ & Übersetzung & Kommentar \\
\hline & canale di Suez & $\begin{array}{l}\text { n.p. }+2 \\
\text { el.n.p. }\end{array}$ & & Sueskanal & \\
\hline \multicolumn{6}{|l|}{ S. 107} \\
\hline \multirow{2}{*}{$\begin{array}{l}\text { Geologia e } \\
\text { rilievi } \\
\text { (199 Wörter) }\end{array}$} & desertiche & agg. & $\mathrm{CO}$ & Wüsten- & \\
\hline & grandi fiumi & $\begin{array}{l}\text { agg. + } \\
\text { s.m. }\end{array}$ & $2 \mathrm{FO}$ & große Flüsse & \\
\hline $\begin{array}{l}\text { S. } 120 \mathrm{f} . \\
\text { Le vie del } \\
\text { petrolio. Una } \\
\text { questione vi- } \\
\text { tale } \\
\text { (62 Wörter) } \\
\text { S. } 120 \mathrm{f} .\end{array}$ & $\begin{array}{l}\text { keine Hervorhe- } \\
\text { bungen }\end{array}$ & & & & \\
\hline $\begin{array}{l}\text { Tra neces- } \\
\text { sità di im- } \\
\text { portare e ri- } \\
\text { schi di tra- } \\
\text { sporto } \\
\text { (150 Wörter) } \\
\text { Una minore } \\
\text { dipendenza } \\
\text { (34 Wörter) }\end{array}$ & $\begin{array}{l}\text { keine Hervorhe- } \\
\text { bungen }\end{array}$ & & & & \\
\hline \multirow[t]{5}{*}{$\begin{array}{l}\text { Civiltà e sto- } \\
\text { ria } \\
\text { (173 Wörter) }\end{array}$} & $\begin{array}{l}\text { Mezzaluna fer- } \\
\text { tile }\end{array}$ & $\begin{array}{l}\text { s.f. + agg. } \\
\text { (n.p.) }\end{array}$ & $\mathrm{CO}+\mathrm{AU}$ (n.p.) & $\begin{array}{l}\text { Fruchtbarer } \\
\text { Halbmond } \\
\text { (Winterregen- } \\
\text { gebiet) }\end{array}$ & $\begin{array}{l}\text { Eigenname, } \\
\text { der den Le- } \\
\text { sern neu sein } \\
\text { wird }\end{array}$ \\
\hline & ebraismo & s.m. & CO TS relig. & Judentum & \\
\hline & cristianesimo & s.m. & $\mathrm{CO}$ & Christentum & \\
\hline & islam & s.m. & CO TS relig. & Islam & \\
\hline & turchi ottomani & $\begin{array}{l}\text { s.m. + } \\
\text { agg. }\end{array}$ & AU + TS.stor. & $\begin{array}{l}\text { ottomanische } \\
\text { Türken }\end{array}$ & \\
\hline \multicolumn{6}{|l|}{ S. 121} \\
\hline \multirow{4}{*}{$\begin{array}{l}\text { Relazioni in- } \\
\text { ternazionali } \\
\text { (314 Wörter) }\end{array}$} & $\begin{array}{l}\text { forte instabilità } \\
\text { politica }\end{array}$ & $\begin{array}{l}\text { agg. + s.f. } \\
+ \text { agg. }\end{array}$ & $\mathrm{FO}+\mathrm{CO}+\mathrm{FO}$ & $\begin{array}{l}\text { starke politi- } \\
\text { sche Instabilität }\end{array}$ & \\
\hline & $\begin{array}{l}\text { popolazione } \\
\text { curda } \\
\text { Afghanistan }\end{array}$ & $\begin{array}{l}\text { s.f. + agg. } \\
\text { n.p. }\end{array}$ & AU + TS etnol. & $\begin{array}{l}\text { kurdische Be- } \\
\text { völkerung } \\
\text { Afghanistan }\end{array}$ & \\
\hline & Palestina & n.p. & & Palästina & \\
\hline & Iraq & n.p. & & Irak & \\
\hline \multicolumn{6}{|l|}{ S. 122} \\
\hline $\begin{array}{l}\text { Territorio e } \\
\text { clima (Tur- } \\
\text { chia) }\end{array}$ & 783562 kmq & simb. & TS metrol. & $783.562 \mathrm{~km}^{2}$ & \\
\hline \multirow[t]{3}{*}{ (180 Wörter) } & Tauro & n.p. & & Taurusgebirge & \\
\hline & Monti del Ponto & n.p. & & Pontusgebirge & \\
\hline & $\begin{array}{l}\text { laghi piuttosto } \\
\text { vasti } \\
\text { varietà climatica }\end{array}$ & $\begin{array}{l}\text { s.m. + avv. } \\
+ \text { agg. } \\
\text { s.f. + agg. }\end{array}$ & & $\begin{array}{l}\text { sehr große } \\
\text { Seen } \\
\text { klimatische } \\
\text { Vielfältigkeit }\end{array}$ & \\
\hline S. 122 & & & & & \\
\hline
\end{tabular}




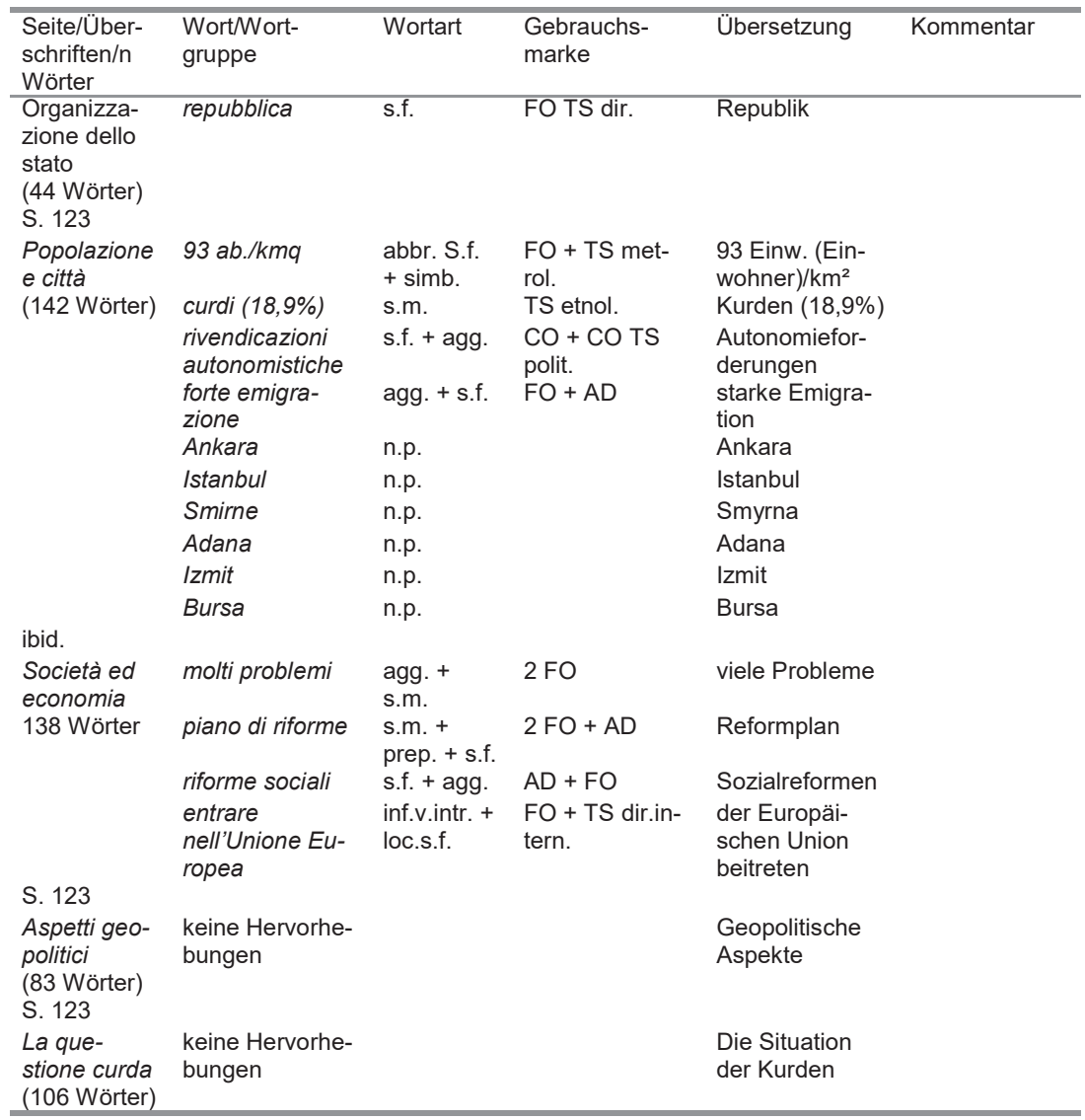

Tabelle 15: Hervorhebungen auf den untersuchten Seiten im Nuovo Geolibro (Forte \& Ubertazzi 2010)

Auf den analysierten Seiten dieses Lehrbuchs befinden sich 15 Texte, von denen ein gutes Drittel (6) keine Hervorhebungen aufweist. In den übrigen Texten sind es mindestens ein Wort und maximal neun. Auch wenn davon 28 aus mehreren graphischen Wörtern bestehen, sind es doch maximal drei, wobei es sich oft um die Elemente eines Eigennamens oder um Substantive mit Adjektiven handelt.

Beachtet man das Verhältnis zwischen den Textüberschriften und den einzelnen Hervorhebungen, so besteht mit einer Ausnahme zwischen allen ein deutlicher Bezug. Lediglich im Text Geologia e rilievi (Geologie und Erhebungen) auf Seite 107 (Forte \& Ubertazzi 2010) sind keine Erhebungen oder 
damit zusammenhängende Ausdrücke hervorgehoben, stattdessen die zwei italienischen Begriffe für Wüsten und große Flüsse. Auf Seite 106 hingegen (ibid.) lautet die Überschrift Posizione e regioni geografiche, und im Text wird sie sowohl mit der fettgedruckten Wortgruppe cinque regioni geografiche wieder aufgegriffen, als auch durch die Hervorhebung der Toponyme der einzelnen Regionen untermauert. Eine ähnliche Situation stellt sich auf derselben Seite im Text zur physischen Beschaffenheit des Landes dar, in dem zunächst das Wort Küsten und dann die einzelnen Küsten hervorgehoben werden. In beiden Fällen sollten die SchülerInnen durch die erste Hervorhebung darauf gestoßen worden sein, dass es sich um wichtige Textinformationen handelt, und dadurch dazu veranlasst werden, selbstständig die Namen der einzelnen Regionen bzw. die Unterschiede der einzelnen Küsten im Text aufzuspüren.

Bevor mit der Vorstellung der letzten beiden Lehrbücher fortgefahren wird, sollen abschließend einige Hervorhebungen miteinander verglichen werden, die aus Texten verschiedener Lehrbücher stammen, die jeweils dasselbe Thema behandeln.

Ein Beispiel sind die Texte in den Lehrbüchern von Bastianelli et al. (2010, Zaino in spalla) und dem zuletzt präsentierten (Nuovo Geolibro, Forte \& Ubertazzi 2010), die sich mit der Einführung in den asiatischen Kontinent befassen. In beiden Texten sind die Namen der fünf Regionen, in die der Kontinent eingeteilt werden kann, hervorgehoben. Beide Texte tragen eine ähnliche Überschrift: Le regioni dell'Asia (Bastianelli et al. 2010, S. 206) und Posizione e regioni geografiche (Forte \& Ubertazzi 2010, S. 106); im Nuovo Geolibro sind außerdem, wie oben dargestellt, erneut die Wortgruppe cinque regioni geografiche sowie die Flächenangabe fettgedruckt, während sich die Hervorhebungen im Text des ersten Erdkundebuchs auf die fünf Regionen beschränken. Dieser Text ist um einiges kürzer als der im Nuovo Geolibro, da er nur 41 Wörter umfasst und Letzterer 130.

Auffällig ist außerdem, dass in den beiden Lehrbüchern unterschiedliche Bezeichnungen für die fünf Regionen gewählt wurden, wobei im Text des ersten Lehrbuchs die Formulierung può essere suddivisa in cinque grandi regioni (Bastianelli et al. 2010, S. 206; m.Ü.: kann in fünf Regionen unterteilt werden) so verstanden werden kann, dass diese Unterteilung nicht als kategorisch aufzufassen ist. Während in diesem Buch die Bezeichnungen Medio Oriente, Asia centro-settentrionale, regione indiana, Asia orientale und Sud-Est asiatico (ibid.) gewählt werden, verwendet das Nuovo Geolibro Asia Occidentale, Asia Centrale, Asia Sudorientale, Sud-Est Asiatico und Asia Orientale.

Vergleicht man hingegen die Hervorhebungen in den Texten der beiden Bücher, die sich mit der Verbreitung der Sprachen in der Welt befassen, das Zaino in spalla (ibid., S. 182) und das Geograficamente (Dinucci \& Dinucci 2008, S. 52 f.), so fällt auf, dass in den beiden Lehrwerken ganz unterschiedliche Aspekte graphisch hervorgehoben werden, auch wenn man berücksichtigt, 
dass die Texte zu diesem Thema inhaltlich verschieden aufgebaut sind. Während das Zaino in spalla zunächst ausführlicher Informationen zur wesentlichen Rolle der Sprache und zu ihrer Verbreitung gibt, erwähnt das andere Lehrwerk knapp die ungefähre Zahl der gesprochenen Sprachen und beginnt sofort mit ihrer Einteilung in verschiedene Sprachfamilien.

Anhand der Hervorhebungen kann schon vermutet werden, dass im ersten Buch ein größeres Basiswissen über die Sprachen und ihre Verbreitung vermittelt wird, während im zweiten Buch das Vermitteln konkreter sprachbezogener Daten im Vordergrund steht. So sind im Zaino in spalla die drei Wortgruppen come organismi viventi (wie lebendige Organismen), continenti interi (ganze Kontinente) und lingue locali (Lokalsprachen) (Bastianelli et al. 2010, S. 182) hervorgehoben, bei denen es sich um Wörter des Basiswortschatzes und eines aus dem allgemeinen Gebrauch handelt. Im Geograficamente gibt es mehr als doppelt so viele (8): circa 6800, famiglie linguistiche (Sprachfamilien), lingua madre (Muttersprache), indoeuropea (indogermanisch) und die substantivierten Adjektive afro-asiatica (Afroasiatische), sino-tibetana (Sinotibetische), uralo-altaica (Uralaltaische) sowie 225 sono ufficiali (225 sind offiziell [Amtssprachen]) (Dinucci \& Dinucci 2008, S. 52).

In diesem Buch sind auf der folgenden Seite weitere sieben Wortgruppen fettgedruckt, deren Wörter größtenteils aus dem Basiswortschatz stammen. Sie befinden sich in zwei Texten, die sich mit den meistgesprochenen Sprachen und dem Risiko des Aussterbens von Sprachen befassen. Insgesamt sind im Geograficamente also 15 Wortgruppen hervorgehoben, während es im Zaino in spalla nur drei sind, zu denen auf der Seite 183 weitere fünf kommen, die in der obenstehenden Tabelle nicht aufgeführt sind, da diese Seite nicht zu den untersuchten Lehrbuchseiten zählt. Sie soll aber an dieser Stelle erwähnt werden, da sie die Sprachfamilien behandelt, die im anderen Lehrwerk hervorgehoben sind.

Auffällig ist hier, dass die beiden Lehrbücher nicht dieselben Sprachfamilien nennen. Es weisen jedoch beide darauf hin, dass sie nur einen Teil der Familien benennen. Unter denen, die in beiden Lehrbüchern Erwähnung finden, lassen sich allerdings Unterschiede in den Bezeichnungen finden. So unterteilt das Lehrbuch Zaino in spalla die im anderen Buch unter dem Namen „Uralaltaische“ zusammengefassten Sprachen in zwei unabhängige Sprachfamilien. Diese unterschiedliche Gewichtung legt die Frage nahe, ob diese Informationen tatsächlich so detailliert an AchtklässlerInnen vermittelt werden sollten. ${ }^{124}$

124 Laut dem Duden handelt es sich bei „uralaltaisch“ um einen selten und früher in der Sprachwissenschaft benutzten Begriff, der aus der Zeit stammt, in denen die beiden Sprachfamilien als eng miteinander verwandt betrachtet wurden (www.duden.de; zuletzt abgerufen am 23.11.2016). 
Im Rahmen dieser Arbeit ist eine gründlichere Untersuchung der Hervorhebungen in den fünf Lehrwerken nicht möglich. Diese Darstellung sollte jedoch verdeutlicht haben, dass unterschiedliche und unterschiedlich viele Ausdrücke, oft ganze Wortgruppen, hervorgehoben werden. In allen analysierten Texten sind dies zumeist Nomen, jedoch besteht in einigen der Bücher die Tendenz, ganze Sätze hervorzuheben, so dass auch Prädikate fettgedruckt sind. Die wenigsten Hervorhebungen befinden sich im ältesten der analysierten Lehrbücher, was darauf hindeuten könnte, dass die Verwendung des Fettdrucks als graphisches Textgestaltungsmittel in den letzten Jahren zugenommen hat. Das könnten allerdings erst weitgreifendere empirische Untersuchungen zu diesem Aspekt tatsächlich belegen. An dieser Stelle kann nur die Vermutung geäußert werden, dass die Zunahme der Hervorhebungen als Indiz dafür gesehen werden kann, dass die LehrbuchautorInnen um eine benutzerfreundlichere Gestaltung ihrer Texte bemüht sind, wobei offenbleibt, wie sehr und wie positiv sie mit dieser Technik den Lernprozess der Schülerinnen und Schüler beeinflussen.

\subsubsection{Das Geograficamente der Klasse III B der Schule Pestalozzi}

Die Tabelle 16 gibt Auskunft über die sprachlichen Elemente der analysierten Seiten im Unterrichtswerk der Autoren Manlio und Federico Dinucci (2008), das in der Klasse III B verwendet wurde.

\begin{tabular}{|c|c|c|c|c|c|c|c|c|c|c|}
\hline $\begin{array}{l}\text { Seite/Kom- } \\
\text { mentar (K) }\end{array}$ & 52 & K1 & 53 & $\mathrm{~K} 2$ & 238 & K3 & $\begin{array}{l}239- \\
241\end{array}$ & K4 & $\begin{array}{l}242 \\
\text { f. }\end{array}$ & K5 \\
\hline n Wörter & 294 & & 304 & & 191 & & 1322 & & 720 & $\begin{array}{l}2 \\
\text { Wör- } \\
\text { ter } \\
\text { nicht } \\
\text { im } \\
\text { GRA- } \\
\text { DIT } \\
\text { (ES) }\end{array}$ \\
\hline Sätze & 9 & & 13 & & 8 & & 58 & & 25 & \\
\hline $\begin{array}{l}\text { kürzester } \\
\text { Satz }\end{array}$ & 7 & & 5 & $\begin{array}{l}\text { Über- } \\
\text { schrift } \\
\text { (Frage); } \\
\text { im Text: } \\
7\end{array}$ & 9 & & 6 & & 6 & \\
\hline $\begin{array}{l}\text { längster } \\
\text { Satz }\end{array}$ & 49 & & 37 & & 32 & & 68 & & 50 & \\
\hline $\begin{array}{l}ø \text { Satz- } \\
\text { länge }\end{array}$ & 23 & & 20 & & 20 & & 21 & & 21 & \\
\hline $\begin{array}{l}\text { Doppel- } \\
\text { punkte }\end{array}$ & 2 & & 4 & & 0 & & 11 & & 2 & \\
\hline $\begin{array}{l}\text { fettge- } \\
\text { druckte } \\
\text { Wörter }\end{array}$ & 10 & $\begin{array}{l}2 \text { Phra- } \\
\text { seme, } \\
3 \text { Bin- } \\
\text { de- } \\
\text { strich- } \\
\text { Wörter }\end{array}$ & 10 & $5+5$ & 16 & $\begin{array}{l}\text { n.p., } 1 \\
\text { Phra- } \\
\text { sem }\end{array}$ & 154 & $\begin{array}{l}6+16+37+19 \\
+76 ; \text { auch } \\
\text { ganze Sätze }\end{array}$ & 49 & $23+26$ \\
\hline
\end{tabular}




\begin{tabular}{|c|c|c|c|c|c|c|c|c|c|c|}
\hline $\begin{array}{l}\text { Seite/Kom- } \\
\text { mentar (K) }\end{array}$ & 52 & K1 & 53 & K2 & 238 & K3 & $\begin{array}{l}239- \\
241\end{array}$ & K4 & $\begin{array}{l}242 \\
f .\end{array}$ & K5 \\
\hline $\begin{array}{l}\text { Substan- } \\
\text { tive u.Ä. }\end{array}$ & 101 & $34 \%$ & 119 & $39 \%$ & 75 & $39 \%$ & 527 & $40 \%$ & 273 & $38 \%$ \\
\hline $\begin{array}{l}\text { Substan- } \\
\text { tive plus } \\
\text { Adj. und } \\
\text { Art. }\end{array}$ & 205 & $70 \%$ & 189 & $62 \%$ & 125 & $65 \%$ & 934 & $71 \%$ & 482 & $67 \%$ \\
\hline Phraseme & 15 & & 7 & & 2 & & 18 & & 19 & \\
\hline el.loc. & 15 & & 7 & & 3 & & 22 & & 22 & \\
\hline $\begin{array}{l}\text { Konjunkti- } \\
\text { onen }\end{array}$ & 18 & $\begin{array}{l}4 \text { Ty- } \\
\text { pes } \\
\text { (FO) }\end{array}$ & 15 & $\begin{array}{l}8 \text { Types } \\
\text { (FO) }\end{array}$ & 10 & $\begin{array}{l}2 \text { Ty- } \\
\text { pes } \\
\text { (FO) }\end{array}$ & 78 & $\begin{array}{l}7 \text { Types } \\
\text { (FO) }\end{array}$ & 36 & $\begin{array}{l}6 \text { Ty- } \\
\text { pes; } 2 \\
\text { loc. } \\
\text { CO } \\
\text { (an- } \\
\text { che } \\
\text { se, } \\
\text { dato } \\
\text { che) }\end{array}$ \\
\hline loc.v. & 2 & & 0 & & 1 & $\begin{array}{l}\text { facenti } \\
\text { parte }\end{array}$ & 0 & & 0 & \\
\hline $\begin{array}{l}\text { konjugierte } \\
\text { Verben }\end{array}$ & 22 & & 26 & & 11 & & 82 & $\begin{array}{l}\text { im 3. und } \\
\text { 26. Satz El- } \\
\text { lipse d. Prä- } \\
\text { dikate }\end{array}$ & 43 & $\begin{array}{l}1 \text { Prä- } \\
\text { dikat } \\
\text { impli- } \\
\text { ziert }\end{array}$ \\
\hline Imperative & 0 & & 0 & & 0 & & 0 & & 0 & \\
\hline $\begin{array}{l}\varnothing \text { konj. } \\
\text { Verben pro } \\
\text { Satz }\end{array}$ & 2,4 & & 2 & & 1,4 & & 2,4 & & 1,7 & \\
\hline Gerundien & 0 & & 0 & & 0 & & 2 & & 1 & \\
\hline Infinitive & 1 & & 6 & & 0 & & 0 & & 0 & \\
\hline Partizipien & 14 & & 8 & & 6 & & 11 & $\begin{array}{l}2 \text { Tokens } \\
\text { part.pres. } \\
\text { (compren- } \\
\text { dente) }\end{array}$ & 8 & \\
\hline FO & 180 & $61 \%$ & 230 & $76 \%$ & 110 & $58 \%$ & 786 & $59 \%$ & 351 & $49 \%$ \\
\hline el.FO & 0 & & 0 & & 0 & & 0 & & 0 & \\
\hline FO TS & 0 & & 0 & & 0 & & 3 & & 5 & \\
\hline $\mathrm{AU}$ & 6 & & 7 & & 16 & & 108 & & 82 & $\begin{array}{l}4 \text { Bin- } \\
\text { de- } \\
\text { strich- } \\
\text { wörter }\end{array}$ \\
\hline AU TS & 0 & & 0 & & 1 & & 1 & & 1 & \\
\hline$A D$ & 4 & & 2 & & 1 & & 34 & & 8 & \\
\hline AD TS & 0 & & 0 & & 0 & & 1 & & 0 & \\
\hline Tot. VdB & 190 & $65 \%$ & 239 & $79 \%$ & 128 & $67 \%$ & 933 & $71 \%$ & 447 & $62 \%$ \\
\hline $\mathrm{BU}$ & 0 & & 0 & & 0 & & 0 & & 0 & \\
\hline $\mathrm{CO}$ & 27 & & 17 & & 21 & & 155 & & 91 & \\
\hline el.CO & 1 & & 0 & & 1 & & 9 & & 12 & \\
\hline CO TS & 1 & & 7 & & 1 & & 8 & & 15 & \\
\hline el.co TS & 8 & & 6 & & 0 & & 0 & & 0 & \\
\hline $\mathrm{TS}$ & 38 & & 22 & & 7 & & 59 & & 51 & \\
\hline el.TS & 6 & & 1 & & 2 & & 11 & & 10 & \\
\hline ES & 0 & & 0 & & 0 & & 4 & $\begin{array}{l}1 \text { doppelte } \\
\text { Marke (per- } \\
\text { mafrost) }\end{array}$ & 0 & \\
\hline
\end{tabular}




\begin{tabular}{|c|c|c|c|c|c|c|c|c|c|c|}
\hline $\begin{array}{l}\text { Seite/Kom- } \\
\text { mentar (K) }\end{array}$ & 52 & K1 & 53 & K2 & 238 & K3 & $\begin{array}{l}239- \\
241\end{array}$ & K4 & $\begin{array}{l}242 \\
\text { f. }\end{array}$ & K5 \\
\hline $\begin{array}{l}\text { o.M. (ohne } \\
\text { n.p. und } \\
\text { sigla: } \\
\text { ES/andere } \\
\text { Sprachen) }\end{array}$ & 8 & $\begin{array}{l}\text { "Was- } \\
\text { ser“ in } \\
\text { ver- } \\
\text { schie- } \\
\text { denen } \\
\text { Spra- } \\
\text { chen }\end{array}$ & 0 & & 0 & & 0 & & 2 & \\
\hline $\begin{array}{l}\text { Tot. restl. } \\
\text { Marken }\end{array}$ & 81 & $28 \%$ & 53 & $17 \%$ & 32 & $17 \%$ & 246 & $19 \%$ & 179 & $25 \%$ \\
\hline $\begin{array}{l}\text { Tot. Mar- } \\
\text { ken insg. }\end{array}$ & 352 & $92 \%$ & 345 & $96 \%$ & 192 & $66 \%$ & 1179 & $90 \%$ & 626 & $87 \%$ \\
\hline
\end{tabular}

Tabelle 16: Untersuchungsergebnisse zu den Seiten aus Geograficamente (Dinucci \& Dinucci 2008)

In diesem Lehrwerk befinden sich nach dem Lehrbuch der III $\mathrm{C}$ an der Pestalozzi (2.890 Wörter) auf den untersuchten acht Seiten insgesamt die meisten Wörter, nämlich 2.831 und damit durchschnittlich 354 auf jeder Seite. Der Anteil an Substantiven, Artikeln und Adjektiven liegt bei 67 Prozent. Die Wörter verteilen sich mit einem Mittelwert von 21 pro Satz auf durchschnittlich 14 Sätze auf jeder Seite, auf die zwei konjugierte Verben sowie insgesamt drei Gerundien, sieben Infinitive und 47 Partizipien entfallen.

69 Prozent der Wörter stammen aus dem Vocabolario di Base (VDB) und durchschnittlich 21 Prozent aus den weniger häufig gebrauchten Bereichen des Wortschatzes. Der höchste Anteil an Ausdrücken aus dem Basiswortschatz findet sich auf der Seite 53 (79\%), welche die Verbreitung der Sprachen weltweit und ihr Risiko, nicht mehr verwendet zu werden, thematisiert, und auf den Seiten 239 bis 241, auf denen Asien sowohl geographisch, als auch wirtschaftlich und gesellschaftlich vorgestellt wird. Hier sind die meisten Wörter fettgedruckt, nämlich insgesamt 154, darunter mehrere Wortgruppen von vier und mehr Wörtern sowie zwei ganze Sätze. Auf den übrigen Seiten liegt der Anteil der Ausdrücke aus dem Grundwortschatz jeweils unter 70 Prozent.

Die wenigsten VDB-Wörter weisen die Seiten 242 f. auf, die eine Einführung in die Region Westasien (Mittlerer Orient) geben. Während mit 28 Prozent die meisten Wörter aus dem Bereich des allgemeinen Wortschatzes (Co) und des spezifisch-technischen Fachwortschatzes (Ts) auf Seite 52 vertreten sind, auf der es um die Sprachfamilien und ihre Verbreitung geht, sind auf den Seiten 53 und 238 (Einführung in den Kontinent Asien, großer Bestand an Toponymen) nur je 17 Prozent aus diesen Wortschatzgruppen verzeichnet. Bezüglich der Seiten, die sich mit den Sprachen dieser Welt befassen, ist interessant, dass die Zusammensetzung des Wortschatzes im Text des Lehrbuchs Zaino in spalla (Bastianelli et al. 2010, S. 182), der sich mit demselben Thema befasst, nicht nur hinsichtlich der fettgedruckten Wörter, sondern generell ganz anders gestaltet ist. Er weist einen sehr hohen Basiswortschatzanteil (80 \%) auf, besteht nur zu 18 Prozent aus den weniger häufig gebrauchten Wörtern und 98 Prozent des Wortvorkommens ist mit einer Gebrauchsmarke versehen. 
Aber auch 91 Prozent der gewählten Wörter zu diesem Thema im Buch Geograficamente haben eine Gebrauchsmarke, und es stammen immerhin 65 Prozent aus dem Bereich des Basiswortschatzes, also drei Prozent mehr als in den Texten mit dem geringsten Anteil am VDB unter den untersuchten Seiten dieses Buches (S. 242 f.).

Durchschnittlich tragen 86 Prozent aller Wörter eine Gebrauchsmarke, wobei der geringste Anteil mit 66 Prozent aufgrund der bereits erwähnten zahlreichen geographischen Eigennamen auf Seite 238 und der höchste mit 96 Prozent auf Seite $53 \mathrm{zu}$ finden ist.

Teil des Wortvorkommens auf den untersuchten Lehrbuchseiten sind auBerdem 14 Fremdwörter (Es) bzw. Wörter aus anderen Sprachen, von denen vier im GRADIT eingetragen sind. Von diesen stellt wieder eines den seltenen Fall dar, mit einer zusätzlichen Marke aufgeführt zu sein, und zwar permafrost (Dinucci \& Dinucci 2008, S. 240), ein englisches Fremdwort, was zum technisch-spezifischen Wortschatz der Geologie gehört. Unter den als solchen markierten Fremdwörtern befindet sich auch die aus dem Englischen stammende Sigle UNESCO (ibid.). Die Gruppe der übrigen zehn Fremdwörter bilden acht Ausdrücke in verschiedenen Sprachen für den Begriff Wasser und die beiden Wörter mesos potamos als etymologische Erklärung für das Toponym Mesopotamien.

Sprachlich auffällig ist ferner, dass im Gegensatz zu den anderen Lehrbüchern der Vansee nicht unter der offiziellen Bezeichnung lago di Van, sondern schlicht unter Van (ibid., S. 242) erscheint, wobei aus dem Kontext zwar erschlossen werden kann, dass es sich um ein Gewässer handelt, die Bezeichnung aber ungenau ist, da auch eine türkische Stadt diesen Namen trägt.

Darüber hinaus sticht ein Verbalphrasem hervor, aber nicht als solches (fare parte; Teil sein, dazugehören), sondern in seinem Einsatz als Partizip Präsens facenti parte (ibid., S. 238). Diese Form wird in ihrer verbalen Funktion in vielen Grammatiken als antiquiert oder bürokratisch und zu vermeiden beschrieben, aber dennoch oft in schriftlichen Texten gebraucht, in denen die Verfasser um einen gewählten Ton und anspruchsvollen Sprachstil bemüht sind (vgl. z.B. Serianni 2006, S. 481 f.; Dardano \& Trifone 2002, S. 291).

\subsubsection{Nuovo Geolibro der Klasse III C an der Pestalozzi}

Als letztes Lehrwerk soll das Nuovo Geolibro (Forte \& Ubertazzi 2010) vorgestellt werden, in dem sich auf den sieben untersuchten Seiten insgesamt 2.890 Wörter befinden, das bedeutet, durchschnittlich 409 Wörter auf jeder Seite, wovon 69 Prozent Substantive inklusive Adjektiven, Artikeln und Präpositionen sind. Strukturiert sind sie in mittleren 15 Sätzen pro Seite mit mindestens sechs Wörtern (ibid., S. 121: Beschreibung zu einer Abbildung, mit Prädikat) und maximal 82 Wörtern auf Seite 107. Auf die Sätze verteilen sich 
durchschnittlich 1,9 konjugierte Verben, zu denen 38 Partizipien, elf Gerundien und 31 Infinitive kommen, von denen zwei passivischer Art sind und einer unter der im Text verwendeten Bedeutung nicht im GRADIT aufgeführt ist (s. Tabelle 17).

Bezüglich der Zusammensetzung aus den verschiedenen Bereichen des Wortschatzes ist zu beobachten, dass insgesamt 1.991 Wörter, also 83 Prozent, Teil des Grundwortschatzes sind, wobei auf jeder Seite durchschnittlich 70 Prozent des Wortvorkommens daraus stammt und die verschiedenen Prozentwerte von einem Minimum von 59 auf Seite 106 bis zu einem Maximum von 81 auf Seite 105 reichen.

Die Seite mit dem geringsten Anteil an Grundwortschatzwörtern befasst sich mit der geographischen Einordnung Asiens und seiner Unterteilung in verschiedene Regionen (hoher Anteil an markenlosen Toponymen), während Seite 105 mit dem größten Basiswortschatz aus einer kurzen Einleitung zu Beginn des Kapitels über Asien sowie fünf Steckbriefen zu ausgewählten Ländern des Kontinents besteht. Außerdem erstreckt sich die Karte Asiens auf Seite 104, um die herum auch Abbildungen zu verschiedenen Ländern angebracht sind, die durch Linien mit den jeweiligen geographischen Positionen der Länder auf der Karte verbunden sind, auch auf Seite 105. Aufgrund des recht hohen Gesamtwortvorkommens auf den untersuchten Seiten in diesem Erdkundebuch ist entschieden worden, die Ländersteckbriefe und die Betitelungen zu den drei Abbildungen nicht mit in die Untersuchung einzubeziehen, so dass der hohe Anteil an Wörtern aus dem Grundwortschatz erklärbar wird, obwohl das Thema der Seite viele Toponyme erwarten lässt (s. Tabelle 2).

Weitere 583 Wörter zählen zum weniger häufig gebrauchten Wortschatz (Co, Es, LE und Ts) und verteilen sich zu durchschnittlich 19 Prozent auf die sieben Seiten. Mit 15 Prozent sind die wenigsten von ihnen auf der bereits erwähnten Seite 105 verzeichnet, während die meisten aufgrund der zahlreichen Ausdrücke aus dem allgemeinen Wortschatz (z.B. sistema montuoso, Gebirgssystem) im Text über Geologie und Erhebungen Asiens und der vielen fachwortschatzsprachlichen Begriffe im Kasten zur Bewegung der Platten der Erdkruste auf Seite 107 entfallen.

Im Mittelwert weisen 89 Prozent aller Wörter eine Marke auf, mit Anteilen von nur 75 Prozent auf Seite 106 und von 91 Prozent auf Seite $122 \mathrm{f}$.

Weitere Informationen zur sprachlichen Gestaltung können Tabelle 17 (K steht für Kommentar) entnommen werden, an die sich einige Bemerkungen zu Auffälligkeiten des untersuchten Wortvorkommens anschließen.

\begin{tabular}{|c|c|c|c|c|c|c|c|c|c|c|}
\hline Seite/K & 105 & $\mathrm{~K}$ & 106 & $\mathrm{~K} 2$ & 107 & K3 & $\begin{array}{l}120 \\
\text { f. }\end{array}$ & K4 & $\begin{array}{l}122 \\
\text { f. }\end{array}$ & K5 \\
\hline n Wörter & 95 & & 510 & & 464 & & 964 & 61 n.p. & 857 & \\
\hline Sätze & 3 & & 15 & & 15 & $\begin{array}{l}\text { 1Satz } \\
\text { nicht } \\
\text { zu } \\
\text { Ende } \\
\text { geführt }\end{array}$ & 37 & & 33 & \\
\hline
\end{tabular}




\begin{tabular}{|c|c|c|c|c|c|c|c|c|c|c|}
\hline Seite/K & 105 & $\mathrm{~K}$ & 106 & K2 & 107 & K3 & $\begin{array}{l}120 \\
\text { f. }\end{array}$ & K4 & $\begin{array}{l}122 \\
\text { f. }\end{array}$ & K5 \\
\hline $\begin{array}{l}\text { kürzes- } \\
\text { ter Satz }\end{array}$ & 21 & & 10 & & 7 & & 6 & $\begin{array}{l}\text { Bildbe- } \\
\text { schrei- } \\
\text { bung; im } \\
\text { Text: } 11\end{array}$ & 8 & \\
\hline $\begin{array}{l}\text { längster } \\
\text { Satz }\end{array}$ & 39 & & 63 & & 82 & & 55 & & 43 & \\
\hline $\begin{array}{l}\varnothing \text { Satz- } \\
\text { länge }\end{array}$ & 30 & & 31 & & 28 & & 23 & & 22 & \\
\hline $\begin{array}{l}\text { Doppel- } \\
\text { punkte }\end{array}$ & 1 & & 3 & & 2 & & 2 & & 2 & \\
\hline $\begin{array}{l}\text { fettge- } \\
\text { druckte } \\
\text { Wörter }\end{array}$ & 0 & & 41 & $17+24$ & 3 & $3+0$ & 15 & $7+8$ & 29 & $9+1+9+9+0$ \\
\hline $\begin{array}{l}\text { Substan- } \\
\text { tive u.Ä. }\end{array}$ & 29 & $31 \%$ & 235 & $46 \%$ & 170 & $37 \%$ & 368 & $38 \%$ & 364 & $42 \%$ \\
\hline $\begin{array}{l}\text { Substan- } \\
\text { tive plus } \\
\text { Adj. und } \\
\text { Art. }\end{array}$ & 62 & $65 \%$ & 365 & $72 \%$ & 317 & $68 \%$ & 661 & $69 \%$ & 612 & $72 \%$ \\
\hline $\begin{array}{l}\text { Phra- } \\
\text { seme }\end{array}$ & 1 & $\begin{array}{l}\text { un } \\
\text { po' }\end{array}$ & 6 & & 10 & & 23 & $\begin{array}{l}2 \text { nicht im } \\
\text { GRADIT, } \\
\text { also o. M. } \\
\text { (via } \\
\text { mare/terra) }\end{array}$ & 24 & $\begin{array}{l}1 \text { Abkür- } \\
\text { zung }\end{array}$ \\
\hline el.loc. & 1 & & 10 & & 10 & & 29 & & 27 & \\
\hline $\begin{array}{l}\text { Konjunk- } \\
\text { tionen }\end{array}$ & 4 & $\begin{array}{l}1 \\
\text { Type } \\
\text { (FO; } \\
e)\end{array}$ & 29 & $\begin{array}{l}6 \text { Ty- } \\
\text { pes } \\
\text { (FO) }\end{array}$ & 26 & $\begin{array}{l}4 \text { Ty- } \\
\text { pes } \\
\text { (FO) }\end{array}$ & 50 & $\begin{array}{l}10 \text { Types } \\
\text { (FO) }\end{array}$ & 47 & $\begin{array}{l}10 \text { Types, } \\
3 \mathrm{CO}(2 \\
\text { pur, dopo), }\end{array}$ \\
\hline loc.v. & 0 & & 1 & $\begin{array}{l}\text { fare } \\
\text { parte } \\
\text { (CO) }\end{array}$ & 1 & $\begin{array}{l}\text { farsi } \\
\text { sentire }\end{array}$ & 0 & & 0 & \\
\hline $\begin{array}{l}\text { konju- } \\
\text { gierte } \\
\text { Verben }\end{array}$ & 6 & & 32 & & 33 & & 67 & & 49 & $\begin{array}{l}1 \text { Prädikat } \\
\text { impliziert } \\
\text { (deriva); im } \\
\text { 18. Satz } \\
\text { fehlt Ge- } \\
\text { rundium } \\
\text { (pur es- } \\
\text { sendo); } \\
\text { Verbal- } \\
\text { periphrase } \\
\text { stare + Ge- } \\
\text { rundium }\end{array}$ \\
\hline $\begin{array}{l}\text { Impera- } \\
\text { tive }\end{array}$ & 0 & & 0 & & 0 & & 0 & & 0 & \\
\hline $\begin{array}{l}\varnothing \text { konj. } \\
\text { Verben } \\
\text { pro Satz }\end{array}$ & 2 & & 2,1 & & 2,2 & & 1,8 & & 1,5 & \\
\hline $\begin{array}{l}\text { Gerun- } \\
\text { dien }\end{array}$ & 1 & & 2 & & 5 & & 1 & & 2 & \\
\hline Infinitive & 1 & & 4 & $\begin{array}{l}2 \\
\text { inf.p. }\end{array}$ & 3 & $\begin{array}{l}1 \text { unter } \\
\text { der Be- } \\
\text { deu- } \\
\text { tung } \\
\text { nicht } \\
\text { im }\end{array}$ & 14 & & 9 & 2 inf.pass. \\
\hline
\end{tabular}




\begin{tabular}{|c|c|c|c|c|c|c|c|c|c|c|}
\hline Seite/K & 105 & K & 106 & K2 & 107 & K3 & $\begin{array}{l}120 \\
f .\end{array}$ & K4 & $\begin{array}{l}122 \\
\text { f. }\end{array}$ & K5 \\
\hline & & & & & & $\begin{array}{l}\text { GRADIT } \\
\text { (spe- } \\
\text { gnersi) }\end{array}$ & & & & \\
\hline $\begin{array}{l}\text { Partizi- } \\
\text { pien }\end{array}$ & 2 & & 11 & & 3 & & 13 & $\begin{array}{l}1 \\
\text { part.pres. } \\
\text { (cre- } \\
\text { scente) }\end{array}$ & 9 & \\
\hline FO & 67 & $71 \%$ & 256 & $50 \%$ & 280 & $60 \%$ & 570 & $59 \%$ & 492 & $57 \%$ \\
\hline el.FO & 0 & & 0 & & 0 & & 0 & & 0 & \\
\hline FO TS & 2 & & 0 & & 2 & & 1 & & 2 & \\
\hline $\mathrm{AU}$ & 8 & & 31 & & 36 & & 94 & & 84 & \\
\hline AU TS & 0 & & 1 & & 2 & & 0 & & 0 & \\
\hline$A D$ & 0 & & 13 & & 11 & & 18 & & 20 & \\
\hline AD TS & 0 & & 0 & & 0 & & 1 & & 0 & \\
\hline Tot. VdB & 77 & $81 \%$ & 301 & $59 \%$ & 331 & $71 \%$ & 684 & $71 \%$ & 600 & $70 \%$ \\
\hline $\mathrm{BU}$ & 0 & & 0 & & 0 & & 0 & & 0 & \\
\hline $\mathrm{CO}$ & 14 & & 61 & & 62 & & 127 & & 103 & \\
\hline el.cO & 1 & & 8 & & 6 & & 22 & & 16 & \\
\hline CO TS & 0 & & 2 & & 12 & & 15 & & 8 & \\
\hline $\begin{array}{l}\text { el.CO } \\
\text { TS }\end{array}$ & 0 & & 0 & & 2 & & 0 & & 3 & \\
\hline TS & 0 & & 13 & & 21 & & 31 & & 44 & \\
\hline el.TS & 0 & & 0 & & 2 & & 3 & & 8 & \\
\hline LE & 0 & & 0 & & 1 & $\begin{array}{l}\text { dipar- } \\
\text { tire } \\
\text { (ge- } \\
\text { braucht } \\
\text { wie di- } \\
\text { partirsi } \\
\text { (CO)) }\end{array}$ & 0 & & 0 & \\
\hline ES & 0 & & 0 & & 0 & & 1 & Intifada & 0 & \\
\hline $\begin{array}{l}\text { o.M. } \\
\text { (loc.avv.) }\end{array}$ & 0 & & 0 & & 0 & & 2 & $\begin{array}{l}\text { via } \\
\text { mare/terra }\end{array}$ & 0 & \\
\hline $\begin{array}{l}\text { Tot. } \\
\text { restl. } \\
\text { Marken }\end{array}$ & 15 & $15 \%$ & 84 & $16 \%$ & 101 & $22 \%$ & 201 & $21 \%$ & 182 & $21 \%$ \\
\hline $\begin{array}{l}\text { Tot. Mar- } \\
\text { ken insg. }\end{array}$ & 92 & $96 \%$ & 385 & $75 \%$ & 432 & $93 \%$ & 883 & $92 \%$ & 780 & $91 \%$ \\
\hline
\end{tabular}

Tabelle 17: Untersuchungsergebnisse zu den Seiten aus Nuovo Geolibro 3 (Forte \& Ubertazzi 2008)

Sprachliche Besonderheiten auf den hier analysierten Lehrbuchseiten sind zum einen das Fehlen mindestens eines Wortes im letzten Satz auf Seite 107 im Kasten zu den Bewegungen der Platten der Erdkruste. Dort ist am Ende des Textes von einem Seebeben die Rede, das über 200.000 Opfer gefordert hat, ohne dass es im Satz, dem auch der Punkt fehlt, ein Wort wie Opfer oder Tote gibt, sondern nur die Zahl 200.000 (Forte \& Ubertazzi 2008, S. 107; Hervorhebung nicht im Original):

I movimenti di una sezione della zona di contatto tra la placca euroasiatica e quella indoaustraliana nell'Oceano Indiano sembra siano stati la causa del tremendo terremoto sottomarino 
- e del conseguente maremoto - verificatosi al largo dell'isola di Sumatra nel dicembre 2004, che ha devastato le coste e le isole di undici paesi del sud-est asiatico arrivando a farsi sentire sino alle coste dell'Africa orientale e provocando oltre 200000

(M.Ü.: Die Bewegungen einer Sektion der Kontaktzone zwischen der eurasischen und der indoaustralischen Platte im Indischen Ozean scheinen der Grund des furchtbaren UnterseeErdbebens gewesen zu sein - und des nachfolgenden Seebebens -, das sich bei der Insel Sumatra im Dezember 2004 ereignet und die Küsten und Inseln von elf Ländern Südostasiens verwüstet hat, sich bis an die Küsten Afrikas bemerkbar machend und über 200.000 [?] verursachend.)

Zum anderen stechen zwei Ausdrücke hervor, da einer mit der Bedeutung gar nicht im GRADIT vertreten ist und der andere zum nur literarisch verwendeten Teil des Wortschatzes zählt. Allerdings trägt er in reflexiver Form die Gebrauchsmarke CO und teilt mit dieser die Bedeutung, bei der es sich um einen normalen Sprachgebrauch handelt, so dass vermutet werden kann, dass sie bei der Erstellung des GRADITs übergangen wurde oder sich der Kreis der Bedeutungen des nicht reflexiven Verbs seitdem erweitert hat. Es handelt sich hierbei, ebenfalls auf Seite 107, um den Ausdruck dipartire (LE, fortgehen, in zwei oder mehr Teile aufteilen) im Textzusammenhang von „Quest'ultimo è collegato all'altopiano del Pamir da cui dipartono verso sud-est i sistemi del Kunlun, del Karakoram, [...]" (Forte \& Ubertazzi 2008, S. 107; m.Ü.: Dieser letzte ist mit der Hochebene des Pamirs verbunden, von wo aus in Richtung Südosten die Gebirgssysteme des Kunlun und des Karakorum „fortgehen/sich aufteilen"). Das ähnliche Wort aus dem Bereich des allgemeinen Wortschatzes ist dipartirsi, was so viel wie „beginnen, sich verzweigen“ bedeutet.

Auf derselben Seite befindet sich im nicht beendeten Kasten ein verschachtelter Satz, der den Infinitiv spegnersi (Co) enthält, der im Textzusammenhang wohl mit „enden“" oder ,abflachen“ zu übersetzen wäre, aber laut GRADIT und dem deutsch-italienischen Wörterbuch DIT (Reiniger 1997) als erste Bedeutung das Ausgehen eines Feuers bezeichnet und sich in den weiteren Bedeutungen auf unterschiedlichste Bereiche wie Licht, Lärm, das Erlöschen von Gefühlen, des Lebens, das Kaputtgehen von technischen und das Ausschalten von elektronischen Gegenständen, aber eben nicht wie im Text auf Berge bezieht (Forte \& Ubertazzi 2008, S. 107; Hervorhebung nicht im Original; nur der nähere Kontext ist von mir übersetzt):

In alcuni casi i margini delle zolle si sovrappongono e si accavallano l'uno all'altro, dando così origine a catene montuose interne ai continenti: l'imponente sistema alpino-himalayano, per esempio, che inizia dai Pirenei per spegnersi con le sue ultime propaggini nella penisola di Kamčatka, attraverso l'arco alpino, i Balcani, i monti della penisola anatolica, i sistemi dell'Hindukush e del Karakoram, la catena himalayana, è la manifestazione esterna e non definitiva dello scontro avvenuto tra il blocco euroasiatico e le placche africana e indoaustraliana.

([...] zum Beispiel das imponierende alpinisch-himalayanische Gebirgssystem, welches von den Pyrenäen aus beginnt, um mit seinen letzten Ausläufern auf der Halbinsel des Karakorums auszugehen.) 
Nun wird jedem erfahreneren Leser, der die Grundbedeutung des Wortes spegnersi und vielleicht auch seine weiteren, figurativen Bedeutungen kennt, mithilfe des Textzusammenhangs klar sein, was die Autoren mit diesem Wort ausdrücken wollen. Jedoch ist davon auszugehen, dass in einem so komplexen Satzgefüge mit einem nicht zu übersehenden Bestand an Worten, die nicht aus dem Bereich des Basiswortschatzes stammen, ein einfacheres Verb aus dem Bereich des fundamentalen Wortschatzes pädagogisch sinnvoller wäre.

Eine gewisse Vorliebe der Autoren für einen literarischen, fast poetischen Stil, der zumindest Lernenden, die sprachlich nicht so bewandert sind, Schwierigkeiten bereiten dürfte, ist auch an anderen Stellen zu beobachten, wie z.B. auf Seite 123, wo im 18. Satz über die wirtschaftliche Lage der Türkei in einem durch die Konjunktion pur (CO; obgleich) ${ }^{125}$ eingeleiteten Nebensatz das erforderliche Gerundium einfach impliziert wird (ibid., S. 123; Hervorhebung nicht im Original):

Le attività produttive, pur in crescita (soprattutto nell'agricoltura), sono arretrate: i disoccupati sono numerosi e lo sviluppo delle industrie e del terziario sono ancora insoddisfacenti, mentre il turismo registra risultati migliori.

(M.Ü.: Die Gewerbe, obgleich [sie] im Wachstum [sind; die italienische Konstruktion mit pur erfordert ein Gerundium: pur essendo in crescita] (vor allem in der Landwirtschaft), sind rückständig: [...].)

Dieser Satz stammt aus einem Text, der auch für die Entwicklung des C-Tests verwendet wurde, in dem die Wörter pur und crescita zu ergänzen waren. Von den 19 Schülern, die am Test teilnahmen, vervollständigten nur zwei die Wörter zu pur, während fünf Lösungsvorschläge falsch und die übrigen akzeptabel waren. ${ }^{126}$ Im Gegensatz zum häufigeren Gebrauch der meisten verwendeten Konjunktionen, die aus dem fundamentalen Wortschatz stammen, zählt die hier verwendete Konzessivkonjunktion zum allgemeinen Wortschatz und wird von den Lernenden eindeutig weniger sicher beherrscht.

Weitere Beispiele für implizierte Wörter sind auf derselben Seite das Prädikat deriva(no) im letzten Satz aus dem Textabschnitt, in dem es um die Situation der Kurden geht (ibid.): „Da ciò [derivano] la loro debolezza e lo scarso favore di cui godono presso l'opinione pubblica dei Paesi che li ospitano." (M.Ü.: Daher [stammen] ihre Schwäche und die geringe Gunst, derer sie sich in der öffentlichen Meinung der Länder, die sie beherbergen, erfreuen) und im siebten Satz auf Seite 122 termica des Phraseologismus escursione termica (Ts meteor.; Temperaturschwankungen, -unterschiede). Im Textzusammenhang geht es um die klimatische Vielfalt in der Region; der Satz lautet (ibid., S. 122): „Nel centro l'altopiano si caratterizza per forti escursioni stagionali e per la scarsità delle precipitazioni.“ (M.Ü.: Im Zentrum ist die Hochebene durch

125 Die Konjunktion pur ist nicht im GRADIT vertreten, sondern nur in der längeren Form seppure (CO).

126 S.a. die Besprechung der C-Testergebnisse in Kapitel 8. 
starke jahreszeitliche Exkursionen und eine Knappheit an Niederschlägen bestimmt).

Auf derselben Seite werden außerdem in der Legende zur thematischen Karte der Wirtschaftssektoren der Türkei (die übrigens keine Überschrift trägt) attività terziarie (Tertiäraktivitäten) erwähnt, wobei im Vorfeld der settore terziario (Ts econ.; Tertiärsektor) nicht genannt wird und terziario im GRADIT nur als Substantiv erscheint und die Bedeutung des aus den Wirtschaftswissenschaften stammenden Begriffs trägt, während es hier adjektivisch verwendet wird. Den Lernenden sollte das Konzept der Wirtschaftssektoren aus den ersten beiden Jahren der Mittelschule bekannt sein, doch ihre rezeptive und produktive lexikalische Kompetenz hinsichtlich des Fachausdrucks wird dadurch gefestigt, dass er ab und zu in genau dieser Form wiederholt wird, beispielsweise indem er in verschiedenen Texten vorkommt. Deshalb wäre es förderlicher, von Aktivitäten des Tertiärsektors (attività del settore terziario) zu sprechen und nicht einfach vorauszusetzen, dass alle Schülerinnen und Schüler in der Lage sind, die Brücke zum Fachwort zu schlagen.

Im Folgenden befasst sich der letzte Teil des Kapitels mit einer über die einzelnen Lehrbücher hinausgehenden Beschreibung der sprachlichen Merkmale und einer Bilanz der Untersuchungsergebnisse, in der vor allem die fachwortschatzsprachlichen Anteile am Wortvorkommen näher beschrieben und miteinander verglichen werden.

\subsection{Lehrbuchübergreifende Betrachtung und Gegenüberstellung der Ergebnisse}

Die Feststellung, dass in einer jeden Unterrichtsstunde Sprachunterricht stattfindet, ist keineswegs neu, auch wenn sie aufgrund der zunehmenden Mehrsprachigkeit an den Schulen aktuell diskutiert und ein gewisses Maß an sprach(bildungs)wissenschaftlicher Ausbildung für angehende Lehrkräfte aller Fächer vermehrt in den Studienplänen verankert wird. ${ }^{127}$ Sie wurde bereits vor langer Zeit geäußert. In Italien zum Beispiel geschah dies durch den Pädagogen und Politiker Giuseppe Lombardo-Radice, der zu Beginn des 20. Jahrhunderts damit beauftragt war, neue Lehrpläne für die Grundschule zu entwickeln, und der sich zusammen mit dem damaligen Bildungsminister Giovanni Gentile um ein Konzept bemühte, wie die italienische Sprache den vielen nur Dialekt sprechenden Schülern vermittelt werden könnte (Lombardo-Radice 1959 (1936), S. 167 128 ):

127 In Italien ist dies noch nicht der Fall, jedoch in vielen deutschen Bundesländern und in anderen europäischen Ländern.

128 Das Zitat ist übernommen aus Gensini (2005), S. 37 f. 
Tutto l'insegnamento, su qualunque materia esso verta (anzi tutta la vita, in quanto fusione in noi della infinita ricchezza di insegnamenti che essa ci offre sotto le forme più diverse) costituisce la nostra educazione linguistica. Giacché la parola non è senza la cosa, se parlare significa esprimere, e se esprimere è lo stesso che aver chiara coscienza del proprio mondo. (M.Ü.: Jeder Unterricht, egal welches Fach er betrifft (besser noch das ganze Leben, in wieweit Fusion in uns des unbegrenzten Reichtums an Unterricht, den es uns in verschiedenen Formen anbietet) bildet unsere Spracherziehung. Denn das Wort besteht nicht ohne die Sache, wenn sprechen ausdrücken bedeutet und wenn ausdrücken mit einem klaren Bewusstsein der eigenen Welt gleichzusetzen ist.)

In den letzten Jahren haben WissenschaftlerInnen die sprachlichen Ursachen von Schwierigkeiten der Lernenden in den naturwissenschaftlichen Fächern und in der Mathematik gezielter erforscht (vgl. z.B. Gogolin \& Schwarz 2004; Bolondi, Brachetti \& Ferretti in Druck). Diese rühren nicht etwa nur von mangelnden Kenntnissen des spezifischen Fachwortschatzes her, sondern ergeben sich auch daraus, dass die generelle, im Unterricht durch die Lehrkraft und in den Lehrwerken vermittelte Bildungssprache vielen Lernenden nicht vertraut genug ist. Dass ihr Erwerb und ihre Vermittlung aber für eine gelungene schulische Ausbildung unabkömmlich ist, gilt natürlich auch für den Erdkundeunterricht. Die hier vorgestellten Analysen einiger Seiten aus den fünf Lehrbüchern, die in den beobachteten Klassen dieser Studie verwendet wurden, haben gezeigt, dass ihre lexikalische Gestaltung zu einem wesentlich geringeren Anteil aus Begriffen des fundamentalen Wortschatzes (Fo) besteht, als es für Texte italienischer Sprache als üblich betrachtet wird (De Mauro 1998a, S. 78).

Allein dadurch wird in den untersuchten Lehrwerken ein sprachliches Umfeld geschaffen, das den Lernenden die Aneignung der fachlichen Konzepte und Inhalte nicht erleichtert. Darüber hinaus sind die vielen niedrigfrequenteren Wörter zudem oft in langen Satzgefügen angeordnet, in denen kompliziertere logische Verknüpfungen auftreten, die sich in hypotaktischen Strukturen äußern. Diese Sätze bestehen oft aus einer Vielzahl von Substantiven, die durch Attribute oder Attributketten ergänzt sind und von wenigen konjugierten Verben regiert werden. Dieser letzte der aufgelisteten und für die Bildungssprache als typisch geltenden Umstände hat zur Folge, dass Prozesse nominalisiert werden und die seitens der Lernenden erforderlichen kognitiven Ansprüche zur Sicherung des Textverständnisses steigen, da beispielsweise die Verursacher von Handlungsabläufen sowie die einzelnen Schritte Letzterer weniger stark expliziert sind.

Auf diese sprachlichen Schwierigkeiten wird in den folgenden drei Kapiteln genauer eingegangen, die untersuchen, wie die Lernenden die aus den Lehrbuchtexten entwickelten C-Tests bearbeiteten und sich die Lehrbuchinhalte aneigneten sowie weitere sprachlichen Aspekte des Unterrichtsgeschehens und der schriftlichen Textproduktion der Lernenden, deren Resultate sprachlich mit thematisch kongruenten Lehrbuchtexten verglichen werden, beleuchten. 
An dieser Stelle hingegen wird lehrbuchübergreifend auf den verwendeten Fachwortschatz als einer Komponente der Bildungssprache Bezug genommen.

\subsubsection{Der Fachwortschatz der untersuchten Lehrbuchseiten}

Wie Tabelle 18 zu entnehmen ist, beläuft sich der Anteil des Fachwortschatzes am Gesamtvorkommen der Wörter auf den untersuchten Seiten je nach Lehrbuch auf fünf bis neun Prozent. In diesem Wert sind auch alle doppelt markierten Wörter enthalten. Betrachtet man nur den rein fachwortschatzsprachlichen Wortanteil, so sind es maximal sieben Prozent im Lehrbuch der Klasse III B und minimal vier Prozent in den Klassen III C und an der Schule Morante. Das entspricht einem durchschnittlichen rein fachsprachlichen Anteil von fünf Prozent und einem von sieben Prozent für alle Wörter, die in irgendeiner Form Ts-markiert sind.

Bei diesen Wörtern handelt es sich teilweise um neu eingeführte Begriffe und Konzepte, die im Text erklärt und manchmal auch in anschließenden Lehrbucheinheiten, die sich mit denselben oder ähnlichen Themen beschäftigen, wiederholt (und manchmal auch erneut erklärt) werden, wie oben im Unterkapitel zu den fettgedruckten Wörtern veranschaulicht wurde.

Andere Fachausdrücke, allen voran die der geographischen Fachsprache, sind desweiteren bereits im Lehrwerk für die vorherige Klassenstufe eingeführt worden und werden deshalb von den Autoren anscheinend als bekannt vorausgesetzt, wie beispielsweise die italienischen Wörter für Äquator und Klimazone. In diesem Kapitel wurde außerdem gezeigt, dass die Autorinnen des Nuovo Geolab (Carazzi \& Pizzetti 2010) auch direkt auf Einführungen von Fachbegriffen in anderen Lehrwerken verweisen, wie es etwa beim Konzept des Bruttoinlandsprodukts der Fall ist (ibid., S. 52).

Generell wird eine Vielzahl von Begriffen zumindest auf den untersuchten Seiten nicht oft bzw. gar nicht und vor allem nicht erklärend wiederholt, obwohl nicht davon ausgegangen werden kann, dass die Lernenden sie beherrschen. Im Rahmen dieser Arbeit ist es weder möglich, für alle Ausdrücke aus den Fachsprachen zu überprüfen, in welcher Lehrwerksausgabe sie zuerst erwähnt wurden und wie sie erklärt sind, noch, das gesamte fachwortschatzsprachliche Wortvorkommen danach zu sortieren, ob es auf den untersuchten Seiten einschließlich Erklärung verwendet wird. Eine derartige Untersuchung wird w.u. nur exemplarisch durchgeführt, doch die Thematik wird in den folgenden Kapiteln wieder aufgegriffen, in denen u.a. aus den schriftlichen und mündlichen Sprachproduktionen der Lernenden auf ihre fachwortschatzsprachliche Kompetenz geschlossen und dargestellt wird, wie fachliche Konzepte des Lehrbuchs im Unterricht verhandelt werden und welche Rolle die Lehrkraft dabei einnimmt. 


\begin{tabular}{lrrrrr}
\hline Klasse & Vespucci & Morante & III A P. & III B P. & III C P. \\
\hline Tot. TS & 153 & 99 & 114 & 209 & 124 \\
Anteil am Wortvorkommen insg. & $6 \%$ & $4 \%$ & $5 \%$ & $7 \%$ & $4 \%$ \\
Tot. CO TS & 16 & 11 & 18 & 32 & 42 \\
Anteil am Wortvorkommen insg. & $0,7 \%$ & $0,5 \%$ & $0,8 \%$ & $1,1 \%$ & $1,3 \%$ \\
Tot. restliche doppelte Markierun- & 11 & 8 & 12 & 12 & 11 \\
gen mit TS & & & & & $0,4 \%$ \\
Anteil am Wortvorkommen insg. & $0,5 \%$ & $0,3 \%$ & $0,5 \%$ & $0,4 \%$ \\
Tot. alle TS-markierten Wörter & 180 & 118 & 144 & 253 & 177 \\
Anteil am Wortvorkommen insg. & $7 \%$ & $5 \%$ & $7 \%$ & $9 \%$ & $6 \%$ \\
\hline
\end{tabular}

Tabelle 18: Fachwortschatz (Ts, Co Ts) aller untersuchten Seiten der Lehrbücher

Als nächstes werden die Zusammensetzungen des technisch-spezifischen Wortschatzes aus seinen verschiedenen Feldern untersucht, wobei es sich fachtypisch bei Erdkunde um eine Disziplin handelt, in der verschiedene Wissenschaften (Geographie, Geologie, Biologie, Wetterkunde, Wirtschaft, Geschichte, Soziologie etc.) aufeinandertreffen, so dass zu erwarten ist, dass sich dies auch im verwendeten Fachwortschatz wiederspiegelt.

Tabelle 19 zeigt für alle fünf Lehrbücher, aus welchen Bereichen die fachwortschatzsprachlichen Wörter stammen und wie viele es jeweils sind.

\begin{tabular}{|c|c|c|c|c|c|c|}
\hline TS-Bereich & Vespucci & Morante & $\begin{array}{r}\text { III A } \\
\text { Pestalozzi }\end{array}$ & $\begin{array}{r}\text { III B } \\
\text { Pestalozzi }\end{array}$ & $\begin{array}{r}\text { III C } \\
\text { Pestalozzi }\end{array}$ & $\begin{array}{r}\text { Tot. } n \\
\text { Tokens }\end{array}$ \\
\hline AD TS bot. & & & 1 & & & 1 \\
\hline AD TS chim. & & & & 1 & & 1 \\
\hline AD TS ecol. & & & & & 1 & 1 \\
\hline AD TS etnol. & & 1 & & & & 1 \\
\hline AU TS archeol. & 1 & & & & & 1 \\
\hline AU TS astron. & 1 & & 1 & & & 2 \\
\hline AU TS econ. & 8 & & 4 & & & 12 \\
\hline AU TS geogr. & & & 1 & 2 & 1 & 4 \\
\hline AU TS geol. & & & & 1 & 2 & 3 \\
\hline AU TS ling. & & & 1 & & & 1 \\
\hline CO TS agr. & & & & 1 & & 1 \\
\hline CO TS amin.az. & & & & & 1 & 1 \\
\hline CO TS archeol. & & 1 & & & & 1 \\
\hline $\begin{array}{l}\text { CO TS astron., te- } \\
\text { lecom. }\end{array}$ & & & & & 1 & 1 \\
\hline $\begin{array}{l}\text { CO TS burocr., } \\
\text { tecn. }\end{array}$ & 1 & & & & & 1 \\
\hline CO TS cartogr. & 1 & & 2 & 1 & & 4 \\
\hline CO TS chim. & 3 & 1 & 3 & 1 & 1 & 9 \\
\hline CO TS dir. & & & 1 & & 1 & 1 \\
\hline CO TS econ. & 3 & & 4 & & 1 & 8 \\
\hline CO TS geofis. & & & & & 3 & 3 \\
\hline CO TS geogr. & 1 & 3 & 2 & 4 & 7 & 17 \\
\hline CO TS geol. & & & & 1 & 1 & 2 \\
\hline CO TS inform. & & & 1 & & & 1 \\
\hline CO TS let. & & 1 & & & & 1 \\
\hline CO TS ling. & & 1 & & 1 & & 2 \\
\hline
\end{tabular}




\begin{tabular}{|c|c|c|c|c|c|c|}
\hline TS-Bereich & Vespucci & Morante & $\begin{array}{r}\text { III A } \\
\text { Pestalozzi }\end{array}$ & $\begin{array}{r}\text { III B } \\
\text { Pestalozzi }\end{array}$ & $\begin{array}{r}\text { III C } \\
\text { Pestalozzi }\end{array}$ & $\begin{array}{r}\text { Tot. } \mathrm{n} \\
\text { Tokens }\end{array}$ \\
\hline CO TS ling., dir. & & & & 6 & & 6 \\
\hline CO TS med. & 1 & & & 1 & & 2 \\
\hline CO TS meteor. & & & & 4 & & 4 \\
\hline CO TS monet. & 2 & & & 1 & & 3 \\
\hline CO TS metall. & & 1 & & & & 1 \\
\hline CO TS polit. & & & & 1 & 2 & 3 \\
\hline CO TS relig. & 1 & 2 & 1 & 8 & 16 & 28 \\
\hline CO TS relig., filos. & & & & 1 & & 1 \\
\hline CO TS relig., polit. & & & & 1 & & 1 \\
\hline CO TS scient. & 3 & 1 & & & 2 & 6 \\
\hline CO TS stat. & & & 4 & & 1 & 5 \\
\hline el.CO TS geofis. & & & & & 2 & 2 \\
\hline el.CO TS ling., dir. & & & & 6 & & 6 \\
\hline el.CO TS polit. & & & & & 2 & 2 \\
\hline el.CO TS stat. & & & & & 1 & 1 \\
\hline el.TS arm. & & & & & 1 & 1 \\
\hline el.TS bot.com. & 2 & 2 & & & & 4 \\
\hline el.TS cartogr. & & & 1 & & & 1 \\
\hline el.TS chim. & 4 & 1 & & 1 & 2 & 3 \\
\hline el.TS dir. & & & & 2 & 1 & 3 \\
\hline el.TS dir.amm. & & & & & 1 & 1 \\
\hline el.TS dir.intern. & & & 2 & & 2 & 4 \\
\hline el.TS ecol. & 2 & & 3 & & & 5 \\
\hline el.TS econ. & 7 & 3 & 6 & & & 16 \\
\hline el.TS econ., polit. & & & 2 & & & 2 \\
\hline el.TS etnol. & & 1 & & & & 1 \\
\hline el.TS fin. & & & & 1 & & 1 \\
\hline el.TS fis. & 4 & & 2 & & & 6 \\
\hline el.TS geogr. & 1 & 1 & 2 & 7 & & 11 \\
\hline el.TS geol. & & & 1 & 1 & 1 & 3 \\
\hline el.TS industr. & & & & & 1 & 1 \\
\hline el.TS ling. & & & & 7 & & 7 \\
\hline el.TS meteor., & 1 & & & 3 & 2 & 6 \\
\hline $\begin{array}{l}\text { geoogr. } \\
\text { el.TS metrol. }\end{array}$ & & 2 & & & 2 & 4 \\
\hline el.TS polit., econ. & 2 & & & & & 2 \\
\hline el.TS sociol. & 1 & & & & & 1 \\
\hline el.TS stat. & 10 & 1 & 7 & 5 & & 23 \\
\hline el.TS stat., polit. & 6 & & & & & 6 \\
\hline el.TS stor. & & 1 & 1 & & 1 & 3 \\
\hline el.TS tecn. & 1 & & 1 & & & 2 \\
\hline el.TS urban. & 2 & 1 & & 2 & 1 & 6 \\
\hline el.TS vulcanol. & & & & 1 & & 1 \\
\hline ES afr. TS etnol. & & 1 & & & & 1 \\
\hline ES ingl. TS geol. & & & & 1 & & 1 \\
\hline ES ingl. TS sport. & & 1 & & & & 1 \\
\hline FO TS arch. & & 1 & & & & 1 \\
\hline FO TS astron. & & 1 & 4 & & 4 & 9 \\
\hline FO TS dir. & 1 & 1 & & 5 & 2 & 9 \\
\hline
\end{tabular}




\begin{tabular}{|c|c|c|c|c|c|c|}
\hline TS-Bereich & Vespucci & Morante & $\begin{array}{r}\text { III A } \\
\text { Pestalozzi }\end{array}$ & $\begin{array}{r}\text { III B } \\
\text { Pestalozzi }\end{array}$ & $\begin{array}{r}\text { III C } \\
\text { Pestalozzi }\end{array}$ & $\begin{array}{r}\text { Tot. } n \\
\text { Tokens }\end{array}$ \\
\hline FO TS econ. & & & & & 1 & 1 \\
\hline FO TS fis. & & & & 2 & & 2 \\
\hline FO TS meteor. & & & & 1 & & 1 \\
\hline FO TS relig. & & 2 & & & & 2 \\
\hline $\begin{array}{l}\text { TS } \\
\text { (nicht im GRADIT) }\end{array}$ & & & & & distretto & 1 \\
\hline TS agr. & 1 & & & & & 1 \\
\hline TS antrop. & & & 1 & 2 & & 3 \\
\hline TS arm. & & & & & 2 & 2 \\
\hline TS astrol. & 1 & 2 & & & & 3 \\
\hline TS biochim. & & & 1 & & & 1 \\
\hline TS biol. & & & & 1 & & 1 \\
\hline TS bot. & & & & 3 & & 3 \\
\hline TS bot., zool., biol. & & & & 1 & & 1 \\
\hline TS bot.com. & 3 & 3 & & 3 & & 9 \\
\hline TS cartogr. & & & 1 & & & 1 \\
\hline TS chim. & 8 & 1 & 2 & 1 & 2 & 14 \\
\hline TS dir. & & 3 & & 3 & 4 & 10 \\
\hline TS dir.amm. & & & & & 1 & 1 \\
\hline TS dir.intern. & & & 2 & & 2 & 4 \\
\hline TS ecol. & 1 & & 8 & 4 & 1 & 14 \\
\hline TS econ. & 34 & 6 & 10 & 2 & 7 & 59 \\
\hline TS econ., ecol. & & & 2 & & & 2 \\
\hline TS econ., polit. & & & 2 & & & 2 \\
\hline TS econ., sociol. & 4 & 1 & 6 & & & 11 \\
\hline TS etnol. & & 15 & 1 & 6 & 7 & 29 \\
\hline TS etnol., stor. & & & & & 1 & 1 \\
\hline TS fis. & 6 & & 2 & & & 8 \\
\hline TS fis., chim. & & & 2 & & & 2 \\
\hline TS fis., mat. & 2 & & 1 & & & 3 \\
\hline TS fin. & & & & 1 & & 1 \\
\hline TS geogr. & 18 & 21 & 14 & 19 & 16 & 88 \\
\hline TS geogr., antrop. & 1 & & & & & 1 \\
\hline TS geogr., idrol. & & & & 1 & & 1 \\
\hline TS geol. & & & 1 & 2 & 10 & 13 \\
\hline TS geol., vulcanol. & & & & 2 & 2 & 4 \\
\hline TS industr. & & & & & 1 & 1 \\
\hline TS ling. & & & 17 & 69 & 6 & 92 \\
\hline TS mar. & & 2 & & & & 2 \\
\hline TS mat. & & & & & 3 & 3 \\
\hline TS meteor. & & & & 1 & 1 & 2 \\
\hline TS meteor., geogr. & 1 & 1 & & 3 & 3 & 8 \\
\hline TS metrol. & 2 & 8 & 1 & 29 & 7 & 47 \\
\hline TS miner. & 1 & 1 & & & 1 & 3 \\
\hline TS monet. & & & & 2 & & 2 \\
\hline TS paleont. & & & 1 & & & 1 \\
\hline TS petrol. & & & & 1 & & 1 \\
\hline TS polit., relig. & & & & 1 & 2 & 3 \\
\hline TS polit., econ. & 2 & & & & & 2 \\
\hline
\end{tabular}




\begin{tabular}{|c|c|c|c|c|c|c|}
\hline TS-Bereich & Vespucci & Morante & $\begin{array}{r}\text { III A } \\
\text { Pestalozzi }\end{array}$ & $\begin{array}{r}\text { III B } \\
\text { Pestalozzi }\end{array}$ & $\begin{array}{r}\text { III C } \\
\text { Pestalozzi }\end{array}$ & $\begin{array}{r}\text { Tot. } n \\
\text { Tokens }\end{array}$ \\
\hline TS pubbl. & & & 1 & & & 1 \\
\hline TS relig. & & 2 & & & 4 & 6 \\
\hline TS relig., polit. & & 2 & & 3 & 1 & 6 \\
\hline TS scient. & & & 1 & & 3 & 4 \\
\hline TS sociol. & 1 & & & & 1 & 2 \\
\hline TS stat. & 18 & 5 & 5 & 3 & 3 & 34 \\
\hline TS stat., polit. & 2 & & & & & 2 \\
\hline TS stor. & & 11 & 1 & 6 & 15 & 33 \\
\hline TS tecn. & 2 & & 3 & & & 5 \\
\hline TS topogr. & & & & & 1 & 1 \\
\hline TS urban. & 2 & 1 & & & 1 & 4 \\
\hline TS urban., archeol. & & 1 & & 2 & & 3 \\
\hline TS vulcanol. & & & & 1 & & 1 \\
\hline TS zool.com. & & & & 1 & & 1 \\
\hline TS zoot. & & & & 1 & & 1 \\
\hline Tot. & 180 & 118 & 144 & 253 & 177 & 868 \\
\hline
\end{tabular}

Tabelle 19: Fachwortschatzbereiche aller untersuchten Seiten der Lehrwerke

Inklusive der doppelt markierten Wörter (z.B. Co Ts econ.) stammen die auf den untersuchten Seiten verwendeten Fachausdrücke aus 105 verschiedenen Bereichen. Zählt man die doppelt markierten zu den reinen Ts-Markierungen, also im Fall des oben gegebenen Beispiels die Co Ts econ.-Wörter zu den Ts econ.-Wörtern, so gehen die Ausdrücke immer noch auf 74 Fachsprachen zurück. Wenn man zuletzt noch die doppelten fachsprachlichen Kategorisierungen (z.B. Ts meteor., geogr.) auflöst und bspw. die drei Markierungen Ts meteor., Ts geogr. und Ts meteor., geogr. nur als zwei Bereiche wertet, bleibt dennoch eine erhebliche Anzahl von Fachsprachen bestehen, nämlich die folgenden 59:

Ts (nicht im GRADIT), Ts agr., Ts amin.az., Ts antrop., Ts arch., Ts archeol., Ts arm., Ts astrol., Ts astron., Ts astron., telecom., Ts biochim., Ts biol., Ts bot., Ts bot.com., Ts burocr., tecn., Ts cartogr., Ts chim., Ts dir., Ts dir.amm., Ts dir.intern., Ts ecol., Ts econ., Ts etnol., stor., Ts fin., Ts fis., Ts geofis., Ts geogr., Ts geogr., antrop., Ts geogr., idrol., Ts geol., Ts industr., Ts inform., Ts let., Ts ling., Ts mar., Ts mat., Ts med., Ts metall., Ts meteor., Ts metrol., Ts miner., Ts monet., Ts paleont., Ts petrol., Ts polit., Ts pubbl., Ts relig., Ts scient., Ts sociol., Ts sport., Ts stat., Ts stor., Ts tecn., Ts topogr., Ts urban., Ts vulcanol., Ts zool.com., Ts zoot.

119 der unterschiedlichen Ts-Markierungen sind in den Lehrbüchern insgesamt maximal mit zehn Vorkommnissen vertreten, davon 51 nur mit einem einzigen und 88 mit maximal drei. Lediglich drei Markierungen sind mit mehr als 50 Vorkommnissen in die Texte gebettet, und zwar die für den sprachwissenschaftlichen (92, allerdings auf den untersuchten Seiten nur in drei Lehrbüchern verwendet), für den geographischen (88) und für den wirtschaftlichen (59) Fachwortschatz. Desweiteren sind, wie der Tabelle zu entnehmen ist, relativ viele Fachwortschatzausdrücke nur einmal auf den untersuchten Seiten 
vertreten (121). Andererseits wiederholen sich bestimmte Wörter in den Texten, wie anhand des Wortschatzes, der nur Ts-markiert ist und somit keine doppelten Gebrauchsmarken aufweist, gezeigt werden soll.

\subsubsection{Der reine Fachwortschatz}

Im Lehrbuch Bersezio (2005) sind auf den untersuchten Seiten 86 solcher verschiedenen Wortvorkommnisse enthalten, die auf nur 58 Worttypen zurückgehen. ${ }^{129}$ Hierbei sind Homographe wie beispielsweise zulu, was sowohl ein Adjektiv als auch ein Substantiv ist, als zwei unterschiedliche Typen gezählt worden. Tabelle 20 listet alle Wörter alphabetisch nach Art des Fachwortschatzes auf.

\begin{tabular}{|c|c|c|c|}
\hline Lemma & Wortart & TS-Bereich & Tokens \\
\hline Capricorno & s.m. & TS astrol. & 2 \\
\hline sesamo & s.m. & TS bot.com. & 1 \\
\hline miglio & s.m. & TS bot.com. & 1 \\
\hline canna & loc.s.f. & TS bot.com. & 1 \\
\hline$d a$ & el.loc.s.f. & el.TS bot.com. & \\
\hline zucchero & el.loc.s.f. & el.TS bot.com. & \\
\hline gas & loc.s.m. & TS chim. & 1 \\
\hline naturale & el.loc. s.m. & el.TS chim. & \\
\hline costituirsi & v.pronom.intr. & TS dir. & 1 \\
\hline contenzioso & s.m. & TS dir. & 1 \\
\hline riconoscere & v.tr. & TS dir. & 1 \\
\hline settore & loc.s.m. & TS econ. & 1 \\
\hline primario & el.loc.s.m. & el.TS econ. & \\
\hline bilancia & loc.s.f. & TS econ. & 1 \\
\hline commerciale & el.loc.s.f. & el.TS econ. & \\
\hline in & loc.agg. & TS econ. & 1 \\
\hline passivo & el.loc.agg. & el.TS econ. & \\
\hline terziario & s.m. & TS econ. & 3 \\
\hline boscimano & s.m. & TS etnol. & 1 \\
\hline gruppo & loc.s.m. & TS etnol. & 1 \\
\hline etnico & el.loc.s.m. & el.TS etnol. & \\
\hline ottentotto & s.m. & TS etnol. & 1 \\
\hline bantu & s.m. & TS etnol. & 1 \\
\hline bantu & agg. & TS etnol. & 2 \\
\hline kraal & s.m. & ES afrik. TS etnol. & 1 \\
\hline zulu & agg. & TS etnol. & 3 \\
\hline zulu & s.m. & TS etnol. & 4 \\
\hline xhosa & s.m. & TS etnol. & 1 \\
\hline curdo & agg. & TS etnol. & 1 \\
\hline catena & loc.s.f. & TS geogr. & 1 \\
\hline montuosa & el.loc.s.f. & el.TS geogr. & \\
\hline Tropico & s.m. & TS geogr. & 3 \\
\hline
\end{tabular}

129 Bei der Berechnung der Typenanzahl sind die Elemente der Phraseme natürlich nicht als Worttypen gezählt worden, sondern nur das erste Wort der Phraseme. 


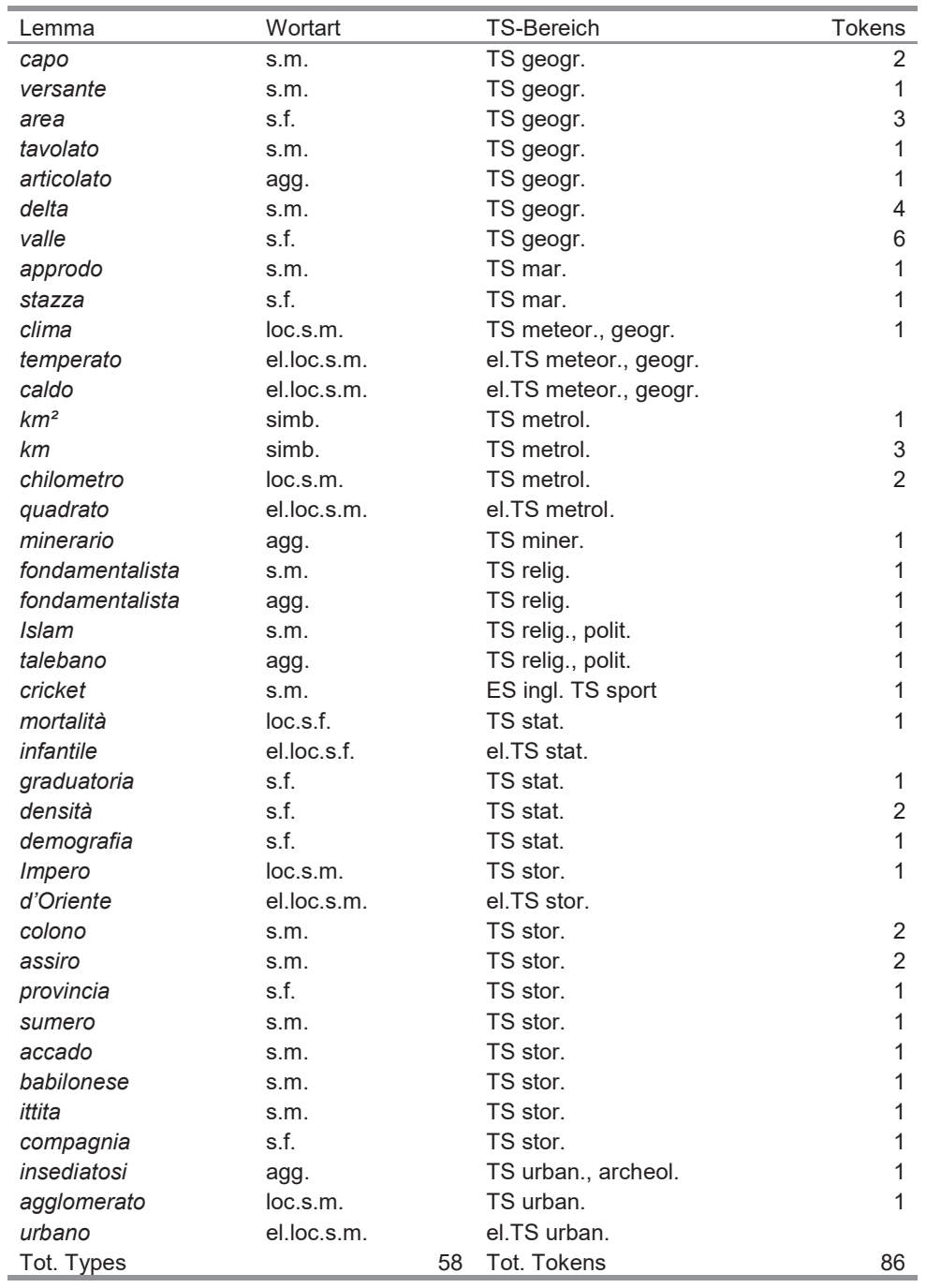

Tabelle 20: Fachwortschatz der untersuchten Seiten im Nuovi territori dell'uomo (Bersezio 2005)

Die am stärksten vertretenen Bereiche des Fachwortschatzes sind der geographische (22 Tokens, 9 Types), der ethnologische (16 Tokens, 10 Types), der wirtschaftliche (11 Tokens, 9 Types) und der geschichtliche (6 Tokens, 4 Types). Fast vier Fünftel (68) aller TS-markierten Wörter sind Substantive, wobei 
die 56 Vorkommnisse einfacher Substantive auf 34 Worttypen und die zwölf Vorkommnisse an Nominalphrasemen auf elf zurückgehen.

Die zweitgrößte Gruppe formen die Adjektive, welche inklusive eines adjektivalen Phrasems mit zwölf Vorkommnissen vertreten sind, die sich auf neun verschiedene Typen belaufen.

Dass sich unter den Wörtern so viele Substantive befinden, spiegelt den Charakter des Fachwortschatzes wieder, der vor allem eine deskriptive Funktion hat (z.B. Giuliani et al. 2005, S. 212), aber es sind auch zwei Verben darunter, welche beide aus dem Fachwortschatz des Rechtswesens stammen (Bersezio 2005, S. 82): costituirsi (u.a. entstehen, sich formen) und riconoscere (offiziell anerkennen).

Auf den untersuchten Seiten des Lehrbuchs Nuovo Geolibro (Forte \& Ubertazzi 2008) der Klasse III C, Pestalozzi sind 74 nur Ts- und nicht doppelt gekennzeichnete Wörter mit insgesamt 108 Vorkommnissen verzeichnet. Um welche Ausdrücke es sich im Einzelnen handelt, ist Tabelle $21 \mathrm{zu}$ entnehmen. Auch hier ist das Wortvorkommen wieder alphabetisch nach Art des Fachwortschatzbereichs sortiert; außerdem wird die Klassifizierung eines Wortes ( $n u$ cleare*) unterhalb der Tabelle kommentiert.

\begin{tabular}{lllr}
\hline Lemma & Wortart & TS-Bereich & Tokens \\
\hline arma & loc.s.f. & TS arm. & 1 \\
chimica & el.loc.s.f. & TS arm. & \\
nucleare* & loc.s.f. & TS arm. & 1 \\
gas & loc.s.m. & TS chim. & 2 \\
naturale & el.loc.s.m. & el.TS chim. & \\
aggressione & s.m. & TS dir. & 1 \\
autodeterminazione & s.f. & TS dir. & 1 \\
Repubblica & loc.s.f. & TS dir. & 1 \\
parlamentare & el.loc.s.f. & el.TS dir. & \\
approvato & part.pass.v.tr. & TS dir. & 1 \\
spesa & loc.s.f. & TS dir.amm. & 1 \\
pubblica & el.loc.s.f. & el.TS dir.amm. & \\
Unione & loc.s.f. & TS dir.intern. & 2 \\
Europea & el.loc.s.f. & el.TS dir.intern. & \\
ambiente & s.f. & TS ecol. & 1 \\
terziario & agg. & TS econ. & 1 \\
terziario & s.m. & TS econ. & 2 \\
crisi & s.f. & TS econ. & 1 \\
consumo & s.m. & TS econ. & 1 \\
compagnia & s.f. & TS econ. & 1 \\
ricchezza & s.f. & TS econ. & 1 \\
etnia & s.f. & TS etnol. & 1 \\
curdo & s.m. & TS etnol. & 4 \\
curdo & agg. & TS etnol. & 2 \\
turco & s.m. & TS etnol., stor. & 1 \\
geopolitico & agg. & TS geogr. & 2 \\
area & s.f. & TS geogr. & 1 \\
struttura & s.f. & TS geogr. & \\
\hline & & & 1
\end{tabular}




\begin{tabular}{|c|c|c|c|}
\hline Lemma & Wortart & TS-Bereich & Tokens \\
\hline indoaustraliano & agg. & TS geogr. & 3 \\
\hline alpino-himalayano & agg. & TS geogr. & 2 \\
\hline emisfero & s.m. & TS geogr. & 1 \\
\hline equatore & s.m. & TS geogr. & 1 \\
\hline dorsale & s.m. & TS geogr. & 2 \\
\hline tavolato & s.m. & TS geogr. & 1 \\
\hline articolato & agg. & TS geogr. & 1 \\
\hline geopolitico & agg. & TS geogr. & 1 \\
\hline pianura & loc.s.f. & TS geol. & 1 \\
\hline alluvionale & el.loc.s.f. & el.TS geol. & \\
\hline zolla & s.f. & TS geol. & 2 \\
\hline orogenetico & agg. & TS geol. & 1 \\
\hline placca & s.f. & TS geol. & 6 \\
\hline vulcanico & agg. & TS geol., vulcanol. & 2 \\
\hline distretto & loc.s.m. & TS industr. & 1 \\
\hline industriale & el.loc.s.m. & el.TS industr. & \\
\hline turco & s.m. & TS ling. & 1 \\
\hline arabo & s.m. & TS ling. & 1 \\
\hline armeno & s.m. & TS ling. & 1 \\
\hline curdo & s.m. & TS ling. & 2 \\
\hline greco & s.m. & TS ling. & 1 \\
\hline media & s.f. & TS mat. & 3 \\
\hline escursione & s.f. & TS meteor. & 1 \\
\hline subtropicale & agg. & TS meteor., geogr. & 1 \\
\hline clima & loc.s.m. & TS meteor., geogr. & 1 \\
\hline temperato & el.loc.s.m. & el.TS meteor., geogr. & \\
\hline clima & loc.s.m. & TS meteor., geogr. & 1 \\
\hline tropicale & el.loc.s.m. & el.TS meteor., geogr. & \\
\hline kmq & simb. & TS metrol. & 5 \\
\hline$m$ & simb. & TS metrol. & 1 \\
\hline $\mathrm{km}$ & simb. & TS metrol. & 1 \\
\hline minerario & agg. & TS miner. & 1 \\
\hline sciita & agg. & TS polit., relig. & 2 \\
\hline Imam & s.m. & TS relig. & 2 \\
\hline sunnita & agg. & TS relig. & 1 \\
\hline Sunna & s.f. & TS relig. & 1 \\
\hline taliban & s.m. & TS relig., polit. & 1 \\
\hline fenomeno & s.m. & TS scient. & 1 \\
\hline morfologico & agg. & TS scient. & 2 \\
\hline modernizzazione & s.f. & TS sociol. & 1 \\
\hline stima & s.f. & TS stat. & 1 \\
\hline densità & s.f. & TS stat. & 2 \\
\hline Impero & loc.s.m. & TS stor. & 1 \\
\hline d'Oriente & el.loc.s.m. & el.TS stor. & \\
\hline ottomano & agg. & TS stor. & 3 \\
\hline sultano & s.m. & TS stor. & 1 \\
\hline mesopotamico & agg. & TS stor. & 1 \\
\hline sumero & s.f. & TS stor. & 1 \\
\hline assiro & s.f. & TS stor. & 1 \\
\hline babilonese & s.f. & TS stor. & 1 \\
\hline
\end{tabular}




\begin{tabular}{lllr}
\hline Lemma & Wortart & TS-Bereich & Tokens \\
\hline fenicio & s.f. & TS stor. & 1 \\
Medioevo & s.m. & TS stor. & 2 \\
califfo & s.m. & TS stor. & 1 \\
ottomani & agg. & TS stor. & 2 \\
altimetrico & agg. & TS topogr. & 1 \\
agglomerato & loc.s.m. & TS urban. & 1 \\
urbano & el.loc.s.m. & el.TS urban. & 108 \\
Tot. Types & & 74 Tot. Tokens \\
\hline
\end{tabular}

Tabelle 21: Fachwortschatz der untersuchten Seiten im Nuovo Geolibro (Forte \& Ubertazzi 2008)

*Nucleare: Dieses Wort ist als Nominalphrasem und nicht als Element davon gewertet, da es im Kontext armi chimiche e nucleari (ibid., S. 121) erscheint und es sich also um zwei verschiedene Phraseologismen handelt, auch wenn das gemeinsame determinierte Hauptwort armi impliziert ist, anstatt im zweiten Phraseologismus wiederholt zu werden.

Eine weitere Tabelle (22) zeigt die vier Fachwortschatzbereiche, aus denen die meisten Wörter stammen (Geographie, Geschichte, Wirtschaft und Linguistik). Außerdem wird ersichtlich, dass der rein fachwortschatzsprachliche Anteil auch auf den untersuchten Seiten in diesem Lehrbuch größtenteils aus Nomen besteht, nämlich zu 66 Prozent. Konjugierte Verben hingegen sind hier unter den Ts-Wörtern nicht vertreten, sondern nur ein dekliniertes Partizip Perfekt (approvato, Ts dir.; verabschieden).

\begin{tabular}{lrr}
\hline Fachsprachen/ & Tokens & Types \\
Wortarten & 16 & 11 \\
\hline Tot. TS geogr. & 15 & 11 \\
Tot. TS stor. & 7 & 6 \\
Tot. TS econ. & 6 & 5 \\
Tot. TS ling. & 57 & 40 \\
Tot. s. (ohne loc.) & 14 & 12 \\
Tot. loc.s. & 29 & 18 \\
Tot. agg. & 7 & 3 \\
Tot. simb. & 7 & \\
\hline
\end{tabular}

Tabelle 22: Stärkste Ts-Bereiche und Zusammensetzung des Ts-Vorkommens nach Wortarten im Nuovo Geolibro (Forte \& Ubertazzi 2008)

Im Lehrbuch Geograficamente (Dinucci \& Dinucci 2008), das in der Klasse III B der Schule Pestalozzi verwendet wird, befinden sich auf den analysierten Seiten 109 Worttypen, die als Fachwortschatz klassifiziert sind (176 Vorkommnisse). Das sind 73 nominale Worttypen (66\%), von denen ein gutes Drittel aus Phraseologismen besteht (20 Types, 25 Tokens). Diese Substantive sind mit insgesamt 111 Vorkommnissen vertreten.

Einen verhältnismäßig großen Anteil an den Ts-gekennzeichneten Wörtern machen hier die Symbole aus: Es sind 30 Vorkommnisse, die auf die vier Typen $\mathrm{km}, \mathrm{m}, \mathrm{km}^{2}$ und ${ }^{\circ} \mathrm{C}$ zurückgehen, wobei sich 28 der Vorkommnisse auf die ersten drei Typen verteilen. Einfach gesagt, besteht ein Sechstel des verwen- 
deten Fachwortschatzes aus Maßeinheiten, welche die Lernenden aus dem Mathematikunterricht und aus ihrer Alltagswelt (abgesehen vielleicht von „ $\mathrm{km}^{26}$ ) gut kennen, so dass ihnen dieser Teil des Fachwortschatzes keine Schwierigkeit bereiten sollte.

Mehr als ein Drittel aller Ts-Wörter stammen aus dem Bereich Linguistik und bezeichnen vor allem Sprachen, von denen viele, ähnlich wie mit den Symbolen, aus Schule und Alltagswelt bekannt sein dürften. Darüber hinaus ist die Anzahl der linguistischen Worttypen um knapp die Hälfte geringer als die ihrer Vorkommnisse (40 zu 69) und sie würde sich noch weiter verringern, wenn man Homographen, die semantisch eng miteinander verwandt sind und sich nur durch unterschiedliche Wortklassenzugehörigkeit unterscheiden (z.B. Substantive für die Einwohner eines Landes und Adjektive für ihre Sprache), nicht als unterschiedliche Typen zählte.

Diese und weitere Informationen sind den Tabellen 23 und 24 zu entnehmen; in der ersten sind zwei Besonderheiten mit einem Sternchen gekennzeichnet und unterhalb der Tabelle erläutert.

\begin{tabular}{|c|c|c|c|}
\hline Lemma & Wortart & TS-Bereich & Tokens \\
\hline sudanese & agg. & TS antrop. & 1 \\
\hline sahariano & agg. & TS antrop. & 1 \\
\hline ambiente & s.m. & TS biol. & 1 \\
\hline conifera & s.f. & TS bot. & 2 \\
\hline lichene & s.m. & TS bot. & 1 \\
\hline specie & s.f. & TS bot.,zool.,biol. & 1 \\
\hline vite & s.f. & TS bot.com. & 1 \\
\hline muschio & s.m. & TS bot.com. & 1 \\
\hline tabacco & s.m. & TS bot.com. & 1 \\
\hline gas & loc.s.m. & TS chim. & 1 \\
\hline naturale & el.loc.s.m. & el.TS chim. & \\
\hline Monarchia & loc.s.f. & TS dir. & 1 \\
\hline assoluta & el.loc.s.f. & el.TS dir. & \\
\hline Monarchia & loc.s.f. & TS dir. & 1 \\
\hline costituzionale & el.loc.s.f. & el.TS dir. & \\
\hline riconosciuto & part.pass. & TS dir. & 1 \\
\hline desertificazione & s.f. & TS ecol. & 1 \\
\hline ambiente & s.m. & TS ecol. & 2 \\
\hline inquinamento & s.m. & TS ecol. & 2 \\
\hline povertà & s.f. & TS econ. & 1 \\
\hline berbero & agg. & TS etnol. & 3 \\
\hline indocinese & agg. & TS etnol. & 1 \\
\hline iranico & agg. & TS etnol. & 1 \\
\hline khoisan & agg. & TS etnol. & 1 \\
\hline reddito & loc.s.m. & TS fin. & 1 \\
\hline nazionale & el.loc.s.m. & el.TS fin. & \\
\hline articolato & agg. & TS geogr. & 1 \\
\hline nivale & agg. & TS geogr. & 1 \\
\hline indogangetico & agg. & TS geogr. & 1 \\
\hline equatoriale & agg. & TS geogr. & 1 \\
\hline
\end{tabular}




\begin{tabular}{|c|c|c|c|}
\hline Lemma & Wortart & TS-Bereich & Tokens \\
\hline cintura & loc.s.f. & TS geogr. & 1 \\
\hline$d i$ & el.loc.s.f. & el.TS geogr. & \\
\hline fuoco & el.loc.s.f. & el.TS geogr. & \\
\hline foresta & loc.s.f. & TS geogr. & 1 \\
\hline boreale & el.loc.s.f. & el.TS geogr. & \\
\hline foresta & loc.s.f. & TS geogr. & 1 \\
\hline temperata & el.loc.s.f. & el.TS geogr. & \\
\hline foresta & loc.s.f. & TS geogr. & 2 \\
\hline pluviale & el.loc.s.f. & TS geogr. & \\
\hline polo & loc.s.m. & TS geogr. & 1 \\
\hline del & el.loc.s.m. & el.TS geogr. & \\
\hline freddo & el.loc.s.m. & el.TS geogr. & \\
\hline area & s.f. & TS geogr. & 2 \\
\hline tavolato & s.m. & TS geogr. & 1 \\
\hline versante & s.m. & TS geogr. & 4 \\
\hline equatore & s.m. & TS geogr. & 1 \\
\hline idrografia & s.f. & TS geogr., idrol. & 1 \\
\hline permafrost & s.m. & ES ingl. TS geol. & 1 \\
\hline pianura & loc.s.f. & TS geol. & 1 \\
\hline alluvionale & el.loc.s.f. & el.TS geol. & \\
\hline zolla & s.f. & TS geol. & 1 \\
\hline vulcanico & agg. & TS geol., vulcanol. & 2 \\
\hline amarico & s.m. & TS ling. & 1 \\
\hline hindi & s.m. & TS ling. & 4 \\
\hline russo & s.m. & TS ling. & 3 \\
\hline spagnolo & s.m. & TS ling. & 4 \\
\hline cinese & loc.s.m. & TS ling. & 2 \\
\hline mandarino & el.loc.s.m. & el.TS ling. & \\
\hline francese & s.m. & TS ling. & 3 \\
\hline tedesco & s.m. & TS ling. & 1 \\
\hline arabo & s.m. & TS ling. & 9 \\
\hline portoghese & s.m. & TS ling. & 2 \\
\hline maleo-indonesiano & conf. + s.m. & TS ling. + TS ling. & $1+1$ \\
\hline giapponese & s.m. & TS ling. & 1 \\
\hline altaico & agg. & TS ling. & 1 \\
\hline amerindiano & agg. & TS ling. & 1 \\
\hline armeno & s.m. & TS ling. & 1 \\
\hline austro-astoatico & agg. & TS ling. & 1 \\
\hline austronesiano & agg. & TS ling. & 1 \\
\hline birmano & s.m. & TS ling. & 1 \\
\hline cinese & s.m. & TS ling. & 1 \\
\hline curdo & s.m. & TS ling. & 2 \\
\hline dravidico & agg. & TS ling. & 1 \\
\hline famiglia & loc.s.f. & TS ling. & 3 \\
\hline linguistica & el.loc.s.f. & el.TS ling. & \\
\hline finlandese & s.m. & TS ling. & 1 \\
\hline germanico & agg. & TS ling. & 1 \\
\hline greco & s.m. & TS ling. & 2 \\
\hline indoeuropeo & agg. & TS ling. & 3 \\
\hline indoeuropeo & s.m. & TS ling. & 1 \\
\hline
\end{tabular}




\begin{tabular}{|c|c|c|c|}
\hline Lemma & Wortart & TS-Bereich & Tokens \\
\hline lingua & loc.s.f. & TS ling. & 2 \\
\hline madre & el.loc.s.f. & el.TS ling. & \\
\hline mongolo & s.m. & TS ling. & 1 \\
\hline neolatine & agg. & TS ling. & 1 \\
\hline рариа & agg. & TS ling. & 1 \\
\hline parlante & s.m. & TS ling. & 2 \\
\hline persiano & s.m. & TS ling. & 2 \\
\hline sino-tibetano & s.m. & TS ling. & 1 \\
\hline sinotibetano & agg. & TS ling. & 1 \\
\hline tibetano & s.m. & TS ling. & 1 \\
\hline turco & s.m. & TS ling. & 2 \\
\hline uralico & agg. & TS ling. & 1 \\
\hline uralo-altaico & s.m. & TS ling. & 1 \\
\hline piovosità & s.m. & TS meteor. & 1 \\
\hline clima & loc.s.m. & $\begin{array}{l}\text { TS meteor., geogr. } \\
\text { el.TS meteor., ge- }\end{array}$ & 1 \\
\hline continentale & el.loc.s.m. & ogr. & \\
\hline continentale & agg. & TS meteor., geogr. & 1 \\
\hline clima & loc.s.m. & $\begin{array}{l}\text { TS meteor., geor. } \\
\text { el.TS meteor., ge- }\end{array}$ & 1 \\
\hline temperato & el.loc.s.m. & ogr. & \\
\hline$k m^{2}$ & simb. & TS metrol. & 13 \\
\hline $\mathrm{km}$ & simb. & TS metrol. & 8 \\
\hline m) & simb. & TS metrol. & 7 \\
\hline$-70^{\circ} \mathrm{C}$ & simb. & TS metrol. & 1 \\
\hline Riyal & s.m. & TS monet. & 1 \\
\hline Dinar & s.m. & TS monet. & 1 \\
\hline lignite & s.f. & TS petrol. & 1 \\
\hline sciita & agg. & TS polit., relig. & 1 \\
\hline sunnita & agg. & TS relig. & 1 \\
\hline maronita & agg. & TS relig. & 1 \\
\hline druso & s.m. & TS relig. & 1 \\
\hline speranza & loc.s.f. & TS stat. & 1 \\
\hline$d i$ & el.loc.s.f. & el.TS stat. & \\
\hline vita & el.loc.s.f. & el.TS stat. & \\
\hline tasso & loc.s.m. & TS stat. & 1 \\
\hline$d i$ & el.loc.s.m. & el.TS stat. & \\
\hline mortalità & el.loc.s.m. & el.TS stat. & \\
\hline infantile & el.loc.s.m. & el.TS stat. & \\
\hline densità & s.f. & TS stat. & 1 \\
\hline sumero & agg. & TS stor. & 1 \\
\hline assiro-babilonese & agg. & TS stor. & 1 \\
\hline ittita & agg. & TS stor. & 1 \\
\hline fenicio & agg. & TS stor. & 1 \\
\hline greco & s.m. & TS stor. & 1 \\
\hline califfo & s.m. & TS stor. & 1 \\
\hline agglomerato & loc.s.m. & TS urban. & 1 \\
\hline urbano & el.loc.s.m. & el.TS urban. & \\
\hline centro & loc.s.m. & TS urban. & 1 \\
\hline urbano & el.loc.s.m. & el.TS urban. & \\
\hline
\end{tabular}




\begin{tabular}{lllr}
\hline Lemma & Wortart & TS-Bereich & Tokens \\
\hline cono & loc.s.m. & TS vulcanol. & 1 \\
vulcanico & el.loc.s.m. & el.TS vulcanol. & \\
zibellino & s.m. & TS zool.com. & 1 \\
acquicoltura & s.f. & TS zoot. & 1 \\
Types & & 106 Tokens & 173 \\
\hline
\end{tabular}

Tabelle 23: Fachwortschatz auf den untersuchten Seiten im Geograficamente (Dinucci \& Dinucci 2008)

Ambiente*: Durch die textkontextbedingte Zuteilung zu unterschiedlichen Fachwortschatzbereichen ist dieses Wort als zwei verschiedene Typen gezählt worden.

Continentale*: Das Adjektiv trägt die Marke des Phrasems clima continentale, da nur seine Bedeutung im Kontext (ibid., S. 242; m.Ü.: das Klima ist kontinental) passend ist.

*Nur eines der beiden Vorkommnisse lautet foresta pluviale tropicale, das andere foreste pluviali ohne den Zusatz tropicali.

\begin{tabular}{lrr}
\hline Fachsprachen/Wortarten & Tokens & Types \\
\hline Tot. TS ling. & 69 & 40 \\
Tot. TS geogr. & 18 & 16 \\
Tot. TS bot./bot.com./bot., zool., biol. & 7 & 6 \\
Tot. TS stor. & 6 & 6 \\
Tot. s. & 81 & 51 \\
Tot. loc.s. & 24 & 21 \\
Tot. agg. & 35 & 30 \\
Tot. simb. & 30 & 4 \\
\hline
\end{tabular}

Tabelle 24: Stärkste Ts-Bereiche und Zusammensetzung des Ts-Vorkommens nach Wortarten im Geograficamente (Dinucci \& Dinucci 2008)

Der Anteil aus dem Fachwortschatz ist auf den untersuchten Seiten im Lehrwerk Zaino in spalla (Bastianelli et al. 2010) etwas geringer als in den anderen untersuchten Büchern: Nur 54 verschiedene Worttypen sind insgesamt 80-mal vertreten. Um welche Ausdrücke es sich handelt, wie oft sie verwendet werden und welchen Bereichen des Ts-Wortschatzes sie entstammen, zeigt die nächste Tabelle (Tab. 25).

\begin{tabular}{lllr}
\hline Lemma & Wortart & TS-Bereich & Vorkommnisse \\
\hline antropico & agg. & TS antrop. & 1 \\
biogas & s.m. & TS biochim. & 1 \\
carta & loc.s.f. & TS cartogr. & 1 \\
tematica & el.loc.s.f. & el.TS cartogr. & 1 \\
carbonio & s.m. & TS chim. & 1 \\
combustibile & s.m. & TS chim. & 1 \\
Unione & loc.s.f. & TS dir. intern. & 2 \\
Europea & el.loc.s.f. & el.TS dir. intern. & 1 \\
fonte & loc.s.f. & TS ecol. & \\
energetica & el.loc.s.f. & el.TS ecol. & 3 \\
fonte & s.f. & TS ecol. & 2 \\
impatto & loc.s.m. & TS ecol. & \\
ambientale & el.loc.s.m. & el.TS ecol. & 1 \\
inquinamento & s.m. & TS ecol. & \\
\hline
\end{tabular}




\begin{tabular}{|c|c|c|c|}
\hline Lemma & Wortart & TS-Bereich & Vorkommnisse \\
\hline libero & loc.s.m. & TS econ. & 2 \\
\hline scambio & el.loc.s.m. & el.TS econ. & \\
\hline crescita & loc.s.f. & TS econ. & 2 \\
\hline economica & el.loc.s.f. & el.TS econ. & \\
\hline Prodotto & loc.s.m. & TS econ. & 1 \\
\hline interno & el.loc.s.m. & el.TS econ. & \\
\hline lordo & el.loc.s.m. & el.TS econ. & \\
\hline ricchezza & s.f. & TS econ. & 1 \\
\hline povertà & s.f. & TS econ. & 2 \\
\hline sostenibile & agg. & TS econ., ecol. & 1 \\
\hline sistema & loc.s.m. & TS econ., polit. & 1 \\
\hline economico & el.loc.s.m. & el.TS econ., polit. & \\
\hline globalizzazione & s.f. & TS econ., sociol. & 1 \\
\hline compatibile & agg. & TS econ., ecol. & 1 \\
\hline sviluppo & s.m. & TS econ., sociol. & 5 \\
\hline indocinese & agg. & TS etnol. & 1 \\
\hline energia & loc.s.f. & TS fis. & 1 \\
\hline elettrica & el.loc.s.f. & el.TS fis. & \\
\hline energia & loc.s.f. & TS fis. & 1 \\
\hline termica & el.loc.s.f. & el.TS fis. & \\
\hline positivo & agg. & TS fis., chim. & 2 \\
\hline valore & s.m. & TS fis., mat. & 1 \\
\hline sub-sahariano & pref.+agg. & TS geogr. & 1 \\
\hline area & s.f. & TS geogr. & 5 \\
\hline tavolato & s.m. & TS geogr. & 1 \\
\hline indogangetico & agg. & TS geogr. & 1 \\
\hline spartiacque & s.m. & TS geogr. & 1 \\
\hline latitudine & s.f. & TS geogr. & 1 \\
\hline Circolo & loc.s.m. & TS geogr. & 1 \\
\hline Polare & el.loc.s.m. & el.TS geogr. & \\
\hline Artico & el.loc.s.m. & el.TS geogr. & \\
\hline Equatore & s.m. & TS geogr. & 1 \\
\hline pianura & loc.s.f. & TS geol. & 1 \\
\hline alluvionale & el.loc.s.f. & el.TS geol. & \\
\hline spagnolo & s.m. & TS ling. & 3 \\
\hline russo & s.m. & TS ling. & 2 \\
\hline sanscrito & agg. & TS ling. & 1 \\
\hline hindi & s.m. & TS ling. & 2 \\
\hline indiano & s.m. & TS ling. & 1 \\
\hline arabo & s.m. & TS ling. & 3 \\
\hline portoghese & s.m. & TS ling. & 2 \\
\hline francese & s.m. & TS ling. & 1 \\
\hline cinese & s.m. & TS ling. & 1 \\
\hline $\mathrm{km}^{2}$ & simb. & TS metrol. & 1 \\
\hline fossile & agg. & TS paleont. & 1 \\
\hline $\log o$ & s.m. & TS pubbl. & 1 \\
\hline negativo & agg. & TS scient. & 1 \\
\hline tasso & loc.s.m. & TS stat. & 1 \\
\hline$d i$ & el.loc.s.m. & el.TS stat. & \\
\hline mortalità & el.loc.s.m. & el.TS stat. & \\
\hline
\end{tabular}




\begin{tabular}{lllr}
\hline Lemma & Wortart & TS-Bereich & Vorkommnisse \\
\hline tasso & loc.s.m. & TS stat. & 1 \\
di & el.loc.s.m. & el.TS stat. & \\
natalità & el.loc.s.m. & el.TS stat. & 3 \\
saldo & loc.s.m. & TS stat. & \\
naturale & el.loc.s.m. & el.TS stat. & 1 \\
lingua & loc.s.f. & TS stor. & \\
franca & el.loc.s.f. & el.TS stor. & 1 \\
macchina & loc.s.f. & TS tecn. & 2 \\
utensili & el.loc.s.f. & el.TS tecn. & 80 \\
cogenerazione & s.f. & TS tecn. & \\
Tot. Types & & Tot. Tokens & \\
\hline
\end{tabular}

Tabelle 25: Fachwortschatz auf den untersuchten Seiten im Zaino in spalla (Bastianelli et al. 2010)

*Fonte (Quelle): Für fonti ist die Marke Ts ecol. des Phrasems fonte energetica (Energiequellen) gewählt worden, da energetiche impliziert ist und diese Gebrauchsmarke somit am treffendsten ist. Allerdings ist das Wort als einfaches Nomen und nicht als ein Phrasem gewertet worden.

*Lingua franca: Dieser Ausdruck trägt die Gebrauchsmarke Ts stor., da er im GRADIT nur geschichtlich definiert ist als ,[lingua] costituita dal lessico italiano e spagnolo con alcune voci arabe, usato in passato per i rapporti commerciali tra Europei e Islamici“" (m.Ü.: aus dem italienischen und spanischen Wortschatz zusammengesetzt[e] [Sprache] mit einigen arabischen Wendungen, die in der Vergangenheit für die Handelsbeziehungen zwischen Europäern und Islamischen genutzt worden ist). Leider gibt es keine Informationen zum Gebrauch der Bedeutungserweiterung des Phrasems für eine Sprache, die heute im internationalen wirtschaftlichen Austausch benutzt wird, und auch in den wichtigsten zweisprachigen deutsch-italienischen Wörterbüchern hat lingua franca keinen Eintrag.

Wie aus Tabelle 26 ersichtlich wird, setzten sich die Fachwortschatzwörter auch in diesem Unterrichtswerk zum größten Teil aus Nomen zusammen: 35 nominale ein- und mehrteilige Worttypen sind mit 48 Vorkommnissen vertreten und machen damit 80 Prozent am Gesamtvorkommen der Ts-gekennzeichneten Typen aus. Bei den restlichen Ausdrücken handelt es sich mit Ausnahme eines Symbols nur um Adjektive. Auf den untersuchten Seiten gibt es nur zwei Verben, die zum Fachwortschatz gezählt werden (die bereits vorgestellten im Lehrwerk von Bersezio (2005)).

Genau wie im Lehrwerk der Klasse III B ist der linguistische Fachwortschatzbereich der am stärksten vertretene, was am gemeinsamen Inhalt zu den Sprachen und ihrer weltweiten Verteilung liegt. Erneut handelt es sich bei den Worttypen um die Bezeichnungen für bestimmte Sprachen (hier sogar ausschließlich), wovon den Lernenden die meisten problemlos geläufig sein sollten (vgl. Tabelle 15). Die anderen Wortschatzbereiche, welche nach dem linguistischen die größten Anteile auf diesen Seiten haben, sind wieder der wirtschaftliche (allerdings hier inklusive doppelter Bereichszuteilungen), der geographische und der ökologische. 


\begin{tabular}{lrr}
\hline Fachsprachen/Wortarten & Tokens & Types \\
\hline Tot. TS ling. & 16 & 9 \\
Tot. TS econ./econ., ecol./econ., sociol./econ., polit. & 12 & 10 \\
Tot. TS geogr. & 11 & 7 \\
Tot. TS ecol. & 7 & 4 \\
Tot. s. (ohne loc.) & 44 & 25 \\
Tot. loc.s. & 23 & 17 \\
Tot. agg. & 11 & 10 \\
Tot. simb. & 1 & 1 \\
\hline
\end{tabular}

Tabelle 26: Stärkste Ts-Bereiche und Zusammensetzung des Ts-Vorkommens nach Wortarten im Zaino in spalla (Bastianelli et al. 2010)

Zuletzt soll nun noch der rein fachwortschatzsprachliche Teil der analysierten Seiten aus dem Lehrwerk Nuovo Geolab (Carazzi \& Pizzetti 2010) vorgestellt werden, das an der Schule Vespucci verwendet wurde. Das sind 48 verschiedene Worttypen, die auf den untersuchten Seiten insgesamt 107-mal vorkommen, wie Tabelle 27 zeigt.

\begin{tabular}{|c|c|c|c|}
\hline Wort & Wortart & TS-Bereich & Tokens \\
\hline Arativo & s.m. & TS agr. & 1 \\
\hline Cancro & s.m. & TS astrol. & 1 \\
\hline caffè & s.m. & TS bot.com. & 1 \\
\hline canna & loc.s.f. & TS bot.com. & 1 \\
\hline$d a$ & el.loc.s.f. & el.TS bot.com. & \\
\hline zucchero & el.loc.s.f. & el.TS bot.com. & \\
\hline tè & s.m. & TS bot.com. & 1 \\
\hline combustibile & s.m. & TS chim. & 1 \\
\hline gas & loc.s.m. & TS chim. & 4 \\
\hline naturale & el.loc.s.m. & el.TS chim. & \\
\hline plutonio & s.m. & TS chim. & 1 \\
\hline uranio & s.m. & TS chim. & 2 \\
\hline fonte & loc.s.f. & TS ecol. & 1 \\
\hline$d i$ & el.loc.s.f. & el.TS ecol. & \\
\hline energia & el.loc.s.f. & el.TS ecol. & \\
\hline bilancia & loc.s.f. & TS econ. & 1 \\
\hline commerciale & el.loc.s.f. & el.TS econ. & \\
\hline indicatore & s.m. & TS econ. & 9 \\
\hline mercato & loc.s.m. & TS econ. & 1 \\
\hline interno & el.loc.s.m. & el.TS econ. & \\
\hline passivo & agg. & TS econ. & 1 \\
\hline povertà & s.f. & TS econ. & 6 \\
\hline prodotto & loc.s.m. & TS econ. & 1 \\
\hline interno & el.loc.s.m. & el.TS econ. & \\
\hline lordo & el.loc.sm. & el.TS econ. & \\
\hline reddito & s.m. & TS econ. & 2 \\
\hline ricchezza & s.f. & TS econ. & 11 \\
\hline settore & loc.s.m. & TS econ. & 1 \\
\hline primario & el.loc.s.m. & el.TS econ. & \\
\hline settore & loc.s.m. & TS econ. & 1 \\
\hline secondario & el.loc.s.m. & el.TS econ. & \\
\hline
\end{tabular}




\begin{tabular}{|c|c|c|c|}
\hline Wort & Wortart & TS-Bereich & Tokens \\
\hline settore & loc.s.m. & TS econ. & 1 \\
\hline terziario & el.loc.s.m. & el.TS econ. & \\
\hline valuta & s.f. & TS econ. & 1 \\
\hline sviluppo & s.m. & TS econ., sociol. & 4 \\
\hline energia & loc.s.f. & TS fis. & 4 \\
\hline nucleare & el.loc.s.f. & el.TS fis. & \\
\hline energia & loc.s.f. & TS fis. & 1 \\
\hline elettrica & el.loc.s.f. & el.TS fis. & \\
\hline trico & agg. & TS fis. & 1 \\
\hline valore & s.m. & TS fis., mat. & 2 \\
\hline area & s.f. & TS geogr. & 10 \\
\hline emisfero & s.m. & TS geogr. & 1 \\
\hline geografia & loc.s.f. & TS geogr. & 1 \\
\hline umana & el.loc.s.f. & el.TS geogr. & \\
\hline Tropico & s.m. & TS geogr. & 1 \\
\hline capo & s.m. & TS geogr. & 1 \\
\hline valle & s.f. & TS geogr. & 1 \\
\hline insediamento & s.m. & TS geogr., antrop. & 1 \\
\hline clima & loc.s.m. & TS meteor., geogr. & 1 \\
\hline mediterraneo & el.loc.s.m. & el.TS meteor., geogr. & \\
\hline $\mathrm{km}$ & simb. & TS metrol. & 2 \\
\hline minerario & agg. & TS miner. & 1 \\
\hline forza & loc.s.f. & TS polit., econ. & 2 \\
\hline lavoro & el.loc.s.f. & el.TS polit., econ. & \\
\hline gruppo & loc.s.m. & TS sociol. & 1 \\
\hline etnico & el.loc.s.m. & el.TS sociol. & \\
\hline densità & s.f. & TS stat. & 1 \\
\hline graduatoria & s.f. & TS stat. & 3 \\
\hline indice & s.m. & TS stat. & 5 \\
\hline mortalità & loc.s.f. & TS stat. & 6 \\
\hline infantile & el.loc.s.f. & el.TS stat. & \\
\hline speranza & loc.s.f. & TS stat. & 2 \\
\hline di & el.loc.s.f. & el.TS stat. & \\
\hline vita & el.loc.s.f. & el.TS stat. & \\
\hline Indice & loc.s.m. & TS stat., polit. & 2 \\
\hline$d i$ & el.loc.s.m. & el.TS stat., polit. & \\
\hline Sviluppo & el.loc.s.m. & el.TS stat., polit. & \\
\hline Umano & el.loc.s.m. & el.TS stat., polit. & \\
\hline pannello & loc.s.m. & TS tecn. & 1 \\
\hline solare & el.loc.s.m. & el.TS tecn. & \\
\hline centro & loc.s.m. & TS urban. & 1 \\
\hline urbano & el.loc.s.m. & el.TS urban. & \\
\hline $\begin{array}{l}\text { agglomerato } \\
\text { urbano }\end{array}$ & $\begin{array}{l}\text { loc.s.m. } \\
\text { el.loc.s.m. }\end{array}$ & $\begin{array}{l}\text { TS urban. } \\
\text { el.TS urban. }\end{array}$ & 1 \\
\hline Tot. Types & 48 & Tot. Tokens & 107 \\
\hline
\end{tabular}

Tabelle 27: Fachwortschatz auf den untersuchten Seiten im Nuovo Geolab (Carazzi \& Pizzetti 2010) 
In diesem Lehrwerk befinden sich unter den Ts-gekennzeichneten Wörtern die meisten Substantive, nämlich abgesehen von drei Adjektiven und zwei Symbolen das gesamte fachwortschatzsprachliche Wortvorkommen (92\% der Worttypen und $95 \%$ der Vorkommnisse). Es besteht fast zu gleichen Teilen aus Phraseologismen und einfachen Nomen (21 zu 23), wobei die Anzahl der einteiligen Wortvorkommnisse fast doppelt so hoch ist wie die der Phraseologismen.

\begin{tabular}{lrr}
\hline Fachsprachen/Wortarten & Tokens & Types \\
\hline Tot. TS econ./econ., sociol. & 39 & 12 \\
Tot. TS stat./stat., polit. & 19 & 6 \\
Tot. TS geogr./geogr., antrop. & 16 & 7 \\
Tot. TS chim. & 9 & 5 \\
Tot. s. (ohne loc.) & 67 & 23 \\
Tot. loc.s. & 35 & 21 \\
Tot. agg. & 3 & 3 \\
Tot. simb. & 2 & 1 \\
\hline
\end{tabular}

Tabelle 28: Stärkste Ts-Bereiche und Zusammensetzung des Ts-Vorkommens nach Wortarten im Nuovo Geolab (Carazzi \& Pizzetti 2010)

\subsubsection{Gemeinsamkeiten des reinen Fachwortschatzes in den verschiedenen Lehrbüchern}

Eine wesentliche Gemeinsamkeit der Zusammensetzung des rein fachwortschatzsprachlichen Anteils in allen untersuchten Erdkundebüchern besteht also in der großen Anzahl an Substantiven. Um einen Eindruck davon zu gewinnen, wie oft die verschiedenen Ts-Nomen in den Texten vorkommen und welche von ihnen in allen oder mehreren Büchern auftreten, werden im Folgenden die Ergebnisse für die Substantive aus den Fachbereichen Geographie und Wirtschaft vorgestellt. Diese Fachwortschatzfelder sind ausgewählt worden, weil sich beide als einzige auf den untersuchten Seiten aller fünf Bücher befinden. ${ }^{130}$

\begin{tabular}{llrr}
\hline Klasse & Lehrbuch & TS geogr. & TS econ. \\
\hline Vespucci & Carazzi \& Pizzetti (2010) & $7(16)$ & $12(39)$ \\
Morante & Bersezio (2005) & $8(21)$ & $4(6)$ \\
IIIA & Bastianelli (2010) & $6(10)$ & $8(15)$ \\
III B & Dinucci \& Dinucci (2008) & $10(15)$ & $1(1)$ \\
III C & Forte \& Ubertazzi (2008) & $6(7)$ & $5(6)$ \\
Tot. Tokens & & 69 & 67 \\
\hline
\end{tabular}

Tabelle 29: Geographischer und wirtschaftlicher Fachwortschatz aller untersuchten Seiten

130 Dieses Ergebnis war aufgrund der Textauswahl zu erwarten, da sie sich in erster Linie auf die Verwendung der Seiten stützte, aus denen die C-Tests entwickelt wurden, und darauf, dass aus jedem Buch sowohl landeskundliche als auch sozioökonomische Texte vertreten sind. Es sei daran erinnert, dass die C-Tests mit Texten entwickelt wurden, die kurz vorher im Unterricht behandelt wurden, so dass es sich zum Teil um sehr unterschiedliche Themen handelt. Um auch für seltener vertretene Texte einen Vergleichspunkt zu haben, wurde außerdem zu jedem Thema aus einem zweiten Lehrwerk ein Text gewählt, der es behandelt. 
Es sind in Tabelle 30 zunächst die Bergriffe aus dem geographischen Fachwortschatz aufgelistet, wobei für jeden Worttyp die deutsche Übersetzung gegeben und seine Vorkommnisse nach Klassen geordnet festgehalten sind.

Anhand der Tabelle lässt sich erkennen, dass die meisten Wörter auf den untersuchten Seiten nur in einem Erdkundebuch vorkommen, einige wenige in mehreren und nur eines in allen fünf Lehrwerken. Hierbei handelt es sich um den Ausdruck area, dessen fachwortschatzsprachliche Bedeutung semantisch sehr nah bei seiner Grundbedeutung liegt, so dass die Lernenden den Begriff wahrscheinlich verstehen und auch korrekt verwenden können, sofern sie die Grundbedeutung kennen. Area ist nicht nur der einzige fachsprachliche Ausdruck, der in allen Büchern vertreten ist, sondern er verzeichnet auch die meisten Vorkommnisse (18).

Dieses Ergebnis der so unterschiedlichen Streuung der Ausdrücke aus den Fachsprachen auf den untersuchten Seiten sowie die weiter oben vorgestellte Tabelle zum kompletten fachwortschatzsprachlich markierten Wortvorkommen der fünf Lehrbücher deuten darauf hin, dass sich die Verwendung des Fachwortschatzes in den Lehrwerken insgesamt sehr stark diversifiziert. Dieser Umstand kann der Tatsache zugeschrieben werden, dass in den Lehrplänen die Wortschatzarbeit zwar für alle Fächer vorgeschrieben ist, aber nicht genauer festgelegt wird, um welchen Wortschatz es sich dabei handeln sollte (MIUR 2012b, S. 30). Eine Konsequenz daraus ist die scheinbar recht willkürliche Wortwahl in den einzelnen Lehrbüchern ein und desselben Schulfachs.

\begin{tabular}{|c|c|c|c|c|c|c|c|}
\hline Lemma & m.Ü. & $\begin{array}{r}\text { Territori } \\
\text { dell'uomo }\end{array}$ & Geolab & $\begin{array}{r}\text { Zaino in } \\
\text { spalla }\end{array}$ & $\begin{array}{r}\text { Geogra- } \\
\text { fica- } \\
\text { mente }\end{array}$ & Geolibro & $\begin{array}{r}\text { To- } \\
\text { kens }\end{array}$ \\
\hline & & (Morante) & (Vespucci) & (III A) & (III B) & (III C) & \\
\hline area & $\begin{array}{l}\text { Gebiet, in dem } \\
\text { bestimmte } \\
\text { Phänomene } \\
\text { auftreten }\end{array}$ & 3 & 10 & 5 & 2 & 1 & 21 \\
\hline articolato & $\begin{array}{l}\text { nicht geradlini- } \\
\text { ger Verlauf } \\
\text { der Küsten } \\
\text { (durch Buch- } \\
\text { ten u.Ä.) }\end{array}$ & 1 & & & 1 & 1 & 3 \\
\hline capo & Kap & 2 & & & & & 2 \\
\hline $\begin{array}{l}\text { catena } \\
\text { montuosa }\end{array}$ & Bergkette & 1 & & & & & 1 \\
\hline $\begin{array}{l}\text { cintura di } \\
\text { fuoco }\end{array}$ & Feuergürtel & 1 & & & & & 1 \\
\hline $\begin{array}{l}\text { circolo po- } \\
\text { lare artico }\end{array}$ & $\begin{array}{l}\text { arktischer Po- } \\
\text { larkreis }\end{array}$ & & & 1 & & & 1 \\
\hline delta & Delta & 4 & & & & & 4 \\
\hline dorsale & $\begin{array}{l}\text { Höhen-, Berg- } \\
\text { rücken }\end{array}$ & & & & & 2 & 2 \\
\hline emisfero & Hemisphäre & & 1 & & & 1 & 2 \\
\hline equatore & Äquator & & & 1 & 1 & 1 & 3 \\
\hline $\begin{array}{l}\text { foresta bo- } \\
\text { reale* }\end{array}$ & $\begin{array}{l}\text { borealer /nor- } \\
\text { discher Wald }\end{array}$ & & & & 1 & & 1 \\
\hline
\end{tabular}




\begin{tabular}{|c|c|c|c|c|c|c|c|}
\hline Lemma & m.Ü. & $\begin{array}{r}\text { Territori } \\
\text { dell'uomo }\end{array}$ & Geolab & $\begin{array}{r}\text { Zaino in } \\
\text { spalla }\end{array}$ & $\begin{array}{r}\text { Geogra- } \\
\text { fica- } \\
\text { mente }\end{array}$ & Geolibro & $\begin{array}{r}\text { To- } \\
\text { kens }\end{array}$ \\
\hline $\begin{array}{l}\text { foresta } \\
\text { temperata* }\end{array}$ & $\begin{array}{l}\text { "gemäßigter" } \\
\text { Wald }\end{array}$ & & & & 1 & & 1 \\
\hline $\begin{array}{l}\text { foresta } \\
\text { pluviale }\end{array}$ & Regenwald & & & & 2 & & 2 \\
\hline $\begin{array}{l}\text { geografia } \\
\text { umana }\end{array}$ & $\begin{array}{l}\text { Humangeo- } \\
\text { graphie }\end{array}$ & & 1 & & & & 1 \\
\hline $\begin{array}{l}\text { insedia- } \\
\text { mento }\end{array}$ & Siedlung & & 1 & & & & 1 \\
\hline idrografia & Hydrographie & & & & 1 & & 1 \\
\hline latitudine & Breite & & & 1 & & & 1 \\
\hline $\begin{array}{l}\text { permafrost } \\
\text { (ES TS) }\end{array}$ & Permafrost & & & & 1 & & 1 \\
\hline $\begin{array}{l}\text { polo del } \\
\text { freddo* }\end{array}$ & Kältepol & & & & 1 & & 1 \\
\hline $\begin{array}{l}\text { spartiac- } \\
\text { que }\end{array}$ & $\begin{array}{l}\text { Wasser- } \\
\text { scheide }\end{array}$ & & & 1 & & & 1 \\
\hline struttura & $\begin{array}{l}\text { eine be- } \\
\text { stimmte Form, } \\
\text { die für eine } \\
\text { Gegend kenn- } \\
\text { zeichnend ist }\end{array}$ & & & & & 1 & 1 \\
\hline tavolato & Hochebene & 1 & & 1 & 1 & 1 & 4 \\
\hline tropico & Wendekreis & 3 & 1 & & & & 4 \\
\hline valle & Tal & 6 & 1 & & & & 7 \\
\hline versante & Hang & 1 & & & 4 & & 5 \\
\hline Tokens & & & & & & & 72 \\
\hline
\end{tabular}

Tabelle 30: Geographischer Fachwortschatz und seine Vorkommnisse auf allen untersuchen Lehrbuchseiten

*Foresta boreale, *foresta temperata, *polo del freddo: außer im GRADIT weder in ein- noch in zweisprachigen deutsch-italienischen Wörterbüchern gefunden. Begriffserklärungen im GRADIT für foresta boreale: , $f$. dei climi temperati, formata spec. da latifoglie e aghifoglie“ (m.Ü.: Wald der gemäßigten Klimazone, vor allem aus Laub- und Nadelbäumen bestehend) und für foresta temperata: ,f. dalla vegetazione simile a quella tropicale ma con piante caduche o sempreverdi con foglie larghe o aghiforme" (m.Ü.: Wald von ähnlicher Vegetation wie der tropische Wald, aber mit Laub- oder immergrünen Pflanzen mit breiten oder nadelförmigen Blättern). Erklärung für polo del freddo: ,località della Terra abitata dove si registrano le temperature invernali più basse" (m.Ü.: bewohnter Ort mit der weltweit niedrigsten Temperatur im Winter).

Die zweitgrößte Anzahl an Vorkommnissen verzeichnet der Begriff valle (7 Tokens), welcher auf den untersuchten Seiten nur in zwei Lehrbüchern erscheint, und an dritter Stelle folgt mit fünf Vorkommnissen das ebenfalls nur in zwei Büchern gebrauchte versante. Weniger oft, aber dafür in vier Büchern, ist das Wort tavolato vertreten (je mit einem Token pro Lehrwerk), und in drei Büchern tritt außerdem der Ausdruck equatore auf.

Auch bei den Nomen aus dem wirtschaftlichen Fachwortschatz ist es so, dass knapp die Hälfte nur ein einziges Mal auftritt (vgl. Tabelle 25). Aus diesem Bereich des Fachwortschatzes stammt kein Substantiv, das auf den untersuchten Seiten aller fünf Erdkundebücher benutzt ist. Maximal beläuft sich die 
Streuung auf die beiden Fälle von insgesamt 13 Vorkommnissen in drei Büchern für den Begriff ricchezza sowie insgesamt neun in drei Büchern für povertà und in zweien für sviluppo. Die meisten Vorkommnisse in ein und demselben Text gibt es dabei für die Wörter ricchezza (11), indicatore (9) und povertà (6) (Nuovo Geolab).

\begin{tabular}{|c|c|c|c|c|c|c|c|}
\hline Lemma & Übersetzung & $\begin{array}{r}\text { Territori } \\
\text { dell'uomo }\end{array}$ & $\begin{array}{l}\text { Nuovo } \\
\text { Geolab }\end{array}$ & $\begin{array}{r}\text { Zaino in } \\
\text { spalla }\end{array}$ & $\begin{array}{r}\text { Geografica- } \\
\text { mente }\end{array}$ & $\begin{array}{r}\text { Geoli- } \\
\text { bro }\end{array}$ & $\begin{array}{r}\text { To- } \\
\text { kens }\end{array}$ \\
\hline & & (Morante) & $\begin{array}{r}\text { (Ve- } \\
\text { spucci) }\end{array}$ & (III A) & (III B) & (III C) & \\
\hline $\begin{array}{l}\text { bilancia } \\
\text { commer- } \\
\text { ciale }\end{array}$ & $\begin{array}{l}\text { Handelsbi- } \\
\text { lanz }\end{array}$ & 1 & 1 & & & & 2 \\
\hline compagnia & Gesellschaft & & & & & 1 & 1 \\
\hline consumo & Konsum & & & & & 1 & 1 \\
\hline $\begin{array}{l}\text { crescita } \\
\text { economica }\end{array}$ & $\begin{array}{l}\text { Wirtschafts- } \\
\text { wachstum }\end{array}$ & & & 2 & & & 2 \\
\hline crisi & Krise & & & & & 1 & 1 \\
\hline $\begin{array}{l}\text { globalizza- } \\
\text { zione }\end{array}$ & $\begin{array}{l}\text { Globalisie- } \\
\text { rung }\end{array}$ & & & 1 & & & 1 \\
\hline indicatore & Indikator & & 9 & & & & 9 \\
\hline $\begin{array}{l}\text { libero } \\
\text { scambio }\end{array}$ & $\begin{array}{l}\text { freier Han- } \\
\text { del }\end{array}$ & & & 2 & & & 2 \\
\hline $\begin{array}{l}\text { mercato in- } \\
\text { terno }\end{array}$ & Binnenmarkt & & 1 & & & & 1 \\
\hline povertà & Armut & & 6 & 2 & 1 & & 9 \\
\hline $\begin{array}{l}\text { prodotto in- } \\
\text { terno lordo }\end{array}$ & $\begin{array}{l}\text { Bruttoin- } \\
\text { landspro- } \\
\text { dukt }\end{array}$ & & 1 & & & 1 & 2 \\
\hline reddito & Einkommen & & 2 & 1 & & & 3 \\
\hline ricchezza & Wohlstand & & 11 & 1 & & 1 & 13 \\
\hline $\begin{array}{l}\text { settore pri- } \\
\text { mario }\end{array}$ & Primärsektor & 1 & 1 & & & & 2 \\
\hline $\begin{array}{l}\text { settore se- } \\
\text { condario }\end{array}$ & $\begin{array}{l}\text { Sekun- } \\
\text { därsektor }\end{array}$ & & 1 & & & & 1 \\
\hline $\begin{array}{l}\text { settore ter- } \\
\text { ziario }\end{array}$ & Tertiärsektor & & 1 & & & & 1 \\
\hline $\begin{array}{l}\text { sistema } \\
\text { economico }\end{array}$ & $\begin{array}{l}\text { Wirtschafts- } \\
\text { system }\end{array}$ & & & 1 & & & 1 \\
\hline sviluppo & Entwicklung & & 4 & 5 & & & 9 \\
\hline terziario & Tertiärsektor & 3 & & & & 2 & 5 \\
\hline valuta & Währung & & 1 & & & & $\begin{array}{r}1 \\
67\end{array}$ \\
\hline
\end{tabular}

Tabelle 31: Wirtschaftlicher Fachwortschatz und seine Vorkommnisse auf allen untersuchen Lehrbuchseiten

Zwar kommen die meisten fachwortschatzsprachlichen Substantive aus den Bereichen Erdkunde und Wirtschaft auf den untersuchten Seiten nur je in einem der Lehrwerke vor, aber es ist zu vermuten, dass ihre Streuung zunimmt, wenn man eine größere Textauswahl oder den Gesamtumfang der Lehrwerke zur Analyse heranzieht. Dennoch bleibt die Tendenz der Lehrbuchautoren erkennbar, den niedrigfrequenteren Wortschatz nicht oder kaum wiederholt zu verwenden und damit eine pädagogische Prämisse der Wortschatzdidaktik un- 
beachtet zu lassen. Aus der Forschung ist nämlich bekannt, dass es einer Vielzahl von Begegnungen mit einem Wort bedarf, damit ihm bezüglich lexikalische Kompetenz erworben werden kann (Nation 1990, S. 44), und dass hierfür eine zyklische Wiederholung ratsam ist (Willis \& Ohashi 2012, S. 134).

Was diese exemplarische Untersuchung eines Teils des Fachwortschatzes jedoch verdeutlicht haben sollte, ist, dass es sich um eine überschaubare Anzahl von Lemmata handelt, welche für die Lernenden eine sprachliche Herausforderung darstellen können. Diese sollte bisweilen nicht überschätzt werden, da sie sich vermutlich in Grenzen hält.

Da gibt es zum einen Fachwörter wie die Bezeichnungen der Sprachen, die vielleicht auch zum Basiswortschatz gezählt werden können und den meisten SchülerInnen geläufig sind. Zum anderen beschreiben die Fachwörter Konzepte wie beispielsweise Handelsbilanz, Bruttoinlandsprodukt oder Wendekreis, die unabhängig von der für sie verwendeten Bezeichnung gelernt und verstanden werden müssen.

$\mathrm{Ob}$ das Verständnis gesichert ist und wie leicht es fällt, einen neuen Begriff aus dem Fachwortschatz zu lernen, hängt stark davon ab, in was für einem sprachlichen Kontext er präsentiert wird: Wird er ausdrücklich erklärt? Wie wird er erklärt? Wird er erneut erklärt, wenn die Einführung bereits vor längerer Zeit erfolgte? Falls eine Definition gegeben wird, entstammen die verwendeten Wörter zur Begriffserklärung dem Basiswortschatz? Denn dann ist eher garantiert, dass der Begriff verstanden wird (vgl. Piemontese 2005, S. 389 f.). An einem konkreten Beispiel wurde im Abschnitt zu den hervorgehobenen Wörtern bereits gezeigt, dass sich das sprachliche Umfeld als sehr komplex erweisen kann (s. lingua madre mit vier satzwertigen Partizipien, darunter zudem Partizipien Präsens, und einem konjugierten Verb im Passiv).

Ferner geht aus den Analyseergebnissen hervor, dass der Anteil des fundamentalen Wortschatzes in allen fünf Lehrbüchern insgesamt verhältnismäßig niedrig, die lexikalische Dichte sehr hoch und die Sätze meist lang sind, was nicht dafürspricht, dass die fachsprachlichen Wörter in Kontexten verwendet werden, für deren Verständnis eine geringe bildungssprachliche Kompetenz ausreichend ist oder gar überhaupt nicht vonnöten wäre. Die folgenden drei Kapitel zeigen an diversen Stellen detailliert, wie sich Verständnisschwierigkeiten sowie eine defizitäre Sprachproduktion vor allem dann ergeben, wenn ein niedrigfrequenter Wortschatz mit komplexeren morphosyntaktischen Strukturen korreliert. 


\section{Ergebnisse des C-Tests}

C-Tests werden in erster Linie dazu eingesetzt, die sprachlichen Fähigkeiten in einer Fremd- oder Zweitsprache in Form eines Screenings zu erfassen, wie es beispielsweise dienlich ist, um zu Beginn eines Sprachkurses die Interessenten in Lerngruppen verschiedener Leistungsniveaus einzuteilen. Dabei wird die Validität eines Tests zuvor anhand erwachsener muttersprachlicher Testpersonen überprüft, die in der Lage sein sollten, mindestens 95 Prozent der Lücken korrekt zu vervollständigen sollten (Raatz \& Klein-Braley 1985, S. 21). Erwartungswidrig liegen die Ergebnisse in dieser für die meisten Teilnehmer muttersprachlichen Erhebung deutlich unter dem angestrebten Wert von 95 Prozent an richtigen Lösungen. ${ }^{131} \mathrm{Da}$ die Texte, aus denen die C-Tests entwickelt wurden, den Lehrwerken entstammen, sagen sie aber vermutlich etwas über die Distanz des verwendeten sprachlichen Registers zum Sprachrepertoire der Lernenden aus und werfen die Frage auf, ob die sprachliche Gestaltung der Lehrbücher dem kognitiven und sprachlichen Entwicklungsstand der Lernenden angemessen ist. Auch wenn es natürlich ein Anliegen der Schule bleibt, auch in den nichtsprachlichen Fächern Sprachbildung zu betreiben und die SchülerInnen Stück für Stück mit dem Gebrauch der Bildungssprache vertraut zu machen. Der geübte Umgang mit dem bildungssprachlichen Register der Lehrwerke ist, wie zuvor schon bemerkt, von hoher Bedeutung für das Erringen guter schulischer Leistungen im Fach Erdkunde, da sich ein Großteil des Unterrichts auf das Erfassen bzw. die Wiedergabe der Lehrbuchtexte beschränkt und alles fachinhaltliche Lernen immer in erster Linie über das Medium Sprache erfolgt.

In diesem Kapitel wird zunächst das Testdesign beschrieben (8.1). Danach erfolgt eine Übersicht über die Ergebnisse der Testbearbeitung in den einzelnen Klassen (8.2), die abschließend in einer ersten Gegenüberstellung mit totalen und prozentualen Fehlerquoten beschrieben werden (8.2.1). Die Unterkapitel 8.3.1, 8.3.2, 8.3.3 und 8.3.4 befassen sich Klasse für Klasse mit einer Fehleranalyse, die das Sprachmaterial der einzelnen Tests sowie die Lösungsvorschläge der Lernenden punktuell untersucht. Hierbei soll auch geprüft werden, wie die Testbearbeitungen mit der unterschiedlichen Textdichte, Wortschatzzusammensetzung, Satzlänge usw. in den Lehrwerken korrelieren. Dabei werden Aspekte, die im Zuge der Lehrbuchanalyse behandelt worden sind, wiederaufgenommen. Die Ergebnisse der einzelnen C-Tests werden danach

131 Maximal finden sich 92 Prozent an richtigen Lösungen, aber durchschnittlich nur 80 Prozent. Auch Grotjahn, Tönshoff und Hohenbleicher (1994, S. 131 f.) haben bei eigenen Untersuchungen mit italienischen C-Tests geringere Werte verzeichnet und, mit Verweis auf ähnliche Umstände in Pilotstudien für das Spanische, darauf hingewiesen, dass der angestrebte Prozentwert der für Muttersprachler ,relativ schwierigen“ Texte (womit eigentlich nicht Geographiebücher für Achtklässler gemeint sein dürften) eventuell herabgesetzt werden sollte. 
unterschieden, ob es sich um zu ergänzende Ausdrücke handelt, die von einigen wenigen Lernenden nicht richtig bearbeitet wurden, oder ob es ich um vermehrt auftretende Falschlösungen handelt. Abschließend werden die sprachlichen Phänomene, welche hauptsächlich zu vermehrten Falschlösungen führten, und ihre möglichen Ursachen klassenübergreifend zusammengefasst (8.4).

\subsection{Das Testdesign}

Der Einsatz von C-Tests hat in den letzten Jahren zugenommen; neben einer Ausdehnung seiner Anwendung auf immer mehr Sprachen wird er auch in Studien zum Bildungsmonitoring verwendet. ${ }^{132}$ Dabei geht es längst nicht mehr nur um die Messung allgemeinsprachlicher Fähigkeiten in einer Fremd- oder Zweitsprache, sondern es werden auch bestimmte Sprachregister getestet, wie beispielsweise Kenntnisse in wirtschaftssprachlichem Deutsch (vgl. Grotjahn 2006). Darüber hinaus findet er im schulischen Rahmen sowohl in der empirischen Unterrichtsforschung (s. Ahrenholz 2013) als auch vereinzelt im Unterricht Anwendung, um die fachsprachlichen, inhaltlichen und allgemeinprachlichen Fähigkeiten von SchülerInnen in der Erst- und in der Zweitsprache zu ermitteln (vgl. Baur \& Spettmann 2007; Baur, Mashkovskaya \& Spettmann 2009; Harsch \& Schröder 2014 ${ }^{133}$ ).

Mit einem C-Test werden ganz verschiedene, miteinander verknüpfte Aspekte der Sprachkompetenz überprüft, da durch das Worttilgungsprinzip die Textredundanz verringert wird und für das richtige Ausfüllen nicht nur der unmittelbare Kontext (beispielsweise das Satzglied, zu dem das getilgte Wort gehört), sondern oftmals auch das weitere sprachliche Umfeld wie das gesamte Satzgefüge und angrenzende Sätze von Bedeutung ist. So kann es etwa sein, dass zur korrekten Vervollständigung eines Pronomens ein Bezugswort ermittelt werden muss, das sich in den vorausgehenden Sätzen befindet. Kontrolliert werden also zum einen lexikogrammatikalische und morphosyntaktische Fertigkeiten und zum anderen auch Lesefähigkeit und Textverständnis. Jedoch ist der C-Test kein Instrument, das eine detaillierte Sprachstandsanalyse ersetzen

132 Z.B. in DESI (Deutsch Englisch Schülerleistungen International) und in der Hamburger KESS-Studie (Kompetenzen und Einstellungen von Schülerinnen und Schülern).

133 In ihrem Aufsatz Der C-Test und sein schulischer Wert: Eine Lanze für mehr C-Tests in der Schule beschreiben die Autoren, welche Vorteile der C-Test als didaktisches Mittel bietet und zeigen auf, wie er aus Sicht der Lehrpersonen wahrgenommen wird. Angaben zum effektiven Einsatz des C-Tests in Schulen finden sich hier nicht. Doch sowohl der Titel als auch die zum Teil kritische Haltung der Lehrenden zum Test lassen vermuten, dass er nur eingeschränkt verwendet wird. 
kann; stattdessen ermöglicht er eine erste Zuordnung der Testpersonen zu verschiedenen Kompetenzstufen und kann so den Ausgangspunkt für genauere sprachbezogene Untersuchungen bilden.

Wie aus der Forschung bekannt ist, ist die Anwendbarkeit des C-Tests im Italienischen wissenschaftlich untersucht worden (vgl. Grotjahn, Tönshoff \& Hohenbleicher 1994). Es können sich bei der Testbearbeitung in dieser Sprache einige Schwierigkeiten ergeben, wie z.B. der veränderte Klang eines Wortes, der eventuell durch das Auslöschen der zweiten Hälfte entsteht. So ist etwa

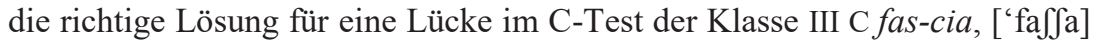
ausgesprochen, obgleich durch die Tilgung des zweiten Wortteils durch innere Phonation in erster Linie die Lesart [fas] suggeriert wird, was die Suchstrategien beeinflussen kann. ${ }^{134}$ Solche tilgungsbedingten Verschiebungen des Lautbilds sind allerdings im Deutschen auf vergleichbare Weise gegeben, beispielsweise bei der Worthalbierung von „Tas-che“ zu „Tas“. In der Regel haben Leser, die sich nicht mehr im Anfängerstadium befinden, die Graphem-Laut-Zuordnung so verinnerlicht, dass sie beim Lesen eines einzelnen Buchstabens berücksichtigen, dass er auch der Anfang einer Graphemgruppe sein kann, die einem anderen Laut zugeteilt ist als das betreffende Graphem alleinstehend. Es liegt auf der Hand, dass diese Fähigkeit in der Erstsprache und bei erfahrenen Lesern besser entwickelt ist als in der Zweit- oder Fremdsprache und bei einer geringen Lesekompetenz.

Ein weiteres Problem kann die anaphorische Referenz von Pronomen und Partizipien darstellen, da sich deren Endung im Italienischen nach dem Genus und Numerus des Bezugsworts richtet und Letzteres von den Testpersonen nicht immer korrekt ermittelt wird. Gerade wenn es nicht im unmittelbaren Textumfeld steht, sondern sich weiter entfernt befindet, ist für eine korrekte Testbearbeitung ein höheres Maß an Textkompetenz erforderlich. Zusätzlich kann die richtige Bearbeitung dadurch erschwert werden, dass das Bezugswort ebenfalls halb getilgt ist. Wie im Deutschen gibt es auch im Italienischen verschiedene Pronomen, die sich nur durch ein grammatikalisches Morph am Wortende unterscheiden, das Genus und Numerus anzeigt (z.B. quest-o (dieser), quest-a (diese) plus entsprechende Pluralmorpheme; quell-o/a (jener/-e)). Wenn also die zweite Worthälfte getilgt ist, kann die Lücke, einmal abgesehen vom Zufallsprinzip, ohne die Bestimmung des Bezugswortes nicht richtig vervollständigt werden. Dies gilt neben den Partizipien, die auch mit einem Genus

134 Im Deutschen wie im Italienischen werden bestimmte Laute durch zwei oder drei Buchstaben gekennzeichnet. Daraus resultierende Probleme beim Lesen oder Vervollständigen von Wörtern, die solche Grapheme enthalten, können an dieser Stelle nicht erörtert werden. Zur Rezeption der geschriebenen Sprache im Italienischen und zum Verhältnis zwischen Orthographie und Phonologie sei z.B. auf Giannelli (1978) und Serianni und Trifone (1994) verwiesen. 
und Numerus anzeigenden Morph enden ${ }^{135}$, gleichermaßen für Enklitika. Einige von ihnen sind außerdem nicht zwingend erforderlich, und bei ihrer Kontraktion mit Verben besteht keine Möglichkeit, das Tilgungsprinzip so zu verändern, dass ihr Vorhandensein im Originaltext ersichtlich wird, ohne dabei die Itemschwierigkeit erheblich zu reduzieren. Etwa im Falle von parlargliene (ihm/ihr/ihnen davon berichten) reicht es nicht aus, einen oder zwei Buchstaben weniger zu tilgen, weil das Wort weiterhin auch einfach nur zu parlargli (ihm/ihr/ihnen berichten) vervollständigt werden kann. ${ }^{136}$

Derartige Komplikationen verhindern allerdings nicht die Anwendbarkeit des Tests im Italienischen (Grotjahn et al. 1996). Die soeben aufgezeigten Referenzprobleme etwa sind im Deutschen bei Pronomen wie dieser, jener und welcher in ähnlichem Ausmaß gegeben, da die Tilgung der zweiten Hälfte verlangt, das Bezugswort korrekt zu identifizieren und das Wort mit dem richtigen Genus und Numerus bezeichnenden Morph enden zu lassen. Wo im C-Test das Italienische bei manchen Wortarten einen höheren Schwierigkeitsgrad aufweist (s. z.B. die variierenden Endungen des Partizips in zusammengesetzten Zeiten), liegen andererseits nur für das Deutsche Kasus anzeigende Morphe vor, die sich am Wortende befinden und auch wieder an Genus und Numerus geknüpft sind, zumal sie manchmal von Kongruenz erfordernden Adjektiven attribuiert werden. Egal welche Teile solcher Statzkonstituenten (Präpositionen, Artikel, Nomen, Adjektive) ergänzt werden sollen, sind dann für eine erfolgreiche Testbearbeitung morphosyntaktische Fähigkeiten und Textkompetenz gefragt.

Darüber hinaus sind im Italienischen lange Wörter zum Teil einfacher, da sie z.B. durch die Verbindung der Adjektivform mit dem Adverbsuffix -mente (vgl. incredibilmente aus incredibile und -mente; velocemente aus veloce und -mente) entstehen. Tendenziell gilt zwar die Regel von Zipf und Guiraud, dass die am häufigsten gebrauchten Wörter einer Sprache die kürzesten sind und lange Wörter seltener gebraucht werden (s. Koesters Gensini 2005), weshalb

135 Wie im Deutschen werden die zusammengesetzten Zeiten mit den Hilfsverben „haben“ und „sein“ gebildet, wobei die Verwendung von „sein“ vorschreibt, dass die Endung des Partizips Genus und Numerus des Subjekts anzeigt. Letzteres gilt bei pronominalen Objekten auch für die Bildung mit haben (z.B.: Anna, c'era? Non l'ho vista; War Ana da? Ich habe sie nicht gesehen.).

136 Auf das Referenzproblem der enklitischen Proformen machen Grotjahn et al. (1994, S. 18 f.) in ihrem Beitrag zur Anwendbarkeit des C-Tests im Italienischen aufmerksam. Während ihre Zielgruppe Personen sind, die Italienisch lernen und deren Fähigkeiten in der Fremdsprache anhand eines C-Tests mit angemessenem Schwierigkeitsgrad ermittelt werden sollen, ist Italienisch für die Testpersonen dieser Untersuchung überwiegend die Erstsprache und bestand kein Anliegen, die Testschwierigkeit durch eine Modifizierung der Textgrundlage herabzusetzen, da das Textverständnis des Lehrbuchs und die Fähigkeit, die dort verwendeten sprachlichen Mittel zu vervollständigen, erhoben werden sollte. Daraus resultiert eine recht große Anzahl von im Text nicht gegebenen, aber logisch und grammatikalisch-syntaktisch richtigen Lösungsvorschlägen. 
sie oft schwieriger erlernt werden und/oder für die Sprecher schlechter verfügbar sind. Die hier genannten Adverbien leiten sich aber wie zahlreiche andere aus Adjektiven ab, die zu den 2.000 am häufigsten gebrauchten Wörtern des Italienischen zählen und deshalb als nicht sehr schwierig eingestuft werden können. Wenn sie von der Tilgung betroffen sind, ist in vielen Fällen der Wortstamm ganz oder größtenteils ersichtlich, so dass keine bzw. kaum lexikalische Fähigkeiten und lediglich geringe morphologisch-grammatikalische Kenntnisse überprüft werden: Den Testeilnehmern müssen nur der Gebrauch des Adverbs und seine Endung in -mente bekannt sein. Dies gilt u.a. auch für den absoluten Superlativ, der zwar textsortenbedingt unterschiedlich, aber insgesamt frequenter als im Deutschen und auch in den C-Tests dieser Erhebung auftritt und mit dem Suffix -issimo gebildet wird (vgl. Grotjahn 1996, S. 124). Beispielsweise ist im Superlativ des Adjektivs sicuro (sicher), sicur-issimo, der Wortstamm vollständig von der Tilgung ausgeschlossen, im Superlativ bravi-ssimo (ausgezeichnet) bleibt sogar der erste Laut des Suffixes vor der Tilgung erhalten und auch in Adverbien einer kürzeren adjektivischen Stammform wie forte-mente (stark) ist der komplette Wortstamm ungetilgt wiedergegeben.

Eine Besonderheit bei der Testherstellung betrifft außerdem die Tilgung apostrophierter Wörter: Folgt den Artikeln und artikulierten Präpositionen ein Wort, das mit einem Vokal beginnt, so wird dieses apostrophiert, so dass beide Wörter dieser Zusammenziehung zwar als ein Wort gezählt werden, aber nur die zweite Hälfte des Wortteils hinter dem Apostroph getilgt wird. ${ }^{137}$

Für die vorliegende Erhebung wurde der C-Test als Verfahren ausgewählt, da sie nicht zum Ziel hatte, die sprachlichen Fähigkeiten der Lernenden möglichst exakt zu ermitteln, sondern weil von verschiedenen Blickwinkeln aus die sprachlichen Herausforderungen betrachtet werden sollten, vor denen die Lernenden im Erdkundeunterricht stehen, und wie sie darauf reagieren. Der Test diente also der Überprüfung ihrer Fertigkeiten im Umgang mit dem Lehrbuch, der primären sprachlichen Grundlage ihres Unterrichts. Da alle Klassen, die an der Studie teilnahmen, unterschiedliche Lehrbücher verwendeten, wurden auch fünf verschiedene C-Test-Versionen entwickelt. Das hat zur Folge, dass die Ergebnisse der einzelnen Klassen nicht direkt miteinander verglichen werden können, doch gilt das Hauptinteresse weder einem Leistungsvergleich zwischen den einzelnen Klassen, noch könnten im Rahmen dieser qualitativen Studie mit einem Testpersonenumfang von 73 Erkenntnisse allgemeingültigen

137 Eine C-Test-Regel für apostrophierte Formen ist zuerst für das Französische entwickelt worden und versteht die mit einem Apostroph verbundenen Wörter als eine Einheit, von der die Hälfte getilgt wird. Im Italienischen bietet sich dieses Verfahren nicht an, weil sich dadurch vergleichsweise mehr kritische Fälle ergeben, in denen entweder das gesamte auf den Apostroph folgende Wort fehlt oder, wenn dieses ausreichend kurz ist, sogar das apostrophierte Wort vervollständigt und mit einem Apostroph versehen werden muss. Grotjahn et al. (1996, S. 122 f.) schlagen deshalb verschiedene Alternativen zum klassischen Tilgungsprinzip vor, von denen die hier gewählte wahrscheinlich die geringste Itemschwierigkeit erzeugt. 
Charakters gewonnen werden. Indes ist es bei den vielen Einflussfaktoren auf die Schülerleistung durchaus möglich, dass die Ergebnisse auch durch die Faktoren Persönlichkeitsmerkmal und sprachdidaktisch-fachliche Kompetenzen der fünf beteiligten Lehrkräfte beeinflusst sind (vgl. Beese \& Benholz 2013, S. 44). Auf der anderen Seite ähnelt sich das Testmaterial, da die fünf Lehrbücher dieselben Themen behandeln und die Textanalyse keine auffälligen Differenzen in der morphosyntaktischen und in der lexikalischen Zusammensetzung (Gebrauchsfrequenz und die an sie gekoppelten Schwierigkeiten) ergeben hat (s. Kapitel 7 zur Lehrbuchanalyse).

Weil schon während der Pilotstudie ersichtlich wurde, dass die Lehrbuchtexte ein Sprachmaterial für die Entwicklung von C-Tests darstellen, das nur mit einer hohen Anzahl von Fehlern ergänzt werden konnte, ist bei der Auswahl der Texte bewusst entschieden worden, auf solche zurückzugreifen, die nur wenige Wochen zuvor im Unterricht behandelt worden waren. ${ }^{138}$

Die fünf C-Tests bestehen jeweils aus fünf kleinen, mit Überschriften versehenen Lückentexten, in denen der erste und der letzte Satz vollständig wiedergegeben sind, um den Einstieg in das Thema und damit das Textverständnis zu erleichtern. In allen anderen Sätzen ist jeweils die Hälfte jedes zweiten Wortes getilgt. Dabei ist den Testpersonen das Tilgungsprinzip nicht bekannt, sie wissen also nur, dass etwas vom Wort fehlt, aber nicht, dass immer genau die Hälfte vervollständigt werden muss.

Alle Texte bestehen aus ca. 25 Lücken bei einer Gesamtzahl von ca. 125 Lücken pro Test und entsprechen damit dem herkömmlichen C-Test (vgl. z.B. Grotjahn 2002). Gegen eine kürzere, auch häufig eingesetzte Version fiel die Wahl, um ein breiteres Wortschatzspektrum und mehr morphosyntaktische und grammatikalische Kenntnisse und Fähigkeiten überprüfen zu können. Dass manche Texte nur 24 oder sogar 27 Lücken aufweisen, rührt daher, dass die Sinnabschnitte eines Textes nicht unnötig zerbrochen werden sollten.

Die C-Tests sind dem Anhang zu entnehmen, weshalb an dieser Stelle nur ein Textbeispiel (C-Test der Klasse Vespucci) vorgelegt wird:

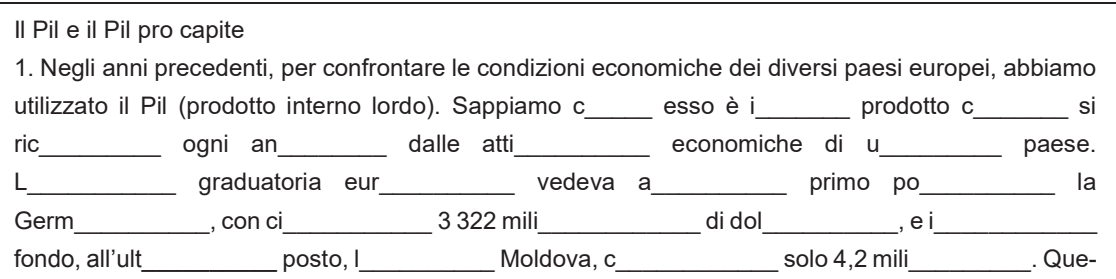

138 In der Pilotstudie wurde ein C-Test aus dem Erdkundebuch einer achten Klasse entwickelt und in dieser durchgeführt. Der geringe Prozentsatz an richtigen Lösungsvorschlägen stieg auch nicht signifikant, als der Test im Zuge einer universitären Lehrveranstaltung einer Gruppe von Studierenden ausgehändigt wurde. 


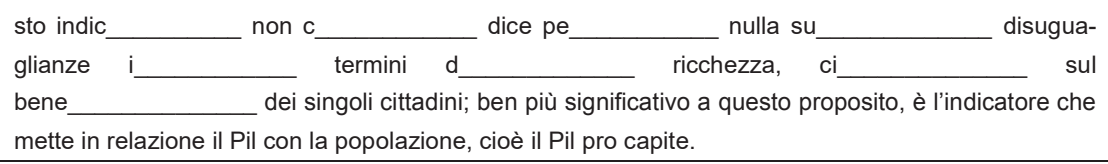

Abbildung 1: Textbeispiel aus dem C-Test der Schule Vespucci

Der Textabschnitt stammt aus dem Lehrwerk Nuovo Geolab von Carazzi und Pizzetti (2010, S. 55) und wurde wenige Wochen vor der Durchführung des CTests ausführlich im Unterricht behandelt. Untenstehend findet sich dasselbe Beispiel ohne Lücken, wobei der getilgte Teil der Wörter markiert ist.

Il Pil e il Pil pro capite

1. Negli anni precedenti, per confrontare le condizioni economiche dei diversi paesi europei, abbiamo utilizzato il Pil (prodotto interno lordo). Sappiamo che esso è il prodotto che si ricava ogni anno dalle attività economiche di un paese. La graduatoria europea vedeva al primo posto la Germania, con circa 3322 milioni di dollari, e in fondo, all'ultimo posto, la Moldova, con solo 4,2 milioni. Questo indicatore non ci dice però nulla sulle disuguaglianze in termini di ricchezza, cioè sul benessere dei singoli cittadini; ben più significativo a questo proposito, è l'indicatore che mette in relazione il Pil con la popolazione, cioè il Pil pro capite.

Abbildung 2: Textbeispiel ohne Lücken

Die Lernenden erhielten eine mündliche Einführung zur Bearbeitung des Tests, im Zuge derer das richtige Ergänzen der Lücken auch gemeinsam in einem Beispielsatz an der Tafel geübt wurde. Sie wurden außerdem dazu angehalten, den Test selbstständig in ungefähr 25 Minuten zu lösen, ohne dabei in Zeitdruck zu geraten. Wer also am Ende etwas mehr Zeit brauchte, dem wurde sie gewährt.

Als Fehler zählten alle morphosyntaktisch und semantisch nicht korrekten Ergänzungen sowie solche, die gegen die Testbearbeitungsregel verstoßen, die Wörter nur zu vervollständigen und nicht durch weitere Ausdrücke zu ergänzen. Als halbe Fehler wurden orthographische wie fehlende oder falsche Akzente und Wiederholungsfehler gewertet. Alle vom Originaltext abweichenden Lösungsvorschläge, die sowohl morphosyntaktisch als auch semantisch angemessen sind, wurden akzeptiert, wobei zwei MuttersprachlerInnen mit akademischen Titeln im Bereich italienische Philologie/Linguistik ihre Richtigkeit überprüften.

Im folgenden Kapitelabschnitt sollen die Ergebnisse der einzelnen C-Tests dargestellt und mit der Zugehörigkeit der getilgten Wörter zu den verschiedenen Wortarten und Bereichen des Gebrauchswortschatzes in Verbindung gebracht werden. 


\subsection{Testergebnisse der fünf Klassen}

\section{Vespucci}

In der Klasse der Schule Vespucci waren am Tag der Testdurchführung elf von 14 SchülerInnen anwesend, die anderen drei fehlten krankheitsbedingt. Eine Schülerin wurde aufgrund einer starken Lernbeeinträchtigung und von der Klasse abweichenden Lernzielen aus der Bewertung ausgenommen, zumal sie nur den ersten der fünf Texte bearbeitet hatte. Der Test besteht aus insgesamt 126 Lücken. Der geringste Anteil an Fehlern liegt mit elf Fehlern bei 8,7 Prozent und der höchste mit 76 Fehlern bei 60,3 Prozent. Durchschnittlich beträgt die Fehlerquote 26,5 Prozent.

Tabelle 1 zeigt alle Ergebnisse im Einzelnen, wobei das letzte Feld angibt, welche Lernenden im Fragebogen als erstrebte weiterführende Schulform ein liceo (Gymnasium) und damit den anspruchsvollsten unter den möglichen Schultypen angaben. Wegen der lehrbuchbedingten Verschiedenartigkeit der fünf $\mathrm{C}$-Tests handelt es sich im Folgenden um eine Illustration der erzielten Leistungen, ohne dass die Fehlerwerte klassenübergreifend direkt miteinander verglichen werden könnten. Im Zuge einer detaillierten Fehleranalyse wird aber auf die jeweilige Itemschwierigkeit eingegangen, so dass ein Überblick über die Gestaltung der einzelnen Tests und ihre Bearbeitung gewährleistet ist.

\begin{tabular}{|c|c|c|c|c|c|}
\hline Lernende & n Fehler & Anteil in \% & Schuljahr wiederholt & $\mathrm{L}_{1}$ nicht it. & Schulwahl liceo \\
\hline a & 11 & 8,7 & & & \\
\hline$b$ & 18 & 14,3 & & & \\
\hline c & 21,5 & 17,1 & & * & * \\
\hline d & 24 & 19 & * & & \\
\hline e & 30 & 23,8 & & * & \\
\hline f & 32 & 25,4 & * & * & \\
\hline g & 39 & 31 & & * & \\
\hline $\mathrm{h}$ & 41 & 32,5 & * & & \\
\hline i & 41 & 33 & & * & \\
\hline j & 76 & 60,3 & & * & \\
\hline $\begin{array}{l}\text { n Lernende/ } \\
\varnothing \text { Fehler (\%) }\end{array}$ & 10 & 26,5 & & & \\
\hline
\end{tabular}

Tabelle 1: Ergebnisse des C-Tests an der Schule Vespucci

\section{Morante}

An der Schule Morante nahmen 13 von 17 SchülerInnen am C-Test teil. Der aus 123 Lücken bestehende Test führte zu einer geringsten Fehlerzahl von 2 und einer höchsten von 54. Diese beiden Werte entsprechen einem Anteil von 1,6 und 43,9 Prozent an Falschlösungen. Die durchschnittliche Fehlerquote liegt bei 19,3 Prozent. Aus Tabelle 2 wird außerdem ersichtlich, dass die mehrsprachigen Lernenden in dieser Klasse, anders als an der zuerst vorgestellten Klasse an der Vespucci, geschlossen die schlechtesten Ergebnisse erzielten. 


\begin{tabular}{|c|c|c|c|c|c|}
\hline Lernende & n Fehler & Anteil in \% & Schuljahr wiederholt & $\mathrm{L}_{1}$ nicht it. & Schulwahl liceo \\
\hline a & 2 & 1,63 & & & * \\
\hline$b$ & 2 & 1,63 & & & \\
\hline c & 13 & 10,6 & & & \\
\hline d & 14 & 11,4 & & & \\
\hline e & 18 & 14,6 & & & \\
\hline$f$ & 19 & 15,4 & & & \\
\hline g & 19 & 15,4 & & & \\
\hline $\mathrm{h}$ & 29 & 23,6 & & * & \\
\hline i & 32 & 26 & & * & \\
\hline j & 33,5 & 27,2 & & * & \\
\hline $\mathrm{k}$ & 37 & 30 & & * & \\
\hline । & 37 & 30 & & * & \\
\hline $\mathrm{m}$ & 54 & 43,9 & & * & \\
\hline $\begin{array}{l}\text { n Ler- } \\
\text { nende/ }\end{array}$ & & & & & \\
\hline$\varnothing$ Fehler & 13 & 19,3 & & & \\
\hline
\end{tabular}

Tabelle 2: Ergebnisse des C-Tests an der Schule Morante

Pestalozzi III A

Diese Klasse bestand aus 19 SchülerInnen, von denen 17 am Test teilnahmen. Im C-Test mussten 125 Wörter vervollständigt werden. Hier liegt das beste Ergebnis bei einem Fehler (0,8 \%). Die höchste Fehlerzahl beträgt 37 mit einer Quote von 29,6 Prozent. Der Mittelwert des Fehleranteils liegt bei 8,7 Prozent.

\begin{tabular}{|c|c|c|c|c|c|}
\hline Lernende & n Fehler & Anteil in \% & Schuljahr wiederholt & $\mathrm{L}_{1}$ nicht it. & Schulwahl liceo \\
\hline a & 1 & 0,8 & & & \\
\hline b & 4 & 3 & & & \\
\hline c & 5 & 4 & & & \\
\hline d & 5 & 4 & & & \\
\hline e & 5 & 4 & & & \\
\hline f & 5 & 4 & & & \\
\hline g & 7 & 5,6 & & & \\
\hline h & 10 & 0,8 & & & \\
\hline i & 10 & 8 & & * & \\
\hline j & 10 & 8 & & & \\
\hline $\mathrm{k}$ & 12 & 9,6 & & & \\
\hline I & 12 & 9,6 & & & \\
\hline $\mathrm{m}$ & 16 & 12,8 & & & \\
\hline$n$ & 16 & 12,8 & & & \\
\hline o & 18 & 14,4 & & & \\
\hline$p$ & 21 & 16,8 & & & \\
\hline $\begin{array}{l}\mathrm{q} \\
\mathrm{n} \text { Lernende/ }\end{array}$ & 37 & 29,6 & * & & \\
\hline$\varnothing$ Fehler & 17 & 8,7 & & & \\
\hline
\end{tabular}

Tabelle 3: Ergebnisse des C-Tests der Klasse III A, Pestalozzi 
Pestalozzi III B

In der Klasse III B der Pestalozzi nahmen 14 von 15 Lernenden an dem aus 124 Lücken bestehenden C-Test teil und bestanden ihn mit einer geringsten Fehlerzahl von 14 (11,3\%) und einer höchsten von 77 (62\%). Der durchschnittliche Fehleranteil liegt bei 29 Prozent.

\begin{tabular}{|c|c|c|c|c|c|}
\hline Lernende & $\mathrm{n}$ Fehler & Anteil in \% & Schuljahr wiederholt & $\mathrm{L}_{1}$ nicht it. & Schulwahl liceo \\
\hline a & 14 & 11,3 & & & * \\
\hline b & 17 & 13,7 & & & * \\
\hline c & 17,5 & 14,1 & & * & * \\
\hline d & 20 & 16,1 & & & * \\
\hline e & 22 & 17,7 & * & & * \\
\hline$f$ & 29 & 23,4 & & & * \\
\hline $\mathrm{g}$ & 32 & 25,8 & & * & * \\
\hline $\mathrm{h}$ & 35 & 28,2 & & & \\
\hline i & 37 & 29,8 & * & * & \\
\hline j & 38 & 30,6 & * & & \\
\hline $\mathrm{k}$ & 52 & 41,9 & & & \\
\hline । & 54 & 43,5 & & * & * \\
\hline $\mathrm{m}$ & 60 & 48,4 & & & \\
\hline$n$ & 77 & 62 & & & * \\
\hline $\begin{array}{l}\text { n Lernende/ } \\
\varnothing \text { Fehler }\end{array}$ & 14 & 29 & & & \\
\hline
\end{tabular}

Tabelle 4: Ergebnisse des C-Tests der Klasse III B, Pestalozzi

Pestalozzi III C

Von den 21 SchülerInnen der Klasse III C waren bei der Durchführung des Tests mit seinen 127 zu ergänzenden Wörtern 19 anwesend und erzielten einen minimalen Fehlerwert von 9 (7 \%) und einen maximalen von 40 (31,5\%). Der durchschnittliche Fehleranteil liegt bei 17,9 Prozent.

\begin{tabular}{|c|c|c|c|c|c|}
\hline Lernende & $\mathrm{n}$ Fehler & Anteil in \% & Schuljahr wiederholt & $\mathrm{L}_{1}$ nicht it. & Schulwahl liceo \\
\hline a & 9 & 7 & & & \\
\hline b & 12 & 9,4 & & & * \\
\hline c & 13 & 10,2 & * & & \\
\hline d & 16,5 & 13 & & & * \\
\hline e & 17 & 13,4 & & & \\
\hline f & 18 & 14,2 & & & * \\
\hline g & 19,5 & 15,4 & & & \\
\hline $\mathrm{h}$ & 20 & 15,7 & & * & * \\
\hline i & 21,5 & 16,9 & & & \\
\hline j & 23,5 & 7,8 & & & \\
\hline $\mathrm{k}$ & 24 & 18,9 & & & \\
\hline । & 25 & 19,7 & & & * \\
\hline $\mathrm{m}$ & 28 & 22 & & & \\
\hline $\mathrm{n}$ & 29 & 22,8 & & & \\
\hline 0 & 31 & 24,4 & & & \\
\hline$p$ & 32 & 25,2 & & & * \\
\hline q & 32 & 25,2 & & * & \\
\hline r & 34 & 26,8 & * & & \\
\hline
\end{tabular}




\begin{tabular}{lrrrrr}
\hline Lernende & $\mathrm{n}$ Fehler & Anteil in $\%$ & Schuljahr wiederholt & $\mathrm{L}_{1}$ nicht it. & Schulwahl liceo \\
\hline $\mathrm{S}$ & 40 & 31,5 & & \\
n Lernende/ & 19 & 17,9 & & \\
$\varnothing$ Fehler & 19 & & & \\
\hline
\end{tabular}

Tabelle 5: Ergebnisse des C-Tests der Klasse III C an der Schule Pestalozzi

\subsubsection{Erste Gegenüberstellung der Ergebnisse in den einzelnen Klassen}

Tabelle 6 stellt alle Klassen bezüglich ihrer minimalen und maximalen Fehlerzahl sowie des durchschnittlichen prozentualen Fehleranteils auf.

\begin{tabular}{lrrr}
\hline Klasse & min. Fehlerzahl & max. Fehlerzahl & $\varnothing$ Fehler in \% \\
\hline Vespucci & 11 & 76 & 26,5 \\
Morante & 2 & 54 & 19,3 \\
III A & 1 & 37 & 8,7 \\
III B & 14 & 77 & 29 \\
III C & 9 & 40 & 17,9 \\
\hline
\end{tabular}

Tabelle 6: Illustration des Testausgangs in allen fünf Lerngruppen

Wie aus der Tabelle ersichtlich wird, bewegen sich die durchschnittlichen Fehleranteile in vier der fünf Klassen zwischen 18 und 29 Prozent. Demnach wurde in diesen Lerngruppen im Mittelwert mehr als jedes vierte zu ergänzen de Wort fehlerhaft gelöst. Nur in der Klasse III A der Schule Pestalozzi liegt der durchschnittliche Fehlerwert mit 8,7 Prozent deutlich tiefer, und zwar besteht zur zweitbesten Klasse (III C) im Mittelwert ein Unterschied von einer doppelten Fehleranzahl. Obwohl in der zweitbesten Klasse mit neun Fehlern weder eine sehr geringe minimale Fehlerzahl noch mit vierzig eine besonders niedrige maximale erzielt wurde, liegt dieses Ergebnis doch elf Prozentpunkte unterhalb der Klasse mit dem schlechtesten Ergebnis und ist die Spannbreite zwischen minimalen und maximalen Fehlerzahlen kleiner als in den anderen Klassen. Das spricht für eine gleichmäßig niedrigere Verteilung der Fehlerzahlen in der Lerngruppe als Ganzer.

Nach dieser ersten Präsentation der C-Testergebnisse in den einzelnen Klassen werden die Lösungsvorschläge der Testteilnehmer nun im Kapitelabschnitt 8.3 detaillierter vorgestellt, indem das sprachliche Material nach Wortklassen sowie Art und Häufigkeit des Gebrauchs beschrieben wird. Darüber hinaus erfolgt eine Einteilung der Falschlösungen in seltenere und häufigere Fehlerquellen und werden Hypothesen über die Ursachen aufgestellt, die zu den nicht korrekten Lösungsvorschlägen führten. 


\subsection{Fehleranalysen}

\subsubsection{Vespucci}

Wie auch bei der Klassifizierung des in den Lehrbüchern untersuchten Wortvorkommens, wird bei der Besprechung der C-Test-Lösungsvorschläge erneut die von Tullio De Mauro (1999/2000; Version 2007) im GRADIT benutzte Wortschatzeinteilung verwendet. Für eine ausführliche Beschreibung und eine tabellarische Übersicht aller von ihm verwendeter Bezeichnungen für die verschiedenen Bereiche des Wortschatzes und ihre Übersetzungen sei auf das Kapitel 7 zur Lehrbuchanalyse verwiesen.

Zunächst zeigen die Tabelle 7 und ein Diagramm (Abb. 3), welchen Bereichen des Gebrauchswortschatzes die Wörter zugeteilt sind, die es im Test der Vespucci zu vervollständigen galt, und welche Anteile sie jeweils am gesamten $\mathrm{zu}$ ergänzenden Wortvorkommen haben. Bei den Phrasemelementen (el.Co/el.Ts) sind im Kommentarfeld diejenigen Wörter fettgedruckt, die ergänzt werden sollten.

\begin{tabular}{lrrlrr}
\hline Marke & $\begin{array}{r}\text { Vorkom- } \\
\text { men }\end{array}$ & in Prozent & Kommentar & n Fehler & $\begin{array}{r}\varnothing \text { Fehler } \\
\text { pro Wort }\end{array}$ \\
\hline AU & 7 & 5,5 & 32 & 4,6 \\
AU TS & 2 & 1,6 & reddito (2) & 1 & 0,5 \\
CO & 18 & 14,3 & 68,5 & 3,8 \\
CO TS & 1 & 0,8 & idrocarburi & 4 & 4 \\
el.CO & 4 & 3,2 & $\begin{array}{l}\text { livelli di vita, più che altro, } \\
\text { in media, per esempio, a }\end{array}$ & 17 & 4,3 \\
& & & persona & \\
el.TS & 2 & 1,6 & speranza di vita (2) & 1 & 0,5 \\
FO & 85 & 67,5 & & 214,5 & 2,52 \\
TS & 3 & 2,4 & indicatore (3) & 10 & 3,3 \\
n.p. & 3 & 2,4 & & 1 & 0,3 \\
n.p./sigla & 1 & 0,8 & & 1 & 1 \\
n getilgte & 126 & 100 & n Fehler & 333 & 2,7 \\
Wörter & & & & & \\
\hline
\end{tabular}

Tabelle 7: Getilgtes Wortvorkommen im C-Test der Vespucci mit Angaben zu den Falschlösungen 


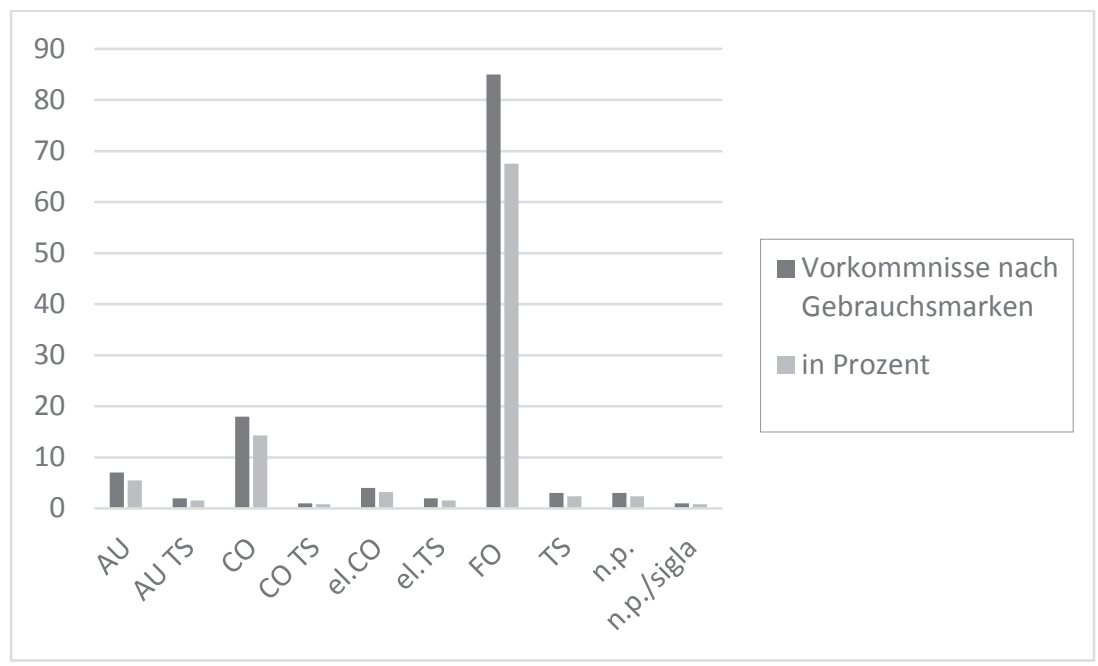

Abbildung 3: Zusammensetzung der getilgten Wörter im C-Test der Schule Vespucci

Mit 67,5 Prozent entstammt der Großteil der zu ergänzenden Wörter dem fundamentalen Wortschatz. ${ }^{139}$ Rechnet man die Wörter des hohen Gebrauchs (AU) und solche mit der doppelten Markierung AU Ts hinzu, kommt man auf einen Anteil von 75 Prozent an getilgten Wörtern aus dem Vocabolario di Base. Dieser Wert, der um vier Prozentpunkte größer ist als der durchschnittliche VDBAnteil auf den untersuchten Seiten des Lehrbuchs (69,4\%), liegt etwas über dem der anderen vier C-Tests (im Mittelwert für alle fünf 71,3\%). Dennoch wurden im Test der Vespucci mit dem höheren zu ergänzenden Wortvorkommen aus dem Bereich des Basiswortschatzes nicht weniger falsche Lösungen produziert als in den anderen Klassen. ${ }^{140}$ Dafür bietet der bereits beschriebene Umstand, dass ein erfolgreiches Schließen der Lücken von der Gestaltung ihres sprachlichen Umfelds abhängt, eine Erklärung.

Der Großteil der übrigen Wörter, insgesamt 18, stammt aus dem Bereich des allgemeinen Wortschatzes (CO) oder ist als Co Ts doppelt markiert. Vier geographische Eigennamen tragen keine Marke und nur drei der 126 getilgten

139 Wie bereits im Kapitel zur Lehrbuchanalyse geschildert, treten die ca. 2.000 frequentesten Wörter der italienischen Sprache zu um die 90 Prozent in Texten jeglicher Art auf. In Fachtexten z.B. geht der Anteil auf ca. 80 Prozent zurück (De Renzo 2011, S. 188), jedoch liegt er in diesen Texten, wie in denen der übrigen C-Tests, die im Verlaufe dieses Kapitel besprochen werden, deutlich unter diesem Wert (vgl. hierzu auch Kap.7).

140 Da die lexikalische Itemschwierigkeit mit seinem sprachlichen Umfeld korreliert, lassen sich aufgrund der Fehlerzahl bei vorliegender Wortschatzzusammensetzung höchstens vorsichtige Vermutungen über einen eventuellen sprachlichen Leistungsrückstand im Vergleich zu den anderen Klassen anstellen. 
Wörter entspringen dem Fachwortschatz, wobei es sich mit indicatore (Indikator) um die Vorkommnisse eines einzigen Worttyps handelt (Carazzi \& Pizzetti 2010, S. 55 f.). Am meisten Falschlösungen lassen sich in diesem C-Test durchschnittlich mit Werten von 4,6 bis 3,8 pro Wort aus den vier Bereichen hoher Gebrauch (AU: 4,6), allgemeiner Wortschatz (Co: 3,8 und el.Co: 4), dem doppelt markierten allgemein-fachwortschatzsprachlichen Vorkommen (CO Ts; 4) finden. Beim Co Ts-Markierten handelt es sich um ein einziges Wort, das fünfmal falsch gelöst wurde (idrocarburi, Kohlenwasserstoff). Das zum Basiswortschatz zählende AU-markierte Wortvorkommen inklusive doppelter Marken (AU TS) ist unter den getilgten Wörtern mit 7,1 Prozent der am drittstärksten vertretene Bereich des Wortschatzes und verzeichnet 33 Falschlösungen. Ungefähr ein Drittel aller Falschlösungen dieses Tests entfällt auf Wörter aus dem allgemeinen Wortschatz, der mit fast 50.000 Lemmata sehr umfangreich ist und in diesem C-Test bei den getilgten Wörtern nach denen aus dem fundamentalen Wortschatz die zweitgrößte Gruppe bildet (14,3\%).

\subsubsection{Texte 1 und 2 des C-Tests (S. 55)}

Die untenstehende Tabelle (Tab. 8) zeigt alle getilgten Wörter der ersten beiden Texte des C-Tests, die der Lehrbuchseite 55 (Carazzi \& Pizzetti 2010) entnommen sind, in ihrem Textkontext mit Wortart und Gebrauchsmarke, wie oft sie fehlerhaft ergänzt wurden und welche Lösungsvorschläge die Lernenden machten. Hierbei steht die Abkürzung acc. für accettabile (akzeptabel), wenn es sich um einen Lösungsvorschlag handelt, der zwar nicht dem Originaltext entspricht, aber sowohl grammatikalisch-syntaktisch als auch logisch stimmig ist. Bei der Erstellung des C-Tests in dieser Klasse ist mir ein Rechtschreibfehler unterlaufen, der ebenfalls in der Tabelle ersichtlich ist (s. Kommentarfeld): Anstelle des im Originaltext verwendeten ogni (jedes) steht dort oggi (heute). Es geht um das Bruttoinlandsprodukt und der ganze Satz lautet „Sappiamo che esso è il prodotto che si ricava ogni anno dalle attività economiche di un paese" (ibid., S. 55; m.Ü.: Wir wissen, dass es das Produkt ist, das jedes Jahr aus den wirtschaftlichen Tätigkeiten eines Landes gewonnen wird). Da ,jedes“ im C-Test durch „,heute“ ersetzt ist, füllten viele Lernende die anschließende Lücke von an- nicht zu anno (Jahr), sondern zu anche (auch), was als Lösungsvorschlag akzeptiert wurde, wie auch annualmente (jährlich). Bei den einzigen zwei nicht akzeptablen Lösungen ist nicht davon auszugehen, dass der Konstruktionsfehler die korrekte Lösung verhinderte, da es sich bei einem Testteilnehmer um einen orthographischen (anualmente ohne den doppelten Konsonanten) und beim anderen um einen logischen Fehler (ancora, noch) handelte: Wie sonst, wenn nicht durch die wirtschaftlichen Tätigkeiten eines Landes, soll sein BIP berechnet werden?

Desweiteren entstanden bei der Testkonstruktion drei nicht vorschriftsmäBige Worttilgungen, von denen bei einer eine längere Ziffer als Wort gewertet 
und dadurch das folgende Wort milioni (Millionen, 0 Falschlösungen) anstelle der Präposition di (von) getilgt wurde, die auch in einem anderen Fall eigentlich von der Tilgung betroffen gewesen wäre. Stattdessen war die Hälfte der darauffolgenden Wörter zu ergänzen, der unbestimmte Artikel un (ein) und das Substantiv dollari (Dollar). Der Test hat an Validität in diesem Fall glücklicherweise nicht eingebüßt, da alle Teilnehmer in der Lage waren, den Artikel richtig zu vervollständigen, und nur einer bei der Lückenfüllung des Substantivs einen Rechtschreibfehler begangen hat. Die dritte falsche Tilgung betrifft die Präposition $a$ im letzten Satz des zweiten Textes. Sie wird im Zuge der Besprechung der häufigsten Fehler auf dieser Lehrbuchseite erklärt.

\begin{tabular}{|c|c|c|c|c|c|}
\hline Fehler & Wort & Wortart & Marke & Kommentar & Lösungsvorschläge \\
\hline $\begin{array}{l}1(28 \\
\text { Lücken) }\end{array}$ & Sappiamo & v.tr. & FO & S. 55 & \\
\hline \multirow[t]{3}{*}{0} & che & cong. & FO & & \\
\hline & esso & pron.pers. & FO & & \\
\hline & $\grave{e}$ & v.intr. & FO & & \\
\hline \multirow[t]{2}{*}{0} & il & art.det. & FO & & \\
\hline & prodotto & s.m. & FO & & \\
\hline \multirow[t]{2}{*}{0} & che & pron.rel. & FO & & \\
\hline & si & pron.pers. & $\mathrm{CO}$ & & \\
\hline \multirow[t]{2}{*}{4} & ricava & v.tr. & $\mathrm{CO}$ & & $\begin{array}{l}\text { ricalcola, riccava (2), } \\
\text { riconosce }\end{array}$ \\
\hline & oggi & avv. & FO & $\begin{array}{l}\text { im Buch steht ogni } \\
\text { anno (Konstrukti- } \\
\text { onsfehler im Test) }\end{array}$ & \\
\hline \multirow[t]{2}{*}{$\begin{array}{l}2+6 \\
\text { acc. }\end{array}$} & anche/anno & cong. & FO & & $\begin{array}{l}\text { anche }(5) \text { (acc.), } \\
\text { anualmente, ancora, } \\
\text { annualmente (acc.) }\end{array}$ \\
\hline & dalle & prep.art. & FO & & \\
\hline \multirow[t]{3}{*}{1,5} & attività & s.f. & FO & & attitudini, attivita \\
\hline & economiche & agg. & FO & & \\
\hline & $d i$ & prep. & FO & Tilgungsfehler & \\
\hline \multirow[t]{2}{*}{0} & un & art.indet. & FO & & \\
\hline & paese & s.m. & FO & & \\
\hline \multirow[t]{2}{*}{0} & $\mathrm{La}$ & art.det. & FO & & \\
\hline & graduatoria & s.f. & TS stat. & & \\
\hline \multirow[t]{2}{*}{1} & europea & agg. & FO & & europeo \\
\hline & vedeva & v.tr. & FO & & \\
\hline \multirow[t]{2}{*}{0} & al & prep.art. & FO & & \\
\hline & primo & agg.num.ord. & FO & & \\
\hline \multirow[t]{2}{*}{0} & posto & s.m. & FO & & \\
\hline & la & art.det. & FO & & \\
\hline \multirow[t]{2}{*}{0} & Germania & n.p.f. & & & \\
\hline & con & prep. & FO & & \\
\hline 3 & circa & avv. & FO & & $\begin{array}{l}\text { cinque milioni, cino- } \\
\text { vanta, cinquanta }\end{array}$ \\
\hline \multirow[t]{2}{*}{9 acc. } & $3322 \mathrm{mi}-$ & s.m. & FO & Tilgungsfehler & milioni (acc.) (9) \\
\hline & di & prep. & FO & & \\
\hline 2 & dollari & s.m. & $\mathrm{CO}$ & & dolari (2) \\
\hline
\end{tabular}




\begin{tabular}{|c|c|c|c|c|c|}
\hline & $e$ & cong. & FO & & \\
\hline \multirow[t]{2}{*}{2} & in & prep. & FO & & i dollari, il \\
\hline & fondo & s.m. & FO & & \\
\hline \multirow[t]{2}{*}{1} & all'ultimo & agg. & $\mathrm{FO}$ & & ultultimo \\
\hline & posto & s.m. & FO & & \\
\hline \multirow[t]{2}{*}{1} & la & art.det. & $\mathrm{FO}$ & & I posto \\
\hline & Moldova & n.p. & $\begin{array}{l}\text { nicht im } \\
\text { GRADIT, nur } \\
\text { als Adj. }\end{array}$ & & \\
\hline \multirow[t]{2}{*}{2} & con & prep. & $\mathrm{FO}$ & & circa (2) \\
\hline & solo & avv. & FO & & \\
\hline \multirow[t]{2}{*}{10 acc. } & 4,2 miliardi & s.m. & $\mathrm{FO}$ & & milioni (acc.) (10) \\
\hline & Questo & agg.dimostr. & $\mathrm{FO}$ & & \\
\hline \multirow[t]{2}{*}{$\begin{array}{l}3+3 \\
\text { acc. }\end{array}$} & indicatore & s.m. & TS econ. & & $\begin{array}{l}\text { indica (3), indice ( } 3 \\
\text { acc.) }\end{array}$ \\
\hline & non & avv. & FO & & \\
\hline \multirow[t]{2}{*}{5} & $c i$ & pron.pers. & $\mathrm{FO}$ & & che, 0 (3), chi \\
\hline & dice & v.tr. & $\mathrm{FO}$ & & \\
\hline \multirow[t]{2}{*}{9} & però & cong. & $A U$ & & per (9) \\
\hline & nulla & pron.indef. & $\mathrm{AU}$ & & \\
\hline \multirow{2}{*}{$\begin{array}{l}1+1 \\
\text { acc. }\end{array}$} & sulle & prep.art. & FO & & 0 (acc.), sulla \\
\hline & $\begin{array}{l}\text { disugua- } \\
\text { glianze }\end{array}$ & s.f. & $\mathrm{CO}$ & & \\
\hline \multirow[t]{2}{*}{4} & in & prep. & FO & & $0(3), i$ tanti \\
\hline & termini & s.m. & FO & & \\
\hline \multirow[t]{2}{*}{2} & $d i$ & prep. & $\mathrm{FO}$ & & della (2) \\
\hline & ricchezza & s.f. & TS econ. & & \\
\hline \multirow[t]{2}{*}{11} & cioè & cong. & FO & & $\begin{array}{l}0 \text { (5), circa, ci può es- } \\
\text { sere, ci dice, ci sono, } \\
\text { ci informano }\end{array}$ \\
\hline & sul & prep.art. & FO & & \\
\hline \multirow[t]{4}{*}{7} & benessere & s.m. & $\mathrm{AU}$ & $\begin{array}{l}\text { letztes getilgtes } \\
\text { Wort in Text } 1\end{array}$ & $\begin{array}{l}0(4), \text { benenessere } \\
(2), \text { che }\end{array}$ \\
\hline & dei & prep.art. & FO & & \\
\hline & singoli & agg. & $\mathrm{AU}$ & & \\
\hline & cittadini & s.m. & $\mathrm{FO}$ & & \\
\hline \multirow{2}{*}{$\begin{array}{l}2(27 \\
\text { Lücken) } \\
9\end{array}$} & Già & avv. & $\mathrm{FO}$ & S. 55 & \\
\hline & $\begin{array}{l}\text { a } \\
\text { livello }\end{array}$ & $\begin{array}{l}\text { loc.avv. } \\
\text { el.loc.avv. }\end{array}$ & $\begin{array}{l}\mathrm{CO} \\
\mathrm{el} . \mathrm{CO}\end{array}$ & Tilgungsfehler & al (5), dal (2), un, il \\
\hline 0 & $\begin{array}{l}\text { europeo } \\
\text { anche }\end{array}$ & $\begin{array}{l}\text { agg. } \\
\text { cong. }\end{array}$ & $\begin{array}{l}\text { FO } \\
\text { FO }\end{array}$ & & \\
\hline \multirow[t]{2}{*}{4} & per & prep. & $\mathrm{FO}$ & & $\begin{array}{l}\text { popolare, } 0 \text {, piccolo, } \\
\text { perché }\end{array}$ \\
\hline & questo & agg.dimostr. & FO & & \\
\hline \multirow[t]{2}{*}{$\begin{array}{l}2+2 \\
\text { acc. }\end{array}$} & indicatore & s.m. & TS econ. & & $\begin{array}{l}0 \text {, indice }(2)(\text { acc.), in- } \\
\text { dicato }\end{array}$ \\
\hline & si & pron.pers. & $\mathrm{CO}$ & & \\
\hline \multirow[t]{2}{*}{3} & posono & v.tr. & $\mathrm{FO}$ & & 0 (2), posono \\
\hline & osservare & v.tr. & $\mathrm{FO}$ & & \\
\hline \multirow[t]{2}{*}{$\begin{array}{l}2+2 \\
\text { acc. }\end{array}$} & forti & agg. & $\mathrm{FO}$ & & forme, folte, fonda- \\
\hline & differenze & s.f. & FO & & \\
\hline
\end{tabular}




\begin{tabular}{|c|c|c|c|c|}
\hline \multirow[t]{2}{*}{3} & tra & prep. & FO & 0 (2), tali \\
\hline & $i$ & art.det. & $\mathrm{CO}$ & \\
\hline \multirow[t]{2}{*}{2} & paesi & s.m. & FO & 0 , pagamenti \\
\hline & primi & agg.num.ord. & $\mathrm{FO}$ & \\
\hline \multirow[t]{2}{*}{0} & in & prep. & FO & \\
\hline & classifica & s.f. & $A D$ & \\
\hline \multirow[t]{2}{*}{4} & come & avv. & FO & $0(2)$, con (2) \\
\hline & per & loc.avv. & $\mathrm{CO}$ & \\
\hline \multirow[t]{2}{*}{2} & esempio & el.loc.avv. & $\mathrm{CO}$ & 0 , eseguendo \\
\hline & la & art.det. & FO & \\
\hline \multirow[t]{2}{*}{0} & Danimarca & n.p. & & \\
\hline & con & prep. & FO & \\
\hline \multirow[t]{2}{*}{3} & più & avv. & FO & $\begin{array}{l}\text { procento, pil, pil pro } \\
\text { capite }\end{array}$ \\
\hline & $d i$ & prep. & FO & \\
\hline \multirow[t]{2}{*}{2} & 57261 dollari & s.m. & $\mathrm{CO}$ & 0 , dolari \\
\hline & $a$ & loc.agg.inv. & $\mathrm{CO}$ & \\
\hline \multirow[t]{2}{*}{6} & persona & el.loc.agg.inv. & el.CO & $0(6)$ \\
\hline & e & cong. & $\mathrm{FO}$ & \\
\hline \multirow[t]{2}{*}{3} & le & art.det. & FO & $0(3)$ \\
\hline & ultime & agg. & $\mathrm{FO}$ & \\
\hline \multirow[t]{2}{*}{3} & posizioni & s.f. & $\mathrm{FO}$ & $0(2), \|$ \\
\hline & occupate & part.pass.v.tr. & $\mathrm{FO}$ & \\
\hline \multirow[t]{2}{*}{6} & dalla & prep.art. & $\mathrm{FO}$ & 0 (3), dalle (3) \\
\hline & già & avv. & FO & \\
\hline \multirow[t]{2}{*}{9} & citata & agg. & $\mathrm{CO}$ & $\begin{array}{l}0 \text { (3), citate (2), città } \\
(3) \text {, citta }\end{array}$ \\
\hline & Moldova & n.p. & $\begin{array}{l}\text { nicht im } \\
\text { GRADIT, nur } \\
\text { als Adj. }\end{array}$ & \\
\hline \multirow[t]{2}{*}{2} & (1248 dollari) & s.m. & $\mathrm{CO}$ & $0(2)$ \\
\hline & e & cong. & FO & \\
\hline 3 & $\begin{array}{l}\text { dalla } \\
\text { Macedonia }\end{array}$ & $\begin{array}{l}\text { prep.art. } \\
\text { n.p. }\end{array}$ & FO & $0(3)$ \\
\hline \multirow[t]{2}{*}{$1 \frac{1 / 2}{2}$} & (3659 dollari) & s.m. & $\mathrm{CO}$ & $\begin{array}{l}\text { 0, dolari (Wiederho- } \\
\text { lungsfehler) }\end{array}$ \\
\hline & Ma & cong. & FO & \\
\hline \multirow[t]{2}{*}{9} & su & loc.avv. & $\mathrm{CO}$ & $\begin{array}{l}0(3), \text { sulla }(5), \text { sola- } \\
\text { mente }\end{array}$ \\
\hline & scala & el.loc.avv. & el.CO & \\
\hline \multirow[t]{2}{*}{9} & $\begin{array}{l}\text { internazio- } \\
\text { nale }\end{array}$ & agg. & $\mathrm{FO}$ & $\begin{array}{l}0(7), \text { interna con, in- } \\
\text { terna ci sono }\end{array}$ \\
\hline & le & art.det. & FO & \\
\hline \multirow[t]{2}{*}{8} & distanze & s.f. & FO & $\begin{array}{l}0 \text { (3), distinzioni (2), } \\
\text { distribuzioni (2), di- } \\
\text { stinzioni che }\end{array}$ \\
\hline & si & pron.pers. & $\mathrm{CO}$ & \\
\hline \multirow[t]{2}{*}{3} & fanno & v.pronom.intr. & $\mathrm{CO}$ & $0(3)$ \\
\hline & ancora & avv. & FO & \\
\hline 4 & più & avv. & FO & 0 (3), posti \\
\hline
\end{tabular}

Tabelle 8: Übersicht über die Texte 1 und 2 des C-Tests an der Vespucci inklusive Lösungsvorschläge 
Elf der insgesamt 54 Lücken bereiteten den Lernenden anscheinend keine Schwierigkeiten, da sie richtig gefüllt wurden. Im weiteren Verlauf werden die Falschlösungen aller fünf Klassen diskutiert, wobei nicht auf jeden Fehler eigens eingegangen werden kann. So bleibt ein Teil der Wörter, die von nur ein, zwei Schülern oder Schülerinnen falsch bearbeitet wurden, unbeachtet, weil sie nicht als typisch anzusehen sind. Zur besseren Orientierung werden für diese Besprechung die Fehlerzahlen unabhängig von der Klassengröße grob in zwei Gruppen der selteneren (1-4) und häufigeren (5 +) Fehler unterteilt.

Ein bis zwei falsche Lösungen gab es für die schon besprochenen Wörter an-no/anche und dol-lari sowie für 15 weitere Wörter, die bis auf fünf CO- und ein Ts-markiertes Wort alle zum fundamentalen Wortschatz zählen.

Die Wörter aus dem allgemeinen Wortschatz (Co) sind die beiden Substantive dol-lari und esempio des Phrasems zum Beispiel. Dollari ist mit vier Vorkommnissen im Test vertreten und wurde über den schon genannten und sich wiederholenden orthographischen Fehler hinaus von zwei Lernenden bei einem Vorkommnis nicht ergänzt. In Hinblick auf das Ausbleiben von Lösungsvorschlägen lässt sich festhalten, dass dollari zwar mit vier Tokens im Test vertreten ist, aber keinmal als vollständiges Wort auftritt, sondern immer ergänzt werden muss. Das Phrasem per esempio hingegen gehört zwar, wie für Phraseme im GRADIT typisch, zum Co-Wortschatz, jedoch stammt das Substantiv aus dem fundamentalen Wortschatz und sollte allen Lernenden auch in seiner phrasematischen Verwendung gerade aus dem Kontext Schule bestens bekannt gewesen sein. Zwei der drei falschen Lösungen bestehen in der fehlenden Ergänzung, während die dritte ohne die Hilfe seines Verursachers nicht erklärbar ist: Anstelle von esempio vervollständigte er das Wort zu einem Gerundium, was sowohl grammatikalisch-syntaktisch als auch logisch völlig fehl am Platz ist. ${ }^{141}$ Das TS-markierte Wort ist indicatore (Indikator), was von zwei Lernenden zum akzeptablen, recht synonyme indice ergänzt wurde und von einem Lernenden fehlerhaft zum Partizip Perfekt des Verbs indicare (anzeigen).

Unter den selten falsch gelösten Wörtern aus dem fundamentalen Wortschatz (Fo) sind zwei Substantive. Zum einen atti-vità (economiche) (wirtschaftliche Tätigkeiten), das jemand zu attitudini (Haltungen, Begabungen, Anlagen u.a.) ergänzte, was grammatikalisch richtig, aber semantisch falsch ist. Es ist wahrscheinlich, dass der/die TestteilnehmerIn nur eine vage oder gar keine Vorstellung der Wortbedeutung von attitudine hatte, dass er/sie das Wort zwar vom Hören oder Lesen kannte und es produzieren konnte, aber das lexikalische Verständnis nicht gegeben war. Bei der anderen Falschlösung handelt es sich um einen orthographischen Fehler, indem das Wort nicht mit einem Akzent versehen wurde. Für das Substantiv paesi gab es neben einer fehlenden

141 Der Lösungsvorschlag lautet im Satzzusammenhang, ,[...] i paesi primi in classifica come per eseguendo la Danimarca [...]“ (Carazzi \& Pizzetti 2010, S. 55; m.Ü.: die in der Rangliste obersten Länder wie ausführend Dänemark). 
Ergänzung den nicht akzeptablen Vorschlag pagamenti (Bezahlung). Semantisch ist der Vorschlag nicht völlig abwegig, da es im Textzusammenhang weiterhin um die Rangliste der Länder nach Größe ihres BIPs geht. Grammatikalisch-syntaktisch ist er jedoch nicht tragbar, und ein Erklärungsversuch, der von Mangel an Konzentration und/oder Textkompetenz absieht, könnte höchstens der sein, dass unter den auf die Lücke folgenden Wörtern class-ifica (Rangliste) aus primi in classifica (die ersten in der Rangliste) nicht als Synonym von graduatoria erkannt wurde. Alles deutet darauf hin, dass dieser Fehler entweder von unzureichender Gewissenhaftigkeit oder Textkompetenz bei der Testbearbeitung herrührt oder durch geringere Kenntnisse in der Zweitsprache Italienisch entstand.

Drei der falsch gelösten Fo-gekennzeichneten Wörter sind Adjektive. Eurорео löste ein/e Lernende/r grammatikalisch falsch, da er keine GenusKongruenz zum Bezugswort herstellte. Dieselbe Fehlerquelle findet sich bei dem zweiten Adjektiv, das in der Zusammenziehung mit einer apostrophierten artikulierten Präposition auftritt (all'ult-imo). Außerdem wurde es von einem weiteren Schüler orthographisch falsch ergänzt, was wahrscheinlich auf einen Mangel an Konzentration zurückzuführen ist, da der nicht getilgte Wortteil wiederholt wurde (all 'ultultimo). Beim dritten Adjektiv handelt es sich um forte (stark, groß), das im schon erwähnten Textzusammenhang nach si possono osservare (man kann beobachten) und vor differenze (Unterschiede) steht. Von vier Lernenden ergänzten es drei zu zwei anderen Adjektiven, von denen eines (fondamentali) akzeptabel ist, während der/die vierte das Substantiv forme (Formen) wählte.

Weitere sechs Wörter betreffen die grammatikalischen Morpheme bestimmter Artikel, Präpositionen, eine artikulierte Präposition und eine Konjunktion. Neben einem grammatikalischen Kongruenzfehler für die Kontraktion aus Artikel und Präposition gab es in den anderen Fällen wieder ein unangemessenes Wechseln der Wortart. Zum Beispiel wurde anstelle der erforderlichen Konjunktion ein Adverb formuliert. Der Fehler beim bestimmten weiblichen Singular-Artikel la hingegen kann insofern als dreifach bezeichnet werden, als dass die Ergänzung $l$-l posto (11/der Platz) semantisch und morphosyntaktisch keinen Sinn ergibt und das zu ergänzende Wort darüber hinaus direkt nach posto steht, das kleine $l$ fälschlicherweise für den Buchstaben $i$ gehalten wurde, obwohl die Lücke nicht am Satzanfang steht, und gegen die Regel verstoßen wurde, dass immer nur ein Wort vervollständigt und nicht mehrere Wörter in die Lücke eingefügt werden sollten.

Von drei Testteilnehmern wurden neun Wörter falsch gelöst, die mit zwei Ausnahmen alle aus dem Bereich des fundamentalen Wortschatzes stammen. Bei den Ausnahmen handelt es sich zum einen um die Form fanno des reflexiven Verbs farsi (Co) mit kopulativer Valenz (hier: „si fanno ancora più grandi“"(Carazzi \& Pizzetti 2010, S. 55; m.Ü.: sie werden noch größer)), die 
von drei Personen nicht ergänzt wurde, und zum anderen um ein weiteres Vorkommnis des Substantivs indicatore. In allen drei Fällen wurde es zur konjugierten Verbform indica vervollständigt, obwohl sich in unmittelbarer Nähe ein nicht getilgtes Prädikat befindet und das zu vervollständigende Wort am Satzanfang steht. Dadurch lässt „Questo indica non ci dice nulla“ (Dies zeigt sagt uns nichts) als Lösungsvorschlag vermuten, dass zur Lückenschließung nur das vorausgehende Wort beachtet wurde. Unter den übrigen Wörtern befindet sich neben einer einfachen und einer artikulierten Präposition, einem Substantiv (posizioni) und zwei Adverbien, die allesamt überwiegend nicht vervollständigt wurden, auch die konjugierte Form pos-sono eines Modalverbs (können), das allen sehr gut bekannt sein dürfte, aber im Text passivierend konstruiert wurde, so dass zwei Lernende (vielleicht in der Zweitsprache beschulte) mit diesem komplexeren Gebrauch des geläufigen Modalverbs vielleicht überfordert waren. ${ }^{142}$

Fünf Wörter wurden von je vier Testteilnehmern nicht richtig bzw. gar nicht ergänzt, hierunter der Infinitiv Präsens rica-vare (gewinnen), der passivierend konstruiert ist und logisch falsch zu ricalcola (berechnet neu) und riconosce (erkennt) ergänzt und überdies zweimal falsch geschrieben wurde (Konsonantenverdopplung). Zum anderen betreffen sie die Vervollständigung der einfachen Präposition in im näheren Textzusammenhang von ,disuguaglianze in termini di ricchezza" (ibid; Ungleichheiten bezüglich des Wohlstands). Zugegebenermaßen ist die Bearbeitung als nicht einfach einzuschätzen, da disuguaglianze kein Ausdruck des Basiswortschatzes ist, sondern zum allgemeinen Wortschatz zählt. Außerdem ist in termini di im GRADIT zwar nicht als Phraseologismus eingetragen, wird seine Bedeutung aber mit dem präpositionalen Phrasem in quanto a erklärt, das zum allgemeinen Wortschatz (CO) gehört. Drei Testteilnehmer füllten die Lücke gar nicht, was ein Indiz dafür sein kann, dass sie den Ausdruck in termini di ${ }^{143}$ nicht kannten.

Für die zu vervollständigende Präposition $p$-er (für) gibt es neben einer fehlenden Vervollständigung auch drei Lösungsvorschläge, allerdings keine akzeptablen (vgl. Tabelle 8). Auffällig ist, dass nicht ein einziger der Vor-

142 Diese passivierende Form kann im Deutschen mit dem unpersönlichen man übersetzt werden; konkret auf das Beispiel bezogen: Man kann große Unterschiede beobachten (,si possono osservare forti differenze" (Carazzi \& Pizzetti 2010, S. 55)). Erschwerend an dieser Konstruktion ist für Lernende mit Italienisch als Zweitsprache, dass sich der Numerus des konjugierten Verbs, anders als im Deutschen, nach dem Akkusativobjekt richtet. Da differenze ein Pluralwort ist, ist das Modalverb in der dritten Person Plural konjugiert.

143 Es ist verwunderlich, dass er im GRADIT mit einem Phrasem (in quanto a) erklärt wird, welches die Marke Co (allgemeiner Wortschatz) trägt, aber selbst nicht als Phrasem eingestuft wurde und die Gebrauchsmarke Fo (fundamentaler Wortschatz) erhielt. Wahrscheinlich ist es ein gutes Beispiel dafür, dass bei der Korpusbildung schriftliche und mündliche Texte Erwachsener berücksichtigt wurden und die daraus gezogenen Schlussfolgerungen über die am häufigsten gebrauchten Wörter der italienischen Sprache für Erwachsene zutreffen, für Kinder oder Jugendliche aber nicht immer in gleicher Weise gelten. 
schläge bezüglich der Wortart mit der vom näheren Text- und Satzzusammenhang geforderten Wortart übereinstimmt. So wurde beispielsweise im Satzzusammenhang von „Già a livello europeo, anche per questo indicatore, si possono osservare forti differenze tra i paesi primi in classifica [...]" (Carazzi \& Pizzetti 2010, S. 55; m.Ü.: Schon auf europäischer Ebene lassen sich auch für diesen Indikator große Unterschiede zwischen den in der Rangliste obersten Ländern beobachten [...]) keine Präposition vorgeschlagen, sondern verschiedene Adjektive. Diese müssten zum einen nach dem folgenden Demonstrativadjektiv questo (dieser, hier: diesen) stehen und sind zum anderen durch den Satzbau ausgeschlossen, weil sie nur auftreten könnten, wenn questo indicatore das Subjekt wäre, was es aber unmöglich ist (und auch demjenigen auffallen müsste, dem nicht alle Wortbedeutungen des Satzes bekannt sind), da das konjugierte Verb in der dritten Person Plural steht.

Ferner ergänzten vier Lernende das Adverb co-me (wie) nicht richtig, das hier ein Beispiel einleitet, so wie es auf die gleiche Weise auch im Deutschen geschieht (wie zum Beispiel). Zwei Testpersonen wählten die Präposition con (mit), welche semantisch sehr nah bei der geforderten liegt und für die „Lexikogrammatik" der Betroffenen, ihr grammatikalisches und lexikalisches Verständnis des Zweitsprachenstadiums, in dem sie sich befinden, richtig klingen mochte (vgl. Roth 2005, S. 81; Halliday 2007).

Ebenso ist möglich, dass der Vorschlag con von muttersprachlichen SchülerInnen produziert wurde, die den Satzzusammenhang nicht im Blick behielten und sich bei der Wahl nur auf den unmittelbaren Textkontext weniger Wörter konzentrierten.

\subsection{Häufige Falschlösungen in den ersten beiden C-Testtexten an der Vespucci}

Im Folgenden wird ein Überblick über die restlichen elf Wörter gegeben, die oft falsch ergänzt erscheinen, nämlich mindestens sechsmal. Sie verteilen sich zu gleichen Anteilen auf drei Sätze, die in der nächsten Abbildung mit ihren Übersetzungen aufgeführt sind, wobei hinter den zu ergänzenden Wörtern (fettgedruckt) in Klammern die Anzahl der Falschlösungen angegeben ist. Außerdem sind zur übersichtlicheren Veranschaulichung die häufigen Falschlösungen markiert worden.

1. Questo indicatore [3 +3 acc.] non ci [5] dice però [9] nulla sulle [1 + 1 acc.] disuguaglianze in [4] termini di [2] ricchezza, cioè [11] sul benessere [7] dei singoli cittadini; [...]

(M.Ü.: Dieser Indikator sagt uns jedoch nichts über die Ungleichheiten bezüglich des Wohlstands, d. h. über das Wohlbefinden der einzelnen Bürger; [...])

2. Già a [9] livello europeo [0], anche per [4] questo indicatore [2 + 2 acc.] si possono [2] osservare forti [2 + 2 acc.] differenze tra [3] i paesi [2] primi in [0] classifica, come [4] per esempio [2] la Danimarca [0] con più [3] di 57261 dollari [2] l'anno a persona [6], e le [3] ultime posizioni [3] 
occupate dalla [6] già citata [9] Moldova (1 248 dollari [2]) e dalla [3] Macedonia (3 659 dollari [2 $1 / 2])$.

(M.Ü.: Schon auf europäischer Ebene lassen sich auch für diesen Indikator große Unterschiede zwischen den in der Rangliste obersten Ländern beobachten, wie zum Beispiel Dänemark mit mehr als 57261 Dollar pro Person, und den letzten von dem schon genannten Moldawien (1 248 Dollar) und Mazedonien (3 659 Dollar) eingenommenen Positionen.)

3. Ma su [9] scala internazionale [9] le distanze [8] si fanno [3] ancora più [4] grandi: [...] (M.Ü.: Aber auf internationaler Ebene/weltweit werden die Abstände noch größer: [...])

Abbildung 4: Sätze auf Seite 55 des Lehrbuchs von Carazzi und Pizzetti (2010) mit den häufigsten Fehlern

Im ersten Satz, der nur bis zum Semikolon abgebildet ist, da er das C-TestEnde formt und sich nach dem Semikolon keine zu ergänzenden Lücken mehr befinden, gibt es sieben Wörter zu vervollständigen, von denen nur drei von allen richtig gelöst wurden und vier häufig falsch. Das allein sagt schon aus, dass dieser Satz den Lernenden Schwierigkeiten bereitete. Das richtige Vervollständigen der ersten Lücken könnte die Tilgung des Subjekts (indicatore) erschwert haben, die das Satzverständnis verkompliziert. Der erste vermehrt auftretende Fehler (fünfmal), die richtige Vervollständigung zu ci (uns), mag daher rühren, dass die SchülerInnen in einem Lehrbuchtext nicht mit einem Personalpronomen der ersten Person Plural rechneten, obwohl zu Beginn des Tests, in den beiden Sätzen, welche dem hier untersuchten vorausgehen, je ein nicht getilgtes Verb in der ersten Person Plural konjugiert ist. Dass sich die fehlenden oder falschen Lösungen der ersten beiden Lücken gegenseitig bedingten, ist nicht anzunehmen, da neun von zehn Testteilnehmern für però per ergänzten, was die Vermutung sehr nahelegt, dass sie bei der Bearbeitung mit einer Art Scheuklappenblick nur den ganz unmittelbaren Kontext beachteten, der durch das folgende nulla gegeben ist. Zusammen formen sie als per nulla ein adverbiales Phrasem, das wahrscheinlich allen bekannt war, auch wenn es dem allgemeinen Gebrauchswortschatz (CO) entstammt. Dass dieses Phrasem morphosyntaktisch fehl am Platz ist, fiel dabei vermutlich niemandem auf; keiner scheint kontrolliert zu haben, ob sich diese Lösung im Satzzusammenhang vertreten lässt. Obwohl però nicht wie die meisten anderen Konjunktionen zum fundamentalen Gebrauchswortschatz zählt, sondern nur die Marke AU (hoher Gebrauch) trägt, sollte dies nicht bedeuten, dass sie den Testteilnehmern weniger geläufig war als andere Konjunktionen. Zumal schon unter jüngsten muttersprachlichen Kindern die tautologische Äußerungsweise ma però sehr verbreitet ist und oft von Erziehenden verbessert wird.

Es ist bemerkenswert, dass es sich beim nächsten, dem mit elf nicht korrekten Lösungen insgesamt am häufigsten falsch gelösten Wort um einen Aus- 
druck handelt, bezüglich dessen den Jugendlichen ein allzu exzessiver Gebrauch vorgeworfen wird: $c i-o \grave{e}$ (das heißt). ${ }^{144}$ Vielleicht ist aber genau das der Grund, warum so viele Lernende dieses Wort nicht wie im Originaltext ergänzten. Möglicherweise führte gerade die an ihnen geübte Kritik über den vehementen Gebrauch des Wortes dazu, dass sie sein Vorkommen in einem Lehrbuchtext nicht erwarteten. Fünf Testteilnehmer ließen die Wortlücke unbearbeitet, einer schlug stattdessen die Präposition circa vor, obwohl auch das Folgewort eine Präposition ist, und vier weitere ergänzten durch mehrgliedrige konjugierte Verbformen bzw. Pronomen und Verben gleich bis zu drei Wörter, obwohl es grammatikalisch-syntaktisch nicht funktionierte und sie vor dem Test angewiesen wurden, immer nur ein Wort zu ergänzen und nie zusätzlich ganz neue, vollständige Wörter in die Lücken zu schreiben. Ähnlich wie bei dem letzten, siebenmal falsch gelösten Wort dieses Satzes bestand die Schwierigkeit beim richtigen Ausfüllen aber wahrscheinlich darin, dass schon die vorherigen Lücken offengeblieben waren oder sich die Lernenden nicht sicher waren, ob sie die richtigen Lösungen gefunden hatten, so dass ihr Kontextverständnis zum Ende des Satzes sicherlich geschwächt war.

Im zweiten Satz wurde gleich die erste Lücke von neun Lernenden nicht richtig bearbeitet, allerdings liegt hier ein grober Fehler in der Testentwicklung vor: Versehentlich ist die Präposition $a$ getilgt worden, obwohl einbuchstabige Wörter bei der Tilgung zu übergehen sind, so dass eigentlich das Folgewort livello zur Hälfte hätte ausgelöscht werden müssen. Abgesehen von den Testteilnehmern, die dieses Hindernis gekonnt überwanden, schlugen sieben Lösungen vor, die in die richtige Richtung gehen, auch wenn sie falsch sind: $a l$ (5) und dal (2). Sie zeigen, dass ihnen das Adverbialphrasem a livello (auf ... Ebene) wahrscheinlich nicht unbekannt war, auch wenn sie es nicht ganz korrekt produzierten.

Während sechs Schüler bei per-sona gar nichts ergänzten (übersahen sie die Lücke oder kannten sie das BIP nur pro capite (pro Kopf) und nicht auch $a$ persona?), gibt es für die weiteren zwei häufig falsch gefüllten Lücken, die gemeinsam betrachtet werden sollten, zumindest einige nicht korrekte Lösungsvorschläge. Es handelt sich hier um eine recht komplizierte Satzkonstruktion, da nicht die Länder Moldawien und Mazedonien, welche die untersten Positionen in der Rangliste einnehmen, das Subjekt des Satzes formen, sondern die Unterschiede, die man zwischen den Positionen verschiedener Länder beobachten kann. Die beiden schlechter bestellten Länder beziehen sich dabei als präpositionale Objekte auf das Partizip mit Adjektivfunktion, occupate, das zum Objekt posizioni gehört. Die Konstruktion hat also passivischen Charakter. Einige in der Zweitsprache Lernende bewiesen eine fortgeschrittene Sprachkompetenz, da ihre Lösungsvorschläge ein Indiz für ihr Wissen darüber sind, dass in solchen Fällen, bei denen sich zwei Objekte aneinanderreihen,

144 Vergleiche hierzu auch die Beispiele im mündlichen Sprachgebrauch der Lernenden im Kapitel 9 zu den Unterrichtsbeobachtungen sowie die Reaktion der Lehrkräfte hierauf. 
auch nur eine artikulierte Präposition und ein Adjektiv, beide dann im Plural, stehen können. Damit ist gemeint, dass der letzte Teil des Satzes auch wie folgt hätte formuliert werden können: le ultime posizioni dalle già citate Moldova e Macedonia. Allerdings ist diese Konstruktion durch die zu ergänzende artikulierte Präposition vor Macedonia ausgeschlossen. Außerdem ist das zweite Land noch nicht genannt worden, weshalb citate logisch falsch ist.

Abschließend wird der letzte Satz dieser ersten beiden C-Testtexte betrachtet, von dessen fünf Lücken zwei drei- und viermal und die anderen drei achtbis neunmal falsch ergänzt wurden. Diese drei Lücken befinden sich gleich am Satzanfang, der nicht mit dem Subjekt beginnt, sondern es mit einer Konjunktion und einem präpositionalen Objekt einleitet. Die Schwierigkeit dieser selteneren Konstruktionsart dürfte sich aber in Grenzen halten, zumal es sich um die Präposition $m-a$ (aber) handelt, die sehr häufig verwendet wird. Weitaus problematischer scheinen hierbei das adverbiale Phrasem s-u scala (auf ... Ebene) und das dazugehörige Adjektiv interna-zionale zu sein. Letzteres sollte allen bekannt gewesen sein, wurde aber trotzdem falsch ergänzt, was vermutlich daran liegt, dass einigen das Bezugswort unklar war: Von den neun Lernenden, die keine korrekte Lösung für $s-u$ anboten, schrieben drei gar nichts und ergänzten fünf zu sulla. Das ist nicht völlig abwegig, zeigt aber, dass sie das Phrasem, welches voraussetzt, dass seine Bestandteile nicht modifiziert werden, nicht oder nicht ausreichend kannten.

Es ist gut möglich, dass den Lernenden die Bearbeitung der letzten häufig falsch gelösten Lücke leichter gefallen wäre, wenn im Text wieder differenze (Unterschiede) und nicht das bedeutungsähnliche Wort dist-anze (Abstände) stünde. „Abstände“ passt zwar besser, da sich die Autoren immer noch auf die Positionen innerhalb der Länderrangliste beziehen, jedoch betrifft die Kernaussage des Textes eben genau die unterschiedlichen Situationen zwischen den einzelnen Ländern. Auch der Lösungsvorschlag distinzioni (Unterscheidung, Unterschied) liegt semantisch unweit der anderen beiden Wörter, ist jedoch nicht akzeptabel. Wie auch bereits in anderen Fällen, ist aber erneut zu betonen, dass die richtige Lösung schon allein dadurch erschwert war, dass die vorausgehenden Lücken vielleicht gar nicht oder falsch vervollständigt wurden, so dass für die entsprechenden Testteilnehmer das Verständnis der Satzaussage beeinträchtigt war.

\subsubsection{Texte 3 und 4 des C-Tests (S. 56)}

Die nächsten beiden Texte des an der Schule Vespucci durchgeführten C-Tests sind der Seite 56 des Lehrbuchs (Carazzi \& Pizzetti 2010) entnommen und beschäftigen sich mit dem unterschiedlichen Wohlstand in verschiedenen Ländern und dem Human Development Index. Zunächst ermöglicht Tabelle 9 einen Überblick über ihren sprachlichen Inhalt und die Lösungsvorschläge. 
Es sei vorweggenommen, dass auch hier wieder zwei Konstruktionsfehler bei der Testentwicklung unterliefen, von denen der erste den Schülern anscheinend keine Schwierigkeiten bereitete: Es hätte die einfache Präposition con (mit) zur Hälfte getilgt werden müssen, stattdessen betrifft die Tilgung erst das folgende Wort, den unbestimmten Artikel $u$ - $n$. Lediglich ein Testteilnehmer vervollständigte ihn nicht.

Der zweite Tilgungsfehler ist gravierender, da er verursacht, dass zwei aufeinanderfolgende Wörter ergänzt werden müssen. Immerhin ist das falsch getilgte Wort eine apostrophierte Zusammensetzung aus einer artikulierten Präposition und einem Indefinitpronomen (nell'al-tro, im anderen), so dass der erste Teil bis zum Apostroph vollständig wiedergegeben ist und nur der zweite Teil zur Hälfte gelöscht wurde. Bei der Besprechung der häufigeren Fehler wird diese Unregelmäßigkeit berücksichtigt.

\begin{tabular}{|c|c|c|c|c|c|}
\hline Fehler & Wort & Wortart & Marke & Kommentar & Lösungsvorschläge \\
\hline $\begin{array}{l}\text { Text } 3 \\
24 \\
\text { Lücken }\end{array}$ & Due & agg.num.card. & FO & & \\
\hline \multirow[t]{2}{*}{2} & paesi & s.m. & FO & & parti (2) \\
\hline & con & prep. & FO & & \\
\hline \multirow[t]{2}{*}{0} & 10 & art.det. & FO & & \\
\hline & stesso & agg.dimostr. & FO & & \\
\hline \multirow[t]{2}{*}{0} & reddito & s.m. & AU TS econ. & & \\
\hline & medio & agg. & $\mathrm{AU}$ & & \\
\hline \multirow[t]{2}{*}{1} & per & prep. & FO & & 0 \\
\hline & abitante & s.m. & $A U$ & & \\
\hline \multirow[t]{2}{*}{1} & possono & v.mod. & FO & & 0 \\
\hline & presentare & inf.v.tr. & FO & & \\
\hline \multirow[t]{2}{*}{1} & situazioni & s.f. & FO & & 0 \\
\hline & completamente & avv. & $\mathrm{CO}$ & & \\
\hline \multirow[t]{2}{*}{2} & diverse & agg. & FO & & diversi, 0 \\
\hline & in & prep. & FO & & \\
\hline \multirow{2}{*}{$\begin{array}{l}2+3 \\
\text { acc. }\end{array}$} & uno & pron.indef. & FO & & una (acc.) (3), un, 0 \\
\hline & $v i$ & avv. & FO & & \\
\hline \multirow[t]{2}{*}{4,5} & può & v.mod. & FO & & puo, possono (3), 0 \\
\hline & essere & $\begin{array}{l}\text { inf.v.pro- } \\
\text { compl. }\end{array}$ & $\mathrm{CO}$ & & \\
\hline \multirow[t]{2}{*}{0} & una & art.indet. & FO & & \\
\hline & distribuzione & s.f. & $A U$ & & \\
\hline \multirow[t]{2}{*}{0} & della & prep.art. & $\mathrm{FO}$ & & \\
\hline & ricchezza & s.f. & TS econ. & & \\
\hline \multirow[t]{3}{*}{4} & molto & avv. & $\mathrm{FO}$ & & 0 , mondiale (3) \\
\hline & squilibrata & agg. & $\mathrm{CO}$ & & \\
\hline & con & prep. & FO & & \\
\hline \multirow[t]{2}{*}{0} & un & art.indet. & FO & Tilgungsfehler & \\
\hline & ristretto & agg. & $\mathrm{CO}$ & & \\
\hline \multirow[t]{2}{*}{1} & numero & s.m. & FO & & numerabile \\
\hline & $d i$ & prep. & FO & & \\
\hline
\end{tabular}




\begin{tabular}{|c|c|c|c|c|c|}
\hline Fehler & Wort & Wortart & Marke & Kommentar & Lösungsvorschläge \\
\hline \multirow[t]{2}{*}{4} & ricchi & s.m. & FO & & $\begin{array}{l}\text { ricchezze, ricchezza } \\
\text { (3) }\end{array}$ \\
\hline & $e$ & cong. & $\mathrm{FO}$ & & \\
\hline \multirow[t]{2}{*}{0} & un & art.indet. & $\mathrm{FO}$ & & \\
\hline & elevato & agg. & $\mathrm{CO}$ & & \\
\hline \multirow[t]{2}{*}{0} & numero & s.m. & $\mathrm{FO}$ & & \\
\hline & $d i$ & prep. & $\mathrm{FO}$ & & \\
\hline 2 & poveri & s.m. & FO & & povertà $(2)$ \\
\hline \multirow[t]{2}{*}{$\begin{array}{l}6+2 \\
\text { acc. }\end{array}$} & nell'altro & pron.indef. & $\mathrm{FO}$ & Tilgungsfehler & $\begin{array}{l}0 \text { (2), nell'altra (6) (2 } \\
\text { acc. in una, nell'altra) }\end{array}$ \\
\hline & la & art.det. & FO & & \\
\hline \multirow[t]{2}{*}{3} & distribuzione & s.f. & $A U$ & & $\begin{array}{l}0 \text {, distrizione, distritri- } \\
\text { buzione }\end{array}$ \\
\hline & può & v.mod. & $\mathrm{FO}$ & & \\
\hline \multirow[t]{2}{*}{0} & essere & inf.v.intr. & $\mathrm{FO}$ & & \\
\hline & più & avv. & $\mathrm{FO}$ & & \\
\hline \multirow[t]{2}{*}{0} & equilibrata & agg. & $\mathrm{CO}$ & & \\
\hline & senza & avv. & FO & & \\
\hline \multirow[t]{2}{*}{3 acc. } & grandi & agg. & $\mathrm{FO}$ & & gravi (acc.) (3) \\
\hline & disuguaglianze & s.f. & $\mathrm{CO}$ & & \\
\hline \multirow[t]{2}{*}{5} & nei & prep.art. & $\mathrm{FO}$ & & $\begin{array}{l}\text { ne, numerabili, nu- } \\
\text { merosi, } 0 \text { (2) }\end{array}$ \\
\hline & livelli & loc.s.m. & $\mathrm{CO}$ & & \\
\hline \multirow[t]{2}{*}{3} & $d i$ & el.loc.s.m. & el.CO & & $0(3)$ \\
\hline & vita & el.loc.s.m. & el.CO & & \\
\hline \multicolumn{6}{|l|}{$\begin{array}{l}4 \\
\text { (S. 56) }\end{array}$} \\
\hline $\begin{array}{l}25 \text { Lü- } \\
\text { cken }\end{array}$ & il & art.det. & $\mathrm{FO}$ & & \\
\hline \multirow[t]{2}{*}{8} & primo & s.m. & $\mathrm{FO}$ & & $\begin{array}{l}\text { pro capite (4), pro- } \\
\text { blema, prodotto (2), } \\
0\end{array}$ \\
\hline & $\grave{e}$ & v.intr. & $\mathrm{FO}$ & & \\
\hline \multirow[t]{2}{*}{2} & la & art.det. & FO & & l'indice, l'isu \\
\hline & speranza & loc.s.f. & TS stat. & & \\
\hline 0 & $\begin{array}{l}\text { di } \\
\text { vita }\end{array}$ & $\begin{array}{l}\text { el.loc.s.f. } \\
\text { el.loc.s.f. }\end{array}$ & $\begin{array}{l}\text { el.TS stat. } \\
\text { el.TS stat. }\end{array}$ & & \\
\hline \multirow[t]{2}{*}{$\begin{array}{l}5+1 \\
\text { acc. }\end{array}$} & indicatore & s.m. & TS econ. & & $\begin{array}{l}\text { indica (2), indica ciò, } \\
\text { indicano, indicatrice, } \\
\text { indice (acc.) }\end{array}$ \\
\hline & che & pron.rel. & $\mathrm{FO}$ & & \\
\hline \multirow[t]{3}{*}{1,5} & già & avv. & $\mathrm{FO}$ & & gia, 0 \\
\hline & conosciamo & v.tr. & $\mathrm{FO}$ & & \\
\hline & $e$ & cong. & $\mathrm{FO}$ & & \\
\hline \multirow[t]{2}{*}{3} & che & pron.rel. & FO & & 0 , ciò, con \\
\hline & misura & v.tr. & $\mathrm{FO}$ & & \\
\hline \multirow[t]{2}{*}{1} & il & art.det. & $\mathrm{FO}$ & & in \\
\hline & numero & s.m. & $\mathrm{FO}$ & & \\
\hline \multirow[t]{2}{*}{1 acc. } & $d i$ & prep. & $\mathrm{FO}$ & & degli (acc.) \\
\hline & anni & s.m. & $\mathrm{FO}$ & & \\
\hline \multirow[t]{2}{*}{4} & che & pron.rel. & $\mathrm{FO}$ & & che ci sono, 0 (3) \\
\hline & in & loc.avv. & $\mathrm{CO}$ & & \\
\hline
\end{tabular}




\begin{tabular}{|c|c|c|c|c|c|}
\hline Fehler & Wort & Wortart & Marke & Kommentar & Lösungsvorschläge \\
\hline \multirow[t]{2}{*}{6} & media & el.loc.avv. & el.CO & & mesi (2), 0 (3), meno \\
\hline & può & v.mod. & FO & & \\
\hline \multirow[t]{2}{*}{7} & aspettarsi & $\begin{array}{l}\text { inf.v.pronom. } \\
\text { intr. }\end{array}$ & $\mathrm{CO}$ & & $\begin{array}{l}\text { aspetta, aspettarla, } \\
0 \text {, aspettare }(4)\end{array}$ \\
\hline & $d i$ & prep. & FO & & \\
\hline \multirow[t]{2}{*}{1} & vivere & inf.v.intr. & FO & & 0 \\
\hline & un & art.indet. & FO & & \\
\hline \multirow[t]{2}{*}{6} & individuo & s.m. & $\mathrm{AU}$ & & $\begin{array}{l}\text { indicato, indicatore } \\
\text { (3), indice, } 0\end{array}$ \\
\hline & nato & part.pass.v.tr. & FO & & \\
\hline \multirow[t]{2}{*}{1} & in & prep. & FO & & 0 \\
\hline & un & art.indet. & FO & & \\
\hline \multirow[t]{3}{*}{1} & determinato & agg. & $\mathrm{CO}$ & & determrminato \\
\hline & anno. & s.m. & FO & & \\
\hline & $A$ & prep. & FO & & \\
\hline \multirow[t]{2}{*}{9} & ciò & pron.dimostr. & FO & & $\begin{array}{l}\text { cui ( } 2), 0 \text { (4), chi, } \\
\text { che, caso }\end{array}$ \\
\hline & si & pron.pers. & $\mathrm{CO}$ & & \\
\hline \multirow[t]{2}{*}{9} & aggiunge & v.pronom.intr. & FO & & $\begin{array}{l}\text { aggisce }(2) \text {, aggiun- } \\
\text { gere, } 0(4) \text {, aggiun- } \\
\text { gono }(2)\end{array}$ \\
\hline & il & art.det. & $\mathrm{FO}$ & & \\
\hline \multirow[t]{2}{*}{1} & dato & s.m. & $A U$ & & 0 \\
\hline & relativo & agg. & $\mathrm{AU}$ & & \\
\hline \multirow[t]{2}{*}{2} & al & prep.art. & FO & & $0(2)$ \\
\hline & livello & s.m. & FO fig. & & \\
\hline \multirow[t]{2}{*}{1 acc. } & $d i$ & prep. & FO & & d' (acc.) \\
\hline & istruzione & s.f. & $\mathrm{AU}$ & & \\
\hline \multirow[t]{2}{*}{1} & della & prep.art. & $\mathrm{FO}$ & & 0 \\
\hline & popolazione & s.f. & $\mathrm{AU}$ & & \\
\hline \multirow[t]{2}{*}{7} & Considerando & ger.v.tr. & FO & & $\begin{array}{l}\text { considerato, consi- } \\
\text { dera, consideriamo } \\
(2) \text {, considerata (2), } \\
0\end{array}$ \\
\hline & $\begin{array}{l}\text { contemporanea- } \\
\text { mente }\end{array}$ & avv. & $\mathrm{CO}$ & & \\
\hline \multirow[t]{2}{*}{2} & reddito & s.m. & $\begin{array}{l}\text { AU TS } \\
\text { econ. }\end{array}$ & & $0(2)$ \\
\hline & speranza & loc.s.f. & TS stat. & & \\
\hline \multirow[t]{3}{*}{1} & $d i$ & el.loc.s.f. & el.TS stat. & & 0 \\
\hline & vita & el.loc.s.f. & el.TS stat. & & \\
\hline & $e$ & cong. & $\mathrm{FO}$ & & \\
\hline 1 & istruzione. & s.f. & $A U$ & & 0 \\
\hline
\end{tabular}

Tabelle 9: Übersicht über die Texte 3 und 4 des C-Tests an der Vespucci inklusive Lösungsvorschläge

Unter den 50 zu komplettierenden Lücken befinden sich nur zehn, die von allen SchülerInnen wie im Originaltext oder durch einen annehmbaren Alternativvorschlag ergänzt wurden. Die höchste Fehlerquote für ein Wort liegt bei neun (cio), doch vor der Analyse der häufigsten Falschlösungen soll erneut zuerst ein Überblick über die geringeren Fehlerquellen erfolgen. Zwei Fünftel (20 
Wörter) wurden von einem oder zwei Testteilnehmern nicht oder falsch ergänzt. Mit wenigen Ausnahmen handelt es sich um Wörter aus dem Bereich des fundamentalen Wortschatzes (Fo), unter denen von einfachen und artikulierten Präpositionen über Artikel, Adjektive und Substantive bis hin zu konjugierten Verben und Infinitiven alle Wortarten vertreten sind. So zum Beispiel ein bestimmter Artikel, der interessanterweise von zwei Lernenden apostrophiert und mit einem Substantiv ergänzt wurde, obwohl das nominale Bezugswort im Text sofort nach der Lücke folgt. Oder eine artikulierte Präposition, die im näheren Kontext zusammen mit livello auftritt und korrekt relativo a-l livello (bezüglich des Grades) lautet. Dadurch könnte es sein, dass die zwei fehlenden Vervollständigungen absichtlich erfolgten, weil die Lernenden an den oben genannten Phraseologismus a livello dachten oder mit relativo a (bezüglich) nicht so vertraut waren.

Die Gruppe der Wörter, die nicht zum fundamentalen Wortschatz zählen, besteht aus fünf: das Substantiv da-to (Datenangabe) aus dem Wortschatz des hohen Gebrauchs (AU), das im Erdkundelehrwerk öfter verwendet wird, das Adjektiv deter-minato (CO; bestimmt), das in einem Fall mit einem Flüchtigkeitsfehler ergänzt wurde (Wiederholung der beiden letzten nicht getilgten Buchstaben), das Phrasemelement $d-i$ aus speranza $d-i$ vita (Ts stat.; Lebenserwartung), das von einem Lernenden nicht vervollständigt wurde, was auch für das Nomen istru-zione (AU; Bildung) gilt. Hierbei handelt es sich um die Wiederaufnahme eines im vorausgehenden Satz verwendeten Begriffs in gekürzter Form. Und zwar taucht er dort ungetilgt im Ausdruck livello d-i istruzione (Bildungsgrad) auf, was das Schließen der Lücke beim zweiten Token des Worttyps erleichterte. Beim letzten Wort handelt es sich um das doppelt gekennzeichnete red-dito (AU TS econ.; Einkommen), das mit zwei getilgten Vorkommnissen in diesem Text des C-Tests erscheint. Auffällig an diesem Wort ist, dass das erste Vorkommnis von allen Teststeilnehmern korrekt und das zweite zweimal nicht vervollständigt wurde. Das bedeutet, dass zwei Testpersonen ein Wort nicht ergänzten, das sie zuvor richtig gelöst hatten. Als möglicher Erklärungsgrund könnte die Distanz zwischen den beiden Tokens herangezogen werden, da sie sich jeweils am Anfang und am Ende des Textes befinden. Allerdings resümiert das Textende die zuvor gegebenen Informationen in Form einer Aufzählung, woraus auch geschlossen werden könnte, dass die Lernenden die vorgestellten Konzepte noch nicht voll und ganz verstanden hatten. Nachteilig könnte sich außerdem das sprachliche Umfeld des zweiten Vorkommnisses ausgewirkt haben, da reddito zwar weit vorne im Satz steht, dieser aber von dem getilgten Gerundium considerando (Fo; betrachtend) eingeleitet wird, mit dem sieben Testteilnehmer Schwierigkeiten zeigten.

Ohne bei den fundamentalen Wörtern auf jeden einzelnen Artikel oder einfache Präpositionen und Rechtschreibfehler wie das Auslassen eines Akzents eingehen zu wollen (s. hierfür Tabelle 9), seien an dieser Stelle nur einige Fehlerquellen kommentiert, die für die Untersuchungsgruppe mit dem in dieser 
Studie größten Anteil an in der Zweitsprache lernenden Schülerinnen und Schülern typisch sein könnten und sprachliche Auffälligkeiten des Lehrbuchs betreffen. Denn es handelt sich um hochfrequent gebrauchte, einfache Wörter, die auch den in der Zweitsprache Lernenden bekannt sind, die sich jedoch in ein sprachlich komplex gestaltetes Umfeld einbetten, für dessen Verständnis die Textkompetenz einiger Lernender anscheinend nicht ausreichte.

So wurde das Adjektiv dive-rso (verschieden), das sich auf situazioni bezieht und durch ein Adverb (completamente, vollständig) verstärkt wird, von einem Lernenden nicht und von einem weiteren mit einer falschen Genus-Endung ergänzt, was wahrscheinlich daran liegt, dass situazioni zwar wie die maskulinen Pluralwörter auf $i$ endet, aber feminin ist. Es ist anzunehmen, dass dieser Fehler von einem Schüler oder einer Schülerin begangen wurde, für den/die Italienisch nicht die Muttersprache ist, obgleich derartige Fehler durchaus auch in der Erstsprache auftreten können und ein Zeichen von Flüchtigkeit in der schriftlichen Sprachproduktion sind.

$\mathrm{Zu}$ Beginn des dritten Textes vervollständigte ein Testteilnehmer das Verb poss-ono (können) und die darauffolgende Lücke situa-zioni (Situation) nicht. Wie bereits erwähnt, geht es um die weltweite Verteilung des Wohlstands, wobei die wirtschaftliche Lage in zwei Ländern bei gleichem Pro-Kopf-Einkommen unterschiedlich sein kann (wörtliche Übersetzung: Zwei Länder mit dem gleichen Pro-Kopf-Einkommen können unterschiedliche Situationen aufweisen). Hierbei wird situazioni aus dem vorausgehenden Satz, der diesen C-Testtext einleitet und deshalb komplett ohne Lücken abgebildet ist, ohne das Adjektiv wirtschaftlich wieder aufgegriffen, so dass ein mangelndes Ausfüllen der Lücke einerseits auf eine schwach ausgebildete Textkompetenz deuten lässt, andererseits aber durch die Komplexität des Satzes verständlich erscheint. Das Subjekt des Satzes, due paesi (zwei Länder), besteht mit seinem mehrgliedrigen Attribut aus neun Wörtern und ist nicht konkret, sondern abstrakt. Ihm folgt ein Verbgefüge aus dem zu ergänzenden Modalverb possono und einem Infinitiv (presentare), was ebenfalls weniger konkret ist, als es bei der Satzformulierung möglich gewesen wäre, und ein Akkusativobjekt (situazioni). Der Satz wäre wahrscheinlich gerade für SchülerInnen, die in der Zweitsprache lernen, einfacher zu verstehen, wenn der eigentliche Aktant des Satzes (die unterschiedlichen wirtschaftlichen Situationen in Ländern mit gleichem Einkommen) auch das Subjekt wäre. Dazu hätte man den mit situazioni endenden ersten Satz durch einen Relativsatz mit einem einfacheren konjugierten Verb verlängern können.

Abgesehen von der gerade beschriebenen Komplexität des Satzgefüges mit einem gewissen Grad an Abstraktheit ist es mit seinen 53 Wörtern auch sehr lang und erklärt nach einem Doppelpunkt die Unterschiede zwischen den Ländern. Diese Erläuterung beginnt mit einem präpositionalen Objekt, das mit einem Indefinitpronomen gebildet ist, von dessen Referenzwort paesi es 13 Wörter entfernt liegt. Es handelt sich um ein getilgtes Pronomen, das nicht nur in 
der im Originaltext gegebenen maskulinen Form ergänzt wurde, sondern u.a. auch in einer akzeptablen femininen, da als weiteres Bezugswort situazioni in Frage kommt. Es folgt erneut ein Verbgefüge, das aus dem Modalverb können und dem aus dem Co-Gebrauchswortschatz stammenden prokomplementarischen (pronominalen) Infinitiv esservi (da sein, geben) gebildet ist, der eine im Gebrauch seltenere Alternative zum frequenteren esserci (Fo) darstellt. Desweiteren wird im ersten Teil der Erklärung nach dem Doppelpunkt mit einem präpositionalen Objekt die unausgeglichene Verteilung des Wohlstands näher beschrieben: „, con un ristretto numero di ricchi e un elevato numero di poveri“ (Carazzi \& Pizzetti 2010, S. 65; m.Ü.: mit einer beschränkten Anzahl von Reichen und einer hohen Anzahl von Armen). Num-ero wurde dabei einmal zu einem Nomen aus dem mathematischen Fachwortschatz ergänzt, das als Lösung im Textkontext nicht annehmbar ist. Dass es Probleme mit dem vertrauten Wort numero gab, könnte durchaus an der Satzlänge und an den vorausgehenden Adjektiven liegen, die beide nicht zum Basiswortschatz des Italienischen gehören. Außerdem wurde das italienische Wort für Reiche in vier Fällen vielleicht daher zu Reichtum/Wohlstand ergänzt, dass dieses Wort mit zwei Vorkommnissen zuvor im Text vertreten ist (im Einleitungssatz und in unmittelbarer Nähe in der Erklärung nach dem Doppelpunkt). Folgerichtig wurde dann auch pov- nicht zu poveri (Arme), sondern povertà (Armut) vervollständigt. Die Lernenden könnten das Wort aus dem mathematischen Fachwortschatz und die beiden zuletzt genannten Nomen auch deshalb gewählt haben, da sie von der etwas geschwollenen Ausdrucksweise und den normaleren bildungssprachlichen Eigenheiten des Textes so beeinflusst waren, dass sie versuchten, eigenständig sprachlich Vergleichbares einzusetzen. Allerdings sind ihre Vorschläge zwar grammatikalisch und syntaktisch richtig, aber semantisch und logisch nicht. ${ }^{145}$ Hier lassen sich deutliche Anzeichen dafür erkennen, dass das Textverständnis erschwert oder sogar verhindert wird, wenn eine überdurchschnittliche Satzlänge, abstrakte Subjekte und Verben und ein gewählter, seltener gebrauchter Wortschatz miteinander korrelieren.

Je drei- bis viermal falsch oder gar nicht aufgelöst wurden die Lücken in sieben Wörtern, von denen sechs Teil des soeben besprochenen langen Satzgefüges sind. In diesem Satz, bei dem mit erhöhten Verständnisschwierigkeiten gerechnet werden darf, gab es Probleme mit dem Wort distri-buzione (Verteilung), das aus dem Wortschatzbereich des hohen Gebrauchs (AU) stammt und von einem Testteilnehmer gar nicht, von einem flüchtig mit der Wiederholung einiger Konsonanten zu distritribuzione und von einem weiteren zu distrizione ergänzt wurde. Dieses Wort existiert im Italienischen nicht; es wurde vielleicht mit restrizione (Einschränkung, Verringerung) verwechselt. Auch das Adjektiv mo-lto (Fo; viel) wurde von vier Lernenden nicht korrekt ergänzt. Das legt nahe, dass ihnen das fachwortschatzsprachliche Bezugswort

145 Das würde im Übrigen eine generell beobachtete Tendenz der schriftlichen Sprachproduktion in Italien Lernender ab dem dritten Jahr der Mittelstufe widerspiegelt (s. Lavinio 2011). 
ricchezza (Wohlstand) nicht ausreichend bekannt war, da sie entweder gar nicht oder zum logisch falschen mondiale (weltweit) ergänzten. Ein zweiter möglicher Erklärungsversuch für das Adjektiv betrifft das im Vorfeld verwendete Indefinitpronomen uno (eines) für das weit entfernt stehende Subjekt due paesi (zwei Länder): Es ist durchaus möglich, dass den Schülern nicht erkenntlich war, worauf es sich bezieht und sie deshalb nicht zögerten, das in diesem Kontext logisch falsche mondiale zu bilden.

Darüber hinaus ergänzten drei Schüler die einfache Präposition di (von) im nominalen Phraseologismus livelli d-i vita (Lebensstandard) nicht, was darauf hindeutet, dass ihnen dieser Ausdruck aus dem allgemeinen Wortschatz (CO) nicht bekannt war.

Außerdem gab es offensichtlich Schwierigkeiten mit dem Modalverb può im bereits angesprochenen Verbgefüge vi p-uò essere (una distribuzione) (es kann (eine Verteilung) geben), in dem es mit einem seltener gebrauchten Verb des allgemeinen Wortschatzes auftritt. Zwei Lernende ergänzten es in der Pluralform, zwei weitere gar nicht und ein/e SchülerIn vergaß lediglich den Akzent. Zum anderen handelt es sich um das ebenfalls schon besprochene substantivierte Adjektiv ric-chi (Reiche).

Schließlich wurde das Relativpronomen $c$-he gar nicht bzw. unpassend zum Demonstrativpronomen ciò (das) und zur einfachen Präposition con (mit) ergänzt. Obwohl ein Relativsatz, in dem das Relativpronomen das Subjekt des Satzes bildet, sicherlich zu den leichteren Nebensätzen zählt, ist dieser Satz wahrscheinlich für die sprachlich nicht so Versierten doch schwieriger, da es der zweite von zwei nebengeordneten Relativsätzen ist, die sich nicht direkt auf das Subjekt (speranza di vita, Lebenserwartung), sondern auf sein Attribut (indicatore, Indikator) beziehen.

\subsection{Häufigste Falschlösungen}

In den C-Testtexten 3 und 4 wurden insgesamt neun Wörter häufig falsch gelöscht. Über die Hälfte (5) gehört zu den am meisten gebrauchten Wörtern des Italienischen (Gebrauchsmarke Fo), zwei zählen zum Basiswortschatz (AU), eines zum allgemeinen Wortschatz (CO) und ein weiteres zum Fachwortschatz (Ts). Das bedeutet nicht automatisch, dass den Schülern die 2.000 häufigsten Wörter des Italienischen nicht bekannt waren und sie diese nicht korrekt verwenden konnten, sondern es kann vielmehr als ein Indiz dafür betrachtet werden, dass in den Lehrbuchtexten eine verdichtete Sprache verwendet wird, in der kompliziertere syntaktische Strukturen das Textverständnis erschweren. Bevor dies im Einzelnen gezeigt wird, gibt Tabelle 10 zunächst einen Überblick über alle Ausdrücke, die von mindestens fünf Lernenden gar nicht oder nicht richtig vervollständigt wurden. Sowohl die Fehler 4-6 als auch die Fehler 7-9 liegen so dicht beieinander, dass sie in der Tabelle zum besseren Verständnis mit ihrem sprachlichen Umfeld aufgelistet sind. 


\begin{tabular}{|c|c|c|c|c|c|c|}
\hline & $\begin{array}{l}\mathrm{n} \\
\text { Fehler }\end{array}$ & Wort & Wortart & $\begin{array}{l}\text { Gebrauchs- } \\
\text { marke }\end{array}$ & $\begin{array}{l}\text { Kom- } \\
\text { mentar }\end{array}$ & Lösungsvorschläge \\
\hline 1) & 5 & nei & prep.art. & $\mathrm{FO}$ & & $\begin{array}{l}\text { ne, numerabili, nu- } \\
\text { merosi, } 0 \text { (2) }\end{array}$ \\
\hline 2) & $\begin{array}{l}5+2 \\
\text { acc. }\end{array}$ & nell'altro & pron.indef. & FO & $\begin{array}{l}\text { Tilgungs- } \\
\text { fehler }\end{array}$ & $\begin{array}{l}0 \text { (3), nell'altra (4) (2 } \\
\text { acc. in una, nell'altra) }\end{array}$ \\
\hline \multirow[t]{3}{*}{ 3) } & $\begin{array}{l}5+1 \\
\text { acc. }\end{array}$ & indicatore & s.m. & TS econ. & & $\begin{array}{l}\text { indica (2), indica ciò, } \\
\text { indicano, indicatrice, } \\
0\end{array}$ \\
\hline & 4 & che & pron.rel. & FO & & che ci sono, 0 (4) \\
\hline & & in & loc.avv. & $\mathrm{CO}$ & & \\
\hline \multirow[t]{2}{*}{ 4) } & 6 & media & el.loc.avv. & el.CO & & mesi (2), 0 (4), meno \\
\hline & & può & v.tr. & $\mathrm{FO}$ & & \\
\hline \multirow[t]{4}{*}{ 5) } & 7 & aspettarsi & $\begin{array}{l}\text { inf.v.pro- } \\
\text { nom.intr. }\end{array}$ & $\mathrm{CO}$ & & $\begin{array}{l}\text { aspetta, aspettarla, } \\
0 \text {, aspettare }(4)\end{array}$ \\
\hline & & $d i$ & prep. & FO & & \\
\hline & 2 & vivere & v.intr. & FO & & $0(2)$ \\
\hline & & un & art.indet. & FO & & \\
\hline \multirow[t]{3}{*}{ 6) } & 6 & individuo & s.m. & $A U$ & & $\begin{array}{l}\text { indicato, indicatore } \\
\text { (3), indice, } 0\end{array}$ \\
\hline & & nato & agg. & FO & & \\
\hline & & $A$ & prep. & FO & & \\
\hline \multirow[t]{2}{*}{ 7) } & 9 & ciò & $\begin{array}{l}\text { pron.di- } \\
\text { mostr. }\end{array}$ & FO & & $\begin{array}{l}\text { cui }(2), 0(5), \text { chi, } \\
\text { che, caso }\end{array}$ \\
\hline & & si & pron.pers. & $\mathrm{CO}$ & & \\
\hline 8) & 9 & aggiunge & $\begin{array}{l}\text { v.pro- } \\
\text { nom.intr. }\end{array}$ & FO & & $\begin{array}{l}\text { aggisce }(2) \text {, aggiun- } \\
\text { gere, } 0(4) \text {, aggiun- } \\
\text { gono (2) }\end{array}$ \\
\hline 9) & 7 & $\begin{array}{l}\text { Conside- } \\
\text { rando }\end{array}$ & ger.v.tr. & FO & & $\begin{array}{l}\text { considerato, consi- } \\
\text { dera, consideriamo } \\
\text { (2), considerata, } 0 \\
\text { (2) }\end{array}$ \\
\hline
\end{tabular}

Tabelle 10: Häufige Fehler in den Texten 3 und 4 des C-Tests an der Vespucci

Dass sechs SchülerInnen die artikulierte Präposition nei nicht richtig vervollständigten, kann man sich abgesehen von Konzentrationsproblemen oder nachlässiger Testbearbeitung nur damit erklären, dass sie am Ende des langen Satzgefüges den Überblick über seinen Inhalt verloren hatten.

Beim zweiten häufigen Fehler wird die Satzstruktur mit dem Indefinitpronomen in uno (in einem) durch nell'altro (im anderen) fortgeführt. Hier wiegt der anfänglich genannte Konstruktionsfehler schwer, weil der Satz lang und kompliziert ist und einigen Lernenden wahrscheinlich schon das Bezugswort des ersten Indefinitpronomens nicht klar war. Drei Lernende ergänzten nichts und weitere vier wählten mit nell'altra (in der anderen) die einzige grammatikalisch korrekte Alternative. Das sagt etwas über ihre grammatikalische Kompetenz aus, aber noch nicht unbedingt über grammatikalisch-syntaktische Fähigkeiten und einen Blick, der über das nähere Textumfeld hinausschweift. Denn nur zwei von vier Schülern bezogen beide Indefinitpronomen auf situazioni und ergänzten sie deshalb mit der femininen Endung, während die anderen die beiden Pronomen mit unterschiedlichen Genus-Endungen ausstatteten bzw. das erste Pronomen gar nicht vervollständigten. 
Weitere häufige Falschlösungen (5) betreffen das bereits erwähnte Attribut zur Lebenserwartung, Indikator (indic-atore), welches zwei nebengeordneten Relativsätzen vorausgeht. Der Ausdruck indicatore stammt aus dem wirtschaftlichen Fachwortschatz und trat schon in den ersten Texten dieses C-Tests auf. Insgesamt vier Lernende vervollständigten das Wort vielleicht zu einer Verbform, weil ihnen der Gebrauch eines Attributs nicht so geläufig war, oder einfach, weil dem halb getilgten Nomen kein Artikel vorausgeht. Eine außergewöhnliche Falschlösung ist die nicht existierende weibliche Form indicatrice, die womöglich produziert wurde, weil das Bezugsnomen des Attributs, speranza di vita, feminin ist. Hier zeigt sich sprachliche Kreativität und ein hohes Maß an Sprachbewusstheit, da das Suffix -trice tatsächlich dazu dient, aus Verben neue Wörter zu bilden, die weibliche Personen bezeichnen. Wem die Wortbildungsregeln einer Sprache bekannt sind, steht also potentiell ein umfangreicherer Wortschatz zur Verfügung (vgl. Koesters Gensini 2009, S. 120).

Im selben Satz kommt ein dritter Relativsatz vor, dessen Pronomen vier Testeilnehmer nicht richtig ergänzten, wobei drei SchülerInnen gar nichts schrieben und eine(r) das Relativpronomen zwar richtig einsetzte, die Lücke aber zusätzlich mit einer konjugierten Verbform erweiterte, die grammatikalisch-syntaktisch nicht vertretbar ist. Auch in diesem Fall ist die Satzkomplexität als Ursache anzunehmen, die sich noch weiter fortsetzt und dazu geführt haben könnte, dass die nächsten beiden Lücken von sieben bzw. neun Personen nicht richtig vervollständigt wurden.

Die erste betrifft den Ausdruck in me-dia (im Durchschnitt), den sechs Lernende nicht als Lösungsvorschlag anboten, wobei sie entweder nichts ergänzten oder aber die logisch abstrusen Lösungen ,,in Monaten“ und „weniger“ lieferten. Übersetzt lautet der Satz damit (vgl. Carazzi \& Pizzetti 2010, S. 56): „Der erste ist die Lebenserwartung, ein Indikator, den wir schon kennen und der die Anzahl an Jahren misst, die *in Monaten ein [...] Individuum erwarten kann zu leben/erwarten kann *weniger zu leben". Media ist ein Wort des hohen Gebrauchs (AU), das als Bestandteil des Phrasems in media zum Co-Wortschatz zählt, obgleich seine Grundbedeutung im Phrasem erhalten bleibt. Auch das siebenmal falsch gelöste reflexive Verb aspet-tarsi stammt aus dem allgemeinen Wortschatz (CO). Dass die Wahl dieses Verbs in einem solchen Satz zusammen mit einem Modalverb für Verständnisschwierigkeiten bei den Testteilnehmern gesorgt haben kann, lassen neben einer nicht gefüllten Lücke die sechs grammatikalisch und lexikalisch falschen verbalen Lösungsvorschläge vermuten. Immerhin wurde zumindest fünfmal die grammatikalisch erforderte Form des Infinitivs gebildet. ${ }^{146}$

146 An dem Infinitiv aspettarsi (erwarten) aus dem Bereich des allgemeinen Wortschatzes (CO) hängt übrigens ein weiterer Infinitiv, der von einem/einer Lernenden nicht vervollständigt wurde, obwohl es sich mit vivere (leben) um ein sehr häufiges Wort handelt, das sicherlich allen bekannt war. 
Sechs Lernende vervollständigten im letzten Teil des Satzes das Wort individuo nicht richtig. Die häufigen falschen Lösungsvorschläge indicatore (3) und indice (1) könnten daher rühren, dass Ersteres mehrmals im Text auftritt und die Schüler daher dazu verleitet hat, es zu benutzen, obwohl es im Satzzusammenhang keinen Sinn ergibt. Das gilt gleichermaßen für indice, was semantisch nah bei Indikator liegt und schon an anderer Stelle anstelle von indicatore ergänzt wurde.

Das folgende Wort c-iò (das) wurde mit neun nicht korrekten Lösungsvorschlägen wie die Lücke in aggi-unge am häufigsten falsch bearbeitet und kann als Beispiel dafür herangezogen werden, dass die Lernenden mit bestimmten sprachlichen Mitteln der Textgestaltung, die der Herstellung von Textkohäsion dienen, wahrscheinlich nicht ausreichend vertraut waren. Weitaus leichter verständlich wäre für sie wohl die Struktur ,A questo dato si aggiunge quello ..." (diesen Fakt ergänzt jener ...). Erneut ist es in diesem sprachlichen Umfeld auch wieder ein reflexives Verb, welches viele SchülerInnen (9) nicht korrekt vervollständigten. Obwohl es eines der äußerst wenigen reflexiven Verben ist, die im GRADIT nicht mit einer CO-Gebrauchsmarke versehen sind, sondern zum Bereich des fundamentalen Wortschatz zählen, füllten vier Lernende die Lücke nicht, zwei ergänzten zu einer konjugierten Form des Verbs agire (handeln), das in reflexiver Form nicht existiert und zudem mit einer Konsonantenverdoppelung falsch geschrieben wurde, und weitere zwei wählten die grammatikalisch-syntaktisch falsche Alternative der Konjugation des Prädikats im Plural.

Die letzte häufige Falschlösung in diesen Texten betrifft ein Gerundium gleich am Satzanfang, wobei zwei Lernende keinen Ergänzungsvorschlag lieferten und weitere fünf die Lücke zu einer konjugierten Form des Verbs bzw. zu seinem Partizip Perfekt vervollständigten, was grammatikalisch-syntaktisch falsch ist. Es zeigt, dass den Lernenden die Form des Gerundiums weniger vertraut ist. Hierzu sei wiederholt, dass für die Mehrheit der Schülerinnen und Schüler dieser Lerngruppe Italienisch nicht die Erstsprache ist. So verwendeten sie in der eigenen Textproduktion keine Gerundien und verstanden bei der Erarbeitung von Texten zwar vielleicht, was mit dieser Form gemeint ist, aber ihre lexikalisch-grammatikalische Kompetenz reichte zur aktiven Verwendung dieser Form anscheinend nicht aus. Dies galt auch, wenn die Produktion durch eine Situation wie die Vorgabe im C-Test unterstützt war.

\subsubsection{Fünfter Text des C-Tests an der Schule Vespucci}

Der letzte Text des C-Tests der Schule Vespucci ist der Lehrbuchseite 68 (Carazzi \& Pizzetti 2010) entnommen und hat die weltweite Produktion und die Hauptverbrauchsstätten von Energieressourcen zum Thema. Er besteht aus 21 Lücken, von denen die Testteilnehmer keine einzige geschlossen richtig vervollständigten. Tabelle 11 zeigt den zu ergänzenden Teil des Textes mit 
Angaben zur Anzahl der Falschlösungen, den Lösungsvorschlägen und der Klassifikation der sprachlichen Elemente nach Wortklassen und Gebrauchsmarken.

\begin{tabular}{|c|c|c|c|c|c|}
\hline Fehler & Wort & Wortart & Marke & Kommentar & Lösungsvorschläge \\
\hline $\begin{array}{l}21 \\
\text { Lücken }\end{array}$ & II & art.det. & FO & S. 68 & \\
\hline \multirow[t]{2}{*}{2} & più & avv. & FO & & pil (2) \\
\hline & alto & agg. & FO & & \\
\hline 2 & $\begin{array}{l}\text { livello } \\
\text { di }\end{array}$ & $\begin{array}{l}\text { s.m. } \\
\text { prep. }\end{array}$ & $\begin{array}{l}\text { FO fig. } \\
\text { FO }\end{array}$ & & 0 , livelli \\
\hline \multirow[t]{2}{*}{5} & consumo & s.m. & $\mathrm{AU}$ & & $\begin{array}{l}0 \text {, consumazione ( } 2) \text {, } \\
\text { consumatori, } \\
\text { considerazione }\end{array}$ \\
\hline & si & pron.pers. & $\mathrm{CO}$ & & \\
\hline \multirow[t]{2}{*}{3} & verifica & v.pronom.intr. & $\mathrm{CO}$ & & 0 , verificano $(2)$ \\
\hline & negli & prep.art. & FO & & \\
\hline \multirow{2}{*}{$\begin{array}{l}1+8 \\
\text { acc. }\end{array}$} & Usa & n.p./sigla & & & 0, USA (acc.) (8) \\
\hline & che & pron.rel. & FO & & \\
\hline \multirow[t]{2}{*}{8} & pur & cong. & $\mathrm{CO}$ & $\begin{array}{l}\text { nicht im GRA- } \\
\text { DIT, Ge- } \\
\text { brauchsmarke } \\
\text { von seppure }\end{array}$ & $\begin{array}{l}0, \text { può (2), possono } \\
\text { (2), per, produttiva- } \\
\text { mente, producendo }\end{array}$ \\
\hline & non & avv. & FO & & \\
\hline \multirow[t]{2}{*}{8} & $\begin{array}{l}\text { raggiun- } \\
\text { gendo }\end{array}$ & ger.v.tr. & FO & & $\begin{array}{l}0, \text { raggiungere }(4) \text {, } \\
\text { raggiungono }(3)\end{array}$ \\
\hline & il $5 \%$ & art.det. & FO & & \\
\hline \multirow[t]{2}{*}{1} & della & prep.art. & FO & & 0 \\
\hline & popolazione & s.f. & $A U$ & & \\
\hline \multirow[t]{2}{*}{2} & mondiale & agg. & FO & & 0, mondo \\
\hline & consumano & v.tr. & FO & & \\
\hline \multirow[t]{2}{*}{2} & un & art.indet. & FO & & $0(2)$ \\
\hline & quarto & s.m. & $\mathrm{CO}$ & & \\
\hline \multirow[t]{2}{*}{2} & dell'energia & s.f. & $\mathrm{CO}$ & & $0(2)$ \\
\hline & disponibile & agg. & $A U$ & & \\
\hline \multirow[t]{2}{*}{1} & $\begin{array}{l}\text { special- } \\
\text { mente }\end{array}$ & avv. & $\mathrm{CO}$ & & 0 \\
\hline & quella & pron.dimostr. & FO & & \\
\hline \multirow[t]{2}{*}{2} & prodotta & part.pass.v.tr. & FO & & 0, produzione \\
\hline & da & prep. & FO & & \\
\hline \multirow[t]{2}{*}{4} & idrocarburi & s.m. & CO TS chim. & & $\begin{array}{l}\text { 0, idrocarburo (2), } \\
\text { idrocarbonio }\end{array}$ \\
\hline & gli & art.det. & $\mathrm{CO}$ & & \\
\hline \multirow[t]{2}{*}{1} & Stati & n.p. & & $\begin{array}{l}\text { mit d'America } \\
\text { als loc.s.m.pl. } \\
\text { im GRADIT }\end{array}$ & 0 \\
\hline & Uniti & el.n.p. & & & \\
\hline \multirow[t]{2}{*}{3} & importano & & $\mathrm{CO}$ & & 0 , impone, impongono \\
\hline & più & avv. & FO & & \\
\hline \multirow[t]{2}{*}{1} & della & prep.art. & FO & & 0 \\
\hline & metà & s.f. & FO & & \\
\hline 3 & $d e l$ & prep.art. & FO & & $0, \mathrm{di}(3)$ \\
\hline
\end{tabular}




\begin{tabular}{|c|c|c|c|c|c|}
\hline Fehler & Wort & Wortart & Marke & Kommentar & Lösungsvorschläge \\
\hline & petrolio & s.m. & $\mathrm{FO}$ & & \\
\hline \multirow[t]{2}{*}{2} & che & pron.rel. & FO & & $0(2)$ \\
\hline & consumano & v.tr. & FO & & \\
\hline \multirow[t]{2}{*}{10} & dipendendo & ger.v.intr. & FO & & $\begin{array}{l}0 \text { (2), dipendente, } \\
\text { dipende }(6) \text {, dipen- } \\
\text { dono }\end{array}$ \\
\hline & più & loc.avv. & $\mathrm{CO}$ & & \\
\hline \multirow[t]{2}{*}{2} & che & el.loc.avv. & el.CO & & $0(2)$ \\
\hline & altro & el.loc.avv. & el.CO & & \\
\hline
\end{tabular}

Tabelle 11: Übersicht über Text 5 des C-Tests an der Vespucci inklusive Lösungsvorschläge

Eine zweisprachige Schülerin ließ diesen Text abgesehen von einer Falschlösung beim ersten getilgten Wort komplett unbearbeitet. Es ist anzunehmen, dass sie bei der Testbearbeitung in Zeitnot geraten war.

Mit zehn hat in diesem Text die meisten Fehler das Gerundium dipendendo (abhängend) verursacht ${ }^{147}$, wobei über die Hälfte der Lücken, nämlich 13, von nur einem oder zwei Lernenden falsch oder gar nicht ergänzt wurden. Von den getilgten Wörtern gehört ein knappes Drittel (8) nicht dem fundamentalen Gebrauchswortschatz (Fo) an, bzw. trägt als Eigenname keine Gebrauchsmarke.

Bei den Eigennamen, die von je einem Testteilnehmer gar nicht ergänzt wurden, handelt es sich um die Sigle Usa (USA) und um Stati aus Stati Uniti (Vereinigte Staaten). Unter den übrigen selten falsch gelösten Wörtern ist neben einem Adverb (specia-lmente, Co; vor allem), einem Nomen (dell'energia, Co; Energie) und einem Relativpronomen (che, Fo) die nicht vervollständigte zweimal im Text auftretende artikulierte Präposition de-lla (von der). Sie wurde von der Klasse eigentlich sicher beherrscht, steht aber im Vorfeld zweier femininer Nomen, die beide nicht die verbreitetste weibliche Singularendung auf $a$ aufweisen: $e$ bei popol-azione (AU; Bevölkerung) und mit Akzent bei metà (Fo; Hälfte).

Wie zu Beginn des Kapitels geschildert, sind die mit dem Suffix -mente aus den Adjektiven gebildeten Adverbien zwar lange Wörter und gelten längere Wörter prinzipiell als seltener gebraucht und schwieriger. Von Adverbien, über die man nur wissen muss, dass sie aus dem entsprechenden Adjektiv und dem Adverbsuffix geformt werden, kann dies jedoch nicht behauptet werden. ${ }^{148} \mathrm{Al}-$

147 Eine Abbildung des fünften Textes mit Kennzeichnung der zu ergänzenden Wörter, Anzahl der Falschlösungen und Übersetzung befindet sich im Abschnitt 8.3.1.3.1 über die häufigsten Fehler dieses Textes.

148 Davon abgesehen, fällt bei einigen Adverbbildungen der letzte Vokal des adjektivischen Wortstamms weg, wie bei dem hier gegebenen specialmente zum Adjektiv speciale, und wird aus der maskulinen Grundform in $o$ beim Adverb ein $a$ (z.B. lento (langsam) und lentamente). Dieses grammatikalische Wissen wird beim Erst- und oft auch Zweitspracherwerb informal ohne große Mühe entwickelt und ist grundsätzlich eher für Fremdsprachenlerner des Italienischen explizit zu erwerben. Es ist davon auszugehen, dass bis auf eine Testperson, die erst 
lerdings ließ ein Schüler bzw. eine Schülerin dieses Wort vielleicht unvollständig, weil sich das Adverb nicht in unmittelbarer Nähe des konjugierten Verbs befindet, wo es leichter als solches zu erkennen wäre.

Das aus dem allgemeinen Gebrauchswortschatz stammende energia war vermutlich allen Lernenden aus dem alltagssprachlichen Gebrauch sowie aus dem Technikunterricht bekannt, ist jedoch in diesem Text etwas schwieriger zu produzieren, da es durch einen Apostroph mit einer artikulierten Präposition zusammengezogen ist. Außerdem bereitete das Wortumfeld zwei Lernenden vielleicht Verständnisprobleme: Consumare (konsumieren, verbrauchen) trägt zwar die Gebrauchsmarke Fo, wurde aber nicht unbedingt von allen Testteilnehmern sicher beherrscht. Zudem ist die Satzaussage nicht schlicht, dass Energie verbraucht wird, sondern un quarto, ein Viertel der Energie. Darüber hinaus folgt dem zu ergänzenden Nomen ein Adjektiv, das vielleicht auch nicht ausreichend beherrscht wurde, da es zum Wortschatzbereich AU gehört. ${ }^{149}$

Das Relativpronomen che ist das einfachste und in der gesprochenen Sprache am häufigsten verwendete Relativpronomen. Es wurde trotzdem von zwei Lernenden nicht vervollständigt, was eventuell daran liegt, dass der Relativsatz nur aus che und dem erneut verwendeten Verb consumare besteht und der anschließende Satzverlauf für die Betroffenen sprachlich zu komplex war. So geht es mit dem elfmal falsch gelösten Gerundium dipendendo und einem Adverbialphrasem aus dem allgemeinen Wortschatz (CO) weiter. Das dreigliedrige Phrasem più c-he altro (vor allem) bereitete vielleicht zwei Lernenden Schwierigkeiten, da sie den Ausdruck nicht kannten.

Darüber hinaus sind weitere Wörter bis zu dreimal falsch gelöst worden, die alle zum fundamentalen Wortschatz zählen. Hier bleibt zu erwähnen, dass beispielsweise livello (Grad) zwar zu den am meisten gebrauchten Wörtern des Italienischen zählt, in einem Satzkontext, in dem es zusammen mit weiteren fünf Wörtern als Subjekt auftritt (,Il più alto livello di consumo“ (Carazzi \& Pizzetti 2010, S. 68; m.Ü.: der höchste Grad an Verbrauch), an das sich als Prädikat das reflexive Verb verificarsi (CO; sich ereignen) anschließt, für sprachlich schwächere Lernende jedoch ganz klar eine Hürde darstellt. ${ }^{150}$ Der Satz weist außerdem eine verschachtelte hypotaktische Struktur auf, in der es

seit kurzem in Italien lebte, alle anderen Lernenden sämtliche Adverbien korrekt bilden konnten.

149 Über das individuelle mentale Lexikon, grammatikalische und morphosyntaktische Fähigkeiten und die Textkompetenz der SchülerInnen dieser Studie ließen sich detailliertere Aussagen treffen, wenn es möglich gewesen wäre, sich nach der Durchsicht der C-Tests mit ihnen einzeln oder in Kleingruppen zusammenzusetzen, um sich die individuellen Lösungsvorschläge kommentieren zu lassen (auch wenn davon ausgegangen werden kann, dass den Lernenden im Nachhinein nicht immer bewusst ist, warum sie welche Wörter wie ergänzt bzw. nicht ergänzt haben). Aufgrund der eh schon reduzierten Unterrichtszeit für das Fach Erdkunde und des straff durchorganisierten Schulalltags im letzten Jahr der Mittelschule stand ein solches Verfahren nicht zur Debatte.

150 Es ist bezeichnend, dass dieses Verb im Textverarbeitungsprogramm Word angestrichen wird, da es zwar nicht falsch, aber ,abgenutzt und übermäßig gebraucht“ sei. 
nicht an komplexeren verbalen Formen wie Gerundien und Partizipien mangelt. Dadurch kommt die Frage auf, ob das Bestreben der Lehrbuchautoren, die SchülerInnen neben der Vermittlung des fachspezifischen Inhalts an die Bildungssprache heranzuführen, bisweilen nicht auf eine zu konzentrierte Weise erfolgt. Viele Jugendliche sind sprachlich wahrscheinlich überfordert und werden deshalb in ihrem Lernprozess eher blockiert als vorangebracht. ${ }^{151}$

Im selben Satz wurden drei weitere Lücken zweimal nicht richtig ergänzt, und zwar die des Adjektivs mond-iale (weltweit), das einmal zum Substantiv mondo (Welt) und einmal gar nicht vervollständigt wurde. Die Substantivergänzung deutet auf unsichere grammatikalische Kenntnisse oder aber auf mangelnde Konzentration hin. Das zweite Wort betrifft einen unbestimmten Artikel vor dem Nomen quarto (Viertel), der in beiden Fällen nicht bearbeitet wurde. Kannten die Betroffenen das Co-gekennzeichnete Wort nicht und konnten sie es nicht einmal als Nomen einordnen und daraus folgern, dass es in der Regel einen Artikel braucht? Das dritte Wort ist ein Partizip und formt einen implizierten Relativsatz. Es hat als direktes Bezugswort ein Indefinitpronomen, und man kann vermuten, dass die Lernenden in der Lage gewesen wären, dieses Partizip zu bilden, wenn es als Teil eines explizierten Relativsatzes und damit einer Passivkonstruktion gebraucht wäre.

Von je drei Lernenden wurden außerdem das bereits besprochene reflexive Verb verificarsi und ein weiteres Verb, impo-rtano (sie importieren), nicht richtig vervollständigt. Letzteres stammt aus dem fundamentalen Wortschatz (Fo) und kommt gerade im Erdkundeunterricht bei der Behandlung der wirtschaftlichen Lage einzelner Länder oder Regionen immer wieder vor, auch schon in den ersten beiden Jahren der Mittelschule. Neben einer Nichtbearbeitung lassen deshalb die beiden unterschiedlich konjugierten Formen des Verbs imporre (durchsetzen, aufzwingen) darauf schließen, dass drei Testteilnehmer die Satzaussage nicht erfasst hatten.

Eine viermal falsch bearbeitete Lücke befindet sich im selben Satz, in dem schon das mittlere Element des Phrasems più che altro zweimal nicht korrekt ergänzt wurde. Sie betrifft eine artikulierte Präposition, die neben einer fehlenden Bearbeitung immerhin dreimal als einfache Präposition ergänzt wurde, auch wenn der Satzzusammenhang außer der Präposition einen Artikel erfordert. Darüber hinaus wurde auch das Wort idrocarburi aus dem allgemeinen (CO) und technisch-spezifischen Wortschatz (Ts chim.) viermal nicht bzw. nicht korrekt vervollständigt. Da es in einem Satz auftritt, in dem es zu mehreren häufigen Falschlösungen kam, wird es mit ihnen zusammen im nächsten Unterkapitel vorgestellt.

151 Vgl. das von Vygotskij (Wygotski 1977 (1934)) eingeführte und dem scaffolding-Ansatz zugrundeliegende Konzept (Collins, Brown \& Newman 1995) der Zone der nächsten Entwicklung, das w.o. vorgestellt wurde. 


\subsection{Häufige Falschlösungen im fünften Text des C-Tests}

Ca. ein Fünftel der zu ergänzenden Wörter (4 von 21) wurde mit mindestens fünf Falschlösungen vermehrt nicht korrekt bearbeitet. Wie Tabelle 12 zeigt, sind zwei von ihnen Fo-gekennzeichnet - wobei es sich allerdings um Verben handelt, die zu den grammatikalisch-syntaktisch anspruchsvolleren Gerundien vervollständigt werden mussten -, stammt eines aus dem Bereich des hohen Gebrauchs (AU) und gehört das letzte nicht zum Basiswortschatz, sondern trägt eine Co-Gebrauchsmarke.

\begin{tabular}{|c|c|c|c|c|c|}
\hline $\begin{array}{l}\mathrm{n} \\
\text { Fehler }\end{array}$ & Wort & Wortart & $\begin{array}{l}\text { Gebrauchs- } \\
\text { marke }\end{array}$ & Kommentar & Lösungsvorschläge \\
\hline 5 & consumo & s.m. & $\mathrm{AU}$ & & $\begin{array}{l}0(2) \text {, consumazione }(2) \text {, } \\
\text { consumatori }\end{array}$ \\
\hline 8 & pur & cong. & $\mathrm{CO}$ & $\begin{array}{l}\text { nicht im GRADIT, } \\
\text { Gebrauchsmarke } \\
\text { von seppure }\end{array}$ & $\begin{array}{l}0 \text { (2), può (2), possono } \\
\text { (2), per, produttiva- } \\
\text { mente, producendo }\end{array}$ \\
\hline 8 & $\begin{array}{l}\text { raggiun- } \\
\text { gendo }\end{array}$ & ger.v.tr. & $\mathrm{FO}$ & & $\begin{array}{l}0(2) \text {, raggiungere (4), } \\
\text { raggiungono (2) }\end{array}$ \\
\hline 10 & $\begin{array}{l}\text { dipen- } \\
\text { dendo }\end{array}$ & ger.v.intr. & FO & & $\begin{array}{l}0 \text { (4), dipendente, } \\
\text { dipende (5), dipendono }\end{array}$ \\
\hline
\end{tabular}

Tabelle 12: Häufigste Fehler in Text 5 des C-Tests, Textauszug aus der Lehrbuchseite 68 (Carazzi \& Pizzetti 2010)

Die Hälfte der Testteilnehmer ergänzte das häufig gebrauchte Nomen consumo (AU) nicht richtig, und vier hatten anscheinend Schwierigkeiten mit dem Wort für Kohlenwasserstoff. Sie sollten es aber sowohl aus dem Erdkunde- als auch aus dem Technikunterricht kennen, da in beiden Fächern im dritten Jahr der Mittelschule das Thema herkömmliche und erneuerbare Energien behandelt wird. Nur zwei Testteilnehmer bildeten die Konjunktion pur aus dem Bereich des allgemeinen Wortschatzes (CO), und mit dem von der Konjunktion geforderten Gerundium des Verbs raggiungere (Fo; erreichen) zeigten fast alle Lernenden Probleme, wie auch mit dem anderen Gerundium, dipendendo.

Vier häufige Falschlösungen verteilen sich auf den schon für andere Fehlerquellen besprochenen grammatikalisch und morphosyntaktisch komplexen Satz. Da sich die Lücken in diesem etwas kürzeren C-Testtext in nur zwei Sätzen befinden, werden sie zur besseren Veranschaulichung der möglichen Ursachen der mehrfachen Falschlösungen einschließlich Überschrift und Einleitungssatz sowie Übersetzung in Abbildung 5 vollständig aufgeführt. Der schlechte Stil der Übersetzung rührt daher, dass sie bewusst sehr wörtlich gehalten ist, um besser einen Eindruck von den verwendeten sprachlichen Mitteln wie z.B. den Gerundien vermitteln zu können. Die halb getilgten Wörter sind fettgedruckt, die häufig falsch gelösten zusätzlich markiert und alle mit ihrer jeweiligen Fehleranzahl versehen.

Le risorse energetiche - paesi consumatori e paesi produttori

Nel campo delle risorse energetiche a livello planetario i principali consumatori, cioè i paesi a economia avanzata che consumano molta energia, non sono autosufficienti. 
II più [2] alto livello [2] di consumo [5] si verifica [3] negli USA [1] che, pur [8] non raggiungendo [8] il 5\% della [1] popolazione mondiale [2], consumano un [2] quarto dell'energia [2] disponibile, specialmente [1] quella prodotta [2] da idrocarburi [4].

Gli Stati [1] Uniti importano [3] più della [1] metà del [4] petrolio che [2] consumano, dipendendo [10] più che [2] altro dal Medio Oriente.

(M.Ü.: Die Energieressourcen - Verbraucherländer und Produzentenländer

Im Bereich der Energieressourcen sind auf Weltebene die prinzipiellen Verbraucher, d.h. die Länder mit einer fortgeschrittenen Wirtschaft, die viel Energie verbrauchen, nicht autark.

Der höchste Grad an Verbrauch ereignet sich in den USA, die, obgleich keine 5\% der Weltbevölkerung erreichend, ein Viertel der verfügbaren Energie konsumieren, vor allem jene mit Kohlenwasserstoff produzierte.

Die Vereinigten Staaten importieren mehr als die Hälfte des Öls, das sie verbrauchen, in erster Linie vom Mittleren Orient abhängend.)

Abbildung 5: Der Lehrbuchseite 68 (Carazzi \& Pizzetti 2010) entnommener C-Testtext mit Angaben zu den Falschlösungen

Beim ersten sehr oft falsch/nicht ergänzten Wort consumo könnte, wie bereits bei der Erörterung der Probleme mit den im Satz seltener falsch gelösten Wörtern betont wurde, das sprachliche Umfeld des Satzes ausschlaggebend gewesen sein. Da einige SchülerInnen das erste reflexive Verb nicht richtig vervollständigten (wobei es vielleicht auch einige gibt, die es zwar korrekt bildeten, aber die Bedeutung nicht genau kannten) und fast alle mit dem folgenden implizierten Konzessivsatz (pur + Gerundium) überfordert waren, war das Textverständnis beeinträchtigt. Vielleicht fand die Hälfte der Testteilnehmer deshalb keine Lösung für das Nomen consumo, obwohl es in Form des Verbs consumare sowohl im Einleitungssatz als auch später im Satzverlauf ein zweites Mal ungetilgt auftritt und als ein weiteres Nomen aus demselben Wortfeld (consumatori) schon in der Überschrift und im Einleitungssatz erscheint. Darum könnte sich auch ein Schüler dazu verleitet haben lassen, das letztgenannte Nomen einzusetzen, auch wenn es an dieser Stelle im Text überhaupt keinen Sinn ergibt.

Die seltener verwendete Konjunktion pur ist auf den analysierten Seiten dieses Lehrbuchs mit drei Vorkommnissen vertreten, aber nur einmal im CTest. Vermutlich ist die Produktion von Konjunktion und Gerundium dadurch zusätzlich erschwert, dass sich der komplexe Nebensatz in einem Relativsatz befindet, an den sich auch noch der nicht explizierte Relativsatz anschließt. Die Schwierigkeit ergab sich für die Testteilnehmer also auf grammatikalischsyntaktischer Ebene durch die ausgeprägte Hypotaxe des Satzes und zugleich auf lexikalischer Ebene, wobei sich die beiden Aspekte gegenseitig bedingen. Trotzdem ist nicht damit zu rechnen, dass die Lernenden in einem andersartigen, weniger kompliziert strukturierten sprachlichen Umfeld dazu in der Lage gewesen wären, einen solchen Nebensatz mit dem Gerundium zu bilden. Wahrscheinlich hätten sie ihn höchstens unter sprachlich etwas einfacheren 
Bedingungen verstanden. ${ }^{152}$ Interessant ist die Vielfalt an Wortarten, auf die sich die Lösungsvorschläge verteilen: Anstelle der Konjunktion boten die SchülerInnen zwei verschiedene konjungierte Formen eines Modalverbs sowie ein Gerundium, ein Adverb und eine Präposition an.

Beim letzten öfter, nämlich viermal falsch gelösten Wort des ersten Satzes ergibt sich aus den Lösungsvorschlägen, dass sich die Testteilnehmer an das Wort wahrscheinlich erinnerten, aber es nicht sicher beherrschten. Es kann im Italienischen im Kontext der Energieproduktion anscheinend nur im Plural auftreten, weshalb die beiden Vorschläge der korrekten Singularform nicht akzeptabel sind. Das Wort idrocarbonio hingegen existiert nicht, obwohl es eine logische Wortbildung wäre, da carbonio Kohlenstoff bedeutet. Wäre dieser Text nicht erst kurz vor der Durchführung des C-Tests im Unterricht behandelt worden, stellten die Lösungsvorschläge für das Co Ts-gekennzeichnete Wort ein durchaus positives Ergebnis für diese Lerngruppe dar.

Sechs der zehn Lösungsvorschläge für das Gerundium dipen-dendo im zweiten mit Lücken versehenen Satz bestehen in der Konjugation desselben Verbs in der dritten Person Präsens. Dieses Mal ist der vorausgehende Teil des Satzes ein wesentlich kürzerer Hauptsatz, welcher prototypgemäß mit dem Subjekt beginnt, dem ein kurzer Relativsatz (Pronomen + Verb) folgt. Hier liegt also ein vergleichsweise reduziertes $\mathrm{Maß}$ an sprachlicher Komplexität vor, was sich auch im Ausfüllen der Lücken wiederspiegelt, bei dem nur wenige Fehler auftreten. Das sprachliche Umfeld bietet also keine Erklärung für diesen Lösungsvorschlag, der ganz augenscheinlich nicht mit dem Pluralsubjekt Stati Uniti kongruent ist, das von einem Pluralartikel begleitet wird, und auch nicht mit dem Prädikat, das logischerweise in der dritten Person Plural formuliert ist. Es ist möglich, dass die Lernenden dipende (hängt ab) produzierten, weil sie diesem Verb vor allem konjugiert in der dritten Person Singular begegnen: Ein Sachverhalt, eine Situation hängt von etwas ab. Desweiteren ist es sehr gut möglich, dass sie den letzten Teil des C-Tests flüchtiger lasen und, obwohl sie ausreichend Zeit zur Vervollständigung des Tests hatten und niemand zur Abgabe gezwungen wurde, nicht mehr kontrollierten, was sie im Einzelnen geschrieben hatten.

Abschließend lässt sich festhalten, dass viele SchülerInnen dieser Lerngruppe die Bearbeitung des C-Tests ziemlich schlecht vollzogen. Betrachtet man das zur Hälfte getilgte Wortvorkommen, so besteht es in allen fünf Tests in erster Linie aus Substantiven und in zweiter aus Adjektiven, wobei Letztere mit elf im Test der Vespucci weniger stark vertreten sind als in denen der anderen vier Klassen (17 bis 20 Adjektive, durchschnittlich 19). Diese grammatikalischen Kategorien führten bei der Bearbeitung jedoch nicht zu den meisten Falschlösungen. Aus Tabelle 13 wird ersichtlich, welchen verschiedenen

152 Ein Konzessivsatz mit pur liegt auch im C-Test der Klasse III C vor, in der ebenfalls fast niemand das Wort wie im Originaltext ergänzte, sondern fünf Lernende die Lücke nicht füllten und zwölf sie zu akzeptablen Alternativen vervollständigten (s.u.). 
Wortarten die halbierten Ausdrücke des Tests angehören und wie viele Fehler sie jeweils verursachten. Allerdings kann hier analog zu den Falschlösungen bei Wörtern bestimmter Gebrauchsmarken nichts oder nicht viel über die Fähigkeiten der Lernenden im Umgang mit bestimmten Wortarten ausgesagt werden, da die Itemschwierigkeit auch aus dem Mikro- und Makrokontext resultiert, in den die getilgten Wörter eingebettet sind. Auf extralinguistischer Ebene stellen außerdem unzureichende Testbearbeitungsstrategien, Flüchtigkeit, fehlender Ehrgeiz u.Ä. mögliche Ursachen für die Falschlösungen dar.

\begin{tabular}{lrlrr}
\hline Wortart & $\mathrm{n}$ Tokens & Kommentar & n Fehler & $\begin{array}{r}\varnothing \text { Fehler } \\
\text { pro Wort }\end{array}$ \\
\hline agg. & 11 & & 27 & 2,5 \\
art.det./indet. & 13 & 9 & 0,7 \\
avv. & 8 & & 22,5 & 2,8 \\
cong. & 5 & cioè (10), però (9), pur (8) & 30 & 6 \\
el.loc. & 7 & & 20 & 2,9 \\
ger. & 3 & dipendendo (10), raggiungendo (8), & 25 & 8,3 \\
& & considerando (7) & & \\
inf. & 3 & & 8 & 2,7 \\
loc. & 2 & a livello (9), su scala (9) & 18 & 9 \\
n.p. & 3 & & 3 & 1 \\
n.p./sigla & 1 & & 0 & 0 \\
part.pass.v.tr. & 1 & & 2 & 2 \\
prep. & 11 & & 19 & 1,7 \\
prep.art. & 11 & & 24 & 2,2 \\
pron. & 4 & ciò (9), ci (5) & 22 & 5,5 \\
pron.rel. & 4 & & 9 & 2,3 \\
s.m./f. & 32 & & 83 & 2,6 \\
v.tr./intr./mod. & 5 & (si) aggiunge (9) & 15,5 & 3,1 \\
v.pronom.intr. & 35 & 15 & 5 \\
\% s.m./f. & 25 & & & \\
\hline
\end{tabular}

Tabelle 13: Getilgtes Wortvorkommen im C-Test der Vespucci nach Wortarten mit Angaben zu den Falschlösungen

pron*: Unter dieser Bezeichnung sind außer den gesondert aufgelisteten Relativpronomen (pron.rel.) alle anderen Arten von Pronomen gesammelt.

Mit 23,3 verzeichnet dieser C-Test von allen fünf die zweithöchsten Fehlerwerte für die drei durchschnittlich am häufigsten falsch bearbeiteten Ausdrücke einer Wortart. ${ }^{153}$ Sie verteilen sich mit 9 auf die ersten Glieder von Phrasemen, mit 8,3 auf drei Gerundien und mit 6 auf Konjunktionen. Zu dieser letzten grammatikalischen Kategorie zählen hauptsächlich Wörter aus dem fundamentalen Wortschatz; die vermehrten Falschlösungen sind neben der von allen Testteilnehmern nicht korrekt bearbeiteten Konjunktion cioè (das ist,

$153 \mathrm{Zu}$ jeder Wortklasse ist angegeben, wie viele Fehler begangen wurden und wie viele davon durchschnittlich auf ein Wort einer bestimmten Wortklasse entfallen. Es ist also nicht mehr ersichtlich, welches Wort wie viele Fehler verursachte, sondern es wird ein genereller Eindruck davon vermittelt, wie schwierig die Bearbeitung der Wörter einer bestimmten Wortart für die Lerngruppe war. 
also) hingegen hier v.a. auf die aus dem Bereich des hohen Gebrauchs (AU) stammende, aber bestimmt bekannte und häufig gebrauchte neunmal falsch gelöste Konjunktion però (aber) und auf die achtmal nicht korrekt bearbeitete pur (obschon) aus dem allgemeinen Wortschatz (CO) zurückzuführen.

Ebenfalls viele Fehler entfallen außerdem auf die Pronomen abzüglich der Relativpronomen (durchschnittlich rund 6 Fehler) und auf die reflexiven Verben (rund 5 Fehler), wobei die meisten im Zusammenhang mit aggiungersi (9; sich hinzubegeben) auftreten, obwohl es sich dabei um eines der wenigen reflexiven Verben handelt, die nicht zum allgemeinen, sondern zum fundamentalen Wortschatz (Fo) zählen.

Nur insgesamt 21 Lücken wurden von allen Testteilnehmern korrekt vervollständigt, während bei allen anderen mindestens ein Fehler auftritt. Viele Lösungsvorschläge ergeben semantisch überhaupt keinen Sinn und morphosyntaktisch nur zum Teil. Daher kann man sich fragen, ob die Lernenden mit der sprachlichen Komplexität ihres Lehrbuchs, aus dem ja die Texte für den $\mathrm{C}$-Test stammen, derart überfordert waren oder ob sie bei besserer Anwendung von Testbearbeitungsstrategien (z.B. das erneute aufmerksame Durchlesen der eigenen Antworten in ihrem Satzzusammenhang und im weiteren Kontext) weniger Fehler produziert hätten. Egal wie die Antwort auf diese Frage ausfällt, zeigt sich doch deutlich, dass den Lernenden die Bearbeitung des Tests nicht leichtgefallen zu sein scheint.

Aus der Fehleranalyse ergibt sich die Hypothese, dass Wörter oft nicht richtig vervollständigt werden konnten, weil sie sich in einem Textumfeld befinden, das aus längeren Satzgefügen besteht, deren Teile sich hypotaktisch strukturieren und manchmal ineinander schachteln, das morphosyntaktische Formen wie beispielsweise Gerundien nutzt, die weder im mündlichen Sprachgebrauch noch in einfacher strukturierten Texten anzutreffen sind, und das sich lexikalischer Mittel bedient, welche die Kernaussagen des Textes nicht immer konkret beim Namen nennen, sondern auf abstraktere, vagere Weise formulieren.

Auch wenn der Anteil an Wörtern, die nicht zum Basiswortschatz zählen, bei den häufig falsch bearbeiteten Wörtern steigt, ist er im Vergleich zu denjenigen aus dem fundamentalen Wortschatz gering. Die Schwierigkeiten der Lernenden mit den 2.000 frequentesten Wörtern der italienischen Sprache dürften dabei nur in Einzelfällen die Wörter an sich betreffen, sondern i.d.R. durch einen verdichteten, komplexen sprachlichen Kontext bedingt sein.

Dieser Umstand wird noch einmal mit der Tabelle 13 veranschaulicht, in der alle häufig falsch gelösten Ausdrücke der fünf Testtexte aufgelistet sind. Von den insgesamt 24 vermehrt falsch bearbeiteten Wörtern zählen fast zwei Drittel (15) zum fundamentalen Wortschatz (Fo) und weitere drei AU-gekennzeichnete zum Basiswortschatz. Auch unter den acht-, neun- und zehnmal nicht korrekt gelösten Items befinden sich Ausdrücke aus dem fundamentalen Wortschatz, so dass deutlich wird, dass die Schwierigkeiten der SchülerInnen 
mit der Testbearbeitung kaum aus einer reinen Auflistung der entsprechenden halbierten Wörter ersichtlich werden, wenn man einmal von bestimmten Wortartenkategorien wie Phrasemen und Gerundien absieht. Stattdessen ist die Ursache für die meisten versäumten und falschen Lösungsvorschläge anzunehmender Weise in der verdichteten und anspruchsvollen sprachlichen Gestaltung des jeweiligen Textkontextes zu suchen.

\begin{tabular}{|c|c|c|c|c|c|}
\hline Text & Fehler & Wort & Wortart & Gebrauchsmarke & Kommentar \\
\hline \multirow[t]{4}{*}{1} & 5 & $c i$ & pron.pers. & FO & \\
\hline & 9 & però & cong. & $\mathrm{FO}$ & \\
\hline & 10 & cioè & cong. & $\mathrm{FO}$ & \\
\hline & 7 & benessere & s.m. & $A U$ & \\
\hline \multirow[t]{7}{*}{2} & 9 & $a$ & loc.avv. & $\mathrm{CO}$ & a livello \\
\hline & 6 & persona & s.f. & FO & a persona \\
\hline & 6 & dalla & prep.art. & FO & \\
\hline & 9 & citata & agg. & $\mathrm{CO}$ & \\
\hline & 9 & su & loc.avv. & $\mathrm{CO}$ & su scala \\
\hline & 9 & $\begin{array}{l}\text { internazio- } \\
\text { nale }\end{array}$ & agg. & FO & \\
\hline & 8 & distanze & s.f. & FO & \\
\hline \multirow[t]{2}{*}{3} & 6 & nell'altro & pron.indef. & FO & \\
\hline & 5 & nei & prep.art. & FO & \\
\hline \multirow[t]{8}{*}{4} & 8 & primo & agg. & $\mathrm{FO}$ & \\
\hline & 5 & indicatore & s.m. & TS econ. & \\
\hline & 6 & media & s.f. & $\mathrm{AU}$ & in media \\
\hline & 7 & aspettarsi & $\begin{array}{l}\text { inf.v.pro- } \\
\text { nom.intr. }\end{array}$ & $\mathrm{CO}$ & \\
\hline & 6 & individuo & s.m. & $A U$ & \\
\hline & 9 & ciò & pron.dimostr. & $\mathrm{FO}$ & \\
\hline & 9 & aggiunge & v.pronom.intr. & $\mathrm{FO}$ & \\
\hline & 7 & $\begin{array}{l}\text { conside- } \\
\text { rando }\end{array}$ & ger.v.tr. & FO & \\
\hline \multirow[t]{3}{*}{5} & 8 & pur & cong. & $\mathrm{CO}$ & $\begin{array}{l}\text { nicht im GRADIT, Gebrauchs- } \\
\text { marke von seppure }\end{array}$ \\
\hline & 8 & $\begin{array}{l}\text { raggiun- } \\
\text { gendo }\end{array}$ & ger.v.tr. & FO & \\
\hline & 10 & dipendendo & ger.v.intr. & FO & \\
\hline
\end{tabular}

Tabelle 14: Häufigste Falschlösungen im C-Test an der Schule Vespucci

Auffällig an den Ergebnissen ist darüber hinaus, dass eine Vielzahl von ausgebliebenen Ergänzungen vorliegt. Bis auf die erste Lücke des fünften Textes ist es in dem Text immer mindestens eine Testperson, welche die Lücken unbearbeitet lässt und Ähnliches gilt fast vollständig für den vierten Text. Insgesamt liegt der Anteil an nicht ergänzten Wörtern mit $132 \mathrm{sehr}$ hoch. Das bedeutet, im Durchschnitt ist jede Lücke des Tests mehr als einmal unausgefüllt geblieben. 


\subsubsection{Morante}

Auch in dieser Lerngruppe war die Unterrichtssprache für viele nicht die Erstsprache: Fast die Hälfte der Testteilnehmer (6 von 13) sprach Italienisch als Zweitsprache und nicht alle von ihnen wurden in Italien eingeschult. Mit gravierenden Problemen, die nicht nur die Bildungs-, sondern auch die Alltagssprache betrafen, war ein chinesischer Schüler konfrontiert, der seit eineinhalb Jahren in Italien lebte und im Unterricht nur zaghaft begann, sich in der Landessprache zu verständigen. Er nahm am C-Test nicht teil.

Die Tabelle 15 veranschaulicht zunächst, welchen Wortschatzbereichen die getilgten Wörter zugeschrieben werden und wie viele Falschlösungen jeweils auf sie entfallen. Im Kommentarfeld sind außerdem einige vermehrt falsch gelöste Ausdrücke mit der entsprechenden Fehleranzahl aufgeführt.

\begin{tabular}{|c|c|c|c|c|c|}
\hline & & & & & $\varnothing$ Fehler pro \\
\hline Marke & Vorkommen & in Prozent & Kommentar & $\mathrm{n}$ Fehler & Wort \\
\hline$A D$ & 1 & 0,8 & atlantica & 0 & 0 \\
\hline $\mathrm{AU}$ & 19 & 15,2 & $\begin{array}{l}\text { rettilinei (7), minoranze (9), } \\
\text { suolo (5) }\end{array}$ & 40 & 2,1 \\
\hline $\mathrm{CO}$ & 16 & 12,8 & $\begin{array}{l}\text { analfabetismo (6), litorali } \\
(5) \text {, desertica (5) }\end{array}$ & 40 & 2,5 \\
\hline el.CO & 4 & 3,2 & al contrario $(4)$ & 9 & 2,3 \\
\hline el.TS & 2 & 1,6 & $\begin{array}{l}\text { (gruppi) etnici (11), (morta- } \\
\text { lità) infantile (0) }\end{array}$ & 0 & 0 \\
\hline FO & 67 & 53,6 & & 167,5 & 2,5 \\
\hline TS & 9 & 7,2 & $\begin{array}{l}\text { zweimal delta (11), approdi } \\
(8), \text { area (5), versante (6), } \\
\text { capricorno (5) }\end{array}$ & 53,5 & 5,9 \\
\hline n.p. & 4 & 3,2 & Kalahari (6) & 6 & 1,5 \\
\hline el.n.p. & 3 & 2,4 & & 1 & 0,3 \\
\hline n Wörter & 125 & 100 & & 317 & 2,5 \\
\hline
\end{tabular}

Tabelle 15: Getilgte Wörter des C-Tests der Morante nach Gebrauchsmarken mit Angaben zu den Falschlösungen

Es ergibt sich für diesen C-Test dieselbe Konstellation der drei am häufigsten vertretenen Wortschatzbereiche wie im Test der Vespucci, nämlich folgen auf den fundamentalen Wortschatz der des hohen Gebrauchs (AU; 15,2 \% des Wortvorkommens) und der allgemeine (CO; 12,8 \%). Die höchsten durchschnittlichen Fehlerwerte bestehen mit 5,9 für die Ts-markierten Wörter und danach mit um mehr als die Hälfte geringeren Werten für den allgemeinen (CO; $2,5)$ und den fundamentalen Wortschatz (FO; 2,5) 


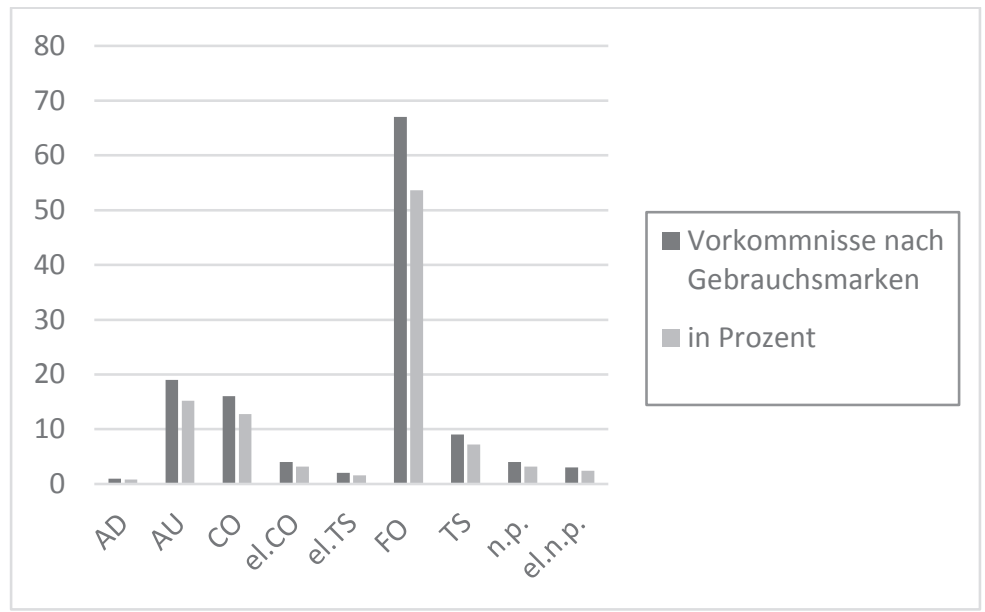

Abbildung 6: Getilgtes Wortvorkommen nach Gebrauchsmarken und ihrem prozentualen Anteil im C-Test, Morante

Nur in zwei der fünf C-Tests ist unter den drei durchschnittlich häufigsten Fehlern pro Wort einer Gebrauchsmarke der fundamentale Wortschatz vertreten. Im nächsten Kapitelabschnitt soll daher auch genauer veranschaulicht werden, wie diese Fehler bei den frequentesten Wörtern des Italienischen zustande gekommen sein könnten.

\subsubsection{Texte 1 bis 3 des C-Tests}

Die Tabelle 16 zeigt die ersten drei C-Testtexte, die das Thema Südafrika behandeln und den Lehrbuchseiten 59 bis 61 (Bersezio 2005) entnommen sind, mit Angaben zur grammatikalischen Kategorie des Wortvorkommens inklusive seiner Gebrauchsmarken und zu den Lösungsvorschlägen der Lernenden sowie den Fehleranzahlen.

\begin{tabular}{|c|c|c|c|c|c|c|}
\hline Text & $\begin{array}{l}\text { n Feh- } \\
\text { ler }\end{array}$ & Wort & Wortart & $\begin{array}{l}\text { Gebrauchs- } \\
\text { marke }\end{array}$ & Kommentar & $\begin{array}{l}\text { Lösungsvor- } \\
\text { schläge }\end{array}$ \\
\hline 1 & & $A$ & prep. & FO & & \\
\hline (S. 59) & 2 & nord & s.m. & $A U$ & & noi (2) \\
\hline 22 & & prevale & v.intr. & $A U$ & & \\
\hline \multirow[t]{7}{*}{ Lücken } & 2 & la & art.det. & FO & & II, lambiente \\
\hline & & savana & & CO TS geogr. & & \\
\hline & 4 & & pron.rel. & FO & & $\operatorname{con}(3), 0$ \\
\hline & & sfuma & v.intr. & $A U$ & & \\
\hline & & a & prep. & FO & & \\
\hline & 1 & sud & s.m. & FO & & 0 \\
\hline & & in & prep. & FO & & \\
\hline
\end{tabular}




\begin{tabular}{|c|c|c|c|c|c|c|}
\hline Text & $\begin{array}{l}\text { n Feh- } \\
\text { ler }\end{array}$ & Wort & Wortart & $\begin{array}{l}\text { Gebrauchs- } \\
\text { marke }\end{array}$ & Kommentar & $\begin{array}{l}\text { Lösungsvor- } \\
\text { schläge }\end{array}$ \\
\hline & 4 & prossimità & s.f. & $\mathrm{CO}$ & & $\begin{array}{l}0(2), \text { prossi- } \\
\text { mita ( } 2)\end{array}$ \\
\hline & & del & prep.art. & FO & & \\
\hline & 4 & Tropico & s.m. & TS geogr. & & 0 (3), Tropicale \\
\hline & & del & prep.art. & $\mathrm{FO}$ & & \\
\hline & 5 & Capricorno & s.m. & TS astrol. & & $0(5)$ \\
\hline & & in & prep. & $\mathrm{FO}$ & & \\
\hline & 0 & una & art.indet. & FO & & \\
\hline & & vasta & agg. & FO & & \\
\hline & 3 & area & s.f. & $\mathrm{AU}$ & & 0 (2), aria \\
\hline & 5 & desertica & agg. & $\mathrm{CO}$ & Tilgungsfehler & deserta (5) \\
\hline & & Namib & n.p. & & nicht im GRADIT & \\
\hline & & $e$ & cong. & FO & & \\
\hline & 6 & Kalahari & n.p. & & nicht im GRADIT & $\begin{array}{l}\text { Kalari, } 0(4) \text {, } \\
\text { Kalahale }\end{array}$ \\
\hline & & che & pron.rel. & FO & & \\
\hline & 2 & si & pron.pers. & $\mathrm{CO}$ & & $0(2)$ \\
\hline & & spinge & $\begin{array}{l}\text { v.pro- } \\
\text { nom.intr. }\end{array}$ & $\mathrm{CO}$ & & \\
\hline & 0 & fino & prep. & FO & & \\
\hline & & alla & prep.art. & FO & & \\
\hline & 2 & costa & s.f. & FO & & 0 , costiera \\
\hline & 0 & atlantica. & agg. & $A D$ & Tilgungsfehler & \\
\hline & & Sul & prep.art. & FO & & \\
\hline & 6 & versante & s.m. & TS geogr. & Tilgungsfehler & verso (4), 0 (2) \\
\hline & 0 & dell‘Oceano & n.p. & & $\begin{array}{l}\text { als n.p. nicht } \\
\text { im GRADIT }\end{array}$ & \\
\hline & 3 & $\begin{array}{l}\text { Atlantico } \\
\text { prevale }\end{array}$ & $\begin{array}{l}\text { el.n.p. } \\
\text { v.intr. }\end{array}$ & $A U$ & & $\begin{array}{l}\text { prevalente- } \\
\text { mente, prende, } \\
\text { precede }\end{array}$ \\
\hline & & un & art.indet. & FO & & \\
\hline & 0 & clima & s.m. & FO & & \\
\hline & & più & avv. & FO & & \\
\hline & $\begin{array}{l}0+ \\
10\end{array}$ & fresco & agg. & FO & & $\begin{array}{l}\text { freddo (acc.) } \\
(10)\end{array}$ \\
\hline & & $e$ & cong. & FO & & \\
\hline & 0 & umido & agg. & $A U$ & & \\
\hline & & $e$ & cong. & FO & & \\
\hline & 2 & quindi & avv. & FO & & $0(2)$ \\
\hline & 0 & l'ambiente & s.m. & FO & Tilgungsfehler & \\
\hline & & della & prep.art. & FO & & \\
\hline & 1 & foresta & loc.s.f. & $\mathrm{CO}$ & & 0 \\
\hline & & temperata. & el.loc.s.f. & el.CO & & \\
\hline (S. 59 f.) & 5 & litorali & s.m. & $\mathrm{CO}$ & & litoranei, lito- \\
\hline & & dell‘Oceano & n.p. & & nicht im GRADIT & \\
\hline & 0 & $\begin{array}{l}\text { Atlantico } \\
\text { sono }\end{array}$ & $\begin{array}{l}\text { el.n.p. } \\
\text { v.intr. }\end{array}$ & FO & & \\
\hline
\end{tabular}




\begin{tabular}{|c|c|c|c|c|c|c|}
\hline Text & $\begin{array}{l}\text { n Feh- } \\
\text { ler }\end{array}$ & Wort & Wortart & $\begin{array}{l}\text { Gebrauchs- } \\
\text { marke }\end{array}$ & Kommentar & $\begin{array}{l}\text { Lösungsvor- } \\
\text { schläge }\end{array}$ \\
\hline & \multirow[t]{2}{*}{7} & rettilinei & agg. & $\mathrm{AU}$ & & $\begin{array}{l}\text { retticolari, retti- } \\
\text { linee }(2), 0(4)\end{array}$ \\
\hline & & $e$ & cong. & FO & & \\
\hline & \multirow[t]{2}{*}{3} & compatti & agg. & $\mathrm{AU}$ & & compatte (2), 0 \\
\hline & & $A l$ & loc.avv. & $\mathrm{CO}$ & & \\
\hline & \multirow[t]{2}{*}{4} & contrario & el.loc.avv. & el.CO & & $\begin{array}{l}0, \text { contorno (2), } \\
\text { continente }\end{array}$ \\
\hline & & le & art.det. & $\mathrm{CO}$ & & \\
\hline & \multirow[t]{2}{*}{0} & coste & s.f. & FO & & \\
\hline & & dell‘Oceano & n.p. & & & \\
\hline & 1 & $\begin{array}{l}\text { Indiano } \\
\text { sono }\end{array}$ & $\begin{array}{l}\text { el.n.p. } \\
\text { v.intr. }\end{array}$ & FO & nicht im GRADIT & Indiani \\
\hline & \multirow[t]{2}{*}{3} & in & loc.avv. & $\mathrm{CO}$ & & 0 (2), irregolari \\
\hline & & gran & agg. & FO & & \\
\hline & \multirow[t]{2}{*}{1} & parte & el.loc.avv. & el.co & & 0 \\
\hline & & paludose & agg. & $\mathrm{CO}$ & & \\
\hline & \multirow[t]{2}{*}{3} & malsane & agg. & $\mathrm{CO}$ & & $\begin{array}{l}\text { malsani, } \\
\text { malsastre, } 0\end{array}$ \\
\hline & & $e$ & cong. & FO & & \\
\hline & \multirow[t]{2}{*}{2} & povere & agg. & FO & & poveri, 0 \\
\hline & & $d i$ & prep. & FO & & \\
\hline & \multirow[t]{2}{*}{8} & approdi & s.m. & TS mar. & & $0(8)$ \\
\hline & & Nell'Oceano & n.p. & & nicht im GRADIT & \\
\hline & \multirow[t]{2}{*}{0} & Indiano & el.n.p. & & & \\
\hline & & $\begin{array}{l}\text { si } \\
\text { trova }\end{array}$ & pron.pers. & CO & & trovano \\
\hline & 1 & lisola & $\begin{array}{l}\text { nom.intr. } \\
\text { s.f. }\end{array}$ & FO & & trovano \\
\hline & \multirow[t]{2}{*}{6,5} & più & avv. & FO & & $\begin{array}{l}0 \text { (5), provi- } \\
\text { dence, piu }\end{array}$ \\
\hline & & vasta & agg. & FO & & \\
\hline & \multirow[t]{2}{*}{2} & quella & $\begin{array}{l}\text { pron.di- } \\
\text { mostr. }\end{array}$ & $\mathrm{FO}$ & & quello, 0 \\
\hline & & del & prep.art. & $\mathrm{FO}$ & & \\
\hline & \multirow[t]{2}{*}{0} & $\begin{array}{l}\text { Madaga- } \\
\text { scar }\end{array}$ & n.p. & & & \\
\hline & & 0 & cong. & FO & & \\
\hline & \multirow[t]{2}{*}{1} & grande & agg. & FO & & 0 \\
\hline & & isola & s.f. & FO & & \\
\hline & \multirow[t]{2}{*}{$\begin{array}{l}5+1 \\
\text { acc. }\end{array}$} & rossa & agg. & $\mathrm{FO}$ & & $\begin{array}{l}\text { rosa }(\text { acc.), } 0 \\
(4), \text { rosso }\end{array}$ \\
\hline & & come & cong. & FO & & \\
\hline & \multirow[t]{2}{*}{$\begin{array}{l}3+3 \\
\text { acc. }\end{array}$} & veniva & v.aus. & $\mathrm{CO}$ & & $\begin{array}{l}0 \text { (2), venne } \\
\text { (acc.) (3), ve- } \\
\text { nita }\end{array}$ \\
\hline & & chiamata & v.tr. & FO & & \\
\hline & \multirow[t]{2}{*}{6} & per & prep. & FO & & $\begin{array}{l}0(4) \text {, prima, } \\
\text { prende }\end{array}$ \\
\hline & & il & art.det. & FO & & \\
\hline & \multirow[t]{2}{*}{5} & colore & s.m. & FO & & 0 (4), colpa \\
\hline & & del & prep.art. & FO & & \\
\hline & 5 & suolo. & s.m. & $A U$ & & suo, 0 (4) \\
\hline
\end{tabular}




\begin{tabular}{|c|c|c|c|c|c|c|}
\hline Text & $\begin{array}{l}\text { n Feh- } \\
\text { ler }\end{array}$ & Wort & Wortart & $\begin{array}{l}\text { Gebrauchs- } \\
\text { marke }\end{array}$ & Kommentar & $\begin{array}{l}\text { Lösungsvor- } \\
\text { schläge }\end{array}$ \\
\hline & & Dal & prep.art. & FO & & \\
\hline & 0 & punto & loc.s.m. & $\mathrm{CO}$ & & \\
\hline & & di & el.loc.s.m. & el.CO & & \\
\hline & 0 & vista & el.loc.s.m. & el.CO & & \\
\hline & & geologico & agg. & CO TS scient. & & \\
\hline & 0 & si & pron.pers. & $\mathrm{CO}$ & & \\
\hline & & tratta & $\begin{array}{l}\text { v.pro- } \\
\text { nom.intr. }\end{array}$ & $\mathrm{CO}$ & & \\
\hline & & $d i$ & prep. & $\mathrm{FO}$ & & \\
\hline \multirow{32}{*}{$\begin{array}{l}3(\mathrm{~S} . \\
61) \\
25 \text { Lü- } \\
\text { cken }\end{array}$} & 2 & Oggi & avv. & $\mathrm{FO}$ & & $\operatorname{ogg}(2)$ \\
\hline & & $i$ & art.det. & $\mathrm{CO}$ & & \\
\hline & 4 & suoi & agg.poss. & FO & & sui $(2), 0$, sud \\
\hline & & territori & s.m. & $A U$ & & \\
\hline & 0 & sono & v.intr. & FO & & \\
\hline & & $\begin{array}{l}\text { scarsa- } \\
\text { mente }\end{array}$ & avv. & $\mathrm{CO}$ & & \\
\hline & 1 & popolati & agg. & $\mathrm{CO}$ & & popolate \\
\hline & & $e$ & cong. & FO & & \\
\hline & 0 & la & art.det. & FO & & \\
\hline & & densità & s.f. & TS stat. & & \\
\hline & & $\grave{e}$ & v.intr. & $\mathrm{FO}$ & & \\
\hline & 3 & bassissima & agg. & FO & & $\begin{array}{l}\text { bassisima (2), } \\
0\end{array}$ \\
\hline & & nelle & prep.art. & FO & & \\
\hline & 2 & aree & s.f. & $A U$ & & 0 , aride \\
\hline & & desertiche & agg. & $\mathrm{CO}$ & & \\
\hline & 3 & dell'interno & s.m. & FO & & 0 (2), intità \\
\hline & & mentre & cong. & FO & & \\
\hline & 1 & cresce & v.intr. & FO & & 0 \\
\hline & & dove & cong. & FO & & \\
\hline & 0 & il & art.det. & FO & & \\
\hline & & clima & s.m. & FO & & \\
\hline & & $\grave{e}$ & v.intr. & FO & & \\
\hline & $\begin{array}{l}1,5 \\
+5 \\
\text { acc. }\end{array}$ & più & avv. & FO & & $\begin{array}{l}\text { prevalente- } \\
\text { mente (acc.) } \\
\text { (5), piu, parti- } \\
\text { colarmente }\end{array}$ \\
\hline & & mite. & agg. & $A U$ & & \\
\hline & 0 & La & art.det. & FO & & \\
\hline & & maggior & agg. & FO & & \\
\hline & 0 & parte & s.f. & FO & & \\
\hline & & della & prep.art. & FO & & \\
\hline & 0 & popolazione & s.f. & $A U$ & & \\
\hline & & appartiene & v.intr. & FO & & \\
\hline & 2 & ai & prep.art. & FO & & a dei, ancora \\
\hline & 0 & $\begin{array}{l}\text { gruppi } \\
\text { etnici } \\
\text { e }\end{array}$ & $\begin{array}{l}\text { loc.s.m. } \\
\text { el.loc.s.m. } \\
\text { cong. }\end{array}$ & $\begin{array}{l}\text { TS etnol. } \\
\text { el.TS etnol. } \\
\text { FO }\end{array}$ & & \\
\hline
\end{tabular}




\begin{tabular}{|c|c|c|c|c|c|c|}
\hline Text & $\begin{array}{l}\text { n Feh- } \\
\text { ler }\end{array}$ & Wort & Wortart & $\begin{array}{l}\text { Gebrauchs- } \\
\text { marke }\end{array}$ & Kommentar & $\begin{array}{l}\text { Lösungsvor- } \\
\text { schläge }\end{array}$ \\
\hline & \multirow[t]{2}{*}{3} & linguistici & agg. & $\mathrm{CO}$ & & $0(3)$ \\
\hline & & bantu. & agg. & TS etnol. & & \\
\hline & \multirow[t]{2}{*}{0} & $\mathrm{La}$ & art.det. & FO & & \\
\hline & & presenza & s.f. & FO & & \\
\hline & \multirow{2}{*}{$\begin{array}{l}11 \\
\text { acc. }\end{array}$} & $d i$ & prep. & FO & & dei (acc.) (11) \\
\hline & & bianchi & s.m. & FO & & \\
\hline & \multirow[t]{2}{*}{2} & $d i$ & prep. & FO & & dalle, dei \\
\hline & & origine & s.f. & $A U$ & & \\
\hline & \multirow[t]{2}{*}{4} & europea & agg. & FO & & $\begin{array}{l}0 \text {, europee (2), } \\
\text { europei }\end{array}$ \\
\hline & & è & v.intr. & FO & & \\
\hline & \multirow[t]{2}{*}{7} & forte & agg. & FO & & $\begin{array}{l}0(6) \text {, formi- } \\
\text { dabile }\end{array}$ \\
\hline & & e & cong. & FO & & \\
\hline & \multirow[t]{2}{*}{6} & $v i$ & avv. & FO & & 0 (5), vasta \\
\hline & & sono & v.procompl. & $\mathrm{CO}$ & & \\
\hline & \multirow[t]{2}{*}{$\begin{array}{l}2+3 \\
\text { acc. }\end{array}$} & anche & cong. & FO & & $\begin{array}{l}0 \text { (2), ancora } \\
\text { (3) (acc.) }\end{array}$ \\
\hline & & consistenti & agg. & $\mathrm{CO}$ & & \\
\hline & 9 & minoranze & s.f. & $A U$ & & $\begin{array}{l}0(4), \text { minori } \\
(3) \text {, minorenni, } \\
\text { minore }\end{array}$ \\
\hline
\end{tabular}

Tabelle 16: Aus dem Lehrbuch entnommene Texte 1 bis 3 (Bersezio 2005, S. 59-61) des CTests an der Morante

Von den insgesamt 73 Lücken vervollständigten alle Lernenden 23 komplett richtig, und weitere 35 wurden ein- bis viermal falsch oder nicht ergänzt. Die Zusammensetzung dieser Wörter nach Wortart und Gebrauchsmarke verhält sich in folgender Weise: Bei den von allen richtig ergänzten Wörtern handelt es sich um zwölf Ausdrücke aus dem fundamentalen Wortschatz (Fo), zwei aus dem des hohen Gebrauchs (AU), einen aus dem der hohen Verfügbarkeit (AD), also insgesamt 15 Wörter aus dem Basiswortschatz (VDB), zwei Eigennamen und zwei Elemente von Eigennamen sowie zwei Co-gekennzeichnete Wörter (allgemeiner Wortschatz) und zwei mit den Marken el.Co und el.Ts etnol. Diese seltener gebrauchten Wörter waren den Schülern wahrscheinlich geläufig, denn eines ist das vielbenutzte Personalpronomen der dritten Person Singular, si, und die beiden anderen Co-gekennzeichneten Wörter stammen aus dem bekannten Phrasem pu-nto di vi-sta (Standpunkt), in dem alle drei Wörter für sich genommen zum fundamentalen Wortschatz zählen. Das fachwortschatzsprachliche Element aus dem Nominalphrasem gruppo etn-ico (Volksgruppe) kannten die Lernenden aus dem Erdkundeunterricht.

Von den ein- bis viermal falsch oder nicht gelösten Wörtern zählt knapp die Hälfte zum fundamentalen Wortschatz (FO; 18 Wörter), während sich die anderen 19 auf die Bereiche des hohen Gebrauchs (AU; 5), des allgemeinen Wortschatzes (CO; 10; el. Co; 2$)$ und des Fachwortschatzes (Ts geogr.; 1) ver- 
teilen, bzw. als Element eines Eigennamens (1) keine Gebrauchsmarke aufweisen. In der Regel wurden Wörter, die nicht zum Basiswortschatz zählen, von mehreren, oft vier Lernenden falsch vervollständigt. So zum Beispiel das von dreien nicht ergänzte und von einem weiteren mit Tropicale (Adjektiv, lexikalisch und grammatikalisch falsch) falsch vervollständigte einzige Fachwort, Tro-pico (Wendekreis), und das Adjektiv dese-rtico (wüstenartig) aus dem allgemeinen Wortschatz, das fünf Lernende zu dem semantisch und graphematisch sehr verwandten Adjektiv deserto (verlassen, öde) ergänzten. Es stammt ebenfalls aus dem allgemeinen Wortschatz, wird also wahrscheinlich nicht häufiger gebraucht als das andere, wodurch der Lösungsvorschlag zeigt, dass einige Schüler bezüglich beider Adjektive zumindest nicht über eine produktive lexikalische Kompetenz verfügten.

Bei den nicht allzu oft falsch gelösten Wörtern treten außerdem grammatikalisch-lexikalische und orthographische Schwächen hervor, die auf geringere Italienischkenntnisse hindeuten. Die Fehler lexikogrammatikalischer Art betreffen zum Beispiel das Adjektiv eur-opea, das als Bezugswort origine (Ursprung) hat, von dem einige Lernende nicht zu wissen schienen, dass es feminin Singular ist (oder sie beachteten es nicht), da sie das Adjektiv zweimal mit der femininen Pluralendung $-e$ und einmal mit der maskulinen versahen. Auch wenn sie die verschiedenen Genus-Endungen gut kannten und diese somit auch verwenden konnten, ohne die Bedeutung eines bestimmten Wortes zu kennen, war die Nutzung dieser Strategie hier jedoch dadurch erschwert, dass vor origine kein Artikel steht. Allerdings wäre dieser durch den Vokalanlaut von origine ohnehin apostrophiert, so dass nicht mehr erkennbar ist, ob es sich um die maskuline oder feminine Form des Artikels handelt.

Unregelmäßigkeiten rein orthographischer Art finden sich u.a. beim Superlativ bassissima und dem Fehlen des Akzents im Wort prossimità. Während das Auslassen von Akzenten auch unter erstsprachig italienischen Altersgenossen sehr verbreitet ist, da der Großteil des Unterrichts und seiner Leistungskontrollen auf Mündlichkeit basiert, wissen im Grunde alle Lernenden, die in Italien die Grundschule besucht haben und nicht an einer Lese-RechtschreibSchwäche leiden, dass der absolute Superlativ auf -issimo mit doppeltem S geschrieben wird. Wohingegen zweisprachige Schülerinnen und Schüler den Superlativ wahrscheinlich erkennen und richtig verwenden können, aber z.T. noch kein korrektes Schriftbild dazu verinnerlicht haben.

Ein anderer orthographischer Fehler lässt darauf schließen, dass sein Verfasser ein Wort bildete, dass er sich entweder ausdachte oder mit einem anderen „falsch abgespeicherten“ verwechselte, nämlich prevare (anstelle von prevale (überwiegt)). Gleiches gilt für das aus dem allgemeinen Wortschatz stam- 
mende Adjektiv malsane (ungesund), das u.a. zu dem Wort malsastre vervollständigt wurde, das ebenfalls nicht existiert. Beide Beispiele verleiten eher dazu, als Urheber einen in der Zweitsprache Lernenden zu vermuten. ${ }^{154}$

Vor der Besprechung der häufigeren Fehlerquellen sei auf ein letztes viermal falsch gelöstes Wort hingewiesen, welches das Element eines Phrasems bildet, das in keinem der vier Fälle produziert wurde und den Betreffenden somit vielleicht unbekannt ist. Es handelt sich um cont-rario aus dem Adverbialphrasem al contrario (Co; im Gegenteil), das einer gar nicht, zwei Lernende zu contorno und einer zu continente ergänzten. Morphosyntaktisch ist der Lösungsvorschlag contorno zwar richtig, aber logisch-semantisch sind er und auch continente nicht akzeptabel. Deshalb zeigt sich außer dem nicht vorhandenen lexikalischen Wissen über das Adverbialphrasem auch ein Mangel an Textkompetenz: Da im Vorfeld eine Beschreibung der Form der Atlantikküstens erfolgt, wird nun der Kontrast zur Form derer des Indischen Ozeans aufgezeigt und lässt Testteilnehmern mit einem gewissen Grad an Textkompetenz beide Lösungsvorschläge „Kontinent“ und „Rand“ unmittelbar unlogisch erscheinen. Allerdings handelt es sich hier um das vielleicht einzige Beispiel unter den selteneren Falschlösungen, bei dem ein komplexerer sprachlicher Zusammenhang die Ursache der Fehlerquelle gewesen sein mag. In den anderen Fällen ist das sprachliche Umfeld durch seine Überschaubarkeit und lexikalische Klarheit bzw. Einfachheit für eine fehlerfreie Lückenfüllung eher förderlich. Die ersten Küsten werden im Text nämlich nicht als coste (Fo), sondern mit dem Synonym litorali aus dem Co-Wortschatz bezeichnet, und auch die beiden Adjektive zur Beschreibung ihrer Form zählen nicht zum fundamentalen Wortschatz, sondern stammen aus dem des hohen Gebrauchs (AU). Darüber hinaus steht das Adverbialphrasem am Satzanfang; hätte es seinen Platz nach dem Subjekt und Prädikat, wäre einigen Lernenden die richtige Lösung womöglich eher geglückt.

Wie auch aus der Tabelle entnommen werden kann, sind im ersten der drei Texte vier Wörter unregelmäßig getilgt worden. In einem Fall hätte das vorangehende Wort um die Hälfte verkürzt werden müssen, so dass die Testteilnehmer durch diesen Fehler in der Testkonstruktion ein zusätzliches vollständiges Wort als Unterstützung für die Lückenbearbeitung hatten, während in den anderen drei Fällen zu früh getilgt wurde. Den meisten Lernenden scheint das keine Schwierigkeiten bereitet zu haben, da sie die Lücke korrekt füllten. Der erste Fall betrifft die zwei aufeinanderfolgenden Lücken in ar-ea dese-rtica (wüstenartiges Gebiet), wobei nur zwei Lernende area nicht vervollständigten und es sich beim dritten Fehler für dieses Wort wohl mehr um einen Rechtschreibfehler als um die bewusste Verwendung des Wortes aria (Luft) handelt.

154 So ist es gut möglich, dass der Fehler prevare vom chinesischen Schüler mit den geringen italienischen Sprachkenntnissen stammt, da -1 und -r in seiner Herkunftssprache bekanntlich keine Phoneme sind. 
Die anderen beiden zu früh getilgten Wörter (l'ambi-ente, Fo und dell'Oceano, n.p.) wurden von allen SchülerInnen richtig vervollständigt.

\subsubsection{Häufige Falschlösungen}

In den ersten drei Texten des C-Tests an der Schule Morante wurden 15 Wörter vermehrt falsch gelöst. Sie sind in der Tabelle 17 nach ihrem Vorkommen in den einzelnen Texten geordnet.

\begin{tabular}{|c|c|c|c|c|c|c|}
\hline Text & $\begin{array}{l}\mathrm{n} \\
\text { Fehler }\end{array}$ & Wort & Wortart & $\begin{array}{l}\text { Gebrauchs- } \\
\text { marke }\end{array}$ & Kommentar & Lösungsvorschläge \\
\hline \multirow[t]{4}{*}{$\begin{array}{l}1 \\
\text { (S. 59) }\end{array}$} & 5 & $\begin{array}{l}\text { Capri- } \\
\text { corno }\end{array}$ & s.m. & TS astrol. & \multirow{4}{*}{$\begin{array}{l}\text { Tropico del } \\
\text { Capricorno } \\
\text { Tilgungsfehler } \\
\text { nicht im } \\
\text { GRADIT }\end{array}$} & $0(5)$ \\
\hline & 5 & desertica & agg. & $\mathrm{CO}$ & & deserta (5) \\
\hline & 6 & Kalahari & n.p. & & & Kalari, 0 (4), Kalahale \\
\hline & 6 & versante & s.m. & TS geogr. & & verso (4), 0 (2) \\
\hline \multirow[t]{8}{*}{$\begin{array}{l}2 \text { (S. } 59 \\
\text { f.) }\end{array}$} & 5 & litorali & s.m. & $\mathrm{CO}$ & \multirow{8}{*}{$\begin{array}{l}\text { Superlativ } \\
\text { più vasta }\end{array}$} & $\begin{array}{l}\text { litoranei, litografia (2), } \\
0, \text { litorale }\end{array}$ \\
\hline & 7 & rettilinei & agg. & $A U$ & & $\begin{array}{l}\text { retticolari, rettilinee } \\
(2), 0(4)\end{array}$ \\
\hline & 8 & approdi & s.m. & TS mar. & & $0(8)$ \\
\hline & 6,5 & più & avv. & $\mathrm{FO}$ & & 0 (5), providence, piu \\
\hline & 5 & rossa & agg. & $\mathrm{FO}$ & & $\begin{array}{l}\text { rosa }(\text { acc.), } 0(4) \text {, } \\
\text { rosso }\end{array}$ \\
\hline & 6 & per & prep. & FO & & 0 (4), prima, prende \\
\hline & 5 & colore & s.m. & FO & & 0 (4), colpa \\
\hline & 5 & suolo & s.m. & $A U$ & & suo, 0 (4) \\
\hline 3 & 7 & forte & agg. & FO & \multirow{3}{*}{ esservi } & 0 (5), formidabile \\
\hline \multirow[t]{2}{*}{ (S. 61) } & 6 & vi & avv. & $\mathrm{FO}$ & & 0 (5), vasta \\
\hline & 9 & $\begin{array}{l}\text { mino- } \\
\text { ranze }\end{array}$ & s.f. & $\mathrm{AU}$ & & $\begin{array}{l}0(4) \text {, minori }(3) \text {, mino- } \\
\text { renni, minore }\end{array}$ \\
\hline
\end{tabular}

Tabelle 17: Häufigste Falschlösungen in den Texten 1 bis 3 des C-Tests an der Schule Morante

Sechs der Wörter zählen zum fundamentalen Wortschatz (Fo), drei weitere als AU-gekennzeichnete zum Basiswortschatz (VDB) und ein Drittel zum Fachwortschatz (3) und zum allgemeinen Wortschatz (2), bzw. trägt als Eigenname keine Marke. Unter diesen häufig falsch gelösten Wörtern befinden sich neben dem Eigennamen sieben Substantive, von denen nur eines aus dem fundamentalen Wortschatz stammt, eine einfache Präposition sowie einige Adjektive und Adverbien. Verben sind hier nicht vertreten, sondern mit dem sechsmal falsch gelösten Adverb vi nur ein Teil des prokomplementaren Verbs esservi (Co; formalere, seltenere Form von esserci: da sein, geben). Es tritt auch im C-Test der Schule Vespucci auftritt und bereitete dort ebenfalls vermehrt 
Schwierigkeiten. Insgesamt sind unter den getilgten Wörtern dieser Texte nur fünf Verben, die alle konjugiert sind und von den meisten Testteilnehmern problemlos vervollständigt wurden. Es gibt also keine komplexeren verbalen Formen wie zum Beispiel Gerundien. Eine leicht kompliziertere Form ist ein passives Verb, das mit dem Hilfsverb venire (Co) anstelle des häufigeren essere gebildet ist, aber nur von zwei Lernenden nicht ergänzt wurde.

Anders als an der Schule Vespucci gibt es hier kein Wort, das von allen 13 Testteilnehmern falsch oder nicht ergänzt wurde, sondern die maximale Fehlerzahl beläuft sich auf neun bei dem Substantiv minor-anze (AU; Minderheiten). Vier Lernende ergänzten es gar nicht und die übrigen nur zu den logisch falschen Wörtern für „Niedrige“ und „Minderjährige“, so dass vermutet werden kann, dass sie das Wort für „Minderheiten“ entweder nicht oder nicht sicher beherrschten oder dass sie in ihrem Textverständnis durch das sprachliche Umfeld dieser Lücke so eingeschränkt waren, dass ihnen eine korrekte Bearbeitung nicht möglich war. Das neunmal falsch gelöste Wort steht nämlich in einem nebengeordneten Satz, der durch die Konjunktion ,,und“ eingeleitet wird und das prokomplementare Verb enthält. Erschwerend ist zusätzlich, dass sich das Subjekt dadurch nicht am Anfang befindet, sondern erst später auftritt, und zwar genau als das getilgte Wort „Minderheiten“. Desweiteren geht ihm ein Adjektiv voraus, das aus dem allgemeinen Wortschatz stammt, nicht unbedingt allen Testteilnehmern bekannt war und deshalb wahrscheinlich keine Verständnishilfe für den Kontext der zu füllenden Lücke darstellte.

Acht Schüler vervollständigten das maritime fachwortschatzsprachliche Wort appr-odi (Anlegestellen) nicht. Hierbei handelt es sich um ein Wort, dem die meisten Lernenden bisher wohl nur in ihrem Erdkundeunterricht begegnet waren, in dem sie es während der Unterrichtsbeobachtungen dieser Studie gemeinsam im Text gelesen und mit der Lehrkraft besprochen hatten. Bezüglich der anderen beiden vermehrt nicht oder falsch ergänzten Wörter aus dem Fachwortschatz lässt sich vermerken, dass bei Trop-ico del Capri-corno (Wendekreis des Steinbocks) fünf das Wort für „Steinbock“ nicht vervollständigten, wobei insgesamt nur vier SchülerInnen das Wort für „Wendekreis“ nicht gebildet hatten. Vielleicht handelt es sich um Testpersonen, in deren Muttersprachen (und teilweise auch ersten Schulsprachen) es, wie im Deutschen, Begriffe für die Wendekreise gibt, welche die Tierkreiszeichen nicht beinhalten, sondern anders geformt sind.

Vers-ante (Hang) hingegen wurde von vier SchülerInnen zu verso ergänzt, das verschiedene Bedeutungen hat, die auch dem fundamentalen Wortschatz entstammen. „Richtung, Seite“ sind davon diejenigen, welche semantisch einigermaßen nah bei versante liegen. Deshalb kann man vermuten, dass die entsprechenden Testteilnehmer ein häufiger gebrauchtes Wort einsetzten, von dem sie annahmen, dass es im Kontext passt, weil ihnen das erforderliche fachsprachliche Wort nicht zur Verfügung stand. 
Auffällig ist, dass fünf Lernende das Adverb $p$-iù, mit dem im Italienischen die Adjektivsteigerungsform des Komparativs und des relativen Superlativs gebildet wird, nicht ergänzten, und ein weiterer ein fiktives Substantiv produzierte, obwohl die Testteilnehmer bei den umliegenden Lücken kaum etwas falsch machten oder ausließen. Das sprachliche Umfeld gestaltete sich natürlich noch simpler mit einer Satzformulierung wie „Madagaskar ist die größte Insel". Dann wären wahrscheinlich alle oder so gut wie alle imstande gewesen, den Superlativ zu bilden.

Bevor die anderen beiden Testtexte untersucht werden, sei noch auf ein Wort hingewiesen, das die SchülerInnen in der überwiegenden Mehrheit (11) abweichend vom Original vervollständigten: Sie machten aus der einfachen Präposition $d i$ eine artikulierte, wahrscheinlich weil Substantive viel häufiger mit einem Artikel auftreten als ohne. Zudem erscheint das Wort als ein Genitivattribut, auf welches sich ein weiteres Genitivattribut mit derselben Präposition bezieht, wobei diese von elf Lernenden wie im Ausgangstext ergänzt wurde. Vielleicht kam ihnen die Konstruktion mit zweifach einfacher Präposition unpassend vor.

\subsubsection{Text 4 und 5 des C-Tests}

Die beiden letzten Texte des C-Tests sind den Lehrbuchseiten 32 und 33 (Bersezio 2005) entnommen und behandeln das Land Ägypten. Wieder dient eine Tabelle (Tab. 18) dem Überblick über die Texte mit einer sprachlichen Klassifizierung und Angaben zu den Fehlerzahlen und Lösungsvorschlägen.

Bei der Konstruktion des vierten C-Testtextes mit 25 Lücken sind erneut drei Wörter unregelmäßig getilgt worden, wodurch in zwei Fällen nicht das übernächste, sondern erst das dritte Wort zur Hälfte gelöscht erscheint, so dass sich in diesen Fällen das Bearbeiten des Tests für die Lernenden eher erleichterte. Hier gibt es nur einen Fehler zu verzeichnen. Anders sieht es mit der dritten Unregelmäßigkeit aus, wodurch die zwei aufeinanderfolgenden Wörter $d$-el (von dem) und de-lta (Ts geogr.; Delta) beide zur Hälfte getilgt wurden. Das Wort de-lta vervollständigte fast keiner (11 von 13) richtig, doch es muss nicht sein, dass es bei regelmäßiger Tilgung zu wesentlich weniger Falschlösungen gekommen wäre. Es handelt sich um ein fachwortschatzsprachliches Wort und zur richtigen Bearbeitung ist ein gewisses Maß an Textkompetenz erforderlich. Eine genauere Besprechung des Wortes wird das w.u. im Rahmen der Analyse der frequentesten Falschlösungen zeigen.

\begin{tabular}{|c|c|c|c|c|c|c|}
\hline Text & $\begin{array}{l}\mathrm{n} \\
\text { Fehler }\end{array}$ & Wort & Wortart & $\begin{array}{l}\text { Gebrauchs- } \\
\text { marke }\end{array}$ & Kommentar & $\begin{array}{l}\text { Lösungsvor- } \\
\text { schläge }\end{array}$ \\
\hline 4 (S. 32) & & tuttavia & cong. & FO & & \\
\hline \multirow{3}{*}{$\begin{array}{l}25 \text { Lü- } \\
\text { cken }\end{array}$} & 1 & soltanto & avv. & FO & & 0 \\
\hline & & una & art.indet. & FO & & \\
\hline & 1 & piccola & agg. & FO & & piccolo \\
\hline
\end{tabular}




\begin{tabular}{|c|c|c|c|c|c|c|}
\hline Text & $\begin{array}{l}\mathrm{n} \\
\text { Fehler }\end{array}$ & Wort & Wortart & $\begin{array}{l}\text { Gebrauchs- } \\
\text { marke }\end{array}$ & Kommentar & $\begin{array}{l}\text { Lösungsvor- } \\
\text { schläge }\end{array}$ \\
\hline & & parte & s.f. & FO & \multirow{6}{*}{$\begin{array}{l}\text { Tilgungsfeh- } \\
\text { ler }\end{array}$} & \\
\hline & 3 acc. & del & prep.art. & FO & & di (acc.) (3) \\
\hline & 1 & territorio & s.m. & $A U$ & & territori \\
\hline & & $\grave{e}$ & v.intr. & FO & & \multirow{5}{*}{ abitato (acc.) (4) } \\
\hline & \multirow[t]{2}{*}{4 acc. } & abitata: & agg. & $\mathrm{CO}$ & & \\
\hline & & la & art.det. & FO & & \\
\hline & \multirow[t]{2}{*}{0} & popolazione & s.f. & $A U$ & & \\
\hline & & infatti & cong. & FO & & \\
\hline & \multirow[t]{2}{*}{9} & vive & v.intr. & FO & & \multirow[t]{2}{*}{$\begin{array}{l}\text { vista (2), viene } \\
\text { (2), vi si è (2), } 0 \\
(2), \text { vi è }\end{array}$} \\
\hline & & concentrata & agg. & $\mathrm{CO}$ & & \\
\hline & \multirow[t]{2}{*}{1} & lungo & prep. & FO & & \multirow[t]{8}{*}{ lungho } \\
\hline & & le & art.det. & FO & & \\
\hline & \multirow[t]{2}{*}{0} & coste & s.f. & FO & & \\
\hline & & del & prep.art. & FO & & \\
\hline & \multirow[t]{2}{*}{0} & $\begin{array}{l}\text { Mediterra- } \\
\text { neo }\end{array}$ & n.p.m. & & & \\
\hline & & e & cong. & FO & & \\
\hline & \multirow[t]{2}{*}{0} & nelle & prep.art. & FO & & \\
\hline & & zone & s.f. & FO & & \\
\hline & \multirow[t]{2}{*}{$\begin{array}{l}4+2 \\
\text { acc. }\end{array}$} & più & avv. & FO & & \multirow[t]{2}{*}{$\begin{array}{l}\text { pianeggianti, } \\
\text { posteriori, palu- } \\
\text { dose, poco } \\
\text { (acc.) (2), piu }\end{array}$} \\
\hline & & ospitali & agg. & $\mathrm{CO}$ & & \\
\hline & 6 & del & prep.art. & FO & & $\begin{array}{l}\text { dalle, } 0(2), \mathrm{da} \text {, } \\
\text { di (2) }\end{array}$ \\
\hline & \multirow[t]{2}{*}{11} & delta & s.m. & TS geogr. & \multirow[t]{18}{*}{$\begin{array}{l}\text { Tilgungsfeh- } \\
\text { ler }\end{array}$} & $\begin{array}{l}0(3) \text {, deserto } \\
\text { (5), detriti, de- } \\
\text { sertiche, deserti }\end{array}$ \\
\hline & & $e$ & cong. & FO & & \\
\hline & \multirow[t]{2}{*}{4} & della & prep.art. & FO & & \multirow[t]{5}{*}{ delle (2), 0 (2) } \\
\hline & & valle & s.f. & TS geogr. & & \\
\hline & 0 & $\begin{array}{l}\text { del } \\
\text { Nilo }\end{array}$ & $\begin{array}{l}\text { prep.art. } \\
\text { n.p. }\end{array}$ & $\mathrm{FO}$ & & \\
\hline & \multirow[t]{2}{*}{0} & dove & cong. & FO & & \\
\hline & & la & art.det. & FO & & \\
\hline & \multirow[t]{2}{*}{0,5} & densità & s.f. & TS stat. & & \multirow[t]{2}{*}{ densita } \\
\hline & & $\grave{e}$ & v.intr. & FO & & \\
\hline & \multirow[t]{3}{*}{6} & spesso & avv. & FO & & \multirow[t]{9}{*}{$\begin{array}{l}0(2), \text { special- } \\
\text { mente }(2), \\
\text { spempre }(2)\end{array}$} \\
\hline & & elevatissima & agg. & $\mathrm{CO}$ & & \\
\hline & & e & cong. & FO & & \\
\hline & \multirow[t]{3}{*}{0} & talvolta & avv. & $A U$ & & \\
\hline & & supera & v.tr. & FO & & \\
\hline & & i 7000 & art.det. & $\mathrm{CO}$ & & \\
\hline & \multirow[t]{2}{*}{0} & abitanti & s.m. & $A U$ & & \\
\hline & & per & prep. & $\mathrm{FO}$ & & \\
\hline & 0 & chilometro & loc.s.m. & TS metrol. & & \\
\hline
\end{tabular}




\begin{tabular}{|c|c|c|c|c|c|c|}
\hline Text & $\begin{array}{l}\mathrm{n} \\
\text { Fehler }\end{array}$ & Wort & Wortart & $\begin{array}{l}\text { Gebrauchs- } \\
\text { marke }\end{array}$ & Kommentar & $\begin{array}{l}\text { Lösungsvor- } \\
\text { schläge }\end{array}$ \\
\hline & & quadrato. & el.loc.s.m. & el.TS metrol. & $\begin{array}{l}\text { Tilgungsfeh- } \\
\text { ler }\end{array}$ & \\
\hline & & Sebbene & cong. & $\mathrm{AU}$ & & \\
\hline & 1 & oltre & avv. & $\mathrm{AU}$ & & 0 \\
\hline & & la & art.det. & FO & & \\
\hline & 3 & metà & s.f. & FO & & $\begin{array}{l}\text { media (2), meta } \\
(2)\end{array}$ \\
\hline & & della & prep.art. & FO & & \\
\hline & 0 & popolazione & s.f. & $\mathrm{AU}$ & & \\
\hline 5 (S. 33) & 2 & il & art.det. & $\mathrm{FO}$ & & $0(2)$ \\
\hline \multirow{29}{*}{$\begin{array}{l}25 \text { Lü- } \\
\text { cken }\end{array}$} & & recente & agg. & $\mathrm{FO}$ & & \\
\hline & 1 & sviluppo & s.m. & $\mathrm{FO}$ & & sviluppati \\
\hline & & delle & prep.art. & FO & & \\
\hline & 1,5 & attività & s.f. & FO & & 0 , attivita \\
\hline & & industriali & agg. & FO & & \\
\hline & 4 & il & art.det. & FO & & $0(2)$, in (2) \\
\hline & & tenore & loc.s.m. & $\mathrm{CO}$ & & \\
\hline & 4 & $d i$ & el.loc.s.m. & el.co & & della (4) \\
\hline & & vita & el.loc.s.m. & el.CO & & \\
\hline & 7 & $d i$ & prep. & $\mathrm{FO}$ & & $\begin{array}{l}\text { della (4), del, } 0 \text {, } \\
\text { da }\end{array}$ \\
\hline & & gran & $\begin{array}{l}\text { agg. var.po- } \\
\text { siz. }\end{array}$ & FO & & \\
\hline & 0 & parte & s.f. & FO & & \\
\hline & & della & prep.art. & FO & & \\
\hline & 0 & popolazione & s.f. & $\mathrm{AU}$ & & \\
\hline & & $\dot{e}$ & v.intr. & FO & & \\
\hline & 11 & basso. & agg. & FO & & $\begin{array}{l}\text { bassa }(8), \text { ba- } \\
\text { sata }(2), 0\end{array}$ \\
\hline & & $\begin{array}{l}\text { Preoccu- } \\
\text { panti }\end{array}$ & agg. & $\mathrm{CO}$ & & \\
\hline & 3 & sono & v.intr. & FO & & $0(3)$ \\
\hline & & i & art.det. & $\mathrm{CO}$ & & \\
\hline & 2 & dati & s.m. & $\mathrm{AU}$ & & $0(2)$ \\
\hline & & relativi & agg. & $A U$ & & \\
\hline & 2 & alla & prep.art. & FO & & alte, 0 \\
\hline & & $\begin{array}{l}\text { disoccupa- } \\
\text { zione }\end{array}$ & s.f. & $A D$ & & \\
\hline & 6 & $\begin{array}{l}\text { all'analfabe- } \\
\text { tismo }\end{array}$ & s.m. & $\mathrm{CO}$ & & $\begin{array}{l}\text { analfabeti (3), } \\
\text { analfabeto, } \\
\text { analfabetita- } \\
\text { zione, analfalfa- } \\
\text { betismo }\end{array}$ \\
\hline & & $e$ & cong. & $\mathrm{FO}$ & & \\
\hline & 0 & alla & prep.art. & FO & & \\
\hline & & mortalità & loc.s.f. & TS stat. & & \\
\hline & 0 & $\begin{array}{l}\text { infantile } \\
\text { ancora }\end{array}$ & $\begin{array}{l}\text { el.loc.s.f. } \\
\text { avv. }\end{array}$ & $\begin{array}{l}\text { el.TS stat. } \\
\text { FO }\end{array}$ & & \\
\hline & $\begin{array}{l}3+2 \\
\text { acc. }\end{array}$ & elevata. & agg. & $\mathrm{CO}$ & & $\begin{array}{l}\text { elevate, eleva- } \\
\text { tissima (acc.), }\end{array}$ \\
\hline
\end{tabular}




\begin{tabular}{|c|c|c|c|c|c|c|}
\hline Text & $\begin{array}{l}\mathrm{n} \\
\text { Fehler }\end{array}$ & Wort & Wortart & $\begin{array}{l}\text { Gebrauchs- } \\
\text { marke }\end{array}$ & Kommentar & $\begin{array}{l}\text { Lösungsvor- } \\
\text { schläge }\end{array}$ \\
\hline & & & & & & $\begin{array}{l}\text { elevati (acc.), } \\
\text { elevatissime, } 0\end{array}$ \\
\hline & & L'agricoltura & s.f. & FO & & \\
\hline & & 1 & art.det. & $\mathrm{CO}$ & & \\
\hline & \multirow[t]{2}{*}{8} & terreni & s.m. & FO & & territori (8) \\
\hline & & coltivati & agg. & $\mathrm{CO}$ & & \\
\hline & \multirow[t]{2}{*}{1} & sono & v.intr. & FO & & 0 \\
\hline & & limitati & agg. & $\mathrm{CO}$ & & \\
\hline & \multirow[t]{2}{*}{8} & all'area & s.f. & TS geogr. & & $\begin{array}{l}0 \text { (3), arrivo, ar- } \\
\text { cipelago (lo- } \\
\text { gisch falsch), } \\
\text { aree, aria, ari- } \\
\text { dità }\end{array}$ \\
\hline & & del & prep.art. & $\mathrm{FO}$ & & \\
\hline & \multirow[t]{2}{*}{11} & delta & s.m. & TS geogr. & & $\begin{array}{l}\text { deserto (7), } 0 \\
\text { (4) }\end{array}$ \\
\hline & & $e$ & cong. & FO & & \\
\hline & \multirow[t]{2}{*}{3} & della & prep.art. & FO & & delle (2), 0 \\
\hline & & valle & s.f. & TS geogr. & & \\
\hline & 0 & $\begin{array}{l}\text { del } \\
\text { Nilo. }\end{array}$ & $\begin{array}{l}\text { prep.art. } \\
\text { n.p.m. }\end{array}$ & FO & & \\
\hline & \multirow[t]{2}{*}{2} & L'irrigazione & s.f. & AU & & 0 , irrigenza \\
\hline & & assicurata & part.pass.v.tr. & FO & & \\
\hline & \multirow[t]{3}{*}{8} & $d a$ & prep. & FO & & $\begin{array}{l}\text { dalle (5), } 0(2) \text {, } \\
\text { delle }\end{array}$ \\
\hline & & dighe & s.f. & AU & & \\
\hline & & $e$ & cong. & FO & & \\
\hline & 1 & canali & s.m. & FO & & 0 \\
\hline
\end{tabular}

Tabelle 18: C-Testtexte 4 und 5 aus dem Lehrbuch von Bersezio (2005, S. 32 f.) inklusive Lösungsvorschläge (Morante)

Von insgesamt 50 Lücken sind 17 komplett richtig gefüllt worden, bzw. nur mit akzeptablen Lösungen: zwölf im vierten und fünf im fünften Text. Weitere 22 Wörter wurden von einer/m bis vier Lernenden nicht oder falsch vervollständigt. Der Großteil von ihnen (15) stammt aus dem Bereich des fundamentalen Wortschatzes; außerdem sind vier weitere Wörter aus dem Grundwortschatz darunter, die mit einer AU-Gebrauchsmarke versehen sind, und zwei Wörter aus dem allgemeinen (CO) sowie eines aus dem Fachwortschatz. Die maximale Fehlerzahl liegt bei Letzteren allerdings lediglich bei 0,5. Insgesamt gibt es nur acht Wörter, die drei- bzw. viermal falsch bearbeitet wurden, so dass als erstes Auswertungsergebnis festgehalten werden kann, dass die Lernenden die beiden letzten Texte mit weniger Fehlern bearbeiteten. Dadurch kann angenommen werden, dass die Textgestaltung sie nicht überforderte, sondern wahrscheinlich ihren sprachlichen Fähigkeiten angemessen war. Bei der Auswertung der vermehrten Falschlösungen wird dies überprüft.

Die von drei bzw. vier Lernenden nicht korrekt gefüllten Lücken betreffen mit einer Ausnahme Ausdrücke aus dem fundamentalen Wortschatz. Der erste 
ist eine artikulierte Präposition, de-lla, die je zweimal nicht bzw. zu delle ergänzt wurde, wofür das Bezugswort valle (Tal) ausschlaggebend gewesen sein könnte. Die häufigste Genus-Endung für feminine Pluralwörter ist $-e$, weshalb die Präposition vielleicht mit einem femininen Pluralartikel komplettiert wurde, obwohl valle ein Singularwort ist und somit zur kleineren Gruppe von femininen Wörtern zählt, die nicht auf - $a$ enden. Es könnte sich aber durchaus auch schlichtweg um Flüchtigkeitsfehler handeln, was dadurch untermauert würde, dass bei einem Vorkommnis derselben Wörter im fünften Text nur noch drei Fehler auftreten (zweimal delle, einmal keine Ergänzung). Wiederum viermal falsch gelöst wurde der maskuline Singularartikel $i$ - $l$ vor tenore di vita (Co; Lebensstandard). Da er zur Präposition in oder gar nicht ergänzt wurde, mag es sein, dass die entsprechenden Testteilnehmer den Ausdruck tenore nicht sicher beherrschten. Dagegen spricht auch nicht, dass niemand das mittlere Wort des Phrasems falsch bearbeitete, da eine Lösung mit etwas Wortbildungskenntnissen oder Sprachgefühl möglich ist, ohne dass dafür die Wortbedeutung bekannt sein muss. ${ }^{155}$

Das einzige Wort, das nicht aus dem Fo-Wortschatz stammt und viermal nicht korrekt ergänzt wurde, ist die einfache Präposition di im Phrasem tenore $d$ - $i$ vita (Lebensstandard), das den vier Lernenden, welche die einfache zu einer artikulierten Präposition ergänzten, wahrscheinlich nicht bekannt war.

Dreimal falsch bzw. nicht vervollständigt wurden die Lücken im Nomen me-tà (Fo; Hälfte) und im Verb so-no (sind). Obwohl Letzteres zu den am allerhäufigsten gebrauchten Verben zählt, wurde es von drei Personen gar nicht ergänzt, was an der Satzkonstruktion liegen könnte: Das Verb steht zwar an zweiter Stelle, aber vor ihm befindet sich nicht wie üblich das Subjekt, sondern ein Co-gekennzeichnetes Adjektiv (preoccupanti, besorgniserregend), das prädikativ gebraucht ist. Wären Subjekt und Prädikatsnomen konvertiert, könnten die SchülerInnen das Verb wahrscheinlich korrekt produzieren. Beim Nomen me-tà fällt auf, dass zwei Testteilnehmer das Wort zu media (Durchschnitt) vervollständigten, was in Hinsicht auf das Attribut della popolazione (der Bevölkerung) unlogisch ist und daher vermuten lässt, dass sie entweder den Test nicht sehr gewissenhaft bearbeiteten oder ihnen die Bedeutung von „Durchschnitt“ nicht ganz klar war.

\subsubsection{Häufigste Falschlösungen in Text 4 und 5}

Insgesamt wurde ein gutes Fünftel (11) der Lücken von mindestens fünf Testteilnehmern nicht richtig bearbeitet. Die betroffenen Wörter verteilen sich

155 Das Italienische neigt im Vergleich zum Deutschen wesentlich weniger stark zur substantivischen Komposita-Bildung. Ihr analogisches Verfahren dazu ist die Verknüpfung von zwei Nomen durch die Präposition di (von), da (aus) oder $a$ (zu) (vgl. Grotjahn, Tönshoff \& Hohenbleicher 1994, S. 120 f.), wie im hier gegebenen Textbeispiel, in ferro da stiro (Bügeleisen) und in forno a gas (Gasofen). 
nicht gleichmäßig auf die beiden Texte, sondern im vierten befinden sich nur vier Wörter mit vermehrten Falschlösungen, während es im fünften Text sieben sind.

Von den Wörtern im vierten Text zählen drei zum fundamentalen Wortschatz (vi-ve (lebt), d-el (des) und spe-sso (oft)), und eines ist das bei der Textkonstruktion versehentlich getilgte Wort de-lta aus dem geographischen Fachwortschatz. Da sich alle vier Wörter im selben Satz befinden, wird er zur besseren Veranschaulichung der möglichen Fehlerursachen zitiert und übersetzt (Bersezio 2005, S. 32; Hervorhebung nicht im Original):

Tuttavia, soltanto una piccola parte dei territori è abitata; la popolazione infatti, vive [9] concentrata lungo le coste del Mediterraneo e nelle zone più ospitali del [6] delta [11] e della valle del Nilo, dove la densità demografica è spesso [6] elevatissima e talvolta supera i 7000 abitanti per chilometro quadrato.

(Dennoch ist nur ein kleiner Teil des Territoriums bewohnt; die Bevölkerung lebt nämlich konzentriert entlang der Küsten des Mittelmeeres und in den gastlicheren Gegenden des Nildeltas und des Niltales, wo die demographische Dichte oft äußerst hoch ist und manchmal 7.0000 Einwohner pro Quadratkilometer übersteigt.)

Es handelt sich um einen recht langen Satz, dessen für die Fehleranalyse interessanter Teil nach dem Semikolon beginnt. Für die Lücken im Vorfeld sind so gut wie keine falschen Ergänzungen zu verzeichnen, wie aus Tabelle 18 ersichtlich wird, die auch für einen Überblick über die Lösungsvorschläge der häufig falsch vervollständigten Wörter empfohlen wird. Von den neun SchülerInnen, die das Prädikat, ,lebt" nicht richtig bildeten, schlugen fünf eine konjugierte Form anderer Verben vor, die logisch (viene concentrata; wird konzentriert, verdichtet) und grammatikalisch-syntaktisch (vi si è/vi è concentrata; dort hat sich/ist konzentriert) nicht funktionieren. Beide verstehen dabei das Kopula-Adjektiv concentrata als Partizip Perfekt, da es dieselbe Form hat und die Lernenden wahrscheinlich eher an die Kopulativbildung mit dem Verb „sein“ oder eine adverbiale Bestimmung der Art in modo concentrato (auf verdichtete Weise) im Zusammenhang mit dem Verb vivere gewöhnt waren. Zwei andere Testeilnehmer vervollständigten das Wort zum Partizip Perfekt vista (gesehen), was vermuten lässt, dass sie sich nicht sonderlich bemühten, den Satz zu verstehen oder seine Länge es ihnen unmöglich machte.

Die nächsten beiden oft falsch gelösten Wörter beinhalten für das erste der artikulierten Präposition neben fehlenden Ergänzungen nur einfache Präpositionen und eine artikulierte, von welchen keine mit den Lösungsvorschlägen der zweiten Lücke im Wort de-lta kongruent ist (außer di deserti). Es ist offensichtlich, dass sich die Schwierigkeit der korrekten Bearbeitung durch die doppelte Worttilgung erheblich vergrößert hat, aber es gibt an späterer Stelle eine zweite Lücke, in der das Wort de-lta zu ergänzen ist, was mehr als die Hälfte der Schülerinnen und Schüler (7) ebenfalls falsch machte. Davon abgesehen geht es um einen kurz vor der Testdurchführung im Unterricht behandelten 
Text, so dass es kaum Verständnis erzeugt, dass viele Lernende die Bevölkerung Ägyptens in der Wüste (deserto) ansiedelten. Falls jemand versuchte, der Satzaussage auf den Grund zu gehen, ist es auch möglich, dass sie für ihn folgende war: „Die Bevölkerung wird [...] in den gastlicheren Gebieten der Wüste konzentriert." Dass die Satzaussage oder auch nur der nächste sprachliche Kontext nicht verstanden oder berücksichtigt wurde, wäre auch ein Erklärungsgrund für das fehlende oder falsche Ergänzen des Worts für „oft“ vor einem absoluten Superlativ. Obwohl dieses Wort so oft benutzt wird und mit Sicherheit allen Testteilnehmern, sogar dem eingangs erwähnten chinesischen, der noch erhebliche Sprachprobleme zeigte, gut bekannt war, füllten zwei Personen die Lücke gar nicht und versuchten weitere zwei, einen absoluten Superlativ durch das Einsetzten des Adverbs specialmente (speziell) noch weiter zu steigern. Wiederum zwei Schüler lasen wohl zu flüchtig und ergänzten dadurch das Wort zu spempre, obwohl es sempre (immer) heißt und in diesen und ähnlichen Kontexten in einem bildungssprachlichen Text nicht zu erwarten ist, sondern eher ein Zeichen schriftlicher Mündlichkeit darstellt.

Im fünften Text resultierte für acht Testteilnehmer das Schließen der Lücke im Adjektiv ba-sso (niedrig) mit der richtigen Genus-Endung schwierig, was daran liegen mag, dass es sich um die letzte von insgesamt sechs Lücken in einem Satz handelt und sich das maskuline Bezugswort dieses Adjektivs direkt am Satzanfang befindet. Alle anderen Substantive hingegen sind weiblich, weshalb vermutlich so viele Testteilnehmer dem Adjektiv eine feminine Endung gaben. Andererseits könnte man auch wissen, dass sich eine Kopula-Bildung wie è bass-o (ist niedrig; wer oder was ist niedrig?) auf das Subjekt beziehen muss, welches sich im Italienischen in einem Aussagesatz in der Regel immer am Satzanfang befindet. Nur zwei der 13 Testteilnehmer schlossen diese Lücke richtig.

Im selben Satz gibt es eine einfache Präposition, $d-i$, welche siebenmal nicht korrekt ergänzt und in vielen Fällen zu einer artikulierten erweitert wurde. Wann ein Artikel erforderlich ist und wann nicht, scheinen vor allem in der Zweitsprache Lernende nicht immer zu wissen, wie sich auch an anderen Stellen und im C-Test der Vespucci gezeigt hat. Hier folgt der Präposition gran parte della popolazione (Großteil der Bevölkerung); wäre es stattdessen nur popolazione oder ein anderes eingliedriges Substantiv, hätte die Entscheidung zugunsten einer einfachen oder artikulierten Präposition wohl kein Problem dargestellt.

Ein ähnlicher Fall ist die einfache Präposition $d-a$ am Ende des letzten Textes, die auch achtmal falsch vervollständigt wurde. Der Lösungsvorschlag $d a-$ lle (von den/der), den fünf Testteilnehmer formulierten, wäre akzeptabel, wenn auch das zweite Nomen feminin wäre, da der Artikel dann beim zweiten Bezugswort entfallen kann. Es endet aber auf $-i$ und ist somit klar als maskulines 
Wort zu erkennen. Deshalb kann davon ausgegangen werden, dass die entsprechenden Testteilnehmer noch keine verlässliche Sprachkompetenz hinsichtlich des Gebrauchs von einfachen/artikulierten Präpositionen erlangt hatten.

Ebenfalls acht Schüler ergänzten das Wort ter-reni (Land, Boden) zu territori (Territorium), was durch die semantisch ähnliche Bedeutung des zweiten Wortes erklärbar ist, und dadurch, dass das Wort territori schon vorher im Test erscheint. Es geht hier aber um Landwirtschaft und Ackerland, weshalb der Lösungsvorschlag nicht akzeptabel ist.

Kurz darauf wurden im gleichen Satz zwei weitere Lücken oft nicht korrekt gefüllt (8- und 11-mal). Sie bilden zusammen eine Sinneinheit, wobei das zweite Wort das bereits genannte Delta bezeichnet, dessen mangelnde produktive Kompetenz die richtige Ergänzung von ar-ea behinderte. So wurde area durch etliche Nomen ersetzt, die logisch falsch und einmal auch grammatikalisch nicht korrekt sind.

Zusammenfassend scheint der zweite Text am schwierigsten gewesen zu sein, in dem bei acht Lücken mindestens fünf keinen passenden Lösungsvorschlag lieferten. Abschließend soll tabellarisch gezeigt werden, aus welchen grammatikalischen Kategorien das zu ergänzende Wortvorkommen zusammengesetzt ist und wie viele Falschlösungen es dafür zu verzeichnen gibt.

\begin{tabular}{|c|c|c|c|c|}
\hline & & & & $\varnothing$ Fehler pro \\
\hline Wortart & Vorkommen & Kommentar & n Fehler & Wort \\
\hline agg. & 20 & basso (11), rettilinei (7), forte (7) & 63 & 3,2 \\
\hline art.det./indet. & 8 & & 8 & 1 \\
\hline avv. & 10 & più (4), spesso (6), vi (6) & 32 & 3,2 \\
\hline cong. & 2 & & 2 & 1 \\
\hline el.loc. & 6 & & 9 & 1,5 \\
\hline el.n.p. & 3 & & 1 & 0,3 \\
\hline ger. & 0 & & 0 & 0 \\
\hline inf. & 0 & & 0 & 0 \\
\hline loc. & 4 & & 4 & 1 \\
\hline n.p. & 3 & & 6 & 2 \\
\hline n.p./sigla & 0 & & 0 & 0 \\
\hline part.pass. & 0 & & 0 & 0 \\
\hline prep. & 7 & $d a(8), d i(7), \operatorname{per}(6), d e l(6)$ & 24 & 3,4 \\
\hline prep.art. & 10 & & 17 & 1,7 \\
\hline pron. ${ }^{*}$ & 3 & & 4 & 1,3 \\
\hline pron.rel. & 1 & & 4 & 4 \\
\hline s.m./f. & 39 & $\begin{array}{l}\text { delta (zweimal 11), minoranze (9), } \\
\text { approdi (8), terreni (8), all'area (8) }\end{array}$ & 120 & 3,1 \\
\hline sigla & 0 & & 0 & 0 \\
\hline v.aus. & 0 & & 0 & 0 \\
\hline v.tr./intr. & 7 & vive (9) & 20 & 2,9 \\
\hline $\begin{array}{l}\text { v.pro- } \\
\text { nom.intr. }\end{array}$ & 1 & & 1 & 1 \\
\hline$\%$ s.m./f. & 31,5 & & & \\
\hline
\end{tabular}

Tabelle 19: Zu ergänzende Wörter im C-Test der Morante nach Wortart mit Angaben zu den Falschlösungen 
In diesem C-Test sind pro Wort durchschnittlich die meisten Fehler mit 3,4 für die Wortart Präpositionen zu verzeichnen. Wie die Fehleranalyse gezeigt hat, zählen sie zwar zum fundamentalen Wortschatz und dürften daher allen Lernenden bestens geläufig gewesen sein, schien ihnen jedoch vor allem der aktive Umgang mit der Unterscheidung einfacher Präpositionen und ihrer kontrahierten Formen aus Präposition und Artikel schwer gefallen zu sein. Ferner ergänzten sie zum Teil andere einfache Präpositionen und zum Teil nichts, was beides anscheinend eher auf die Komplexität des sprachlichen Kontextes des getilgten Wortes und eine unzureichende Textkompetenz zurückzuführen ist. Ein nicht ausreichendes Textverständnis ist vermutlich auch die Ursache für die vermehrten Falschlösungen bei vielen Adverbien und dem Adjektiv basso (niedrig) aus dem fundamentalen Wortschatz, wobei sich die beiden Wortarten mit durchschnittlich 3,2 Fehlern pro Wort den Platz der zweithäufigsten Falschlösungen teilen. In diesem Bereich sind allerdings auch Lösungsschwierigkeiten mehr lexikalischer Art zu beobachten.

An dritter Stelle folgen mit einen Wert von 3,1 die Substantive, unter denen sich Ausdrücke aus dem Fachwortschatz befinden, welche die höchsten Fehlerzahlen erzielten (z.B. delta), aber auch Wörter aus dem Basiswortschatz, die ebenfalls zu sehr vielen Falschlösungen führten.

\subsubsection{A der Pestalozzi}

Der C-Test in der Klasse III A hat 123 Lücken und wurde aus Texten der Lehrbuchseiten 116, 118 und 149 (Bastianelli et al. 2010) entwickelt. Unter den 17 der insgesamt 19 SchülerInnen, die am Test teilnahmen, war nur ein Lernender mit nicht italienischer Erstsprache. In dieser Lerngruppe wurden die besten Ergebnisse erzielt und die wenigsten häufigen Fehler begangen, wobei die Leistungsdifferenz im Vergleich zu den anderen vier Klassen signifikant groß ist (vgl. Gegenüberstellung der Ergebnisse in 8.4 und Tabelle 6 zu Beginn des Kapitels). Auch wenn die Ergebnisse der fünf verschiedenen C-Tests nicht direkt miteinander verglichen werden können, darf angenommen werden, dass in dieser Klasse auch bei einer Distribution ein und desselben Tests in allen fünf Lerngruppen mit einem gewissen Abstand die wenigsten Fehler produziert worden wären.

Zunächst illustrieren zwei Tabellen die Zusammensetzung der zu ergänzenden Ausdrücke nach ihrer Wortschatzbereichszugehörigkeit, den unterschiedlichen grammatikalischen Kategorien und der Anzahl der auf sie jeweils entfallenen Falschlösungen.

\begin{tabular}{lrrlrr}
\hline Marke & Vorkommen & in Prozent & Kommentar & n Fehler & $\varnothing$ Fehler \\
\hline $\mathrm{AD}$ & 1 & 0,8 & apparecchiature (7) & 7 & 7 \\
$\mathrm{AU}$ & 6 & 4,9 & valutate (12), esportano (3) & 17 & 2,8 \\
$\mathrm{CO}$ & 18 & 14,6 & $\begin{array}{l}\text { serve (10), gli (8), manufatti (7), } \\
\text { individuale (4) }\end{array}$ & 47 & 2,6 \\
& & & & \\
\hline
\end{tabular}




\begin{tabular}{|c|c|c|c|c|c|}
\hline CO TS & 1 & 0,8 & & 0 & 0 \\
\hline el.CO & 3 & 2,4 & da parte di (4) & 5 & 1,7 \\
\hline el.TS & 6 & 4,9 & tasso di mortalità (6) & 9 & 1,5 \\
\hline FO & 72 & 58,5 & & 88 & 1,2 \\
\hline TS & 8 & 6,5 & macchine utensili (5) & 10 & 1,25 \\
\hline n.p. & 6 & 4,9 & Bangladesh (3), Australia (3) & 6 & 1 \\
\hline el.n.p. & 2 & 1,6 & & 1 & 0,5 \\
\hline n Wörter & 123 & & $\mathrm{n}$ Fehler & 190 & 1,5 \\
\hline
\end{tabular}

Tabelle 20: Getilgte Wörter des C-Tests der III A nach Gebrauchsmarken mit Angaben zu den Falschlösungen

Abgesehen vom Eindruck eines höheren sprachlichen und fachinhaltlichen Leistungsstands dieser Klasse im Vergleich zu den anderen vier in die Erhebung einbezogenen, der während der Unterrichtshospitationen entstand, ist von besseren Fähigkeiten der SchülerInnen auch dadurch auszugehen, dass dieser C-Test weniger Fehler verursacht hat, obwohl sein zu vervollständigendes Wortvorkommen im Vergleich zu den anderen C-Tests zum geringsten Teil aus Ausdrücken besteht, die aus dem Vocabolario di Base stammen. Hier liegt der Anteil aus dem VDB nämlich nur bei 64,2 Prozent und damit deutlich unter dem durchschnittlichen Wert von 71,3 Prozent.

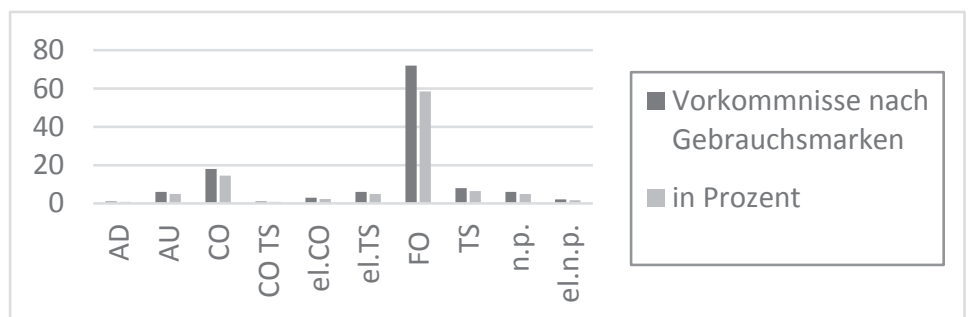

Abbildung 7: Getilgtes Wortvorkommen im C-Test der Klasse III A (Pestalozzi) nach Gebrauchsmarken und ihren prozentualen Anteilen

\begin{tabular}{lrlrr}
\hline Wortart & Vorkommen & Kommentar & n Fehler & $\varnothing$ Fehler pro Wort \\
\hline agg. & 17 & precise (6) & 27 & 1,6 \\
art. det./indet. & 12 & 13 & 1,1 \\
avv. & 5 & & 10 & 2 \\
cong. & 2 & ma (3) & 4 & 2 \\
el.loc. & 9 & tasso di mortalità (6) & 14 & 1,6 \\
el.n.p. & 2 & & 1 & 0,5 \\
ger. & 1 & aumentando & 3 & 3 \\
inf. & 1 & distinguere & 4 & 4 \\
loc. & 5 & 8 & 1,6 \\
n.p. & 6 & 6 & 1 \\
part.pass. & 2 valutate (12), misurata (3) & 15 & 7,5 \\
prep. & 8 & tra (6) & 9 & 1,1 \\
prep.art. & 5 & 6 & 1,2 \\
pron. & 6 & 3 & 0,5 \\
\hline
\end{tabular}




\begin{tabular}{lrlrr}
\hline Wortart & Vorkommen & Kommentar & n Fehler & $\varnothing$ Fehler pro Wort \\
\hline pron.rel. & 4 & & 8 & 2 \\
s.m./f. & 25 & apparecchiature (7), manufatti & 28 & 1,1 \\
sigla & & $(7)$ & 0 & 0 \\
v.aus./tr./intr. & 9 & & 18 & 2 \\
v.pronom.intr. & 3 & serve (servirsi, 10) & 11 & 3,7 \\
\% s.m./f. & 20 & & & \\
\hline
\end{tabular}

Tabelle 21: Getilgtes Wortvorkommen des C-Tests der III A (Pestalozzi) nach Wortarten mit Angaben zu den Falschlösungen

pron*: Unter dieser Bezeichnung sind außer den gesondert aufgelisteten Relativpronomen (pron.rel.) alle anderen Arten von Pronomen gesammelt.

Die meisten Fehler entfallen mit zwölf pro Wort auf einen Ausdruck aus dem Bereich des hohen Gebrauchs (valutate, bewertet), während die durchschnittlichen Falschlösungen pro Vorkommnis für die folgenden zwei Gebrauchsmarken zwar insgesamt die höchsten aller Gebrauchsmarken sind, aber mit 2,8 für den hohen Gebrauch (AU) und 2,6 für den allgemeinen Wortschatz (CO) weitaus niedriger liegen als in den anderen Klassen.

Die unter den getilgten Ausdrücken am stärksten vertretenen Wortarten sind Substantive, Adjektive und Artikel. Sie zählen nicht zu den drei Wortarten, bei denen durchschnittlich die meisten Fehler produziert wurden. An erster Stelle stehen die Partizipien mit 7,5 pro Wort, von denen es im Text zwei getilgte gibt und eines zwölf Falschlösungen verursachte. Auch an zweiter und dritter Stelle folgen komplexere Verformen, und zwar ein Infinitiv mit vier und ein Gerundium mit drei Fehlern, während die Fehlerquoten mit Ausnahme der reflexiven Verben für alle anderen Wortarten unter durchschnittlich zwei pro Token liegen. Bei den Adjektiven ist es vor allem das in Tabelle 21 ersichtliche, welches zu vermehrten Falschlösungen führte. Der nächste Kapitelabschnitt wird zeigen, wie die Ursachen hierfür wahrscheinlich auf lexikalischer Ebene zu suchen sind. Im Folgenden wird die Bearbeitung der ersten beiden C-Testtexte untersucht, die sich mit den sozioökonomischen Unterschieden zwischen Ländern auf Weltebene beschäftigen.

Von den drei reflexiven Verben verursachte eines zehn Fehler, so dass die durchschnittliche Fehlerquote pro Wort dieser Kategorie bei 3,7 liegt.

\subsubsection{Text 1 und 2 des C-Tests der III A an der Pestalozzi}

\begin{tabular}{lllllll}
\hline Text & Fehler & Wort & Wortart & Marke & Kommentar & Lösungsvorschläge \\
\hline 1 & 0 & $\begin{array}{l}\text { crescita } \\
\text { econo- }\end{array}$ & $\begin{array}{l}\text { loc.s.f. } \\
\text { el.loc.s.f. }\end{array}$ & TS econ. & el.TS econ. & \\
$\begin{array}{l}\text { (S. 118) } \\
\text { mica }\end{array}$ & & part.pass.v.tr. & FO & misura (2), si misura \\
cken & 3 & con & $\begin{array}{l}\text { prep. } \\
\text { art.det. }\end{array}$ & FO & FO & $0(2)$ \\
& 2 & il & & & \\
\hline
\end{tabular}




\begin{tabular}{|c|c|c|c|c|c|c|}
\hline Text & Fehler & Wort & Wortart & Marke & Kommentar & Lösungsvorschläge \\
\hline & \multirow[t]{3}{*}{1} & $\begin{array}{l}\text { Prodotto } \\
\text { Interno }\end{array}$ & $\begin{array}{l}\text { loc.s.m. } \\
\text { el.loc.s.m. }\end{array}$ & $\begin{array}{l}\text { TS econ. } \\
\text { el.TS econ. }\end{array}$ & & Internazionale \\
\hline & & $\begin{array}{l}\text { Lordo } \\
\text { (PIL) }\end{array}$ & $\begin{array}{l}\text { el.loc.s.m. } \\
\text { sigla }\end{array}$ & el.TS econ. & & \\
\hline & & e & cong. & FO & & \\
\hline & \multirow[t]{2}{*}{0} & le & art.det. & $\mathrm{FO}$ & & \\
\hline & & condizioni & s.f. & $\mathrm{FO}$ & $\begin{array}{l}\text { kein Phra- } \\
\text { sem }\end{array}$ & \\
\hline & \multirow[t]{2}{*}{2} & $d i$ & prep. & FO & & della (2) \\
\hline & & vita & s.f. & FO & & \\
\hline & \multirow[t]{2}{*}{2} & della & prep.art. & $\mathrm{FO}$ & & deella (2), delle \\
\hline & & $\begin{array}{l}\text { popola- } \\
\text { zione }\end{array}$ & s.f. & $A U$ & & \\
\hline & \multirow[t]{2}{*}{12} & valutate & part.pass.v.tr. & $A U$ & & $\begin{array}{l}\text { valutata }(8), 0(2), \text { valu- } \\
\text { tano, valutando }\end{array}$ \\
\hline & & sulla & prep.art. & FO & & \\
\hline & \multirow[t]{2}{*}{1} & base & s.f. & FO & & 0 \\
\hline & & del & prep.art. & FO & & \\
\hline & \multirow[t]{2}{*}{0} & reddito & s.m. & $A U$ & & \\
\hline & & medio & agg. & $A U$ & & \\
\hline & \multirow[t]{2}{*}{4} & $\begin{array}{l}\text { indivi- } \\
\text { duale }\end{array}$ & agg. & $\mathrm{CO}$ & & $\begin{array}{l}\text { individuo (3), indivi- } \\
\text { duano }\end{array}$ \\
\hline & & (la & art.det. & FO & & \\
\hline & \multirow[t]{3}{*}{1} & ricchezza) & s.f. & TS econ. & & ricchezza pro capite \\
\hline & & $\begin{array}{l}\text { l'istru- } \\
\text { zione }\end{array}$ & s.f. & $\mathrm{AU}$ & & \\
\hline & & e & cong. & FO & & \\
\hline & \multirow[t]{2}{*}{1} & la & art.det. & FO & & le condizioni di \\
\hline & & salute. & s.f. & FO & & \\
\hline & \multirow[t]{2}{*}{0} & Sono & v.aus. & FO & & \\
\hline & & perciò & cong. & $\mathrm{FO}$ & & \\
\hline & \multirow[t]{2}{*}{$\begin{array}{l}2+1 \\
\text { acc. }\end{array}$} & indicati & v.tr. & FO & & $\begin{array}{l}\text { individuate, individuati } \\
\text { (acc.), } 0\end{array}$ \\
\hline & & come & avv. & FO & & \\
\hline & \multirow[t]{2}{*}{1} & Paesi & s.m. & FO & & 0 \\
\hline & & sviluppati & agg. & $\mathrm{CO}$ & & \\
\hline & \multirow[t]{2}{*}{8} & gli & art.det. & $\mathrm{CO}$ & & $0(2)$, grandi (4), già (2) \\
\hline & & Stati & s.m. & FO & & \\
\hline & \multirow[t]{2}{*}{0} & che & pron.rel. & $\mathrm{FO}$ & & \\
\hline & & hanno & v.tr. & FO & & \\
\hline & \multirow[t]{2}{*}{1} & $\begin{array}{l}\text { un'econo- } \\
\text { mia }\end{array}$ & s.f. & $A U$ & & econeconomia \\
\hline & & progredita & agg. & $\mathrm{CO}$ & & \\
\hline & \multirow[t]{2}{*}{3} & che & pron.rel. & $\mathrm{FO}$ & & $0(3)$ \\
\hline & & si & pron.pers. & $\mathrm{CO}$ & & \\
\hline & \multirow[t]{2}{*}{10} & serve & $\begin{array}{l}\text { v.pro- } \\
\text { nom.intr. }\end{array}$ & $\mathrm{CO}$ & & servono (6), segue, 0 (3) \\
\hline & & di & prep. & FO & & \\
\hline & \multirow[t]{3}{*}{4} & moderne & agg. & $\mathrm{FO}$ & & moderate, 0 (3) \\
\hline & & tecnologie & s.f. & $\mathrm{CO}$ & & \\
\hline & & e & cong. & FO & & \\
\hline & 4 & che & pron.rel. & FO & & come, cure, 0 (2) \\
\hline
\end{tabular}




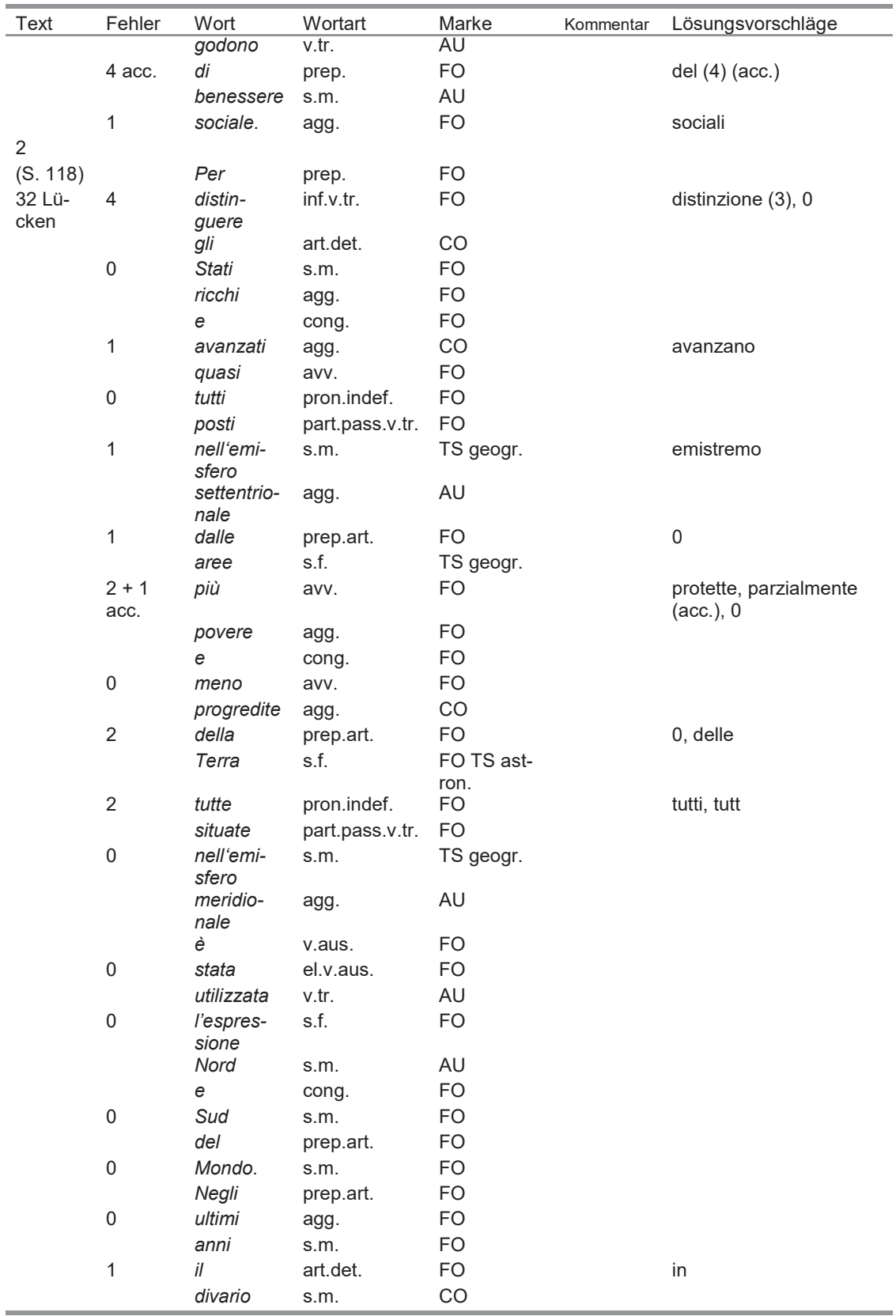




\begin{tabular}{|c|c|c|c|c|c|c|}
\hline Text & Fehler & Wort & Wortart & Marke & Kommentar & Lösungsvorschläge \\
\hline & \multirow[t]{2}{*}{6} & tra & prep. & FO & & $\begin{array}{l}\text { terrestre (2), tutti (2), } \\
\text { ttra, } 0\end{array}$ \\
\hline & & $i$ & art.det. & $\mathrm{CO}$ & & \\
\hline & \multirow[t]{3}{*}{0} & Paesi & s.m. & FO & & \\
\hline & & ricchi & agg. & FO & & \\
\hline & & $e$ & cong. & FO & & \\
\hline & \multirow[t]{2}{*}{0} & quelli & pron.dimostr. & FO & & \\
\hline & & poveri & agg. & FO & & \\
\hline & \multirow[t]{2}{*}{1} & si & pron.pers. & $\mathrm{CO}$ & & 0 \\
\hline & & $\dot{e}$ & v.aus. & FO & & \\
\hline & \multirow[t]{2}{*}{1} & $\begin{array}{l}\text { notevol- } \\
\text { mente }\end{array}$ & avv. & $\mathrm{CO}$ & & notevotevolmente \\
\hline & & allargato & $\begin{array}{l}\text { v.pro- } \\
\text { nom.intr. }\end{array}$ & $\mathrm{CO}$ & & \\
\hline & \multirow[t]{2}{*}{3} & $\begin{array}{l}\text { aumen- } \\
\text { tando }\end{array}$ & ger.v.tr. & FO & & $\begin{array}{l}\text { aumentato, aumento, } \\
\text { aumenta }\end{array}$ \\
\hline & & $d i$ & prep. & $\mathrm{FO}$ & & \\
\hline & \multirow[t]{2}{*}{$1 \mathrm{acc}}$. & cinque & agg. & FO & & cinquanta (acc.) \\
\hline & & volte. & s.f. & FO & & \\
\hline & \multirow[t]{2}{*}{0} & In & prep. & FO & & \\
\hline & & questo & agg.dimostr. & FO & & \\
\hline & \multirow[t]{2}{*}{2} & periodo & s.m. & FO & & però, percorso \\
\hline & & i & art.det. & $\mathrm{CO}$ & & \\
\hline & \multirow[t]{2}{*}{1} & commerci & s.m. & FO & & commercianti \\
\hline & & si & pron.pers. & $\mathrm{CO}$ & & \\
\hline & \multirow[t]{3}{*}{0} & sono & v.aus. & FO & & \\
\hline & & sviluppati & $\begin{array}{l}\text { v.pro- } \\
\text { nom.intr. }\end{array}$ & $\mathrm{CO}$ & & \\
\hline & & a & loc.avv. & $\mathrm{CO}$ & & \\
\hline & \multirow[t]{2}{*}{0} & livello & el.loc.avv. & el.CO & & \\
\hline & & $\begin{array}{l}\text { internazio- } \\
\text { nale }\end{array}$ & agg. & $\mathrm{FO}$ & & \\
\hline & \multirow[t]{2}{*}{3} & ma & cong. & FO & & molti (3) \\
\hline & & hanno & v.aus. & $\mathrm{FO}$ & & \\
\hline & 5 & $\begin{array}{l}\text { avvantag- } \\
\text { giato }\end{array}$ & v.tr. & CO estens. & I & $\begin{array}{l}0 \text {, avvantagiato }(3), \\
\text { avvantantaggiato }\end{array}$ \\
\hline
\end{tabular}

Tabelle 22: Text 1 und 2 des Tests der Klasse III A, Pestalozzi (Textgrundlage Bastianelli et al. 2010, S. 118)

Von den 56 Lücken der ersten beiden Texte füllten alle Testteilnehmer 19 komplett richtig. Dabei sind nicht nur Wörter des fundamentalen Wortschatzes betroffen, sondern auch red-dito (Einkommen) aus dem Bereich des hohen Gebrauchs, zwei Teile des reflexiven Verbs svilupparsi (CO; sich entwickeln) und ein Co-gekennzeichnetes adverbiales Phrasemelement (liv-ello aus a livello, auf [...] Ebene) sowie drei fachwortschatzsprachliche Ausdrücke. Aus dem Bereich der Wirtschaft sind das cres-cita (Wachstum) aus crescita economia (Wirtschaftswachstum) und ricc-hezza (Wohlstand), wobei das letzte doch von einer Person insofern falsch ergänzt wurde, als dass sie dem Wort noch das zweigliedrige Phrasem pro capite (pro Kopf) nachstellte. Das dritte, emis-fero 
(Hemisphäre) ${ }^{156}$, ist ein geographisches Fachwort. Es ist sehr unwahrscheinlich, dass die beiden bisher vorgestellten Lerngruppen dazu imstande gewesen wären, dieselben 22 Wörter komplett fehlerfrei zu ergänzen.

Bei 30 Wörtern treten ein bis vier Fehler auf oder die Vervollständigung bleibt aus. Darunter befinden sich größtenteils Wörter aus dem fundamentalen Wortschatz, wobei auch von den vier viermal nicht korrekt bearbeiteten Wörtern nur eines aus einem anderen Bereich des Wortschatzes stammt, nämlich aus dem allgemeinen (CO). Das ist das Adjektiv indiv-iduale (individuell), das in vielen Fällen zum verwandten Substantiv individuo vervollständigt wurde, was darauf hindeutet, dass die entsprechenden Testteilnehmer nicht genau auf den sprachlichen Kontext achteten, sondern bei der Vervollständigung den Inhalt des Textabschnitts nur grob im Sinn hatten oder das Umfeld ganz ignorierten und nur das getilgte Wort beachteten.

Ebenfalls viermal falsch oder nicht gelöst wurde das Adjektiv mod-erne (Fo), das immerhin nicht zu anderen Wortarten ergänzt wurde. Das Adjektiv an sich scheint nicht gerade schwierig zu sein, das sprachliche Umfeld zu einem gewissen $\mathrm{Ma} ß$ aber schon (übersetzt lautet der Kontext „eine fortgeschrittene Wirtschaft, die sich moderner Technologien bedient [...]"), weil das vorausgehende Verb aus dem allgemeinen Wortschatz stammt und das Subjekt abstrakter Natur ist. Auch die nächste Lücke im selben Satz wurde viermal nicht richtig vervollständigt; sie betrifft das einfache Relativpronomen $c$-he (FO). Vielleicht fiel einigen Lernenden hier die Bearbeitung nicht leicht, da es um ein Wort im letzten von insgesamt drei Relativsätzen in ein und demselben Satzgefüge geht.

Desweiteren wurde der Infinitiv disti-nguere (Fo; unterscheiden) von einer/einem gar nicht und von dreien zum Substantiv distinzione (AU; Unterscheidung) ergänzt. Es handelt sich hier um einen sehr langen implizierten Finalsatz gleich zu Beginn des Satzgefüges, von dem der Infinitiv nach der einleitenden Präposition per (für, hier: um zu) das zweite Wort ist. Lässt man einmal den Kontext der beiden Wörter außer Acht, so könnte nach der Präposition auch das als Lösungsvorschlag angebotene Substantiv stehen, allerdings wäre ein anschließendes Komma erforderlich und würde man eigentlich, was viel wichtiger ist, von muttersprachlichen Lernenden der achten Klasse erwarten, dass sie in der Lage sind, auch einen längeren Satz komplett zu lesen, zu verstehen und zu bemerken, dass ein Prädikat fehlt.

Bezüglich der nicht so häufigen Fehler fällt darüber hinaus auf, dass auch in dieser Klasse einige Probleme mit der Entscheidung zu haben schienen, ob eine Präposition, wenn ein Substantiv folgt, artikuliert wird oder ohne Artikel stehen kann, wobei es sich vermutlich oft auch nur um Flüchtigkeitsfehler handelte. Außerdem wussten alle bis auf eine(n) Lernende(n) die Sigle PIL (BIP)

156 Dieses Wort tritt mit zwei Tokens im C-Test auf und verzeichnet bei einem davon einen Fehler. Ein Lernender oder eine Lernende ergänzte das Wort also in einem Fall richtig und im anderen nicht. 
aufzulösen: Das mittlere Wort wurde einmal zu internazionale anstelle von interno vervollständigt, also so ziemlich zu seinem Gegenteil. Dass die Lernenden ansonsten keine Probleme mit der Auflösung zeigten, spricht dafür, dass sie im Unterricht aufgepasst und den Begriff verinnerlicht hatten. Ob sie allerdings auch hätten erklären können, was damit gemeint ist, ist eine andere Frage. ${ }^{157}$

\subsubsection{Häufigste Falschlösungen in den C-Testtexten 1 und 2 der III A}

In den ersten beiden Texten wurden in dieser Lerngruppe nur fünf Wörter vermehrt falsch gelöst. Drei von ihnen verteilen sich auf den ersten Text und zählen nicht zum fundamentalen Wortschatz. Es sind das Partizip Perfekt valutate (bewertet, 12) aus dem Bereich des hohen Gebrauchs (AU) und mit einem maskulinen Pluralartikel und dem konjugierten Teil des reflexiven Verbs servirsi (sich bedienen) zwei aus dem des allgemeinen Wortschatzes (CO). Wie schon zuvor für die Klasse der Schule Morante beobachtet, lag die Schwierigkeit der Partizip- wie Adjektivergänzung mit einer kongruenten Endung wahrscheinlich darin, dass das Bezugswort nicht in unmittelbarer Nähe steht, sondern sich zwischen ihm und dem Partizip zwei weitere Nomen befinden, beide feminin und im Singular. So versahen sieben Schüler das Partizip mit einer femininen Singularendung, was jedoch nicht automatisch bedeutet, dass sie nicht in der Lage waren, für ein Partizip im Satz das Bezugswort zu finden. Es ist möglich, dass sie die Bedeutung von Kontext und Textkompetenz für eine erfolgreiche Testbearbeitung unterschätzten und zu sehr die Lücken und ihre nächste Umgebung im Blick hatten. Zwei weitere Lernende bildeten anstelle des Partizips verbale Formen (eine konjugierte und ein Gerundium), die sowohl logisch als auch grammatikalisch-syntaktisch falsch sind.

Das zehnmal falsch bearbeitete, konjugierte reflexive Verb servirsi (CO; sich bedienen) wurde vermutlich daher so oft falsch gelöst, dass es sich am Ende eines längeren und schwierigeren Satzgefüges befindet, das drei Relativsätze und einen im Passiv konstruierten Hauptsatz beinhaltet. Dass sechs von zehn Lernenden zu servono vervollständigten, dem Prädikat also eine Pluralendung der dritten Person verliehen, deutet darauf hin, dass sie als Bezugswort des Relativsatzes im Hauptsatz fälschlicherweise paesi (Länder) und nicht economia (Wirtschaft) nahmen, obwohl es sich in größerer Nähe zum letzten Relativsatz befindet. Allerdings handelt es sich um den mittleren von drei Relativsätzen, von denen der erste und der letzte auf paesi Bezug nehmen, so dass die beiden nicht getilgten Prädikate in der dritten Person Plural konstruiert sind.

157 Vergleiche hierzu die Schülerdefinitionen des Begriffs an den Schulen Vespucci und Morante, die in Kapitel 9 präsentiert werden. 
Dass acht Lernende einen einfachen bestimmten Artikel nicht richtig ergänzten, liegt womöglich daran, dass er das Subjekt des Satzes begleitet, das aber nicht traditionell am Anfang des Satzes steht, der sich stattdessen in Form eines passiven Prädikats und eines präpositionalen Objekts komplexer präsentiert.

Dei beiden übrigen häufigen Fehlerquellen, die sich im zweiten Text befinden, betreffen Verben. Es ist zum einen das fünfmal falsch bearbeitete Partizip Perfekt avvantaggiato (CO; bevorteilt) aus dem Prädikat im Perfekt, wobei es sich neben einer ausbleibenden Ergänzung ausschließlich um Rechtschreibfehler handelt (unterlassene Verdoppelung des Konsonanten $g$ sowie den Flüchtigkeitsfehler avvantantaggiato), und zum anderen ein sechsmal nicht korrekt vervollständigtes reflexives Verb, das in dem Satzgefüge mit den drei Relativsätzen im zweiten von ihnen auftritt und zweimal logisch nicht richtig konjugiert, sondern dem folgenden im Plural stehenden Verb angeglichen wurde. Auch das dreimalige Offenlassen der Lücke deutet darauf hin, dass die Jugendlichen möglicherweise mit der syntaktischen Struktur etwas überfordert waren und die Satzaussage nicht erfassten.

Ferner ist ein sechsmal falsch gelöstes Wort im zweiten Text die Präposition $t$-ra (zwischen), die fünf Personen nicht zu einer solchen vervollständigten (einer weiteren unterläuft nur ein Rechtschreibfehler). Außer mangelnder Gewissenhaftigkeit oder Konzentration bei der Testbearbeitung kann ein Erklärungsgrund nur sein, dass sie das vorausgehende Substantiv divario (Unterschied, Schere) aus dem Bereich des allgemeinen Wortschatzes nicht sicher genug beherrschten.

\subsubsection{Texte 3 bis 5 des C-Test der Klasse III A}

Die anderen drei C-Testtexte sind den Lehrbuchseiten 149 und 116 (Bastianelli et al. 2010) entnommen und beschäftigen sich mit unterschiedlichen demographischen Entwicklungen verschiedener Länder und den Weltwirtschaftsmächten. Tabelle 23 gibt zunächst Auskunft über die Art des Wortvorkommens in den drei Texten und die Fehleranzahlen und Lösungsvorschläge der Testteilnehmer.

\begin{tabular}{|c|c|c|c|c|c|c|}
\hline Text & $\begin{array}{l}\mathrm{n} \\
\text { Fehler }\end{array}$ & Wort & Wortart & Marke & $\begin{array}{l}\text { Kommen- } \\
\text { tar }\end{array}$ & $\begin{array}{l}\text { Lösungsvor- } \\
\text { schläge }\end{array}$ \\
\hline $\begin{array}{l}3 \\
\text { (S. 149) }\end{array}$ & & Questo & $\begin{array}{l}\text { agg.di- } \\
\text { mostr. }\end{array}$ & $\mathrm{FO}$ & & \\
\hline 22 & 1 & incremento & s.m. & $\mathrm{CO}$ & & 0 \\
\hline Lücken & & $\dot{e}$ & v.intr. & FO & & \\
\hline & 2 & $\begin{array}{l}\text { dovuto } \\
\text { alle }\end{array}$ & $\begin{array}{l}\text { agg. } \\
\text { prep.art. }\end{array}$ & $\begin{array}{l}\mathrm{CO} \\
\mathrm{FO}\end{array}$ & & 0 , dovuta \\
\hline & 1 & $\begin{array}{l}\text { migliori } \\
\text { condizioni }\end{array}$ & $\begin{array}{l}\text { agg. } \\
\text { s.f. }\end{array}$ & $\begin{array}{l}\text { FO } \\
\text { FO }\end{array}$ & & migliorare \\
\hline & 1 & $d i$ & prep. & FO & & della \\
\hline
\end{tabular}




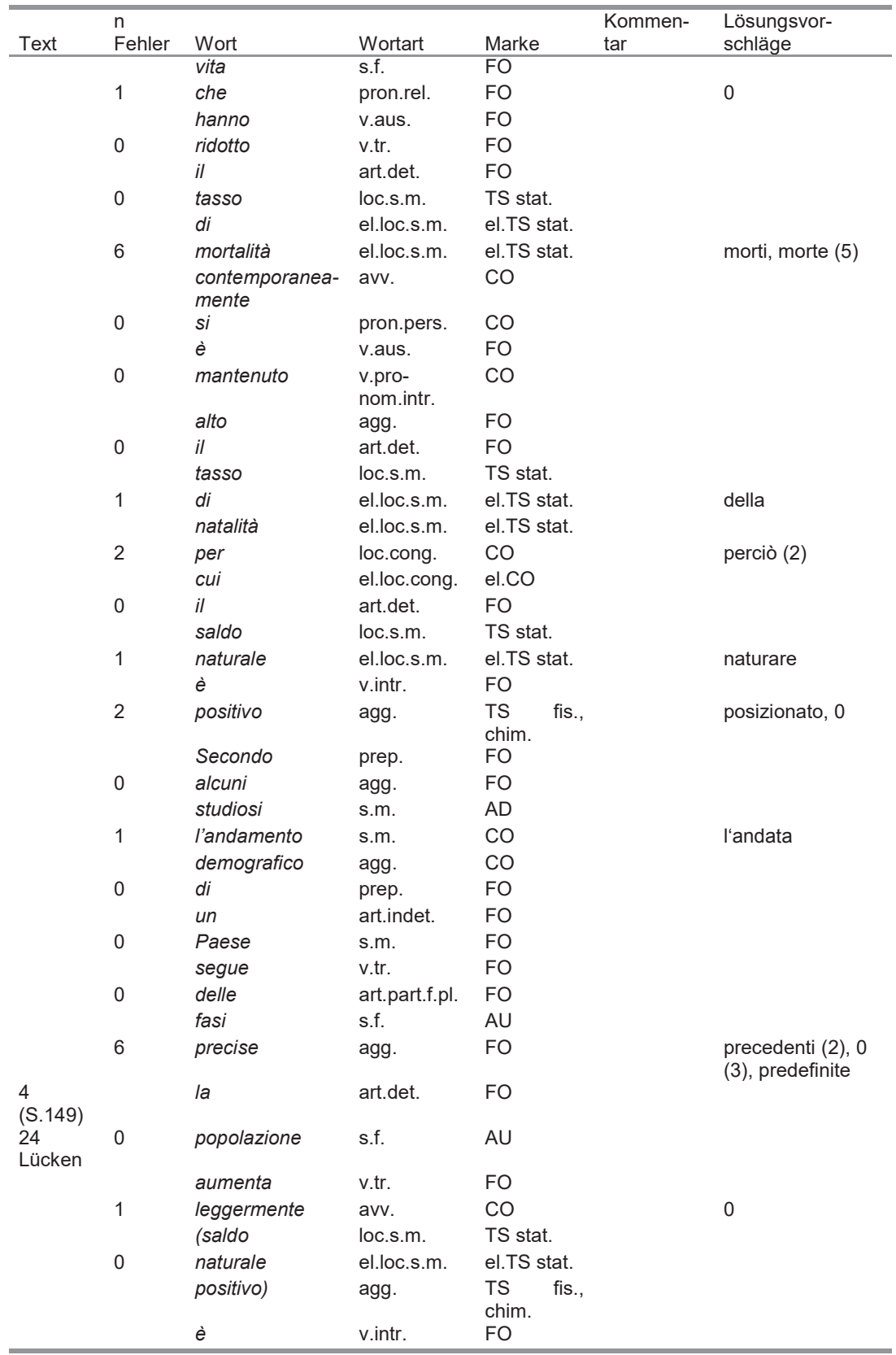




\begin{tabular}{|c|c|c|c|c|c|c|c|}
\hline Text & \multirow{2}{*}{$\begin{array}{l}\mathrm{n} \\
\text { Fehler } \\
6\end{array}$} & Wort & Wortart & \multicolumn{2}{|l|}{ Marke } & $\begin{array}{l}\text { Kommen- } \\
\text { tar }\end{array}$ & $\begin{array}{l}\text { Lösungsvor- } \\
\text { schläge }\end{array}$ \\
\hline & & quanto & avv. & FO & & & quato, quando (5) \\
\hline & & si & pron. & $\mathrm{CO}$ & & & \\
\hline & 1 & verifica & $\begin{array}{l}\text { v.pro- } \\
\text { nom.intr. }\end{array}$ & $\mathrm{CO}$ & & & verificano \\
\hline & & attualmente & avv. & $A U$ & & & \\
\hline & 0 & nei & prep.art. & $\mathrm{FO}$ & & & \\
\hline & & Paesi & s.m. & FO & & & \\
\hline & 0 & sottosviluppati & agg. & $\begin{array}{l}\text { co } \\
\text { econ. }\end{array}$ & TS & & \\
\hline & & come & avv. & FO & & & \\
\hline & 0 & $\begin{array}{l}\text { la } \\
\text { Sierra }\end{array}$ & $\begin{array}{l}\text { art.det. } \\
\text { n.p. }\end{array}$ & FO & & & \\
\hline & 1 & $\begin{array}{l}\text { Leone } \\
\text { in }\end{array}$ & $\begin{array}{l}\text { el.n.p. } \\
\text { prep. }\end{array}$ & $\mathrm{FO}$ & & & Lerale \\
\hline & 0 & Africa & n.p.f. & & & & \\
\hline & 0 & $\begin{array}{l}\text { Haiti } \\
\text { in } \\
\text { America }\end{array}$ & $\begin{array}{l}\text { n.p. } \\
\text { prep. } \\
\text { n.p.f. }\end{array}$ & FO & & & \\
\hline & 1 & centrale & agg. & FO & & & centro \\
\hline & & il & art.det. & $\mathrm{FO}$ & & & \\
\hline & 3 & Bangladesh & n.p. & & & & 0 (2), Banglades \\
\hline & & in & prep. & FO & & & \\
\hline & 0 & Asia & n.p. & & & & \\
\hline & & in & prep. & FO & & & \\
\hline & 0 & una & art.indet. & $\mathrm{FO}$ & & & \\
\hline & & fase & s.f. & $A U$ & & & \\
\hline & 2 & successiva & agg. & FO & & & $\begin{array}{l}\text { succeccita, suc- } \\
\text { cede }\end{array}$ \\
\hline & & grazie & loc.prep. & $\mathrm{CO}$ & & & \\
\hline & 1 & ai & el.loc.prep. & el.CO & & & a molti \\
\hline & & progressi & s.m. & FO & & & \\
\hline & 0 & in & prep. & FO & & & \\
\hline & & campo & s.m. & $\mathrm{FO}$ & & & \\
\hline & 2 & medico & agg. & FO & & & medio, 0 \\
\hline & & diminuisce & v.intr. & $A U$ & & & \\
\hline & 0 & la & art.det. & FO & & & \\
\hline & & mortalità & s.f. & $\mathrm{CO}$ & & & \\
\hline & & e & cong. & $\mathrm{FO}$ & & & \\
\hline & 3 & aumenta & v.tr. & FO & & & $\begin{array}{l}\text { aumeta, au- } \\
\text { mente, aumen- } \\
\text { tata }\end{array}$ \\
\hline & & la & art.det. & $\mathrm{FO}$ & & & \\
\hline & 1 & durata & s.f. & $A U$ & & & durarata \\
\hline & & media & agg. & $A U$ & & & \\
\hline & 1 & della & prep.art. & $\mathrm{FO}$ & & & deella \\
\hline & & vita & s.f. & FO & & & \\
\hline & 1 & mentre & cong. & FO & & & 0 \\
\hline $\begin{array}{l}5 \\
(\mathrm{~S} .116)\end{array}$ & & Stati & n.p. & & & & \\
\hline
\end{tabular}




\begin{tabular}{|c|c|c|c|c|c|c|}
\hline Text & $\begin{array}{l}\mathrm{n} \\
\text { Fehler }\end{array}$ & Wort & Wortart & Marke & $\begin{array}{l}\text { Kommen- } \\
\text { tar }\end{array}$ & $\begin{array}{l}\text { Lösungsvor- } \\
\text { schläge }\end{array}$ \\
\hline 21 & 0 & Uniti & el.n.p. & & & \\
\hline \multirow[t]{35}{*}{ Lücken } & & $e$ & cong. & FO & & \\
\hline & 0 & Canada & n.p.m. & & & \\
\hline & & Unione & loc.s.f. & $\begin{array}{l}\text { TS dir. in- } \\
\text { tern. }\end{array}$ & & \\
\hline & 0 & Europea & el.loc.s.f. & $\begin{array}{l}\text { el.TS dir. } \\
\text { intern. }\end{array}$ & & \\
\hline & & Giappone & n.p.m. & & & \\
\hline & 3 & Australia & n.p.f. & & $\begin{array}{l}\text { logisch } \\
\text { falsch }\end{array}$ & Austria (3) \\
\hline & & $e$ & cong. & FO & & \\
\hline & 0 & Nuova & n.p.f. & & & \\
\hline & & Zelanda & el.n.p. & & & \\
\hline & 0 & Questi & $\begin{array}{l}\text { pron.di- } \\
\text { mostr. }\end{array}$ & FO & & \\
\hline & & Stati & s.m. & & & \\
\hline & 1 & producono & v.tr. & FO & & produttori \\
\hline & & ed & cong. & FO & & \\
\hline & $\begin{array}{l}3+1 \\
\text { acc. }\end{array}$ & esportano & v.tr. & $\mathrm{AU}$ & & $\begin{array}{l}\text { esportatori, } \\
\text { espongono, e- } \\
\text { sportatano, e- } \\
\text { sportanto }\end{array}$ \\
\hline & & soprattutto & avv. & FO & & \\
\hline & 7 & manufatti & s.m. & $\mathrm{CO}$ & & $\begin{array}{l}\text { manutenzione, } \\
\text { manutenzioni, } 0 \\
\text { (5) }\end{array}$ \\
\hline & & $a d$ & prep. & FO & & \\
\hline & 2 & alto & agg. & FO & & $0(2)$ \\
\hline & & contenuto & s.m. & $\mathrm{AU}$ & & \\
\hline & 1 & tecnologico & agg. & $\mathrm{CO}$ & & tecnonologico \\
\hline & & automobili & s.f. & FO & & \\
\hline & $\begin{array}{l}5+3 \\
\text { acc. }\end{array}$ & macchine & loc.s.f. & TS tecn. & $\begin{array}{l}\text { Phrasem } \\
\text { allerdings } \\
\text { angemes- } \\
\text { sener }\end{array}$ & $\begin{array}{l}\text { macchinari (3) } \\
(\text { acc.) } 0(5)\end{array}$ \\
\hline & & utensili & el.loc.s.f. & el.TS tecn. & & \\
\hline & 1 & prodotti & s.m. & FO & & 0 \\
\hline & & chimici & agg. & $A U$ & & \\
\hline & 7 & apparecchiature & s.f. & $A D$ & $\begin{array}{l}\text { Lösungs- } \\
\text { vorschlag } \\
\text { appare- } \\
\text { cemi } \\
\text { unleserlich }\end{array}$ & $\begin{array}{l}\text { apparecchi (5), } \\
\text { apparecchiazioni, } \\
0\end{array}$ \\
\hline & & elettroniche & agg. & $A D$ & & \\
\hline & & $e$ & cong. & FO & & \\
\hline & 4 & importano & v.tr. & FO & & $\begin{array}{l}\text { importanti (3), im- } \\
\text { porta }\end{array}$ \\
\hline & & minerali & s.m. & $\mathrm{FO}$ & & \\
\hline & 1 & fonti & loc.s.f. & TS ecol. & & forme \\
\hline & & energetiche & el.loc.s.f. & el.CO & & \\
\hline & & $e$ & cong. & FO & & \\
\hline & 0 & prodotti & s.m. & FO & & \\
\hline & & alimentari & agg. & $A U$ & & \\
\hline
\end{tabular}




\begin{tabular}{|c|c|c|c|c|c|c|}
\hline Text & $\begin{array}{l}\mathrm{n} \\
\text { Fehler }\end{array}$ & Wort & Wortart & Marke & $\begin{array}{l}\text { Kommen- } \\
\text { tar }\end{array}$ & $\begin{array}{l}\text { Lösungsvor- } \\
\text { schläge }\end{array}$ \\
\hline & \multirow[t]{2}{*}{1} & $\mathrm{La}$ & art.det. & FO & & 0 \\
\hline & & forte & agg. & FO & & \\
\hline & \multirow[t]{2}{*}{1} & importazione & s.f. & $\mathrm{CO}$ & & importanza \\
\hline & & $d i$ & prep. & FO & & \\
\hline & \multirow[t]{2}{*}{1} & petrolio & s.m. & FO & & 0 \\
\hline & & $d a$ & loc.prep. & $\mathrm{CO}$ & & \\
\hline & \multirow[t]{2}{*}{4} & parte & el.loc.prep. & el.co & & $\begin{array}{l}\text { parti (2), } \\
\text { parecchi, } 0\end{array}$ \\
\hline & & $d i$ & el.loc.prep. & el.CO & & \\
\hline
\end{tabular}

Tabelle 23: Texte 3 bis 5 des C-Tests der Klasse III A, Pestalozzi (Textgrundlage Bastianelli et al. 2010, S. 116, 149)

Von insgesamt 67 Lücken füllten alle Testteilnehmer 27 korrekt. Auch hierunter sind erneut nicht nur Wörter des fundamentalen Wortschatzes, sondern neben etlichen Eigennamen auch zwei Co-gekennzeichnete Wörter, eines mit der doppelten Marke Co Ts econ. und drei fachwortschatzsprachliche Phrasemelemente vertreten.

Weitere 34 Wörter wurden mit ein bis vier Fehlern vervollständigt, wobei heraussticht, dass in diesen drei Texten nur zwei Ausdrücke viermal und nur vier weitere dreimal falsch gelöst wurden. Der Großteil der selten falsch ergänzten Wörter zählt zum fundamentalen Wortschatz, aber es sind auch Wörter aus dem allgemeinen Wortschatz (CO), dem des hohen Gebrauchs (AU), zwei fachwortschatzsprachliche Phrasemelemente und Eigennamen darunter. Aufgrund der überwiegend sehr geringen Zahl an Falschlösungen werden nur die sechs Ausdrücke besprochen, die von drei bzw. vier SchülerInnen falsch ergänzt wurden. Das sind im vierten Text das Toponym Bangl-adesh (zweimal nicht und einmal ohne $h$ vervollständigt) sowie das konjugierte Verb aum-enta (Fo; zunehmen) und das Toponym Austr-alia, die konjugierten Verbformen impo-rtano und espo-rtano (AU; exportieren) und das Phrasemelement pa-rte aus da parte di (CO; vonseiten) im fünften Text.

Australien wird als eines der Länder in der Aufzählung der weltweit stärksten Wirtschaftsmächte genannt und von drei SchülerInnen zu Austria (Österreich) ergänzt, was im Erdkundeunterricht einen groben logischen Fehler darstellt, da kurz vorher in der Liste die Europäische Union als Ganze genannt ist. Aber vielleicht handelt es sich hier in erster Linie um ein Konzentrationsproblem.

Die gleiche Ursache mögen auch die drei falschen Vorschläge für das Verb aumenta haben, da es sich dabei zum einen um zwei fiktive Formen handelt und damit recht sicher um Rechtschreibfehler, und zum anderen um ein morphosyntaktisch nicht korrektes Partizip Perfekt desselben Verbs. Ebenso verhält es sich mit den Vorschlägen für das Verb esportano, die orthographische Fehler und eine logisch grammatikalisch nicht korrekte Änderung der Wortart zum Substantiv esportatori (Exporteure) beinhalten. Im selben komplexeren 
Satz wurde anschließend das Prädikat importano in vielen Fällen zum verwandten Adjektiv importanti ergänzt, da es sich vor einem Substantiv befindet, das fälschlicherweise als weiteres Glied einer Aufzählung betrachtet wurde. Diese Lösung funktioniert nicht, weil nach einer neuen Aufzählung, die eben durch das Prädikat importano eingeleitet wird, eine weitere Konjunktion $e$ (und) folgt.

Bei den Falschlösungen für parte scheint in allen vier Fällen nicht erkannt worden zu sein, dass es sich bei da parte di um einen Phraseologismus handelt.

\subsubsection{Häufige Falschlösungen in den C-Testtexten 3 bis 5 der Klasse III A}

In diesen drei Texten des Tests sind insgesamt nur sechs Wörter oft falsch gelöst worden. Um welche Wörter es sich handelt, ist in Tabelle 24 ersichtlich.

\begin{tabular}{|c|c|c|c|c|c|c|}
\hline Text & Fehler & Wort & Wortart & Marke & $\mathrm{K}$ & Lösungsvorschläge \\
\hline 3 & $\begin{array}{l}6 \\
6\end{array}$ & $\begin{array}{l}\text { tasso } \\
\text { di } \\
\text { mortalità } \\
\text { precise }\end{array}$ & $\begin{array}{l}\text { loc.s.m. } \\
\text { el.loc.s.m. } \\
\text { el.loc.s.m. } \\
\text { agg. }\end{array}$ & $\begin{array}{l}\text { TS stat. } \\
\text { el.TS stat. } \\
\text { el.TS stat. } \\
\text { FO }\end{array}$ & & $\begin{array}{l}\text { morti, morte (5) } \\
\text { precedenti (2), } 0 \text { (3), } \\
\text { predefinite }\end{array}$ \\
\hline 4 & 6 & quanto & avv. & $\mathrm{FO}$ & & quato, quando (5) \\
\hline 5 & $\begin{array}{l}7 \\
5+3 \\
\text { acc. } \\
7\end{array}$ & $\begin{array}{l}\text { manufatti } \\
\text { macchine } \\
\text { utensili } \\
\text { apparecchia- } \\
\text { ture } \\
\text { elettroniche }\end{array}$ & $\begin{array}{l}\text { s.m. } \\
\text { loc.s.f. } \\
\text { el.loc.s.f. } \\
\text { s.f. }\end{array}$ & $\begin{array}{l}\text { CO } \\
\text { TS tecn. } \\
\text { el.TS tecn. } \\
\text { AD } \\
\text { AD }\end{array}$ & $\begin{array}{l}\text { Phrasem } \\
\text { unbekannt }\end{array}$ & $\begin{array}{l}\text { manutenzione, manu- } \\
\text { tenzioni, } 0 \text { (5) } \\
\text { macchinari (3) (acc.), } 0 \\
\text { (5) } \\
\text { apparecchi (4), ap- } \\
\text { parecchiazioni, appare- } \\
\text { cemi (? unleserlich), } 0\end{array}$ \\
\hline
\end{tabular}

Tabelle 24: Häufige Falschlösungen in den Texten 3 bis 5 des C-Test der Klasse III A (Textgrundlage Bastianelli et al. 2010, S. 116, 149)

Von den 17 Testteilnehmern lösten in diesen beiden Texten maximal sieben ein Wort falsch. Die Ausdrücke, die vermehrt Schwierigkeiten bereiteten, sind vom fundamentalen bis zum fachwortschatzsprachlichen ganz verschiedenen Bereichen des Wortschatzes zugeordnet. Anhand der Lösungsvorschläge lässt sich erkennen, dass die Fehlerquellen vermutlich hauptsächlich auf lexikalische Probleme zurückzuführen sind. Möglicherweise kannten die Lernenden die gefragten Begriffe entweder nicht oder fielen sie ihnen bei der Testbearbeitung und für den entsprechenden Textzusammenhang nicht ein. Letzteres gilt wahrscheinlich für das Adjektiv pre-ciso (genau) aus dem fundamentalen Wortschatz. Obwohl angenommen werden kann, dass alle Testteilnehmer dieses Wort sicher beherrschten, ergänzten drei die Lücke zu anderen Adjektiven, die zwar grammatikalisch akzeptabel sind, aber logisch nicht, und drei weitere 
produzierten in diesem Kontext keine passende Adjektivergänzung. Ähnliches gilt für das siebenmal falsch vervollständigte apparec-chiature, das vier Lernende zu apparecchi (Apparate) ergänzten, was logisch akzeptabel ist, grammatikalisch aber nicht, da das dazugehörende Adjektiv eine Feminin-Endung besitzt. Einem weiteren Lernenden war dadurch eventuell bewusst, dass es nicht zu apparecchi ergänzt werden konnte. Also wählte er das nicht existierende apparecchiazioni, was zu verraten scheint, dass er das erforderte Wort verstand, wenn er es rezipierte, es aber nicht produzieren konnte.

Die beiden Phraseme für „Sterberate“ (Ts stat.) und „Werkzeugmaschine“ (Ts tecn.) hingegen waren vielen anscheinend einfach nicht bekannt, wobei die Lösungsvorschläge aus dem statistischen Wortschatz vermuten lassen, dass ihre Verfasser die Bedeutung des Konzepts verstanden hatten, auch wenn sie den richtigen Ausdruck nicht produzierten. Für den zweiten Phraseologismus wurde auch das verwandte macchinari (Maschinerien) als Lösung akzeptiert, da es sich bei utensile um ein Adjektiv handelt, so dass sich für das Syntagma eine sinnverwandte Bedeutung ergibt, auch wenn der Gebrauch des Phrasems macchine utensili angemessener erscheint.

\subsubsection{B der Pestalozzi}

Die Texte des C-Tests, der in dieser Klasse durchgeführt wurde, sind den Lehrbuchseiten 238, 241 f. und 53 (Dinucci \& Dinucci 2008) entnommen und behandeln landeskundliche und sozioökonomische Aspekte Asiens sowie die verschiedenen Sprachfamilien der Welt und ihre Verbreitung. Nach einer tabellarischen Übersicht über die Zugehörigkeit der getilgten Wörter zu den verschiedenen Bereichen des Gebrauchswortschatzes, die grammatikalischen Kategorien und die Falschlösungen werden zunächst die Fehler in den ersten drei Texten über Asien und im Anschluss daran die der anderen beiden zum asiatischen Klima und den Sprachen vorgestellt.

Die drei am häufigsten vertretenen Wortschatzbereiche im getilgten Wortvorkommen dieses C-Tests sind nach dem fundamentalen (Fo; $66 \%$ ) der allgemeine $(\mathrm{CO} ; 13,6)$ und, ohne Gebrauchsmarke, der der Eigennamen (n.p.; 8,8\%), wobei sich sein Anteil nur um 2,4 Prozentpunkte von dem des hohen Gebrauchs (AU; 6,4 \%) unterscheidet. Die meisten Falschlösungen pro Wort einer Gebrauchsmarke entstanden mit sechs beim getilgten Vorkommen aus dem allgemeinen Wortschatz (CO und el.CO). Desweiteren wurden auch im Test dieser Klasse bei den wenigen AD-gekennzeichneten Wörtern der hohen Disponibilität verhältnismäßig viele Falschlösungen produziert (durchschnittlich 3,7). Die sich im nächsten Kapitelabschnitt anschließende ausführliche Untersuchung der einzelnen Falschlösungen wird in vielen Fällen Hypothesen 
aufstellen, inwiefern die Ursachen dafür im lexikalischen oder im textgrammatikalischen Bereich zu suchen sind, bzw. wie sehr sich die jeweiligen sprachlichen Aspekte gegenseitig bedingt haben könnten.

\begin{tabular}{lrrrrr}
\hline Marke & Vorkommen & Prozent & Kommentar & n Fehler & $\begin{array}{r}\varnothing \\
\text { Fehler }\end{array}$ \\
\hline AD & 3 & 2,4 & bovini (6), asiatici (2), trasformazione & 11 & 3,7 \\
AU & 8 & 6,4 & orientale (6), colture (9) & 21 & 2,6 \\
CO & 17 & 13,6 & articolazioni (12), impetuoso (10), essa & 102 & 6 \\
& & & (10), ostacolato (10), nomade (9) & & \\
el.CO & 1 & 0,8 & a causa di (6) & 6 & 6 \\
FO & 83 & 66,4 & separano (14) & 322 & 3,9 \\
TS & 1 & 0,8 & densità (3,5) & 3,5 & 3,5 \\
n.p. & 11 & 8,8 & Alaska (6), Iran (5) & 28 & 2,5 \\
el.n.p. & 1 & 0,9 & 0 & 0 \\
n Wörter & 125 & 100 & n Fehler & 493,5 & 3,9 \\
\hline
\end{tabular}

Tabelle 25: Getilgte Wörter des C-Tests der Klasse III B (Pestalozzi) nach Gebrauchsmarken mit Angaben zu den Falschlösungen

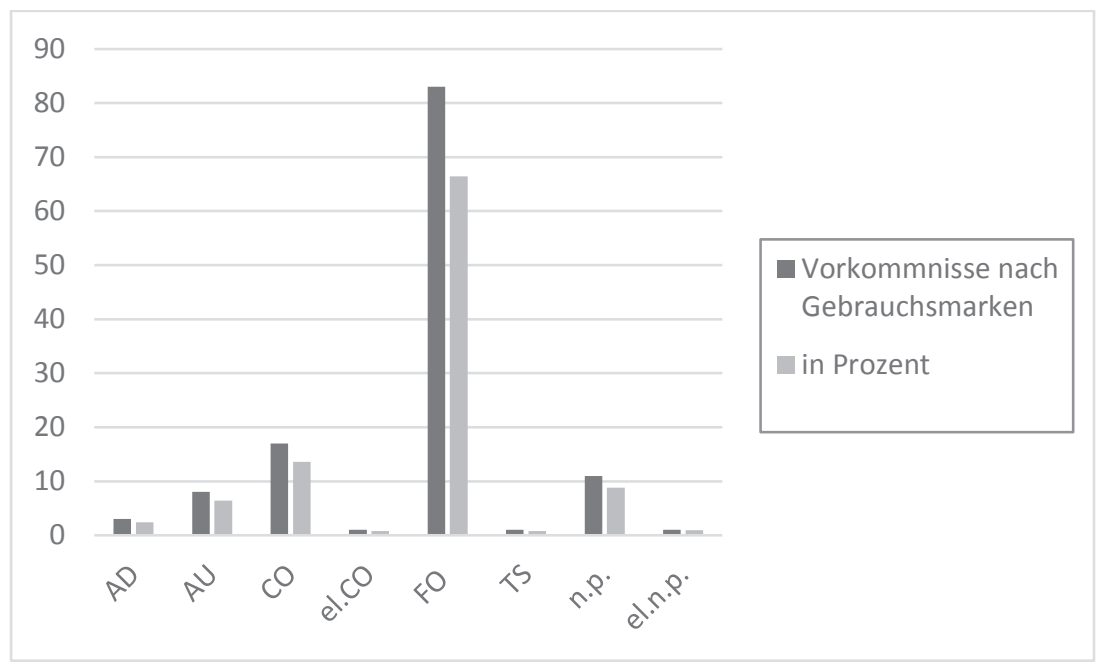

Abbildung 8: Getilgtes Wortvorkommen nach Gebrauchsmarken und ihrem prozentualen Anteil im C-Test der Klasse III B, Pestalozzi

Aus welchen Wortarten die zu ergänzenden Ausdrücke bestehen und wie hoch die Fehlerquote jeweils für sie ist, ist Tabelle $26 \mathrm{zu}$ entnehmen. Wie in allen fünf C-Tests, gehören die meisten zu ergänzenden Ausdrücke zu den Wortarten der Substantive und Adjektive. An dritter Stelle folgen hier mit 14 die zusammengesetzten Präpositionen mit Artikel, wobei auch die Adverbien mit 13 und die Eigennamen mit elf recht stark vertreten sind. Schaut man, welche 
Wortarten durchschnittlich die meisten Fehlerzahlen pro Wort verzeichnen, so sind es jedoch nicht die drei am häufigsten vertretenen Kategorien, sondern ein Relativpronomen (13), konjugierte Verben $(7,8)$ und ein Partizip (7). Die möglichen Ursachen für die vermehrten Falschlösungen werden im Folgenden genauer analysiert; es sei nur vorweggenommen, dass zahlreiche Falschlösungen für die Partizipien zusammengesetzter Verbformen im Passiv vorliegen (neben der in Tabelle 26 angegebenen weitere mit 8 Fehlern), während die Schwierigkeit beim Partizip avviato (FO; in die Wege leiten), Teil eines aktivischen Prädikats im Perfekt, eher auf lexikalischer Ebene zu suchen sein wird.

\begin{tabular}{|c|c|c|c|c|}
\hline Wortart & Vorkommen & Kommentar & n Fehler & $\varnothing$ Fehler pro Wort \\
\hline agg. & 18 & $\begin{array}{l}\text { impetuoso (10), diverse } \\
(10), \text { nomade (9), suo } \\
(10)\end{array}$ & 87 & 4,8 \\
\hline art. det./indet. & 4 & & 6 & 1,5 \\
\hline avv. & 13 & non (5) & 43 & 3,3 \\
\hline cong. & 1 & quando (6) & 6 & 6 \\
\hline el.loc. & 1 & a causa di & 6 & 6 \\
\hline el.n.p. & 1 & & 0 & 0 \\
\hline inf. & 1 & & 1 & 1 \\
\hline n.p. & 11 & Alaska (6), Iran (5) & 28 & 2,5 \\
\hline part.pass. & 1 & unita (7) & 7 & 7 \\
\hline prep. & 9 & lungo (7), con (7) & 35 & 3,9 \\
\hline prep.art. & 14 & dalle $(6)$ & 40 & 2,9 \\
\hline pron. ${ }^{*}$ & 3 & essa (10) & 15 & 5 \\
\hline pron.rel. & 1 & cui & 13 & 13 \\
\hline s.m./f. & 32 & articolazioni (12) & 113,5 & 3,5 \\
\hline v.aus. & 4 & vengono (8) & 17 & 4,3 \\
\hline v.tr./intr. & 9 & $\begin{array}{l}\text { separano (14), avviato } \\
(10), \text { ostacolato }(10), \\
\text { bagnata }(9)\end{array}$ & 70 & 7,8 \\
\hline v.pronom.intr. & 1 & concentrano & 6 & 6 \\
\hline \% s.m./f. & 26,4 & & 493,5 & \\
\hline
\end{tabular}

Tabelle 26: Getilgtes Wortvorkommen des C-Tests der III B (Pestalozzi) nach Wortarten mit Angaben zu den Falschlösungen

pron*: Unter dieser Bezeichnung sind außer den gesondert aufgelisteten Relativpronomen (pron.rel.) alle anderen Arten von Pronomen gesammelt.

\subsubsection{C-Testtexte 1 bis 3 der Klasse III B}

Wie gehabt, ermöglicht eine Tabelle (27) den Überblick über die Art des Wortvorkommens in den ersten Texten des C-Test und über die Anzahl und Art der Lösungsvorschläge von 14 Schülerinnen und Schülern der Klasse, während einer bei der Durchführung des Tests fehlte. Für vier Testteilnehmer ist Italienisch eine Zweitsprache, und einer von ihnen sowie zwei italienische Testteilnehmer haben ein Schuljahr wiederholt. 


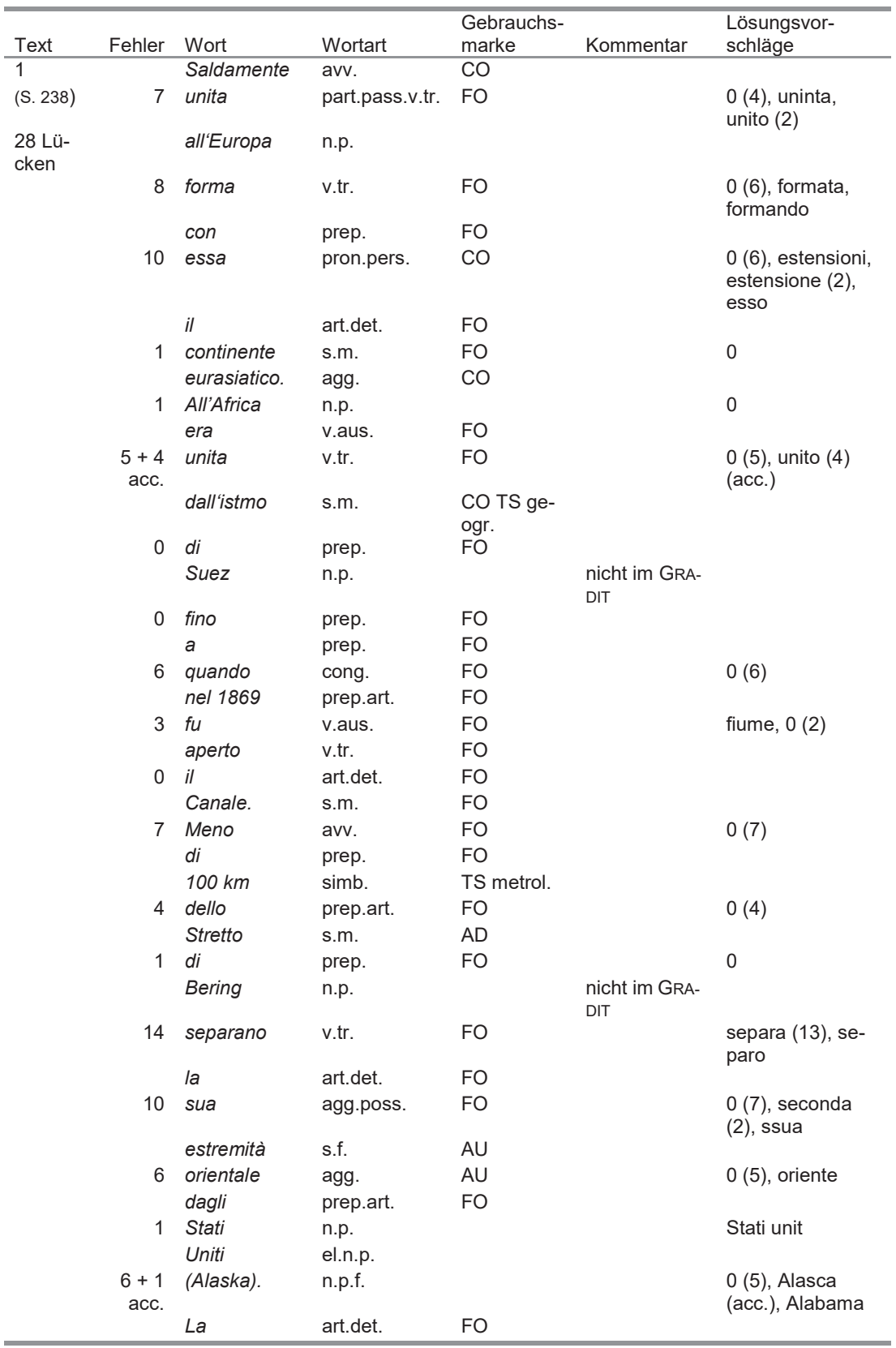




\begin{tabular}{|c|c|c|c|c|c|c|}
\hline Text & Fehler & Wort & Wortart & $\begin{array}{l}\text { Gebrauchs- } \\
\text { marke }\end{array}$ & Kommentar & $\begin{array}{l}\text { Lösungsvor- } \\
\text { schläge }\end{array}$ \\
\hline & 4 & costa & s.f. & FO & & $\begin{array}{l}0(2) \text {, coltivazi- } \\
\text { one, costo }\end{array}$ \\
\hline & & $\begin{array}{l}\text { settentrio- } \\
\text { nale }\end{array}$ & agg. & $A U$ & & \\
\hline & 3 & $\begin{array}{l}\text { general- } \\
\text { mente }\end{array}$ & avv. & $\mathrm{CO}$ & & genera una (2), 0 \\
\hline & & bassa & agg. & FO & & \\
\hline & & $e$ & cong. & FO & & \\
\hline & 9 & con & prep. & FO & & $0(9)$ \\
\hline & & rare & agg. & $A U$ & & \\
\hline & 12 & articolazioni & s.f. & $\mathrm{CO}$ & $\begin{array}{l}\text { richtige Lö- } \\
\text { sung durch- } \\
\text { gestrichen }\end{array}$ & $\begin{array}{l}0(10) \text {, articolata } \\
(2)\end{array}$ \\
\hline & & è & v.aus. & FO & & \\
\hline & 9 & bagnata & v.tr. & FO & & $\begin{array}{l}0(3), \text { bagnato } \\
(4), \text { bagnate }(2)\end{array}$ \\
\hline & & dalle & prep.art. & $\mathrm{FO}$ & & \\
\hline & 4 & acque & s.f. & $\mathrm{FO}$ & & 0 (3), acqua \\
\hline & & quasi & avv. & FO & & \\
\hline & 4 & sempre & avv. & FO & & semi (4) \\
\hline & & ghiacciate & agg. & $\mathrm{CO}$ & & \\
\hline & 2 & $\begin{array}{l}\text { del } \\
\text { Mar }\end{array}$ & $\begin{array}{l}\text { prep.art. } \\
\text { n.p. }\end{array}$ & FO & & dal, 0 \\
\hline & 0 & Glaciale & el.n.p. & & $\begin{array}{l}\text { nicht im GRA- } \\
\text { DIT }\end{array}$ & \\
\hline & & Artico & el.n.p. & & & \\
\hline 2 & & & & & & \\
\hline (S. 241) & & Grazie & loc.prep. & $\mathrm{CO}$ & & \\
\hline \multirow[t]{20}{*}{$\begin{array}{l}25 \text { Lü- } \\
\text { cken }\end{array}$} & $\begin{array}{r}2+4 \\
\text { acc. }\end{array}$ & soprattutto & avv. & FO & & $\begin{array}{l}\text { sopratutto (acc.) } \\
(4), 0(2)\end{array}$ \\
\hline & & allo & el.loc.prep. & el.CO & & \\
\hline & 1 & sviluppo & s.m. & FO & & svillupo \\
\hline & & agricolo & agg. & FO & & \\
\hline & 12 & $\begin{array}{l}\text { di } \\
\text { Cina }\end{array}$ & $\begin{array}{l}\text { prep. } \\
\text { n.p.f. }\end{array}$ & FO & & della (11), da \\
\hline & & $e$ & cong. & FO & & \\
\hline & 4 & India & n.p.f. & & & 0 (3), Inghilterra \\
\hline & & la & art.det. & FO & & \\
\hline & 2 & regione & s.f. & $A U$ & & reggione, 0 \\
\hline & & $\grave{e}$ & v.intr. & FO & & \\
\hline & 1 & al & prep.art. & FO & & 0 \\
\hline & & primo & agg.num.ord. & FO & & \\
\hline & 1 & posto & s.m. & FO & & 0 \\
\hline & & nel & prep.art. & FO & & \\
\hline & 2 & mondo & s.m. & FO & & 0 , moondo \\
\hline & & come & avv. & $\mathrm{FO}$ & & \\
\hline & 8 acc. & produzione & s.m. & $\mathrm{AU}$ & & $\begin{array}{l}\text { produttore (8) } \\
\text { (acc.) }\end{array}$ \\
\hline & & di & prep. & FO & & \\
\hline & 0 & riso & s.m. & $A U$ & & \\
\hline & & grano & s.m. & FO & & \\
\hline
\end{tabular}




\begin{tabular}{|c|c|c|c|c|c|c|}
\hline Text & Fehler & Wort & Wortart & $\begin{array}{l}\text { Gebrauchs- } \\
\text { marke }\end{array}$ & Kommentar & $\begin{array}{l}\text { Lösungsvor- } \\
\text { schläge }\end{array}$ \\
\hline & \multirow[t]{3}{*}{$\begin{array}{r}5+9 \\
\text { acc. }\end{array}$} & tè & s.m. & FO & $\begin{array}{l}\text { zwei richtige } \\
\text { Lösungen, oft } \\
\text { tabacco }\end{array}$ & $\begin{array}{l}0(3), \text { tabacco }(9) \\
\text { (acc.), tomatos, } \\
\text { timo }\end{array}$ \\
\hline & & cotone & s.m. & $A U$ & & \\
\hline & & $e$ & cong. & FO & & \\
\hline & \multirow[t]{2}{*}{10} & diverse & agg. & FO & & 0 (8), divano (2) \\
\hline & & altre & agg. & FO & & \\
\hline & \multirow[t]{3}{*}{9} & colture. & s.f. & $A U$ & $\begin{array}{l}\text { oft coltivazi- } \\
\text { oni (AD) }\end{array}$ & $\begin{array}{l}0 \text { (5), coltivazioni } \\
\text { (4) }\end{array}$ \\
\hline & & Sviluppato & agg. & FO & & \\
\hline & & è & v.intr. & FO & & \\
\hline & \multirow[t]{2}{*}{1} & anche & avv. & FO & & 0 \\
\hline & & l'allevamento & s.m. & FO & & \\
\hline & \multirow[t]{3}{*}{6} & bovini & s.m. & $A D$ & & bovivi, bovino (5) \\
\hline & & ovini & s.m. & $A D$ & & \\
\hline & & $e$ & cong. & FO & & \\
\hline & \multirow[t]{2}{*}{$\begin{array}{r}4+1 \\
\text { acc. }\end{array}$} & caprini & s.m. & $\mathrm{CO}$ & & $\begin{array}{l}0 \text {, caprino (3), } \\
\text { capre (acc.) }\end{array}$ \\
\hline & & soprattutto & avv. & FO & & \\
\hline & 1 & $\begin{array}{l}\text { in } \\
\text { India }\end{array}$ & $\begin{array}{l}\text { prep. } \\
\text { n.p.f. }\end{array}$ & FO & & 0 \\
\hline & \multirow[t]{3}{*}{7} & suini & s.m. & $\mathrm{CO}$ & & $0(7)$ \\
\hline & & soprattutto & avv. & FO & & \\
\hline & & in & prep. & FO & Tilgungsfehler & \\
\hline & \multirow[t]{2}{*}{0} & Cina. & n.p.f. & & & \\
\hline & & La & art.det. & FO & & \\
\hline & \multirow[t]{2}{*}{0} & Cina & n.p. & & & \\
\hline & & è & v.intr. & FO & & \\
\hline & \multirow[t]{2}{*}{3} & nettamente & avv. & $\mathrm{CO}$ & & $0(3)$ \\
\hline & & al & prep.art. & FO & & \\
\hline & 0 & primo & agg. & FO & & \\
\hline & \multirow[t]{2}{*}{0} & posto & s.m. & FO & Tilgungsfehler & \\
\hline & & mondiale & agg. & FO & & \\
\hline & \multirow[t]{2}{*}{0} & anche & avv. & FO & & \\
\hline & & nella & prep.art. & FO & & \\
\hline & \multirow[t]{3}{*}{1} & pesca. & s.f. & $A U$ & & pepesca \\
\hline 3 & & & & & & \\
\hline (S. 241) & & Più & avv. & FO & & \\
\hline \multirow[t]{10}{*}{$\begin{array}{l}25 \\
\text { Lücken }\end{array}$} & \multirow[t]{2}{*}{4} & recente & agg. & $\mathrm{FO}$ & & $\begin{array}{l}\text { recnte, recente- } \\
\text { mente, } 0 \text { ( } 2 \text { ) }\end{array}$ \\
\hline & & è & v.intr. & FO & & \\
\hline & \multirow[t]{2}{*}{10} & l'impetuoso & agg. & $\mathrm{CO}$ & oft impero & $\begin{array}{l}\text { imperiale, } 0 \text { (3), } \\
\text { impero (4), impe- } \\
\text { ono, impei }\end{array}$ \\
\hline & & sviluppo & s.m. & FO & & \\
\hline & \multirow[t]{2}{*}{0} & industriale & agg. & FO & & \\
\hline & & della & prep.art. & $\mathrm{FO}$ & & \\
\hline & \multirow[t]{2}{*}{0} & Cina & n.p.f. & & & \\
\hline & & soprattutto & avv. & FO & & \\
\hline & \multirow[t]{2}{*}{1} & delle & prep.art. & FO & & 0 \\
\hline & & province & s.f. & FO & & \\
\hline
\end{tabular}




\begin{tabular}{|c|c|c|c|c|c|c|}
\hline Text & Fehler & Wort & Wortart & $\begin{array}{l}\text { Gebrauchs- } \\
\text { marke }\end{array}$ & Kommentar & $\begin{array}{l}\text { Lösungsvor- } \\
\text { schläge }\end{array}$ \\
\hline & \multirow[t]{2}{*}{2} & costiere & agg. & $\mathrm{CO}$ & & $0(2)$ \\
\hline & & in & prep. & FO & & \\
\hline & \multirow[t]{2}{*}{13} & cui & pron.rel. & FO & $\begin{array}{l}\text { nur eine rich- } \\
\text { tige Lösung }\end{array}$ & $\begin{array}{l}0(10), \text { come, } \\
\text { cina, costa }\end{array}$ \\
\hline & & si & pron.pers. & $\mathrm{CO}$ & & \\
\hline & \multirow[t]{2}{*}{6} & concentrano & $\begin{array}{l}\text { v.pro- } \\
\text { nom.intr. }\end{array}$ & $\mathrm{CO}$ & & $\begin{array}{l}\text { concentra (2), } \\
\text { concedono, } 0 \text { (2), } \\
\text { concede }\end{array}$ \\
\hline & & le & art.det. & FO & & \\
\hline & \multirow[t]{2}{*}{1} & produzioni & s.f. & FO & & produzione \\
\hline & & destinate & agg. & $\mathrm{CO}$ & & \\
\hline & \multirow[t]{2}{*}{2} & $\begin{array}{l}\text { all'esporta- } \\
\text { zione. }\end{array}$ & s.f. & $A U$ & & $\begin{array}{l}\text { all'esporto, all'e- } \\
\text { sportazioni }\end{array}$ \\
\hline & & Anche & cong. & FO & & \\
\hline & \multirow[t]{2}{*}{6} & altri & agg. & FO & & $\begin{array}{l}0 \text { (4), alcuni, } 1 \\
\text { unleserlich }\end{array}$ \\
\hline & & paesi & s.m. & FO & & \\
\hline & \multirow[t]{2}{*}{6} & (India & n.p.f. & & & $\begin{array}{l}\text { Industrializzati, } 0 \\
\text { (4), Inghilterra; } \\
\text { Inndia }\end{array}$ \\
\hline & & Indonesia & n.p. & & $\begin{array}{l}\text { nicht im GRA- } \\
\text { DIT }\end{array}$ & \\
\hline & \multirow[t]{2}{*}{$\begin{array}{r}5+3 \\
\text { acc. }\end{array}$} & Iran & n.p. & & $\begin{array}{l}\text { nicht im GRA- } \\
\text { DIT, oft Iraq }\end{array}$ & $\begin{array}{l}0 \text { (3), Iraq } 2 \\
\text { (acc.), Iraq//ran } \\
\text { (acc.) Irlandesi, } \\
\text { Irlanda }\end{array}$ \\
\hline & & $e$ & cong. & FO & & \\
\hline & \multirow[t]{2}{*}{2} & Turchia) & n.p.f. & & & 0, Turkia \\
\hline & & hanno & v.aus. & FO & & \\
\hline & \multirow[t]{2}{*}{10} & avviato & v.tr. & FO & & $\begin{array}{l}0 \text { (7), avverito, } \\
\text { avvertito, avvisi }\end{array}$ \\
\hline & & negli & prep.art. & FO & & \\
\hline & \multirow[t]{2}{*}{1} & ultimi & agg. & FO & & ulteriori \\
\hline & & decenni & s.m. & $\mathrm{CO}$ & & \\
\hline & \multirow[t]{2}{*}{$\begin{array}{r}8+3 \\
\text { acc. }\end{array}$} & progetti & s.m. & FO & & $\begin{array}{l}\text { programmi (acc.) } \\
\text { (3), } 0 \text { (3), pro- } \\
\text { gressi (5) }\end{array}$ \\
\hline & & $d i$ & prep. & FO & & \\
\hline & \multirow[t]{3}{*}{1} & sviluppo & s.m. & FO & & 0 \\
\hline & & industriale. & agg. & FO & & \\
\hline & & Ma & cong. & FO & & \\
\hline & \multirow[t]{2}{*}{3} & nella & prep.art. & FO & $\begin{array}{l}\text { Tilgungsfeh- } \\
\text { ler }\end{array}$ & nel, 0 (2) \\
\hline & & maggioranza & s.f. & $A U$ & & \\
\hline & \multirow[t]{2}{*}{1} & $d e i$ & prep.art. & FO & & di \\
\hline & & paesi & s.m. & FO & & \\
\hline & 2 & asiatici & agg. & $A D$ & & $0(2)$ \\
\hline & \multirow[t]{2}{*}{1} & l'industria & s.f. & $\mathrm{FO}$ & $\begin{array}{l}\text { Tilgungsfeh- } \\
\text { ler }\end{array}$ & l'industriali \\
\hline & & $\grave{e}$ & v.intr. & FO & & \\
\hline & \multirow[t]{2}{*}{1} & limitata & v.aus. & FO & & limitato \\
\hline & & alle & v.tr. & FO & & \\
\hline & 4 & attività & s.f. & FO & & $0(4)$ \\
\hline
\end{tabular}




\begin{tabular}{|c|c|c|c|c|c|c|}
\hline Text & Fehler & Wort & Wortart & $\begin{array}{l}\text { Gebrauchs- } \\
\text { marke }\end{array}$ & Kommentar & $\begin{array}{l}\text { Lösungsvor- } \\
\text { schläge }\end{array}$ \\
\hline & & di & s.f. & $\mathrm{FO}$ & & \\
\hline & 3 & $\begin{array}{l}\text { trasforma- } \\
\text { zione }\end{array}$ & s.f. & $A D$ & & $\begin{array}{l}0(2) \text {, trasforma- } \\
\text { zioni }\end{array}$ \\
\hline
\end{tabular}

Tabelle 27: Texte 1 bis 3 des C-Tests der Klasse III B, Pestalozzi (Textgrundlage Dinucci \& Dinucci 2008, S. 238, 241)

Bei der Testkonstruktion wurden im zweiten und dritten Text je zwei Wörter unregelmäßig getilgt (vgl. Tabelle 27). Während die Tilgung im ersten Fall das vorausgehende Wort hätte treffen müssen, so dass die Testpersonen ein zusätzliches nicht getilgtes Wort zur Verfügung hatten, wurden im zweiten Fall zwei aufeinanderfolgende Wörter getilgt, doch erfreulicherweise ließ keiner die betroffenen Lücken offen oder füllte sie falsch aus.

Im dritten Text ergibt sich bezüglich der fehlerhaften Konstruktion der getilgten Wörter die gleiche Situation, wobei beim zweiten Fall, in dem die getilgten Wörter direkt nacheinander stehen, drei Lernende das erste nicht richtig lösten und einer das zweite so falsch ergänzte, dass die Ursache dafür nicht allein die unregelmäßige Tilgung gewesen sein kann.

Der erste Text besteht aus 28 Lücken und der zweite und dritte aus je 25 . Von diesen 78 Lücken hat die Lerngruppe geschlossen lediglich 13 richtig vervollständigt. Für ein Wort gab es dabei achtmal einen akzeptablen Alternativvorschlag. Die 13 Wörter stammen überwiegend aus dem Bereich des fundamentalen Wortschatzes, eins aus dem des hohen Gebrauchs (ri-so (Reis)) und zwei sind die geographischen Eigennamen Glac-iale aus Mar Glaciale Artico (Nördliches Eismeer) und $\mathrm{Ci}$-na (insgesamt drei Vorkommnisse).

38 Wörter wurden von ein bis vier Lernenden nicht oder falsch vervollständigt. Zwei Drittel davon (25) zählen zum fundamentalen Wortschatz; unter den restlichen befinden sich Eigennamen, vier Wörter des hohen Gebrauchs (AU), zwei der hohen Disponibilität (AD) und vier Co-gekennzeichnete.

In den drei Texten treten keine fachwortschatzsprachlichen Ausdrücke auf, sondern nur das als Co Ts geogr. doppelt markierte is-tmo (Isthmus), das zudem nicht von der Worttilgung betroffen ist. 13 Wörter wurden drei- bzw. viermal nicht korrekt ergänzt; da acht davon aus dem fundamentalen Wortschatz stammen, dürften die Wörter an sich bekannt gewesen sein. Das erste dieser Fo-gekennzeichneten Wörter ist das Hilfsverb $f$ - $u$, das in einer Passivkonstruktion auftritt. Es könnte am Passiv und am Vergangenheitstempus des passato remoto liegen, dass einige Schüler dieses häufig auftretende Verb nicht richtig vervollständigten. Obwohl es, anders als weiter südlich in Italien, in der gesprochenen Sprache in Rom nicht verwendet wird, war es den Lernenden als geläufiges Erzähltempus in Geschichten und aus den Lehrbuchtexten dennoch bekannt. Dass vier das ihnen sicherlich vertraute co-sta (Küste) nicht richtig vervollständigten, könnte daher rühren, dass diesem Subjekt rare artico-lazioni (seltene Gliederungen, Auffächerungen) zugeschrieben sind. Dieses 
Wort wurde zwölfmal nicht korrekt vervollständigt und diente somit in vielen Fällen nicht als Verständnishilfe für das Ausfüllen der Lücke in costa. Das könnte außerdem dazu geführt haben, dass drei Lernende die darauffolgende Lücke im Adverb genera-lmente (CO; generell) nicht bzw. falsch vervollständigten. Ein weiterer Fall betrifft auch in dieser Klasse eine artikulierte Präposition, die sehr wohl bekannt war, aber, wie schon für die anderen Testgruppen beobachtet, Probleme bei der Wahl zwischen einfacher und artikulierter Präposition bereitete. Hier ist es so, dass das Folgewort mit -st beginnt und die Präposition deshalb auf -lo enden muss, was manche nicht beachteten, obwohl sie die Regel wahrscheinlich beherrschten.

Weitere zwei Aspekte der seltener falsch gelösten Wörter sollen noch erwähnt werden. Da ist zum einen das aus dem allgemeinen Wortschatz (Co) stammende cap-rini (gastronomische und zoologische Bezeichnung für Tiere aus der Familie der Ziegen), das nur ein Lernender nicht vervollständigte und drei andere zu caprino ergänzten. Es erscheint als Teil einer Aufzählung zur betriebenen Tierzucht (allevamento), die nach einem Doppelpunkt beginnt. Wie sich auch an dem fünfmal zu bovino (Rind) ergänzten Glied der Aufzählung erkennen lässt, ignorierten viele den Doppelpunkt nach allevamento und bildeten die Tiernamen als Adjektive zum Subjekt Zucht. Das scheint dadurch möglich, dass die entsprechenden Adjektive und Substantive Homographe sind, allerdings verbietet es der Doppelpunkt. Nach ihm kann nur eine Aufzählung mit Substantiven folgen, was auch durch ihr mittleres, nicht getilgtes Glied, ein Substantiv in Pluralform, vorgegeben ist.

Außerdem wurden von insgesamt fünf $(2+3)$ SchülerInnen zwei Wörter überhaupt nicht vervollständigt, deren Gebrauchsmarken nachdenklich stimmen: Sowohl asia-tico (asiatisch) als auch trasfor-mazione ((Produkt-)Verarbeitung) sind AD-gekennzeichnet, also als Wörter, die uns immer zur Verfügung stehen sollten, da sie zu unserer Alltagswelt gehören und oft gedacht werden, auch wenn sie selten schriftlich oder mündlich produziert werden (vgl. De Mauro 2003, S. 162).

\subsubsection{Frequente Fehlerquellen in den ersten drei Texten des Tests in der} Klasse III B

Gut ein Drittel (27) aller getilgten Wörter dieser Texte wurde mindestens fünfmal falsch oder gar nicht vervollständigt. Sie sind in Tabelle 28 aufgeführt.

\begin{tabular}{|c|c|c|c|c|c|c|}
\hline Text & n Fehler & Wort & Wortart & Marke & Kommentar & Lösungsvorschläge \\
\hline \multirow[t]{4}{*}{$\begin{array}{l}1 \\
\text { (S. 238) }\end{array}$} & 7 & unita & part.pass.v.tr. & FO & & $\begin{array}{l}0 \text { (4), uninta, unito } \\
\text { (2) }\end{array}$ \\
\hline & 8 & forma & v.tr. & FO & & $\begin{array}{l}0(6), \text { formata, for- } \\
\text { mando }\end{array}$ \\
\hline & 10 & essa & pron.pers. & $\mathrm{CO}$ & & $\begin{array}{l}0(6), \text { estensioni, } \\
\text { estensione ( } 2) \text {, esso }\end{array}$ \\
\hline & $\begin{array}{r}5+4 \\
\text { acc. }\end{array}$ & unita & v.tr. & FO & & (5), unito (3) (acc.) \\
\hline
\end{tabular}




\begin{tabular}{|c|c|c|c|c|c|c|}
\hline Text & n Fehler & Wort & Wortart & Marke & Kommentar & Lösungsvorschläge \\
\hline & 6 & quando & cong. & $\mathrm{FO}$ & & $0(6)$ \\
\hline & 7 & Meno & avv. & FO & & $0(7)$ \\
\hline & 14 & separano & v.tr. & FO & & separa (13), separo \\
\hline & 10 & sua & agg.poss. & FO & & $\begin{array}{l}0(7), \text { seconda }(2), \\
\text { ssua }\end{array}$ \\
\hline & 6 & orientale & agg. & $\mathrm{AU}$ & & 0 (5), oriente \\
\hline & $\begin{array}{r}6+1 \\
\text { acc. }\end{array}$ & (Alaska) & n.p.f. & & & $\begin{array}{l}0 \text { (5), Alasca (acc.), } \\
\text { Alabama }\end{array}$ \\
\hline & 9 & con & prep. & FO & & $0(9)$ \\
\hline & 12 & $\begin{array}{l}\text { articola- } \\
\text { zioni }\end{array}$ & s.f. & $\mathrm{CO}$ & $\begin{array}{l}\text { eine richtige } \\
\text { Lösung } \\
\text { durchgestri- } \\
\text { chen }\end{array}$ & 0 (10), articolata (2) \\
\hline & 9 & bagnata & v.tr. & FO & & $\begin{array}{l}0(3), \text { bagnato }(4), \\
\text { bagnate }(2)\end{array}$ \\
\hline \multirow{6}{*}{$\begin{array}{l}2 \\
\text { (S. 241) }\end{array}$} & 12 & $d i$ & prep. & $\mathrm{FO}$ & & della (11), da \\
\hline & 8 acc. & $\begin{array}{l}\text { produ- } \\
\text { zione }\end{array}$ & s.m. & $\mathrm{AU}$ & & produttore (8) (acc.) \\
\hline & $\begin{array}{r}5+9 \\
\text { acc. }\end{array}$ & tè & s.m. & FO & $\begin{array}{l}\text { nur } 2 \text { richtige } \\
\text { Lösungen }\end{array}$ & $\begin{array}{l}0(3), \text { tabacco }(7) \\
\text { (acc.), tomatos, timo }\end{array}$ \\
\hline & 10 & diverse & agg. & FO & & 0 (8), divano (2) \\
\hline & 9 & colture & s.f. & $A U$ & $\begin{array}{l}\text { oft coltivazi- } \\
\text { oni (AD) }\end{array}$ & 0 (5), coltivazioni (4 \\
\hline & 7 & suini & s.m. & $\mathrm{CO}$ & & $0(7)$ \\
\hline \multirow[t]{8}{*}{$\begin{array}{l}3 \\
\text { (S. 241) }\end{array}$} & 10 & $\begin{array}{l}\text { l'impe- } \\
\text { tuoso }\end{array}$ & agg. & $\mathrm{CO}$ & oft impero & $\begin{array}{l}\text { imperiale, } 0 \text { (3), im- } \\
\text { pero (4), impeono, } \\
\text { impei }\end{array}$ \\
\hline & 13 & cui & pron.rel. & $\mathrm{FO}$ & & $\begin{array}{l}0(10), \text { come, cina, } \\
\text { costa }\end{array}$ \\
\hline & 6 & $\begin{array}{l}\text { concen- } \\
\text { trano }\end{array}$ & $\begin{array}{l}\text { v.pro- } \\
\text { nom.intr. }\end{array}$ & $\mathrm{CO}$ & & $\begin{array}{l}\text { concentra }(2), \text { con- } \\
\text { cedono, } 0(2), \text { con- } \\
\text { cede }\end{array}$ \\
\hline & 6 & altri & agg. & FO & & $\begin{array}{l}\text { (4), alcuni, } 1 \text { unleser- } \\
\text { lich }\end{array}$ \\
\hline & 6 & (India & n.p.f. & & & $\begin{array}{l}\text { Industrializzati, } 0 \text { (4), } \\
\text { Inghilterra }\end{array}$ \\
\hline & $\begin{array}{r}5+3 \\
\text { acc. }\end{array}$ & Iran & n.p. & & $\begin{array}{l}\text { nicht im GRA- } \\
\text { DIT, oft Iraq }\end{array}$ & $\begin{array}{l}0 \text { (3), Iraq } 2 \text { (acc.), } \\
\text { Iraq/Iran (acc.) Irlan- } \\
\text { desi, Irlanda }\end{array}$ \\
\hline & 10 & avviato & v.tr. & $\mathrm{FO}$ & & $\begin{array}{l}0(8) \text {, avverito, av- } \\
\text { vertito }\end{array}$ \\
\hline & $\begin{array}{r}8+3 \\
\text { acc. }\end{array}$ & progetti & s.m. & $\mathrm{FO}$ & & $\begin{array}{l}\text { programmi (acc.) (3), } \\
0(3) \text {, progressi (5) }\end{array}$ \\
\hline
\end{tabular}

Tabelle 28: Falschlösungen von mindestens fünf Lernenden in den ersten drei C-Testtexten der Klasse III B, Pestalozzi

Sie verteilen sich recht gleichmäßig auf die drei Texte und beinhalten 16 Wörter aus dem fundamentalen Wortschatz. Zuzüglich der Wörter des hohen Gebrauchs (AU) befinden sich darunter 19 Wörter aus dem Basiswortschatz. Zusammenfassend liegen die Ursachen für die vermehrten Falschlösungen bei diesen Wörtern, die den meisten Lernenden geläufig gewesen sein sollten, schätzungsweise in unzureichender grammatikalischer Kompetenz und im mangelnden Textverständnis, das durch lexikalische und grammatikalisch- 
syntaktische Komplexitäten des sprachlichen Umfelds geschwächt war und dazu führte, dass auch sehr bekannte Wörter für die Lückenfüllung nicht bereitstanden. Für diese zweite Ursache kommt erschwerend hinzu, dass sich manche Lücken gegenseitig bedingen und Lernende, welche die ersten Lücken nicht vervollständigen konnten, aufgrund eines noch eingeschränkteren Textverständnisses die folgenden Lücken nicht mit den vorgesehenen Wörtern aus dem Basiswortschatz ergänzen konnten.

Beispiele für annehmbare Rückstände in der grammatikalischen Kompetenz zeigen sich einmal mehr im Fall der Präposition $d$ - $i$, welche nicht artikuliert wird, da sie für zwei durch die Konjunktion ,und“" miteinander verbundene Eigennamen gilt. Stattdessen bildeten elf Lernende eine artikulierte Präposition feminin Singular, die sich also nicht auf beide Eigennamen beziehen kann. Außerdem vervollständigten acht gleich im ersten Satz des Tests das Prädikat nicht korrekt (6 davon gar nicht), wobei zwei es zu einer nicht konjugierten Form desselben Verbs ergänzten. Aber schon bei diesem Beispiel zeigt sich, dass die verschiedenen Ursachen für die fehlerhafte Bearbeitung wahrscheinlich in einer wechselseitigen Beziehung zueinander stehen. Bei dem Satz handelt es sich weder morphosyntaktisch noch lexikalisch um eine einfache Konstruktion; die beiden Lücken vor und nach dem Prädikat füllten ebenfalls sieben bzw. zehn Lernende nicht korrekt. Übersetzt lautet der Satz ,fest mit Europa verbunden, formt es [Asien] mit ihm den eurasiatischen Kontinent" (vgl. Dinucci \& Dinucci 2008, S. 238). Er beginnt also mit einem satzwertigen Partizip, das im Test zu ergänzen ist. Hier besteht im Italienischen die zusätzliche Schwierigkeit, dass die Endung dem Genus des Bezugswortes angepasst werden muss. Im Folgenden greift ein CO-markiertes Pronomen (essa) Europa auf; außerdem ist das Subjekt des Satzes nicht expliziert, sondern nur im Prädikat enthalten, das ergänzt werden musste. Unter Verwendung derselben lexikalischen Mittel könnte die Komplexität des Satzes verringert werden, indem man ihn mit einem expliziten Subjekt beginnen lässt und einen Relativsatz nutzt: Es/Asien formt mit Europa, mit dem es fest verbunden ist, den eurasiatischen Kontinent. So wäre den Jugendlichen das Lückenfüllen vermutlich leichter gefallen.

Auffällig ist, dass keiner der ersten zu ergänzenden Sätze mit dem Subjekt beginnt; nach dem soeben besprochenen Satz starten sie mit einer adverbialen Bestimmung. Diese Form der Satzkonstruktion erschwerte mit großer Wahrscheinlichkeit das Füllen der Lücken, auch wenn es sich dabei um ein stilistisches Mittel handelt, dessen Bedeutung sich die Lernenden bewusst waren, wie an ihren eigenen Texten zu erkennen ist (obgleich sie es nicht oft verwendeten; s. Kapitel 10). Nur kann es problematisch für das Textverständnis werden, wenn sich die komplizierten morphosyntaktischen Strukturen und lexikalischen Mittel in einem Satz verdichten.

Ein weiteres Beispiel für ein derartiges sprachliches Umfeld und die Schwierigkeiten beim Ergänzen von hochfrequent gebrauchten Wörtern ist der 
letzte Satz des ersten Textes mit der schon erwähnten nordasiatischen Küste. Mit einer Passivkonstruktion informiert er über ihre Umspülung vom (fast immer vereisten) Wasser des Nordpolarmeeres und ihre allgemeine Flachheit mit seltenen Auffächerungen, die attributiv formuliert ist. Wie schon erwähnt, kannten zwölf Lernende die Wortbedeutung Auffächerung von artico-lazioni nicht oder produzierten das Wort zumindest nicht und lieferten neun keine Ergänzung für die einfache Konjunktion c-on (mit). Es ist anzunehmen, dass auch dieser Satz weniger Schwierigkeiten bereitet hätte, wenn zur Reduzierung seiner Komplexität der Inhalt des Attributs als Relativsatz mit einem weiteren Prädikat formuliert wäre. Hier zeigt sich ein wesentliches Merkmal der Bildungssprache: Enorm viele Informationen werden nicht prädikativ zum Ausdruck gebracht, sondern konzentrieren sich in Form von Nominalisierungen, Attributen, adverbialen Bestimmungen um ein Minimum an Prädikaten - und wenn dem verbale Formen hinzugefügt werden, sind es oft nicht konjugierte, also Infinitive, Partizipien und Gerundien.

Ein Wort wurde von allen Testteilnehmern (14) falsch ergänzt, und zwar sep-ara (trennen). 13-mal wurde es in der dritten Person Plural vervollständigt, weil das vorausgehende Subjekt meno di $100 \mathrm{~km}$ dello Stretto di Bering (weniger als $100 \mathrm{~km}$ der Meeresenge von Bering) dazu verleitet haben wird. Im Italienischen ist es möglich, das Verb nicht nach dem grammatikalischen, sondern nach dem logischen Subjekt zu konjugieren, beispielsweise bei la metà degli alunni non hanno fatto i compiti (die Hälfte der Schüler haben die Hausaufgaben nicht gemacht), wobei das logische Subjekt „die Schüler" sind und das grammatikalische „die Hälfte“. Beim Testbeispiel ist es allerdings nicht gestattet, das Verb nur nach „100 km“ zu konjugieren, da meno kein Substantiv ist, sondern unweigerlich zu der Kilometerangabe gehört (s. Serianni 2006, S. 154).

Neben den schon besprochenen Wörtern artico-lazioni und $d$-i (je 12 Falschlösungen) gibt es ein 13-mal und vier zehnmal falsch vervollständigte Wörter. Da ist zum einen das Relativpronomen cui (FO; denen), das als indirektes Objekt fungiert und das vorausgehende province costiere (Küstenprovinzen) wiederaufnimmt. Zehn Lernende füllten die Lücke gar nicht. Drei weitere Vorschläge sind weder logisch akzeptabel noch grammatikalisch korrekt im Fall der Präposition come (wie) und des Substantivs coste und orthographisch sowie syntaktisch falsch im Fall von cina (china). Bei dieser Lösung wäre abgesehen vom Rechtschreibfehler anstelle des Kommas ein anderes Satzzeichen (zumindest ein Semikolon) erforderlich. Derartige Relativsätze sind aber nicht selten und werden von den Lernenden wahrscheinlich auch ohne Schwierigkeiten richtig gebildet, weshalb die Vermutung naheliegt, dass ihr Textverständnis zu eingeschränkt war, um die Lücke füllen zu können. Eventuell liegt es an den lexikalischen Gestaltungsmitteln des Relativsatzes mit einem reflexiven Prädikat aus dem allgemeinen Wortschatz, einem weite- 
ren Co-gekennzeichneten Wort und einem Substantiv aus dem Wortschatzbereich des hohen Gebrauchs (AU). Allerdings wurden sowohl die im Relativsatz folgenden Lücken als auch die vorausgehenden von den meisten Schülern richtig vervollständigt.

Zehnmal falsch gelöst wurden das bereits angesprochene Pronomen es-sa (sie) gleich zu Beginn des C-Tests und das Possessivpronomen s-ua (ihre) direkt hinter dem 14-mal falsch ergänzten separano. Die Ursache kann bei diesem Wort aus dem fundamentalen Wortschatz nicht in mangelnder lexikalischer Kompetenz liegen. Vielleicht ist sie in Verständnisschwierigkeiten mit dem Bezugswort estremità orientale (Au; östliches Ende) ${ }^{158}$ in einem kurzen, sonst größtenteils mit Wörtern aus dem fundamentalen Wortschatz gebildeten Satz zu suchen. Es erstaunt, dass die Hälfte der Testeilnehmer die Lücke gar nicht und zwei weitere mit dem grammatikalisch zwar korrekten, aber logisch nicht akzeptablen seconda (zweite) ergänzten.

Ebenfalls zehnmal nicht richtig vervollständigt wurden zwei Wörter im dritten Text, wobei es sich beim ersten sowohl um ein lexikalisches Problem, als auch um mangelnde morphosyntaktische Kompetenz bzw. Konzentrationsdefizite zu handeln scheint, da nur ein Lernender das Wort impe-tuoso (heftig, vehement) aus dem allgemeinen Wortschatz (CO) als ein anderes, logisch nicht akzeptables Adjektiv schrieb, drei es gar nicht, zwei es zu nicht existierenden und nicht klar einer bestimmten Wortart zuzuordnenden Wörtern und vier es zum morphosyntaktisch falschen Substantiv impero (Reich) ergänzten.

Das weitere sehr oft nicht richtig vervollständigte Wort ist das Partizip Perfekt avv-iato (u.a. in die Wege geleitet, begonnen), ein Bestandteil des Prädikats im Perfekt im vorletzten Satz. Acht Schülerinnen und Schüler ergänzten das Wort nicht, weitere zwei zu einem fiktiven, wohl mit avverato (verwirklicht) verwechselten Wort und zum logisch nicht annehmbaren avvertito (gespürt). Es handelt sich bei avviato um ein Verb aus dem fundamentalen Wortschatz, was aber nicht bedeuten muss, dass es alle aus dem mentalen Lexikon abrufen und aktiv gebrauchen konnten. Auf der Satzkomplexität können die vielen Falschlösungen eigentlich nicht basieren, da es ein sehr überschaubarer, einfach strukturierter Satz ist. Allerdings besteht das vom Verb geforderte Akkusativobjekt (wer oder was in die Wege geleitet worden ist; ins Deutsche übersetzt ändert es seine Funktion aufgrund des Passivgebrauchs) aus drei Wörtern (prog-etti di svil-uppo, Entwicklungsprojekte), von denen die beiden Lexeme ebenfalls je zur Hälfte getilgt sind (das erste davon achtmal falsch

158 Darauf deutet auch hin, dass fünf Lernende die Lücke von orientale (AU) nicht füllten und ein weiterer falsch. Darüber hinaus existiert anders als im Deutschen für „östlich“ kein vom Substantiv (est) abgeleitetes Adjektiv, sondern nur die zu orientale mögliche Alternative, das Nomen als Lokaladverbiale a est (im Osten) zu verwenden. Das gilt für alle vier Himmelsrichtungen, so dass die SchülerInnen hier ein größeres Wortfeld zu erlernen haben als etwa ihre deutschsprachigen Altersgenossen. 
gelöst). Die Bearbeitung der beiden Lücken beim Partizip und beim ersten Lexem des direkten Objekts bedingt sich also gegenseitig.

Ohne alle 27 vermehrt fehlenden oder falschen Vervollständigungen analysieren zu wollen, soll noch auf einige weitere hingewiesen werden, die aus verschiedenen Gründen auffällig sind. Es geht um mehrere Lücken im zweiten Text, der sich mit dem Primärsektor Asiens beschäftigt. Über die Hälfte der Testteilnehmer erbrachte für produ-zione (Produktion) den akzeptablen Vorschlag produttore (Hersteller), und dies nicht etwa, weil das zweite Wort häufiger benutzt oder den Lernenden besser bekannt wäre (beide tragen die Gebrauchsmarke AU), sondern wahrscheinlich, weil es weniger abstrakt als das im Original stehende ist.

Zwei Lücken weiter befindet sich im selben Satz erneut eine von vielen gewählte Ergänzung, die sich vom Wort des Originaltextes unterscheidet, aber annehmbar ist. Hier werden die Produkte genannt, für deren Anbau China weltweit das Primat besitzt, wobei neun Lernende eines nicht zu Tee, sondern zu Tabak ergänzten. Das ist unter sprachlichen Gesichtspunkten absolut akzeptabel, unter inhaltlichen jedoch nur eingeschränkt, da China nicht gerade für seinen Tabakanbau berühmt ist, der andere klimatische Bedingungen erfordert als der von Tee, aber der Lösungsvorschlag wurde akzeptiert. Auch hier sind sicherlich beide Begriffe lexikalisch bestens geläufig, so dass die vorschriftsmäBige Bearbeitung dieser Lücke nicht so sehr von sprachlichen Fähigkeiten abhing wie von fachlichem Wissen. Eigentlich sollten sie bereits gelernt haben, welche Produkte in China vorwiegend angebaut werden.

Drei weitere Lücken in diesem Satz sind von Interesse: Bei der ersten handelt es sich um den Ausdruck col-ture (Pflanzung, Anbau) aus dem Wortschatzbereich des hohen Gebrauchs (AU), welcher der Hälfte der Klasse vielleicht nicht unbekannt war, aber von ihr nicht produziert wurde. Neben fünf unterlassenen Lückenfüllungen findet sich viermal derselbe Ergänzungsvorschlag. Er belegt, dass die Lernenden den sprachlichen Kontext verstanden, und lässt vermuten, dass ihnen das gewählte Synonym coltivazioni aus dem AD-Bereich vertrauter war als colture, sie jedoch noch keine gefestigte lexikalische Kompetenz hinsichtlich der kollokationalen Verwendung der Wörter besaßen.

Beachtenswert ist außerdem, dass acht Testteilnehmer das Adjektiv diverse (verschieden) überhaupt nicht und zwei weitere zu divano (Sofa) ergänzten. Liegt es daran, dass es das erste Wort nach einer Konjunktion ist, welches vor einem anderen Adjektiv steht, so dass die Lernenden ein Verb einsetzen wollten, wobei ihnen mit diesen Anfangsbuchstaben kein passendes einfiel?

Definitiv lexikalischer Art erscheint das Problem mit der Worttilgung bei suini (Co; Schweine), dem letzten Glied der schon erwähnten Aufzählung zur Viehzucht. Wie bei Rindern, Schafen und ähnlichen Zuchttieren gibt es, anders als im Deutschen, im Italienischen relative Synonyme für diese Tiere, die im 
gastronomischen und zoologischen Bereich mit den im Text verwendeten Ausdrücken bezeichnet sind. Es handelt sich hierbei um textsortenspezifische und registerbedingte Differenzen im Gebrauch. Im Alltag bezeichnet das sprachliche Umfeld der Lernenden, die das Wort nicht ergänzen können, Schweinefleisch wahrscheinlich nicht als carne suina, sondern als carne di maiale, da maiale (AU) die häufiger gebrauchte Bezeichnung für "Schwein“ ist.

\subsubsection{Texte 4 und 5 des C-Tests der Klasse III B}

Die den Lehrbuchseiten 242 und 38 (Dinucci \& Dinucci 2008) entnommenen Texte 4 und 5 des C-Tests bestehen aus insgesamt 47 Lücken, von denen lediglich drei zu Beginn des fünften von allen richtig ergänzt wurden. In Tabelle 29 sind zusammen mit den Angaben zur Art der Wörter und ihrer Häufigkeit sowie den Lösungsvorschlägen der Testpersonen die Teile der beiden Texte aufgelistet, in denen sich die getilgten Wörter befinden.

\begin{tabular}{|c|c|c|c|c|c|c|}
\hline Text & Fehler & Wort & Wortart & Marke & Kommentar & Lösungsvorschläge \\
\hline $\begin{array}{l}4 \\
\text { (S. 242) }\end{array}$ & & $\dot{E}$ & v.intr. & $\mathrm{FO}$ & & \\
\hline \multirow[t]{27}{*}{$\begin{array}{l}22 \text { Lü- } \\
\text { cken }\end{array}$} & 4 & $\begin{array}{l}\text { complessiva- } \\
\text { mente }\end{array}$ & avv. & $\mathrm{CO}$ & & $\begin{array}{l}\text { complessi, } 0 \text {, com- } \\
\text { plesso (2) }\end{array}$ \\
\hline & & arido & agg. & $\mathrm{AU}$ & & \\
\hline & 3 & negli & prep.art. & $\mathrm{FO}$ & & $\begin{array}{l}\text { nemmeno, nei, } \\
\text { neve }\end{array}$ \\
\hline & & altopiani & s.m. & $A D$ & & \\
\hline & 3 & interni & agg. & $\mathrm{FO}$ & & inti, 0 (2) \\
\hline & & dove & cong. & $\mathrm{FO}$ & & \\
\hline & 2 & la & art.det. & $\mathrm{FO}$ & & $0(2)$ \\
\hline & & vegetazione & s.f. & $\mathrm{AU}$ & & \\
\hline & 3 & principale & agg. & $\mathrm{FO}$ & & $\begin{array}{l}\text { principalmente (2), } \\
0\end{array}$ \\
\hline & & è & v.intr. & FO & & \\
\hline & 3 & quella & agg.dimostr. & $\mathrm{FO}$ & & questa, quello, 0 \\
\hline & & della & prep.art. & $\mathrm{FO}$ & & \\
\hline & 5 & steppa & s.f. & $\mathrm{CO}$ & & $\begin{array}{l}\text { sterpe, steppe, } \\
\text { stessa, } 0 \text { (2) }\end{array}$ \\
\hline & & con & prep. & FO & & \\
\hline & 4 & erbe & s.f. & $\mathrm{FO}$ & & 0 (3), erbacei \\
\hline & & basse & agg. & $\mathrm{FO}$ & & \\
\hline & & e & cong. & $\mathrm{FO}$ & & \\
\hline & 1 & cespugli & s.m. & $A U$ & & cespugliose \\
\hline & & spinosi & agg. & $\mathrm{CO}$ & & \\
\hline & & $e$ & cong. & $\mathrm{FO}$ & & \\
\hline & 6 & nei & prep.art. & $\mathrm{FO}$ & & 0 (5), non \\
\hline & & deserti & s.m. & $\mathrm{AU}$ & & \\
\hline & & $A$ & loc.prep. & $\mathrm{CO}$ & & \\
\hline & 6 & causa & el.loc.prep. & el.CO & & $0(6)$ \\
\hline & & dell'aridità & s.f. & $\mathrm{CO}$ & & \\
\hline & 3 & grandi & agg. & FO & & grande (2), grani \\
\hline & & distese & s.f. & $\mathrm{AU}$ & & \\
\hline
\end{tabular}




\begin{tabular}{|c|c|c|c|c|c|c|}
\hline Text & Fehler & Wort & Wortart & Marke & Kommentar & Lösungsvorschläge \\
\hline & 7 & con & prep.art. & FO & & $0(6)$, carenti \\
\hline & & scarsissima & agg. & FO & & \\
\hline & 3,5 & densità & s.f. & TS stat. & & $\begin{array}{l}0 \text { (2), densita, } \\
\text { densificate }\end{array}$ \\
\hline & & abitativa & agg. & $\mathrm{CO}$ & & \\
\hline & 8 & vengono & v.aus. & $\mathrm{CO}$ & $\begin{array}{l}\text { ersetzt es- } \\
\text { sere }\end{array}$ & $\begin{array}{l}0(4), \text { veniva, } \\
\text { venne, vennero, } \\
\text { venivano }\end{array}$ \\
\hline & & lasciate & v.tr. & FO & & \\
\hline & 3 & al & prep.art. & FO & & $0(3)$ \\
\hline & & pascolo & s.m. & $A U$ & & \\
\hline & 9 & nomade & agg. & $\mathrm{CO}$ & & $\begin{array}{l}0 \text { (6), nomi, nomi- } \\
\text { nando, nome }\end{array}$ \\
\hline & & specialmente & avv. & $\mathrm{CO}$ & & \\
\hline & 3 & degli & prep.art. & FO & & dei, degl', 0 \\
\hline & & ovini & s.m. & $A D$ & & \\
\hline & 7 & Lungo & prep. & FO & & 0 (5), Lulu, Lula \\
\hline & & le & art.det. & FO & & \\
\hline & 6 & coste & s.f. & FO & & $0(3)$, coi, cose (2) \\
\hline & & del & prep.art. & FO & & \\
\hline & 3 & Mediterraneo & n.p.m. & & & $\begin{array}{l}\text { Mediteranneo (2), } \\
0\end{array}$ \\
\hline & & si & pron.pers. & $\mathrm{CO}$ & & \\
\hline & 2 & ha & v.tr. & FO & & $0(2)$ \\
\hline \multirow{22}{*}{$\begin{array}{l}5 \\
\text { (S. 53) } \\
25 \text { Lü- } \\
\text { cken }\end{array}$} & & Circa & avv. & FO & & \\
\hline & 1 & la & art.det. & FO & & 0 \\
\hline & & metà & s.f. & FO & & \\
\hline & 0 & delle & prep.art. & FO & & \\
\hline & & lingue & s.f. & FO & & \\
\hline & 0 & del & prep.art. & FO & & \\
\hline & & mondo & s.m. & FO & & \\
\hline & $\begin{array}{r}4+5 \\
\text { acc. }\end{array}$ & rischia & v.tr. & FO & & $\begin{array}{l}\text { risultano, rischiano } \\
\text { (acc.) (5), rischio, } \\
0, \text { rispetto }\end{array}$ \\
\hline & & inoltre & avv. & FO & & \\
\hline & 2 & $d i$ & prep. & FO & & devono, 0 \\
\hline & & scomparire & inf.v.intr. & FO & & \\
\hline & 2 & si & pron.pers. & $\mathrm{CO}$ & & soltanto, 0 \\
\hline & & tratta & $\begin{array}{l}\text { v.pro- } \\
\text { nom.intr. }\end{array}$ & $\mathrm{CO}$ & & \\
\hline & $\begin{array}{r}2+2 \\
\text { acc. }\end{array}$ & soprattutto & avv. & $\mathrm{CO}$ & & $\begin{array}{l}\text { sopratutto (acc.) } \\
(2), 0(2)\end{array}$ \\
\hline & & delle & prep.art. & FO & & \\
\hline & 0 & lingue & s.f. & FO & & \\
\hline & & usate & agg. & FO & & \\
\hline & 3 & per & prep. & FO & & $\begin{array}{l}\text { prima, pakistano, } \\
0\end{array}$ \\
\hline & & parlare & inf.v.tr. & FO & & \\
\hline & & $e$ & cong. & FO & & \\
\hline & 5 & non & avv. & FO & & 0 (4), nel \\
\hline & & per & prep. & FO & & \\
\hline
\end{tabular}




\begin{tabular}{|c|c|c|c|c|c|c|}
\hline Text & Fehler & Wort & Wortart & Marke & Kommentar & Lösungsvorschläge \\
\hline & \multirow[t]{2}{*}{1} & scrivere & inf.v.tr. & FO & & 0 \\
\hline & & Le & art.det. & FO & & \\
\hline & \multirow[t]{2}{*}{8} & cause & s.f. & FO & & $0(7)$, catene \\
\hline & & sono & v.intr. & FO & & \\
\hline & \multirow[t]{2}{*}{6} & varie & agg. & FO & & $\begin{array}{l}\text { vaste, } 0(3) \text {, vaie, } \\
\text { vaarie }\end{array}$ \\
\hline & & in & prep. & FO & & \\
\hline & \multirow[t]{2}{*}{2} & alcuni & agg. & FO & & alcune, 0 \\
\hline & & casi & s.m. & FO & & \\
\hline & \multirow[t]{2}{*}{3} & la & art.det. & FO & & II, 0 (2) \\
\hline & & lingua & s.f. & FO & & \\
\hline & \multirow[t]{2}{*}{4} & non & avv. & FO & & $0(3)$, neolatina \\
\hline & & viene & v.aus. & $\mathrm{CO}$ & & \\
\hline & \multirow[t]{2}{*}{$\begin{array}{r}7+5 \\
\text { acc. }\end{array}$} & più & avv. & FO & & $\begin{array}{l}0 \text { (4), proprio } \\
\text { (acc.) (4), parlata } \\
\text { (2), praticamente, } \\
\text { particolarmente } \\
\text { (acc.) }\end{array}$ \\
\hline & & usata & v.tr. & FO & & \\
\hline & \multirow[t]{2}{*}{$\begin{array}{r}6+3 \\
\text { acc. }\end{array}$} & dalle & prep.art. & FO & $\begin{array}{l}\text { dai (4), da } \\
\text { molti (3) }\end{array}$ & $\begin{array}{l}0 \text { (3), dai (3), da } \\
\text { molti (3) }\end{array}$ \\
\hline & & giovani & agg. & FO & & \\
\hline & \multirow[t]{2}{*}{$\begin{array}{r}4+6 \\
\text { acc. }\end{array}$} & generazioni & s.f. & FO & $\begin{array}{l}\text { falscher } \\
\text { Doppel- } \\
\text { punkt, des- } \\
\text { halb alcuni } \\
\text { OK }\end{array}$ & $\begin{array}{l}\text { generi, general- } \\
\text { mente (acc.) (6), } \\
\text { genere (2), } 0\end{array}$ \\
\hline & & in & prep. & FO & & \\
\hline & \multirow[t]{2}{*}{$\begin{array}{r}3+4 \\
\text { acc. }\end{array}$} & altri & s.m. & FO & & $\begin{array}{l}\text { alcuni (4) (acc.), } \\
\text { allevamento, alla, } \\
0\end{array}$ \\
\hline & & il & art.det. & FO & & \\
\hline & \multirow[t]{2}{*}{10} & suo & agg.poss. & FO & & $\begin{array}{l}\text { solo (4), } 0(4) \text {, sin- } \\
\text { goli, secondo }\end{array}$ \\
\hline & & uso & s.m. & FO & & \\
\hline & 5 & viene & v.aus. & $\mathrm{CO}$ & & $\begin{array}{l}0 \text { (3), vivile, visino } \\
\text { (? unleserlich) }\end{array}$ \\
\hline & \multirow[t]{2}{*}{8} & proibito & v.tr. & FO & Tilgungsfehler & $0(7)$, proibiti \\
\hline & & od & $\begin{array}{l}\text { cong.var. } \\
\text { posiz. }\end{array}$ & FO & & \\
\hline & \multirow[t]{2}{*}{10} & ostacolato & v.tr. & $\mathrm{CO}$ & & $\begin{array}{l}\text { ostacolare (3), } \\
\text { ostacoli (6), } 0\end{array}$ \\
\hline & & perché & cong. & FO & & \\
\hline & \multirow[t]{3}{*}{1} & non & avv. & FO & & 0 \\
\hline & & sono & v.intr. & FO & & \\
\hline & & riconosciuti & agg. & $\mathrm{CO}$ & & \\
\hline
\end{tabular}

Tabelle 29: Texte 4 und 5 des C-Tests der Klasse III B an der Schule Pestalozzi

Von den 47 Lücken des zweiten Testteils wurden nur vier von allen fehlerfrei vervollständigt. Mit 27 wurde etwas mehr als die Hälfte der getilgten Wörter von bis zu vier Testpersonen nicht oder falsch ergänzt, wobei der Anteil mit 15 von 22 im vierten Text höher liegt als im fünften (12 von 25). Von den 
ersten 15 zählen fünf nicht zum fundamentalen Wortschatz und wurden von mindestens drei Lernenden falsch vervollständigt. Doch auch unter den Wörtern aus dem fundamentalen Wortschatz gibt es viele mit drei Falschlösungen und eines mit vier. Von den Wörtern, die nicht zu den häufigsten 2.000 des Italienischen zählen und die von mindestens drei Falschlösungen betroffen sind, stammen zwei aus dem Bereich des allgemeinen Wortschatzes, nämlich das Adverb compless-ivamente (insgesamt) und das Substantiv ste-ppa (Steppe). Ersteres wurde einmal nicht und dreimal zum logisch und morphosyntaktisch falschen Nomen complesso (Komplex) und seiner Pluralform ergänzt. Das zweite wurde zweimal gar nicht, einmal zum Adjektiv/Pronomen stessa (dieselbe), einmal in der Pluralform steppe und einmal zu dem mit der Marke LE (literarischer Gebrauch) versehenen Nomen sterpe (gleichbedeutend mit sterpo, Co; Reisig) vervollständigt. Wahrscheinlich lag also für einige das Lösungsproblem im Lexikalischen, zumal vorher im Satz das Subjekt vegetazione und das Prädikat è (die Vegetation ist) ungetilgt auftreten, so dass eigentlich klar ist, dass in der Satzaussage etwas zur Flora folgen wird.

Die anderen beiden Ausdrücke sind den-sità (Bevölkerungsdichte) aus dem statistischen Fachwortschatz und der geographische Eigenname Medite-rraneo (Mittelmeer). Während Letzterer nur von drei Lernenden falsch vervollständigt wurde, aber zwei einen bei diesem Wort verbreiteten Fehler der Verdoppelung des falschen Konsonanten begingen, wurde das statistische Fachwort (das im Erdkundeunterricht bei der Behandlung eines jeden Landes benutzt wird) von zweien gar nicht ergänzt und von einem nur zum logisch und grammatikalisch falschen Adjektiv/Partizip densificato (verdichtet). Desweiteren ist noch ein Rechtschreibfehler, ein fehlender Akzent, zu verzeichnen.

Auch in diesem Text wurde eine artikulierte Präposition nicht richtig gebildet. Anstelle des erforderten de-gli (da das maskuline Bezugsnomen im Plural steht und beginnt mit einem Vokal) kam eine Testperson auf die originelle, aber nicht korrekte Idee, die zusammengesetzte Präposition zu apostrophieren. Eine weitere vervollständigte sie gar nicht und eine dritte falsch zu dei. Es befanden sich in der Klasse III B keine Schülerinnen und Schüler, die nicht ihre gesamte Schulzeit in Italien verbracht hatten, so dass es zu derartig elementaren Fehlern eigentlich nicht hätte kommen sollen.

Im fünften Text wurden sechs Wörter von drei oder vier Lernenden falsch bzw. nicht vervollständigt, die alle aus dem fundamentalen Wortschatz stammen. Generell beschränkt sich das Wortvorkommen in diesem Text auf die Gebrauchsmarken FO und CO, wovon die zweite nur fünf getilgte Wörter und zwei ausgeschriebene kennzeichnet. Unter den selteneren Falschlösungen (2) sind zwei Ausdrücke des allgemeinen Wortschatzes betroffen, wohingegen sich unter den frequenteren Falschlösungen ( 3 und mehr) viele fundamentale Wörter befinden.

Bei der Testkonstruktion dieses Textes unterliefen drei Fehler, von denen zwei die Tilgung zweier aufeinanderfolgender Wörter betreffen und das dritte 
ein Semikolon, das unglücklicherweise, für einen Doppelpunkt gehalten, als solcher im Test erscheint. Dieser Doppelpunkt hat zur Folge, dass die Ergänzung von al-tri (anderen) zu alcuni (einigen) akzeptiert wurde, so dass nur wenige Lernende mit der Worttilgung nach dem falschen Satzzeichen Schwierigkeiten zeigten. Anders sieht es mit einer der beiden aufeinanderfolgenden Worttilgungen aus, die von fünf und acht Lernenden nicht korrekt bearbeitet wurden. Da sie sich in einem Satz befinden, in dem acht von insgesamt 14 Lücken vermehrt falsch gelöst wurden, wird er bei der Besprechung der häufigeren Fehlerquellen vollständig und übersetzt wiedergegeben.

\subsubsection{Vermehrte Fehlerquellen im zweiten Teil des C-Tests der Klasse III B}

In den Texten 4 und 5 wurden von mindestens fünf Testpersonen insgesamt 17 Wörter nicht richtig ergänzt, die sich zu etwa gleichen Teilen (9 im 2. Text) auf die beiden Texte verteilen. Sie sind alle der Tabelle $30 \mathrm{zu}$ entnehmen.

\begin{tabular}{|c|c|c|c|c|c|c|}
\hline Text & Fehler & Wort & Wortart & Marke & $\mathrm{K}$ & Lösungsvorschläge \\
\hline 4 & 5 & steppa & s.f. & $\mathrm{CO}$ & & $\begin{array}{l}\text { sterpe, steppe, stessa, } 0 \\
\text { (2) }\end{array}$ \\
\hline \multirow[t]{7}{*}{ (S. 242) } & 6 & nei & prep.art. & FO & & $0(5)$, non \\
\hline & 6 & causa & el.loc.prep. & el.CO & & $0(6)$ \\
\hline & 7 & con & prep.art. & $\mathrm{FO}$ & & $0(6)$, carenti \\
\hline & 8 & vengono & v.aus. & $\mathrm{CO}$ & & $\begin{array}{l}0(4) \text {, veniva, venne, ven- } \\
\text { nero, venivano }\end{array}$ \\
\hline & 9 & nomade & agg. & $\mathrm{CO}$ & & $\begin{array}{l}0(6), \text { nomi, nominando, } \\
\text { nome }\end{array}$ \\
\hline & 7 & Lungo & prep. & FO & & 0 (5), Lulu, Lula \\
\hline & 6 & coste & s.f. & FO & & $0(3)$, coi, cose (2) \\
\hline 5 & 5 & non & avv. & FO & & 0 (4), nel \\
\hline \multirow[t]{8}{*}{ (S. 53) } & 8 & cause & s.f. & FO & & $0(7)$, catene \\
\hline & 6 & varie & agg. & FO & & vaste, 0 (3), vaie, vaarie \\
\hline & $\begin{array}{r}7+5 \\
\text { acc. }\end{array}$ & più & avv. & FO & & $\begin{array}{l}0(4) \text {, proprio (acc.) }(4), \\
\text { parlata }(2), \text { praticamente, } \\
\text { particolarmente (acc.) }\end{array}$ \\
\hline & $\begin{array}{r}6+3 \\
\text { acc. }\end{array}$ & dalle & prep.art. & $\mathrm{FO}$ & & 0 (3), dai (3), da molti (3) \\
\hline & 10 & suo & agg.poss. & $\mathrm{FO}$ & & $\begin{array}{l}\text { solo (4), } 0 \text { (4), singoli, se- } \\
\text { condo }\end{array}$ \\
\hline & 5 & viene & v.aus. & $\mathrm{CO}$ & \multirow{3}{*}{$\begin{array}{l}\text { Tilgungs- } \\
\text { fehler }\end{array}$} & $\begin{array}{l}0 \text { (3), vivile, visino (? unle- } \\
\text { serlich) }\end{array}$ \\
\hline & 8 & proibito & v.tr. & $\mathrm{FO}$ & & $0(7)$, proibiti \\
\hline & 10 & ostacolato & v.tr. & $\mathrm{CO}$ & & $\begin{array}{l}\text { ostacolare (3), ostacoli } \\
(6), 0\end{array}$ \\
\hline
\end{tabular}

Tabelle 30: Häufige Falschlösungen in den C-Testtexten 4 und 5 der Klasse III B, Pestalozzi

In den meisten Fällen ließen viele Testteilnehmer die Lücke offen. Anhand der Tabelle ist schnell zu erkennen, dass ein großer Teil der Wörter (10) aus dem 
fundamentalen Wortschatz stammt; außerdem handelt es sich um je acht grammatikalische Morpheme und Lexeme. Beides deutet darauf hin, dass die Lernenden aufgrund der grammatikalisch-syntaktischen Gestaltung der Sätze und einer hohen lexikalischen Verdichtung anscheinend nur ein eingeschränktes Textverständnis entwickelten und deshalb oft nicht imstande waren, einfache grammatikalische Morpheme oder häufig gebrauchte Lexeme richtig zu ergänzen. Abgesehen von zwei Ausdrücken konzentrieren sich die vermehrt nicht korrekten Wortergänzungen im vierten Text auf zwei Satze, welche deshalb in Abbildung 9 wiedergegeben und übersetzt sind. Dort sind die zu vervollständigenden Wörter fettgedruckt und mit einer Angabe zur Anzahl der Falschlösungen versehen. Die häufigen Falschlösungen sind markiert.

1. A causa [6] dell'aridità, grandi [3] distese con [7] scarsissima densità [3,5] abitativa vengono [8] lasciate al [3] pascolo nomade [9] specialmente degli [3] ovini.

M.Ü.: Aufgrund der Dürre werden große Weiten mit einer äußerst geringen Wohndichte als nomadisches Weideland vor allem für (die) Schafe belassen.

2. Lungo [7] le coste [6] del Mediterraneo [3] si ha [2] un clima temperato, con sufficiente umidità che ben si presta alle coltivazioni ortofrutticole e della vite e dell'olio.

M.Ü.: Entlang der Küsten des Mittelmeers herrscht [wörtlich: hat man] ein gemäßigtes Klima mit ausreichend Feuchtigkeit, das sich gut für den Anbau von Obst und Gemüse, Wein und Öl eignet.

Abbildung 9: Häufige Falschlösung im vierten C-Testtext der Klasse III B, der Lehrbuchseite 242 (Dinucci \& Dinucci 2008) entnommene Sätze

Der erste Satz beginnt mit einem präpositionalen Phrasem aus dem Bereich des allgemeinen Wortschatzes, dessen getilgten, lexikalischeren Teil sechs Testteilnehmer nicht ergänzten. Natürlich kann es daran liegen, dass sie das Phrasem nicht kannten, jedoch ist anzunehmen, dass das Satzumfeld den Ausschlag dazu gab. Denn bei allen im Satz folgenden Substantiven handelt es sich um Wörter, die nicht aus dem fundamentalen Wortschatz stammen (einige auch nicht aus dem VDB) und zudem durch Adjektive und Adverbien angereichert sind, die ebenfalls seltenere Gebrauchsmarken tragen. Als zusätzliche relative Schwierigkeit steht das Prädikat im Passiv, wobei als Hilfsverb das im Vergleich zu essere weniger häufige venire (CO; kommen) auftritt, das zu ergänzen war. Im Verhältnis zu den umliegenden häufig falsch gelösten Wörtern wurde diese Prädikatslücke von weniger Testteilnehmern (4) gar nicht ergänzt, doch weitere vier Personen schlossen sie nicht in der für diese Textsorte erforderlichen Zeitform des Präsens, sondern in Vergangenheitstempora und in der falschen Person (dritte Singular; vgl. Tabelle 29).

Dass bei der vorausgehenden Worttilgung sechs Lernende für die einfache Präposition c-on (mit) keinen bzw. einen logisch und morphosyntaktisch falschen Lösungsvorschlag hatten, kann nicht an der Präposition selbst liegen, sondern nur am näheren Textzusammenhang, der offenbar unverständlich war. Anders verhält es sich mit den neun Falschlösungen für nom-ade, da dieses Wort aus dem allgemeinen Wortschatz (CO) stammt und möglicherweise nicht von allen Testeilnehmern produktiv beherrscht wurde. Während sechs Ler- 
nende nichts ergänzten, vervollständigten weitere drei zu logisch und grammatikalisch falschen Nomen sowie einem Gerundium, was erneut auf ein geringes Textverständnis als Folge des sprachlichen Umfelds deutet. Gleichzeitig kann man sich bei derartigen Lösungsvorschlägen fragen, ob die Lernenden den Kontext überhaupt beachteten.

Der zweite abgebildete Satz ist der letzte des vierten Textes und deshalb nur am Anfang mit Lücken versehen. Wie im Fall des vorherigen Satzes beginnt auch er wieder nicht mit der Nennung des Subjekts, sondern mit einer Lokaladverbiale. Sie wird von der Präposition lu-ngo aus dem fundamentalen Wortschatz eingeleitet, die fünf Lernende nicht vervollständigten. Zwei weitere ergänzten zu den Phantasiewörtern oder Eigennamen Lulu und Lala, wobei es sich um einen Scherz handelt, den sie wohl unterdrückt hätten, wäre ihnen ein passendes Wort in den Sinn gekommen. Obwohl abgesehen von einem Testteilnehmer alle erkannten, dass in diesem Satz das Mittelmeer auftritt, ergänzten trotzdem sechs das häufig gebrauchte Wort coste nicht richtig, was womöglich mit der satzeinleitenden Präposition zusammenhängt, welche die Hälfte nicht vervollständigte. Dadurch scheint ihr Textverständnis insgesamt zu brüchig gewesen zu sein.

Im fünften Text über die Verbreitung der Sprachen treten die häufigsten Falschlösungen mit Ausnahme des Adverbs non, das von vier Lernenden gar nicht und von einem falsch ergänzt wurde, im letzten Satz auf, der mit einer Tilgungsunregelmäßigkeit und einem falschen Satzzeichen behaftet ist und hier zur besseren Veranschaulichung ebenfalls abgebildet wird (die halbierten Wörter sind wieder fettgedruckt und die häufig falsch gelösten markiert).

Le cause [8] sono varie [6]: in alcuni [2] casi la [3] lingua non [4] viene più [7] usata dalle [6] giovani generazioni [4]; in altri [3] il suo [10] uso viene [5] proibito [8] od ostacolato [10] perché non [1] sono riconosciuti i diritti della minoranza nazionale che la parla.

M.Ü.: Die Gründe sind verschiedene: In einigen Fällen wird die Sprache von den jungen Generationen nicht mehr benutzt; in anderen wird ihr Gebrauch verhindert oder verboten, weil die Rechte der nationalen Minderheit, die sie spricht, nicht anerkannt werden.

Abbildung 10: Der Lehrbuchseite 242 (Dinucci \& Dinucci 2008) entnommener letzter Satz im fünften C-Testtext der Klasse III B mit Anzahl der Falschlösungen

Obwohl im vorausgehenden Satz nur eine Lücke von fünf und die anderen von weniger Testteilnehmern gar nicht bzw. nicht korrekt vervollständigt wurden, das Textverständnis also auszureichen schien, ergänzten sieben das erste getilgte Wort des abgebildeten Satzes nicht und einer zum logisch falschen Nomen catene (Ketten). Auch das folgende Prädikatsnomen varie (Fo; verschieden) wurde vermehrt gar nicht oder unzureichend vervollständigt, was wahrscheinlich in den meisten Fällen durch die vorausgehende nicht gefüllte Lücke bedingt wurde. Es fällt auf, dass die Hälfte der Klasse mit dem einfachen Adverb $p$-iù (FO; mehr) Probleme zeigte, obwohl Subjekt und Prädikat in diesem recht kurzen Satz nicht von der Worttilgung betroffen sind. Das gilt auch für die anschließende zusammengesetzte Präposition, bei der sechs entweder gar 
nichts oder zwei Wörter ergänzten. Weitere drei bildeten die artikulierte Präposition dai (von den), die akzeptabel ist, wenn in der nächsten Lücke eine Vervollständigung zu einem maskulinen Nomen im Plural oder zum Adverb generalmente (generell) erfolgte. Diese grammatikalisch korrekte Alternativlösung wurde gleich von mehreren Lernenden erbracht. Bei ihnen sind es also keine jungen Generationen, die eine Sprache nicht mehr sprechen, sondern die Jungen generell (vgl. Tabelle 29 und Abb. 10). Dass hierbei das Adverb generalmente an einer Position im Satz steht, wo es eher alltagssprachlich und mündlich gebraucht wird und von den Verfassern des Schulbuchs nicht verwendet würde, führte nicht dazu, den Lösungsvorschlag abzulehnen, da er zwar stilistisch unpassend ist, morphosyntaktisch und logisch aber nicht.

Das Semikolon nach dem Wort generazioni wurde bei der Testentwicklung versehentlich in einen Doppelpunkt verwandelt, der die Satzstruktur mit der ordnenden Reihung ,in einigen Fällen [...]; in anderen [...]“ aufbricht. Deshalb wurde entschieden, anstelle von ,anderen“ als Lösung „einigen“ zuzulassen.

Bemerkenswert ist, dass zehn Lernende das Possessivpronomen $s$-uo nicht richtig vervollständigten. Sein Bezugswort uso (Gebrauch) stammt aus dem fundamentalen Wortschatz, aber neben vier ganz ausbleibenden Ergänzungsvorschlägen erfolgten zwei grammatikalisch falsche und viermal der logisch falsche zum Adverb solo (nur). Auch in diesem Satz liegt wieder eine Passivkonstruktion vor, die mit dem Hilfsverb venire konstruiert ist und mehreren SchülerInnen Schwierigkeiten zu bereiten schien. Diese rührten sicherlich auch von der doppelten Worttilgung her, die gleichermaßen das Partizip der Passivkonstruktion trifft, das von der Hälfte der Testteilnehmer gar nicht und von einem weiteren falsch konjugiert vervollständigt wurde. Da dieses Partizip allerdings öfter falsch gelöst wurde als das dazugehörige Hilfsverb, kann es auch sein, dass es trotz seiner Zugehörigkeit zu den häufigsten Wörtern des Italienischen im Moment der Testbearbeitung produktiv nicht zur Verfügung stand.

Etwas anders sieht es mit dem nächsten Partizip aus, das mit zehn Falschlösungen neben dem Possessivpronomen suo das am häufigsten nicht korrekt bearbeitete Wort der letzten beiden C-Testtexte ist. Sechs Lernende vervollständigten es zum Substantiv ostacoli (AU; Hindernisse) und drei ergänzten es zum Infinitiv des richtigen Verbs (behindern), so dass alle Lösungsvorschläge ein recht hohes Maß an textgrammatikalischer Schwäche aufweisen. Niemandem scheint aufgefallen zu sein, dass dem Satz bei diesen Lösungsvorschlägen das Prädikat fehlt.

Zusammenfassend scheint insbesondere für diese Lerngruppe die Beobachtung angebracht, dass die Bearbeitung der Lücken oft zu sehr mit einer puren Fokussierung auf das getilgte Wort unter Ausblendung des Kontextes erfolgte. Dies zeigen beispielsweise die Lösungsvorschläge beim zuletzt besprochenen 
Wort, aber auch allevamento (Zucht) in einem Text über Sprachen oder divano (Sofa) anstelle von diverse (verschiedene [andere Anbausorten]).

\subsubsection{C der Pestalozzi}

Unter den 19 Schülerinnen und Schülern, die am Test teilnahmen, befinden sich je zwei, die ein Schuljahr der Mittelschule wiederholten und die eine andere Erstsprache als Italienisch besaßen. Dieser C-Test wurde aus Texten der Lehrbuchseiten 105, 106 (Einführung in den Kontinent Asien, morphologische Aspekte) und 121 bis 123 (internationale Beziehungen und Konflikte Westasiens sowie Landeskunde und sozioökonomische Aspekte der Türkei) (Forte \& Ubertazzi 2008) entwickelt. Welchen Bereichen des Gebrauchswortschatzes die zu ergänzenden Ausdrücke zugeteilt sind und wie viele Falschlösungen auf die einzelnen Wortschatzbereiche entfallen, ist Tabelle 31 und Abbildung 11 zu entnehmen.

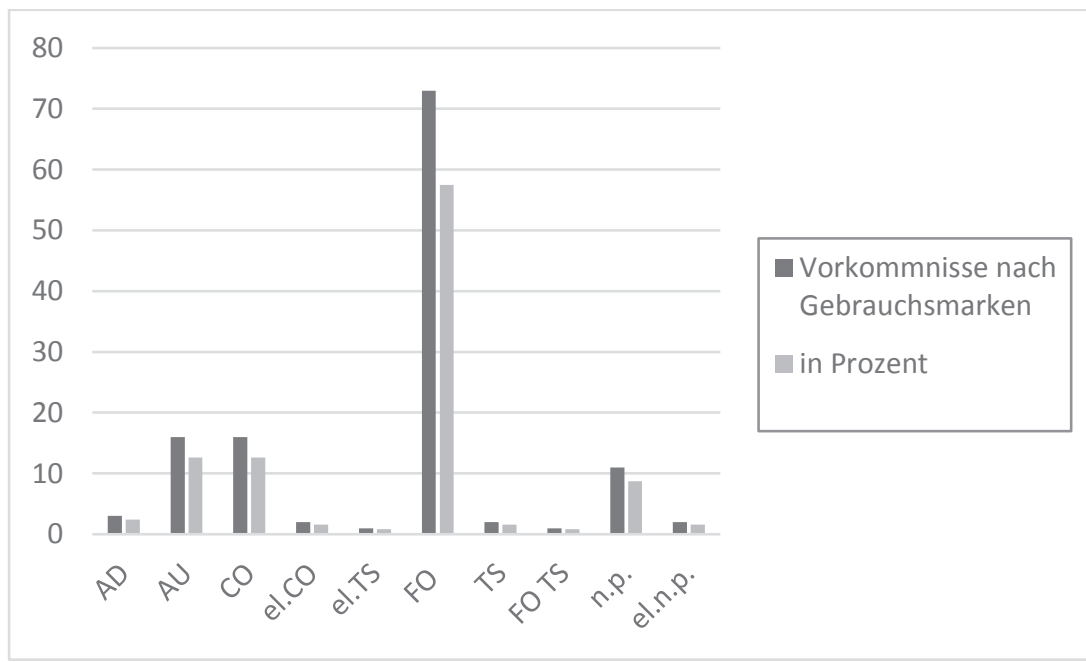

Abbildung 11: Zusammensetzung der getilgten Wörter im C-Test der III C (Pestalozzi)

\begin{tabular}{lrrlrr}
\hline Marke & $\begin{array}{r}\text { Vorkom- } \\
\text { men }\end{array}$ & in \% & Kommentar & n Fehler & $\varnothing$ Fehler \\
\hline AD & 3 & 2,4 & riforme (12), registra (9) & 22 & 7,3 \\
AU & 16 & 12,6 & feroce (10), disoccupati (6), spicca (6) & 38 & 2,4 \\
CO & 16 & 12,6 & $\begin{array}{l}\text { privatizzazioni (18), popolamento (15), uti- } \\
\text { lizzo (14), focolaio (11), apre (13) }\end{array}$ & 114 & 7,1 \\
el.CO & 2 & 1,6 & a causa della (6), da parte dei (6) & 12 & 6 \\
el.TS & 1 & 0,8 & 2 & 2 \\
FO & 73 & 57,5 & contadine (19), su (17), priva (12), subita \\
& & & 202,5 & 2,8 \\
\hline
\end{tabular}




\begin{tabular}{|c|c|c|c|c|c|c|}
\hline TS & 2 & 1,6 & curda (11), dorsale (12) & & 23 & 11,5 \\
\hline FO TS & 1 & 0,8 & & & 0 & 0 \\
\hline n.p. & 11 & 8,7 & & & 31,5 & 2,9 \\
\hline el.n.p. & 2 & 1,6 & Monti del Ponto (17) & & 18 & 9 \\
\hline n Wörter & 127 & & & n Fehler & 463 & 3,6 \\
\hline
\end{tabular}

Tabelle 31: Getilgte Wörter des C-Tests der III C (Pestalozzi) nach Gebrauchsmarken und mit Angaben zu den Falschlösungen

Nach den Wörtern aus dem fundamentalen Wortschatz (FO) stammen in diesem C-Test die meisten zur Hälfte getilgten Wörter mit je 16 aus den Bereichen allgemeiner Wortschatz (C0) und hoher Gebrauch (AU), so dass der Anteil an getilgten Wörtern aus dem Basiswortschatz (VDB) 72,5 Prozent beträgt. AuBerdem stark vertreten sind mit elf die Eigennamen. Auf die mehrteiligen Eigennamen entfallen pro Wort durchschnittlich neun Fehler (eines führte zu 17 Falschlösungen), während im Mittelwert am häufigsten die beiden fachwortschatzsprachlichen Ausdrücke falsch gelöst wurden (11 und 12), von denen einer aus dem geographischen Fachwortschatz stammt.

Die punktuelle Fehleranalyse im nächsten Kapitelabschnitt wird u.a. zeigen, wie die zahlreichen Falschlösungen für das zweite und dritte in der Tabelle angegebene Wort aus dem Bereich des hohen Gebrauchs wahrscheinlich in nur einem Fall (registra) auch auf mangelnder lexikalischer Kompetenz und ansonsten eher auf Nichtbeachtung bzw. geringem Verständnis der mikrokontextuellen Ebene beruht. Dies gilt in ähnlicher Weise für die Falschlösungen bei den Ausdrücken aus dem allgemeinen Wortschatz.

Welchen Wortarten die getilgten Wörter angehören und wie ihre Falschlösungen ausfallen, ist in Tabelle 32 illustriert.

\begin{tabular}{lrlrr}
\hline Wortart & $\begin{array}{r}\text { Vorkom- } \\
\text { men }\end{array}$ & Kommentar & n Fehler & $\begin{array}{r}ø \text { Fehler } \\
\text { pro Wort }\end{array}$ \\
\hline agg. & 20 contadine (19), priva (12), curda (11), & 82 & 4,1 \\
art.det./indet. & 9 & & 6 & 0,7 \\
avv. & 3 anche (7), qui (5), spesso (5) & 17 & 5,7 \\
cong. & 1 & 5 & 5 \\
el.loc. & 3 & a causa della (6), da parte dei (6) & 14 & 4,7 \\
el.n.p. & 2 & Monti del Ponto (17) & 18 & 9 \\
inf. & 1 & & 1 & 1 \\
loc. & 2 & zweimal catene montuose & 0 & 0 \\
n.p. & 11 & Siria (10), Tibet (8) & 31,5 & 2,9 \\
part.pass. & 3 & orlato (10), subita (10) & 22 & 7,3 \\
prep. & 11 & su (17), di (9), da (5) & 34 & 3,1 \\
prep.art. & 5 & & 5 & 1 \\
pron.* & 2 la (5), esso (5) & 10 & 5 \\
pron.rel. & 4 & che (10), cui (7), che (5) & 23 & 5,8 \\
s.m./f. & 36 & privatizzazioni (18), popolamento (15), & 135,5 & 3,8 \\
v.aus. & & utilizzo (14), dorsale (12), riforme (12) & 1 & 1 \\
v.tr./intr./mod. & 1 & & 1 & 3,3 \\
\hline
\end{tabular}




\begin{tabular}{lrlrr}
\hline & $\begin{array}{r}\text { Vorkom- } \\
\text { men }\end{array}$ & Kommentar & n Fehler & $\begin{array}{r}\varnothing \text { Fehler } \\
\text { pro Wort }\end{array}$ \\
\hline v.pronom.intr. & 4 & apre (13), innalzano (9), trova (5) & 28 & 7 \\
$\varnothing$ s.m./f. & 28,3 & & & \\
\hline
\end{tabular}

Tabelle 32: Getilgtes Wortvorkommen des C-Tests der III C (Pestalozzi) nach Wortarten mit Angaben zu den Falschlösungen

pron*: Unter dieser Bezeichnung sind außer den gesondert aufgelisteten Relativpronomen (pron.rel.) alle anderen Arten von Pronomen gesammelt.

Die meisten zu ergänzenden Wörter sind auch in diesem C-Test Substantive und Adjektive, und mit je elf Vorkommnissen folgen Präpositionen und Eigennamen. Auf Letztere und ein Partizip Perfekt entfallen durchschnittlich die meisten Fehler, da sie beide mit Wörtern vertreten sind, die zehn und mehr (17) Fehler verursachten. Bei den Partizipien gab vermutlich eher das sprachliche Umfeld und im Falle von orlato (umsäumt) auch die lexikalischer Ebene den Ausschlag, beim Toponym Monti del Ponto (Pontusgebirge) hingegen ohne Zweifel mangelnde lexikalische Kompetenz. Komplikationen sowohl im Verständnis des Mikro- als auch des Makrokontextes scheinen bei den reflexiven Verben die zahlreichen Falschlösungen verursacht zu haben. Bei si apre (Co; sich öffnen) könnten damit Schwächen in der lexikalischen Kompetenz verwoben sein.

Eine genauere Analyse im Kapitelabschnitt 8.3.5.4 wird das sprachliche Umfeld des reflexiven Verbs beschreiben. An dieser Stelle sei die Hypothese aufgestellt, dass es zwar wahrscheinlich gut bekannt war, in einem derartigen Kontext aber nicht erwartet wurde. Selbiges gilt höchstwahrscheinlich für das Adjektiv contadine (Fo; bäuerlich, der Landbevölkerung angehörend) bzw. eher für sein nicht getilgtes Bezugswort, das die Lernenden zur Produktion eines Adjektivs veranlasste, das als sein Attribut des Öfteren im Erdkundeunterricht gebraucht wurde (s. 8.3.5.2).

Im Bereich der Adjektive scheinen sich bei den sehr häufigen Falschlösungen nicht nur Schwierigkeiten mit dem Verständnis von Mikro- und Makrokontext, sondern womöglich auch eine unzureichende lexikalische Kompetenz (z.B. feroce, AU; grausam) zu offenbaren.

Im Weiteren erfolgt zunächst eine Lösungsbesprechung der ersten drei Texte und im Anschluss daran die der übrigen zwei.

\subsubsection{Texte 1 bis 3 des C-Tests der Klasse III C}

In Tabelle 33 sind alle Wörter der Sätze, in denen die Testteilnehmer Lücken füllen sollten, mit ihrer Art und Gebrauchsmarke sowie mit der Anzahl der Falschlösungen und den Lösungsvorschlägen der Schülerinnen und Schüler aufgeführt.

\begin{tabular}{|c|c|c|c|c|c|c|}
\hline Text & Fehler & Wort & Wortart & Marke & Kommentar & Lösungsvorschläge \\
\hline 1 & & Abitata & part.pass.v.tr. & FO estens. & & \\
\hline
\end{tabular}




\begin{tabular}{|c|c|c|c|c|c|c|}
\hline Text & Fehler & Wort & Wortart & Marke & Kommentar & Lösungsvorschläge \\
\hline (S. 105) & 0 & $d a$ & prep. & FO & & \\
\hline 27 & & moltissimo & agg. & FO & & \\
\hline \multirow[t]{40}{*}{ Lücken } & 1 & tempo & s.m. & FO & & territorio \\
\hline & & $\dot{e}$ & v.aus. & FO & & \\
\hline & 1 & stata & v.intr. & FO & & stato \\
\hline & & la & art.det. & FO & & \\
\hline & 4 & culla & s.f. & CO fig. & & cui, cultura (2), 0 \\
\hline & & delle & prep.art. & FO & & \\
\hline & $\begin{array}{r}0+2 \\
\text { acc. }\end{array}$ & prime & agg. & FO & & $\begin{array}{l}0 \text {, principali (acc.) } \\
\text { (2) }\end{array}$ \\
\hline & & civiltà & s.f. & $\mathrm{FO}$ & & \\
\hline & 4 & urbane & agg. & $\mathrm{AU}$ & & $\begin{array}{l}\text { urbanistiche (3), ur- } \\
\text { banizzate }\end{array}$ \\
\hline & & in & prep. & FO & & \\
\hline & 0 & Asia & n.p. & & & \\
\hline & & sono & v.aus. & FO & & \\
\hline & 0 & nate & v.intr. & FO & & \\
\hline & & le & art.det. & $\mathrm{FO}$ & & \\
\hline & 0 & grandi & agg. & FO & & \\
\hline & & religioni & s.f. & $A U$ & & \\
\hline & $\begin{array}{r}5+2 \\
\text { acc. }\end{array}$ & $q u i$ & avv. & FO & & $\begin{array}{l}\text { queste (4), quindi, } \\
\text { qua (acc.) (2) }\end{array}$ \\
\hline & & vivono & v.intr. & FO & & \\
\hline & 1 & $d a$ & prep. & FO & & dopo \\
\hline & & millenni & s.m. & FO & & \\
\hline & 1 & le & art.det. & FO & & II \\
\hline & & più & avv. & FO & & \\
\hline & 0 & numerose & agg. & FO & & \\
\hline & & masse & s.f. & CO estens. & & \\
\hline & 19 & contadine & agg. & FO & alle falsch & continentali (19) \\
\hline & & della & prep.art. & FO & & \\
\hline & 0 & Terra. & s.f. & $\begin{array}{l}\text { FO TS ast- } \\
\text { ron. }\end{array}$ & & \\
\hline & & II & art.det. & FO & & \\
\hline & 0 & rilievo & s.m. & $\mathrm{AU}$ & & \\
\hline & & $e$ & cong. & FO & & \\
\hline & 0 & il & art.det. & FO & & \\
\hline & & clima & s.f. & FO & & \\
\hline & 1 & hanno & v.aus. & FO & & ha contribuito \\
\hline & & contribuito & v.intr. & $\mathrm{CO}$ & & \\
\hline & & a & prep. & FO & & \\
\hline & 1 & delimitare & inf.v.tr. & $\mathrm{CO}$ & & delimitatare \\
\hline & & grandi & agg. & FO & & \\
\hline & $\begin{array}{r}7+1 \\
\text { acc. }\end{array}$ & aree & s.f. & $A U$ & & $\begin{array}{l}0(4) \text {, aretratezze, } \\
\text { arrivi } \\
(2, \text { davon } 1 \text { acc. mit } \\
\text { popoli), armi }\end{array}$ \\
\hline & & $d i$ & prep. & FO & & \\
\hline & $\begin{array}{r}15+1 \\
\text { acc. }\end{array}$ & $\begin{array}{l}\text { popola- } \\
\text { mento }\end{array}$ & s.m. & $\mathrm{CO}$ & & $\begin{array}{l}\text { popolazione }(7) \text {, po- } \\
\text { polazioni }(5) \text {, popoli } \\
(2 \text { davon } 1 \text { acc.), } 0 \\
\text { (2) }\end{array}$ \\
\hline
\end{tabular}




\begin{tabular}{|c|c|c|c|c|c|c|}
\hline Text & Fehler & Wort & Wortart & Marke & Kommentar & Lösungsvorschläge \\
\hline \multirow{39}{*}{$\begin{array}{l}2 \\
(\mathrm{~S} .106) \\
24 \\
\text { Lücken }\end{array}$} & & $e$ & cong. & FO & & \\
\hline & \multirow[t]{2}{*}{1,5} & civiltà & s.f. & FO & & civilta (3) \\
\hline & & $\begin{array}{l}\text { caratteriz- } \\
\text { zate }\end{array}$ & part.pass.v.tr. & $\mathrm{CO}$ & & \\
\hline & \multirow[t]{2}{*}{$1 \mathrm{acc}}$. & $d a$ & prep. & FO & & dai (acc.) \\
\hline & & modi & s.m. & FO & & \\
\hline & \multirow[t]{2}{*}{2} & diversi & agg. & FO & & diverse, divivere \\
\hline & & $d i$ & prep. & FO & & \\
\hline & \multirow[t]{2}{*}{$\begin{array}{r}14+2 \\
\text { acc. }\end{array}$} & utilizzo & s.m. & $\mathrm{CO}$ & & $\begin{array}{l}\text { utilizzare (10), uti- } \\
\text { lità, utilizzazione } \\
\text { (acc.) (2), 0, utili- } \\
\text { zare, utilmente }\end{array}$ \\
\hline & & delle & prep.art. & FO & & \\
\hline & \multirow[t]{2}{*}{1} & risorse & s.f. & $A U$ & & 0 \\
\hline & & $e$ & cong. & FO & & \\
\hline & \multirow[t]{2}{*}{9} & $d i$ & prep. & FO & & $\begin{array}{l}\text { 0, delle, dell' (4), de, } \\
\text { della, d' }\end{array}$ \\
\hline & & $\begin{array}{l}\text { organizza- } \\
\text { zione. }\end{array}$ & s.f. & $A U$ & & \\
\hline & & $\mathrm{La}$ & art.det. & $\mathrm{FO}$ & & \\
\hline & \multirow[t]{2}{*}{2} & prima & agg. & FO & & prima fascia, 0 \\
\hline & & $\grave{e}$ & v.aus. & FO & & \\
\hline & \multirow[t]{2}{*}{2} & costituita & v.tr. & FO & & costiera (2) \\
\hline & & dal & prep.art. & FO & & \\
\hline & \multirow[t]{2}{*}{2} & vasto & agg. & FO & & $0(2)$ \\
\hline & & Bassopiano & n.p. & & $\begin{array}{l}\text { als n.p. } \\
\text { nicht im } \\
\text { GRADIT }\end{array}$ & \\
\hline & \multirow[t]{2}{*}{1} & siberiano & el.n.p. & & & siberico \\
\hline & & un & art.indet. & FO & & \\
\hline & \multirow[t]{3}{*}{0} & territorio & s.m. & $A U$ & & \\
\hline & & $\begin{array}{l}\text { pianeg- } \\
\text { giante }\end{array}$ & agg. & $A D$ & & \\
\hline & & e & cong. & FO & & \\
\hline & \multirow[t]{2}{*}{5} & spesso & avv. & FO & & $\begin{array}{l}0 \text { (3), specialmente } \\
\text { (2) }\end{array}$ \\
\hline & & paludoso & agg. & $\mathrm{CO}$ & & \\
\hline & \multirow[t]{2}{*}{2} & attraversato & part.pass.v.tr. & FO & & $\begin{array}{l}\text { attraversata, attra- } \\
\text { versa }\end{array}$ \\
\hline & & da & prep. & FO & & \\
\hline & \multirow[t]{3}{*}{1} & numerosi & agg. & FO & & numerose \\
\hline & & fiumi. & s.m. & FO & & \\
\hline & & $A$ & prep. & FO & & \\
\hline & \multirow[t]{2}{*}{4} & sud & s.m. & $\mathrm{FO}$ & & $\begin{array}{l}\text { seconda }(2), 0, \text { si- } \\
\text { nistra }\end{array}$ \\
\hline & & $d i$ & prep. & $\mathrm{FO}$ & & \\
\hline & \multirow[t]{2}{*}{$\begin{array}{r}5+3 \\
\text { acc. }\end{array}$} & esso & pron.pers. & $\mathrm{FO}$ & & $\begin{array}{l}\text { essa (3) (acc.), } 0 \\
\text { (2), est (3) }\end{array}$ \\
\hline & & si & pron.pers. & $\mathrm{CO}$ & & \\
\hline & \multirow[t]{2}{*}{1} & estende & $\begin{array}{l}\text { v.pro- } \\
\text { nom.intr. }\end{array}$ & $\mathrm{CO}$ & & 0 \\
\hline & & un'ampia & agg. & FO & & \\
\hline & 0 & fascia & s.f. & $\mathrm{AU}$ & & \\
\hline
\end{tabular}




\begin{tabular}{|c|c|c|c|c|c|c|}
\hline Text & Fehler & Wort & Wortart & Marke & Kommentar & Lösungsvorschläge \\
\hline & \multirow{3}{*}{0} & centrale & agg. & FO & & \\
\hline & & montuosa & agg. & $\mathrm{CO}$ & & \\
\hline & & costituita & part.pass.v.tr. & FO & & \\
\hline & \multirow[t]{2}{*}{$\begin{array}{r}4+3 \\
\text { acc. }\end{array}$} & dagli & prep.art. & FO & & $\begin{array}{l}\text { molti (3), } 0 \text { (acc.) } \\
\text { (3), dai }\end{array}$ \\
\hline & & altipiani & var.s.m. & $A D$ & & \\
\hline & \multirow[t]{2}{*}{10} & che & pron.rel. & FO & & $\begin{array}{l}\text { centrali (5), carsici, } \\
0 \text { (3), continentali }\end{array}$ \\
\hline & & si & pron.pers. & $\mathrm{CO}$ & & \\
\hline & \multirow[t]{2}{*}{9} & innalzano & $\begin{array}{l}\text { v.pro- } \\
\text { nom.intr. }\end{array}$ & $\mathrm{CO}$ & & innalza (8), innalsa \\
\hline & & dal & prep.art. & FO & & \\
\hline & \multirow[t]{2}{*}{8} & Tibet & n.p.m. & & & $\begin{array}{l}0(4), \text { Ticino }(2), \\
\text { Tigre, Tiro }\end{array}$ \\
\hline & & alla & prep.art. & FO & & \\
\hline & \multirow[t]{4}{*}{6} & Mongolia & n.p. & & $\begin{array}{l}\text { n.p. nicht im } \\
\text { GRADIT }\end{array}$ & $\begin{array}{l}0 \text { (4), Mongolio, } \\
\text { Mongagnola }\end{array}$ \\
\hline & & tra & prep. & FO & & \\
\hline & & i 1000 & art.det. & $\mathrm{CO}$ & & \\
\hline & & e 1500 & cong. & FO & & \\
\hline & \multirow[t]{2}{*}{0} & metri & s.m. & FO & & \\
\hline & & $e$ & cong. & FO & & \\
\hline & \multirow[t]{2}{*}{5} & $d a$ & prep. & FO & & di (2), 0, delle (2) \\
\hline & & imponenti & agg. & $A U$ & & \\
\hline & \multirow[t]{2}{*}{0} & catene & loc.s.f. & $\mathrm{CO}$ & & \\
\hline & & montuose & el.loc.s.f. & el.CO & & \\
\hline & \multirow[t]{2}{*}{1} & tra & prep. & $\mathrm{FO}$ & & troveremo \\
\hline & & cui & pron.rel. & FO & & \\
\hline & \multirow[t]{2}{*}{6} & spicca & v.tr. & $A U$ & & $\begin{array}{l}0 \text { (3), spizza, spira, } \\
\text { spiccano }\end{array}$ \\
\hline & & l'alta & agg. & FO & & \\
\hline & 12 & dorsale. & s.m. & TS geogr. & & dormita, 0 (11) \\
\hline \multirow[t]{2}{*}{$\begin{array}{l}3 \\
\text { (S. 121) }\end{array}$} & \multirow[t]{2}{*}{11} & focolaio & s.m. & CO fig. & $\begin{array}{l}\text { kein Phra- } \\
\text { sem im } \\
\text { GRADIT }\end{array}$ & focolare (7), 0 (4) \\
\hline & & $d i$ & prep. & FO & & \\
\hline \multirow[t]{12}{*}{$\begin{array}{l}26 \\
\text { Lücken }\end{array}$} & \multirow[t]{2}{*}{$\begin{array}{r}2+8 \\
\text { acc. }\end{array}$} & tensioni & s.f. & $A U$ & & $\begin{array}{l}\text { tensini, tensione } \\
\text { (acc.) (8), } 0\end{array}$ \\
\hline & & si & pron.pers. & $\mathrm{CO}$ & & \\
\hline & \multirow[t]{2}{*}{5} & trova & $\begin{array}{l}\text { v.pro- } \\
\text { nom.intr. }\end{array}$ & $\mathrm{CO}$ & & $\begin{array}{l}\text { tratta (2), trovano, } 1 \\
\text { unleserlich, traversa }\end{array}$ \\
\hline & & nella & prep.art. & FO & & \\
\hline & \multirow[t]{2}{*}{0} & regione & s.f. & $A U$ & & \\
\hline & & compresa & agg. & $\mathrm{CO}$ & & \\
\hline & \multirow[t]{2}{*}{0} & tra & prep. & FO & & \\
\hline & & la & art.det. & $\mathrm{FO}$ & & \\
\hline & \multirow[t]{3}{*}{0} & Turchia & n.p. & & & \\
\hline & & l'Iran & n.p. & & & \\
\hline & & $e$ & cong. & FO & & \\
\hline & 3 & I'Iraq & n.p. & $\mathrm{FO}$ & & Irlanda (2), Irun \\
\hline
\end{tabular}




\begin{tabular}{|c|c|c|c|c|c|c|}
\hline \multirow{3}{*}{\multicolumn{2}{|c|}{$\begin{array}{r}\text { Fehler } \\
7\end{array}$}} & Wort & Wortart & Marke & Kommentar & Lösungsvorschläge \\
\hline & & cui & pron.rel. & FO & & $\begin{array}{l}0, \text { cosidetta (3), cre- } \\
\text { sciente, crescenti, } \\
\text { consistente }\end{array}$ \\
\hline & & popolazione & s.f. & $\mathrm{AU}$ & & \\
\hline \multirow{2}{*}{\multicolumn{2}{|c|}{11}} & curda & agg. & TS etnol. & & $\begin{array}{l}0 \text { (4), cui, curiana, } \\
\text { culturale (5) }\end{array}$ \\
\hline & & presente & agg. & FO & & \\
\hline \multirow{2}{*}{\multicolumn{2}{|c|}{1}} & con & prep. & $\mathrm{FO}$ & & come \\
\hline & & minoranze & s.f. & $\mathrm{CO}$ & & \\
\hline \multirow{2}{*}{\multicolumn{2}{|c|}{7}} & anche & avv. & FO & & antiche (6), 0 \\
\hline & & in & prep. & $\mathrm{FO}$ & & \\
\hline \multirow{2}{*}{\multicolumn{2}{|c|}{10}} & Siria & n.p. & & & $\begin{array}{l}\text { Siberia (6), Sicilia, } 0 \\
\text { (3) }\end{array}$ \\
\hline & & $e$ & cong. & FO & & \\
\hline \multirow{3}{*}{\multicolumn{2}{|c|}{0}} & in & prep. & FO & & \\
\hline & & Armenia & n.p. & & & \\
\hline & & $\grave{e}$ & v.intr. & $\mathrm{FO}$ & & \\
\hline \multirow{2}{*}{\multicolumn{2}{|c|}{12}} & priva & agg. & FO & & $\begin{array}{l}\text { prarte, presente, } \\
\text { privo, propria (2), } \\
\text { pro, protetta, pro- } \\
\text { prietaria, } 0(2) \text {, pro- } \\
\text { prio, prriva }\end{array}$ \\
\hline & & $d i$ & prep. & $\mathrm{FO}$ & & \\
\hline \multirow{2}{*}{\multicolumn{2}{|c|}{0}} & un & art.indet. & FO & & \\
\hline & & proprio & agg. & FO & & \\
\hline \multirow{2}{*}{\multicolumn{2}{|c|}{1}} & Stato & s.m. & FO & & Stata \\
\hline & & a & loc.prep. & $\mathrm{CO}$ & & \\
\hline \multirow{2}{*}{\multicolumn{2}{|c|}{6}} & causa & el.loc.prep. & el.CO & & $\begin{array}{l}0(3) \text {, capo }(2), \text { ca- } \\
\text { ausa }\end{array}$ \\
\hline & & della & el.loc.prep. & el.CO & & \\
\hline \multirow{2}{*}{\multicolumn{2}{|c|}{$\begin{array}{r}10+2 \\
\text { acc. }\end{array}$}} & feroce & agg. & $\mathrm{AU}$ & & $\begin{array}{l}0 \text { (8), ferte, ferrea } \\
(\text { acc.) (2), feroci }\end{array}$ \\
\hline & & $\begin{array}{l}\text { persecu- } \\
\text { zione }\end{array}$ & s.f. & $\mathrm{CO}$ & & \\
\hline \multirow{2}{*}{\multicolumn{2}{|c|}{10}} & subita & part.pass.v.tr. & FO & & $\begin{array}{l}0(6) \text {, subcontinen- } \\
\text { tale, subordi- } \\
\text { nata, subordinante, } \\
\text { subite }\end{array}$ \\
\hline & & $d a$ & loc.prep. & $\mathrm{CO}$ & & \\
\hline \multirow{2}{*}{\multicolumn{2}{|c|}{6}} & parte & el.loc.prep. & el.CO & & paarte, parti, 0 (4) \\
\hline & & dei & el.loc.prep. & el.CO & & \\
\hline \multirow{2}{*}{\multicolumn{2}{|c|}{1}} & governi & s.m. & $\mathrm{FO}$ & & governo \\
\hline & & dei & prep.art. & $\mathrm{FO}$ & & \\
\hline \multirow{2}{*}{\multicolumn{2}{|c|}{8}} & Paesi & s.m. & $\mathrm{FO}$ & & $\begin{array}{l}\text { Papa, } 0 \text { (2), Palesti- } \\
\text { nesi (3), Parteci- } \\
\text { panti (2) }\end{array}$ \\
\hline & & confinanti & agg. & $\mathrm{CO}$ & & \\
\hline \multirow{2}{*}{\multicolumn{2}{|c|}{1}} & che & pron.rel. & FO & & 0 \\
\hline & & non & avv. & FO & & \\
\hline & 2 & $\begin{array}{l}\text { vogliono } \\
\text { riconoscere }\end{array}$ & $\begin{array}{l}\text { v.mod. } \\
\text { inf.v.tr. }\end{array}$ & $\begin{array}{l}\text { FO } \\
\text { FO }\end{array}$ & & voglare, 0 \\
\hline \multirow{2}{*}{\multicolumn{2}{|c|}{0}} & il & art.det. & FO & & \\
\hline & & suo & agg.poss. & $\mathrm{FO}$ & & \\
\hline
\end{tabular}




\begin{tabular}{|c|c|c|c|c|c|c|}
\hline lext & Fehler & Wort & Wortart & Marke & Kommentar & Lösungsvorschläge \\
\hline
\end{tabular}

Tabelle 33: Wortvorkommen und Lösungsvorschläge der ersten drei Texte des C-Tests der Klasse III C, Pestalozzi

In den ersten drei Texten befinden sich insgesamt 77 Lücken, von denen 22 richtig gefüllt wurden. Der Großteil der betroffenen Wörter stammt aus dem Bereich des fundamentalen Wortschatzes (FO), aber es handelt sich dabei auch um das als Fo Ts astron. doppelt markierte Nomen Terra (Erde), vier Ausdrücke des hohen Gebrauchs (AU), ein Phrasemelement aus dem allgemeinen Wortschatz (catene aus catene montuose, Co; Gebirgsketten) und einen geographischen Eigennamen (Turchia).

Von den übrigen Wörtern wurden 28 von bis zu vier SchülerInnen gar nicht oder nicht korrekt vervollständigt. Auch sie zählen überwiegend zum fundamentalen Wortschatz. Häufig bearbeiteten lediglich ein oder zwei Lernende eine Lücke nicht/nicht richtig. Nur vier Wörter wurden viermal nicht korrekt ergänzt: zwei Ausdrücke aus dem fundamentalen Wortschatz (Fo), ein figurativer aus dem allgemeinen (CO) und einer aus dem des hohen Gebrauchs (AU). Die beiden letztgenannten befinden sich im ersten Satz von Text 1, culla (Co fig.; Wiege) und urbane (AU; städtisch). Während ein Schüler bei culla die Lücke offen ließ, ein anderer zu einem morphosyntaktisch falschen Relativpronomen und zwei weitere zum logisch falschen Nomen cultura (Kultur) ergänzten, handelt es sich bei den vier nicht akzeptablen Lösungsvorschlägen für urbane um semantisch verwandte Wörter. Deshalb kann davon ausgegangen werden, dass den Lernenden die Bedeutungsnuancen zwischen den Adjektiven urbano, urbanizzato (verstädtert) und urbanistico (urbanistisch, städtebaulich) nicht bekannt waren. Davon abgesehen ist es jedoch interessant, dass sie als Lösungsvorschläge Wörter anboten, die seltener gebraucht werden als das im Originaltext verwendete „urban“: Eines trägt die doppelte Gebrauchsmarke CO Ts und das andere ist als urbanistisches Fachwort verzeichnet.

Das Fo-gekennzeichnete Nomen sud steht mit der Präposition $a$ (hier: im) als Lokaladverbiale am Satzanfang. Ihm folgt di esso (von ihm), wobei sich das Pronomen auf die sibirische Tiefebene aus dem vorausgehenden Satz bezieht. Schätzungsweise verleitete die darin enthaltene Präposition di zwei Testteilnehmer dazu, die Lücke in sud als Element des Präpositionalphrasems $a$ seconda di (CO; abhängend von) zu vervollständigen, was aber logisch keinen Sinn ergibt. Dadurch kann hier ein mangelndes Textverständnis unterstellt werden, bzw. kommt einmal mehr der Verdacht auf, dass sich die Lernenden beim Füllen der Lücken zu sehr auf das getilgte Wort und den unmittelbaren sprachlichen Kontext beschränkten und den Rest des Satzes oder weiteren $\mathrm{Zu}$ sammenhangs außer Acht ließen.

Die Ergänzung zu sinistra (links) deutet auf Unaufmerksamkeit im Unterricht hin, denn die Schülerinnen und Schüler aller beobachteten Klassen wur- 
den immer wieder darauf hingewiesen, dass die geographische Einordnung eines Landes mit der Nennung der Himmelsrichtungen und nicht mit „links“ und „rechts“ zu erfolgen hat. ${ }^{159}$

Die artikulierte Präposition da-gli (von den) wurde viermal nicht richtig ergänzt, wobei man anhand der Lösungsvorschläge keine eindeutige Aussage über die Art der Schwierigkeiten der SchülerInnen bei der Entscheidung darüber treffen kann, ob eine Präposition ohne den Artikel vor einem bestimmten Nomen stehen kann und welcher der richtige Artikel ist. Drei Lernende beachteten die Regel nicht, die Lücken nicht mit zusätzlichen ganzen Wörtern zu füllen. Die ansonsten logisch und grammatikalisch korrekten Lösungen sind aus diesem Grund nicht vertretbar. Darüber hinaus füllten drei Lernende die Lücke nicht, was akzeptiert werden kann, da der nicht getilgte Teil des Wortes eine einfache Präposition ist, die keinen Artikel erfordert. Es ist aber nicht klar, ob die Lernenden die Lücke bewusst offenließen, sie übersahen oder ob sie vielleicht nicht wussten, was sie ergänzen sollten. So bleibt noch ein Lösungsvorschlag zu erwähnen, der definitiv falsch ist (dai) und auf eine nicht muttersprachliche oder sehr unkonzentrierte Testperson hinweist, weil bei dieser Präpositionsergänzung durch das mit einem Vokal beginnende Bezugswort der Artikel gli erforderlich ist, wie jedes erstsprachig italienische Kind spätestens im Grundschulalter lernt.

\subsubsection{Häufigste Falschlösungen der Klasse III C in Text 1 bis 3}

Fast ebenso viele Lücken wie die seltener falsch gelöste wurden von mindestens fünf SchülerInnen nicht richtig gefüllt, nämlich 27. Die meisten davon entfallen auf den dritten Text (12), gefolgt vom zweiten (10), wobei insgesamt 15 Lücken von mindestens 8 Lernenden falsch oder nicht ergänzt wurden. Unter den Wörtern befindet sich außerdem gleich im ersten Text eines, das niemand richtig, sondern alle zu continentali (kontinental) ergänzten. Das zu vervollständigende Adjektiv ist contadine (bäuerlich) und befindet sich hinter dem Nomen masse (Massen). Es gehört zwar zum fundamentalen Wortschatz, wurde aber in der Kombination mit dem Nomen aus dem allgemeinen Wortschatz vielleicht aus den folgenden zwei Gründen nicht gewählt. Zum einen ist es ein ungewöhnlicher, recht abstrakter Ausdruck zur Bezeichnung einer zahlreichen Landbevölkerung, zumal auch das animierte Nomen contadino (Bauer, Landarbeiter) existiert. Zum anderen ergänzten die Lernenden wahrscheinlich

159 Dennoch konnte der Gebrauch dieser beiden Richtungsadverbien bei mündlichen Abfragungen immer wieder beobachtet werden. Er wurde jedes Mal von der Lehrkraft beanstandet. Wahrscheinlich waren die Lernenden so an das alltagssprachliche „links“ und ,rechts“ gewöhnt, dass es ihnen viel Konzentration abverlangte, sich anders auszudrücken. Obgleich im schriftlichen Sprachgebrauch mehr Zeit zum Überdenken der Wortwahl besteht, war den zwei Testteilnehmern anscheinend auch dort die registerunangemessene Verwendung nicht bewusst. 
spontan zu masse continentali (Kontinentalmassen), weil dies ein phraseologischer, nicht als solcher im GRADIT verzeichneter Ausdruck ist, dem sie des Öfteren im Lehrbuch und im Unterricht begegneten. Der Lösungsvorschlag ist allerdings logisch falsch und erneut ein gutes Beispiel dafür, dass die Jugendlichen den Kontext bei der Testbearbeitung vermutlich viel zu wenig berücksichtigten. Denn es geht um einen kurzen Satz, dessen Prädikat vivono (Fo; leben) nicht getilgt ist und ein animiertes Subjekt benötigt, weshalb ihr Lösungsvorschlag unpassend ist.

Auf den letzten Satz von Text 1 entfallen mit einmal 15 und einmal 14 falschen Lösungen die nächsthäufigsten Falschlösungen, und es kommen mit sieben und neun noch zwei weitere große Fehlerquellen hinzu. Die folgende Abbildung veranschaulicht, um welchen Satz es sich handelt und welche getilgten Wörter wie oft nicht korrekt ergänzt wurden.

II rilievo [0] e il [0] clima hanno [1] contribuito a delimitare [1] grandi aree [7] di popolamento [15] e civiltà [3] caratterizzate da modi diversi [2] di utilizzo [14] delle risorse [1] e di [9] organizzazione del territorio, che esploreremo suddividendole in cinque settori: occidentale, centrale, centromeridionale, sudorientale, orientale.

M.Ü.: Die Erhebungen und das Klima haben dazu beigetragen, große Gebiete der Besiedlung und Kultur/Zivilisation zu begrenzen, welche durch verschiedene Arten der Verwendung der Ressourcen und der Organisation des Territoriums charakterisiert sind, die wir, sie in fünf Sektoren unterteilend, erkunden werden: westlicher, zentraler, zentral-südlicher, südöstlicher, östlicher.

Abbildung 12: S. 122 des Lehrbuchs (Forte \& Ubertazzi 2008) entnommener Satz mit Hervorhebung der getilgten Wörter, Fehleranzahl und Übersetzung

Die Schwierigkeiten bei der Bearbeitung dieses Satzes sind wahrscheinlich zweierlei Art und zeigen sich auch in der übersetzten Version. Es sind einerseits bestimmte Ausdrücke, die aus den seltener benutzten Bereichen des Wortschatzes stammen, und andererseits morphosyntaktische Strukturen. So liegt hier ein impliziter Relativsatz vor, dem also das Relativpronomen fehlt und der nicht durch Kommata vom Rest des Satzgefüges abgetrennt, sondern nur am Partizip caratterizzate zu erkennen ist. Desweiteren beziehen sich auf das präpositionale Objekt da modi diversi zwei Attribute, von denen das erste noch ein weiteres einschließt. Auf diese lange Kette folgt ein Relativsatz, der ein Gerundium enthält. Während die ersten Lücken des Satzes einschließlich des etwas schwierigeren Co-gekennzeichneten Wortes delimitare von fast allen Testteilnehmern gefüllt wurden, bereiteten die anderen getilgten Wörter anscheinend dadurch größere Schwierigkeiten, dass der Satz erheblich an Komplexität gewinnt. Es ist außerdem möglich, dass sie delimitare zwar richtig vervollständigten, aber die Bedeutung nicht genau kannten oder sie in diesem Satz nicht verstanden. Vor allem aber bedingen sich die beiden getilgten Wörter aree und popolamenti gegenseitig. Während das erste von vier Lernenden überhaupt nicht ergänzt wurde, vervollständigten 15 das zweite entweder zu einer Form von popolazione (Bevölkerung) oder popoli (Volk). Das sind Nomen aus derselben Wortfamilie, die aber im Gegensatz zu popolamento (CO) aus dem Basiswortschatz stammen und zum fundamentalen Wortschatz (Fo) 
und zu dem des hohen Gebrauchs (AU) zählen. Logisch ergeben beide keinen Sinn, wobei eine akzeptable Kombination von zwei Alternativvorschlägen vorliegt, und zwar arrivi di popoli (Ankünfte von Völkern).

Die beiden anderen häufig falsch gelösten Wörter sind Teil der Attribute von „verschiedene Arten“ und zeigen im ersten Fall, dass den Testpersonen hier eine Infinitivkonstruktion viel geläufiger war als die attributive (11 Lösungsvorschläge). Neben logisch falschen Ergänzungen zu einem anderen Nomen und einem Adverb bildeten zwei Lernende das in vielen seiner Bedeutungen zu utilizzo synonyme Nomen utilizzazione. Es ist als Wort der hohen Disponibilität $(\mathrm{AD})$ gekennzeichnet und somit verbreiteter als das im Text vorgesehene. Bezüglich der Präposition $d-i$ zeigt sich an den Lösungsvorschlägen, dass viele wahrscheinlich durch die vorausgehende, nebenordnende Konjunktion $e$ dazu verleitet wurden, das folgende Attribut wie das vorherige zu konstruieren und somit fälschlicherweise auf utilizzo anstatt auf modi diversi zu beziehen. Die häufigen Falschlösungen lassen die Schlussfolgerung zu, dass die Schüler bei der Bearbeitung dieser Lücke einmal mehr das sprachliche Umfeld ausgeblendet haben könnten, so dass die Lösung kompliziert wurde.

Im zweiten Text konzentrieren sich neun häufige Falschlösungen auf einen langen Satz (35 Wörter), der mit der bereits erwähnten Lokaladverbiale $a$ sud di esso (im Süden von ihm) beginnt. Hier ist das Bezugswort des Pronomens nicht eindeutig, so dass drei SchülerInnen zur weiblichen Form essa ergänzten, was dadurch akzeptabel ist, dass sich das Bezugswort Bassopiano siberiano in einem Satz befindet, der mit dem femininen la prima (die erste) beginnt und damit die grandi aree di popolamento (großen Gebiete der Besiedlung) aufgreift. Von weiteren fünf Lernenden vervollständigten drei hingegen das Wort logisch falsch zu est („Im Süden von Osten“).

Es folgt ein Relativsatz, in dem zehn Lernende das einfache Relativpronomen $c$-he (Fo) nicht vervollständigten, sondern stattdessen überwiegend verschiedene Adjektive bildeten, die sich zwar grammatikalisch richtig auf das vorausgehende Substantiv beziehen, aber dennoch syntaktisch falsch sind. Es steht nämlich nicht im Nominativ und nach der Lücke folgt ein reflexives Prädikat, das ein Subjekt zwischen sich und dem Substantiv, das hierfür nicht in Frage kommt, da es Teil eines präpositionalen Objekts ist, erfordert. Als mögliche Alternative zur Adjektiv- anstelle der Pronomenbildung gäbe es die Nennung des Substantivs an späterer Stelle, doch auch diese bleibt aus, so dass wieder zwei oft herangezogene Erklärungsversuche für das Lösungsverhalten der Lernenden in Frage kommen: Sie könnten entweder nur das allernächste sprachliche Umfeld des zu ergänzenden Relativpronomens berücksichtigt haben oder damit überfordert gewesen sein, über den langen Satz mit etlichen Wörtern, die nicht aus dem Basiswortschatz stammen, den Überblick zu behalten. Dass über die Hälfte der Testteilnehmer das Relativpronomen nicht an- 
gemessen vervollständigte, hat wohl auch dazu geführt, dass das folgende Prädikat nicht in der erfragten Pluralform, sondern neunmal falsch im Singular konjugiert wurde.

Ebenfalls problematisch stellt sich die anschließende Lücke im Eigennamen Ti-bet dar, welcher u.a. recht willkürlich zu Ticino (Tessin) vervollständigt wurde. Außerdem vervollständigten sechs den nächsten Eigennamen, Mong-olia, nicht richtig.

Im selben Satz befinden sich vier weitere häufige Fehlerquellen, von denen die erste die einfache Präposition $d-a$ (von) betrifft, die fünf Lernende nicht korrekt ergänzten. Hier braucht es schon einen relativ hohen Grad an grammatikalisch-textueller Kompetenz, um zu bemerken, dass es sich um ein präpositionales Objekt handelt, das einem anderen, vorausgehenden durch die davorstehende Konjunktion ,und“ nebengeordnet ist. Das zweite präpositionale Objekt liegt allerdings elf Wörter zurück, da an ihm noch der schon angesprochene Relativsatz hängt, der von so vielen Lernenden nicht als solcher erkannt wurde. Daher ist es nicht verwunderlich, dass sechs Lernende desweiteren am Satzende keine richtige Prädikatsbildung vornahmen. Das letzte zu ergänzende Wort hätten sie vielleicht bei einem ausgeprägteren Verständnis der Satzaussage produzieren können. Es geht hier um das fachwortschatzsprachliche dorsale (Berghang, -rücken), das von zwölf Personen, also mehr als der Hälfte der Testteilnehmer, gar nicht und von einer weiteren zum logisch nicht korrekten dormita (Schlaf) vervollständigt wurde.

Im dritten Text wurden zwölf Wörter falsch oder nicht bearbeitet, mehr als die Hälfte davon (sieben) von mindestens acht Testteilnehmern. Alle Lücken dieses Textes befinden sich in einem einzigen langen, syntaktisch verschachtelten Satz. Gleich zu Beginn zeigten elf Lernende Schwierigkeiten bei der Vervollständigung des ersten Elements im Ausdruck foco-laio di tens-ioni (Spannungsherd), der zwar im GRADIT nicht als Phrasem verzeichnet, aber dennoch ein recht feststehender Ausdruck ist, da nur der Numerus des zweiten Nomens variiert werden kann. Diese Variante mit tensione (Spannung) wählten acht SchülerInnen, während nur zwei es zu einem fiktiven Wort (vielleicht ein orthographischer Flüchtigkeitsfehler, da nur ein Buchstabe ausgelassen wurde) bzw. gar nicht ergänzten. Anders ist es mit focolaio (Brandherd, Herd), das von vier Testteilnehmern gar nicht und von sieben zu focolare (Herd, Feuerstelle) vervollständigt wurde. Dieses zweite, ebenfalls aus dem allgemeinen Wortschatz stammende Nomen wird zwar nicht in der Kombination mit di tensione verwendet, ist in vielen seiner Bedeutungen aber mit dem ersten deckungsgleich und wird auch im übertragenen Sinn gebraucht, wie z.B. in focolare di epidemia (Seuchenherd).

Auch in diesem Text wurde ein reflexives Verb (Co; trovarsi; sich befinden) fünfmal falsch bearbeitet, wobei die entsprechenden Lösungsvorschläge bis auf einen unleserlichen aus vier Ergänzungen zu anderen reflexiven Verben bestehen, die logisch-semantisch nicht in den Satz passen. Eines ist zusätzlich 
grammatikalisch falsch. Das Verb steht direkt nach dem Ausdruck für „Brandherd", womit die Satzstruktur also nicht einfacher sein könnte. Deshalb könnte es sich bei den Testteilnehmern, die das Prädikat nicht richtig vervollständigten, um diejenigen handeln, die für focolaio gar nichts ergänzt hatten und somit das zum Prädikat gehörende Subjekt nicht kannten.

Erneut erscheint ein Relativpronomen als häufige Fehlerquelle (sieben Falschlösungen), jedoch betrifft es diesmal cui (Fo; deren) und damit eine schwierigere Nebensatzkonstruktion, in der das Relativpronomen nicht das Subjekt des Satzes ist. Darüber hinaus befinden sich zwischen dem Pronomen und seinem Bezugswort sieben, also wieder nicht gerade wenig Wörter.

In diesem Textabschnitt geht es um die kurdischen Minderheiten, doch das als ethnologisches Fachwort gekennzeichnete Adjektiv cur-da (kurdisch) wurde von fast zwei Dritteln der Testteilnehmer (11) nicht richtig vervollständigt. Mit Si-ria (Syrien) war ein weiterer geographischer Eigenname anscheinend für viele schwierig, weil zehn Personen ihn entweder nicht (3) oder aber zu den logisch nicht annehmbaren Toponymen Siberia (6) und Sicilia (Sizilien) (1) ergänzten, wobei Sibirien immerhin eine Region Asiens ist. Davor wurde zudem die frequente Konjunktion anche (auch) siebenmal falsch bearbeitet. Sechs ergänzten die Konjunktion zu antiche und bezogen sie als adjektivisches Attribut auf das vorausgehende Wort minoranze.

Obwohl das Prädikatsnomen pr-iva (bar, beraubt) zum fundamentalen Wortschatz zählt, ist es mit hoher Wahrscheinlichkeit doch nicht so geläufig wie viele andere Fo-gekennzeichnete Wörter und vervollständigten zwölf Testteilnehmer es nicht richtig. Lediglich zwei von ihnen kamen der korrekten Lösung nahe, indem der eine nur flüchtig auch den letzten nicht getilgten Buchstaben ergänzte und der andere das richtige lexikalische Wort mit einer falschen Genus-Endung versah. Zu der vermehrten Falschlösung des Adjektivs trugen wahrscheinlich die Satzlänge und die grammatikalisch-syntaktische Komplexität bei, die auch ein Grund für die vermehrten Falschlösungen im Vorfeld sein könnten, so dass beides zu einer ausgesprochenen Einschränkung des Satzverständnisses geführt haben wird. Nach dem Prädikatsnomen wurde außerdem ein Phrasemelement vermehrt nicht oder nicht korrekt vervollständigt, und zwar das präpositionale Phrasem a cau-sa di (CO; aufgrund von), wobei einem Lernenden nur ein, wenn auch grober, Rechtschreibfehler ( $c a$ ausa) unterlief.

Fer-oce (wild, heftig) ist ein weiteres Adjektiv, das zehn Testteilnehmer nicht richtig bearbeiteten. Es entstammt dem Wortschatzbereich des hohen Gebrauchs (AU) und wurde von acht SchülerInnen gar nicht ergänzt, was eventuell an mangelnder lexikalischer Kompetenz lag. Seinem Bezugswort aus dem allgemeinen Wortschatz folgt ein Partizip Perfekt (sub-ita, Fo; erlitten), das ebenfalls von zehn Testteilnehmern nicht korrekt ergänzt wurde. Nur eine Person vervollständigte die Lücke zum gesuchten Partizip, allerdings mit der fe- 
mininen Pluralendung -e. Dazu wurde sie wahrscheinlich durch das vorausgehende Wort persecuzione verleitet, das auf -e endet, aber im Singular steht. Alle anderen falschen Lösungsvorschläge blieben entweder ganz aus (6) oder bestehen aus logisch falschen Adjektiven (3).

Die letzte vermehrte Fehlerquelle betrifft das Wort Pa-esi (Fo; Länder), das zwei von acht Testteilnehmern nicht vervollständigten, obwohl es sehr häufig gebraucht wird, und das die anderen zu logisch abstrusen Begriffen ergänzten. Dass ein so langer, komplexer Satz mit seinen zahlreichen unausgefüllten oder falsch bearbeiteten Lücken zu einer beträchtlichen Einschränkung des Textverständnisses führt, ist klar. Dennoch kann man sich fragen, wie jemand auf Lösungsvorschläge wie Papa (Papst), Palestinesi oder Partecipanti (Teilnehmer; warum mit Majuskel?) kommt - in einer nächsten sprachlichen Umgebung von ,[Verfolgung erlitten seitens] der Regierungen der“. Wurden Energien hier einzig dafür aufgebraucht, Silben zu finden, die das getilgte Wort zu einem existierenden Ausdruck vervollständigten?

\subsubsection{Texte 4 und 5 des C-Test der Klasse III C}

Der vierte und fünfte C-Testtext befassen sich landeskundlich (Text 4) und sozioökonomisch (Text 5) mit der Türkei. Das Wortvorkommen der Sätze, in denen sich die getilgten Wörter befinden, ist in Tabelle 34 nach Wortart, Gebrauchsmarke, Fehleranzahl und Lösungsvorschlägen klassifiziert.

\begin{tabular}{|c|c|c|c|c|c|c|}
\hline Text & Fehler & Wort & Wortart & Marke & Kommentar & Lösungsvorschläge \\
\hline 4 & 6 & bagnata & v.tr. & FO & & bagnato (5), bagno \\
\hline (S. 122) & & $a$ & prep. & FO & & \\
\hline 25 & 0 & nord & s.m. & $\mathrm{AU}$ & & \\
\hline \multirow[t]{16}{*}{ Lücken } & & dal & prep.art. & $\mathrm{FO}$ & & \\
\hline & 0 & Mar & n.p. & & $\begin{array}{l}\text { nicht im } \\
\text { GRADIT }\end{array}$ & \\
\hline & & Nero & el.n.p. & & & \\
\hline & & $a$ & prep. & $\mathrm{FO}$ & & \\
\hline & 0 & ovest & s.m. & $A U$ & & \\
\hline & & $d a l$ & prep.art. & $\mathrm{FO}$ & & \\
\hline & 1 & Mar & n.p. & & $\begin{array}{l}\text { nicht im } \\
\text { GRADIT }\end{array}$ & Mare \\
\hline & & Egeo & el.n.p. & & & \\
\hline & & $e$ & cong. & FO & & \\
\hline & & $a$ & prep. & $\mathrm{FO}$ & & \\
\hline & 0 & sud & agg. & FO & & \\
\hline & & dal & prep.art. & $\mathrm{FO}$ & & \\
\hline & 0,5 & Mar & n.p. & & $\begin{array}{l}\text { nicht im } \\
\text { GRADIT }\end{array}$ & Mare (Wdhg.) \\
\hline & & $\begin{array}{l}\text { Mediterra- } \\
\text { neo. }\end{array}$ & el.n.p. & & & \\
\hline & $\begin{array}{r}4+3 \\
\text { acc. }\end{array}$ & Due & agg.num.card. & $\mathrm{FO}$ & & $\begin{array}{l}0 \text { (2), Da (2), Delle } \\
\text { (acc.) (3) }\end{array}$ \\
\hline & & possenti & agg. & $\mathrm{CO}$ & & \\
\hline
\end{tabular}




\begin{tabular}{|c|c|c|c|c|c|c|}
\hline Text & Fehler & Wort & Wortart & Marke & Kommentar & Lösungsvorschläge \\
\hline & 0 & $\begin{array}{l}\text { catene } \\
\text { montuose }\end{array}$ & loc.s.f. & $\mathrm{CO}$ & & \\
\hline & 5 & la & $\begin{array}{l}\text { el.IOC.s.t. } \\
\text { pron.pers. }\end{array}$ & $\begin{array}{l}\text { El.cu } \\
\text { FO }\end{array}$ & & li lo (3) le \\
\hline & & circondano & pron.pers. & $\begin{array}{ll}\mathrm{FO} \\
\mathrm{FO}\end{array}$ & & li, lo (3), le \\
\hline & 3 & il & vil. & FO & & imonti j monti del \\
\hline & & & & & & (2) \\
\hline & & Tauro & n.p. & & $\begin{array}{l}\text { nicht im } \\
\text { GRADIT }\end{array}$ & \\
\hline & & $a$ & prep. & FO & & \\
\hline & 0 & sud & s.m. & FO & & \\
\hline & & $e$ & cong. & FO & & \\
\hline & & $i$ & art.det. & $\mathrm{CO}$ & & \\
\hline & 1 & Monti & n.p. & & nicht im & monsoni \\
\hline & & del & el.n.p. & FO & & \\
\hline & 17 & Ponto & el.n.p. & & & $\begin{array}{l}0 \text { (13), Ponte (3), } \\
\text { Polo }\end{array}$ \\
\hline & & $a$ & prep. & FO & & \\
\hline & 0 & nord. & s.m. & $\mathrm{AU}$ & & \\
\hline & & Verso & prep. & $\mathrm{AU}$ & & \\
\hline & 1 & occidente & s.m. & $A U$ & & 0 \\
\hline & & l'altopiano & s.m. & $A D$ & & \\
\hline & 10 & orlato & part.pass.v.tr. & $\mathrm{CO}$ & & $0(10)$ \\
\hline & & dai & prep.art. & $\mathrm{FO}$ & & \\
\hline & 4 & monti & s.m. & FO & & $0(4)$ \\
\hline & & si & pron.pers. & FO & & \\
\hline & $\begin{array}{r}13+1 \\
\text { acc. }\end{array}$ & apre & v.pronom.intr. & $\mathrm{CO}$ & & $\begin{array}{l}\text { appoggia (acc.), } \\
\text { aprono, appogiano } \\
(2), 0(7) \text {, appiana, } \\
\text { appoggiano, ap- } \\
\text { paiono }\end{array}$ \\
\hline & & sul & prep.art. & FO & & \\
\hline & 2 & $\begin{array}{l}\text { Mediterra- } \\
\text { neo } \\
\text { con }\end{array}$ & $\begin{array}{l}\text { n.p.m. } \\
\text { prep. }\end{array}$ & & & $0(2)$ \\
\hline & 0 & una & art.indet. & FO & & \\
\hline & & serie & s.f. & FO & & \\
\hline & 0 & $d i$ & prep. & FO & & \\
\hline & & vallate & s.f. & $A U$ & & \\
\hline & 5 & che & pron.rel. & FO & & $0(5)$ \\
\hline & & danno & v.tr. & FO & & \\
\hline & 0 & alla & prep.art. & FO & & \\
\hline & & costa & s.f. & FO & & \\
\hline & 1 & un & art.indet. & FO & & $0(1)$ \\
\hline 5 & & & & & & \\
\hline (S. 123) & & Le & art.det. & FO & & \\
\hline 25 & 0 & attività & s.f. & FO & & \\
\hline Lücken & & produttive & agg. & $\mathrm{CO}$ & & \\
\hline & $\begin{array}{r}5+12 \\
\text { acc. }\end{array}$ & pur & cong. & $\mathrm{CO}$ & $\begin{array}{l}\text { Marke von } \\
\text { seppure }\end{array}$ & $\begin{array}{l}0(2), \text { poco }(\text { acc. }) \\
(5), \text { poche }(\text { acc. })(2), \\
\text { prima (acc.), però, }\end{array}$ \\
\hline
\end{tabular}




\begin{tabular}{|c|c|c|c|c|c|c|}
\hline Text & Fehler & Wort & Wortart & Marke & Kommentar & Lösungsvorschläge \\
\hline & & & & & & $\begin{array}{l}\text { poste, particolar- } \\
\text { mente (acc.), più } \\
\text { (acc.) (3), parevano }\end{array}$ \\
\hline & & in & prep. & FO & & \\
\hline & \multirow[t]{2}{*}{1} & crescita & s.f. & $A D$ & & crescità (2) \\
\hline & & soprattutto & avv. & FO & & \\
\hline & \multirow[t]{2}{*}{0} & $\begin{array}{l}\text { nell'agricol- } \\
\text { tura }\end{array}$ & s.f. & FO & & \\
\hline & & sono & v.intr. & FO & & \\
\hline & \multirow[t]{2}{*}{1} & arretrate & agg. & $A U$ & & arrese \\
\hline & & $i$ & art.det. & $\mathrm{CO}$ & & \\
\hline & \multirow[t]{2}{*}{6} & disoccupati & s.m. & $A U$ & & $\begin{array}{l}0 \text { (3), disocupati (2), } \\
\text { disocupa }\end{array}$ \\
\hline & & sono & v.intr. & FO & & \\
\hline & \multirow[t]{2}{*}{2} & numerosi & agg. & FO & & numeri, numerose \\
\hline & & e & cong. & FO & & \\
\hline & \multirow[t]{2}{*}{0} & lo & art.det. & FO & & \\
\hline & & sviluppo & s.m. & FO & & \\
\hline & \multirow[t]{3}{*}{0} & delle & prep.art. & FO & & \\
\hline & & industrie & s.f. & FO & & \\
\hline & & $e$ & cong. & FO & & \\
\hline & \multirow[t]{2}{*}{1} & $d e l$ & prep.art. & FO & & 0 \\
\hline & & terziario & s.m. & TS econ. & & \\
\hline & \multirow[t]{2}{*}{1} & sono & v.intr. & FO & & 0 \\
\hline & & ancora & avv. & FO & & \\
\hline & \multirow[t]{2}{*}{8} & $\begin{array}{l}\text { insoddisfa- } \\
\text { centi }\end{array}$ & agg. & $\mathrm{CO}$ & & $\begin{array}{l}\text { insoddisfatti (3), in- } \\
\text { soddisfazione, } 0 \text {, in- } \\
\text { soddisfatte, insoddi- } \\
\text { sfacente, insoddisfa- } \\
\text { cienti }\end{array}$ \\
\hline & & mentre & cong. & FO & & \\
\hline & \multirow[t]{2}{*}{1} & il & art.det. & FO & & 0 \\
\hline & & turismo & s.m. & $A U$ & & \\
\hline & \multirow[t]{2}{*}{9} & registra & v.tr. & $A D$ & & $\begin{array}{l}\text { regionale dà, regio- } \\
\text { nale }(3), 0(2) \text {, regi- } \\
\text { nale, regia, regime }\end{array}$ \\
\hline & & risultati & s.m. & FO & & \\
\hline & \multirow[t]{2}{*}{4} & migliori. & agg. & FO & & $\begin{array}{l}\text { migliorato (2), mi- } \\
\text { gliore, } 0\end{array}$ \\
\hline & & Negli & prep.art. & FO & & \\
\hline & \multirow[t]{2}{*}{0} & anni & s.m. & FO & & \\
\hline & & più & avv. & FO & & \\
\hline & \multirow[t]{2}{*}{0} & recenti & agg. & FO & & \\
\hline & & un & art.indet. & FO & & \\
\hline & \multirow[t]{2}{*}{8} & piano & s.m. & FO & & $\begin{array}{l}0(5), \text { picco }(2), \\
\text { piunto }\end{array}$ \\
\hline & & $d i$ & prep. & $\mathrm{FO}$ & & \\
\hline & \multirow[t]{2}{*}{$\begin{array}{r}12+4 \\
\text { acc. }\end{array}$} & riforme & s.f. & $A D$ & & $\begin{array}{l}0(6) \text {, rifiuti, riferi- } \\
\text { mento (3), riforza- } \\
\text { mento, rifacimento, } \\
\text { riforma (acc.) (4) }\end{array}$ \\
\hline & & basato & $\begin{array}{l}\text { part.pass.v.pro- } \\
\text { nom.intr. }\end{array}$ & $A D$ & & \\
\hline
\end{tabular}




\begin{tabular}{|c|c|c|c|c|c|c|}
\hline \multirow[t]{12}{*}{ Text } & Fehler & Wort & Wortart & Marke & Kommentar & Lösungsvorschläge \\
\hline & 17 acc. & su & prep. & & & sui (acc.) (17) \\
\hline & & tagli & s.m. & $\begin{array}{l}\text { CO TS a- } \\
\text { min.az. }\end{array}$ & & \\
\hline & 0 & alla & prep.art. & FO & & \\
\hline & & spesa & loc.s.f. & $\begin{array}{l}\text { TS } \\
\text { dir.amm. }\end{array}$ & & \\
\hline & 2 & pubblica & el.loc.s.f. & $\begin{array}{l}\text { el.TS } \\
\text { dir.amm. }\end{array}$ & & $\begin{array}{l}\text { pubblicaria, pub- } \\
\text { bliclo }\end{array}$ \\
\hline & & e & cong. & $\mathrm{FO}$ & & \\
\hline & 18 & $\begin{array}{l}\text { privatizza- } \\
\text { zioni }\end{array}$ & s.f. & $\mathrm{CO}$ & & $\begin{array}{l}0(7) \text {, privatizzan- } \\
\text { dole, privativa- } \\
\text { mente, privatizzata } \\
\text { (6), privatizzati (2), } \\
\text { privatizzazione (1) }\end{array}$ \\
\hline & & hanno & v.aus. & FO & & \\
\hline & 3 & consentito & v.tr. & FO & & $\begin{array}{l}\text { conseguenza, con- } \\
\text { seguenze, } 0\end{array}$ \\
\hline & & al & prep.art. & FO & & \\
\hline & $\begin{array}{r}3+4 \\
\text { acc. }\end{array}$ & Paese & s.m. & FO & & $\begin{array}{l}\text { Parlamento (4) } \\
\text { (acc.), Palestina, } \\
\text { Papa, } 0\end{array}$ \\
\hline
\end{tabular}

Tabelle 34: Wortvorkommen und Lösungsvorschläge der Texte 4 und 5 des C-Tests der Klasse III C, Pestalozzi

In den beiden Texten befinden sich je 25 Lücken, von denen insgesamt 18 richtig ergänzt wurden. Neben Wörtern aus dem fundamentalen Wortschatz (Fo) handelt es sich hierbei um das Element Mar aus dem geographischen Eigennamen Mar Nero (Schwarzes Meer), um zwei Bezeichnungen (mit 3 Vorkommnissen) für Himmelsrichtungen, die dem Wortschatz des hohen Gebrauchs (AU) zugeordnet sind, und um ein Phrasemelement aus dem allgemeinen Wortschatz, das bereits im zweiten Text von allen richtig vervollständigt wurde: catene aus catene montuose (Co; Gebirgskette).

Von den übrigen Lücken wurden 19 von ein bis vier Lernenden nicht bzw. nicht richtig gefüllt und 14 von fünf oder mehr. Mit Ausnahme von Mar (Meer), das in zwei geographischen Eigennamen vorkommt und je einmal falsch ergänzt wurde (Mar Egeo und Mar Mediterraneo, Ägäisches Meer, Mittelmeer), Monti aus Monti del Ponto (Pontusgebirge), den AU-gekennzeichneten Wörtern occidente (Westen) und arretrate (rückständig), einem Wort der hohen Disponibilität (AD; crescita, Wachstum) und dem Phrasemelement spesa aus dem verwaltungsrechtssprachlichen spesa pubblica (öffentliche Ausgaben, Staatsausgaben) zählen die von weniger Falschlösungen betroffenen Wörter zum fundamentalen Wortschatz (Fo).

Nur drei Wörter, die alle aus dem fundamentalen Wortschatz stammen, wurden viermal nicht oder falsch vervollständigt. Im vierten Text ist es einmal das Zahlwort due (zwei), das direkt am Satzanfang steht und von mehreren SchülerInnen zu Präpositionen ergänzt wurde, von denen die artikulierte delle sowohl logisch als auch morphosyntaktisch akzeptabel ist. Überdies wurde 
mo-nti (Berge) von vier Lernenden gar nicht vervollständigt, was kaum an mangelnder lexikalischer Kompetenz gelegen haben wird, sondern wahrscheinlich damit zusammenhängt, dass die angrenzenden Lücken, die zehnbzw. 13-mal nicht korrekt bearbeitet wurden, das Textverständnis zu sehr reduziert hatten (s.a. 8.3.5.4). Das dritte viermal falsch gelöste Wort ist der Komparativ migl-iori im fünften Text. Hier unterliefen neben einer nicht erfolgten Ergänzung drei grammatikalische Fehler, da die gebildeten Adjektive nicht wie erfordert in der maskulinen Pluralform, sondern im Singular gebildet wurden, obwohl es sich bei dem Bezugswort um ein angrenzendes und somit nicht getilgtes Wort aus dem Bereich des fundamentalen Wortschatzes handelt, das allen bekannt gewesen sein dürfte (risultati, Ergebnisse).

8.3.5.4 Häufigste Falschlösungen in den Texten 4 und 5 des C-Tests der III C

Eine ganze Reihe der 14 Wörter, die vermehrt fehlerhaft bearbeitet wurden, ist gleich von sehr vielen Lernenden nicht korrekt ergänzt worden. Um welche Wörter es im Einzelnen geht und zu wie vielen und welchen Falschlösungen sie führten, ist in Tabelle 35 ersichtlich.

\begin{tabular}{|c|c|c|c|c|c|c|}
\hline Text & $\begin{array}{r}n \\
\text { Fehler }\end{array}$ & Wort & Wortart & Marke & $\begin{array}{l}\text { Kommen- } \\
\text { tar }\end{array}$ & Lösungsvorschläge \\
\hline 4 & 6 & bagnata & v.tr. & $\mathrm{FO}$ & & bagnato (5), 0 \\
\hline \multirow[t]{5}{*}{ (S. 122) } & & la & pron.pers. & FO & & li, lo (3), le \\
\hline & 17 & Ponto & el.n.p. & & $\begin{array}{l}\text { Monti del } \\
\text { Ponto }\end{array}$ & 0 (13), Ponte (3), Polo \\
\hline & 10 & orlato & agg. & $\mathrm{CO}$ & & $0(10)$ \\
\hline & $\begin{array}{r}13+1 \\
\text { acc. }\end{array}$ & apre & $\begin{array}{l}\text { v.pro- } \\
\text { nom.intr. }\end{array}$ & $\mathrm{CO}$ & & $\begin{array}{l}\text { appoggia (acc.), } \\
\text { aprono, appogiano (2), } \\
0 \text { (7), appiana, appog- } \\
\text { giano, appaiono }\end{array}$ \\
\hline & 5 & che & pron.rel. & $\mathrm{FO}$ & & $0(5)$ \\
\hline 5 & $\begin{array}{r}5+12 \\
\text { acc. }\end{array}$ & pur & cong. & $\mathrm{CO}$ & $\begin{array}{l}\text { Marke von } \\
\text { seppure }\end{array}$ & $\begin{array}{l}0(2), \text { poco }(\text { acc.) }(5), \\
\text { poche }(\text { acc.) }(2), \text { prima } \\
\text { (acc.), però, particolar- } \\
\text { mente (acc.), più (acc.) } \\
\text { (3), parevano, poste }\end{array}$ \\
\hline \multirow[t]{5}{*}{ (S. 123) } & 6 & disoccupati & s.m. & $A U$ & & $\begin{array}{l}0 \text { (3), disocupati (2), } \\
\text { disocupa }\end{array}$ \\
\hline & 8 & $\begin{array}{l}\text { insoddisfa- } \\
\text { centi }\end{array}$ & agg. & $\mathrm{CO}$ & & $\begin{array}{l}\text { insoddisfatti (3), insod- } \\
\text { disfazione, } 0 \text {, insoddi- } \\
\text { sfatte, insoddisfacente, } \\
\text { insoddisfacienti }\end{array}$ \\
\hline & 9 & registra & v.tr. & $A D$ & & $\begin{array}{l}\text { regionale dà, regionale } \\
\text { (3), } 0(2) \text {, reginale, re- } \\
\text { gia, regime }\end{array}$ \\
\hline & 8 & piano & s.m. & $\mathrm{FO}$ & & 0 (4), picco (3), piunto \\
\hline & & $d i$ & prep. & FO & & \\
\hline
\end{tabular}




\begin{tabular}{|c|c|c|c|c|c|c|}
\hline \multirow[t]{4}{*}{ Text } & $\begin{array}{r}n \\
\text { Fehler }\end{array}$ & Wort & Wortart & Marke & $\begin{array}{l}\text { Kommen- } \\
\text { tar }\end{array}$ & Lösungsvorschläge \\
\hline & $\begin{array}{r}12+4 \\
\text { acc. }\end{array}$ & riforme & s.f. & $\mathrm{AD}$ & & $\begin{array}{l}0(6) \text {, rifiuti, riferimento } \\
(3) \text {, riforzamento, rifaci- } \\
\text { mento, riforma (acc.) } \\
\text { (4) }\end{array}$ \\
\hline & & basato & agg. & $A D$ & & \\
\hline & 18 & $\begin{array}{l}\text { privatizza- } \\
\text { zioni }\end{array}$ & s.f. & $\mathrm{CO}$ & & $\begin{array}{l}0(7) \text {, privatizzandole, } \\
\text { privativamente, priva- } \\
\text { tizzata }(6) \text {, privatizzati } \\
(2) \text {, privatizzazione }(1)\end{array}$ \\
\hline
\end{tabular}

Tabelle 35: Häufige Falschlösungen im vierten und fünften Text des C-Test der Klasse III C, Pestalozzi

Unter den oft falsch gelösten Wörtern befinden sich Verben, Nomen, je ein Element eines Eigennamens und ein Pronomen, aber auch Adjektive, eine Konjunktion und eine Präposition. Ein Drittel von ihnen ist Teil des fundamentalen Wortschatzes; zählt man noch die Ausdrücke aus dem Bereich des hohen Gebrauchs (AU) und der hohen Disponibilität (AD) hinzu, kommt man auf einen Anteil von zwei Dritteln an Wörtern aus dem Basiswortschatz, während die restlichen Co-markiert sind. Sieben wurden von mindestens acht Lernenden nicht oder falsch ergänzt, und auch davon stammt noch fast die Hälfte aus dem Basiswortschatz ( 2 AD, 1 FO). Im Folgenden sollen diese mindestens achtmal falsch gelösten Wörter vorgestellt werden.

Mit 13 nicht erfolgten Ergänzungen und vier falschen wurde erneut ein Teil eines geographischen Eigennamens nicht korrekt bearbeitet, nämlich Po-nto aus Monti del Ponto (Pontusgebirge) im vierten Text. Ohne geographisches Fachwissen, das auf der Verankerung des Toponyms im mentalen Lexikon basiert, ist hier keine korrekte Lösung möglich.

Ponto ist nicht das einzige Wort, dessen Ursache für die ausgebliebene oder falsche Bearbeitung auf lexikalischer Ebene gesucht werden kann. Genauso verhält es sich wahrscheinlich mit dem Adjektiv orl-ato (umsäumt) aus dem allgemeinen Wortschatz (CO), für das zehn Lernende keine Ergänzung erbringen. Orlato tritt auch in einem Lehrbuchtext der Klasse an der Schule Morante auf und wurde dort vom Lehrer ausführlich mit seinen Schülern besprochen, weil das Wort vielen unbekannt war. $\mathrm{Zu}$ wissen, ob dieses gemeinsame Erarbeiten des Wortschatzes dazu ausgereicht hätte, die Klasse Morante mehr richtige Lösungen für den besprochenen Ausdruck finden zu lassen als die Klasse III C, wäre interessant.

Von lexikalischer Schwierigkeit war für einige Lernende anscheinend auch die Vervollständigung des Co-markierten Adjektivs insoddisfacenti (unzufriedenstellende), das von einem gar nicht ergänzt wurde, von einem anderen zum Nomen insoddisfazione (so dass hier das Problem auf morphosyntaktischer Ebene zu lokalisieren ist) und von drei weiteren zu zwei Formen des ähnlichen Adjektivs insoddisfatto (unzufrieden). Immerhin zwei Lernende produzierten 
das richtige Lexem, allerdings mit einem grammatikalischen und einem orthographischen Fehler.

Bei weiteren häufigen Falschlösungen spielte die lexikalische Komponente wahrscheinlich eine Rolle, jedoch nicht die einzige. So kann zum Beispiel angenommen werden, dass vielen die Konjunktion pur bekannt war, allerdings eher rezeptiv als produktiv. Eigentlich erfordert sie ein Gerundium; im vorliegenden Satz hingegen ist dieses nicht expliziert, was den Testteilnehmern höchstwahrscheinlich die korrekte Vervollständigung erschwerte. Bemerkenswert ist, dass zwar nur zwei Testpersonen das Wort pur bildeten, weitere zwölf aber insgesamt drei akzeptable Lösungsvorschläge mit Adverbien und einem Adjektiv fanden, die von ihnen wohl auch dadurch, dass sie alle zum fundamentalen Wortschatz gehören, eher produziert werden konnten als die Konjunktion im Originaltext.

Ebenfalls sehr oft (13-mal) wurde ap-re des zweiteiligen, reflexiven Verbs aprirsi (sich öffnen) nicht korrekt gelöst, wobei sieben Testteilnehmer die Lücke gar nicht vervollständigten. Auch wenn das Verb zum allgemeinen Wortschatz (CO) zählt, war es sicherlich allen Schülerinnen und Schülern bekannt und kann es bei Beherrschung der Wortbildungsregeln auch leicht erschlossen werden, da es sich aus dem sehr häufigen Verb aprire (FO; öffnen) und dem Reflexivpronomen si (sich) zusammensetzt. Dass so viele es im Test nicht bildeten, liegt also vermutlich am Kontext, in den sich das getilgte Wort eingliedert: Im Satz gehen dem Prädikat inklusive des nicht von der Tilgung betroffenen Subjekts sieben Wörter voraus. Darauf bezieht sich das bereits erwähnte Adjektiv orlato, das zehn Lernende gar nicht ergänzten. Bei der Ausformulierung des Prädikats kannten also viele Testteilnehmer ein Detail der Satzaussage nicht. Aber die wesentliche Ursache für die vermehrte Schwierigkeit mit dem Prädikat ist schätzungsweise darin zu suchen, dass ihnen bei ,die von den Bergen gesäumte Hochebene“ „sich öffnen“ gar nicht in den Sinn kam. Ferner zählt das Wort für „Hochebene“ im GRADIT zwar zu den Wörtern der hohen Disponibilität (AD), also zu denen, die wir oft denken, aber selten produzieren und rezipieren, fällt es aber in der Mittelschule im Fach Erdkunde gewöhnlich bei der morphologischen Beschreibung eines Landes, in dem sich Berge und Hochebenen befinden, und ist es aus demselben Grund auch an vielen Stellen der Lehrbücher verzeichnet. Es handelt sich also fast um ein Fachwort, zumindest ein fachtypisches Wort. Auf die Lösungsvorschläge bezüglich des Prädikats zurückkommend, fällt auf, dass das Wort mit und ohne Rechtschreibfehlern zu drei anderen Verben ergänzt wurde, von denen nur zwei reflexiv gebraucht wurden (das Reflexivpronomen ist im Satz aber ungetilgt gegeben). Keines dieser Verben ist logisch annehmbar und viele sind in der dritten Person Plural konjugiert, also auch morphosyntaktisch unangemessen.

Weiter ist das Prädikat regi-stra (verzeichnen) zu erwähnen, das von acht Lernenden falsch und von zwei weiteren gar nicht vervollständigt wurde. Es 
befindet sich in einem sehr kurzen Satz, der zu einem längeren Satzgefüge gehört und in dem weder das Subjekt noch das direkte Objekt von der Worttilgung betroffen ist. Außerdem darf man davon ausgehen, dass den Testteilnehmern die beiden Ausdrücke bekannt waren (turismo (AU; Tourismus) und risultati (FO; Ergebnisse)), so dass ihnen vielleicht das zu ergänzende Prädikat ebenfalls geläufig, aber anscheinend aus ihrem mentalen Lexikon nicht so leicht abrufbar war. Vermutlich führte der Umstand, dass ihnen kein passendes Verb, das mit regi- beginnt, einfiel, dazu, dass sie stattdessen als Lösungsvorschläge u.a. sechsmal Adjektive und Nomen lieferten, die sowohl semantisch als auch grammatikalisch nicht korrekt sind. Davon abgesehen ist das Kuriose an dem zu ergänzenden Wort, dass es dem Wortschatzbereich der hohen Disponibilität zugeteilt ist.

In diesem Satz gibt es gleich zwei AD-gekennzeichnete Wörter, die zu vervollständigen sind, und ein weiteres, nicht getilgtes aus demselben Wortschatzbereich. Insgesamt sind im C-Test der Klasse III C fünf Wörter der hohen Disponibilität vertreten, von denen drei zur Hälfte getilgt sind, so dass der Anteil an Wörtern dieser Art damit etwas höher liegt als in den anderen vier C-Tests. Schaut man sich die fünf Wörter an, stellt sich die Frage, ob sie tatsächlich so selten produziert und rezipiert werden und man verhältnismäßig häufiger an sie denkt: crescita (Wachstum), registrare (registrieren), riforma (Reform) und basato (basierend) im fünften Text und altopiano (Hochebene) im vierten.

Alle übrigen mindestens achtmal falsch gelösten Wörter befinden sich im letzten Satz von Text 5, welcher deshalb zur besseren Veranschaulichung der Fehlerart und ihrer möglichen Ursachen in Abbildung 13 wiedergegeben und übersetzt ist (die halbierten Wörter sind hervorgehoben und die oft falsch gelösten markiert).

Negli anni [0] più recenti [0] un piano [8] di riforme [12] basato su [17acc.] tagli alla [0] spesa pubblica [2] e privatizzazioni [18] ha consentito [3] al Paese [3] di uscire dalla crisi scoppiata nel 2001 e dal 2002 l'economia è in ripresa.

M.Ü.: In den letzten Jahren hat ein auf Kürzungen der Staatsausgaben und Privatisierungen basierender Reformplan dem Land ermöglicht, aus der 2001 ausgebrochenen Krise herauszukommen, und seit 2002 erholt sich die Wirtschaft.

Abbildung 13: Dem Lehrbuch entnommener Satz (Forte \& Ubertazzi 2008, S. 123) mit Hervorhebung der getilgten Wörter, Fehlerzahl und Übersetzung

Während der Satz mit zwei von allen Testteilnehmern richtig vervollständigten Lücken beginnt, wirft sofort danach sein Subjekt pi-ano di rif-orme anscheinend viele Probleme auf. Diese beschränken sich im ersten Teil noch auf vier fehlende Ergänzungen sowie weitere vier logisch nicht akzeptable Alternativvorschläge, umfassen im zweiten Teil, dem Wort der hohen Disponibilität (AD) riforme, aber sechs ausbleibende Ergänzungen sowie vier alternative Nomen, die alle logisch-semantisch nicht funktionieren (man beachte z.B. rifiuti (Müll)), obwohl einige in die richtige Richtung gehen. Als Lösung akzeptiert 
werden kann hingegen die von vier SchülerInnen erbrachte Ergänzung zur Singularform riforma.

Privatizzazioni ist das mit 18 fehlenden oder nicht korrekten Ergänzungen am häufigsten falsch gelöste Wort im zweiten Teil des C-Tests. Dieser hohe Fehlerwert kann vermutlich zum Teil mit mangelnder lexikalischer Kompetenz des Co-markierten Wortes erklärt werden, wenn es sich auch um eine wirtschaftspolitische Praxis handelt, über welche die Schülerinnen und Schüler im Fach Erdkunde unterrichtet worden waren. Die zahlreichen aus ganz anderen und morphosyntaktisch falschen Wortarten bestehenden Lösungsvorschläge (Adjektive, ein Adverb und ein Gerundium) deuten aber vor allem darauf hin, dass viele Testteilnehmer einerseits vom Inhalt des Satzes, in dem sie vermehrt das dreigliedrige Subjekt nicht vervollständigen konnten, nur sehr wenig aufgenommen hatten. Andererseits reichten ihre sprachlichen Fähigkeiten anscheinend nicht aus, um zu erkennen, dass privatizzazioni eines von zwei präpositionalen Objekten ist, die zusammen mit dem Adjektiv „basierend“ den Reformplan näher bestimmen. In der Tat ist dies eine syntaktisch und grammatikalisch komplizierte Konstruktion, die durch einen einfachen Relativsatz ersetzt werden könnte, um das morphosyntaktische Gewicht des Satzes zu reduzieren und so das Verständnis wichtiger Textaussagen besser zu garantieren.

\subsection{Klassenübergreifende Zusammenfassung der Testergebnisse}

Generell ist bei der Analyse der Lösungsvorschläge deutlich geworden, dass die Bearbeitung der getilgten Wörter neben offensichtlichen lexikalischen Lücken vermutlich sehr oft aufgrund unzureichender Textkompetenz nicht oder falsch erfolgte. In vielen Fällen ist anzunehmen, dass die grammatikalische und syntaktische Komplexität der Sätze das Textverständnis zu stark beeinträchtigte. Dadurch konnten bei der Bearbeitung einer Lücke des Öfteren diverse in einem Satz vorausgehende Lücken schon nicht vervollständigt werden und waren somit bei der Verständnissicherung nicht hilfreich. Dieses Problem ergibt sich natürlich nicht nur in den hier untersuchten Texten, die zur Hälfte aus getilgten Wörtern bestehen. Ganz allgemein können Fehlinterpretationen im Verständnisprozess weitere Fehlinterpretationen nach sich ziehen. Auf der anderen Seite erwecken die Lösungsvorschläge nicht selten den Eindruck, dass sich die Lernenden bei der Testbearbeitung allein auf das jeweilige zu vervollständigende Wort konzentrierten, ohne den Makrokontext angemessen zu berücksichtigen, egal wie komplex er sich gestaltete. Nur so scheinen manche logisch und auch grammatikalisch völlig unpassenden Ergänzungen erklärbar zu sein. Dies könnte als Indiz dafür ausgelegt werden, dass die Lernenden nicht ausreichend für die Relevanz textbezogener Fähigkeiten sensibilisiert waren. 
Beispielsweise waren sie anscheinend oft nicht imstande, die Bezüge zwischen einzelnen Textkomponenten nachzuvollziehen. Das zeigte sich konkret daran, dass sie in einigen Fällen bei der Vervollständigung eines Relativpronomens oder einer Partizipialendung keine Kongruenz zum Bezugswort herstellten. Diese sprachbezogenen Schwierigkeiten der Lernenden stimmen vor allem nachdenklich, wenn man bedenkt, dass der Großteil des beobachteten Erdkundeunterrichts auf der Erarbeitung von Texten und der Kontrolle darüber, ob die Inhalte von den Schülerinnen und Schülern wiedergegeben werden konnten, beruhte. Im folgenden Kapitel zu den Unterrichtsbeobachtungen wird daher der Frage nachgegangen, ob und wie die Lehrkräfte den textbezogenen Verständnisprozess der Lernenden unterstützten und überprüften oder ob sich ihre Fragen zu den erarbeiteten Texten nur auf leichter zu erwerbendes Oberflächenwissen beschränkten.

Die Untersuchung der Falschbearbeitungen in den einzelnen C-Tests führt mithilfe der zur Erklärung des Antwortverhaltens herangezogenen Hypothesen zu dem Ergebnis, dass Falschlösungen vor allem aus der Fokussierung des getilgten Wortes unter Ausblendung des größeren Zusammenhangs, der grammatikalisch-syntaktischen Komplexität der Satzgefüge und aus Mängeln in der lexikalischen Kompetenz resultieren. Darüber hinaus sind für eine klassenübergreifende Fehlertypologie orthographische Unkorrektheiten zu verzeichnen, die vor allem durch eine allzu flüchtige Testbearbeitung aufgetreten sein werden, zumal nicht selten einzelne Buchstaben oder ganze Silben des nicht getilgten Teils der Wörter wiederholend ergänzt wurden. Unter die Kategorie der flüchtigen Bearbeitung und des Mangels an Konzentration fallen außerdem Ergänzungen vollständiger Wörter, die für die Bearbeitung vor der Aushändigung der Tests ausdrücklich ausgeschlossen worden waren.

Wenn man aufgrund der Gleichaltrigkeit der Testpersonen und ihrer in fast allen Fällen vergleichbaren schulischen Laufbahn davon ausgehen kann, dass ein beträchtlicher Teil ihres mentalen Lexikons sehr ähnlich beschaffen sein wird, erklären sich Bearbeitungsdifferenzen auf lexikalischer Ebene zwischen den einzelnen Klassen primär dadurch, dass in den fünf Tests innerhalb der verschiedenen Wortschatzbereiche unterschiedliche Wörter auftreten und ihre korrekte Vervollständigung eben nicht nur und des Öfteren nicht einmal erstranging von diesen Wörtern selbst, sondern vom Verständnis des sprachlichen Umfelds abhängt, in dem sie erscheinen. Es lässt sich aber dennoch beobachten, dass alle fünf Testgruppen einen durchschnittlichen Fehlersatz pro Wort aus einem bestimmten Bereich erzielten, der für die Ausdrücke aus dem Basiswortschatz und die Eigennamen am niedrigsten ist und mit den seltener gebrauchten Wörtern zunimmt. Eine Ausnahme hierzu bilden nur die auch zum Basiswortschatz zählenden Ausdrücke der hohen Disponibilität (AD), für die eine durchschnittlich höhere Fehlerzahl zu verzeichnen ist. Allerdings sind 
diese Wörter nur in drei der fünf Tests vertreten. Dass die Einstufung bestimmter Vorkommnisse als AD-Wörter Fragen aufwirft, wurde z.B. unter Punkt 8.3.5.4 (Besprechung der häufigsten Fehler der Klasse III C) erläutert.

Dass die Lernenden zum ersten Mal einen C-Test vervollständigten, wird zu den nicht besonders guten Resultaten beigetragen haben. Auch wenn sie wussten, dass er keinen Einfluss auf ihre Bewertung im Fach Erdkunde haben und er der Lehrkraft nicht vorgelegt werden würde, erweckten die Testteilnehmer dennoch auch - auch auf die anwesenden Lehrkräfte - den Eindruck, bei der Einführung in den Test aufmerksam zugehört zu haben und bei der Testdurchführung gewissenhaft und konzentriert vorzugehen. Deshalb darf angenommen werden, dass sie mit dem sprachlichen Niveau des Testmaterials überfordert waren und insgesamt bessere Ergebnisse erzielt hätten, wenn die zugrundeliegenden Lehrbuchtexte vor allem morphosyntaktisch einfacher strukturiert wären. In diesem Zusammenhang soll noch einmal betont werden, dass das Kriterium der Textauswahl lediglich war, wenige Wochen zuvor im Unterricht erarbeitete Texte für die Konstruktion der Texte zu verwenden und nicht, aus den Lehrbüchern besonders anspruchsvolle herauszufiltern.

Im Zuge der Lehrbuchanalyse in Kapitel 7 wurden nicht nur die Seiten, denen die C-Testtexte entnommen sind, sondern für jedes Lehrbuch auch noch weitere Seiten auf ihre Zusammensetzung und die Komplexität ihres Sprachmaterials hin untersucht (pro Buch insgesamt 6-8), wobei die einzelnen Befunde eines sich ähnelnden Schwierigkeitsgrads der sprachlichen Gestaltung die Vermutung nahelegen, dass in den Lehrbuchtexten grundsätzlich ein anspruchsvolles, den Sprachstand der Lernenden oft übersteigendes Register verwendet wird. Das soll an dieser Stelle noch einmal an der bloßen Länge der Sätze festgemacht werden, in denen sich die getilgten Wörter der fünf Tests befinden. Wie schon bei der Lehrbuchanalyse gilt dabei als Satz alles bis zu einem Punkt und als Wort das graphische Wort, also das, was sich zwischen zwei Leerzeichen befindet, mit Ausnahme von Zahlen und Symbolen, die keinen eigenen Eintrag im GRADIT haben (z.B. das Prozentzeichen).

In der folgenden Tabelle (Tab. 36) steht SL für Satzlänge, und für den dritten und vierten C-Testtext der Klasse III A sind die Werte zusammen ermittelt, weil sich ein sehr langer Satz, der nach einem Doppelpunkt eine Aufzählung verschiedener Gliederungspunkte enthält, über beide Texte erstreckt.

\begin{tabular}{lrrrrrr}
\hline Text & Satz & Vespucci & Morante & III A & III B & III C \\
\hline Text 1 & 1 & 17 & 30 & 30 & 9 & 30 \\
& 2 & 24 & 17 & 24 & 32 & 39 \\
$\varnothing$ SL & 3 & 41 & & & 52 & \\
Text 2 & & 27 & 24 & 27 & 31 & 35 \\
& 1 & 45 & 8 & 34 & 28 & 18 \\
& 2 & 26 & 16 & 20 & 15 & 36 \\
& 3 & & 22 & 45 & 23 & \\
$\varnothing \mathrm{SL}$ & 4 & & 22 & & & \\
\hline
\end{tabular}




\begin{tabular}{lrrrrrr}
\hline Text & Satz & Vespucci & Morante & III A & III B & III C \\
\hline Text 3 & 1 & 53 & 25 & 32 & 20 & 55 \\
& 2 & & 12 & 123 & 17 & \\
$\varnothing$ SL & 3 & & 18 & & 22 & \\
Text 4 & & 53 & 18 & 78 & 20 & 55 \\
& 1 & 32 & 46 & s. Text 3 & 23 & 18 \\
& 2 & 13 & 47 & & 17 & 40 \\
$\varnothing$ SL & 3 & 15 & & & 25 & \\
Text 5 & 1 & 20 & 47 & & 22 & 29 \\
& 2 & 18 & 26 & 57 & 23 & 31 \\
& 3 & & 13 & 24 & 37 & 32 \\
& 4 & & 13 & & & \\
$\varnothing$ SL & & 23 & 18 & 41 & 30 & 32 \\
$\varnothing \mathrm{SL}$ alle Texte & & 28 & 22 & 43 & 25 & 33 \\
\hline
\end{tabular}

Tabelle 36: Länge der Sätze mit Worttilgungen in den fünf C-Tests

Die Sätze sind im Lehrbuch an der Schule Morante am kürzesten, aber selbst dort erreichen sie eine Durchschnittslänge von 22 graphischen Wörtern. Sie steigt auf ein Maximum von 43 Wörtern im Lehrbuch der Klasse III A und liegt im Mittelwert für alle fünf C-Tests bei 30 .

Es wurde ermittelt, dass der Großteil der getilgten Wörter in diesen Sätzen, wie in quasi allen Texten konzeptioneller Schriftlichkeit (Weber 1973, S. 153), aus Substantiven besteht, in den Tests gefolgt von Adjektiven. Die Testergebnisse der fünf Klassen zeigen, dass die Schülerinnen und Schüler unter den getilgten Wörtern nicht nur diese Lexeme, sondern auch Funktionswörter wie Präpositionen und Konjunktionen häufig falsch oder gar nicht lösten. Vor allem für Letztere besteht die Unmöglichkeit einer Fehlerdiagnose, die sich nur auf das getilgte Wort bezieht und weder Mikro- und Makrokontext der zu ergänzenden Ausdrücke berücksichtigt, wie auch Grotjahn, Tönshoff und Hohenbleicher (1994, S. 139) unterstreichen ${ }^{160}$, noch die Testteilnehmer unmittelbar nach dem Test zu ihren Lösungsvorschlägen interviewt. Dennoch können für beide Wortgruppen klassenübergreifend einige einschlägige Aspekte zum Lösungsverhalten skizziert werden.

Prinzipiell ist bei der Bearbeitungskompetenz hinsichtlich der Lexeme die sprachliche von der inhaltlich-fachlichen Komponente kaum zu isolieren. Es gibt in allen fünf Tests aber einige Wörter, die eher vervollständigt werden können, wenn die Testpersonen über das spezifische fachliche Wissen verfügen und es lexikalisch abgespeichert haben, wobei sich dieses für das Unterrichtsfach Erdkunde als „weiche Naturwissenschaft“ (Lee 1996, S. 27), das auch gesellschaftswissenschaftliche Aspekte behandelt, auf ganz unterschied-

160 Das zeigt etwa auch das als Ts econ. gekennzeichnete Wort indicatore, das mit drei Vorkommnissen im C-Test der Schule Vespucci vertreten ist und höchstwahrscheinlich aufgrund des unterschiedlichen sprachlichen Umfelds zu divergierenden Falschlösungen geführt hat. 
liche Bereiche ausdehnt (z.B. Morphologie, Geologie, Wetterkunde, Soziologie, Geschichte, Politik, Wirtschaft). Hierzu zählen nicht nur geographische Eigennamen und als Fachwortschatz gekennzeichnete Wörter, sondern auch fachtypische Ausdrücke aus dem allgemeinen Wortschatz. Ein gutes Beispiel für Letztere ist masse continentali (Kontinentalmassen), das von den Lernenden fast ausnahmslos vervollständigt wurde, obwohl es weder in der Textgrundlage erscheint, noch semantisch passt. ${ }^{161}$ Auch für die korrekte Vervollständigung einiger Toponyme war fachinhaltliches Wissen erforderlich, wenn man einmal von Zufallstreffern absieht, wie es sie in erster Linie bei den Ländernamen gegeben haben kann, die den Testteilnehmern aus dem Erdkundeunterricht des zweiten Jahres der Mittelschule und auch aus anderen Kontexten bekannt waren. ${ }^{162}$ Bei Begriffen wie „Pontusgebirge“ hingegen ist es sehr unwahrscheinlich, dass sie den Testteilnehmern außerhalb des Geographieunterrichts begegneten. Aufgrund seiner selteneren Frequenz ist es nicht verwunderlich, dass der Eigenname von vielen Lernenden anscheinend nicht aktiv beherrscht wurde, obwohl sie womöglich über eine passive lexikalische Kompetenz verfügten, die es ihnen erlaubte, das Gebirge geographisch richtig einzuordnen, wenn sie seinen Namen hörten. Ähnlich verhält es sich vermutlich für weitere Toponyme wie beispielsweise im selben C-Test (III C) für diejenigen unbekannterer Länder, in denen kurdische Minderheiten leben, und im Test der III A für die der ärmeren Länder der Welt in Afrika und Asien. Ein weiteres Beispiel für eine Lücke, die ohne fachinhaltliche Kenntnisse nur schwer gefüllt werden konnte, ist die in $t$-è im Test der III C, die in zahlreichen Fällen zu tabacco vervollständigt wurde.

Tabelle 37 zeigt, welche fachwortschatzsprachlichen Ausdrücke in den einzelnen Tests zu ergänzen waren. ${ }^{163}$ Sie machen nur einen kleinen Teil der Nomen, Adjektive und Phrasemelemente aus und sind zudem, wie aus den Angaben zu den Falschlösungen ersichtlich wird, nur teilweise stark, in vielen Fällen geringfügig fehlerbehaftet und in manchen Fällen gar nicht von falschen Lösungen betroffen.

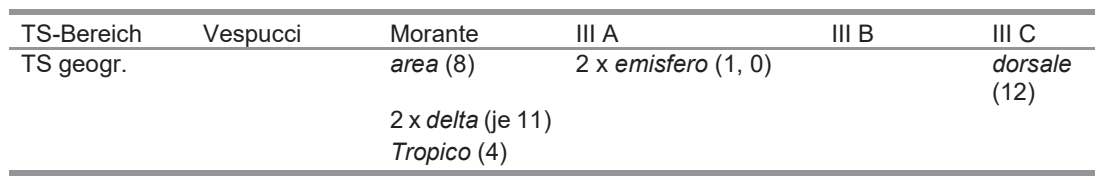

161 Das eigentlich zu ergänzende Adjektiv, contadine (bäuerlich), ist den meisten SchülerInnen wahrscheinlich bekannt, befindet sich aber durch das Bezugswort für masse und in einem Text ihres Erdkundebuchs in einem atypischen Kontext.

162 Voraussichtlich bekannte Eigennamen sind neben einigen weiteren z.B. Germ-ania und Dani-marca im Zusammenhang mit dem BIP im C-Test der Vespucci; Oceano Atla-ntico in dem der Morante; As-ia, Afr-ica und Stati Un-iti im C-Test der III A (Pestalozzi), St-ati Uniti und $C i$-na im Test der III B und As-ia und M-ar Mediterraneo in dem der III C.

163 Eine tabellarische Übersicht über alle vorkommenden fachwortschatzsprachlichen Bereiche mit ihren Übersetzungen ins Deutsche befindet sich in Kap. 7 auf S. 218 und im Anhang. 


\begin{tabular}{|c|c|c|c|c|c|}
\hline TS-Bereich & Vespucci & Morante & III A & III B & III C \\
\hline \multirow{2}{*}{\multicolumn{6}{|c|}{ TS dir. amm. }} \\
\hline & & & & & \\
\hline TS dir. intern. & & & Unione Europea (0) & & \\
\hline TS ecol. & & & fonti energetiche (1) & & \\
\hline TS econ. & $\begin{array}{l}3 \times \text { indicatore } \\
(3,2,5)\end{array}$ & & $\begin{array}{l}\text { Prodotto Interno } \\
\text { Lordo (1) } \\
\text { crescita economica } \\
(0) \\
\text { ricchezza (1) }\end{array}$ & & \\
\hline TS etnol. & & $\begin{array}{l}\text { gruppi etnici } \\
(0)\end{array}$ & & & $\begin{array}{l}\text { curda } \\
\text { (11) }\end{array}$ \\
\hline TS fis., chim. & & & positivo (2) & & \\
\hline TS mar. & & approdi (8) & & & \\
\hline TS metrol & & chilometro (0) & & & \\
\hline TS stat. & $\begin{array}{l}2 \times \text { speranza di } \\
\text { vita }(2+1)\end{array}$ & densità $(0,5)$ & tasso di natalità $(0)$ & densità $(3,5)$ & \\
\hline & & & $\begin{array}{l}2 \times \text { saldo naturale }(1 \\
+0)\end{array}$ & & \\
\hline TS tecn. & & & $\begin{array}{l}\text { macchine utensili } \\
\text { (5) }\end{array}$ & & \\
\hline n Tokens & 5 & 10 & 14 & 1 & 2 \\
\hline n Types & 2 & 9 & 12 & & \\
\hline
\end{tabular}

Tabelle 37: Getilgte Ts-Wörter der fünf C-Tests mit Angaben zu den Falschlösungen

In allen fünf Klassen führten die kontrahierten Formen aus Präpositionen und Artikeln zu vermehrten Falschlösungen, was kaum an mangelnder lexikalischer und grammatikalischer Kompetenz, sondern eher an der Nicht-Anwendung morphosyntaktischer Fähigkeiten aufgrund der unzureichenden Beachtung des sprachlichen Umfelds gelegen haben wird. Es gibt jedoch weitere grammatikalische Kategorien, die als halbierte Wörter zahlreiche Fehler verursachten, wie Gerundien (s. Vespucci) und Partizipien (s. Vespucci, III B und III C). Desweiteren erwiesen sich in allen beobachteten Klassen Relativ- und andere Pronomen sowie Konjunktionen als problematisch, wenn sie sich in langen, hypotaktisch strukturierten Sätzen summieren. Das gilt in ähnlicher Weise für die artikulierten Präpositionen, die sich manchmal auf zwei Substantive beziehen und des Öfteren in langen Attributketten auftreten.

Unter Berücksichtigung des Bildes des Leistungsniveaus in den einzelnen Klassen, das sich in den Unterrichtsbeobachtungen zeigte, und seiner Einschätzung seitens der Lehrkräfte lassen sich vorsichtig bestimmte Korrelationen zwischen den in den Lehrbüchern verwendeten sprachlichen Mitteln, der Sprachkompetenz der Lernenden und den Testergebnissen aufzeigen: Die Untersuchung in diesem Kapitel hat veranschaulicht, dass im C-Test der Klasse III A über fast alle grammatikalischen Kategorien und die verschiedenen Bereiche des Wortschatzes hinweg die wenigsten Fehler produziert wurden, obwohl die Satzgefüge besonders lang sind und der Wortschatz weniger aus dem Vocabolario di Base stammt als in den anderen Tests. Das deutet auf solide 
und womöglich überdurchschnittliche sprachliche Fähigkeiten im Vergleich zu den anderen Klassen hin. Auf der anderen Seite wurden die meisten Fehler in der Klasse III C produziert, auch wenn sich unter den Testteilnehmern der Schulen Vespucci und Morante wesentlich mehr in der Zweitsprache lernende Schülerinnen und Schüler befinden, von denen einige ihre Schullaufbahn nicht in Italien begonnen hatten. Dass sie sprachlich kompetenter wirkten als ihre Altersgenossen in der III C, die fast ausnahmslos einsprachig italienisch waren, kann nicht behauptet werden. Deshalb deutet sich hier an, wie sehr die durch die jeweiligen Lehrbücher bedingte Komplexität des Testmaterials mit den sprachlichen Fähigkeiten der Lernenden korreliert: Mit bündigeren, einfacher strukturierten Sätzen im Lehrbuch von Bersezio (2005) geringer ausfallend, führt sie zu besseren Testergebnissen als beispielsweise in der Klasse III C. Dort sind die dem Lehrbuch von Forte und Ubertazzi (2008) entnommenen Texte mit längeren und morphosyntaktisch komplizierter strukturierten Sätzen wahrscheinlich zu einem guten Teil für die schlechteren Ergebnisse verantwortlich. 


\section{Sprache im Fachunterricht: die Unterrichtsbeobachtungen}

In diesem Kapitel wird das während der empirischen Studie beobachtete sprachliche Handeln in fünf achten Klassen in den Blick genommen. Zunächst erfolgt eine Beschreibung zeitorganisatorischer Aspekte der Unterrichtsgestaltung (9.1.1) und der Räumlichkeiten, in denen der Erdkundeunterricht stattfand (9.1.2). Danach werden die drei vorherrschenden Formen der Unterrichtsgestaltung erläutert (9.1.3): Lehrervortrag/Frontalunterricht (9.1.3.1), Abfragungen (9.1.3.2) und Gruppenarbeit (9.1.3.3), wobei Letztere nur in einer Klasse regelmäßig durchgeführt wurde und somit einen innovativen Kontrast zur ansonsten traditional gehaltenen Unterrichtsgestaltung darstellt. In einzelnen Unterkapiteln zu den dominierenden Typen der Unterrichtsgestaltung werden ferner ein in der Formulierung von Designedly Incomplete Utterances bestehendes sprachlich interessantes Gestaltungsmerkmal des Lehrervortrags vorgestellt, das (9.1.3.1.1), die Rolle der Lehrkräfte als Sprachmodelle beschrieben, welche vor allem in den Lehrervorträgen hervortrat (9.1.3.1.2), und die in den Abfragungen gestellten Fragen danach untersucht, ob zu ihrer Beantwortung die Aktivierung von Oberflächenwissen ausreichte oder tieferes Wissen erforderlich war (9.1.3.2.1).

Obwohl aus der Forschung bekannt ist, dass sich die einzelsprachlichen Fähigkeiten von Mehrsprachigen gegenseitig beeinflussen, so dass ein $\mathrm{Zu}-$ wachs an Kompetenzen in der Erstsprache für die Entwicklung in der Zweitsprache von Vorteil sein kann (z.B. Cummins 1981; Ricento 2013, S. 358), und die beobachtete Sprachförderung im Erdkundeunterricht in allen fünf Klassen auch an in der Zweitsprache lernende Schülerinnen und Schüler gerichtet war, konzentrierte sie sich dort so gut wie ausschließlich auf den Sprachausbau in der Unterrichtssprache Italienisch. Die Lehrpersonen gaben ihren zweisprachigen Lernenden im Erdkundeunterricht nur selten die Gelegenheit, ihre Erstsprachen zu nutzen. Über den Umgang mit der Mehrsprachigkeit und Multikulturalität der Lernenden informiert das Unterkapitel 9.1.4.

An diese Vorstellung der Rahmenbedingungen des beobachteten sprachlichen Handelns mit einigen ersten Sprachbeispielen schließen sich die eigentlichen linguistischen Untersuchungen an (9.2). Sie konzentrieren sich zum einen auf sprachorientierte und -fördernde Lehrerbeiträge im Frontalunterricht (9.2.1), die sprachlichen Herausforderungen der Lernenden in den Abfragungen (9.2.2) und die sprachbezogene Interaktion der am Unterrichtsgeschehen beteiligten Akteure (9.2.3). Im Unterrichtsgespräch bildete die gemeinsame Texterschließung einen Schwerpunkt. Sie wird untersucht, indem der Frage nachgegangen wird, wie die Lehrkräfte sich vergewisserten, ob die Lernenden Inhalt und Sprache der Texte verstehen, wie sie dem Schülerverständnis potentiell schwierige Textpassagen zugänglicher machten und wie die Lernenden 
die an sie gerichteten Aufgaben meisterten (9.2.3.1). Zum anderen fokussiert Kapitel 9.2 das gemeinsame Erarbeiten des (Fach-)Wortschatzes und der mit ihm verbundenen Konzepte, deren mündliche und schriftliche Wiederholungen Rückschlüsse auf den Lernerfolg ermöglichen (9.2.4. und 9.2.4.1). Darüber hinaus wird abschließend die Gestaltung des Lehrerfeedbacks beschrieben und auf seine Bedeutung für die Sprachförderung eingegangen (9.2.5).

Eine derartige Untergliederung im zweiten Teil des Kapitels ermöglicht eine Akzentuierung spezifischer Komponenten der Sprachbildung, die jedoch in der beobachteten Praxis durchaus zusammen auftraten, so dass es auch in ihrer Darstellung zu Überschneidungen kommt.

\subsection{Rahmenbedingungen des beobachteten sprachlichen Handelns}

Die folgenden Ausführungen sind als Einleitung zu den sprachlichen Analysen w.u. zu betrachten und dienen mittels einer Beschreibung des Kontextes, in dem die Lehrenden und ihre Schülerinnen und Schüler gemeinsam sprachliches und inhaltliches Wissen im Sach-Fachunterricht demonstrieren und konstruieren, einer präziseren Verortung des analysierten Handelns.

Zu den Faktoren, die sich auf schulischen Lernerfolg auswirken, zählen u.a. auch zeitorganisatorische Aspekte wie der Stundenplan und das Zeitmanagement im Unterricht sowie die räumliche Gestaltung der Lernumgebung (Hattie 2013; A. Helmke 2009²; Hammond \& Gibbons 2005). Hattie rechnet den Stundenplan neben der Klassengröße und einer leistungsdifferenzierten Klassenbildung zu den strukturellen Aspekten, die zwar wichtig sind, aber einen recht geringen Einfluss auf die Lernleistungen haben und dennoch ,zu den am heftigsten diskutierten Problemen im Bildungsbereich geworden" sind (Hattie 2013, S. 41). Während die Festlegung des Stundenplans von den Lehrpersonen nur sehr bedingt beeinflussbar ist - oft werden ihnen bestimmte Wochenstunden in einer Klasse zugeteilt, wobei für die beobachteten FachlehrerInnen jedoch der Vorteil bestand, dass sie im ihnen zugeteilten Zeitraum die zehn Stunden für Italienisch, Geschichte und Erdkunde in Eigenregie festlegen konnten -, können das Zeitmanagement und die Raumgestaltung einen nicht zu unterschätzenden Beitrag zum besseren Gelingen schulischen Lernens leisten.

\subsubsection{Zeitliche Organisation des Erdkundeunterrichts}

Von den fünf beobachteten Lehrkräften wählte nur eine zur Erteilung des Erdkundeunterrichts eine erste Stunde, in der die SchülerInnen in der Regel konzentrierter sind als in den letzten Stunden eines Schultages. Tabelle 1 zeigt, an 
welchen Wochentagen und in welchen Stunden der Unterricht im Fach Erdkunde während des beobachteten Zeitraumes erteilt wurde.

\begin{tabular}{|c|c|c|c|c|c|}
\hline Uhrzeit & Montag & Dienstag & Mittwoch & Donnerstag & Freitag \\
\hline $08.00-09.00$ & & III A & & & \\
\hline $09.00-10.00$ & & III C & & III A & \\
\hline $10.00-11.00$ & & III B & & & \\
\hline $11.00-12.00$ & & & & & \\
\hline $12.00-13.00$ & & & & Morante & III B \\
\hline $13.00-14.00$ & & & & Morante & \\
\hline $14.00-16.00$ & Vespucci & & & & \\
\hline
\end{tabular}

Tabelle 1: Durchführung des Erdkundeunterrichts in den beobachteten Klassen

Die Klasse an der Schule Vespucci hatte als einzige auch nachmittags Unterricht, während der Schultag für alle anderen Klassen immer um 14.00 Uhr endete. ${ }^{164}$ Sowohl an der Schule Vespucci als auch an der Schule Morante wurde das Fach Erdkunde also in den letzten beiden Unterrichtsstunden erteilt.

Unter zeitorganisatorischen Gesichtspunkten relevanter ist jedoch die tatsächlich zur Vermittlung fachkundlichen und sprachbezogenen Wissens genutzte Zeit, da bekanntlich für guten Unterricht nicht nur die Qualität, sondern immer auch seine Quantität entscheidend ist: Ein von Treiber (1982) entwickeltes Modell zur Veranschaulichung von Zeitfaktoren für den Lernerfolg illustriert, dass die planmäßig festgelegte Unterrichtszeit in einem Fach zum einen durch Stundenausfall wegen Lehrererkrankungen, Schulveranstaltungen u.Ä. und zum anderen durch Aktivitäten im Unterricht, die nicht der Lehrstoffbehandlung dienen, reduziert wird. Aus dem tatsächlichen Unterrichtsangebot ergibt sich eine Nutzung durch die Schülerin X, welche sowohl anwesend als auch auf das Unterrichtsgeschehen konzentriert sein muss ${ }^{165}$, damit es zu einem Ertrag kommen kann, also ihrem Verständnis eines Lehrstoffsegments und einer diesbezüglich von ihr erbrachten Leistung (Treiber 1982 zit. in A. Helmke $2009^{2}$, S. 80 f.). Verschiedenen empirischen Studien zufolge besteht zunächst eine positive, lineare Korrelation zwischen dem Ausmaß an Unterrichtszeit und erbrachter Schülerleistung, ab einem gewissen Punkt ist der Leistungszuwachs allerdings nur noch minimal. Generell kommt es immer auf die konkrete Zeitnutzung an, d.h. wie die zur Verfügung stehende Unterrichtszeit durch eine gute Unterrichtsorganisation effizient genutzt wird. Dabei werden der Unterrichtsbeginn und Übergänge als besonders sensible Phasen ange-

164 Der Unterricht begann in den fünf Klassen jeden Schultag um 8.00 Uhr und endete um 14.00 bzw. 16.00 Uhr. Eine italienische Schulstunde dauert 60 Minuten. Pausen gibt es an der Mittelschule in der Regel zwei von je 15 Minuten, wobei die Unterrichtsstunden vor und nach der Pause früher enden bzw. beginnen.

165 Dies ist die entscheidendste zeitbezogene Variabel, die allerdings schwer messbar ist. Denn aktive Lernzeit kann für einen Schüler auch gegeben sein, obwohl dieser den Eindruck erweckt, unkonzentriert zu sein, indem er etwa in seinem Rucksack kramt oder aus dem Fenster schaut (s. Helmke 2009², S. 81). 
sehen (s.a. A. Helmke 2009², S. 185). Hattie hält in den von ihm zusammengestellten Metastudien zum erfolgreichen Lernen fest, dass die SchülerInnen verschiedenen Untersuchungen nach ca. 40 bis 50 Prozent der Unterrichtszeit mit Aktivitäten beschäftigt sind, wobei sie diese Zeit nicht komplett produktiv nutzen, und die übrige Zeit v.a. damit verbringen, der Lehrperson zuzuhören oder so zu tun, als ob. ${ }^{166}$ Daraus resultiert die weit verbreitete Auffassung, dass der Schlüssel zur Verbesserung des Lernens in einer produktiveren Gestaltung der Unterrichtszeit liegt, anstatt einfach den Schultag oder das Schuljahr zu verlängern (Hattie 2013, S. 218 f.).

Während dieser empirischen Studie wurde beobachtet, dass die Lehrkräfte sowohl unterschiedlich pünktlich mit dem Unterricht begannen, als auch die für den Erdkundeunterricht bestimmte Zeit zur Vermittlung geographischer Fachinhalte unterschiedlich intensiv nutzten. Einige von ihnen gingen nämlich auch anderen Aufgaben nach, wie etwa der Aufsicht über die Rückgabe und das Entleihen von Büchern aus der Schulbibliothek, der Planung von Ausflügen und Klassenfahrten, dem Einsammeln von Geld und dem Beenden von Unterrichtseinheiten der vorausgehenden Stunde in anderen Fächern (z.B. Italienisch). Wahrscheinlich ist es kein Zufall, dass nur in der leistungsstärksten Klasse der Unterricht in einer ersten Stunde stattfand, die Lehrerin immer pünktlich war und sie die zur Verfügung stehende Zeit so gut wie ausschließlich und von allen beobachteten Lehrpersonen am intensivsten für den Erdkundeunterricht nutzte. ${ }^{167}$

\subsubsection{Lernförderliche Nutzung der Raumgestaltung}

Der Erdkundeunterricht aller beobachteten Klassen fand in den jeweiligen Klassenzimmern statt, wo außer Musik und Sport auch alle übrigen Fächer unterrichtet wurden. Es handelt sich um Räumlichkeiten, deren Dimension je nach Klassenstärke variiert und die mit Ausnahme eines Raums im Erdgeschoss in den oberen Stockwerken liegen, wodurch sie sehr lichtdurchflutet sind. ${ }^{168}$ Auffällig an ihnen war die fast vollkommen fehlende persönliche Aus-

166 In den empirischen Untersuchungen nimmt die „,beschäftigte Zeit“ in den oberen Klassenstufen ab und ist in Mathematik höher als in Englisch und sozialwissenschaftlichen Fächern, am niedrigsten wenn die Lehrperson vorliest oder ein Film geschaut wird und am höchsten bei Gruppenarbeit oder im Labor. Je stärker sich die Lernenden ,herausgefordert fühlen und je größer die akademische Anforderung an die Lernenden ist, je mehr die Lernenden am Unterricht beteiligt werden, desto weniger geneigt sind sie, anderen Beschäftigungen nachzugehen (Yair 2000, S. 256 zit. in Hattie 2013, S. 219).

167 Daneben ist das Unterrichtstempo eine weitere zeitliche Größe, die den Lernerfolg bedingt. Hierauf wird w.u. in den sprachlichen Analysen eingegangen.

168 Besonders in einer Klasse war der Lichteinfall so intensiv, dass während der wärmeren Monate die Rollläden heruntergelassen waren und bei künstlicher Beleuchtung gearbeitet wurde. 
gestaltung. Die elementare Inneneinrichtung mit Tafel, Pult, Tischen und Stühlen war kaum durch etwas ergänzt, was die Räume als die der jeweiligen Klassen hätte ausweisen können: Es gab keine Pflanzen und die Wände und Fenster waren nicht mit selbstgemachten Bildern oder Plakaten geschmückt. Während in einer Klasse im Laufe der Unterrichtsbeobachtungen eine von den Eltern finanzierte Pinnwand angeschafft wurde, um Informationsmaterial (bspw. zu den verschiedenen weiterführenden Schulen) anheften zu können, zierten ansonsten nur in einem Klassenzimmer zwei Plakate von Jugendbuchverlagen, die zum Lesen einladen, in zwei Räumen geographische Karten und in einem ein Werbekalender die Wände.

Dieser Umstand wird erwähnt, weil es hierbei nicht nur um eine Gemütlichkeit erzeugende und eine positive Lernatmosphäre schaffende Dekoration des Raumes geht, den die Lernenden auch während der Pausen nicht verlassen durften und in dem sie folglich jeden Werktag um die sechs Stunden verbrachten. Es ist auch Ausdruck eines Unterrichts, in dem weder Wandplakate produziert werden, auf denen wichtige Lernziele oder fachliche Inhalte festgehalten werden, noch die Schülerinnen und Schüler alleine oder in Gruppenarbeit Referate halten, an die später dafür entwickelte Poster erinnern. Dass gerade Lernende, für die sprachliches und fachliches Lernen eine größere Herausforderung darstellt, von derartigen Informationsträgern profitieren können, steht außer Frage. Wandplakate mit Merksprüchen, Formelsammlungen oder nützlichen Sprachbausteinen für darstellendes und erklärendes Referieren tragen zu einer lernförderlichen message abundancy bei (Gibbons 2003), durch die Schülerinnen und Schüler kognitiv entlastet werden und Lerninhalte mit größerer Wahrscheinlichkeit länger und besser behalten. Deshalb zählen Hammond und Gibbons sie als semiotische Unterstützung zu den lernunterstützenden Aspekten des scaffolding auf Makroebene (Hammond \& Gibbons 2005, S. 17).

Ein weiterer wesentlicher Aspekt der Raumnutzung ist die Anordnung der Tische und die Bewegung der Lehrperson im Klassenzimmer. Die fünf Klassenzimmer waren in dieser Hinsicht sehr ähnlich gestaltet: Das Lehrerpult stand vor der Tafel, und die Tische der Lernenden waren in Reihen dorthin ausgerichtet. Dabei standen in der Regel jeweils drei Tische auf gleicher Höhe mit Platz dazwischen, um bei Gefahren wie einem Brandfall ein zügiges Evakuieren des Raumes zu ermöglichen. Wahrscheinlich führte die starke Konzentration auf die Auslegung der Lehrbuchinhalte und die Praxis des Abfragens dazu, dass die Lehrpersonen während des Unterrichts fast ausschließlich an ihrem Pult hinter dem aufgeschlagenen Lehrbuch saßen, anstatt sich im Klassenraum zu bewegen und ihren Unterricht auch einmal aus dem Hintergrund oder von der Seite des Raumes aus zu führen. Eine größere Mobilität

Hierbei wurden sie oft auch dann nicht wieder hochgezogen, wenn die Bewölkung zunahm bzw. die Sonne nicht mehr direkt ins Klassenzimmer schien. 
wurde nur bei Herrn Cese beobachtet, der die Lehrbuchinhalte weniger textgebunden vermittelte und sich dabei gelegentlich durch den Raum bewegte. Desweiteren kam es lediglich in zwei der fünf Klassen - in einer davon nur sporadisch - zu einer Variation in der Unterrichtsgestaltung, indem die Lernenden in Einzel- bzw. Gruppenarbeit beschäftigt waren und die beiden Lehrerinnen durch den Raum gingen, um den Prozess zu begutachten und einige SchülerInnen individuell zu unterstützen. Diese sowie die weit stärker eingesetzten herkömmlichen Methoden der Unterrichtsgestaltung werden im nächsten Kapitelabschnitt vorgestellt.

\subsubsection{Die beobachteten Formen der Unterrichtsgestaltung}

In allen fünf Klassen bestand der Erdkundeunterricht im Beobachtungszeitraum größtenteils aus Lehrervorträgen, gemeinsamer Textarbeit und Abfragungen. Neben der hierbei erfolgten sprachlichen Förderung der Lernenden auf der Mikroebene ist weiter oben bereits unterstrichen worden, dass ein sprachbezogenes scaffolding der Lernenden auch auf der Makroebene angesiedelt werden kann, wenn Lehrpersonen ihren Unterricht gründlich planen, eine effiziente Reihenfolge der Aufgabenstellungen ausloten, die Vorkenntnisse der SchülerInnen kennen und berücksichtigen und ihnen die angestrebten Lernziele offenlegen (s. Hammond \& Gibbons 2005). Hinsichtlich dieses letzten Aspekts wurde beobachtet, dass die Lehrpersonen ihre Lernenden nur sehr selten im Vorfeld über die anvisierten Lernziele und den Stundenablauf, geschweige denn den Ablauf einer ganzen Unterrichtseinheit, informierten. Diesbezügliche Hinweise reduzierten sich auf eine gelegentliche Bemerkung der Lehrkraft zu Stundenbeginn, dass sie in dieser Stunde beispielsweise abfragen oder mit der Behandlung eines neuen Kontinents beginnen würde. Hierbei kann festgehalten werden, dass die Lehrenden bei der Behandlung der Lerninhalte im Großen und Ganzen die im Lehrbuch vorgegebene Reihenfolge einhielten und ihre Unterrichtsgestaltung kaum variierten, so dass die Lernenden im dritten Schuljahr mit derselben Fachlehrkraft auch ohne explizite Informationen zum Stundenverlauf und zu den Lernzielen erahnen konnten, was sie im Erdkundeunterricht erwartete. Darüber hinaus starteten die Unterrichtsbeobachtungen nicht sofort zu Beginn des Schuljahrs, weshalb nicht bekannt ist, ob die Lehrkräfte ihren Klassen anfangs das Programm für das gesamte Jahr vorstellten.

Lediglich Frau Rea in der Klasse III A an der Schule Pestalozzi informierte ihre Lernenden vor den Weihnachtsferien, womit sie sich im Fach Erdkunde in den Monaten Januar und Februar beschäftigen würden und welches Material benötigt und mitgebracht werden sollte. Es fallen in den Bereich des Makroscaffolding ebenfalls die von ihr unternommenen Anmerkungen, dass es sich bei bestimmten, im Unterricht behandelten und wiederholten Gegenständen 
(bspw. den Klimazonen) um Grundkenntnisse im Fach Erdkunde handele, die erworben und langfristig behalten werden müssen. Um die Bedeutung der Grundkenntnisse im Fach Erdkunde zu veranschaulichen, nutzte sie auch einen Vergleich mit den Grundrechenarten, die im Fach Mathematik als Basis für den Lernstoff dienen, immer wieder angewandt werden müssen und deshalb nicht vergessen oder verlernt werden dürfen.

Im Folgenden werden Einblicke in das sprachbezogene Handeln während der drei vorherrschenden Unterrichtsgestaltungsformen vermittelt.

\subsubsection{Der Lehrervortrag}

Die Prädilektion der italienischen Lehrpersonen im Allgemeinen und der fünf beobachteten Lehrerinnen und Lehrer im Spezifischen für das Unterrichtssetting Lehrervortrag ist keine landestypische Eigenheit: Der Lehrervortrag gilt weiterhin als die mit Abstand verbreitetste Art der Unterrichtsgestaltung (Hattie 2013, S. 216 mit Verweis auf Cotton 1998). So hören die Schüler ihren Lehrern durchschnittlich 85 Prozent der Unterrichtszeit zu, bzw. bemühen sich mehr oder weniger stark darum, den Anschein zu erwecken (Hattie 2013, S. 35). Auch wenn die aktive Beteiligung der SchülerInnen für den Lernerfolg entscheidend ist, wäre es allerdings aus mehreren Gründen falsch, diese herkömmliche Unterrichtsmethode reduktionistisch mit schlechtem Unterricht gleichzusetzen. A. Helmke betont zum Beispiel, dass das, was gemeinhin als solcher eingestuft wird, zu guten Resultaten führen kann und andersherum (A. Helmke $2009^{2}$, S. 25, Hervorhebung im Original):

Abgesehen von der bereits angesprochenen Tatsache, dass es viele simultan wirkende Einflussgrößen gibt, ist ein perfekter Zusammenhang zwischen Prozess- und Produktqualität auch aus methodischen Gründen nicht zu erwarten: Der Zusammenhang zwischen Merkmalen der Unterrichtsqualität und spezifischen Wirkungen ist niemals deterministisch, sondern immer nur probabilistisch, das heißt, er hat Wahrscheinlichkeitscharakter.

Darüber hinaus gibt es nicht den guten Unterricht. Jeder Unterricht übt auf die einzelnen Lernenden eine unterschiedliche Wirkung aus (A. Helmke 2009², S. 34). Gerade Leistungsschwächere wünschen sich oft einen stark lehrergesteuerten Unterricht und profitieren davon (ibid.):

So hat die Forschung gezeigt (vgl. die Übersicht bei Klauer \& Leutner, 2007), dass ein hochstrukturierter, lehrerzentrierter Unterricht mit vielen förderlichen Hinweisen und unterstützenden Maßnahmen von selbstbewussten, selbstsicheren und leistungsstärkeren Schülerinnen und Schülern als bevormundend und demotivierend empfunden wird, während diese Art von „direkter Instruktion“ von leistungsängstlichen Schülerinnen und Schülern geschätzt wird und für ihren Lernfortschritt geradezu nötig ist. Man spricht deshalb auch von „,differenziellem Profit“" oder von „Wechselwirkungen“ zwischen Lehrmethode und Schülermerkmalen [...]: Ein und derselbe Unterricht in einer Klasse kann für einige Schüler gut, für andere schlecht sein - je nach den kognitiven und motivationalen Eingangsvoraussetzungen auf Schülerseite. 
Es geht also nicht darum, bestimmte Unterrichtsmethoden kategorisch anzuprangern, sondern es ergibt sich aus den Wechselwirkungen zwischen den Unterrichtssettings und den Schülerleistungen vielmehr die Notwendigkeit, Methoden zu variieren und den Unterricht nicht immer auf dieselbe Weise zu gestalten, will man möglichst alle Lernenden effizient fördern (s.a. A. Helmke 2009², S. 145; S. 260 f.). Deshalb sieht Oser in der Fähigkeit der Lehrpersonen, ihren Unterricht abwechslungsreich und methodisch vielfältig durchzuführen, eine wesentliche Grundvoraussetzung für den Lernerfolg (Oser 2001, S. 230 f.). Desweiteren unterschätzen Lehrpersonen in der Regel ihre eigene Sprechzeit, wie bspw. im Zuge der DESI-Studie erhoben werden konnte (T. Helmke et al. 2008, S. 352).

In den beobachteten Klassen nutzten die Lehrpersonen die Methode des Vortragens primär zur Vermittlung neuer Lerninhalte, wobei sie sich an deren Aufbereitung in den Lehrwerken orientierten. Dieses Vorgehen resultiert einerseits vermutlich insofern aus der Traditionsverbundenheit, als dass sie den Unterricht als Schüler selbst vorrangig auf diese Weise erfuhren und es nicht untypisch ist, dass Lehrpersonen ,lediglich reproduzieren, [...] in gleicher Art unterrichten wie die Lieblingslehrpersonen aus ihrer eigenen Schulzeit" (Hattie 2013, S. 131 f.). Andererseits ergibt es sich aus dem Umstand, dass die Lehrbuchtexte oft als so komplex eingestuft werden, dass sie den Lehrpersonen zur eigenständigen Erarbeitung durch die Lernenden ungeeignet erscheinen (Bertocchi 2003, S. 22; Lavinio 2003, S. 16; Piemontese \& Cavaliere1997).

Aus der Unterrichtsforschung ist bekannt, dass Lehrkräfte sogenannte Handlungsketten aufbauen, wenn sich ihr Einsatz bei der Bewältigung des Berufsalltags bewährt hat. Von diesen Mustern weichen sie nicht mehr ab, es sei denn, etwas ganz Außergewöhnliches passiert (A. Helmke 2009², S. 313) und daran wird sich voraussichtlich auch nichts ändern, ,[o]hne Feedback seitens der Schülerinnen und Schüler, in einer noch immer vorherrschenden individualistischen Berufskultur von Lehrkräften, ohne Verständigung über Unterricht und Unterrichtsprobleme unter Kollegen, von gemeinsamer Unterrichtsvorbereitung und -durchführung ganz zu schweigen“ (ibid.).

Alternativ zum Lehrervortrag wurden neue Lehrbuchinhalte auch gemeinsam gelesen, wobei die Lehrperson das laute Vorlesen der Lernenden immer wieder unterbrach, um den Text ergänzende oder erklärende Informationen zu liefern oder textbezogene Fragen zu stellen. Bei diesem Vorgehen wurde deutlich, dass die verbreitete Praxis unter Lehrpersonen, sich in der Klasse einen Experten oder Assistenten zu suchen, auf den sie sich verlassen können und der mit großer Wahrscheinlichkeit eine Antwort auf die gestellten Fragen weiß (Harren 2015, S. 82), sogar auf das Vorlesen ausgedehnt wird: So sollte in einer Klasse fast ausschließlich ein bestimmter Schüler neue Lehrbuchtexte vorlesen, wozu mir seine Klassenkammeraden erklärten, dass ihm diese Aufgabe so oft übertragen wurde, da er gut und vor allem schnell vorlas. 
In ihrer Länge waren die Lehrervorträge im Beobachtungszeitraum unterschiedlich ausgeprägt; in einigen Fällen erstreckten sie sich über die ganze Unterrichtsstunde. In drei der fünf Klassen (III A und III C der Pestalozzi und Vespucci), vierhielten sich die Lernenden während der Redezeit der Lehrperson grundsätzlich still und folgten dem Vortrag in ihr Erdkundebuch schauend, vorgestellte Textpassagen anstreichend und Notizen an den Rand schreibend. Den Lehrpersonen dieser Klassen war ein vergleichsweise autoritativer Unterrichtsstil gemein, und den konzentriert wirkenden Schülerinnen und Schülern schienen die Ausführungen der Lehrkraft eine als nützlich empfundene Unterstützung bei der Vorbereitung auf die bevorstehenden Abfragungen zu sein. Desweiteren mussten sie während der Vorträge auch mit gelegentlichen Fragen rechnen.

In den anderen beiden Klassen war die institutionelle Kommunikationssituation weniger stark durch ein hierarchisches Gefälle zwischen der Lehrkraft und den Lernenden geprägt. Das ging nicht mit einer aktiveren Mitarbeit der Schülerinnen und Schülern, sondern mit einem verstärkten Aufkommen von Nebengesprächen unter Banknachbarn ${ }^{169}$ und der Ausübung anderer Tätigkeiten wie der Vorbereitung auf Abfragungen in anderen Fächern und der Anfertigung von Hausaufgaben einher. Dieses Verhalten zeigten vereinzelt auch SchülerInnen der Klasse III C - vor allem wenn sich der Lehrervortrag an Abfragungen anschloss und somit kaum zu befürchten war, in derselben Unterrichtsstunde noch geprüft zu werden. Nur in den Klassen III A und an der Vespucci schienen die Lernenden permanent dem aus dem Lehrervortrag bestehenden Unterrichtsgeschehen zu folgen.

\subsection{Ein individuelles stilistisches Merkmal: Designedly Incomplete Utterances}

Eine Auffälligkeit in einer der beiden lautstärkeren Klassen bestand in der Praxis der Lehrerin Frau Cese, in ihren Vortrag sogenannte DiUs einzubauen, Designedly Incomplete Utterances (Harren 2015, S. 55). Diese Bezeichnung wurde laut Harren von Koshik für einen bestimmten Typ von Äußerungen eingeführt (Koshik 2002, S. 279), der zuerst von Lerner (1995, S. 120 ff.) beschrieben worden war. Mit der Verwendung von DIUs werden verschiedene Absichten verfolgt. Sie bestehen aus einer Art Wortspiel aus Elizitierungen der

169 Gegenstand dieser Nebengespräche kann allerdings das Klären von Verständnisproblemen sein, was Lernende eher untereinander vornehmen, als sich an die Lehrkraft zu wenden, wobei sie dann nicht selten als Unruheherde wahrgenommen werden (s. Harren 2015, S. 78). Auch wenn in der Unterrichtsforschung v.a. Lehreräußerungen untersucht werden, ist dank einer in A. Helmke (2015, S. 192) erwähnten Studie von Nuthall (1997) bekannt, dass nur 14 Prozent aller Schüleräußerungen auf dem Sprechen mit Lehrern oder im Chor basieren. Der Rest betrifft Gespräche mit Gleichaltrigen (69 \%), Selbstgespräche (15\%) sowie Summen und Stöhnen (2\%). 
Lehrkräfte, die ihre Äußerungen unterbrechen, um sie von den Lernenden vervollständigen zu lassen. Sie dienen also hauptsächlich der Wissenselizitierung, wobei es sich nicht wie gewöhnlich um vollständige ,ungerichtete“ (Mazeland 1983, S. 8) Fragen, sondern um abgebrochene Aussagesätze handelt. Sie sorgen für verstärkte Aufmerksamkeit, weil sie jederzeit eingesetzt werden können und ihr Vervollständigen allen Schülerinnen und Schülern Konzentration auf den Lehrervortrag abverlangt.

Aufmerksamkeit für die Äußerungen des Gesprächspartners ist in jeder Kommunikationssituation eine wesentliche Voraussetzung für ihr Gelingen, kann jedoch gerade bei Kindern und Jugendlichen noch nicht gleichermaßen vorausgesetzt werden wie bei Erwachsenen (Harren 2015, S. 77). Nach Einschätzungen der Lehrerin, die in ihren Gesprächsbeiträgen auffallend oft DIUs formulierte, waren ihre Schüler im Vergleich zu anderen Achtklässlern, die sie bisher unterrichtet hatte, noch besonders kindlich: Sie hatten Schwierigkeiten, komplexere Konzepte zu verstehen und lasen trotz Ermunterung kaum Zeitungen oder schauten Nachrichten. Womöglich ist in dieser Beurteilung des Entwicklungsstands der Grund zu sehen, warum Frau Cese so viele zu vervollständigende Äußerungen in ihre Vorträge streute. Diese DIUs wurden von den Schülern fast immer vervollständigt, waren allerdings zumeist auch sehr leicht zu ergänzen, da in der Regel ein Wort als Antwort genügte und von der Lehrkraft angedeutet wurde. Üblicherweise nannte sie die erste Silbe des zu vervollständigenden Wortes, wie die beiden folgenden Beispiele veranschaulichen: ${ }^{170}$

Auszug aus Unterrichtsmitschrift 1, Lehrervortrag Frau Cese (FrC), Asien, DIUs

FrC [spricht über die politische Geschichte Chinas]: Cosa succede, in Cina si sono aperte le fron? (Was passiert, in China haben sich geöffnet* die Gren?)

Klasse: Tiere! (Zen!)

FrC: La manodopera economica in Cina consente un'emissione di prodotti a basso? (Die günstigen Arbeitskräfte in China erlauben die Ausgabe von Produkten zu einem niedrigen?)

Klasse: Prezzo! (Preis!)

FrC [erklärt, dass sich das ändern wird]: Cina, India e Russia stanno emer? (China, Indien und Russland sind dabei emporzu?)

170 In den Transkriptauszügen wurden für die Akteure und ihre Schulen Aliasnamen verwendet. Zur besseren Lesbarkeit der Beispiele aus der beobachteten Unterrichtskommunikation wurden auch für die Lernenden Aliasnamen und nicht wie in den Kapiteln 8 und 10 einfache Buchstaben eingesetzt. Für eine Zuordnung der SchülerInnen zu ihrer Bezeichnung mit bloßem Buchstaben s. Anhang. Die Redner werden vor ihren Gesprächsbeiträgen mit Abkürzungen genannt, die jeweils im Titel angegeben sind. In den Auszügen ist zunächst die italienische Originalversion angeführt, an die sich in runden Klammern meine Übersetzung anschließt. Unverständliche Wörter sind durch ein Fragezeichen ([?]) angezeigt, besonders betonte Ausdrücke sind unterstrichen und zusätzliche Informationen (z.B. gleichzeitiges Reden, Unterbrechen und Pausen) in eckigen Klammern ergänzt. Die fettgedruckten Wörter dienen der Lokalisierung des sprachlichen Phänomens, aufgrund dessen die Wahl des Auszugs erfolgte. 
Abbildung 1: Auszug Unterrichtsmitschrift III A, DIUs (I)

*Für die Transkription wurde diese syntaktisch falsche Schreibweise gewählt, da es im Italienischen keine Satzklammer gibt, sondern die Formen eines mehrteiligen Prädikats beieinanderstehen und die Kenntnis des Prädikatsteils, das die semantische Bedeutung hauptsächlich trägt, den Lernenden die Vervollständigung des folgenden, halb getilgten Wortes erleichtert.

In dem hier wiedergegebenen Auszug aus einem Lehrervortrag von Frau Cese zögern die Schülerinnen und Schüler nicht, die abgebrochenen Wörter zu vervollständigen. Das Anheben der Stimme der Lehrkraft (im Transkript durch die Fragezeichen wiedergegeben) hilft ihnen zudem zu bemerken, dass sie zu einem Gesprächsbeitrag aufgefordert werden. Bei dem ersten Wort, frontiera (Grenze) handelt es sich um einen Ausdruck aus dem Vokabular des hohen Gebrauchs (AU im GRADIT). Nach der zweiten DIU sollen die Schüler zwar ein ganzes Wort ergänzen, doch gehört es zu einer Kollokation (prodotti) a basso prezzo ((Produkte) zu einem niedrigen Preis), die sie - nicht nur im Erdkundeunterricht - schon oft gehört und gelesen haben werden, auch wenn sie selbst eher das im Mündlichen gebräuchlichere prodotti economici verwendet hätten. Das dritte zu ergänzende Wort stellt ebenfalls eine Wiederholung eines Ausdrucks dar, der ihnen sowohl aus dem Geschichts- als auch aus dem Erdkundeunterricht geläufig war und darüber hinaus zum Basiswortschatz zählt (AU).

Vor allem im zweiten Turn der Lehrkraft zeigt sich eine Ausdrucksweise, die im Register der Bildungssprache verortet werden kann und deren Produktion vielen Lernenden wahrscheinlich nicht leichtfiele. Abgesehen vom Spielcharakter der Designedly Incomplete Utterances hat dieses Verfahren für die Lernenden den Vorteil, dass sie so an der Formulierung bildungssprachlicher Äußerungen beteiligt werden - zwar nur geringfügig, aber dennoch aktiv. Das erhöht die Wahrscheinlichkeit, dass sie sich die behandelten Begriffe und Konzepte besser merken.

Das zweite Beispiel für eine bewusst unvollständig gelassene Lehreräußerung erfolgt im Zuge eines Lehrervortrags, der sich an ein Unterrichtsgespräch zum Thema wirtschaftlich motivierte und geopolitische Konflikte (Israel und Palästina, Mittlerer Orient) anschließt und die Positionierung Italiens bei internationalen Konflikten umreißt. Der folgende Transkriptauszug gibt einen Ausschnitt daraus wieder.

Auszug aus Unterrichtsmitschrift 2, Lehrervortrag Frau Cese (FrC), Position Italiens in internationalen Konflikten, DIU

FrC: Noi siamo filoamericani. Abbiamo sempre dato la nostra adesione agli americani. Abbiamo i nostri contingenti dell'esercito in quelle terre accanto agli eserciti USA, perché gli americani ci hanno aiutato tantissimo a riprenderci dopo la seconda guerra? (Wir sind philoamerikanisch. Wir haben unsere Unterstützung immer den Amerikanern gegeben. Wir haben unsere Truppenkontingente in jenen Ländern neben den 
Heeren der USA, weil die Amerikaner uns enorm geholfen haben, uns zu erholen nach dem zweiten? Krieg*)

Klasse: Mondiale! (Welt-!)

Abbildung 2: Auszug aus Unterrichtsmitschrift III B, DIUs (II)

*Dem deutschen Kompositum „Weltkrieg“ entspricht im Italienischen das Phrasem guerra mondiale, bei dem das erste substantivische Element „Krieg“ von einem Adjektiv genauer bestimmt wird. Die merkwürdige Schreibweise in der deutschen Übersetzung rührt von der Absicht her, die italienische Phrasemstruktur beizubehalten.

Auch in dieser zweiten Gesprächssituation wird es für die Lernenden einfach gewesen sein, das Wort zu ergänzen, was man an ihrer sofortigen und vielstimmigen Antwort erkennen kann. Das Phrasem guerra mondiale ist zwar ein Ausdruck des allgemeinen Wortschatzes (CO), also des weitläufigen peripheren Bereichs des Basiswortschatzes, doch sie kannten es seit langem aus dem Unterricht und hatten es mit großer Wahrscheinlichkeit auch außerhalb der Schule schon gehört. Während der Rest des Nebensatzes gleichermaßen allgemeinverständlich ist, weil er mit Ausnahme von riprendersi (Co; sich erholen) nur aus Wörtern des fundamentalen Wortschatzes (Fo) besteht, ist der erste Teil der Lehreräußerung unter lexikalischen Gesichtspunkten etwas komplexer gestaltet, was an den Begriffen filoamericano aus dem allgemeinen Wortschatz, adesione (Unterstützung) aus dem des hohen Gebrauchs (AU) und an contingente (Kontingent) aus dem militärischen Fachwortschatz liegt, wobei diese Bedeutung aus dem Kontext erschlossen werden konnte.

Neben Frau Cese formulierten noch zwei weitere beobachtete Lehrkräfte DIUs, und zwar Frau Rea (III A) und Frau Dante (Vespucci). Ihre Verwendung des kommunikativen Gestaltungsmittels erfolgte nicht nur seltener, sondern unterschied sich auch darin, dass sie sich nicht auf den Lehrervortrag konzentrierte, sondern größtenteils als unterstützende Maßnahme aufkam, wenn auf die Frage nach einem bestimmten Begriff keine sofortige Antwort erfolgte oder die Beherrschung eines Begriffs, den die Lehrerin verwendete, als ungewiss eingestuft wurde.

Wie in diesen Auszügen aus dem Unterrichtsgespräch waren die beobachteten Lehrpersonen in ihren Vorträgen allgemein um eine präzise Ausdrucksweise mit bildungssprachlichen Zügen bemüht, wobei sie sich den Verständnisfähigkeiten der Lernenden anpassten. So erklärten sie beispielsweise bestimmte Begriffe, die sie verwendeten, oder vergewisserten sich, ob die Klasse sie verstanden hatte und erklären konnte. Mit der bedeutenden Rolle der Lehrenden als Sprachmodelle für die SchülerInnen, der sich die beobachteten LehrerInnen bewusst zu sein schienen, beschäftigt sich der nächste Kapitelabschnitt. 


\subsection{Die Lehrperson als Sprachmodell}

Sprache und ihr Ausbau sind eines unserer individuellsten Persönlichkeitsmerkmale, aber sie wird immer ,als die Sprache der Anderen“ gelernt (Maas 2008, S. 265 mit Rückgriff auf Bühlers Symbolfeld (1934)). Diese Orientierung an der Sprache der Anderen beruht bei Kindern und Jugendlichen während der Schulzeit vor allem auf der Beobachtung und Nachahmung des sprachlichen Handelns der Eltern. Gleich an zweiter Stelle folgen als wichtigstes Sprachmodell die Lehrkräfte ${ }^{171}$, weshalb es so wichtig ist, dass sie positive sprachliche Vorbilder abgeben (A. Helmke 2009², S. 113, Hervorhebung im Original):

Lehrkräfte, deren sprachliche Klarheit zu wünschen übrig lässt [...], üben damit einen doppelt negativen Einfluss aus: Zum einen erschweren sie den Lernprozess der Schüler, zum anderen signalisieren sie unbewusst, dass sie der Beherrschung der Muttersprache keine besondere Bedeutung beimessen. In einem Spiegelinterview (34/2007) äußerte sich Hartmut von Hentig zu diesem Thema wie folgt:

„Hentig: Was mir unter anderem auffällt, wenn ich die jungen Leute im Bus quasseln höre, sind die Stereotypen ,toll' und , super ' und , scheiß ' und, kannste vergessen ' - in stundenlanger Wiederholung. Sie wollen etwas ausdrücken und können es nicht. Das tut mir leid.

Spiegel: Was soll die Schule dagegen tun?

Hentig: Der Lehrer muss selber eine gute Sprache sprechen und die Schüler auf ihre eigene Sprache aufmerksam machen: ,Meinst du das wirklich?' Und: ,Versuch es mal genauer zu sagen!' In jedem Unterricht! An der Bielefelder Laborschule habe ich immer verlangt: Deutschunterricht sei immer. Sprache werde in jedem Augenblick geübt, selbst im Sport. “

Weiter betont A. Helmke, dass die meisten Lehrkräfte sich noch nie angehört haben, wie sie selbst im Unterricht reden und agieren, wofür sich Audio- oder Videoregistrationen eignen. Viele sind sich nämlich nicht bewusst, wo ihre sprachlichen Stärken liegen und welche Marotten sie eventuell haben: So war eine Lehrerin nach dem Schauen einer Videoaufnahme ihres Unterrichts sehr überrascht, dass sie um die hundert Mal den Ausdruck ,okay“ gebraucht hatte (A. Helmke 2009², S. 194). ${ }^{172}$ Während derartige sprachliche Eigenheiten und

171 Womit nicht in Abrede gestellt werden soll, dass schon im Grundschulalter auch Peers einen nicht unerheblichen Einfluss auf die Sprachentwicklung eines Kindes haben und dieser vor allem während der Pubertät an Bedeutung gewinnt. Gerade in dieser Phase sollten Jugendliche die Registerdifferenzierung jedoch bis zu einem gewissen Grad erkannt haben und ein Register der Sprechsituation angemessen wählen können.

172 Desweiteren stellt die Stimme für die Lehrpersonen wohl das Hauptwerkzeug zur Ausübung ihres Berufes dar, weshalb es verwundert, dass Lehrkräfte mit diesbezüglichen Auffälligkeiten oder Problemen nur selten ein Stimmtraining in Anspruch nehmen. Eine Studie des Bereichs Sprechwissenschaft/Sprecherziehung an der Leipziger Universität hat ergeben, dass von 5.357 angehenden Lehrkräften 44 Prozent stimmliche Auffälligkeiten zeigten und 17 Prozent so aus dem Rahmen fielen, dass ihnen eine fachärztliche Untersuchung nahegelegt wurde. Neben 16 Prozent der StudienteilnehmerInnen, die lispelten, gab es auch einige die 
dialektale Färbungen zwar von den Schülerinnen und Schülern herausgefiltert und nicht übernommen werden, ist die Klarheit der Lehrersprache sowohl für den allgemeinen Lernerfolg als auch in Bezug auf ihre Modellfunktion im Sprachausbau der Lernenden entscheidend (ibid., S. 194 f.). Dieser leicht nachvollziehbare Umstand wurde bereits vor langer Zeit erkannt und herausgestellt, so zum Beispiel von Schnell auf recht schonungslose Art (1850, S. 61 zit. in A. Helmke $2009^{2}$, S. 191):

Ein Lehrer und Erzieher muß daher, wenn auch nicht Schönredner und Phrasenmacher, so doch ein Meister des Wortes sein; er muss das Wort in seiner Gewalt haben, wie ein geschickter Fechtmeister das Schwerdt; er muß durch seine Sprache seine Gedankenwelt beherrschen, und sich selbst und die Jugend. Dazu gehört nicht, daß er viele Worte mache, weit und breit sei in seiner Rede; es gehört dazu im Gegenteil eine klare und kurze, eine deutliche und bündige Sprache, wie sie sich jeder Lehrer anzueignen vermag durch Fleiß und Ausdauer, durch festen Willen und Uebung. Viele Lehrer scheinen es freilich gar nicht zu wissen, welches einflussreiches Mittel ihnen in der Sprache zu Gebote steht, weil sie sonst nicht so viel sprechen, sich nicht halb todt reden, nicht die Sprache zu ihrem und der Kinder Verderben mißbrauchen würden. Andere nehmen beim Sprechen Eigenheiten an, wodurch sie selbst den Kindern unangenehm, auffällig und der leichtfertigen Jugend sogar lächerlich werden. Das muß man sagen, wenn man das Näseln, Nuscheln und Stoßen mancher Lehrer hört. Wenn man sich nicht von dergleichen Unarten überzeugen könnte, so wäre es kaum glaublich, daß gebildete Männer, geschweige Lehrer, ihre Sprache und Bildung so zu verunstalten mögen.

Während sich die weiter oben geschilderten Richtlinien für einen verständlichen Sprachgebrauch auf die Gestaltung der Lehrbuchtexte konzentrierten, soll ihnen an dieser Stelle eine Konzeption hinzugefügt werden, die aufgrund ihrer Prägnanz und Plausibilität ${ }^{173} \mathrm{sehr}$ weit und auch in Italien (s. z.B. Fratter \& Jafrancesco 2003, S. 48 f.) rezipiert worden ist, die Hamburger Verständlichkeitsformel von Langer, Schulz von Thun und Tausch (1974). Sie besitzt für eine große Bandbreite von Kommunikationssituationen (schriftliche Texte, z.B. in Lehrbüchern und von Schülern, und Aufgaben sowie mündliche Lehrer- und Schüleräußerungen) Gültigkeit und beruht auf den folgenden vier Punkten (A. Helmke 2009², S. 196; Hervorhebung im Original):

- Einfachheit (kurze, einfache Satzstrukturen; vertraute Sprache, geläufige Wörter; erklärte Fachwörter) versus Kompliziertheit;

- Kürze/Prägnanz (knappe Darstellung; aufs Wesentliche konzentriert) versus Langatmigkeit/fehlende Präzision (optimal: mittlere Ausprägung);

stotterten, näselten oder polterten (Deutsche Gesellschaft für Sprechwissenschaft und Sprecherziehung zit. in A. Helmke $2009^{2}$, S. 194).

173 An dem Modell ist allerdings kritisiert worden, dass es Verständlichkeit als textimmanentes Kriterium auffasst und die entscheidende Bedeutung der Interaktion zwischen Leser/Hörer und dem sprachlichen Produkt außer Acht lässt (z.B. Tergan \& Mandl 1983 zit. in A. Helmke 2009², S. 196): Je größer etwa sein inhaltsbezogenes Vorwissen ist, desto einfacher wird das Verständnis, desto eher kann eine Sprachproduktion beispielsweise auch nicht erklärte Fachwörter enthalten usw. 
- Ordnung/Gliederung (gegliedert; folgerichtig; geordnet; übersichtlich; gute Unterscheidung von Wesentlichem und Unwesentlichem; sichtbarer roter Faden) versus Ungegliedertheit und Zusammenhangslosigkeit;

- Zusätzliche Stimulanz: anregende, stimulierende Zutaten wie wörtliche Rede, lebensnahe Beispiele (nur wirksam bei hoher Ausprägung des Faktors Gliederung/Ordnung).

Die unter Berücksichtigung der mutmaßlichen sprachlichen Kompetenz der Gesprächspartner erwogene Einfachheit der Darstellung garantiert hierbei, dass ihre Kürze nicht in Komplikationen ausartet, die sich durch eine gesteigerte informationelle Dichte ergeben können. Werden alle vier Punkte in der schriftlichen und mündlichen Kommunikation ausreichend beachtet, kann mit einer größeren Motivation des Lesers/Zuhörers gerechnet werden, der Darstellung zu folgen. Diesbezüglich leiten Fratter und Jafranesco vor allem aus dem Aspekt der zusätzlichen Stimulanz ab (Fratter \& Jafrancesco 2003, S. 49; Hervorhebung im Original): "[E'] bene che l'insegnante eviti il più possibile le lezioni frontali e una modalità di tipo passivo, stimolando invece la partecipazione attiva alla lezione per mezzo di tecniche del Cooperative Learning“ (M.Ü.: Es ist ratsam, dass die Lehrkraft Frontalunterricht und passive Modalitäten so gut wie möglich vermeidet und stattdessen die aktive Mitarbeit an der Unterrichtsstunde mittels Techniken des Cooperative Learnings anregt). Viele Studien belegen, dass die Eigenaktivität ein entscheidender Schlüssel zum Lernerfolg ist (z.B. Hattie 2013, S. 42). Allerdings wurde w.o. darauf hingewiesen, dass gerade leistungsschwache Lernende von einem lehrergesteuerten Unterricht profitieren und seine Darstellungen und Erklärungen benötigen. Hinzukommt die wesentliche Funktion der Lehrkraft als Sprachmodell, die sie vor allem beim Vortragen wahrnehmen kann.

Der beobachtete Sprachgebrauch der fünf in die Studie involvierten Lehrkräfte berücksichtigte viele der beschriebenen verständnis- und sprachfördernden Merkmale, was sicherlich auch der Tatsache geschuldet ist, dass alle auch Italienischlehrkräfte waren. Es war auffällig, wie sie sich um eine dem Bildungskontext Schule angemessene Sprachverwendung bemühten, ohne unüberwindbar weit von den sprachlichen und kognitiven Fähigkeiten der SchülerInnen entfernt zu sein. Das erreichten sie, indem sie nicht zu lange Sätze bildeten und den Kenntnisstand der Lernenden reflektierten. Seltener gebrauchte Wörter und neue und komplexere Konzepte erklärten sie und/oder erkundigten sich bei den Lernenden, ob ihre Bedeutung bekannt war. Um Lerninhalte besser zu veranschaulichen und dadurch den Lernenden zu helfen, sie besser und mit den angemessenen lexikalischen Mitteln im mentalen Lexikon abzuspeichern und als Grundlage vor allem für mündliche Darstellungen nutzbar zu machen, gaben sie konkrete Beispiele und stellten Bezüge zur Lebenswelt der Lernenden her. Außerdem sprachen sie langsam und deutlich und zeigten keine besonderen sprachlichen Eigentümlichkeiten.

Der Umgangston war überwiegend freundlich, bisweilen scherzhaft und bei drei Lehrkräften auch durchaus römisch gefärbt. Dass die anderen beiden 
keine sprachlichen Merkmale des Regiolekts offenbarten, lag wahrscheinlich am Verständnis ihrer spracherzieherischen Vorbildrolle und an der institutionsbezogenen Registerwahl. Davon abgesehen, stammen sie beide nicht aus Rom, so dass gewisse römische Färbungen deshalb schlichtweg nicht zu ihrem produktiven Sprachrepertoire gehörten.

Bisweilen konnte sogar registriert werden, wie sich in den Redebeiträgen einiger Lehrpersonen regionalsprachliche mit jugendsprachlichen Zügen vermischten, so zum Beispiel bei Prof.ssa Mancuso, die des Öfteren das in der Jugendsprache sehr gebräuchliche und in den zentral-südlichen Regiolekten verwendete mo anstelle der standardsprachlichen adesso, ora (jetzt) aus dem fundamentalen Wortschatz formulierte, ferner die gekürzte und kontrahierte Form vabbè für va bene (in Ordnung) und das soziolektal inflationäre c'ha anstelle der hochsprachlichen Form des Verbs avere (haben) ohne die Partikel $c i$ gebrauchte und die Schülerinnen und Schüler darüber hinaus gelegentlich jugendsprachlich-römisch mit regà anredete, wie die Abbildung 3 veranschaulicht.

Transkriptauszug 1, PIL (BIP), Frau Mancuso (FrM), Nicholas (Nich) - Regiolekt (I)

Nich: Prodotto interno lordo. (Bruttoinlandsprodukt.)

FrM: Prodotto interno lordo. L'analisi della parola l'abbiamo fatta l'anno scorso, quindi non ci torniamo, eh, che vuol dire? [Mehrere Schüler sprechen durcheinander, unverständlich] Aspetta, uno mi parla. Fabio, non stai a dire [ugs. Verbkonstruktion] niente? lo a questi mo li metto il voto oggi, eh. Perché non c'ho neanche un voto a geografia. (Bruttoinlandsprodukt. Die Wortanalyse haben wir schon letztes Jahr gemacht, also kommen wir darauf nicht zurück, eh, was bedeutet es? [Mehrere Schüler sprechen durcheinander, unverständlich] Warte, einer spricht mit mir. Fabio, du sagst nichts?! Nu geb' ich denen da heute eine Note, eh. Weil ich nicht eine einzige Note in Erdkunde habe.

Abbildung 3: Transkriptauszug Regiolekt Frau Mancuso

In diesem Auszug aus einem Unterrichtsgespräch zum Bruttoinlandsprodukt verwendet die Lehrkraft gleich zwei regionale Formen, die verstärkt im Sprachgebrauch vieler Jugendlicher untereinander auftreten. Zum einen ist es die Verlaufsform stare a dire anstelle der hochsprachlichen Konstruktion mit einem Gerundium, stare dicendo (dabei sein zu sagen), und zum anderen das oben geschilderte Wort mo für das sprachlich neutralere adesso oder ora. Überdies verwendet sie das Verb avere auf nicht hochsprachkonforme Weise mit der Partikel $c i$.

Auch Frau Cese „verschluckte“ hin und wieder auf typisch römische Art die letzte Silbe der Infinitive, $-r e^{174}$, äußerte einmal perdoname, wie es im römischen Dialekt, mehr noch im jugendlichen römischen Soziolekt anstelle des

174 So z.B. Frau Cese: „Questo vel'ho spiegato però per 3 anni e voi non vi ricordate niente. Ve lo dovete ricorda'! [Ergänzt dann] Re." (Das habe ich aber drei Jahre lang erklärt und ihr erinnert euch an nichts. Ihr müsst das „,behalt“! [Ergänzt den lokalen, v.a. jugendlichen Sprachgebrauch mit der hochsprachlich erforderten Endung] En.). Vermutlich verwendete 
hochsprachlichen perdonami (Verzeih mir) üblich ist, und betitelte einen ihrer Schüler als collega (Kollege), wie es wiederum v.a. in der Jugendsprache typisch ist. In den beiden letztgenannten Fällen ließ die prompte Reaktion der Lernenden erkennen, dass sie der Sprachgebrauch ihrer Lehrerin merklich überraschte:

Auszug aus Unterrichtsmitschrift 3, Frau Ceses (FrC), Luca (Luc), Verwendung jugendsprachlicher/soziolektaler Ausdrucksmittel I

FrC: [Fragt zwei Lernende zu den Hausaufgaben ab (Abwandlungen/Untergruppen der monotheistischen Religionen - koptische Christen, Mormonentum usw.). In der Klasse ist es unruhig].

Luc: [Unverständlich]

FrC: Non sento il collega qua! (Ich höre den Kollegen hier nicht).

Klasse [lacht, wiederholt]: I/ collega! (Den Kollegen!)

FrC: Il vostro collega. (Euren Kollegen).

Abbildung 4: Auszug aus der Unterrichtsmitschrift Jugendsprache/Soziolekt Frau Cese (I)

Es ist sehr gut möglich, dass die Lehrerin dieses sprachliche Gestaltungsmittel bewusst wählte, um die Aufmerksamkeit der Lernenden zu gewinnen und die Ruhe im Klassenzimmer wiederherzustellen. Damit reagierte die Lehrerin, wie es sich in verschiedenen Studien als am effektivsten herausgestellt hat, um mit Störungen im Unterrichtsverlauf umzugehen, nach dem low profile-Ansatz (Borich 20074); das bedeutet, dass Störungen unverzüglich unterbunden werden, wenn sie nicht im Vorfeld zu vermeiden waren, und dass man kein großes Aufsehen darum macht (s. A Helmke 2009², S. 188).

Auch im Falle von perdoname ist es wahrscheinlich, dass Frau Cese den nicht hochsprachkonformen Ausdruck nicht unbedacht formulierte, sondern dass sie damit ganz bewusst versuchte, einen Schüler auf bestimmte, aber freundliche Art auf seine sprachliche und konzeptuelle Vagheit hinzuweisen:

Auszug aus Unterrichtsmitschrift 4, Frau Ceses (FrC), Marco (Mar), Federico (Fed) Verwendung jugendsprachlicher/soziolektaler Ausdrucksmittel II

Mar [während einer Abfragung zur Sklaverei in den amerikanischen Südstaaten]: Di prendere la sua terra. Di venderla. (Sein Land zu nehmen. Es zu verkaufen.)

FrC: Perdoname! Che significa? Che vuoi dire? ([Regiolekt/Jugendsprache]: Entschuldige mich. Was bedeutet das? Was willst du sagen?)

Fed: Si dice, perdonami. (Es heißt, entschuldige mich.)

FrC [ignoriert Fed und wendet sich wieder an Mar]: II fenomeno schiavitù, dove si situa in America? (Das Phänomen Sklaverei, wo ist es angesiedelt in Amerika?)

[Fed schweigt, Sch E antwortet unaufgefordert.]

Abbildung 5: Auszug aus der Unterrichtsmitschrift Jugendsprache/Soziolekt Frau Cese (II)

sie das Wort wie unter den Schülern üblich, um sich ihrer Aufmerksamkeit zu versichern. Allerdings fügte sie nach einer Weile die Standardendung hinzu, als ob sie daran erinnern wollte, dass in der Schule in der Hochsprache kommuniziert wird. 
Dass jugendlicher Sprachgebrauch allerdings nicht grundsätzlich im Unterrichtsgespräch geduldet wurde, wird w.u. anhand einiger Beispiele aus den Transkripten gezeigt, die veranschaulichen, dass die Lehrkräfte gegen manche dieser Sprachgewohnheiten systematisch und recht vehement vorgingen, wie es insbesondere bei dem weit verbreiteten Gebrauch von tipo (eine Art) und cioè (das bedeutet, soll heißen; oft als Verzögerungsmittel bei Formulierungsschwierigkeiten aufgrund lexikalischer oder konzeptueller Lücken verwendet) beobachtet werden konnte (s. 9.2.5).

Zusammenfassend lässt sich über die beobachteten Lehrkräfte mit Blick auf ihre Rolle als Sprachmodell festhalten, dass sie unter spracherzieherischen Gesichtspunkten davon profitierten, dass sie auch Sprachen unterrichteten. Dadurch legten sie im Fachunterricht viel Wert auf einen angemessenen sprachlichen Ausdruck und forderten diesen auch von den Schülern gemäß ihrer Fähigkeiten ein.

\subsubsection{Die Abfragungen - le interrogazioni}

Mit Ausnahme einer der fünf Klassen, in der die Lernenden regelmäßig zur Eigenarbeit angeregt wurden, wie im nächsten Unterkapitel zur Gruppenarbeit erläutert wird, verging kaum eine beobachtete Erdkundestunde, ohne dass die Lehrkräfte mehrere SchülerInnen mündlich prüften. Bei dieser Praxis sind zwischen den einzelnen Klassen einige Unterschiede zu verzeichnen. So bekam die Klasse III A an der Schule Pestalozzi manchmal die Gelegenheit, sich vor Beginn der Abfragung in ca. fünf Minuten darauf vorzubereiten. Grundsätzlich bestanden in allen Klassen vor einer Abfragung die Hausaufgaben darin, im Lehrbuch die Seiten zu lesen, die in der vorherigen Stunde im Unterricht behandelt worden waren. ${ }^{175}$ Verschiedene Lehrpersonen erkundigten sich nach freiwilligen Prüflingen, doch nur in der Klasse III B an der Schule Pestalozzi gab es gelegentlich welche. Während Herr Tigre (Morante) und Frau Rea (III A, Pestalozzi) die SchülerInnen vorrangig einzeln abfragten, wurden in den anderen drei Klassen immer zwei bis vier Schüler gleichzeitig geprüft. Dies erfolgte nach Angaben der entsprechenden Lehrkräfte, um etwas Zeit einzusparen und den weniger selbstbewussten Lernenden einen Teil ihrer Prüfungsangst zu nehmen, zumal sie in vier der fünf Klassen für die Prüfung ans Pult treten mussten. Nur in der Klasse III C konnten sie auf ihren Plätzen sitzen bleiben und bei landeskundlichen Fragen die Karten in ihren Büchern verwenden, während in den anderen Klassen an Wandkarten gearbeitet wurde.

Beim gleichzeitigen Abfragen mehrerer Lernender wurde eine nicht beantwortete Frage an den/die nächste(n) Schüler(in) weitergereicht, bzw. wurden

175 Nur in der Klasse III A an der Schule Pestalozzi wurde einmal beobachtet, dass die Lernenden als Hausaufgabe einen noch nicht behandelten Lehrbuchtext lesen sollten und sein Inhalt in der darauffolgenden Erdkundestunde in die Abfragungen einbezogen wurde, nachdem sich die Lehrkraft zuvor erkundigt hatte, ob es Schwierigkeiten beim Textverständnis gab. 
nach den ersten Antworten weitere Abgefragte gebeten, die gegebenen Informationen zu vervollständigen. Die Betroffenen hatten also den gesamten Prüfungszeitraum über (manchmal 10-15 min, manchmal 1 h), mit einer Frage zu rechnen. Das galt gleichermaßen auch für den Rest der Klasse, wenn keiner der Geprüften die Antwort wusste, was für eine größere Aufmerksamkeit aller sorgte. Ähnlich wie beim Lehrervortrag war es in den Klassen an den Schulen Morante und Pestalozzi (III A und III C) während der Abfragungen ziemlich still und erweckten die Lernenden einen konzentrierteren Eindruck als ihre Altersgenossen in den übrigen beiden Klassen, die sich relativ laut miteinander unterhielten und gelegentlich von der Lehrkraft zurechtgewiesen wurden, die hauptsächlich auf ihre Prüfungen konzentriert war. Alle fünf Lehrkräfte benoteten die Abfragungen, welche die Hauptbewertungsgrundlage für die mündliche Leistung im Erdkundeunterricht darstellten.

Vor allem in einer Klasse, der III B an der Schule Pestalozzi, konnte beobachtet werden, wie die Schülerinnen und Schüler während der Lehrervorträge und Unterrichtsgespräche immer wieder dazu angehalten wurden, sich Notizen $\mathrm{zu}$ machen, da diese für ein erfolgreiches Bestehen der Abfragungen und als Vorbereitung für die Abschlussprüfung am Schuljahresende nützlich seien. Auch in drei weiteren Klassen (III A und III C Pestalozzi und Vespucci) wurden einige SchülerInnen bei der Anfertigung von Notizen und dem Markieren von Textpassagen im Geographiebuch beobachtet. Da sie hierzu von ihren Lehrkräften nicht aufgefordert wurden, kann davon ausgegangen werden, dass sie diese Lern- und Vorbereitungsstrategie entweder in den vorherigen Schuljahren vermittelt bekommen oder eigenständig erworben hatten.

Die Befragungen einzelner Schülerinnen und Schüler während der Erkundungsstudie und der Erhebung ergaben, dass ihre Technik, sich den Lernstoff anzueignen, darin bestand, die entsprechenden Lehrbuchseiten zuhause zu lesen (oft laut) und die Inhalte mündlich und laut vorzutragen, wobei sich einige auch von Familienmitgliedern abfragen ließen. ${ }^{176}$

In der beobachteten Klasse an der Schule Morante waren die interrogazioni vorwiegend auf Aspekte der physischen Geographie konzentriert, da es dem Lehrer, wie er erklärte, darum ging, alle Schülerinnen und Schüler dazu zu befähigen, einen geographischen Raum nach physischen Gesichtspunkten einschließlich Erklärungen zu ihrer Entstehung (z.B. Alter der Gebirge, Bewegung der Kontinentalplatten; zum Anbau geeignetes Land und die physischen und meteorologischen Gründe dafür) vorzustellen. In den ersten beiden Schuljahren war das bereits erprobt worden und spätestens jetzt sollten die Lernenden einen bestimmten Ablauf verinnerlichen und selbst befolgen lernen. Den meisten beobachteten Prüflingen aller fünf Klassen gelang es allerdings nicht, eigenständig eine Region vorzustellen und sich dazu länger zu äußern. Sie schienen darauf angewiesen zu sein, sich in ihrer Darstellung von punktuellen

176 Aus dieser Praxis ergibt sich wohl der Zeitaufwand der SchülerInnen zur Bewerkstelligung der Erdkundehausaufgaben (s. Kap. 6). 
Lehrerfragen leiten zu lassen, auf die sie eher kurze Antworten gaben, wie das Unterrichtsbeispiel in Abbildung 6 zeigt.

Transkriptauszug 2, Abfragung physische Geographie Herr Tigre (HrT), Giada (Gia), Sonja Castaldo (Son)

HrT: Vediamo un po' quali informazioni riuscite a tirare fuori dalla carta. Tanto per cominciare, vi ho messo anche tutte e due le carte. Allora, vediamo un po'. Che possiamo dire? (Schauen wir mal ein bisschen, welche Informationen ihr schafft, aus der Karte zu entnehmen. Um mal zu beginnen, ich habe euch alle beiden Karten gegeben. Also, schauen wir mal. Was können wir sagen?)

Gia: Che l'Asia è attaccata all'Europa. (Dass Asien an Europa gehaftet ist.)

$\mathrm{HrT}$ : Oh, prima informazione importante. L'Asia è attaccata all'Europa. Quindi se io chiedessi a Castaldo una definizione di continente. Che cos'è un continente in geografia? (Oh, erste wichtige Information. Asien ist an Europa gehaftet. Also, wenn ich Castaldo nach einer Definition von Kontinent fragen würde? Was ist ein Kontinent in Erdkunde?)

Son: $\dot{E}$ una vasta porzione di territorio emerso. (Es ist ein großes Stück aufgetauchter Erde.)

HrT: Emerso, quindi separato da tutti gli altri, circondato dai mari. Quindi l'Asia è un continente? (Aufgetaucht, also getrennt von allen anderen, umgeben von Meeren. Also ist Asien ein Kontinent?)

Gia: Certo. (Sicher.)

Son: Però non è proprio l'Asia, il continente. Il continente è l'Eurasia. (Aber der Kontinent ist eigentlich nicht Asien. Der Kontinent ist Eurasien.)

HrT: Quindi l'Asia non è un continente, il continente è appunto l'Eurasia. Quindi è l'unico caso in cui la definizione geografica di continente non funziona. Allora, gli diamo una definizione storica. Perché l'Europa e l'Asia? Perché l'Europa e l'Asia hanno avuta ciascuna delle storie in comune. I popoli europei e i popoli asiatici hanno avuto tra loro molti contatti e quindi diamo una definizione di continente che è storica. Che altro possiamo dire? (Also Asien ist kein Kontinent, der Kontinent ist eben Euroasien. Also ist es der einzige Fall, für den die geographische Definition eines Kontinents nicht funktioniert. Also geben wir ihm eine geschichtliche Definition. Warum Europa und Asien? Weil Europa und Asien jeweils eine gemeinsame Geschichte haben. Die europäischen und die asiatischen Völker hatten viele Kontakte untereinander und daher geben wir eine Definition von Kontinent, die geschichtlich ist. Was können wir noch sagen?)

Gia: Che in questa parte dell'Asia (Dass in diesem Teil von Asien)

HrT: [unterbricht sie]: In tanto, guarda la carta generale! Nella carta generale, in termini di grandezza, che cosa possiamo dire a colpo? (Erst mal schau dir die allgemeine Karte an! Auf der allgemeinen Karte, hinsichtlich der Größe, was können wir da sofort sagen?)

Gia: Non ho capito, professore. (Ich habe nicht verstanden, Lehrer.)

$\mathrm{HrT}$ : Guardando la carta generale e, confrontando $i$ vari continenti in termini di grandezza, che possiamo dire dell'Asia? (Auf die allgemeine Karte blickend und die verschiedenen Kontinente der Größe nach vergleichend, was können wir über Asien sagen?)

Gia: Che è più grande. (Dass es größer ist.)

Son: $\dot{E}$ il più vasto continente e occupa un terzo delle terre emerse. (Es ist der größte Kontinent und er nimmt ein Drittel des aufgetauchten Landes ein.)

HrT: Aah. Che vuol dire "terre emerse"? (Aah. Was bedeutet „aufgetauchte Länder“?)

Gia: Che so' tutti, cioè che son' tutte cose separate. (Dass sie alle, das heißt, dass alles getrennte Sachen sind.) 
HrT: No, che vuol dire "terre emerse"? Cosi e basta. Che vuol dire "terra emersa"? Emersa da che cosa? (Nein, was bedeutet „aufgetauchte Länder“? So und Schluss. Was bedeutet „aufgetauchtes Land“? Aufgetaucht aus was?)

Klasse: Dal mare. (Aus dem Meer.)

HrT: Dal mare. Quindi le terre emerse sono tutte terre che sono sopra il livello del mare. Anche il fondo del mare è terra, ma sotto il livello del mare, ok? Ci siamo? A questo punto, io, un'altra cosa che segnalerei subito, considerando l'intera sfera terrestre, da che parte sta? (Aus dem Meer. Also, die aufgetauchten Länder sind alles Länder über dem Meeresspiegel. Auch der Meeresgrund ist Land, aber unter dem Meeresspiegel, ok? Haben wir das [verstanden]? An diesem Punkt, ich, eine andere Sache, auf die ich sofort aufmerksam machen würde, bezüglich der Erdkugel, wo ist es?)

Son: $A$ sinistra. (Links.)

HrT: Scusate, adesso prendo un po' in giro Castaldi. Questa carta indica una sfera. Perché abbiamo, secondo voi, in una Scuola Terza media degli Stati Uniti, avranno una carta cosi? Che carta avranno? (Entschuldigt, jetzt nehme ich Castaldi ein bisschen auf den Arm. Diese Karte zeigt eine Kugel. Warum haben wir, eurer Meinung nach, in der dritten Klasse einer Mittelschule werden sie eine solche Karte haben? Was für eine Karte werden sie haben?)

Klasse: No, l'America sarebbe in mezzo. (Nein, Amerika wäre in der Mitte).

HrT: Quindi l'Asia non sta a destra. (Also ist Asien nicht rechts.)

Abbildung 6: Abfragung Herr Tigre, Asien

Nur in einem Ausnahmefall wurde in der Klasse III A beobachtet, wie die mündlich geprüften SchülerInnen in ihrem Vortrag nicht durch Fragen unterstützt wurden, sondern ohne weitere Hilfe frei reden sollten. Dies geschah aus einem bestimmten Grund, und zwar handelte es sich bei den Prüflingen um diejenigen, die nicht an einem schriftlichen Test teilgenommen hatten, so dass dieser von ihnen unter möglichst ähnlichen Bedingungen in Form einer mündlichen Prüfung nachgeholt werden sollte.

Desweiteren waren vor allem die Abfragungen in der Klasse an der Schule Morante durch längere Redeanteile des Lehrers geprägt, wie auch im oben stehenden Beispiel zu erkennen ist. Dadurch kam es nicht selten zu einer Vermischung der Unterrichtsformen mündliches Prüfen, fragend-entdeckendes Unterrichtsgespräch und Lehrervortrag, während in den anderen vier Klassen die Redeanteile zwischen den Prüflingen und den Lehrkräften gleichmäßiger verteilt waren. So bauten Letztere ihren zweiten Part in der Drei-Turn-Sequenz Frage-Antwort-Lehrerreaktion nicht so sehr wie Herr Tigre aus, sondern griffen nur in einigen wenigen Fällen erklärend ein, wenn die Reaktion der Abgefragten und der Klasse vermuten ließ, dass der Lernstoff noch nicht verstanden worden war. Bevor im zweiten Teil des Kapitels auf sprachliche Einzelheiten des Lehrer- und Schülerhandelns während der Prüfungen eingegangen wird, soll an dieser Stelle beschrieben werden, wie die gestellten Fragen geartet waren. 


\subsection{Oberflächenwissen versus tieferes Wissen}

Neben Oberflächenwissen, das im Fach Erdkunde beispielsweise aus Faktenwissen bezüglich der geographischen Einordnung eines Landes, seiner physischen Beschaffenheit und seiner soziopolitischen Organisation (Hauptstadt, Amtssprache, Währung, ...) besteht, wird in den Lehrplänen auch die Anregung zu tieferen Denkprozessen gefordert. Danach sollen Hintergründe verstanden werden, warum zum Beispiel die wirtschaftliche oder politische Lage eines Landes auf eine bestimmte Art und Weise gestaltet ist, und Kompetenzen erworben werden, um verschiedene geographische Regionen miteinander $\mathrm{zu}$ vergleichen, Daten aus unterschiedlichen Informationsträgern wie Texten, Karten, Schaubildern, Tabellen und dergleichen gewinnen und miteinander in Verbindung setzen zu können (vgl. Kap. 4.2.1 und 4.2.2). Obwohl die Notwendigkeit der Vermittlung dieses tieferen Wissens immer wieder betont wird, ergibt Hatties Zusammenstellung von Metaanalysen zur Unterrichtsgestaltung, dass die meisten Lehrkräfte ( $80 \%$ und mehr) nur Fragen zum Oberflächenwissen stellen (Hattie 2013, S. 34).

Ein erheblicher Teil des Alltagslebens im Klassenzimmer dreht sich um das Mitteilen von Wissen und dafür ist Oberflächenwissen ausreichend. Schülerinnen und Schüler lernen schnell, dass das Lernen mithilfe von Oberflächenstrategien oder -methoden (z.B. die Durchsicht der Arbeiten des vergangenen Jahres zum Wiederholen des Stoffs) zum Erfolg führt. Dagegen behaupten Lehrpersonen, dass sie einem tiefen Lehrverständnis den Vorzug geben. Gleichzeitig wenden sie Oberflächen-Unterrichtsmethoden an, was sie meist damit rechtfertigen, dass diese erforderlich seien, um die Lernenden auf wichtige Examen und Prüfungen vorzubereiten. Diese Betonung des Oberflächen-Ansatzes bedeutet, dass den Klassen selten über entsprechende Anforderungen die Gelegenheit gegeben wird, tiefes Denken unter Beweis zu stellen.

Hierbei steht außer Frage, dass der Erwerb von Oberflächenwissen wichtig ist, auch weil tiefere Denkprozesse ohne es kaum angeregt werden können. Es kommt also eher auf Ausgewogenheit zwischen der Vermittlung und Festigung beider Wissensformen und der mit dem tieferen Wissen verbundenen Fertigkeiten an (s.a. Hattie 2013, S. 35 f.; A. Helmke 2009², S. 40). Eine Möglichkeit $\mathrm{zu}$ einer solchen Ausbalancierung ist in den Abfragungen gegeben, im Zuge derer neben der Kontrolle von Faktenwissen auch Fragen gestellt werden können, die darüber hinausgehen und den Einsatz von sogenannten Schlüsselkompetenzen erfordern (ibid.). Als eine solche Schlüsselkompetenz gilt das mündliche Ausdrucksvermögen, welches die Lernenden durch ihre Beiträge am Unterrichtsgespräch unter Beweis stellen und mithilfe eines systematischen und wiederholten Übens der oraten Darstellung und mit dem entsprechenden 
Lehrerfeedback ausbauen können. ${ }^{177}$ Die während der Erhebung beobachtete Vorgehensweise bestand dabei darin, bisweilen von faktenbezogenen zu komplexeren Fragen überzugehen. Wenn die abgefragten SchülerInnen diese - oft trotz Unterstützung der Lehrkräfte - nicht ausschöpfend beantworteten, wurden sie nicht selten dafür gescholten, dass sie sich darauf beschränkten, die Lehrbuchtexte auswendig zu lernen. Als Beispiele hierfür werden im Folgenden einige Auszüge aus unterschiedlichen Abfragungen in verschiedenen Klassen angeführt.

Auszug aus Unterrichtsmitschrift 5, Abfragung über Amerika, Frau Cese (FrC), Marco (Mar), Chiara (Chi), Federico (Fed) - Auswendiglernen

Mar: Di prendere la sua terra. Di venderla. (Sein Land zu nehmen. Es zu verkaufen.)

FrC: Perdoname! Che significa? Che vuoi dire? (Entschuldige mich. Was bedeutet das? Was willst du sagen?)

Fed: Si dice, perdonami. (Es heißt, entschuldige mich.)

FrC [ignoriert Fed und wendet sich wieder an Mar]: /l fenomeno schiavitù, dove si situa in America? (Das Phänomen Sklaverei, wo ist es angesiedelt in Amerika?)

[Mar schweigt, Sch E antwortet unaufgefordert.]

[...]

Chi: Le colonie tedesche venivano spartite tra gli inglesi, ... (Die deutschen Kolonien wurden aufgeteilt unter den Engländern, ...)

[Chi weiß nicht weiter, FrC hilft]: Allora, hai detto le colonie vennero spartite tra? (Also, du hast gesagt, die Kolonien wurden aufgeteilt unter?)

Chi: Le colonie europee, eh, le potenze europee. (Den europäischen Kolonien, eh, den europäischen Mächten.)

FrC: Quali sono? (Welche sind das?)

[Chi zählt sie alle auf.]

FrC [zu Fed]: Il pigmeo valeva? (Der Pygmäe zählte?)

Fed: Poco, quasi niente. Invece, con la visione moderna abbiamo capito che le culture sono altre, si dice diverse, valgono in maniera uguale, ma gli europei si sono arricchiti. (Wenig, fast nichts. Mit der modernen Sicht hingegen haben wir verstanden, dass Kulturen andere sind, man sagt, unterschiedliche, sie zählen gleich viel, aber die Europäer haben sich bereichert.)

FrC: Questi confini africani che vedi sono quelli veri? (Diese afrikanischen Grenzen, die du siehst, sind das die echten?)

Fed: Crearono dei confini che mettevano a disagio le persone e questo creò, appunto, molti conflitti tra i popoli. (Sie schufen Grenzen, welche die Personen in Unannehmlichkeiten brachten, und das schuf eben viele Konflikte zwischen Völkern.)

Mar: [unverständlich; nur FrC und die anderen beiden Prüflinge hören ihn.]

FrC: Che vuol dire? (Was bedeutet das?)

Mar: [antwortet nicht; auch Chi bleibt still.]

FrC: Chi governava le colonie? [Stille] Eppure qui c'è scritto molto chiaro. Non è linguaggio difficile! (Wer regierte die Kolonien? [Stille]. Und doch steht es hier sehr klar geschrieben. Es ist keine schwierige Sprache!)

Chi: Allora, in Asia venne colonizzata l'India, dagli inglesi. E appunto, nelle scuole, erano tutti inglesi, c'era l'insegnamento dell'inglese. (Also, in Asien wurde Indien kolonisiert,

$177 \mathrm{Zu}$ dieser Schlüsselqualifikation s.a. das Unterkapitel zur Gruppenarbeit (9.1.3.3) sowie zum Lehrerfeedback die im ganzen Kapitel vorgestellten Transkriptauszüge und das ihm gewidmete Unterkapitel 9.2.5. 
von den Engländern. Und eben, in den Schulen waren alle Engländer, es gab den Englischunterricht.)

FrC [unterbricht sie]: Era un governo diretto o indiretto? (War es eine direkte oder indirekte Regierungsform?)

Fed: Da una parte [...] [erzählt von der Regierungsform, vom Salz- und Opiumhandel nach China]. Sono materie grezze che facevano lavorare a destinazione, era più economico. In India instaurarono anche tre università che si potevano permettere solo le persone agiate. (Auf der einen Seite [...]. Es sind Rohstoffe, die sie am Zielort verarbeiten ließen, das war billiger. In Indien errichteten sie auch drei Universitäten, die sich nur die wohlhabenden Personen leisten konnten.)

FrC [zu Mar]: Allora, tesoro mio, bello, te l'ho detto sempre. Studiare a memoria non va bene. Ce l'hai messa tutta, ma non va bene. (Also, mein Lieber [wörtlich: mein Schatz, Guter], ich habe es dir immer gesagt. Auswendiglernen ist nicht gut. Du hast alles gegeben, aber das geht so nicht.)

Abbildung 7: Abfragung Frau Cese, zum Auswendiglernen

Das nächste Beispiel stammt aus der Klasse III C, in der Prof.ssa Dante unterrichtete. Hier und in den anderen beiden beobachteten Klassen an der Schule Pestalozzi war das Audioregistrieren nicht gestattet, weshalb Teile des Unterrichtsgespräches nicht vollständig protokolliert werden konnten.

Auszug aus Unterrichtsmitschrift 6, Abfragung Frau Dante (FrD), Margherita (Mar), Lea - Auswendiglernen

[Die Erdkundestunde beginnt damit, dass ein Italienischtest aus der Stunde davor zuende geschrieben wird.]

FrD: Allora, sentiamo invece in geografia [Pause] Margherita e Lea. (Also, hören wir in Erdkunde hingegen Margherita und Lea.)

FrD: L'Asia centrale. (Zentralasien).

Mar: L'Asia centrale... [spricht sehr schnell, zählt alles Mögliche zur Morphologie, aber auch Geschichte, Brauchtum und Gepflogenheiten auf. Formuliert einen semantisch merkwürdigen Satz und wird daraufhin von Frau Dante unterbrochen].

FrD: Hai imparato a memoria quello che c'è scritto sul libro, vero? Ma forse non I'hai capito bene. [FrD beginnt zu erklären.] (Du hast das auswendiggelernt, was im Buch geschrieben ist, richtig? Aber vielleicht hast du es nicht richtig verstanden. [FrD beginnt zu erklären.])

Abbildung 8: Abfragung Frau Dante, zum Auswendiglernen

Während Marco während der ersten Abfragung, in die mehrere Lernende involviert waren (s. Abb. 7), kaum zu Wort kommt, keine Antwort gibt und zweimal etwas sagt, dass er wahrscheinlich auswendig gelernt hat und auf Bitte der Lehrkraft nicht erklären kann, unterbricht Frau Dante eine kaum strukturierte und konfuse Schilderung Margheritas und unterstellt ihr, dass sie vielleicht gelernt habe, ohne das Gelesene richtig zu verstehen. Daraufhin erklärt Frau Dante ihr und dem Rest der Klasse die Zusammenhänge, bevor sie mit der Abfragung weiterfährt. Auf diese Weise werden zwar sowohl Schüler C als auch Margherita dafür kritisiert, dass ihre Lernmethode nicht den Vorstellun- 
gen der Lehrkraft entspricht, doch für Margherita ergibt sich durch das Lehrerhandeln eine günstigere Lerngelegenheit, indem Prof.ssa Dante ihr als Vorbild dient, wie der Inhalt sprachlich besser formuliert und strukturiert werden kann.

Bei einer Abfragung in einer anderen Stunde merkt Frau Cese wieder an, dass eine Schülerin auswendig gelernt habe, aber schwächt die Kritik an dieser Lerntechnik ab, was damit zusammenhängen wird, dass die Schülerin imstande war, auf alle Lehrerfragen zufriedenstellend zu antworten, ihre Ausführungen durch Detailwissen wie exakte Zahlenangaben auszubauen und dabei in ihrer Darstellung verständlich zu sein. So beurteilt Frau Dante: „Hai uno studio menomonico. È più difficile, perché, appunto, ti devi ricordare tante cose“ (Du hast einen mnemonischen Lernstil. Das ist schwieriger, weil du dir eben viele Sachen merken musst).

In dieser mündlichen Prüfung unterbricht die Lehrkraft die Schülerin nach einer Weile mit den Worten: „Però ti posso dire una cosa? Lavoriamo sulle cartine. Se no, diventa una geografia delle nozioni“" (Aber kann ich dir etwas sagen? Lass uns mit den Karten arbeiten. Sonst wird das eine FaktenwissenGeographie). Hier zeigt sich also, dass die Lehrkraft von der Darstellung des Oberflächenwissens der Schülerin zu kognitiv anspruchsvolleren Aufgaben übergehen möchte, für deren Bearbeitung tieferes Wissen aktiviert werden muss.

Generell ließ sich bei den Abfragungen beobachten, dass die Lehrkräfte oft mit offenen Fragen begannen, so z.B. ,Stelle die geographische Region X vor“. Als Herr Tigre hingegen eine mündliche Prüfung mit der Frage an eine Schülerin eröffnete, was sie am Erarbeiten des afrikanischen Kontinents besonders beeindruckt habe, blieb eine Antwort der Schülerin aus. Wenn die Prüfungen durch offene Fragen eingeleitet wurden, unterbrachen die Lehrpersonen die Darbietungen der SchülerInnen häufig, um ihr Wissen mit punktuelleren Fragen genauer zu überprüfen und/oder um etwas mehr Struktur in die Darstellungen zu bringen, da sich die Lernenden oft sprunghaft und ohne Methode von einem Thema zum nächsten bewegten. Es kann festgehalten werden, dass sich die meisten Fragen auf Faktenwissen wie die Glaubensangehörigkeit der Landesbevölkerung, die Namen der höchsten Gebirge u.Ä. bezogen. Das Verfügen über bestimmte geographische Begriffe und Konzepte, die Gegebenheiten und Phänomene bezeichnen, die nicht nur lokal, sondern in verschiedenen Regionen auftreten, wurde dabei regelmäßig überprüft, wie es z.B. bei Fachausdrücken wie altopiano (AD; Hochebene), pianura alluvionale (Ts geol.; Schwemmebene) oder dem Monsun (monsone, Co Ts meteor.) in allen beobachteten Klassen der Fall war.

Dass die Lernenden auch tiefere Denkprozesse tätigen mussten, war eher die Ausnahme und wurde vor allem im Vorfeld einer Abfragung in der Klasse III A an der Schule Pestalozzi beobachtet: Die Klassenlehrerin Frau Rea erteilte 
in der Stunde vor den interrogazioni den Auftrag, in Einzelarbeit ein Histogramm (Säulendiagramm) anzufertigen, für das die Lernenden Daten aus zwei verschiedenen Schaubildern in ihrem Lehrwerk verwenden sollten.

Obwohl das Verstehen und Anfertigen von Diagrammen und Tabellen in den Lehrplänen als Aufgabe des Edkundeunterrichts verankert ist, wurde eine Beschäftigung mit ihnen nur in den Klassen von Herrn Tigre und Frau Dante beobachtet. Beide beschränkten sich darauf, die Lernenden mit viel Unterstützung Informationen aus den entsprechenden Lehrbuchgraphiken gewinnen zu lassen. Nur in der Klasse III A fand eine systematische Förderung diesbezüglicher Kompetenzen statt, die auch das Erstellen von Diagrammen umfasste. Hier sollte die Klasse etwa in einem Diagramm die Geburtenrate verschiedener Länder und den Bildungsstand der weiblichen Bevölkerung zueinander in Bezug setzen, die im Lehrbuch in zwei unterschiedlichen thematischen Planisphären illustriert waren. Zudem sollten nur die Daten bestimmter Länder extrahiert werden, was dadurch erschwert wurde, dass nicht alle Ländernamen auf den Karten verzeichnet waren. Die Lernenden mussten also wissen, wo sich die Länder, für die sie Angaben ermitteln sollten, befinden. Dabei half die Lehrkraft mit Hinweisen für noch nicht im Unterricht behandelte Länder wie Afghanistan. Desweiteren verlangte sie eine sorgfältige und präzise Diagrammanfertigung mit einer Säulenrangfolge, die nach der Höhe des Bildungsstands geordnet werden musste, und einer Legende, in der neben den Ländernamen auch aufgeführt werden sollte, dass für beide Datensätze unterschiedliche Maßeinheiten gelten.

Die SchülerInnen konnten während der Unterrichtsstunde mit der Aufgabe beginnen und sollten sie zuhause beenden. Während sie sich an die Arbeit machten, ging die Lehrkraft von Tisch zu Tisch, half einzelnen Lernenden und ermahnte andere, die ohne Heft zum Unterricht erschienen waren oder das Diagramm nicht auf kariertem, sondern auf liniertem Papier anfertigten. Zwischendurch richtete sie Empfehlungen zur Bearbeitung an die ganze Klasse, etwa ausreichend Platz zwischen den Säulen für die einzelnen Länder und unter dem Histogramm zu lassen. Ein Schüler, der das Säulendiagramm bereits am unteren Rand der Seite gezeichnet hatte, entgegnete, er könne Nummern in die Säulen schreiben und in der Legende erklären, für welche Länder die jeweiligen Zahlen stehen. Daraufhin erklärte Prof.ssa Rea der ganzen Klasse, warum das keine Lösung sei, da ein Diagramm unmittelbar verständlich sein müsse. Zwischendurch wurden die Lernenden von ihr daran erinnert, alleine zu arbeiten und nicht auf den Banknachbarn zu achten, sondern sich bei Erklärungsbedarf an sie zu richten, da sie sichergehen wollte, dass wirklich alle die Fähigkeit zur Anfertigung eines Diagramms erwarben. Die hier wiedergegebenen Ratschläge, welche die Lehrkraft teils an einzelne SchülerInnen, teils an die gesamte Klasse richtete, stellten das scaffolding für eine gelingende Ausführung des Arbeitsauftrages durch die Lernenden dar. 
Die Unterrichtseinheit, die sich mit dem Erstellen und Interpretieren von Diagrammen und anderen graphischen Informationsträgern befasste, wird hier auszugshaft ausführlicher beschrieben, weil sie verschiedene Kompetenzen wie das Extrapolieren und Konvertieren von Daten aus graphischen Darstellungen und ihre Verknüpfung und Überführung in ein neu zu entwickelndes Diagramm erfordert, wobei die Schüler dazu angehalten wurden, konzentriert und zügig zu arbeiten. Überdies wird sie geschildert, weil in anderen Klassen weder ähnlich komplexe Anforderungen gestellt wurden, noch überhaupt im Geographieunterricht schriftlich gearbeitet wurde, wenn man einmal von den gelegentlichen Aufforderungen absieht, Notizen zur Präsentation der Lehrbuchtexte anzufertigen. ${ }^{178}$

Diese komplexere Art der Aktivierung von Tiefenwissen wurde zudem während einer Abfragung, die auf die Diagrammerstellung folgte, fortgesetzt. So sollte ein Schüler das eigenständig erarbeitete Wissen über den Zusammenhang zwischen der Geburtenrate und dem Bildungsstand des weiblichen Bevölkerungsanteils mit Informationen über die weltweite wirtschaftliche Entwicklung und den Wohlstand, die in einem weiteren Schaubild des Lehrbuchs präsentiert waren, in Verbindung setzen.

\subsubsection{Gruppenarbeit}

So wie in der gerade geschilderten Unterrichtssituation, in der die SchülerInnen das Säulendiagramm in Einzelarbeit anfertigen sollten, fand der Unterricht im gesamten beobachteten Zeitraum ohne Partnerarbeit, sondern immer nur im Klassenplenum statt. Während der Lehrervorträge wurden die SchülerInnen nur selten mit Fragen aktiv am Unterrichtsgespräch beteiligt, was in den Abfragungen natürlich zunahm, doch nur wenige betraf. Von diesem vorherrschenden Muster wich eine Klasse allerdings ab.

Obwohl hinlänglich bekannt ist, dass die Gruppenarbeit als schülerzentrierte Arbeitsform ein didaktisches Gestaltungsmittel ist, das in Hinblick auf die Leistung im Allgemeinen und die Förderung selbstregulierten Lernens und sozialer Kompetenzen im Speziellen als sehr wirksam gilt (A. Helmke $2009^{2}$, S. 211 f.) und durch eine Steigerung der Selbstregulierung gleichzeitig die Belastung der Lehrpersonen im Unterricht abnimmt (Klippert 2006), konnte diese Praxis nur in einer Klasse (Vespucci) beobachtet werden. Hier entschied sich die Lehrkraft dafür, die zehnte Stunde ihres für Italienisch, Geschichte und Erdkunde bestimmten Zeitkontingents dafür zu nutzen, den Schülerinnen und Schülern im Rahmen des Erdkundeunterrichts eine Unterrichtsform zu eröffnen, in der sie das Lernen lernten.

178 Eine Ausnahme hierzu bildet ein in der Klasse der Schule Vespucci gemeinsam angefertigtes und w.o. (Kap. 7) vorgestelltes Tafelbild. 
Es sei angemerkt, dass als Argument gegen die Gruppenarbeit in den anderen Klassen sogar die geltenden Sicherheitsnormen angeführt wurden, die es in vielen Klassenzimmern angeblich nicht gestatten, die Tische zu Gruppentischen zusammenzustellen, weil dadurch bei Notfällen das Evakuieren der nicht sehr geräumigen Klassenzimmer erschwert sei. Aber auch aus der Forschung im deutschsprachigen Raum ist bekannt, dass Gruppenarbeit wesentlich weniger praktiziert wird, als es sinnvoll wäre, obwohl die Lehrer den positiven Nutzen kennen (A. Helmke 2009², S. 212). Die Gründe hierfür sind vielfältig und zum Beispiel im Kontrollverlust der Lehrperson, im Zeitaufwand, der für die Arbeitsform erforderlich ist, und in der dabei entstehenden Unruhe zu sehen. Nicht zuletzt spielt auch eine Rolle, dass Gruppenarbeit in der Lehrerausbildung nicht unbedingt thematisiert wird - und wenn, dann nur abstrakt und verbal (ibid., S. 214). A. Helmke verweist auf eine Studie von Dann, Diegritz und Rosenbusch (2002), in der Lehrkräfte nach ihren Unterrichtsstunden, in denen es Arrangements wie Kleingruppenarbeit gegeben hatte, dazu befragt wurden, was sie diesbezüglich beschäftigte, und fasst die Ergebnisse folgendermaßen zusammen (A. Helmke 2009², S. 212):

Dann et al. verwenden bei ihrer Erklärung das Konzept des „,widersprechenden Imperativs“ im Sinne von inneren Handlungsaufforderungen: Einerseits wird Kleingruppenarbeit für richtig und wichtig gehalten, andererseits erzeugt diese Lehrform bei Lehrkräften Konflikte zwischen ihrer traditionellen Lehrerrolle (wie sie im lehrergesteuerten Unterricht dominiert: Strukturierung, Kontrolle) und dem völlig entgegengesetzten Rollenprofil, das der Kleingruppenarbeit zugrunde liegt (Akzeptanz von Unübersichtlichkeit, Sichzurücknehmen).

Der Konflikt, in dem sich die Lehrpersonen bei der Durchführung von Gruppenarbeit und ähnlichen innovativeren Formen des Unterrichts befinden, besteht für die Autoren zwischen dem Eingreifen in das Geschehen und dem Abgeben von Kontrolle. Eine häufige Entscheidung zugunsten des Eingreifens (Strukturierung der Gruppe, Überwachung des Arbeitsprozesses und inhaltliche Einmischung, Intervention bei Streitigkeiten usw.) führe dazu, dass das Unterrichtsarrangement spürbar an Qualität verliert (ibid., S. 213).

Wenn Gruppenarbeit hingegen sinnvoll eingesetzt wird, so hat diese Form des kooperativen Lernens für die SchülerInnen neben der schon erwähnten Förderung ihrer fachlichen Leistungen und des selbstregulierten Lernens noch weitere Vorteile. Zum einen sind soziale Kompetenzen wie das Miteinander, das Zusammenarbeiten, die gegenseitige Unterstützung, das Nachfragen, Erklären und das Übernehmen von Verantwortung sowohl eine Voraussetzung für erfolgreiche Gruppenarbeit als auch durch diese ergiebig optimierbar. Darüber hinaus hat sich gezeigt, dass kooperatives Lernen gerade für Leistungsschwächere überproportional förderlich ist, so dass es im Gegensatz zu LehrLern-Settings, die den Lernenden viel Freiraum lassen und Vorgaben und Rückmeldungen durch die Lehrkraft nur geringfügig beinhalten, die Leistungsdifferenzen zwischen den Lernenden nicht weiter vergrößert (vgl. ibid., S. 249, S. 214 mit Verweis auf Rohrbeck et al. 2003). Die beobachtete Klasse, 
in der regelmäßig in Gruppen gearbeitet wurde, wies nach Angaben ihrer Lehrkraft ein niedrigeres Leistungsniveau auf und umfasste mehrere in der Zweitsprache lernende SchülerInnen, die ihre Schulzeit in den Herkunftsländern begonnen hatten und sich vor allem mit den in der Schule verwendeten sprachlichen Registern des Italienischen noch schwertaten. Mit diesen Schwierigkeiten standen sie nicht alleine da, sondern sie wurden auch von einigen autochthonen Lernenden geteilt, die teilweise bereits ein Schuljahr wiederholt hatten und weiterhin z.B. beim Leseverstehen Probleme aufwiesen.

Der Einsatz der Gruppenarbeit wirkte in der beobachteten Klasse wohl durchdacht und diente primär der Festigung und dem (Über-)Lernen von Konzepten, die zuvor im lehrergesteuerten Frontalunterricht eingeführt worden waren oder ihn vertieften. W.o. wurde bereits herausgestellt, dass diese Form der Unterrichtsgestaltung für leistungsschwächere Lerngruppen vorteilhaft ist, da sie von einer stärker anleitenden Unterrichtsführung und Gelegenheiten zum Wiederholen des Lernstoffs profitieren. So erscheint gleichsam die beobachtete, relativ ausgeprägte Kontrolle der Lehrkraft über die Gruppenarbeit als zweckmäßig: Frau Mancuso stellte die unter dem Namen isole (Inseln) eingeführten Gruppen recht leistungshomogen zusammen, was ihr ein individuelleres Zuschneiden der Lerninhalte gestattete, als es im Frontalunterricht möglich ist. So hatten die leistungsstärkeren Schülerinnen und Schüler im gleichen Zeitraum ein umfangreicheres $\mathrm{Ma} \beta$ an Textmaterial zu bearbeiten, wodurch sowohl ihre Unterforderung als auch Überforderung vermieden werden konnte. Auch die „Kontrollgänge“ $\mathrm{zu}$ den verschiedenen Inseln schienen ihren lernunterstützenden Zweck zu haben, weil der Schulalltag so vom Frontalunterricht bestimmt war, dass die Lernenden stark an Hilfestellungen der Lehrperson und die Reaktion auf ihre Beiträge gewöhnt waren.

Es wurde jedoch beobachtet, dass sich die SchülerInnen im Laufe des Schuljahrs immer mehr an die Unterrichtsform gewöhnten und zunehmend selbstständig, aktiv und konzentriert arbeiteten, selbst wenn die Lehrerin über den gesamten Zeitraum der Gruppenarbeit das Klassenzimmer verließ. Desweiteren wurden die Gruppen in der beobachteten Zeitspanne nicht grundsätzlich nach dem Kriterium vergleichbarer Leistungen gebildet, sondern ab und $\mathrm{zu}$ bewusst heterogener zusammengesetzt. Sofern das Leistungsgefälle zwischen den Gruppenmitgliedern nicht zu ausgeprägt war, sondern sich die Leistungen der Stärkeren innerhalb der Zone der nächsten Entwicklung der Schwächeren befanden, bot die Gruppenarbeit allen Beteiligten und insbesondere den Schwächeren eine förderliche Lerngelegenheit.

In der beobachteten Klasse an der Schule Vespucci setzen sich die einzelnen Gruppen bei einer Gesamtklassengröße von 14 aus drei bis vier Lernenden zusammen. Solche Kleingruppen verhindern im Gegensatz zu größeren eher, 
dass sich arbeitsscheue oder schüchternere Schüler dem Arbeitsprozess entziehen ${ }^{179}$, und sorgen für eine gleichmäßige Beteiligung aller Gruppenmitglieder. Zum Zeitpunkt, an dem die Unterrichtsbeobachtungen starteten, hatten die Lernenden diese Form des Lernens bereits so routiniert, dass sie in der Regel nicht viel Zeit mit dem Zusammenstellen der Tische verloren und zügig mit der Arbeit begannen.

Ihr auch in Partnerarbeit durchführbarer Auftrag ist aus der Fachliteratur als ein „Lehren durch Lernen“-Szenario der scripted cooperation bekannt (vgl. A. Helmke 2009², S. 209): Die SchülerInnen erarbeiteten gemeinsam einen Text und versuchten den Inhalt zu behalten, indem sie ihn zuerst still lasen und dann reihum je einen Absatz zusammenfassten und den anderen Gruppenmitgliedern vortrugen. Jene hatten dabei die Aufgabe zu kontrollieren, ob die Textaussage des Abschnitts in ihren wesentlichen Elementen vollständig und richtig wiedergegeben wurde, wobei sie gegebenenfalls eine ergänzende oder korrigierende Funktion übernahmen. Da zum Schluss alle Gruppenmitglieder jeden Absatz vor der Klasse richtig wiedergeben können mussten, war ihre Motivation gefördert, sich nicht zurückzuziehen, sondern die Vorträge der anderen Gruppenmitglieder mitzuverfolgen und sich an den anschließenden Besprechungen zu beteiligen.

In der kleinen Gruppe von Peers trauen sich zudem vor allem leistungsschwächere und schüchterne Lernende eher als vor der Lehrkraft und der gesamten Klasse, etwas beizutragen oder einzugestehen, dass sie einen bestimmten Aspekt noch nicht verstanden haben (z.B. Häussermann \& Piepho 1996, S. 215). Hierzu ist anzumerken, dass das beobachtete Klassenklima als positiv empfunden, niemand als Außenseiter wahrgenommen wurde und unter den Lernenden ein freundlicher Umgangston herrschte. So kam es auch in den Gruppenarbeitsphasen nicht zu Streitereien oder Gereiztheit. Der bisweilen stockenden, unvollständigen oder inhaltlich falschen Vortragsweise einer oder eines Einzelnen begegnete die Gruppe geduldig.

Während der Gruppenarbeit schaute die Lehrerin von Tisch zu Tisch und vergewisserte sich, wie die SchülerInnen vorankamen. Ab und zu griff sie auch direkt in den Lernprozess ein, indem sie jemanden bat, einen Begriff oder ein Konzept zu erläutern. Da die Erklärungen nicht selten sehr knapp ausfielen, versuchte sie anhand weiterer Fragen herauszufinden, ob die wesentlichen Aspekte des Konzepts bekannt waren. Es ging ihr dabei vor allem um die inhaltliche Ebene, denn sie verlangte nicht, dass eine Antwort in ganzen Sätzen erfolgte oder dass die mit ihrer Hilfe gemeinsam erarbeiteten Einzelheiten einer Erklärung als Ganzes von dem entsprechenden Schüler wiederholt wurden.

Dass auch einige der einsprachig italienischen SchülerInnen dieser Lerngruppe über geringere sprachliche Fähigkeiten verfügten, wurde u.a. deutlich,

179 Auch in größeren Gruppen kann dies vermieden werden, indem jedes Mitglied eine bestimmte Aufgabe zugeteilt bekommt und z.B. alle Lernenden nach der Arbeitsphase in der Lage sein sollen, die Gruppenergebnisse vorzustellen (s. A. Helmke 2009², S. 211). 
als die Ergebnisse der Gruppenarbeit vorgestellt werden sollten: Auf die Aufforderung etwa, ihren Absatz zum Konzept des HDI (Human Development Index) vorzustellen, reagierte eine Schülerin mit den Worten „Non lo so ancora

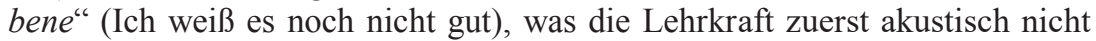
verstand, wiederholen ließ und dann lediglich mit einem ,ah“ kommentierte, bevor sie eine andere Schülerin aufrief. Defizite in der Lesekompetenz schienen der Grund zu sein, warum diese Schülerin der Lehreraufforderung nicht nachkam, denn während der Gruppenphase verweigerte sie den Arbeitsauftrag nicht, sondern las ihren Textabschnitt. Auch wenn der Rest der Klasse angab, die Aufgabe fertiggestellt zu haben, reichte ihr die Zeit zur Erarbeitung nicht. Das lässt auf eine geringere Lesefähigkeit schließen, was bei einem langsameren Lesetempo mit einem Bedürfnis nach mehr Zeit einhergeht, um das Gelesene zu elaborieren. Diese Schülerin wollte ihren Absatz in der geschilderten Unterrichtssituation weder im Klassenplenum noch in der Gruppe vorstellen, da sie sich dazu noch nicht ausreichend vorbereitet fühlte, weshalb sie von den anderen übergangen wurde. Es zeigte sich auch in anderen Stunden, dass sie sehr langsam arbeitete und versuchte, sich der mündlichen Darstellung des Gelesenen zu entziehen. Allerdings redeten die anderen Gruppenmitglieder ihr dann gut zu, es wenigstens zu versuchen und unterstützten sie, wenn sie beim Vortragen nicht weiterwusste oder die Sachverhalte unvollständig bzw. falsch darstellte.

Die Gruppenarbeit ist somit eine geeignete Form der Unterrichtsgestaltung, um die aktivere Mitarbeit aller zu garantieren, und unter sprachförderlichen Gesichtspunkten lässt sich für die Organisation dieser Arbeitsform an der Vespucci festhalten, dass die SchülerInnen durch das Lesen und Verstehen von Textpassagen, bei dem sie sich gegenseitig unterstützten, grundlegende rezeptive sprachliche Fähigkeiten trainierten und ausbauten. Zudem gab das erforderte Vortragen eines/einer jeden innerhalb der Gruppen allen Raum, an der mündlichen Ausdrucksfähigkeit zu feilen. Das orate Darstellen hebt Weinert (2002) als eine der Schlüsselkompetenzen unter diesen ganz besonders hervor (Weinert 2002 in A. Helmke 2009², S.44):

Der mündliche sprachliche Ausdruck ist wahrscheinlich die bedeutendste Schlüsselqualifikation, die es gibt. Sie ist unverzichtbar für sehr viele Berufsbereiche und wichtig in vielen Situationen.

Sowohl die Gruppenarbeitsphasen als auch die Abfragungen verleihen der mündlichen Darstellungskraft großes Gewicht und bieten somit eine Gelegenheit, die Lernenden in der Entwicklung und im Ausbau ihrer produktiven Sprachkompetenz zu unterstützen. Auf diese Weise ging der Unterricht durch die Zentralität des oraten Ausdrucksvermögens in allen fünf Klassen entscheidend über das hauptsächliche Vermitteln von Faktenwissen hinaus. Wie sich die Lehrperson sprachfördernd verhielten, vor welchen Herausforderungen die 
Lernenden standen und welche Rolle hierbei das Lehrbuch einnahm, beschreibt der zweite Teil des Kapitels, dem jedoch noch ein letzter Absatz über den Umgang mit der Mehrsprachigkeit im Unterricht vorausgehen soll.

\subsubsection{Mehrsprachigkeit im Unterricht}

Die fünf beobachteten Lehrkräfte schienen allesamt nach der Auffassung zu handeln, dass ihre Schülerinnen und Schüler das Medium der Unterrichtssprache am besten beherrschen lernen, wenn sie ausschließlich ihm ausgesetzt sind. Es zeigte sich also in ganz deutlicher Form der monolinguale Habitus, wie er in öffentlichen Einrichtungen vieler westlicher Nationalstaaten seit ein paar Jahrhunderten fest verankert ist (vgl. Gogolin 1994). Dieser ist im Bildungswesen mit der falschen, aber weit verbreiteten Ansicht gepaart, dass die Lernenden für gute Sprachkenntnisse so zeitaufwendig wie möglich in der Unterrichtssprache verkehren sollten und das Pflegen einer anderen Sprache im Elternhaus der Entwicklung der Fähigkeiten in der Bildungssprache im Wege stehe. Dabei ist längst erwiesen, dass von der im Elternhaus gesprochenen Sprache keine Rückschlüsse auf die schulischen Leistungen und Literalität in der Unterrichtssprache geschlossen werden können (z.B. Maas 2008, S. 84) und dass ein zeitliches Mehr allein nicht automatisch die Entwicklung besserer sprachlicherer Kompetenzen garantiert (Cummins 1979, S. 20 ff.; S. 41f.). Außerdem bestehen die Sprachsysteme Mehrsprachiger nicht mit festen Grenzen abgeschottet voneinander, sondern sind im Gegenteil miteinander verwoben. Auch wenn im Fremdsprachenunterricht sowie im Umgang mit in der Zweitsprache lernenden Schülern danach gehandelt wird, die Sprache so zu vermitteln, wie der Erwerb der Muttersprache oft erfolgt, nämlich indem man ihr in hohem Maße und ausschließlich oder überwiegend ausgesetzt ist, ist inzwischen unbestritten, dass sich sprachliche Kenntnisse in verschiedenen Sprachen gegenseitig beeinflussen. Zwar kann sich dieser Transfer z.B. auf phonologischer Ebene oder auch durch lexikogrammatikalisch falsche Übertragungen von der einen in die andere Sprache negativ manifestieren, jedoch besteht er genauso sehr oder noch mehr auch im positiven Sinne - und das auf verschiedenen sprachlichen Ebenen: Je größer das Sprachrepertoire, desto häufiger kann bei der Worterschließung in einer weniger gut beherrschten Sprache daraus geschöpft werden. Gleichzeitig stärkt es außerdem die Sprachbewusstheit und die metasprachlichen Fertigkeiten. Mehrsprachige vergleichen verschiedene Sprachen, beobachten automatisch, welche Unterschiede und Gemeinsamkeiten es in den Ausdrucksmöglichkeiten gibt, und entwickeln so ein umfangreiches Wissen darüber, wie Sprache funktioniert. Darüber hinaus ermöglicht nach der von Cummins (1981) aufgestellten Interdependenzhypothese eine hohe Sprachkompetenz in einer Sprache, das in ihr Gelernte (bspw. 
über literate Textgestaltung oder Strategien des Leseverstehens) auf eine andere Sprache zu übertragen. Aus diesen Gründen ist es für die Entwicklung bildungssprachlicher Fähigkeiten kontraproduktiv, den Gebrauch der Erstsprachen strikt zu unterbinden. Dabei wird und kann bei der wachsenden Anzahl der Erstsprachen in den Klassenzimmern ${ }^{180}$ auch nicht vorausgesetzt werden, dass die Lehrperson über Kenntnisse in allen verfügt.

Dass die Schülerinnen und Schüler untereinander in ihren nicht italienischen Erstsprachen sprachen, wurde so gut wie nie beobachtet, was wohl in erster Linie daher rührt, dass selten zwei dieselbe Erstsprache sprachen und Banknachbarn waren. Da die meisten in Italien eingeschult worden waren und ihre BICS für die Gespräche untereinander bestens ausreichten, verhielten sie sich gemäß der gängigen Praxis von Mehrsprachigen so, dass sie in gemischten Gruppen diejenige Sprache sprachen, die alle verstanden, also Italienisch.

Anders war es lediglich um den chinesischen Schüler an der Morante bestellt, dessen Italienischkenntnisse noch so gering waren, dass die Lehrkraft des Öfteren eine andere erstsprachig chinesische Schülerin als Dolmetscherin einspannte. Bei diesem Schüler handelt es sich - neben einem weiteren in einer an der Vespucci beobachteten Situation - auch um den einzigen in allen fünf Klassen, von dem die Lehrkraft im Erdkundeunterricht ausdrücklich den Gebrauch der Erstsprache erwünscht hat. So wurde ihm beispielsweise bei der Einführung in den Kontinent Asien die Hausaufgabe gestellt, schriftlich festzuhalten, was er an der Landschaft und Kultur seiner Heimat besonders mag. Im Unterricht nahm seine „Sprachassistentin“, eine Mitschülerin, dann für die Klasse eine mündliche Stehgreifübersetzung seines Textes ins Italienische vor.

An der Schule Vespucci hingegen forderte die Lehrerin während des gemeinsamen Erstellens eines Netzdiagramms zur gesellschaftlichen Entwicklung einen Schüler, der vor ca. zwei Jahren aus Peru nach Italien gezogen war (im Folgenden Rodrigo genannt), auf, einen Begriff zu nennen, den er damit verband. Da er keine Antwort gab, bat sie ihn, zunächst den spanischen Ausdruck für „Entwicklung“ zu nennen. ${ }^{181}$ Auch danach blieb er zunächst wieder still und übersetzte einen bereits genannten Ausdruck (crescita, Wachstum) ins Spanische. Dadurch dass die Lehrerin nicht von ihm abließ, nannte er schließlich einen eigenen Begriff, der im Spanischen und Italienischen identisch ist

180 Gogolin, Siemund, Schulz und Davydova (2013) weisen auf die europäische linguistische und kulturelle „Superdiversität“ vor allem in Großstädten hin, welche die Hauptattraktion einer Migration darstellen, die sich seit den frühen 1990er Jahren zunehmend verändert. Während in den Jahrzehnten davor Zuwanderungen aus einigen wenigen Ländern erfolgten, sind es nun kleinere Gruppen von Migranten, die sich in allen europäischen Ländern niederlassen und beispielsweise in Deutschland aus nahezu allen Teilen der Welt stammen, nämlich aus 190 der weltweit 193 von den Vereinten Nationen als solche definierten Staaten. Das führt zu einer entsprechend hohen Zahl von Erstsprachen, die auf europäischem Boden vertreten sind (vgl. Gogolin et al. 2013, S. 3-5).

181 Die SchülerInnen der beobachteten Klassen lernten als zweite Fremdsprache nach Englisch Spanisch. 
(aumento, Steigerung), so dass unklar blieb, welche Sprache er verwendete. In diesem Fall war vermutlich nicht die Unterrichtssprache der Grund für sein zögerliches Antworten, sondern die Tatsache, dass ihm sprachunabhängig in Kürze kein passendes Wort einfiel. Da die Lehrerin den Auftrag aber nicht an jemanden weiterreichte, sondern auf seine Antwort wartete, gelang es ihm schließlich, einen Beitrag zu leisten. Ohne dass es jemand bemerkte, übersetzte er den italienischen Ausdruck sviluppo (Fo; Entwicklung) mit einem Wort, das es im Spanischen nicht gibt und das mit dem italienischen fast identisch ist esvilupo. Er schien also Schwierigkeiten damit zu haben, sich das Wort in seiner Muttersprache wachzurufen, was nicht ungewöhnlich ist.

Transkriptauszug 3, Frau Mancuso (FrM), Rodrigo (Rod), Larissa (Lar), Unterstützungslehrkraft Frau Sole (FrS) - Einbezug der Erstsprache

FrM: Vabbè [ugs. für va bene], "un passo avanti". Bello. Un passo [Pause, sie schreibt die Wörter an die Tafel] avanti. Poi? Rodrigo, tu, eh. [Pause]. In spagnolo, Rodrigo, dimmelo in spagnolo. [Pause] Sviluppo, come si dice sviluppo in spagnolo? (In Ordnung. „Ein Schritt vorwärts“ Schön. Ein Schritt [Die Wörter an die Tafel schreibend, Pause] voran. Dann? Rodrigo, du, ne. Auf Spanisch, Rodrigo, sag es mir auf Spanisch. [Pause] Entwicklung, wie sagt man Entwicklung auf Spanisch?

Lar: Sviluppos. [erfundenes Wort mit italienischem Stamm und spanischer Endung]

FrM: Eh? (Hä?)

Rod: Esvilupo. [fiktives spanisches Wort für Entwicklung]

FrM: Es...?

Rod, andere [gleichzeitig]: Esvilupo. [erfundenes Spanisch für Entwicklung]

FrM: Esvilupo, ok. Dimmi una parola in spagnolo che ti fa pensare a sviluppo, ah, a esvilupo? [Rodrigo bleibt still, jemand kichert.] E' inutile che ridete. [Pause] Eh? [Etwas unruhig, jemand lacht.] Dai, tu! Eh? (Esvilupo, ok. Sag mir ein Wort auf Spanisch, das dich an sviluppo, äh, an esvilupo denken lässt. [Rodrigo bleibt still, jemand kichert.] Es ist unnötig, dass ihr lacht. [Pause] Eh? [Etwas unruhig, jemand lacht]. Los, du! Eh?)

Rod: Crece... crecida. (Wachs... Wachstum).

FrM: Crecida. [Stille] Poi? [Schreit:] Oh, dai! (Crecida. Dann? Oh, komm schon!)

Rod: Aumento. (Steigerung.)

FrM: Aumento. (Steigerung.)

[fast zeitgleich] FrS: America.

FrM: Aumento. Bravo! Aumento. Che ha detto? (Steigerung. Toll! Steigerung. Was hat sie gesagt?)

FrS: America.

FrM: Aumento. Aumento, non "aumenca". Bravo! Aumento. Che schifo qua, questa lavagna. (Steigerung. Steigerung, nicht aumenca (Kunstwort). Toll! Wie eklig hier, diese Tafel.)

Lar: Aumentos. [künstliches Wort mit italienischem Stamm und spanischer Endung]

FrM: Aumentos. Come si dice aumento in spagnolo? (Aumentos. Wie sagt man aumento auf Spanisch?)

Rod: Aumento.

FrM: Allora, vedete, abbiamo trovato delle parole. Forza! Sviluppo uguale rinnovo. Rinnovamento, rinnovare, dai, rinnovare. Crescita, poi, un passo avanti. Poi? Aumento. Fantastico. Un'altra e ci fermiamo qua. Un'altra? (Also, seht ihr, wir haben Wörter gefunden. Los! Entwicklung gleich Erneuerung. Erneuerung [Synonym], erneuern, 
los, erneuern. Wachstum, dann ein Schritt vorwärts. Dann? Steigerung. Phantastisch. Ein anderes und dann hören wir hier auf. Ein anderes?)

Abbildung 9: Auszug aus einem Unterrichtsgespräch an der Schule Vespucci, Mehrsprachigkeit

Der während der Unterrichtsbesuche beobachtete Sprachgebrauch der Lernenden untereinander und der von der Lehrkraft fast ausschließlich geforderte deuten darauf hin, dass die nicht italienischen Erstsprachen in dem Moment aus den Klassenzimmern wichen, in dem die alltagssprachlichen Fähigkeiten der Lernenden im Italienischen ausgeprägt genug waren, um in dieser Sprache kommunizieren zu können. Abgesehen vom aktiven Einbezug der Erstsprachen bietet dabei gerade auch das Fach Erdkunde zahlreiche Möglichkeiten, sie zu thematisieren und auf diese Weise das Selbstbild der Lernenden in Hinblick auf ihr erstsprachliches und erstkulturelles Kapital positiv und (sprach)lernförderlich zu beeinflussen. So beschäftigen sich die Klassen im achten Schuljahr mit der Sprachenvielfalt in der Welt, was nahelegt, die mehrsprachigen Schüler über ihr Sprachrepertoire und die in den Herkunftsländern ihrer Familien gesprochenen Sprachen zu befragen. Stattdessen wurde in der Klasse III B an der Schule Pestalozzi beobachtet, dass eine mehrsprachige Schülerin genau zu diesem Thema abgefragt wurde, ohne ihre eigenen Erfahrungen mit der Mehrsprachigkeit einbringen zu dürfen. ${ }^{182}$

Etwas anders sieht es mit nicht sprachbezogenen Schilderungen über ihre Herkunftsländer (oder die der Eltern) aus, die vereinzelt in zwei der fünf Klassen bei der Behandlung der Kontinente Asien, Afrika und Amerika ausdrücklich erwünscht waren. Neben dem bereits erwähnten, an den chinesischen Schüler gerichteten Arbeitsauftrag an der Schule Morante wurde so auch an der Vespucci ein ecuadorianischer Schüler zum Schulsystem in seinem Herkunftsland und zu Unterschieden in der Unterrichtsgestaltung befragt und eine Schülerin nach einer Reise in das afrikanische Herkunftsland ihrer Eltern gebeten, ihre Eindrücke vorzustellen.

Der Verzicht der Lernenden auf die Verwendung ihrer Erstsprachen ist wahrscheinlich Ausdruck eines starken Wir-Gefühls und Zusammenhalts auf Klassenebene: Wir sprechen Italienisch, denn so verstehen wir uns alle. Diese Geschlossenheit im Klassenverband, anstatt sich in kleinere Gruppen aufzuteilen, kann auf das Alter zurückzuführen sein und wurde vermutlich durch die gemeinsame Erfahrung, sich auf ihre erste bedeutende Abschlussprüfung vorzubereiten, und durch das Bewusstsein, dass der Klassenverband danach aufgelöst und sich alle auf verschiedene weiterführende Schulen verteilen würden, noch verstärkt.

182 Natürlich ist es möglich, dass vor dem Beginn der Unterrichtsbeobachtungen, bspw. am Anfang der Mittelschule, als die Klassen sich kennenlernten, über die Mehrsprachigkeit der Lernenden geredet wurde. Aber selbst wenn dem so ist, hätte es sich m.E. angeboten, dieses Thema in einer Unterrichtseinheit über Sprachenvielfalt und Mehrsprachigkeit wieder aufzunehmen. 
Cummins (2013, S. 298 f.) listet mit Verweis auf Lucas und Katz (1994) eine Reihe von Unterrichtsaktivitäten auf, bei denen die Erstsprachen genutzt werden können, ohne dass die Lehrkräfte Kenntnisse darin benötigen. Die Lehrkräfte können beispielsweise den Gebrauch zweisprachiger Wörterbücher zur Erschließung schwieriger Texte gestatten, zumal das Vertrautmachen im Umgang mit Wörterbüchern in den Lehrplänen verankert ist. Außerdem können die Lernenden dazu ermutigt werden, zuhause in ihrer Erstsprache über Hausaufgaben und Schulstoff zu sprechen und von Familienmitgliedern Unterstützung zu erhalten. Diesbezüglich ist nämlich beobachtet worden, dass Eltern, welche die Unterrichtssprache nicht oder kaum beherrschen, zwar hohe Ansprüche an den schulischen Erfolg ihrer Kinder stellen, sich aber oft kaum nach dem erkundigen, was ihre Kinder in der Schule lernen (Maas 2008, S. 650 f.). Wenn nicht immer alles an der Sprache festgemacht wird, nach dem Motto „Du kannst mir bei den Hausaufgaben oder beim Üben für eine Klassenarbeit nicht helfen, du kennst ja die Unterrichtssprache nicht", haben Eltern durchaus die Gelegenheit, eigenes Wissen an ihre Kinder weiterzugeben, das Aushandeln der Lerninhalte in beiden Sprachen zu unterstützen und damit sowohl einen förderlichen Beitrag zu einer breiteren und tieferen Verankerung des Wissens im Gedächtnis ihrer Kinder zu leisten, als auch deren Sprachausbau in der Erstsprache voranzutreiben.

Ferner können für die Schulbibliothek Bücher in den unterschiedlichen Erstsprachen angeschafft und die Lernenden ermutigt werden, sie zu lesen. Das Auswerten der Fragebögen hat allerdings ergeben, dass sich das freiwillige Lesen bei vielen Lernenden auf ein absolutes Minimum beschränkte, was sicher nicht zuletzt daran lag, dass sie täglich bereits für mehrere Schulfächer zuhause viel lesen mussten.

Wenn auf diesem Weg die Weiterentwicklung der Kompetenz in der Erstsprache vorangetrieben wird, kann sich das nur positiv auf die bildungssprachlichen Fähigkeiten der SchülerInnen auswirken und somit den schulischen Leistungen generell dienen (s. Cummins 2013, S. 358). Es steht dabei außer Frage, dass von einer derartigen Motivation, also dem Anregen zum freiwilligen Lesen, was kognitiv nicht überfordert, aber die Lesefähigkeiten verbessert, zum Gebrauch von Wörterbüchern und zum häuslichen Austausch über den Schulstoff, auch die einsprachigen Lernenden profitieren.

\subsection{Das sprachliche Handeln im Fokus}

In diesem zweiten Teil des Kapitels wird das Agieren der am Erdkundeunterricht beteiligten Akteure genauer in den Blick genommen und anhand von Beispielen aus den Unterrichtsbeobachtungen skizziert. Zunächst gilt das Interesse 
den Momenten, in denen die beobachteten Lehrpersonen sprachförderlich unterrichteten und die Aufmerksamkeit auf sprachliche Aspekte lenkten.

\subsubsection{Beobachtetes sprachförderliches Lehrerhandeln im Frontalunterricht}

Zwar kann auch im Fachunterricht Sprachliches fokussiert und dabei von der Vermittlung fachinhaltlicher Lerngegenstände getrennt werden, wie es beispielsweise bei der Verbesserung des allgemeinen, nicht fachbezogenen Ausdrucksvermögens und einiger Grammatikfehler beobachtet wurde ${ }^{183}$, doch meist geht eine Sprachförderung mit der Vermittlung von Fachinhalten einher. Eine wesentliche sprachdidaktische Funktion aller beobachteten Lehrkräfte bestand darin, den Schülerinnen und Schülern durch eine mündliche Vorstellung der Lehrbuchinhalte den Zugang zu den Texten zu erleichtern. Sie bilden die primäre Grundlage, anhand der die Abfragungen mithilfe der unterstützenden Erläuterungen und Erklärungen der Lehrkraft vorbereitet wurden. Diese Abfragungen machten im Beobachtungszeitraum das Hauptfeld der aktiven Schülerbeteiligung aus. So wurden abgesehen von sehr wenigen Ausnahmen ${ }^{184}$ in den fünf Klassen Lerninhalte nicht in schriftlichen Texten festgehalten, weder in Form von gemeinsam oder von der Lehrkraft erstellten Tafelbildern noch in Form einer an die Lernenden gerichteten Aufgabe. Die schriftliche Basis, auf der die Inhalte gelernt werden sollten, waren also die Lehrbuchtexte zuzüglich der auf sie bezogenen Markierungen und Anmerkungen und die Notizen, die sie zum Lehrervortrag festhalten konnten.

Bei der Präsentation der Lehrbuchinhalte reduzierten die Lehrkräfte die Komplexität der Textaussagen, indem sie kürzere Sätze mit einem geringeren Grad an Informationsdichte formulierten, niedrigfrequente Wörter durch häufiger gebrauchte Äquivalente ersetzten, Fachwörter und Konzepte erklärten, dabei auch auf die Lebenswelt der Lernenden Bezug nahmen und sich zwischendurch vergewisserten, ob bestimmte Ausdrücke und Konzepte bekannt waren.

183 Allerdings waren auch in den beobachteten Momenten, in denen beispielsweise Grammatikregeln wiederholt wurden, die Auslöser konkrete, fachbezogene Schüleräußerungen. Vgl. hierzu v.a. Kap. 9.2.4 und 9.2.5.

184 In der Klasse der Schule Vespucci erstellte die Lehrkraft zusammen mit den Schülern das oben erwähnte Netzdiagramm zum Begriff sviluppo sociale (Fo; soziale Entwicklung), um das mit dem Human Development Index bezeichnete Konzept des gesellschaftlichen Entwicklungsstandes zu erklären, und eine Tabelle, in der Informationen aus einem Lehrbuchtext zu den verfügbaren Ressourcen, dem technischen Entwicklungsstand und dem Bevölkerungswachstum verschiedener Länder vorgestellt werden. Herr Tigre (Morante) hingegen hielt an der Tafel Begriffe fest, die seine Lernenden als Hausaufgabe in einem Lehrbuchtext identifiziert, herausgeschrieben und nachgeschlagen hatten, weil sie kompliziert oder unbekannt waren (s.u.). 
In mehreren Klassen (Morante, III A und III C der Pestalozzi) wurde beim Vorstellen einer neuen geographischen Region beobachtet, dass die Lehrkraft die SchülerInnen dazu aufforderte, auf der Karte im Buch mitzuverfolgen, was sie beschrieb. Dadurch sorgte sie dafür, dass die neuen Lerninhalte nicht nur in sprachlicher, sondern auch in graphischer Form präsentiert und miteinander verknüpft wurden. Diese semiotische Vielfalt hat sowohl das Potential, ein besseres Verständnis des Referierten zu fördern, als auch die Wahrscheinlichkeit der Abspeicherung der Lerninhalte im Langzeitgedächtnis zu erhöhen (Hammond \& Gibbons 2005). Frau Dante etwa begann bei der Vorstellung des Kontinents Asien mit der geographischen Definition des Begriffs „Kontinent“ und der Unterteilung Eurasiens in zwei, Europa und Asien. Sie folgte dabei der Präsentation im Lehrbuch, vereinfachte ihre Darstellung jedoch auf lexikogrammatikalischer und syntaktischer Ebene. Dabei verzichtete sie keineswegs auf die Verwendung von Fachausdrücken wie beispielsweise emisfero boreale (Ts geogr.; Nordhalbkugel), wies aber darauf hin, wenn sie Wörter aufgrund ihrer Bearbeitung in vorherigen Schuljahren als bekannt voraussetzte, und erkundigte sich, ob die Bedeutung klar war. Die SchülerInnen sollten ihren Vortrag nicht nur auf der Karte im Buch mitverfolgen, sondern erhielten auch eine wiederholende Einweisung in das Kartenlesen, nachdem sie keine Antwort auf die Frage gegeben hatten, was man bei der Konsultation von Karten im Hinterkopf behalten müsse. Die Lehrkraft erinnerte daran, dass eine Karte immer ridotta, aprossimativa, simbolica (reduziert - mit Verweis auf die Skala, annähernd und symbolisch) ist.

Indem sie den neuen Kontinent nach physischen Gesichtspunkten vorstellte, lieferte sie den Lernenden ein sprachliches Modell, wie die mündliche Darstellung aufgebaut sein sollte. Das im Lehrplan festgehaltene Ziel der Entwicklung von Fertigkeiten im Kartenlesen und -vorstellen wurde in allen fünf Klassen verfolgt, wobei die Lehrkräfte zwar unterschiedlich detaillierte Kompetenzen erwarteten, doch allesamt darum bemüht waren, bestimmte Sprachbausteine zu vermitteln, deren Verwendung auch kontrolliert wurde. Mehrere Lehrkräfte betonten zum Beispiel, dass ein Land oder Kontinent nicht „an (Meere) grenze“ (confinare con, AU), sondern ,,auf (Meere) gehe“ (affacciarsi $s u$, Fo) und besser noch „,von (ihnen) umspült sei“ (essere baganto da, Fo). Diese bevorzugten Ausdrücke werden laut GRADIT zwar häufiger gebraucht als das erste Verb, dennoch verwendeten es viele SchülerInnen in ihren mündlichen Darstellungen, was daran liegen könnte, dass es ihnen geläufiger war, zumal confinare auch für die Positionsbestimmung eines Landes im Vergleich zu den umliegenden Ländern benutzt wird und im Gegensatz zum letztgenannten Verb nicht im Passiv formuliert werden muss. Lexikalische Aufmerksamkeit galt im Weiteren den Himmelsrichtungsbezeichnungen zur Beschreibung der geographischen Lage von Ländern, Städten, Gebirgen usw. anstelle der von den Schülern in vielen Fällen eher verwendeten Richtungsangaben links, rechts, oben und unten oder einfach nur qua (hier). 
Auf textstruktureller Ebene waren die Lehrkräfte um das Einhalten einer vorgegebenen Reihenfolge der geographischen Einordnung und der physischen Beschaffenheit einer Region bedacht. Das machten sie den Schülerinnen und Schülern in ihren Vorträgen vor und überprüften mit Fragen, ob sich die Inhalte im Gedächtnis verankert hatten. Während der mündlichen Präsentationen der Lernenden korrigierten sie das, was von diesen Vorgaben abwich.

Auf das konkrete Unterrichtsbeispiel von Frau Dantes Einführung in den asiatischen Kontinent zurückkommend, ging die Lehrerin nach der geographischen Einordnung zur Beschaffenheit des Territoriums über, erklärte dabei auch den Begriff Geologie und bezeichnete diese als Hilfswissenschaft der Geographie. Es ging ihr nicht nur darum, den Lernenden die Beschaffenheit der Region zu vermitteln, sondern auch um ihre Entstehungsgeschichte. Hieran zeigt sich, wie, ebenso wie in den anderen beobachteten Klassen, reines Faktenwissen mit tieferem Fachwissen verbunden wurde, das auf unterschiedliche geographische Räume und Phänomene angewandt werden kann: Die Lernenden sollten beispielsweise imstande sein zu erklären, wie ein Gebirge entstanden war und woraus sein Alter abzuleiten ist oder welche physischen und klimatischen Faktoren dazu führen, dass sich an einem bestimmten Ort eine Wüste befindet.

Desweiteren stellte Prof.ssa Dante die verschiedenen Klimazonen vor und bat die Lernenden, das auf einer entsprechenden thematischen Karte nachzuvollziehen. Obwohl es sich bei dem Begriff „Monsunklima“ nach ihren Angaben um eine Wiederholung handelte, beschrieb sie es erneut ausführlich und wiederholte dabei auch, was das Phrasem vento periodico (Ts meteor.; periodischer Wind) bedeutet. Ihr Vorgehen entspricht der zyklischen Ausrichtung des Lernprozesses, bei dem Inhalte mehrmals wiederholt und gegebenenfalls nach und nach vertieft werden, damit sie mit größerer Wahrscheinlichkeit im Langzeitgedächtnis abgespeichert werden.

Der Monsun wurde in den fünf Klassen vor bzw. während des Beobachtungszeitraums eingeführt und regelmäßig thematisiert. Alle Lehrkräfte betonten dabei die Bedeutung des bis dahin allen unbekannten Adjektivs ,periodisch“, was dazu führte, dass fast alle Lernenden es in ihren Definitionen des Windes verwendeten. Dabei reduzierten sie ihre Beschreibung des Lerngegenstands allerdings in der Regel auf diese Eigenschaft, anstatt auch weitere wesentliche Informationen wie die Richtung und seinen Einfluss auf die Landwirtschaft zu nennen (obwohl sie ebenfalls wiederholt von den Lehrkräften vorgestellt worden waren). Hier zeigt sich die Gefahr, die aus dem Erlernen von Fachwörtern zu bestimmten Konzepten erwachsen kann: Bisweilen beschränkten sich die Lernenden auf das Erlernen der Fachbegriffe und schafften 
es nicht, ein Konzept vollständig zu erklären. Das ging einher mit der Akzeptanz des Fachbegriffs als Antwort, ohne weiter nachzuhaken, ob das Konzept verstanden worden war und vollständig erklärt werden konnte. ${ }^{185}$

Der hier beschriebene Lehrervortrag beanspruchte eine ganze Unterrichtsstunde, die Frau Dante mit der Erteilung der Hausaufgabe, die entsprechenden Seiten im Lehrbuch zu lesen und die Inhalte zu lernen, beendete. Bei allen beobachteten Lehrkräften zeichnete sich der Sprachgebrauch im Lehrervortrag durch eine deutliche Aussprache und eine langsame bis mäßige Redegeschwindigkeit aus. Darüber hinaus bestanden natürlich Unterschiede, die zum einen auf den individuellen Sprachstil und zum anderen vermutlich darauf zurückzuführen waren, wie sehr eine Lehrkraft bei ihrem Vortrag an den Lehrbuchtext gebunden war und wie sie das sprachliche und kognitive Leistungsniveau der Klasse bewertete. Diese Hypothese ist der Tatsache geschuldet, dass zwei der drei Lehrkräfte an der Schule Pestalozzi, nämlich Frau Dante und Frau Cese, ihren Blick sowohl beim Vortragen als auch beim Abfragen vorrangig auf das Lehrbuch richteten und viele Konzepte daraus wortgetreu verwendeten oder ihre Formulierung von den Schülerinnen und Schülern in den Abfragungen erwarteten. Die dritte Lehrkraft an dieser Schule, Frau Rea, verhielt sich lehrbuchungebundener, gebrauchte aber dennoch eine ähnliche, wenn nicht gewähltere Sprache als ihre beiden Kolleginnen und hatte die höchsten sprachlichen und kognitiven Anforderungen an die Lernenden, was an verschiedenen Stellen im weiteren Verlauf dieses Kapitels verdeutlicht wird.

Frau Mancuso, die sich mal mehr, mal weniger an den Lehrbuchtexten orientierte, und Herr Tigre, der sie nur beim gemeinsamen Lesen in der Klasse beachtete, bildeten einfachere Sätze, die durch weniger syntaktische Komplexität und eine ausgeprägtere Verwendung des fundamentalen Wortschatzes gekennzeichnet waren, formulierten öfter Beispiele und stellten Bezüge zur Lebenswelt der Schüler her, die zudem leicht verständlich waren. Beiden ist außerdem gemein, dass sie Schüleräußerungen regelmäßig wiederholten, was auf der einen Seite unterstützt, dass keine Teile des Unterrichtsgesprächs überhört werden (bzw. inhaltlich nicht genau Verstandenes beim erneuten Hören eventuell klarer wird) und auf der anderen Seite das Unterrichtstempo entschleunigt. In diesen beiden Klassen war der Anteil von SchülerInnen mit einer geringeren Sprachkompetenz und einem anderen Geburtsland als Italien höher.

185 S. hierzu auch das Bespiel des Ozonlochs w.u. Eine ähnliche Situation ergab sich außerdem in einer Abfragung in der Klasse III C, Pestalozzi, bei der eine Schülerin nicht auf den Fachausdruck zur Bezeichnung des Klimas in Italien kam. Hier half die Lehrkraft entschieden mit („Come si chiama il nostro mare?“ - „Mediterraneo.“ „Quindi abbiamo un clima?“ - „Mediterraneo.“ („Wie heißt unser Meer?“ - „Mittelmeer (Mediterraneo).“ „Also haben wir welches Klima?“" - „Mediterranes.“) und verzichtete darauf, sich die Merkmale dieses Klimas nennen zu lassen. 


\subsubsection{Sprachliche Herausforderungen und ihre Bewerkstelligung in den Abfragungen}

Die während der interrogazioni beobachteten Lernenden schienen darum bemüht, die gelernten Inhalte vollständig wiederzugeben und ihnen eine angemessene sprachliche Form zu verleihen. Es ist bereits darauf hingewiesen worden, dass die meisten Abfragungen in allen Klassen mit offeneren Fragen der Lehrkräfte an die Prüflinge begannen und ihre mündliche Darstellung nach einer Weile unterbrochen wurde, um anhand punktuellerer Fragen gezielt zu überprüfen, ob sie über das nötige Hintergrundwissen verfügten und Konzepte genauer erklären konnten. Bisweilen erfolgte dieser Übergang vom freien Sprechen zu einem aus kurzen Fragen und Antworten bestehenden Prüfungsgespräch auch aus dem Grund, dass die Lernenden Zusammenhänge falsch oder unverständlich darstellten (vgl. Auszug aus Unterrichtsmitschrift 6). Hierbei ist anzunehmen, dass die Lernenden teilweise Inhalte präsentierten, die sie bei der Lehrbuchtexterarbeitung nicht richtig verstanden hatten, wie z.B. Prof.ssa Dante hinsichtlich des Redebeitrags einer abgefragten Schülerin vermutend äußerte (ibid.). Desweiteren schienen sich solche sprachlichen Ungereimtheiten aus dem Bestreben zu ergeben, eine Äußerung möglichst bildungssprachlich zu gestalten, ohne dass die hierzu benötigten lexikogrammatikalischen Mittel ausreichend beherrscht wurden. Beispielhaft ist hier etwa eine Schüleräußerung in der Klasse III A der Schule Pestalozzi zu nennen, in der die Gewohnheiten indischer Autofahrer vorgestellt wurden.

Auszug aus Unterrichtsmitschrift 7, Frau Rea (FrR), Pierpaolo (Pie), Abfragung Indien spärliche Verkehrsregeln

Pie: Le leggi automobilistiche, non so come si dice. (Die Automobilgesetze, ich weiß nicht, wie man es sagt.)

FrR: Il codice stradale! Adesso lo sai. (Die Verkehrsregeln! Jetzt weißt du es.)

Pie: No, lo sapevo anche prima. Il codice stradale è scarso. (Nein, ich wusste es auch vorher. Die Verkehrsregeln sind spärlich.)

FrR: Che vuol dire scarso? (Was bedeutet spärlich?)

Pie: E' scarso. (Sie sind spärlich.)

FrR: Un codice, una legge non può essere scarso! E che ne sai, hai la patente? (Ein Kodex, ein Gesetz kann nicht spärlich sein! Und was weißt du davon, hast du den Führerschein?)

Abbildung 10: Abfragung Frau Rea, Pierpaolo, III A, Pestalozzi, Bestrebungen um bildungssprachlichen Ausdruck

Da im Folgenden nicht weiter auf seinen Beitrag eingegangen wurde, bleibt offen, was er meinte. Vielleicht wollte er sagen, dass die Verkehrsregeln nur spärlich befolgt werden, da er eventuell gehört hatte, dass auf den indischen Straßen viele Menschen bei Verkehrsunfällen sterben. Oder er könnte sich auf volle Straßen in den Städten bezogen haben, welche von verschiedenen Ver- 
kehrsmitteln, Menschen und Tieren geteilt werden. Der einzige Satz im Lehrbuch, der das Thema Autofahren behandelt, lautet: „La rete stradale è abbastanza sviluppata, ma risulta ancora inadeguata rispetto alle dimensioni del Paese" (Bastianelli et al. 2010, S. 229; m.Ü.: Das Straßennetz ist ziemlich entwickelt, aber erscheint hinsichtlich der Dimensionen des Landes noch unangemessen). Pierpaolo versuchte hier also, etwas aus seinem persönlichen Wissen bildungssprachlich zu präsentieren.

Positiv an solchen Bemühungen der Lernenden ist ihre Gewissheit darüber zu betrachten, dass der Kontext Schule und im Spezifischen die Erdkundelehrkraft von ihnen ein Vortragen im bildungssprachlichen Register erwarteten. Dieses unterscheidet sich grundlegend vom Sprachgebrauch, den sie untereinander und im außerschulischen Umfeld meist pflegten, dennoch strebten sie seine Aneignung an. Indem sie morphosyntaktische und lexikalische Gestaltungsmittel verwendeten, obwohl sie sie noch nicht sicher beherrschten, versuchten sie, sich in ihrem sprachlichen Raum zu bewegen, der sich aufgrund der Registererweiterung vergrößerte, und erhielten durch das Lehrerfeedback hilfreiche Hinweise, wie erfolgreich ihre Versuche dabei waren.

Transkriptauszug 3, Abfragung zur Globalisierung, Frau Mancuso (FrM), Ava, Unterstützungslehrkraft Frau Sole (FrS) - Schülerbeitrag mit bildungssprachlichen Zügen

FrM [nachdem sie wiederholt hat, was globo im Wort globalizzazione (Globalisierung)bedeutet]: Che vuol dire, allora, globalizzazione? (Was bedeutet also Globalisierung?)

Ava: Eeh, gli scambi economici di beni e servizi e viene chiamato, eh, viene di solito con il termine di globalizzazione. Eeeh, eh, sarebbe un commercio internazionale in cui tutti i prodotti vengono, eh, vengono venduti in tutti i paesi. Eeeh, eeh il motore di, eh, a proposito della globalizzazione è il commercio internazionale. (Eeeh, der wirtschaftliche Austausch von Gütern und von Dienstleistungen, und er wird genannt, eh, er wird normalerweise mit dem Begriff Globalisierung. Eeeh, eh, das wäre ein internationaler Handel, in dem alle Produkte in alle Länder verkauft werden. Ehh, eeh, der Motor von, eh, bezüglich der Globalisierung ist der internationale Handel.)

FrM: Eh, eh [zustimmend, zum Weiterreden anregend]

Ava: Eeh, poi, le, i prodotti vengono venduti, soprattutto in paesi dove la manodopera è molto, eh, costa molto meno. (Eeeeh, dann, die [Art. f. PI.], die [Art. m. PI.] Produkte werden vor allem in Länder verkauft, wo die Arbeitskräfte viel weniger kosten.)

FrM: Vedi che già non si capisce. Se tu mi dici, i prodotti vengono venduti nei paesi in cui la manodopera costa meno, questa frase non significa niente. [Pause, Stille] Perché è sbagliato il verbo. I prodotti non vengono venduti, i prodotti vengono? (Schau [pass auf], dass man es jetzt schon nicht versteht. Wenn du mir sagst, die Produkte werden in Ländern verkauft, in denen die Arbeitskraft weniger kostet, hat dieser Satz keinen Sinn. Weil das Verb falsch ist. Die Produkte werden nicht verkauft, die Produkte werden?)

[Schülerin sieht die Lehrerin fragend an.]

Ava: Prodotti. (Produziert.)

FrM: Prodotti! Eh, eh, allora, se no, se no, non ci capisco niente. Allora, spiegami che vuol dire questa differenza. (Produziert! He, he, also, wenn nicht, wenn nicht, dann verstehe ich nichts davon. Also erklär mir, was dieser Unterschied bedeutet.)

Ava: Che vengono venduti, cioè, prodotti [kurze Unterbrechung durch Unterstützungslehrerin, die das Klassenzimmer betritt] (Die verkauft werden, das heißt, produziert werden.) 
FrS: Buongiorno. (Guten Tag.)

FrM: Se tu mi dici che i prodotti vengono venduti, come hai detto? I prodotti vengono? (Wenn du mir sagst, dass die Produkte verkauft werden; wie hast du gesagt? Die Produkte werden?)

Ava: Venduti. (Verkauft.)

FrM: Venduti. (Verkauft.)

Ava: In un paese. (In einem Land.)

FrM: In un paese dove la manodopera è scarsa. Quindi si può dire, che lei il concetto ce I'ha. Ma sbaglia il verbo, sbaglia il verbo e quindi dice fischio per fiasco. I prodotti vengono, i prodotti! Le merci vengono prodotte, e allora è molto diverso in, eeh, paesi, nei quali la manodopera è a buon prezzo. Che vuol dire questo? Spie, dammi un esempio. [Stille, Pause]. Facciamo un esempio. L'abbiamo fatto l'altra volta, l'esempio. [Stille, Pause] (In einem Land, wo es wenig Handarbeit gibt. Also kann man sagen, dass sie das Konzept hat. Aber sie vertut sich mit dem Verb, vertut sich mit dem Verb und sagt also „fischio" statt „fiasco“. Die Produkte, die Produkte! Die Waren werden produziert, und also ist das sehr verschieden, eh, in Ländern, in denen die Handarbeit wenig kostet. Was bedeutet das? Erkl, gib mir ein Beispiel. Machen wir ein Beispiel. Wir haben das letztes Mal entwickelt, das Beispiel.)

Ava: Eeh.

FrM: Là, là, ti ricordi che l'altra volta siamo andati ognuno a vedere da dove è questo giubbottino? ( $\mathrm{Da}$, da [zeigt auf einen Anorak], erinnerst du dich, dass wir letztens alle schauen gegangen sind, woher dieser Anorak ist?)

Ava: Aah!

Abbildung 11: Transkriptauszug Abfragung Frau Mancuso, Avas Versuch einer bildungssprachlichen Darstellung

Ava ist eine Schülerin, die vor einigen Jahren mit ihrer Familie aus Bangladesch, wo sie die ersten Schuljahre absolviert hatte, nach Italien kam. Italienisch war für sie also eine Zweitsprache, die sie erst seit einigen Jahren lernte, was wahrscheinlich erklärt, wie Frau Mancuso zu ihrer Aussage kommt, die Schülerin vertue sich mit einem Verb und habe das Konzept verstanden. Im Folgenden soll Avas Versuch, das Konzept der Globalisierung zu erklären, sprachlich analysiert werden.

Ava beginnt mit einer Definition von Globalisierung, indem sie auf den wirtschaftlichen Austausch zwischen den Ländern aufmerksam macht und darauf, dass ,alle Produkte in allen Ländern verkauft werden“. Der internationale Handel treibe die Globalisierung an. Bildungssprachlich an dieser Darstellung ist, das ein Phänomen geschildert wird, ohne dass die Akteure, die dafür verantwortlich sind, genannt werden: Die Subjekte in Avas ersten Sätzen sind der wirtschaftliche Austausch, der internationale Handel und die Produkte. Das erste Subjekt, scambi economici di beni e di servizi besteht zudem aus einer Nominalkette, die sich aus dem Substantiv scambio (Austausch), das dem fundamentalen Wortschatz, aber auch dem wirtschaftlichen Fachwortschatz angehört, zusammensetzt, das vom Adjektiv economico (Fo; wirtschaftlich) und den nominalen Attributen di beni (Fo; von Gütern) und di servizi (Fo; Dienstleistungen) ergänzt wird.

Zwei von drei der zuerst verwendeten Prädikate konstruiert sie im Passiv, was vor allem im mündlichen Sprachgebrauch seltener verwendet wird als das 
andere Genus verbi. Allerdings ist ihr erster Satz grammatikalisch eigentlich nicht korrekt: „Eeh, gli scambi economici di beni e servizi e viene chiamato, eh, viene di solito con il termine di globalizzazione." Während man den Satzanfang, gli scambi economici di beni e di servizi als prädikatslose Antwort auf die Lehrerfrage „Was bedeutet Globalisierung?“ werten kann, folgt auf diesen Pluralnumerus ein in der dritten Person Singular konjugiertes Prädikat. Zwar ist es im Italienischen möglich, wie im vorliegenden Satz das Subjekt nicht zu explizieren, doch es bleibt unklar, was als Subjekt intendiert ist. Die Zuhörer müssen dadurch inferieren, dass sie wahrscheinlich etwas wie Questo fenomeno/fatto viene chiamato... (Dieses Phänomen/dieser Sachverhalt wird ... genannt) im Sinn hat. Derartige Inferenzen sind in der mündlichen Kommunikation oft erforderlich und erschweren im vorliegenden Fall das Verständnis auch nicht besonders, doch das bildungssprachliche Register verlangt eine gröBere Präzision.

Desweiteren scheint die Schülerin mit der lexikalischen Wahl ihres Prädikats nicht zufrieden, denn sie wiederholt das vorausgehende Hilfsverb viene (wird) und vervollständigt es nicht zum Prädikat. Dies geschieht wahrscheinlich in Ermangelung einer besseren Alternative, jedoch hätte sie, um einen morphosyntaktisch korrekten Satz zu bilden, zumindest das zuerst genannte Prädikatslexem chiamato (genannt) wiederholen müssen.

Das langgezogene „eeeh“ am Anfang ihres nächsten Satzes drückt aus, dass sie Zeit zum Überlegen und Formulieren braucht, bis sie ein längeres Satzgefüge bildet - diesmal grammatikalisch korrekt -, das typisch bildungssprachliche Züge aufweist. Da gibt es zum einen das nicht animierte Subjekt commercio internazionale mit seiner genaueren Bestimmung durch das adjektivische Attribut internazionale und der Erläuterung durch einen Relativsatz und zum anderen den erneuten Gebrauch des Passivs.

Ihr Satzgefüge konstruiert sie um zwei Prädikate, von denen das erste im Konjunktiv steht (sarebbe, es wäre), wodurch sie sich von ihrer Äußerung distanziert. Durch dieses Stilmittel kann, ähnlich wie mit dem Gebrauch des Indikativ Präteritums, signalisiert werden, dass man etwas wiedergibt, das nicht von einem selbst stammt, sondern man irgendwo gehört oder gelesen hat. Noch stärker vielleicht das Präteritum, doch auch der Konjunktiv dient den Lernenden außerdem dazu auszudrücken, dass sie sich nicht sicher sind, ob sie etwas korrekt erinnern. ${ }^{186}$

Auch der folgende Satz wird wieder von einem langgezogenen ,eeh“ eingeleitet und ist Ausdruck ihres Zögerns. Ferner ist das eingeschobene a proposito (bezüglich) überflüssig und eher störend, stellt aber wahrscheinlich den Anfang ihrer Reparatur des vorausgehenden Wortes di dar. Avas Aussage würde an Kohäsion und Folgerichtigkeit gewinnen, wenn die Wortstellung im Satz anders gewählt wäre: Questo commercio internazionale è il motore della

186 Siehe hierzu auch das Unterkapitel 9.2.5 zum Lehrerfeedback. 
globalizzazione (Dieser internationale Handel ist der Motor der Globalisierung). Denn in ihrem Redebeitrag fungiert „Motor der Globalisierung“ als Rhema, also als neue Information, während der internationale Handel das Thema darstellt, das an den vorausgehenden Satz anknüpft.

Prinzipiell muss zu ihrer mündlichen Darbietung angemerkt werden, dass die Klasse das Konzept Globalisierung vor dieser Abfragung gemeinsam besprochen und auch die entsprechende Lehrbuchseite gelesen hatte. Avas Antwort auf die Lehrerfrage entspricht zunächst wortgetreu dem Satzanfang zu Beginn eines neuen Absatzes auf der Lehrbuchseite, und auch die Ausdrücke termine [...] di globalizzazione, motore della globalizzazione und commercio internazionale werden dort verwendet. Letzterer und globalizzazione sind zudem fettgedruckt, woraus die Schülerin schließen kann, dass sie zur Vorstellung des Konzepts wichtig sind und deshalb verwendet werden sollten. Dass sie bei der Formulierung von „Il motore della globalizzazione è il commercio commerciale" nach di zögert, liegt vielleicht daran, dass im Lehrbuchtext das Subjekt vom Adjektiv principale (prinzipiell) ergänzt wird, welches ihr im Moment der Abfragung nicht einfällt. Darauf deutet auch das von ihr eingeschobene und überflüssige a proposito (bezüglich) hin; es kann sich aber auch einfach um eine Reparaturstrategie handeln, weil ihr danach formuliertes nominales Attribut anstelle der einfachen Präposition di die Kontraktion aus Artikel und Präposition, della, erfordert. Im Übrigen dürfte die Wortstellung des Lehrbuchtextsatzes der Schülerin eine textkohärentere Formulierung wie die oben von mir vorgestellte erschweren. ${ }^{187}$

Nachdem Prof.ssa Mancuso Ava dazu auffordert, mit der Vorstellung des Konzepts fortzufahren und sie dadurch darauf hinweist, dass noch nicht alle wesentlichen Punkte zum Thema Globalisierung genannt worden sind, geht Ava zur Beschreibung des für die Globalisierung charakteristischen Outsourcens der Produktion von Gütern oder ihren Teilprozessen über. Auch hier verwendet Ava wieder einen passenden wirtschaftlichen Fachbegriff, nämlich manodopera (AD, Ts econ.; Arbeitskraft). Erneut bildet sie ein längeres Satzgefüge mit einem Relativsatz und verwendet zwei nicht animierte Subjekte (Produkte + Arbeitskraft) wodurch das erste eine passivische Prädikatskonstruktion erfordert.

Auch hier zeigen sich Avas Schwierigkeiten, Sprachliches angemessen zu formulieren bzw. sich an weitere Einzelheiten des Konzepts zu erinnern, an mehreren Stellen. Auf der einen Seite wählt sie zunächst einen femininen Pluralartikel, den sie durch den maskulinen korrigiert, um prodotti (Produkte) anschließen zu können, und verbessert das letzte Prädikat, è (FO; ist), in costa (Fo; kostet), wahrscheinlich, weil ihr kein passendes adjektivisches Prädikatsnomen zu ,ist“" einfällt. Auf der anderen Seite äußert sie wieder verschiedene

187 Auch dieser Satz steht wieder am Anfang eines neuen Absatzes und enthält zwei fettgedruckte Wörter, die zusammen ein Konzept bilden (commercio internazionale). Beides verdeutlicht den Lesern wahrscheinlich seine Wichtigkeit und erleichtert das Behalten. 
eeh und poi (dann), um Zeit zu gewinnen. Das entscheidende Problem an ihrem Satz ist die inhaltliche Fehlerhaftigkeit, da sie erklärt, dass die Produkte dort verkauft werden, wo die Arbeitskräfte sehr wenig kosten. ${ }^{188}$ Auf diesen wesentlichen semantischen Fehler (die Produkte werden dort produziert und nicht verkauft) reagiert die Lehrerin direkt, wobei sie unterstreicht, dass der Schülerin das Konzept klar sei, aber sie sich bei der Wahl des Verbs vertue und dadurch ihre Aussage unverständlich mache. Ob der Schülerin das Konzept des Outsourcens und seine Rolle in der Globalisierung wirklich klar sind, bleibt fraglich, zumal sich die Lehrkraft auf ein gemeinsam erarbeitetes Beispiel der Produktion von Kleidungsstücken der Schüler beruft, woran Ava sich nicht sofort erinnert und das sie auch später nicht selbstständig wiederholen kann. Deshalb ist es aus lern- und sprachförderlichen Gründen vorteilhaft, dass Prof.ssa Mancuso es für sie wiederholt.

Der Absatz auf der Lehrbuchseite, der sich mit der Globalisierung und dem Outsourcen auseinandersetzt, enthält die Begriffe für „Produktion“" und „Arbeitskraft", von denen Ava in ihrer Schilderung nur den zweiten erwähnt. Aber zusätzlich verwendet sie anstelle des mit dem ersten Substantiv nominal formulierten Prozesses „Produktion“ sein Resultat, die Produkte. Es ist anzunehmen, dass der Textabschnitt ihr nicht dabei behilflich war, das im Unterricht gemeinsam Erarbeitete besser zu verstehen und/oder zu behalten, da er nicht gerade einfach formuliert ist. Zur besseren Veranschaulichung soll er hier wiedergegeben werden (Carazzi \& Pizzetti 2010, S. 59, Fettdruck im Original):

Il fenomeno della globalizzazione investe anche la fase della produzione, che sempre più spesso viene trasferita in paesi (e continenti) diversi da quello in cui ha sede la ditta produttrice, là dove sono minori i costi della manodopera. Tale processo ha preso il nome di delocalizzazione.

(M.Ü.: Das Phänomen der Globalisierung betrifft auch die Phase der Produktion, die immer öfter in Länder (und Kontinente) transferiert wird, die von dem verschieden sind, in dem die produzierende Firma niedergelassen ist, dort wo die Kosten der Arbeitskraft geringer sind. Dieser Prozess hat den Namen Standortverlagerung angenommen.)

Die Subjekte sind abstrakt. Auf paesi bezieht sich ein mehrteiliges Attribut, das in der deutschen Übersetzung mit zwei Relativsätzen wiedergegeben wird, wobei es im Italienischen vom Adjektiv diversi (verschieden) regiert wird (diversi da quello in cui ha sede la ditta produttrice) und der Bezug des Demonstrativpronomens quello nicht leicht erkennbar ist, da es sich im Numerus von seinem Bezugswort paesi unterscheidet. Acht der 46 Wörter zählen außerdem nicht zum fundamentalen Wortschatz, also mehr als jedes sechste Wort, unter ihnen auch Wörter verschiedener Fachsprachen. Auch das erste Prädikat, investe, stammt von einem Verb, das im GRADIT zwar AU-markiert ist, den

188 Es könnte auch damit zusammenhängen, dass ihr der gelesene und von ihr verwendete Begriff manodopera nicht geläufig war. Diese Vermutung ist naheliegend, weil sich in einer anderen Unterrichtseinheit in derselben Klasse herausstellte, dass die Lernenden die Bedeutung dieses Begriffs nicht kannten. Hierzu s. Kap. 9.2.3.1. 
Lernenden jedoch primär in seiner Bedeutung von ,,anfahren“ bekannt gewesen sein sollte, während viele die im Text gemeinte Bedeutung wahrscheinlich nur aus dem Kontext erahnen konnten. Von den neun im GRADIT verzeichneten Bedeutungen, von denen vier mit einer Ts-Marke ausgestattet sind, passt übrigens keine einzige zu der hier synonym zu concerne (betreffen) verwendeten.

Das nächste Beispiel stammt aus einer Abfragung in der Klasse III B der Schule Pestalozzi und veranschaulicht die Bestrebungen einer Schülerin (Pamela) bildungssprachliche Ausdrücke zu verwenden. Da in dieser Klasse die Audioaufnahmen nicht gestattet waren, kann der Gesprächsauszug nicht vollständig wortgetreu wiedergegeben werden.

Auszug aus Unterrichtsmitschrift 8, Abfragung Frau Cese (FrC), Pamela (Pam), bildungssprachorientierter Versuch

Pam: Le città vicino alla costa sono molto soggette di inondazioni. (Die Städte nahe der Küste unterliegen sehr Überschwemmungen.)

FrC: Grammatica! Siamo qui proprio per parlare bene. Soggette a inondazioni, non di. (Grammatik! Wir sind hier tatsächlich, um gut zu sprechen. Soggette a Überschwemmungen, nicht di.)

Pam: [fährt fort.]

FrC [unterbricht sie]: Amore bello, non possiamo parlare cosi agli esami! (Liebes, so können wir in den Abschlussprüfungen nicht sprechen!)

Abbildung 12: Abfragung Frau Cese, Pamela, Bildungssprachversuche

Das Adjektiv soggetto erfordert als Prädikatsnomen die Präposition $a$ und nicht die von Pamela verwendete di. Es gehört zum allgemeinen Wortschatz (CO), weshalb es nicht verwunderlich ist, dass Pamela die Präposition falsch wählt: Wahrscheinlich versteht sie zwar die Bedeutung des Wortes, aber es ist nicht Teil ihres aktiven Wortschatzes. Die Abfragung vorbereitend, sollten die SchülerInnen Lehrbuchseiten lesen, auf denen der Ausdruck im selben Kontext der Überschwemmungen auftritt (Dinucci \& Dinucci 2008, S. 254). Dass sie ihn verwendet, zeigt ihre Bereitschaft, sich in der Situation der mündlichen Abfragung eines gewählteren Sprachregisters zu bedienen. Sie ist sich also bewusst, dass ein solcher Sprachgebrauch von ihr erwartet wird. Das wird auch von der Lehrerin unterstrichen, die sofort darauf hinweist, dass die Schule der Ort sei, an dem ,gut" gesprochen werden soll, und dass es in der Abschlussprüfung verlangt werde. Es geht also nicht nur um eine inhaltlich vollständige Darstellung, sondern auch um ein gutes Ausdrucksvermögen, wobei sich beide oft gegenseitig bedingen.

Ein Beispiel für einen missglückten, bzw. unterlassenen Versuch, den angemessenen Ausdruck für etwas zu verwenden, das ein Schüler (Tommaso) während einer Abfragung über Indien bei derselben Lehrerin schildert, ist in Abbildung 13 wiedergegeben. 
Auszug aus Unterrichtsmitschrift 9, Abfragung Frau Cese (FrC), Tom (Tommaso), bildungssprachliche Schüleräußerung mit Registerwechsel

FrC: Una persona famosa? (Eine berühmte Person?)

Tom: Gandhi.

FrC: Chi era? (Wer war das?)

Tom: Fece ottenere l'indipendenza all'India. Divise l'Asia orientale in India e Pakistan. Non è che faceva la guerra. (Er ließ Indien die Unabhängigkeit erhalten. Er teilte Ostasien in Indien und Pakistan. Es ist nicht, dass er Krieg führte.)

FrC: No, non faceva la guerra. Era una persona pacifista, faceva una rivoluzione non? (Nein, er führte keinen Krieg. Er war eine pazifistische Person, er führte eine Revolution durch, eine nicht?) [Pause, Stille]

FrC: Violenta. Dovete ricordarvi le cose, che parlo a fa'? (Gewaltsame. Ihr müsst euch die Sachen merken, wozu red' ich?)

Abbildung 13: Auszug aus Unterrichtsmitschrift, Abfragung Frau Cese, Registerwechsel eines Schülers aufgrund von Wortfindungsschwierigkeiten

Tommaso weiß auf die Lehrerfrage zu antworten und unternimmt dies zunächst auch auf sprachlich angemessene Weise. Bildungssprachlich an seiner Äußerung ist generell die Verwendung eines Vergangenheitstempus, des passato remoto, das in Mittelitalien im Mündlichen so gut wie gar nicht gebraucht wird (Lorenzetti 2002, S. 75 f.). Es ist aber das gängige Erzähltempus in Märchen und Geschichten und erscheint auch in den Lehrbuchtexten oft, um weiter zurückliegende Ereignisse zu schildern. Somit passt Tommaso seinen Sprachgebrauch mit der Verwendung dieser Zeitform dem des bildungssprachlichen schriftlichen Textes an und wählt zudem die Lexeme far ottenere l'indipendenza (die Unabhängigkeit erhalten lassen) zur Schilderung eines Sachverhalts, anstatt ihn mit einfacheren lexikalischen und morphosyntaktischen Mitteln auszudrücken.

Der Begriff rivoluzione non violenta (gewaltlose Revolution), an den sich der Schüler im Folgenden nicht erinnert, wurde zuvor im Unterricht besprochen und wird auf den Lehrbuchseiten verwendet, welche die SchülerInnen vor der Abfragung lesen sollten. Möglicherweise fällt ihm auch das aus dem allgemeinen Wortschatz (CO) stammende Adjektiv pacifista nicht ein, wodurch er eine Konstruktion wählt, die eine dazu gegenteilige Haltung verneint. An dieser Stelle zeigt sich in der mündlichen Darstellung des Schülers aufgrund seiner lexikalischen Lücke ein Bruch in der Registerverwendung. Zwar demonstriert Tommaso, dass er sich das Phrasem nicht eingeprägt hat, er sich aber an den Inhalt erinnert und diesen mit einer eigenen Formulierung, non è che faceva la guerra, umschreiben kann. Diese stellt jedoch aufgrund ihrer Vagheit zum vorher gewählten bildungssprachlichen Register einen Kontrast dar. Anstatt den Schüler dazu zu bewegen, seine Aussage selbst zu präzisieren und zu vervollständigen, übernimmt die Lehrkraft umgehend eine genauere Erklärung mit den angemessenen Begriffen pacifista und rivoluzione non violenta und schärft den Schülern ein, sie zu lernen. 
Nicht aus einer Abfragung, aber dennoch aus einer Prüfungssituation stammt das folgende Beispiel, auf das vor der Wiedergabe eines schriftlichen Tests in der Klasse III A, Pestalozzi im Unterrichtsgespräch Bezug genommen wird. Es betrifft die im Test verlangte Beschreibung des Treibhauseffekts und den dazu von den Lernenden verwendeten Begriff „Ozonloch“. Die Lehrkraft kommentiert folgendermaßen: „'Il buco dell'ozono protegge dai raggi del sole.' Attenti! Questo è un fenomeno tipico del linguaggio italiano! Non è il buco! State attenti all'uso del linguaggio" (,Das Ozonloch schützt vor den Sonnenstrahlen. 'Aufgepasst! Das ist ein typisches Phänomen der italienischen Sprache! Es ist nicht das Loch! Achtet auf den Gebrauch der Sprache!). Mit ihrer Äußerung „Das ist ein typisches Phänomen des italienischen Sprachgebrauchs" bezieht sich die Lehrkraft wahrscheinlich auf die Angewohnheit mancher Sprecher, sich um eine gewählte Ausdrucksweise zu bemühen und dabei inhaltlich-semantische Ungenauigkeiten oder Fehler zu produzieren. ${ }^{189}$

Ein letztes Beispiel ist ebenfalls nicht einer mündlichen Abfragung entnommen, soll an dieser Stelle aber dennoch wiedergegeben werden, da es eine Selbstkorrektur durch eine Schülerin (Arianna) der Klasse III D (Pestalozzi) in einer weniger formalen Unterrichtssituation enthält. Wie andere Lernende hilft sie einer Mitschülerin, die auf der Wandkarte Sri Lanka zeigen soll: „Guarda a sinistra delle Maldive. Eh, a nord-est, eh sì, a nord-ovest" (Schau links von den Malediven. Eh, nordöstlich, eh, ja, nordwestlich). Hier wird deutlich, dass es den Schülern leichter fällt, zur Lokalisierung die Richtungsadverbien wie „links“ zu verwenden, wie sie es auch in alltagssprachlichen Kontexten tun. Obwohl die Unterrichtssituation weniger formal ist, da die Mitschülerin, die das Land auf der Karte zeigen soll, sich nicht in einer Abfragung befindet und die anderen ihr helfen können, ohne auf eine Redeturnzuweisung durch die Lehrerin warten zu müssen, ist sich Arianna doch bewusst, dass ihre spontane Sprachverwendung in der Unterrichtssituation unerwünscht ist. Sie verbessert sich sofort selbst und unterliegt dabei der nicht untypischen Verwechselung der Himmelsrichtungen West und Ost. Sowohl ihr erster Ausdruck „links“ als auch die falsche Bezeichnung der Himmelsrichtung sind wahrscheinlich dem Eifer des Gefechts geschuldet, weil sie das Land entdeckt hat und ihrer Mitschülerin schneller behilflich sein möchte als die anderen.

189 Vgl. in diesem Zusammenhang der Pseudo-Klarheit, die bei einer gehobenen Wortwahl entstehen kann, den Dr. Fox-Effekt. Dieser Ausdruck geht auf ein 1970 durchgeführtes Experiment zurück, in dem ein Schauspieler als Dr. Fox ein gebildetes Publikum mit einem inhaltlich leeren bzw. nicht korrekten, doch sprachlich brillanten Vortrag täuschte (A. Helmke 2009, S. 199). 


\subsubsection{Sprachbezogene Interaktion im beobachteten Erdkundeunterricht}

Wenn die Unterrichtszeit nicht für Lehrervorträge oder Abfragungen genutzt wurde, diente sie vorrangig einem fragend-leitenden Unterrichtsgespräch, das i.d.R. aus der gemeinsamen Erarbeitung von Lehrbuchtexten bestand. Gerade bei dieser letzten Unterrichtsform waren die Lehrkräfte oft sprachförderlich tätig und dabei vorrangig auf das Stärken der Lesekompetenz und Wortschatzarbeit bedacht, während zugleich das mündliche Ausdrucksvermögen trainiert wurde.

\subsubsection{Gemeinsame Textarbeit}

Eine nützliche Strategie zur Erleichterung des Textverständnisses ist die Konzentration auf seine Überschrift und eventuelle Unterüberschriften, bevor mit dem Lesen begonnen wird. Anhand der darin enthaltenen Informationen können die Leser und Leserinnen Hypothesen aufstellen, wovon der Text handelt, und bauen somit eine Erwartungshaltung auf. Gleichzeitig ermöglicht ein Klassengespräch über die Überschriften von Texten die Aktivierung relevanten Vorwissens, was sich ebenfalls förderlich auf das Leseverstehen auswirkt. Ein solches Vorgehen wurde in einer Klasse beobachtet, und zwar in der III A an der Schule Pestalozzi. Hier begann Frau Rea beispielsweise eine Erdkundestunde mit der gemeinsamen Erarbeitung eines Lehrbuchtextes zur Weltwirtschaft. Sie las selbst die Überschrift vor und fragte dann die SchülerInnen, worum es im Text gehen werde.

Auszug aus einem Unterrichtsgespräch (10) zur Weltwirtschaft, Frau Rea (FrR), Arianna (Ari), Pierpaolo (Pie), Luca (Luc) - Textarbeit

FrR: Aprite il libro a pagina 116. [Pause, alle schlagen die Seite auf]. "Il sistema economico mondiale. Le potenze economiche mondiali." Secondo voi, di che cosa tratterà questo testo? (Schlagt Seite 116 im Buch auf. [Pause]. „Das Weltwirtschaftssystem. Die wirtschaftlichen Weltmächte." Was wird dieser Text eurer Meinung nach behandeln?)

Ari: La globalizzazione. (Die Globalisierung.)

Pie: L'economia dei paesi più importanti. Cioè, dei paesi più potenti, dal punto di vista economico. (Die Wirtschaft der wichtigsten Länder. Das heißt, der mächtigsten Länder, in wirtschaftlicher Hinsicht.)

FrR: Bene. Luca, leggi la prima frase. (Gut. Luca, lies den ersten Satz vor).

Luc: Oggi l'economia mondiale è dominata da pochi stati che sono i maggiori produttori di merci e di servizi del mondo: Stati Uniti e Canada, Unione Europea, Giappone, Australia e Nuova Zelanda. (Heutzutage wird die Weltwirtschaft von wenigen Staaten dominiert, welche die Hauptproduzenten von Gütern und Dienstleistungen weltweit sind: Vereinigte Staaten und Kanada, Europäische Union, Japan, Australien und Neuseeland.)

FrR: Bene. Quali parole in questa prima frase sono le più importanti? (Gut. Was sind die wichtigsten Wörter in diesem ersten Satz?) 
Klasse: [ruft verschiedene Wörter durcheinander, auch die von Frau Rea anvisierten.]

FrR: Sì. Sottolineate "oggi" e "pochi stati". Come vedete, l'informazione principale di questa frase non è contenuta nelle parole a grassetto! (Ja. Unterstreicht "heutzutage“ und "wenige Länder“. Wie ihr seht, ist die Hauptinformation dieses Satzes nicht in den fettgedruckten Wörtern enthalten!)

Abbildung 14: Auszug aus Unterrichtsgespräch Klasse III A, Pestalozzi, Textarbeit

Neben dem Aufbauen einer Erwartungshaltung zum Inhalt des Textes, welche die Inferenzenbildung beim Lesen und somit das Textverständnis leiten kann, ist eine solche Herangehensweise an die schulische Textarbeit besonders für Lernende mit geringeren Sprachfähigkeiten von Vorteil, zumal sie eventuell schon bei der Überschrift auf Wörter stoßen, deren Bedeutung ihnen nicht oder nicht genau bekannt ist. Im weiteren Verlauf wird der Text anfangs Satz für Satz laut vorgelesen, wonach in jedem Satz die Hauptinformation bzw. Schlüsselwörter unterstrichen werden sollen. Die Strategie des Auffindens von Schlüsselwörtern dient ebenfalls der Festigung des Textverständnisses und ist außerdem hilfreich, wenn in einem späteren Schritt die wesentlichen Textaussagen wiedergegeben werden sollen. Nicht nur in dieser, sondern auch in zwei anderen Klassen wurde das Üben dieser Technik beobachtet, wobei allerdings außer Frau Rea nur Frau Mancuso (Vespucci) die SchülerInnen zum Bestimmen von Schlüsselwörtern anhielt, während Frau Cese (III B, Pestalozzi) vorgab, welche Wörter zu unterstreichen waren. In der Klasse von Frau Dante hingegen strichen Lernende Textpassagen oder einzelne Wörter an, ohne dass diese gemeinsam identifiziert worden waren oder sie dazu aufgefordert wurden. Es ist möglich, dass Frau Dante diese Technik im Vorfeld der Studie bereits hatte üben lassen, so dass ihre Klasse sie jetzt selbstständig anwandte.

Am Gesprächsbeispiel in Abbildung 14 ist darüber hinaus interessant, dass die Lehrkraft darauf aufmerksam macht, dass die in den Lehrbüchern fettgedruckten Wörter nicht immer die Hauptinformation eines Satzes oder Textabschnittes enthalten. Es wurde auch in anderen Momenten beobachtet, dass Frau Rea und Frau Mancuso gegen den Fettdruck in den Büchern wetterten und darauf hinwiesen, dass es sich beim Aufspüren wesentlicher Textaussagen eigentlich um eine Aufgabe des Lesers handele, die ihm durch derartige Gestaltungsmittel nicht abgenommen werden dürfe. Sich nicht auf den vorgegebenen Fettdruck zu verlassen, sondern sich selbst beim Lesen immer wieder zu fragen, was die entscheidenden Textinformationen sind, dazu riet Frau Rea ihren Lernenden auch deshalb, weil in Texten für erfahrenere Leser an den weiterführenden Schulen keine Wörter fettgedruckt seien.

Der Auszug aus dem Unterrichtsgespräch stammt aus einer Doppelstunde, von der die erste Stunde komplett der gemeinsamen Texterarbeitung gewidmet war. Auf die vorgestellte Weise wurden dreieinhalb Lehrbuchseiten zu den Themen Weltwirtschaft, ungleiche Verteilung des Wohlstands und Kinderarbeit behandelt. Dabei unterbrach die Lehrerin das Vorlesen der Schülerinnen und Schüler immer wieder, um sich anhand von Fragen zu vergewissern, ob 
das Gelesene verstanden wurde, wobei sich diese Fragen teilweise auch an diejenigen richteten, die gerade mit dem Vorlesen an der Reihe waren.

Diese Fragen, die entweder ungerichtet der gesamten Klasse oder einem bestimmten Schüler gestellt wurden, bezogen sich oft auf die Bedeutung bestimmter Wörter oder Mehr-Wort-Inhaltseinheiten kürzeren Ausmaßes. So wollte Frau Rea z.B. vom Vorleser (Costa) eines längeren Absatzes die Bedeutung des darin enthaltenen Wortes divario (CO; Unterschied, Gefälle) wissen. Offensichtlich war ihm dieses Wort aus dem allgemeinen Wortschatz - also dem umfangreichen Teil des Gebrauchswortschatzes, der seltener in der gesprochenen und geschriebenen italienischen Sprache verwendet wird als der Grundwortschatz und über den Lernende dieser Klassenstufe erst zu einem kleinen Teil verfügen -, nicht bekannt. Der Schüler nutzte zur Bedeutungserschließung nicht den Textzusammenhang, obwohl es hilfreich gewesen wäre, da im vorausgehenden Satz distinguere Stati ricchi [...] dalle aree povere (reiche Staaten [...] von den armen Gebieten unterscheiden) und Nord e Sud del Mondo (Norden und Süden der Welt) erwähnt werden und der zu erklärende Ausdruck genau dieses Thema aufgreift und zum Ausgangspunkt für die anschließende neue Textinformation macht.

Halliday (1993, S. 109 ff.) hat dieses Phänomen ausführlich beschrieben und ihm den Namen grammatische Metapher gegeben. Es ist ein bildungstexttypisches Merkmal geschriebener Sprache, dass beschriebene Sachverhalte für die weitere Ausführung der Darstellung am Satzanfang aufgegriffen und im Nominalstil konzentriert werden. Im Textbeispiel aus dem Lehrbuch wird das Verständnis dieses inhaltlichen Zusammenhangs sowohl aus dem Grund erschwert, dass es sich dabei um ein Substantiv handelt, das eben nicht im Grundwortschatz enthalten ist, als auch dadurch, dass die Autoren es nicht mit einem Demonstrativadjektiv einleiten: Stünde im Text nicht il divario tra i Paesi ricchi e poveri (das Gefälle zwischen den reichen und den armen Ländern), sondern questo divario, wäre der inhaltliche Zusammenhang durch die explizitere Verknüpfung der beiden Sätze klarer.

$\mathrm{Zu}$ einem umfassenderen Textverständnis gelangte Costa vermutlich auch nicht, da er sich beim Vorlesen auf eine korrekte Aussprache und Betonung konzentrierte, was ihm Mühe bereitete, wie sein Zögern und Verlesen an verschieden Stellen anzeigen. Dadurch standen ihm weniger kognitive Kapazitäten zur Sicherung des Textverständnisses zur Verfügung als einem kompetenteren Vorleser. In Abbildung 15 wird der kurze Wortwechsel zwischen ihm und der Lehrkraft wiedergegeben.

Auszug aus einem Unterrichtsgespräch (11) zwischen Frau Rea (FrR) und Costa (Cos), anderer Schüler (Sch)

[Costa liest einen Textabschnitt vor und beantwortet anschließend einigermaßen befriedigend einige an inn gerichtete Fragen der Lehrkraft.]

FrR: Che vuol dire "divario"? (Was bedeutet „divario“?)

[Pause, Costa antwortet nicht.] 
FrR: Ti viene in mente un sinonimo? (Fällt dir ein Synonym ein?)

Cos: Litigio. (Streit.)

FrR: No. E' diff ...? (Nein, es ist Unter..?) [Stille, Costa schweigt]

Sch: Differenza (Unterschied.)

Abbildung 15: Auszug aus Unterrichtsgespräch, Frau Rea, Costa, Textarbeit - Wortbedeutung

Anstatt ihn nach Erschließungshilfen im Text suchen zu lassen, gibt Prof.ssa Rea ihm die Hälfte eines Synonyms des unbekannten Wortes vor, das ein anderer Schüler nach einer kurzen Pause, in der Costa nicht auf die Frage antwortet, vervollständigt, ohne dazu aufgefordert worden zu sein. ${ }^{190}$

Auf eine längere Nominalkette, nämlich esporto di manufatti di alto contenuto tecnologico (Export von hoch technologischen Geräten), der vorausgehenden, gemeinsam gelesenen Seite geht sie an späterer Stelle ein, um diesen Oberbegriff von den Lernenden inhaltlich füllen zu lassen, sich zu vergewissern, ob die Schüler wissen, wie man Produzent oder Erfinder von hoch technologischen Gegenständen wird, und mit thematisch affinem Wissen aus der Stunde, in der das oben vorgestellte Diagramm zur Bildung der Frauen und der Geburtenrate entwickelt werden sollte, zu kombinieren: „Secondo voi, in Arabia Saudita, le donne possono lavorare per lo sviluppo dell'alta tecnologia?“" (Können die Frauen in Saudi-Arabien eurer Meinung nach in der HightechEntwicklung arbeiten?). Es zeigte sich, dass die Lernenden Beispiele für hoch technologische Produkte nennen konnten und sich an den Grad der Schulbildung der Frauen in Saudi-Arabien erinnerten. Sie wussten jedoch nicht, welche schulische Bildung (Hochschulreife, Studium, Spezialisierung) zur Entwicklung von Hightech-Produkten erforderlich ist. Bei der gemeinsamen Lehrbuchlektüre wurde also auch textbezogenes Hintergrundwissen überprüft und erweitert.

Bereits vermitteltes relevantes Wissen wiederholte Frau Rea auch an anderen Stellen, indem sie kontrollierte, ob die SchülerInnen es noch abrufen konnten. So wurde es zusätzlich gefestigt, auf neue Kontexte angewandt und dabei in den Dienst einer gründlicheren Auseinandersetzung mit den neuen Texten gestellt. In der beschriebenen Unterrichtsituation der gemeinsamen Textbehandlung fragte sie beispielsweise nach einem bestimmten Fachausdruck, der im Text nicht genannt wird, aber mit seinem Inhalt zusammenhängt: , ,Come si chiama questo tipo di economia che garantisce a stento la sopravvivenza?“" (Wie heißt diese Art von Wirtschaft, die mit Mühe und Not das Überleben

190 Wie in der w.o. beschriebenen Unterrichtssituation, in der eine Schülerin ein Land auf der Wandkarte zeigen sollte, durften die SchülerInnen in allen beobachteten Klassen einen Redebeitrag äußern, ohne auf die Erteilung des Rederechts warten zu müssen, sofern die Lehrkraft ihre Frage nicht gezielt an einen bestimmten Lernenden gerichtet hatte. Auch dann mussten die anderen SchülerInnen ihren Wunsch, etwas beizutragen, nicht durch ein Handzeichen anzeigen, sondern konnten sich direkt äußern. Nur wenn es allzu laut wurde, weil alle durcheinanderredeten, forderten die Lehrkräfte sie auf, sich zu melden und abzuwarten, dass ihnen das Rederecht erteilt wurde. 
garantiert?). Hierauf antwortete die Klasse korrekt mit economia di sussistenza (Ts econ.; Subsistenzwirtschaft).

Darüber hinaus versuchte die Lehrerin, das Textverständnis der Lernenden zu verbessern und Inhalte fester in ihrem Gedächtnis zu verankern, indem sie Bezüge zu ihrer Lebenswelt herstellte. Nicht immer verfügte die Klasse jedoch über das nötige Wissen, um solche Bezüge unter der Anleitung der Lehrkraft zu entwickeln und zum besseren Verständnis zu nutzen, obwohl sie sich auch auf im Unterricht behandelte Gegenstände stützten. So wollte Frau Rea etwa die Bruttoinlandsprodukte von ärmeren Ländern der Welt mit dem Italiens vergleichen lassen, kannte aber niemand in der Klasse den Umfang des italienischen BIPs. ${ }^{191}$

Die Bezüge wurden desweiteren auch zu Inhalten hergestellt, die in einem anderen Fach behandelt worden waren. So wies sie, ähnlich wie ihre beobachteten Kollegen in anderen Situationen, die Schülerinnen und Schüler darauf hin, dass sie über das Thema Kinderarbeit bereits ausführlich im Geschichtsunterricht gesprochen hatten, und ließ wiederholen, durch welche Merkmale sie gekennzeichnet ist. Gleichzeitig versuchte Frau Rea, eine Vorstellung davon zu vermitteln, was sich hinter der neutralen Sprache des Lehrbuchtextes für die Betroffenen verbirgt, indem sie zunächst fragte, wer jüngere Geschwister oder Cousins und Cousinen hatte und wie diese ihren Tag verbrachten. Sie verglich die Schülerangaben dann mit dem Alltag vier- oder fünfjähriger Kinder, die ein Gelände von Steinen und Gestrüpp (CO; sterpaglie) befreien müssen.

Unterrichtsgespräch (12) Frau Rea (FrR), verschiedene Schüler (Sch), Vera (Ver), Bezug zur Lebenswelt der Lernenden

Ver: Che sono le sterpaglie? (Was sind die sterpaglie?)

FrR: Come, che sono le sterpaglie?! (Wie, was sind sterpaglie?!)

Sch: Erba, rami, piante inutili. (Gras, Äste, unnützliche Pflanzen.)

FrR: $E$ a otto anni? Cosa fanno i bambini qui? (Und mit acht Jahren? Was machen die Kinder hier?)

Sch: Vanno a scuola, in terza elementare. (Sie gehen zur Schule, in die dritte Klasse.)

FrR: Mentre i bambini in altri posti del mondo hanno mai visto una scuola. E di pomeriggio, dopo la scuola? Cosa fanno i bambini? (Während die Kinder an anderen Orten der Welt nie eine Schule gesehen haben. Und am Nachmittag, nach der Schule?)

Sch: Danza, sport. (Tanzen, Sport.)

FrR: A dodici anni, molti bambini lavorano già sotto un padrone. Adesso, Vera non sa neanche che cosa sono le sterpaglie, mentre questi bambini lavorano tanto. (Mit zwölf Jahren arbeiten viele Kinder schon unter einem Dienstherrn. Jetzt weiß Vera nicht mal, was Gestrüpp ist, während diese Kinder hart arbeiten.)

Abbildung 16: Auszug aus Unterrichtsgespräch Frau Rea, Bezug zur Lebenswelt der Schüler

191 Ähnlich erging es bspw. Herrn Tigre an der Schule Morante, als er die Länge des Flusses Zambesi veranschaulichen wollte und die Lernenden nach dem Namen und der Länge des längsten Flusses Italiens fragte, worauf sie keine Antwort gaben. 
Ähnlich verhielt sich die Lehrerin in einer anderen Schulstunde, in der bei der Behandlung des Lehrbuchthemas Konflikte in Afrika die Begriffe pulizia etnica (CO; ethnische Säuberung) und genocidio (CO; Genozid) aufkamen. Um den Schülerinnen und Schülern die im Lehrbuch angegebene Zahl der Toten und damit die Bedeutung des Völkermords begreiflicher zu machen, stellte sie eine Rechenaufgabe:

Auszug aus einem Unterrichtsgespräch (13) Frau Rea (FrR), verschiedene Schüler (Sch) - Genozid

FrR: La pulizia etnica può portare fino al genocidio. Questo termine significa "sterminio di un popolo". Dove è successo nel giro di due mesi? (Die ethnische Säuberung kann bis zum Genozid führen. Dieser Begriff bedeutet „Ausrottung eines Volkes“. Wisst ihr, wo das innerhalb von zwei Monaten geschehen ist?)

[Pause, Stille]

Sch1: In Ruanda.

FrR: Si. Cercate sulla mappa, dove si trova questo paese. (Ja. Sucht auf der Karte, wo sich das Land befindet.)

[Klasse sucht Ruanda auf der Karte im Buch.]

FrR: Avete idea quante persone bisogna uccidere al giorno per arrivare a un milione e mezzo in due mesi? (Habt ihr eine Idee, wie viele Menschen man am Tag töten muss, um auf eineinhalb Millionen in zwei Monaten zu kommen?)

[Die Lernenden rechnen.]

Sch2: 22.000 al giorno. (22.000 am Tag.)

FrR: Il genere umano è molto inventivo per quanto riguarda la crudeltà. C'è anche lo stupro etnico, veniva praticato anche qui in Europa, per esempio durante la guerra balcanica. (Das Menschengeschlecht ist sehr erfinderisch, was die Grausamkeit anbelangt. Da ist auch der ethnische Missbrauch, der wurde auch hier in Europa praktiziert, zum Beispiel während des Balkankriegs)

Abbildung 17: Unterrichtsgespräch Frau Rea, Genozid

Auf dieselbe Art bemühte sich Frau Mancuso an der Vespucci, ihren Schülern die Implikationen einer neutral gehaltenen Formulierung im Lehrbuch zu vergegenwärtigen, deren Bewusstmachung bei der einfachen Lehrbuchlektüre durch die Verwendung komplizierterer Lexeme zusätzlich erschwert wurde. Welche sprachlichen Gestaltungsmittel im Textzusammenhang zur Kolonialisierung und zur Sklaverei Schwierigkeiten bereiteten, geht aus dem Transkriptauszug in Abbildung 18 hervor. Vor allem handelt es sich um ein mangelndes Verständnis der Satzaussage praticare razzie di manodopera (Raubzüge [auf der Suche] nach Arbeitskraft praktizieren), was primär auf falsches lexikalisches Wissen über den Ausdruck manodopera zurückzuführen ist.

Die drei in der Satzaussage enthaltenen Lexeme zählen allesamt nicht zum fundamentalen Wortschatz; das erste ist darüber hinaus das einzige, das als Wort des hohen Gebrauchs (AU) zum Grundwortschatz gerechnet wird. Razzia ist ein Wort des allgemeinen Wortschatzes (CO) und manodopera eines der hohen Disponibilität und der Wirtschaftsfachsprache zugleich (AD Ts econ.). Diese für italienische Sach- und Lehrbuchtexte typische Wahl eines gehobenen 
Sprachregisters und die Tendenz, Sachverhalte nicht mithilfe einer Verbalphrase, sondern nominalisiert auszudrücken, stellen für viele Lernende dieser Altersstufe offensichtlich eine erhebliche Schwierigkeit dar.

Sie würden die Textaussage leichter verstehen, wenn sie zum Beispiel „, diversi popoli africani e $i$ dominatori arabi sequestravano/rubavano uomini da impiegare come manodopera nelle coltivazioni o nei servizi domestici“ (die verschiedenen afrikanischen Völker und arabischen Herrscher entführten/stahlen Menschen, um sie als Arbeitskräfte in den Bewirtschaftungen und häuslichen Diensten einzusetzen) lautete. Dieser Alternativvorschlag greift einen Großteil der im Text gegebenen Lexeme aus dem gehobeneren Sprachregister auf, erklärt aber insbesondere manodopera besser, indem es direkt mit uomini (da impiegare) in Verbindung gesetzt wird. Anstatt wie im Lehrbuchtext sofort den Beutezug nach Arbeitskräften zu erwähnen, wird so zuerst geschildert, dass Menschen gestohlen werden, und dann ergänzt, aus welchem Grund dies geschieht.

Dass die Lernenden das Wort mandopera nicht sicher beherrschten, ist im Übrigen zwar zum einen damit erklärbar, dass sie es aufgrund der lexikalischen Komplexität der umliegenden, nicht aus dem fundamentalen Wortschatz stammenden Wörter nicht aus dem Kontext erschließen konnten. Erschwerend kam aber zum anderen noch hinzu, dass sie es mit einem sowohl im Wortlaut als auch von der semantischen Bedeutung seiner Bestandteile her ähnlichen Wort verwechselten, das sie ebenfalls erst in der Mittelstufe gelernt haben dürften und noch nicht sicher beherrschten, nämlich manufatto (CO; handgefertigter Gegenstand).

Von den beiden Bestandteilen der Determinativkomposita sind jeweils die bestimmenden ersten nahezu identisch, mano (Fo; Hand) sowie sein lateinischer Ursprung manu im zweiten Kompositum, und die folgenden Determinata liegen semantisch sehr nah beieinander: Opera (Fo) bedeutet „menschliches Werk" und fatto in der Hauptbedeutung als Nomen „Tatsache, Fakt" und als Partizip Perfekt des Verbs fare ,gemacht“.

Transkriptauszug 4, Frau Mancuso (FrM), Diego (Die), Olga (Olg), Ava - neutrale und komplexe Lehrbuchsprache

FrM: Andiamo a fare il discorso della schiavitù. [Liest vor]: "Già negli anni precedenti al Cinquecento" [unterbricht das Lesen]. E quello che vi ho detto io. Devi anticipare. Non è soltanto il problema della schiavitù. Non è soltanto un problema dell'Ottocento. [Liest weiter:] "Già negli anni precedenti al 1500, i diversi popoli africani e i dominatori arabi praticavano razzie di manodopera da impiegare nelle coltivazioni o nei servizi domestici." Che vuol dire, Rodrigo, "praticavano razzie di manodopera"? (Lasst uns das Gespräch der Sklaverei führen. [Liest vor:] „Schon in den Jahren vor dem 16. Jahrhundert" [unterbricht das Lesen]. Das ist das, was ich euch gesagt habe. Du musst weiter zurückgehen. Es ist nicht nur das Problem der Sklaverei. Es ist nicht nur ein Problem des 19. Jahrhunderts. [Liest weiter:] „Schon in den Jahren vor 1500 praktizierten die verschiedenen afrikanischen Völker und die arabischen Herrscher Raubzüge [auf der Suche] nach Arbeitskräften zur Nutzung in den Bewirtschaftungen 
oder für Dienste im Haushalt.“ Was bedeutet, Rodrigo, „sie praktizierten Raubzüge [auf der Suche] nach Arbeitskräften"?)

Rod: Che venivano prodotti solo per loro. No? (Dass sie nur für sie produziert wurden, nicht?)

FrM: "Praticare razzie di manodopera." Che vu, chi me lo sa dire? Tranne Lorenzo, che non mi risponde, pure Nina, mi dovete dire che vuol dire „praticavano razzie di manodopera”! („Raubzüge [auf der Suche] nach Arbeitskräften praktizieren.“ Was be, wer kann es mir sagen? Außer Lorenzo, der mir nicht antwortet, auch Nina, ihr müsst mir sagen, was „Raubzüge [auf der Suche] nach Arbeitskräften praktizieren“ bedeutet!

[Pause, Stille]

Klasse: [?] [unverständlich]

FrM: E dai! Che cosa, Olga? (Los! Was, Olga?)

Olg: C'erano i saccheggi. (Es gab Plünderungen.)

FrM: Eeh [zustimmend], ma che, di che cosa? Praticare razzia vuol dire fare un saccheggio? Vuol dire che vai là e rubi, vero? Una razzia è un furto. Ma di che? (Eeh, aber was, von was? Einen Raubzug praktizieren bedeutet eine Plünderung zu machen? Es bedeutet, dass du dort hingehst und stiehlst, richtig? Ein Raubzug ist ein Diebstahl. Aber von was?)

Klasse: [?] Di cose fatte a mano. ([?] Von handgemachten Sachen.)

FrM: No! Quello e manufatto, le cose fatte a mano. Qua usano la parola "manodopera" per dire "uomini". Uomini che lavoravano, quindi praticavano razzie di uomini in grado di lavorare. Eh, Olga? Quindi, praticavano razzie di uomini da impiegare nelle coltivazioni o nei servizi domestici e allora la, la, il serv, lo schiavo nero era proprio il tipico domestico ecc. C'è un, c'è un bellissimo romanzo, eeeh, che si chiama "Via col vento". Della, no, poi ve lo dico la prossima volta, mo, non me lo ricordo. Vabbè, insomma, avete mai sentito nominare "Via col vento". (Nein! Das ist „manufatto" [handgefertigter Gegenstand]. Hier benutzen sie das Wort „manodopera“, um „Menschen“ zu sagen. Menschen, die arbeiteten, also praktizierten sie Raubzüge auf der Suche nach arbeitsfähigen Menschen, eh, Olga? Also, sie praktizierten Raubzüge nach Menschen, um sie in den Bewirtschaftungen oder den häuslichen Diensten einzusetzen und also die, die, der [nicht korrekter Art. m.] Skla, der schwarze Sklave war echt der typische Sklave, etc. Es gibt, es gibt einen sehr schönen Roman, eeeh, er heißt „Vom Winde verweht“. Von der, nein, das sage ich euch dann nächstes Mal, jetzt erinnere ich mich nicht. Vabbè [hier ungefähr: egal], also, ihr habt nie von „Vom Winde verweht“ gehört.

Klasse: Si. (Doch.)

FrM: Avete visto il film in televisione? (Habt ihr den Film im Fernsehen gesehen?)

Ava: No, no, io ho sentito il nome. (Nein, nein, ich habe den Namen gehört.)

Fr M: [...] [Sie beschreibt die Handlung des Romans und stellt die Sklavin „Mami“ vor, die im Haushalt arbeitete]. Quindi di solito, venivano usate per fare, spesso diventavano domestici. Eh, [liest weiter] "tali individui, individui venivano ridotti in schiavitù ed esistevano anche forme di commercio di schiavi." Questo lo abbiamo capito. Commercio di uomini. [Sie liest weiter vor und wiederholt mit den Schülerinnen und Schülern, was sie dazu schon im Geschichtsunterricht gelernt haben.] Quindi, qual è il sistema? I sovrani locali, che facevano? Proprio i sovrani davano un quantitativo di schiavi, di uomini ai sovra, ai portoghesi e poi questi li portavano in patria dove venivano venduti. Commercio. Hai presente quando vendi cavalli, quando vendi, insomma, è la stessa cosa, cioè scendevano questi poveracci e si guardava la bocca, le mani, come era la complessione fisica e poi si vendeva. "Gli spagnoli" [...] [liest weiter]. Quindi si è capito, che vuol dire manodopera? (Also, normalerweise wurden sie benutzt, um zu machen, oft wurden sie Haushaltskräfte. Eh [liest weiter] „diese Individuen, Indivi- 
duen wurden versklavt und es existierten auch Formen von Sklavenhandel." Das haben wir verstanden. Menschenhandel. [Sie liest weiter vor und erinnert die SchülerInnen an das, was sie dazu schon im Geschichtsunterricht behandelt haben.] Also, was ist das System? Die lokalen Herrscher, was machten sie? Genau diese Herrscher gaben eine bestimmte Menge an Sklaven, an Menschen den Herrsch, den Portugiesen und dann brachten diese sie in die Heimat, wo sie verkauft wurden, kurzum, es ist die gleiche Sache, das heißt, diese armen Tropfe gingen herunter [wahrscheinlich „vom Schiff“ impliziert] und man guckte sich den Mund an, die Hände, wie der physische Körperbau war, und dann verkaufte man. „Die Spanier“ [...] [liest weiter]. Also, hat man verstanden, was Arbeitskraft bedeutet?

Klasse: Uomini. (Männer.)

FrM: E? O, facciamo la parola. Manodopera, quindi, che ti su, che ti fa venire in mente? Eh? (Und? Oder machen wir das Wort. Arbeitskraft, also, was sugg[eriert], woran lässt es dich denken? Eh?

Olg: Schiavismo. [Sklaverei.]

FrM: No. [Nein.]

Rod: Lavoro. [Arbeit.]

FrM: Mh. [Pause]

Nora: Cioè, che da soli [?], progettano le cose? (Das heißt, dass sie alleine [?], die Dinge planen?)

FrM [hört Nora wahrscheinlich nicht und liest weiter vor]: „II litorale centrale in Africa prese il nome di costa degli schiavi..." („Die zentrale Küste in Afrika bekam den Namen Sklavenküste...")

Abbildung 18: Transkriptauszug zu Unterrichtsgespräch Frau Mancuso, neutrale und komplexe Lehrbuchsprache, lexikalische Schwierigkeiten

Nachdem Prof.ssa Mancuso die Schüler darauf aufmerksam gemacht hatte, dass sie das Wort manodopera mit manufatto verwechselten, fuhr sie zum einen mit der Lektüre fort und versuchte zum anderen, das Konzept der Versklavung besser $\mathrm{zu}$ veranschaulichen, indem sie auf einen Roman hinwies. Sie stellte den Bezug zu „Vom Winde verweht“ wegen der Sklaven und ihrer Besitzer her, allerdings kannten die Schüler weder das Buch noch seine Verfilmung, bestenfalls den Titel. Deshalb umriss die Lehrkraft den Inhalt. Als sie zu einem späteren Zeitpunkt des Gesprächs erneut nach der Bedeutung des Wortes manodopera fragte, zeigte sich, dass die Klasse diesen Ausdruck immer noch nicht richtig erklären konnte. Hierbei ist es normal, dass der Aneignungsprozess eines neuen Wortes allmählich erfolgt und es mehrere Male in günstigen Kontexten auftreten muss, damit Wissen über seine Bedeutung und Verwendung gefestigt werden kann. Doch der durch Fragen unterbrochene Lehrervortrag Frau Mancusos, in den sich die Worterarbeitung eingliederte, könnte aufgrund seiner Weitschweifigkeit eine bessere Verankerung des Ausdrucks im mentalen Lexikon erschwert haben.

Hier und bei zwei weiteren beobachteten Lehrkräften war eine Tendenz festzustellen, bei Begriffs- und Konzepterklärungen weit auszuholen, was gerade für schwächere SchülerInnen weniger förderlich ist als eine klare und einfachere Strukturiertheit des sprachlichen Lehrerhandelns, wie es u.a. im Hamburger Verständlichkeitsmodell und im Zuge des von Gibbons und Hammond (2005) vorgestellten Konzept des scaffolding betont wird. Auf der anderen 
Seite konnte vor allem in dieser und in der Klasse an der Schule Morante beobachtet werden, wie sich die Lehrkräfte in der mündlichen Interaktion darum bemühten, einen der Klasse bekannten Wortschatz zu verwenden und sowohl langsam und deutlich zu sprechen, als auch Schüleräußerungen zu wiederholen, um das Unterrichtstempo zu entschleunigen und die Lernenden in ihrem mündlichen Ausdrucksvermögen und Lernprozess zu unterstützen.

So wiederholte Frau Mancuso z.B. im folgenden Beispiel eines Unterrichtsgesprächs systematisch die Schüleräußerungen, forderte dazu auf, ausführlicher zu antworten und baute am Ende des Transkriptauszugs selbst einen Schülerbeitrag aus.

Transkriptauszug 5, Frau Mancuso (FrM), Lorenzo (Lor), Rodrigo (Rod), Ava - Mikroscaffolding PIL

FrM: Allora, già da ora, che vuol dire PIL?

Klasse: [?]

FrM: Chi è che mi dice che cos'è il PIL? (Also, schon ab jetzt, was bedeutet BIP? Wer sagt mir, was das BIP ist?)

Lor: Prodotto interno lordo. (Bruttoinlandsprodukt.)

FrM: Prodotto interno lordo. L'analisi della parola l'abbiamo fatta l'anno scorso, quindi non ci torniamo, eh, che vuol dire? [?] [Mehrere Schüler sprechen durcheinander, unverständlich]. Aspetta, uno mi parla. Fabio, non stai a dire niente? lo a questi mo li metto il voto oggi, eh. Perché non c'ho neanche un voto a geografia. (Bruttoinlandsprodukt. Die Wortanalyse haben wir schon letztes Jahr gemacht, also kommen wir darauf nicht zurück, eh, was bedeutet es? [?] [Mehrere Schüler sprechen durcheinander, unverständlich]. Warte, einer spricht mit mir. Fabi, du sagst nichts?! Nun gebe ich denen heute eine Note, eh. Weil ich nicht eine einzige Note in Erdkunde habe.)

Lor: Quanto produce un paese. (Wie viel ein Land produziert.)

FrM: Quanto produce un paese. E quanto produce? Che cosa? (Wie viel ein Land produziert. Und wie viel produziert es? Was?)

Lor: La ricchezza di un Paese. (Den Reichtum eines Landes.)

FrM: La ricchezza di un Paese. Dai, dai, Rodrigo. E che vuol dire? (Den Reichtum eines Landes. Los, los, Rodrigo. Und was bedeutet das?)

Rod: Ehm, i prodotti. (Ehm, die Produkte.)

FrM: I prodotti? Le merci? (Die Produkte? Die Waren?)

Ava: La vendita. (Der Verkauf.)

FrM: Hm. La vendita, lo scambio che fa quel paese di tutte le cose che ha dentro, che ha e la quantità di industrie. Mio dio. Per esempio, noi in Italia abbiamo un'importante industria sider, eh, eh, di acciaio. [...] (Hm. Der Verkauf, den Austausch, den das Land mit allen Sachen hat, die es drinnen hat, die es hat und die Anzahl der Industrien. Mein Gott. Zum Beispiel haben wir in Italien eine wichtige Eis, eh, eh, Stahlindustrie. [...])

Abbildung 19: Unterrichtsgespräch Frau Mancuso, Mikro-scaffolding - PIL

In seinem Handbuch zur Unterrichtsqualität betont Andreas Helmke (2009, S. 228 f.), dass es bei dem Qualitätsmerkmal Unterrichtstempo und Wartezeit zwar auf eine Optimierung der zeitlichen Komponente ankommt, Probleme sich für die Schülerleistungen aber häufiger aus einem zu schnellen Tempo ergeben. Helmke schildert zwei kritische Wartezeiten, von denen die erste den 
Zeitraum betrifft, den die Lehrperson eine Schülerantwort abwartet, und die zweite den, der zwischen einem Schülerbeitrag und dem Kommentar durch die Lehrperson vergeht. Gerade für die erste Wartezeit sollten drei bis vier Sekunden angesetzt werden, wohingegen es in den meisten Fällen nur eine Sekunde ist. Helmke verweist auf die Studienergebnisse von Borich (2007, S. 319) und aus der DESI-Erhebung (T. Helmke et al. 2008, S. 371-381), die jedoch gezeigt haben, dass Schüler sich häufiger freiwillig melden, längere Antworten geben und ihre Beiträge selbstständig ergänzen oder verbessern, wenn ihnen sowohl zum Formulieren vor der Antwort als auch zu ihrem anschließenden Überdenken mehr Zeit gegeben wird. Und je häufiger das geschieht, desto positiver beeinflusse es das Antwortverhalten der Lernenden. Wartet man nämlich mit dem Kommentar zu einem Schülerbeitrag, verstehe der/die Lernende, dass die Antwort entweder zu einsilbig ist und ergänzt werden muss oder dass sie korrigiert werden sollte. Geht man hingegen direkt zu einer neuen Frage über, so vermittele es dem/der Betreffenden den Eindruck, dass die karg ausformulierte Antwort genügte.

Auf Prof.ssa Mancusos wiederholtes Nachfragen, was das Bruttoinlandsprodukt eines Landes ist, gaben die SchülerInnen nur einsilbige und unvollständige Antworten. Die Lehrkraft wiederholte die Äußerungen jeweils und regte dazu an, den Begriff genauer zu definieren. Da die Schüler es aber unterließen, unternahm die Lehrkraft am Ende des Transkriptauszugs selbst einen Versuch einer Definition des Begriffs, den sie mit einem Beispiel aus der italienischen Industrie abschloss.

Nicht vollständig wiedergegeben, betrifft ihr Beispiel ein Stahlwerk, das sich zur Zeit des Unterrichtsgesprächs in den Schlagzeilen befand, weil es von der Schließung bedroht war. Hierbei brach sie das Wort siderurgico (CO; Eisen-/Stahl-) nach den ersten beiden Silben ab und korrigierte ihre Äußerung zu di acciaio (AU; aus Stahl). Das erste Wort war korrekt gewählt und wird in Lehrbuchtexten über die wirtschaftliche Situation eines Landes oft verwendet, so dass es den Schülerinnen und Schülern bekannt gewesen sein dürfte. Da Prof.ssa Mancuso den Ausdruck aber in ,,aus Stahl“ verbesserte, schien sie Bedenken zu haben, ob er wirklich allen geläufig war. Sie entschied sich für einen Begriff aus dem Wortschatz des hohen Gebrauchs, der frequenter verwendet wird und dadurch wahrscheinlich besser beherrscht wurde.

Darüber hinaus reduzierte Frau Mancuso bei der gemeinsamen Texterarbeitung des Öfteren die zu lesenden Texte, indem sie während des Unterrichts Abschnitte identifizierte, die ihres Erachtens weniger themenrelevante Informationen enthielten und/oder sprachlich zu kompliziert gestaltet waren. So gab sie den Schülern auch während der Unterrichtseinheit zur Geschichte Afrikas und der Sklaverei, aus welcher der Transkriptauszug 5 stammt, vor, bestimmte Textteile, z.B. die ersten beiden Absätze, welche die Überschrift I regni africani (Die afrikanischen Reiche) tragen, nicht zu beachten. Sie las sie der Klasse 
vor und entschied dann, dass sie nicht gelernt werden mussten, fasste sie aber dennoch zusammen.

Zum besseren Verständnis werden im Folgenden zunächst die beiden Absätze aus dem Lehrbuchtext und anschließend der Transkriptauszug (Abb. 20) wiedergegeben, in dem die Lehrkraft ihren Lernenden die Absätze vorstellt und sich auch kurz an die Beobachterin (Stephanie) wendet, um auf die Textschwierigkeit aufmerksam zu machen (Carazzi \& Pizzetti 2010, S. 117; Hervorhebung im Original):

La storia

I regni africani

La storia dell'Africa è stata spesso trascurata dalla cultura europea, per due ragioni: la scarsità di documenti scritti e la poca considerazione nei confronti di popolazioni reputate primitive.

Nel periodo corrispondente al nostro Medioevo la storia del continente è invece testimoniata dagli storici di lingua araba.

(M.Ü.: Die Geschichte Afrikas ist von der europäischen Kultur oft vernachlässigt worden, aus zwei Gründen: die Knappheit an geschriebenen Dokumenten und die geringe Wertschätzung gegenüber den als primitiv erachteten Bevölkerungen.

In der unserem Mittelalter entsprechenden Phase ist die Geschichte des Kontinents hingegen von den Geschichtswissenschaftlern arabischer Sprache bezeugt.)

Transkriptauszug 6, Frau Mancuso (FrM), Nora (Nor), Ava, Beobachterin (Ste), Olga (Olg), Vereinfachung/Erklärung der Lehrbuchtexte

FrM: Eeh, allora, „La storia dell'Africa è stata spesso trascurata dalla cultura europea. Per due ragioni. "Uno: "a scarsità di documenti scritti e la poca consideraz", eh, due: "la poca considerazione nei confronti delle popolazioni reputate primitive." Qua dentro, su tre righe ci sta scritto il mondo. Ci sta dicendo una cosa gigantesca. Sta affrontando in tre righe due problemi pazzeschi, hm? II primo, lo sappiamo, cioè, il secondo problema, eh, la poca considerazione nei confronti di popolazioni considerate primitive, che vuol dire? Dai, forza, che vuol dire? (Eeh. also, „Die Geschichte Afrikas ist von der europäischen Kultur oft vernachlässigt worden.“ Eins: „die Knappheit an geschriebenen Dokumenten und die geringe Wertsch“, eh, zwei: "die geringe Wertschätzung gegenüber den als primitiv erachteten Bevölkerungen." Hier drin, in drei Zeilen steht die Welt geschrieben. Es sagt uns etwas Gigantisches. Es nimmt in drei Zeilen zwei unglaubliche Probleme in Angriff, hm? Das erste, das wissen wir, also, das zweite Problem, eh, die geringe Wertschätzung gegenüber den als primitiv erachteten Bevölkerungen, was bedeutet das? Los, auf geht's! Was bedeutet das?)

Nor: Che nessuno gli aiuta. (Dass ihnen niemand hilft.)

FrM: No. (Nein.)

Ava: Le popolazioni, cioè, che non vanno a scuola. Quindi, cioè [Pause]. (Die Bevölkerungen, also, dass sie nicht zur Schule gehen. Also, das heißt [Pause].)

FrM: Ti vuoi spieg, ehm? Che me importa a me di stare a pensare a uno che io considero una specie di insetto. Perché mai io devo stare a preoccupare di osservare, di studiare gli usi e i costumi di un africano che per me non è niente? Vi ricordate l'atteggiamento dei colonialisti nei confronti di queste popolazioni qua. Ma si preoccupano per caso delle loro cultura? Ma che li importa? Nulla. Una parola abbiamo fatto per capire. Una parola. Tutto questo sta dentro a un ombrello, quale era la parola? Ra? (Willst du dich erklären, ehm? Was bedeutet es mir [ugs.], mich damit aufzuhalten, an jemanden zu denken, den ich für eine Art Insekt halte. Warum bloß sollte ich mich 
darum scheren, die Sitten und Gebräuche eines Afrikaners zu beobachten, zu erforschen, der für mich ein Nichts ist? Ihr erinnert euch an die Haltung der Kolonialisten gegenüber diesen Bevölkerungen hier. Sorgen sie sich vielleicht um ihre Kultur? Aber was kümmert es sie? Gar nichts. Ein Wort haben wir gemacht, um zu verstehen. Ein Wort. All das befindet sich unter einem Schirm, welches war das Wort? Ra?)

Klasse: Zzismo. (Ssismus).

FrM [nickt]: Due. E questo è un problema tipicamente africano. L'Africa, tutt, moltissime tribù, pop, o diciamo, gruppi africani, ha una cultura essenzialmente orale. Vale a dire che la trasmissione scritta dei contenuti culturali e meno frequenti che in Europa, che negli Stati Uniti. È evidente che questo non vuol dire che è più brutta, che è inferiore, che non vale niente, però, è meno presente, meno gestibile. Un conto è avere un foglio dove c'è scritto o un libr, libri, libri, libri, chilometri, fiumi di libri, come per esempio la nostra cultura. Un conto è sapere che la cultura ce l'hai, pazzesca, ma sta tutto dentro la testa delle persone. É più difficile, no? E un po' il problema dei rom. I rom, che so', i rom non hanno una cultura scritta. Questo li rende a noi abbastanza grandi [?], come possiamo, aiutatemi a dire, un po' sconosciuti, non sa i, non scrive. Dopodiché, i rom non hanno una cultura? (Zwei. Und das ist ein typisch afrikanisches Problem. Afrika, all, sehr viele Volksstämme, Bevö, oder sagen wir afrikanische Gruppen, haben eine hauptsächlich mündliche Kultur. Das bedeutet, die schriftliche Überlieferung der kulturellen Inhalte ist weniger häufig als in Europa, als in den Vereinigten Staaten. Es ist offensichtlich, dass das nicht bedeutet, dass sie hässlicher ist, dass sie minderwertig ist, dass sie nichts zählt, aber sie ist weniger präsent, weniger überschaubar. Es ist eine Sache, ein Blatt zu haben, auf dem geschrieben steht oder ein $\mathrm{Bu}$, Bücher, Bücher, Bücher, Kilometer, Flüsse an Büchern, wie zum Beispiel unsere Kultur. Eine Sache ist es zu wissen, dass du eine Kultur hast, eine unglaubliche, aber alles ist komplett in den Köpfen der Personen. Das ist schwieriger, nicht? Es ist ein bisschen das Problem der Roma. Die Roma, soweit ich weiß, die Roma haben keine geschriebene Kultur. Das führt dazu, dass sie uns ziemlich große [?], wie können wir, helft mir, es zu formulieren, etwas unbekannt, er kennt die nicht, er schreibt nicht. Danach haben die Roma keine Kultur?)

Klasse: Si. (Ja.)

FrM: Come si esprimerà la cultura rom? C'avranno i loro usi, i loro costumi, le loro tradizioni. Come fanno a sapere quali sono? (Wie wird sich die Kultur der Roma ausdrücken? Sie werden ihre Gebräuche, ihre Sitten, ihre Traditionen haben. Wie wissen sie, welche es sind?)

Olg: Attraverso, parlando? (Durch, sprechend?)

FrM: Eh, trasmettendosi le informazioni a voce. Ma, vi rendete conto che poi, però, rimane meno. Allora, quindi abbiamo, due problemi abbiamo individuato. La scarsità di documenti scritti e la poca considerazione nei confronti di popolazioni reputate primitive. "Nel periodo corrispondente al nostro medioevo, la storia del continente è invece testimoniata dai storici di lingua araba. A quel tempo, nel continente africano si svilupparono diversi regni." Tu mi devi spiegare, Stephanie, eh, scusami, queste tre righe. (Eh, sich die Informationen mündlich überliefernd. Aber, versteht ihr, dass es bedeutet, dass dann aber wenig bleibt. Also haben wir, wir haben zwei Probleme ausfindig gemacht. Die Knappheit an schriftlichen Dokumenten und die geringe Wertschätzung gegenüber den für primitiv gehaltenen Bevölkerungen. „In der unserem Mittelalter entsprechenden Phase ist die Geschichte des Kontinents hingegen von den Geschichtswissenschaftlern arabischer Sprache bezeugt. Zu jener Zeit entwickelten sich auf dem afrikanischen Kontinent verschiedene Reiche." Du musst mir erklären, Stephanie, eh, entschuldige, diese drei Zeilen.

Ste: Io? (Ich?)

FrM: No, guardate qua, poi lo dico a te. "Nel periodo corrispondente al nostro medioevo, la storia del continente è invece testimoniata dai storici di lingua araba." [Pause] 
Quindi, allora voi, queste informazioni le imparate, le incamerate e poi dite, ma che vuol di [ugs.], cioè, che vuol dire? [Pause, Stille] Infatti, la saltiamo. Bene? Quello che dobbiamo sapere in questo momento è che la difficoltà, la storia dell'Africa è stata trascurata per due ragioni fondamentali: mancanza di testi scritti e, eehm, diffide, diffidenza, senso di superiorità dei conquistatori nei confronti di quella cultura, per cui quella cultura non mi interessa, cioè andiamo a sottolineare questo. (Nein, schaut hier, dann sage ich es dir. „In der unserem Mittelalter entsprechenden Phase ist die Geschichte des Kontinents hingegen von den Geschichtswissenschaftlern arabischer Sprache bezeugt." [Pause] Sodann, also, ihr lernt diese Informationen, ihr saugt sie auf und dann sagt ihr, aber was bedeutet das [ugs.], also, was heißt das? [Pause, Stille]. In der Tat, wir überspringen sie. Gut? Das, was wir in diesem Moment wissen müssen, ist, dass die Schwierigkeit, die Geschichte Afrikas ist aufgrund von zwei wesentlichen Gründen vernachlässigt worden: Mangel an schriftlichen Texten und, eehm, misstraut, Misstrauen, Überlegenheitssinn der Eroberer gegenüber jener Kultur, weshalb mich jene Kultur nicht interessiert, das heißt wir unterstreichen dies.)

Abbildung 20: Frau Mancuso, Vereinfachung/Erklärung der Lehrbuchtexte

Generell bleibt zu erwähnen, dass Frau Mancuso mit dieser wiederholt beobachteten Praxis, Abschnitte aus den Lehrbuchtexten auszuklammern, alleine dastand, während die anderen Lehrperson im Zeitraum der Unterrichtsbeobachtungen die Reihenfolge der Lehrbuchtexte einhielten, sie vollständig behandelten und lesen ließen. Vor allem bei Frau Rea wurde außerdem beobachtet, dass sie sogar anhand von Fragen zum Inhalt kontrollierte, ob die teils aus mehreren Sätzen bestehenden Bildbeschriftungen mitgelesen oder übersprungen worden waren.

\subsubsection{Wortschatzarbeit}

Bei der Behandlung neuer Lehrbuchtexte vergewisserten sich die Lehrkräfte, ob alle Begriffe verstanden wurden. Das machten sie, indem sie die Lernenden dazu aufforderten, einen Ausdruck zu erklären, ein Konzept zu definieren oder zu der Schilderung eines Sachverhalts das passende Fachwort zu nennen. ${ }^{192}$ Bisweilen erläuterten sie einige Begriffe auch von sich aus, wenn sie Erklärungsbedarf vermuteten. Außerdem fragten einige die Klasse direkt, ob Wörter unklar waren. In diesen Fällen lieferten sie die Erklärung entweder selbst oder gaben die Schülerfrage nach einem Ausdruck an das Klassenplenum weiter. Dabei ging es nicht nur um geographische Konzepte, sondern auch um allgemeinsprachliche Ausdrücke, die nicht bekannt waren. So fragte beispielsweise eine Schülerin an der Morante nach der Bedeutung von imponente (AU; imposant), was die Lehrkraft folgendermaßen kommentierte, bevor sie das Wort erklärte: „Qui non stiamo al linguaggio geografico purtroppo. Stiamo al lin-

192 Hierbei nutzten sie für Fachwörter, die gekannt werden sollten, auch die oben vorgestellte Technik der Designedly Incomplete Utterances (Kap. 9.1.3.1.1). 
guaggio italiano." (Hier sind wir leider nicht bei der geographischen Fachsprache. Hier sind wir bei der italienischen Sprache). Auch in anderen Klassen wurde mehrmals beobachtet, dass die Lehrkraft auf sprachliche Schwierigkeiten einging und unterstrich, dass sie nicht oder vielmehr nicht nur die Aneignung des Geographiewortschatzes, sondern die allgemeinsprachliche lexikalische Kompetenz beträfen. Auf der anderen Seite resultierten die Ausdrucksfehler der SchülerInnen auch aus der unzureichenden Beherrschung von niedrigfrequenten und fachsprachlichen Wörtern. Solche Fehler begangen sie vor allem dann, wenn es sich um Begriffe handelte, die mit einem semantisch ähnlichen bzw. ähnlich klingenden Wort verwechselt werden konnten. So hielten einige megalopoli (CO; Megalopolis) für metropoli (AU; Metropole), gebrauchten esportare (AU; exportieren) anstelle von deportare (CO; deportieren) und bezeichnen einzelne Berge als catena montuosa (Co; Gebirgskette). Die Lehrkräfte wiesen fast immer auf einen derartigen falschen Gebrauch hin und erklärten die richtige Bedeutung der entsprechenden Wörter, bzw. nannten das passendere Wort, das die Lernenden in ihren Darstellungen durch ein ähnlich klingendes, aber semantisch falsches Wort ersetzt hatten.

In der Klasse an der Schule Morante forderte Prof. Tigre seine Lernenden zudem dazu auf, bei der häuslichen Lektüre in den Texten acht unbekannte oder komplizierte Wörter auszuwählen, zu unterstreichen, ihre Bedeutung in einem Wörterbuch nachzuschlagen und sie schriftlich festzuhalten. Im Beobachtungszeitraum hielt nur er die Lernenden explizit (in Form einer Hausaufgabe) zum Nachschlagen im Wörterbuch an, um das Textverständnis zu sichern. Aber auch in anderen Klassen konnte beobachtet werden, dass ein einsprachiges Wörterbuch stets vorhanden war und gelegentlich zur Klärung von Begriffsbedeutungen genutzt wurde, wie beispielsweise in der III B an der Schule Pestalozzi für den Begriff crogiuolo (CO, Ts; Schmelztiegel). Die Gewöhnung an den Umgang mit Wörterbüchern ist für diese Altersstufe im Lehrplan vorgesehen, jedoch handelte es sich bei den in den Klassen vorhandenen Nachschlagewerken um sehr umfangreiche, für erwachsene Leser geschriebene, deren Lemmata zahlreiche Unterbedeutungen enthielten, so dass die Lernenden zum Teil lange Abschnitte vorlesen mussten, bis sie zu der für sie nützlichen Wortbedeutung kamen. In diesen Momenten erweckten die anderen nicht den Eindruck, aufmerksam zuzuhören, und einige der Wörterbuchdefinitionen waren sprachlich so komplex, dass ihr Verständnis selbst bei einer gröBeren Konzentration auf ihren Inhalt fraglich bliebe. Das veranschaulicht die folgende Beschreibung des Unterrichtsverlaufs an der Schule Morante.

Bei der Besprechung der Wörterbuch-Hausaufgabe sammelte Herr Tigre die genannten Begriffe nach und nach an der Tafel und hielt dahinter die Anzahl der Schüler und Schülerinnen fest, die dasselbe Wort unterstrichen hatten. Die Ausdrücke wurden zunächst im Klassengespräch beschrieben, bevor die Wörterbuchdefinition vorgelesen wurde, oder umgekehrt. Hierbei zeigte sich, dass Wörterbuchdefinitionen zusätzliche Ausdrücke enthalten können, die den 
Lernenden unbekannt sind, so dass auch sie im Unterricht behandelt wurden. Transkriptauszug 7 gibt einen Teil der von dieser Methode bestimmten Art der Wortschatzerarbeitung wieder.

Transkriptauszug 7, Herr Tigre (HrT), Asia (Asi), Lev, nicht identifizierter Schüler (Sch), Luca Carnieri (Luc), Giorgia (Gio) - Textarbeit: unbekannte Wörter und ihre Definitionen

$\mathrm{HrT}$ : Facciamo rapidamente un lavoro di verifica? Quali sono le parole che voi avete scelto? Dunque. La consegna era: tra le pagine che avete studiato, otto termini che vi hanno colpito. Cominciamo da Asia? Qual è il primo termine che ti ha colpito? (Machen wir schnell eine Kontrollarbeit? Was sind die Wörter, die ihr ausgewählt habt? Die Aufgabe war: acht Ausdrücke auf den Seiten, die ihr gelernt habt, die euch aufgefallen sind. Fangen wir mit Asia an?)

Asi: Meridionale. (Meridional.)

HrT: Merid. Lev, cosa vuol dire "meridionale”? (Merid. Lev, was bedeutet „meridional“?)

Lev: Eeh. Sud. (Eeh, Süden.)

HrT: Vuol dire sud? (Heißt es Süden?)

Lev: Eh, eh, meridione. (Eh, eh, Meridian.)

HrT: Meridione vuol dire sud. Meridionale, che cosa vuol dire? [Pause, Stille] $E$ un aggettivo relativo al sud. Ok? Quindi, del sud. Che definizione hai trovato? (Meridian bedeutet Süden. Merdional, was heißt das? [Pause, Stille] Es ist ein Adjektiv bezogen auf Süden. Ok? Was für eine Definition hast du gefunden?)

Asi: Eeh, posto a sud, cioè a mezzogiorno. (Eeh, im Süden gelegen, das heißt, im Mezzogiorno).

HrT: Posto a sud, cioè a mezzogiorno. Ok. Ehm, dunque, dovete sapere che il sud si può dire anche con "mezzogiorno". Perché? (Im Süden gelegen, das heißt im Mezzogiorno. Ok. Ehm, also, ihr müsst wissen, dass man den Süden auch „Mezzogiorno“ nennen kann. Warum?

Sch: Aaah.

HrT: Perché il sud e il mezzogiorno sono la stessa cosa? (Warum sind der Süden und der Mezzogiorno dieselbe Sache?)

Luc: Perché il sole (Weil die Sonne)

HrT [unterbricht inn]: Perché Carnieri, stare zitto, per esempio. Prego. (Weil Carnieri, still sein, zum Beispiel. Bitte.)

Gio: Ha detto lui, perché il sole (Er hat [es] gesagt, weil die Sonne)

HrT [übernimmt das Wort]: Perché il mezzogiorno è il momento in cui il percorso apparente del sole che è un arco di circonferenza, nel suo percorso apparente il sole arriva al punto di massima e comincia a scendere. Ok? Perché parlo di "percorso apparente del sole"? [Wendet sich an Giorgia] (Weil der Mezzogiorno der Moment ist, in dem der scheinbare Kurs der Sonne, der ein Kreisbogen ist, in ihrem scheinbaren Kurs erreicht die Sonne den Höhepunkt und beginnt zu sinken. Ok? Warum spreche ich von „scheinbarer Kurs der Sonne“? [Wendet sich an Giorgia])

Gio: [?]

$\mathrm{HrT}$ : E proprio questo, perché ho usato il termine "apparente"? (Es ist genau das, warum habe ich den Ausdruck „apparente" (scheinbar) gebraucht?)

Gio: Appartiene. (Es gehört.)

HrT: Cosa vuol dire? Quello è "appartenente”. (Was soll das heißen? Das ist „appartenente“ [gehören]).

Gio: Quello che sembra. (Das, was scheint.)

HrT: Quindi io ho detto: quello che sembra il viaggio del sole, perché quello che sembra? Chi lo sa? (Also ich habe gesagt: Das, was die Reise der Sonne scheint, warum das, was es scheint? Wer weiß es?)

Gio: Perché sembra. (Weil es scheint.) 
HrT: E perché sembra e non è? (Und warum scheint es und ist nicht?)

Gio: Perché? (Warum?)

HrT: Perché? Guardate, se io faccio la domanda a una, gli altri sono invitati a pensare.

(Warum? Schaut, wenn ich einer die Frage stelle, sind die anderen eingeladen nachzudenken.)

Klasse: Perché la terra non è ferma. (Weil die Erde nicht stillsteht.)

Gio: Ma mi ha chiesto un'altra cosa. (Aber Sie haben mich eine andere Sache gefragt.)

HrT: Allora, ricostruiamo la sequenza. Tu mi hai chiesto perché sud e mezzogiorno sono uguali, ok? Allora, sono uguali perché la parola mezzogiorno, cioè le ore 12 e la e coincide con il momento in cui il sole [zeichnet Bild von der Erde und der (scheinbaren) Sonnenlaufbahn an die Tafel] noi abbiamo, noi siamo qui? Bene. Qui c'è il sole che sorge. Poi, fa quest'arco qui per poi tramontare [Klasse unruhig]. Per poi tramontare. Quando il sole è a mezzogiorno si trova sulla verticale. Ok? Poi vi ho detto, quindi qui indica esattamente il sud. Basta guardare il sole e tu sai, dove è il sud. Ok? Bene. Poi ho detto "percorso apparente" del sole perché è vero che il sole sorge e tramonta nella nostra esperienza, ma in realtà, non è il sole che sorge e tramonta perché il sole sta fermo ed è la terra che rota. Quindi il movimento è quello della terra. Ok? Ci siamo? Perché abbiamo cominciato a parlare di sud? Seconda definizione, Tina? (Also, rekonstruieren wir den Ablauf. Du hast mich gefragt, warum Süden und Mezzogiorno gleich sind, ok? Also, sie sind gleich, weil das Wort Mezzogiorno, das heißt zwölf Uhr und die und es fällt zusammen mit dem Moment, in dem die Sonne, [zeichnet Bild von der Erde und der Sonnenlaufbahn an die Tafel] wir haben, wir sind hier? Gut. Hier ist die Sonne, die aufgeht. Dann, um diesen Bogen hier zu machen, um dann unterzugehen [Klasse unruhig]. Um dann unterzugehen. Wenn die Sonne um zwölf Uhr ist, befindet sie sich auf der Vertikale. Ok? Gut. Dann habe ich „scheinbarer Lauf" der Sonne gesagt, denn es ist wahr, dass die Sonne nach unserer Erfahrung auf- und untergeht, aber in Wirklichkeit ist es nicht die Sonne, die auf- und untergeht, weil die Sonne stillsteht und es die Erde ist, die rotiert. Also ist die Bewegung die der Erde. Ok? Haben wir das? Warum haben wir begonnen, über den Süden zu sprechen? Zweite Definition, Tina?)

Abbildung 21: Transkriptauszug Herr Tigre, Textarbeit - unbekannte Wörter und ihre Erklärungen

In der Definition, welche die Schülerin für das Adjektiv meridionale (AU) gefunden hatte, taucht ein anderes Wort auf, das der Lehrkraft erklärungsbedürftig erschien, nämlich Mezzogiorno. Es hat verschiedene Bedeutungen, von denen die erste als „Mittag/12.00 Uhr“ dem fundamentalen Wortschatz (Fo) angehört. Die weiteren Bedeutungen, auch die hier intendierte, gehören zu weniger frequenten Wörtern, und zwar als „Moment, in dem die Sonne den oberen Meridian eines bestimmten Ortes passiert" zum astronomischen Fachwortschatz (TS astron.), als „Süden“ zum allgemeinen Wortschatz (CO) und als „meridionaler Teil einer geographischen Region; Süditalien“ ebenfalls zum allgemeinen Wortschatz (CO).

Die Lehrkraft nutzte die Gelegenheit der Erklärung, warum man den Süden auch Mezzogiorno nennt, zu einer Wiederholung der scheinbaren Sonnenlaufbahn um die Erde. Hierbei ergab sich eine weitere lexikalische Schwierigkeit bei der Bedeutung des Wortes „scheinbar“ (apparente, Co), die Giorgia, eine mehrsprachige Schülerin mit gut entwickelten BICS im italienischen Regiolekt Roms, nicht kannte. Darüber hinaus verwechselte sie das Wort mit dem sehr 
ähnlich klingenden appartenente (Fo; gehörend). An dieser Stelle ließ Prof. Tigre das bereits behandelte Konzept nicht erklären, obwohl ein Schüler (Luca) es versuchte. Er unterbrach ihn und äußerte daraufhin den Wunsch, die Antwort von anderen zu hören. Die aktive Beteiligung der Schülerinnen und Schüler an der Erarbeitung einer Erklärung des Konzepts beschränkte sich hier auf eine recht kurze Äußerung in Form einer Antwort auf die Frage, warum die Bewegung der Sonne um die Erde nur eine scheinbare ist: „Perché la terra non è ferma." (Weil die Erde nicht stillsteht.). Anstatt die Lernenden dazu aufzufordern, die Darstellung weiter auszuführen, übernahm es der Lehrer selbst und unterstützte seine Erklärung mit einer Zeichnung an der Tafel.

Im weiteren Gesprächsverlauf wurden noch andere nachgeschlagene Ausdrücke aus den Lehrbuchtexten vorgestellt und besprochen, u.a. foresta temperata (Ts geogr.; Biodiversität), was die Arbeit an einer ganzen Reihe von Lexemen nach sich zog. So wiederholte der Lehrer mit den Schülern zunächst die Merkmale der gemäßigten Klimazone, fragte sie dann nach anderen Waldtypen und wies die antwortenden Lernenden darauf hin, dass sie unterschiedliche Klassifikationssysteme verwendeten (biologisch, geographisch). Der Beitrag foresta a latifoglie (Ts bot.; Laubwald) diente ihm darüber hinaus als Anlass, die Bezeichnungen für Laub- und Nadelwälder und die Bedeutung des lateinischen Latus zu wiederholen, was auch der Ursprung des italienischen Wortes (Lazio) für die Region Latiens und damit die Heimat der Lernenden ist. Nach einer Weile bat er die Schülerin, die das Wort notiert hatte (Sania), ihre herausgeschriebene Definition vorzutragen, wobei sie auf weitere Wörter stießen, die erklärt wurden, wie z.B. bioma (TS ecol., Biom).

An diesem Beispiel wird deutlich, wie die Wortschatzarbeit immer mehr einen Eigencharakter annimmt, indem sie sich vom Lehrbuchtext loslöst. Während die Mehrzahl der besprochenen Wörter zumindest noch im entfernteren Sinne mit dem Erdkundeunterricht in Zusammenhang zu bringen ist, gehen andere, wie das letztgenannte „Biom“ deutlich darüber hinaus. Weiter wurde beobachtet, dass die SchülerInnen entweder gar nicht zum Zug kamen, ihre Definitionen mit eigenen Worten zusammenzufassen, oder nicht dazu in der Lage waren, sondern nur die von ihnen gefundenen und herausgeschriebenen vorlesen konnten. Die abgeschriebenen Darstellungen schienen allerdings allesamt weiterer Worterklärungen zu bedürfen, um als Verständnishilfe zu dienen.

Deshalb stellt sich die Frage, ob diese Art der Beschäftigung mit Wörtern, die nur zu einem gewissen Grad beherrscht werden und die nicht in ihrem konkreten Verwendungskontext betrachtet und zumindest ansatzweise mit ihm erschlossen werden, förderlich ist. Anzunehmen ist, dass es sich um einen neuen Aufgabentyp handelte, mit dem die SchülerInnen erst hätten vertraut gemacht werden müssen, so dass sie mit der nötigen Unterstützung durch die Lehrkraft und etwas Übung angeleitet worden wären, die Begriffe besser mit eigenen Worten zu erklären. Anstatt für die nicht oder nicht komplett beherrschten 
Ausdrücke nur Definitionen zu suchen, die lediglich abgeschrieben werden, hätte die Konzentration auf den semantischen Gehalt gelenkt werden sollen, damit die Lernenden sich mit den Wörtern produktiver und sinnvoller beschäftigen und die Wahrscheinlichkeit eines nachhaltigeren Behaltens vergrößert wird.

Dass gerade dies ein generelles Problem bei der Erweiterung des mentalen Lexikons ist, wurde bereits an verschiedenen Stellen umrissen. Das mehrmalige Wiederholen bestimmter Begriffe und Konzepte durch Fragen der Lehrkraft dient als Kontrolle, ob die jeweiligen Ausdrücke beherrscht werden und hilft den Schülern gleichzeitig, das Wissen darüber zu festigen und besser zu verankern. Dass aber auch eine gemeinsame, umfassende Behandlung wichtiger Konzepte nicht in jedem Fall ausreichend ist, um eine solide Abspeicherung im mentalen Lexikon zu garantieren, zeigte sich u.a. in den zwei Klassen an der Schule Morante und an der Pestalozzi, in denen die Lernenden dazu aufgefordert wurden, zu intensiv behandelten Konzepten schriftliche Definitionen zu formulieren.

\subsubsection{Mündliche und schriftliche Wortdefinitionen der Lernenden}

In der beobachteten Klasse an der Schule Morante wurden im Erdkundeunterricht verschiedene Wörter behandelt, die mit einem zeitlichen Abstand von einer Woche schriftlich definiert werden sollten. Im Folgenden wird zuerst die Behandlung der Ausdrücke im Unterrichtsgespräch vorgestellt, um einen Eindruck der gemeinsamen Erarbeitung zu vermitteln, woran sich eine Besprechung der Testergebnisse anschließt.

Gleich in der ersten beobachteten Stunde bildete die Wortschatzarbeit in dieser Klasse einen Unterrichtsschwerpunkt: Die Lernenden sollten als Hausaufgabe einige Lehrbuchseiten erarbeiten und wurden von Herrn Tigre gefragt, ob es Ausdrücke gab, die sie nicht verstanden hatten. Da ihm keine genannt wurden, ist es möglich, dass sich die SchülerInnen nicht erinnerten und vielleicht über Wörter, die sie nicht gut oder gar nicht kannten, hinweggelesen hatten. Erst als der Lehrer sie fragte, ob sie also beruhigt einer Abfragung entgegensehen könnten, weil die Lehrbuchexte ihnen ja keine Verständnisschwierigkeiten bereitet hatten, gab es eine Wortmeldung.

Sie bildete die Einleitung für ein beobachtetes typisches Beispiel im Umgang mit unbekannten Wörtern in dieser Klasse und betrifft das Wort acquitrino, das eine Schülerin nicht kannte und deshalb dem Lehrer nannte. Dieser italienische Ausdruck für „Morast/Sumpf“ stammt aus dem allgemeinen Gebrauchswortschatz (CO) und wird daher seltener verwendet, so dass es nicht ungewöhnlich ist, dass die Schülerin ihn nicht beherrschte. Daraufhin wurde die Klasse aufgefordert, das Wort zu erklären, doch niemand meldete sich.

Mindestens zwei Gründe für dieses Verhalten der Lerngruppe sind als wahrscheinlich zu betrachten. Auf der einen Seite taucht der Ausdruck im Text 
mit der Konjunktion „und“ nebengeordnet zu palude (Moor, Sumpf) auf, einem Wort, das acquitrino semantisch sehr ähnlich ist, aber aus dem Wortschatzbereich der hohen Disponibilität (AD) stammt, also im Gegensatz zu $a c$ quitrino zum Vocabolario di Base zählt und deshalb mit größerer Wahrscheinlichkeit bekannt war. Daher mag es sein, dass sie beim Lesen des Textes durch die Satzkonstruktion mit der Nebenordnung und aus der BedeutungserschlieBung unter Einbezug des Kontextes eine Vorstellung von acquitrino als etwas hatten, dass dem Moor ähnelt, und/oder ihr Textverständnis hier als ausreichend gesichert bewerteten. Nun, als es um ein genaueres Definieren des Wortes ging, blieben sie entweder still, weil sie es vermutlich nicht konnten, oder, weil sie dem Unterrichtsgeschehen gerade nicht aufmerksam genug folgten.

Ein Schüler, der aufgrund der Nichtbeteiligung der Klasse zur Antwort aufgefordert wurde, gab an, das Wort ebenfalls nicht zu kennen, woraufhin sich Prof. Tigre die Stelle nennen ließ, an der es im Text erscheint. Es sollte also versucht werden, die Wortbedeutung anhand des Kontextes gemeinsam zu erschließen. In diesen führte der Lehrer seine Schülerinnen und Schüler ein, indem er vorgab, dass die entsprechende Textpassage vom Flussbecken des Kongos handelt. Zunächst fragte er nach der Bedeutung des Co-gekennzeichneten Wortes bacino (Becken) und wies darauf hin, dass es schon in der vorherigen Erdkundestunde besprochen worden war. Die Lernenden schwiegen, also wurde einer von ihnen, Luca, von der Lehrkraft aufgefordert und wegen der ausbleibenden Antwort weiter zu einem Beitrag ermutigt, woraufhin der Schüler als Erklärung schließlich formulierte: „Dovrebbe dì gli affluenti“ (Müsste heißen, die Zuläufe [lokale Färbung durch Auslöschung des Infinitivsuffixes re]).

Herr Tigre kommentierte diese Schüleräußerung folgendermaßen: „'Dovrebbe dì gli affluenti.' Secondo me, l'hai capito ma l'hai detto in un italiano per dire pessimo è poco. Che cosa è un bacino?" (,Müsste heiß[en], die Zuläufe.' Meiner Meinung nach hast du es verstanden, aber Du hast es in einem Italienisch gesagt, das milde ausgedrückt grottenschlecht ist. Was ist ein Becken?)

Er wiederholte also die Schülerdefinition mit den (sprach)lernförderlichen Implikationen, die schon w.o. für diese auch von der Lehrkraft Mancuso verwendete Methode des scaffolding beschrieben worden sind (s. Kap. 9.2.3), und bewertete sie im Anschluss. Dabei bezog er sich zwar auf die sprachliche Ebene, die seiner Ansicht nach ,,mindestens sehr schlecht“ war, brachte allerdings gleichzeitig eine positive Erwartungshaltung gegenüber der Schülerleistung zum Ausdruck. Er bekundete nämlich die Annahme, dass der befragte Schüler, dessen Muttersprache übrigens Italienisch ist, die Wortbedeutung kenne. Ob das tatsächlich der Fall war, bleibt an dieser Stelle offen. Eindeutig ist nur, dass Luca seinem Wissen keine sprachlich angemessene Form verliehen hatte. 
Auf Lucas Schweigen hin bat der Lehrer ihn, nach vorne an eine große Wandkarte zu kommen und das Flussbecken des Kongos zu zeigen. Luca wurde also die Möglichkeit verschafft, seinem Wissen auf nonverbale Art Ausdruck zu verleihen; jedoch kam er auch dieser Aufforderung nicht nach. Deshalb deutete die Lehrkraft selbst auf ein Gebiet auf der Karte und fragte, ob es das Flussbecken sei, was der Schüler falsch beantwortete. Also erklärte Herr Tigre ihm anhand einfacher Fragen an der Landkarte, was ein Flussbecken ist, während es in der Klasse unruhig wurde. Prof. Tigre bemerkte dies und wandte sich wieder an die ganze Klasse, indem er erklärte:

Ci siamo, ragazzi? Il bacino del fiume Congo è tutto questo. Perché? Perché qui, questi sono i punti più alti. L'acqua che cade di qua finisce sul Congo. [...] Ci siamo? Allora, il bacino del fiume Congo è questo. Bene. Nel bacino del fiume Congo ci sono molte [...]

(Haben wir das, Jungs und Mädels? Das Becken des Flusses Kongo ist all das hier. Warum? Weil hier, dies sind die höchsten Punkte [zeigt ein Gebirge]. Das Wasser, das hier runterfällt, endet auf dem Kongo. [...] Haben wir das verstanden? Gut. Im Flussbecken des Kongos gibt es viele $[\ldots]$.)

Herr Tigre wollte also eine Überleitung zum eingangs genannten Wort mit Erklärungsbedarf, aquitrino, schaffen, wurde jedoch von einem Schülerkommentar, der nichts mit dem Thema zu tun hatte, unterbrochen.

Die geschilderte Situation verdeutlicht auf der einen Seite, dass sich die SchülerInnen ihrer Wortlücken vermutlich nicht bewusst waren oder sie nicht als schlimm empfanden, da sie von sich aus nicht der Aufforderung nachkamen, unbekannte Wörter zu nennen. Außerdem folgten sie der Erklärung des Lehrers nur streckenweise. Hierzu muss gesagt werden, dass diese sehr umfangreich und etwas weitschweifend war, zumal sie sich in dem bisher geschilderten Teil ja nur auf den näheren Kontext des zu erklärenden Wortes beschränkte und noch gar nicht beim Wort aquitrino selbst angelangt war.

Wäre die Aufmerksamkeit der Schüler über den gesamten Zeitraum des Lehrervortrags aufrechterhalten worden, hätten seine Erklärungsstrategien einer gefestigteren Verankerung des Wortes im mentalen Lexikon und einer leichteren Abrufbarkeit. Denn die Informationsverarbeitung konnte auf eine breitere Weise geschehen, indem das Wort bacino unter Einbezug des Kontextes beschrieben und außerdem gegebene Definitionen wiederholt wurden. Zusätzlich regte Prof. Tigre die Informationsverarbeitung über zwei verschiedene Sinneskanäle an: Die Worterklärung erfolgte verbal und wurde daneben visuell durch das Mitlesen im Text und die Erklärung an der Landkarte unterstützt.

Dadurch dass Luca eine Definition für Flussbecken geben und selbiges an der Karte zeigen sollte, ist die Wahrscheinlichkeit relativ groß, dass er den Ausdruck verinnerlichte. Der Rest der Klasse hingegen arbeitete nicht aktiv genug mit dem Wort, als dass von einer gesicherten Abspeicherung im mentalen Lexikon ausgegangen werden könnte (vgl. A. Helmke 2009, S. 62 f.).

Für eine solche Verankerung ist es beispielsweise auch hilfreich, eine Verknüpfung zu bereits Gelerntem herzustellen, wie es der Lehrer in derselben 
Unterrichtsstunde mit dem Wort creolo (CO; Kreole) macht, indem er auf die Erklärung des Ausdrucks durch einen Polizisten verweist, zu der es während eines kürzlich erfolgten Klassenausflugs kam. Sie besuchten ein Konzert des Polizeiorchesters und erhielten dort vor der Aufführung eine kurze Einführung in die Jazzmusik, bei der die Rede auch auf Kreolen kam. So erschienen das Wort und seine Bedeutungserklärung in zwei verschiedenen Kontexten, die miteinander verknüpft wurden, und konnten daher tiefer verarbeitet werden und eher im Gedächtnis bleiben.

Dass jedoch weder dies noch die ausführlichen Erläuterungen des Lehrers und seine Wiederholungen zum Behalten des Wortes führten, zeigte ein kurzer „Vokabeltest" in der nächsten Erdkundestunde, in dem die Schüler dazu aufgefordert waren, vier Wörter zu erklären, die im Unterricht besprochen worden waren. Darunter befindet sich auch creolo: Nur ein einziger Schüler erhielt für seine Wortdefinition Punkte, wobei auch er die volle Punktzahl nicht erreichte. Hier verdeutlicht sich wie bei allem Wissen, dass es nicht ausreicht, die Definition eines neuen Wortes zu verstehen, sondern in den meisten Fällen „ein wiederholtes Bewusstmachen, eine Herstellung von Verbindungen zu anderen Informationen und ein Anwenden des Wissens folgen [muss], anderenfalls werden die aufgenommenen Informationen vergessen oder können nicht abgerufen werden" (A. Helmke 2009², S. 200).

In derselben Unterrichtsstunde fragte der Lehrer erneut Luca nach der Bedeutung des Wortes autoconsumo aus dem wirtschaftlichen Fachwortschatz (Ts econ.), das im Titel zu einem Abschnitt der gelesenen Seiten steht (vgl. Abbildung 22). Er verbesserte den sprachlichen Ausdruck der Schülerantwort nicht, aber da sie unvollständig war, versuchte er mit einer weiteren Frage, eine ausführlichere Definition zu erhalten. Die Hilfestellung des Lehrers ist groß, da es sich um eine leicht zu beantwortende Frage handelte und nur dadurch die vom Schüler begonnene Definition für autoconsumo vervollständigt wurde. Möglicherweise war Luca aber gar nicht bewusst, dass es noch immer um die Erklärung des Begriffs autoconsumo ging, weil Herr Tigre Lucas Definitionsangebot nur mit der Interjektion beh (also, naja etc.) kommentierte und direkt eine weitere Frage stellte, die er mit „Und wenn hingegen“ einleitete. Nach deren knapper, aber korrekter Beantwortung verzichtete der Lehrer zunächst darauf, die Begriffsdefinition vervollständigt zu wiederholen, und fragte stattdessen, ob die Bedeutung klar sei. Dadurch dass ein darum gebetener Schüler sie nicht wiederholte, forderte der Lehrer Luca auf, den Ausdruck erneut zu erklären. Dieser wiederholte seine anfänglich gegebene Definition fast wortwörtlich und ergänzte sie also nicht um den durch die Lehrerfrage entwickelten zweiten Part der Erläuterung. Nun präzisierte Herr Tigre Lucas Äußerung, wiederholte aber den eigenen Aspekt der Definition nicht, sondern schlug einen anderen Weg der Worterklärung ein, indem er eine Übersetzung des Präfixes auto- lieferte. Dieses Vorgehen bei Fachwörtern mit lateinischen und griechi- 
schen Wurzeln wurde im Übrigen bei allen Lehrkräften beobachtet. So wiederholten sie Begriffe mit ihrer Klasse, indem sie diese in ihre Bestandteile zerlegen ließen, die einzeln übersetzt wurden. Beispiele hierfür sind Wörter wie democrazia (AU; Demokratie), demografico (Co; demographisch), pro capite (Co; pro Kopf).

Prof. Tigre bediente sich demnach einer Vermittlungsstrategie, welche die Wortzusammensetzung und Regelmäßigkeiten der Wortbildung berücksichtigt und so den Vorteil hat, dass die Lernenden sich weitere, mit demselben Präfix gebildete Wörter erschließen können, wenn ihnen dessen Bedeutung erst einmal klar ist und sie ihm bezüglich über eine gewisse lexikalische Kompetenz verfügen. Dieser Prozess wurde vom Lehrer unterstützt, indem er die Klasse fragte, was ein Automobil ist, woraufhin lebhaft verschiedene Antworten durcheinandergerufen wurden, bis der Lehrer das Wort selbst nannte. Danach wiederholte er die Definition für autoconsumo und schloss so die Behandlung des Wortes ab:

Transkriptauszug 8, Herr Tigre (HrT), Luca (Luc), Textarbeit - Wortschatz

HrT: Luca, un'agricoltura per l'autoconsumo. Che vorrà dire quel titolo? (Luca, eine Landwirtschaft für den Eigenbedarf. Was wird dieser Titel sagen wollen?)

Luc: Agricoltura per soddisfare la popolazione che ci sta. (Landwirtschaft, um die Bevölkerung, die dort ist, zu befriedigen.)

HrT: Beh, e se invece un'agricoltura, oltre ad essere per l'autoconsumo, per che cosa può essere utilizzata? (Beh [naja, also], und wenn hingegen eine Landwirtschaft, abgesehen vom Eigenverbrauch, wofür kann sie noch gebraucht werden?)

Luc: Per la vendita. (Für den Verkauf.)

HrT: Per la vendita. E chiaro quindi il concetto di autoconsumo? (Für den Verkauf. Ist also das Konzept Eigenverbrauch klar?)

[HrT fordert einen Schüler auf, der nicht antwortet.]

HrT: Luca, gli ripeti, per favore, allora quando viene detto che l'agricoltura è destinata all'autoconsumo, cosa vuol dire, Luca? (Luca, wiederholst du ihm bitte, was es also heißt, wenn gesagt wird, dass die Landwirtschaft für den Eigenbedarf bestimmt ist, Luca?)

Luc: Per soddisfare la popolazione che è là. (Um die Bevölkerung zu befriedigen, die dort ist.)

HrT: La popolazione che coltiva. Auto vuol dire per sé o da sé. Che vuol dire la parola automobile? (Die Bevölkerung, die anbaut. Auto bedeutet für sich oder von sich. Was bedeutet das Wort Automobil?)

Klasse: [?] [Sie rufen Antworten durcheinander, unverständlich.]

HrT: Non ho capito. Mobile. Si, qualcosa che si muove da sé, senza bisogno, per esempio, di cavalli, perché ha un motore. Automobile vuol dire qualcosa che si muove da sola. Autoconsumo vuol dire è destinata al consumo di sé stessi. Per sé stessi. Ok? E non è l'agricoltura destinata all'esportazione. (Ich habe es nicht verstanden. Mobil. $\mathrm{Ja}$, etwas, das sich von sich aus bewegt, ohne zum Beispiel Pferde zu brauchen, weil es einen Motor hat. Automobil will sagen, etwas, das sich von alleine bewegt. Autokonsum bedeutet, es ist für den eigenen Konsum bestimmt. Für sich selbst. Ok? Und es ist nicht die Landwirtschaft, die für die Ausfuhr bestimmt ist.

Abbildung 22: Transkriptauszug Herr Tigre, Textarbeit - Wortschatz 
In der nächsten Erdkundestunde wurde auch „Autokonsum“ in dem oben erwähnten „Vokabeltest“" abgefragt, der aus zwei Versionen zu je vier Wörtern bestand, um zu vermeiden, dass die 14 bei der Durchführung anwesenden Lernenden von ihren Banknachbarn abschrieben. Alle acht Wörter entstammen behandelten Texten des Erdkundebuchs und waren in der vorherigen Unterrichtsstunde gemeinsam erläutert worden, wobei es sich bei einigen um die Wiederholung bereits zuvor besprochener Begriffe handelte.

Die zu definierenden Wörter der ersten Gruppe waren:

- popolazione non attiva (Co TS stat.; nicht erwerbstätige Bevölkerung);

- monocoltura (Ts agr., Ts econ.; Monokultur);

- tasso di crescita (Co; Wachstumsrate);

- acrocoro (Ts geogr.; Hochebene).

Und die der zweiten Gruppe:

- popolazione attiva (Co Ts stat.; erwerbstätige Bevölkerung);

- creolo (Co; Kreole);

- autoconsumo (Ts econ.; Eigenverbrauch);

- altopiano (AD; Hochebene).

Autoconsumo erklärten drei Schüler der zweiten Gruppe gar nicht und ein weiterer erhielt nur 1,5 von 2,5 möglichen Punkten. Unter ihnen war auch Luca, dessen Definition sich stark an die von ihm im Unterricht gegebene anlehnte, ohne dass sie die dort erfolgte Ergänzung durch den Lehrer zum Ausdruck bringt: „Il consumo autonomo di una persona o stato ad esempio, l'agricoltura che soddisfa solo quella persona o quello stato" (Der autonome Verbrauch einer Person oder Staates, zum Beispiel die Landwirtschaft, die nur jene Person oder jenen Staat befriedigt). Schätzungsweise aufgrund des sprachlichen Ausdrucks, der im Verhältnis zur Klasse recht gut war, erhielt Luca für seine Definition dennoch die volle Punktzahl.

Insgesamt konnten die Lernenden im Test zehn Punkte erreichen, wobei sich das beste Ergebnis auf neun Punkte beläuft und das schlechteste auf null. ${ }^{193}$ Bevor auf weitere Einzelheiten der Testbearbeitung eingegangen wird, gibt Tabelle 2 zunächst einen Überblick über die erzielten Ergebnisse (L in der ersten Spalte steht für Lernende und $\mathrm{P}$ in der ersten und letzten für Punkte).

\begin{tabular}{|c|c|c|c|c|c|c|c|c|c|}
\hline L & $\begin{array}{c}\text { popolazione } \\
\text { non attiva }\end{array}$ & $\begin{array}{l}\text { mono- } \\
\text { coltura }\end{array}$ & $\begin{array}{l}\text { tasso di } \\
\text { crescita }\end{array}$ & $\begin{array}{l}\text { acro- } \\
\text { coro }\end{array}$ & $\begin{array}{r}\text { popolazi- } \\
\text { one } \\
\text { attiva }\end{array}$ & creolo & $\begin{array}{l}\text { auto- } \\
\text { con- } \\
\text { sumo }\end{array}$ & $\begin{array}{r}\text { alto- } \\
\text { piano }\end{array}$ & P \\
\hline a & 0 & 0 & 0 & 0 & & & & & 0 \\
\hline$b$ & & & & & 0 & 0 & 0 & 1,5 & 1,5 \\
\hline c & 0 & 0 & 2 & 0 & & & & & 2 \\
\hline d & 0 & 0 & 0 & 2,5 & & & & & 2,5 \\
\hline
\end{tabular}

193 Der chinesische Schüler mit geringen Italienischkenntnissen war bei der Testdurchführung abwesend. 


\begin{tabular}{|c|c|c|c|c|c|c|c|c|c|}
\hline L & $\begin{array}{r}\text { popolazione } \\
\text { non attiva }\end{array}$ & $\begin{array}{l}\text { mono- } \\
\text { coltura }\end{array}$ & $\begin{array}{l}\text { tasso di } \\
\text { crescita }\end{array}$ & $\begin{array}{l}\text { acro- } \\
\text { coro }\end{array}$ & $\begin{array}{r}\text { popolazi- } \\
\text { one } \\
\text { attiva }\end{array}$ & creolo & $\begin{array}{l}\text { auto- } \\
\text { con- } \\
\text { sumo }\end{array}$ & $\begin{array}{r}\text { alto- } \\
\text { piano }\end{array}$ & $\mathrm{P}$ \\
\hline e & 1 & 0 & 1,5 & & & & & & 2,5 \\
\hline$f$ & & & & & 2,5 & 0 & 0 & 0 & 2,5 \\
\hline g & 1 & 0 & 1,5 & 2 & & & & & 4,5 \\
\hline $\mathrm{h}$ & & & & & 2,5 & 0 & 0 & 2,5 & 5 \\
\hline i & & & & & 2,5 & 0 & 1,5 & 1,5 & 5,5 \\
\hline j & & & & & 2,5 & 0 & 2,5 & 1,5 & 6,5 \\
\hline $\mathrm{k}$ & 0 & 2,5 & 1,5 & 2,5 & & & & & 6,5 \\
\hline I & 2,5 & 2,5 & 0 & 2,5 & & & & & 7,5 \\
\hline $\mathrm{m}$ & 2,5 & 1,5 & 1 & 2,5 & & & & & 7,5 \\
\hline Luca & & & & & 2,5 & 2 & 2,5 & 2 & 9 \\
\hline$\varnothing n P$ & & & & & & & & & 4,5 \\
\hline
\end{tabular}

Tabelle 2: Ergebnisse eines Worterklärungstests in der Klasse der Schule Morante

Durchschnittlich erzielten die Lernenden nicht mehr als 4,5 Punkte, das heißt, niemand erhielt für alle Worterklärungen die volle Punktzahl. Insgesamt bekamen nur zwei der 14 Lernenden für ihre vier Definitionen jeweils mehr als null Punkte, während die übrigen mindestens mit einer der vier Definitionen keine Punkte erzielten. Das Wort, das insgesamt am schlechtesten definiert wurde, ist „Kreole“: Nur ein Schüler gab eine Definition und erhielt dafür zwei Punkte, die anderen bekamen null Punkte. Am zweitschlechtesten fallen die Ergebnisse für monocoltura in der ersten Gruppe und autoconsumo in der zweiten mit jeweils insgesamt 6,5 Punkten aus. Fast doppelt so viele Punkte wurden hingegen bei den am besten definierten Wörtern erzielt, nämlich zwölf für acrocoro in der ersten und 12,5 für popolazione attiva in der zweiten Gruppe. Dass das aus der geographischen Fachsprache stammende acrocoro insgesamt besser definiert wurde als sein allgemeinsprachliches und ebenfalls im Unterricht verwendetes Synonym altopiano, ist damit zu erklären, dass die SchülerInnen als Definition das Synonym altopiano nannten, während die Lernenden der anderen Gruppe größtenteils versuchten, den Begriff altopiano ohne den Rückgriff auf ein Synonym zu erläutern. Außerdem ist es möglich, dass sie sich so gut an die Wortbedeutung erinnerten, da sie in der Unterrichtsstunde, in der es eingeführt wurde, seinen merkwürdigen Klang kommentierten und eine Schülerin bemerkte, dass es sich nach einer Krankheit anhörte.

Um einen Einblick zu vermitteln, was für Definitionen die SchülerInnen formulierten, sollen nun für alle acht Wörter eine Definition, die mit null Punkten, und eine, die mit der höchsten Punktzahl bewertet wurde, sowie eine eigentümliche zusätzliche Definition für den Begriff acrocoro, die zwei Punkte einbrachte, vorgestellt werden.

popolazione non attiva

È la popolazione che non è attiva, cioè che non si distribuisce nelle zone. (Ist die Bevölkerung, die nicht aktiv ist, das heißt, die sich nicht in den Zonen verteilt.) (0 Punkte) 
Popolazione che non può lavorare per un qualsiasi motivo. (Bevölkerung, die aus verschiedenen Gründen nicht arbeiten kann.) (2.5 Punkte)

monocoltura

La coltivazione della persona che ha il terreno (es. fattoria, ranch) e non una ditta che lavora in questo campo. (Die Bewirtschaftung der Person, die das Land hat (Bsp. Bauernhof, Ranch) und nicht ein Unternehmen, das in diesem Feld arbeitet.) (0 Punkte)

Coltivano nel terreno solo un tipo di frutta. (Sie bauen auf dem Feld nur eine Sorte Frucht an.) (2,5 Punkte)

tasso di crescita

È la crescita economica di quel paese. (Ist das wirtschaftliche Wachstum jenes Landes.) (0 Punkte)

Il tasso di crescita indica l'aumento di una popolazione. (Die Wachstumsrate zeigt den Zuwachs einer Bevölkerung an.) (2 Punkte)

acrocoro

unterlassene Definitionen (0 Punkte)

Altopiano, un terreno che supera il livello del mare. (Hochebene, ein Gebiet, das über dem Meeresspiegel liegt.) (2,5 Punkte)

Acropolo se non mi sbaglio è una definizione per l'altopiano o come dire un piccolo aggettivo. (Acropolo [falsch geschriebenes/fiktives Wort für acrocoro (Hochplateau)] wenn ich mich nicht irre, ist eine Definition für Hochebene oder wie zu sagen ein kleines Adjektiv.) (2 Punkte)

popolazione attiva

unterlassene Definition (0 Punkte)

Popolazione attiva vol [sic] dire le persone che possono lavorare per esempio in italia [sic] le persone che possono lavorare è da secidi [sic] anni ai sesanta [sic] anni. (Erwerbstätige Bevölkerung heißt die Personen, die arbeiten können zum Beispiel in Italien die Personen, die arbeiten können, ist von 16 bis 60 Jahren.) (2,5 Punkte)

\section{Creolo (einzige Definition)}

Figlio di madre straniera e padre nativo di quel paese ad esempio Simón Bolívar. (Sohn einer ausländischen Mutter und eines eingeborenen Vaters aus dem Land zum Beispiel Simón Bolívar.) (2 Punkte)

autoconsumo

Autoconsumo vol [sic] dire le cose che consumi per es. (Eigenverbrauch heißt die Sachen, die du verbrauchst z.B.) (1,5 Punkte)

Consumo per se stesso. (Verbrauch für sich selbst.) (2,5 Punkte)

altopiano

L'altopiano è un rilievo, una montagna pianeggiante. (Die Hochebene ist eine Erhebung, ein flacher Berg.) (1,5 Punkte)

L'altopiano è il sinonimo di Acrocoro [sic]. (Die Hochebene ist das Synonym von Hochplateau.) (2,5 Punkte)

Abbildung 23: Definitionsbeispiele aus dem Worterklärungstest an der Schule Morante 
Diese Beispiele einiger Schülerdefinitionen verdeutlichen, dass die Lehrkraft bei der Bewertung weder die orthographische und grammatikalische Korrektheit (s. z.B. die Definition für popolazione attiva) noch den sprachlichen Ausdruck oder die Verwendung eines möglichst bildungssprachlichen Registers berücksichtigte. Stattdessen standen inhaltliche Gesichtspunkte klar im Vordergrund. Die angewandte Bewertungspraxis bestand aus der Punktvergabe pro gegebener Definition und einer daraus resultierenden Gesamtbewertung in Form eines maximalen Ergebnisses mit einem doppelten Pluszeichen und einem minimalen mit einem doppelten Minuszeichen. Auch wenn die Lehrkraft Aspekte der sprachlichen Gestaltung wie die Rechtschreibung oder den Ausdruck bei der Bewertung bewusst nicht berücksichtigte, wurden sie auch nicht verbessert oder unterstrichen. Die Klasse bekam die Tests einige wenige Minuten zur Ansicht ausgehändigt, bevor sie wieder eingesammelt wurden. Dies entspricht dem üblichen Vorgehen bei der Rückgabe schriftlicher Tests und Klassenarbeiten: Sie dürfen das Schulgebäude nicht verlassen. Ferner wurden in den beobachteten Klassen grundsätzlich keine schriftlichen Berichtigungen angefertigt, sondern erfolgte nur eine Besprechung der Ergebnisse vor der kurzen Testaushändigung, bei der in der Regel die Aufgaben, die vermehrt falsch bearbeitet worden waren, vorgestellt und durch die Lehrkraft oder von ihr gemeinsam mit den Schülern gelöst wurden.

Bei diesem Wortschatztest fällt auf, dass die Definitionsversuche der Lernenden auf einem recht niedrigen sprachlichen Niveau erfolgten und sehr kurz gehalten waren. Allerdings bedienten sich die SchülerInnen zum Teil typischer Definierungsstrategien wie der Nennung eines synonymen Begriffs oder der ergänzenden Erläuterung anhand eines Beispiels. Auch wenn Herr Tigre nach eigenen Angaben in seinen Fächern Italienisch, Erdkunde und Geschichte noch nie verlangt hatte, ein Wort schriftlich zu definieren, hatten die Schüler und Schülerinnen ein gewisses $\mathrm{Ma} ß$ an Übung im mündlichen Erläutern und im Definieren, was ihnen bei der Bewältigung der Aufgabe nützte. Eine sprachund lernförderliche Einführung in die Unterschiede des schriftlichen und mündlichen Definierens war ihnen nicht erteilt worden, und auch nach der Testrückgabe wurden keine Musterbeispiele schriftlicher Definitionen der acht Wörter entwickelt, geschweige denn von allen schriftlich festgehalten.

$\mathrm{Da}$ in den Unterrichtsgesprächen bisweilen deutlich wurde, dass Lernende einzelne Begriffe und Konzepte, die im Erdkundeunterricht der Mittelschule jedes Jahr mehrmals wiederholt wurden, nicht behielten, räumte der Lehrer selbst ein, dass es wahrscheinlich lernförderlich wäre, bestimmte fachinhaltliche Aspekte schriftlich festhalten zu lassen. Doch gleichzeitig erklärte er, dass es äußerst schwierig sei, eine einmal eingeschlagene Unterrichtspraxis, an die sich die einzelnen Akteure gewöhnt haben, umzugestalten. In seinem Unterricht war es den Schülern in den sogenannten mündlichen Fächern Geschichte und Erdkunde selbst überlassen, ob und wie sie ein Heft führten, weshalb viele in der Klasse ein gemeinsames Heft für die beiden Fächer anlegten. 
W.o. ist schon darauf hingewiesen worden, dass in den fünf Klassen sehr wenig geschrieben wurde. Nur eine Lehrkraft hielt die SchülerInnen dazu an, bestimmte Begriffe in einem Glossar festzuhalten, allerdings kontrollierte sie es nicht. Als ich aus Interesse an den so festgehaltenen Wörtern vier oder fünf Lernende fragte, ob ich das Glossar einmal sehen dürfe, stellte sich heraus, dass zumindest die von mir Befragten gar kein Glossar angelegt hatten.

Lediglich in der Klasse III A an der Schule Pestalozzi bestand die Lehrkraft Frau Rea von Zeit zu Zeit auf der schriftlichen Formulierung von Lerninhalten. So wurde beispielsweise beobachtet, wie sie ihre Schüler zu Stundenbeginn bei der Einführung in einen neuen Kontinent informierte, dass als Hausaufgabe eine schriftliche Vorstellung des Kontinents anzufertigen sei. Das hatte neben einer sprachförderlichen Bedeutung - die Arbeit an allen vier rezeptiven und produktiven Sprachfähigkeiten spielt im allgemeinen Sprachausbau eine große Rolle - auch den didaktischen Vorteil, dass die Klasse motivierter war, dem vom Lehrervortrag bestimmten Unterrichtsgeschehen aufmerksam zu folgen. In dieser Klasse sollten die SchülerInnen nicht nur gelegentlich Inhalte schriftlich fixieren, sondern bekamen von der Lehrkraft auch hilfreiche Anweisungen, wie sie dabei vorzugehen hatten. In der oben vorgestellten Unterrichtseinheit besprach die Lehrerin auch, wie die schriftliche Präsentation des Kontinents inhaltlich strukturiert sein sollte. Darüber hinaus konnte in dieser Klasse beobachtet werden, dass sich in Momenten der Abfragung oder des Unterrichtsgesprächs, in denen Einzelne Schwierigkeiten bei der Erklärung bestimmter Begriffe zeigten, alle aufgefordert wurden, diese Begriffe in Eigenarbeit und schriftlich zu erläutern. Hierbei diktierte die Lehrkraft manchmal den Beginn einer Definition, was als Orientierungshilfe für die Verwendung eines bildungssprachlichen Registers bei der schriftlichen Begriffserklärung angesehen werden kann.

Weiter wurde, die Klasse an der Schule Morante und die III A an der Pestalozzi vergleichend, beobachtet, dass die Lernenden der Schule Morante nicht auf die Lehrerfrage reagierten, ob sie beim Lesen der Lehrbuchtexte auf unbekannte Wörter gestoßen waren, während Lernende der Klasse III A die Lehrkraft aus Eigeninitiative nach der Bedeutung bestimmter Wörter fragten. Vermutlich ist das mit dem unterschiedlichen Führungsstil der Lehrkräfte zu erklären. So erfolgten in beiden Klassen regelmäßig Abfragungen, bei denen Frau Rea strenger als ihre vier beobachteten Kollegen bewertete und als einzige auch sehr schlechte Noten verteilte. Die Prüfung manchmal sogar abbrechend, verwies sie die Lernenden auf ihren Platz, wenn sie bestimmte Unterrichtsgegenstände nicht erklären konnten. So erweckte es den Eindruck, dass ihre Lernenden intensiver darum bemüht waren, beim häuslichen Aufarbeiten der Lehrbuchtexte zu überprüfen, ob sie sie vollständig verstanden hatten, weshalb sie sich bei etwaigen Zweifeln öfter fragend an die Lehrkraft wandten. 
Bevor sich Kapitel 10 mit weiteren schriftlichen Produktionen der SchülerInnen (Texte zur Vorstellung geographischer Regionen in zwei Klassen) befasst, werden im letzten Unterkapitel Aspekte des sprachbezogenen Feedbacks der Lehrkräfte vorgestellt, von denen einige schon an verschiedenen Stellen des Kapitels geschildert wurden. Hier wird auch auf Gegenstände der Wortschatzarbeit wie die Angemessenheit und Verbesserung des Ausdrucks und die Vermittlung von kollokationalem Wissen eingegangen.

\subsubsection{Sprachbezogenes Feedback der beobachteten Lehrpersonen}

Alle fünf Lehrkräfte reagierten im Zeitraum der Unterrichtsbeobachtungen auf sprachliche Kriterien der Schüleräußerungen. Sowohl in den Abfragungen als auch im Unterrichtsgespräch gaben sie den Lernenden zu verstehen, dass ihre Darstellung erweitert werden müsse, um verständlicher und/oder vollständiger zu sein. Die Arbeit am sprachlichen Ausdrucksvermögen ging also über die Verwendung lexikalischer Gestaltungsmittel und die Wahl des Sprachregisters hinaus.

Um die Lernenden zu weiteren Ausführungen zu bewegen, bedienten sie sich eines verbalen und nonverbalen Feedbacks. Letzteres äußerte sich in der Regel in einem zustimmenden Kopfnicken und dem Ausbleiben einer weiteren Frage, bzw. war das Lehrerfeedback auf ein sprachliches Minimum reduziert, indem es nur aus einem „Eh, eh“ bestand (s. z.B. Transkriptauszug 3). Die hierarchisch gestaltete Rollenverteilung im institutionellen Rahmen des Unterrichtsgesprächs bestimmt das Handeln der beteiligten Akteure. Die Lehrkraft leitet die Unterrichtsgespräche, indem sie das Rederecht, das ihr jederzeit zusteht und nach einem Schülerbeitrag automatisch an sie zurückgeht, direkt an einzelne Lernende übergibt oder mit ungerichteten Fragen allen die Möglichkeit einräumt, einen Gesprächsbeitrag zu leisten. In diesem Fall erfolgte der Sprecherwechsel teilweise, ohne dass „Redewillige“ ihren Wunsch, etwas zu äußern, zuvor durch ein Handzeichen ankündigen und auf die Zuteilung des Rederechts warten mussten.

Der klassische Gesprächsverlauf in der Unterrichtsdiskussion ist dabei durch die Drei-Turn-Sequenz Lehrerfrage, Schülerantwort, Korrektur/Lehrerfeedback festgelegt, so dass ein Ausbleiben des verbalen Lehrerkommentars ausreichte, um den Schülerinnen und Schülern zu signalisieren, dass ihre Beiträge vervollständigt werden sollten. In anderen Fällen wurden sie auch etwas direkter dazu aufgefordert, indem die Lehrkraft das Feedback darauf beschränkte, die Schüleräußerung zu wiederholen und lediglich durch ein „Was noch?" zu ergänzen, wenn es danach still blieb. Darüber hinaus erfolgten konkretere Aufforderungen, ein Konzept weiter auszuführen, wie es im Auszug aus einer Abfragung in der Klasse III B der Pestalozzi in Abbildung 24 ersichtlich wird. 
Auszug einer Mitschrift (14), Abfragung Frau Cese (FrC), Linda (Lin) - Aufforderung zum Konzeptausbau

FrC: E il clima? (Und das Klima?)

Lin: Ci sono i monsoni che sono venti periodici. [Pause] (Es gibt die Monsune, die periodische Winde sind. [Pause])

FrC: Da dove arrivano? (Woher kommen sie?)

Lin: Ci sono venti che spirano dal mare alla terra. (Es gibt Winde, die vom Meer zum Land wehen.)

FrC: Cosa portano? (Was bringen sie?)

Lin: Umidità. E quando vanno dalla terra al mare, fa una stagione secca. [Fährt fort, über Entwaldungen und Tsunamis zu reden.] (Feuchtigkeit. Und wenn sie vom Land zum Meer wehen, macht es eine trockene Jahreszeit. [Fährt fort, über Entwaldungen und Tsunamis zu reden.])

FrC: Quando si crea uno tsunami? (Wann entwickelt sich ein Tsunami?)

Lin: Quando c'è un terremoto dentro al mare. (Wenn es im Meer drinnen ein Erdbeben gibt.)

FrC: Diciamo meglio. (Sagen wir es besser.)

Lin: Quando l'epicentro di un terremoto sta sotto il mare [...]. (Wenn das Epizentrum eines Erdbebens unter dem Meer ist [...].)

Abbildung 24: Abfragung Frau Cese, Aufforderung zum genaueren Erklären

Aus dem kurzen Auszug geht hervor, dass Linda die Antworten auf die Lehrerfragen wusste und nannte, sich dabei aber, wie in allen Klassen fast ausnahmslos beobachtet, recht kurzfasste. Nachdem Prof.ssa Cese Linda erst bei einer gründlicheren Erklärung der Monsunwinde unterstützte, indem sie eine weitere Frage zu diesem Thema an die Schülerin richtete, um ihr beim Ausbau der Erläuterung zu helfen, forderte sie später nur: „Diciamo meglio“ (Sagen wir es besser). Mit dieser Äußerung erreichte sie, dass die Schülerin ihre mündliche Darstellung der Tsunamis sowohl sprachlich als auch inhaltich verbesserte. Sie gab genauere Informationen, benutzte Fachwörter wie „Epizentrum“ (Ts geofis.) und formulierte insgesamt einen längeren Beitrag, so dass die Lehrkraft mit der Erläuterung zufrieden war und die Schülerin nach einer Weile mit einem knappen Lob und dem Hinweis unterbrach, die Abfragung mit einem anderen Prüfling weiterzuführen („Bene, fermati, sentiamo Roberta." (Gut, das reicht. Hören wir Roberta.)).

Oft blieb ein explizites Feedback aus. Stattdessen forderten die Lehrpersonen ihre Gesprächspartner entweder dazu auf, einen verwendeten Fachausdruck zu erklären oder für ein vorgestelltes Konzept den entsprechenden Fachausdruck zu ergänzen. Auch diese Lehrerreaktion signalisierte den Lernenden, dass ihre Gesprächsbeiträge akzeptiert wurden und im weiteren Verlauf zu vervollständigen waren.

Dass sich die fünf Lehrkräfte ihrer spracherzieherischen Funktion bewusst waren, zeigte sich u.a. auch daran, dass sie wiederholt auf registerbezogene und stilistische Unangemessenheiten reagierten und dadurch versuchten, Einfluss auf das mündliche Ausdrucksvermögen der Lernenden zu nehmen. Dies geschah zum Beispiel, indem sie Äußerungen mit den Sätzen „Parliamo in 
italiano!“ (Sprechen wir Italienisch!) oder „Parliamo bene!“ (Sprechen wir gut!) kommentierten. Solche Formulierungen im Imperativ der ersten Person Plural stellen eine indirektere Kritik am Sprachverhalten dar und sind dadurch auf der einen Seite für die Betroffenen gesichtswahrender. Auf der anderen Seite wird dem Rest der Klasse damit signalisiert, dass die Ermahnung, sich angemessen auszudrücken, auch an sie gerichtet ist. Gleichsam verwiesen die Lehrkräfte von Zeit zu Zeit auf die bevorstehende Abschlussprüfung und die Wichtigkeit, dort besonders auf den sprachlichen Ausdruck zu achten: ,Agli esami non possiamo parlare cosi" (In der Abschlussprüfung können wir so nicht sprechen). Ferner wurde beobachtet, wie die Lehrkräfte darauf aufmerksam machten, dass es sich bei sprachlichen Schwächen nicht immer nur um Schwierigkeiten mit dem geographischen Fachwortschatz handelte, sondern diese allgemeiner im Bereich der italienischen Sprache lagen (s. 9.2.4).

Besonders vehement begegneten sie typisch jugendlichen und stark umgangssprachlichen Phänomenen im Sprachgebrauch wie der intensiven Verwendung der Ausdrücke cioè (das heißt, also) und tipo (Art, Sorte, Typ). Bei cioè handelt es sich um ein Wort, das seine Bedeutung oft verliert und stattdessen als Füllwort gebraucht wird, um beim Nachdenken Zeit zu schinden, ohne durch Stillschweigen aufzufallen:

Transkriptauszug 9, Textarbeit zur Unterernährung, Frau Mancuso (FrM), Ava - cioè

FrM: Ava, che vuol dire quello che abbiamo letto? (Ava, was bedeutet das, was wir gelesen haben?)

Ava: Che, cioè, eh il mondo è male, affamato, no? E proprio perché, cioè, le risorse ci sono ma sono maldistribuite perché ad esempio nei paesi più sviluppati sono più cibo, quando invece non occorrono così tanto. Mentre tipo Africa che è un paese povero, (Dass, also, eh, die Welt ist schlecht, hungrig, nicht? Es ist tatsächlich, weil, also, die Ressourcen sind da, aber sie sind schlecht verteilt, weil zum Beispiel in den entwickelteren Ländern mehr Essen sind [sic], wenn nicht so viel benötigt werden. Während tipo [zum Beispiel] Afrika, das ein armes Land ist)

FrM [unterbricht sie]: Date le soluzioni però! Queste tre righe danno tre soluzioni. Dimmi la prima, Lorenzo la seconda e Ania la terza. Prima soluzione! (Gebt mir aber die Lösungen! Diese drei Zeilen geben drei Lösungen. Nenn mir die erste, Lorenzo die zweite und Ania die dritte.)

Ava: Che bisognerebbe sviluppare l'agricoltura. (Dass man die Landwirtschaft entwickeln müsste.)

FrM: Nelle zone? (In den Gebieten?)*

Ava: Eh, cioè [Pause, Stille] (Eh, also [Pause, Stille]).

FrM: Nelle zone più? (In den Gebieten mehr?)

Ava: Povere. (Armen.)

Abbildung 25: Textarbeit Klasse Vespucci, cioè

*Bei diesem und dem folgenden Redebeitrag der Lehrerin handelt es sich um eine Designedly Incomplete Utterance, welche die Schülerin vervollständigen soll. Für ihre Wiedergabe ist anstelle des korrekten in den ärmeren Gebieten eine wörtliche Übersetzung ins Deutsche gewählt worden (im Italienischen stehen Adjektive, wie hier, auch nach ihren Bezugswörtern und wird der Komparativ mit dem Adverb più und dem Positiv gebildet). 
Das Wort tipo, mit dem ausgedrückt wird, dass ein Gegenstand oder Sachverhalt für seine Klasse kennzeichnend, mustergültig ist, und das Objekte und Personen konzeptionell nach ihren wesentlichen gemeinsamen Merkmalen gruppiert, wird inzwischen gerade von jungen Leuten im weniger formalen und mündlichen Sprachgebrauch synonym zu „Beispiel/par excellence“ verwendet, um eine oft beschreibende, beispielhafte Ergänzung einzuleiten oder um das, was im Folgenden gesagt wird, einzuschränken, da einem keine bessere Beschreibung oder kein treffender Ausdruck einfällt.

Transkriptauszug 10, Abfragung Herr Tigre (HrT), Giorgia (Gio) - tipo (I)

HrT: Hm? Chi lavora, chi no, ok. Poi? [Stille] Poi c'è tutto l'ambito relativo alla cultura. Che cos'è? É tutto l'ambito relativo anche alle etnie. Perché in un continente cosi vasto le persone hanno tutte le stesse fattezze? No. Ci sono, come si chiamano persone che hanno aspetti differenti? (Hm? Wer arbeitet und wer nicht, ok? Dann? [Stille] Dann gibt es den ganzen Bereich bezüglich der Kultur. Was ist das? Es ist der ganze Bereich auch bezüglich der Ethnien. Weil in einem so großen Kontinent die Personen alle die gleichen Gesichtszüge haben? Nein, es gibt, wie heißen die Personen, die ein verschiedenes Aussehen haben?)

Gio: Oh dio, ce ne stanno due, sa. Ehm, $i$ [Stille]. (Oh Gott, es gibt dort zwei, wissen Sie. Ehm, die [Stille].

HrT: Allora, come si chiamano le, i vari e differenti gruppi di cultura ecc.? (Also wie heißen die, die unterschiedlichen und verschiedenen Kulturgruppen etc.?)

Gio: Ci stanno $i$ [Stille]. (Es gibt die [Stille].)

HrT: Sono le etnie, no? Che cos'è un'etnia? (Das sind die Ethnien, nicht? Was ist eine Ethnie?

Gio: Sono, tipo, delle persone, che hanno lo stesso aspetto. (Das sind, tipo, Personen, die dasselbe Aussehen haben.)

Abbildung 26: Transkriptauszug Klasse Morante, tipo (I)

Im nächsten Auszug aus derselben Abfragung taucht das Wort gleich mehrmals auf. U.a. wird es auch von der Lehrkraft beim Wiederholen der Schülerantwort verwendet, wahrscheinlich um die Schülerin humorvoll auf ihre unpassende Ausdrucksweise aufmerksam zu machen:

Transkriptauszug 11, Abfragung Herr Tigre (HrT), Giorgia (Gio) - tipo (II)

HrT: [...] No. Eeeh, senti, fermiamoci soltanto a un aspetto, se no, non usciamo. La religione. Allora, abbiamo detto che la regione, dove è più sviluppata, dove è più sviluppato, eehm, l'islamismo, eehm, (Nein. Eeh, hör mal, konzentrieren wir uns nur auf einen Aspekt, sonst kommen wir da nicht raus. Die Religon. Also, wir haben gesagt, dass die Religion, wo sie entwickelter ist, wo der, eehm, Islamismus entwickelter ist, eehm, )

Gio [unterbricht inn]: E poi che (Und dann, dass)

$\mathrm{HrT}$ [ergreift wieder das Wort]: Oltre a questa zona li (Abgesehen von dieser Gegend dort)

Gio [unterbricht ihn erneut]: Aspetta [sic] un attimo. Che quella parte là sono, tipo, i più popolati del mondo, di tutto il pianeta. Cioè (Warte [sic] einen Moment. Dass dieser Teil dort, sie sind, tipo, die bevölkerungsreichsten der Welt, des ganzen Planeten. Das heißt) 
HrT [unterbricht sie]: Aah. Però qui stiamo passando su un altro discorso. Qui stiamo parlando di demo? (Aah. Aber hier sind wir dabei, zu einem anderen Diskurs überzugehen. Hier sprechen wir gerade von Demo?)

Gio: Grafia. (Graphie.)

HrT: Di demografia. Cioè, che volevi dire? Quale parte là? (Über Demographie. Also, was wolltest du sagen? Welcher Teil dort?)

Gio: Tipo, I'India. (Tipo, Indien.)

HrT: Tipo, l'I, quali sono le parti più densamente popolate del continente? (Tipo, I, was sind die am stärksten besiedelten Teile des Kontinents?)

Gio: Eeh, vicino al mare. (Eeh, in der Nähe vom Meer.)

$\mathrm{HrT}: \mathrm{Hm}$ ? (Hm?)

Gio: Vicino alle coste. (In der Nähe der Küsten.)

HrT: Mhm. Quindi qui [Pause, zeigt Gebiete auf der Wandkarte]. Quindi qui. (Mhm. Also hier [Pause, zeigt Gebiete auf der Wandkarte]. Also hier.)

Gio: Là, ci sta il deserto. (Da ist die Wüste.)

$\mathrm{HrT}$ : Allora, non vicino a tutte le coste. (Also nicht in der Nähe aller Küsten.)

Gio: No, tutte no. (Nein, nicht alle.)

HrT Perché, per esempio nemmeno qui, però anche queste so' coste. Vicino a quali coste? (Denn zum Beispiel auch nicht hier, aber auch dies sind Küsten. In der Nähe welcher Küsten?)

Gio: Tipo, Oceano Pacifico. (Tipo, Pazifischer Ozean.)

HrT: Tipo, per esempio c'è una carta tematica là sotto che puoi tranquillamente leggere, eh. Guarda quante sorprese. (Tipo, zum Beispiel gibt es eine thematische Karte da unten, die du ruhig lesen kannst, eh. Schau, wie viele Überraschungen.)

Abbildung 27: Transkriptauszug Abfragung, Klasse an der Morante, tipo (II)

In ähnlichen Situationen in den anderen Klassen fiel die Reaktion der Lehrkräfte auf derartige Züge des mündlichen Ausdrucksvermögens nicht so entspannt aus, wie oben in den Abbildungen 26 und 27 wiedergegeben. Nachdem Prof.ssa Cese z.B. einen abgefragten Schüler dazu aufgefordert hatte, das Wort nicht so oft zu verwenden, hob sie bei einem weiteren Gebrauch durch eine andere Schülerin in derselben Unterrichtsstunde entschieden die Stimme und entgegnete Folgendes: „Ma io voglio sapere perché nel vostro linguaggio c'è tutto questo "tipo". Ma chi è questo tipo? Tipo è un intercalare giovanilistico che a scuola non va proprio usato!" (Aber ich will wissen, warum es in eurer Sprache dieses ganze tipo gibt. Aber wer ist denn dieser tipo/Typ? Tipo ist ein gezwungen jugendliches Füllwort, das in der Schule absolut nicht zu benutzen ist!) Zwar schlug auch sie einen scherzhaften Ton an und fuhr damit fort („Tipo, go out!"), dennoch merkte man, dass es ihr mit der Kritik sehr ernst war, was auch an der emphatischen Betonung und langsameren Aussprache der Wörter „absolut nicht zu nutzten ist" deutlich wurde.

Prof.ssa Rea ging noch weiter und kritisierte nicht nur sprachliche Ungenauigkeiten im Ausdruck und den übertriebenen Gebrauch des Wortes tipo, sondern auch die Eröffnung eines Schülerbeitrages mit dem Wort „vielleicht“:

Auszug aus Mitschrift (15), Unterrichtsgespräch Frau Rea (FrR), Tommaso (Tom), Ludovico (Lud) - mündlicher Ausdruck (forse)

Tom: Marittimità e continentalità. (Maritimität und Kontinentalität.) 
FrR: Questi fattori, come influenzano il clima? (Diese Faktoren, wie beeinflussen sie das Klima?)

Lud: Forse (Vielleicht)

FrR [unterbricht ihn]: Forse? Ancora questo forse! Ė un alibi! [Stille]. O sì o no, quindi? (Vielleicht? Immer noch dieses „vielleicht“! Das ist ein Alibi! [Stille] Entweder ja oder nein, also?)

Lud: Allora niente, mi ritiro. (Also nichts, ich mache einen Rückzieher.)

Abbildung 28: Gesprächsauszug III A, Pestalozzi, mündliches Ausdrucksvermögen - forse

Dieses Kritikverhalten an der mündlichen Darstellungsweise wurde in einer ähnlichen Situation in abgeschwächter Form auch in der Klasse III B der Schule Pestalozzi beobachtet, wo ein Schüler die Antwort auf eine an ihn gerichtete Lehrerfrage mit dem Wort erano (es waren) begann. Weiter oben wurde bereits darauf hingewiesen, dass Lernende den Imperfekt bzw. den Konjunktiv verwendeten, um sich von ihren Aussagen zu distanzieren, wenn sie sich nicht sicher waren, ob das Gesagte stimmte, oder weil sie sich nicht genau erinnerten. Im nächsten Beispiel aus einem Unterrichtsgespräch (Abb. 29) unterbricht die Lehrkraft den befragten Schüler, Vincenzo, sofort und weist darauf hin, dass sie ,es immer noch sind“, um ihn so indirekt auf die Unangemessenheit der verwendeten Zeitform aufmerksam zu machen, bzw. darauf, dass man so nicht zu verstehen geben sollte, dass man sich an etwas nicht erinnert.

Transkriptauszug 12, Unterrichtsgespräch Herr Tigre (HrT), Vincenzo (Vin), Letizia (Let), Lisa (Lis) - erano

HrT: Guardiamo da questa parte qua. Abbiamo studiato l'altra volta i monsoni. Che cosa sono i monsoni? [Pause, Stille] Monsoni. (Schauen wir in diese Richtung hier. Wir haben neulich die Monsune gelernt. Was sind die Monsune? [Pause, Stille] Monsune.)

Vin: Erano (Es waren)

HrT (unterbricht inn): Sono ancora. [Pause.] Ne abbiamo pure parlato più volte. (Darüber haben wir auch mehrmals gesprochen.)

Vin: Venti (Winde)

HrT: Venti, sono dei venti? Ne abbiamo anche aggiunto un altro aggettivo. (Winde, es sind [was für] Winde? Wir haben auch ein anderes Adjektiv beigefügt.)

Vin: Freddi. (Kalte.)

HrT: No. [Pause, Stille] (Nein. [Pause])

Let: Periodici. (Periodische)

HrT: Periodici. [Pause, Stille] Che vuol dire che sono dei venti periodici? (Periodische.

[Pause, Stille]. Was bedeutet, dass es periodische Winde sind?)

Let: $\dot{E}$ che, che non ci stanno sempre. (Es ist, dass, dass sie nicht immer da sind.)

HrT: Che? (Was?)

Let: Che non ci stanno sempre. (Dass sie nicht immer da sind.)

HrT: Che non ci stanno sempre, ma? Ma? Ma ci stanno? (Dass sie nicht immer da sind, aber? Aber? Aber sie sind dort?)

Let: In vari periodi. (In verschiedenen Perioden.)

HrT: Con una certa regolarità, ok? Bene. E chiaro, questo? Eh, ci siamo, Michela? Poi abbiamo distinto questi monsoni in due tipi. Abbiamo detto che esistono i monsoni di mare e i monsoni di terra, ok? Quindi monsoni sono dei venti periodici che per, diciamo, sei mesi soffiano dal mare verso la terra, e per sei mesi soffiano dalla terra 
verso il mare. Questa ciclicità, il fatto che soffiano una volta dal mare verso la terra, una volta dalla terra verso il mare, ha delle conseguenze? (Mit einer gewissen Regelmäßigkeit, ok? Gut. Ist das klar? Eh, haben wir das, Michela? Dann haben wir diese Monsune in zwei Sorten unterschieden. Wir haben gesagt, dass es die Meeresmonsune und Landmonsune gibt, ok? Also sind Monsune periodische Winde, die, sagen wir, sechs Monate vom Meer in Richtung Land wehen, und sechs Monate lang wehen sie vom Land in Richtung Meer. Diese Zyklizität, die Tatsache, dass sie einmal vom Meer in Richtung Land wehen, einmal vom Land zum Meer, hat sie Konsequenzen?)

Lis: Non ho capito, professore. (Ich habe nicht verstanden, Lehrer.)

Abbildung 29: Transkriptauszug Unterrichtsgespräch Herr Tigre, indirekt geäußerte Wissenslücke

Nach der sofortigen Unterbrechung mit der Kritik am sprachlichen Ausdruck machte Herr Tigre eine Pause, um Vincenzo zu ermöglichen, auf seine Frage zu antworten. Er wartete also ab, ob der Schüler die Antwort wusste und gab ihm noch mehr Zeit. Denn er wies ihn darauf hin, dass sie dieses Thema schon mehrmals besprochen hatten und forderte nicht einfach jemand anderen auf, die Frage zu beantworten, oder übernahm es selbst. So formulierte Vincenzo schließlich eine aus einem Wort bestehende Äußerung, die korrekt, aber nicht ausschöpfend war, weshalb Prof. Tigre als Tipp zur Ergänzung gab, dass sie ein bestimmtes Adjektiv in Bezug auf diese Winde verwendet hatten. Im weiteren Verlauf zeigte sich, dass Vincenzo in diesem Unterrichtsgespräch nicht der einzige Schüler war, der einsilbig antwortete, so dass davon ausgegangen werden kann, dass niemand in der Lage oder bereit war, die Monsunwinde zusammenhängend und vollständig vorzustellen, weshalb der Lehrer es dann selbst übernahm.

Abschließend ist im Zusammenhang mit diesen vorgestellten Merkmalen der Schülersprache, die von den Lehrkräften im Unterricht thematisiert wurden, auch der Umstand zu betrachten, dass Frau Rea regelmäßig die Bedeutung einer präzisen Ausdrucksweise sowohl im Schriftlichen als auch im Mündlichen betonte, wie es etwa aus der Besprechung einer schriftlichen Hausaufgabe hervorgeht. Sie ist auszugsweise in Abbildung 31 wiedergegeben.

Auszug aus Mitschrift (16), Hausaufgabenbesprechung, Frau Rea (FrR), Camilla (Cam), Ines (Ine) - Betonung des präzisen Ausdrucks

FrR: La Rift Valley? (Rift Valley?)

Ine: Una falda nella terra terrestre. [Pause, Stille] (Ein Hang in der Erderde. [Pause, Stille])

Cam: Una ricca frattura nella crosta terreste. (Ein reichlicher Bruch in der Erdkruste.)

FrR: La parola, il termine preciso, giusto sono importanti. La descrizione deve essere precisa perché non siamo a letteratura, siamo a geografia, anzi siamo a geologia. (Das Wort, der präzise, der richtige Begriff sind wichtig. Die Beschreibung muss präzise sein, denn wir sind nicht in Literatur [Teil des Italienischunterrichts], sondern in Erdkunde, besser noch, wir sind in Geologie.)

Abbildung 30: Hausaufgabenbesprechung Frau Rea, III A, Pestalozzi - Betonung der Wichtigkeit, sich präzise auszudrücken 
Dadurch dass Frau Rea nicht verbal auf Ines Äußerung reagierte, sondern nur mit einem Schweigen, wusste die Klasse, dass an dem Schülerbeitrag etwas zu verbessern war. Während Ines sich nicht korrigierte, unternahm ihre Mitschülerin Camilla einen neuen Versuch, das Rift Valley zu beschreiben, womit die Lehrerin einverstanden war. Auch das verbalisierte sie nicht, sondern hob direkt die Wichtigkeit einer genauen Ausdrucksweise hervor.

Generell kann anhand einer Bilanz der Unterrichtsbeobachtungen in allen Klassen festgehalten werden, dass sie die sprachlichen Fähigkeiten strenger bewertete und öfter kommentierte als die anderen vier Lehrpersonen. Wenn ihre Fragen nicht zufriedenstellend beantwortet wurden, obwohl die Lerninhalte bereits im Unterricht behandelt worden waren, beschränkte sich ihr Feedback nicht, wie es bei den anderen vier Lehrpersonen beobachtet wurde, auf ein „falsch“ bzw. ,richtig“ oder das Elizitieren von zusätzlichen Informationen, sondern äußerte sie ihre Unzufriedenheit über die Schülerbeiträge sehr deutlich.

Auszug aus Mitschrift (17), Abfragung Ostasien, Frau Rea (FrR), Ludovico (Lud) - negatives Feedback

FrR: Poi ci sono due zone? (Dann gibt es zwei Zonen?)*

Lud: Desertiche. (Wüsten[zonen].)

FrR: Sono deserti? (Das sind Wüsten?)

Lud: Freddi. (Kalte.)

FrR: Perché sono deserti? (Warum sind es Wüsten?)

Lud: Perché non ci sono forme di vita. (Weil es keine Lebensformen gibt.)

FrR: Perché non piove! Ancora con queste risposte! (Weil es nicht regnet! Noch immer mit diesen Antworten!)

Abbildung 31: Abfragung Frau Rea, III A, Pestalozzi, Unzufriedenheit mit Schüleräußerung (I)

*In diesem und im nächsten Redebeitrag der Lehrperson ist im Deutschen die Wortstellung des Italienischen beibehalten worden, da es sich um zu vervollständigende Designedly Incomplete Utterances handelt, die sonst im Deutschen nicht wiedergegeben werden können.

Frau Rea schien von ihren Schülerinnen und Schülern, die insgesamt bessere sprachliche Fähigkeiten zeigten als die Lernenden in den anderen Klassen, mehr zu erwarten. ${ }^{194}$

Welchen Einfluss Erwartungen der Lehrpersonen hinsichtlich der Schülerleistungen haben, ist in den 1970er und 1980er Jahren intensiv untersucht worden und auch zuletzt wieder ins Interesse gerückt (vgl. Hattie 2013, S. 146). Zwar sind diese Einflüsse nur moderat, dennoch legt die von Rosenthal und

194 Beispiele hierfür sind neben den in den anderen Unterkapiteln beschriebenen (s. v.a. Wortschatzarbeit 9.2.4) ihre Korrektur der Schüleräußerung „Il settore primario è avanzato.“ (Der Primärsektor ist fortgeschritten.) mit „Si dice meccanizzato.“ (Man sagt mechanisiert.) und ihr Kommentar bei der Rückgabe eines Erdkundetests: „Non si dice della popolazione che è alta, ma ,la popolazione è numerosa' (Man sagt nicht, dass die Bevölkerung hoch ist, sondern , die Bevölkerung ist zahlreich"). 
Rubin (1978) vorgenommene Zusammenfassung der Ergebnisse aus 345 Experimenten die Schlussfolgerung nahe, ,dass es wahrscheinlich ist, dass Lernende die von den Lehrpersonen, erwarteten " Ergebnisse erzielen, ungeachtet des Wahrheitsgehalts dieser Erwartungen" (Hattie 2013, S. 146). Hierbei ist durch neuere Forschungen (Weinstein 2002) nachgewissen worden, dass Unterschiede in den Lernleistungen zwischen Klassen bestehen, in denen die Lehrpersonen eine höhere Erwartungshaltung gegenüber dem Lernerfolg bestimmter SchülerInnen haben, und solchen, in denen sie davon ausgehen, dass sich jeder Schüler verbessern kann und alle gefördert werden (Hattie 2013, S. 148):

Es gibt Unterschiede im Klassenzimmer, in denen [sic] Lehrpersonen Talente heraussuchen, um ihnen einen gesonderten Bildungsweg zu eröffnen (z.B. Schulen mit Tracking) verglichen mit Klassenzimmern, in denen Leistungskulturen darauf abzielen, das Talent in jedem Kind zu entwickeln. Es gibt Unterschiede in Klassen, in denen Lehrpersonen überzeugt sind, dass man Lernleistung ändern kann (Dweck, 2006). [...]

Zweitens haben Rubie-Davies et al. (Rubie, 2003, 2006, 2007; Rubie-Davies, Hattie \& Hammilton 2006) der Erwartungsforschung eine weitere Dimension hinzugefügt, die Anlass zur Sorge gibt: Wenn Lehrpersonen geringere Erwartungen hegen, dann tun sie dies gegenüber allen Lernenden einer Klasse - das ist sicherlich ein Lehrereffekt.

Abschließend bleibt zu erwähnen, dass sich das sprachbezogene Feedback der Lehrkräfte nicht nur auf die Bedeutungen einzelner Begriffe der SchülerInnen bezog, sondern auch ihre Kollokation in den Blick nahm. Hierauf wurde w.o. bereits hingewiesen, beispielsweise mit der Schilderung zur Sprachförderung bei der Ländereinordnung bezüglich des „,von Meeren Umspültseins“ und der Bezeichnung der Himmelsrichtungen. So reagierte Prof.ssa Cese in einer Abfragung auch auf die Äußerung „La coltivazione è piena di $[\ldots]$ [. (Der Anbau ist voll von [...]) mit „Piena magari non diciamo.“ (Voll sagen wir lieber nicht), wodurch eine andere Schülerin unaufgefordert das angemessenere ricca [di] (reich [an]) vorschlug.

Außerdem wurde beobachtet, dass sich Schwierigkeiten der SchülerInnen mit registerkongruenten Kollokationen vor allem dann ergaben, wenn sie sich um einen bildungssprachlichen Ausdruck bemühten. Dies führte zu Beiträgen wie

- Si coltiva cibo. (Man baut Essen an.)

- Alle volte il clima raggiunge anche i 50 gradi. (Manchmal erreicht das Klima auch 50 Grad.)

- Il codice stradale è scarso. (Die Verkehrsregeln sind spärlich.)

die von den Lehrkräften umgehend kommentiert wurden. Derartige Kollokations- und Ausdrucksschwierigkeiten zeigten die Lernenden z.T. schon auf einem niedrigeren sprachlichen Niveau, wie etwa „I treni sono velocissimi e non hanno quello che guida" (Die Züge sind extrem schnell und haben den nicht, der fährt) und „Ci stanno meno ricchi, ma più poveri“" (Es gibt weniger Reiche, 
aber mehr Arme). Gleichsam enthielten Äußerungen manchmal elementare grammatikalische Fehler, die von den Lehrkräften direkt verbessert wurden. Dabei blieb ihr Feedback nicht nur auf die Korrektur beschränkt, sondern bestand auch aus allgemeinen Fragen zur Grammatik, wie die an eine Schülerin gerichtete Frage nach der Regel zum Artikelgebrauch: ${ }^{195}$

Auszug aus Mitschrift (18), Abfragung Frau Rea (FrR), Tania (Tan) - Grammatikkorrektur

Tan: Ci sta il Zambesi. (Es gibt den [falscher Artikel] Zambesi.)

FrR: Lo Zambesi. E sai perché "Io"? (Der Zambesi. Und weißt du, warum Io?)

Tan: Perché comincia con la zeta. (Weil es mit Zett anfängt).

Abbildung 32: Abfragung Frau Rea, III A, Pestalozzi, Unzufriedenheit mit Schüleräußerung (II)

Insgesamt ist bezüglich des Korrekturverhaltens der Lehrkräfte festzuhalten, dass sie Verbesserungen entweder selbst formulierten oder sich mit Fragen wie „E' corretto quello che ha detto Ava?" an die Klasse wandten und somit um eine erhöhte Schülerbeteiligung bemüht waren. Auf dieser Linie wurden auch unangekündigte Verbesserungsvorschläge der Lernenden positiv entgegengenommen. Aufgrund der Bedeutung der Eigenaktivität im Lernprozess ist davon auszugehen, dass auf Korrektur oder Bestätigung beschränktes Feedback weniger förderlich ist als die Technik, die Lernenden mit scaffolding zu einer Verbesserung oder Vervollständigung ihrer Beiträge zu bewegen.

Im folgenden Kapitel, das die Vorstellung der Ergebnisse dieser Erhebung beendet, werden verschiedene schriftliche Arbeiten der Lernenden aus zwei Klassen präsentiert.

195 Falscher Artikelgebrauch konnte auch bei SchülerInnen an der Morante beobachtet werden. Er ist wahrscheinlich als nachlässige Aussprache ihrem lokalen Soziolekt zuzuschreiben, wie z.B. in „Con l'occhi a mandorla.“ (Mit den [falscher Art.] Mandelaugen), worauf der Lehrer erwiderte: „Occhi. Oh! Gli occhi a mandorla.“ (Augen. Oh! Die Mandelaugen.). 


\section{Schriftliche Textproduktionen der Lernenden}

In diesem Kapitel werden Textproduktionen der Lernenden aus zwei der fünf Klassen, die an der Studie teilgenommen haben, vorgestellt. Diese Auswahl ist getroffen worden, um die lexikalischen und morphosyntaktischen Kompetenzen der Lernenden detaillierter erfassen und beschreiben zu können. Außerdem beruht sie auf der Annahme, dass die Textproduktionen eines guten Drittels der Lernenden, die in dieser Studie beobachtet wurden, die durchschnittlichen Fähigkeiten aller beobachteten Lernenden ausreichend widerspiegeln. Bei den Texten handelt es sich zum einen um schriftliche Leistungskontrollen über die indische Region, die in der Klasse an der Schule Morante durchgeführt worden waren, und zum anderen um von mir erbetene Ländervorstellungen der Klasse an der Schule Vespucci.

Im Folgenden werden zunächst die Texte über die indische Region aus der Klasse an der Morante präsentiert (10.1), wobei erst auf die graphischen strukturellen Gestaltungsmittel sowie das Schriftbild der Texte eingegangen wird (10.1.1) und danach eine Analyse des verwendeten Wortschatzes erfolgt (10.1.2), wie sie bereits im Zuge der Untersuchungen zu Lehrbuchseiten und C-Tests angewandt worden ist. In eigenen Unterkapiteln werden dabei der allgemeinsprachliche (10.1.2.1), der fachwortschatzsprachliche (10.1.2.2) sowie der phrasematische Wortgebrauch (10.1.2.3) vorgestellt. Ferner werden einige grammatikalische und syntaktische Eigenschaften der Texte in den Blick genommen (10.1.3), wie die Art der Satzverknüpfungen (10.1.3.1), der Prädikatsgebrauch (10.1.3.2.1) und die Verwendung infiniter Verbformen (10.1.3.2.2), also Infinitive und sprachlich komplexerer Gestaltungsmittel wie Gerundien und Partizipien. Hierbei wird darauf geachtet, wie die Texte dem Schreibanlass textsortenspezifisch und bildungssprachlich gerecht werden (10.1.3.3) und wie solche Bemühungen mit stilistischen Zügen konzeptioneller (und umgangssprachlicher) Mündlichkeit kontrastieren (10.1.3.4). Da die Vorbereitung der Lernenden auf diesen Test in der Behandlung einiger Lehrbuchtexte im Unterricht und ihrer Wiederholung als Hausaufgabe bestand, bildeten die Lehrbuchseiten zur indischen Region die Grundlage für das Erstellen der Schülertexte und werden deshalb ebenfalls vorgestellt (10.2). Auch sie werden nach den Merkmalen ihrer Wortschatzzusammensetzung (10.2.1) und ihrer grammatikalisch-syntaktischen Gestaltung (10.2.2) untersucht und im Anschluss daran mit den Schülertexten verglichen, um Gemeinsamkeiten und Unterschiede herauszustellen (10.3).

Das Unterkapitel 10.4 stellt die Schülertexte aus der Klasse an der Vespucci vor. Hier hatten die Lernenden freie Wahl, über welches Land oder welche geographische Region sie schreiben wollten, und konnten auch zwei Länder oder Regionen gegenüberstellend beschreiben. Nach Informationen zum Schriftbild (10.4.1) werden in mehreren Unterkapiteln die Zusammensetzung 
des Wortschatzes (10.4.2) und grammatikalisch-syntaktische Eigenschaften (10.4.3) beschrieben. Ein Abgleich mit den entsprechenden Lehrbuchtexten erfolgt nicht, da die SchülerInnen ganz unterschiedliche Länder und Kontinente wählten. Stattdessen wird auf einige inhaltliche und sprachliche Unterschiede zu den Texten ihrer Altersgenossen an der Schule Morante eingegangen (10.5).

\subsection{Schülertexte der Klasse an der Morante. Die indische Region}

In der beobachteten Klasse an der Schule Morante führte die Lehrkraft einen schriftlichen Test durch, der nicht angekündigt worden war, jedoch insofern von den Schülern vorbereitet wurde, als dass die Hausaufgabe in der sorgfältigen Vorbereitung auf eine mündliche Abfragung zur indischen Region bestand.

Die Aufgabenstellung zur Textproduktion lautete folgendermaßen:

1) Componi un testo in cui descrivi i caratteri fisici, demografici ed economici della regione indiana, sapendo che su questo scritto alcuni tuoi compagni dovranno studiare per preparare l'interrogazione di geografia.

2) Andresti a vivere in India? Motiva la tua risposta.

(M.Ü.: 1) Erstelle einen Test, in dem du die physischen, demographischen und wirtschaftlichen Eigenschaften der indischen Region beschreibst, wobei du weißt, dass einige deiner Mitschüler anhand dieses Textes eine Erdkundeabfragung vorbereiten müssen.

2) Würdest du nach Indien gehen, um dort zu leben? Begründe deine Entscheidung.)

Die 13 Anwesenden, von denen fünf zweisprachig waren, wobei Italienisch für einen davon die Erstsprache darstellte, sollten den Test in insgesamt 70 Minuten bearbeiten und konnten als Hilfsmittel eine im Klassenzimmer aufgestellte Wandkarte zur indischen Region benutzen.

Der Test wurde von Prof. Tigre mit dem italienischen Notenspektrum von 1 bis 10 , in dem 10 die beste Note ist, bewertet und teilweise durch einfache sowie doppelte Plus- und Minuszeichen bzw. halbe Punkte noch weiter differenziert. In die Benotung flossen Kriterien zu fünf verschiedenen Aspekten ein:

1. Inhalt: 1. physische Merkmale

2. demographische Merkmale

3. wirtschaftliche Merkmale (drei Teilnoten)

2. Textaufbau und Fähigkeit zur Zusammenfassung

3. Gebrauch eines spezifischen Wortschatzes

4. Globales Verständnis der Unterschiede zwischen den beiden kulturellen Kontexten 


\section{Individualisierung und Ausdruck des persönlichen Standpunktes.}

Die Endnote berechnet sich aus dem Durchschnitt aller sieben Teilnoten. Die durchschnittliche Gesamtnote beträgt 6 , wobei die Noten von 4,5 bis zu 8 reichen und über die Hälfte der Lernenden Noten von 6,5 bis 7+ erzielt. Die folgende Tabelle zeigt die Notenverteilung im Einzelnen.

\begin{tabular}{lcccccccccccc}
\hline Note & 4,5 & $5-$ & 5 & 5,5 & $6+/ 6,5$ & $7-$ & 7 & $7+$ & $8=$ & 8 & $\varnothing$ \\
\hline $\begin{array}{l}\text { Ler- } \\
\text { nende }\end{array}$ & H & C & K & B & A, E, M & । & F, L & J & D & G & 6 \\
\hline
\end{tabular}

Tabelle 1: Notenspiegel der Textproduktionen in der Klasse Morante

Auf die aus sprachlicher Sicht interessantere Teilnote für den Wortschatz wird an späterer Stelle eingegangen, während sich das folgende Unterkapitel zunächst mit dem Schriftbild und der strukturellen Gestaltung befasst.

\subsubsection{Schriftbild und graphische strukturelle Gestaltungsmittel}

Die Texte umfassen in der Regel eine gute Din A4-Seite und bestehen aus durchschnittlich 343 Wörtern, wobei der längste Text mit 819 Wörtern doppelt so lang ist wie die übrigen und der kürzeste Text nur 115 Wörter enthält. Ignoriert man den auffallend langen Text, bestehen sie aus durchschnittlich 304 Wörtern.

Bezüglich ihrer graphischen Gestaltung fällt auf, dass der Großteil nicht mit einer Überschrift versehen ist: Neun Texte weisen keine Überschrift auf, und von den vier mit Überschrift trägt einer lediglich den Titel Compito di geografia (Erdkundetest). Außerdem ist bezeichnend, dass sie nur sehr geringfügig oder gar nicht in Absätze untergliedert sind.

Zum Teil sind die Texte recht unleserlich geschrieben und enthalten bis zur Unkenntlichkeit übermalte Wörter und Sätze sowie eingeschobene Ergänzungen zwischen den Zeilen. Fast die Hälfte der Texte ist in Druckschrift oder aus einem Mix aus Druck- und Schreibschrift verfasst, und in einem Text ist es nur in seltenen Fällen möglich, die Buchstaben $\mathrm{A}$ und $\mathrm{O}$ voneinander $\mathrm{zu}$ unterscheiden. Diese Aufmachung sowie das häufige Fehlen der Interpunktion, der Akzente und des Großbuchstabens am Satzanfang - während stattdessen Wörter mit Majuskeln auftreten, die keine Großschreibung erfordern - legen den Schluss nahe, dass die Lernenden in der schulischen schriftsprachlichen Praxis nicht so gefördert wurden, dass sie formal korrekte Texte mit leicht lesbarer Handschrift verfassten, bzw. dass auf diese äußeren Gestaltungsmerkmale nicht viel Wert gelegt wurde. Möglich ist aber auch, dass sie aus Gründen schlechter Zeiteinteilung ihren Text nicht mehr säuberlich abschreiben oder zumindest abschließend durchlesen und einige Rechtschreibfehler korrigieren konnten. 
Auf orthographische Fehler wird nicht näher eingegangen, da die lexikalischen und grammatikalisch-syntaktischen Kompetenzen der SchülerInnen im Zentrum des Interesses stehen und die Rechtschreibfehler das Textverständnis nicht gravierend einschränken. Sie sind aber in den Zitaten der Lernenden übernommen worden. Das nächste Unterkapitel beschreibt, wie der verwendete Wortschatz strukturiert ist.

\subsubsection{Zusammensetzung des Wortschatzes}

Wie es für einen jeden italienischen Text üblich ist, bestehen die 13 Schülertexte überwiegend aus Wörtern des Basiswortschatzes (VDB) und entfällt der Großteil auf Ausdrücke des fundamentalen Wortschatzes (Fo). In allen Texten sind auch Begriffe aus dem allgemeinen Wortschatz (CO) zu finden, die nach den Basiswörtern durchschnittlich die größte Gruppe ausmachen. Außerdem liegen Toponyme, die in einem geographischen Text zu erwarten sind und im GRADIT nicht mit einer Gebrauchsmarke ausgestattet sind, und Wörter aus verschiedenen Fachsprachen vor. Ihr Anteil reicht von einem einzigen Wort in einem Text bis zu 21 in einem anderen und liegt durchschnittlich bei sechs.

Tabelle 2 gibt einen Überblick über die Gestaltung der Texte in Bezug auf ihre Länge und Unterteilung in Sätze, den Wortschatz nach Gebrauchsmarken, die Toponyme und die prozentualen Anteile des fundamentalen und des Basiswortschatzes am Gesamttextvolumen. Zusätzlich sind in der Spalte ganz rechts die Mittelwerte aller aufgeführten Texteigenschaften angegeben. ${ }^{196}$

\begin{tabular}{|c|c|c|c|c|c|c|c|c|c|c|c|c|c|c|}
\hline Schüler & A & B & C & D & $E$ & $\mathrm{~F}$ & G & $\mathrm{H}$ & I & $\mathrm{J}$ & $\mathrm{K}$ & L & $\mathrm{M}$ & $\varnothing$ \\
\hline n Wörter & 389 & 205 & 198 & 369 & 377 & 490 & 305 & 115 & 381 & 819 & 220 & 304 & 289 & 343 \\
\hline n Sätze & 17 & 13 & 15 & 24 & 19 & 16 & 21 & 7 & 16 & 43 & 8 & 19 & 8 & 17 \\
\hline $\begin{array}{l}\text { längster } \\
\text { Satz }\end{array}$ & 55 & 38 & 31 & 55 & 43 & 55 & 45 & 24 & 56 & 32 & 43 & 29 & 66 & 44 \\
\hline $\begin{array}{l}\varnothing \text { Satz- } \\
\text { länge }\end{array}$ & 23 & 16 & 13 & 15 & 20 & 31 & 15 & 16 & 29 & 19 & 29 & 16 & 36 & 21 \\
\hline$A D$ & 7 & 1 & 1 & 2 & 5 & 4 & 3 & 1 & 2 & 11 & 1 & 2 & 2 & 3 \\
\hline AD TS & 0 & 0 & 0 & 0 & 0 & 0 & 0 & 1 & 0 & 0 & 0 & 0 & 0 & 0 \\
\hline $\mathrm{AU}$ & 32 & 14 & 8 & 21 & 31 & 44 & 20 & 6 & 35 & 73 & 12 & 28 & 22 & 27 \\
\hline AU TS & 0 & 0 & 0 & 1 & 0 & 1 & 1 & 0 & 2 & 1 & 0 & 0 & 0 & 0 \\
\hline $\mathrm{CO}$ & 31 & 18 & 19 & 32 & 42 & 42 & 34 & 11 & 35 & 95 & 20 & 29 & 29 & 34 \\
\hline CO TS & 0 & 1 & 1 & 2 & 6 & 4 & 3 & 0 & 1 & 9 & 1 & 1 & 0 & 2 \\
\hline el.CO & 6 & 2 & 7 & 0 & 4 & 6 & 2 & 1 & 4 & 9 & 2 & 8 & 2 & 4 \\
\hline $\begin{array}{l}\text { el.CO } \\
\text { TS }\end{array}$ & 0 & 0 & 0 & 0 & 0 & 9 & 1 & 0 & 0 & 3 & 0 & 0 & 0 & 1 \\
\hline el.TS & 1 & 0 & 2 & 3 & 1 & 5 & 4 & 2 & 0 & 11 & 0 & 2 & 2 & 3 \\
\hline ES & 0 & 0 & 0 & 1 & 0 & 0 & 0 & 0 & 0 & 0 & 0 & 0 & 0 & 0 \\
\hline FO & 252 & 133 & 105 & 259 & 242 & 329 & 192 & 77 & 263 & 527 & 142 & 195 & 191 & 223 \\
\hline
\end{tabular}

196 Die TestteilnehmerInnen sind in der Kopfzeile mit Großbuchstaben genannt und o. M. bedeutet ohne (Gebrauchs-)Marke. Zu den Kürzeln der Gebrauchsmarken s. Übersicht in Kap. 7 bzw. im Anhang. 


\begin{tabular}{|c|c|c|c|c|c|c|c|c|c|c|c|c|c|c|}
\hline Schüler & A & B & C & D & $\mathrm{E}$ & $\mathrm{F}$ & G & $\mathrm{H}$ & 1 & $\mathrm{~J}$ & $\mathrm{~K}$ & $\mathrm{~L}$ & M & $\varnothing$ \\
\hline FO TS & 1 & 0 & 0 & 0 & 0 & 0 & 0 & 0 & 1 & 0 & 0 & 0 & 0 & 0 \\
\hline LE & 0 & 0 & 0 & 0 & 0 & 1 & 0 & 0 & 0 & 0 & 0 & 0 & 0 & 0 \\
\hline OB & 0 & 0 & 0 & 1 & 0 & 0 & 0 & 0 & 0 & 0 & 0 & 0 & 0 & 0 \\
\hline TS & 7 & 1 & 5 & 4 & 5 & 11 & 6 & 2 & 5 & 23 & 2 & 8 & 5 & 6 \\
\hline n.p. & 40 & 24 & 33 & 33 & 30 & 21 & 31 & 11 & 28 & 45 & 33 & 21 & 25 & 29 \\
\hline el.n.p. & 12 & 11 & 17 & 10 & 10 & 10 & 8 & 3 & 5 & 12 & 6 & 10 & 11 & 10 \\
\hline sigla & 0 & 0 & 0 & 0 & 0 & 0 & 0 & 0 & 0 & 0 & 0 & 0 & 0 & 0 \\
\hline o. M. & 0 & 0 & 0 & 0 & shikh & arya & 0 & 0 & 0 & arya & 0 & 0 & 0 & 0 \\
\hline $\begin{array}{l}n \mathrm{FO} \text { in } \\
\%\end{array}$ & 64,8 & 65 & 53 & 70 & 64,2 & 65,5 & 63 & 67 & 69 & 64,3 & 65 & 64 & 66 & 65 \\
\hline $\mathrm{n} \mathrm{VdB}^{*}$ & 290 & 148 & 114 & 283 & 281 & 385 & 216 & 85 & 303 & 612 & 155 & 224 & 215 & 255 \\
\hline $\begin{array}{l}n \mathrm{VdB} \text { in } \\
\%\end{array}$ & 74 & 72 & 58 & 77 & 75 & 77,7 & 71 & 74 & 80 & 74,7 & 71 & 74 & 74 & 73 \\
\hline $\begin{array}{l}\text { n n.p. } \\
\text { inkl. } \\
\text { el.n.p. in } \\
\%\end{array}$ & 13 & 17 & 25 & 12 & 11 & 6,5 & 13 & 12 & 8,7 & 7 & 18 & 10 & 13 & 13 \\
\hline $\begin{array}{l}\text { n n.p. } \\
\text { inkl. } \\
\text { el.n.p. }\end{array}$ & 52 & 35 & 50 & 43 & 40 & 32 & 39 & 14 & 33 & 57 & 39 & 31 & 36 & 39 \\
\hline
\end{tabular}

Tabelle 2: Klasse Morante, Länge der Texte und Sätze sowie Gebrauchsmarken und Anteile der Wortschatzbereiche VDB und FO am Gesamttext

*inkl. doppelter Marken mit Ts

Wie schon bei der Lehrbuchanalyse gilt auch hier wieder, dass als Wort die graphische Einheit zählt, die durch zwei Leerstellen von den anderen abgetrennt ist, und als Satz alles bis zu einem Punkt. Während die durchschnittliche Satzlänge bei 21 Wörtern liegt, weisen viele Texte einzelne Sätze auf, die weit darüber hinausgehen. So bestehen in fünf Texten die längsten Sätze aus mindestens 55 Wörtern, in einem Fall sogar aus 66.

Durchschnittlich machen die Wörter aus dem Basiswortschatz (VDB) 73 Prozent aller Wörter eines Textes aus. Ein Maximum gebrauchte die zweisprachige Schülerin I mit 80 Prozent, ein Minimum von 58 die einsprachige Schülerin C. Auffallend oft werden konjugierte Formen der Verben essere (Fo; sein), esserci (AU; geben/da sein) und avere (Fo; haben) verwendet. Einen nicht unerheblichen Anteil am totalen Wortaufkommen stellen auch die Toponyme, nämlich im Mittelwert 39 Wörter und damit 12,7 Prozent der Gesamtzahl. Hierbei handelt es sich um maximal 26 verschiedene, um mindestens acht und durchschnittlich um 19 Worttypen.

\subsubsection{Allgemeiner Wortschatz (Co)}

Durchschnittlich 38 Wörter, also ungefähr ein Zehntel des gesamten Textes $(11,1 \%)$, sind mit den Gebrauchsmarken Co und el.Co versehen. Am meisten Ausdrücke aus dem allgemeinen Wortschatz verwendete mit 95 der einsprachige Schüler J, der auch den mit Abstand längsten Text verfasste. Er kommt auf einen prozentualen Anteil Co-gekennzeichneter Wörter von 12,7, womit 
er leicht über dem Durchschnitt liegt. Am wenigsten Co-Wörter benutzte der einsprachige Schüler H, nämlich zwölf, was einem Anteil am Gesamtwortvolumen von 10,4 Prozent entspricht und somit nah am Mittelwert liegt.

Neben den bestimmten maskulinen Pluralartikeln $i$ und gli sowie dem Personalpronomen der dritten Person Singular si (man, sich), die im GRADIT mit einer Co-Marke verzeichnet sind und deren korrekte Verwendung wenig Schwierigkeiten bereiten sollte, befinden sich unter den Ausdrücken des allgemeinen Wortschatzes Nomen, Adjektive, Adverbien, Verben und einige Phraseme. Letztere sind im GRADIT so gut wie nie mit einer Marke des Basiswortschatzes gekennzeichnet, sondern tragen entweder eine Co- oder eine TsMarke.

Zur Gruppe der Nomen, Adjektive und Verben zählen einige aus dem Erdkundeunterricht und der Lektüre des Lehrwerks sicherlich gut bekannte Ausdrücke wie catena montuosa (Gebirgszug/Bergkette), arcipelago (Archipel/Inselgruppe), disabitato (unbewohnt), coltivato (angebaut), sviluppato und die reflexiven Verben trovarsi (sich befinden) und prolungarsi (sich verlängern, sich erstrecken). W.u. wird darauf eingegangen, dass sich ihr im Vergleich zu den Texten der anderen Klasse frequenteres Vorkommen zumindest teilweise mit der wortgetreuen Wiedergabe von Ausschnitten aus den zu lernenden Lehrbuchtexten erklären lässt.

\subsubsection{Fachsprachlicher Wortschatz (Ts)}

Die von den Lernenden gebrauchten Ausdrücke aus den verschiedenen Fachsprachen machen einen geringeren Anteil aus: Durchschnittlich lassen sich in den Texten sechs Fachwörter finden, bei einem niedrigsten Vorkommen von nur einem solchen Wort in den Texten der Schüler B und K und dem höchsten Vorkommen von 23 in dem des Schülers J. Da es sich insgesamt um wenige Worttypen handelt, sind alle mit weiteren Informationen zu ihrer Verwendung in Tabelle 3 abgebildet. ${ }^{197}$

\begin{tabular}{lllr}
\hline Wort & Wortart & Marke & Tokens \\
\hline densità & s.f. & TS stat. & $8(7)$ \\
clima & loc.s.m. & TS meteor., geogr. & 5 \\
monsonico & el.loc.s.m. & TS meteor., geogr. & \\
$k m^{2}$ & simb. & TS metrol. & $5(4)$ \\
$m$ & simb. & TS metrol. & $5(3)$ \\
pianura & loc.s.f. & TS geol. & 5 \\
alluvionale & el.loc.s.f./agg. & TS geol./TS geol., petr. & 6 \\
vento & loc.s.m. & TS meteor. & 4 \\
periodico & el.loc.s.m. & el.TS meteor. & \\
\hline
\end{tabular}

197 In der Spalte der Tokens ist in Klammern angegeben, in wie vielen Schülertexten sie vorkommen, sobald die Anzahl der Tokens von der Anzahl der Texte abweicht, in denen sie erscheinen. 


\begin{tabular}{|c|c|c|c|}
\hline Wort & Wortart & Marke & Tokens \\
\hline centro & loc.s.m. & TS urban. & 3 \\
\hline urbano & el.loc.s.m. & el.TS urban. & \\
\hline chilometro & loc.s.m. & TS metrol. & 3 \\
\hline quadrato & el.loc.s.m. & el.TS metrol. & \\
\hline minerario & agg. & TS miner. & 3 \\
\hline settore & loc.s.m. & TS econ. & 3 \\
\hline terziario & el.loc.s.m. & el.TS econ. & \\
\hline bauxite & s.f. & TS petr. & 2 \\
\hline demografia & s.f. & TS stat. & 2 \\
\hline Dravida & agg. & TS etnol. & 2 \\
\hline hindi & s.m. & TS ling. & 2 \\
\hline lingua & loc.s.f. & TS ling., dir. & $2(1)$ \\
\hline ufficiale & el.loc.s.f. & el.TS ling., dir. & \\
\hline manganese & s.m. & TS chim. & 2 \\
\hline settore & loc.s.m. & TS econ. & 2 \\
\hline secondario & el.loc.s.m. & el.TS econ. & \\
\hline settore & loc.s.m. & TS econ. & 2 \\
\hline primario & el.loc.s.m. & el.TS econ. & \\
\hline agenti & loc.s.m. & TS geol., geogr. & 1 \\
\hline atmosferici & el.loc.s.m. & el.TS geol., geogr. & \\
\hline barriera & loc.s.f. & TS geogr. & 1 \\
\hline corallina & el.loc.s.f. & el.TS geogr. & \\
\hline Bollywood & s.m. & TS cinem. & 1 \\
\hline canna & loc.s.f. & TS bot.com. & 1 \\
\hline da & el.loc.s.f. & el.TS bot.com. & \\
\hline zucchero & el.loc.s.f. & el.TS bot.com. & \\
\hline delta & s.m. & TS geogr. & 1 \\
\hline erosione & s.f. & TS geol. & 1 \\
\hline gas & loc.s.m. & TS chim. & 1 \\
\hline naturale & el.loc.s.m. & el.TS chim. & \\
\hline gruppo & loc.s.m. & TS sociol. & 1 \\
\hline etnico & el.loc.s.m. & el.TS sociol. & \\
\hline lignite & s.f. & TS petr. & 1 \\
\hline morfologia & s.f. & TS geol. & 1 \\
\hline morfologicamente & avv. & TS scient., ling., sociol. & 1 \\
\hline placca & s.f. & TS geol. & 1 \\
\hline portata & s.f. & TS fis., idraul. & 1 \\
\hline sedimento & s.m. & TS fis., geol., petr. & 1 \\
\hline Tot. Wörter & & & 33 \\
\hline Tot. Tokens & & & 93 \\
\hline
\end{tabular}

Tabelle 3: Fachsprachliches Wortvorkommen in den Schülertexten der Morante

Insgesamt wurden 33 fachsprachliche Wörter gebraucht, wobei sich darunter auch Phraseme befinden (z.B. die italienischen Ausdrücke für „Monsunklima“ und „Primärsektor"), die an dieser Stelle als ein Wort und nicht wie bei den Wortschatzberechnungen w.o. als zwei bzw. drei graphische Einheiten gezählt wurden. In den Texten der Lernenden erscheinen 93 Ts-markierte Tokens, 
doch nur wenige Ausdrücke treten mehr als dreimal auf, und zwar insgesamt sieben.

Am häufigsten wurde der Ausdruck densità (Bevölkerungsdichte) aus dem statistischen Fachwortschatz gebraucht, nämlich achtmal in sieben verschiedenen Texten. Dieses Wort kannten die Lernenden bereits aus den ersten Jahren der Mittelschule aus ihrem Unterrichtswerk zur demographischen Beschreibung von Ländern und Regionen. Ebenfalls häufiger, nämlich jeweils fünfmal, erscheinen die Ausdrücke bzw. Maßeinheiten clima monsonico (Monsunklima), pianura alluvionale (Schwemmebene) aus den meteorologisch/geographischen und der geologischen Fachsprache sowie $\mathrm{km}^{2}$ und $\mathrm{m}$ aus der Metrologie. Das Monsunklima war in den Stunden vor der Testdurchführung ausführlich im Unterricht behandelt worden, dennoch verwendete den Ausdruck weniger als die Hälfte der TestteilnehmerInnen. Von den zu diesem Thema behandelten Informationen und Zusammenhängen erwähnten die Lernenden, wenn überhaupt, nur einzelne Aspekte: Vier schrieben über die periodischen Winde (venti periodici) und zwei von ihnen ergänzten Informationen über die daraus resultierende Niederschlagsmenge. Nur einer der beiden Schüler (J) fuhr danach mit der Vorstellung des Tertiärsektors fort und beschrieb den Einfluss des Klimas auf diesen. Die drei anderen Schüler, welche die Monsunwinde erwähnten, lieferten nur vereinzelte Informationen darüber. Zum Beispiel nannten sie ihren Namen, ohne auf das Klima oder auf die Konsequenzen für die Landwirtschaft einzugehen.

In drei Texten kommen die Ausdrücke centro urbano (Stadt) aus der Urbanistik, chilometro quadrato (Quadratkilometer) aus der Metrologie und minerario (Bergbau-, montan) aus der Fachsprache des Bergbaus vor. Centro urbano ist ein Begriff, der im Lehrbuch bei der Behandlung demographisch-urbanistischer Gesichtspunkte oft verwendet wird, so wie auch minerario bei der Vorstellung der Bodenschätze einer Region oder eines Landes. Die Maßeinheit $m$ hingegen sollte den Schülerinnen und Schülern sowohl aus dem Erdkundeals auch aus dem Mathematikunterricht schon seit mehreren Schuljahren gut bekannt sein.

Auch ein weiterer fachwortschatzsprachlicher Ausdruck, der von drei Schülern verwendet wurde, war den Lernenden bereits in den ersten Jahren der Mittelschule wiederholt begegnet, und zwar der aus der Wirtschaftssprache stammende Ausdruck settore terziario (Tertiärsektor).

Neben acht Wörtern, die insgesamt jeweils zweimal erscheinen, gibt es eine ganze Reihe von fachsprachlichen Wörtern (14), auf die nur ein Schüler zurückgriff. Darunter sind Begriffe aus verschiedenen fachsprachlichen Sektoren wie der Geologie (z.B. die italienischen Ausdrücke für atmosphärische Einflüsse, Erosion und Morphologie), der Geographie (bspw. It. für Korallenriff und Delta), der Soziologie (It. für Volksgruppe) und der Botanik (It. für Zuckerrohr). Insgesamt stammen die einmal verwendeten Ts-gekennzeichneten 
Wörter aus zwölf verschiedenen Fachsprachen, während alle Ts-Wörter auf 18 Fachsprachen rekurrieren.

Mit insgesamt sechs Wörtern gehören die meisten verwendeten Begriffe aus einer Fachsprache zur geologischen, gefolgt von der geographischen, wirtschaftlichen und metrologischen Fachsprache mit jeweils drei Wörtern. Unter den Ts-Wörtern sind elf in den Schülertexten, die auf den entsprechenden Seiten im Unterrichtswerk nicht verwendet werden, wohingegen von den 52 fachsprachlichen Wörtern der Lehrbuchtexte nur 21, also weniger als die Hälfte, von den Schülern aufgegriffen wurden.

Die fachsprachlichen Wörter der SchülerInnen, die nicht auf den Lehrbuchseiten zur indischen Region vorkommen, sind:

- die Maßeinheit $m$ sowie das Adjektiv alluvionale (Ts geol., petr.; Schwemm-), wobei es in den Schüler- und Lehrbuchtexten als Phrasemelement in pianura alluvionale auftritt und von der Schülerin, die es nicht als Phrasemelement verwendete, im semantisch gleichen Zusammenhang mit pianura (Ebene) gebraucht wurde;

- settore primario und settore secondario (Ts econ.; Primär- und Sekundärsektor), die an anderen Stellen des Buchs auftreten und auch aus dem Unterricht bekannt sind;

- agenti atmosferici (Ts geol., geogr.; atmosphärische Einflüsse), das im Unterricht bei der Beschreibung des Klimas wiederholt verwendet wurde;

- Bollywood (Ts cinem.), das die Schülerin wahrscheinlich außerhalb des schulischen Unterrichts gelernt hatte;

- morfologia und morfologicamente (Ts geol.; Morphologie und morphologisch), die im Unterricht bei der Behandlung der Beschaffenheit eines geographischen Gebiets und auf anderen Seiten des Lehrbuchs verwendet werden;

- placca (Ts geol.; Platte), das im Unterricht bei der Besprechung der Entstehung von Gebirgen und seismischen Zonen gebraucht wurde und wahrscheinlich auch an anderen Stellen des Lehrbuchs auftritt;

- portata (Ts fis., idraul.; Wassermenge), das während der Unterrichtsbeobachtungen gebraucht wurde und wahrscheinlich auch im Lehrbuch verwendet wird;

- vento periodico (Ts meteor.; periodischer Wind), das bei der Besprechung der Monsunwinde hier wie in allen anderen Klassen mehrmals hervorgehoben wurde.

Nur an drei Stellen benutzten zwei SchülerInnen darüber hinaus Ausdrücke, die im GRADIT mit einer Gebrauchsmarke verzeichnet sind und weder zum Basiswortschatz noch zum Fachwortschatz zählen. Das sind das Adjektiv inabitato (LE; unbewohnt) bei Schüler F, das seltener als sein Synonym disabitato aus dem allgemeinen Wortschatz ist, und die Phrasemabkürzung etc., die im GRADIT als OB (veraltet) gekennzeichnet ist und seltener verwendet wird 
als ihr Synonym ecc., sowie das Fremdwort shopping (Es ingl.) im Text der Schülerin D.

Ferner erwähnten zwei Schüler den Eigennamen einer lokalen Volksgruppe, arya, der im Lehrbuch erscheint, aber im GRADIT nicht aufgeführt ist, und die Schülerin E eine Religion mit dem Namen shikh, der nicht annähernd so im Buch genannt wird und auch nicht im GRADIT eingetragen ist. Es ist anzunehmen, dass sie ihn mit dem Wort sheik (It. sceicco, Co; Scheich) verwechselte.

Prof. Tigre bewertete die Angemessenheit und Präzision des verwendeten Wortschatzes mit Noten von $5+$ bis 8,5 , wobei die Durchschnittsnote 7 beträgt. Viele SchülerInnen erhielten für die Wortschatzverwendung eine Note, die besser als ihre Gesamtnote war; im Durchschnitt liegt sie bei 6 . Nur zwei einsprachige und eine mehrsprachige Schülerin erhielten bessere Gesamt- als Wortschatznoten. Die drei schlechtesten Noten im Wortschatz wurden übrigens nicht an mehrsprachige Lernende vergeben, sondern an einsprachige.

\subsubsection{Phraseme}

Da Phraseme als Mehrworteinheiten weniger gebräuchlich als Begriffe aus dem Basiswortschatz sind und deshalb sowohl von SchülerInnen, für die Italienisch nicht die Erstsprache darstellt, als auch von den einsprachigen potentiell schwieriger zu erwerben sind, zeigt Tabelle 4, welche in den Texten auftreten. Neben den Wortarten und Gebrauchsmarken der Phraseme wird über ihr Vorkommen in den Schülertexten und auf den Lehrbuchseiten zur indischen Region informiert. ${ }^{198}$

\begin{tabular}{|c|c|c|c|c|c|}
\hline Phrasem & Wortart & Marke & $\begin{array}{l}\text { Kommentar/ } \\
\text { Übersetzung }\end{array}$ & Tokens & im LB \\
\hline agenti atmosferici & loc.s.m. & $\begin{array}{l}\text { TS geol., } \\
\text { geogr. }\end{array}$ & $\begin{array}{l}\text { atmosphärische } \\
\text { Einflüsse }\end{array}$ & 1 & \\
\hline (al) massimo & loc.avv. & $\mathrm{CO}$ & $\begin{array}{l}\text { maximal; bei } \mathrm{SCH} \\
\text { ohne al }\end{array}$ & 1 & \\
\hline anche se & loc.cong. & $\mathrm{CO}$ & auch wenn & 1 & ja \\
\hline a punta & loc.agg. & $\mathrm{CO}$ & spitz & 1 & \\
\hline a volte & loc.avv. & $\mathrm{CO}$ & manchmal & 2 & \\
\hline all'incirca & loc.avv. & $\mathrm{CO}$ & ungefähr & 1 & \\
\hline barriera corallina & loc.s.f. & TS geogr. & Korallenriff & 1 & ja \\
\hline canna da zucchero & loc.s.f. & $\begin{array}{l}\text { TS } \\
\text { bot.com. }\end{array}$ & Zuckerrohr & 1 & ja \\
\hline catena montuosa & loc.s.f. & $\mathrm{CO}$ & $\begin{array}{l}\text { Gebirgszug; } 5 \mathrm{SCH} \\
\text { catena, davon } 3 \\
\text { auch catena mon- } \\
\text { tuosa }\end{array}$ & $12(8)$ & nur catena \\
\hline centro urbano & loc.s.m. & TS urban. & Stadt & 3 & ja \\
\hline $\begin{array}{l}\text { chilometro quad- } \\
\text { rato }\end{array}$ & loc.s.m. & TS metrol. & Quadratkilometer & 3 & ja \\
\hline
\end{tabular}




\begin{tabular}{|c|c|c|c|c|c|}
\hline Phrasem & Wortart & Marke & $\begin{array}{l}\text { Kommentar/ } \\
\text { Übersetzung }\end{array}$ & Tokens & im LB \\
\hline clima monsonico & loc.s.m. & $\begin{array}{l}\text { TS meteor., } \\
\text { geogr. }\end{array}$ & Monsunklima & 5 & ja \\
\hline da solo & loc.agg. & $\mathrm{CO}$ & allein & 3 & ja \\
\hline di più & loc.avv. & $\mathrm{CO}$ & mehr & 1 & \\
\hline etc. & abbr.loc.avv.lat. & OB & etc. & 1 & \\
\hline fare capo & loc.v. & $\mathrm{CO}$ & $\begin{array}{l}\text { an der Spitze ste- } \\
\text { hen }\end{array}$ & 1 & ja \\
\hline fare parte & loc.v. & $\mathrm{CO}$ & Teil sein & 3 & \\
\hline gas naturale & loc.s.m. & TS chim. & Erdgas & 1 & ja \\
\hline grazie a & loc.prep. & $\mathrm{CO}$ & dank & $6(4)$ & ja \\
\hline gruppo etnico & loc.s.m. & TS sociol. & Volksgruppe & 1 & ja \\
\hline in abbondanza & loc.avv. & $\mathrm{CO}$ & $\begin{array}{l}1 \text { in grande ab- } \\
\text { bondanza, wie in } \\
\text { gran parte (LB) als } \\
\text { Phrasem mit einge- } \\
\text { schobenem Adj. } \\
\text { gewertet }\end{array}$ & $2(1)$ & \\
\hline lingua ufficiale & loc.s.f. & $\begin{array}{l}\text { TS ling., } \\
\text { dir. }\end{array}$ & Amtssprache & $2(1)$ & ja \\
\hline mezzo di trasporto & loc.s.m. & $\mathrm{CO}$ & Verkehrsmittel & 1 & \\
\hline oltre a & loc.prep. & $\mathrm{CO}$ & außer/neben & 1 & \\
\hline per esempio & loc.avv. & $\mathrm{CO}$ & zum Beispiel & 3 & ja \\
\hline $\begin{array}{l}\text { per quanto ri- } \\
\text { guarda }\end{array}$ & loc.avv. & $\mathrm{CO}$ & bezüglich & 1 & ja \\
\hline per questo & loc.cong. & $\mathrm{CO}$ & deshalb & 2 & \\
\hline pianura alluvionale & loc.s.f. & TS geol. & Schwemmebene & 5 & ja \\
\hline popolazione attiva & loc.s.f. & $\begin{array}{l}\text { CO TS } \\
\text { stat. }\end{array}$ & $\begin{array}{l}\text { erwerbstätige Be- } \\
\text { völkerung }\end{array}$ & 2 & ja \\
\hline pro capite & loc.s.m.inv. & $\mathrm{CO}$ & $\begin{array}{l}\text { pro Kopf; } \\
\text { PIL impliziert }\end{array}$ & 2 & ja \\
\hline repubblica federale & loc.s.f. & $\mathrm{CO}$ & Bundesrepublik & 2 & ja \\
\hline servizi igienici & loc.s.m.pl. & $\mathrm{CO}$ & Sanitäranlagen & 1 & \\
\hline settore primario & loc.s.m. & TS econ. & Primärsektor & 2 & $\begin{array}{l}\text { settore } \\
\text { agricolo }\end{array}$ \\
\hline settore secondario & loc.s.m. & TS econ. & Sekundärsektor & 2 & \\
\hline settore terziario & loc.s.m. & TS econ. & Tertiärsektor & 3 & $\begin{array}{l}\text { settore dei } \\
\text { servizi }\end{array}$ \\
\hline $\begin{array}{l}\text { stagione dei mon- } \\
\text { soni }\end{array}$ & loc.s.f. & $\begin{array}{l}\text { CO TS me- } \\
\text { teor. }\end{array}$ & $\begin{array}{l}\text { Monsunzeit; nicht } \\
\text { im GRADIT, wie } \\
\text { stagione delle pi- } \\
\text { ogge gewertet }\end{array}$ & 1 & ja \\
\hline $\begin{array}{l}\text { stagione dei mon- } \\
\text { soni e delle piogge }\end{array}$ & loc.s.f. & $\begin{array}{l}\text { CO TS me- } \\
\text { teor. }\end{array}$ & $\begin{array}{l}\text { nicht im GRADIT; } \\
\text { analog zu den an- } \\
\text { deren als Phrasem } \\
\text { gewertet }\end{array}$ & 1 & \\
\hline $\begin{array}{l}\text { stagione delle } \\
\text { piogge }\end{array}$ & loc.s.f. & $\begin{array}{l}\text { CO TS me- } \\
\text { teor. }\end{array}$ & Regenzeit & 2 & ja \\
\hline tra l'altro & loc.avv. & $\mathrm{CO}$ & unter anderem & 1 & \\
\hline un po' & loc.avv. & $\mathrm{CO}$ & etwas & 1 & \\
\hline vento periodico & loc.s.m. & TS meteor. & periodischer Wind & 5 & \\
\hline vicino a & loc.prep. & $\mathrm{CO}$ & nahe & $3(2)$ & ja \\
\hline visto che & loc.cong. & $\mathrm{CO}$ & $\begin{array}{l}\text { angesichts der Tat- } \\
\text { sache, dass }\end{array}$ & 1 & \\
\hline vuol dire & loc.v. & $\mathrm{CO}$ & das bedeutet & 1 & \\
\hline
\end{tabular}




\begin{tabular}{lllccrr}
\hline & & & Kommentar/ & & & \\
Phrasem & Wortart & Marke & Übersetzung & Tokens & im LB & \\
\hline Tot. & & & & 44 & 96 & 21 \\
\hline
\end{tabular}

Tabelle 4: Phraseme in den Schülertexten der Morante

Von den 44 Phrasemen, die sich größtenteils aus zwei Elementen, zum Teil auch aus dreien zusammensetzen (z.B. mezzo di trasporto, Transportmittel; per quanto riguarda, bezüglich), wird fast exakt die Hälfte auch im Lehrbuch verwendet und zählen 25 zum allgemeinen Wortschatz (CO). Hierunter befinden sich einige auch im Mündlichen recht gebräuchliche Ausdrücke wie per esempio (zum Beispiel), voler dire (bedeuten), a volte (manchmal) und da solo (allein).

Die vier Phraseme stagione delle piogge, stagione delle piogge e dei monsoni, stagione dei monsoni (Regenzeit, Monsunzeit) und popolazione attiva (erwerbstätige Bevölkerung) tragen die doppelte Marke Co T und wurden nicht nur im Unterricht gebraucht, sondern erscheinen auch im Lehrbuch (nicht alle auf den Seiten zur indischen Region). Von den 13 Phrasemen aus den fachsprachlichen Wortschätzen, die größtenteils schon w.o. vorgestellt wurden, befinden sich neun auf den entsprechenden Seiten im Unterrichtswerk. Das letzte Phrasem, et cetera, das abgekürzt zu etc. erscheint und die Gebrauchsmarke Oв trägt, ist ebenfalls bereits präsentiert worden.

Was die Wortarten der Phraseme betrifft, so handelt es sich bei der Mehrheit, nämlich bei 23, um substantivische Mehrworteinheiten. An zweiter Stelle folgen zehn adverbiale Phraseme und an dritter Stelle mit jeweils drei Worttypen verbale, präpositionale und konjunktionale Phraseme. Unter den Verben sind fare capo, fare parte und voler dire vertreten, die alle zum allgemeinen Wortschatz gehören und von denen das erste auch im Lehrbuch erscheint.

Die drei ebenfalls Co-gekennzeichneten präpositionalen Phraseme sind das sechsmal verwendete grazie a (dank), das wie vicino a (nahe) auch auf den entsprechenden Lehrbuchseiten verwendet wird, und oltre a (außerdem, abgesehen von), das dort nicht in dieser Form, sondern stattdessen in der bedeutungsähnlichen Einwortform oltre (Fo) erscheint.

Die konjunktionalen Phraseme sind anche se (auch wenn), per questo (deshalb) und visto che (angesichts der Tatsache, dass). Sie stammen alle aus dem allgemeinen Wortschatz, und nur das erste wird auf den Lehrbuchseiten zur indischen Region gebraucht. Desweiteren finden sich in den Schülertexten die zwei adjektivischen Phraseme a punta (spitz) und da solo (allein) aus dem allgemeinen Wortschatz, die zwei präpositionalen Phraseme oltre a und grazie a, beide ebenfalls Co-gekennzeichnet, und das konjunktionale Phrasem per questo $(\mathrm{CO})$.

Das in den Schülertexten am häufigsten verwendete Phrasem ist catena montuosa (Gebirgszug/Bergkette) aus dem allgemeinen Wortschatz, das in acht Texten mit insgesamt zwölf Vorkommnissen auftritt. Auf den Lehrbuchseiten über die indische Region wird es nicht benutzt, sondern stattdessen das 
Wort catena (FO; Kette), das im GRADIT mit vielen verschiedenen Bedeutungen aufgelistet ist, unter denen sich auch die des Phrasems catena montuosa befindet, die wie dieses Co-gekennzeichnet ist. Eine korrekte Bedeutungserschließung von catena ist auf den Lehrbuchseiten und in den Schülertexten durch den thematischen Kontext gesichert. Wie der Tabelle zu entnehmen ist, verwendeten fünf Lernende das Wort catena anstelle des Phrasems, bzw. drei von ihnen gebrauchten beide Ausdrücke, was auch stilistische Gründe haben könnte, um Wortwiederholungen zu vermeiden.

Das zweithäufigste Phrasem der Schülertexte wurde halb so oft verwendet (6-mal). Es ist die bereits vorgestellte Präposition grazie a (dank).

An dritter Stelle folgen die w.o. illustrierten substantivischen Phraseme fachwortschatzsprachlichen Gebrauchs pianura alluvionale (Ts geol.; Schwemmebene), vento periodico (Ts meteor.; periodischer Wind) und clima monsonico (Ts meteor., geogr.; Monsunklima) mit jeweils fünf Vorkommnissen.

Während sich auf den entsprechenden Seiten des Unterrichtswerks unter den Phrasemen bevorzugt nominale (28 von 57) und adverbiale (12) befinden, erscheinen dort auch die drei konjunktionalen Phraseme tant'è che (CO; soweit, dass), al posto di (Co; anstelle von) und anche se (CO; auch wenn) sowie die präpositionalen Phraseme a forma di (in Form von), accanto a (neben), in seguito (anschließend), invece di (anstatt), rispetto a (hinsichtlich) und vicino $a$ (neben), die allesamt aus dem allgemeinen Wortschatz (CO) stammen. Ihr spärlicherer Gebrauch spiegelt die semantisch und in der Frequenz allgemein eingeschränktere Verwendung von satzverknüpfenden Elementen in den Schülertexten wider, wie im Folgenden beschrieben wird.

\subsubsection{Lexikogrammatikalische und morphosyntaktische Gestaltung}

Viele längere Sätze in den Schülertexten bestehen nicht aus einem komplexen hypotaktischen Satzgefüge mit expliziter Kohäsion, sondern oft aus parataktischen Sätzen, die mit der Konjunktion „und“ verbunden sind und im Schreiben Geübtere in kürzere Satzgefüge unterteilen würden. Das verdeutlicht ein Beispiel aus dem Text von Schüler F (Rechtschreibfehler im Original):

Infine parlando dell'India diciamo che è una Repubblica Federale ed è composta da 28 Stati e da 7 territori amministrativi e ci sono molti gruppi come il gruppo Arya a nord, Dravida a Sud e altri che hanno culture differenti e fino a adesso hanno trovato 16 gruppo [sic] e ci sono anche migliaia e migliaia di dialetti.

(Schließlich von Indien sprechend, sagen wir, dass es eine Bundesrepublik ist und aus 28 Staaten und aus 7 Verwaltungsbezirken zusammengesetzt ist, und es gibt viele Gruppe [sic] wie die Gruppe Arya im Norden, Draviden im Süden und andere, die verschiedene Kulturen haben, und bis jetzt haben sie 16 Gruppen gefunden, und es gibt auch Abertausende von Dialekten.) 


\subsubsection{Satzverknüpfungen}

Die Lernenden gebrauchten in ihren Texten zwar bis zu 67 Konjunktionen bei einem Mittelwert von 27 pro Text und einer geringsten Anzahl von sechs, jedoch beschränken sich die verwendeten Worttypen auf höchstens 15, mindestens zwei und durchschnittlich sechs. Die am häufigsten verwendeten Konjunktionen sind das parataktische $e$ (FO; und), gefolgt von che (FO; dass) und anche (Fo; auch). Diese Konjunktionen, die zu den allgemein am häufigsten gebrauchten zählen, erzeugen oft eine schwächere Kohäsion zwischen den einzelnen Textaussagen, als es spezifischere Verknüpfungsmittel wie beispielsweise kausale und temporale Konjunktionen ermöglichen.

Außerdem verwendeten vier Lernende mit insgesamt sechs Vorkommnissen ein che, das aus dem alltagssprachlichen Mündlichen kommt und zwischen der Konjunktion „dass“ und den Relativpronomen oszilliert. Dieses Verknüpfungsmittel trägt den Namen polyvalentes che, weil es verschiedene syntaktische Funktionen übernehmen kann; allerdings ist sein Gebrauch formalsprachlich nicht normgemäß. ${ }^{199}$ Die nächsten Beispiele aus drei Schülertexten veranschaulichen die Verwendung dieses umgangssprachlichen Mittels, das im Deutschen am ehesten noch mit dem ähnlich generischen und weniger formalen Bindewort „wo“ übersetzt werden kann (Hervorhebung nicht im Original):

Schülerin A: Altri fiumi importanti sono il Gange, l'Indo e il Brahmaputra, che il Gange insieme al Brahmaputra sfociano nel Golfo del Bengala, mentre l'Indo sfocia nel Mar Arabico.

(Andere wichtige Flüsse sind der Ganges, der Hindus und der Brahmaputra, wo der Ganges zusammen mit dem Brahmaputra in den Golf von Bengalen münden [sic], während der Hindus ins Arabische Meer mündet.)

Schüler F: Nel Nord si trovano la catena dell'Himalaya e del Karakoram dove ci stanno le vette più alte del mondo come l'Everest e il K2 che arrivano ad un'altezza di 8000 metri e queste montagne hanno la cima a punta, cioè che sono giovani e l'erosione ancora non le hanno [sic] spianate.

(Im Norden befinden sich die Gebirgszüge des Himalayas und des Karakorums, wo es die höchsten Gipfel der Welt gibt, wie den Everest und den K2, die eine Höhe von 8.000 Metern erreichen, und diese Berge haben spitze Gipfel, das heißt, dass sie jung sind und die Erosion sie noch nicht geebnet haben $[\mathrm{sic}]$.)

199 Das che polivalente ist seit dem Beginn der Untersuchungen des italiano popolare (De Mauro 1970; also des vom Standard abweichenden, aber aus der Bemühung heraus, ihn zu produzieren, resultierenden Sprachgebrauchs von Teilen der Bevölkerung mit geringerer Bildung, der bereits im 19. Jahrhundert beobachtet werden kann (z.B. Romani, S. 407)) als Substandard-Varietät mit geringem sozialen Status beschrieben worden. Neue Untersuchungen unterstreichen, dass es sich dabei keineswegs um ein neues Phänomen handelt, sondern dass es die gesamte italienische Sprachgeschichte begleitet hat. Desweiteren wurden für das che polivalente einzelne Untertypen ausgemacht, welche auf einer Frequenz- und Akzeptanz-Skala unterschiedliche Ränge einnehmen (Lorenzetti 2002, S. 79-81). 
Schülerin I: Una parte della regione Indiana che è il Deccan che ha una lunga storia che tanto tempo fa faceva parte del continente Africano che è andato a spostarsi verso l'asia [sic] e con le placchè convergenti e andato asbattere ecco perché in quella regione rioviamo [sic] i monti più alti della terra.

(Ein Teil der indischen Region, welcher der Dekkan ist, der eine lange Geschichte hat, wo er vor langer Zeit zum afrikanischen Kontinent gehörte, der sich in Richtung asien [sic] verschoben hat, und mit den konvergierenden Erdplatten ist er zusammengestoßen, deshalb finden wir in dieser Region die höchsten Berge der Welt.)

Im Beispiel aus dem Text der Schülerin A wird das polyvalente che anstelle des erforderlichen Relativpronomens di cui (von denen) verwendet, was sich damit erklären lässt, dass das Pronomen im Relativsatz weder das Subjekt noch das Akkusativobjekt, sondern ein präpositionales Objekt darstellt. Diese Funktion hat das Pronomen deutlich seltener als eine Subjekt- oder Akkusativobjektfunktion, weshalb die mehrsprachige Schülerin die Verknüpfung anscheinend nicht formal korrekt bilden konnte. An späterer Stelle verwendete sie erneut ein polyfunktionales che anstelle des Relativpronomens di cui. Es steht in einem Satz, in dem sie ein anderes Relativpronomen mit der Funktion eines präpositionalen Objekts, in cui (in denen), standardsprachlich formuliert. So gibt es offenbar auch innerhalb der Gruppe der Relativpronomen mit Funktion als präpositionales Objekt Unterschiede in der Frequenz ihrer Verwendung und der daraus hervorgehenden Erwerbsbedingungen. ${ }^{200}$

Das längere Satzgefüge von F beginnt mit einem Hauptsatz, an den sich ein mit dove (wo) eingeleiteter Relativsatz anschließt, von dem ein weiterer Relativsatz abhängt. Danach geht es parataktisch mit einem durch die Konjunktion und angeschlossenen Hauptsatz weiter, auf den die beiden Konjunktionen cioè und che folgen, um einen kurzen Nebensatz anzubinden, an den sich ein weiterer durch und verbundener Satz anschließt. Der gemeinsame Gebrauch der beiden Konjunktionen cioè und che ist formal nicht korrekt, stattdessen müsste es etwa ciò significa che (das bedeutet, dass) heißen oder che significa che (was bedeutet, dass), bzw. das polyvalente che könnte ganz entfallen.

Desweiteren gehen auf das umgangssprachlich Mündliche der Gebrauch von starci mit der Bedeutung von esserci (da sein, geben) und die fehlende Kongruenz zwischen dem Numerus von Subjekt und Prädikat am Ende des Satzgefüges zurück, die vielleicht eine Folge seiner Länge ist. So müsste das

200 Der Satz lautet: „L'agricoltura è molto sviluppata nelle zone pianeggianti e dove scorrono $i$ fiumi, e vengono coltivati vari cose: il riso, che l'India è la migliore produttrice, dopo la Cina, è coltivato ovunque ma di più nelle zone in cui c'è pioggia abbondante; il tè, la cannella ed il cotone, coltivati in India ed in Sri Lanka. "(Die Landwirtschaft ist sehr entwickelt in den ebenen Gebieten und wo die Flüsse fließen, und es werden verschiedene Sachen angebaut: Der Reis, ,wo“ Indien die beste Produzentin ist nach China, wird überall angebaut, aber mehr in den Gebieten, in denen es viel Regen gibt; der Tee, der Zimt und die Baumwolle, in Indien und in Sri Lanka angebaut.) 
letzte Prädikat in der dritten Person Singular konjugiert werden und nicht im Plural. ${ }^{201}$

Im Textauszug der Schülerin I wird ein che als generischer Konnektor gebraucht, das von der Standardsprache abweicht. Normgerechter wäre eine Unterteilung des langen Satzgefüges, bei der das erste Stück genau bis zum che ein eigenständiges Gefüge bilden könnte, so dass danach das che entfiele und ein neues Gefüge begänne. Alternativ könnte das che erhalten bleiben, indem ihm die Funktion eines Relativpronomens verliehen und der so entstehende Relativsatz mithilfe der Konjunktion ,und“ dem vorausgehenden nebengeordnet würde, wobei das che des nächsten Relativsatzes entfallen sollte, so dass endlich der Hauptsatz fortgeführt wird: „Una parte della regione Indiana che è il Deccan che ha una lunga storia e che tanto tempo fa faceva parte del continente africano è andato a spostarsi verso l'Asia [...] " (Ein Teil der indischen Region, welcher der Dekkan ist, der eine lange Geschichte hat und der vor langer Zeit zum afrikanischen Kontinent gehörte, hat sich nach Asien verschoben $[\ldots])$.

\subsubsection{Verben}

In den nächsten beiden Unterkapiteln werden zuerst Informationen über die verwendeten finiten Verbformen gegeben, woran sich eine Beschreibung der infiniten Verbformen (Partizipien, Gerundien und Infinitive) anschließt.

\subsection{Prädikate}

Bezüglich der verwendeten Prädikate lässt sich der Tabelle 5 entnehmen, dass sich in den Texten durchschnittlich 37 Prädikate befinden (s. letzte Spalte für alle Mittelwerte), die sich mit einer Anzahl von 2,3 auf die einzelnen Sätze verteilen. ${ }^{202}$

201 Am Ende seines Textes wiederholt der Schüler diesen von der Norm abweichenden Gebrauch der beiden Konjunktionen cioè und che neben weiteren logisch-semantischen und grammatikalischen Fehlern, die an dieser Stelle nicht besprochen werden (Hervorhebung nicht im Original): „Un altro motivo sarebbe l'autosufficienza alimentare, cioè che alcuni campi non possono essere coltivati durante la Stagione dei Monsoni e gli agricoltori non annaffiano e $i$ campi prendono l'acqua dalle precipitazioni e se i campi non vengono annaffiati ci sarebbe un periodo di carestia" (Ein anderer Grund wäre Nahrungsmittelautonomie, das heißt, dass einige Felder während der Monsunzeit nicht bestellt werden können und die Landwirte nicht bewässern und die Felder das Wasser von den Niederschlägen nehmen, und wenn die Felder nicht bewässert werden, gäbe es eine Periode der Hungersnot).

202 Neben der Gesamtzahl der Verben und Informationen zu ihren Gebrauchsmarken sind in der Tabelle auch Angaben zu der totalen und der prozentualen Anzahl der Prädikate essere (Fo; sein), esserci (AU; da sein) und starci (CO; da sein) enthalten. Da der Ausdruck starci allerdings, wie im Mündlichen üblich, synonym zu esserci und nicht in seiner eigentlichen Be- 


\begin{tabular}{lrrrrrrrrrrrrrr}
\hline SCH & $\mathrm{A}$ & $\mathrm{B}$ & $\mathrm{C}$ & $\mathrm{D}$ & $\mathrm{E}$ & $\mathrm{F}$ & $\mathrm{G}$ & $\mathrm{H}$ & $\mathrm{I}$ & $\mathrm{J}$ & $\mathrm{K}$ & $\mathrm{L}$ & $\mathrm{M}$ & $\varnothing$ \\
\hline $\begin{array}{l}\text { Prädi- } \\
\text { kate }\end{array}$ & 40 & 26 & 22 & 43 & 45 & 62 & 37 & 11 & 42 & 98 & 29 & 35 & 32 & 40 \\
$\begin{array}{l}\text { Wort- } \\
\text { typen }\end{array}$ & 15 & 11 & 13 & 17 & 17 & 25 & 16 & 8 & 17 & 45 & 19 & 16 & 15 & 18 \\
loc.v. & 0 & 0 & 0 & 0 & 0 & 0 & 0 & 0 & 1 & 1 & 1 & 0 & 2 & 0,4 \\
v.aus. & 7 & 1 & 1 & 3 & 5 & 7 & 3 & 1 & 5 & 10 & 5 & 1 & 2 & 4 \\
v.mod. & 0 & 0 & 0 & 4 & 0 & 3 & 0 & 0 & 0 & 1 & 0 & 0 & 1 & 1 \\
Passiv & 6 & 1 & 1 & 1 & 5 & 5 & 0 & 1 & 3 & 7 & 0 & 1 & 2 & 3 \\
essere & 21 & 12 & 7 & 18 & 20 & 13 & 12 & 3 & 16 & 29 & 2 & 12 & 7 & 13 \\
\% es- & 53 & 46 & 32 & 42 & 44 & 21 & 32 & 27 & 38 & 30 & 7 & 34 & 22 & 33 \\
sere & & & & & & & & & & & & & & \\
es- & 3 & 0 & 3 & 2 & 0 & 10 & 2 & 1 & 1 & 6 & 5 & 4 & 6 & 3 \\
serci & & & & & & & & & & & & & & \\
$\%$ es- & 60 & 46 & 46 & 47 & 44 & 37 & 38 & 36 & 40 & 36 & 24 & 46 & 41 & 42 \\
sere + & & & & & & & & & & & & & & \\
es- & & & & & & & & & & & & & & \\
serci & & & & & & & & & & & & & \\
n & 0 & 3 & 0 & 1 & 1 & 1 & 0 & 0 & 0 & 0 & 0 & 0 & 0 & 0 \\
starci & & & & & & & & & & & & & & \\
AD & $4(2)$ & 1 & 1 & 0 & 1 & $3(2)$ & $2(1)$ & 0 & 0 & 0 & $1(1)$ & 0 & 0 & 1 \\
AU & 3 & 3 & 3 & 4 & 3 & 12 & 2 & 2 & 2 & 3 & 5 & 5 & 7 & 4 \\
& $(1)$ & $(1)$ & $(1)$ & $(3)$ & $(1)$ & $(3)$ & $(1)$ & $(2)$ & $(2)$ & $(2)$ & $(1)$ & $(1)$ & $(2)$ & $(2)$ \\
CO & 4 & 1 & 2 & 3 & 2 & 5 & 7 & 2 & 5 & 11 & 6 & 0 & 9 & 4 \\
& $(3)$ & & $(2)$ & $(1)$ & & $(2)$ & $(5)$ & $(2)$ & $(4)$ & $(4)$ & $(6)$ & & $(6)$ & $(3)$ \\
FO & 34 & 22 & 15 & 38 & 21 & 49 & 28 & 6 & 40 & 69 & 20 & 28 & 18 & 29 \\
& $(10)$ & $(9)$ & $(7)$ & $(14)$ & $(8)$ & $(18)$ & $(10)$ & $(9)$ & $(15)$ & $(30)$ & $(14)$ & $(15)$ & $(9)$ & $(13)$ \\
$\varnothing$ pro & 2,4 & 2 & 1,5 & 1,8 & 1,4 & 3,9 & 1,8 & 1,6 & 2,6 & 2 & 3,6 & 1,8 & 4 & 2,3 \\
Satz & & & & & & & & & & & & & & \\
\hline
\end{tabular}

Tabelle 5: Prädikate der Schülertexte an der Morante

Der Großteil der Prädikate in den Schülertexten besteht aus Wortformen des fundamentalen Wortschatzes im Indikativ Präsens aktiv (z.B.: vivere (leben), andare (gehen), coltivare (anbauen), potere (können)). Diese vorherrschende Tempus- und Moduswahl ist für einen darstellenden und erklärenden Text sortenspezifisch. Nur durchschnittlich zwei Prädikate sind im Passiv konstruiert, und nur fünf Prädikate (in 4 Texten) bestehen aus Phrasemen. Die geringste Anzahl an unterschiedlichen Worttypen wurde mit neun im kürzesten Text verzeichnet, in dem sich insgesamt elf Prädikate zählen lassen, und die meisten unterschiedlichen Worttypen im längsten Text, nämlich 48 bei 98 Prädikaten.

Das durchschnittliche Wortvorkommnis geht auf 18 verschiedene Worttypen zurück. Wie dem unteren Teil der Tabelle, der die Gebrauchsmarken der Prädikate illustriert, zu entnehmen ist, entspricht dem nicht etwa eine ungefähr

deutung von ,enthalten können“, ,reinpassen“ verwendet wurde, ist er nicht mit der im GRADIT angegeben CO-Gebrauchsmarke, sondern mit der AU-Marke des Verbs esserci versehen worden. Bei den Gebrauchsmarken ist nach den Wortvorkommnissen in Klammern die Anzahl der einzelnen Worttypen angegeben. Bezüglich der Summe der verschiedenen Worttypen ist bei essere und avere (Fo; haben) danach unterschieden worden, ob es sich um ein Voll- oder ein Hilfsverb (v.aus.) handelt und im letzteren Fall, ob es der Tempus- (Perfekt) oder der Passivbildung dient. 
zweimalige Verwendung aller Verben, sondern werden einige wenige Verben mit gesteigerter Frequenz wiederholt. So gebrauchten die Lernenden auffallend oft das semantisch schwache „sein“, das einen durchschnittlichen Anteil von 33 Prozent an der Gesamtzahl der Prädikate hat. Dabei stellt es in vier Texten mit Werten von 42 bis 60 Prozent ungefähr jedes zweite Prädikat und wird nur in einem Text viel seltener (sieben Prozent bei zwei Vorkommnissen) als in allen anderen verwendet. Zählt man auch die mit Formen von „da sein“ (esserci) gebildeten Prädikate hinzu, die in der Bedeutung zu „sein“ ähnlich sind, machen die beiden Verben zusammen einen durchschnittlichen Anteil von 42 Prozent aus, der in der knappen Hälfte der Texte (6 von 13) mindestens 46 Prozent entspricht und in einem Fall sogar bis zu 63 Prozent reicht.

Das Verb essere wurde oft prädikativ gebraucht, weshalb die Sätze gleichförmig mit der Nennung des Subjekts beginnen, woran sich nach dem Prädikat ein Prädikatsnomen anschließt, das teils durch ein Adjektiv, teils durch ein Substantiv gebildet ist. Auf diese Weise entstanden Texte, die durch eine Aneinanderreihung von Fakten bei steter Wiederholung ein und desselben Prädikats charakterisiert sind. Hierdurch wirken sie zum einen recht eintönig auf den Leser, zum anderen werden so auch eventuelle Bezüge zwischen den einzelnen Textaussagen nicht expliziert. Ein Bespiel hierfür ist der folgende Auszug aus dem Text des Schülers B (Hervorhebung nicht im Original):203

L'isola più grande della regione indiana è lo Sri Lanka e poi ci sta l'arcipelago delle Maldive dove ci vanno molti turisti da tutto il mondo per il paesaggio suggestivo.

Nella catena montuosa dell'Himalaia, la montagna più alta è l'Everest invece nel Karakoran ci sta il K2.

La densità della popolazione nella regione indiana è di 320 abitanti per km².

Il clima nella regione indiana è: a sud della penisola del deccan [sic] il clima è tropicale ma attenuato; a nord della penisola il clima è tropicale e monsonico e infine il clima tra le montagne è molto freddo.

La distribuzione di popolazione è irregolare.

(Die größte Insel der indischen Region ist Sri Lanka, und dann gibt es die Inselgruppe der Malediven, wo viele Touristen aus aller Welt hinfahren wegen der suggestiven Landschaft.

In der Bergkette des Himalaya ist der höchste Berg der Everest, im Karakoran hingegen gibt es den K2.

Die Dichte der Bevölkerung in der indischen Region ist 320 Einwohner pro km².

Das Klima in der indischen Region ist: Im Süden der Halbinsel dekkan [sic] ist das Klima tropisch, aber gemäßigt, im Norden der Halbinsel ist das Klima tropisch und monsunisch, und das Klima zwischen den Bergen schließlich ist sehr kalt.

Die Verteilung der Bevölkerung ist unregelmäßig.)

In diesem Textauszug kommen neben den Prädikatsformen von essere nur zwei weitere Prädikate vor. Sie gehen beide auf das Verb starci zurück, dessen

203 Die in diesem und dem folgenden Zitat enthaltenen Rechtschreibfehler und die Gliederung in Absätze entsprechen den Originaltexten. Die Hervorhebung hingegen dient der Veranschaulichung der vorgestellten Prädikativverwendung und Wortwiederholung. 
Verwendung als Synonym zu esserci stark durch den informalen mündlichen Sprachgebrauch der Lernenden geprägt ist.

Ähnliche Texte entstanden nicht nur durch die vermehrt auftretenden Prädikativa, sondern beispielsweise auch bei einem wiederholten Gebrauch von esserci (da sein), starci (hier: da sein) oder trovarsi (sich befinden), wie das folgende Textbeispiel der Schülerin D veranschaulicht (Hervorhebung nicht im Original):

L'India si trova nel continente Euro-Asiatico ed è posta nel Centro-Meridione dell'Asia. L'India confina a Nord-Ovest con il Pakistan a Nord-Est con la Cina e con il Bangladesh. Troviamo anche un'isola importante che si chiama Sri Lanka. L'India è bagnata a Ovest dal Mar Arabico a Sud con il Oceano Indiano e a Est con il Golfo del Bengala. A Nord si trova uno dei monti più alti del mondo che è l'Himalaia, oltre all'Himalaia c'è l'Evereste [sic] e il K2. nel Nord dell'India dove si trova l'Himalaia è molto montuoso, e da lì in giù è collinare e pianeggiante. Verso Nord-Ovest troviamo il deserto del Tahar. Il clima è di tipo Monsonico sul Tropicale. I fiumi più importanti sono l'Indo, il Gange e il Brahamputra. Il fiume più sacro è Gange dove molti indiana vanno lì per purificarsi. La capitale dell'India è New Delhi. La popolazione indiana è 20 volte più di quella italiana. La popolazione è più tosto sovrapopolata [sic], e gran parte della popolazione vive nei villaggi. La città più popolata è Mumbai che ha allincirca [sic] undici mila abitanti per chilometro. Le lingue parlate in India sono molte tra cui possono essere addirittura $16 \mathrm{ma}$ ci possono essere anche migliaia di dialetti.

(Indien befindet sich auf dem euroasiatischen Kontinent und ist in Südzentralasien gelegen. Indien grenzt im Nordwesten an Pakistan und im Nordosten an China und an Bangladesch. Wir finden auch eine wichtige Insel, die Sri Lanka heißt. Indien ist im Westen vom Arabischen Meer umspült und im Süden mit dem Indischen Ozean und im Osten mit dem Golf von Bengalen. Im Norden befindet sich einer der höchsten Berge der Welt, der das Himalaya ist, außer dem Himalaya gibt es den Everest und den K2. Im Norden von Indien, wo sich das Himalaya befindet, ist es sehr gebirgig, und von dort nach unten ist es hügelig und flach. Gegen Nordwest finden wir die Thar-Wüste. Das Klima ist von monsunischer auf tropische Art. Die wichtigsten Flüsse sind der Hindus, der Ganges und der Brahmaputra. Der heiligste Fluss ist der Ganges, wo viele Inder hingehen, um sich zu läutern. Die Hauptstadt Indiens ist New Delhi. Die indische Bevölkerung ist 20-mal mehr als die italienische. Die Bevölkerung ist ziemlich überbevölkert, und ein Großteil der Bevölkerung lebt auf den Dörfern. Die bevölkerteste Stadt ist Mumbai, die circa elftausend Einwohner pro Kilometer hat. Die gesprochenen Sprachen in Indien sind viele, darunter können sogar 16 sein, aber es kann dort auch tausende von Dialekten geben.)

Neben dem dreimal wiederholten trovarsi und dem ähnlichen trovare (finden) in unmittelbarer Textnähe sind auch in diesem Auszug vor allem Kopulaformen des Verbs essere und das semantisch ähnlich leere Verb avere (haben) vertreten. Eine der wenigen Varianten bei der Wahl der Prädikatsworttypen zeigt sich im letzten Satz, in dem die Schülerin zweimal zusätzlich zu da sein das Modalverb potere (können) als Prädikat verwendet. Diese Wahl ist jedoch stilistisch fragwürdig. Desweiteren geht aus dem Textauszug hervor, dass Schülerin D den von den Erdkundelehrkräften erwünschten Ausdruck essere bagnato da (von ... umspült sein) verwendet. Jedoch fiel sie aus der Konstruktion, wie man an der Fortführung der Aufzählung der verschiedenen Meere mit 
der Präposition con (mit) anstelle von $d a$ (von) sieht und was darauf hindeutet, dass sie beim Schreiben den ihr geläufigeren Ausdruck confina con im Sinn hatte.

Die geringe Abwechslung im Textaufbau, die durch die vielen Prädikativa entsteht, wird zudem dadurch verstärkt, dass die Lernenden oft Subjekte wiederholten, anstatt mit Synonymen und Pronomen Kohäsion zu erzeugen.

Auch enthalten die Texte bisweilen dort Prädikate, wo geübtere Schreibende dieselben Inhalte nominal als Attribute formulieren würden. Das führt zwar zu einer höheren Textdichte, entspricht damit jedoch der üblichen Weise, schriftliche Texte zu verfassen. Stattdessen verhält es sich beispielsweise beim folgendem Satzgefüge des Schülers K so, dass nicht nur die Prädikatsverwendung vermeidbar ist, sondern es im Ganzen und insbesondere aufgrund der lexikogrammatikalischen Gestaltung durch einen weniger formalen, dem mündlichen Sprachgebrauch entlehnten Aufbau gekennzeichnet ist:

Io non andrei a vivere in India perché avendo una densità altissima è una città caotica e c'è molta gente, certo andrei sicuramente a vivere nelle Maldive o nelle altre isole, ma in India mi piacerebbe andare a visitarla, ma non a viverci.

(Ich würde nicht losziehen, um in Indien zu leben, weil es, eine äußerst hohe Bevölkerungsdichte habend, eine chaotische Stadt ist, und es gibt viele Leute. Klar, ich würde sicherlich losziehen, um auf den Malediven zu leben oder auf den anderen Inseln, aber nach Indien würde ich gern losziehen, um es zu besichtigen, aber nicht, um dort zu leben.)

Nicht typisch für den mündlichen Sprachgebrauch, sondern vielmehr für den schriftlichen ist die Verwendung des Gerundiums avendo in der ersten Zeile. Dieses bildungssprachliche Element trifft aber sofort mit einem kurzen Hauptsatz aufeinander, der das konzeptionell mündliche Prädikat $c$ 'è enthält und den sprachlich Gewandtere nicht einmal durch ein Attribut ersetzen würden, sondern ganz ausließen, da er inhaltlich eine Wiederholung darstellt. Positiv ist zu vermerken, dass der Schüler die Aufgabenstellung beachtete und seine Entscheidung, in Indien nicht leben zu wollen, begründete. Allerdings ist innerhalb seiner Aussage keine Kohäsion gegeben, da er synonym zu Indien den Ausdruck „Stadt" gebraucht. Außerdem sticht die eher im Mündlichen übliche, verstärkende Verwendung zweier semantisch fast gleichbedeutender Wörter, nämlich certo und sicuramente, hervor und ist auch die den Satz abschließende Aussage morphosyntaktisch nicht korrekt. Als solche ist sie eher im mündlichen Sprachgebrauch anzutreffen. Ferner wird die Präposition $a$ anstelle der eigentlich erforderlichen per verwendet.

Bezüglich der Gebrauchswortschatzbereiche, aus denen die Prädikate in den Schülertexten stammen, ist der Tabelle zu entnehmen, dass sich darunter keine fachsprachlichen Verben befinden. ${ }^{204}$ In der guten Hälfte der Texte sind ein bis zwei AD-gekennzeichnete Verben verzeichnet, nämlich:

204 Unter den als fachsprachlich gekennzeichneten Wörtern macht die Gruppe der Verben aber nur einen kleinen Anteil aus; größtenteils sind es Substantive, die fachsprachlich sind. 
- sfociare (münden);

- spianare (ebnen);

- evaporare (verdunsten);

- annaffiare (gießen);

- basarsi (sich stützen auf).

Von diesen Verben erscheinen nur annaffiare und evaporare nicht auf den Lehrbuchseiten zur indischen Region. Anstelle des ersten Verbs findet sich dort irrigare, das zum allgemeinem Wortschatz zählt und der Schüler wahrscheinlich zwar gehört bzw. gelesen hatte und verstand oder aus dem Kontext erschloss, allerdings nicht aktiv produzierte. Stattdessen verwendete er das ihm auch aus dem Alltag geläufigere annaffiare.

Die AU-gekennzeichneten Prädikate gehören in den einzelnen Schülertexten maximal zu drei verschiedenen Worttypen, in der Regel nur zu einem:

- esserci (da sein);

- $\quad$ starci (hier: da sein);

- scaricare (entladen);

- confinare (grenzen an);

- utilizzare (gebrauchen);

- prevalere (vorherrschen);

- coincidere (zusammenfallen);

- alimentare (ernähren, speisen);

- coinvolgere (verwickeln);

- esportare (exportieren).

Von diesen zehn Verben werden sechs auch auf den Lehrbuchseiten zur indischen Region verwendet, nämlich esserci, confinare, utilizzare, prevalere, alimentare und esportare. Das erste Verb erscheint jedoch auf den Seiten im Unterrichtswerk wesentlich seltener als in den Schülertexten und wird zudem oft durch sein weniger gebräuchliches Synonym esservi aus dem allgemeinen Wortschatz (CO) ersetzt.

Verben aus dem allgemeinen Wortschatz wurden von den Lernenden durchschnittlich viermal pro Text verwendet, was im Einzelnen von einem Minimum von null bis zu einem Höchstmaß von elf Vorkommnissen von vier verschiedenen Worttypen und einem von sechs Worttypen mit neun verschiedenen Vorkommnissen reicht. Insgesamt handelt es sich um Tokens der folgenden 22 Ausdrücke: 205

allargarsi (sich erweitern); attaccare (angreifen); colonizzare (kolonisieren); convergere (konvergieren); costituire (bilden, darstellen); delimitare (begrenzen); estendersi (sich ausbreiten); fare capo (abhängen von, sich an jm. wenden), fare parte (Teil sein); formarsi (sich bilden); innovare (erneuern); prolungarsi (sich verlängern); protendersi (überhängen, sich

205 Die in der folgenden Aufzählung hervorgehobenen Wörter werden auch auf den Lehrbuchseiten zur indischen Region verwendet. 
vorbeugen); rendersi (sich ... machen); ricordarsi (sich erinnern); sfoggiare (zur Schau stellen); sollevarsi (sich erheben); spirare (wehen); spostarsi (sich verlagern. rücken); trovarsi (sich befinden); unirsi (sich zusammenschließen); venire (kommen, hier: Hilfsverb in der Konstruktion einfacher (nicht zusammengesetzter Tempora im Passiv).

Davon wird fast die Hälfte (10) auch auf den entsprechenden Seiten im Erdkundebuch verwendet, und es ist anzunehmen, dass ein weiterer Teil dieser Wörter wie z.B. delimitare, spirare und unirsi aus anderen behandelten Lehrbuchtexten und dem (Erdkunde-)Unterricht bekannt war.

Bei anderen Co-gekennzeichneten Wörtern handelt es sich hingegen um Verben, deren Gebrauch man als häufiger einschätzen könnte und die man deshalb eher dem Basiswortschatz zuteilen würde. Sie sind allen oder den meisten Lernenden bekannt und werden auch mündlich im Schulalltag verwendet, wie ricordarsi, spostarsi und rendersi. Diese reflexiven Verben sind wie fast alle der pronominalen Kategorie im GRADIT so gut wie ausnahmslos Co-gekennzeichnet, leiten sich aber von nicht reflexiven Verben wie ricordare, spostare und rendere ab. Diese sind in vielen Fällen gleichbedeutend, bzw. weisen nur Nuancen im Bedeutungsunterschied auf, und zählen zum fundamentalen Wortschatz, weshalb die Aneignung ihrer pronominalen Fast-Synonyme leichtfallen sollte.

Das letzte Wort der Aufzählung, venire, ist bei der Passivbildung gerade im Mündlichen sehr gebräuchlich, und wurde beispielsweise während der Abfragungen im Erdkundeunterricht benutzt.

\subsection{Nicht flektierte Verbformen}

Komplexere verbale Gestaltungsmittel wie Gerundien oder Partizipien als Teil implizierter Relativsätze, wie sie als Merkmal der Lehrbuchtexte im Zuge der Lehrbuchanalyse vorgestellt wurden, lassen sich in den Schülertexten kaum finden.

Die Lernenden verwendeten pro Text durchschnittlich ein Gerundium, wobei über die Hälfte der Texte (7) gar kein Gerundium aufweist, in vier Texten eines erscheint, in einem Text drei und in einem weiteren neun. Dieser an Gerundien reiche Text veranschaulicht sehr deutlich, dass sein Gebrauch im Unterrichtswerk und in den Schülertexten deutlich differiert: Schüler F formulierte viermal das Gerundium parlando di (von ... sprechend), das nicht nur von ihm, sondern auch von vielen MitschülerInnen und in den anderen beobachteten Klassen während der mündlichen Abfragungen oft verwendet wurde, in einem formalen schriftlichen Text aber unpassend ist. Darüber hinaus tauchen in seinem Text Gerundien als Bestandteil einer zusammengesetzten Zeitform auf, die dem Präsens nahesteht und einen zeitlichen Verlauf zum Ausdruck bringt, z.B. sta crescendo (ist dabei zu wachsen; Hervorhebung nicht im Original): 
Parlando del Settore Secondario ci sono molte miniere di Carbone, Ferro, Manganese e Bauxite e ci sono anche industrie petrolifere, meccaniche, tessili e chimiche, mentre parlando del Settore Terziario possiamo dire che il turismo sta crescendo e la rete stradale è un po' diminuita.

(Vom Sekundärsektor sprechend, gibt es viele Kohle-, Eisen-, Mangan- und Bauxit-Minen und es gibt auch Erdölindustrien, mechanische, Textil- und chemische, während wir, vom Tertiärsektor sprechend, sagen können, dass der Tourismus dabei ist zu wachsen und das Straßennetz etwas geschrumpft ist.)

Bildungssprachlicher geprägte Verwendungen von Gerundien liegen hingegen in den Texten von J und L vor, wie die folgenden Beispiele veranschaulichen, von denen die ersten beiden aus dem Text von $\mathrm{J}$ und das dritte aus dem von $\mathrm{L}$ stammen (Hervorhebungen nicht in den Originalen):

Quando l'India si è resa indipendente (1946) la lingua ufficiale divenne l'Hindi, ma i popoli si ribellarono rivendicando la propria lingua e per questo motivo ancora oggi la lingua più parlata è l'inglese.

(Als Indien sich unabhängig machte (1946), wurde die Amtssprache Hindu, aber die Völker begehrten auf, indem sie für ihre eigene Sprache eintraten, und aus diesem Grund ist die heute noch am meisten gesprochene Sprache Englisch.)

Solo 16 sono le lingue ufficiali, considerando che sono presenti migliaia di dialetti.

(Nur 16 sind die Amtssprachen, wenn man berücksichtigt, dass es Tausende von Dialekten gibt.)

Per quanto riguarda l'economia, è basata sull'agricoltura ed essendoci numerose piogge, si coltivano prodotti come il riso che ha bisogno di moltissima acqua.

(Was die Wirtschaft betrifft, basiert sie auf der Landwirtschaft und ,zahlreiche Regenfälle gebend“", baut man Produkte wie Reis an, der sehr viel Wasser benötigt.)

Auch wenn das Gerundium im letzten Beispiel zwar dem Schreibanlass angemessen verwendet wird, so klingt die Konstruktion dennoch nicht sehr bildungssprachlich, da sie das Akkusativobjekt numerose piogge regiert, das stilistisch treffender mit dem Lexem precipitazioni formuliert wäre (essendo numerose le precipitazioni).

Insgesamt befinden sich in den Schülertexten außerdem 15 Partizipien Perfekt, die auf folgende neun Verben zurückgehen:

- sovraffollare (Co; überlaufen, überfüllen);

- unire (Fo; vereinigen, verbinden; 2 Tokens);

- formare (Fo; formen; 3 Tokens);

- delimitare (CO; begrenzen);

- considerare (Fo; berücksichtigen, ansehen);

- coltivare (Fo; anbauen)

- parlare (Fo; sprechen; 2 Tokens);

- raggruppare (AU; gruppieren; 2 Tokens);

- usare (Fo; benutzen; 2 Tokens);

- utilizzare (AU; gebrauchen). 
Beim ersten handelt es sich um einen Gebrauch mit adjektivischer Funktion. Es ist durch ein Attribut ergänzt, das als überflüssig zu erachten ist, da es den semantischen Gehalt des partizipialen Adjektivs wiederholt (Schüler B; Hervorhebung nicht im Original): „L'India è una regione sovraffollata dalla popolazione (1 miliardo e 41 milioni di persone)“ (Indien ist eine von der Bevölkerung überlaufene Region (1 Milliarde und 41 Millionen Personen).

Das zweite Partizip wurde von Schüler H und Schülerin A in fast identischer Weise verwendet und greift eine Formulierung aus dem Lehrbuch auf (Hervorhebung nicht im Original):

Schüler H: La regione indiana ha la forma di un triangolo rovesciato unito al resto dell'Asia dalla catena dell'Himalaya dove prevale un clima freddo. (Die indische Region hat die Form eines umgekehrten Dreiecks, das mit dem Rest von Asien durch die Bergkette des Himalayas verbunden ist, wo ein kaltes Klima vorherrscht.)

Schülerin A: La regione ha una forma di un grandissimo triangolo rovesciato, unito dal resto dell'Asia, dalla catena dell'Himalaya dove fa freddo. (Die Region hat die Form eines riesengroßen umgekehrten Dreiecks, das mit dem Rest Indiens durch die Bergkette des Himalayas verbunden ist, wo es kalt ist.)

Bersezio (2005, S. 115): La regione indiana ha la forma di un immenso triangolo rovesciato, unito al resto dell'Asia dalla catena himalaiana, dove prevale un clima nivale. (M.Ü.: Die indische Region hat die Form eines immensen umgekehrten Dreiecks, das mit dem Rest Asiens durch die Bergkette des Himalayas verbunden ist, wo ein nivales Klima vorherrscht.)

Abbildung 1: Partzipvervendung von unito in verschieden Texten an der Schule Morante

Bei diesem Ausschnitt aus dem Lehrbuch handelt es sich um den ersten Satz des ersten kurzen Textes auf Seite 115, der neben einer Karte der indischen Region abgebildet ist und diese beschreibt. Es ist also nicht verwunderlich, dass er sich den beiden Lernenden eingeprägt hatte. Auch überrascht es wenig, dass die beiden die drei Lexeme immenso (Fo), himalaiano und nivale (Ts geogr.) durch Ausdrücke ersetzten, die ihnen wahrscheinlich geläufiger waren, bzw. im Falle der Bergkette durch eine Wortart, die ihnen in diesem Kontext vertrauter war, nämlich Himalaya als Substantiv.

Das dritte Partizip Perfekt ist ebenfalls den Lehrbuchseiten entnommen und wurde von den Lernenden J, G und F in einem sehr ähnlichen Zusammenhang gebraucht. Von den im Unterrichtswerk enthaltenen Informationen über die Schwemmebene gaben sie neben den mit der Partizipialkonstruktion formulierten auch die bezüglich ihrer Lage und Größe wieder (Hervorhebungen nicht in den Originalen):

Schüler J: Poi troviamo una delle più grandi pianure alluvionali del mondo, e cioè formate dal depositamento dei detriti del fiume Gange; nella zona fra Pakistan ed India troviamo il deserto del Thar. (Dann finden wir eine der größten Schwemmebenen der Welt, und das heißt, die sind durch die Ablagerung von Geröll des Flusses Ganges entstanden, in der Gegend zwischen Pakistan und Indien finden wir die Wüste vom Thar.) 
Schülerin G: Lungo il corso del fiume Gange si estende una pianura più vasta pianura [sic] alluvionali della terra formata dai sedimenti delle acque. (Entlang des Flussverlaufes des Ganges breitet sich eine Ebene größte Schwemmebene [sic] der Erde aus, die von den Ablagerungen des Wassers geformt wurde.)

Schüler F: lo personalmente non ci andrei a vivere in India perché nella Pianura del Ghange [sic] che è una pianura alluvionale cioè formata dai detriti del fiume Ghange [sic] ci sono molte zone paludose e poi perché il $70 \%$ della popolazione vive nelle campagne dove ci sono solo sentieri e non ci sono le fogne. (Ich persönlich würde nicht dorthin losziehen, um in Indien zu leben, weil es in der Ebene des Ghanges [sic], die eine Schwemmebene ist, das heißt, die von den Ablagerungen des Flusses Ghanges [sic] wurden, viele Sumpfgegenden gibt, und dann leben 70 Prozent der Bevölkerung auf dem Land wo es nur (nicht gepflasterte) Wege gibt und es keine Abwasserkanäle gibt.)

Bersezio $(2005, \text { S. 116 })^{206}$ : Lungo il corso del Gange si estende una delle più vaste pianure alluvionali della Terra, formata dai sedimenti depositati dalle acque. (M.Ü: Entlang des Verlaufes des Ganges breitet sich eine der größten Schwemmebenen der Welt aus, die durch die abgelagerten Sedimente des Gewässers entstanden ist.)

Abbildung 2: Textauszüge zum Partizip Perfekt formata/formate, Morante

Im ersten Textauszug äußern sich die Bemühungen des Schülers um einen bildungssprachlichen Ausdruck in der Verwendung der Partizipialkonstruktion und der grammatikalischen Metapher depositamento, d.h. der Nominalisierung eines Prozesses, der als Teil des impliziten Relativsatzes zusammen mit dem Partizip eine Erklärung für die Entstehung des im Vorfeld Genannten bildet. Diesen Bestrebungen steht die Verwendung einiger sprachlicher Mittel entgegen, die der konzeptionellen Mündlichkeit zuzuordnen sind, bzw. Unerfahrenheit beim Schreiben offenbaren. So existiert das Nomen depositamento in der italienischen Sprache nicht, auch wenn es verständlich ist. Ferner verrät es, dass der Schüler Kenntnisse über die Wortbildungsregeln besitzt, weil das Suffix -mento Verben in Nomen verwandelt und einen Prozess zum Ausdruck bringt. Im Falle des Verbs depositare (CO) ist das daraus geformte Nomen jedoch deposito (AU). Es ist möglich, dass der Schüler es nicht verwendete, da es ihm vor allem in seiner Bedeutung von „Lager“ bekannt war und er es deshalb für kontextunangemessen erachtete.

Auch der unter den Lernenden verbreitete und bereits weiter oben vorgestellte Gebrauch des Ausdrucks cioè ist in einem formalen schriftsprachlichen Text deplatziert, zumal ihn die Lehrkräfte selbst im Mündlichen kritisierten. Darüber hinaus wiederholte der Schüler das Prädikat troviamo, das er außerdem in der ersten Person Plural konstruierte. Zwar werden Verben auch in den untersuchten Lehrbuchtexten manchmal in der ersten Person Plural konjugiert, jedoch weniger häufig als in den Textproduktionen der SchülerInnen und nicht im Zusammenhang mit diesem Prädikat, das in den Lehrbüchern vorzugsweise in der dritten Person Singular des reflexiven Verbs trovarsi (sich befinden)

206 Die Hervorhebung von pianure alluvionali entspricht dem Original. 
auftritt. Der letzte Teil des Satzgefüges enthält außerdem eine völlig neue Information, die zu den vorausgehenden keine engere Bindung hat, so dass sie besser in einem neuen Satz oder Satzgefüge erscheinen oder zumindest durch ein Semikolon abgetrennt werden sollte.

Wie aus den anderen beiden Textauszügen und ihren Übersetzungen zu entnehmen ist, tragen auch diese Züge konzeptioneller Mündlichkeit. Schülerin $\mathrm{G}$ ist mehrsprachig, lebt aber seit langem in Italien. Ihre Wiederholung des Ausdrucks pianura und die damit verbundene verletzte Grammatikalität des Satzes deuten eher darauf hin, dass sie ihren Text nicht noch einmal durchgelesen hatte, da sie diesen Fehler sonst vielleicht bemerkt und verbessert hätte.

Die beiden nächsten Partizipien, die von den Verben considerare und delimitare stammen, erscheinen beide im Text der Schülerin L, doch nur das erste geht auch auf ein Partizip in Verbindung mit dem Adjektiv sacro auf den entsprechenden Lehrbuchseiten zurück. Dort handelt es sich allerdings um eine zusammengesetzte Prädikatsform im Passiv und auch die sprachliche Gestaltung im zweiten Textauszug weicht deutlich vom Unterrichtswerk ab (Hervorhebung nicht im Original).

Schülerin L: I fiumi sono abbondanti grazie al clima monsonico e hanno una foce a delta. I più importanti sono il Gange, considerato il fiume sacro, l'Indo e il Brahmaputra. (Die Flüsse sind dank des Monsunklimas üppig und haben eine Deltamündung. Die wichtigsten sind der Ganges, der als der heilige Fluss betrachtet wird, der Hindus und der Brahmaputra.)

Infine troviamo la penisola del Deccan, delimitata dai Ghati occidentali, che si affacciano sul Mar Arabico, e i Ghati orientali che si affacciano sul Golfo del Bengala. (Schließlich finden wir die Dekkan-Halbinsel, die durch die Westghats begrenzt ist, die auf den Golf von Bengalen gehen.)

Abbildung 3: Implizite Relativsätze einer Schülerin an der Morante

Die beiden Formen des Partizips von sprechen (parlare) befinden sich in den Texten der mehrsprachigen Schülerin D und des einsprachigen Schülers J im Zusammenhang mit der Schilderung der Sprachen, die in Indien gesprochen werden. Beide haben adjektivischen Charakter und sind so auch auf den Lehrbuchseiten vertreten.

Auch die Partizipien coltivati (angebaut) und raggrupate (gruppierte) erscheinen in den Texten der Schülerin A wie auf den Lehrbuchseiten (Hervorhebung nicht in den Originalen):

Schülerin A: [...] vengono coltivati vari cose: il riso, che l'India è la migliore produttrice, dopo la Cina, è coltivato ovunque ma di più nelle zone in cui c'è pioggia abbondante; il tè, la cannella ed il cotone, coltivati in India ed in Sri Lanka. (Der Reis, von dem Indien die beste Produzentin ist nach China, wird überall angebaut, aber am meisten in den Gebieten, in denen es reichlichen Regen gibt; der Tee, der Zimt und die Baumwolle, angebaut in Indien und Sri Lanka.) 
Bersezio (2005, S. 118): Tra gli altri prodotti vengono largamente esportati il tè, la cannella e il cotone, coltivati in India e nello Sri Lanka. (M.Ü: Neben den anderen Produkten werden der Tee, der Zimt und die Baumwolle stark exportiert, die in Indien und in Sri Lanka angebaut werden.)

Schülerin A: La regione indiana occupa una gran parte dell'Asia centro-meridionale e si prolunga fin nell'Oceano Indiano dove si trovano tante isole: le più importanti sono quella dello Sri Lanka e quelle raggruppate nell'Arcipelago delle Maldive. (Die indische Region nimmt einen Großteil Zentral-Südasiens ein und erstreckt sich bis in den Indischen Ozean, wo sich viele Inseln befinden: Die wichtigsten sind die von Sri Lanka und die im Archipel der Malediven gruppierten.

Bersezio (2005, S. 114): La regione indiana occupa una vasta parte dell'Asia centro-meridionale e si prolunga fin nell'Oceano Indiano, dove si trovano varie isole: le più importanti sono quella dello Sri Lanka, che è anche la più vasta, e quelle raggruppate nell'Arcipelago delle Maldive, splendido atollo corallino che si solleva qualche metro sul livello del mare in prossimità dell'Equatore. (M.Ü: Die indische Region nimmt einen Großteil Zentral-Südasiens ein und erstreckt sich bis in den indischen Ozean, wo sich viele Inseln befinden: Die wichtigsten sind die von Sri Lanka, die auch die größte ist, und die im Archipel der Malediven gruppierten, prächtiges Korallenriff, das sich einige Meter über den Meeresspiegel in der Nähe des Äquators erhebt.).

Abbildung 4: Wortgetreue Übernahme des Lehrbuchtextes einschließlich der Partizipien coltivati und raggruppate, Schülerin A

Beim Verwendungskontext des zweiten Partizips im Lehrbuch handelt es sich um die ersten Sätze der Lehrbucheinheit über die indische Region, die von der Schülerin A abzüglich zweier Zusätze in Form eines Relativsatzes und einer Apposition wortwörtlich übernommen wurden.

Das Partizip usato (gebraucht) hingegen erscheint im Text derselben Schülerin in einem Kontext, der die Lehrbuchinformationen so weit verkürzt, dass ihr Sinn entstellt wird: Die Schülerin benutzt das Partizip im Zusammenhang mit den Bodenschätzen Indiens. Sie schreibt, dass sie wenig genutzt werden, während das Lehrbuch angibt, dass sie kaum im Land verarbeitet, sondern fast vollständig exportiert werden.

Auch beim zweiten Gebrauch einer Form desselben Partizips (Schülerin D) entspricht der Inhalt wieder einem vom Lehrbuch vorgegebenen, und zwar bei der Information über Englisch als die in Indien am meisten gesprochene Sprache.

Das letzte Partizip, utilizzato, ist fast bedeutungsgleich zu usato und wird ebenfalls bei der Beschreibung des Sprachgebrauchs in den vorgestellten Ländern verwendet (Schülerin E).

Bezüglich der Infinitive ist anzumerken, dass sie größtenteils in Verbindung mit Modalverben und am Schluss der Texte auftreten, wo die Lernenden bewerten sollten, ob sie in Indien leben möchten. Hier haben sehr viele den Ausdruck andare a vivere/visitare (gehen/losziehen um zu leben/zu besichtigen) u.Ä. und Formen wie mi piacerebbe di vedere (ich würde mir gerne ... ansehen) gewählt. Darüber hinaus werden Infinitive verwendet, die wie si può dire che (man kann sagen, dass) und possiamo dire che (wir können sagen, 
dass) stark durch Mundsprachlichkeit geprägt sind, und andere, die vermutlich aus der Bemühung um eine stilistische Textaufwertung resultieren. Das sind z.B. [il continente africano] è andato a spostarsi ([der afrikanische Kontinent] hat sich verschoben) und la distribuzione della popolazione [...] arriva a toccare anche (Die Bevölkerungsverteilung [...] erreicht). Diese Konstruktionen sind nur schwer ins Deutsche zu übersetzen und erfahren auch im Italienischen gegenüber ihrer Reduzierung auf konjugierte Verbformen von spostarsi und toccare ohne die Infinitive andare und arrivare keine wesentliche Bedeutungsveränderung.

\subsubsection{Bildungssprachlichkeit und wortgetreue Übernahme aus Lehrbuchtexten}

Anhand dieser Auszüge aus einigen Schülertexten zur Verwendung von Partizipien und Gerundien wird eine generelle Tendenz im Schreibstil deutlich: Viele Schülerinnen und Schüler waren anscheinend nicht in der Lage, bei der Aneignung von Lerninhalten mithilfe des Unterrichtswerks die dort gegebenen Informationen zu paraphrasieren, sondern gaben sie teilweise wortwörtlich wieder. Das geschah sowohl während der mündlichen Abfragungen als auch in den hier analysierten schriftlichen Textproduktionen, bei denen theoretisch mehr Zeit war, ihre Äußerungen vorzubereiten bzw. zu überarbeiten.

Das fällt besonders im längsten Schülertext auf, da sein Verfasser nicht nur ein gutes Gedächtnis, sondern anscheinend auch einen gefestigteren Wortschatz hatte, der es ihm erlaubte, sich das Gelesene einzuprägen und präzise wiederzugeben. Einige MitschülerInnen ersetzten stattdessen weniger geläufige Ausdrücke und morphosyntaktische Konstruktionen, die zwar verstanden, aber anscheinend nicht aktiv, produzierend beherrscht wurden, durch einfachere Gestaltungsmittel. Es folgen ein Auszug aus dem Text von J mit einer dem Lehrbuch (Bersezio 2005, S. 125) ähnelnden Passage und sein Pendant aus dem Unterrichtswerk:

L'India è una Repubblica federale composta da 28 Stati e 7 [territori] amministrati direttamente dal governo [quasi O-Ton]. Tra i gruppi etnici più diffusi a Nord c'è il gruppo Arya e a Sud il gruppo Dravida. Solo 16 sono le lingue ufficiali, considerando che sono presenti migliaia di dialetti. Quando l'India si è resa indipendente (1946) la lingua ufficiale divenne l'Hindi, ma i popoli si ribellarono rivendicando la propria lingua e per questo motivo ancora oggi la lingua più parlata è l'inglese.

Al momento dell'unificazione sono stati altri problemi come per esempio ridisegnare i confini e Paesi come il Kashmir ed il Punjab minacciavano la secessione [quasi O-Ton].

(Indien ist eine 28 Staaten und 7 direkt von der Regierung verwaltete umfassende Bundesrepublik. Unter den am weitesten verbreiteten ethnischen Gruppen gibt es im Norden die Gruppe der Aryer und im Süden die drawidische. Nur 16 sind die Amtssprachen, wenn man bedenkt, dass Tausende von Dialekten vorliegen. Als Indien sich unabhängig gemacht hat (1946), wurde die Amtssprache Hindi, aber die Völker lehnten sich auf und verteidigten die eigene Sprache, und aus diesem Grund ist noch heute die am meisten gesprochene Sprache Englisch. 
Im Moment der Vereinigung waren es andere Probleme wie zum Beispiel die Grenzen neu zu zeichnen, und Länder wie Kaschmir und Punjab drohten mit der Sezession.)

Bersezio (2005, S. 125; Hervorhebung im Original): L'India è una repubblica federale che comprende 28 Stati e 7 Territori amministrati direttamente dal governo centrale. [...]

Vi sono molti popoli diversi tra loro che hanno lingue, culture, usanze e religioni talora assai differenti. Per esempio, le lingue, che appartengono al gruppo arya a nord e al gruppo dravida a sud, sono tante e diverse: quelle riconosciute ufficialmente sono sedici, ma esistono anche migliaia di dialetti. Raggiunta l'indipendenza (1947), il governo aveva deciso che l'hindi fosse la lingua ufficiale di tutto il Paese, affiancata dall'inglese. Molti Stati però si opposero, rivendicando il diritto di usare ciascuno la propria lingua. Così oggi l'inglese continua a essere la lingua più usata.

Al momento dell'indipendenza non ci fu solo il problema della lingua: i nuovi confini interni furono ridisegnati tra mille difficoltà e alcuni Stati, come il Punjab e il Kashmir, minacciarono la secessione.

(M.Ü.: Indien ist eine Bundesrepublik, die 28 Statten und 7 Gebiete, welche direkt von der Zentralregierung verwaltet werden, umfasst.

Es gibt dort viele voneinander verschiedene Völker, die Sprachen, Kulturen, Bräuche und Religionen haben, die zum Teil stark differieren. Zum Beispiel sind die Sprachen, die der Gruppe der Aryer im Norden und der drawidischen Gruppe im Süden angehören, viele und verschieden: Die offiziell anerkannten sind 16, aber es existieren auch Tausende von Dialekten. Die Unabhängigkeit erreicht (1947), hatte die Regierung entschieden, dass Hindi die Amtssprache des ganzen Landes sei, flankiert von Englisch. Viele Staaten widersetzten sich allerdings, indem sie sich auf das Recht beriefen, jeder seine eigene Sprache zu benutzen. So ist Englisch heute weiter die meistgebrauchte Sprache. Im Moment der Unabhängigkeit gab es nicht nur das Problem der Sprache: Die neuen inneren Grenzen wurden unter tausend Schwierigkeiten wiedergezeichnet und einige Staaten wie Punjab und Kaschmir drohten mit der Sezession.)

Ein weiteres Beispiel für die enge Gebundenheit an den Lehrbuchtext veranschaulicht der nächste Auszug aus dem Text von G. Es handelt sich um eine Schülerin mit nicht italienischer Erstsprache, die jedoch ihre gesamte Schulzeit in Italien verbracht hatte. Auch sie übernahm Textpassagen wortwörtlich und selbst dann, wenn sie derart in einen veränderten Kontext eingefügt wurden, dass sie logisch-semantisch nicht mehr korrekt waren. Bspw. macht sie exakte Höhenangaben zu bestimmten Bergen, behält jedoch das Verb übertreffen aus dem Lehrbuch bei:

A nord della regione si trovano le catene montuosi l'Himalaya e il Karakoram. In queste si trovano le montagne più alte della Terra che sono l'Everest [che] supera i 8846 metri di altezza e il K2 che supera i 8611 metri di altezza. Le due catene si sono sollevate quando il Deccan si è staccato dall'Africa e si è spostata in Asia e adesso si chiama India. La regione indiana ha il clima monsonico. La zona meridionale è secca e compaiono gli ambienti tipici della savana.

(Im Norden der Region befinden sich die Bergketten Himalaya und Karakorum. In diesen befinden sich die höchsten Berge der Erde, die der Everest sind, der die 8.846 Höhenmeter übertrifft und der K2, der die 8.611 Höhenmeter übertrifft. Die zwei Bergketten haben sich erhoben, als der Dekkan sich von Afrika abgetrennt hat und sich nach Asien verschoben hat, und jetzt heißt er Indien. Die indische Region hat das Monsunklima. Die südliche Gegend ist trocken und es erscheint die typische natürliche Umwelt der Savanne.) 
Bersezio (2005, S. 115; Hervorhebung im Original): A nord della regione indiana si trovano i sistemi montuosi più alti della Terra: 1'Himalaia e il Karakoram. In Nepal vi sono infatti tutte le quattordici cime che superano gli 8000 metri di altezza, tra cui 1'Everest e il K2.

Le due catene si sono sollevate nella stessa epoca delle Alpi [...].

Il centro della regione e soprattutto il settore occidentale è occupato dalle pianure, con clima monsonico e conseguente manto forestale fitto. La zona meridionale è più secca e compaiono gli ambienti tipici della savana.

(M.Ü.: Im Norden der indischen Region befinden sich die höchsten Gebirge der Erde: das Himalaya und der Karakorum. In Nepal sind nämlich alle 14 Gipfel, die die 8.000 Höhenmeter übertreffen, unter ihnen der Everest und der K2.

Die beiden Bergketten haben sich in der gleichen Epoche der Alpen erhoben [...].

Das Zentrum der Region und vor allem der westliche Sektor ist von den Ebenen eingenommen, mit Monsunklima und daraus folgendem dichten Waldmantel. Die südliche Gegend ist trockener und es erscheint die typische natürliche Umwelt der Savanne.)

Ein letztes Beispiel zeigt noch einmal, wie der Schülertext dem Lehrbuchtext fast wortwörtlich entspricht (Schülerin A):

La regione indiana occupa una gran parte dell'Asia centro-meridionale e si prolunga fin nell'Oceano Indiano dove si trovano tante isole: le più importanti sono quella dello Sri Lanka e quelle raggruppate nell'Arcipelago delle Maldive.

La regione indiana è costituita: dall'India, dal Pakistan. dal Bangladesh. La regione ha una forma di un grandissimo triangolo rovesciato, unito dal resto dell'Asia, dalla catena dell'Himalaya dove fa freddo. Al centro ed a ovest della regione si trovano delle pianure che hanno un clima monsonico; e nella parte meridionale verso il Deccan ed a ovest verso il Deserto del Thar il clima è quasi sempre arido e quest'ultimo è un'area disabitata.

(Die indische Region nimmt einen Großteil Zentralsüdasiens ein und verlängert sich bis zum Indischen Ozean, wo sich viele Inseln befinden: Die wichtigsten sind die von Sri Lanka und die im Archipel der Malediven gruppierten.

Die indische Region ist zusammengesetzt: aus Indien, aus Pakistan, aus Bangladesch. Die Region hat eine Form eines riesengroßen umgekehrten Dreiecks, das mit dem Rest Asiens durch die Bergkette des Himalayas verbunden ist, wo es kalt ist. Im Zentrum und im Westen der Region befinden sich Ebenen, die ein Monsunklima haben; im südlichen Teil in Richtung des Dekkans und im Westen in Richtung der Tharwüste ist das Klima fast immer trocken und Letztere ist eine unbewohnte Gegend.)

Bersezio (2005, S. 114 f.): La regione indiana occupa una vasta parte dell'Asia centro-meridionale e si prolunga fin nell'Oceano Indiano, dove si trovano varie isole: le più importanti sono quella dello Sri Lanka, che è anche la più vasta, e quelle raggruppate nell'Arcipelago delle Maldive, splendido atollo corallino che si solleva qualche metro sul livello del mare in prossimità dell'Equatore.

La regione indiana ha la forma di un immenso triangolo rovesciato, unito al resto dell'Asia dalla catena himalaiana, dove prevale un clima nivale. Il centro della regione e soprattutto il settore occidentale è occupato dalle pianure, con clima monsonico e conseguente manto forestale fitto. La zona meridionale è più secca e compaiono gli ambienti tipici della savana.

(M.Ü.: Die indische Region nimmt einen umfangreichen Teil Zentralsüdasiens ein und verlängert sich bis zum Indischen Ozean, wo sich viele Inseln befinden: Die wichtigsten sind die von Sri Lanka, die auch die ausgedehnteste ist, und die im Archipel der Malediven gruppierten, prächtiges Korallenriff, dass sich einige Meter über dem Meeresspiegel in der Nähe des Äquators erhebt. 
Die indische Region hat die Form eines immensen umgekehrten Dreiecks, das mit dem Rest Asiens von der Bergkette des Himalayas verbunden ist, wo ein nivales Klima vorherrscht. Das Zentrum der Region und vor allem der Westen ist von den Ebenen eingenommen, mit Monsunklima und daraus resultierendem dichten Waldmantel. In der südlichen Gegend ist es trockener, und es erscheint die typische natürliche Umwelt der Savanne.)

Der Text der Schülerin A geht in diesem Stil weiter, auch wenn sich gelegentlich zeigt, dass seine Verfasserin zusammenfassen kann, da Lehrbuchpassagen übergangen und andere miteinander verknüpft werden.

Das in allen Klassen beobachtete Auswendiglernen und die zumindest während des Beobachtungszeitraums nicht erfolgte Vermittlung und Verfestigung von Strategien der Textparaphrase sind zwar aus didaktischer Sicht anfechtbar, schienen jedoch gerade denjenigen, deren Erstsprache nicht die Unterrichtssprache ist, und generell denen, die über geringere Sprachkompetenzen verfügen, dazu zu dienen, ihren Wortschatz systematisch zu erweitern. Wie lange sie bestimmte, weniger frequente bzw. fachwortschatzsprachliche Ausdrücke und Sprachbausteine behalten und verwenden können, steht allerdings auf einem anderen Blatt.

Wie w.u. bei der Vorstellung der schriftlichen Tests in der Klasse der Vespucci illustriert wird, benutzte die Klasse an der Morante durch die Aneignung der Lehrbuchtexte in ihren eigenen Texten mehr Ausdrücke, die über den Basiswortschatz hinausgehen, mehr Passivkonstruktionen und weniger Modalverben als die Klasse an der Vespucci. Diese konnte bei der Textverfassung nicht auf eine unmittelbar vorausgehende Erarbeitung eines Lehrbuchtextes zurückgreifen, zumal sie sich im Unterricht zuletzt mit geoökonomischen und -sozialen Inhalten befasst hatte.

Hiermit sei also betont, dass die in den Schülertexten der Morante vorherrschenden bildungssprachlicheren Ausdrücke und Konstruktionen zu einem guten Teil direkt aus dem Lehrbuch übernommen wurden und keine eigenständigen Produktionsleistungen darstellen.

Darüber hinaus lassen sich vereinzelt weitere Merkmale eines formaleren schriftsprachlichen Stils erkennen, die zumindest auf den zu lernenden Lehrbuchseiten nicht erscheinen und deshalb nicht einfach auswendig gelernt worden sein können. Das sind zum Beispiel die passende Verwendung des Adjektivs omogeneo (homogen) aus dem allgemeinen Wortschatz (CO) in gleich zwei Schülertexten (Hervorhebung nicht in den Originalen):

Schülerin A: La popolazione è sovrappopolata. E soprattutto in India la popolazione è di quasi un miliardo di abitanti e la distribuzione del popolo non è omogenea perché nelle zone aride c'è poca abitazione, mentre vicino ai fiumi e alle pianure la popolazione è tantissima. (Die Bevölkerung ist überbevölkert. Und vor allem in Indien beträgt die Bevölkerung fast eine Milliarde Einwohner, und die Verteilung des Volkes ist nicht homogen, weil es in den trockenen Gebieten wenig Behausung gibt, während in der Nähe der Flüsse und in den Ebenen die Bevölkerung sehr zahlreich ist.)

Schüler J: La regione indiana è sovrappopolata e certe zone, come Delhi, toccano una densità di 4000 abitanti per chilometro quadrato. La popolazione non è distribuita in 
modo omogeneo, nelle aree desertiche e montuose la popolazione è assente. (Die indische Region ist übervölkert und bestimmte Gebiete wie Delhi berühren eine Dichte von 4.000 Einwohnern pro Quadratkilometer. Die Bevölkerung ist nicht in homogener Weise verteilt, in den Wüstengebieten und in den Bergen ist die Bevölkerung abwesend.)

Abbildung 5: Korrekte Verwendung eines bildungssprachlichen Begriffs, der nicht auf den entsprechenden Lehrbuchseiten erscheint

Schülerin A ist mehrsprachig und erst seit einigen Jahren in Italien. Von ihr sind w.o. bereits Textauszüge vorgestellt worden, die eine starke Anlehnung an Passagen aus dem Unterrichtswerk aufweisen. Auf einige Mängel in der Sprachkompetenz im Italienischen deuten ihr erster Satz in Abbildung 5 sowie die schwache Verknüpfung der ersten drei Informationen durch die zweifach eingesetzte Konjunktion $e$ (und) hin. Zwar hatte sie sich das im Lehrbuchtext verwendete Adjektiv sovrappopolato aus dem allgemeinen Wortschatz (Co) eingeprägt und seine Bedeutung anscheinend verstanden, jedoch verfügte sie ihm bezüglich nicht über ausreichendes kollokationales Wissen, da sie es nicht im Verwendungskontext physischer Räume, sondern als adjektivisches Prädikatsnomen zu „Bevölkerung“ gebrauchte. ${ }^{207}$

Umso auffälliger ist ihr treffender Gebrauch des Adjektivs omogeneo im Fortgang des Textauszugs, dem sie darüber hinaus noch eine detaillierte Beschreibung der Bevölkerungsverteilung anschloss. Dass es sich bei dieser Schülerin um eine geübtere Schreiberin handelte, die zum Verfassen auf Italienisch wahrscheinlich auch die im erstsprachlichen Schulunterricht in ihrem Heimatland Ägypten erworbene Textkompetenz nutzte, zeigt sich beispielsweise an der Verwendung struktureller Gestaltungsmittel. So nahm sie eine inhaltlich-konzeptionell folgerichtige Gliederung ihres Textes in Absätze vor, die jeweils mit einer Überschrift versehen sind, und gebrauchte das Semikolon, was außer ihr nur zwei weitere Lernende taten, deren Erstsprache allerdings Italienisch ist.

Darüber hinaus ist auf lexikalischer Ebene hervorzuheben, dass der Schüler $\mathrm{K}$ für die Beschreibung des Kleidungsstils der indischen Frauen ein Co-gekennzeichnetes Verb benutzte, das vielen seiner Mitschüler wahrscheinlich nicht geläufig war, sfoggiare (zur Schau stellen): ,[...] le donne sfoggiano vestiti meravigliosi di tutti i colori" ${ }^{\text {" }}([\ldots .$.$] die Frauen stellen wunderbare Kleider in$ allen Farben zur Schau).

Auch die mehrsprachige Schülerin D gebrauchte zur geographischen Einordnung Indiens eine prädikative Konstruktion mit dem aus dem allgemeinsprachlichen Wortschatz stammenden und damit seltener genutzten Adjektiv

207 A ist übrigens nicht die einzige Schülerin, die das Adjektiv sovrappopolata falsch gebraucht, sondern auch Schülerin D, ebenfalls mehrsprachig, sowie Schüler B bei einem semantisch verwandten Adjektiv: „L'India è una regione sovraffollata dalla popolazione (1 miliardo e 41 milioni di persone) "(Indien ist eine von der Bevölkerung überlaufene Region (1 Milliarde und 41 Millionen Menschen)). 
posto (gelegen) als Prädikatsnomen: „L'India si trova nel continente EuroAsiatico ed è posta nel Centro-Meridione dell'Asia" (Indien befindet sich im eurasischen Kontinent und ist im Zentrum und Süden Asiens gelegen).

Zuletzt sei noch auf die Verwendung eines Mittels zur expliziten Textkohäsion hingewiesen, mit dem die Wiederholung bereits genannter Subjekte vermieden werden kann. Die Strategie, sie durch ein Demonstrativpronomen zu ersetzen, wurde nämlich kaum angewandt. Die Texte von J (w.u. vorgestellt) und A weisen solche Pronomen auf (Schülerin A, Hervorhebung nicht im Original): ,Il sistema ferroviario è estremamente sviluppato; il treno costituisce il principale mezzo di trasporto. Quello aereo non è molto sviluppato, ma è in crescita grazie al turismo" (Das Eisenbahnwesen ist extrem entwickelt; der Zug bildet das hauptsächliche Verkehrsmittel. Das der Flugzeuge ist nicht sehr entwickelt, aber befindet sich dank des Tourismus im Wachstum). Die Wiedergabe ist im Deutschen nur eingeschränkt möglich, da es sich bei aereo um ein Adjektiv handelt, das es im Deutschen nicht gibt. Das Demonstrativpronomen quello hat drei mögliche Bezugswörter, die logisch-semantisch alle in Frage kommen, und zwar sistema, mezzo di trasporto und mezzo.

\subsubsection{Schwierigkeiten mit dem Register und konzeptionelle Mündlichkeit}

Bemühungen um einen bildungssprachlichen Ausdruck, die ihr Ziel verfehlten, waren häufiger zu beobachten. So übernahm sogar der einsprachige Schüler F, der die zweitbeste Note im Teilbereich Wortschatz (8) erhielt, einen Ausdruck aus dem Lehrbuch und reduzierte ihn so, dass er das eigentlich Gemeinte in sein Gegenteil kehrte: „La Regione Indiana è molto povera ed il [PIL] Pro Capite è di 481 dollari annui e ci sono persone che vivono di povertà" (Die indische Region ist sehr arm, und das Pro-Kopf-[Bruttoinlandsprodukt] beträgt 481 Dollar jährlich, und es gibt Menschen, die von Armut leben).

Es handelt sich hierbei nur um einen Präpositionswechsel von in zu di, der aus dem im Lehrbuchtext gegebenen „in Armut leben“ „von Armut leben“ macht. Darüber hinaus verkürzten er und einige andere den Ausdruck Pro Kopf-Bruttoinlandsprodukt zu „Pro Kopf“, da sie dieses lateinische Phrasem bisher nur aus dem Zusammenhang mit dem BIP kannten, so dass ihnen der Bezug wahrscheinlich eindeutig erschien und sie ihn deshalb nicht explizierten. Wie in allen Fällen sprachlicher Ungenauigkeit oder nicht korrekter Ausdrucksweise in den vorliegenden Schülertexten, bleibt auch hier die intendierte Aussage zwar verständlich, sind derartige Reduzierungen dem Schreibanlass jedoch nicht angemessen. Das gilt auch für die schon kritisierte Verwendung der nebenordnenden Konjunktion ,und“", die in den Schülertexten die vorherrschende Verknüpfungsweise von Sätzen darstellt.

Schüler F schrieb außerdem an anderer Stelle, das Straßennetz habe abgenommen (la rete stradale è diminuita), während das Lehrbuch mit ,[a]nche la rete stradale è molto estesa, ma solo per la metà è asfaltata“ (Bersezio 2005, 
S. 127; m.Ü.: Auch das Straßennetz ist sehr weitläufig, aber nur zur Hälfte asphaltiert) ganz andere Angaben macht.

Einen ungewollt komischen Ton nimmt eine Formulierung des Schülers J genau dort an, wo er ein bereits genanntes Konzept mit einem Demonstrativpronomen angemessen wieder aufgriff: „E' molto sviluppato l'allevamento di bovini, infatti ne troviamo in grande abbondanza, però questi ultimi vengono usati solo per l'estrazione del latte, per motivi religiosi“" (Die Rinderhaltung ist sehr entwickelt, in der Tat finden wir davon reichlich, aber Letztere werden nur zur Extraktion der Milch gebraucht, aus religiösen Gründen). Als bildungssprachlich können an dieser Formulierung das Passiv, die grammatikalische Metapher estrazione del latto anstelle einer Wiedergabe des Prozesses mit einem Prädikat, das Demonstrativpronomen und der kausale Zusatz per motivi religiosi betrachtet werden.

Der bildungssprachlich orientierte Formulierungsversuch beruht auf einer Lehrbuchinformation, die ebenfalls sehr technisch gehalten ist (Bersezio 2005, S. 126): „In India vengono allevati moltissimi bovini, un quinto del totale mondiale; ma, per motivi religiosi, viene utilizzata solo la loro produzione di latte“ (M.Ü.: In Indien werden sehr viele Rinder gehalten, ein Fünftel der weltweiten Gesamtmenge, aber aus religiösen Gründen wird nur ihre Milchproduktion genutzt).

Weitere Auffälligkeiten der Schülertexte zeigen sich auf lexikalischer Ebene bei den Bemühungen um die Verwendung angemessener Lexeme, wenn diese innerhalb eines Satzes wiederholt werden bzw. es zur Verwendung von Ausdrücken kommt, die zwar zu unterschiedlichen Wortarten, aber zur selben Wortfamilie zählen (Hervorhebung nicht in den Originalen):

Schülerin I: L'india è abitata da circa un miliardo di abitanti, nelle aree più abitate per esempio il Bumbai che ha circa undici milioni di abitanti, e l'India ha una densità di $501 \mathrm{ab} / \mathrm{km}^{2}$ (abitanti $\times \mathrm{km}^{2}$ ). (Indien ist von zirka einer Milliarde Einwohnern bewohnt, in den meist bewohnten Gebieten zum Beispiel Mumbai, das zirka elf Millionen Ein-

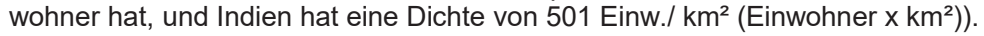

Schülerin L: Per quanto riguarda l'economia, è basata sull'agricoltura ed essendoci numerose piogge, si coltivano prodotti come il riso che ha bisogno di moltissima acqua. Se qualche anno piove di meno, sarebbe un vero problema perché ci sarebbe una carestia che impedirebbe la produzione di prodotti e il cibo non basterebbe per tutti. (Was die Wirtschaft betrifft, basiert sie auf der Landwirtschaft und, da es zahlreichen Regen gibt, baut man Produkte wie den Reis an, der sehr viel Wasser benötigt. Wenn es ein Jahr weniger regnet, wäre es ein ehrliches Problem, weil es eine Hungersnot gäbe, die die Produktion von Produkten verhinderte, und das Essen würde nicht für alle reichen).

Abbildung 6: Wiederholungen von Lexemen aus derselben Wortfamilie

Neben der aus Wortwiederholungen resultierenden Ausdrucksschwäche ist der Textauszug der mehrsprachigen Schülerin I auch von Nichtgrammatikalität geprägt, da sie nach dem ersten Hauptsatz einen zweiten beginnt, den sie allerdings nach der Unterbrechung durch einen Relativsatz nicht zu Ende führt, 
sondern durch einen weiteren nebengeordneten Hauptsatz ergänzt. Das ist im Mündlichen, gerade in nicht allzu formalen Situationen, oft zu beobachten und zeigt, dass die Lernenden zum Teil so schreiben, wie sie sprechen. Außerdem ist davon auszugehen, dass sich die Schülerin ihren Text nicht noch einmal durchgelesen hatte, da sie die syntaktische Fehlkonstruktion ansonsten vermutlich bemerkt hätte. Ferner ist ihr Auflösen der Abkürzung $a b / \mathrm{km}^{2}$ dem Schreibanlass ebenfalls nicht komplett angemessen, da sie das Wort per nicht ausschreibt, sondern durch ein Symbol (x) ersetzt, wie es für viele bei Mitschriften im Unterricht und auch in Vorlesungen sowie beim Verfassen von SMS üblich ist. ${ }^{208}$

Der erste Teil des Textauszugs der einsprachigen Schülerin L wurde schon w.o. (Gebrauch des Gerundiums) vorgestellt. Beim zweiten Teil handelt es sich um einen Konditionalsatz, dessen Inhalt problemlos verstanden werden kann, obwohl er logisch-semantische Fehler enthält und entschieden vom bildungssprachlichen Register abweicht. So ist es nicht die Hungersnot, die den Anbau von Nahrungsmitteln verhindern würde, sondern der ausbleibende Niederschlag. Der Schülerin gelingt es offenbar innerhalb des Konditionalsatzes nicht, die kausalen und konsekutiven Zusammenhänge angemessen zu formulieren. Auf lexikalischer Ebene ist zudem der Ausdruck produzione dei prodotti durch Vagheit und die Wiederholung des Wortstamms gekennzeichnet.

Ausgesprochen konzeptionell mündlich sind in vielen Fällen die schon erwähnten langen Satzgefüge, deren einzelne Aussagen oft nur schwach durch die nebenordnende Konjunktion und verbunden sind. Sie erinnern an einen Gedankenfluss (stream of consciousness), wie er eher in der spontanen gesprochenen Sprache oder beispielsweise beim Tagebuchschreiben erfolgt, während er in darstellenden Texten vermieden werden sollte. Auch das Nichtbeenden von begonnen Satzkonstruktionen, d.h. die daraus entstehende Nichtgrammatikalität der Satzgefüge, die in einigen Textproduktionen vorliegt, wiederholte Redewendungen wie parlando di (von ... sprechend), possiamo dire che (wir können sagen, dass), e poi (und dann) und das Füllwort cioè sind Merkmale konzeptueller Mündlichkeit. In drei Texten haben Lernende darüber hinaus das allzu generische Substantiv cosa (Fo; Sache) benutzt (Hervorhebung nicht in den Originalen):

Schülerin D: Io non andrei mai a vivere in India, ma magari forse per le vacanze ci andrei per vedere varie cose come il Taj Mahal che è una delle 4 meraviglie del mondo oppure ci sono molte altre cose da vedere, ma non solo questo, vorrei sopratutto andare a Mumbai perché mi hanno detto che è bella. Poi ci sta "Bollywood" non si sa mai, se puoi incontrare un attore che ti piace, e la cosa più importante da fare quando si va in un nuovo paese: scattare foto e far shopping. (Ich würde nie nach Indien gehen, um dort zu leben, aber möglicherweise vielleicht würde ich für die Ferien dort

208 Dasselbe Symbol gebraucht auch der Schüler J an zwei Stellen seines Textes anstelle des Wortes per. 
hingehen, um verschiedene Sachen zu sehen wie den Taj Mahal, der eines der 4 Weltwunder ist, oder es gibt viele andere Sachen zu sehen, aber nicht nur das, ich würde vor allem nach Mumbai gehen wollen, weil sie mir gesagt haben, es ist schön. Dann gibt es Bollywood, man weiß ja nie, ob du einen Schauspieler treffen kannst, der dir gefällt, und die wichtigste Sache, die es zu tun gibt, wenn man in ein neues Land fährt: Photos machen und shoppen.)

Schülerin M: lo in India ci andrei a vedere i monumenti, le cose belle da visitare che ci sono ma a vivere penso che non ci andrei mai perche [sic] si vive male, non ci sono le fogne né gli acquedotti e poi d'estate fa troppo caldo ed io non lo posso sopportare. (Ich würde nach Indien fahren um die Monumente zu sehen, die schönen Sachen, die es zu besichtigen, die es gibt, aber um zu leben, denke ich, würde ich dorthin niemals fahren, weil man schlecht lebt, es gibt keine Abwasserkanäle, noch Aquädukte und dann ist es im Sommer zu heiß und ich kann das nicht aushalten.)

Schülerin A: L'agricoltura è molto sviluppata nelle zone pianeggianti e dove scorrono $i$ fiumi, e vengono coltivati vari cose: il riso, che l'India è la migliore produttrice, dopo la Cina, è coltivato ovunque ma di più nelle zone in cui c'è pioggia abbondante; il tè, la cannella ed il cotone, coltivati in India ed in Sri Lanka. Die Landwirtschaft ist sehr entwickelt in den ebenen Gegenden und wo die Flüsse fließen, und es werden verschiedene Sachen angebaut: der Reis, der (von dem) Indien die beste Produzentin nach China ist, wird überall angebaut, aber mehr in den Gebieten wo es reichlichen Regen gibt, der Tee, der Zimt und die Baumwolle, die in Indien und Sri Lanka angebaut werden.)

Schülerin E: Sarebbe un'esperienza bellissima scoprire nuove cose su altre persone o altri luoghi. (Es wäre eine wunderschöne Erfahrung, neue Sachen über andere Personen zu entdecken oder andere Orte.)

Abbildung 7: Gebrauch des generischen Substantivs cosa in den Schülertexten der Morante

In vielen Fällen könnte das Substantiv cosa/cose einfach weggelassen werden. Teilweise stand den Lernenden wahrscheinlich kein spezifischeres Nomen als Alternative zur Verfügung, wie z.B. im Satz von A (Sachen anbauen). Im letzten Beispiel hingegen ist außer dem generischen Substantiv auch das Prädikat schlecht gewählt, denn mit dem Verb „entdecken“ wäre eine Kollokation wie in „die Unterschiede von Land und Leuten entdecken“ angemessener.

Die Texte in Abbildung 7 enthalten darüber hinaus noch weitere Merkmale des Umgangssprachlichen und Mündlichen, die im Folgenden kurz vorgestellt werden sollen. Das sind im Textauszug der mehrsprachigen Schülerin D die emphatische Formulierung, die durch die zwei semantisch sehr verwandten Wörter magari und forse entstand, und die Konstruktionen ,poi ci sta“ (dann gibt es) und „non si sa mai, se puoi incontrare..." (man weiß ja nie, ob du ... treffen kannst). Hier ist der Gebrauch der zweiten Person Singular nicht registertypisch und sind die Konjunktion se und das Modalverb potere unpassend gewählt (entweder non si sa se si incontra (man weiß nicht, ob man trifft) oder non si sa mai, si potrebbe incontrare (man weiß ja nicht, man könnte treffen)).

Im Textauszug der einsprachigen Schülerin M ist das im umgangssprachlichen Mündlichen oft zu findende Adverb ci (dort) fehl am Platz, da der Satz 
schon eine Lokaladverbiale enthält (Indien). Außerdem gehen die Wiederholung des Prädikats (andarci), auch hier e poi (und dann) sowie der vermeidbare Relativsatz che ci sono ([die schönen Sachen], die es gibt) auf konzeptionelle Mündlichkeit zurück.

Auch im Text der mehrsprachigen Schülerin A ist ein Prädikat enthalten, das in einem bildungssprachlicheren Text in diesem Verwendungskontext nicht erschiene, nämlich scorrono. Der Nebensatz „wo die Flüsse fließen“ wäre beispielsweise zu einem präpositionalen Objekt verdichtet, wie „entlang der Flüsse". Desweiteren ist das Relativpronomen di cui durch das nur im umgangssprachlichen Mündlichen zulässige, polyvalente che wiedergegeben. Das Adjektiv migliore ist ebenfalls nicht gerade treffend gewählt, da es ein Attribut bildet, das in diesem Verwendungskontext Angaben zur Quantität und nicht zur Qualität liefern sollte.

Als nächstes wird die sprachliche Gestaltung der Lehrbuchseiten zur indischen Region geschildert, um daran einen Vergleich zu den Schülertexten anschließen zu können.

\subsection{Die Lehrbuchseiten zur indischen Region}

Im Lehrbuch von Bersezio (2005) befassen sich die Seiten 114-121 mit der indischen Region, die Seiten 122-127 mit Indien und die Seiten 128 f. mit der Vorstellung der anderen Staaten der Region in Form von Steckbriefen und kurzen Texten einer Länge von jeweils zwei Absätzen. Mit Ausnahme der Seiten 120 f. (kürzere Absätze mit Informationen zu Landschaftsmotiven auf abgebildeten Photos), die in den Schülertexten nicht erwähnt werden, und unter Auswahl nur derjenigen Staaten auf den Seiten 128 f., über die in den Schülertexten berichtet wird, ist ihr Wortvorkommen vollständig analysiert worden. Zunächst erfolgt eine Beschreibung des verwendeten Wortschatzes und im Anschluss daran wird auf einige grammatikalische und syntaktische Eigenschaften der Texte eingegangen.

\subsubsection{Zusammensetzung des Wortschatzes}

Tabelle 6 gibt einen Überblick über die Gestaltung der Texte, indem sie Angaben über ihre Länge und Unterteilung in Sätze, den Wortschatz nach Gebrauchsmarken, Toponyme, die prozentualen Anteile des fundamentalen und des Basiswortschatzes am Gesamttextvolumen sowie die Gesamtanzahl der Wörter einzelner Wortschatzbereiche liefert. 


\begin{tabular}{|c|c|c|c|c|c|c|c|c|c|}
\hline Seite(n) & $114 \mathrm{ff}$. & 117 & 118 & 119 & $122-124$ & 125 & $126 \mathrm{f}$. & $128 \mathrm{f}$. & Tot./ø \\
\hline n Wörter & 512 & 371 & 403 & 372 & 867 & 298 & 478 & 560 & 3.861 \\
\hline n Sätze & 24 & 16 & 18 & 13 & 40 & 14 & 28 & 40 & 193 \\
\hline längster Satz & 55 & 37 & 50 & 38 & 47 & 42 & 44 & 36 & 43,6 \\
\hline ø Satzlänge & 19,7 & 22,6 & 21,5 & 27,8 & 21,3 & 18,9 & 16,8 & 13,9 & 20 \\
\hline$A D$ & 6 & 2 & 4 & 7 & 13 & 1 & 13 & 17 & 63 \\
\hline AD TS & 1 & 2 & 0 & 0 & 0 & 0 & 0 & 0 & 3 \\
\hline $\mathrm{AU}$ & 29 & 34 & 44 & 47 & 79 & 27 & 53 & 61 & 374 \\
\hline AU TS & 0 & 0 & 1 & 1 & 0 & 0 & 1 & 0 & 3 \\
\hline $\mathrm{CO}$ & 87 & 61 & 46 & 44 & 119 & 35 & 54 & 67 & 513 \\
\hline CO TS & 6 & 1 & 4 & 8 & 5 & 2 & 6 & 10 & 42 \\
\hline el.CO & 7 & 3 & 7 & 6 & 16 & 2 & 7 & 7 & 55 \\
\hline el.CO TS & 4 & 0 & 1 & 0 & 1 & 0 & 0 & 2 & 8 \\
\hline el.FO & 0 & 0 & 0 & 0 & 1 & 0 & 0 & 0 & 1 \\
\hline el.TS & 3 & 2 & 6 & 0 & 2 & 1 & 4 & 7 & 25 \\
\hline ES & 0 & 0 & 0 & 0 & 1 & 0 & 0 & 1 & 3 \\
\hline ES TS & 0 & 0 & 0 & 0 & 1 & 0 & 0 & 0 & 1 \\
\hline FO & 303 & 217 & 256 & 232 & 549 & 216 & 314 & 346 & 2433 \\
\hline LE & 0 & 0 & 0 & 0 & templi & 0 & 0 & 0 & 1 \\
\hline TS & 12 & 10 & 12 & 6 & 9 & 6 & 7 & 17 & 79 \\
\hline FO TS & 1 & 0 & 0 & 0 & 0 & 0 & 0 & 1 & 2 \\
\hline n.p. & 38 & 34 & 17 & 15 & 56 & 7 & 16 & 20 & 203 \\
\hline el.n.p. & 15 & 4 & 5 & 6 & 13 & 0 & 3 & 4 & 50 \\
\hline sigla & 0 & 0 & 0 & 0 & 0 & 0 & 0 & 0 & 0 \\
\hline ohne Marke & 0 & newar & 0 & 0 & dun & arya & 0 & 0 & 3 \\
\hline n FO in \% & 59 & 58,5 & 63,5 & 62,4 & 63,3 & 72,5 & 65,3 & 61,8 & \\
\hline$n \mathrm{VdB}^{*}$ & 339 & 255 & 304 & 287 & 641 & 244 & 379 & 424 & \\
\hline $\mathrm{n}$ VdB in \% & 66,2 & 68,7 & 75,4 & 77,2 & 73,9 & 81,9 & 79,3 & 75,7 & \\
\hline
\end{tabular}

Tabelle 6: Zusammensetzung des Wortschatzes auf den Lehrbuchseiten zur indischen Region (Bersezio 2005)

*inkl. doppelter Marken mit Ts

Da die Wortschätze nach Texteinheiten untersucht wurden, die sich zum Teil über mehrere Seiten erstrecken und von sehr unterschiedlicher Länge sind, ergibt es keinen Sinn, Durchschnittswerte bezüglich ihrer Gesamtwortvolumen, der verwendeten Gebrauchsmarken usw. zu berechnen. Für den an späterer Stelle folgenden Vergleich mit den Schülertexten werden allerdings Textauszüge der durchschnittlichen Länge der Schülertexte herangezogen, so dass genauere Angaben über die unterschiedliche Zusammensetzung des Wortschatzes gegeben werden können.

Zunächst ist von Interesse, dass die Texte aus Sätzen bestehen, die eine Länge von durchschnittlich 20 Wörtern erreichen, wobei die längsten Sätze von einem Minimum von 37 Wörtern (Seite 117) bis zu einem Maximum von 50 (Seite 118) reichen. Der Anteil von Wörtern aus dem fundamentalen Wortschatz, die, wie für italienische Texte gleich welcher Art üblich, mit Abstand die größte Gruppe ausmachen, liegt bei Werten von 58,5 Prozent auf der Seite 117 bis zu 72,5 Prozent auf der Seite 125 und durchschnittlich bei 63,2 Prozent. 
Zu den Wortvorkommnissen aus dem Basiswortschatz zählen auch die ADund die AU-gekennzeichneten Wörter sowie diejenigen, die eine dieser beiden Marken zuzüglich einer fachwortschatzsprachlichen tragen (AU TS, AD Ts). Der Anteil der Wörter aus dem hohen Gebrauch (AU) ist mit 374 ca. sechsmal so groß wie der der hohen Disponibilität (AD; 63 Wörter), was auch nicht verwundert, da es sich bei Letzteren bekanntlich um Wörter handelt, die eher gedacht, als schriftlich oder mündlich formuliert werden.

Unter den verwendeten Ausdrücken, die nicht zum Basiswortschatz zählen, bilden eine zu vernachlässigende kleine Gruppe die drei Exotismen (2 Es, 1 ES Ts), zu der weitere drei Ausdrücke gezählt werden können, die zwar Exotismen sind, aber keinen Eintrag im GRADIT haben, weshalb sie in der Tabelle unter der Rubrik o.M. (ohne Marke) geführt werden, und das Wort templi (Tempel) aus dem literarischen Wortschatz (LE), das anstelle seines Synonyms tempi aus dem allgemeinen Wortschatz (CO) verwendet wurde.

\subsubsection{Allgemeinsprachlicher Wortschatz (Co)}

Die größte Gruppe wird von den 555 Co- und Co Ts-gekennzeichneten Wörtern aus dem allgemeinen Wortschatz geformt, der an den Basiswortschatz angrenzt. Er besteht aus um die 50.000 Wörtern, die jedem erwachsenen italienischen Sprecher, der eine mittlere oder höhere Schulbildung genossen hat, unabhängig von seinem Beruf und seiner geographischen Herkunft innerhalb Italiens zur Verfügung stehen sollten. Es ist anzunehmen, dass von diesen Wörtern einige näher am Kern des Gebrauchswortschatzes, dem Basiswortschatz (VDB), angesiedelt sind als andere. Das bedeutet, dass die knapp 50.000 Wörter wahrscheinlich unterschiedlich häufig gebraucht werden und eine feinere Unterteilung der Co-Wörter möglich wäre. Da die beobachteten Lernenden sich noch nicht am Ende ihrer mittleren Schulausbildung befanden und einzelne Untersuchungen ergeben, dass selbst Abiturienten nicht über den gesamten getesteten Bereich des Co-Wortschatzes verfügten (De Renzo 2008), kann davon ausgegangen werden, dass sie diesen großen Wortschatzbereich nur teilweise beherrschten. Tabelle 7 illustriert die Zusammensetzung der verwendeten CO- und Co Ts-gekennzeichneten Ausdrücke nach Wortarten.

\begin{tabular}{lrrr}
\hline Wortart & $\mathrm{n}$ & davon CO TS & davon loc. \\
\hline agg. & 160 & 11 & 5 \\
art.det. & 62 & 0 & 0 \\
avv. & 30 & 0 & 17 \\
cong. & 6 & 0 & 4 \\
inf. & 4 & 0 & 0 \\
part.pass. & 3 & 0 & 0 \\
prep. & 11 & 0 & 11 \\
pron.dimostr./pron.pers. & $34(33$ si) & 0 & 0 \\
s.m./s.f. & 188 & 31 & 15 (7 CO TS) \\
v.aus. (venire) & 6 & 0 & 0 \\
v.tr./v.intr. & 18 & 0 & 3 \\
\hline
\end{tabular}




\begin{tabular}{lrrr}
\hline Wortart & $\mathrm{n}$ & davon CO TS & davon loc. \\
\hline v.procompl. (esservi) & 5 & 0 & 0 \\
v.pronom.intr. & 26 & 0 & 0 \\
loc. & 55 & 7 & \\
\hline
\end{tabular}

Tabelle 7: Zusammensetzung des Co-gekennzeichneten Wortvorkommens auf den Lehrbuchseiten

Daraus geht hervor, dass es sich bei der Wortart mit den meisten Co-/Co Tsgekennzeichneten Tokens mit 188 um die der Nomen handelt, dichtgefolgt von den Adjektiven mit 160. Zu diesen beiden Wortarten zählen auch alle Co Tsgekennzeichneten Wörter, nämlich elf Adjektive und 31 Substantive, und 20 Co- und Co Ts-gekennzeichnete Phraseme. Das sind die fünf Adjektivphraseme da solo (allein), in alto (oben), in atto (stattfindend), a parte (separat) und pro capite (pro Kopf), von denen die SchülerInnen nur das letzte benutzten, wobei das erste aus einer Bildbeschreibung stammt und in den Schülertexten keinen Verwendungszweck erfüllt.

Bei den 15 substantivischen Phrasemen handelt es sich um folgende:209

popolazione attiva (Co Ts stat.; erwerbstätige Bevölkerung; 3 Tokens); guerra civile (CO; Bürgerkrieg; 3 Tokens); via di comunicazione (CO; Verkehrsweg); repubblica federale (CO; Bundesrepublik; 2 Tokens); clima tropicale (CO; Tropenklima); livello del mare (Co Ts geogr., Meeresspiegel); sistema montuoso (CO Ts geogr.; Gebirgssystem); clima tropicale monsonico (CO Ts meteor., geogr.: Monsun-Tropenklima); potere politico $(\mathrm{CO}$; politische Macht); venditore ambulante (CO Ts burocr.; Straßenhändler); fare capo (CO; vorstehen; 2 Tokens); aver luogo (Co; stattfinden).

Lediglich vier dieser Phraseme erscheinen auch in den Schülertexten.

Jeweils maximal elf Co-gekennzeichnete Ausdrücke sind Konjunktionen (6), Präpositionen (11), prokomplementäre Verben (5) und Hilfsverben (6). Bei Letzteren handelt es sich ausschließlich um Vorkommnisse des zur Passivbildung benötigten venire, das auch die Lernenden verwendeten (aber seltener), und des Prädikats esservi, das sie lediglich in der gebräuchlicheren Variante esserci (AU) benutzten. Desweiteren sind unter den nicht flektierten Verbformen vier Co-gekennzeichnete Infinitive und drei Partizipien.

Bei den Partizipien handelt es sich um Formen der Verben stimolare (anregen), solcare (zerfurchen) und penalizzare (benachteiligen). Das erste wird gleich in der Überschrift auf Seite 119 in attributiver Funktion verwendet: „Un'economia frenata e stimolata dagli stranieri“ (M.Ü.: Eine von den Ausländern gebremste und angeregte Wirtschaft). Es erscheint in einem Umfeld von Wörtern aus dem Basiswortschatz und zusammen mit einem weiteren Partizip, frenata, das wie economia zu den Wörtern des hohen Gebrauchs (AU) zählt, während die anderen Teil des fundamentalen Wortschatzes sind. Dieses Substantiv, auf das sich das Attribut bezieht, steht an erster Stelle und war aus

209 In der Aufzählung sind die auch in den Schülertexten verwendeten Phraseme hervorgehoben. 
dem Unterricht gut bekannt. Die Überschrift sollte also keine Verständnisschwierigkeiten bereiten und dient dazu, Interesse für den Text zu wecken.

Das zweite Partizip, solcate, erscheint in einem wesentlich komplizierteren Kontext, denn es ist ebenfalls Bestandteil eines Attributs, doch von seinem Bezugswort montagne (Berge), vor dem bereits ein adjektivisches Attribut steht, durch ein Beispiel und einen eingeschobenen Relativsatz getrennt (Bersezio 2005, S. 122):

Nel Kashmir si trovano sia rilievi collinari incisi da ampie valli dal fondo pianeggiante e fertile, dette dun, fittamente popolate, sia gigantesche montagne, come il Nanda Devi che raggiunge quota 7817 metri, solcate da profonde valli.

(M.Ü.: Im Kaschmir befinden sich sowohl hügelige, von weiten Tälern ebenen und fruchtbaren Bodens eingeschnittene Erhebungen, dun genannt, die dicht besiedelt sind, als auch riesige Berge wie der Nanda Devi, der eine Höhe von 7817 Metern erreicht, von tiefen Tälern gekerbt.)

Desweiteren befindet es sich am Satzende als letztes einer ganzen Reihe von Partizipien. Auch lexikalisch ist dieser Satz komplexer gestaltet, denn nur etwas weniger als jedes zweite Wort des 35 Wörter langen Satzes zählt zum fundamentalen Wortschatz, während die übrigen aus den Wortschätzen der hohen Disponibilität (AD), des hohen Gebrauchs (AU) und neun von ihnen aus dem allgemeinen Wortschatz (CO) stammen. Darüber hinaus sind unter ihnen ein Exotismus (dun) sowie zwei Eigennamen, von denen noch am ehesten das Toponym Kashmir bekannt sein dürfte.

Dieser Satz ist außerdem exemplarisch dafür, wie die Lehrbuchtexte eine ganze Fülle von Informationen innerhalb einer Satzeinheit verdichten. Dass die Schüler dazu imstande gewesen wären, eigenständig einen solchen Satz zu bilden, kann ausgeschlossen werden. Auch bleibt fraglich, ob sie beim Lesen alle enthaltenen Informationen verstehen und behalten könnten. Das Partizip aus dem allgemeinen Wortschatz liefert zur Hauptinformation, dass es Hügel, Berge und Täler gibt, eine sekundäre Ergänzung, und zwar, wo sich die Hügel befinden. Wem das Verb unbekannt ist, der kann seine Bedeutung nicht aus dem Kontext erschließen, was jedoch das Verständnis der Hauptaussage nicht beeinträchtigt. ${ }^{210}$

Von den vier Co-gekennzeichneten Infinitiven essersi unito (sich vereint haben), decollare (abheben), barattare (eintauschen) und rifornirli (versorgen) befindet sich der erste in einem mit der Konjunktion dopo (Co) eingeleiteten temporalen Nebensatz (Bersezio 2005, S. 122):

Il Gange, fiume sacro degli induisti e meta di immense folle di pellegrini, sfocia nel Golfo del Bengala, dopo essersi unito con il fiume Brahmaputra e aver formato un unico grande ramo.

210 Zur Besprechung der übrigen Co-gekennzeichneten Partizipien s. w.u. das Unterkapitel 8.2.2.3. 
(M.Ü.: Der Ganges, heiliger Fluss der Hindus und Ziel immenser Pilgermassen, mündet in den Golf von Bengalen, nachdem er sich mit dem Fluss Brahmaputra vereint und einen einzigen großen Zweig geformt hat.)

Es handelt sich hier um einen von zwei Infinitiven der Vergangenheit, der eine Handlung versprachlicht, die sich in Vorzeitigkeit zum im Hauptsatz formulierten Geschehen abspielt. Infinitive der Vergangenheit werden seltener formuliert als der Infinitiv Präsens und sind in den Schülertexten, in denen temporale Zusammenhänge generell nur sehr selten expliziert sind, gar nicht vertreten. Daraus kann aber nicht geschlossen werden, dass die Lernenden die im Lehrbuch gegebene Satzkonstruktion und seine Aussage nicht verstehen. Obgleich anzunehmen ist, dass zumindest einige Schwierigkeiten hatten, das Zeitverhältnis korrekt zu interpretieren, zumal das direkte Objekt des zweiten Infinitivs (Zweig) synonym zu „Fluss“ gebraucht wird, was das Verständnis zusätzlich erschwert, da dieses Wort primär mit einem anderen Verwendungskontext assoziiert wird.

Auch der zweite Co-gekennzeichnete Infinitiv, decollare, erscheint in einem Zusammenhang, der sich lexikalisch wohl nicht leicht ausnimmt. Darüber hinaus drückt der Infinitiv nicht die primäre, sondern die damit verwandte figurative Wortbedeutung aus (ibid., S. 128): „Nonostante gli aiuti statunitensi, l'economia pakistana stenta a decollare“" (M.Ü.: Trotz der amerikanischen Hilfen hat die pakistanische Wirtschaft Mühe anzulaufen).

Barattare (CO) war vermutlich nicht allen SchülerInnen geläufig. Vertrauter sollte ihnen das aus dem fundamentalen Wortschatz stammende, ähnlich gebrauchte cambiare gewesen sein. Der Infinitiv erscheint in einem literarischen Zitat von Milarepa, das weitere Verständnishürden beinhaltet, die vor allem lexikalischer Art sind (ibid., S. 114): „Nelle solitarie pietraie himalaiane c'è un curioso mercato: puoi barattare il vortice della vita con una beatitudine senza confini." (M.Ü.: In den einsamen Halden des Himalayas gibt es einen eigenartigen Markt: Du kannst den Strudel des Lebens mit einer grenzenlosen Glückseligkeit tauschen.)

In diesem Satz befindet sich das aus dem geologischen Fachwortschatz stammende Wort pietraie (Halden), das allerdings mit dem Wort pietra (Stein) aus dem fundamentalen Wortschatz verwandt ist, was bei der Bedeutungserschließung hilft. Darüber hinaus enthält er den Co-gekennzeichneten Infinitiv sowie zwei weitere Ausdrücke aus dem allgemeinen Wortschatz, den figurativ verwendeten für „Strudel“ und den für „Glückseligkeit“. Beim Wort für „Strudel“" besteht zudem die Möglichkeit, dass die Lernenden es mit dem ähnlich lautenden Wort vertice (Gipfel) verwechselten, das sie aus dem Erdkundeunterricht kannten und in einem Textzusammenhang wie diesem auch eher erwarten durften. Falls sie statt „Strudel des Lebens“ „Gipfel/Höhepunkt des Lebens" lasen, war die Satzaussage noch schwieriger zu verstehen. 
Weil das Zitat als solches gekennzeichnet ist, ist davon auszugehen, dass sie sich nicht lange mit der Bedeutungserschließung des Satzes aufhielten, sondern ihr Interesse mehr den eigentlichen Texten galt, deren Inhalt sie verstehen und behalten sollten.

Der letzte Infinitiv aus dem allgemeinen Wortschatz wird zur Beschreibung der Bewässerung der Felder gebraucht, wobei provvedere und (ri)fornire zwei fast bedeutungsgleiche Verben sind, was die Infinitivkonstruktion in ,[i]l $70 \%$ dei campi non viene irrigato e solo le piogge monsoniche provvedono a rifornirli d'acqua" (Bersezio 2005, S. 126; m.Ü.: 70 Prozent der Felder werden nicht bewässert und nur die Monsunniederschläge tragen Sorge dafür, sie wieder mit Wasser zu versorgen) eigentlich überflüssig macht.

In diesem Satzgefüge ist das erste Prädikat im Passiv gebildet und stammt außerdem aus dem allgemeinen Wortschatz, war also voraussichtlich nicht allen bekannt. Dass es hier Verständnisprobleme gab, kann nicht ausgeschlossen werden, auch weil w.o. geschildert wurde, dass die Monsunwinde lediglich in einem Schülertext im Zusammenhang mit der Landwirtschaft erwähnt wurden (und nur indirekt). Zu eventuellen Verständnisschwierigkeiten wird außerdem beigetragen haben, dass in beiden Hauptsätzen mit ,70 Prozent der Felder“ und „Niederschläge“ nicht animierte Subjekte vorliegen und Ersteres im zweiten Satz mit einem Pronomen wiederholt wird.

Die Co-gekennzeichneten Präpositionen sind Phraseme, während die EinWort-Präpositionen auf den Seiten des Lehrbuchs allesamt zum fundamentalen Wortschatz zählen. Die verwendeten präpositionalen Phraseme sind a forma $d i$ (in Form von), a causa di (wegen), accanto a (neben), al posto di (anstelle von), grazie a (dank; 2 Tokens), in seguito a (nach (zeitlich); 2 Tokens), rispetto $a$ (hinsichtlich) und vicino $a$ (nahe bei). Nur drei davon wurden auch in den Schülertexten verwendet, und zwar a forma di, grazie a und vicino a. Dass die Ausdrücke a causa di, in seguito a und rispetto a sowie der Großteil der im Folgenden illustrierten Konjunktionen aus dem Co-Wortschatz dort nicht vorkommen, ist bezeichnend. Denn es spiegelt die Tendenz der Lernenden zur karg explizierten Verknüpfung von Inhaltseinheiten wider, die sich i.d.R. auf die Verbindung von Informationen mit „und“, „dass“ und einigen Relativpronomen beschränkte.

Von den sechs Konjunktionen aus dem allgemeinen Wortschatz sind zwei einfacher Art (dopo (nachdem) und pur (auch wenn, obgleich)). Erstere dient der Einleitung eines impliziten temporalen Nebensatzes, der hier mit einem Infinitiv Perfekt gebildet ist, während derartige Zeitangaben sonst in den Lehrbuch- und in den Schülertexten überwiegend mit der Präposition dopo (nach) aus dem fundamentalen Wortschatz ausgedrückt sind. Zusammen mit der Konzessivkonjunktion handelt es sich also um zwei sprachliche Gestaltungsmittel, die zwar voraussichtlich verstanden, in den Schülertexten allerdings nicht verwendet wurden und möglicherweise nicht aktiv produziert werden konnten. 
Die vier konjunktionalen Phraseme sind anche se (obwohl; 3 Tokens) und tant'è che (so dass).

Von den 30 Vorkommnissen an Co-gekennzeichneten Adverbien sind über die Hälfte (17) Mehrworteinheiten, die auf folgende 25 Adverbien zurückgehen:

a.C. (v.Chr.; 2 Vorkommnisse); a capo (am Anfang, an der Spitze); a lato (seitlich); ai piedi (zu Füßen); al contrario (im Gegenteil); da sempre (seit jeher, 2 Vorkommnisse); di fatto (tatsächlich); direttamente (direkt); economicamente (wirtschaftlich, finanziell), esclusivamente (exklusiv); estremamente (extrem); facilmente (leicht); fittamente (dicht); formalmente (formal); in genere (generell); in parte (zum Teil, 4 Vorkommnisse); in particolare (insbesonders); ovunque (überall); per esempio (zum Beispiel); per quanto riguarda (hinsichtlich); periodicamente (periodisch); pressoché (beinahe); ripetutamente (wiederholt); specialmente (besonders); ufficialmente (offiziell).

Nur sechs davon erscheinen in den Schülertexten: in dreien per esempio, in zweien ovunque und in jeweils einem die Adverbien direttamente, estremamente und specialmente. Vermutlich sind auch alle anderen bekannt, zumal fast die Hälfte (11) auf Adjektive aus dem fundamentalen Wortschatz zurückgeht, die mit dem Suffix -mente die entsprechenden Adverbien bilden. Ferner befinden sich sehr gebräuchliche wie per esempio und ovunque darunter, von denen eher eine Zuteilung zur Gebrauchsmarke Fo zu erwarten wäre. Insgesamt sind Adverbien in den Schülertexten stärker vertreten als in den Lehrbuchtexten, 7,8 Prozent (63 Worttypen) gegenüber 4,9 Prozent (61 Worttypen).

Ähnlich wie mit per esempio und anderen verhält es sich auch mit den Cogekennzeichneten Artikeln und Pronomen. Das sind die beiden maskulinen Pluralformen des bestimmten Artikels, gli und $i$, deren Verwendung sich nach dem Anfangslaut des Folgeworts richtet und die überwiegend korrekt benutzt wurden. Außerdem betrifft es das Demonstrativpronomen ciò (dies) und das Personalpronomen der dritten Person Singular si, wobei die SchülerInnen ciò nicht verwendeten, es aber allen bekannt gewesen sein sollte, und $s i$ sowohl in den Lehrbuch- als auch in den Schülertexten als Bestandteil der reflexiven Verben und in Verbindung mit unpersönlichen Prädikaten gebraucht wird, wie zum Beispiel im Text von $\mathrm{J}$ in ,si esportano riso, $[\ldots]$ “. (man exportiert Reis, ...).

\subsubsection{Fachwortschatz (Ts)}

Wie der Tabelle 8 zu entnehmen ist, setzt sich der Fachwortschatz der Lehrbuchseiten zur indischen Region größtenteils aus Nomen zusammen. Unter den insgesamt 78 Ts-markierten Worttypen befinden sich nur sechs Adjektive, das Symbol $\mathrm{km}^{2}$ und das Partizip Perfekt riconosciuto (Ts dir.; anerkannt). Alle anderen 46 fachwortschatzsprachlichen Ausdrücke sind Nomen, die z.T. 
zwei- oder mehrmals gebraucht werden und zu etwas weniger als der Hälfte aus Phrasemen bestehen.

\begin{tabular}{lllll}
\hline Wort- u. TS-Arten & $\mathrm{n}$ Types & davon loc. & Tokens & einmal vorkommende Wörter \\
\hline simb. & 1 & 0 & 1 & $k m^{2}$ \\
agg. & 6 & 0 & 12 & \\
part.pass. & 1 & 0 & 1 & riconosciuto \\
s.f./s.m. & 46 & 20 & 63 & \\
TS bot.com. & 1 & 1 & 1 & canna da zucchero \\
TS chim. & 2 & 1 & 5 & \\
TS dir. & 6 & 4 & 6 & \\
TS dir.intern. & 1 & 0 & 2 & \\
TS dir.lav. & 1 & 1 & 1 & lavoro nero \\
TS econ. & 2 & 1 & 3 & \\
TS etnol. & 6 & 0 & 9 & \\
TS fis. & 2 & 2 & 2 & \\
TS fis., geol. & 1 & 0 & 1 & \\
TS geogr. & 7 & 3 & 8 & \\
TS geogr., antrop. & 1 & 0 & 3 & \\
TS geol. & 5 & 1 & 8 & \\
TS ling. & 1 & 1 & 1 & hindi \\
TS ling., dir. & 1 & 1 & 1 & lingua ufficiale \\
TS meteor., geogr. & 1 & 1 & 1 & clima monsonico \\
TS metrol. & 2 & 0 & 3 & \\
TS miner. & 1 & 0 & 4 & minerarie \\
TS petr. & 2 & 0 & 3 & \\
TS polit. & 1 & 0 & 1 & aristocrazia \\
TS relig. & 1 & 0 & 2 & bramino \\
TS sociol., urban. & 2 & 1 & 2 & \\
TS stat. & 2 & 0 & 4 & \\
TS stor. & 2 & 0 & 4 & \\
TS tecn. & 1 & 1 & 1 & energia idroelettrica \\
TS urban. & 2 & 1 & 2 & \\
\hline
\end{tabular}

Tabelle 8: Fachwortschatz auf den Lehrbuchseiten zur indischen Region (Bersezio 2005)

Insgesamt stammen die Ausdrücke aus 25 verschiedenen Fachgebieten. Darunter ist mit zehn die Rechtssprache einschließlich einer doppelten Markierung von lingua ufficiale (Amtssprache) als linguistisch und juristisch am stärksten vertreten, gefolgt vom geographischen Fachwortschatz inklusive einer doppelten Ts-Markierung als geographisch und anthropologisch mit acht.

Die juristischen Ausdrücke sind das oben genannte Partizip und die sieben Nomen monarchia costituzionale (konstitutionelle Monarchie), repubblica parlamentare/presidenziale (parlamentarische/präsidiale Republik), zona franca (Freihandelszone) und autonomia (Autonomie), die aus dem Geschichts- und Erdkundeunterricht bekannt gewesen sein sollten, sowie protettorato (Protektorat, 2 Tokens) aus dem internationalen Recht und lavoro nero (Schwarzarbeit) aus dem Arbeitsrecht.

Von diesen Begriffen verwendeten die SchülerInnen, abgesehen vom ersten (Amtssprache) mit zwei Vorkommnissen in einem Text, keinen, wie auch 
sonst keine anderen aus diesem fachwortschatzsprachlichen Bereich. Eine Erklärung hierfür kann darin bestehen, dass sie sich in den Erdkundestunden des beobachteten Schuljahrs intensiv mit der physisch-geographischen Beschreibung einzelner Länder und Regionen befasst hatten, weshalb diese auch in den Textproduktionen den größten Raum einnimmt und andere Fachbereiche vernachlässigt werden.

Die auf den Lehrbuchseiten gebrauchten Ausdrücke aus der geographischen Fachsprache sind das Adjektiv ,nival“ sowie die Nomen barriera corallina (Korallenriff), foresta tropicale (Tropenwald), valle fluviale (Flusstal), delta (Delta), Equatore (Äquator), versante (meridionale) (Südseite) und insediamento (Ts geogr., antrop.; Niederlassung). Letzteres kommt auf den entsprechenden Seiten dreimal und delta zweimal vor.

Von den acht Worttypen verwendeten die Lernenden in ihren Texten lediglich zwei. Der erste, barriera corallina, stammt von einem Schüler, der darüber hinaus als einziger zusätzlich den Ausdruck atollo corallino (Korallenatoll) ${ }^{211}$ aus dem allgemeinsprachlichen Wortschatz gebrauchte.

Anstelle des Tropenwaldes taucht nur in einem einzigen Text das generischere foresta (Fo; Wald) auf, und delta bei einer Schülerin. Davon abgesehen wird nur ein weiteres geographisches, im Lehrbuch auf den Seiten zur indischen Region nicht gebrauchtes Fachwort von einer Schülerin verwendet, und zwar das Phrasem agenti atmosferici. Die Lernenden verfassten ihre Texte also mit sehr wenigen Fachwörtern und nur in drei Ausnahmefällen mit einem bzw. zwei Wörtern aus dem geographischen Fachwortschatz.

An dritter Stelle folgen in den Lehrbuchtexten mit jeweils sechs Worttypen die fachsprachlichen Bereiche der Geologie und der Ethnologie, Letzterer mit den drei Adjektiven indoeuropeo (indoeuropäisch), tamil (tamilisch, 4 Tokens) und dravida (drawidisch) und den drei Nomen etnia (Ethnie), Dravidi (Draviden) und sherpa (Scherpa). Hiervon sind in den Schülertexten nur das Adjektiv dravida (2 Tokens in 2 Texten) und indirekt etnia, nämlich das ebenfalls fachsprachliche gruppo etnico (Volksgruppe), vertreten.

Die geologischen Fachwörter sind pianura alluvionale (Schwemmebene), erosione (Erosion), pietraia (Halde), risorgiva (Karstquelle) und conoide (Konoid), von denen das erste in fünf Schülertexten und das zweite in einem verwendet wurde. Die anderen geologischen Fachwörter gebrauchten die Lernenden nicht, und es ist fraglich, ob sie dazu über rezeptive, geschweige denn produktive Kompetenz verfügten. Stattdessen erscheinen in den Schülertexten zwei weitere geologische Fachwörter mit jeweils einem Vorkommnis: das von der Lehrkraft im Unterricht oft benutzte morfologia (Morphologie) und sedimento (Ts fis., geol., petr.; Sediment).

211 Hierbei handelt es sich um eine recht tautologische Formulierung, da der Begriff „Atoll“ ein kreisförmiges Korallenriff bezeichnet. Allerdings wird das Substantiv mit genau diesem adjektivischen Attribut auch im Lehrbuch verwendet (s. Zitat w.o.). 
Die übrigen 22 Fachbereiche stellen nur einen oder maximal zwei Worttypen; hierunter sind allerdings vier Ausdrücke, die mit drei bzw. vier Vorkommnissen vertreten sind. Es sind das Adjektiv minerario (Boden-) aus dem Fachwortschatz der Mineraltechnik, das viermal als Attribut zu risorse aufritt und im Deutschen mit „Bodenschätze“ wiedergegeben wird, sowie die jeweils dreimal vertretenen Phraseme/Substantive gas naturale (Erdgas) aus der Fachsprache der Chemie, densità (Bevölkerungsdichte) aus der Statistik und compagnia (Kompanie) aus der geschichtlichen Fachsprache.

Die Beschreibung des Wortschatzes abschließend, werden nun noch die verwendeten Phraseme vorgestellt.

\subsubsection{Phraseme}

Die Lehrbuchseiten zur indischen Region enthalten 60 Mehrworteinheiten mit insgesamt 79 Vorkommnissen, wobei gut 60 Prozent von ihnen, nämlich insgesamt 38, nur einmal und die restlichen zwei- bis viermal verwendet werden. Tabelle 9 informiert, um welche Phraseme es sich handelt.

\begin{tabular}{llll}
\hline Phrasem & Wortart & Gebrauchsmarke & Vorkommnisse \\
\hline a.C. & abbr.loc.avv. & CO & 2 \\
a capo & loc.avv. & CO & 1 \\
a causa di & loc.prep. & CO & 1 \\
a forma di & loc.prep. & CO & 1 \\
a lato & loc.avv. & CO & 1 \\
a parte & loc.agg.inv. & CO & 1 \\
accanto a & loc.prep. & CO & 1 \\
ai piedi & loc.avv. & CO & 1 \\
al contrario & loc.avv. & CO & 1 \\
al posto di & loc.cong. & CO & 1 \\
anche se & loc.cong. & CO & 3 \\
avere luogo & loc.v. & CO & 1 \\
barriera corallina & loc.s.f. & TS geogr. & 1 \\
canna da zucchero & loc.s.f. & TS bot.com. & 1 \\
centrale idrica & loc.s.f. & TS fis. & 1 \\
centro urbano & loc.s.m. & TS urban. & 1 \\
chilometro quadrato & loc.s.m. & TS metrol. & 1 \\
clima monsonico & loc.s.m. & TS meteor., geogr. & 2 \\
clima tropicale monsonico & loc.s.m. & TS meteor., geogr. & 1 \\
clima tropicale & loc.s.m. & CO & 1 \\
da sempre & loc.avv. & CO & 2 \\
da solo & loc.agg. & CO & 1 \\
di fatto & loc.avv. & CO & 1 \\
energia elettrica & loc.s.f. & TS fis. & 1 \\
energia idroelettrica & loc.s.f. & TS tecn. & 1 \\
fare capo & loc.v. & CO & 2 \\
foresta tropicale & loc.s.f. & TS geogr. & 1 \\
gas naturale & loc.s.m. & TS chim. & 3 \\
grazie a & loc.cong. & CO & 2 \\
\hline
\end{tabular}




\begin{tabular}{llll}
\hline Phrasem & Wortart & Gebrauchsmarke & Vorkommnisse \\
\hline gruppo etnico & loc.s.m. & TS sociol., urban. & 1 \\
guerra civile & loc.s.f. & CO & 3 \\
in atto & loc.agg.inv. & CO & 1 \\
in alto & loc.agg. & CO & 1 \\
in genere & loc.avv. & CO & 1 \\
in parte & loc.avv. & CO & 4 \\
in particolare & loc.avv. & CO & 1 \\
in seguito & loc.prep. & CO & 2 \\
invece di & loc.prep. & CO & 1 \\
lavoro nero & loc.s.m. & TS dir.lav. & 1 \\
lingua ufficiale & loc.s.f. & TS ling., dir. & 1 \\
livello del mare & loc.s.m. & CO TS geogr. & 1 \\
monarchia costituzionale & loc.s.f. & CO TS dir. & 1 \\
per esempio & loc.avv. & CO & 1 \\
per quanto riguarda & loc.avv. & CO & 1 \\
pianura alluvionale & loc.s.f. & TS geol. & 2 \\
popolazione attiva & loc.s.f. & CO TS stat. & 3 \\
potere politico & loc.s.m. & CO & 1 \\
pro capite & loc.agg. & CO & 1 \\
repubblica federale & loc.s.f. & CO & 2 \\
repubblica parlamentare & loc.s.f. & TS dir. & 1 \\
repubblica presidenziale & loc.s.f. & TS dir. & 1 \\
rispetto a & loc.prep. & CO & 1 \\
settore dei servizi & loc.s.m. & TS econ. & 1 \\
sistema montuoso & loc.s.m. & CO TS geogr. & 1 \\
tant'è che & loc.cong. & CO & 1 \\
valle fluviale & loc.s.f. & TS geogr. & 1 \\
venditore ambulante & loc.s.m. & CO TS burocr. & 1 \\
via di comunicazione & loc.s.f. & CO & 1 \\
vicino a & loc.prep. & CO & 1 \\
zona franca & loc.s.f. & TS dir. & 1 \\
\hline
\end{tabular}

Tabelle 9: Phraseme der Lehrbuchseiten zur indischen Region (Bersezio 2005)

Nur acht Phraseme bestehen aus mehr als zwei graphischen Wörtern mit jeweils einem Token:

- per quanto riguarda (CO; bezüglich);

- al posto di (CO; anstelle von);

- a causa di (CO; aufgrund von);

- a forma di (CO; in Form von);

- canna da zucchero (Ts bot.com; Zuckerrohr);

- via di comunicazione (CO; Verkehrsweg);

- livello del mare (CO; Meeresspiegel);

- settore dei servizi (Ts econ.; Dienstleistungssektor).

Da alle 60 Phraseme aus dem allgemeinwortschatz- und fachsprachlichen Gebrauch stammen, der w.o. vorgestellt worden ist, wird im Folgenden auf eine 
nähere Beschreibung verzichtet und lediglich auf ihre Verteilung auf die beiden Wortschatzbereiche und die einzelnen Gebrauchsmarken eingegangen. Unter den Worttypen befinden sich fünf Adjektive mit jeweils einem Token, das abgekürzte Adverbialphrasem a.C. (CO; v.Chr.) mit zwei Vorkommnissen sowie elf ausgeschriebene, vier konjunktionale, sieben präpositionale und zwei verbale Phraseme, die komplett aus dem allgemeinsprachlichen Gebrauch stammen. Die am stärksten vertretene Gruppe bilden mit den übrigen 30 Phrasemen die Substantive, die insgesamt 39-mal vorkommen und somit die Hälfte aller vorhandenen Phraseme und 49,4 Prozent aller Vorkommnisse stellen. Ungefähr jedes Vierte davon ist Co-gekennzeichnet (5) oder als Co Ts doppelt markiert (5), während die anderen 20 Mehrworteinheiten aus verschiedenen Fachsprachen stammen.

Am totalen Wortvolumen machen die Phraseme einen Anteil von 4,2 Prozent aus.

\subsubsection{Lexikogrammatikalische und syntaktische Eigenschaften}

In dieser Sektion des Kapitels werden zunächst die verwendeten Konjunktionen (10.2.2.1) vorgestellt, woran sich eine Beschreibung der Verben (10.2.2.2) anschließt, die sich wieder in die finiten Formen der Prädikate (10.2.2.2.1) sowie den Passivgebrauch (10.2.2.2.1.1) und die infiniten Formen der Partizipien, Gerundien und Infinitive (10.2.2.2.2) untergliedert.

\subsubsection{Satzverknüpfungen}

Viele der untersuchten Sätze sind durch Konjunktionen miteinander verbunden. Wie Tabelle $10 \mathrm{zu}$ entnehmen ist, gibt es nur zwei Texteinheiten (S: 126 f. und S. 128 f.), auf denen nicht durchschnittlich mindestens eine Konjunktion auf einen Satz entfällt.

\begin{tabular}{lrrrr}
\hline Seite $(\mathrm{n})$ & n Konjunktionen & n Types & davon nicht FO & $\varnothing$ Konj. pro Satz \\
\hline S. $114 \mathrm{ff}$. & 27 & 5 & 0 & 1,13 \\
S. 117 & 22 & 5 & 1 (benché, AU) & 1,38 \\
S. 118 & 20 & 5 & 1 (anche se, CO) & 1,11 \\
S. 119 & 25 & 8 & 2 & 1,92 \\
S. 122 ff. & 40 & 11 & 3 & 1 \\
S. 125 & 22 & 6 & 1 (però) & 1,57 \\
S. 126 f. & 22 & 6 & 1 (anziché) & 0,79 \\
S. 128 f. & 14 & 3 & 2 & 0,35 \\
\hline
\end{tabular}

Tabelle 10: Konjunktionen der Lehrbuchseiten zur indischen Region (Bersezio 2005)

In allen anderen untersuchten Texteinheiten sind es mindestens eine Konjunktion pro Satz, auf S. 119 sogar 1,92 im Durchschnitt. Wie in den Schülertexten ist die am häufigsten verwendete Konjunktion $e$ (Fo; und), doch in sieben der 
acht untersuchten Lehrbuchauszüge erscheinen auch Konjunktionen, die nicht zum fundamentalen Wortschatz zählen. Abgesehen von den Seiten 128 f. werden mindestens fünf verschiedene konjunktionale Worttypen verwendet.

\begin{tabular}{llll}
\hline Konjunktionen & Gebrauchsmarke & Vorkommnisse & Übersetzungen \\
\hline Tot. & & 192 & \\
anche & FO & 12 & auch \\
anche se & CO & 3 & auch wenn \\
anziché & AU & 1 & anstatt \\
che & FO & 2 & dass \\
dopo & CO & 1 & nachdem \\
dove & FO & 13 & wo \\
e & FO & 129 & und \\
infatti & FO & 2 & in der Tat \\
ma & FO & 10 & aber \\
mentre & FO & 1 & während \\
perché & FO & 2 & weil \\
però & AU & 1 & jedoch \\
pur & CO & 1 & auch \\
quando & FO & 1 & wenn, als \\
sebbene & AU & 1 & obwohl \\
sia & FO & 6 & sei es; sowohl... als auch \\
tant'è che & CO & 1 & so sehr, dass \\
tuttavia & FO & 3 & jedoch, dennoch \\
\hline
\end{tabular}

Tabelle 11: Worttypen der Konjunktionen mit Vorkommnissen

129, also 67 Prozent aller Konjunktionen, stellt das parataktische Bindewort „und“ (Fo). An zweiter Stelle folgt mit sehr großem Abstand die lokale Relativkonjunktion „wo“(Fo) mit 13 Vorkommnissen. Am dritthäufigsten wird mit zwölf Vorkommnissen die Konjunktion anche (Fo) verwendet. Ebenfalls häufiger erscheinen noch die adversative Konjunktion ,aber“ (Fo) mit zehn Vorkommnissen und „sei es“ bzw. „sowohl ... als auch“ (sia, Fo) mit sechs Tokens. Alle übrigen Konjunktionen treten maximal dreimal, meist nur einmal auf. Insgesamt handelt es sich um 18 verschiedene Worttypen, darunter u.a. drei weitere adversative, vier konzessive und eine konsekutive. Die letztgenannten, in den Schülertexten nicht vertretenen führen zur Bildung von Satzgefügen mit komplizierteren Verknüpfungen zwischen den einzelnen Satzaussagen, die möglicherweise nur z.T. verstanden wurden. Die adversativen Konjunktionen gehören zum Basiswortschatz: Mentre, tuttavia und $m$ sind Fo-gekennzeichnet und però trägt die Marke AU.

Beispielsweise im folgenden Satzgefüge, in dem sich die Präposition mentre befindet, könnten die Verknüpfungen zwischen den einzelnen Informationen noch deutlicher expliziert sein. Die Konjunktion leitet auch nicht den einzigen untergeordneten Satz des Gefüges ein, denn vom Adversativsatz hängt ein weiterer Nebensatz ab (Bersezio 2005, S. 123): 
L'artigianato e l'agricoltura tradizionali decaddero all'inizio del Novecento, mentre si sviluppavano i centri urbani che avevano una posizione migliore per i commerci e le vie di comunicazione.

(M.Ü.: Das traditionelle Handwerk und die traditionelle Landwirtschaft verfielen zu Beginn des 20. Jahrhunderts, während sich die Stadtzentren entwickelten, welche einen besseren Standort für den Handel und die Verkehrswege hatten.)

Auch lexikalisch bereitet dieses 27 Wörter lange Satzgefüge potentiell einige Schwierigkeiten, da fast die Hälfte der Ausdrücke (13) nicht aus dem fundamentalen Wortschatz stammt, sondern das erste Prädikat AU-gekennzeichnet ist und nur ein weiteres Wort aus diesem Bereich kommt, während die anderen dem allgemein- bzw. fachwortschatzsprachlichen Teil entstammen.

Noch mehr kann sich das Textverständnis für weniger geübte Leser erschweren, wenn die Konjunktionen seltener verwendet werden oder sie komplexere grammatisch-syntaktische Strukturen schaffen. Im nächsten Textauszug etwa wird mit sebbene ein Konzessivsatz eingeleitet, in dem das Prädikat nicht expliziert ist (ibid., S. 124):

La zona, sebbene paludosa e con fondali bassi, godeva di un'ottima posizione, vicino all'area più ricca dell'India: la Pianura del Gange e le piantagioni del Bengala.

(M.Ü.: Das Gebiet, obgleich sumpfig und mit tiefen Gründen, genoss eine optimale Position nahe der reichsten Gegend Indiens: die Ganges-Ebene und die Plantagen Bengalens.)

Abgesehen vom implizierten Prädikat entspringen auch in diesem Satz wichtige Inhaltswörter Bereichen des Gebrauchswortschatzes, die über den fundamentalen hinausgehen. So folgen auf die Konjunktion ein Adjektiv und ein Substantiv aus dem allgemeinen Wortschatz, und an den Konzessivsatz schließt das Prädikat des Hauptsatzes an, welches aus dem Bereich des hohen Gebrauches (AU) stammt.

Generell ist davon auszugehen, dass das Textverständnis umso stärker beeinträchtigt ist, je mehr sich die Schwierigkeiten auf lexikogrammatikalischer und morphosyntaktischer Ebene summieren. So entstehen auf den Lehrbuchseiten zur indischen Region bisweilen Satzgefüge mit hoher lexikalischer Dichte, ausgeprägter Hypotaxe, implizierten Prädikaten und niedrigfrequenten Lexemen wie die w.o. bei den passivischen und partizipialen Konstruktionen vorgestellten, mit benché und pur eingeleiteten Konzessivsätze.

Auch wenn die Art der Verknüpfungen zwischen den einzelnen Informationen weniger Verständnisschwierigkeiten hervorruft, weil es sich nicht um kompliziertere wie konzessive, konsekutive, kausale oder konditionale handelt, erfordern manche Satzgefüge allein durch ihre Länge und Fülle an Informationen eine anspruchsvollere Verständnisleistung, wie der letzte Textauszug verdeutlicht (ibid., S. 118; Hervorhebung im Original):

Il riso, base dell'alimentazione in tutta la regione, è coltivato ovunque, ma specialmente nelle zone dove le precipitazioni sono abbondanti, tanto da garantire spesso un secondo raccolto (come accade nel Bengala indiano e nel Bangladesh); il frumento e la segala predominano dove il clima è più asciutto, come in Pakistan. 
(M.Ü.: Der Reis, Nahrungsgrundlage in der ganzen Region, wird überall angebaut, aber vor allem in den Gebieten, wo die Niederschläge reichhaltig sind, so sehr, dass sie oft eine zweite Ernte garantieren (wie es im indischen Bengalen und in Bangladesch geschieht); der Weizen und der Roggen herrschen vor, wo das Klima trockener ist, wie in Pakistan).

In diesem 50 Wörter langen Satzgefüge gibt es keine nebenordnenden Konjunktionen, die ganze Sätze miteinander verknüpfen, und die hypotaktischen beschränken sich auf zwei lokale Relativsätze und einen Adversativsatz. Darüber hinaus enthält das Gefüge jedoch einen komplexeren satzwertigen Infinitiv, der zusammen mit dem Adverb tanto einen Konsekutivsatz bildet, einen Vergleichssatz, der als Zusatz aufgeführt ist und in Klammern steht, sowie einen weiteren Vergleich. Ein Drittel der Wörter stammt nicht aus dem fundamentalen Wortschatz, sondern ist entweder AU- oder Co-markiert bzw. ein Toponym.

Hierbei handelt es sich um Wörter, welche wahrscheinlich alle SchülerInnen verstanden, da sie im Erdkundeunterricht häufiger und z.T. auch aktiv von ihnen selbst verwendet wurden. Lediglich das Verb predominare (Co; vorherrschen) war vielleicht nicht allen bekannt und wurde in ihren Texten auch nicht verwendet.

Andere Ausdrücke, die nicht zum Basiswortschatz zählen, wurden wahrscheinlich verstanden, im produktiven Gebrauch aber durch frequentere Synonyme wie beispielsweise precipitazioni und specialmente ersetzt ${ }^{212}$ : Während das italienische Wort für „Niederschläge“ nur in drei Texten vorkommt, erscheint in fünf mit insgesamt neun Vorkommnissen das Synonym pioggia aus dem fundamentalen Wortschatz, und in einem weiteren wird zur Darstellung eines diesbezüglichen Sachverhalts keine grammatikalische Metapher benutzt, sondern der Schüler drückt ihn verbal mit einem von piovere (FO; regnen) gebildeten Prädikat aus.

Selbst wenn die Lernenden den oben stehenden Satz vollständig verstanden, weisen ihre Texte keine derart ausgeprägte Verdichtung auf. Eine mehrsprachige Schülerin verwendete zwar precipitazioni und als einzige specialmente, verkürzte und vereinfachte das Satzgefüge aus dem Lehrbuchtext aber erheblich (Schülerin G):

Il settore agricolo: il riso è la base dell'alimentazione in tutta la regione. Si coltiva specialmente nelle zone dove le precipitazioni sono abbondanti.

(Der Landwirtschaftssektor: Der Reis ist die Nahrungsgrundlage der ganzen Region. Man baut ihn vor allem in den Gebieten an, wo die Niederschläge reichhaltig sind.)

Lexikalisch und semantisch sind ihre Sätze mit denen des Lehrbuchs identisch; sie reduzierte aber die informationelle Dichte und ersetzte ein Prädikativum

212 Hierzu kann auch segala gerechnet werden, das in den Schülertexten entweder durch ein Hyperonym ersetzt wird, ganz entfällt oder aber in drei Texten in der gebräuchlicheren Variante mit der Endung auf -e, segale, erscheint. 
durch die Formulierung si coltiva, die den Aktanten ebenfalls nicht klar bezeichnet, ihn aber im Gegensatz zum Lehrbuchtext mit dem Prädikativum ins Spiel bringt. Die erste Information über den Reis (Nahrungsgrundlage der Region), die im Lehrbuchtext in Form einer Apposition erfolgt, formulierte sie als eigenständigen Hauptsatz. Manche Informationen ließ sie aus, wie beispielsweise, dass der Reis überall angebaut wird. Außerdem ergab sich daraus der Verzicht auf den durch $m a$ eingeleiteten Adversativsatz. Dessen Inhalt gab sie nicht hypotaktisch zum Vorausgehenden, sondern in Form eines eigenständigen Hauptsatzes wieder, wobei sie ebenfalls den komplexeren Zusatz wegließ, der mithilfe des Adverbs tanto und dem Infinitiv einen Konsekutivsatz formt.

\subsubsection{Verben}

\subsection{Prädikate}

Auf den Lehrbuchseiten befinden sich insgesamt 314 Prädikate, von denen die meisten im Indikativ Präsens aktiv konjugiert sind. Im Vergleich zu den Schülertexten ist das verwendete Spektrum an Zeitformen, Modi und hinsichtlich des Genus verbi jedoch deutlich größer. So gibt es mehr im Passiv konstruierte Prädikate, und die abgedeckten Zeitformen reichen nach Häufigkeit geordnet vom Präsens über das Perfekt und das passato remoto bis zum Imperfekt. Diese größere Zeitenvielfalt ist nicht nur auf die höhere Schreibkompetenz des Lehrbuchautors zurückzuführen, sondern resultiert v.a. aus dem Textinhalt: Die Themenwahl hat zur Folge, dass geschichtliche Hintergrundinformationen vorgestellt werden, was in den Vergangenheitstempora geschieht, während diese Aspekte in den Schülertexten keine Erwähnung finden.

Jeder Satz bzw. jedes Satzgefüge verfügt durchschnittlich über 1,6 Prädikate, bei einem Minimum von 1,4 auf den Seiten 117 und $126 \mathrm{f}$. und einem Maximum von 2 auf Seite 125. Bei einer durchschnittlichen Satzlänge von 20 Wörtern bedeutet das, dass jedes Prädikat im Mittelwert mindestens acht Wörter regiert. Allerdings ist eine Eigenschaft der sprachlichen Gestaltung der Lehrbuchtexte nicht nur eine im Vergleich zu den Schülertexten größere Fülle an Attributen, Objekten und adverbialen Bestimmungen, sondern auch eine regere Verwendung von nicht flektierten Verbformen, wie sie sich beispielsweise in den Formulierungen von satzwertigen Infinitiven und Partizipien sowie Gerundiva finden lassen. Tabelle 12 illustriert die Prädikatsverteilung auf die untersuchten Lehrbucheinheiten mit einer Auswahl an Merkmalen (z.B. Worttypen und Wortvorkommnisse, Hilfs- und Modalverben, Passivgebrauch, Zuordnung zu den einzelnen Feldern des Gebrauchswortschatzes).

\begin{tabular}{lrrrrrrrrr}
\hline Prädikate & S. $114 \mathrm{ff}$. & S. 117 & S. 118 & S. 119 & S. $122 \mathrm{ff}$. & S. 125 & S. $126 \mathrm{f}$. & S. $128 \mathrm{ff}$ & $\mathrm{n} / \%$ \\
\hline $\begin{array}{l}\text { Vorkom- } \\
\text { men }\end{array}$ & 41 & 24 & 32 & 24 & 64 & 32 & 39 & 58 & 314 \\
\hline
\end{tabular}




\begin{tabular}{|c|c|c|c|c|c|c|c|c|c|}
\hline Prädikate & S. $114 \mathrm{ff}$. & S. 117 & S. 118 & S. 119 & S. $122 \mathrm{ff}$. & S. 125 & S. $126 \mathrm{f}$. & S. $128 \mathrm{f}$. & $n / \%$ \\
\hline Worttypen & 26 & 16 & 22 & 23 & 43 & 27 & 28 & 34 & \\
\hline n loc.v. & 0 & 0 & 0 & 0 & 1 & 0 & 2 & 0 & \\
\hline n v.mod. & 1 & 1 & 0 & 0 & 0 & 0 & 1 & 1 & 4 \\
\hline n v.aus. & 10 & 5 & 9 & 6 & 9 & 4 & 11 & 10 & 64 \\
\hline n Passiv & 5 & 3 & 4 & 2 & 4 & 2 & 7 & 7 & 34 \\
\hline n essere & 9 & 7 & 11 & 2 & 18 & 8 & 14 & 25 & 94 \\
\hline$\%$ essere & 22 & 29,1 & 34,4 & 8,3 & 28,1 & 25 & 35,9 & 43,1 & 29,9 \\
\hline $\begin{array}{l}\mathrm{n} \text { esserci } \\
\% \text { essere }\end{array}$ & 1 & 0 & 0 & 0 & 1 & 1 & 1 & 0 & 4 \\
\hline + esserci & 24,4 & 29,1 & 34,4 & 8,3 & 29,7 & 28,1 & 38,5 & 43,1 & 31,2 \\
\hline n esservi & 1 & 1 & 0 & 0 & 2 & 1 & 0 & 0 & 5 \\
\hline$A D$ & 1 & 0 & 0 & 0 & 1 & 0 & 1 & 1 & \\
\hline $\mathrm{AU}$ & 4 & 0 & $6(5)$ & $5(4)$ & 7 & 2 & $8(7)$ & $6(5)$ & \\
\hline $\mathrm{CO}$ & $13(6)$ & $7(6)$ & 3 & 4 & $14(13)$ & 4 & $7(3)$ & 3 & \\
\hline $\begin{array}{l}\text { FO } \\
\varnothing \text { pro }\end{array}$ & $33(15)$ & $22(12)$ & $28(14)$ & $21(16)$ & $46(20)$ & $30(21)$ & $32(14)$ & $61(21)$ & \\
\hline Satz & 1,6 & 1,4 & 1,8 & 1,8 & 1,6 & 2 & 1,4 & 1,45 & 1,6 \\
\hline n Sätze & 25 & 16 & 18 & 13 & 40 & 16 & 28 & 40 & 25 \\
\hline
\end{tabular}

Tabelle 12: Prädikate der Lehrbuchseiten zur indischen Region (Bersezio 2005)

Der Großteil der Prädikate stammt aus dem fundamentalen Wortschatz (Fo), gefolgt vom allgemeinsprachlichen (CO). Hierunter befinden sich sechs Formen des zur Passivkonstruktion benötigten Hilfsverbs venire sowie die vier intransitiven Verben predominare (überwiegen), digradare (abflachen), convergere (zusammenlaufen), stazionare (stationieren) und diese elf transitiven Verben:

individuare (erkennen), cingere (umschließen), insanguinare (mit Blut bedecken), bonificare (trockenlegen), costeggiare (entlanggehen), ridisegnare (wieder zeichnen), dedicare (widmen), irrigare (bewässern), ostacolare (behindern), devastare (zerstören).

Desweiteren sind auf den Lehrbuchseiten fünf Vorkommnisse des prokomplementären Verbs esservi (da sein) und 25 Vorkommnisse reflexiver Verbformen vertreten. Dabei handelt es sich um die folgenden 17 Worttypen: ${ }^{213}$

accrescersi (sich vergrößern), alternarsi (sich abwechseln), ampliarsi (sich ausdehnen), basarsi (basieren), dividersi (sich teilen), estendersi (sich erstrecken), insediarsi (sich niederlassen), mescolarsi (sich vermengen), mettersi (a capo) (sich (an die Spitze) stellen), opporsi (sich widersetzen), prolungarsi (sich verlängern), rompersi (kaputtgehen), sollevarsi (sich erheben), svilupparsi (sich entwickeln), trattarsi (sich handeln um), trovarsi (sich befinden).

Das letzte Verb ist mit acht Tokens das am stärksten vertretene reflexive Verb und kommt auch in den Schülertexten vermehrt vor, nämlich insgesamt 16mal. Die anderen Verben sind auf den Lehrbuchseiten entweder ein- oder zweimal verzeichnet; lediglich svilluparsi (sich entwickeln) kommt dreimal vor. Neben trovarsi werden nur drei weitere in zwei bis drei Schülertexten gebraucht.

213 Die Hervorhebung zeigt an, welche Verben auch die Lernenden in ihren Texten gebrauchten. 
Aus dem Bereich der hohen Disponibilität (AD) stammen die vier Tokens der Verben spianare (ebnen), sfociare (münden) und basarsi (basieren auf), die auch in einigen Schülertexten vorkommen: das erste und das dritte in zwei Texten und das zweite in vier mit insgesamt sechs Tokens.

Verben aus dem Bereich des hohen Gebrauchs (AU) sind in einer untersuchten Lehrbucheinheit gar nicht vertreten, in einer weiteren nur mit zwei und in den übrigen mit vier bis acht Tokens. Insgesamt handelt es sich um diese Prädikate, wobei ihre Hervorhebung anzeigt, dass sie auch in den Schülertexten auftreten:

assorbire (absorbieren, aufnehmen), determinare (bestimmen), incidere (durchschneiden), causare (verursachen), impiegare (aufwenden, beschäftigen), praticare (praktizieren), esportare (exportieren), portare (tragen, hier: führen $\mathrm{zu}$ ), abolire (abschaffen), attirare (anziehen), dominare (dominieren), alimentare (nähren), regnare (regieren), spiccare (hervorstechen), governare (regieren), esscerci (da sein, geben), provenire (stammen), prevalere (vorherrschen), emigrare (emigrieren), utilizzare (benutzen), confinare (grenzen an), stentare (sich abmühen), decadere (verfallen), godere (genießen), emergere (auftauchen), facilitare (erleichtern).

Acht der 26 Verben wurden auch von den Lernenden verwendet, wobei confinare in fünf Texten zur geographischen Einordnung der indischen Region benutzt wird und portare in unterschiedlichen Verwendungskontexten in zwei Texten insgesamt dreimal auftritt. Die anderen Verben sind nur in je einem Text vertreten, esportare allerdings mit vier Tokens.

Von allen Prädikaten in den Lehrbuchtexten gehen 29,9 Prozent (94) auf das Verb essere zurück, wobei die vorherrschende morphosyntaktische Konstruktion das Prädikativum ist.

Textbeispiele hierfür sind „I monti non sono mai stati un vero impedimento alle comunicazioni e sono attraversati da alcuni valichi, che comunque toccano i 5000 metri di altezza" (Bersezio 2005, S. 114; M.Ü.: Die Berge sind nie ein wirkliches Kommunikationshindernis gewesen und sind von einigen Gebirgspässen durchsetzt, die trotzdem eine Höhe von 5.000 Metern erreichen), „L'agricoltura è l'attività prevalente e occupa due terzi degli indiani“" (ibid., S. 126; M.Ü.: Die Landwirtschaft ist die vorherrschende Aktivität und beschäftigt zwei Drittel der Inder) und auch (ibid., S. 120):

La montagna più sacra di tutte è il Kangchenjunga (fotografia a sinistra), che supera gli 8500 metri di altezza e il cui nome significa "Cinque fratelli della grande neve". È una divinità protettrice e creatrice e molti indiani fanno risalire le proprie origini a una donna e a un uomo plasmati dai suoi ghiacci.

(M.Ü.: Der heiligste Berg von allen ist der Kangchendzönga (Photo links), der die 8.500 Höhenmeter überragt und dessen Name „Fünf Geschwister des großen Schnees“ bedeutet. Er ist eine beschützende und schaffende Gottheit, und viele Inder leiten die eigenen Ursprünge von einer Frau und einem Mann her, die aus seinem Eis geformt sind.)

So wie aus diesen Beispielen ersichtlich wird, handelt es sich auf den Lehrbuchseiten meist um Satzgefüge, in denen nicht einfach ein Subjekt durch ein 
Prädikativum ergänzt, sondern dieses bspw. von einem oder mehreren Relativsätzen näher bestimmt und durch weitere Informationen in Form von parataktisch verbundenen Sätzen ergänzt wird. Darüber hinaus bestehen die Lehrbuchtexte nicht aus einer Aneinanderreihung von Prädikativa, da zwischen ihnen i.d.R. Prädikate liegen, die mit anderen Verben als essere gebildet sind.

Einen im Vergleich zu den Schülertexten wesentlich geringeren Anteil an den Verben machen die modalen und die prokomplementären Prädikate von esserci und esservi aus.

\subsection{Passivgebrauch}

Insgesamt sind 34 Prädikate passivisch konstruiert, also durchschnittlich etwas mehr als jedes zehnte Verb und ungefähr drei Passivformen auf jeder untersuchten Lehrbuchseite.

Es ist davon auszugehen, dass sich das Textverständnis durch Passivbildungen vor allem dann für einige erschwert, wenn sie in Sätzen mit einer hohen lexikalischen Dichte und einem lexikalischen Umfeld auftreten, das nicht aus dem Basiswortschatz besteht, und wenn die Verben selbst nicht aus dem Basiswortschatz stammen. In Abbildung 8 sind einige Textbeispiele aufgeführt, in denen sich diese sprachlichen Merkmale konzentrieren.

1) Lungo il corso del Gange si estende una delle più vaste pianure alluvionali della Terra, formata dai sedimenti depositati dalle acque. L'intera pianura è cinta verso nord da una zona di risorgive e di terreni paludosi. A ovest è limitata dal Deserto di Thar, tra India e Pakistan, un'area pressoché disabitata (Bersezio 2005, S. 116; Hervorhebung im Original; m.Ü.: Entlang des Flusses Ganges erstreckt sich eine der weitläufigsten Schwemmebenen der Welt, die von den vom Wasser abgelagerten Sedimenten geformt ist. Die gesamte Ebene ist gegen Norden von einem Gebiet von Karstquellen und Sumpfböden umgürtet. Im Westen ist sie durch die Tharwüste begrenzt, zwischen Indien und Pakistan, eine nahezu unbewohnte Gegend.)

2) Nelle città del Bangladesh, Dacca, la capitale, e Chittagong, la situazione è ancora più drammatica, benché tre abitanti su quattro vivano nelle campagne, che tuttavia vengono periodicamente invase da inondazioni, talvolta catastrofiche. (ibid., S. 117; Hervorhebung im Original; m.Ü.: In den Städten von Bangladesch, Dhaka, der Hauptstadt, und Chittagong ist die Situation noch dramatischer, obwohl drei von vier Einwohnern auf dem Land leben, das trotzdem periodisch von Überschwemmungen befallen wird, manchmal katastrophalen.)

3) Lo sviluppo economico della regione indiana è stato fortemente condizionato dagli interventi stranieri che, nel corso del tempo, hanno operato sia da stimolo in alcuni settori sia da freno in altri. (ibid., S. 119; m.Ü.: Die wirtschaftliche Entwicklung der indischen Region ist stark durch die ausländischen Eingriffe beeinflusst worden, die im Laufe der Zeit sowohl als Stimulation in einigen Sektoren als auch als Bremse in anderen gewirkt haben.)

4) Al momento dell'indipendenza non ci fu solo il problema della lingua: i nuovi confini interni furono ridisegnati tra mille difficoltà e alcuni Stati, come il Punjab e il Kashmir, minacciarono la secessione. (ibid., S. 125; Hervorhebung im Original; m.Ü.: Im Moment der Unabhängigkeit gab es nicht nur das Problem der Sprache: Die neuen Grenzen wurden unter tausend Schwierigkeiten neugezeichnet, und einige Staaten wie Punjab und Kaschmir drohten mit der Sezession.) 
5) Una vasta zona di risorgive ha creato terreni paludosi, i terai, che oggi sono stati in parte bonificati. (ibid., S. 122; m.Ü.: Ein weitläufiges Gebiet von Karstquellen hat Sumpfböden geschaffen, die Terai, welche heute zum Teil trockengelegt worden sind.)

Abbildung 8: Passivkonstruktionen auf den Lehrbuchseiten zur indischen Region

Im ersten Beispiel aus der physischen Beschreibung der Region befinden sich zwei aufeinanderfolgende Passivformen, von denen die erste von einem Verb aus dem allgemeinen (CO) und die zweite aus dem fundamentalen Wortschatz (Fo) stammt. Die Verbformen, die den passiven Prädikaten vorausgehen und folgen, sind nicht flektiert: das Partizip formata, Bestandteil eines impliziten Relativsatzes, und das adjektivische Partizip disabitata, das attributiv gebraucht ist.

Neben dem Co-gekennzeichneten Passiv gibt es zudem in den oben abgebildeten Sätzen des ersten Beispiels weitere Ausdrücke, die nicht zum fundamentalen bzw. zum Basiswortschatz zählen: Von den insgesamt 51 Wörtern sind nur 31 Fo-gekennzeichnet, also 60 Prozent, während von den übrigen eines die doppelte Marke Fo Ts trägt, drei aus dem des hohen Gebrauchs (AU), sechs aus dem des allgemeinen Wortschatzes (CO) und drei aus fachsprachlichen Wortschätzen stammen, darunter auch ein Phrasem. Abgesehen von Verständnisschwierigkeiten, die sich hier aus mangelnder lexikalischer Kompetenz ergeben können, ist zudem keiner der Aktanten animiert. Das hat zwar keineswegs stilistische Gründe, da es sich eben um eine physisch-geographische Beschreibung handelt, jedoch würde es zur Erleichterung des Textverständnisses beitragen, wenn bei der Wahl dieser grammatikalisch komplexeren Mittel des Passivs und der Partizipien zumindest die lexikalische Gestaltung des Satzgefüges stärker im fundamentalen Wortschatz verortet wäre. Beispielsweise könnte das passivische Prädikat è cinta durch è circondata (FO; umgeben) ersetzt werden, was häufiger gebraucht wird und zweifellos bekannter ist als das bedeutungsähnliche Verb aus dem allgemeinen Wortschatz.

Desweiteren könnte das adjektivisch-partizipiale Attribut am Schluss in einen Relativsatz aufgelöst werden, um dem Satz etwas von seiner Dichte zu nehmen und zumindest einen animierten Aktanten einzuführen: ,[...] Deserto di Thar, tra India e Pakistan, un'area in cui/dove non vive quasi nessuno" (Tharwüste zwischen Indien und Pakistan, ein Gebiet, in dem/wo fast niemand lebt).

Im zweiten Beispiel stammt das passivisch gebrauchte Verb invadere zwar aus dem fundamentalen Wortschatz, wird aber nicht in einer seiner Hauptbedeutungen, sondern figurativ verwendet. Hier können eventuelle Verständnisschwierigkeiten neben der lexikalischen Gestaltung des Kontextes auch aus den lexikogrammatikalischen und morphosyntaktischen Gegebenheiten entstehen. Denn die Passivkonstruktion befindet sich innerhalb eines komplexen Satzgefüges, in dem das Subjekt des Hauptsatzes, la situazione, nicht animiert ist, eine adverbiale Bestimmung mit zwei Präpositionen auftritt und ein zweiter 
Hauptsatz durch die nebenordnende Konjunktion benché (AU; obgleich) mit dem ersten verbunden ist. Darauf folgen die semantisch verwandte Konjunktion tuttavia (FO; trotzdem) und ein Relativsatz, in dem sich schließlich das passivische Verb befindet und an den noch eine Apposition angeschlossen ist. Vor allem die Häufung der konzessiven bzw. adversativen Verknüpfungen der Satzaussagen, welche die produktiven Satzverbindungsleistungen der Lernenden übersteigen, dürfte dazu geführt haben, dass das Satzgefüge mit all seinen inhaltlichen Nuancen nicht vollständig verstanden wurde. Auch hier gilt, dass eine bessere Garantie für das Textverständnis aller SchülerInnen eine stärker aus dem Basiswortschatz schöpfende lexikalische Gestaltung wäre.

Das dritte potentiell kompliziertere Passivbeispiel stammt aus dem ersten Satz eines Textes, dessen Überschrift bereits wegen der beiden darin vorhandenen Partizipien vorgestellt worden ist. Der Satz mit der Passivkonstruktion greift die Überschrift auf und führt diese weiter aus. Neben dem seltenen Auftreten des Verbs condizionare aus dem allgemeinen Wortschatz (Co) trägt auch hier die Verwendung nicht animierter Aktanten zu eventuellen Verständnishürden bei. Der Text, aus dem dieser Ausschnitt stammt, lässt die Auffassung vieler Lehrkräfte nachvollziehen, dass die Texte der Unterrichtswerke nicht eigenständig von den Lernenden erarbeitet und verstanden werden können, sondern dass es ihrer Erklärungshilfen bedarf. Hier wird die zu erbringende Verständnisleistung dadurch anspruchsvoller, dass es sich um einen komplexeren Sachverhalt handelt, der mit nur einem konkreten Beispiel illustriert und zudem im Passiv gebildet ist.

Im vierten Textausschnitt liegt eine Passiv-Konstruktion vor, die mit einem Verb (ridisegnare) aus dem allgemeinen Wortschatz gebildet ist, das für ein gesichertes Textverständnis bekannt sein muss. Darüber hinaus ist im Ausschnitt nicht direkt expliziert, was im Moment der indischen Unabhängigkeit neben der Sprachwahl ein weiteres Problem darstellt, da der Ausschnitt inhaltlich damit beginnt, dass die Sprachwahl nicht das einzige Problem ist, woraufhin nach einem Doppelpunkt die Grenzziehung thematisiert wird. Die Lernenden müssen also nicht nur den Bezug herstellen, dass es sich dabei um ein weiteres Problem handelt, sondern auch noch verstehen, warum die Grenzziehung überhaupt ein Problem darstellt.

Im letzten Textauszug ist bonificare (trockenlegen) ein im Passiv gebrauchtes Verb aus dem allgemeinen Wortschatz (CO), das voraussichtlich nicht allen bekannt ist und auch nicht leicht aus dem Kontext erschlossen werden kann, zumal von den 18 Wörtern, die diesen Satz bilden, nur elf aus dem fundamentalen Wortschatz (Fo) stammen. Eines ist als fach- und fremdsprachliches Wort doppelt markiert, ein weiteres fachsprachlicher Herkunft und die anderen fünf Wörter rekurrieren auf vier verschiedene Worttypen aus dem allgemeinsprachlichen Wortschatz (CO), von denen ein zweigliedriges Phrasem als Adverbiale zwischen dem Hilfsverb und dem Partizip auftritt. 
Auch eventuelle Wortbildungskenntnisse helfen hier nur bedingt weiter, da das im Wortstamm enthaltene Adjektiv zum einen in seiner ursprünglichen lateinischen Variante bon(us) und nicht in der heutigen italienischen Form mit dem Doppelkonsonanten buono (gut) erscheint, und zum anderen selbst bei erfolgreicher Identifizierung dieses Wortelements nicht automatisch auf die Bedeutung des Verbs geschlossen werden kann. Es ist anzunehmen, dass ein Teil der SchülerInnen diesen Satz ohne Verständnishilfen wie eine Erklärung im Text, der Lehrkraft oder in einem Glossar in Textnähe nicht versteht.

In anderen Fällen ist eine Bedeutungserschließung anhand morphologischer Kenntnisse leichter möglich, wie etwa bei dem ebenfalls im Passiv formulierten und Co-gekennzeichneten Prädikat è insanguinato, das in seine Bestandteile des präpositionalen Präfixes in- (FO; in/ein) und das Verb sanguinare (AD; bluten) zergliedert, bzw. in dem das Substantiv sangue (FO; Blut) erkannt werden kann, was geläufiger ist als das davon abgeleitete Verb sanguinare. Zudem kennen die Lernenden wahrscheinlich weitere Verben, die nach demselben Prinzip gebildet werden, wie z.B. innervorsire (Co; nervös machen), imbalsamare (CO; einbalsamieren), imbarcare (AU; einschiffen), infastidire (CO; belästigen) und indebolire (AU; schwächen).

\subsection{Nicht flektierte Verbformen}

Auf den untersuchten Lehrbuchseiten sind 33 partizipiale Vorkommnisse zu verzeichnen, die auf 31 Worttypen zurückgehen. 29 davon sind mit einem Token und zwei mit jeweils zweien vertreten. 19 dieser Partizipien werden von 18 Verben aus dem fundamentalen Wortschatz gebildet, neun von acht Verben des hohen Gebrauchs (AU), eines vom reflexiven Verb basarsi (basieren auf) aus dem Gebrauchswortschatz der hohen Disponibilität (AD), eines vom fachsprachlichen Wort riconoscere (Ts dir.; anerkennen) und vier von Verben aus dem allgemeinen Wortschatz (CO). Von diesen letzten sind zwei oben bereits vorgestellt worden, teilweise zusammen mit weiteren Partizipien aus anderen Gebrauchsbereichen. Die anderen beiden lauten in der flektierten Form, in der sie im Text erscheinen, penalizzato (benachteiligt) und riconosciute (anerkannt).

Das erste kann Verständnisschwierigkeiten bereiten, die sich auf die Aussage des gesamten Satzes erstrecken, da die angrenzenden Wörter ebenfalls aus dem allgemeinen Wortschatz stammen. Darunter ist auch die seltener gebrauchte Konjunktion pur ((auch) wenn), die sowohl eine konditionale als auch adversative Funktion übernehmen kann: „Il turismo, pur penalizzato dall'instabilità politica, è in crescita" (Bersezio 2005, S. 129; m.Ü.: Der Tourismus, befindet sich, auch wenn er durch die politische Instabilität benachteiligt ist, im Wachstum). Es handelt sich hierbei um eine morphosyntaktisch kompliziertere Konstruktion, die ein Gerundium erfordert, das jedoch nur impliziert ist (pur essendo penalizzato). Ob diese Konstruktion mit ihrem adversativen 
Gehalt erkannt und/oder verstanden wird, ist fraglich, zumal das ähnlich klingende Adverb pure ,auch“bzw. ,ruhig/bloß" bedeutet und diesen adversativen Charakter also nicht aufweist. Jedenfalls wurde in keinem Schülertext eine Konstruktion mit dieser Konjunktion gefunden.

Auch die Bedeutung des Partizips contesa ist vermutlich nicht allen bekannt. Zwar stammt es von einem Verb aus dem Basiswortschatz, ist aber als Wort des hohen Gebrauchs (AU) seltener als Wörter aus dem fundamentalen Wortschatz zu finden und aus dem Kontext nicht oder nur eingeschränkt erschließbar. Außerdem befindet es sich in einem impliziten Relativsatz, der in Klammern angeführt ist, was dazu einlädt, ihn als weniger relevante Ergänzung aufzufassen und zu ignorieren. Warum diese zwischen Klammern stehende Ergänzung erwähnt wird, erschließt sich ohnehin nicht eindeutig, da es inhaltlich um eine geophysische Beschreibung Indiens geht, mit der sie nur sehr entfernt zu tun hat (ibid., S. 122):

L'ambiente naturale

Il territorio dell'India è diviso in tre regioni molto differenti: la zona himalaiana comprende a nord-ovest la regione del Kashmir (contesa con il Pakistan) e a nord-est quella del Sikkim. (M.Ü.: Die natürliche Umwelt

Das Gebiet Indiens ist in drei sehr unterschiedliche Regionen unterteilt: Das Himalaya-Gebiet beinhaltet im Nordwesten die Region Kaschmir (umstritten mit Pakistan) und im Nordosten die des Sikkim.)

Selbst dann, wenn die Partizipien von Verben aus dem fundamentalen Wortschatz stammen, werden sie wahrscheinlich nicht immer leicht verstanden, was neben dem lexikalischen Umfeld auch an den grammatikalisch-syntaktischen Strukturen liegt, die mit den Partizipien gebildet werden. So erscheint etwa im folgenden Textauszug das Partizip sorta (FO; entstanden) als Teil eines impliziten Relativsatzes, d.h. ihm fehlt das Prädikat (ibid., S. 124; Hervorhebung nicht im Original):

Delhi, la capitale, è la città più ricca di storia, sorta in un punto di importanza strategica, che domina il passaggio della Valle dell'Indo a quella del Gange.

(Delhi, die Hauptstadt, ist die an Geschichte reichste Stadt, die an einem Punkt strategischer Bedeutung entstanden ist, der den Übergang vom Tal des Hindus zu dem des Ganges beherrscht.)

Obwohl in diesem 28 Wörter langen Satzgefüge fast alle verwendeten Begriffe aus dem fundamentalen Wortschatz (FO) stammen - Ausnahmen bilden lediglich die drei Eigennamen der Stadt und der beiden Flüsse sowie das Adjektiv strategico (Co fig.) und das Prädikat dominare (AU) -, ist es relativ komplex. Es beginnt recht einfach mit einem kurzen Hauptsatz und dem Prädikat ,ist““ als Teil eines Prädikativums. Allerdings enthält der kurze Hauptsatz bereits eine Apposition (la capitale) sowie einen Superlativ, der zusammen mit einem präpositionalen Objekt ein Attribut zum Prädikatsnomen formt. Das darauffolgende Partizip weist eine feminine Singularendung auf, so dass theoretisch als 
Bezugswort außer dem Subjekt des Hauptsatzes und seiner Apposition ebenfalls das präpositionale Objekt ,,an Geschichte“ in Frage kommt. Was die Satzaussage des impliziten Relativsatzes anbelangt, so wird darin oder danach nicht direkt mit einer kausalen Bestimmung oder einem Kausalsatz erklärt, warum es sich um einen strategischen Punkt handelt, sondern nur mit einem weiteren Relativsatz, der von dem impliziten abhängt. Darin ist außerdem das AU-gekennzeichnete Prädikat enthalten, das eventuell nicht alle kennen.

Das letzte Beispiel eines schwer zu interpretierenden Partizips, das von einem wahrscheinlich bekannten Verb aus dem fundamentalen Wortschatz stammt, bildet zugleich die Überleitung zu einer weiteren auf den untersuchten Lehrbuchseiten verwendeten Kategorie nicht flektierter Verbformen, den Gerundien. Im nächsten Textauszug schließt sich nämlich eines an das Partizip an (Bersezio 2005, S. 125; Hervorhebung im Original):

Raggiunta l'indipendenza (1947), il governo aveva deciso che l' hindi fosse la lingua ufficiale di tutto il Paese, affiancata dall'inglese. Molti Stati però si opposero, rivendicando il diritto di usare ciascuno la propria lingua.

(M.Ü.: Die Unabhängigkeit erreicht (1947), hatte die Regierung entschieden, dass Hindi die Amtssprache des ganzen Landes sei, begleitet von Englisch. Aber viele Staaten widersetzten sich, indem sie für das Recht eintraten [wörtlich: für das Recht eintretend], jeder die eigene Sprache zu benutzen.)

23 der 32 Wörter des Textausschnitts stammen aus dem fundamentalen (Fo), die übrigen aus dem allgemeinen Wortschatz (CO), dem des hohen Gebrauchs (AU) und das Phrasem „Amtssprache“ aus den Fachsprachen des Rechtswesens und der Sprachwissenschaft. Es handelt sich mit den übrigen acht Wörtern also um einen relativ geringen Anteil an Ausdrücken, die nicht Fo-gekennzeichnet sind. Dennoch machen sie zusammen ein Viertel des gesamten Wortvorkommens aus und konzentrieren sich unter ihnen ein Prädikat, ein Partizip und ein Gerundium. Aufgrund dessen, dass sie einen Großteil der Satzglieder regieren, hängt von ihrem Verständnis erheblich das des gesamten Textausschnitts ab.

Raggiunta als Bestandteil eines satzwertigen Partizips am Anfang des Ausschnitts hat auch temporalen Charakter, indem es Vorzeitigkeit zur ebenfalls vorzeitigen Handlung des nächsten Satzabschnittes ausdrückt. Ungeübteren Lesern würde es das Textverständnis erleichtern, wenn ein Temporalsatz das satzwertige Partizip ersetzte.

Daran schließt sich zunächst ein weiteres Partizip an, mit dem erneut ein impliziter Relativsatz geformt wird, der sich auf das Prädikatsnomen „Amtssprache" bezieht, aber nicht direkt an dieses anschließt, da noch ein Attribut dazwischenliegt. Darüber hinaus steht das Prädikat des Prädikativums nicht im Indikativ, sondern im Konjunktiv. Auf diesen relativ komplexen Satz folgt ein weiterer, dessen Verb aus dem allgemeinen Wortschatz (Co) stammt, ,sich widersetzen“. Von diesem hängt der Gerundivsatz ab, der modalen Charakter aufweist; er gibt die Art und Weise an, wie das Widersetzen erfolgte. Einmal 
mehr sind im Übrigen die Aktanten nicht direkt animiert, sondern stehen als „Staaten“ und „Regierung" stellvertretend für die darin agierenden Menschen.

Auf den Lehrbuchseiten zur indischen Region erscheint noch ein weiteres Gerundium (ibid, S. 117):

Verso il 1500 a.C., ondate successive di popolazioni di pastori provenienti da nord-est invasero le stesse regioni e si mescolarono agli antichi abitanti, creando una complessa sovrapposizione di gruppi etnici diversi.

(M.Ü.: Gegen 1500 v.Chr. invadierten sukzessive Völkerwellen von aus Nordosten stammenden Hirten dieselben Regionen und mischten sich unter die Ureinwohner, eine komplexe Überlagerung verschiedener Volksgruppen schaffend.)

Der 30 Wörter lange Textauszug besteht zu über einem Drittel aus Wörtern, die nicht zum fundamentalen Wortschatz zählen. Das lexikalische Umfeld des Fo-gekennzeichneten Gerundiums gestaltet sich also recht komplex. Sieben Ausdrücke stammen aus dem allgemeinen Wortschatz (CO), unter ihnen das Prädikat „mischten sich“. Auch das erste, nicht animierte Subjekt ist ein figurativ gebrauchtes Wort aus dem Co-Bereich (Wellen), was die Lernenden jedoch aufgrund ihrer Kenntnisse der Wortbildungsmechanismen als bedeutungsähnlich zu onda (Welle) aus dem fundamentalen Wortschatz erkennen können. Ebenfalls aus dem allgemeinen Wortschatz stammt das Akkustativobjekt im Gerundivsatz, das zu Verständnisschwierigkeiten des gesamten Satzes führen kann, wenn es nicht beherrscht wird.

Wie im Falle des ersten Gerundiums werden auf diese Weise viele Informationen innerhalb des Satzgefüges verdichtet, die eher und vollständiger zugänglich wären, wenn sie durch etwas frequentere Lexeme oder aber mit einer finiten Verbform anstelle des Gerundiums und einer expliziteren Verknüpfung mit dem Textkontext wiedergegeben wären.

Desweiteren sind auf den Lehrbuchseiten zur indischen Region 26 Infinitive vertreten, die größtenteils aus dem fundamentalen Wortschatz stammen. Mit dieser infiniten Verbkategorie werden Konstruktionen gebildet, die überwiegend unkompliziert sind und nur in bestimmten Fällen vermehrt zu Verständnisschwierigkeiten führen können.

Beispielsweise erscheinen auf den Seiten vier Modalverben, die einen Infinitiv nach sich ziehen, wie sie auch in den Schülertexten verwendet werden. Ebenfalls leichter zu verstehen sind Konstruktionen mit bestimmten Verben, auf die meistens ein Infinitiv folgt, und die sowohl im Schriftlichen als auch im Mündlichen häufig gebraucht werden. Das sind auf den untersuchten Seiten etwa riuscire a (imstande sein) plus Infinitiv oder cercare di (versuchen) plus Infinitiv mit jeweils zwei Vorkommnissen.

Etwas anspruchsvoller gestaltet sich die Verständnisleistung bei Infinitivkonstruktionen mit der Präposition per, (hier: um zu), die eine finale Bedeutung annimmt. Derartige Finalsätze liegen mit drei Infinitiven vor.

1) La rete ferroviaria indiana è gigantesca e fu realizzata nell'Ottocento dal Regno Unito per facilitare le importazioni di cotone. (Bersezio 2005, S. 127; m. Ü.: Das indische 
Eisenbahnnetz ist gigantisch und wurde im 18. Jahrhundert vom Vereinigten Königreich errichtet, um die Baumwollimporte zu erleichtern.)

2) Si tratta in genere di città che si sono sviluppate sotto la dominazione britannica, spesso per sostenere le attività commerciali dei colonizzatori. (ibid., S. 124; m.Ü.: Es handelt sich in der Regel um Städte, die sich unter der britischen Herrschaft entwickelt haben, oft um die kommerziellen Aktivitäten der Kolonisatoren zu unterstützen.)

3) Ci sono ancora comunità di raccoglitori che praticano un'agricoltura itinerante basata sull'incendio delle foreste per aprire $i$ campi destinati alla semina dei cereali: consumato il campo, la comunità emigra (ibid., S. 126; Hervorhebung im Original, m.Ü.: Es gibt noch Sammler-Gemeinschaften, die eine Wanderlandwirtschaft betreiben, die auf dem Brand der Wälder beruht, um Felder zu eröffnen, die für die Saat von Getreide bestimmt sind: Wenn das Feld verbraucht ist, emigriert die Gemeinschaft.)

Abbildung 9: Finalsätze mit Infinitiven auf den Lehrbuchseiten zur indischen Region

Der erste Finalsatz mit Infinitiv stammt zwar aus einem eher kurzen Satzgefüge mit insgesamt 19 Wörtern, die allerdings nur zur Hälfte zum fundamentalen Wortschatz zählen, so dass sich bereits die lexikalische Ebene etwas komplex gestaltet. Bei den übrigen Ausdrücken handelt es sich um Toponyme und Wörter aus dem allgemeinen Wortschatz (CO) und dem des hohen Gebrauchs (AU), woraus auch das Verb stammt, das im Infinitiv erscheint. Das Akkusativobjekt des Finalsatzes ist ebenfalls ein Co-markierter Ausdruck, importazioni, der im Erdkundeunterricht jedoch wiederholt benutzt wird.

Auch im Satzgefüge des zweiten Finalsatzes zählen neun von 22 Wörtern nicht zum fundamentalen Wortschatz. Zu Letzterem gehört zwar das Verb im Infinitiv, sostenere, das Textverständnis ist aber neben der lexikalischen Komplexität und dem Finalsatz dadurch erschwert, dass der Aktant des Satzes nicht animiert ist und zwei Handlungen vollzieht, unter denen einige sich wahrscheinlich nichts Genaues vorstellen können.

Beim dritten Finalsatz mit Infinitiv handelt es sich insofern um das anspruchsvollste sprachliche Umfeld, als dass mit 29 Wörtern das Satzgefüge recht lang ist und 13 Ausdrücke nicht aus dem fundamentalen Wortschatz stammen. Es ist fraglich, ob allen klar ist, was mit dem Aktanten des Satzgefüges, den Sammlergemeinschaften, bezeichnet wird. Zwar wird ihre Lebensweise im Satzgefüge vorgestellt, doch geschieht es sowohl auf lexikalischer als auch auf grammatikalisch-syntaktischer Ebene auf recht komplizierte Weise. So gliedert sich der Finalsatz zwischen zwei Partizipialkonstruktionen ein, wobei er vom ersten Partizip abhängt und das zweite nach sich zieht. Im letzten Teil des Satzes ist eine größere Verstehensleistung vonnöten, da die Erklärung des Begriffs agricultura itinerante nicht direkt daran angeschlossen und beispielsweise mit einem Doppelpunkt gekennzeichnet wird, sondern dieses Satzzeichen erst vor dem letzten Teil der Beschreibung verwendet wird.

Unter den Infinitivkonstruktionen der Lehrbuchseiten zur indischen Region ist außerdem ein weiterer mit finalem Charakter zu finden (Bersezio 2005, S. 115): 
Le due catene si sono sollevate nella stessa epoca delle Alpi e sono così alte e aguzze perché sono "giovani" e l'erosione non ha avuto ancora il tempo di spianarle.

(M.Ü.: Die zwei Gebirgszüge haben sich in derselben Phase erhoben wie die Alpen und sind so hoch und spitz, weil sie ,jung“ sind und die Erosion noch keine Zeit gehabt hat, sie zu ebnen.)

Hier wird der satzwertige Infinitiv nicht von der Präposition per, sondern von di eingeleitet. Das Verb stammt aus dem Basiswortschatz (AD, hohe Disponibilität), ist aber trotzdem vielleicht nicht allen Lernenden bekannt. Zusätzlich ist es enklitisch konstruiert, da es ein Personalpronomen an sich bindet, welches das Subjekt des Satzgefüges aufgreift. Dieses steht allerdings ganz am Satzanfang, während das Pronomen zusammen mit dem Infinitiv das letzte Wort bildet und dazwischen verschiedene Satzglieder, drei hypotaktisch verbundene Hauptsatze und ein Kausalsatz liegen.

\subsection{Vergleich der Schülertexte mit den Lehrbuchseiten}

Die obigen Beschreibungen zeigen, dass sich die Schüler- und die Lehrbuchtexte auf allen sprachlichen Ebenen deutlich voneinander unterscheiden, wenn man einmal von den Textstellen absieht, die wortwörtlich aus den Lehrbuchtexten übernommen sind. Doch selbst dann reduzierten die SchülerInnen die informationelle Dichte und morphosyntaktische Komplexität der Lehrbuchtexte.

Sie verwendeten mehr Ausdrücke aus dem Basiswortschatz und formulierten Texte mit geringerer lexematischer Varianz. Das heißt, sie gebrauchten insgesamt weniger unterschiedliche Worttypen und wiederholten bereits genannte Begriffe, anstatt sie durch Synonyme zu ersetzen.

Der Wortschatz ihrer Texte ist zu einem größeren Teil aus dem häufiger gebrauchten fundamentalen Wortschatz (Fo) zusammengesetzt als der in den Lehrbuchtexten und beinhaltet demzufolge auch weniger Phraseologismen. Desweiteren sind Informationen öfter als Handlungen als in Form von Zuständen bzw. mit grammatikalischen Metaphern wiedergegeben. Daraus folgt, dass sie mehr Prädikate enthalten. So weisen die Schülersätze durchschnittlich 2,3 Prädikate auf, während es in den Lehrbuchtexten nur 1,6 sind. Gleichzeitig sind die nicht flektierten Verbformen seltener und auch weniger komplex gestaltet. Beispielsweise findet man den Großteil der Infinitive in den Schülertexten bei der Verwendung von Modalverben. Die zahlreicher vertretenen Modalverben gehen allerdings zumindest teilweise auf die Aufgabenstellung zurück, welche die Texte inhaltlich von denen des Lehrbuchs unterscheidet: 
Viele Modalverben erscheinen am Schluss, wo die SchülerInnen angeben sollten, ob sie sich vorstellen konnten, in der indischen Region zu leben. ${ }^{214}$

Zwar differieren die Texte der Lernenden in ihrer durchschnittlichen Satzlänge nur um drei Wörter von denen des Lehrbuchs (22 zu 25 Wörter), jedoch entstanden ihre langen Sätze oft durch parataktische Nebenordnungen bzw. Schwierigkeiten mit der Zeichensetzung. Diese äußerten sich darin, dass die Lernenden Satzaussagen innerhalb eines Gefüges nebenordneten, die geübtere Schreibende in unabhängigen Sätzen oder Satzgefügen formulieren würden, die durch eine satzbeendende Interpunktion voneinander getrennt wären.

Außerdem unterscheiden sich die satzverknüpfenden Elemente wie Konjunktionen und präpositionale/adverbiale Bestimmungen dadurch, dass in den Schülertexten eine geringere semantische Spannbreite von Verknüpfungen abgedeckt ist. Konjunktionale Verknüpfungen beschränken sich größtenteils auf parataktische Anordnungen bzw. auf die hypotaktischen Relativsätze. Gründe, Folgen, Konditionen oder Gegensätze werden von den Lernenden grundsätzlich seltener und somit auch geringfügiger mit entsprechenden Nebensätzen oder grammatikalischen Metaphern ausgedrückt.

Ferner ähneln die Schülersätze einander strukturell erheblich mehr als die der Lehrbuchseiten, weil sie vorwiegend mit dem Subjekt beginnen, das überdies im weiteren Verlauf eher wiederholt, als durch Pronomen oder andere Synonyme ersetzt wird.

Die Lehrbuch- und die Schülertexte unterscheiden sich auch auf inhaltlicher Ebene, was vor allem an den sehr unterschiedlichen Längen liegt. Die Lernenden mussten zwangsläufig die in den Lehrbuchtexten gegebenen Informationen reduzieren, doch zeigt sich bei ihnen die Tendenz, die unterschiedlichen inhaltlichen Aspekte weniger ausbalanciert darzustellen, als es in den Lehrbuchtexten erfolgt. Die Konzentration im Erdkundeunterricht auf die physischen Aspekte geographischer Räume spiegelt sich in ihren Texten dadurch wider, dass sie diesem Gegenstand mehr Platz einräumten als der Vorstellung wirtschaftlicher, demographischer und geschichtlicher Aspekte.

Abschließend zeigt Tabelle 13 im Einzelnen, wie sich die Texte der Lernenden und des Unterrichtswerks auf lexikalischer Ebene und in ihrer Gliederung in Sätze voneinander unterscheiden. Um eine bessere Vergleichbarkeit zu gewährleisten, als es ihre unterschiedlichen Längen zulässt, da sie sich auf die prozentuale Zusammensetzung des Wortschatzes aus den verschiedenen Gebrauchsbereichen auswirken, sind von den einzelnen Lehrbuchseiten zwölf ungefähr gleichlange Textauszüge untersucht worden, welche der durchschnittlichen Länge der Schülertexte entsprechen. ${ }^{215}$

214 S. in diesem Zusammenhang Christie (2012), die im Schreibstil von Jugendlichen u.a. eine Zunahme an Modalität ausmacht, die mit einer ausgeprägteren Versprachlichung der Einstellungen und Haltungen zum Textgegenstand einhergeht (ibid., S. 188).

215 Ausgenommen aus dieser Berechnung ist der eine Schülertext, der sich in seinem Umfang signifikant von allen anderen unterscheidet. 


\begin{tabular}{lrr}
\hline$\varnothing$ & Schülertexte & Lehrbuchseiten \\
\hline n Wörter & 304 & 298 \\
n Sätze & 15 & 12 \\
längster Satz & 45 & 39 \\
$\varnothing$ Satzlänge & 22 & 25 \\
AD & 3 & 5 \\
AD TS & 0 & 0 \\
AU & 23 & 29 \\
AU TS & 0 & 0 \\
CO & 29 & 39 \\
CO TS & 2 & 3 \\
el.CO & 4 & 4 \\
el.CO TS & 1 & 1 \\
el.FO & 0 & 0 \\
el.TS & 2 & 2 \\
ES & 0 & 0 \\
ES TS & 0 & 0 \\
FO & 198 & 188 \\
FO TS & 0 & 0 \\
LE & 0 & 0 \\
OB & 0 & 0 \\
TS & 5 & 6 \\
n.p. & 28 & 16 \\
el.n.p. & 9 & 4 \\
sigla & 0 & 0 \\
o. M. & 0 & 0 \\
n FO in \% & 05,1 \\
n VdB* & 0,1 & 74,5 \\
n VdB in \% & 224 & 20 \\
n n.p. inkl. el.n.p. in \% & 13,7 & \\
n n.p. inkl. el.n.p. & 13 & 0 \\
\hline & 37 & \\
Tal & & 0 \\
\hline
\end{tabular}

Tabelle 13: Mittelwerte sprachlicher Merkmale der Schülertexte und der Lehrbuchseiten zur indischen Region (Bersezio 2005)

*inkl. doppelter Marken mit Ts

Aus der Tabelle geht hervor, dass die Schülertexte im Mittelwert zwar die längsten Sätze aufweisen, dennoch liegt ihre durchschnittliche Länge mit 22 Wörtern unter der der Lehrbuchseiten (25 Wörter).

Hinsichtlich der Wortschatzbeschreibung mithilfe der Angaben über seinen Gebrauch anhand des GRADITs ist festzuhalten, dass in beiden Textgruppen die doppelten Markierungen abgesehen von der Markierung Co Ts, die in den Schülertexten durchschnittlich zwei Wörter pro Text tragen und auf den Lehrbuchseiten drei, keine große Rolle spielen. Während die Lernenden durchschnittlich zehn Wörter mehr aus dem fundamentalen Wortschatz verwendeten, liegen die Werte für die beiden anderen Marken des Basiswortschatzes (AD und $\mathrm{AU}$ ) sowie für die allgemeinsprachliche (CO) und die fachsprachliche (Ts) in den Lehrbuchtexten höher. Überwiegend handelt es sich um relativ kleine Unterschiede; die Wortschatzbereiche des hohen Gebrauchs (AU) und des allgemeinsprachlichen (CO) sind im Durchschnitt auf den Lehrbuchseiten 
aber deutlich stärker vertreten: In den Schülertexten finden sich im Mittelwert 23 AU-gekennzeichnete Wörter, wohingegen es in den Texten des Lehrbuchs 29 sind, und der Abstand zwischen den beiden Textgruppen besteht für die Cogekennzeichneten aus zehn Wörtern.

Aus dieser Verteilung ergibt sich, dass der prozentuale Anteil der Fo-markierten Wörter am Gesamtwortvolumen in den Texten der Lernenden bei 65 und in denen des Lehrbuchs bei 63 liegt, der Unterschied also zwei Prozentpunkte beträgt. Anders verhält es sich mit dem prozentualen Anteil der aus dem Basiswortschatz (VDB) stammenden Ausdrücke, von denen sich mit 75 Prozent etwas mehr in den Lehrbuchtexten befinden als in denen der Schülerinnen und Schüler. Das widerlegt auf den ersten Blick die Annahme, dass in den Lehrbuchtexten mehr niedrig frequente Wörter verwendet werden als in den Schülertexten, ist aber damit zu erklären, dass dort durchschnittlich mehr Eigennamen erscheinen, die keine Marken tragen. Das sind hauptsächlich Toponyme, die im Mittelwert zahlreicher vorkommen, weil die Lernenden sich vor allem auf die physische Beschreibung der Region konzentrierten und sie dabei benötigten, während die Lehrbuchseiten auch andere Aspekte wie die sozioökonomischen und geschichtlichen ausführlicher behandeln. Subtrahiert man den Anteil der Eigennamen vom Gesamtwortvolumen, so dass dieses nur noch aus Wörtern besteht, die eine Gebrauchsmarke tragen, ergeben die prozentualen Werte des Fo-gekennzeichneten Wortaufkommens und des verwendeten Basiswortschatzes ein anderes Bild: Die Schülertexte setzen sich durchschnittlich zu 74 Prozent aus Wörtern aus dem fundamentalen Wortschatz und zu 84 Prozent aus dem Basiswortschatz zusammen, während der Fo-markierte Anteil der Lehrbuchseiten bei knapp 68 Prozent und der des Basiswortschatzes bei 80 liegt.

Dass die Werte immer noch recht nah beieinanderliegen - sie unterscheiden sich beim fundamentalen Wortschatz um sechs Prozentpunkte und beim Basiswortschatz um vier -, rührt wahrscheinlich daher, dass die Lernenden sich auf den Test vorbereiten konnten, indem sie die entsprechenden Lehrbuchseiten wiederholt gelesen hatten und dann viele der dort verwendeten Wörter und Formulierungen in ihre Texte übernahmen.

In der Klasse an der Schule Vespucci fand vor der Textproduktion keine gezielte Erarbeitung einer geographischen Region statt, da sie sich mit globalen sozioökonomischen Aspekten befasste. Das nächste Unterkapitel wird beschreiben, wie ihre Texte gestaltet sind und dabei zeigen, dass sie ohne eine so starke Anlehnung an das Lehrbuch aus einem höheren Anteil von Ausdrücken aus dem fundamentalen und dem Basiswortschatz bestehen. 


\subsection{Schülertexte der Klasse an der Vespucci}

Die Lernenden an der Schule Vespucci bekamen die Aufgabe, für mich einen Text über ein Land oder eine geographische Region ihrer Wahl zu verfassen, wie er in einem Erdkundebuch ihrer Klassenstufe formuliert sein könnte. Auf ihre Anfrage wurde ihnen außerdem freigestellt, zwei Länder oder Regionen miteinander zu vergleichen. Sie wussten, dass die Lehrkraft den Text nicht benoten würde und sollten ihn mit einem persönlichen Kommentar darüber abschließen, ob sie sich vorstellen konnten, eine Zeit lang in der von ihnen präsentierten Gegend zu leben.

Die Wahl der Region war ihnen freigestellt, um möglichen Schreibblockaden entgegenzuwirken, die aus mangelnden Kenntnissen über ein Thema resultieren können. Insgesamt waren zehn SchülerInnen bei der Durchführung anwesend. Darunter befand sich auch eine Schülerin mit Verzögerungen in der geistigen Entwicklung, die keinen Text verfasste, sondern gemeinsam mit der Unterstützungslehrkraft ein Schaubild mit Stichpunkten zu einem Land ihrer Wahl erstellte.

Die Tabelle 14 stellt die vorgestellten Regionen der übrigen neun Schülerinnen und Schüler dar.

\begin{tabular}{lll}
\hline Lernende & Thema & Kommentar \\
\hline A & Brasilien & \\
B & Afrika & \\
C & Bangladesch & Herkunftsland ihrer Eltern \\
D & England & \\
E & Nigeria und Toronto & \\
F & Afrika & \\
G & Ecuador & Herkunftsland ihrer Eltern \\
H & New York und Afrika & \\
I & Rumänien & Herkunftsland der Schülerin \\
\hline
\end{tabular}

Tabelle 14: Geographische Gebiete der Schülertexte (Vespucci)

Fast die Hälfte (4) wählte Afrika bzw. ein afrikanisches Land aus, was neben persönlichen Interessen daran liegen könnte, dass in dieser Klasse in den Stunden vor der Textproduktion globale sozioökonomische Aspekte behandelt wurden, die oft anhand der afrikanischen Situation vorgestellt wurden.

Zwei Schülerinnen berichteten über die Herkunftsländer ihrer Eltern, Bangladesch und Peru, und eine weitere Schülerin schrieb über Rumänien, das Land, in dem sie selbst bis zu ihrem zehnten Lebensjahr aufgewachsen war. Unter den Lernenden befanden sich außerdem noch drei Mehrsprachige (Schülerin $\mathrm{D}$, Schüler E und F), die nicht über die Herkunftsländer ihrer Familien schrieben. 
Schülerin D wählte als einzige ein Land, das bereits im zweiten Jahr der Mittelschule behandelt worden war (England). Schüler A und Schülerin H hingegen entschieden sich mit Brasilien und New York für noch nicht im Unterricht behandelte Regionen.

\subsubsection{Schriftbild und graphische strukturelle Gestaltungsmittel}

Die Texte haben meist die Länge von einer Din A4-Seite und enthalten durchschnittlich 328 Wörter, wobei der längste Text aus 486 Wörtern und der kürzeste aus 225 besteht. Sie tragen oft eine Überschrift, aber es sind auch welche ohne Überschrift darunter. Viele sind nicht oder nur geringfügig in Absätze unterteilt, während das Schriftbild im Großen und Ganzen leserlich und ordentlich ist. Auch die Lernenden dieser Klasse schrieben jedoch vorrangig in Druckschrift bzw. einem Mix aus Druck- und Schreibschrift, vernachlässigten gelegentlich Interpunktion (auch satzbeendend) und Majuskeln am Satzanfang und versahen viele Wörter nicht mit den erforderlichen Akzenten. Hinzu kommen orthographische Fehler, die sich vor allem bei zwei Lernenden häufen, die nicht in Italien eingeschult worden waren und erst seit einigen Jahren Italienisch lernten. Weil das Beherrschen der Rechtschreibung nicht im Interesse dieser Arbeit stand, zumal die Fehler das Textverständnis nicht beeinträchtigen, werden sie zwar in den Textzitaten beibehalten, aber darüber hinaus nicht weiter kommentiert.

\subsubsection{Zusammensetzung des Wortschatzes}

Das Wortvolumen der Schülertexte verteilt sich auf durchschnittlich zwölf Sätze mit einer Länge von 26 Wörtern. Alle Texte weisen aber auch Sätze auf, die noch deutlich länger sind, wobei das Maximum von 34 Wörtern bei Schülerin I bis zu 84 Wörtern bei Schülerin G reicht. Auch hier bestehen die Texte allerdings z.T. aus sehr langen Satzgefügen, in denen nur eine schwache explizite Kohäsion zwischen den einzelnen Aussagen besteht. Aus Tabelle 15 können neben genauen Angaben zur Text- und Satzlänge Informationen über die Zusammensetzung des verwendeten Wortschatzes entnommen werden.

\begin{tabular}{lrrrrrrrrrr}
\hline Lernende & $\mathrm{A}$ & $\mathrm{B}$ & $\mathrm{C}$ & $\mathrm{D}$ & $\mathrm{E}$ & $\mathrm{F}$ & $\mathrm{G}$ & $\mathrm{H}$ & $\mathrm{I}$ & $\varnothing$ \\
\hline n Wörter & 330 & 354 & 352 & 274 & 313 & 261 & 486 & 358 & 225 & 328 \\
n Sätze & 18 & 12 & 13 & 15 & 9 & 11 & 10 & 11 & 13 & 12 \\
längster Satz & 46 & 47 & 69 & 38 & 78 & 46 & 84 & 50 & 34 & 55 \\
$\varnothing$ Satzlänge & 18,3 & 29,5 & 27 & 18,2 & 34,4 & 23,6 & 32,2 & 34,8 & 17,2 & 26,1 \\
AD & 5 & 2 & 1 & 3 & 4 & 2 & 2 & 2 & 3 & 3 \\
AU & 8 & 21 & 4 & 13 & 19 & 11 & 15 & 23 & 8 & 14 \\
AU TS & 1 & 0 & 0 & 0 & 0 & 0 & 0 & 0 & 0 & 0 \\
CO & 22 & 24 & 30 & 14 & 22 & 20 & 25 & 21 & 13 & 21 \\
\hline
\end{tabular}




\begin{tabular}{lrrrrrrrrrr}
\hline Lernende & $\mathrm{A}$ & $\mathrm{B}$ & $\mathrm{C}$ & $\mathrm{D}$ & $\mathrm{E}$ & $\mathrm{F}$ & $\mathrm{G}$ & $\mathrm{H}$ & $\mathrm{I}$ & $\varnothing$ \\
\hline CO TS & 0 & 2 & 5 & 0 & 4 & 4 & 1 & 2 & 3 & 2 \\
el.CO & 9 & 7 & 13 & 4 & 11 & 2 & 8 & 4 & 3 & 7 \\
el.CO TS & 0 & 0 & 0 & 0 & 0 & 2 & 0 & 0 & 0 & 0 \\
el.TS & 11 & 2 & 0 & 2 & 6 & 5 & 5 & 7 & 2 & 4 \\
FO & 251 & 286 & 286 & 211 & 228 & 204 & 406 & 276 & 181 & 259 \\
TS & 8 & 1 & 0 & 3 & 4 & 3 & 3 & 4 & 2 & 3 \\
FO TS & 0 & 0 & 0 & 0 & 0 & 0 & 0 & 0 & 0 & 0 \\
n.p. & 13 & 9 & 13 & 25 & 14 & 8 & 20 & 11 & 10 & 14 \\
el.n.p. & 0 & 0 & 0 & 0 & 0 & 0 & 0 & 7 & 0 & 1 \\
sigla & 2 & 0 & 0 & 0 & 0 & 0 & 0 & 0 & 0 & 0 \\
n FO in \% & 76,1 & 80,8 & 81,3 & 77 & 72,8 & 78,2 & 83,5 & 77,1 & 80,4 & 79 \\
n VdB* & 265 & 309 & 290 & 227 & 251 & 217 & 423 & 301 & 192 & 266 \\
n VdB in \% & 80,3 & 87,3 & 82,7 & 82,8 & 80,2 & 83,1 & 87 & 84,1 & 85,3 & 81,1 \\
n n.p. und el.n.p. & 13 & 9 & 13 & 25 & 14 & 8 & 20 & 11 & 10 & 14 \\
\% n.p. und el.n.p. & 3,9 & 2,5 & 3,7 & 9,1 & 4,5 & 3 & 4,1 & 3 & 4,4 & 4,3 \\
\hline
\end{tabular}

Tabelle 15: Text- und Satzlängen, Gebrauchsmarken und VDB- und Fo-Anteile am Gesamtvolumen der Schülertexte (Vespucci)

*inkl. doppelter Marken mit Ts

Der Großteil der verwendeten Wörter stammt aus dem Bereich des fundamentalen Wortschatzes, der einen prozentualen Anteil am Gesamtwortvolumen von mindestens 76 Prozent ausmacht und bis zu 84 Prozent reicht, während er durchschnittlich bei 79 Prozent liegt. Am zweithäufigsten wurde bei der Verfassung der Texte auf den Bereich des allgemeinsprachlichen Gebrauchswortschatzes (CO) zurückgegriffen, aus dem ca. 21 Wörter pro Text stammen.

Auch wenn der Anteil an allgemeinsprachlichen Wörtern in den Texten recht hoch ist, entspringt der verwendete Wortschatz dennoch hauptsächlich dem VDB (Basiswortschatz). So beträgt dieser durchschnittlich 81 Prozent am Gesamtwortvolumen, wobei die Schüler A und E ein Minimum von 80 Prozent und die Schülerinnen B und $\mathrm{H}$ ein Maximum von 87 erzeugten.

Am dritthäufigsten wurden Wörter des hohen Gebrauchs (AU) verwendet, auch wenn sie, durchschnittlich mit 14 Ausdrücken vertreten, in den einzelnen Texten sehr unterschiedlich ausgeprägt erscheinen. So gibt es im Text der Schülerin C nur vier AU-Wörter, während die Schülerin H 23 gebrauchte.

Ebenfalls 14 Ausdrücke im Mittelwert bilden außerdem geographische Eigennamen ohne Marke, wobei in zwei Texten über 20 und in drei Texten nur acht bis zehn erscheinen. Die durchschnittlich 14 Vorkommnisse der einzelnen Toponyme gehen auf vier Worttypen zurück.

\subsubsection{Allgemeinsprachlicher Wortschatz (Co)}

Insgesamt handelt es sich bei den verwendeten Co-Wörtern um 88 Worttypen, die mit 226 Vorkommnissen vertreten sind. Die meisten Tokens entfallen mit 31 auf das Pronomen si, das in Verbindung mit reflexiven Verben gebraucht wird. An zweiter Stelle folgt der bestimmte maskuline Pluralartikel $i$ mit 30 
Vorkommnissen. Letzterer erscheint in acht der neun Texte und das Pronomen in allen. Die anderen Wörter aus dem allgemeinen Wortschatz sind in weniger Texten und mit weitaus geringeren Vorkommnissen enthalten, wie die Tabelle 16 zunächst für diejenigen Ausdrücke veranschaulicht, die mindestens vier Vorkommnisse haben.

\begin{tabular}{|c|c|c|c|c|}
\hline Wort & Wortart & Tokens & $\begin{array}{l}\text { Lernende, } \\
\text { die es verwenden }\end{array}$ & Übersetzung \\
\hline un po' & loc.avv. & 7 & $\mathrm{D}, \mathrm{G}, \mathrm{C}$ & etwas \\
\hline anche se & loc.cong. & 6 & $\mathrm{~B}, \mathrm{C}, \mathrm{D}, \mathrm{G}, \mathrm{I}$ & auch wenn \\
\hline gli & art.det. & 6 & $B, E, F, G, I$ & die \\
\hline sviluppato & agg. & 6 & $A, B, D, F, H$ & entwickelt \\
\hline per questo & loc.cong. & 5 & $A, E$ & deshalb \\
\hline $\begin{array}{l}\text { a causa di } \\
\text { economica- }\end{array}$ & loc.prep. & 4 & $B, E$ & aufgrund von \\
\hline mente & avv. & 4 & $\mathrm{C}, \mathrm{E}$ & wirtschaftlich \\
\hline $\begin{array}{l}\text { per esempio } \\
\text { per quanto ri- }\end{array}$ & loc.avv. & 4 & $B, E, F, H$ & zum Beispiel \\
\hline guarda & loc.avv. & 4 & C & bezüglich \\
\hline tasso & s.m. & 4 & $A, D, H$ & $\begin{array}{l}\text { Rate } \\
\text { Hilfsverb „kommen“ zur }\end{array}$ \\
\hline venire & v.aus. & 4 & $A, C, I$ & Passivkonstruktion \\
\hline
\end{tabular}

Tabelle 16: Co-Wörter mit mindestens vier Tokens in den Schülertexten (Vespucci)

Darunter sind neben dem zweiten bestimmten maskulinen Pluralartikel und dem von venire gebildeten Hilfsverb, das bei der Passivkonstruktion anstelle von essere (sein) verwendet werden kann, ein Adjektiv, ein Substantiv und ein präpositionales, drei adverbiale und zwei konjunktionale Phraseme.

Genauso viele Wörter wurden dreimal verwendet:

abituarsi (sich gewöhnen; B, G, I); accesso (Zugang; F, H); ad esempio (zum Beispiel; C, G, $\mathrm{H}$ ); andare a scuola (zur Schule gehen; B, G); diffuso (verbreitet; C, F); mortalità (Sterblichkeit; B, F, I); nutrimento (Ernährung, C); percentuale (Anteil; I).

Die folgenden 14 Wörter haben maximal zwei Vorkommnisse:

affitto (Miete; G); all 'incirca (ungefähr; G, I); andare in giro (herumlaufen; A); determinato (bestimmt; C, H); di più (mehr; D, F); industrializzazione (Industrialisierung; B, H); mantenersi (für seinen Unterhalt sorgen; H); piantagione (Plantage; A, B); potabile (trinkbar; F); preoccuparsi (sich sorgen; E); pulito (sauber; G); riguardo (bezüglich; D, H); svilupparsi (sich entwickeln; E); zanzariera (Insektennetz; C, F).

Ferner wurden weitere 50 Wörter, also mehr als die Hälfte aller Co-markierten Ausdrücke, nur ein einziges Mal gebraucht.

Von allen Worttypen aus dem allgemeinsprachlichen Wortschatz stellen mit 24 die größte Gruppe die Verben in finiter, infiniter sowie phrasematischer Form. Danach folgen mit 20 die Adverbien. Ebenfalls zahlreich sind die Substantive mit 19 vertreten. Außerdem zählen auch 14 Adjektive, fünf präpositionale, drei konjunktionale Phraseme sowie die beiden Artikel und das Pronomen dazu. 
Insgesamt befinden sich unter den Worttypen 25 Phraseme, d.h. mit knapp 30 Prozent $(28,4)$ aller gebrauchten Ausdrücke aus dem allgemeinsprachlichen Wortschatz machen die Mehrworteinheiten einen erheblichen Teil des CoWortschatzes aus.

\subsubsection{Fachwortschatz (Ts)}

Fachwortschatzsprachliche Begriffe wurden kaum verwendet, nämlich durchschnittlich nur drei, wobei Schülerin C gar keinen, zwei weitere einen bzw. zwei und nur Schüler A mit acht deutlich mehr gebrauchte. Im Ganzen handelt es sich in den Schülertexten um 14 verschiedene fachsprachliche Worttypen mit 26 Vorkommnissen. Darunter befinden sich mit Ausnahme des Adjektivs negativo (TS scient.) nur Substantive in Form von Ein- und Mehrworteinheiten, die in Tabelle 17 aufgelistet sind.

\begin{tabular}{|c|c|c|c|c|c|}
\hline Wort & Wortart & $\begin{array}{l}\text { Fachspra- } \\
\text { che }\end{array}$ & Tokens & $\begin{array}{l}\text { Verwendung in } \\
\text { Schülertexten }\end{array}$ & Übersetzung \\
\hline cacao & s.m. & TS bot.com. & 1 & A & Kakao \\
\hline caffè & s.m. & TS bot.com. & 1 & A & Kaffee \\
\hline $\begin{array}{l}\text { densità } \\
\text { economia di mer- }\end{array}$ & s.f. & TS stat. & 1 & 1 & Bevölkerungsdichte \\
\hline $\begin{array}{l}\text { cato } \\
\text { economia di sus- }\end{array}$ & loc.s.f. & TS econ. & 2 & $\mathrm{~A}, \mathrm{H}$ & Marktwirtschaft \\
\hline sistenza & loc.s.f. & TS econ. & 1 & A & Bedarfswirtschaft \\
\hline elemento & s.m. & TS chim. & 1 & E & Element \\
\hline fecondità & s.f. & $\begin{array}{l}\text { TS stat. } \\
\text { TS stat., po- }\end{array}$ & 3 & $A, D$ & $\begin{array}{l}\text { Fruchtbarkeitsrate } \\
\text { Human Development }\end{array}$ \\
\hline indice di sviluppo & loc.s.m. & lit. & 1 & A & $\begin{array}{l}\text { Index } \\
\text { Säuglingssterblich- }\end{array}$ \\
\hline mortalità infantile & loc.s.f. & TS stat. & 1 & G & keit \\
\hline $\begin{array}{l}\text { negativo } \\
\text { prodotto interno }\end{array}$ & agg. & TS scient. & 1 & B & negativ \\
\hline lordo & loc.s.m. & TS econ. & 1 & A & $\begin{array}{l}\text { Bruttoinlandsprodukt } \\
\text { natürliche Bevölke-- }\end{array}$ \\
\hline saldo naturale & el.loc.s.m. & TS stat. & 2 & $\begin{array}{l}B, F \\
A, D, E, F, G, H,\end{array}$ & rungs-entwicklung \\
\hline speranza di vita & loc.s.f. & TS stat. & 9 & & Lebenserwartung \\
\hline tasso di natalità & loc.s.m. & TS stat. & 1 & $F, G, H$ & Geburtenrate \\
\hline
\end{tabular}

Tabelle 17: Fachwortschatzsprachliche Ausdrücke in den Schülertexten (Vespucci)

Die von den Schülern verwendeten Ts-markierten Ausdrücke stammen aus fünf verschiedenen Fachsprachen. Am stärksten ist die der Statistik vertreten, aus der sieben Worttypen stammen, von denen einer die meisten Vorkommnisse hat: Der Ausdruck „Lebenserwartung“ wurde von sieben Lernenden mit insgesamt neun Vorkommnissen benutzt. Es handelt sich hierbei um einen Begriff, der im Erdkundeunterricht vor der Textproduktion intensiv behandelt worden war und der auch im Lehrbuch erscheint (Carazzi \& Pizzetti 2010, S. 56). 
Desweiteren stammen aus diesem fachsprachlichen Gebiet die Ausdrücke für „Bevölkerungsdichte“, „Fruchtbarkeitsrate“, „Human Development Index“, „,natürliche Bevölkerungsentwicklung“ und „Geburtenrate“. Auch sie waren im Vorfeld der Textproduktion im Zuge der Bearbeitung von Lehrbuchtexten besprochen worden. Außer dem letzten, der in drei Schülertexten erscheint, wurden alle höchstens von zwei Lernenden benutzt.

Diese spärliche Verwendung fachsprachlicher Ausdrücke, die entschieden geringer ausfällt als im Lehrbuch, legt nahe, dass die Lernenden wenig Erfahrung mit dem Verfassen schriftlicher Texte im Fach Erdkunde hatten. Denn die Präsenz von Fachausdrücken ist ein textsortenspezifisches Merkmal darstellender Texte im Fachunterricht (wie u.a. auch aus der Analyse der Lehrbuschseiten hervorgegangen ist), das die Schüler im Wesentlichen ignorierten. Dabei ist auch denkbar, dass sie kaum fachsprachliche Begriffe gebrauchten, weil ihre produktiven Wortkompetenzen dafür nicht ausreichten.

Die Begriffe aus anderen Fachsprachen kommen fast ausschließlich nur einmal vor; drei Worttypen stammen aus der Wirtschaftssprache, zwei aus der Botanik und je einer aus der Chemie sowie der Wissenschaft.

\subsubsection{Phraseme}

Der Tabelle 18 ist zu entnehmen, dass in den Schülertexten 38 verschiedene Phraseme mit insgesamt 84 Vorkommnissen vertreten sind. Einige davon wurden w.o. bereits bei der Beschreibung des allgemeinen und des Fachwortschatzes vorgestellt.

\begin{tabular}{lrllll}
\hline Wortart & Worttypen & CO & CO TS & TS & Verwendung in Schülertexten \\
\hline agg. & 1 & 1 & 0 & 0 & H \\
avv. & $12(28)$ & 12 & 0 & 0 & A-I \\
cong. & $3(12)$ & 3 & 0 & 0 & A, B, C, D, E, G \\
prep. & $5(11)$ & 5 & 0 & 0 & A, B, C, D, E, H \\
s.f./s.m. & $10(23)$ & 0 & 2 & 8 & A, B, D; E, F, G, H, I \\
v. & $5(8)$ & 5 & 0 & 0 & A, B, C; F, G \\
Tot. & $38(84)$ & & & & \\
\hline
\end{tabular}

Tabelle 18: Phraseme in den Schülertexten (Vespucci)

Die meisten Mehrworteinheiten sind adverbiale Phraseme, von denen in allen Texten welche auftreten. Sie stammen ausnahmslos aus dem allgemeinsprachlichen Wortschatz. Darunter befinden sich Begriffe wie per esempio (zum Beispiel), un po' (etwas) a volte (manchmal) sowie di più (mehr), die den Schülern gut bekannt waren und auch im Mündlichen oft von ihnen verwendet wurden.

An zweiter Stelle folgen die Substantive, von denen keines aus dem allgemeinsprachlichen Wortschatzbereich stammt, sondern die überwiegend zu den Fachsprachen zählen und in zwei Fällen die doppelte Markierung Co Ts tragen. Diese beiden letzten Substantive sind die Schultypbezeichnungen scuola 
primaria und scuola secondaria (Co Ts scol; Primar- und Sekundarstufe) mit je einem Token. Mit Ausnahme der Schülerin C gebrauchten alle Lernenden dieser Klasse mindestens ein substantivisches Phrasem.

Sowohl die phrasematischen Verben als auch die Präpositionen sind mit fünf Worttypen vertreten, die alle dem allgemeinsprachlichen Wortschatz zugeteilt sind. Sie wurden ungefähr in der Hälfte der Texte verwendet, wobei jeder Worttyp höchstens in zwei Texten erscheint.

Von den drei mehrteiligen Konjunktionen, die alle Co-markiert sind, gibt es zwölf Vorkommnisse, die sich auf die Mehrheit der Texte verteilen. Es handelt sich um anche se (auch wenn; 6 Tokens), per questo (deshalb; 5 Tokens) und per cui (deswegen). Alle drei dienen der Herstellung kausaler und konzessiver Beziehungen, die auch in diesen Schülertexten deutlich seltener expliziert sind als nebenordnende Verknüpfungen mit der Konjunktion ,und“.

Die Konzessivkonjunktion ,auch wenn“ schienen die SchülerInnen den ebenfalls aus dem allgemeinsprachlichen Wortschatz stammenden synonymen Konjunktionen/Präpositionen „sebbene“ und „nonostante“ vorzuziehen. Vielleicht liegt es daran, dass sie aus zwei Wörtern zusammengesetzt ist, die beide aus dem fundamentalen Wortschatz stammen, und sich ihre Bedeutung direkt daraus übertragen lässt. Von den beiden nicht phrasematischen mit gleicher Bedeutung wird nur die zweite in einem Text als Präposition verwendet.

Zuletzt befindet sich unter den Mehrworteinheiten noch eine adjektivische aus dem allgemeinen Wortschatz, a persona (pro Person).

Fast zwei Drittel der verwendeten Phraseme besteht aus zwei Wörtern, die übrigen aus drei und eines sogar aus vier. Es ist der Ausdruck indice di sviluppo umano, der aus dem Erdkundeunterricht bekannt war und der im Lehrbuch bspw. bei der Vorstellung der einzelnen Länder wiederholt verwendet wird.

Unter den dreigliedrigen Phrasemen sind Verben, Substantive, Adverbien und Präpositionen vertreten, die oft aus mindestens einer Präposition plus Substantiven oder Verben plus Substantiven bestehen. Abgesehen von dem vielbenutzten Nomen „Lebenserwartung“ und von tasso di natalità in drei Texten, erscheinen die anderen in maximal zweien. Bei den dreiteiligen Phrasemen handelt es sich um folgende:

a causa di (aufgrund von, Co), andare a scuola (zur Schule gehen, Co); andare in giro (herumgehen, CO); economia di mercato (Marktwirtschaft, Ts econ.); economia di sussistenza (Bedarfswirtschaft, Ts econ.); mettere a confronto (vergleichen, Co); per quanto riguarda (bezüglich, Co); più che altro (vor allem, Co); prodotto interno lordo (Bruttoinlandsprodukt, Ts econ.); speranza di vita (Lebenserwartung, Ts stat.); tasso di natalità (Geburtenrate, Ts stat.).

Die Lernenden verwendeten alle Phraseme treffend, was die Hauptsache ist, doch z.B. wurde all'incirca als ein einziges, nicht apostrophiertes Wort und rispetto a (bezüglich, Schülerin D) ohne Präposition geschrieben. D gebrauchte das Phrasem jedoch zweimal und davon einmal mit der Präposition, so dass 
nicht klar ist, ob es sich beim Auslassen der Präposition um einen Flüchtigkeitsfehler handelte oder ihre lexikalische Kompetenz nicht gefestigt genug war.

\subsubsection{Grammatikalische und syntaktische Eigenschaften}

\subsubsection{Satzverknüpfungen}

Die drei gerade vorgestellten phrasematischen Konjunktionen anche se, per questo und per cui zählen zu insgesamt 22 konjunktionalen Worttypen der Schülertexte, die mit 272 Vorkommnissen vertreten sind. Tabelle 19 illustriert, um welche Konjunktionen es sich handelt und wie sie sich auf die Texte verteilen.

\begin{tabular}{|c|c|c|c|c|c|c|c|c|c|c|c|}
\hline Lernende & A & B & C & D & $E$ & $\mathrm{~F}$ & G & $\mathrm{H}$ & I & Tot. & $\varnothing$ \\
\hline n Wörter & 330 & 354 & 352 & 274 & 313 & 261 & 486 & 358 & 225 & & 328 \\
\hline n Sätze & 18 & 12 & 13 & 15 & 9 & 11 & 10 & 11 & 13 & & 12 \\
\hline längster & 46 & 47 & 69 & 38 & 78 & 46 & 84 & 50 & 34 & & 55 \\
\hline $\begin{array}{l}\text { Satz } \\
\varnothing \text { Satz- } \\
\text { länge }\end{array}$ & 18,3 & 29,5 & 27 & 18,2 & 34,4 & 23,6 & 32,2 & 34,8 & 17,2 & & 26,1 \\
\hline cong. & 31 & 39 & 26 & 26 & 27 & 29 & 49 & 25 & 20 & & 30 \\
\hline $\begin{array}{l}\text { Types } \\
\text { cong. }\end{array}$ & 11 & 11 & 11 & 9 & 5 & 10 & 13 & 9 & 8 & 22 & 10 \\
\hline $\begin{array}{l}\varnothing \text { cong. } \\
\text { pro Satz }\end{array}$ & 0,6 & 1 & 0,8 & 0,6 & 0,6 & 0,9 & 1,3 & 0,8 & 0,6 & & 0,8 \\
\hline anche & 0 & 4 & 0 & 1 & 3 & 2 & 0 & 4 & 2 & 16 & 1,8 \\
\hline $\begin{array}{l}\text { anche se } \\
\text { (CO) }\end{array}$ & 0 & 2 & 1 & 1 & 0 & 0 & 1 & 0 & 1 & 6 & 0,7 \\
\hline che & 2 & 1 & 6 & 3 & 0 & 3 & 6 & 1 & 2 & 24 & 2,7 \\
\hline cioè & 0 & 0 & 3 & 0 & 0 & 0 & 0 & 0 & 0 & 3 & 0,4 \\
\hline come & 1 & 1 & 0 & 0 & 0 & 0 & 0 & 0 & 0 & 2 & 0,2 \\
\hline dove & 0 & 0 & 1 & 0 & 0 & 2 & 1 & 1 & 1 & 6 & 0,7 \\
\hline$e$ & 8 & 10 & 3 & 10 & 12 & 13 & 21 & 11 & 7 & 95 & 10,6 \\
\hline infatti & 0 & 0 & 0 & 0 & 0 & 1 & 0 & 1 & 0 & 2 & 0,2 \\
\hline ma & 3 & 7 & 2 & 2 & 0 & 2 & 6 & 0 & 3 & 25 & 2,8 \\
\hline mentre & 1 & 0 & 0 & 0 & 0 & 0 & 2 & 4 & 0 & 7 & 0,8 \\
\hline 0 & 2 & 3 & 2 & 1 & 0 & 0 & 1 & 1 & 0 & 10 & 1,1 \\
\hline oppure & 0 & 0 & 0 & 0 & 0 & 0 & 1 & 0 & 0 & 1 & 0,1 \\
\hline $\begin{array}{l}\text { per cui } \\
\text { (CO) }\end{array}$ & 0 & 1 & 0 & 0 & 0 & 0 & 0 & 0 & 0 & 1 & 0,1 \\
\hline $\begin{array}{l}\text { per } \\
\text { questo } \\
\text { (CO) }\end{array}$ & 2 & 0 & 0 & 0 & 3 & 0 & 0 & 0 & 0 & 5 & 0,6 \\
\hline perché & 7 & 4 & 3 & 5 & 7 & 3 & 6 & 1 & 3 & 39 & 4,3 \\
\hline quando & 0 & 0 & 1 & 0 & 0 & 0 & 0 & 0 & 0 & 1 & 0,1 \\
\hline perciò & 0 & 1 & 0 & 0 & 0 & 0 & 1 & 0 & 0 & 2 & 0,2 \\
\hline $\begin{array}{l}\text { però } \\
\text { (AU) }\end{array}$ & 1 & 0 & 0 & 2 & 0 & 1 & 0 & 0 & 0 & 4 & 0,4 \\
\hline quando & 0 & 0 & 0 & 0 & 0 & 1 & 1 & 0 & 1 & 3 & 0,3 \\
\hline quindi & 1 & 0 & 0 & 0 & 0 & 0 & 1 & 0 & 0 & 2 & 0,2 \\
\hline
\end{tabular}




\begin{tabular}{lrllllllllrr}
\hline Lernende & A & B & C & D & E & F & G & H & I & Tot. & $\varnothing$ \\
\hline se & 3 & 5 & 2 & 1 & 2 & 1 & 1 & 1 & 0 & 16 & 1,8 \\
sia & 0 & 0 & 2 & 0 & 0 & 0 & 0 & 0 & 0 & 2 & 0,2 \\
\hline
\end{tabular}

Tabelle 19: Konjunktionen in den Schülertexten (Vespucci)

Fast alle Konjunktionen stammen aus dem Bereich des fundamentalen Wortschatzes (FO). Die drei phrasematischen gehören zum allgemeinen (CO) und però (jedoch) zu dem des hohen Gebrauchs (AU). In den einzelnen Texten kommen durchschnittlich zehn unterschiedliche Konjunktionen mit 30 Vorkommnissen vor, also etwas weniger als die Hälfte der insgesamt verwendeten konjunktionalen Worttypen (22). Am wenigsten unterschiedliche Worttypen befinden sich im Text des Schülers E (5) und am meisten in dem der Schülerin G (13).

In allen Texten wurde mit deutlichem Abstand am häufigsten die Konjunktion ,und“ verwendet, nämlich insgesamt 95- und durchschnittlich elfmal. Danach folgt mit 39 Vorkommnissen und einer durchschnittlich viermaligen Verwendung ,weil““. An dritter Stelle sind mit fast identischen Werten die Konjunktionen „aber“ (25 Tokens, Ø 2,8-mal) und „dass“ (24 Tokens, Ø 2,7-mal) vertreten. Im Mittelwert ungefähr einmal gebrauchten die Lernenden außerdem noch die Konjunktionen ,auch“ (16 Tokens, Ø 1,8-mal), ,auch wenn“ und „wo“ (je 6 Tokens, Ø 0,7-mal), „während“ (7 Tokens, Ø 0,8-mal), ,oder“ (10 Tokens, Ø 1,1-mal), „deshalb“ (5 Tokens, Ø 0,6-mal) und „wenn“ (16 Tokens, $\varnothing$ 1,8-mal). „Während“ wurde allerdings nur in drei Texten (einmal mit vier Vorkommnissen) verwendet.

Durchschnittlich entfallen auf jeden Satz 0,8 Konjunktionen, wobei es in mehreren Texten nur 0,6 sind und der Wert mit 1,3 im Text von G am größten ist. Diese Schülerin hat mit 84 Wörtern auch das längste Satzgefüge gebildet, das hier wiedergegeben werden soll, um zu veranschaulichen, wie in langen Sätzen zum Teil Gedankenflüsse versprachlicht werden, ohne dem Schreibanlass auf angemessene Weise gerecht zu werden - zumal sich Komplikationen bei der Beachtung der Kongruenz im Numerus der Prädikate und Substantive ergaben (Schülerin G):

La capitale del Perù è Lima, si parla lo spagnolo e in alcune parti del Perù ci sono dei paesini più poveri, dove la gente non ha cibo, vive per la strada oppure dei poveri che hanno una casa ma non ha l'acqua calda e non si può permettere un bagno pulito o delle stanze, e c'è gente che è malata e quando vanno in ospedale e gli dicono che deve fare un'operazione gli chiedono i soldi, ma purtroppo molta gente non ha soldi.

(Die Hauptstadt von Peru ist Lima, man spricht Spanisch und in einigen Teilen Perus gibt es kleine, ärmere Dörfer, wo die Leute kein Essen haben, auf der Straße leben oder wo die Armen, die ein Haus haben, aber hat [sic] kein warmes Wasser und kann sich kein sauberes Bad(-ewasser) leisten oder Zimmer, und es gibt Leute, die krank sind und wenn sie ins Krankenhaus gehen und sie ihnen sagen, dass sie eine Operation machen muss [sic], aber leider haben viele Leute kein Geld.) 
G wurde in Italien geboren und hatte peruanische Eltern. Sie gab im Fragebogen an, zuhause Spanisch und Italienisch zu sprechen und in Italien eingeschult worden zu sein. Es kann also davon ausgegangen werden, dass sie sich den Satz nach dem Verfassen nicht noch einmal durchgelesen hatte, weil sie sonst die Kongruenzfehler bemerkt hätte. Der Züge konzeptioneller Mündlichkeit aufweisende Satz legt den Schluss nahe, dass er von jemandem stammt, der nicht viel Schreibkompetenz und -übung hat, obgleich man ohne Schwierigkeiten versteht, was gemeint ist. Er erweckt den Eindruck, dass er so aufgeschrieben wurde, wie seine Verfasserin ihn auch mündlich produziert hätte. Die mangelnde Kongruenz ergibt sich daraus, dass sie einerseits la gente (Leute), im Italienischen ein Singularwort, und andererseits $i$ poveri (die Armen) formulierte und den Numerus bei der Konjugation des Prädikats nicht immer beachtete.

Neben der übermäßigen Satzlänge mit seinen vielen verschiedenen Informationen sind für einen darstellenden Text, wie sie ihn verfassen sollte, außerdem die Verwendung des Diminutivs paesini (Dörfchen/kleine Dörfer), das Nicht-Explizieren des Substantivs (Ärzte) bei gli dicono (sie sagen ihnen) und das wertende Adjektiv purtroppo nicht typisch.

Mit 78 Wörtern fast genauso lang ist ein Satzgefüge im Text von E, dessen Erstsprache ebenfalls Spanisch war und der erst im Alter von elf Jahren, knapp drei Jahre vor der Erhebung, mit seiner Familie von Ecuador nach Italien gezogen war (Schüler E):

Invece toronto [sic] che sarebbe una piccola città del Canada, ha una buona economia è una buona speranza di vita e una natalita [sic] alta, questo paese può svilupparsi più velocemente perché non si deve preoccupare per la loro economia perché con quello che hanno possono aiutare il popolo e anche lo sviluppo della nazione, il loro clima è fresco e non si devono preocupare [sic] per l'agricoltora [sic] come l'Africa che a causa del troppo sole non possono coltivare molti prodotti.

(Toronto hingegen, das eine kleine Stadt Canadas wäre, hat eine gute Wirtschaft und eine gute Lebenserwartung, und die Geburtenrate ist hoch, dieses Land kann sich schneller entwickeln, weil es sich nicht um ihre Wirtschaft sorgen muss, weil sie mit dem, was sie haben dem Volk und auch der Entwicklung der Nation helfen können, ihr Klima ist frisch, und sie müssen sich nicht um die Landwirtschaft sorgen wie in Afrika, das aufgrund der zu vielen Sonne nicht viele Produkte anbauen können [sic]).

Auch dieser Textauszug ist sprachlich-inhaltlich verständlich, aber durch verschiedene Merkmale mangelnder schriftlicher Textproduktionskompetenz gekennzeichnet. Damit sind nicht die orthographischen Fehler gemeint, die sich z.T. aus Interferenzen mit der Erstsprache ergeben und auch im Auslassen von Akzenten bestehen, wie es bei etlichen einsprachig italienischen Schülern ebenfalls beobachtet werden konnte. Vielmehr geht es darum, dass der Schüler ganz unterschiedliche Themenkomplexe in einem Satzgefüge miteinander verbindet, die von der wirtschaftlichen Lage Kanadas über die wirtschafts- und 
sozialpolitische zum Klima und zur Landwirtschaft inklusive eines Vergleichs mit Afrika reichen.

Auch dieser Schüler hält die Kongruenz zwischen Subjekt und Prädikat in dem langen Satzgefüge nicht aufrecht und zeigt darüber hinaus noch weitere Schwierigkeiten mit der Kohäsion. So beginnt er mit der Stadt Toronto, schildert grammatikalisch ihre sozioökonomische Lage und bezieht sich dabei aber eigentlich auf das Land Kanada. Dieses greift er im Folgenden auch wieder richtig mit dem Synonym questo paese (dieses Land) auf, benutzt aber sein Possessivpronomen dann nicht im Singular, sondern im Plural und konstruiert später die Prädikate ebenfalls im Plural, so dass als Subjekt wahrscheinlich die Einwohner Kanadas impliziert sind.

Nicht bildungssprachlich, sondern typisch für den mündlichen Sprachgebrauch der SchülerInnen, wie es in allen Klassen während der Abfragungen beobachtet werden konnte, ist die Verwendung des Konjunktivs sarebbe (wäre). Ausgeprägt hingegen ist sein Argumentationsstil, da er gleich drei gelieferte Fakten begründet, wobei zwei davon mit einem kausalen Nebensatz und einer sogar mit einem kausalen präpositionalen Objekt formuliert sind, also als grammatikalische Metapher im Nominalstil (aufgrund der zu vielen Sonne).

Derart lange Satzgefüge bildeten auch einsprachige Lernende, wie ein letzter Textauszug verdeutlicht (Schülerin $\mathrm{H}$ ):

Nel primo, ad esempio c'è una forte economia di mercato, mantenuta con delle tasse molto alte con cui vengono ripagati anche i cittadini del proprio lavoro, nel secondo caso l'economia è molto scarsa ed è di sussistenza, il popolo deve quindi mantenersi autonomamente con ciò che c'è nel proprio territorio.

(Im ersten zum Beispiel gibt es eine starke Marktwirtschaft, die mit sehr hohen Steuern unterhalten wird, mit denen auch die Bürger von der eigenen Arbeit belohnt werden, im zweiten Fall ist die Wirtschaft gering und für den Bedarf, das Volk muss sich also selbst unterhalten mit dem was es auf dem eigenen Grund und Boden gibt.)

Hier ist abgesehen von der Länge des Satzgefüges, das geübtere Schreibende nach sussistenza beenden oder vom Folgenden zumindest durch ein Semikolon abtrennen würden, die Kohäsion mit dem Vorausgehenden vernachlässigt. Denn der Satz vor diesem Textauszug lautet „Zwischen dieser Stadt und diesem Land gibt es viele Unterschiede“, so dass das Genus von nel primo (maskulin), nicht mit dem zuerst genannten femininen übereinstimmt. Allerdings folgt später im Satz nel secondo caso (im zweiten Fall), wodurch der Leser seine Intentionshypothese korrigieren und nel primo zu nel primo caso vervollständigen kann.

Desweiteren zeigt der Textauszug Bemühungen um eine angemessene Wortwahl, der jedoch Ausdrucksschwierigkeiten gegenüberstehen. So stammen zwar 14 von 50 Wörtern nicht aus dem fundamentalen Wortschatz, sondern sind AU-, CO- und Ts-markiert, und ist außerdem ein satzwertiges Partizip enthalten (dessen Verb allerdings nicht treffend gewählt ist). Dennoch liegen 
Schwächen im Ausdruck vor, wie sich vor allem im Relativsatz im passivischen Prädikat und dem präpositionalen Objekt, das es regiert, aber auch beim Adjektiv scarsa als Prädikatsnomen zu economia zeigt, das besser durch poco sviluppata (kaum entwickelt) ersetzt wäre.

\subsubsection{Bildungssprachliche Formulierungsbestrebungen}

Derartige Kontraste zwischen Ausdrucksschwächen bei gleichzeitiger Verwendung bildungssprachlicher Lexeme und morphosyntaktischer Strukturen finden sich in unterschiedlicher Ausprägung in allen Schülertexten wieder und sind ein Zeichen dafür, dass die Lernenden sehr wohl ein Gespür dafür hatten, wie ein darstellender Text im Kontext Schule gestaltet werden sollte, sich dieses Register aber recht stark von ihrem alltagssprachlichen unterschied. Das Ausmaß der entstandenen Gegensätzlichkeit rührt wahrscheinlich auch von einer unzureichenden Praxis im schriftlichen Verfassen her, wodurch sie für Registerdifferenzen weniger sensibilisiert waren. Indem sie Konstruktionen und Lexeme gebrauchten, die sie produktiv nicht sicher beherrschten, trieben sie aber ihren Sprachausbau voran, anstatt aufgrund von Vermeidungsstrategien Texte ausschließlich mit dem lexikogrammatikalischen und morphosyntaktischen Material zu formulieren, das sie sicher beherrschten. Im Folgenden sollen einige Beispielsätze diese Bemühungen veranschaulichen.

1) II Brasile non si può paragonare alla Germania perché la Germania ha un'economia molto più sviluppata rispetto al Brasile, le persone con difficoltà economiche o con malattie, vengono aiutate senza nessun problema perché è un paese civile. (Schüler A; Brasilien kann man nicht mit Deutschland vergleichen, weil Deutschland gegenüber Brasilien eine viel entwickeltere Wirtschaft hat, denn Personen mit finanziellen Schwierigkeiten oder mit Krankheiten wird ohne Probleme geholfen, weil es ein ziviles Land ist.)

2) Per quanto riguarda economicamente è molto indietro perché ora se lo stato aiutasse il paese ora non ci sarebbero tutti questi problemi. (Schülerin C; Bezüglich wirtschaftlich liegt es sehr zurück, weil jetzt, wenn der Staat dem Land helfen würde, gäbe es diese ganzen Probleme nicht.)

3) Nei paesi più sviluppati il diritto alla scuola è molto grande invece nel continente africano ci sono molti bambini che sono analfabeti e che lo saranno anche quando diventeranno vecchi (Schüler F; In den entwickelteren Ländern ist das Recht auf Schule sehr groß, im afrikanischen Kontinent hingegen gibt es viele Kinder, die Analphabeten sind und die es auch sein werden, wenn sie alt werden.)

4) II tasso di natalità, è molto differente, per esempio a New York il tasso è di circa 2 bambini a persona, mentre in Africa è di una differenza molto notevole che dimostra che nei Paesi più poveri la popolazione è molto più numerosa. (Schülerin $\mathrm{H}$; Die Geburtenrate ist sehr unterschiedlich, zum Beispiel in New York ist die Rate ca. 2 Kinder pro Person, während in Afrika der Unterschied sehr bemerkenswert ist, was zeigt, dass in den ärmeren Ländern die Bevölkerung viel zahlreicher ist.)

Abbildung 10: Bildungssprachliche Versuche 
Im ersten Beispiel ist der Satzeinstieg für einen bildungssprachlichen Text nicht sehr typisch, doch bildungssprachliche Bestrebungen sind v.a. auf lexikalischer Ebene zu beobachten. So formuliert der Schüler nicht ,arme oder kranke Menschen“, sondern „Menschen mit finanziellen Schwierigkeiten oder Krankheiten", wobei im Italienischen die Präposition di anstelle von con angemessener wäre. Hiermit kontrastiert „ohne Probleme“, was eher im Alltagsund Umgangssprachlichen verwendet wird, wobei der Ausdruck in einem Satz auftritt, der passivisch und damit wieder eher bildungs- als alltagssprachlich konstruiert ist. Schließlich wird im letzten Teil, im kausalen Nebensatz, das Adjektiv „,zivil“ (Fo) unangemessen verwendet. Auch wenn es sich hier um ein Wort des fundamentalen Wortschatzes handelt, waren dem Schüler seine genauen Bedeutungen und Verwendungskontexte anscheinend nicht ausreichend bekannt.

Im zweiten Beispiel erfordert das Phrasem per quanto riguarda aus dem allgemeinen Wortschatz ein Substantiv. Stattdessen verwendet die mehrsprachige Schülerin C, die zwar in Italien geboren und eingeschult wurde, ein Adverb. Danach benutzt sie eine kausale Konjunktion, nach der eine als Nebensatz formulierte Begründung der zuvor gegebenen Satzaussage folgen müsste. Sie bildet aber keinen Kausalsatz, sondern fährt mit einem Konditionalsatz fort. Darin verwendet sie in beiden Teilen den korrekten Modus, doch es besteht keine explizite Kohäsion mit der vorherigen Äußerung. Darüber hinaus sind in diesem Beispiel der Ausdruck ora, den sie sogar wiederholt, und die Gegenüberstellung der Begriffe „Staat" und „Volk“ unangemessen (mit „Staat" ist wahrscheinlich die Regierung intendiert).

Im dritten Beispiel zeigt auf lexikalischer Ebene diritto alla scuola Bemühungen um einen bildungssprachlichen Ausdruck, die ihr Ziel etwas verfehlen. In diesem Kontext ist auch das adjektivische Prädikatsnomen grande nicht gut gewählt. Positiv hervorzuheben ist hingegen die Verwendung der adversativen adverbialen Verknüpfung invece, die für Kohäsion zwischen den beiden unterschiedlichen Satzaussagen sorgt. Allerdings schildert der Schüler nicht explizit, wie es um die Schulpflicht und ihre Wahrung bestellt ist, sondern beschreibt umständlich die Situation, aus der dies zu schließen ist.

Im letzten Beispiel geht es um einen Vergleich der Geburtenraten zwischen den Vereinigten Staaten und Afrika. Dafür stehen der Schülerin das richtige fachwortschatzsprachliche Phrasem und weitere bildungssprachliche lexikalische Mittel zur Verfügung, allerdings formuliert sie gleichzeitig molto notevole, was in einem darstellenden Text mit bildungssprachlichen Charakter übertrieben anmutet und außerdem anstelle einer exakten Angabe verwendet wird, die in diesem Kontext zu erwarten wäre. Diese bleibt nicht nur aus, sondern wird noch nicht einmal durch eine ungenauere ersetzt, weshalb der Leser die beabsichtigte Aussage nur indirekt aus dem weiteren Satzverlauf (,,und das zeigt...") folgern kann. 


\subsubsection{Prädikate}

Die SchülerInnen verwendeten in ihren Texten durchschnittlich 43 Prädikate, die auf 20 Worttypen zurückgehen. Am wenigsten Prädikate (28) und Worttypen (11) befinden sich im Text der Schülerin I, am meisten mit 66 Verben von 30 Worttypen in dem der Schülerin G. Diese und weitere Angaben sind der Tabelle $20 \mathrm{zu}$ entnehmen.

\begin{tabular}{lrrrrrrrrrr}
\hline Lernende & $\mathrm{A}$ & $\mathrm{B}$ & $\mathrm{C}$ & $\mathrm{D}$ & $\mathrm{E}$ & $\mathrm{F}$ & $\mathrm{G}$ & $\mathrm{H}$ & $\mathrm{I}$ & $\varnothing$ \\
\hline n Prädikate & 44 & 52 & 49 & 43 & 35 & 37 & 66 & 33 & 28 & 43 \\
Worttypen & 18 & 23 & 23 & 16 & 18 & 23 & 30 & 14 & 11 & 20 \\
n loc.v. & 2 & $2(1)$ & 1 & 0 & 0 & 1 & 1 & 0 & 0 & 1 \\
v.aus. & 3 & 1 & 2 & 2 & 5 & 3 & 3 & 1 & 3 & 3 \\
v.mod. & 2 & 4 & 5 & 2 & 9 & 2 & 8 & 4 & 1 & 4 \\
n Passiv & 2 & 0 & 1 & 0 & 0 & 0 & 0 & 1 & 0 & 0 \\
n essere & 17 & 9 & 20 & 17 & 0 & 11 & 5 & 13 & 16 & 12 \\
$\%$ essere & 38,6 & 17,3 & 40,8 & 39,5 & 0 & 29,7 & 7,6 & 39,4 & 57,1 & 30 \\
n esserci & 0 & 5 & 2 & 3 & 2 & 2 & 11 & 6 & 0 & 3 \\
$\%$ essere & & & & & & & & & & \\
esserci & 38,6 & 60,9 & 44,9 & 46,5 & 5,7 & 35,1 & 24,2 & 57,6 & 57,1 & 41,2 \\
n starci & 0 & 0 & 0 & 0 & 1 & 0 & 0 & 0 & 0 & 0 \\
AD & 0 & 0 & 0 & 1 & 0 & 0 & 0 & 0 & 0 & 0 \\
AU & 1 & $5(1)$ & $1(2)$ & $4(2)$ & $3(2)$ & $3(2)$ & $11(1)$ & $1(6)$ & 0 & $3(2)$ \\
CO & $5(4)$ & $4(3)$ & 2 & 1 & $3(3)$ & $3(3)$ & $3(3)$ & 0 & $2(2)$ & $3(2)$ \\
& 40 & 43 & 46 & 38 & 13 & 34 & 55 & 28 & & 36 \\
FO & $(13)$ & $(19)$ & $(19)$ & $(12)$ & $(34)$ & $(18)$ & $(26)$ & $(13)$ & $29(9)$ & $(18)$ \\
$\varnothing$ pro Satz & 2,4 & 4,3 & 3,8 & 2,9 & 3,9 & 3,4 & 6,6 & 3 & 2,2 & 3,6 \\
n Sätze & 18 & 12 & 13 & 15 & 9 & 11 & 10 & 11 & 13 & \\
\hline
\end{tabular}

Tabelle 20: Prädikate der Schülertexte (Vespucci)

Auf jeden Satz entfallen damit im Mittelwert 3,7 Prädikate. Die meisten stammen mit durchschnittlich 36 von 18 verschiedenen Worttypen aus dem fundamentalen Wortschatz (FO). Zu dem des hohen Gebrauchs (AU) und dem allgemeinsprachlichen (CO) gehören im Durchschnitt drei Vorkommnisse von zwei Worttypen. Bei Ersteren handelt es sich um konjugierte Formen von:

corrompere (bestechen, 1 Vorkommnis); esserci (da sein/geben, 31 Vorkommnisse); starci (da sein/geben, 1 Vorkommnis); ispirare (inspirieren); governare (regieren, 1 Vorkommnis); abbassare (verringern, 2 Vorkommnisse).

Als einziges Verb aus dem Wortschatz der hohen Disponibilität (AD) gebraucht die Schülerin D außerdem confrontare (gegenüberstellen, vergleichen).

Einige Prädikate erscheinen in Verwendungskontexten, in denen geübtere Schreibende auf prädikative Formulierungen verzichtet hätten, wie zum Beispiel in „Il tasso di natalità è alto e quindi vuol dire che la popolazione aumenta" (Schüler F; Die Geburtenrate ist hoch und das heißt also, dass die Be- 
völkerung zunimmt), wo die Formulierung vuol dire che eine überflüssige Verstärkung des Adverbs quindi darstellt, die getrost entfallen kann. Auch der nächste Textauszug käme mit weniger Prädikaten aus (Schülerin G):

Invece ci sono persone che hanno dei soldi che guadagnano ogni mese, ad esempio $€ 1000$ al mese e diciamo che può bastare per pagare l'affitto e per mangiare, poi ci sono i diciamo "ricchi" perché hanno una casa più grande e ricevono all'incirca $€ 2000$ al mese e basta per vivere.

(Hingegen gibt es Personen, die Geld haben, das sie jeden Monat verdienen, zum Beispiel $€ 1.000$ [sic] im Monat und sagen wir, dass kann reichen, um die Miete zu bezahlen und zu essen, dann gibt es die, sagen wir, „Reichen“, weil sie ein größeres Haus haben und ungefähr $€ 2.000$ [sic] im Monat bekommen, und das reicht zum Leben.)

Schreibkundigere würden vor allem die Konstruktion „Personen, die Geld haben, das sie jeden Monat verdienen“" auf maximal ein Prädikat verkürzen oder die Aussage als präpositionales Objekt wiedergeben (Personen mit einem Einkommen von). Außerdem vermieden sie die Formulierung „sagen wir“ und gebrauchten wahrscheinlich auch die letzten beiden Prädikate nicht, sondern stattdessen eine Konstruktion wie „Reichere, die ein größeres Haus und ein Einkommen von ungefähr 2.000 Euro haben und gut leben“.

Ähnlich wie in den Texten an der Morante benutzte auch diese Klasse sehr oft das Verb essere, um damit ein Prädikativum zu bilden. Durchschnittlich fast ein Drittel aller konjugierten Verben eines Textes stellen solche Formen, wobei „sein“" nur in seiner intransitiven Form und nicht als Hilfsverb gezählt wurde. Während die Schülerin E es gar nicht und Schüler G nur fünfmal verwendete, rekurriert im Text der Schülerin I mehr als jedes zweite Prädikat da$\operatorname{rauf}(57 \%)$.

Eine im Alter von zehn Jahren mit ihrer Familie nach Italien gezogene Schülerin begann ihren Text über Rumänien folgendermaßen (Schülerin G):

La densità della popolazione rumena e [sic] nella norma e [sic] un paese con difficoltà economiche non molto basse ma nemeno [sic] alte. La speranza di vita e [sic] intorno 70 per gli uomini e 80 per le donne. La percentuale di trovare un lavoro non e [sic] molto alta. Il confronto tra la Romania e l'Italia: e [sic] che il guadagno del'Italia [sic] e [sic] maggiore del guadagno della Romania.

(Die rumänische Bevölkerungsdichte ist in der Norm, es ist ein Land mit nicht sehr niedrigen wirtschaftlichen Schwierigkeiten, aber auch nicht hohen. Die Lebenserwartung ist bei 70 für die Männer und bei 80 für die Frauen. Der Anteil, eine Arbeit zu finden, ist nicht sehr hoch. Der Vergleich zwischen Rumänien und Italien: Es ist, dass das Einkommen in Italien größer ist als das Einkommen Rumäniens.)

Sie ist nicht die einzige, welche die konjugierte Form è (er/sie/es ist) nicht mit einem Akzent versah, wodurch sie graphisch nicht von der Konjunktion $e$ (und) zu unterscheiden ist. Dennoch versteht man ihren Text, weil sie die Wörter richtig gebraucht und die Regeln der Grammatik beachtet. Als einzige Ausnahme hierzu ist das Subjekt percentuale (Anteil) zu betrachten, das im Textzusammenhang keinen Sinn ergibt und eventuell mit probabilità (Wahrscheinlichkeit) verwechselt wurde. 
Alle Sätze dieses Auszugs sind prädikativ konstruiert und beginnen mit dem Subjekt. Lediglich eine Abweichung davon bildet die Formulierung e che nach dem Doppelpunkt, bei der zwei Lesarten denkbar sind. Entweder ist es eine Redewendung, die im Mündlichen vorherrscht, oder aber man streicht den Doppelpunkt, so dass sich ein mit der Konjunktion che eingeleiteter Subjektsatz ergibt.

Formal ist der Textauszug zwar einigermaßen korrekt, aber er erscheint dadurch monoton, dass die Sätze immer auf dieselbe Wiese konstruiert sind. Dieses Vorgehen ist nicht nur in den Texten mehrsprachiger Lernender zu beobachten, die erst seit einigen Jahren Italienisch lernen, sondern auch einigen einsprachig italienischen MitschülerInnen gemein, wie bspw. dieser Auszug zeigt (Schüler A):

Il tasso di fecondità è alto, ma non è molto ricco come paese. Le persone hanno un reddito basso. Le industrie non sono molte, ma è molta la produzione di piantagioni di cacao e di caffè. La situazione non è delle migliori perché solo le persone importanti (le persone che sono a capo di questo paese) sono ricche. Il cibo non è in grandi quantità. La speranza di vita non è alta.

(Die Fertilitätsrate ist hoch, aber es ist nicht sehr reich als Land. Die Personen haben ein niedriges Einkommen. Die Industrien sind nicht viele, aber viel ist die Produktion von Kakao- und Kaffeeplantagen. Die Situation ist keine der besten, weil nur die wichtigen Personen (die Personen, die an der Spitze dieses Landes sind) reich sind. Das Essen ist nicht in großen Mengen. Die Lebenserwartung ist nicht hoch.)

Zwar reihen sich hier nicht ausschließlich Prädikativa aneinander, sondern es wurden ein Adversativsatz eingefügt und ein Prädikat mit dem Verb avere (haben) gebildet, dennoch tauchen viele Prädikativa auf und beginnen alle Sätze gleichförmig mit dem Subjekt. Auch ist das Verb avere nicht etwa bedeutungstragender als essere (sein). Es ist möglich, dass der Schüler die Äußerungen so formulierte, weil er sich um einen bildungssprachlichen Stil bemühte, wie er ihn aus dem Lehrbuch kannte. Vor allem die Sätze „Il cibo non è in grandi quantità" und „è molta la produzione di piantagioni di cacao e di caffè"legen diese Vermutung nahe.

\subsubsection{Modalverben}

Im Mittelwert verwendeten die Lernenden vier Modalverben, wobei in zwei Texten mit acht und neun doppelt so viele auftreten. Damit gebrauchten sie deutlich mehr Modalverben als die Lernenden der Schule Morante: Während es dort nur insgesamt neun sind und neun Texte gar keines aufweisen, erscheinen sie in allen Texten der Vespucci. Zum Teil kann der stärkere Gebrauch auf inhaltliche Aspekte der Textgestaltung zurückgeführt werden, da die Beschreibung sozioökonomischer Aspekte einen größeren Raum einnimmt, wohingegen sich die Texte der Morante vorrangig mit der physisch-geographischen 
Beschaffenheit der Region befassen. Die Darstellung dieser Fakten erfordert nämlich keine Modalverben.

Der Gebrauch der Modalverben ist in einigen Fällen vermeidbar. So finden sich etwa in den Texten der Lernenden D und E die Formulierungen possiamo parlare di (wie können von ... sprechen) und possiamo dire che (wir können sagen, dass), die auch während der mündlichen Abfragungen beobachtet wurden, in einem schriftlichen, darstellenden Text aber nicht angemessen sind. Ähnlich verhält es sich mit der Formulierung diciamo che può bastare (sagen wir, dass es reichen kann) im Text der Schülerin G, wobei sowohl das Prädikat als auch das Modalverb und somit die gesamte Konstruktion textsortenspezifischer mit einem Adjektiv wie sufficiente (ausreichend) formuliert wäre.

Desweiteren verwendet ein Schüler Modalverben, die Ausdrucksschwierigkeiten erkennen lassen, die wahrscheinlich darauf zurückzuzuführen sind, dass der Schüler erst seit wenigen Jahren Italienisch lernte (Schüler E):

La speranza di vita è molto bassa e la natalina [sic] è alta, anche per questo l'Africa ha molti problemi perché l'economia è bassa e la natalita [sic] è alta. L'Africa non si può sviluppare se con la loro economia bassa devono spendere tutto nel popolo e per aumentare l'economia devono avere più soldi per aiutare il popolo è anche la nazione.

(Die Lebenserwartung ist sehr gering und die Geburtenrate ist hoch, auch deshalb hat Afrika viele Probleme, weil die Wirtschaft niedrig ist, und die Geburtenrate ist hoch. Afrika kann sich nicht entwickeln, wenn sie mit ihrer niedrigen Wirtschaft alles ins Volk ausgeben müssen und um die Wirtschaft steigern zu können, müssen sie mehr Geld haben, um dem Volk zu helfen und auch der Nation.)

Ohne auf den Wahrheitsgehalt dieser Textaussage einzugehen, zeigen sich in dem Auszug mehrere sprachliche Schwächen, die sich auf lexikalischer Ebene ansiedeln. Zum einen sind es Schwierigkeiten mit dem fachwortschatzsprachlichen Wort natalità (Geburtenrate), das zweimal ohne Akzent und bei der ersten Verwendung auch mit einem falschen Konsonanten erscheint. Das gemeinte Wort kann aber eindeutig erschlossen werden, so dass daraus keine Verständnisschwierigkeiten entstehen. Zum anderen ergibt sich der dreifache Gebrauch eines Modalverbs wahrscheinlich aus dem Umstand, dass dem Schüler keine angemesseneren lexikalischen Mittel zur Verfügung standen, um seinen Gedanken zu versprachlichen, der in etwa lauten könnte: L'Africa non si può sviluppare se le sue risorse non bastano per coprire tutte le spese statali e per promuovere l'economia. (Afrika kann sich nicht entwickeln, wenn seine Ressourcen nicht ausreichen, alle Staatsausgaben abzudecken und die Wirtschaft anzukurbeln.)

Während gegen die Verwendung der ersten beiden Modalverben nichts einzuwenden ist, könnte das dritte vermieden werden, ist das Adjektiv basso als Attribut zu economia nicht treffend gewählt, das Possessivpronomen loro inkohärent, weil es im Numerus vom Bezugswort abweicht, und die Präposition per (für) anstelle von nel (ins) erforderlich. Auch die Gegenüberstellung von „Volk" und „Nation“ ist unpassend. 
Neben den illustrierten Verwendungskontexten der als ersetzbar zu betrachtenden Modalverben zeigen sich in den Schülertexten auch legitimere Verwendungszwecke, die vorrangig im Schlussteil der Texte im persönlichen Kommentar verortet sind. Modale Formulierungen wie

Sinceramente io in Bangladesh ci vorrei andare per vedere i problemi, vorrei aiutarli, vorrei portare i bambini ad un futuro migliore. L'Italia è il paese dove ci vorrei restare per l'aiuto che ci danno.

(Ehrlich gesagt würde ich gerne nach Bangladesch fahren, um die Probleme zu sehen, ich würde ihnen helfen wollen und ich würde die Kinder zu einer besseren Zukunft bringen. Italien ist das Land, wo ich bleiben möchte wegen der Hilfe, die sie uns geben.)

oder

Io se dovessi andare a vivere in Perù ci andrei ma solo per le vacanze così per stare con la mia famiglia, ma più che altro per aiutarli.

(Wenn ich nach Peru ziehen müsste, würde ich dort hingehen, aber nur für die Ferien, so, um mit meiner Familie zusammen zu sein, aber vor allem um ihnen zu helfen.)

sind hier vollkommen angemessen.

\subsubsection{Passivgebrauch}

In den Texten dieser Lerngruppe liegen so gut wie keine Passivkonstruktionen vor. Lediglich ein Drittel der SchülerInnen (3) verwendeten maximal zwei passivische Prädikate. So ist zum Beispiel ein Prädikat aus dem Wortschatz des hohen Gebrauchs (AU), corrompere (bestechen), passivisch formuliert (Schüler A):

In questo paese io avrei paura ad andare in giro, perché non sei mai sicuro, alcune persone vanno in giro con pistole o fucili e i poliziotti se vedono qualcosa di pericoloso non fanno niente perché vengono corrotti da questi "mafiosi" che gli danno dei soldi.

(In diesem Land hätte ich Angst herumzulaufen, weil du nie sicher bist, einige Personen laufen mit Pistolen oder Gewehren herum und die Polizisten, wenn sie etwas Gefährliches sehen, machen nichts, weil sie von diesen "Mafiosi" bestochen werden, die ihnen Gelb geben.)

Hier kann der Passivgebrauch zwar als Bemühung um das verlangte schriftsprachliche Register betrachtet werden, jedoch befindet sich das Prädikat in einem langen Satzgefüge mit vielen verschiedenen Informationen, die besser auf mehrere Sätze verteilt wären. Zusätzlich wird das phraseologische Verb „herumlaufen“ in unmittelbarer Nähe wiederholt und ist auf lexikalisch-stilistischer Ebene die Formulierung „sie machen nichts“ schwach. Der abschließende Relativsatz deutet darauf hin, dass corrompere im mentalen Lexikon des Schülers noch nicht gut verankert war, da er den Begriff erklären wollte, indem er die Bedeutung im Text beschrieb. Dem Text merkt man generell Bemühungen um einen angemessenen Ausdruck an, wie bereits ein anderer Auszug w.o. 
zeigte, in dem sich eine zweite passivische Konstruktion in einem sehr langen Satzgefüge befindet (s. Abb. 10).

Die anderen beiden Lernenden verwenden jeweils nur ein Prädikat im Passiv. Auch im Text der mehrsprachigen Schülerin C ist ein Bestreben um das passende schriftsprachliche Register zu erkennen, welches mit oraten Merkmalen kontrastiert $(\mathrm{C})$ :

Per quanto riguarda il nutrimento è ancora più grave perché i cibi che loro mangiano non vengono protetti adeguatamente, cioè quando i bengalesi ad esempio vanno a comprare il pesce si capisce che è stato troppo tempo fuori, le mosche restano appresso a quei pesci per molte ore.

(Bezüglich der Ernährung ist es noch gravierender, weil die Nahrungsmittel, die sie essen, nicht angemessen geschützt werden, das heißt, wenn die Bengalen zum Beispiel Fisch kaufen gehen, versteht man, dass er zu lange draußen gewesen ist, die Fliegen bleiben an diesen Fischen viele Stunden lang haften.)

Die Schülerin beginnt mit dem bildungssprachlichen phrasematischen Einstieg per quanto riguarda $(\mathrm{CO})$ und konstruiert eine Passivform auf korrekte Weise, verwendet aber gleichzeitig typische Ausdrucksweisen des umgangssprachlichen Mündlichen wie cioè (das heißt) und si capisce che (man versteht, dass). Auch è stato troppo tempo fuori (war zu lange draußen) ist für einen schriftlichen Text im schulischen Kontext der achten Klasse ausdrucksschwach.

Die letzte Passivkonstruktion stammt von einer Schülerin, die erst seit einigen Jahren Italienisch lernte (Schülerin I):

È un paese che sta incontrando dificoltà [sic] economiche abastanza [sic] alte ma non come quelle del Africa e di questo paese a me piacciono le tradizioni e come vengono afrontate [sic].

(Es ist ein Land, das ziemlich hohen wirtschaftlichen Schwierigkeiten begegnet, aber nicht wie denen Afrikas, und an diesem Land gefallen mir die Traditionen und wie sie angegangen werden.)

Formal ist gegen ihre Passivkonstruktion nichts einzuwenden, aber auf lexikalischer Ebene ist das Verb, auf das sie zurückgeht, nicht gut gewählt, da es eher in Kollokationen mit negativ konnotierten Substantiven wie „Probleme angehen“ auftritt, während sie es mit „Traditionen“ gebraucht. Letztere stehen in ihrem Text aber in einem positiven Licht, wie aus dem Textfortgang erschlossen werden kann, in dem sie das rumänische Weihnachtsfest beschreibt.

\subsubsection{Nicht flektierte Verbformen}

In den Texten lassen sich außer den Infinitiven, die zu einem guten Teil aus dem verstärkten Modalverbgebrauch resultieren, kaum infinite Verbformen finden. So verwendeten nur eine einsprachig italienische Schülerin (H) ein Partizip Perfekt und sieben von neun Lernenden ein bis maximal drei Gerundien bei einem Durchschnittswert von einem Gerundium pro Text.

Tabelle 21 zeigt die genauen Werte für die einzelnen Texte an. 


\begin{tabular}{lrrrrrrrrrr}
\hline Lernende & $\mathrm{A}$ & $\mathrm{B}$ & $\mathrm{C}$ & $\mathrm{D}$ & $\mathrm{E}$ & $\mathrm{F}$ & $\mathrm{G}$ & $\mathrm{H}$ & $\mathrm{I}$ & $\varnothing$ \\
\hline ger. & 0 & 1 & 1 & 0 & 1 & 3 & 2 & 1 & 1 & 1 \\
inf./inf.loc. & 8 & 13 & 17 & 10 & 16 & 6 & 32 & 10 & 3 & 14 \\
part.pass. & 0 & 0 & 0 & 0 & 0 & 0 & 1 & 0 & 0 & 0 \\
\hline
\end{tabular}

Tabelle 21: Partizipien, Gerundien und Infinitive der Schülertexte (Vespucci)

Das Partizip Perfekt stammt vom Verb mantenere (Fo; unterhalten), das in dem bereits zitierten Verwendungskontext semantisch nicht treffend gewählt ist (Schülerin H):

Nel primo, ad esempio c'è una forte economia di mercato, mantenuta con delle tasse molto alte con cui vengono ripagati anche i cittadini del proprio lavoro [...].

(Im ersten zum Beispiel gibt es eine starke Marktwirtschaft, die mit sehr hohen Steuern unterhalten wird, mit denen auch die Bürger von ihrer eigenen Arbeit belohnt werden [...]).

Diesem Satzgefüge ist ein Bestreben um das angemessene Register anzumerken. So ist der Wortschatz einerseits präzise, bisweilen sogar fachsprachlich (economia di mercato) und erscheinen die morphosyntaktisch komplexeren Konstruktionen eines impliziten Relativsatzes mit dem Partizip Perfekt, an den sich ein passivisches Prädikat anschließt. Andererseits liegen lexikalisch-semantische Ungenauigkeiten bzw. Ausdrucksschwächen vor: Anstelle des Verbs mantenere wäre basarsi (AD; basieren) angemessener und im zweiten Relativsatz kann die intendierte Satzaussage aufgrund der Verbwahl und des Objekts, das es regiert, lediglich erahnt werden.

Von den zehn Gerundien in sieben Schülertexten dienen drei der Prädikatsbildung mit der periphrastischen Verlaufsform stare plus Gerundium (C, E, I). Die sieben infiniten Gerundivformen verteilen sich auf insgesamt vier Texte von je zwei einsprachigen und zwei mehrsprachigen Lernenden. Diese Gerundien gehen auf sechs unterschiedliche Worttypen zurück und werden mit einer Ausnahme logisch-formal korrekt gebraucht.

So formuliert beispielsweise Schülerin B:

Un altra [sic] causa naturale dell'Africa è che le persone muoiono anche a causa delle malattie non avendo ospedali e strutture per curarsi.

(Ein anderer natürlicher Grund Afrikas ist, dass die Personen auch aufgrund von Krankheiten sterben, da sie keine Krankenhäuser und Strukturen haben, um sich zu pflegen/auszukurieren.)

Während das Gerundium richtig und textsorten- sowie registerangemessen verwendet ist - wie auch die nominale Konstruktion a cause delle malattie -, liegen die Schwächen des Textauszugs abgesehen von einem orthographischgrammatikalischen Fehler auf lexikalischer Ebene bei dem Ausdruck ,natürlicher Grund“ und lexikogrammatikalisch bei „sich zu pflegen“ was besser durch das Hilfsverb ,lassen“ ergänzt wäre (sich pflegen zu lassen).

Schüler B formulierte gleich drei infinite Gerundien korrekt, von denen sich zwei im folgenden Textauszug befinden (B): 
Alcuni scienziati pensano che mettendo delle zanzariere nelle case dei paesi africani, per le malarie [sic] e dando loro medicine e acqua potabile, la mortalità si abbassa.

(Einige Wissenschaftler denken, dass die Sterberate sinkt, wenn man für die Malarien Moskitonetze in die Häuser der afrikanischen Dörfer hängt und ihnen Medizin und Trinkwasser gibt.)

Der Schüler beachtete allerdings nicht, dass das von ihm gewählte Prädikat des Hauptsatzes im Nebensatz einen Konjunktiv erfordert. Überdies stünde malarie registerangemessener im Singular und durch eine infinite Verbform ergänzt oder mit einer anderen Präposition: per prevenire la malaria (um Malaria vorzubeugen) bzw. contro la malaria (gegen Malaria).

Formal richtig, aber lexikalisch-semantisch falsch formulierte die einsprachige Schülerin H außerdem mit einem Gerundium Folgendes: „Si dovrebbe aiutare $i$ vari Paesi stabilendo un'economia stabile" (Man müsste den verschiedenen Ländern helfen, indem man eine stabile Wirtschaft etabliert). Abgesehen vom störenden Gleichklang durch die Verwendung desselben Wortstammes im ersten und letzten Ausdruck von stabilire un'economia stabile wäre lexikogrammatikalisch und semantisch anstelle des Gerundiums ein Infinitiv passender, so dass sich ergäbe: Man müsste [...] helfen, eine stabile Wirtschaft zu etablieren.

\subsection{Inhaltlich-sprachlicher Vergleich der Texte beider Klassen}

Dieses Kapitel abschließend, sollen einige Merkmale der Textgestaltung beider Klassen miteinander verglichen werden, auch wenn auf inhaltlicher Ebene ein solcher Vergleich nicht direkt möglich ist, da sich in der Klasse Vespucci nur ein Text mit einem Land der indischen Region beschäftigt. Der Schreibauftrag jedoch war für beide Klassen nahezu identisch: Ein Unterschied bestand lediglich in dem Umstand, dass der Klasse an der Morante die vorzustellende Region von der Lehrkraft vorgeschrieben wurde, während die andere sie frei wählen konnte. Die Schreibprodukte beider Klassen nun zueinander in Bezug zu setzen, erfolgt mit Blick auf die Analyseergebnisse, aus denen zu folgern ist, dass durch eine intensive Beschäftigung mit den Lehrbuchtexten als Vorbereitung auf eine angekündigte Abfragung sprachlich und inhaltlich bessere Texte produziert werden als ohne eine solche Vorbereitung. Jedoch wurde w.o. darauf aufmerksam gemacht, dass die so entstandenen Schreibprodukte bildungssprachliche Ausdrücke und Strukturen enthalten, die wortgetreu aus dem Lehrbuch übernommen sind. Das stärkt den sowohl in der Forschung (Brasca et al. 2014, S. 77) als auch von den beobachteten Lehrkräften geäußerten Verdacht, die SchülerInnen lernten die Lehrbuchtexte auswendig, was wiederum zwei Fragen aufwirft: Verstanden sie, was sie versprachlichten, und wären sie 
in der Lage, die Lerninhalte mit eigenen Worten darzustellen und auswendiggelernte Begriffe und Strukturen auf andere Kontexte anzuwenden?

Auch an der Vespucci waren Lehrbuchtexte zu den in den Schülertexten beschriebenen Regionen erarbeitet worden, nur nicht unmittelbar vor dem Schreibauftrag. Dadurch kann die Qualität der so entstandenen Schreibprodukte auch als Indiz dafür angesehen werden, dass ein Gutteil der auswendiggelernten Formulierungen und Inhalte keinen Einzug in das Langzeitgedächtnis der Schülerinnen und Schüler gefunden hatte.

Den Texten beider Klassen ist gemein, dass die inhaltliche Schwerpunktsetzung aus den im Unterricht behandelten Themen resultierte. So schilderten die Schüler der Klasse Morante sehr ausführlich die physische Geographie der indischen Region, während sie in den Texten der Klasse Vespucci kaum oder gar kein Gewicht erhielt. Stattdessen verwendeten die Schülerinnen und Schüler hier unterschiedliche Aspekte aus dem im Vorfeld länderunabhängig behandelten sozioökonomischen Themenkomplex und ergänzten sie durch persönliches Wissen. Somit kamen sie der Aufgabenstellung nur zum Teil nach, da sie in der Regel keine geographische Einordnung vornahmen, geophysische Gesichtspunkte nur minimal schilderten und auch keine Informationen über die soziopolitische Ordnung des Landes oder des Kontinents gaben. Ihren Texten fehlen allgemeinere Informationen über die vorgestellte Region, denn sie befassen sich gleich mit einem sehr spezifischen Thema und folglich nur mit einem kleinen Teil dessen, was zu einer Länderbeschreibung gehört.

Sowohl auf inhaltlicher als auch auf sprachlicher Ebene wurde der Klasse an der Schule Morante die Textverfassung gewissermaßen erleichtert, da ihnen eine Abfragung über die Region angekündigt worden war, so dass sie sich mithilfe der Lehrbuchtexte gezielt darauf vorbereiten konnten. Das spiegelt sich auf inhaltlicher Ebene in der umfassenderen Vorstellung der Region nach unterschiedlichen Gesichtspunkten (physisch, gesellschaftlich und wirtschaftlich) wider. Auf sprachlicher zeigt es sich in einer gesteigerten Verwendung von Ausdrücken, die nicht aus dem Basiswortschatz stammen, und komplexerer grammatikalischer Strukturen. Wie sich die Texte in dieser Hinsicht konkret unterscheiden, ist zunächst in Tabelle 22 illustriert.

\begin{tabular}{lrr}
\hline$\varnothing$ pro Klasse & Vespucci & Morante \\
\hline $\mathrm{n}$ Wörter & 328 & 343 \\
$\mathrm{n}$ Sätze & 12 & 17 \\
längster Satz & 55 & 44 \\
$\varnothing$ Satzlänge & 26 & 21 \\
$\mathrm{AD}$ & 3 & 3 \\
$\mathrm{AU}$ & 14 & 27 \\
$\mathrm{AU}$ TS & 0 & 0 \\
CO & 21 & 34 \\
CO TS & 2 & 2 \\
el.CO & 7 & 4 \\
el.CO TS & 0 & 1 \\
el.TS & 4 & 3 \\
\hline
\end{tabular}




\begin{tabular}{lrr}
\hline$\varnothing$ pro Klasse & Vespucci & Morante \\
\hline FO & 259 & 223 \\
TS & 3 & 6 \\
FO TS & 0 & 29 \\
n.p. & 14 & 10 \\
el.n.p. & 1 & 0 \\
sigla & 0 & 0 \\
$\mathrm{n}$ FO in \% & 79 & 65 \\
$\mathrm{n} \mathrm{VdB}$ & 275 & 255 \\
$\mathrm{n}$ VdB in \% & 84 & 73 \\
\hline
\end{tabular}

Tabelle 22: Klassenvergleich - Text- und Satzlängen, Gebrauchsmarken *inkl. doppelter Marken mit Ts

Aus der Tabelle geht hervor, dass die Texte ungefähr gleichlang sind. Mit 343 Wörtern sind sie an der Morante etwas länger als die der anderen Klasse. Schließt man allerdings den einen wesentlich längeren Text an der Morante aus der Berechnung aus, so ergibt sich für die Klasse ein Durchschnittswert von 306 Wörtern, der dann etwas unter dem der anderen Klasse liegt. Die Klasse der Schule Vespucci bildete mit 26 Wörtern durchschnittlich fünf Wörter längere Sätze als die andere Klasse.

Bezüglich der Gebrauchsmarken lässt sich festhalten, dass die SchülerInnen der Vespucci deutlich mehr Wörter aus dem fundamentalen Wortschatz verwendeten. So nehmen die Fo-gekennzeichneten Ausdrücke 79 Prozent am Gesamttextvolumen ein, was einer Differenz von 14 Prozentpunkten zur anderen Klasse entspricht. Dementsprechend ist auch das Ausmaß der Wörter aus dem Basiswortschatz größer, und zwar um 20 Ausdrücke. Prozentual ergibt sich mit 84 zu 73 Prozent ein Unterschied von elf Punkten.

Hinsichtlich der Prädikate ist interessant, dass die Klassen in etwa gleichviele verwendeten, nämlich an der Vespucci durchschnittlich ca. 43 und an der Morante 40. An erster entfallen davon jedoch im Mittelwert 3,6 auf einen Satz und an zweiter nur 2,3. Sehr ähnlich ist es wieder um den Anteil des Verbs essere (sein) an den Prädikaten bestellt, der an der Vespucci mit 30 Prozent knapp unter dem der Schule Morante (33 Prozent) liegt. Identisch hingegen ist die durchschnittliche Verwendung des Verbs esserci (da sein, geben).

Was die lexikogrammatikalische Komplexität der verwendeten Prädikate betrifft, so fällt die Klasse Vespucci hinter die Klasse Morante zurück: In Ersterer wurden deutlich mehr Modalverben, weniger Passivformen und mehr Verben aus dem Bereich des fundamentalen Wortschatzes formuliert, wie aus Tabelle 23 ersichtlich wird.

\begin{tabular}{lrr}
\hline Klassen & Vespucci & Morante \\
\hline n Prädikate & 43 & 40 \\
Worttypen & 20 & 18 \\
n loc.v. & 1 & 0,4 \\
v.aus. & 3 & 6 \\
v.mod. & 4 & 1 \\
n Passiv & 0 & 2 \\
\hline
\end{tabular}




\begin{tabular}{lrr}
\hline Klassen & Vespucci & Morante \\
\hline n essere & 12 & 13 \\
$\%$ essere & 30 & 33 \\
n esserci & 3 & 3 \\
$\%$ essere + esserci & 41,2 & 42 \\
n starci & 0 & 0 \\
AD & 0 & 1 \\
AU & $3(2)$ & $4(2)$ \\
CO & $3(2)$ & $4(3)$ \\
FO & $36(18)$ & $29(13)$ \\
$\varnothing$ pro Satz & 3,6 & 2,3 \\
\hline
\end{tabular}

Tabelle 23: Klassenvergleich - Prädikate

Als Analyseergebnis der Textproduktion kann für beide Klassen festgehalten werden, dass sie sich um ein angemessenes schriftsprachliches Register bemühten, was ihnen besonders gelang, wenn sie sich in ihren Formulierungen stark am Lehrbuch orientierten. Gleichzeitig weisen ihre Texte aber sowohl in der graphischen Gestaltung als auch auf sprachlicher Ebene deutliche Schwächen auf. So verfielen sie beim Schreiben an verschiedenen Stellen in ein umgangssprachliches und konzeptionell mündliches Register und drängt sich insgesamt der Eindruck auf, dass es ihnen vor allem an Übungsmöglichkeiten im Schriftsprachlichen fehlte. Ihre Texte würden auf inhaltlicher und sprachlicher Ebene viel gewinnen und auch eine bessere äußere Form erhalten, wenn die SchülerInnen einzelne Schritte und Aspekte der Textproduktion und des Paraphrasierens gezielt und kontinuierlich erproben könnten. 


\section{Fazit}

Die vorgestellte Erhebung wurde mit dem Ziel durchgeführt, die sprachlichen Herausforderungen auszumachen, denen Lernende im Fachunterricht begegnen, und den Fragen nachzugehen, wie sie ihnen gerecht werden und welche Unterstützung sie dabei von den Erdkundelehrkräften erfahren. Dazu wurde mit einer Anzahl von fünf Klassen an drei Schulen ein überschaubarer Rahmen gewählt, um das sprachliche Handeln möglichst genau und multiperspektivisch untersuchen zu können. Während der Unterrichtsbeobachtungen, die sich fast über ein ganzes Schuljahr erstreckten, erwies sich, dass der Schwerpunkt in der Unterrichtsgestaltung auf der Arbeit mit dem Lehrbuch lag.

Abgesehen von einer Klasse, in der die Textinhalte regelmäßig in Gruppenarbeit angeeignet und wiederholt wurden, nachdem sie von der Lehrkraft in Anlehnung an das Lehrbuch vorgestellt worden waren, herrschte überall ein lehrerzentrierter Frontalunterricht vor. Die LehrerInnen fassten den Stoff mit eigenen Worten zusammen oder ließen die Texte vorlesen und gaben im Anschluss daran Erklärungen, um das Textverständnis zu fördern. Außerdem überprüften sie kontinuierlich anhand mündlicher Leistungskontrollen, ob die Schülerinnen und Schüler die Inhalte kannten und verstanden. Demzufolge konzentrierte sich das sprachförderliche Lehrerhandeln auf ihre Rolle als sprachliches Vorbild in den Lehrervorträgen und auf die sprachbezogene Korrektur von Schülerbeiträgen, zu denen es hauptsächlich in den Abfragungen kam.

Dadurch dass der Großteil des Unterrichtsgeschehens durch Mündlichkeit geprägt war, gerieten vor allem der Wortschatz der Lernenden (eventuelle Lücken oder inhaltliche bzw. registerspezifische Unangemessenheit) und grobe grammatikalische, syntaktische und andere Abweichungen von der Standardsprache in den Fokus der Sprachförderung. Denn komplexere sprachliche Zusammenhänge von Mehrworteinheiten, wie sie z.B. die logisch-semantische Organisation eines Gesprächsbeitrags bestimmen und die Vertextungs- und Kontextualisierungskompetenzen voraussetzen, können im Mündlichen nicht so leicht und unverzüglich erfasst und bewertet werden wie lexikalische Phänomene oder grobe Verstöße (Harren 2015, S. 176 mit Verweis auf Fiehler 2009). Darüber hinaus wurden den Schülerinnen und Schülern ohnehin oft punktuelle Fragen gestellt, auf die sie mit wenigen Worten oder in kurzen Sätzen antworteten. Eine Erweiterung des methodisch-didaktischen Repertoires wäre nicht zuletzt für den Sprachausbau der Lernenden wünschenswert, da Sprachförderung dann besonders greift, wenn sie sich ausgewogen auf alle vier Kompetenzbereiche (hören, sprechen, lesen und schreiben) erstreckt (Piemontese 2014, S. 9; Ehlich 2007, S. 25; Stefinlongo 2002, S. 15 f.). 
Die Erdkundehausaufgaben bestanden in allen Klassen überwiegend aus einer wiederholenden Beschäftigung mit den Lehrbuchtexten, was der Aneignung und Festigung der Lerninhalte und der Vorbereitung auf die Abfragungen diente. Jede Klasse verwendete ein anderes Erdkundebuch, dessen sprachliche Gestaltung auszugsweise und dennoch detailliert untersucht wurde. Als Ergebnis dieser Lehrmittelanalyse ist festzuhalten, dass ihre sprachliche Gestaltung sowohl auf lexikalischer als auch auf morphosyntaktischer und textueller Ebene durchweg als komplex einzustufen ist. Das in den Blick genommene Wortvorkommen setzt sich in einem erheblichen Ausmaß aus Begriffen zusammen, die seltener gebraucht werden als der Grundwortschatz (VDB), so dass es deutlich unter dem gemeinhin für einen italienischen Text üblichen Wert von um die 96 Prozent an VDB-Wörtern (De Mauro, 1999/2000, Bd. 1, S. XX) liegt. Der Bestand an Ausdrücken aus dem Grundwortschatz reicht in den untersuchten Lehrbuchtexten von 59 bis zu 81 Prozent pro Seite bzw. Seiteneinheit und siedelt sich oft bei ca. 70 Prozent an.

Der Sprachgebrauch der Lernenden unterschied sich auf lexikalischer Ebene deutlich von dem der Lehrbücher, da sie in der Unterrichtskommunikation und beim Verfassen schriftlicher Texte entschieden seltener auf niedrigfrequente und fachwortschatzsprachliche Begriffe zurückgriffen. In diesem Zusammenhang zeigen die Analyseergebnisse der Schülertexte, insbesondere in der Klasse an der Schule Vespucci, in der die Texte ohne eine vorausgehende lehrbuchbasierte Vorbereitung auf eine angekündigte Lernkontrolle entstanden waren, dass das Wortvorkommen im Durchschnitt zu 84 Prozent aus Begriffen des Basiswortschatzes stammt. Außerdem enthalten die Texte mehr Wörter aus dem fundamentalen Wortschatz (Fo), also den 2.000 meistgebrauchten Wörtern, und weniger verschiedene Worttypen. So ist beispielsweise das Vorkommen des hochfrequenten Verbs essere (sein) sehr hoch.

Auch wenn die Lehrkräfte und Lernenden die sprachlichen Herausforderungen primär am Wortschatz festmachten - beispielsweise der Schüler aus der Klasse III C an der Pestalozzi, der im Fragebogen angab, die in Erdkunde verwendeten Begriffe gerne ausgetauscht zu sehen, weil er sie sich nicht einprägen konnte-, sollte die Untersuchung des Wortschatzes nicht losgelöst vom Textzusammenhang erfolgen, in dem er auftritt (s.a. Bates \& Goodman 1997). Die Analyse der Lehrbücher ergab, dass fachsprachliche, andere seltener gebrauchte wie auch allgemeinsprachliche Begriffe, die im fachlichen Diskurs eine ganz präzise, den Lernenden manchmal unbekannte Bedeutung annehmen, in einem sprachlichen Umfeld auftreten, das aus Strukturen besteht, die ebenfalls weniger geläufig sind und Schwierigkeiten bereiten können. Gemeinsam mit den Wortbedeutungen muss ein kollokationales Wissen erworben werden, um die lexikalische Kompetenz zu stärken. Außerdem zeigt sich hier ganz deutlich, dass sich die einzelnen sprachlichen Fähigkeiten wie grammatikalische und lexikalische gegenseitig bedingen und unbedingt gemeinsam entwickelt werden sollten. 
Wie aus der C-Testbearbeitung ersichtlich und durch die Beobachtungen der Unterrichtskommunikation bekräftigt, schien zumindest für einige beim Textverständnis eine kaum überwindbare Hürde die Tatsache darzustellen, dass das niedrigfrequente Wortvorkommen in den Lehrbuchtexten nicht in einfach strukturierten Sätzen angeordnet war. Stattdessen bestanden die einzelnen Sätze oft aus mehr als 20 Wörtern, waren in hypotaktischen Gefügen angeordnet und mittels grammatikalischer Strukturen gebildet, die sich durch nicht konjugierte Verbformen wie Partizipien und einen stark ausgeprägten Nominalstil kennzeichneten. Eine Konsequenz daraus waren z.B. lange Nominalketten und Aneinanderreihungen von Attributen sowie (teilweise impliziten) Relativsätzen, die zusammen mit der ausgeprägten Hypotaxe dazu führten, dass die Übersicht über die inhaltlichen Bezüge zwischen den einzelnen Satzkonstituenten teilweise bzw. ganz verloren ging.

So identifizierten die SchülerInnen etwa im C-Test die Bezugswörter für anaphorische Textgestaltungsmittel wie Pronomen nicht korrekt. Gleichzeitig formulierten auch sie schriftliche Sätze von einer beträchtlichen Durchschnittslänge (21-26 Wörter), hielten dabei jedoch morphosyntaktische Regeln wie die Kongruenz zwischen dem Numerus des Subjekts und dem des Prädikats nicht immer ein. Einerseits erweckten ihre Schreibprodukte den Eindruck, den Sprachstil der Lehrbuchtexte zu imitieren, andererseits deutet viel darauf hin, dass ihre Gestaltungsmittel wie z.B. die langen Satzgefüge auf mangelnde Übung im Umgang mit der Schriftsprache zurückzuführen sind. Denn in vielen Fällen schrieben die Schülerinnen und Schüler so, wie sie redeten, und fehlten sowohl textuelle Grundfähigkeiten wie eine gewisse Sicherheit im Umgang mit der Zeichensetzung als auch morphosyntaktische Kompetenzen: Sie schrieben im wahrsten Sinne des Wortes oft „ohne Punkt und Komma“ und stellten nur in einem sehr geringen Umfang Kohäsion her.

Die Auswertung der C-Tests zeigte, dass sich vermehrte Falschlösungen vor allem dort konzentrierten, wo eine Lehrbuchstelle auf unterschiedlichen sprachlichen Ebenen, etwa auf lexikogrammatikalischer und morphosyntaktischer, komplex gestaltet war. Hieran wurde gut ersichtlich, dass die Schwierigkeiten im Umgang mit den schulsprachlichen Registern nicht allein im lexikalischen Bereich verortet waren, sondern dort, wo mehrere registerspezifische Sprachkomponenten aufeinandertrafen, welche die Schülerinnen und Schüler in ihrer Alltagssprache nicht benutzten und auch dann, wenn sie sich um bildungssprachlichere Formulierungen bemühten, nur mit Einschränkungen zu bilden imstande waren.

Während es ein wichtiges Ziel der schulischen Sprachbildung bleibt, die Wortschatzerweiterung quantitativ und qualitativ voranzutreiben, unterstreichen die Ergebnisse dieser Erhebung noch einmal, dass der Sprachausbau eine sehr facettenreiche Aufgabe darstellt, die dann bessere Aussichten auf Erfolg hat, wenn ihr die LehrerInnen in allen Fächern nachkommen und die Sprachkompetenz der Lernenden gleichmäßig in der mündlichen und der schriftlichen 
Produktion und Rezeption vorangetrieben wird. Nur auf diese Weise kann der spazio linguistico (De Mauro 1980), in dem sich die Lernenden kompetent bewegen, über das alltagssprachliche Register hinaus vergrößert werden.

Ferner ließ bei der C-Testauswertung die Nicht- oder Falschbearbeitung einer Lücke in vielen Fällen die Hypothese zu, dass nur das unmittelbare sprachliche Umfeld des getilgten Worts und nicht auch der weitere Zusammenhang beachtet wurde. Im beobachteten Unterricht zeigten die Schülerinnen und Schüler ebenfalls ein eher schwaches Textverständnis. Allerdings bekamen sie auch nur eingeschränkt die Möglichkeit, es zu verbessern, was vor allem daher bedenklich ist, dass sich das Unterrichtsgeschehen auf die Vermittlung der Lerninhalte anhand des Lehrbuchs konzentrierte. Wenn seine sprachliche Gestaltung jedoch so komplex ist, dass SchülerInnen sich die Texte nicht selbstständig aneignen können, ist für sie innerhalb der ,Zone der nächsten Entwicklung“" (Wygotski 1977 (1934)) sowohl fachlicher als auch sprachlicher Lernerfolg kaum möglich.

Während der Abfragungen beschränkte sich das Überprüfen des Lernerfolgs hauptsächlich auf Oberflächenwissen. Dafür wurden die Lehrbuchtexte geradezu auswendig gelernt, wie auch die in der Klasse an der Morante verfassten Schülertexte zeigten. Beim Abfragen und im Unterrichtsgespräch waren alle fünf Lehrkräfte zwar immer auch sprachbildend tätig, konkrete gemeinsame Textarbeit fand jedoch nur sehr selten und nicht überall statt. Meist war es die Lehrkraft, die den Schülerinnen und Schülern die Lehrbuchtexte paraphrasierte. Ebenfalls nicht bzw. nur sehr eingeschränkt und nur in zwei Klassen konnte darüber hinaus ein schriftliches Arbeiten beobachtet werden. Auch da, wo die Lernenden in Form von Hausaufgaben dazu angehalten wurden, Zusammenfassungen der Lehrbuchtexte zu schreiben, was in den meisten Klassen nicht der Fall war, wurden die Schreiberzeugnisse weder im Unterricht besprochen noch von der Lehrkraft kontrolliert.

Wie eingangs betont, unterrichteten die beobachteten Fachlehrkräfte ihre Klassen in Erdkunde, in Geschichte und in Italienisch. Dass sie also auch Sprachlehrkräfte waren, führte wahrscheinlich zu ihrer konstanten Aufmerksamkeit für die sprachlichen Fähigkeiten und ihren Ausbau. Die Gestaltung der Lehrmittel war ihnen dabei ebenso wenig eine große Hilfe wie die auf die Lehrtätigkeit vorbereitenden Ausbildungsbedingungen und der eng gesteckte Lehrplan. Insgesamt ist der Eindruck entstanden, dass sie die Bedeutung der Sprachförderung im Fachunterricht erkannt hatten und dieser Aufgabe ihren Fähigkeiten entsprechend nachkamen.

Die auf Sprache zielende Grundhaltung der Lehrkräfte und ihr (sprach)pädagogisches Handeln stellen m.E. eine gute Basis dar, auf der mit Weiterbildungsmaßnahmen gezielt aufgebaut werden könnte, um die Sprachförderung noch effizienter werden zu lassen. Es wäre in anknüpfenden Studien interessant zu untersuchen, wie sich solche Maßnahmen auf den Umgang der Lehrenden und der Lernenden mit den sprachlichen Anforderungen im Fachunterricht 
niederschlagen würden. In diesem Zusammenhang von Weiterbildungsmaßnahmen, die auch die Unterrichtserfahrung der Lehrkräfte und ihre bereits vorhandenen sprachförderlichen Kompetenzen nutzen, könnten Interventionsstudien durchgeführt werden, die konkrete Aspekte der Sprachbildung und ihre Vermittelbarkeit im Fachunterricht ins Visier nehmen. Beispielsweise ist dabei an die Förderung der mündlichen Darstellungskompetenz, die über ein Aufsagen auswendiggelernten Oberflächenwissens hinausgeht, und somit auch an die produktive und rezeptive schriftsprachliche Textkompetenz zu denken. Gerade sie bedarf einer intensiveren Zuwendung auch in den nicht sprachlichen Fächern und Lehrkräfte und Schülerinnen und Schüler würden vermutlich viel dadurch gewinnen, dass sie unter Anleitung in Teilkompetenzen zerlegt und ausgebaut wird.

Außerdem könnte der Frage nach dem Umgang mit den sprachlichen Herausforderungen im Fachunterricht auch nachgegangen werden, indem untersucht wird, ob vielleicht andere Erhebungspopulationen in lehrbuchbasierten C-Tests, in der Unterrichtskommunikation und in Schriftproduktionen bessere sprachliche Leistungen erbringen und welche Faktoren ihrer Unterrichtspraxis damit verbunden sind.

Neben weiteren Studien und der Notwendigkeit, Lehrkräfte in der Aus- und Fortbildung für eine durchgängige schulische Sprachbildung - gerade auch im Fachunterricht - zu qualifizieren, bleibt die Kritik an den italienischen Lehrwerken berechtigt (s. Kap. 3.2). Hier wäre es mehr als wünschenswert, dass Schulbuchautoren und -verlage bei der sprachlichen Gestaltung von Lehrmitteln konsequent darauf achten, dass sie den Fähigkeiten der Schüler und Schülerinnen gerecht werden und neben der fachlichen auch zur sprachlichen Bildung beitragen. 


\section{Literaturverzeichnis}

J. Achterberg (2005), Zur Vitalität slavischer Idiome in Deutschland. Eine empirische Studie zum Sprachverhalten slavophoner Immigranten, Otto Sagner, München.

K. Adamzik (2004), Textlinguistik. Eine einführende Darstellung, Niemeyer, Tübingen.

B. Ahrenholz (2013), Sprache im Fachunterricht untersuchen, in Ch. Röhner, B. Hövelbrinks (Hrsg.), Fachbezogene Sprachförderung in Deutsch als Zweitsprache. Theoretische Konzepte und empirische Befunde zum Erwerb bildungssprachlicher Kompetenzen, Beltz Juventa, Weinheim und Basel, S. 87-98.

B. Ahrenholz (2010a), Bildungssprache im Sachunterricht der Grundschule, in B. Ahrenholz (Hrsg.), Fachunterricht und Deutsch als Zweitsprache, Narr, Tübingen, S. 15-37.

B. Ahrenholz (Hrsg.) (2010b), Fachunterricht und Deutsch als Zweitsprache, Narr, Tübingen.

B. Ahrenholz (Hrsg.) (2006), Kinder mit Migrationshintergrund. Spracherwerb und Fördermöglichkeiten, Fillibach, Freiburg.

B. Ahrenholz, I. Oomen-Welke (Hrsg.) (2007), Deutsch als Zweitsprache. Handbuch Deutschunterricht in Theorie und Praxis (hg. v. Winfried Ulrich, Band VIII), Schneider-Hohengehren, Baltmannsweiler, S. 430-441.

G. Alfieri (2005), La lettura linguistica dei testi letterari nella scuola secondaria: apparato didattico di antologie ed edizioni scolastiche di classici, in C. Lavinio (Hrsg.), Educazione linguistica e educazione letteraria. Intersezioni e interazioni, Franco Angeli, Mailand, S. 394-421.

M.L. Altieri Biagi (2005), Creatività, parola ambigua, in C. Lavinio (Hrsg.), Educazione linguistica e educazione letteraria. Intersezioni e interazioni, Franco Angeli, Mailand, S. 67-85.

L. Altichieri, G.F. Colmelet, V. Deon (1997), Informazione e informatività nei manuali scientifici, in R. Calò, S. Ferreri (Hrsg.), Il testo fa scuola. Libri di testo, linguaggi ed educazione linguistica, La Nuova Italia, Florenz, S. 181-200.

W. Ament (1899), Die Entwicklung von Sprechen und Denken beim Kinde, Leipzig.

L. Amenta, F. Cappadonna (GISCEL Sizilien) (2014), Le competenze lessicali tra autovalutazione e uso: un 'indagine nelle scuole di Palermo, in A. Colombo, G. Pallotti (Hrsg.), L'italiano per capire. Quaderni del GISCEL, Aracne, Rom, S. 169-180.

M. Angvik, B. Von Borries (Hrsg.) (1997), Youth and History. A Comparative Survey on Historical Consciousness and Political Attitudes among Adolescents, Körber Stiftung, Hamburg. 
P. Arpai et al. (GiSCEL Piemont) (1997), Il difficile alfabeto del libro di scuola, in R. Calò, S. Ferreri (Hrsg.), Il testo fa scuola. Libri di testo, linguaggi ed educazione linguistica, La Nuova Italia, Florenz, S. 241-260.

G.I. Ascoli (1981), Relazione sul II ${ }^{\circ}$ tema proposto al IX Congresso Pedagogico Italiano, in M. Raichich (Hrsg.), Scuola, cultura e politica da De Sanctis a Gentile, Nistri-Lischi, Pisa, S. 425-431 (Erstausgabe 1874).

G.I. Ascoli (1975), Scritti sulla questione della lingua, C. Grassi (Hrsg.), Einaudi, Turin (Erstausgabe 1873).

L. Atzeni, L. Milia (GISCEL Sardinien) (2008), Il cammino delle parole. La costruzione del senso e del linguaggio nell'apprendimento delle scienze, in M. Barni, D. Troncarelli, C. Bagna (Hrsg.), Lessico e apprendimenti. Il ruolo del lessico nella linguistica educativa, F. Angeli, Mailand, S. 288294.

G. Augst (1984), Kinderwort: Der aktive Kinderwortschatz (kurz vor der Einschulung), P. Lang, Frankfurt a.M.

L.F. Bachman (1990), Fundamental Considerations in Language Testing, Oxford University Press, Oxford.

L.F. Bachman, A. S. Palmer (2010), Language Assessment in Practice: Developing Language Assessments and Justifying their Use in the Real World, Oxford University Press, Oxford.

L.F. Bachman, A.S. Palmer (1996), Language Testing in Practice: Designing and Developing Useful Language Tests, Oxford University Press, Oxford.

M. Bakhtin (1981), The dialogic imagination, University of Texas Press, Austin.

P.L. Baldi $\left(2008^{2}\right)$, Le parole della mente. Lessico mentale e processi linguistici, Franco Angeli, Mailand.

H. Balhorn, H. Brügelmann (Hrsg.) (1987), Welten der Schrift in der Erfahrung der Kinder, Faude, Konstanz.

E. Banfi (Hrsg.) (1993a), L'altra Europa linguistica. Varietà di apprendimento e interlingue nell'Europa contemporanea, La Nuova Italia, Scandicci (Florenz).

E. Banfi (1993b), Italiano come L2, in E. Banfi (Hrsg.), L'altra Europa linguistica. Varietà di apprendimento e interlingue nell'Europa contemporanea, La Nuova Italia, Scandicci (Florenz), S. 35-102.

P. Barki, S. Gorelli, S. Maccetti, M.P. Sergiacomo, B. Strambi (2003), Valutare e certificare l'italiano di stranieri. I livelli iniziali, Guerra Edizioni, Perugia.

M. Barni, D. Troncarelli, C. Bagna (2008) (Hrsg.), Lessico e apprendimenti. Il ruolo del lessico nella linguistica educativa, F. Angeli, Mailand.

M. Barni, A. Villarini (Hrsg.) (2001), La questione della lingua per gli immigrati stranieri. Insegnare, valutare e certificare l'italiano L2, F. Angeli, Mailand. 
M.D. Barrett (1999), Vocabulary in Language Acquisition, in B. Spolsky (Hrsg.), Concise Dictionary of Educational Linguistics, Elsevier Science Ltd., Oxford, S. 244-248.

U. Bartolini, C. Tagliavini, A. Zampolli (1971), Lessico di frequenza della lingua italiana contemporanea, IBM, Mailand.

C. Bascetta (1964), La lingua dei libri di testo, Armando Editore, Rom.

E. Bastianelli, S. Rancati, P. Maestri (2010), Zaino in spalla 3. Il mondo e $i$ paesi extraeuropei, Sansoni, Mailand.

E. Bates, J.C. Goodman (1997), On the inseparability of grammar and the lexicon: Evidence from acquisition, aphasia, and real-time processing, in Language and Cognitive Processes, 12, S. 507-584.

G. Battimelli (1997), Linguaggi scientifici e linguaggi di manuali: Il caso della Fisica, in R. Calò, S. Ferreri (Hrsg.), Il testo fa scuola. Libri di testo, linguaggi ed educazione linguistica, La Nuova Italia, Florenz, S. 169-179.

G. Battisti (1988), Didattica della geografia. Intervista a Gianfranco Battisti, M. Faccioli (Hrsg.), in Riforma della scuola, 3, S. 60-62.

R.S. Baur, A. Mashkovskaya, M. Spettmann (2009), Der C-Test als Instrument zur Ermittlung allgemeinsprachlicher und fachsprachlicher Fähigkeiten am Berufskolleg, in A. Berndt, K. Kleppin (Hrsg.), Sprachlehrforschung. Theorie und Empirie. Festschrift für Rüdiger Grotjahn, Peter Lang Frankfurt a.M., S. 23-38.

R.S. Baur, M. Spettmann (2007), Sprachstandsmessung und Sprachförderung mit dem C-Test, in B. Ahrenholz, I. Oomen-Welke (Hrsg.), Deutsch als Zweitsprache. Handbuch Deutschunterricht in Theorie und Praxis (hg. v. Winfried Ulrich, Band VIII), Schneider Hohengehren, Baltmannsweiler, S. 430-441.

M. Becker-Mrotzek (Hrsg.) (2009), Mündliche Kommunikation und Gesprächsdidaktik. Deutschunterricht in Theorie und Praxis (Handbuch zur Didaktik der deutschen Sprache und Literatur in elf Bänden, Bd. 3), Schneider Hohengehren, Baltmannsweiler.

M. Beese, C. Benholz (2013), Sprachförderung im Fachunterricht. Voraussetzungen, Konzepte und empirische Befunde, in Ch. Röhner, B. Hövelbrinks (Hrsg.), Fachbezogene Sprachförderung in Deutsch als Zweitsprache. Theoretische Konzepte und empirische Befunde zum Erwerb bildungssprachlicher Kompetenzen, Beltz Juventa, Weinheim, Basel, S. 37-56.

C. Bereiter, M. Scardamaglia (1995), La psicologia della composizione scritta, La Nuova Italia, Florenz.

K. Berendes, N. Dragon, S. Weinert, B. Heppt, P. Stanat (2013), Hürde Bildungssprache? Eine Annäherung an das Konzept „Bildungssprache“ unter Einbezug aktueller empirischer Forschungsergebnisse, in A. Redder, S. Weinert (Hrsg.), Sprachförderung und Sprachdiagnostik - Interdisziplinäre Perspektiven, Waxmann, Münster, New York, S. 17-41. 
A. Berndt, K. Kleppin (Hrsg.) (2009), Sprachlehrforschung. Theorie und Empirie. Festschrift für Rüdiger Grotjahn, Peter Lang Frankfurt a.M.

B. Bernstein (1990), The structuring of pedagogic discourse, Vol 4: Class, codes, and control, London, Routledge.

B. Bernstein (1971), Class, codes and control. Vol. 1: Theoretical studies towards a sociology of language, Routledge, London.

B. Bernstein (1964), Elaborated and Restricted Codes: Their Social Origins and Some Consequences, in American Anthropologist, 66 (6), Teil 2, S. 55-69.

B. Bernstein (2010), A public language: Some sociological implications of a linguistic form, in British Journal of Sociology, 61, S. 53-69 (Erstausgabe 1959, British Journal of Sociology, 10, S. 311-326).

M. Berretta (1990), Apprendimento di lingue seconde con input sub standard: l'analisi di un caso, in G. Berruto, A. Sobrero (Hrsg.), Studi di sociolinguistica e dialettologia italiana offerti a Corrado Grassi, Congedo, Galatina, S. 151-177.

G. Berruto (1993), La varietà del repertorio, in A. Sobrero (Hrsg.), Introduzione all'italiano contemporaneo. La variazione e gli usi, Laterza, RomBari, S. 3-36.

G. Berruto, A. Sobrero (Hrsg.) (1990), Studi di sociolinguistica e dialettologia italiana offerti a Corrado Grassi, Congedo, Galatina.

L. Bersezio (2005), I nuovi territori dell'uomo. 3B, Regioni e paesi extraeuropei, De Agostini; Novara.

D. Bertocchi (2003), La comprensione dei libri di testo nella classe plurilingue, in R. Grassi, A. Valentini, R. Bozzone Costa (Hrsg.), L'italiano per lo studio nella scuola plurilingue: tra semplificazione e facilitazione. Atti del Convegno-Seminario "Alunni stranieri nella scuola: l'italiano per lo studio”, Guerra Edizioni, Perugia, S. 21-31.

D. Bertocchi (2009), Non solo italiano: ipotesi per un'educazione linguistica integrata verso l'Europa, in G. Favaro, N. Papa (Hrsg.), Non uno di meno. Le ragazze e i ragazzi stranieri nella scuola superiore, F. Angeli, Mailand, S. 183-193.

R. Bertozzi (2014), Lo scenario nazionale della presenza di alunni con cittadinanza non italiana, in M. Colombo, V. Ongini (Hrsg.), Alunni con cittadinanza non italiana. L'eterogeneità dei percorsi scolastici. Rapporto nazionale 2012/2013 (Quaderni ISMU 1/2014), Fondazione ISMU, Mailand, S. 12-44.

B.J. Biddle, T.L. Good, I.F. Goodson (Hrsg.) (1997), Handbook of Teachers and Teaching, Vol. 2, Kluwer Academic Publishers, Dordrecht.

C. Boisio (2003), L'italiano per lo studio: la lingua, "filtro" del contenuto? Una proposta didattica, in R. Grassi, A. Valentini, R. Bozzone Costa 
(Hrsg.), L'italiano per lo studio nella scuola plurilingue: tra semplificazione e facilitazione. Atti del Convegno-Seminario "Alunni stranieri nella scuola: l'italiano per lo studio", Guerra Edizioni, Perugia, S. 137-155.

G. Bolondi, L. Brachetti, F. Ferretti (in Druck), Correlazioni tra componenti della competenza linguistica e capacità di lavoro su un testo matematico: gli studenti del Liceo Scientifico alle prese con le prove dell'Esame di Stato, Beitrag zur GISCEL-Tagung 2014 Educazione linguistica e apprendimento/insegnamento delle discipline matematico-scientifiche.

G.D. Borich $\left(2007^{4}\right)$, Observation skills for effective teaching, Merrill Prentice Hall, Upper Saddle River, NJ.

B. von Borries (2012), Erwartungen an, Erfahrungen mit und Wirkungen von Geschichtsschulbüchern - empirische Befunde, in J. Doll et al. (Hrsg.), Schulbücher im Fokus. Nutzungen, Wirkungen und Evaluation, Waxmann, Münster, New York, S. 43-65.

L. Bosi, A. Busi, A. Candiotto, A. Chiantera, A. Colombo, F. Forni, S. Loiero, L. Manzoni, M.P. Miglio, G. Padoan, M.E. Sogos, D. Vignola (GISCEL Emilia Romagna) (2014), La comprensione delle relazioni causali nella scuola di base, in A. Colombo, G. Pallotti (Hrsg.), L'italiano per capire. Quaderni del GISCEL, Aracne, Rom, S. 93-107.

C. Bosisio (2003), L'italiano per lo studio: la lingua, "filtro" del contenuto? Una proposta didattica, in R. Grassi, A. Valentini, R. Bozzone Costa (Hrsg.), L'italiano per lo studio nella scuola plurilingue: tra semplificazione e facilitazione. Atti del Convegno-Seminario "Alunni stranieri nella scuola: l'italiano per lo studio", Guerra Edizioni, Perugia, S. 137-160.

R. Bozzone Costa (2003), Come lavorare sulle caratteristiche linguistiche dei testi disciplinari, in R. Grassi, A. Valentini, R. Bozzone Costa (Hrsg.), L'italiano per lo studio nella scuola plurilingue: tra semplificazione e facilitazione. Atti del Convegno-Seminario "Alunni stranieri nella scuola: l'italiano per lo studio", Guerra Edizioni, Perugia, S. 113-135.

L. Brasca, M. Fransoni, M. G. Gozio, O. Mandelli, S. Pozzi, L. Preti \& G. Ravizza (GISCEL Lombardei) (2014), Strategie di lettura per comprendere un testo: dalla superficie alla profondità dei significati, in A. Colombo, G. Pallotti (Hrsg.), L'italiano per capire. Quaderni del GISCEL, Aracne, Rom, S. 77-92.

M. Brenzinger (1997), Language contact and language displacement, in F. Coulmas (Hrsg.), The handbook of sociolinguistics, Blackwell, Malden, MA, S. 273-285.

M.E. Brisk (2005), Bilingual Education, in E. Hinkel (Hrsg.), Handbook of Research in Second Language Learning and Research, Lawrence Erlbaum Associates, Publishers, Mahwaw, New Jersey, S. 7-24.

L.A. Brown (1994), The advancement of learning, in Educational Researcher, $23(8)$, S. 4-12. 
H. Brügelmann, H. Balhorn, I. Füssenich (Hrsg.) (1995), Am Rande der Schrift. Zwischen Sprachenvielfalt und Analphabetismus, DGLS-Jahrbuch Bd. 6, Libelle Verlag, Lengwil, S. 250-262.

J.S. Bruner (1970), Der Prozess der Erziehung, Berlin Verlag, Berlin 1970.

P.E. Bryant (1999), Literacy: Phonological Awareness, in B. Spolsky (Hrsg.), Concise Dictionary of Educational Linguistics, Elsevier Science Ltd., Oxford, S. 169-171.

K. Bühler (1982), Sprachtheorie. Die Darstellungsfunktion der Sprache, Fischer, Stuttgart, New York (Uni-Taschenbücher 1159) (Erstausgabe 1934).

BMBF (Hrsg.) (2008), Referenzrahmen zur altersspezifischen Sprachaneignung - Forschungsgrundlagen, Bd. 29/II, Referat Bildungsforschung, Bonn, Berlin

BMBF (Hrsg.) (2007), Anforderungen an Verfahren der regelmäßigen Sprachstandsfeststellung als Grundlage für die frühe und individuelle Förderung von Kindern mit und ohne Migrationshintergrund, Bildungsforschung Bd. 11, Referat Öffentlichkeitsarbeit, Bonn, Berlin.

BMBF (Hrsg.) (2005), Migrationshintergrund von Kindern und Jugendlichen. Wege zur Weiterentwicklung der amtlichen Statistik, BMBF Referat Publ. Internetred (Deutschland. Das von morgen.14), Berlin, Bonn.

F.A. Butler, C. Lord, R. Stevens, M. Burrego, A.L. Bailey (2004), An approach to operationalizing academic language for language test purposes: Evidence from fifth-grade science and math (CSE Report 626), University of California, Los Angeles CA.

A. Cacchione (2014), Tra scritto e parlato: I pericoli dell'interferenza, in M.E. Piemontese, P. Sposetti (Hrsg.), La scrittura dalla scuola superiore all'università, Carocci, Rom, S. 65-82.

D. Cacia (2014), Una lingua per la storia: confronto diacronico sull'italiano dei manuali di storia per la scuola primaria, in A. Colombo, G. Pallotti (Hrsg.), L'italiano per capire. I quaderni del GISCEL 1, Aracne, Rom, S. 311-322.

R. Calò, S. Ferreri (Hrsg.) (1997), Il testo fa scuola. Libri di testo, linguaggi ed educazione linguistica, La Nuova Italia, Florenz.

M. Carazzi, L. Pizzetti (2010), I continenti e gli stati del mondo. Manuale e Dossier 3, Edizioni Scolastiche Bruno Mondadori, Mailand, Turin.

Carnegie Council on Advancing Adolescent Literacy (2009/2010), Time to act: An agenda for advancing adolescent literacy for college and career success, unter https://www.carnegie.org/publications/time-to-act-anagenda-for-advancing-adolescent-literacy-for-college-and-career-success/ (Stand: 15.12.2016).

W. Carr, S. Kemmis (1985), Becoming critical: Knowing through Action Research, Deakin University Press, Victoria. 
W. Castellino, S.C. Sgroi (2005), Educazione letteraria e programmi scolastici, C. Lavinio (Hrsg.), Educazione linguistica e educazione letteraria. Intersezioni e interazioni, Franco Angeli, Mailand, S. 359-374.

L. Cavaliere (1993), Leggibilità e comprensione di sussidiari per le scuole elementari, Magisterarbeit in der Sprachphilosophie, Facoltà di Lettere e filosofia, Università degli studi di Roma "La Sapienza", Rom.

C.B. Cazden (1999), Socialization, in B. Spolsky (Hrsg.), Concise Encyclopaedia of Educational Linguistics, Elsevier Science Ltd., Oxford, S. 6365.

Cede (1999), Richerche internazionali, im Internet unter www.cede.it/ricerche-internazionali (Stand: 10.11.2014).

Centro Studi e Ricerche IDOS (Hrsg.) (2014), Dossier Statistico Immigrazione 2014. Rapporto UNAR. Dalle discriminazioni ai diritti; http://www.dossierimmigrazione.it/docnews/file/2014_Scheda\%20breve\%20Dossier.pdf (Stand: 12.01.2016).

V. Cesareo (2014), Introduzione, in M. Colombo, V. Ongini (Hrsg.), Alunni con cittadinanza non italiana. L'eterogeneità dei percorsi scolastici. Rapporto nazionale 2012/2013, Quaderni ISMU 1/2014, Fondazione ISMU, Mailand, S. 7-11.

A. Chiantera (2005), Teoria e didattica della punteggiatura moderna, in C. Lavinio (Hrsg.), Educazione linguistica e educazione letteraria. Intersezioni e interazioni, Franco Angeli, Mailand, S. 200-207.

M. Chini (Hrsg.) (2009), Plurilinguismo e immigrazione nella società italiana. Repertori, usi linguistici e fenomeni di contatto. Numero monografico di "Studi italiani di linguistica teorica e applicata", XXXVIII/1, S. 3-8.

M. Chini (2005), Che cos'è la linguistica acquisizionale, Carocci, Rom.

M. Chini (2000), Interlingua: modelli e processi di apprendimento, in A. De Marco (Hrsg.), Manuale di glottodidattica. Insegnare una lingua straniera, Carocci, Rom, S. 45-69.

C. Chlosta, T. Ostermann (2005), Warum fragt man nach der Herkunft, wenn man die Sprache meint? Ein Plädoyer für eine Aufnahme sprachbezogener Fragen in demographische Untersuchungen, in BMBF (Hrsg.), Migrationshintergrund von Kindern und Jugendlichen. Wege zur Weiterentwicklung der amtlichen Statistik, Referat Publ. Internetred (Deutschland. Das von morgen. 14), Berlin, Bonn, S. 55-65.

F. Christie (2012), Language Education throughout the School Years: A Functional Perspective, in Language Learning Monograph Series, 62, Suppl. 1.

F. Christie (Hrsg.) (1990), Literacy for a changing world, Australian Council for Educational Research, Hawthorn.

H. Clahsen (1982), Spracherwerb in der Kindheit: Eine Untersuchung zur Entwicklung der Syntax bei Kleinkindern, G. Narr, Tübingen. 
H. Clahsen (1988), Normale und gestörte Kindersprache. Linguistische Untersuchungen zu Syntax und Morphologie, J. Benjamins, Amsterdam.

H. Clahsen, M. Rothweiler, A Woest (1990), Lexikalische Ebenen und morphologische Entwicklung: Eine Untersuchung zum Erwerb des deutschen Pluralsystems im Rahmen der Lexikalischen Morphologie, in M. Rothweiler (Hrsg.), Spracherwerb und Grammatik. Linguistische Untersuchungen zum Erwerb von Syntax und Morphologie, (= LB-Sonderheft 3), Westdeutscher Verlag Opladen, S. 105-126.

N. Cloud, E. Genesee, E. Hamayan. (2000). Dual language instruction: A handbook for enriched education, Heinle \& Heinle, Boston.

E. Coelho (1998), Teaching and learning in multicultural schools, Multilingual Matters, Clevedon.

A. Collins, J.S. Brown, S.E. Newman (2005), L'apprendistato cognitivo, per insegnare a leggere, scrivere e a far di conto, in C. Pontecorvo, A.M. Aiello, C. Zucchermaglio (Hrsg.), I contesti sociali dell'apprendimento. Acquisire conoscenze a scuola, nel lavoro, nella vita quotidiana, Ambrosiana, Mailand, S. 181-231.

G.F. Colmelet, D. Lovision et al. (GISCEL Venetien) (2008), Piazza, bella piazza... Lessico e rappresentazioni mentali, in M. Barni, D. Troncarelli, C. Bagna (Hrsg.), Lessico e apprendimenti. Il ruolo del lessico nella linguistica educativa, F. Angeli, Mailand, S. 317-327.

A. Colombo, G. Pallotti (Hrsg.) (2014), L'italiano per capire. Quaderni del GISCEL, Aracne, Rom.

M. Colombo (2014), Gli esiti scolastici degli alunni con cittadinanza non italiana, in M. Colombo, V. Ongini (Hrsg.), Alunni con cittadinanza non italiana. L'eterogeneità dei percorsi scolastici. Rapporto nazionale 2012/2013, Quaderni ISMU 1/2014, Fondazione ISMU, Mailand, S. 6990.

M. Colombo, V. Ongini (Hrsg.) (2014), Alunni con cittadinanza non italiana. L'eterogeneità dei percorsi scolastici. Rapporto nazionale 2012/2013, Quaderni ISMU 1/2014, Fondazione ISMU, Mailand.

M. Compagni, M. Lunardon, F. Mastella, V. Sofia (Sektion Giscel Venetien, Gruppe Verona) (1997), Insospettati luoghi di fraintendimento lessicale, in R. Calò, S. Ferreri (Hrsg.), Il testo fa scuola. Libri di testo, linguaggi ed educazione linguistica, La Nuova Italia, Florenz, S. 261-293.

B. Cope, M. Kalantzis (Hrsg.) (1999), Multiliteracies. Literacy learning and the design of social futures, Routledge, London.

A. Corda, C. Marello (2004), Lessico. Insegnarlo e impararlo, Guerra Edizioni, Perugia.

D. Corno (1991), Il ragionar testuale: il testo come risultato del processo di comprensione, in P. Desideri (Hrsg.), La centralità del testo nelle pratiche 
didattiche. Quaderni del GISCEL/10, La Nuova Italia, Scandicci (Florenz), S. 45-67.

D.J. Corson (1999), Language Across the Curriculum, in B. Spolsky (Hrsg.), Concise Dictionary of Educational Linguistics, Elsevier Science Ltd., Oxford, S. 323-325.

D.J. Corson (1997), Encyclopaedia of Language and Education, Kluwer Academic Publishers, Dordrecht.

F. Coulmas (Hrsg.) (1997), The handbook of sociolinguistics, Blackwell, Malden, MA.

J. Cummins (2013), Current research on language transfer. Implications for language teaching policy and practice, in P. Siemund et al. (Hrsg.), Multilingualism and Language Diversity in Urban Areas. Acquisition, identities, space, education, John Benjamins, Amsterdam, Philadelphia, S. 289304.

J. Cummins (2002), Foreword, in P. Gibbons (2002), Scaffolding language, scaffolding learning. Teaching Second Language Learners in the Mainstream Classroom, Heinemann, Portsmouth, S. V-VIII.

J. Cummins (1981), Bilingualism and Minority Language Children, OISE Press Edition, Toronto.

J. Cummins (1980) The construct of language proficiency in bilingual education, in J.E. Alatis (Hrsg.), Current Issues in Bilingual Education, Georgetown University Press, Washington, D.C., S. 81-103.

J. Cummins (1979a) Cognitive/academic language proficiency, linguistic interdependence, the optimum age question and some other matters, in Working Papers on Bilingualism, 19, S. 121-129.

J. Cummins (1979b), Linguistic Interdependence and the Educational Development of Bilingual Children, in Review of Educational Research, 49 (2), S. 222-251.

P. Czerniewska (1999), Writing: Process Approach, in B. Spolsky (Hrsg.), Concise Dictionary of Educational Linguistics, Elsevier Science Ltd., Oxford, S.475-477.

H.D. Dann, T. Diegritz, H.S. Rosenbusch (2002), Gruppenunterricht im Schulalltag. Ergebnisse eines Forschungsprojektes und praktische Konsequenzen, in Pädagogik, 54 (1), S. 11-15.

T. De Mauro (2012), Linguistica educativa: ragioni e prospettive, in S. Ferreri (Hrsg.), Linguistica educativa: atti del XLIV Congresso internazionale di studi della Società di linguistica italiana (SLI), Viterbo, 27-29.09.2010, Bulzoni, Rom, S. 3-22.

T. De Mauro (2010²), La cultura degli italiani, F. Erbani (Hrsg.), Laterza, Rom, Bari (Erstausgabe 2004). 
T. De Mauro (2003), Nuove parole Italiane dell'Uso, VII (Ergänzung zu De Mauro 1999/2000).

T. De Mauro (1999/2000), GRADIT - Grande dizionario italiano dell'uso, UTET, Turin.

T. De Mauro (1998a), Linguistica elementare, Laterza, Bari.

T. De Mauro (1998b), Passato e futuro dell'educazione linguistica. Intervista a Tullio De Mauro di Silvana Ferreri, in S. Ferreri, A.S. Guerriero (Hrsg.), Educazione linguistica vent'anni dopo e oltre. Che cosa ne pensano De Mauro, Renzi, Simone, Sobrero, La Nuova Italia, Scandicci (Florenz), S. 13-35.

T. De Mauro (1997), Se una notte d'inverno un editore..., in R. Calò, S. Ferreri (Hrsg.), Il testo fa scuola. Libri di testo, linguaggi ed educazione linguistica, La Nuova Italia, Scandicci (Florenz), S. 25-32.

T. De Mauro (1995a), Idee per il governo. La scuola, Saggi tascabili Laterza, Bari.

T. De Mauro (1995b), Capire le parole, Laterza, Rom, Bari.

T. De Mauro (19912), Guida all'uso delle parole, Editori Riuniti, Rom (2. Auflage, Erstausgabe 1980).

T. De Mauro (1986), Ai margini del linguaggio, Editori Riuniti, Rom.

T. De Mauro (1985), Appunti e spunti in tema di (in)comprensione, in Linguaggi, 3, S. 22-32.

T. De Mauro (1980), Guida all'uso delle parole, Editori Riuniti, Rom.

T. De Mauro (1979), Scuola e linguaggio, Editori Riuniti, Rom.

T. De Mauro (1979²), Storia linguistica dell'Italia unita, Laterza, Bari (Erstausgabe 1963).

T. De Mauro (1976a), Ipotesi e problemi per la costruzione di una educazione linguistica democratica che utilizzi i prodotti e $i$ valori della tradizione orale, in Scuola e città, XXVII, S. 341-346 (zit. Ausgabe: De Mauro 1979, S. 224-240).

T. De Mauro (1976b), Linguaggio, scuola e stratificazione sociale, in Unità sindacale, 3, S. 1-6 (zit. Ausgabe: De Mauro 1979, S. 75-87).

T. De Mauro (1975a), Il plurilinguismo nella scuola e nella società italiana, in La radio nella scuola oggi. Linguaggio, ascolto, utilizzazione, ERI, Turin, 1975, S. 38-54 (zit. Ausgabe: De Mauro 1979, S. 124-137).

T. De Mauro (1975b), La tutela del patrimonio e dei diritti linguistici delle popolazioni italiane, in Città e regione, II, S. 131-144 (zit. Ausgabe: De Mauro 1979, S. 204-218).

T. De Mauro (1975c), Le scienze linguistiche nella scuola media superiore: una proposta, in Pratica educativa, Beiheft zu Riforma della Scuola, XXI, 5, S. 2-4 (zit. Ausgabe: De Mauro 1979, S. 179-188). 
T. De Mauro (1975d), Dieci tesi per l'educazione linguistica democratica, in Cidi, Atti del convegno sugli organi collegiali, Rom, 14.-16. März 1975 (Text anschließend mit R. Simone überarbeitet und dem GISCEL in der Versammlung des 26. Aprils 1975 überlassen, abgedruckt in Scuola e città XXVI (1976), S. 433-436; zit. Ausgabe: De Mauro 1979, S. 138-152).

T. De Mauro (1970), Per lo studio dell'italiano popolare unitario, in A. Rossi (Hrsg.), Lettere di una tarantata, De Donati, Bari, S. 43-75.

T. De Mauro (1963), Storia linguistica dell'Italia unita, Laterza, Bari.

T. De Mauro, I. Chiari (Hrsg.), Parole e numeri. Analisi quantitative dei fatti di lingua, Aracne, Rom.

T. De Mauro, E. Piemontese, M. Vedovelli (Hrsg.) (1986), Leggibilità e comprensione, in Linguaggi, III/3, S. 28-38.

C. De Santis, F. Gatta (2013), Notizie dalla scuola. Le competenze grammaticali e testuali degli studenti madrelingua all 'uscita dalla scuola secondaria. I risultati di un 'indagine, in Studi di grammatica italiana, XXXIXXXII, S. 411-441.

V. Deon (1997), I manuali di storia fra divulgazione, parafrasi e storia generale, in R. Calò, S. Ferreri (Hrsg.), Il testo fa scuola. Libri di testo, linguaggi ed educazione linguistica, La Nuova Italia, Florenz, S. 41-60.

DESI-Konsortium (Hrsg.) (2008), Unterricht und Kompetenzerwerb in Deutsch und Englisch. Ergebnisse der DESI-Studie, Beltz, Weinheim.

F. De Renzo (2011), Lessico di base e indice di leggibilità per l'analisi e la produzione di testi per la didattica dell'italiano L2, in C. Diadori, P. Gennai, S. Semplici (Hrsg.), Progettazione editoriale per l'italiano L2, Guerra edizioni, Perugia, S. 183-202.

F. De Renzo (2005), Nuove considerazioni sul vocabolario di base e di alta disponibilità, in T. De Mauro, I. Chiari (Hrsg.), Parole e numeri. Analisi quantitative dei fatti di lingua, Aracne, Rom, S. 215-232.

F. De Renzo (GISCEL Kalabrien) (2008), La conoscenza del vocabolario comune negli studenti dalle elementari alla maturità, in M. Barni, D. Troncarelli, C. Bagna (Hrsg.), Lessico e apprendimenti. Il ruolo del lessico nella linguistica educativa, F. Angeli, Mailand, S. 187-201.

P. Desideri (Hrsg.) (1991), La centralità del testo nelle pratiche didattiche. Quaderni del GISCEL/10, La Nuova Italia, Scandicci (Florenz).

C. De Santis, F. Gatta (2013), Notizie dalla scuola. Le competenze grammaticali e testuali degli studenti madrelingua all'uscita dalla scuola secondaria. Risultati di un'indagine, in Studi di grammatica italiana (Accademia della Crusca), Vol. XXXI-XXXII, S. 411-441.

C. Diadori, P. Gennai, S. Semplici (Hrsg.) (2011), Progettazione editoriale per l'italiano L2, Guerra edizioni, Perugia, S. 183-202. 
M. Dinucci, F. Dinucci (2008), Geograficamente 3. Noi cittadini del mondo, Zanichelli, Bologna.

M. Döbert, P. Hubertus (Hrsg.) (2000), Ihr Kreuz ist die Schrift. Analphabetismus und Alphabetisierung in Deutschland, Bundesverband Alphabetisierung e.V., Münster und Ernst Klett Verlag, Stuttgart.

J. Doll, K. Frank, D. Fickermann, K. Schwippert (Hrsg.) (2012), Schulbücher im Fokus. Nutzungen, Wirkungen und Evaluation, Waxmann, Münster, New York.

M. Döll, İ. Dirim (2011), Mehrsprachigkeit in der Sprachdiagnostik, in S. Fürstenau, M. Gomolla (Hrsg.), Migration und schulischer Wandel: Mehrsprachigkeit, VS Verlag, Wiesbaden, S. 153-167.

Duden (1999), Das große Wörterbuch der deutschen Sprache in zehn Bänden. (3., völlig bearbeitete und erweiterte Auflage), Dudenverlag, Mannheim, Leipzig, Wien u.a.

P.A. Duff (2005), ESL in Secondary Schools: Programs, Problematics and Possibilities, in E. Hinkel (Hrsg.), Handbook of Research in Second Language Learning and Research, Lawrence Erlbaum Associates, Publishers, Mahwaw, New Jersey, S. 45-63.

C. S. Dweck (2006), Mindset. The New Psychology of Success, Random House, New York.

A. Eckhardt (2008), Sprache als Barriere für den schulischen Erfolg. Potentielle Schwierigkeiten beim Erwerb schulbezogener Sprache für Kinder mit Migrationshintergrund, Waxmann, Münster, New York.

D. Edwards, J. Potter (1992), Discursive Psychology, Sage, Newbury Park, CA.

J.R. Edwards (1999), Educational Failure, in B. Spolsky (Hrsg.), Concise Encyclopaedia of Educational Linguistics, Elsevier Science Ltd., Oxford, S. 192-200.

K. Ehlich (2009), Sprachaneignung - Was man weiß, und was man wissen müsste, in D. Lengyel, H.H. Reich, H.-J. Roth, M. Döll (Hrsg.), Von der Sprachdiagnose zur Sprachförderung, FörMig Edition Band 5, Waxmann, Münster, New York, S. 15-24.

K. Ehlich (2007), Sprachaneignung und deren Feststellung bei Kindern mit und ohne Migrationshintergrund: Was man wei $\beta$, was man braucht, was man erwarten kann, in BMBF (Hrsg.), Anforderungen an Verfahren der regelmäßigen Sprachstandsfeststellung als Grundlage für die frühe und individuelle Förderung von Kindern mit und ohne Migrationshintergrund, Bildungsforschung Bd. 11, Referat Öffentlichkeitsarbeit, Bonn, Berlin, S. 11-77.

K. Ehlich (1999), Alltägliche Wissenschaftssprache, in Info DaF, 26/1, S. 324. 
K. Ehlich, J. Rehbein (Hrsg.) (1983), Kommunikation in Schule und Hochschule, G. Narr, Tübingen.

F. Erbani (Hrsg.) (2010²), La cultura degli italiani, Laterza, Rom, Bari (Erstausgabe 2004).

M. Faccioli (1988a), A scuola di geografia. Prima parte, in Riforma della Scuola, 1, S. 55-58.

M. Faccioli (1988b), A scuola di geografia. Seconda parte, in Riforma della Scuola, 3, S. 55-58.

N. Fairclough (1992), Discourse and social change, Polity Press, Cambridge.

R. Fasold (1984), The sociolinguistics of society, Blackwell, Oxford.

G. Favaro (2015), La lingua per riuscire. L'apprendimento dell'italiano dello studio nella classe eterogenea, Tagungsbeitrag zu "Insegnare e studiare in italiano", Bozen, 15.01.2015; http://www.bildung.suedtirol.it/download_file/view/3192/902/ (Stand: 15.12.2016).

G. Favaro (2010a), Una lingua "seconda e adottiva". L'italiano delle seconde generazioni, in Italiano LinguaDue, 1, 2010.

G. Favaro (2010b), Le ragazze e i ragazzi delle "terre di mezzo"; http://srvapl.istruzione.it/dgstudente/intercultura/allegati/rel_favaro_padova.pdf (Stand: 16.12.2016).

G. Favaro (2003), L'italiano per lo studio: i bisogni degli apprendenti, le risorse e $i$ modelli organizzativi della scuola, in R. Grassi, A. Valentini, R. Bozzone Costa (Hrsg.), L'italiano per lo studio nella scuola plurilingue: tra semplificazione e facilitazione. Atti del Convegno-Seminario "Alunni stranieri nella scuola: l'italiano per lo studio", Guerra Edizioni, Perugia, S. 13-20.

G. Favaro (2001), Bambini e ragazzi non italofoni a scuola: modelli organizzativi e di accoglienza, in F. Gattullo, P. Leone (Hrsg.), Italiano lingua seconda, in Lend lingua e nuova didattica (Monographie), 3, 2001, S. 5166.

G. Favaro (Hrsg.) (1999), Imparare l'italiano, imparare in italiano, Guerini e associati, Mailand.

G. Favaro, N. Papa (Hrsg.) (2009), Non uno di meno. Le ragazze e i ragazzi stranieri nella scuola superiore, F. Angeli, Mailand.

A. Felici, F. Giarè, A. Villarini (1994), Spazio linguistico, rapporto italiano/dialetto e attività metalinguistica nell'apprendimento spontaneo dell'italiano L2, in A. Giacolone Ramat, M. Vedovelli (Hrsg.), Italiano. Lingua seconda/lingua straniera. Atti del XXVI congresso della Società di Linguistica Italiana, Bulzoni, Rom, S. 479-518.

S. Ferreri (Hrsg.) (2012), Linguistica educativa: atti del XLIV Congresso internazionale di studi della Società di linguistica italiana (SLI), Viterbo, 27.-29.09.2010, Bulzoni, Rom. 
S. Ferreri (2006), Alfabeti e analfabeti, in La vita scolastica, LX, 12, S. 13-18. S. Ferreri (2005), L'alfabetizzazione lessicale. Studi di linguistica educativa, Aracne, Rom.

S. Ferreri (Hrsg.) (2002), Non uno di meno, La Nuova Italia, Florenz.

S. Ferreri (1998), Per una introduzione: spunti in tema di educazione linguistica, in S. Ferreri, A. S. Guerriero (Hrsg.), Educazione linguistica vent'anni dopo e oltre. Che cosa ne pensano De Mauro, Renzi, Simone, Sobrero, La Nuova Italia, Scandicci, Florenz, S.1-9.

S. Ferreri, A. S. Guerriero (Hrsg.) (1998), Educazione linguistica vent'anni dopo e oltre. Che cosa ne pensano De Mauro, Renzi, Simone, Sobrero, La Nuova Italia, Scandicci, Florenz.

M. Fibbi, M. Vedovelli (1988), Problemi sociolinguistici dell'immigrazione straniera a Rom, in A. Giacolone Ramat (Hrsg.), L'italiano tra le altre lingue: strategie di acquisizione, il Mulino, Bologna, S. 21-39.

R. Fiehler (2009), Mündliche Kommunikation, in. M. Becker-Mrotzek (Hrsg.), Mündliche Kommunikation und Gesprächsdidaktik. Deutschunterricht in Theorie und Praxis. (Handbuch zur Didaktik der deutschen Sprache und Literatur in elf Bänden, Bd. 3), Schneider Hohengehren, Baltmannsweiler, S. 25-51.

T. Fiorucci (1982), Si raccomanda un periodare breve: leggibilità dei libri di base secondo l'indice di Flesch, in EDA, 3, II, S. 39-55.

N.A. Flanders (1970), Analyzing Teaching Behavior, Addison-Wesley, Reading, MA.

R. Flesch (1948), A new readability yardstick, in Journal for Applied Psychology, 32 (3), S. 221-233.

L. Flower, J. R. Hayes (1980), A cognitive process theory of writing, in College Composition and Communication, 31, S. 365-381.

M. Foucault (1980), Power/Knowledge: Selected interviews and other writings 1972-1977, Harvester Press, Brighton.

G. Forte, T. Ubertazzi (2008), Nuovo Geolibro 3, De Agostini, Novara.

M. Fortino, C. Stancati, F. Vercillo (1997), Il linguaggio e l'argomentazione filosofica nel liceo, in R. Calò, S. Ferreri (Hrsg.), Il testo fa scuola. Libri di testo, linguaggi ed educazione linguistica, La Nuova Italia, Florenz, S. 201-215.

N. Francis (2000), The shared conceptual system and language processing in bilingual children: Findings from literacy assessment in Spanish and $\mathrm{Na}$ huatl, in Applied Linguistics, 21 (2), S. 170-204.

D. Fraser, R. Openshaw (Hrsg.) (2003), Informing our practice. Special volume, Kanuka Grove Press, Palmerston North.

I. Fratter, E. Jafrancesco (2003), Selezione, facilitazione, e comprensione dei testi scritti e parlati delle discipline di studio in ambito scolastico, in 
R. Grassi, A. Valentini, R. Bozzone Costa (Hrsg.), L'italiano per lo studio nella scuola plurilingue: tra semplificazione e facilitazione. Atti del Convegno-Seminario "Alunni stranieri nella scuola: l'italiano per lo studio”, Guerra Edizioni, Perugia, S. 39-57.

H. Fröhler (2008), Texte verfassen - Texte beurteilen. Eine zeitgemäße Didaktik des Schreibens für die Schulstufen 2 bis 9, HF Verlag, Wien.

S. Fürstenau, M. Gomolla (Hrsg.) (2011), Migration und schulischer Wandel: Mehrsprachigkeit, VS Verlag, Wiesbaden, S. 153-167.

D. Galbraith (1999), Writing as a Knowledge-Constituting Process, in M. Torrance, D. Galbraith (Hrsg.), Studies in Writing, vol. IV. Knowing What to Write. Conceptual Processes in Text Production, Amsterdam University Press, Amsterdam, S. 137-157.

P. Gallin, U. Ruf (1990), Sprache und Mathematik in der Schule. Auf eigenen Wegen zur Fachkompetenz, Kallmeyer, Seelze.

V. Gallina (Hrsg.) (2006), Letteratismo e abilità per la vita, Indagine nazionale sulla popolazione italiana 16-65 anni, Armando Editore, Rom.

Ch. Gantefort (2013), Schriftliches Erzählen mehrsprachiger Kinder. Entwicklung und sprachenübergreifende Fähigkeiten (Interkulturelle Bildungsforschung, Vol. 21), Waxmann, Münster, New York.

Ch. Gantefort, H.-J. Roth, (2010), Sprachdiagnostische Grundlagen für die Förderung bildungssprachlicher Fähigkeiten, in Erziehungswissenschaft, Vol. 13, S. 573-591.

H. Gardner (1993), Educare al comprendere, Feltrinelli, Mailand (Originalausgabe: The unschooled mind. How children think and schools should teach, Basic Books, New York, 1991).

S. Gass, C. Madden (Hrsg.) (1985), Input in Second Language Acquisition, Newbury House, New York.

F. Gattullo, P. Leone (2001a), Insegnare italiano L2 in Italia. Seminario Nazionale LEND Bologna, 15 aprile 2000, in F. Gattullo, P. Leone (Hrsg.), Italiano lingua seconda, in Lend lingua e nuova didattica (Monographie), 3, S. 4-5.

F. Gattullo, P. Leone (Hrsg.) (2001b), Italiano lingua seconda, in Lend lingua e nuova didattica (Monographie), 3.

M. Geat (Hrsg.) (2010), Identità e valori europei. Il sentimento europeo attraverso la narrazione, Aracne, Rom.

J.P. Gee $\left(1996^{2}\right)$, Social linguistics and Literacies: Ideologies in discourse, (Erstausgabe 1990, Bristol), Tayler \& Francis, London.

M. Gelmini (2009), Ministerialdekret 37/2009; http://hubmiur.pubblica.istruzione.it/alfresco/d/d/workspace/SpacesStore/670cf38b-4c3c-4003-af8b0c9e17ee5d4d/dm37 09.pdf (Stand: 03.12.2016). 
S. Gensini (2010), La comunicazione interculturale nella società e nella scuola. Appunti su criticità e prospettive, in M. Geat (Hrsg.), Identità e valori europei. Il sentimento europeo attraverso la narrazione, Aracne, Rom, S. 151-172.

S. Gensini (2005), Breve storia dell'educazione linguistica dall'Unità a oggi, Carocci, Rom.

S. Gensini (1991), Il problema semiotico del testo fra ricerca e didattica, in P. Desideri (Hrsg.), La centralità del testo nelle pratiche didattiche. Quaderni del GISCEL/10, La Nuova Italia, Scandicci (Florenz), S. 69-91.

S. Gensini, M. Vedovelli (1981), Lingua, linguaggi e società: proposta per un aggiornamento, L. Manzuoli, Florenz.

A. Giacolone Ramat (Hrsg.) (1988), L'italiano tra le altre lingue: strategie di acquisizione, il Mulino, Bologna.

A. Giacolone Ramat, M. Chini (Hrsg.) (1998), Strutture testuali e principi di organizzazione dell'informazione nell'apprendimento linguistico, in Studi di linguistica teorica e applicata, XXVII, 1.

L. Giannelli (1978), Ortografia e sistema fonologico: proposte per l'insegnamento della scrittura, Rivista italiana di dialettologia, 2, S. 82-101.

P. Gibbons (2003), Mediating language learning: Teacher interactions with ESL students in a content-based classroom, in TESOL Quarterly, 37 (2), S. 247-273.

P. Gibbons (2002), Scaffolding language, scaffolding learning. Teaching Second Language Learners in the Mainstream Classroom, Heinemann, Portsmouth.

H.W. Giese (1987), Warum wird der Analphabetismus gerade heute zu einem Problem?, in H. Balhorn, H. Brügelmann (Hrsg.), Welten der Schrift in der Erfahrung der Kinder, Faude, Konstanz, S. 260-266.

GISCEL (2004), Idee per un curricolo linguistico democratico; http://www.giscel.it/sites/default/files/documenti/16 Idee\%20per\%20un\%20curricolo.pdf (Stand: 18.12.2016).

Giscel (1975), Dieci tesi per un'educazione linguistica democratica; http://www.giscel.it/?q=content/dieci-tesi-leducazione-linguistica-democratica (Stand 07.12.2016).

GISCEL Sicilia (1997), La varietà di registro nei manuali di educazione linguistica, in R. Calò, S. Ferreri (Hrsg.), Il testo fa scuola. Libri di testo, linguaggi ed educazione linguistica, La Nuova Italia, Florenz, S. 147-168.

A. Giuliani, C. Iacobini, A.M. Thornton (2005), La nozione di vocabolario di base alla luce della stratificazione diacronica del lessico dell'italiano, in T. De Mauro, I. Chiari (Hrsg.) (2005), Parole e numeri. Analisi quantitative dei fatti di lingua, Aracne, Rom, S. 193-213. 
P. Giuliano (2014), La competenza narrativo-testuale degli adolescenti: soggetti emarginati e soggetti privilegiati, in A. Colombo, G. Pallotti (Hrsg.), L'italiano per capire. Quaderni del GISCEL, 1, Aracne, Rom, S. 257-275.

I. Gogolin (2009a), Zweisprachigkeit und die Entwicklung bildungssprachlicher Fähigkeiten, in I. Gogolin., U. Neumann (Hrsg.), Streitfall Zweisprachigkeit - The Bilingualism Controversy, VS Verlag für Sozialwissenschaften, Wiesbaden, S. 263-280.

I. Gogolin (2009b), „Bildungssprache“ - The importance of teaching language in every school subject, in T. Tajmel, K. Starl (Hrsg.), Science Education Unlimited. Approaches to Equal Opportunities in Learning Science, Waxmann, Münster, New York, S. 91-102.

I. Gogolin (1994), Der monolinguale Habitus der multilingualen Schule, Waxmann, Münster, New York.

I. Gogolin, P. Siemund, M.E. Schulz, J. Davydova (2013), Multilingualism, language contact, and urban areas. An introduction, in P. Siemund et al. (Hrsg.), Multilingualism and Language Diversity in Urban Areas. Acquisition, identities, space, education, John Benjamins, Amsterdam, Philadelphia, S. 1-15.

I. Gogolin, İ. Dirim, Th. Klinger, I. Lange, D. Lengyel, U. Michel, U. Neumann, H.H. Reich, H.-J. Roth \& K. Schwippert (2011), Förderung von Kindern und Jugendlichen mit Migrationshintergrund FörMig: Bilanz und Perspektiven eines Modellprogramms, Waxmann, Münster, New York.

I. Gogolin, I. Lange (2010), Durchgängige Sprachbildung. Eine Handreichung, Waxmann, Münster, New York.

I. Gogolin, U. Neumann (Hrsg.) (2009), Streitfall Zweisprachigkeit - The Bilingualism Controversy, VS Verlag für Sozialwissenschaften, Wiesbaden.

I. Gogolin, U. Neumann, H.-J. Roth (2007), Abschlussbericht über die italienisch-deutschen, portugiesisch-deutschen und spanisch-deutschen Modellklassen; https://www.ew.uni-hamburg.de/ueber-die-fakultaet/personen/gogolin/pdf-dokumente/bericht2007.pdf (Stand: 16.12.2016).

I. Gogolin, M. Krüger-Protratz et al. (Hrsg.) (2005), Migration und sprachliche Bildung, (Interkulturelle Bildungsforschung, 15.) Waxmann, Münster, New York.

I. Gogolin, I. Schwarz (2004), „,Mathematische Literalität“ in sprachlich-kulturell heterogenen Schulklassen, in Zeitschrift für Pädagogik (ZfPäd), 50 (6), S. 835-848.

D. Graf, K.-H. Berck (1993), Begriffslernen im Biologieunterricht-mangelhaft. Wirrwarr von Begriffen überfordert Schüler und Schülerinnen, in Spiegel der Forschung, 2, S. 24-28. 
A. Gramsci (1975), Quaderni del carcere, (kommentierte Ausgabe des Istituto Gramsci, V. Gerratana (Hrsg.)), Einaudi, Turin.

R. Grassi, A. Valentini, R. Bozzone Costa (Hrsg.) (2003), L'italiano per lo studio nella scuola plurilingue: tra semplificazione e facilitazione. Atti del Convegno-Seminario "Alunni stranieri nella scuola: l'italiano per lo studio”, Guerra Edizioni, Perugia.

D.H. Graves (1983), Writing: Teachers and Children at Work, Heinermann Educational, London.

K.R. Gregg (1999), Second language acquisition: History and theories, in B. Spolsky (Hrsg.), Concise Encyclopaedia of Educational Linguistics, Elsevier Science Ltd., Oxford, S. 577-584.

F. Groeben (1978), Die Verständlichkeit von Unterrichtstexten, Aschendorff, Münster.

R. Grotjahn (Hrsg.) (2010), Der C-Test: Beiträge aus der aktuellen Forschung, Peter Lang, Frankfurt a.M., Berlin, Bern u.a.

R. Grotjahn (Hrsg.) (2006), Der C-Test: Theorie, Empirie, Anwendungen, Peter Lang, Frankfurt a.M., Berlin, Bern u.a.

R. Grotjahn (Hrsg.) (2002), Der C-Test. Theoretische Grundlagen und praktische Anwendungen. Bd. 4, AKS-Verlag, Bochum.

R. Grotjahn (Hrsg.) (1994), Der C-Test. Theoretische Grundlagen und praktische Anwendungen. Bd. 2, Brockmeyer, Bochum.

R. Grotjahn, W. Tönshoff, H. Hohenbleicher (1994), Der C-Test im Italienischen. Theoretische Überlegungen und empirische Analysen, in R. Grotjahn (Hrsg.), Der C-Test. Theoretische Grundlagen und praktische Anwendungen. Bd. 2, Brockmeyer, Bochum, S. 115-149.

A.R. Guerriero (2014), Orientamenti didattici e pratiche di scrittura nella scuola secondaria superiore, in M.E. Piemontese, P. Sposetti (Hrsg.), La scrittura dalla scuola superiore all'università, Carocci, Rom, S. 162-173.

A.R. Guerriero (Hrsg.) (1988), L'educazione linguistica e i linguaggi delle scienze. Quaderni del Giscel, La Nuova Italia, Florenz.

M. Gyger, B. Heckendorn (Hrsg.) (1999), Erfolgreich integriert? Fremd- und mehrsprachige Kinder und Jugendliche in der Schweiz, Berner LehrmittelVerlag, Bern.

M.A.K. Halliday (2007), Language and Education. Collected works of M.A.K. Halliday, Bd. 9, J. Webster (Hrsg.), Continuum, London, New York.

M.A.K. Halliday (2002), On grammar. Collected works of M.A.K. Halliday, Bd. 1, J. Webster (Hrsg.), Continuum, London, New York.

M.A.K. Halliday (1990), On the Concept of "Educational Linguistics", zit. Ausgabe: ead. (2007), Language and Education. Collected works of M.A.K. Halliday, Bd. 9, J. Webster (Hrsg.), Continuum, London, New York, S. 354-367. 
M.A.K. Halliday (1993), Towards a Language Based Theory of Learning, in Linguistics and Education, 5, S. 93-116.

M.A.K. Halliday (1985), An introduction to functional grammar, Arnold, London.

J. Hammond, P. Gibbons (2005), Putting scaffolding to work: The contribution of scaffolding in articulating ESL education, in Prospect, 20 (1), S. 6-30.

W.F. Hanks (1996), Language and communicative practices, Westview, Boulder, CO.

I. Harren (2015), Fachliche Inhalte sprachlich ausdrücken lernen. Sprachliche Hürden und interaktive Vermittlungsverfahren im naturwissenschaftlichen Unterrichtsgespräch in der Mittel- und Oberstufe, Verlag für Gesprächsforschung, Mannheim.

C. Harrison (1999), Readability, in B. Spolsky (Hrsg.), Concise Encyclopaedia of Educational Linguistics, Elsevier Science Ltd., Oxford, S.428-431.

C. Harsch, K. Schröder (2014), Der C-Test und sein schulischer Wert. Eine Lanze für mehr C-Tests in der Schule, in R. Grotjahn (Hrsg.), Der C-Test: Aktuelle Tendenzen/The C-Test: Current Trends, P. Lang, Frankfurt a.M., S. 315-324.

J. Hattie (2013), Lernen sichtbar machen, Schneider-Verlag Hohengehren, Baltmannsweiler (Originalausgabe 2008, Visible learning, Routledge, Tayler \& Francis, London).

U. Häussermann, H.-E. Piepho (1996), Aufgaben-Handbuch. Deutsch als Fremdsprache. Abriss einer Aufgaben- und Übungstypologie, Iudicium Verlag, München.

M. Hawkins (2005), ESL in Elementary Education, in E. Hinkel (Hrsg.), Handbook of Research in Second Language Learning and Research, Lawrence Erlbaum Associates, Publishers, Mahwaw, New Jersey, S. 25-43.

M. Hawkins (2004), Researching English language and literacy development in schools, in Educational Researcher, 33 (3), 365-384.

J. R Hayes, L.S. Flower, (1980), Identifying the organization of writing processes, in L.W. Gregg, E.R. Steinberg, (Hrsg.), Cognitive processes in writing, Erlbaum, Hillsdale.

C. Heinze, E. Matthes (Hrsg.) (2010), Das Bild im Schulbuch, Klinkhardt, Bad Heilbrunn.

A. Helmke (2009²), Unterrichtsqualität und Lehrerprofessionalität. Diagnose, Evaluation und Verbesserung des Unterrichts, 2. aktualisierte Auflage, Klett-Kallmeyer, Seelze-Velber.

T. Helmke, A. Helmke, F.-W. Schrader, W. Wagner, G. Nold, K. Schröder (2008), Die Videostudie des Englischunterrichts. Unterricht und Kompetenzerwerb in Deutsch und Englisch, in DESI-Konsortium (Hrsg.), Ergebnisse der DESI-Studie, Beltz, Weinheim, S. 345-363. 
E. Hinkel (Hrsg.) (2005a), Handbook of Research in Second Language Learning and Research, Lawrence Erlbaum Associates, Publishers, Mahwaw, New Jersey.

E. Hinkel (2005b), Analysis of Second Language Text, in E. Hinkel (Hrsg.), Handbook of Research in Second Language Learning and Research, Lawrence Erlbaum Associates, Publishers, Mahwaw, New Jersey, S. 615-628.

P. Hubertus (1995), Wo steht die Alphabetisierungsarbeit heute?, in H. Brügelmann, H. Balhorn, I. Füssenich (Hrsg.), Am Rande der Schrift. Zwischen Sprachenvielfalt und Analphabetismus, DGLS-Jahrbuch Bd. 6, Libelle Verlag, Lengwil, S. 250-262.

INVALSI (2015), Rilevazioni nazionali degli apprendimenti 2014-2015. La rilevazione degli apprendimenti nelle classi II e V primaria, nella classe III (Prova nazionale) della scuola secondaria di primo grado e nella II classe di scuola secondaria di secondo grado; http://www.invalsi.it/invalsi/doc_evidenza/2015/034_Rapporto_Prove_INVALSI_2015.pdf (Stand: 20.12.2016).

ISTAT (2012), Italia in 150 anni. Sommario di statistiche storiche 1861-2010, Capitolo 7:

Istruzione; http://www3.istat.it/dati/catalogo/20120118_00/cap_7.pdf 20.12.2016).

E. Jafrancesco (2002), L'abilità di lettura: leggibilità di un testo e proposte di facilitazione, in Didattica \& Classe Plurilingue (Associazione I.L.S.A), 1, 2002 (ohne Seitenangabe); www.associazione-ilsa.it/bollettiniprecedenti/dcp.../art eja.rtf (Stand 20.12.2016).

F. Kaeding (1896), Häufigkeitswörterbuch der deutschen Sprache, Selbstverlag, Steglitz bei Berlin.

Ch. Klein-Brayley, U. Raatz (Hrsg.) (1985), Fremdsprachen und Hochschule, 13/14: Thematischer Teil: C-Tests in der Praxis, AKS Bochum.

W. Knapp (2007), Didaktische Konzepte Deutsch als Zweitsprache, in B. Ahrenholz, I. Oomen-Welke (Hrsg.), Deutsch als Zweitsprache. Voraussetzungen für die Förderung von Kindern und Jugendlichen mit Migrationshintergrund, Schneider Verlag Hohengehren, Baltmannsweiler, S. 133-148.

S.E. Koesters Gensini (2010), Per la misurazione del lessico ricettivo: riflessioni metodologiche e qualche dato empirico, in Bollettino di italianistica. Rivista di critica, storia letteraria, filologia e linguistica, IV, 1, S. 110122.

S.E. Koesters Gensini (2009), Le parole del tedesco. Tipi, struttura, relazioni, uso, Carocci, Rom.

S.E. Koesters Gensini (2005), Lunghezza e frequenza delle parole nel lessico e nel testo: il caso del tedesco, in T. De Mauro, I. Chiari (Hrsg.), Parole e numeri. Analisi quantitative dei fatti di lingua, Aracne, Rom. 
H. Korte (1993²), Einführung in die Geschichte der Soziologie, VS Verlag für Wissenschaften (UTB für Wissenschaft), Wiesbaden (2. Auflage).

I. Koshik (2002), Designedly Incomplete Utterances: A pedagogical practice for eliciting knowledge displays in error correction sequences, in Research on Language and Social Interaction, 35, 3, S. 277-309.

G. Kress, T. van Leeuwen (2001), Multimodal discourse. The modes and media of contemporary communication, Edward Arnold, London.

M. La Grassa, D. Troncarelli (2014), Comprendere le scienze attraverso i manuali scolastici, in A. Colombo, G. Pallotti (Hrsg.), L'italiano per capire. Quaderni del GISCEL, 1, Aracne, Rom, S. 293-309.

K. Langer, F. Schulz von Thun, R. Tausch (1974), Verständlichkeit in Schule, Verwaltung, Politik und Wissenschaft (2. Auflage), Reinhardt, München.

J.P. Lantolf (2005), Sociocultural and Second Language Learning Research: an Exegesis, in E. Hinkel (Hrsg.) (2005), Handbook of Research in Second Language Learning and Research, Lawrence Erlbaum Associates, Publishers, Mahwaw, New Jersey, S. 335-353.

A. Laudanna, C. Burani (Hrsg.) (1993), Il lessico: processi e rappresentazioni, La Nuova Italia Scientifica, Rom.

B. Laufer, P. Nation (1995), Vocabulary Size and Use: Lexical Richness in L2 Written Production, in Applied Linguistics, 16 (3), S. 307-322.

J. Lave (1996), Teaching, as learning, in practice, in Mind, Culture, and Activity: An International Journal, 3 (3), S. 149-164.

C. Lavinio (2014), Le discipline e i loro linguaggi: perché una tavola rotonda, in A. Colombo, G. Pallotti (Hrsg.), L'italiano per capire. Quaderni del GISCEL, Aracne, Rom, S. 365-379.

C. Lavinio (2011), Testi scritti e testi orali: differenze, interazioni, intersezioni, in M. T. Calzetti, L. Panzeri Donaggio (Hrsg.), Educare alla scrittura. Quaderni di LEND; http://www.lend.it/italia/images/archiveslend/documenti/archivio quaderni/LAVINIO $\% 20$ testi $\% 20$ scritti $\% 20 \mathrm{e} \% 20$ testi\%20orali.\%20Differenze\%20Interazioni\%20Intersezioni.pdf (Stand: 20.12.2016).

C. Lavinio (Hrsg.) (2005a), Educazione linguistica e educazione letteraria. Intersezioni e interazioni, Franco Angeli, Mailand.

C. Lavinio (2005b), Plurilinguismo e colori regionali dell'italiano nella scrittura letteraria, in C. Lavinio (Hrsg.), Educazione linguistica e educazione letteraria. Intersezioni e interazioni, Franco Angeli, Mailand, S. 150-162.

C. Lavinio (1991), Il lavoro sul testo descrittivo, in P. Desideri (Hrsg.), La centralità del testo nelle pratiche didattiche. Quaderni del GISCEL/10, La Nuova Italia, Scandicci (Florenz), S. 93-119.

A. Lee (1996), Gender, Literacy, Curriculum. Re-Writing School Geography, Tayler and Francis, London. 
J. Leisen (2010), Handbuch Sprachförderung im Fach, Verus Verlag, Bonn.

J. Leisen (1998), Sprache(n) im Physikunterricht, in PdN-Ph, 2 (47), S. 2-4.

J. Lemke (1995), Textual politics: Discourse and social dynamics, Taylor \& Francis, Bristol, PA.

D. Lengyel, H.H. Reich, H.-J. Roth, M. Döll (Hrsg.) (2009), Von der Sprachdiagnose zur Sprachförderung, FörMig Edition Band 5, Waxmann, Münster, New York, S. 15-24.

E.H. Lenneberg (1967), Biological Foundations of Language, John Wiley \& Sons, New York.

M.P. Lewis, G.F. Simons, Ch.D. Fennig (Hrsg.) (2016), Ethnologue: Languages of the World. Nineteenth edition, SIL International, Dallas, Texas; http://www.ethnologue.com (Stand: 12.01.2017).

G. Lieber (2012), ,Ich mag es, wenn ich noch selbst überlegen kann. “-Schulbuchillustrationen, Leerstellen und kindliches Bildinteresse, in J. Doll et al. (Hrsg.), Schulbücher im Fokus. Nutzungen, Wirkungen und Evaluation, Waxmann, Münster, New York, S. 67-82.

G. Lieber (Hrsg.) (2008), Lehren und Lernen mit Bildern. Ein Handbuch zur Bilddidaktik, Schneider Verlag Hohengehren, Baltmannsweiler.

P.M. Lightbown (1985), Great expectations: Second-language research and classroom teaching, in Applied Linguistics, 6, S. 173-189.

G. Lombardo-Radice (1959), Lezioni di didattica e ricordi di esperienza magistrale, Sandron, Florenz (33. überarbeitete Ausgabe; Erstausgabe 1936).

G. Lombardo-Radice (1931), Orientamenti pedagogici per la scuola italiana. Nuova ed. aumentata dei saggi "Accanto ai maestri" (decennio 19191930), Bd. 2, Paravia, Turin.

L. Lorenzetti (2002), L'italiano contemporaneo, Carocci editore, Rom.

T. Lucas, A. Katz (1994), Reframing the debate. The roles of native languages in English-only programs for language minority students, in TESOL Quaterly, 28 (4), S. 537-562.

P. Lucisano, E. Piemontese (1988), GULPEASE - Una formula per la previsione della difficoltà dei testi in lingua italiana, in Scuola e città, 3 (31), La Nuova Italia, Scandicci (Florenz).

L. Lumbelli (1989), Fenomenologia dello scrivere chiaro, Editori Riuniti, Rom.

L. Lumbelli (1984a), Effetti paradossali del farsi capire, in Riforma della Scuola, 9-10, S. 13-26.

L. Lumbelli (1984b), Per una diagnosi della comprensibilità, in Riforma della Scuola, 5, S. 23-24.

A. R. Luria (1973), The Working Brain, Basic Books, New York. 
U. Maas (2008), Sprache und Sprachen in der Migrationsgesellschaft. Die schriftkulturelle Dimension, V\&R unipress, Universitätsverlag Osnabrück, Göttingen.

M. MacLure (1999), Home and school language, in B. Spolsky (Hrsg.), Concise Encyclopaedia of Educational Linguistics, Elsevier Science Ltd., Oxford, S. 202-204.

H. Maier, F. Schweiger (1999), Mathematik und Sprache, Öbv \& hpt, Wien.

J.R. Martin (1990), Literacy in science: Learning to handle text as technology, in F. Christie (Hrsg.), Literacy for a changing world, Australian Council for Educational Research, Hawthorn S. 79-117.

I. Matozzi (2014), Competenze linguistiche per la storia o competenze storiche per l'italiano, in A. Colombo, G. Pallotti (Hrsg.), L'italiano per capire. Quaderni del GISCEL, 1, Aracne, Rom, S. 381-397.

H. Mazeland (1983), Sprecherwechsel in der Schule, in K. Ehlich, J. Rehbein (Hrsg.), Kommunikation in Schule und Hochschule, G. Narr, Tübingen, S. 77-101.

S.L. McKay (2005), Sociolinguistics and Second Language Learning, in E. Hinkel (Hrsg.), Handbook of Research in Second Language Learning and Research, Lawrence Erlbaum Associates, Publishers, Mahwaw, New Jersey, S. 281-299.

T.F. McNamara (2001), Language Assessment as Social Practice: Challenges for Research, in Language Testing, 18 (4), S. 330-349.

T.F. McNamara (1999), Validity in language testing. The challenge of Sam Messick's legacy, $1^{\text {st }}$ Messick Memorial Lecture, Language Testing Research Colloquium (LTRC), Tsukuba, Japan, Juli.

T.F. McNamara (1997), 'Interaction' in second language performance assessment: whose performance?, in Applied Linguistics, 18, S. 446-466.

T.F. McNamara (1996), Measuring second language performance, Longman, London, New York.

P. Meara, P.M. Lightbown, R.H. Halter (Hrsg.) (1997), Classrooms as lexical environments, in Language Testing Research 1,1, S. 28-47.

J. Meibauer, M. Rothweiler (1999), Das Lexikon im Spracherwerb, A. Francke Verlag, Tübingen und Basel.

N. Mercer (1999), Classroom Language, in B. Spolsky (Hrsg.), Concise Encyclopaedia of Educational Linguistics, Elsevier Science Ltd., Oxford, S. 315-319.

S. Messick (1988), The once and future issues of validity. Assessing the meaning and consequences of measurement, in H. Wainer, H. L. Braun (Hrsg,), Test Validity, Lawrence Earlbaum Associates, Hillsdale, NJ, S. 33-45. 
A. Miglietta, A.A. Sobrero (2008), Il primo approccio al lessico tecnico-scientifico, in M. Barni, D. Troncarelli, C. Bagna (Hrsg.), Lessico e apprendimenti. Il ruolo del lessico nella linguistica educativa, F. Angeli, Mailand, S. 202-217.

L. Milani (1958), Esperienze pastorali, Libreria editrice fiorentina, Florenz.

L. Milani/Scuola di Barbiana (1967), Lettere a una professoressa, Libreria editrice fiorentina, Florenz.

L. Milia, P. Depau, M. T. Lecca (2014), I colloqui pluridisciplinari della secondaria di primo grado, in A. Colombo, G. Pallotti (Hrsg.), L'italiano per capire. Quaderni del GISCEL, 1, Aracne, Rom, S. 277-289.

MIUR -Ufficio di Statistica (2015), "Anticipazione sui principali dati della scuola statale" as 2015/16; http://hubmiur.pubblica.istruzione.it/web/istruzione/rilevazioni (Stand: 22.01.2017).

MIUR (2015), Libri di testo, Dipartimento per l'istruzione. Direzione Generale per gli Ordinamenti Scolastici e per l'Autonomia Scolastica; http://hubmiur.pubblica.istruzione.it/web/istruzione/libri (Stand: 31.12.2016).

MIUR (2014), MIURAOODGOS prot. 2581. Oggetto: Adozioni libri di testo anno scolastico 2014/2015, Dipartimento per l'istruzione. Direzione Generale per gli Ordinamenti Scolastici e per l'Autonomia Scolastica; http://www.istruzione.it/allegati/2014/NOTA ADOZIONI_LIBRI TESTO.pdf (Stand: 31.12.2016).

MiUR (2012a), L'obbligo scolastico, Ministero dell'Istruzione, dell'Università e della Ricerca. Ufficio Relazioni con il Pubblico; http://www.istruzione.it/urp/obbligo scolastico.shtml (Stand: 31.12.2016).

MIUR (2012b), Indicazioni nazionali per il curricolo della scuola d'infanzia e del primo ciclo d'istruzione; http://www.indicazioninazionali.it/documenti_Indicazioni_nazionali/indicazioni_nazionali_infanzia_primo_ciclo.pdf (Stand: 04.11.2016).

J. Moffett (1968), Teaching the Universe of Discourse, Houghton-Miffin, London.

U. Moser, M. Stamm, J. Hollenweger (2005), Für die Schule bereit? Lesen, Wortschatz, Mathematik und soziale Kompetenzen beim Schuleintritt, Sauerländer, Oberentfelden.

A. Müller (1997), Wissen und Schreiben. Ergebnisse aus der Aufsatzstudie Ost, Peter Lang, Frankfurt a.M., Berlin, Bern u.a.

D. Murray $\left(1987^{2}\right)$, Write to Learn, Holt, Rinehart and Winston, New York (Erstausgabe 1984).

I.S.P. Nation (2006), How Large a Vocabulary is Needed for Reading and Listening?, in The Canadian Modern Language Review, 63 (1), S. 59-81.

I.S.P. Nation (2001), Learning Vocabulary in Another Language, Cambridge University Press, Cambridge. 
I.S.P. Nation (1990), Teaching and Learning Vocabulary, Heinle \& Heinle, Boston.

C. Neugebauer, C. Nodari (1999), Aspekte der Sprachförderung, in M. Gyger, B. Heckendorn (Hrsg.), Erfolgreich integriert? Fremd- und mehrsprachige Kinder und Jugendliche in der Schweiz, Berner Lehrmittel-Verlag, Bern, S. 161-175.

M.A. Nippold (2007), Later Language Development: School-Age Children, Adolescents and Young Adults, PRO-ED, Austin, 3. Auflage (Erstausgabe 1988).

C. Nodari (2010), Fachdingsda - Fächerorientierter Grundwortschatz für das 5.-9. Schuljahr, in B. Ahrenholz (Hrsg.), Fachunterricht und Deutsch als Zweitsprache, Narr, Tübingen, S. 279-281.

D. Nunan (2005), Classroom Research, in E. Hinkel (Hrsg.), Handbook of Research in Second Language Learning and Research, Lawrence Erlbaum Associates, Publishers, Mahwaw, New Jersey, S. 225-240.

G. Nuthall (1997), Understanding Student Thinking and Learning in the Classroom, in B. J. Biddle, T. L. Good, I. F. Goodson (Hrsg.), Handbook of Teachers and Teaching, Vol. 2, Kluwer Academic Publishers, Dordrecht, S. 681-768.

L. Oakes (2001), Language and National Identity, John Benjamins Publishing Company, Amsterdam, Philadelphia.

OECD (2014), Ein Profil der Schülerleistungen in Mathematik in PISA 2012 Ergebnisse: Was Schülerinnen und Schüler wissen und können (Bd. 1, überarbeitete Ausgabe Februar 2014): Schülerleistungen in Lesekompetenz, Mathematik und Naturwissenschaften, W. Bertelsmann Verlag, Bielefeld.

OECD, Statistics of Canada (2000), Literacy in the Information Age. Final Report of the International Adult Literacy Survey, Organisation for Economic Co-Operation and Development, Paris und Minister of Industry, Canada.

OECD, Definition literacy; http://www.oecd.org/edu/innovation-education/adultliteracy.htm (Stand: 31.12.2016).

F. Oser (2001), Standards. Kompetenzen von Lehrpersonen, in F. Oser, J. Oelkes (Hrsg.), Die Wirksamkeit der Lehrerbildungssysteme, Ruegger, Zürich, S. 215-342.

F. Oser, J. Oelkes (Hrsg.) (2001), Die Wirksamkeit der Lehrerbildungssysteme, Ruegger, Zürich.

M. Ott (1997), Deutsch als Zweitsprache. Aspekte des Wortschatzerwerbs. Eine empirische Längsschnittuntersuchung zum Zweitspracherwerb, Peter Lang, Frankfurt a.M., Berlin, Bern u.a.

T.S. Paribakht, M. Wesche (1996), Enhancing vocabulary acquisition through reading. Ahierarchy of text-related exercise types, in The Canadian Modern Language Review, 52 (2), S. 155-178. 
E. Passaponti (1980), Comprensione del testo nella scuola primaria, in Riforma della scuola, 2/3, S. 51-54.

A. Pennycook (2001), Critical applied linguistics: A critical introduction, Lawrence Erlbaum Associates, Mahwah, NJ.

C. Perdue (1993), Adult language acquisition: cross-linguistic perspectives. Vol. 2: The results, Cambridge University Press, Cambridge.

K. Perera (1999), Educational Linguistics, in B. Spolsky (Hrsg.), Concise Encyclopaedia of Educational Linguistics, Elsevier Science Ltd., Oxford, S. 17-21.

M. Perlemann-Balme (2010), Testen und Prüfen von Sprachkenntnissen, in H.J. Krumm, P. Kosta, Ch. Fandrych, B. Hufeisen und C. Riemer (Hrsg.), Deutsch als Fremd- und Zweitsprache, Part 2, De Gruyter, Berlin, New York, S. 1272-1279.

F. Petermann (2010), Set 5-10. Sprachstandserhebungstest für Kinder im Alter zwischen 5 und 10 Jahren, Hogrefe, Göttingen.

A. Peyer (2011), Sätze untersuchen. Lerntheoretische Sprachreflexion und grammatisches Wissen, Klett/Kallmeyer, Seeze.

T. Pica (2005), Second Language Acquisition Research and Applied Linguistics, in E. Hinkel (Hrsg.), Handbook of Research in Second Language Learning and Research, Lawrence Erlbaum Associates, Publishers, Mahwaw, New Jersey, S. 263-280.

M.E. Piemontese (2014), Introduzione, in M.E. Piemontese, P. Sposetti (Hrsg.), La scrittura dalla scuola superiore all'università, Carocci, Rom, S. 9-14.

M.E. Piemontese (2005), Misurazioni quantitative degli stili verbali e indici di leggibilità, in T. De Mauro, I. Chiari (Hrsg.), Parole e numeri. Analisi quantitative dei fatti di lingua, Aracne, Rom, S. 377-397.

M.E. Piemontese (1996), Capire e farsi capire, Tecnodid, Neapel.

M.E. Piemontese (1993), Criteri e proposte di semplificazione, in Codice di stile delle comunicazioni scritte a uso delle pubbliche amministrazioni, Dipartimento per la Funzione Pubblica - Presidenza del Consiglio dei Ministri, S. 27-33.

M.E. Piemontese, P. Sposetti (Hrsg.) (2014), La scrittura dalla scuola superiore all'università, Carocci, Rom.

M.E. Piemontese, L. Cavaliere (1997), Leggibilità e comprensibilità di sussidiari per le scuole elementari, in R. Calò, S. Ferreri (Hrsg.), Il testo fa scuola. Libri di testo, linguaggi ed educazione linguistica, La Nuova Italia, Florenz, S. 221-240.

M.E. Piemontese, M. T. Tiraboschi (1990), Strumenti e metodologie di ricerca al servizio del diritto a capire testi di rilievo pubblico, in E. Zuanelli (Hrsg.), Il diritto all'informazione in Italia. Ricerche promosse dalla Presidenza del Consiglio dei Ministri, Dipartimento per l'informazione e l'editoria, Istituto Poligrafico e Zecca dello Stato, Rom, S. 225-246. 
M. Pienemann (1989), Is Language Teachable? Psycholinguistic Experiments and Hypotheses, in Applied Linguistics, 10 (1), S. 52-79.

M.A. Pinto (1980), Svantaggio linguistico, ambiente sociale, educazione critica a B. Bernstein, Bulzoni Editori, Rom.

C. Pontecorvo, A.M. Aiello, C. Zucchermaglio (Hrsg.) (2005), I contesti sociali dell 'apprendimento. Acquisire conoscenze a scuola, nel lavoro, nella vita quotidiana, Ambrosiana, Mailand.

T. Quehl (2009), Sprachbildung im Sachunterricht der Grundschule, in D. Lengyel, H.H. Reich, H.-J. Roth, M. Döll (Hrsg.), Von der Sprachdiagnose zur Sprachförderung, FörMig Edition Bd. 5, Waxmann, Münster, New York.

U. Raatz, Ch. Klein-Brayley (1985), How to develop a C-Test, in Ch. KleinBrayley, U. Raatz (Hrsg.). Fremdsprachen und Hochschule, 13/14: Thematischer Teil: C-Tests in der Praxis, AKS Bochum, S. 20-22.

M. Raicich (1981), Scuola, cultura e politica da De Sanctis a Gentile, NistriLischi, Pisa.

A. Redder, S. Weinert (Hrsg.) (2013), Sprachförderung und Sprachdiagnostik - Interdisziplinäre Perspektiven, Waxmann, Münster, New York.

H.H. Reich (2008), Die Sprachaneignung von Kindern in Situationen der Zwei- und Mehrsprachigkeit, in BMBF (Hrsg.), Referenzrahmen zur altersspezifischen Sprachaneignung - Forschungsgrundlagen, Bd. 29/II, Referat Bildungsforschung, Bonn, Berlin, S. 163-169.

H.H. Reich, H.-J. Roth, U. Neumann (Hrsg.) (2007), Sprachdiagnostik im Lernprozess. Verfahren zur Analyse von Sprachständen im Kontext von Zweisprachigkeit, FörMig Edition Bd. 3, Waxmann, Münster, New York.

I. Reid (Hrsg.) (1987), The place of genre in learning: Current debates, Deakin University Press, Geelong.

A. Reiniger (Hrsg.) (1997), DIT. Langenscheidts Handwörterbuch Italienisch (Italienisch-Deutsch, Deutsch-Italienisch) in Zusammenarbeit mit Paravia, (Leitung des Redaktionsteams F. Cicoira), Langenscheidt, Berlin u.a.

L. Renzi (1998), La questione della grammatica. Intervista a Lorenzo Renzi di Michele A. Cortelazzo, in S. Ferreri, A.S. Guerriero (Hrsg.), Educazione linguistica vent'anni dopo e oltre. Che cosa ne pensano De Mauro, Renzi, Simone, Sobrero, La Nuova Italia, Scandicci (Florenz), S. 51-72.

L. Renzi (1997), Uno sguardo d'insieme, in R. Calò, S. Ferreri (Hrsg.), Il testo fa scuola. Libri di testo, linguaggi ed educazione linguistica, Florenz, S. 503-508.

L. Revelli (2008), Cent'anni di esercizi. La didattica del lessico e dei significati in Valle d'Aosta attraverso i quaderni di scuola (1899-2000), in M. Barni, D. Troncarelli, C. Bagna (Hrsg.), Lessico e apprendimenti. Il ruolo del lessico nella linguistica educativa, F. Angeli, Mailand, S. 339347. 
Th. Ricento (2013), Measuring success when English isn't your native language. Perspectives from Canada, in P. Siemund et al. (Hrsg.), Multilingualism and Language Diversity in Urban Areas. Acquisition, identities, space, education, John Benjamins, Amsterdam, Philadelphia, S. 349-368.

F. Richadieu (1969), La Lisibilité, Danoel, Paris.

B. Rogoff (1994), Developing understanding of the idea of community of learners, in Mind, Culture and Language, 1 (4), S. 209-229.

Ch. Röhner, B. Hövelbrinks (Hrsg.) (2013), Fachbezogene Sprachförderung in Deutsch als Zweitsprache: Theoretische Konzepte und empirische Befunde zum Erwerb bildungssprachlicher Kompetenzen, Beltz Juventa, Landsberg.

C. A. Rohrbeck, M. D. Ginsburg-Block, J. W. Fantuzzo, T. R. Miller (2003), Peer assisted learning interventions with elementary school students: a meta-analytic review, in Journal of Educational Psychology, 95 (2), S. 240-257.

G. Romani (1827), Opuscoli sulla lingua italiana, Silvestri, Mailand.

R. Rosenthal, D. B. Rubin (1978), Issues in summarizing the first 345 studies of interpersonal expectancy effects, in Behavioral and Brain Sciences, 1 (3), S. 410.

A. Rossi (Hrsg.) (1970), Lettere di una tarantata, De Donati, Bari, S. 43-75.

H.-J. Roth (2005), Warum weint die Katze? - Einige Überlegungen zur Systematik der gesprochenen Kindersprache im Kontext von Zweisprachigkeit, in I. Gogolin, M. Krüger-Protratz et al. (Hrsg.), Migration und sprachliche Bildung (= Interkulturelle Bildungsforschung, 15.), Waxmann, Münster, New York, S. 81-102.

H.-J. Roth (2002), Die Ausgangssprachen mehrsprachiger Kinder berücksichtigen, in Th. Fitzner (Hrsg.), Alphabetisierung und Sprachenlernen. Eine Fachtagung, Evangelische Akademie Bad Boll ed Ernst Klett Verlag, Stuttgart, S. 121-145.

M. Rothweiler (Hrsg.) (1990), Spracherwerb und Grammatik. Linguistische Untersuchungen zum Erwerb von Syntax und Morphologie, (= LB-Sonderheft 3), Westdeutscher Verlag Opladen, S. 105-126.

C.M. Rubie-Davies (2007), Classroom interactions. Exploring the practices of high and low expectation teachers, in British Journal of Educational Psychology, 77, S. 289-306.

C.M. Rubie-Davies (2006). Teacher expectations and student self-perceptions. Exploring relationships, in Psychology in the Schools, 43, S. 537-552.

C.M. Rubie (2003), Planning for learning. Instructional decisions that impact on opportunities to learn, in D. Fraser, R. Openshaw (Hrsg.), Informing our practice. Special volume, Kanuka Grove Press, Palmerston North. 
C.M. Rubie-Davies, J.A.C. Hattie, R.J. Hamilton (2006), Expecting the best for New Zealand students. Teacher expectations and academic outcomes, in British Journal of Educational Psychology, 76, S. 429-444.

F. Salvatori (1988), Geografia come scienza, in Riforma della scuola, 3, S. $58 \mathrm{f}$.

E. Sapir (1962), Culture, Language and Personality: Selected Essays, D. Mandelbaum (Hrsg.), University of California Press, Berkely, CA.

E.A. Schegloff, E. Ochs, S.A. Thompson (1996), Introduction, in E.A. Schegloff, E. Ochs, S.A. Thompson (Hrsg.), Interaction and grammar, Cambridge University Press, Cambridge, S. 1-51.

A. Schmidt, N. Altwicker (1986) (Hrsg.), Max Horkheimer: Werk und Wirkung, Fischer Taschenbuch, Frankfurt a.M.

N. Schmitt, D. Schmitt, C. Clapham (2001), Developing and exploring the behaviour of two new versions of the Vocabulary Levels Test, in Language Testing, 18 (1), S. 55-88.

S. Schmölzer-Eibinger (2011), Lernen in der Zweitsprache: Grundlagen und Verfahren der Förderung von Textkompetenz in mehrsprachigen Klassen, 2., durchgesehene Auflage, G. Narr, Tübingen.

S. Schmölzer-Eibinger, G. Weidacher (2007), Textkompetenz. Eine Schlüsselkompetenz und ihre Vermittlung, G. Narr, Tübingen.

H. Schneider, M. Becker-Mrotzek, A. Sturm, S. Jambor-Fahlen, U. Neugebauer, Ch. Efing, N. Kernen (2013), Wirksamkeit von Sprachförderung. Expertise, Bildungsdirektion Zürich (Hrsg.), Mercator-Institut für Sprachförderung und Deutsch als Zweitsprache (Universität Köln).

K. F. Schnell (1850), Die Schuldisziplin - Als wissenschaftlich geordnete Kunde, Verlag von Karl Wiegandt, Berlin.

Department of Education and Science (1975), A language for Life (The Bullock Report),D.E.S., London; http://www.educationengland.org.uk/documents/bullock/bullock1975.html (Stand: 02.01.2017).

L. Serianni (2010), L'ora di italiano. Scuola e materie umanistiche, Laterza, Rom, Bari.

L. Serianni (2007), Grammatica italiana: italiano comune e lingua letteraria, UTET, Turin.

L. Serianni (2006), Prima lezione di grammatica, Laterza, Rom, Bari.

L. Serianni, P. Trifone (Hrsg.) (1994), Storia della lingua italiana, II, Scritto e parlato, Einaudi, Turin.

P. Siemund, I. Gogolin, M.E. Schulz, J. Davydova (Hrsg.) (2013), Multilingualism and Language Diversity in Urban Areas. Acquisition, identities, space, education, John Benjamins, Amsterdam, Philadelphia.

R. Simone (1998), Educazione linguistica minimalista? Intervista a Raffaele Simone di Anna Rosa Guerriero, in S. Ferreri, A.S. Guerriero (Hrsg.), Educazione linguistica vent'anni dopo e oltre. Che cosa ne pensano De 
Mauro, Renzi, Simone, Sobrero, La Nuova Italia, Scandicci, Florenz, S. 37-56.

R. Simone (1997), Quel che cercai di fare, quel che feci, e che cosa ne derivò. Riflessioni vent'anni dopo su un libro di testo da parte del suo autore in R. Calò, S. Ferreri (Hrsg.), Il testo fa scuola. Libri di testo, linguaggi ed educazione linguistica, Florenz, S. 477-487.

R. Simone (1974), Libro d'italiano, La Nuova Italia, Florenz.

T.R. Sizer (1995), Making the Grade, in Washington Post Education Review (2.4.), S. 12.

T.R. Sizer (1992), Horace's School: Redesigning the American High School, Houghton Mifflin, New York.

D.J. Slobin (1987), Thinking for speaking, in J. Aske et al. (Hrsg.), Papers from the $13^{\text {th }}$ Annual Meeting of the Berkeley Linguistics Society, BLS, Berkeley, S. 435-462.

A.A. Sobrero (1998), La lingua nella scuola del domani, Intervista a Alberto A. Sobrero di Anna Rosa Guerriero, in S. Ferreri, A.S. Guerriero (Hrsg.), Educazione linguistica vent'anni dopo e oltre. Che cosa ne pensano De Mauro, Renzi, Simone, Sobrero, La Nuova Italia, Scandicci (Florenz), S. 57-72.

A.A. Sobrero (1993), Introduzione all'italiano contemporaneo. La variazione e gli usi, Editori Laterza, Rom, Bari.

J. Söhn (2005), Zweisprachiger Schulunterricht für Migrantenkinder. Ergebnisse der Evaluationsforschung zu seinen Auswirkungen auf Zweitspracherwerb und Schulerfolg. AKI-Forschungsbilanz, Berlin; http://edoc.vifapol.de/opus/volltexte/2009/1558/pdf/iv05 akibilanz2.pdf (Stand: 02.01.2017).

B. Spolsky (Hrsg.) (1999), Concise Encyclopaedia of Educational Linguistics, Elsevier Science Ltd., Oxford.

B. Spolsky (1995), Measured Words: The Development of Objective Language Testing, Oxford University Press, Oxford.

B. Spolsky (1981), Some ethical questions about language testing, in Ch. Klein-Brayley, D. K. Stevenson (Hrsg.), Practice and Problems in Language Testing, Peter Lang, Frankfurt a.M., Berlin, Bern u.a., S. 5-30.

H. Steffens (2005), Warum brauchen Wirtschaft und Grundbildung einander? Drei Thesen, in A. Müller (Hrsg.), Alphabetisierung. Kultur. Wirtschaft, Bundesverband Alphabetisierung e.V., Ernst Klett, Stuttgart, S. 31-32.

A. Stefinlongo (2002), I giovani e la scrittura. Attitudini, bisogni, competenze di scrittura delle nuove generazioni, Aracne, Rom.

C. Stern, W. Stern (1928), Die Kindersprache, Barth, Leipzig (Neuauflage: 1978, Wissenschaftliche Buchgesellschaft, Darmstadt). 
M. Swain (2005), The Output Hypothesis: Theory and Research, in E. Hinkel (Hrsg.), Handbook of Research in Second Language Learning and Research, Lawrence Erlbaum Associates, Publishers, Mahwaw, New Jersey, S. 471-483.

M. Swain (1985), Communicative competence: Some roles of comprehensible input and comprehensible output in its development, in S. Gass, C. Madden (Hrsg.), Input in Second Language Acquisition, Newbury House, New York, S. 235-256.

P. Szlabewski-Çavus (2002), Warum Deutschlernen für Ausländerinnen meist gar nicht soleicht ist und was daraus für ein Unterrichtsangebot erfolgt, in Th. Fitzner (Hrsg.), Alphabetisierung und Sprachenlernen. Eine Fachtagung, Evangelische Akademie Bad Boll und Ernst Klett Verlag, Stuttgart, S. $75 \mathrm{f}$.

T. Tajmel, K. Starl (Hrsg.) (2009), Science Education Unlimited. Approaches to Equal Opportunities in Learning Science, Waxmann, Münster, New York.

S.O. Tergan, H. Mandl (1983), Neuere Ansätze zur Textverständlichkeit, in Unterrichtswissenschaft, 11, S. 56-72.

F.H. Thöneböhn (1995), Rezeption und Verwendung des geographischen Schulbuches in der Sekundarstufe 1. Interviewstudie zum Umgang von Lehrern mit dem geographischen Schulbuch bei curricularen Entscheidungen, bei der Unterrichtsplanung und im Unterricht, Dissertation, Fakultät für Geowissenschaften an der Ruhr-Universität Bochum, Bochum.

A.M. Thornton (1984), Leggibilità dei manuali, in Riforma della Scuola, Nr.4, S. $50-51$.

O. Togeby (1971), Sproq og laeseproces, Den Gjellerny, Kopenhagen.

P. Togliatti (1973), Opere, Bd. 3 (1944-1945), E. Ragionieri (Hrsg.), Editori Riuniti und Istituto Gramsci, Rom.

M. Torrance, D. Galbraith (Hrsg.) (1999), Studies in Writing, vol. IV. Knowing What to Write. Conceptual Processes in Text Production, Amsterdam University Press, Amsterdam.

B. Treiber (1982), Lehr- und Lernzeiten im Unterricht, in B. Treiber, F. Weinert (Hrsg.), Lehr-Lernforschung. Ein Überblick in Einzeldarstellungen, Urban und Schwarzenberg, München u.a., S. 12-36.

B. Treiber, F. Weinert (Hrsg.) (1982), Lehr-Lernforschung. Ein Überblick in Einzeldarstellungen, Urban und Schwarzenberg, München u.a.

E. Tschirner, R. L. Jones (2006), Frequency Dictionary of German, Routledge, London-New York.

R. Vacca (1972), Per una critica quantitative: Romanzi a chilometri, in Il Messaggero, 14.12.1972.

R. Vacca, V. Franchina (1986), Taratura di indici di Flesch su testo bilingue italiano inglese di unico autore, in Linguaggi, 2-3. 
L. van Lier (1994), Forks and hope: Pursuing understanding in different contexts, in Applied Linguistics, 15 (3), S. 328-346.

L. van Lier (1996), Interaction in the language curriculum: Awareness, autonomy and authenticity, Longman, London.

M. Vedovelli (2002), Guida all'italiano per stranieri. La prospettiva del Quadro comune europeo per le lingue, Carocci editore, Rom.

M. Vedovelli (1993), Note per una sociolinguistica dei movimenti migratori in Europa, in E. Banfi (Hrsg.) (1993a), L'altra Europa linguistica, La Nuova Italia, Scandicci (Florenz), S. 1-34.

G. Vico (2008), La scienza nuova, BUR, Mailand (10. Ausgabe, Erstausgabe 1977; Originalausgabe 1725).

P. Villari (1909), I dialetti e la lingua, in La Nuova Antologia, 44, S. 385-395.

A. Villarini (2001), La didattica dell 'italiano agli immigrati stranieri in Italia e a Roma, in M. Barni, A. Villarini (Hrsg.), La questione della lingua per gli immigrati stranieri. Insegnare, valutare e certificare l'italiano L2, Franco Angeli, Mailand, S. 9-69.

M. Voghera (2008), La grammatica nei testi, in A. Ledgeway, A.L. Lepschy (Hrsg.), Didattica della lingua italiana: testo e contesto, Guerra Edizioni, Perugia, S. 181-195.

H.J. Vollmer (2011), Schulsprache -Was ist das?, Handreichung zum Vortrag beim DGFF-Kongress Hamburg, 29.09.2011.

H.J. Vollmer, E. Thürmann (2010), Zur Sprachlichkeit des Fachlernens: Modellierung eines Referenzrahmens für Deutsch als Zweitsprache, in B. Ahrenholz (Hrsg.), Fachunterricht und Deutsch als Zweitsprache, Narr, Tübingen, S. 107-132.

L.S. Vygotsky (1978), Mind in society. The development of higher psychological processes, Harvard University Press, Cambridge M.A.

L.S. Vygotsky (1986), Thought and language, MIT Press, Cambridge, M.A. (Originalausgabe 1934).

K. Wagner ( Hrsg.) (1985), Wortschatz-Erwerb, P. Lang, Frankfurt.

A. Weber (1973), Dialektik der Aufsatzbeurteilung, Verlag Ludwig Auer, Donauwörth.

F.E. Weinert (2000), Lernen und Lernen für die Zukunft-Ansprüche an das Lernen in der Schule, in Pädagogische Nachrichten Rheinland-Pfalz, 2, S. 1-16.

R.S. Weinstein (2002), Reaching Higher: The Power of Expectations in Schooling, Harvard University Press, Harvard.

G. Wells (1999), Dialogic inquiry: Towards a sociocultural practice and theory of education, Cambridge University Press, Cambridge.

G. Wells (1996), Using the tool-kit of discourse in the activity of learning and teaching, in Mind, Culture and Language, 3 (2), S. 74-101.

J.V. Wertsch (1998), Mind as action, Oxford University Press, Oxford. 
H. Wiese, I. Wiese (1998). Mathematik und Sprache - ein Problem aus dem Bereich der ,gemischten Zahlen', in M. Neubrand (Hrsg.), Beiträge zum Mathematikunterricht 1998. Vorträge auf der 32. Tagung für Didaktik der Mathematik vom 2. bis 6. März 1998 in München, Franzbecker Hildesheim, S.647-650.

H. Willenberg (2007a), Der vergessene Wortschatz, in H. Willenberg (Hrsg.), Kompetenzhandbuch für den Deutschunterricht, Schneider Hohengehren, Baltmannsweiler, S. 148-156.

H. Willenberg (Hrsg.) (2007b), Kompetenzhandbuch für den Deutschunterricht, Schneider Hohengehren, Baltmannsweiler.

M. Willis, Y. Ohashi (2012), A model of L2 vocabulary learning and retention, in The Language Learning Journal, 40 (1), S. 125-137.

L. Wittgenstein (2003), Tractatus logico-philosophicus, Logisch-philosophische Abhandlung, Suhrkamp, Frankfurt a.M. (Erstausgabe 1921, Annalen der Naturphilosphie, Bd. 14, S. 185-262).

L. Wittgenstein (2001), Philosophische Untersuchungen. Kritisch-genetische Edition, J. Schulte (Hrsg.), Wissenschaftliche Buchgesellschaft, Frankfurt (Erstausgabe 1953, posthum).

L. Wittgenstein (1977), Wörterbuch für Volksschulen (Schriften der Österreichischen Wittgensteingesellschaft), A. Hübner, E. Leinfellner, W. Leinfellner (Hrsg.), öbv, Wien (Erstausgabe 1926).

L. Wong-Fillmore (1985), When does teacher talk work as input?, in S. Gass, C. Madden (Hrsg.), Input in second language acquisition, Newbury House, Rowley, MA, S. 89-114.

D. Wood, J. Bruner, G. Ross (1976), The Role of Tutoring in Problem Solving, in Journal of Child Psychology and Psychiatry, 17 (2), S. 89-100.

L.S. Wygotski (1977), Denken und Sprechen, S. Fischer, Frankfurt a.M. (Originalausgabe 1934, erste deutsche Ausgabe 1964, Berlin).

G. Yair (2000), Educational battlefields in America: The tug-of-war over students' engagement with instruction, in Sociology of Education, 23, S. 247269.

M.L. Zambelli (Hrsg.) (1994), La rete e i nodi. Il testo scientifico nella scuola di base, La Nuova Italia, Florenz.

K. M. Zeichner (1986), Teacher socialisation research and the practice of teacher training, in Educational Society, 3, S. 25-37.

E. Zuanelli (Hrsg.) (1990), Il diritto all informazione in Italia. Ricerche promosse dalla Presidenza del Consiglio dei Ministri, Dipartimento per l'informazione e l'editoria, Istituto Poligrafico e Zecca dello Stato, Rom.

J. Zuengler, K. Cole (2005), Language Socialisation and Second Language Learning, in E. Hinkel (Hrsg.), Handbook of Research in Second Language Learning and Research, Lawrence Erlbaum Associates, Publishers, Mahwaw, New Jersey, S. 301-316. 


\section{Anhang}

\section{Terminologie der sprachlichen Analysen}

\subsection{Abkürzungen der Wortarten}

\begin{tabular}{|c|c|c|c|}
\hline Abkürzung & übersetzte Bedeutung & $\begin{array}{l}\text { Abkürzungen, Ge- } \\
\text { brauchsmarken }\end{array}$ & übersetzte Bedeutung \\
\hline s.m. & maskulines Substantiv & part.pres.v.tr./intr. & Partizip Präsens \\
\hline s.f. & feminines Substantiv & pron.dimostr. & $\begin{array}{l}\text { Demonstrativprono- } \\
\text { men }\end{array}$ \\
\hline pl. & Plural & pron.pers. & Personalpronomen \\
\hline art.det. & bestimmter Artikel & pron.rel. & Relativpronomen \\
\hline art.indet. & unbestimmter Artikel & pron.indef. & Indefinitpronomen \\
\hline agg. & Adjektiv & conf. & Konfix \\
\hline agg.num.ord. & Ordinalzahl & var. (s.m./f.) & $\begin{array}{l}\text { Variante (eines No- } \\
\text { mens) }\end{array}$ \\
\hline agg.num.card. & Kardinalzahl & var.posiz. & $\begin{array}{l}\text { positionsbedingte Va- } \\
\text { riante }\end{array}$ \\
\hline agg.dimostr. & Demonstrativadjektiv & el.inf.p. & $\begin{array}{l}\text { Element eines Infinitiv } \\
\text { Passivs }\end{array}$ \\
\hline agg.poss. & Possessivadjektiv & el.loc. & $\begin{array}{l}\text { Element eines Phra- } \\
\text { sems }\end{array}$ \\
\hline agg.indef. & indefinites Adjektiv & el.n.p. & $\begin{array}{l}\text { Element eines Eigen- } \\
\text { namens }\end{array}$ \\
\hline n.p. & Eigenname & el.CO/el.FO, etc. & $\begin{array}{l}\text { Element eines Phra- } \\
\text { sems aus dem ent- } \\
\text { sprechenden Teil des } \\
\text { WS }\end{array}$ \\
\hline sigla & Sigle & FO & $\begin{array}{l}\text { fundamentaler Wort- } \\
\text { schatz }\end{array}$ \\
\hline n.p./sigla & Eigenname / Sigle & $A U$ & hoher Gebrauch \\
\hline prep. & Präposition & $A D$ & hohe Disponibilität \\
\hline avv. & Adverb & $\mathrm{CO}$ & $\begin{array}{l}\text { allgemeiner Wort- } \\
\text { schatz }\end{array}$ \\
\hline abbr. & Abkürzung & ES & Exotismus/Fremdwort \\
\hline simb. & Symbol & $\mathrm{BU}$ & niedriger Gebrauch \\
\hline loc.agg. & adjektivales Phrasem & LE & literarischer Gebrauch \\
\hline loc.cong. & $\begin{array}{l}\text { konjunktionales Phra- } \\
\text { sem }\end{array}$ & ES & Exotismus \\
\hline loc.s.f./m. & nominales Phrasem & TS & $\begin{array}{l}\text { technisch-spezifischer } \\
\text { WS }\end{array}$ \\
\hline $\begin{array}{l}\text { loc.avv. } \\
\text { loc.v. }\end{array}$ & $\begin{array}{l}\text { adverbiales Phrasem } \\
\text { verbales Phrasem }\end{array}$ & $\begin{array}{l}\text { (FO, AU etc.) fig. } \\
\text { (FO, AU etc.) estens. }\end{array}$ & $\begin{array}{l}\text { figurative Bedeutung } \\
\text { ausgeweitete Bedeu- }\end{array}$ \\
\hline v.tr. & transitives Verb & Beispiele für TS: & \\
\hline v.intr. & intransitives Verb & TS geogr. & geographischer WS \\
\hline inf.v.tr./intr. & Infinitiv Präsens & TS econ. & wirtschaftlicher WS \\
\hline inf.p.v.tr. & Infinitiv Passiv & TS dir.intern. & $\begin{array}{l}\text { WS internationales } \\
\text { Recht }\end{array}$ \\
\hline
\end{tabular}




\begin{tabular}{llll}
\hline Abkürzung & übersetzte Bedeutung & $\begin{array}{l}\text { Abkürzungen, Ge- } \\
\text { brauchsmarken }\end{array}$ & übersetzte Bedeutung \\
\hline inf.pass.v.tr./intr. & Infinitiv Perfekt & TS ling. & linguistischer WS \\
ger.v.tr./intr. & Gerundium & TS scient. & $\begin{array}{l}\text { naturwissenschaftli- } \\
\text { cher WS } \\
\text { geschichtlicher WS }\end{array}$ \\
part.pass.v.tr./intr. & Partizip Perfekt & TS stor. & gesh \\
\hline
\end{tabular}

Tabelle 1: Terminologie der sprachlichen Analysen

\subsection{Abkürzungen der verwendeten Fachsprachen nach dem Gradit (De Mauro 1999/2000)}

\begin{tabular}{ll}
\hline Technisch-spezifischer Wortschatz & Übersetzung \\
\hline TS agr. & Agrarwissenschaft \\
TS amin.az. & Betriebsverwaltung \\
TS antrop. & Anthropologie \\
TS archeol. & Archäologie \\
TS arm. & Waffen, Bewaffnungen \\
TS astrol. & Astrologie \\
TS astron. & Astronomie \\
TS biochim. & Biochemie \\
TS biol. & Biologie \\
TS bot. & Botanik \\
TS bot.com. & allgemeiner Begriff aus der Botanik \\
TS burocr. & Bürokratie \\
TS cartogr. & Kartographie \\
TS chim. & Chemie \\
TS dir. & Rechtswissenschaften \\
TS dir. intern. & internationales Recht \\
TS dir.amm. & Verwaltungsrecht \\
TS dir. Lav. & Arbeitsrecht \\
TS ecol. & Ökologie \\
TS econ. & Wirtschaft \\
TS polit. & Politik \\
TS sociol. & Soziologie \\
TS etnol. & Ethnologie \\
TS fin. & Finanzwesen \\
TS fis. & Physik \\
TS mat. & Mathematik \\
TS geofis. & Geophysik \\
TS geogr. & Geographie \\
TS idrol. & Hydrologie \\
TS geol. & Geologie \\
TS industr. & Industrie \\
TS inform. & Informatik \\
TS let. & Literatur \\
TS ling. & Linguistik \\
TS mar. & Marine \\
TS metall. & Hüttenwesen \\
TS meteor. & Wetterkunde \\
TS metrol. & Maß- und Gewichtslehre \\
\hline
\end{tabular}




\begin{tabular}{ll}
\hline Technisch-spezifischer Wortschatz & Übersetzung \\
\hline TS miner. & Mineralogie \\
TS monet. & Währungen \\
TS paleont. & Paläontologie \\
TS pubbl. & Werbung \\
TS relig. & Religion \\
TS scient. & (natur-)wissenschaftlicher Wortschatz \\
TS sport & Sport \\
TS stat. & Statistik \\
TS stor. & Geschichte \\
TS tecn. & Technik \\
TS topogr. & Topographie \\
TS urban. & Urbanistik \\
TS vulcanol. & Vulkanologie \\
TS zool.com. & allgemeiner Begriff aus der Zoologie \\
TS zoot. & Viehzucht \\
\hline
\end{tabular}

Tabelle 2: Im GRADIT (De Mauro 1999/2000) verwendete Abkürzungen der Fachsprachen

\section{C-Tests}

\section{$2.1 \quad$ C-Test an der Schule Morante}

\section{La regione meridionale (Africa)}

\section{L'identità geografica}

La situazione climatico - ambientale della regione dell'Africa meridionale è molto varia. A no prevale 1 savana $\mathrm{c}$ sfuma a s__ in pross $\overline{\text { vasta ar_ }}$ del Tro_ dese $\operatorname{del} \overline{\text { Capri }} \overline{\text { (Namib }}$ e Kala_ , che s_ spinge fi_ alla co atla . Sul vers dell'Oce Atlantico pre un $\mathrm{cl}$ più fre umi qui l'ambi della fore temperata. L'area più ospitale è comunque l'estrema propaggine meridionale, dove prevalgono un clima e un ambiente mediterranei.

\section{Coste e isole}

Le coste sono in genere poco articolate. I lito Atla sono retti e comp co dell'Oceano Indi . Al cont dell'Oceano pa paludose, mals sono i , le app 


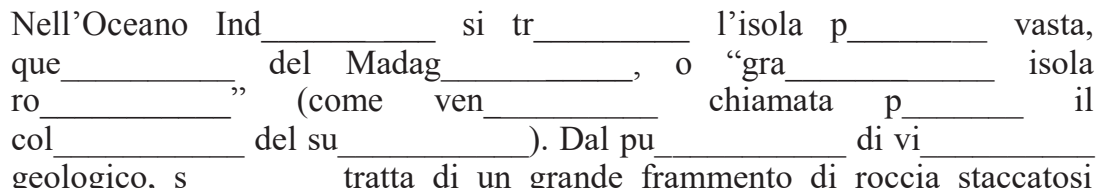
dall'Africa e dall'India, quando anticamente queste erano unite.

\section{L'identità demografica}

L'Africa meridionale era originariamente popolata da boscimani (san) e ottentotti (khoi-khoin), che i bantu spinsero progressivamente verso le aree inospitali dell'interno. Ancora og scarsamente popo i su

ar desertiche dell'int

i

L clima è $\mathrm{p}$ territori so maggior pa

a

$\mathrm{L}$ gruppi etn presenza d è fo e 1 densità è bassi nelle , mentre cre dove

eur mite.

mino di origine asiatica. Il Madagascar è abitato dai malgasci, che derivano dalla fusione di genti diverse di origine indonesiana.

\section{Egitto - Demografia e modelli urbani}

La popolazione dell'Egitto sfiora i 68 milioni di abitanti. Tuttavia, solt_ una pic abi_: la popo lu_le co zone $\mathrm{p}$ valle $\mathrm{d}$ spe per chilo elevatissima e talv parte $\mathrm{d}$ infatti, vi terri della popol appartiene e lingui bantu. bianchi $\mathrm{d}$ origine sono an consistenti

Sebbene ol quadrato.

nelle campagne, in villaggi o in gruppi sparsi di case (ezbahin), il Paese vanta alcune delle città più popolose del continente: Il Cairo, capitale del Paese, deve la propria fortuna alla presenza del Nilo e alla vicinanza al mare. Oggi è un'importante metropoli, con un agglomerato urbano di oltre 10 milioni di abitanti.

\section{Risorse ed economia}

L'economia dell'Egitto è ancora basata sul settore primario; nonostante i__recente svil__ delle atti__ industriali, i tenore d_ vita d_ gran pa della popol è ba_ Preoccupanti so da_relativi al_ disoccupazione (9\%), all'analfa $(4 \overline{4 \%)}$ e al_ mortalità infa__ (29 \%) ancora ele

\section{L'agricoltura}


I ter coltivati so limitati all'ar del de e de_ valle d Nilo. L'irrig assicurata

d dighe e can , e i fertilizzanti chimici permettono di realizzare fino a tre raccolte l'anno.

Le colture vengono distinte secondo la stagione: quelle invernali (frumento, legumi), quelle estive (cotone, canna da zucchero, riso, arachidi, sesamo) e quelle autunnali (miglio, mais).

\subsection{C-Test an der Schule Vespucci}

\section{Il Pil e il Pil pro capite}

1. Negli anni precedenti, per confrontare le condizioni economiche dei diversi paesi europei, abbiamo utilizzato il Pil (prodotto interno lordo). Sappiamo c__ esso è i__ prodotto c__ si ric__ oggi an_ dalle atti_ economiche di u_ paese. $\mathrm{L} \_$graduatoria eur_ vedeva a primo po_ la Germ_ con ci_ 3322 mili di dol $\quad$ e i all'ult_ posto, 1 Moldova, $\mathrm{c}_{\text {___ }}$ solo 4,2 mili_. Questo indic_ non c_ dice pe_nulla su disuguaglianze i trormini d_ ricchezza, ci_ sul bene_dei singoli cittadini; ben più significativo a questo proposito, è l'indicatore che mette in relazione il Pil con la popolazione, cioè il Pil pro capite.

2. Il Pil pro capite ci indica in pratica il reddito medio degli abitanti di un paese.

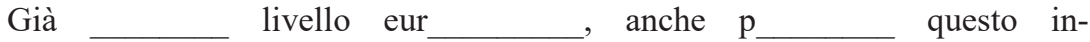
dic_ per ese_la Dani con p di 57261 posi_occupate da_r già cit_ Moldova (1 248 dol__ e da___ Macedonia (3 659 dol__ Ma $\mathrm{s}$ scala interna le dist si fa ancora $p \quad$ grandi: negli Stati Uniti il Pil pro capite è di oltre 45000 dollari, nella Repubblica del Congo 166.

\section{L'Isu}

$3+4$. Il reddito e la ricchezza non riescono però a dire tutto di una situazione economica. Due pa_ con l__ stesso red___ medio $\mathrm{p} \_$abitante pos___ presentare situa___ completamente div_: in $\mathrm{u}_{\text {_ }} \quad$ vi $\mathrm{p}$ essere $\mathrm{u}$ distribuzione de ricchezza mo_ squilibrata, con u_ ristretto num_ 


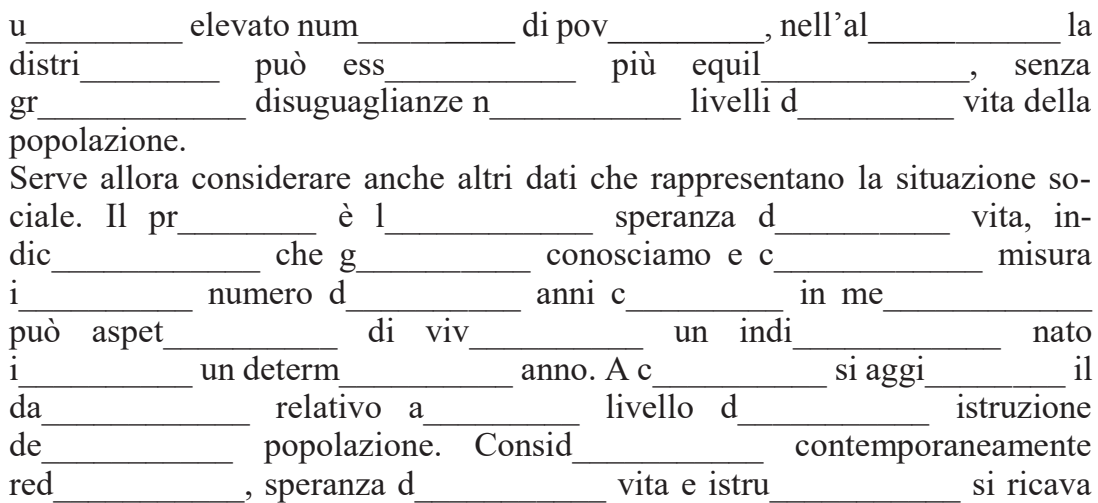

l'Isu (Indice di Sviluppo Umano). Esso colloca i vari paesi in una scala di punteggi che va da 0 a 1: i valori vicini a 1 segnalano una condizione di sviluppo, i valori tendenti a zero indicano situazioni di povertà.

\section{Le risorse energetiche -paesi consumatori e paesi produttori}

5. Nel campo delle risorse energetiche a livello planetario i principali consumatori, cioè i paesi a economia avanzata che consumano molta energia non sono autosufficienti.

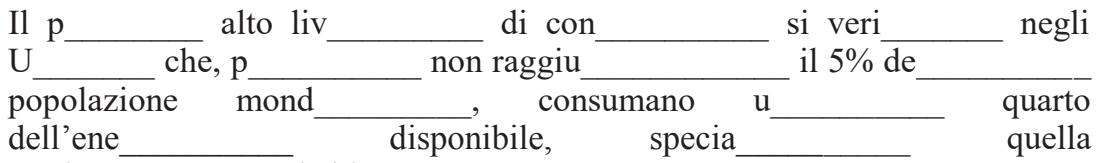

prod da idroc

Gli St_Uniti impo__ più de___ metà $\mathrm{d} \_$petrolio c_ consumano, dipen_ più c_ altro dal Medio Oriente. Anche Europa e Giappone sono importatori di petrolio, così come lo è la Cina, pur disponendo di grandi giacimenti di carbone.

\subsection{C-Test der Klasse III A an der Schule Vespucci}

\section{Sviluppo e sottosviluppo tra Nord e Sud del Mondo}

a) Oggi si usano i termini "sviluppo" e sottosviluppo per riferirsi a due caratteristiche di uno Stato. La cres economica, misu

i Prodotto Int Lordo (PIL), e 1_ condizioni
d_ vita de popolazione valu_ sulla ba_ del red_ medio indiv__ (la ricc_ $)$, l'istruzione e l__ salute.

$\begin{array}{llll}\text { So } & \text { perciò indi } & \text { come Pa } & \text { sviluppati } \\ \text { Stati c } & \text { hanno un'econ } & \text { progredita }\end{array}$


c c godono d

si se

di $\bmod$

tecnologie e

Sono invece segnalati come Paesi sottosviluppati gli Stati con una crescita economica molto lenta, in cui i sistemi produttivi sono molto arretrati e la povertà è diffusa.

b) Tra questi due estremi si pongono i Paesi a sviluppo intermedio, che negli ultimi decenni hanno avviato moderni sistemi produttivi e nei quali le condizioni di vita della popolazione sono migliorate.

Per disti_ gli St tu_ posti nell'emis ricchi e avan__, quasi

$\mathrm{p} \quad$ povere e me tu_ situate nell'emis

utilizzata l'espre "Nord e S

Negli ult anni i settentrionale, da aree ricchi e que gato, aumen per progredite de

meridionale, è st del Mo divario $t$ poveri $\mathrm{s}$ di cin , infatti, i comm

liv internazionale, $\mathrm{m}$ è notevo i $\mathrm{Pa}$ volte. I si so sviluppati a hanno avvant Terra, i Paesi ricchi e parzialmente quelli in via di sviluppo, perché quelli poveri non hanno né le conoscenze, né i mezzi per trarre beneficio dal nuovo sistema economico mondiale.

\section{Le dinamiche demografiche oggi}

c) + d) Negli ultimi cinquant'anni l'aumento della popolazione nei Paesi in via di sviluppo è il fenomeno demografico più importante. Questo in-

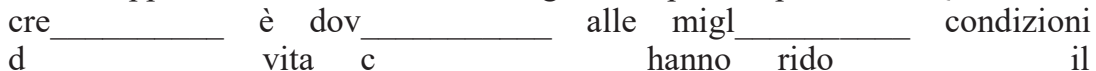
ta $\quad \begin{array}{llll}\text { vita } & \mathrm{c} & \text { hanno rido } & \text { il } \\ \text { di mort } & \text { è contemporaneamente } \mathrm{s}\end{array}$

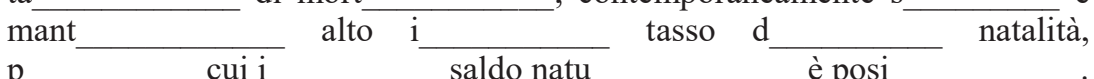
Secondo alc studiosi, l'anda d un $\mathrm{Pa}$ segue de fasi pre

- inizialmente la natalità è alta ma simile alla mortalità, perché quest'ultima è molto elevata a causa delle precarie condizioni di vita; la popol

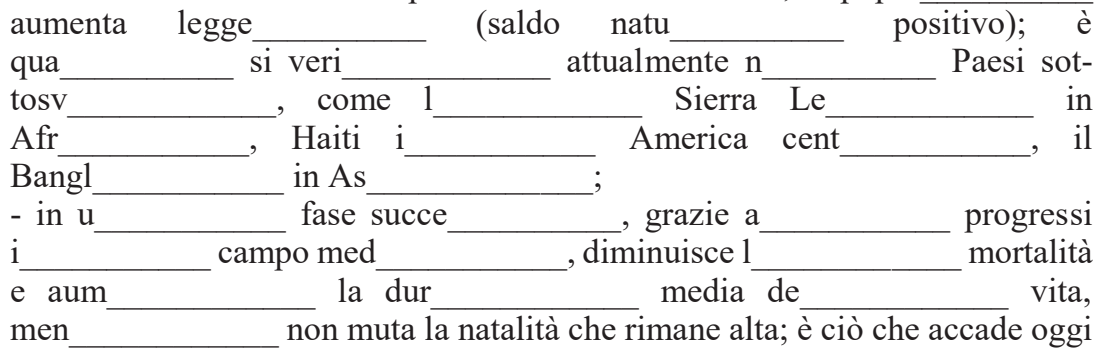


in Senegal, uno dei Paesi in via di sviluppo, in cui si registra una morte ogni quattro nascite e di conseguenza un incremento demografico molto sostenuto.

\section{Le potenze economiche mondiali}

e) Oggi l'economia mondiale è dominata da pochi Stati che sono i maggiori produttori di merce e di servizi del mondo: Stati Un Can_ Unione Eur_ Giappone, Aust $\mathrm{Nu}$ Zelanda. Que_ Stati produ

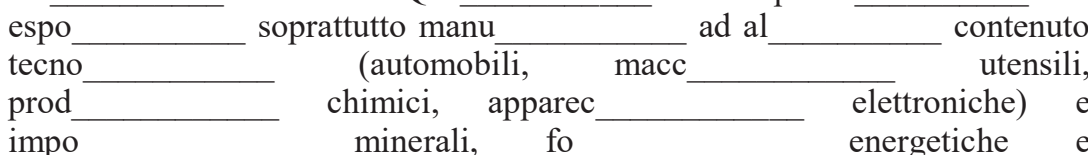
prod alimentari.
$\mathrm{L}$ forte import di petr da pa

di questi Paesi ha arricchito anche alcuni Stati produttori, come l'Arabia Saudita e gli Emirati Arabi Uniti.

\subsection{C-Test der Klasse III B an der Schule Vespucci}

\section{Asia}

L'Asia è la regione più vasta del mondo, estesa su una superficie di 44 milioni di $\mathrm{km}^{2}$, con uno sviluppo costiero di 70 mila $\mathrm{km}$. Saldamente un all'Europa, fo con es

All'Afr era un il conti eurasiatico. fi nale. Me sepa a qua , nel 1869, f dall'istmo Suez Uniti (Ala la $\mathrm{s}$ di $100 \mathrm{~km}$ del estremità orie Stretto d aperto $i$ Ca-

La co settentrionale, genera bassa e c rare artico , è bag giacciate $\mathrm{d}$ dalle ac quasi sem Mar Glac dagli St Bering

l'Oceano Pacifico bagna coste molto articolate con penisole e vaste insenature, bordati da lunghi festoni di isole interessate da irruzioni vulcaniche e terremoti, facenti parte della cosiddetta cintura di fuoco.

\section{Asia: economia e società}

a) L'agricoltura rappresenta la base primaria per la maggior parte delle popolazioni asiatiche. Grazie sopra allo svil d Cina e In , la reg è a agricolo po_ nel mo_ come produ_ di ri $\quad$ grano, $\mathrm{t} \_$, cotone $\mathrm{e}$ div $\longrightarrow$ altre col . Sviluppato è an l'allevamento: bov 
ovini e cap soprattutto $\mathrm{i}$ prattutto in $\mathrm{Ci}$ . $\mathrm{La} \overline{\mathrm{Ci}}$ India; su , sopr_ po_ mondiale an_ nella pe__ nelle acque marine e interne e nell'acquicoltura (allevamento di pesci e molluschi).

b) Il processo di industrializzazione ha da tempo raggiunto un alto livello in Giappone, in Siberia (regione nella parte asiatica della Russia), nella Corea del Sud, a Taiwan, in Thailandia e a Singapore. Più rec l'impe_ sviluppo indusi_della $\mathrm{Ci}$

de province cost in $\mathrm{c}$ , soprattutto

le produ___ destinate all'esport___ Anche al___ paesi
(In_, Indonesia, Ir__ e Tur__ hanno avv_ negli ult industriale. 18 Ma ne asia l'indu decenni prog di svil

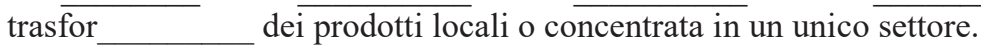
Nonostante il forte sviluppo economico, oltre i tre quarti della popolazione dell'Asia meridionale e circa la metà di quella dell'Asia orientale vivono in condizioni di povertà: sono circa due miliardi di persone, tra cui oltre $700 \mathrm{mi}-$ lioni sono in condizioni di povertà estrema.

\section{Asia: clima}

Quasi ovunque il clima è continentale, con elevate temperature estive e inverni freddi. E' compless vegetazione princ arido ne

con er

ca

den

nom

del Medite basse e cesp dell'aridità, gra abitativa ven specialmente de si h altopiani int della ste , dove è que spinosi, e $\mathrm{n}$ deserti. A distese $\mathrm{c}$ scarsissima lasciate a ovini. Lu pascolo dità che ben si presta alle coltivazioni ortofrutticole e della vite e dell'olio. Lungo il Mar Rosso e l'Oceano Indiano, soggetto al monsone estivo che genera umidità, è sviluppata la coltivazione di frumento, cotone, tabacco e ortofrutta.

\section{Lingue che rischiano di scomparire}

Tra le circa 6800 lingue del mondo, più di 6500 sono parlate ciascuna da meno di un milione di persone, cioè da gruppi molto ristretti di popolazione.

Circa 1

tre d metà de lingue $d$ mondo ris inollin scomparire: $\mathrm{s}$ tratta sopra delle usate $\mathrm{p}$ parlare e $\mathrm{n}$ per scri

\begin{tabular}{|c|c|c|c|}
\hline Le ca & sono va & in alc & casi 1 \\
\hline $\begin{array}{l}\text { gua } \\
\text { ner }\end{array}$ & $\begin{array}{c}\text { viene } \mathrm{p} \\
: \text { in al }\end{array}$ & $-{ }_{\mathrm{S}}$ usata da & $\begin{array}{l}\text { giovani } \\
\text { vi }\end{array}$ \\
\hline
\end{tabular}
ritti della minoranza nazionale che la parla. 
Per evitare l'estinzione delle lingue, occorre che gli stati favoriscono il multilinguismo, ossia l'uso di più lingua oltre quella ufficiale.

\subsection{C-Test der Klasse III C an der Schule Vespucci}

\section{Asia}

\section{Uno sguardo sul continente}

L'Asia, il continente più vasto e più popolato della Terra, è un po' il "cuore" del mondo per la sua storia. Abitata d st la cu

As delle pr lunghissimo te , è

$\mathrm{q}$ vivono, $\mathrm{d}$ nume masse cont

Il ril e i della $\mathrm{Te}$

$\lim$ grandi ar

terizzate d modi div

clima ha civiltà urb

e d di popol di util civ delle ris in cinque settori: occidentale, centrale, centromeridionale, sudorientale, orientale.

\section{Aspetti fisici}

Dal punto di vista morfologico, il continente asiatico può essere suddiviso in tre fasce orizzontali. La pr è costi dal va Bassopiano sibe , un terri pianeggiante e spe paludoso, attrav da nume

es si est fiumi. A $\mathrm{s}$ un'ampia fas di

mont inna , costituita da dal $\mathrm{Ti}$ altipiani

me e d $\mathrm{t}$ cui spi

$\mathrm{d} \longrightarrow$ imponenti cat
dell'Himalaya. La terza

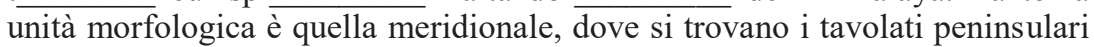
dell'Arabia e del Deccan.

\section{Asia Occidentale - Relazioni internazionali}

La regione attraversa da diversi decenni una fase di forte instabilità politica, con conseguenti tensioni e conflitti che hanno pesanti ripercussioni anche a livello internazionale.

Un foco di tens si tr nella reg

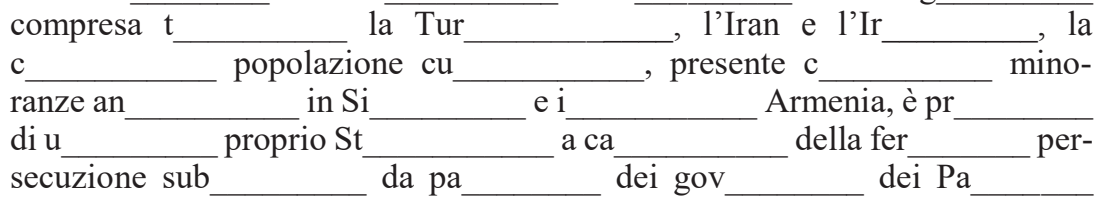


confinanti, c dir non $\operatorname{vogl}$ riconoscere $\mathrm{i}$ suo

In Afghanistan si susseguono scontri armati e attentati provocati dall'intervento militare degli Stati Uniti a seguito del clamoroso attentato dell'11 settembre 2001 a New York.

\section{Turchia - Territorio e clima}

Estesa su $783562 \mathrm{kmq}$, dei quali solo una piccola parte è geograficamente "europea", la Turchia confina a nord-est con la Georgia e l'Armenia, a est con l'Iran, a sud con l'Iraq e la Siria, a nord-ovest con la Grecia e la Bulgaria. E' bag a no dal $\mathrm{M}$

$\mathrm{M} \quad$ Egeo e a s

D i possenti cat Tauro, a s dal $\mathrm{M}$ Nero, a ov dal - Verso occi

a no mo si ap rie d u aspetto fortemente frastagliato. Mediterraneo. circondano: del Po montuose 1 , e i Mo , l'altopiano orl dai con u se-

I corsi d'acqua di una certa importanza sono pochi, sia per la scarsità delle precipitazioni, sia per l'assenza di pianure di una certa ampiezza; sono presenti laghi piuttosto vasti come il lago Tuz e il lago di Van.

\section{Turchia - Società ed economia}

In Turchia, l'economia e la società hanno molti problemi. Le atti produttive, $\mathrm{p}$ in cres

sono arre luppo de : i disoc industrie e d sono nume (soprattutto nell'agric insoddi , mentre $\mathrm{i}$ migl

Negli an basato $\mathrm{s}$ vati più rec tagli al ha conse sono nume terziario so e 1 sviancora turismo regi risultati

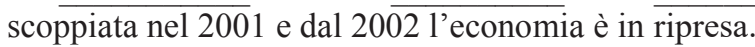

La società non asseconda gli orientamenti laici delle classi dirigenti.

\section{$3 \quad$ Zuordnung der Schüler-Aliasnamen aus Kapitel 9}

\subsection{Klasse an der Vespucci}

Ava: Schülerin C

Larissa: Schülerin D

Lorenzo: Schüler I 
Nicholas: Schüler A

Nora: Schülerin B

Olga: Schülerin J

Rodrigo: Schüler H

\subsection{Klasse an der Morante}

Asia: Schülerin $\mathrm{H}$

Giada: Schülerin M

Giorgia: Schülerin C

Letizia: Schülerin I

Lev: Schüler G

Lisa: Schülerin K

Luca: Schüler A

Sonia: Schülerin B

Vincenzo: Schüler F

\subsection{Klasse III A der Pestalozzi}

Arianna: Schülerin B

Camilla: Schülerin F

Costa: Schüler L

Ines: Schülerin $\mathrm{H}$

Luca: Schüler D

Ludovico: Schüler C

Vera: Schülerin G

Pierpaolo: Schüler M

Tania: Schülerin M

\subsection{Klasse III B der Pestalozzi}

Chiara: Schülerin G Federico: Schüler E

Luca: Schüler I

Marco: Schüler K

Pamela: Schülerin J

Tommaso: Schüler B 


\subsection{Klasse III C der Pestalozzi}

Lea: Schülerin E

Linda: Schülerin G

Margherita: Schülerin I 


\section{Textproduktionen der Lernenden zu geographischen Regionen}

4.1 Textbeispiele aus der Klasse an der Schule Morante

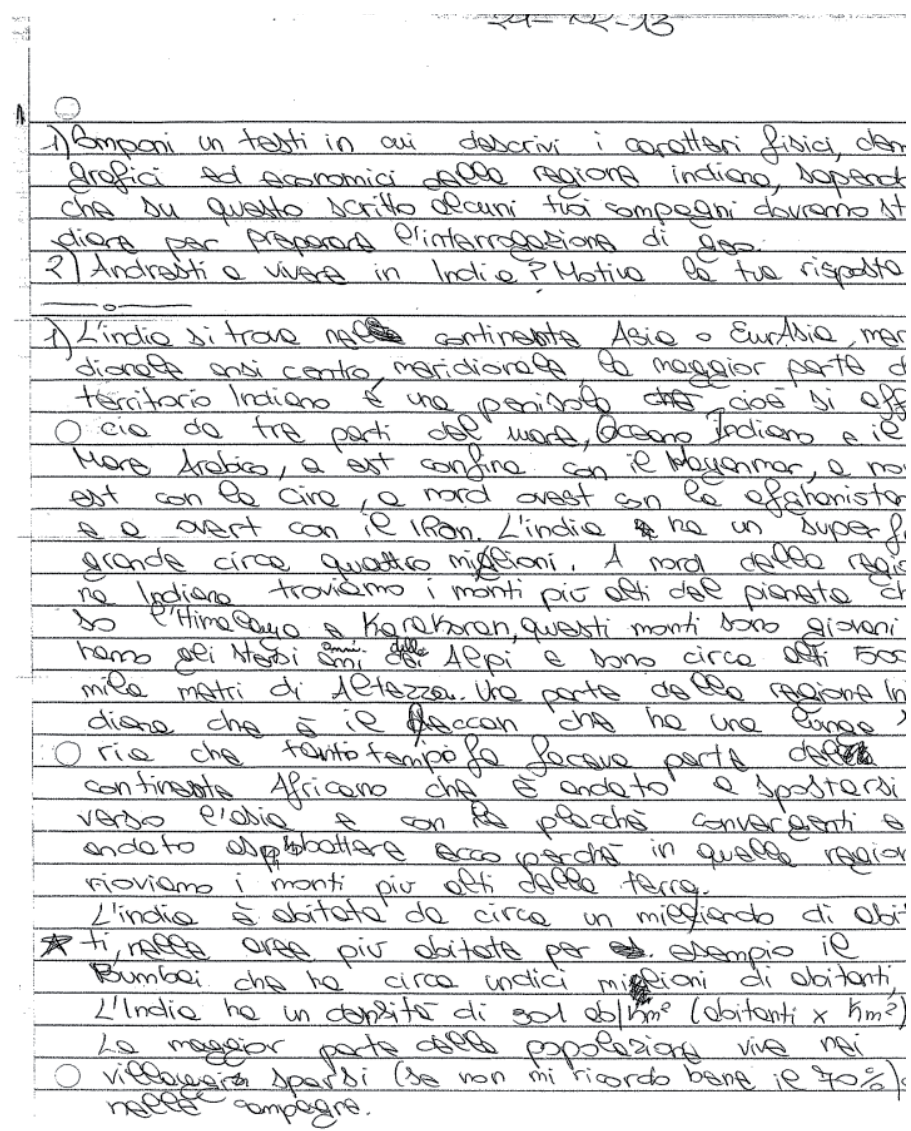

Abbildung 1: Text der Schülerin M (Seite 1) 
di meno, un vere prolilema,

perché ci barelble una f carestio

che impeditudele la, produrione

di prodoltj e, il cilo non basterelle

per tutti

Q' industrias non és molto rrviluppoto

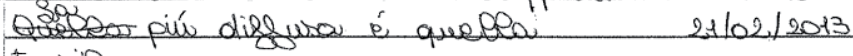

tossiede.

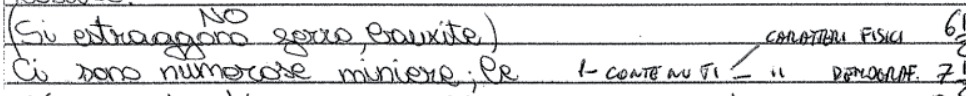
piú importanti tono queple

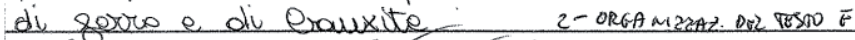

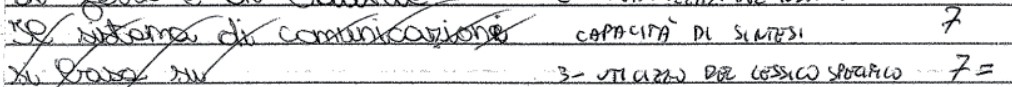

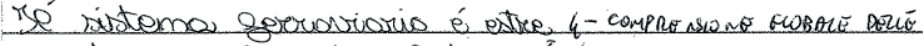

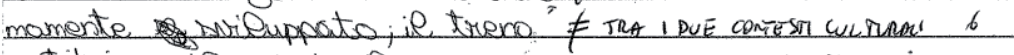

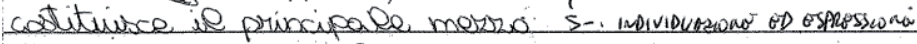

div trosporto. Queres apres Dor persomate pundo di uLSA

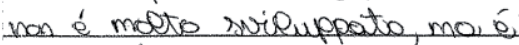

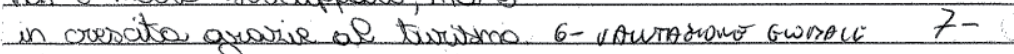

2 Io andrei a vinere in 'Srdivo

por il esel mare, peró ci sono

comungus numgrosi virantaggi

Abbildung 2: Textproduktion der Schülerin M (Seite 2) 


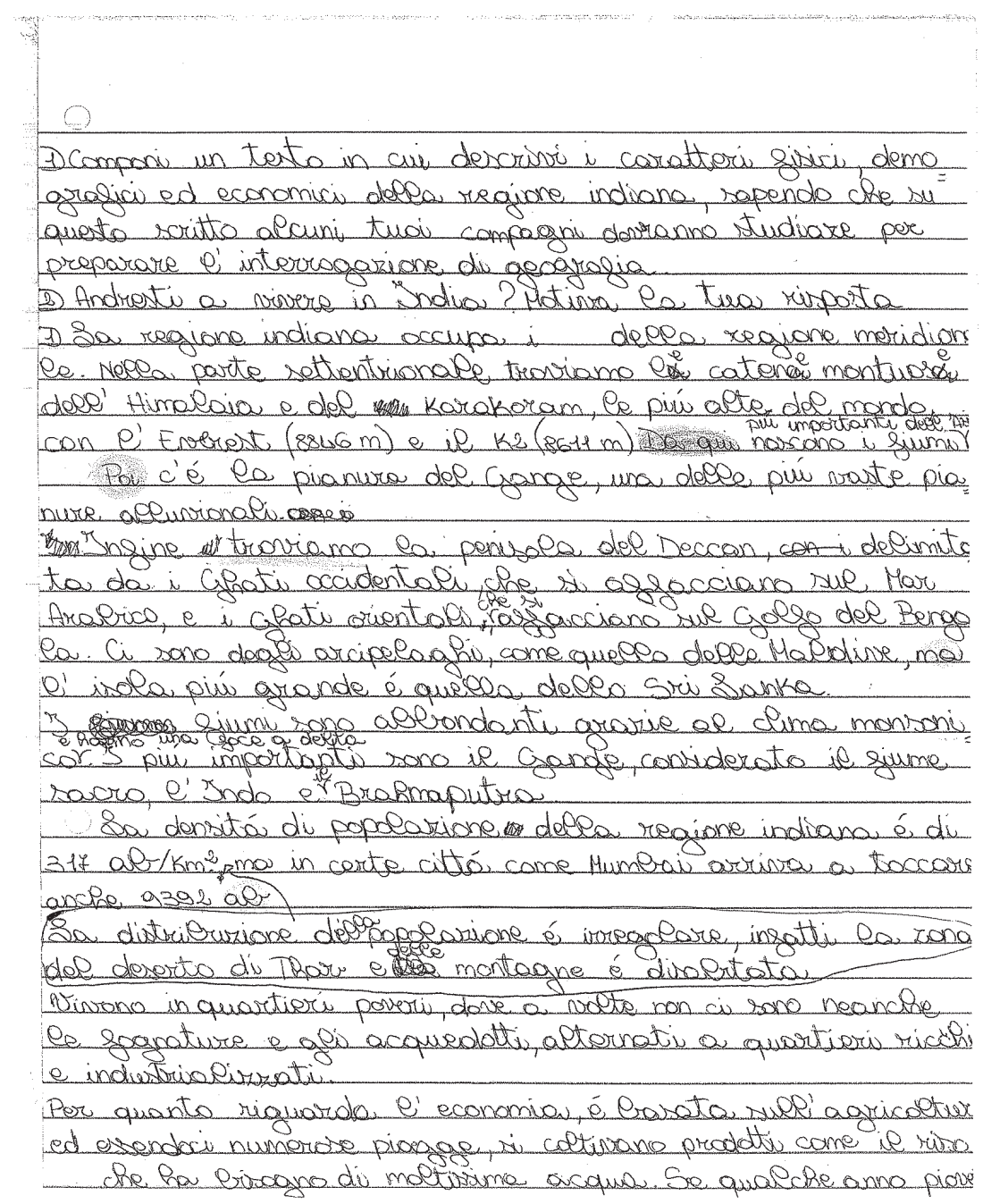

Abbildung 3: Textproduktion der Schülerin B (Seite 1) 
div mene, un vere prollema, perché ci barelle una f corestio che impeditiple la, produrione di prodolty e il cilo non fosterelde pere tutti

Q' induntries non és molto sriluppato.

Ques púu dillura é quegea 21/02/2013

tossive

(Si estragano sores, eravite)

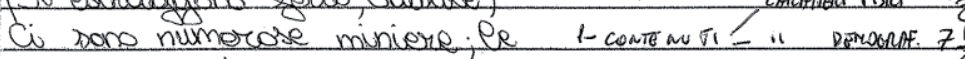
piú importantin sono queple

di sorow e di erawixite 111 ecomamici 8 -

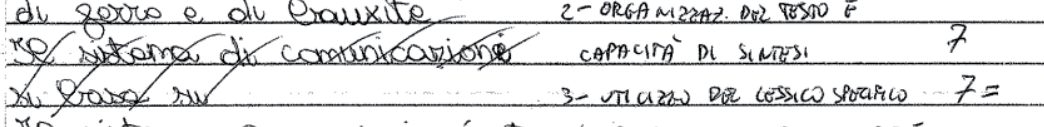

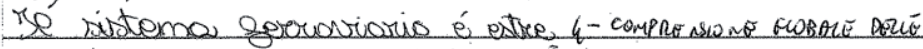

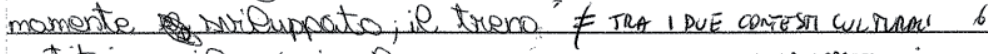

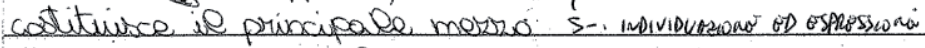
div trasporto. Quepe ares Doz persomte pundo di visina 6 han é molto suiluppoto, mo í.

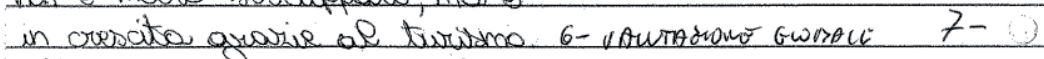
2) Io andrei a vinere in Indivo por il bel mare, peró ci sono comunque numgroi siantagai

Abbildung 4: Textproduktion der Schülerin B (Seite 2) 


\subsection{Textbeispiel aus der Klasse an der Schule Vespucci}

Inghitiema

$3 \mathrm{c} \mathrm{in} / \mathrm{01} / \mathrm{i3}$

L'Inghitierna e' un paese che mipiace perche' e' molio ricca e mi piace la

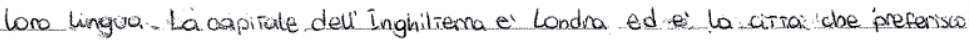
di più. L'Inghilserna e la lina sono paesi diversi e sono anche un po rivali perche l'Inghitierna nan vuole prodorin' provenienti dallo Cina la lina é un patse molio grande e la sua cevnomia è un po piu alía rispeitro a quella. inglese, ma ci sono alcune citrai della lina che sono ancona arretrate la Cina ha un tasso of fecondita molio alio rispeito Linghiliema. Invece se confrantramo l'Africa e l'Inghilierna seno toialmenie diverse. Nella Storia LInghitierna e stéra una delle prime a colomizzare, ll Africa Riguando all economia sano molio ma molio diverse. Anche se linghiliema e' un parse e l'Africa e' un continenie, possiamo dire che l'inghilierna e' molio più sviluppaia pero. "Africa ha molte piv risorse e da li i paesi piu richi vanno a prendene le loro ricchezze. It tassó di feconditai dell Africa é alisssima pero muciono ianiissimi bambinś per la fame o per le malaitie In Inghitiena non li sono questi problemi perche ci sano le medicine, invece, in Afriva tempersone non si possono permetiene le medicine percho sono cosiose. Bisogna abbassare i usii per quesie. persone cosi si ha più spenanza di vita. Io vorrei vivere in Inghilierna perche: posso trovare piu speranza degli aliri paesi. e poi hai più possibilitai che un giorno puoi diveniare importante. Ame piace molíissimo questo paese, e grazie ad alumi caniantingles mi hanno ispirato ad essere piv fiduciosa. Spero dhe un giomo possa andarci in questo paese per cercare un lavoro importante.

Abbildung 5: Textproduktion der Schülerin E 